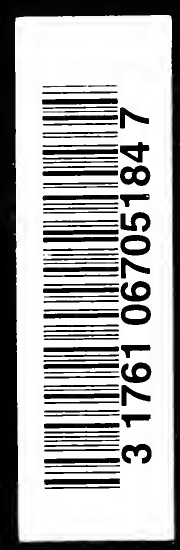

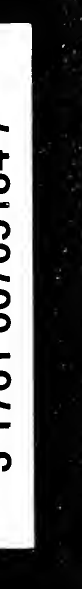

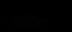

$$
\text { . }
$$
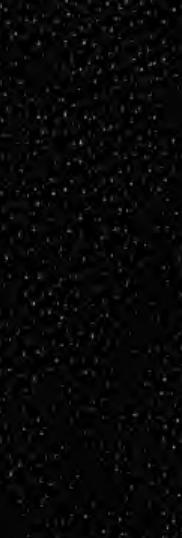


Digitized by the Internet Archive in 2007 with funding from Microsoft Corporation 


\section{A TREATISE ON}

\section{DISEASES OF THE NOSE AND THROAT}

BY

\section{ERNEST L. SHURLY, M D.}

Vice-President and Professor of Laryngology and Clinical Medicine, Detroit College of Medicine; Laryngologist and late Chief of Staff, Harper Hospital; Consulting

Laryngologist and Chief of Laryngological Clinic of St. Mary's Hospital; Consulting Laryngologist to the Woman's Hospital and Foundlings'

Home: Member of the American Laryngological Association; of the American Climatological Association : of the American

Medical Association; of the Michigan State Medical Society; of the American Academy of Ophthalmology and Oto-laryngology, etc.

SECOND EDITION, REVISED

NEW YORK AND LONDON

D. APPLETON AND COMPANY 
Copyright, 1900, 1905, BY

v. APPLETON AND COMPANY

PRINTED AT THE APPLETON PRESS NEW YORK, U. S. A. 
TO HIS PRECEPTOR,

EDWARD M. MOORE, M. D.,

WHOSE BRILLIANT INTELLECT, WONDERFUL ERUDITION, MODEST DEMEANOUR, UNSWERVING INTEGRITY, UNABATING COURAGE AND BENEVOLENCE HAVE

ALWAys EXCITED THE VENERATION OF THOSE WHO KNOW HIM, THIS BOOK IS GRATEFULLY DEDICATED BY THE AUTHOR. 



\section{PREFACE TO THE SECOND EDITION}

THE sale of the first edition of this book having been effected and demands for it still being made upon the publishers, the author has felt justified in offering another edition of the work to the medical public. While the plan of the book remains the same, new facts of noteworthy importance have been added.

Although the critics and other friends of the author have called attention to the brevity of the chapter on Diseases of the Accessory Sinuses, especially relating to the surgical treatment of the same, the author still thinks that any extension or particularization on this subject would not be in consonance with the scope of this work-which purports to be a reference book for general practitioners and students-because surgical work in these regions ought not to be attempted by unskilled and inexperienced persons.

A few of the illustrations have been changed to more important ones, and a number of new ones have been added. The author regrets that he has been unable to reproduce in extcnso all of the ideas and innovations which have appeared from time to time concerning methods and technique since the publication of the first edition.

E. L. S. 



\section{INTRODUCTION}

THIS volume has been prepared for the perusal of the general practitioner and medical student rather than for the specialist in laryngology. It has therefore been the intention of the author to present more particularly the ideas and facts pertaining to the various subjects from the standpoint of practical experience or observation. To this end he has refrained from speculating upon unimportant theories and superfine distinctions, while at the same time endeavouring to give both sides of important controversies.

The therapy of the diseases described has received especial attention in as concise a manner as possible, while at the same time affording a sufficient range for indicating different plans of treatment. For the convenience of ready reference, an extensive formulary and chapter on local treatment will be found in the latter part of the book.

The chapters and subjects have been arranged according to a classification based upon the general nature of the disease treated rather than according to the anatomical region involved. The operations on the external nose and maxillæ have been excluded, as they belong to the domain of general surgery.

An honest endeavour has been made to give proper credit for the researches and writings of others, and those quoted have been duly credited. If in any instance this has been omitted, it has occurred through unintentional error, and not with any desire to plagiarize. 
The author desires to acknowledge his indebtedness to the excellent works of J. Solis-Cohen, Bosworth, Allen, M. Mackenzie, Lennox Browne, Ingals, Kyle, Burnett, Randall and De Schweinitz, Francotte, Schech, Hajek, Butlin, Rotch, Burow, Grünwald, Schnitzler, Türck, Luschka, Price Brown, McDonald, Woakes, Ziemssen, and others; also to the many excellent monographs from which he has gleaned much valuable information. The author is under great obligation to his associate, Dr. P. M. Hickey, for his assistance in preparing the anatomical chapters and the illustrations. He would also express his thanks to Drs. W. S. Anderson, C. G. Jennings, B. R. Shurly, S. G. Miner, Thaddeus Walker, and Wadsworth Warren, for courtesies rendered.

32 Adams Avenue, W., Detroit, Mich. 


\section{CONTENTS}

\section{CHAPTER I}

Anatomy of the Upper Air Passages

External nose ; Nasal chambers ; Accessory sinuses ; Nasopharynx ; Oropharynx ; Laryngo-pharynx ; Larynx ; Physiology of the upper air passages ; Olfaction; Function of the pharynx and larynx; Voice production.

\section{CHAPTER II}

\section{Examination of the Upper Air Passages .}

Physical laws involved in laryngoscopy ; Illumination ; Instruments ; Anterior rhinoscopy; Median rhinoscopy; Pharyngoscopy; Posterior rhinoscopy ; Obstacles to posterior rhinoscopy ; Digital examination of the nasopharynx ; Laryngoscopy ; Auto-laryngoscopy ; Obstacles to laryngoscopy.

\section{CHAPTER III}

Acute Rhinitis, Pharyngitis, and Laryngitis .

Pathology ; Etiology ; Symptomatology ; Treatment. Acute pharyngitis ; Etiology ; Symptomatology ; Treatment. Uvulitis ; Symptomatology ; Treatment. Acute laryngitis; Etiology ; Symptomatology; Treatment. Edematous laryngitis ; Etiology ; Symptomatology ; Differential diagnosis ; Treatment. Membranous laryngitis ; Acute glandular fever of children; Herpes of the upper air passages; Abscess of the septum.

\section{CHAPTER IV}

Acute Infectious Diseases of the Upper Air Passages.

Influenza ; Etiology ; Symptomatology ; Complications ; Differential diagnosis ; Treatment. Pandemic influenza ; Etiology; Symptomatology ; Course ; Sequelæ ; Prognosis ; Treatment.

\section{CHAPTER V}

\section{Diphtheria}

Definition ; History ; Etiology ; Pathology ; Classification ; Anomalous cases ; Complications; Mixed infection; Prophylactic treatment ; Local treatment; Constitutional treatment; Action and dosage of antitoxin; Preparation of the antitoxin. 


\section{CHAPTER VI}

\section{Fibrinous Rhinitis}

Etiology ; Pathology ; Symptomatology ; Diagnosis ; Prognosis ; Course ; Treatment. Acute follicular tonsilitis; Etiology ; Prognosis; Treatment. Acute peritonsilitis; Etiology; Predisposing causes; Age; Diathesis ; Occupation ; Climate; Season; Hygienic surroundings; Habits ; Exciting causes ; Pathology ; Treatment.

\section{CHAPTER VII}

\section{Erysipelas of the Upper Air Passages}

Erysipelas of the nose ; Etiology; Age ; Prognosis ; Treatment. Acute retropharyngeal abscess; Etiology; Age ; Symptomatology ; Diagnosis ; Treatment. Acute infectious phlegmonous pharyngitis ; Etiology ; Symptomatology ; Diagnosis ; Treatment.

\section{CHAPTER VIII}

\section{Diseases of the Nasal Passages, Pharynx, and Larynx de-} pending upon the Exanthemata and Essential Fevers 163

Scarlet fever ; Treatment. Measles ; Treatment. Rötheln ; Variola ; Typhoid fever; Typhus fever; Malarial fevers; Rheumatism; Treatment.

\section{CHAPTER IX}

\section{Neuroses of the Upper Air Passages.}

Neuroses of the nose and nasopharynx ; Parosmia ; Hyperosmia ; Anosmia ; Prognosis ; Treatment. Hay fever ; Etiology ; Exciting causes ; Age ; Sex ; Race ; Location ; Social condition ; Symptomatology ; Pathology ; Complications; Treatment. Nasal hydrorrhœa; Etiology; Symptomatology ; Treatment. Sialorrhœa ; Treatment. Xerostomia ; Etiology ; Symptomatology ; Diagnosis ; Course ; Appearances ; Treatment. Hyperæsthesia of the nasal passages and nasopharynx; Treatment. Hyperæsthesia of the pharynx; Treatment. Hyperæsthesia of the larynx; Treatment. Paræsthesia of the nasal passages; Treatment. Paræsthesia of the pharynx; Symptoms; Age; Sex; Etiology: Prognosis; Treatment. Paræesthesia of the larynx ; Etiology ; Symptoms ; Laryngoscopic examination; Prognosis; Treatment. Anæsthesia and Analgesia; Treatment. Spasmodic affections of the upper air passages; Etiology: Sex; Age ; Social condition: Occupation; Nasal passages; Pharynx ; Soft palate ; Larynx; Spasm of the glottis; Symptomatology; Prognosis; Treatment. Paralysis of the muscles of the upper air passages; Paralysis of the muscles of the nose, soft palate, and pharynx: Symptoms; Treatment. Paralysis and paresis of the larynx; Etiology ; Sex ; Location ; Diagnosis ; Prognosis; Treatment. Functional aphonia; Etiology ; Age ; Sex ; Pathology ; Symptomatology; Diagnosis; Prognosis; Treatment. Neuralgia of the upper air passages; Nose and nasal passages; Neuralgia of the pharynx and larynx; Treatment. 


\section{CHAPTER $\mathrm{X}$}

\section{Chronic Infectious Diseases of the Upper Air Passages}

Hereditary syphilis ; Symptomatology ; Primary syphilis of the upper air passages ; Secondary syphilis ; Tertiary syphilis ; Symptoms ; Diagnosis ; Pathology ; Sequelæ; Treatment. Tuberculosis of the upper air passages ; Definition; General etiology ; Latency ; Heredity ; Contagion ; Inoculation; Predisposing causes ; Social condition; Food; Tuberculosis of the nasal passages; Etiology; Diagnosis; Prognosis; Tuberculosis of the nasopharynx ; Etiology ; Tuberculosis of the pharynx ; Diagnosis ; Prognosis; Tuberculosis of the larynx; Diagnosis; Prognosis; Treatment. Lupus of the upper air passages; Symptomatology ; Appearances (Nose, Pharynx, and Larynx); Etiology ; Pathology; Differential diagnosis ; Treatment. Rhinoscleroma ; Etiology ; Clinical history ; Diagnosis ; Prognosis ; Treatment.

\section{CHAPTER XI}

\section{Chronic Diseases of the Upper Air Passages .}

Chronic rhinitis ; Hypertrophic rhinitis ; Etiology ; Symptomatology ; Anterior hypertrophies ; Posterior hypertrophies ; Pathology ; Classification ; Diagnosis; Treatment. Chronic atrophic rhinitis ; Etiology ; Symptomatology ; Pathology ; Prognosis ; Treatment. Ozena ; Etiology ; Pathology ; Treatment. Chronic follicular pharyngitis ; Etiology ; Pathology ; Symptomatology; Objective signs; Appearances; Hypertrophic pharyngitis ; Atrophic pharyngitis; Etiology ; Pathology ; Symptomatology ; Diagnosis ; Prognosis ; Treatment. Pharyngitis ulcerosa ; Pathology ; Treatment. Hypertrophy of the tonsils; Etiology ; Symptomatology ; Pathology ; Diagnosis ; Prognosis ; Treatment. Adenoids ; Etiology ; Symptomatology ; Diagnosis ; Pathology ; Prognosis ; Treatment. Lingual tonsil ; Acute inflammation ; Symptomatology ; Chronic enlargement ; Symptomatology; Treatment. Chronic laryngitis; Etiology; Symptomatology ; Laryngoscopic appearances ; Pachydermia laryngis ; Pathology ; Diagnosis; Course ; Treatment. Hyperplastic laryngitis.

\section{CHAPTER XII}

\section{Local Diseases of the Nose.}

Perforation of the cartilaginous septum ; Etiology ; Treatment. Mycosis ; Etiology ; Pathology ; Symptomatology ; Diagnosis ; Prognosis ; Treatment. Varix ; Etiology ; Symptomatology ; Diagnosis ; Treatment. Leucoplakia ; Etiology ; Pathology ; Symptomatology ; Diagnosis ; Prognosis ; Treatment.

\section{CHAPTER XIII}

Diseases of the Accessory Cavities of the Nose, Antrum of Highmore, Frontal and Sphenoidal Sinuses, and Ethmoid Cells

Acute sinusitis ; General etiology ; Symptomatology ; Diagnosis ; Treatment ; Chronic diseases of the accessory cavities.; General etiology ; Eth- 
moid cells; Antrum of Highmore ; Frontal sinus ; Sphenoidal sinus ; DifPAGE ferential diagnosis ; Treatment.

\section{CHAPTER XIV}

\section{Neoplasms of the Upper Air Passages}

Classification; General etiology ; Benign neoplasms of the nasal passages and pharynx; Malignant neoplasms of the nasal passages and pharynx; Neoplasms of the accessory cavities; Treatment. Chemical escharotics; Neoplasms of the larynx ; Cysts of the larynx; Malignant neoplasms of the larynx; Symptomatology; Laryngoscopic appearances; Treatment of benign neoplasms; Treatment of malignant neoplasms; Intubation for neoplasms.

\section{CHAPTER XV}

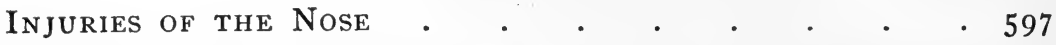

External injuries; Treatment. Injuries to the pharynx and larynx ; Treatment. Epistaxis ; Etiology ; Treatment.

\section{CHAPTER XVI}

Deformities of the UPper Air Passages . . . . 6 i6

Deformities of the nose; Deformities of the nasal cavities; Deviation of the septum; Etiology ; Symptoms ; Diagnosis ; Cartilaginous deformities : Results of septal operations; Synechia; Treatment. Deformities of the pharynx and larynx.

\section{CHAPTER XVII}

\section{Foreign Bodies in the Upper Air Passages . . . 650}

Foreign bodies in the nasal passages: Etiology ; Symptoms; Parasites in the nose; Symptoms; Diagnosis; Treatment. Foreign bodies in the pharynx, larynx, and esophagus ; Symptomatology ; Diagnosis ; Treatment.

\section{CHAPTER XVIII}

\section{Operations in the Pharyngeal and Laryngeal Regions}

ANd Methods of Local Treatment . $\cdot$ •
Preliminary measures; Position of the patient ; Aseptic precautions ; Anæsthesia; Pharyngotomy, lateral and subhyoid; Esophagotomy; Tracheotomy ; Operation ; After treatment. Intubation ; Indications for ; Operation; Difficulties; Thyreotomy ; Laryngectomy ; Partial laryngectomy ; Methods of local treatment ; Classification; Pigments ; Sprays ; Douches ; Powders ; Injections ; Inhalations ; Curettement ; Electrolysis ; Cauterization ; Formulary. 


\section{COLOURED PLATES}

\section{Plate I}

FIG.

I. Nasal polyp. (Grünwald.)

2. Adenoid vegetations of the nasopharynx. (Grünwald.)

3. Polypoid hypertrophy of the posterior ends of the inferior turbinals. (Grünwald.)

4. Pharyngomycosis. (Grünwald)

5. Chronic follicular pharyngitis with lateral hypertrophy. (Grünwald.)

6. Hypertrophy of the tonsils. (Grünwald.)

\section{Plate II}

I. Hard wart. (Grünwald.)

2. Lupus; cicatrized loss of substance of epiglottis; growths on the posterior wall of the larynx, on the posterior surface of the epiglottis, and on the vocal cords. (Türck.)

3. Catarrhal erosive ulcers of decubital origin. (Grünwald.)

4. Papilloma. (Grünwald.)

5. Erysipelas of the larynx. (Grünwald.)

6. Normal larynx during deep respiration. (Schnitzler.)

\section{Plate III}

I. First stage of tubercular infiltration. (Grünwald.)

2. Multiple tubercle infiltration in early stage. (Grünwald.)

3. Mixture of destructive and retrograde tubercular infiltration. (Grünwald.)

4. Tubercular infiltration and ulceration. (Grünwald.)

5. Diffuse tubercular infiltration of the mucous membrane. (Grünwald.)

6. Tuberculous and early (?) tertiary syphilis. (Grünwald.)

\section{Plate IV}

I. Hyperplastic tuberculosis. (Grünwald.)

2. Diffuse tubercular infiltration of the whole larynx and tumour. (Grünwald.)

3. Tubercular tumours. (Grünwald.)

4. Tuberculosis, lupoid form. (Grünwald.)

5. Syphilitic ulcer on the upper part of the posterior wall of the larynx. (Türck.)

6. Lupus (laryngeal) ; ulcers of epiglottis commencing to cicatrize; hypertrophy of the mucous glands at base of tongue. (Grünwald.) 
FIG.

\section{Plate V}

r. A normal pharynx.

2. Solitary ulcer of faucial pillar. (Siegel.)

3. Acute follicular pharyngitis.

4. Varix of lingual tonsil.

5. Peritonsilitis of the right side of the fauces, showing congestion and swelling of the tissues.

6. Acute follicular tonsilitis.

\section{Plate VI}

I. Mass of threads in tonsillar crypt. (Wright.)

2. Mycelial threads. (Wright.)

3. Hyperæmia of the larynx. (Schnitzler.)

4. Acute laryngeal catarrh. (Schnitzler.) 


\section{ILLUSTRATIONS IN THE TEXT}

FIG.

PAGE

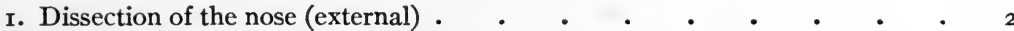

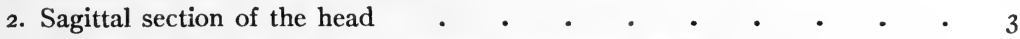

3. Coronal section of the head, showing turbinated bones $\quad$ • $\quad$. 4

4. Coronal section of the head, showing turbinated bones and ethmoidal sinus . 5

5. Sagittal section of the head, showing frontal and ethmoidal sinuses . . 6

6. Coronal section through maxillary sinuses and turbinated bones $\quad$. 7

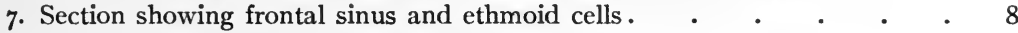

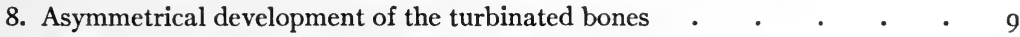

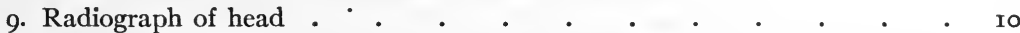

Io. Sagittal section of the head (the middle turbinated bone so elevated as to

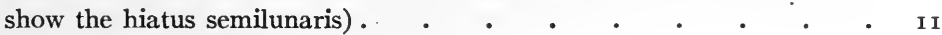

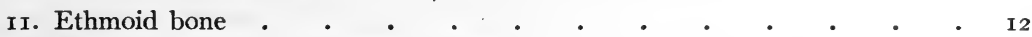

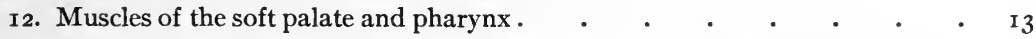

I3. Coronal section through sphenoidal sinus and posterior end of nasal cavity . I4

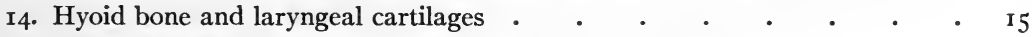

I5. Photomicrograph showing section of arytenoid cartilage $\quad$ • . $\quad$ • $\quad$ I5

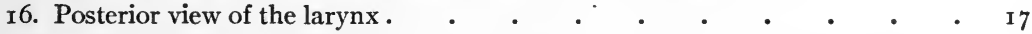

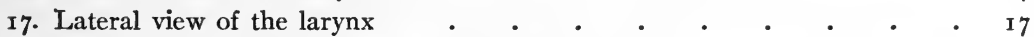

r8. Section of vocal cord showing internal fibres of the thyro-arytenoid muscle . 19

I9. Adult larynx opened in the posterior median line . . . . . . 22

20. Vertical section through the olfactory mucosa of a rabbit $(\times 560)$. $\quad$. 24

$2 \mathrm{r}$. Vertical section through the olfactory region of a young rat $\left(x_{480}\right)$. 25

2ra, b, c. Position of vocal cords in taking low, middle, and high notes . $\quad . \quad 3$ I

22. Head mirror supported by the teeth - . . . . . . 35

23. Bosworth's nasal speculum . • . • . • . • . 36

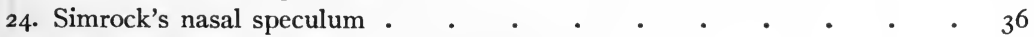

25. De Vilbliss's self-retaining nasal speculum . • • • • • • 36

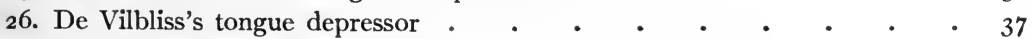

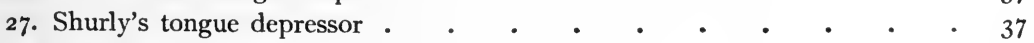

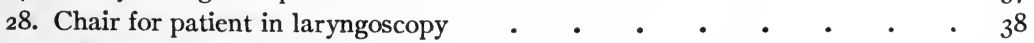

29. Position for anterior rhinoscopy • • • • • • • • • • • 39

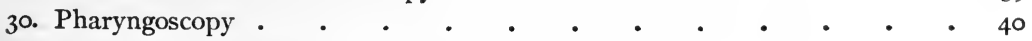

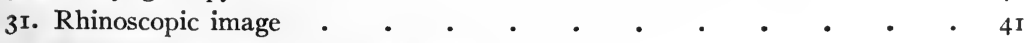

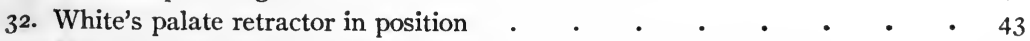

33. Method of holding laryngeal mirror . • • • • • • • • $\quad$ • 44

34. Enlarged sketch of normal laryngoscopic image in respiration , • $\quad 45$

35A. Larynx of child in quiet breathing . . . . . . . . 46

35B. Larynx of child during phonation . . . . . . . $\quad$. 46 
FIG.

36A. Adult larynx and trachea in deep inspiration . . . . . 46

36B. Usual view of normal larynx . . . . . . . . . . 46

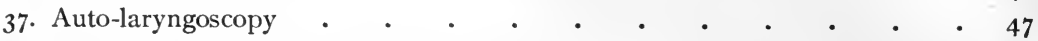

38. Kilian's method of examining the posterior wall of the larynx . . $\quad 48$

39. Position for autoscopy . . • . . . . . . . . 49

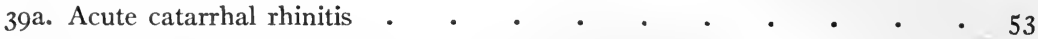

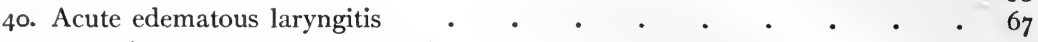

4I. Klebs-Loeffler bacillus (Stengel) • • • • • • • • • • 95

4ra. Photomicrograph of exudate from a case of pharyngeal diphtheria . $\quad 98$

$4 \mathrm{Ib}$. Photomicrograph of vertical section through tracheotomy wound on the fifth day

42. Leland's tonsil knife .

43. Left recurrent paralysis. Abduction .

44. Bilateral paralysis of lateral crico-arytenoids

45. Left recurrent paralysis. Abduction .

46. Bilateral paralysis of internal thyro-arytenoids and arytenoids

47. Bilateral paralysis of external tensors . . . . . . . . . 223

48. Bilateral recurrent paralysis . . . . . . . . . 223

49. Paralysis of left posterior crico-arytenoid. Inspiration . . . 223

5. Relaxed or cadaveric position . . . . . . . . . . . $\quad$. 223

51. Diagram showing outline of the rima glottidis in paralysis . . . 223

52. Symmetrically crescent-shaped condylomata of soft palate . . . . 248

53. Deep secondary syphilitic ulceration of the soft palate and fauces $\quad$ - 248

54. Deep syphilitic ulceration of soft palate and fauces . . . . . $\quad$. 249

55. Sequestrum of bone from case of nasal syphilis . . . . . . $\quad$. 250

56. Tertiary syphilitic ulceration and perforation at junction of soft and hard palate • • . . . • • • • • • . $25 \mathrm{I}$

57. Double perforation (syphilitic) of palate . . . . . . . 252

58. Syphilitic perforation of palate . . . . . . . . $\quad$ • 252

59. Syphilitic ulceration of right pillar and perforation of palate $\quad . \quad$. 252

60. Healed cicatrix of case illustrated in Fig. $59 \quad$ • . . . . $\quad$. 252

61. Syphilitic ulceration of the larynx with perichondritis . . . . $\quad 253$

62. Cicatrix after loss of part of soft palate and uvula $\quad . \quad$. $\quad . \quad 265$

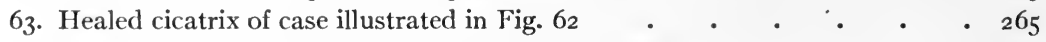

64. Section through interarytenoid space of normal larynx.$\quad \cdot \quad \cdot \quad \cdot 302$

65. Acute inflammatory cdema of tuberculous larynx . . . . . . $\quad$. 303

66. Superficial tubercular ulcers of the vocal cords and of the free edges of the

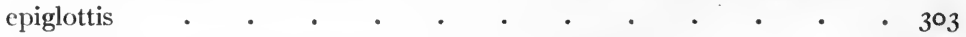

67. Tubercular ulceration of the glottis in the form of a girdle . . . . 303

68. Unusual manifestation of tuberculosis of the larynx . . . . . . 303

69. A pale larynx with swollen and roughened posterior wall and cicatricial changes about the left vocal cord . . . . . . . . . . 303

70. Unusual manifestations of tuberculosis of the larynx . . . . $\quad$. 303

7I. Tuberculosis of the larynx-Granular hyperplasia . . . . . 303

72. Tubercular tumour . . . . . . . . . . . . 303

73. Tubercular ulceration and granulomata on the posterior wall of the larynx . 304

74. Tubercular ulceration of the posterior wall of the larynx . . . $\quad 304$

75. Tubercular perichondritis of the arytenoid . . . . . . . 304

76. Tubercular perichondritis of the arytenoid . . . . . . . 304 
FIG.

PAGE

77. Extensive tubercular ulceration of the larynx-Necrosis of cricoid with considerable new tissue formation .

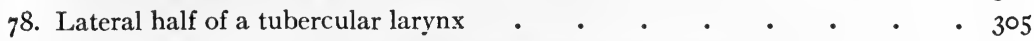

79. Lupus vulgaris of the right nasal passage and ala of the nose $\quad \cdot \quad \cdot 3^{\text {t9 }}$

8o. Lupus of the mucous membrane . • • • • • • • 328

8r. Rhinoscleroma • • • • • • • • • • •

82. Posterior rhinoscopic image of rhinoscleroma $\quad$. $\quad$. $\quad . \quad$. $\quad$ • 334

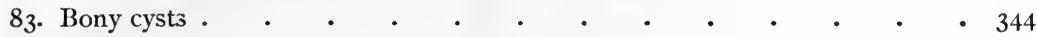

84. Bony cysts . . . . • . . . . . . . . . 345

85. Microscopical appearances of tissue in chronic hypertrophic nasal inflammation . . . . . . . . . . . . . 346

86. Photomicrograph of section from posterior hypertrophy $\quad$. . . $\quad$ - 347

87. Posterior hypertrophies of the right and left inferior turbinals . . . 348

88. Post-rhinoscopic image showing hypertrophied turbinal . $\quad . \quad$ • 349

89. Shurly's nasal speculum with ivory slide, right and left $\quad . \quad$ • $\quad$ • 354

90. Martin's snare . • . . • . . • • • . • . 356

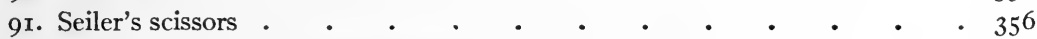

92. Hickey's snare for posterior hypertrophies . • • • . • 357

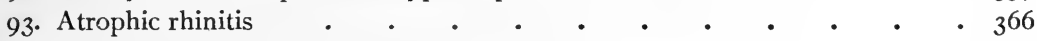

94. Atrophic pharyngitis (with enlarged follicles) . . . . . $\quad$ • 399

95. Exsected hypertrophied tonsil • • • • • • • • • 408

96. Section of hypertrophied faucial tonsil . . . . . . . 409

97. Tiemann \& Co.'s tonsillotome . • • • • . . . • 4II

98. Mackenzie's tonsillotome . . • . . . . . . • 4II

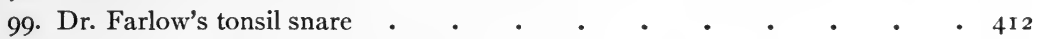

Iоo. Typical facial appearance of a mouth breather .. . . . . . 424

Ior. The Gottstein curette applied for the removal of the pharyngeal tonsil . $\quad$ - 429

102. Post-nasal forceps . . . . . . . . . . . 431

Iо3. Gottstein curette. • • • • • • • • • • 43 I

104. Edema of the glottis-unilateral . . . . . . . . $45^{6}$

I05. Chronic laryngeal catarrh, affecting principally the vocal cord on the left side . . . . . . . . . . . . . 456

I06. Chronic laryngeal catarrh, showing circumscribed swelling of one vocal cord $44^{6}$

107. Chronic inflammation of the vocal cords . . . . . . . 456

ro8. Ulcers of the vocal cords . . . . . . . . . . 456

I09. Chronic laryngeal catarrh, with swelling of the ventricular bands . $\quad .456$

I ro. Chronic laryngeal catarrh, with thickened mucus adhering to the vocal cords $45^{6}$

rr I. Chronic laryngeal catarrh, with paresis of vocal cords . . . . . 456

I 2 . Chorditis vocalis hypertrophica superior . • • • • . 457

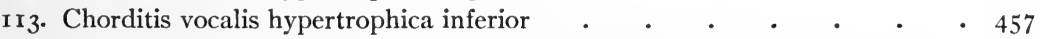

r14. Perichondritis of the left half of the cricoid cartilage with unopened abscess . 457

Ir5. Perichondritis of the posterior part and left half of the cricoid cartilage . $\quad 457$

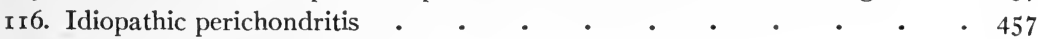

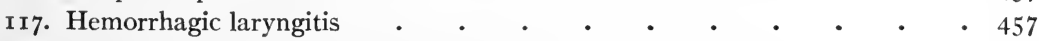

Ir8. Horizontal section through ethmoid cells . . . . . . . 500

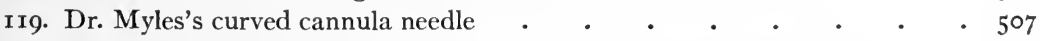

r20. Lamp for transillumination . . . . . . . . $\quad$. 507

I2 I. Hajek's straight cannula inserted for puncture of the antrum wall in the in-

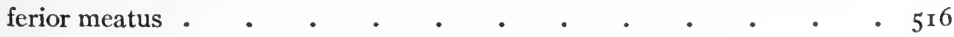


FIG.

122. Hand drill, with guard

123. Buck's nasal and ear curette

124. Section of nasal polypus

125. Section of papillary fibroma of the nose

126. Angioma of the nose .

127. Section of papilloma of the uvula

I 28. Sarcoma in the left nasal passage

I 28a. Photomicrograph of round-celled sarcoma of nose

129. Carcinoma of antrum and secondary growth in orbit

130. Carcinoma of the larynx and the pharynx, involving cervical glands

I31. Bosworth's snare

132. Jarvis's wire snare

133. Simrock's polypus forceps.

I34. Upson's syringe for hypodermic injections

135. Congenital papillomata

I36. Fibroma of the vocal cord .

I37. Fibro-myxoma

138. Myxoma of the epiglottis

I39. Subglottic myxoma

140. Cyst of the larynx

I4I. Epithelioma of the larynx .

142. Author's case of epithelioma of the larynx removed by the operation of laryngectomy .

143. Epithelioma of the larynx-Laryngectomy .

144. Sarcoma of the epiglottis

145. Primary lupus of the larynx

146. Supposed case of enchondroma

I47. Schrötter's laryngeal lancet and forceps

148. Fauvel's laryngeal polypus forceps

149. Mackenzie's lateral throat forceps

150. Mackenzie's antero-posterior throat forceps

I5. Gibbs's laryngeal écraseur or snare

152. Jarvis's laryngeal applicator

I53. Apparatus for repairing a broken nose.

154. An eligible method of repairing a broken nose

I55. Gunshot wound of the larynx

156. Injury to larynx from contusion .

I57. Case of paralysis of left vocal band following an incised wound of the neck

I58. Case of paralysis of left vocal band following an incised wound of the neck

159. Stenosis of the larynx following typhus fever

r60. Rubber tampon for plugging the nose .

r6r. Hopkins's bridge

I62. Coronal section of dry specimen, showing bony spur

163. Spud for dissecting up the septal mucous membrane

I64. Operation for removal of spur of septum

165. Steele's septum forceps

r66. Asch's curved gouge for breaking up adhesions between septum and turbinate

167. Cartilage scissors for operation of deflected septum 
FIG.

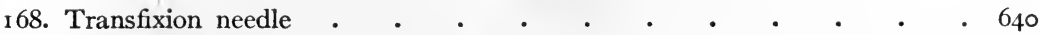

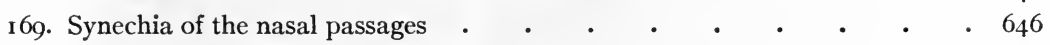

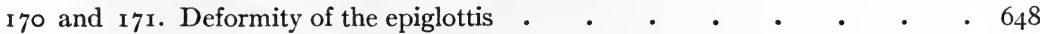

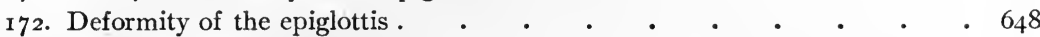

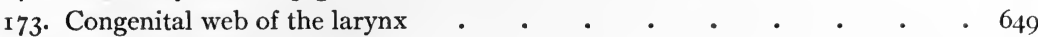

r74. Bifid uvula ..$\quad$. . . . . . . . . 649

175. A pin which was removed from the larynx two years after having been

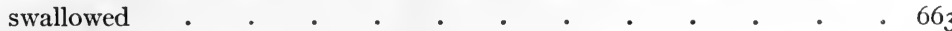

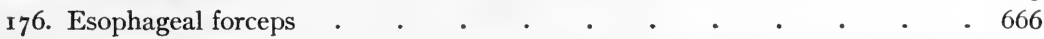

I76a. Radiograph of thorax of five-year-old child showing coin impacted in the esophagus

177. Trousseau's dilator, to facilitate the introduction of cannula $\quad . \quad$. $\quad .676$

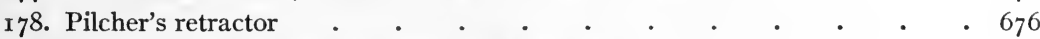

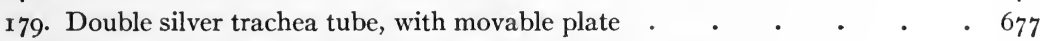

I80. Position of child, operator, and assistants, for intubation . • . . 680

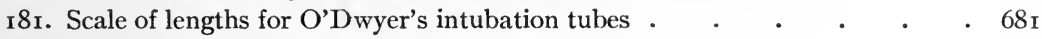

r82. O'Dwyer's mouth gag.

ı83. O'Dwyer's intubation tube and introducer . . . . . . . . . . 681

I84. Insertion of finger to locate epiglottis and arytenoids . • • • . 682

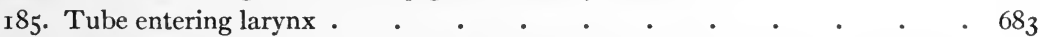

186. Illustrating the error of depressing the handle of the introducer, resulting in the tube entering the esophagus instead of the larynx $\quad$ • $\quad$ • $\quad$ • $\quad$ - 684

187. Removal of the introducer with the finger holding tube in the larynx $\quad$. 685

188. Tube in the larynx, with the string attached . . . . . . . . 686

189. Withdrawal of the string, with the finger on the tube to hold it in position 687

190. Tube in situ. Showing the head of the tube upon the vocal cords, and the position of its distal end in the trachea . . . . . . .

I9r. Method of removing the tube. The beak of the extractor, guided by the finger, is just entering the tube

I92. Diphtheritic cast of trachea and bronchi (natural size)

690

690

I94. Feeding intubated child with nursing bottle, the head being lower than the shoulders

195. Feeding intubated child by suction of fluids through rubber catheter . . 69I

ı96. Phillips's electric photophore, with adjustment for focusing light . 693

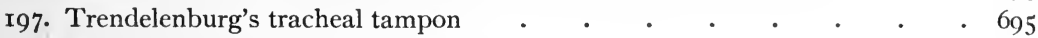

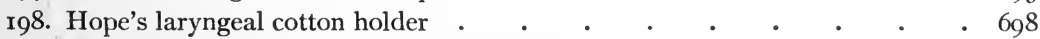

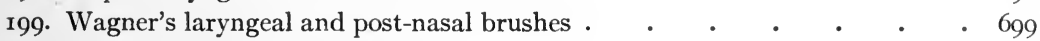

200 and 20r. Shurly's cotton forceps . . . . . . . . . . . . 700

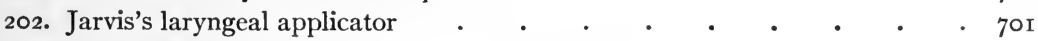

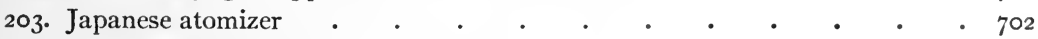

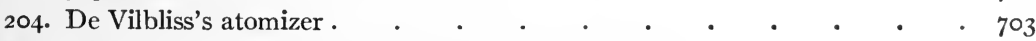

205. Hard-rubber pharyngeal and posterior nares syringe $\quad$. $\quad$ • . 704

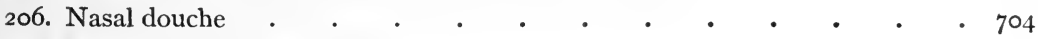

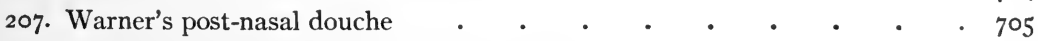

208. De Vilbliss's powder blower . . • • • • • • . . . . 706

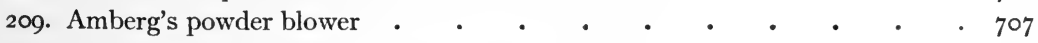

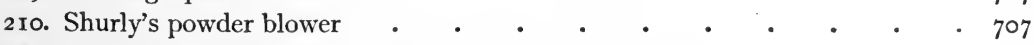

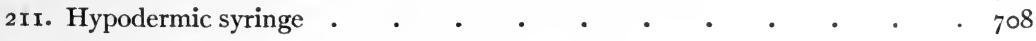


FIG.

2 I2. Tobold's laryngeal syringe

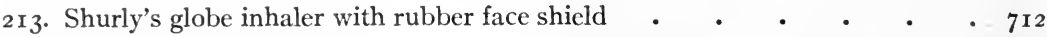

2r4. Shurly's hot water inhaler.

2 I5. Tyndale's antiseptic oro-nasal respirator

2 I6. Tobold's pneumatic apparatus

2 I 7. Cohen's laryngeal curette

2 I 8. Mackenzie's laryngeal lancet

2 I9. Needles for electrolysis

220. Dawson's universal cautery electrode.

22r. C. H. Knight's electric tonsil snare

222. Nasal electrode

223. Mackenzie's laryngeal electrode .

224. Caldwell's throat X-ray tube

225. Dr. B. R. Shurly's self-retaining metal drainage tube for antrum .

226. Author's tonsil scissors 


\title{
DISEASES OF THE NOSE AND THROAT
}

\author{
PART I
}

\section{CHAPTER I \\ ANA TOMY OF THE UPPER AIR PASSAGES}

THE component parts of the upper air passages are the nose, pharynx, and larynx. The nose is divided into the external nose, the nasal fossæ, and accessory sinuses. The external nose consists of the rigid or bony structure, supplemented with cartilages and the coverings of muscles and integument. The rigid structure is composed of the nasal bones and the nasal processes of the superior maxillaries; the nasal bones, two in number, form the bridge of the nose and articulate with each other, the frontal bones, and the superior maxillaries. The cartilages of the nose are the septal, the four lateral, and the accessory, usually four in number (Fig. I). The septal cartilage, sometimes denominated the triangular, and by some authors the quadrilateral, forms the anterior part of the nasal septum, and one of the boundaries of the nasal vestibule. The upper and lower lateral cartilages form on each side the alæ of the nose. The accessory or sesamoid cartilages are posterior to the two lateral and serve as a flexible attachment to the maxillary bones. The muscles of the nose are the levator labii superioris alæque nasi, the dilators-anterior and posterior -the compressor nasi, the depressor alæ nasi, and pyramidalis.

The anterior nares open into the vestibule, which is the part of the internal nose whose walls are cartilaginous. Posterior are the nasal fossæ proper, extending back to the posterior nares and separated one from the other by the nasal septum. The septum is divided into an anterior or cartilaginous part and a posterior or bony part formed by the perpendicular plate of the ethmoid and by the vomer (Fig. 2). 
The components of the upper and posterior boundaries of the nasal chambers are the nasal bones, the body of the sphenoid, and the cribriform plate of the ethmoid. The outer wall is

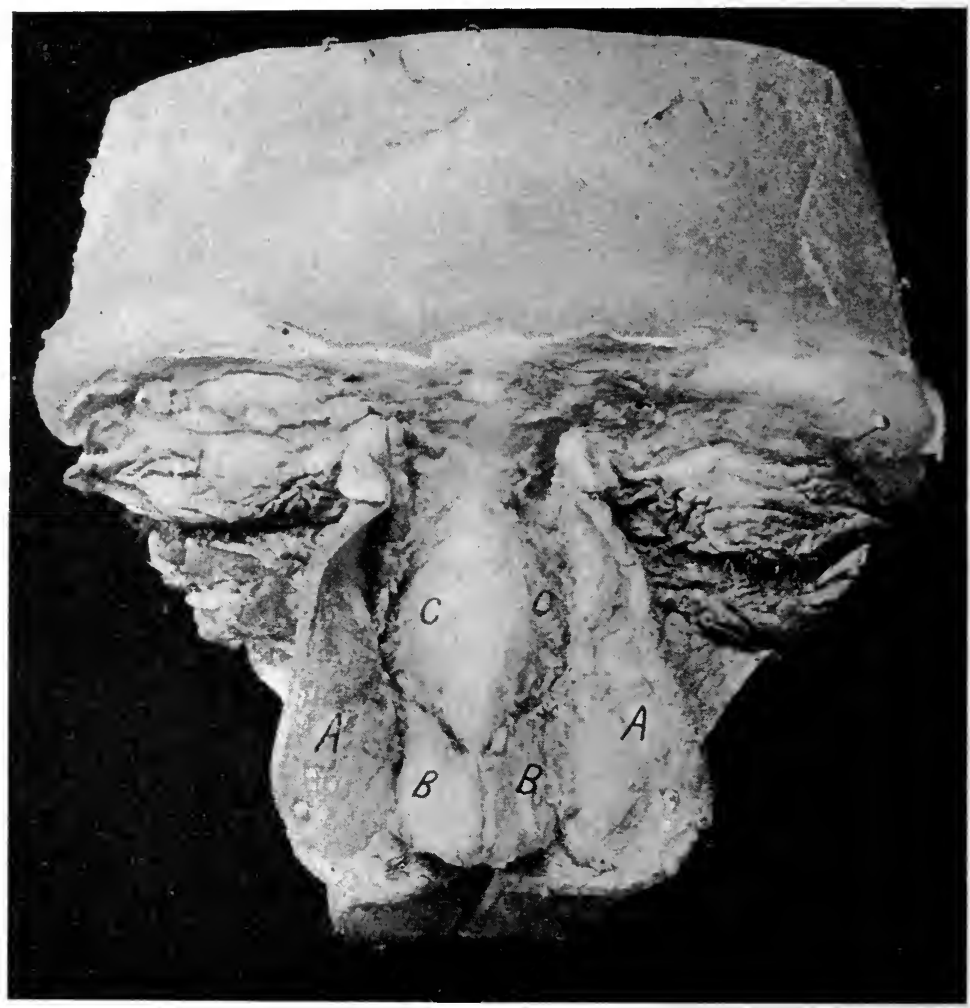

FIG. I.-Dissection of external nose. $A, A$, reflected integument; $B, B$, alar (or lateral) cartilages; $C, C$, nasal bones.

made up of parts of the superior maxillary, lachrymal, palatal, ethmoidal, and sphenoidal bones. The floor of these passages is formed by the palatine processes of the superior maxillaries, and by the horizontal plates of the palate bones. Encroaching on the lumen of each cavity are three small bones known as the turbinals, so named from their scroll-like appearance (Figs. 3, $4,5,6$, and 7). The superior turbinated bone projects from the lateral mass of the ethmoid, forming a distinct ridge posteriorly, while anteriorly it merges into the middle turbinal, which is also a part of the ethmoid. The middle turbinal is a broad, thin, bony plate curling downward upon itself, and 
has on its anterior inferior surface a slight projection known as the agger nasi; opposite to this upon the septum will usually be found a corresponding small protuberance; these two points may be taken as marking the line between the respiratory and olfactory parts of the nasal tract. The inferior turbinated bone is an independent structure articulating anteriorly with the nasal process of the superior maxillary, centrally with the lachrymal and ethmoidal bones, and posteriorly with the lower crest of the palate bone. These supplementary bones, which are nearly parallel with and approximately equi-

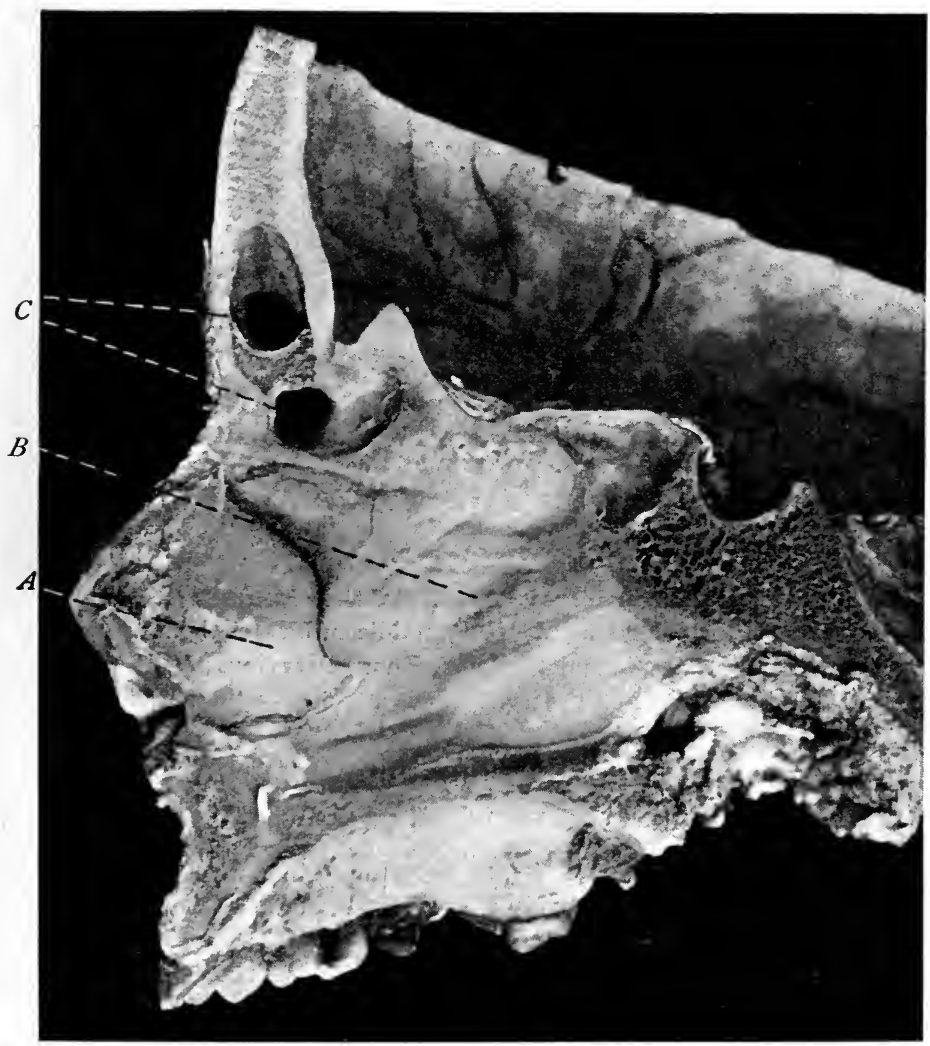

FIG. 2.-Sagittal section of head. $A$, quadrilateral cartilage ; $B$, perpendicular plate of the ethmoid bone ; $C$, frontal sinus.

distant from each other, subdivide the nasal passages into three channels called meatuses, the superior meatus lying between the superior and middle turbinals, the middle meatus 
between the middle and inferior turbinals, the inferior meatus between the inferior turbinal and the floor of the nose (Fig. 8). The superior meatus contains the opening of the posterior

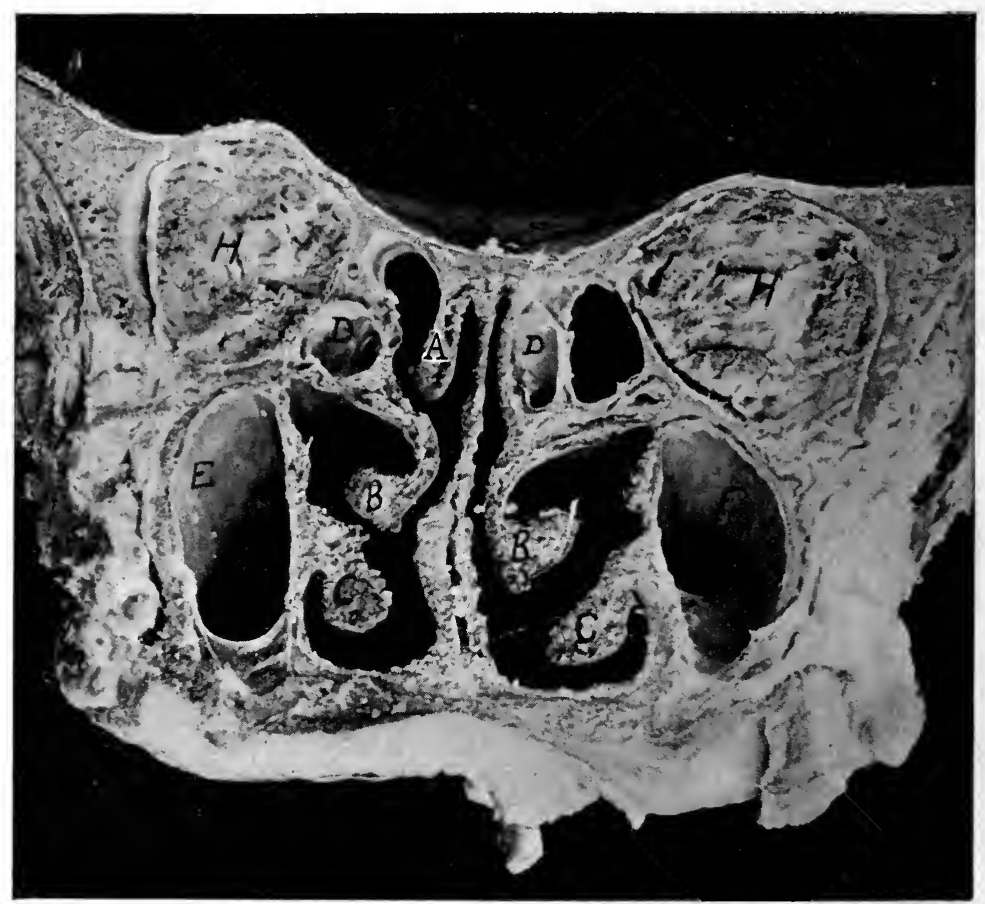

Fig. 3. $-A$, superior turbinated bone; $B, B$, middle turbinated bones ; $C$, $C$, inferior turbinated bones ; $D, D$, ethmoidal cells ; $E, E$, maxillary sinus ; $H, H$, cavity of orbit. (Superior turbinated bone on the right side is attached to the middle turbinate.)

ethmoidal cells, the middle communicates with the anterior ethmoidal cells, and has upon its external wall the hiatus semilunaris, the convexity of whose curve is directed forward and downward; at its anterior end is the opening into the infundibulum, communicating with the frontal sinus, while the ostium maxillare, or opening into the antrum of Highmore, is at the posterior part, where is also found the prominence known as the bulla ethmoidalis, formed by the bulging of one of the ethmoidal cells. In the inferior meatus is the opening of the lachrymal duct (Figs. 9 and ro).

Each nasal chamber has communicating cavities, the accessory sinuses-the frontal, ethmoidal (anterior and posterior) maxillary, and sphenoidal. The frontal sinuses are of varying size 
and shape, and lie between the two tables of the frontal bone at the root and to the inside of the nose; they are rudimentary during infancy. Each opens into the middle meatus through the infundibulum, though there is often a channel of communication of one frontal sinus with the other. While the ethmoidal sinuses are dignified with the name of cavities, they are in reality an aggregation of bony cells divided into the anterior and posterior groups. The anterior cells open into the middle meatus by the ostia ethmoidalia; the posterior cells communicate with the superior meatus (Fig. II). The maxillary sinus, more commonly known as the antrum of Highmore, is an irregularly pyramidal cavity in each superior maxillary bone. The upper boundary is the floor of the orbit, the

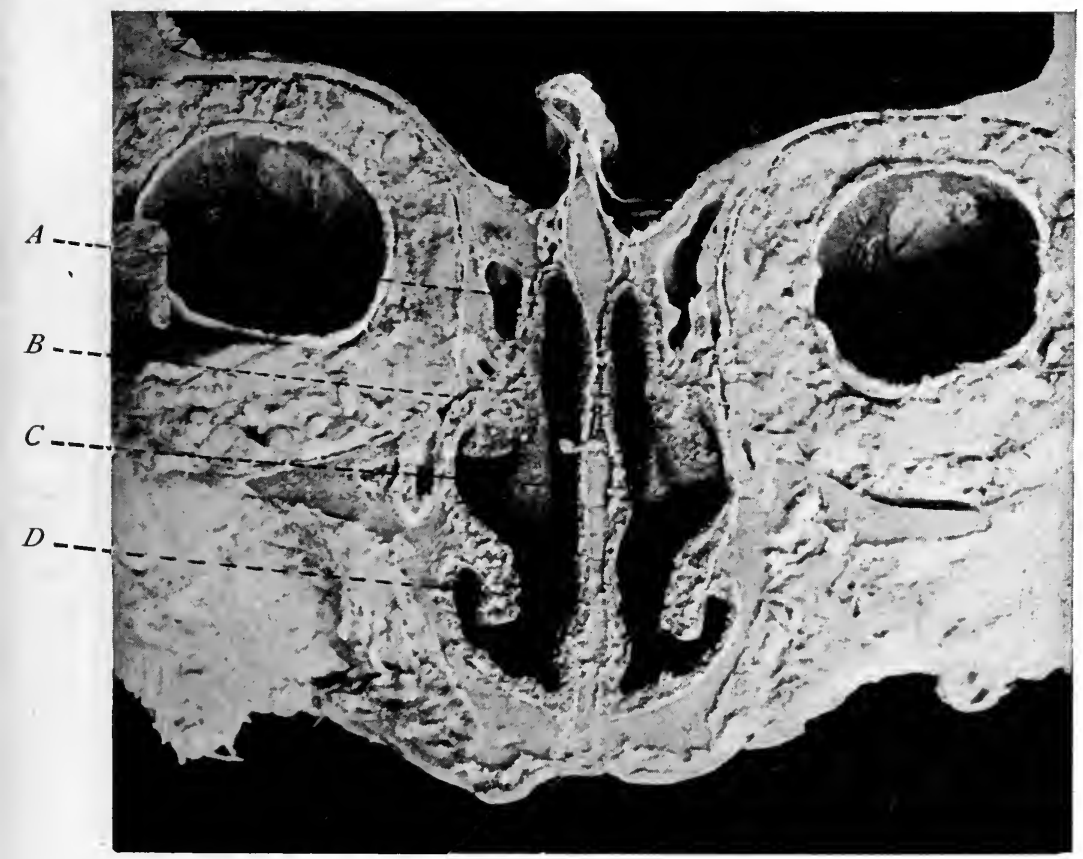

FIG. 4.- $A$, ethmoidal sinus ; $B$, opening from the maxillary sinus; $C$, middle turbinated bone; $D$, inferior turbinated bone.

lower boundary is the roof of the mouth, and the inner boundary is the outer wall of the nasal cavity. It communicates with the middle meatus by the ostium maxillare. The sphenoidal sinuses are two rounded cavities, often asymmetrical, in the 
body of the sphenoid, separated the one from the other by a thin bony plate. Each sinus opens into the superior meatus of its respective side.

The lining of the vestibule at the opening of the anterior nares is a continuation of the integument; this gradually changes until it assumes completely the character of a mucous membrane at the junction of the vestibule with the nasal fossa. In this vestibular lining are found sebaceous glands and numerous minute hairs called vibrissæ, which serve as a filtre against the ingress of gross particles in the inspired air. The Schnei-

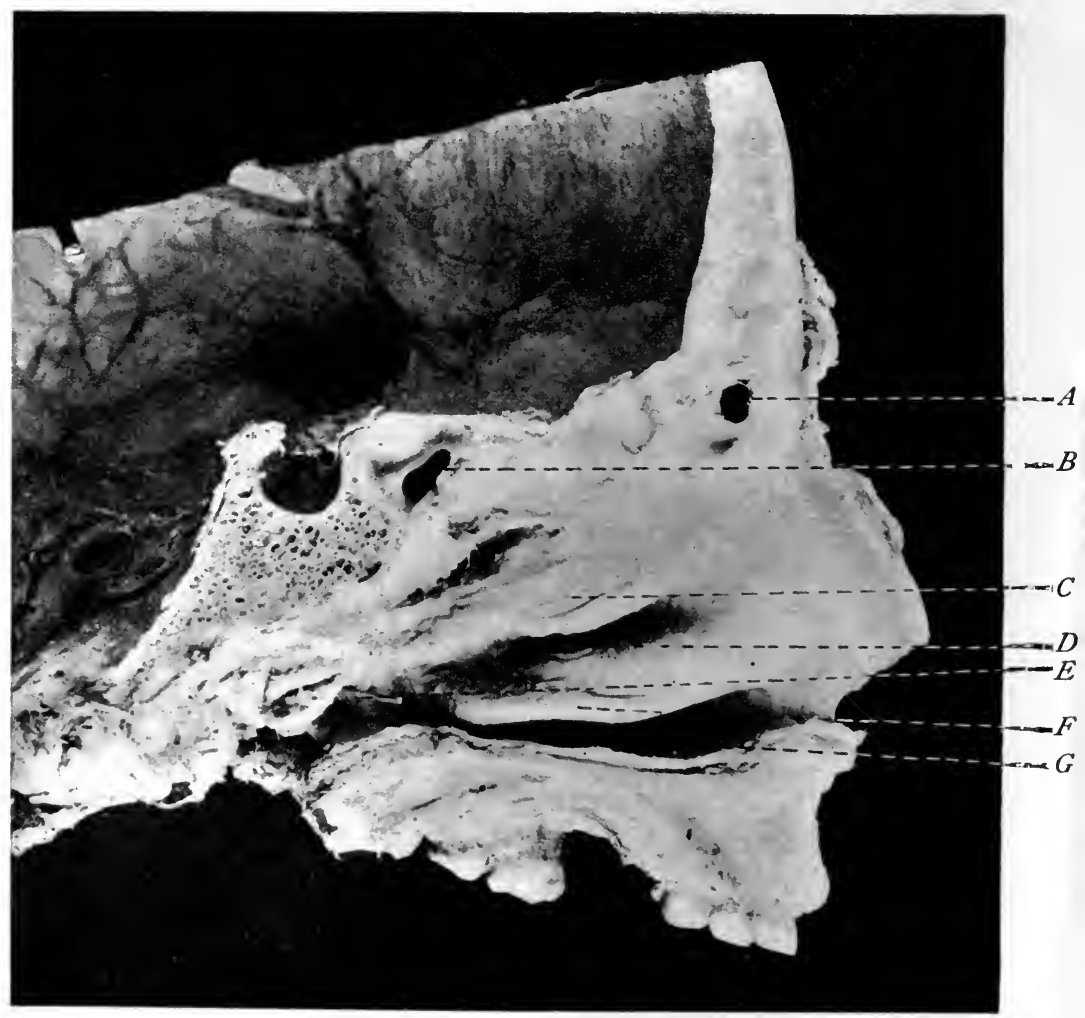

FIG. 5.- $A$, frontal sinus ; $B$, ethmoidal sinus ; $C$, middle turbinated bone ; $D$, middle meatus ; $E$, bristle passed into Eustachian tube ; $F$, inferior turbinated bone ; $G$, inferior meatus.

derian or mucous membrane of the nasal fossæ shares the general character of mucous membranes elsewhere in the air passages. The columnar epithelium is ciliated in the respiratory tract 
and non-ciliated in the olfactory tract. The membrane lining the sinuses is continuous with that of the nasal fossæ, though its secretion has more of a serous nature. The covering of

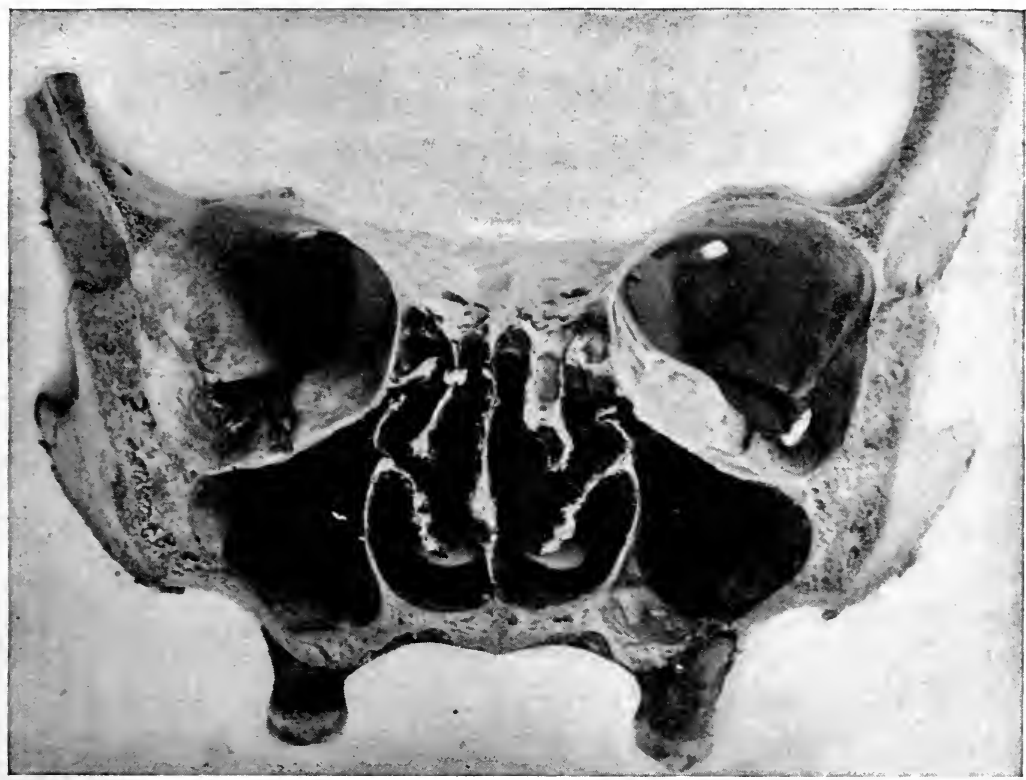

Fig. 6.-Coronal section (dry specimen) through maxillary sinuses and turbinated bones.

the turbinated bones and the posterior parts of the septum deserves more extended consideration, as an understanding of its peculiar structure explains many of the facts of its special pathology. Beneath the epithelial layer is the submucous connective tissue of varying thickness, whose lower layers form the periosteum and perichondrium. Within this connective tissue are imbedded the tubular mucous glands. The special points are the existence of venous sinuses or plexuses capable of distention and of containing large quantities of blood. When thus engorged the thickness of the membrane is greatly increased, so that it has been likened to an erectile tissue, from its property of intumescence. According to Bresgen there is one plexus for the inferior turbinated body, one for the free border of the middle portion of the middle turbinated body, and one for the posterior end of each of the turbinated bodies.

Olfactory impulses are conveyed to the brain by the olfactory nerve which passes through the cribriform plate of the 
ethmoid; the terminal filaments have their endings in the membrane covering the superior turbinal, the upper third of the middle turbinal, and the upper third of the septum. The nerves of sensation are the nasal, the anterior palatine, the naso-palatine, the spheno-palatine, and the Vidian.

The blood supply is derived from the nasal artery and the

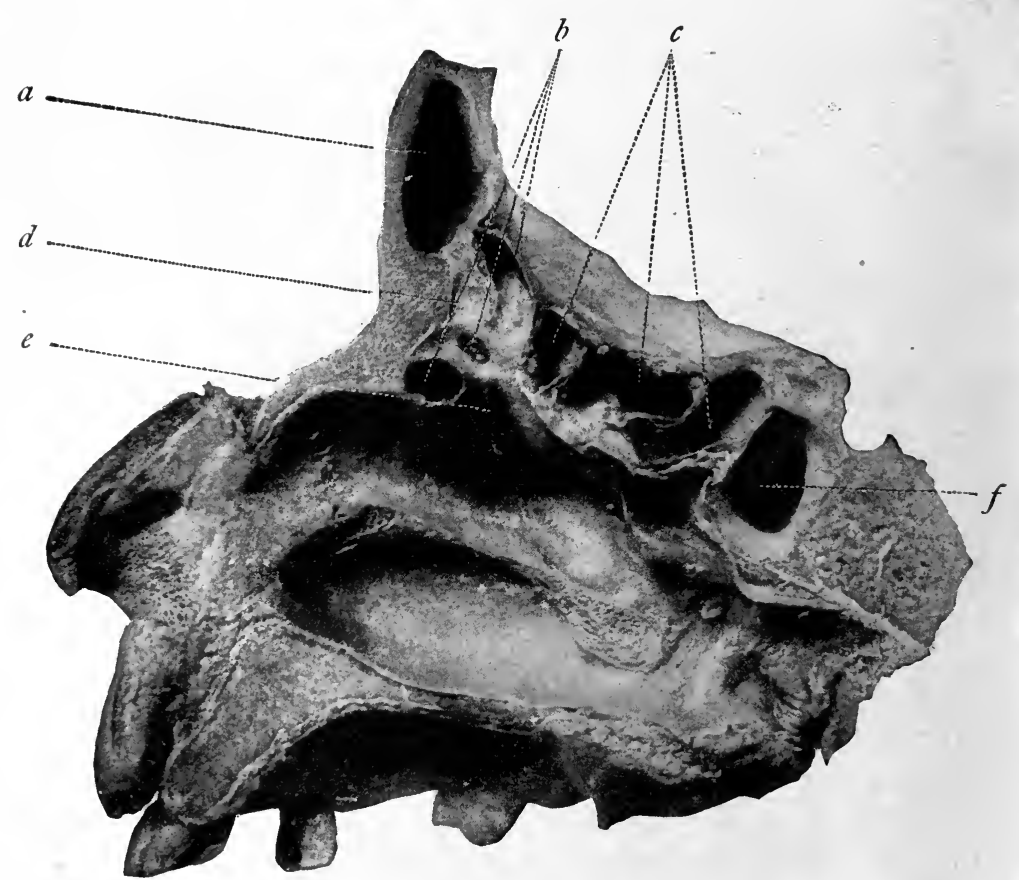

FIG. 7.-Section showing frontal sinus and ethmoid cells. $a$, frontal sinus; $b$, anterior ethmoidal cells ; $c$, posterior ethmoidal cells ; $d$, naso-frontal canal ; $e$, infundibulum ; $f$, sphenoidal sinus. (Hartmann's Atlas.)

posterior dental branch of the internal maxillary and the anterior and posterior ethmoidal branches of the ophthalmic arteries. The lymphatics of this region communicate with the general lymphatic system by means of two trunks passing near the orifice of the Eustachian tube.

The Naso-pharynx.-This division of the upper respiratory tract extends from the openings of the posterior nares to the lower border of the palate; it is hood-shaped, with the upper 
boundary formed by the basilar process of the occipital bone and the posterior wall by the spinal column. The communication of the nasal passages with the naso-pharynx is effected by two oval openings-the posterior nares-or, as they are sometimes called, "the choanæ." On each lateral wall of this cavity are the openings of the Eustachian tubes and the fossæ of Rosenmuiller. In the upper and central part of the nasopharynx, known as the vault, is found a mass of lymphoid tissue-the pharyngeal or Luschka's tonsil-constituting part of the pharyngeal lymphoid ring. The lining membrane is covered with ciliated epithelium, and contains numerous mucous glands whose secretion assists in the lubrication of the pharynx.

Below the naso-pharynx is situated the oro-pharynx extending from the border of the soft palate to the level of the

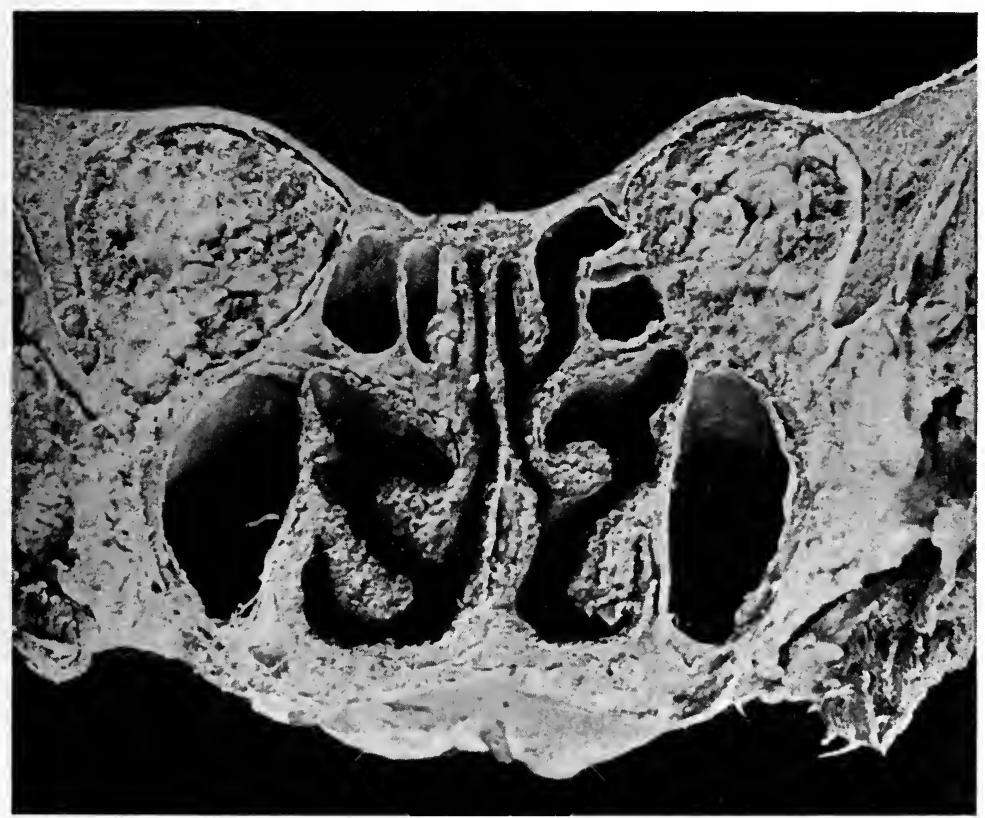

FIG. 8.-Asymmetrical development of the turbinated bones.

greater horn of the hyoid bone. In this division are included the velum palati, the uvula, the anterior and posterior pillars, the faucial tonsils, and the posterior wall of the pharynx. The velum palati or soft palate is attached to the edge of the hard 
palate, with its lower border hanging free, from which in the median line depends the uvula; during deglutition the nasopharynx is shut off from the oro-pharynx by the approxi-

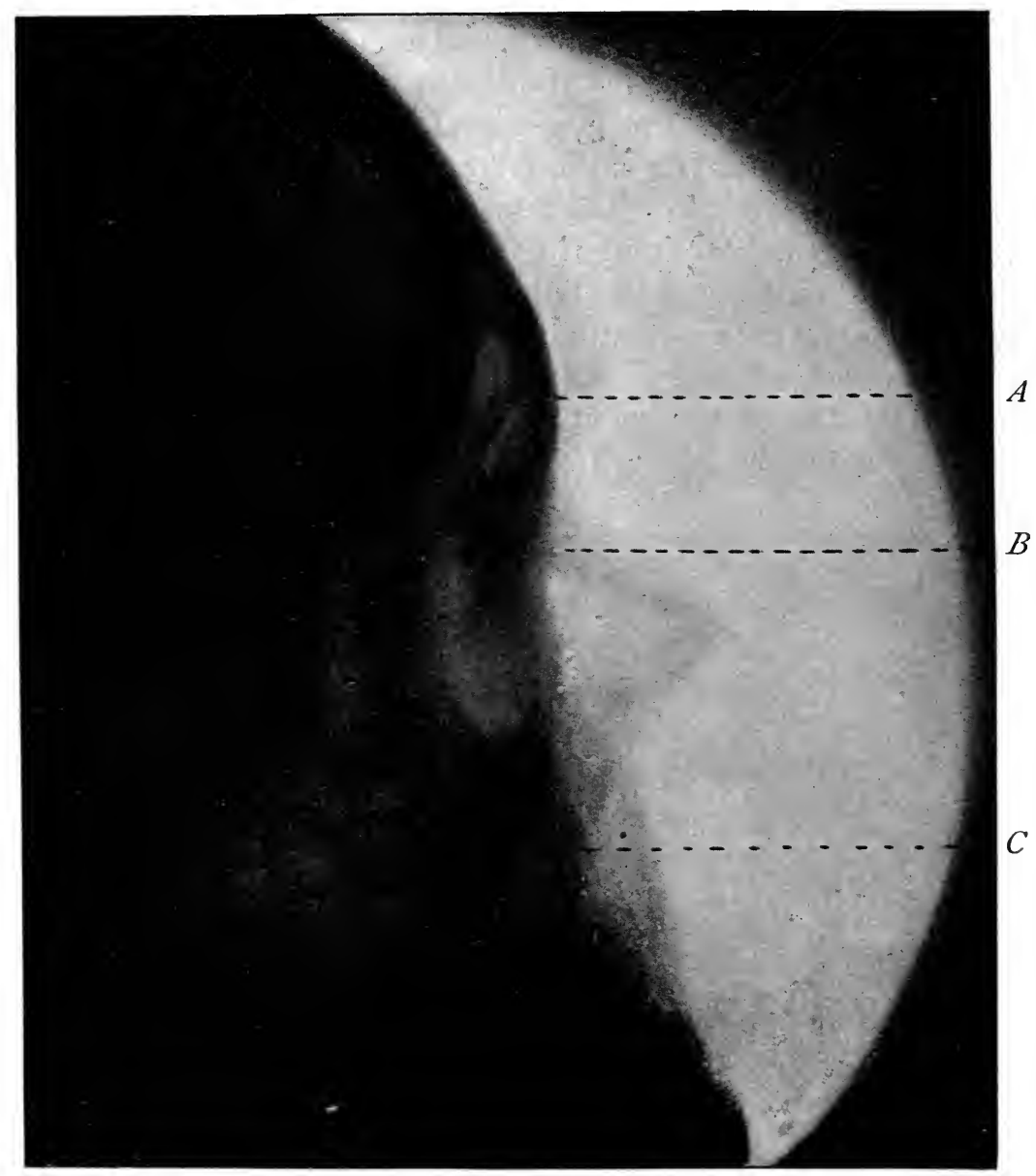

FIG. 9.-Radiograph of head showing patency of frontal sinus. $A$, frontal sinus ; $B$, nasal bone ; $C$, antrum.

mation of this curtain-like fold to the posterior wall of the pharynx. The soft palate is covered with squamous epithelium upon its lower or buccal surface, and columnar, ciliated epithelium upon its upper and posterior surface. Beneath these epithelial layers are mucous and lymphoid glands. The soft palate on either side of the uvula curves downward and outward in two folds, known as the anterior and posterior pil- 
lars or palatine arches; in the anterior pillars are found the palato-glossal, and in the posterior pillars the palato-pharyngeal muscles. Between these are situated the faucial tonsils, ovoidal in shape, composed of masses of lymphoid tissue. The surface of the tonsils is covered with squamous epithelium, and presents the depressions or openings, eight to ten in number, which are known as lacunæ or crypts. These two faucial

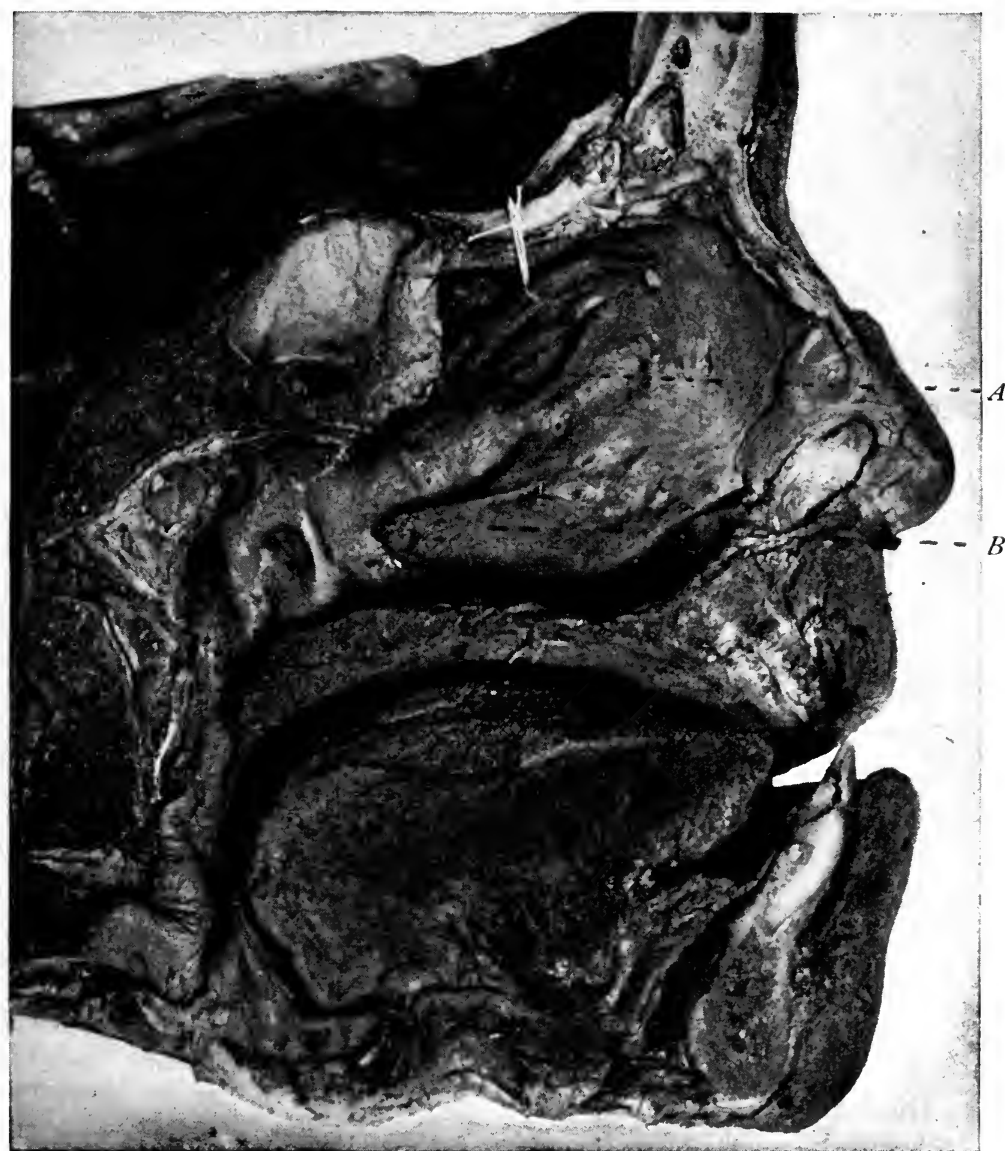

FIG. 10.-Sagittal section of head (the middle turbinated bone so elevated as to show the hiatus semilunaris). $A$, hiatus semilunaris ; $B$, inferior turbinated bone.

tonsils with the tonsil of Luschka form the prominent parts of the pharyngeal lymphoid ring.

The laryngo-pharynx extends from the level of the greater horn of the hyoid bone down to the lower border of the cricoid 
cartilage, where it divides, passing on into the trachea in front and the esophagus behind.

Muscles of the Soft Palate.-The tensor palati or dilator tubæ arises from the scaphoid

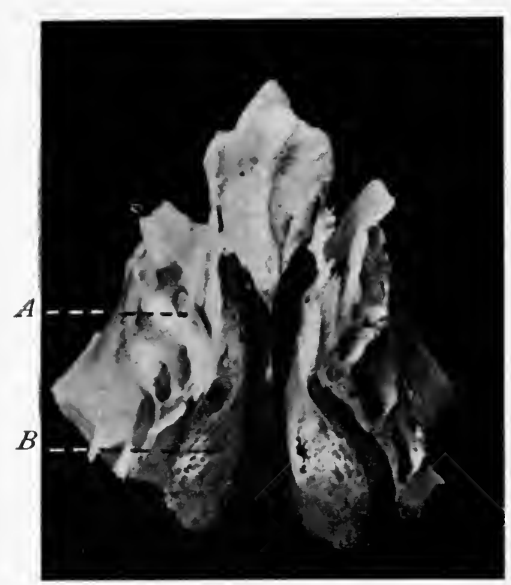

FIG. II.-Ethmoid bone. $A$, ethmoid cells ; $B$, middle turbinated bone. fossa, from the great wing of the sphenoid, from the anterior cartilaginous portion of the Eustachian tube, and from the membranous portion ; its outer border gradually becomes tendinous, and unites with the aponeurosis of the internal pterygoid muscle. Its broad tendinous portion winds round the hamular process and passes into the aponeurosis of the palate with an attachment also to the posterior border of the hard palate. The action of this muscle is to open the orifice of the tube by separating the cartilaginous from the membranous portion.

The levator palati arises from the apex of the petrous portion of the temporal bone, from the commencement of the external plate of the cartilage of the Eustachian tube, dividing into two portions at the pharyngeal opening, one of which is attached to the posterior nasal spine and periosteum of the hard palate, while the larger unites in the median line of the soft palate with the same muscle of the opposite side. During deglutition this muscle raises the soft palate and assists the action of the tensor palati in opening the orifice of the Eustachian tube.

The palato-glossi, or constrictors of the isthmus of the fauces, arise from the soft palate on each side of the uvula, and, passing obliquely downward and outward, are inserted into the base of the tongue.

The palato-pharyngei arise from the soft palate by two fasciculi, passing downward, backward, and outward, and, blending with the inferior constrictors and the stylo-pharyngei, are inserted into the posterior borders of the thyroid cartilage. There is often united with this muscle on each side a fasciculus, from the corresponding Eustachian tube, called the salpingo-pharyngeus. 
The azygos uvulæ (levator uvulæ) consists of a pair of muscular bundles arising from the posterior nasal spine of the palate bone and from the palatal aponeurosis, which, passing downward, have their insertion in the uvula.

The muscles of the pharynx, besides those of the soft palate already mentioned, are the superior, middle, and inferior constrictors and the stylo-pharyngei. The inferior con-

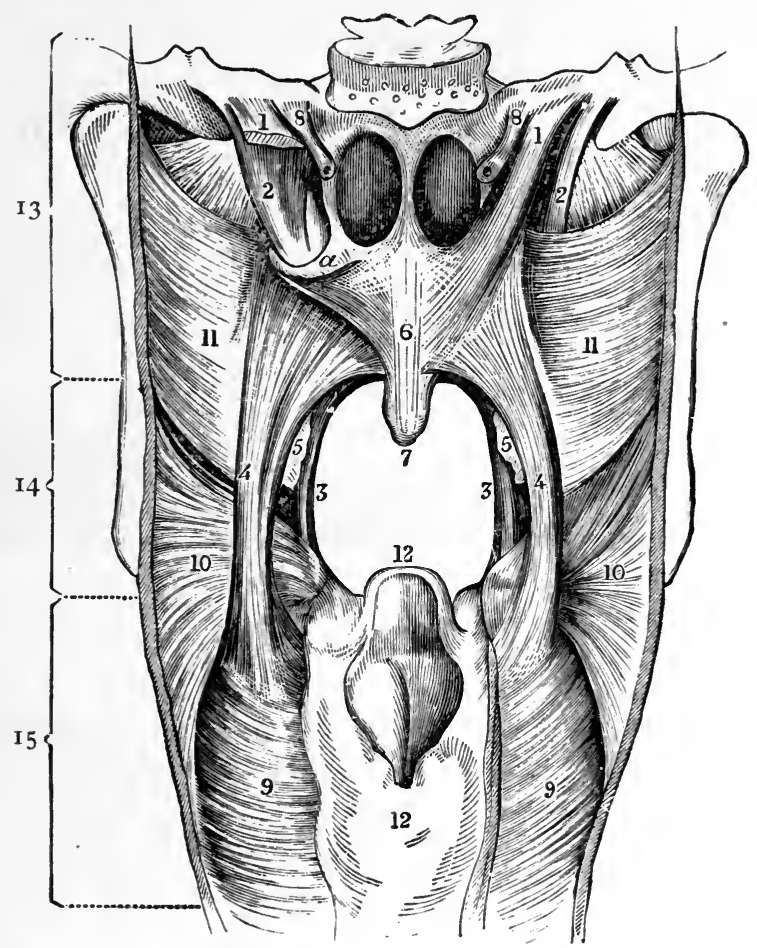

FIG. 12.-Muscles of the soft palate and pharynx. Pharynx laid open from behind. I, I, levatores palati, the left being cut short near to its origin ; 2,2 , tensores palati, the left showing its reflected tendon and relation to the hamular process, $a ; 3,3$, palato-glossi, anterior pillars of the fauces ; 4, 4, palato-pharyngei, posterior pillars of the fauces; 5,5 , tonsils; 6 , azygos uvula ; 7 , uvula; 8,8 , Eustachian tubes ; 9, 9, inferior constrictors; IO, Io, middle constrictors; II, II, superior constrictors ; 12, I2, epiglottis and larynx, not laid open; 13, cephalo-pharynx or nasopharynx ; I4, hyo-pharynx or oro-pharynx ; I5, laryngo-pharynx. (Gray, Browne, and Burnett.)

strictor is attached to the cricoid and thyroid cartilages, and extends backward and inward to the fibrous raphe in the posterior wall of the pharynx. The middle constrictor, fan-like in 
shape, arises from the greater and lesser horns of the hyoid bone, and from the stylo-hyoid ligament, and extends to the posterior pharyngeal raphe. The superior constrictor, quad-

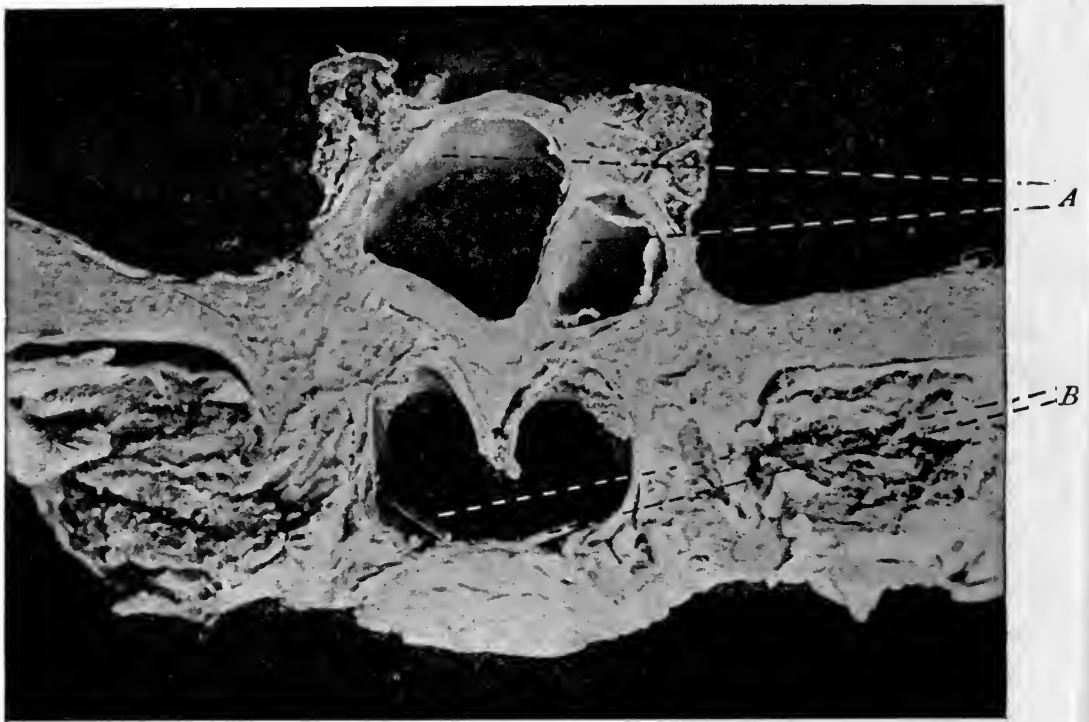

FIG. I3.-Coronal section through sphenoidal sinus and posterior end of nasal cavity. $A$, sphenoidal sinus (asymmetric); $B$, bristle passed into the Eustachian tube, uvula above.

rilateral in shape, arises from the lower third of the posterior edge of the internal pterygoid plate and its hamular process, from the adjacent part of the palate bone and the reflected tendon of the tensor palati muscle, from the pterygo-maxillary ligament and from a portion of the alveolar process of the inferior maxillary, and is also inserted into the median raphe, with an additional insertion in the pharyngeal spine of the basilar process of the occipital bone. The stylo-pharyngeus arises from the styloid process of the temporal bone, and has its insertion into the posterior border of the thyroid cartilage, though some of its fibres join and merge with those of the constrictors.

The action of these constrictors is to cause an undulatory constriction of the pharynx from above downward and thus propel particles of food down into the esophagus.

The lymphatics of the pharynx have a peculiar distribution, somewhat circular in shape, which has led to the term pharyngeal lymphoid ring, with the pharyngeal, lingual, and two fau- 
cial tonsils, marking, as it were, the division into quadrants. The pharyngeal tonsil forms the superior part of the circle, and is connected with the faucial tonsil by a slender chain of glands running down on each lateral edge of the posterior wall in close relation with the posterior pillars. Below, the circle is completed by the follicles on the base of the tongue and in the glosso-epiglottic folds; here the distribution is in a broad layer. Upon the posterior wall of the pharynx are numerous solitary follicles which become conspicuous when enlarged, as in follicular pharyngitis.

The arterial supply of the pharynx is derived from the facial, the internal maxillary, and the ascending pharyngeal arteries; the blood supply of the tonsils comes from the dorsalis linguæ, the ascending palatine and descending palatine, and from the ascending pharyngeal.

The veins on the posterior surface of the pharynx form a plexus continuous with those of the nasal mucous membrane; the anterior plexus communicates with the internal jugular by means of the pharyngeal vein. The nerves of this region are, first, the motor, derived from the lower division of the fifth, the facial, and branches from Meckel's ganglion; second, the sensory nerves, derived from the third division of the fifth, with branches from the vagus and the glossopharyngeal.

The Larynx.-The larynx or

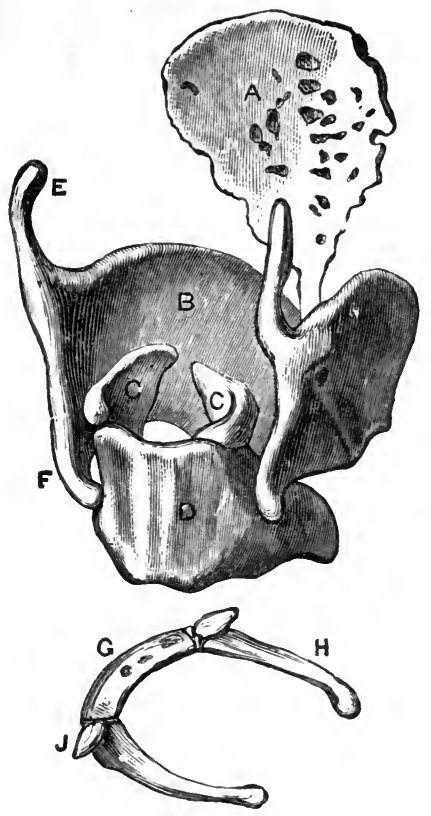

FIG. I4.-Hyoid bone and laryngeal cartilages. $G$, body of the hyoid bone; $H$, large cornu ; $J$, small cornu; $A$, epiglottis ; $B$, thyroid cartilage; $C$, arytenoid cartilage ; $D$, cricoid cartilage ; $E$, upper cornu, and $F$, lower cornu, of the thyroid cartilage. (Ellis, Seiler.) voice box is that part of the respiratory tract situated just above the trachea, and contains the specialized structures for phonation, with their protective coverings. The supporting framework of the larynx are the following cartilages: the thyroid, the cricoid, the arytenoids, the epiglottis, and two pairs of small cartilages-the cartilages of Santorini and Wrisberg (see Fig. I4). 
The thyroid cartilage (the shield) is the largest of these, and consists of two quadrilateral plates or wings united along their anterior border at an acute angle, forming a projection in the median line (more marked in males than females) known as the pomum Adami, or Adam's apple; between the skin and this projection is oftentimes placed a small bursa. The posterior border of each wing is prolonged superiorly and inferiorly into projections known as the superior and inferior horns; the latter rest upon and articulate with the cricoid cartilage. At the upper part of the union of the two alæ is a deep depression

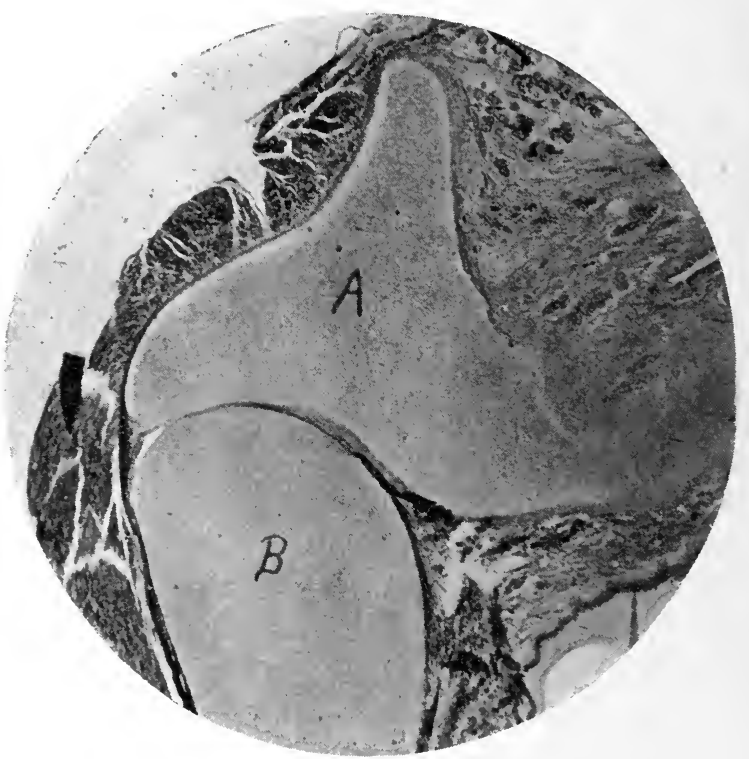

FIG. I5.- $A$, arytenoid cartilage ; $B$, cricoid cartilage.

(Photomicrograph by T. P. Whittier.)

known as the thyroid notch, which permits of the thyroid passing behind the hyoid bone in deglutition.

The arytenoids are pyramidal in shape, with their bases, which are concave and smooth, resting upon the upper surface of the posterior portion of the cricoid, and with their apices directed backward and inward (Fig. I5). They present three surfaces: an anterior, posterior, and internal; at the junction of the anterior and internal surfaces are projections known as the vocal processes, to which are attached the posterior ends of the vocal cords; at the junction of the anterior and posterior 
surfaces project the muscular processes, to which are attached the posterior and lateral crico-arytenoid muscles.

The cricoid cartilage ("the signet ring") is in reality the upper ring of the trachea, but so modified as to furnish support to the larynx proper. The anterior portion of the cricoid, hoop-like and narrow, can be readily felt under the skin ; the posterior or "seal" part is broad, and presents upon its

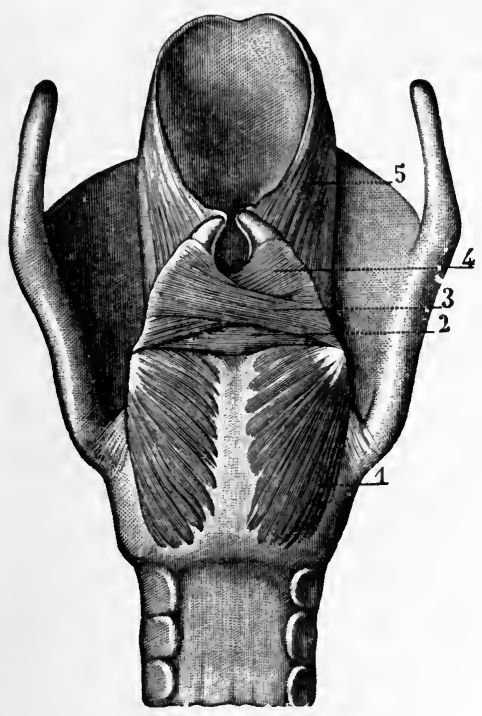

FIG. 16.

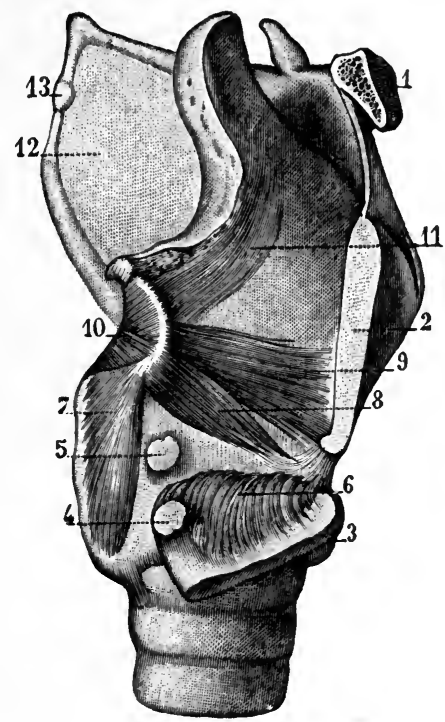

FIG. I7.

FIG. I6.--Posterior view of the larynx. (From Ranney, after Sappey.) I, posterior crico-arytenoid muscle; 2, 3, 4, different fasciculi of the arytenoid muscle; 5, aryteno-epiglottidean muscle.

FIG. I7.-Lateral view of the larynx. (From Ranney, after Sappey.) I, body of the hyoid bone; 2, vertical section of the thyroid cartilage; 3 , horizontal section of the thyroid cartilage turned downward to show the deep attachment of the cricothyroid muscle ; 4 , facet of articulation of the small cornu of the thyroid cartilage with the cricoid cartilage; 5 , facet on the cricoid cartilage; 6 , superior attachment of the crico-thyroid muscle; 7 , posterior crico-arytenoid muscle; 8 , Io, arytenoid muscle; 9 , thyro-arytenoid muscle; II, aryteno-epiglottidean muscle; 12, middle thyro-hyoid ligament; I3, lateral thyro-hyoid ligament.

lateral oblique surface two facets covered with synovial membrane for articulation with the inferior horns of the thyroid; upon the superior border are two smooth, oval, convex surfaces which articulate with the arytenoid cartilages. The epiglottis, which contains considerable fibrous tissue, rendering it flexible, is shaped like a leaf, and is attached to the receding 
angle of the thyroid cartilage just below the thyroid notch by the thyro-epiglottic ligament, and to the hyoid bone by the hyo-epiglottic ligament. At its base on each side are attached the ary-epiglottic folds. The cartilages of Santorini, also known as the cornicula laryngis, are located in the membrane over the summit of each arytenoid. The cartilages of Wrisberg are also placed in the aryteno-epiglottic folds.

The thyro-hyoid ligament, or membrane, more properly speaking, connects the thyroid cartilage with the hyoid bone, extending from the upper border of the former to the upper posterior border of the latter. The lateral hyoid ligaments extend from the superior horns of the thyroid cartilage to the greater horns of the hyoid bone. The hyo-epiglottic ligament connects the epiglottis near its apex to the posterior surface of the body of the hyoid bone. The thyro-epiglottic ligament connects the base of the epiglottis with the thyroid cartilage. The glosso-epiglottic ligament, consisting of a median portion and two lateral folds, serves to attach the epiglottis to the base of the tongue. The crico-thyroid membrane or median ligament connects the thyroid with the cricoid cartilage; it is thick and elastic, and forms the anterior covering of the larynx below the thyroid. The anterior insertion of the true vocal cords is blended on the sides of this membrane, which explains the frequent impairment of the voice following the operation of thyrotomy. In the median line it is subcutaneous, but on the sides it is covered by the crico-thyroid muscles. Its surface is crossed from side to side by an anastomosis between the crico-thyroid arteries. Laterally its inner surface is in relation with the thyro-arytenoid and lateral crico-arytenoid muscles. The lateral crico-thyroid ligaments keep in coaptation the articular surfaces of the inferior horns of the thyroid with the sides of the cricoid cartilage. The cricoarytenoids are the capsular ligaments of the crico-arytenoid joints.

Muscles of the Larynx.-The laryngeal muscles may be divided into those governing the vocal cords and into those controlling the movements of the epiglottis and laryngeal vestibule. The former may be subdivided into muscles which approximate and tighten the vocal cords and into those which relax and separate them. The crico-thyroid muscles, triangular in shape, take their origin from the front and sides of the 
cricoid cartilage, and, passing obliquely upward and backward, are inserted into the inner portion of the lower border of the thyroid cartilage; a second set of bundles is inserted near the base of the inferior horns of the thyroid cartilage. The action of these muscles is to draw the cricoid up and backward, thus increasing the tension of the vocal cords. The two posterior crico-arytenoids, fan-like in shape, arise from the posterior surface of the cricoid cartilage, and are inserted into the outer angle of the bases of the arytenoid car. tilages; their contraction rotates the arytenoids so that the

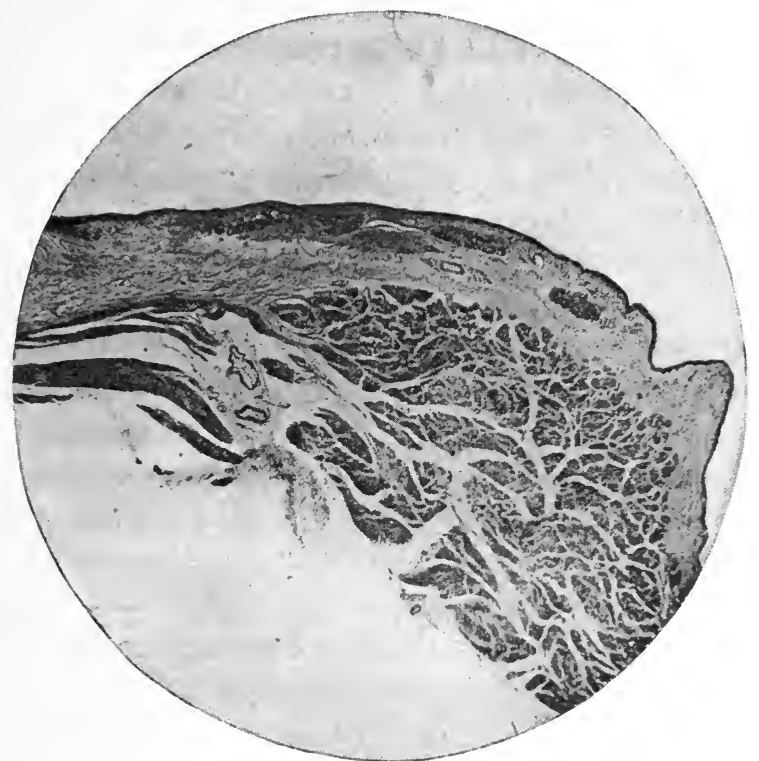

Fig. 18.-Section of vocal cord showing internal fibres of the thyro-arytenoid muscle.

(Photomicrograph by T. P. Whittier.)

vocal processes of the latter are turned outward; for this reason they are called "the glottis openers." The lateral crico-arytenoids arise from the upper border of the sides of the cricoid cartilage, and are inserted into the outer angle or muscular process of the arytenoids in front of the insertion of the posterior crico-arytenoids; their contraction turns the arytenoids, so that the vocal processes are approximated. The thyro-arytenoid muscles arise from the internal portion of the thyroid cartilage along the median line, and passing backward, immediately beneath the outer side of the vocal 
cords, are inserted into the base and anterior surfaces of the arytenoid cartilages; they are divided into an internal and external set of bundles, the former attached to the vocal cords throughout their entire length and inserted into the external surfaces of the vocal processes, the latter inserted into the anterior face of the arytenoid cartilage. The contraction of the internal fibres approximates the edges of the cords, while the external fibres bring about their relaxation. The arytenoid muscle, quadrilateral in shape, extends between the posterior surfaces and outer borders of the arytenoid cartilages; it has three sets of fibres-two oblique and one transverse. Each set of oblique fibres crosses from the apex of one cartilage to the base of the other, forming an $\mathrm{X}$; the transverse or deep fibres lie horizontally. The action of this muscle approximates the arytenoids, thus adducting the cords and closing the glottis. The thyro-epiglottic muscles have their origin from the inner surface of the thyroid cartilages; some of the fibres are lost in the ary-epiglottic folds, while the rest are inserted in the sides of the epiglottis; their action is to depress the epiglottis. The aryteno-epiglottic muscles take their origin from the posterior surface of the base of the arytenoids; some of the fasciculi end in the ary-epiglottic folds, while the others pass upward and are inserted into the margin of the epiglottis. These two last muscles close the opening of the larynx during deglutition.

According to E. Meyer, there is direct connection between the fasciculi of the crico-thyroid muscle and the inferior constrictor of the pharynx. According to Jelenffy and Meyer the posterior crico-arytenoid muscle is divided into three distinct portions. Jelenffy states also that there is a connection between the posterior crico-arytenoid and the arytenoideus muscle, and also a similar connection between the fasciculi of the other muscles.

The mucous membrane of the larynx, continuous with that of the respiratory tract above and below, is thrown into folds according to the structures which it covers. Over the aryepiglottic muscles it forms the ary-epiglottic folds, and over the superior thyro-arytenoid ligaments it forms the ventricular bands. Above the ventricular bands the epithelium is squamous, with the exception of the lower half of the lingual surface of the epiglottis, where it is columnar and ciliated, as is 
the membrane below the ventricular bands. The epithelium of the vocal cords, however, is tessellated. The mucous membrane of the larynx contains many tubular mucous glands.

The superior laryngeal artery supplies the muscles and mucous membrane of the upper part of the larynx. The inferior laryngeal or crico-thy roid artery divides into two branches, one of which passes through the cricoid membrane and supplies the mucous membrane below the cords; the other branch passes beneath the border of the thyroid cartilage and forms an anastomosis with the superior laryngeal. The posterior laryngeal artery, derived from the inferior thyroid, passes upward with the recurrent laryngeal nerve and divides near the lower edge of the thyroid cartilage, one branch anastomosing with the superior laryngeal, while the other supplies the posterior crico-arytenoid muscle. The veins of the larynx empty into the internal jugular through the superior, middle, and inferior thyroid veins.

The larynx is connected with the central nervous system through the inferior or recurrent laryngeal and the superior laryngeal nerves. The latter has two branches-the external, which is the motor nerve for the crico-thyroid muscles, and the internal, which pierces the thyro-hyoid membrane with the superior laryngeal artery, and is the sensory nerve for the mucous membrane of the larynx. On the right side the recurrent laryngeal nerve arises in front of the subclavian artery, while on. the left side it arises on the level with the arch of the aorta; the two nerves enter the larynx near the articulation of the inferior horns of the thyroid cartilage with the cricoid, and are motor for all the laryngeal muscles except the crico-thyroid.

The opening into the larynx is guarded by the epiglottis, on whose posterior surface near the base is a rounded eminence known as the cushion of the epiglottis. Between the base of the tongue and the epiglottis are two depressions-the lingual sinuses-separated from each other by the frenum of the epiglottis. From each side of the base of the epiglottis, extending backward and inward, are the aryteno-epiglottic folds; exterior to these are the pyriform sinuses. These aryepiglottic folds extend to the apex of each arytenoid cartilage and contain the cartilages of Wrisberg and Santorini. Below are found the ventricular bands or false vocal cords formed by a reduplication of the lining membrane over the superior 
thyro-arytenoid ligaments. Immediately below are the ventricles of the larynx (ventricles of Morgagni), two narrow spaces from whose anterior portion extends a pouch called the sacculus laryngis (Fig. 19). The membrane lining the ventricles is continued downward, and, changing its character, covers the true vocal cords. When the cords are viewed from above, or as ordinarily seen in the mirror, they appear as two narrow, white, glistening bands; transverse section of the cords show that their real shape is that of a prism whose oblique surface is directed downward. The structure of the cords is squamous epithelium covering the ligamentous bor-

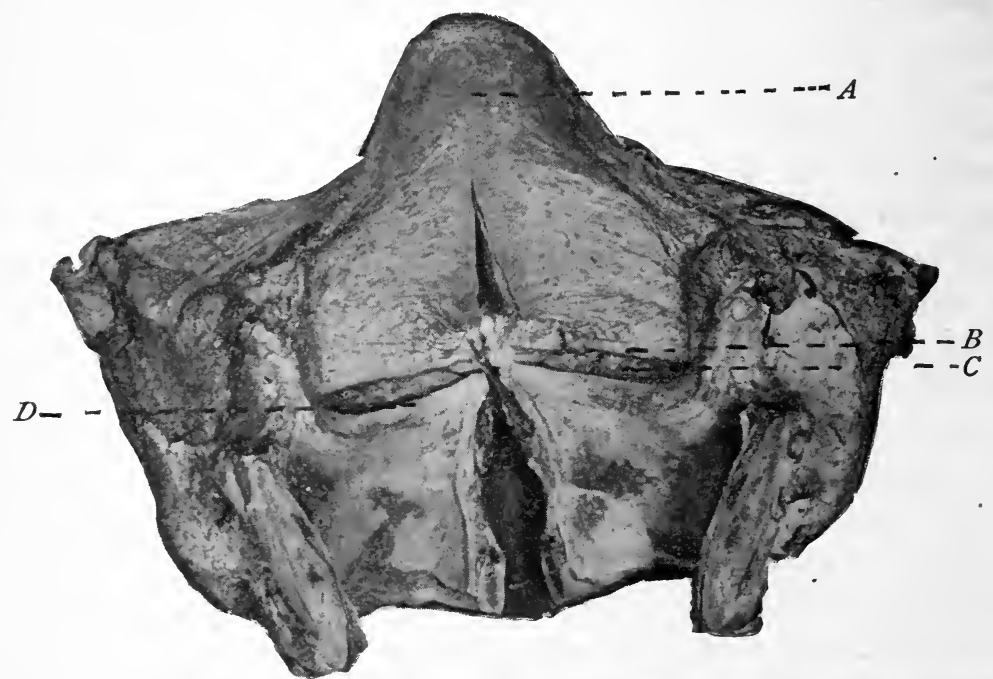

FIG. I9.-Adult larynx opened in the posterior median line. $A$, epiglottis, posterior surface ; $B$, ventricular band ; $C$, ventricle of the larynx $; D$, vocal cord.

ders of the internal thyro-arytenoid muscles. The opening between the two vocal cords is known as the glottis or rima glottidis.

\section{Physiology of the Upper Air Passages}

The nose is the seat of a special sense-olfaction-and besides takes part in three other important functions-viz., respiration, phonation, and the aeration of the middle ear. The apparatus for the former function lies almost entirely in the superior meatus and the upper posterior part of the middle meatus of the nasal fossa (the olfactory region); while the respiratory and 
vocal functions are dependent to a great extent upon the inferior and middle meati (the respiratory region). The mucous membrane lining the nasal chambers is somewhat different in the two regions: in the olfactory region it is endowed with special anatomic elements and its epithelium is of the nonciliated variety. In the lower portion it is columnar and ciliated. The olfactory region is funnel-shaped, the large end opening into the naso-pharynx behind, and the smaller end presenting anteriorly.

Olfaction.-The sense of smell depends especially upon the ultimate division into filaments of the olfactory nerve and the peripheral exposure of the same; these filaments, descending through the cribriform plate of the ethmoid bone, are distributed to the mucosa, which lines the upper part of the septum and covers the superior turbinated bone and attic of the nose. The histologic arrangement of the mucous membrane and nerve filaments is unique, each one being partially inclosed, so to speak, at its peripheral end. According to Stöhr, "The nasal mucosa consists of an olfactory epithelium and of a tunica propria ; in the olfactory epithelium two forms of cells occurthe one form is cylindrical in its upper half, and contains yellowish pigment and minute granules, often arranged in longitudinal rows; the lower half is more slender, the edge is serrated and indented, the inferior end is forked and is said to unite with the similar ends of neighbouring cells to form a protoplasmic network; these elements are called sustentacular cells; their nuclei are generally oval and usually lie at the same level. In vertical sections they are seen to occupy a narrow belt-the zone of the oval nuclei. The second form of cells possess a spherical nucleus, and only in the vicinity of the latter an appreciable amount of protoplasm; from this a slender ciliated cylinder, the attenuated cell body, extends upward, while from the opposite pole a very delicate process continues directly into the axis cylinder of a nerve fibre. These cells, the olfactory, are ganglion cells, and their lower process is a centripetal nerve fibre. Their round, nucleated nucleoli lie at different levels, the zone of the round nuclei. At the border of the epithelium, toward the connective tissue, is a protoplasmic network furnished with nuclei, the so-called basal cells (Fig. 20). The surface of the epithelium is covered by an extremely delicate homogeneous membrane-the membrana 
limitans olfactoria. It is pierced by the ciliated extremities of the olfactory cells, and is covered by a peculiar substance re-

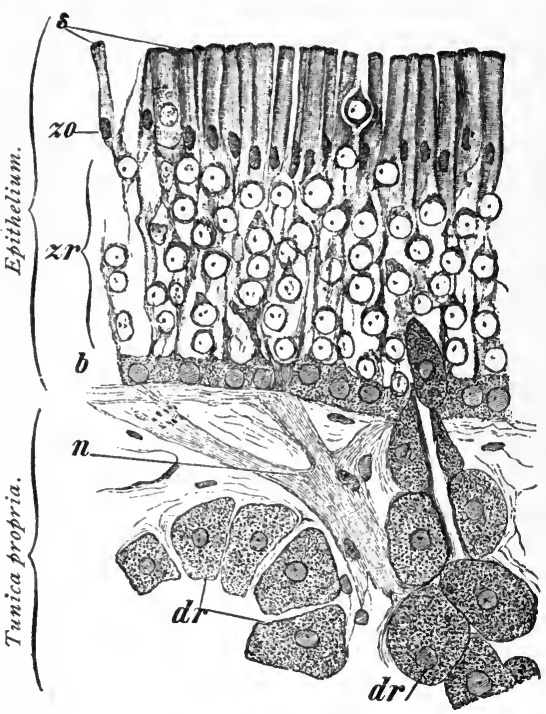

FIG. 20.-Vertical section through the olfactory mucosa of a rabbit $(\times 560)$. $s$, cuticular border; $z o$, zone of oval; $z r$, zone of round nuclei; $b$, basal cells; $d r$, portions of olfactory glands; on the right the lower portion of the excretory duct is shown; $n$, branch of the olfactory nerve (Stöhr). garded by some authors as a cuticular formation similar to the basal border of the intestinal epithelium, by others as delicate cilia, by still others interpreted as minute particles of discharged mucus (Fig. 21). The tunica propria consists of a loose feltwork of rigid connective-tissue fibres intermingled with delicate elastic fibres, which in some animals toward the epithelium is condensed to a structureless membrane. Numerous glands - the so-called olfactory (Bowman's) - are imbedded in the tunica propria. They are either simple or (for example, in man) branched tubules, in which an excretory duct (situated in the epithelium), a body, and a fundus may be distinguished. The cells of the body of the glands are pigmented. The glands until recently were regarded as serous glands, but latterly they have been pronounced mucous glands. The olfactory glands advance beyond the territory of the olfactory mucous mem. brane, and are found in portions of the respiratory mucous membrane. The tunica propria also carries the ramifications of the nerves. The branches of the olfactory nerve are accompanied by processes of the dura, and consist throughout of non-medullated fibres that readily separate into their component fibrillæ. The fibres are inferior processes of the olfactory cells grouped in bundles, which pass in horizontal arches from the epithelium, descending into the tunica propria, and by union with neighbouring bundles form the branches of the olfactory nerve. The terminal ramifications of the olfactory nerve lie within the tunica propria." The peripheral processes 
of the olfactory cell were observed by Schultze to be surmounted by a short projection, which is surmounted by a bunch of fine, hairlike processes (J. M. Bleyer).

The greater part of the mucous membrane of the nasal channels is supplied with nerves of common sensation derived from branches of the fifth pair. The function of smell depends, of course, upon the contact of the odorous substance with the peripheral nerve ends in the olfactory apparatus, and for this purpose the upper nasal passages must be open to the air, and also be in a tolerably healthy state. The substance to be recognised must be volatile, as no absolutely liquid or solid substance can be recognised by the sense of smell alone, although common sensation and the sense of taste may assist. The nasal passages must be free to allow of the ingress of air with which the volatile substance must be mixed, or no sensation of smell will be produced. There have been many theories put forth in explanation of the mechanism of olfaction. Among them three only deserve mention, according to $\operatorname{McCoy}(\mathrm{A}$. W.)-viz., the mechanical-that the particles impinged upon the mucous membrane excite mechanical irritation; the chemical-that a process of oxidation of the substance is necessary, so that only those which are oxidizable are capable of being smelled, and those which are not thus oxidized remain inodorous; the molecular or vibratory theorythat substances of light molecular weight and of rapid vibratory movement are odorous, while, on the contrary, those of heavier molecular weight and slow vibration are inodorous.

Tyndall thought that bodies were odorous in proportion to their capacity for absorbing heat. Ogle's theory is that the pigment of the olfactory region plays an important part by receiving the waves of

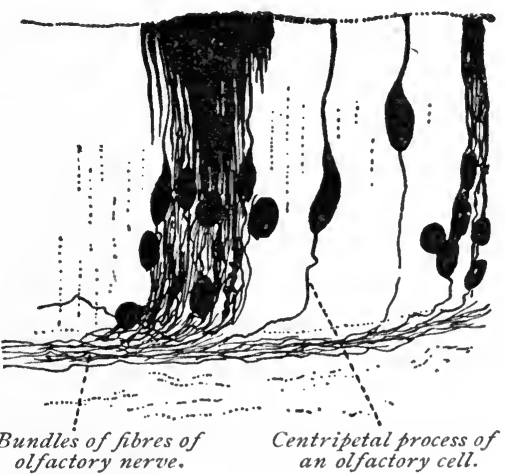

FIG. 21.-Vertical section through the olfactory region of a young rat $(\times 480)$. odour very much as the choroid coat of the eye and the aural ampullæ receive waves of light and sound respectively (McCoy). 
The lymph vessels form a coarse-meshed network lying in the deeper strata and are in close connection with the subarachnoid space. Medullated twigs of the trifacial nerve may be found in the respiratory as well as in the olfactory mucosa. The glands of Bowman open by fine ducts lined with flattened cells which extend to the surface between the olfactory epithelial cells.

The respiratory function of the nose is a very important one. The coarse material floating in the air to be respired, if not strained out by the vibrissæ at the vestibule of the nose, is lodged upon the mucous-covered surface of the irregular nasal passages and thus prevented from going into the lungs. If the material be large or otherwise of a character which the lining mucous membrane is unaccustomed to, acts of sneezing are excited, immediately followed by an abundant secretion of serous fluid, both of which phenomena are natural efforts for the expulsion of the foreign material.

Another and perhaps the most important function of the respiratory nasal region is the warming and moistening of the air to be respired. This is accomplished largely through the presence of the inferior and middle turbinated bodies, the vascular mechanism of which is calculated to impart to the air in a short time a great amount of heat. The vascular arrangement, according to Jonathan Wright, is as follows :

The larger arterioles, well supplied with muscular coats, lie in the deepest layers of the mucous membrane close to the bone. They give off capillaries supplying the periosteum, glands, and epithelial layer of mucous membrane. These capillaries are collected into veins which dilate into venous sinuses, the larger lacunæ, which are deeper, and the superficial lacunæ or "cortical" network, communicate with them. These lacunæ, again, empty into the veins which accompany the primary arterioles into the periosteal layer. The capillaries do not empty directly into the venous sinuses nor into the radical veins accompanying the arterioles.

It is obvious, therefore, that the turbinated bodies constitute Nature's stoves, so to speak, for warming the respired air. The very varied temperature to which the intranasal apparatus is exposed in our northern climates during the winter season is certainly a great tax upon the tonicity of its bloodvessels, especially under the prevailing custom of living in 
overheated houses, offices, stores, or workshops. It is therefore not astonishing that Nature so often produces an extra secretion of mucus for the purpose of coating the surface of the nasal passages. Although it is estimated that from five to seven thousand grains of fluid are secreted and exhaled by the glandular apparatus of the nose, yet it is doubtful if Nature designed this secretion for the moistening of the respired air, inasmuch as the atmosphere is generally well provided with moisture, excepting in dry, mountainous districts, where, of course, all the body fluids are constantly suffering more or less loss. In conjunction with the cavity of the pharynx the nasal fossæ act as resonance spaces for the modification of tone. This is not an unimportant function, as we all know the difficulty experienced in the articulation of certain letters ( $\mathrm{N}, \mathrm{M}$, etc.), and the peculiar deadness of tone (erroneously called talking through the nose) which is noticeable when any considerable obstruction to the nasal passages exists.

Pharynx.-The chief function of the naso-pharynx is undoubtedly that of an air chamber or resonance chamber contributing to voice production by furnishing an inclosed space for the amplification and modification of sound waves, and to audition by furnishing to the middle ear the necessary amount of warm, dry air for the maintenance of the equipoise of the tympanum.

Its mucous secretion in the normal state is designed to diminish the friction of the inspiratory current of air, and its shape and capacity furnishes a chamber for the retention of a certain quantity of air in a more or less passive state.

Oro-pharynx and Laryngo-pharynx.-These regions also contribute to voice production somewhat, but are mainly concerned in the function of deglutition.

By the co-ordinate action of the soft palate and pharyngeal muscles the sound waves are modified and directed in their emission so as to produce certain desirable effects. These effects are particularly necessary for the production of the musical voice, for without a tolerably free pharynx artistic vocalization is well-nigh impossible.

In deglutition the bolus, after proper mastication, is carried by the tongue to the isthmus of the fauces, where it is grasped, as it were, by the palato-glossi and palato-pharyngei muscles (the anterior and posterior pillars of the fauces), which approach 
toward the median line for the purpose. At the same time the free edge of the soft palate, by the action of its levator muscles, is fixed against the posterior wall of the pharynx, thus shutting off the naso-pharynx, while the larynx is raised up under the tongue and the epiglottis pushed down over the laryngeal aperture, as may be seen by the fluoroscope.

$\mathrm{Up}$ to this point the whole act has been more or less voluntary, but now it becomes automatic, as the bolus, being pushed fairly into the cavity of the pharynx, is taken by the constrictor muscles, and by their successive and co-ordinate contractions passed down to the esophagus and stomach. When the anterior and posterior faucial pillars are free there is not much contact between the bolus of food and the palatal tonsils; but when the pillars are adherent to the tonsils or adjacent glandular tissue, the tonsils are always more or less smeared and their crypts more or less filled with food.

Tonsils. - The function of the faucial tonsils is still a mooted question. It has not yet been definitely settled whether the purposes of these glands are absorptive or excretory. It is claimed by some, and these claims are based on experiment, that the tonsils can and do absorb micro-organisms into their interior spaces. This is denied by others who have likewise experimented in the same direction. Many practitioners believe that they act as sentinels to entrap and retain microorganisms and thus save the system from infection.

One function long ago attributed to them was that of assisting in the preparation of the bolus of food through the office of their secretion, and another of assisting in the lubrication of the throat by the same agency. It seems as though $E$. Frankel had shown that these organs are capable of at least arresting noxious material, and by a copious production of leucocytes and the rapid extrusion of the same through the follicles do neutralize and eject it. Physiologically, they may be considered more important during childhood, as they normally undergo absorption soon after the period of puberty.

Soft Palate and Uvula.-The soft palate and uvula constitute a flexible valve for partitioning the naso-pharynx from the oro-pharynx, and in co-operation with the base of the tongue the pharynx from the buccal cavity. The posterior raphe of the soft palate is continuous with the uvula, the muscles of which, acting independently, are capable of fixing this 
appendix to the median line against the middle of the posterior wall of the pharynx in such a way as to leave one crescentic aperture on each side. This position becomes necessary in the production or modulation of certain tones, and especially in the formation of certain elements of articulation. The part played by the soft palate in deglutition has already been alluded to. Besides these special uses of the soft palate and uvula another one, which is frequently overlooked, relates to the drainage from the posterior nares. It will be remembered that the posterior surface of the soft palate is convex and is continuous with the floor of the nose, therefore the secretion from the posterior portion of the nasal passages in the normal state flows over this surface, and is guided by the pointed uvula, as well as the free edge of the soft palate, to the posterior wall of the pharynx, whence it flows into the lower pharynx or esophagus, or to the dorsum of the tongue.

Larynx. - This highly important organ, sometimes called the "voice box," is the seat of two important functionsnamely, respiration and phonation. As all respiration must be carried on through its aperture, the rima glottidis, it has been truly designated "the portal of life."

During deglutition the principal function of the epiglottis is to close the aperture of the larynx and thus prevent the ingress of particles of food. Besides its function in deglutition it also has an important part in voice production, acting, so to speak, as a damper to the expiratory blast. Its numerous muciparous glands also serve in lubricating the parts.

The larynx proper, as we have already seen, is partly a cartilaginous and partly a membranous cylinder, provided with four projecting folds, composed of ligamentous and muscular tissue covered by mucous membrane, which lie in an anteroposterior direction one above the other, and are called the ventricular bands and the vocal bands (vocal cords) (Fig. I9). They are attached anteriorly to the receding angle of the thyroid cartilage, and posteriorly to the anterior inferior angle of the movable arytenoid cartilages. The upper part of the larynx is inclosed by a firm ligamentous structure upon which a series of muscular fibres running in different directions are attached. This is called the vestibule of the larynx. The muscular fibres, acting synchronously, constitute a sphincter capable of either closing or enlarging this vestibule of the 
larynx. In respiration both the upper aperture of the larynx and the glottis (rima glottidis), which is bounded by the vocal bands, remain in a passively open state. The movements of the vocal bands being very slight, this function is ordinarily an automatic one.

Voice Production.-This natural reed instrument is one of the wonders of Nature on account of its capacity for the production of the choicest refinement of the musical scale. Of course the larynx should not get the whole credit for the consummation of the artistic voice, inasmuch as the pharynx, nasal and buccal cavities, with their muscular apparatus, are certainly important adjuncts. The steps in voice production are as follows: Primarily an inspiration is taken which thoroughly fills the trachea and larger bronchial tubes. The air contained is thus under control of the respiratory musclesfrequently the diaphragm-the contractions of which are so modified as to keep up the desired pressure on the contained air.

The tone originates through the vibrations of the vocal bands or cords; the sound wave thus started, acting upon the column of air occupying the larynx and trachea is modified first, by the degree of compression to which it is subjected in the laryngeal cavity, and afterward in the pharyngeal and buccal cavities.

Phonation.-The position of the vocal cords in phonation is always one of more or less closure. In emitting the high notes, a mere slit is observed between the vocal cords, the blast of air as it proceeds in its passage becomes amplified or modulated by the physical condition in the upper structures. The deeper or lower tones arise from a more extensive and slower vibration of the vocal cords, while the high tones conversely arise from more rapid vibrations emanating from only the edges of the vocal cords.

The tones of the human voice are divided into registers, called middle and lower registers, and the quality of the human voice is usually classified in the male as the bass, baritone, and tenor, and the female as soprano, mezzo-soprano, and contralto. The essential quality or timbre of the musical voice depends largely upon the anatomical peculiarities of the individual. The use of the human voice for the exposition of the musical scale has been used by men for many ages. According to Chapman the human voice was first used for the rendition of 
music. Since that time all civilized nations have paid a great deal of attention to its artistic cultivation, until at the present time vocal music is one of the glories of mankind, not only in a purely æsthetic sense, but as an admirable element of social education. The wonderful attachments and interlacing of the muscular fibres of the laryngeal muscles render the vocal cords and other apparatus of the organ capable of assuming various shapes and degrees of tension.

It is estimated that the vocal cords of some artists have the power of assuming over two hundred different states of tension.
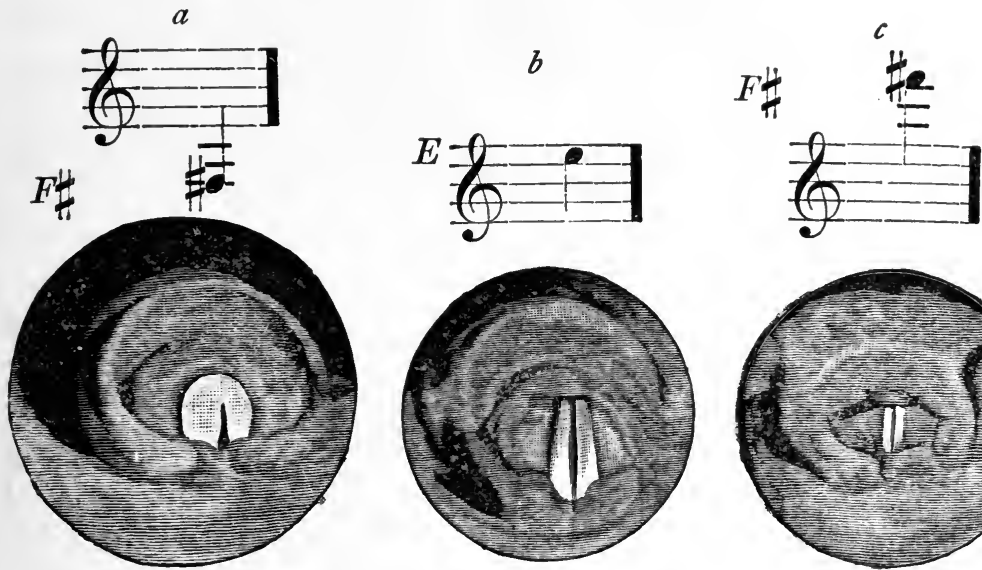

FIGS. 2I $a, b, c$.-Position of vocal cords in taking low, middle, and high notes.

(Burnet.)

The organ is also sometimes capable of anomalous performances resulting in abnormal tones. There are a few cases on record of "whistling larynx." Dr. Mungar (C. E.), of Waterbury, Conn., reports a case of " a woman nineteen years of age with a larynx perfectly normal in appearance and function, who can whistle at any time without the use of lips, tongue, pharynx, soft or hard palate, or cheeks."

The objection that this is not a whistle can not hold, as a proficient singing master has assured me that the sound is purely a whistle, and not a singing or speaking note. This is, then, a unique case of the production of the whistle in the larynx unaided by the lips, tongue, cheeks, or palate.

The falsctto voice in males is chiefly characterized by a high pitch and a thin, piping quality. Its resonance is limited to the 
region of the head, and therefore it lacks volume and strength. There is also a manifest effort in its production, which makes it appear forced and unnatural. Dr. Makuen (G. H.) has reported five cases (Jour. Am. Med. Assoc., March 4, I899).

The range of the musical voice is said to be about two octaves. The voice of the female may be one octave higher than that of the male. The respiratory function of the larynx is automatic, while the vocal function is voluntary. There has been much experimentation and discussion over the enervation of this organ, but space will not permit of a detailed history of the many interesting observations made by Hooper, Donalson, Delavan, Horsely, Semon, Onodi, Lermoyez, and many others. Suffice it to say that there is little doubt concerning a cortical centre which presides over the voluntary or voice function of the larynx, and a bulbar centre which presides over the respiratory or automatic function of the larynx.

Undoubtedly, however, through communicating branches, and by means of insulation of nerve filaments, these two centres may be connected for the purpose of performing a mixed function when such a requirement arises.

A paper read recently by Lermoyez before the Paris Academy of Medicine contains a very complete résumé of the interesting physiological work which has been done in different parts of the world concerning the detection of the cortical centre of the larynx.

Observation has probably established the existence of this independent cortical centre for phonation, and, furthermore, that each centre controls both vocal cords. There is still considerable controversy as to the occurrence of the crossed paralyses of the vocal cords due to cerebral lesions. Experimentation upon dogs shows that the entire brain above the corpora quadrigemina may be removed without impairment of the voice. When the brain is completely divicled at the upper part of the medulla loss of voice ensues. Onodi maintains that these results indicate the existence of a supposed cerebral centre of phonation situated between the root of the vagus and the corpora quadrigemina. The conclusion reached by some that it is impossible to localize even an approximate voice centre is probably incorrect.

W. Kattwinkel (American Journal of the Medical Sciences, April, I898, page 463), after the examination of two hundred cases, concludes: ( I) That the centre for word-forming is in 
Broca's convolution; (2) that the centre for co-ordination of speech (articulation) is chiefly in the third right frontal convolution; (3) both centres are united by association, the fibres passing through the corpora striata; (4) the reflex centre for the pharynx and the larynx is in the corpus striatum, especially on the right side, where the deglutition centre also lies. In acts of coughing and vomiting the soft palate serves the purpose of shutting off the naso-pharynx and guiding the expelled material directly into the buccal cavity. 
THE examination of the upper air passages is embraced by the art of rhinoscopy (anterior, median, and posterior), pharyngoscopy, and laryngoscopy. Like many other arts where the application of previous well-known physical laws was involved, the development of the proper technic was a matter of growth, to which a number of careful observers and experimenters contributed their share. Among those to whom credit is especially due may be mentioned Garcia, Turck, Czermak, and the modern English, German, and American workers in this special field.

Physical Laws involved in Laryngoscopy.-The optical principle involved in laryngoscopy is the law that the angle of reflection is equal to the angle of incidence. The realization and application of this optical law form the basis for the placing of the mirror to obtain views of cavities which are not in the straight line of vision. Owing to the obliquity of the mirror there is a reversal of the image in an antero-posterior direction; structures in the patient which are in reality anterior appear posterior in the mirror, and vice versa. It should be borne in mind that there is no lateral reversal of the image.

Illumination. - In making these examinations one of the first essentials is proper illumination, which may be either direct or reflected. Direct light, as sunlight, or the rays from an electric or other artificial illuminant, may be allowed to shine directly into the nose or throat; the disadvantages are the shadows cast by the observer's head and the inconvenience of easily adjusting the direction of the light rays. Accordingly, a concave mirror with a central opening is usually employed to concentrate and, at the same time, to direct the rays of light into the cavity which we wish to examine. Such a reflector is frequently called the head mirror from the fact of 
its being worn upon the examiner's forehead, and is held in place either by an elastic headband, a spectacle frame, or attached to a rubber support between the teeth (Fig. 22). The exact position of the head mirror is of some importance, as when it is worn upon the forehead the reflected rays are not parallel with the line of vision, although the examiner has no obstacle in the way of binocular sight. If the reflector is worn in front of one eye, the reflected rays become practically parallel with the line of vision; although, if the mirror is not accurately adjusted so that its central opening is in front of the pupil of the eye, monocular vision results with its attendant disadvantages of all objects appearing in the same focal plane.

While sunlight is the most satisfactory illuminant, it is often not at our disposal when most needed, so that some constant and brilliant artificial illuminant is necessary. The oxy-hydrogen or calcium light, while very powerful, is expensive and difficult of manipulation. The electricarc lamp gives such an intense light that its continued use is trying to the eyes. The incandescent electric bulb, while furnish-

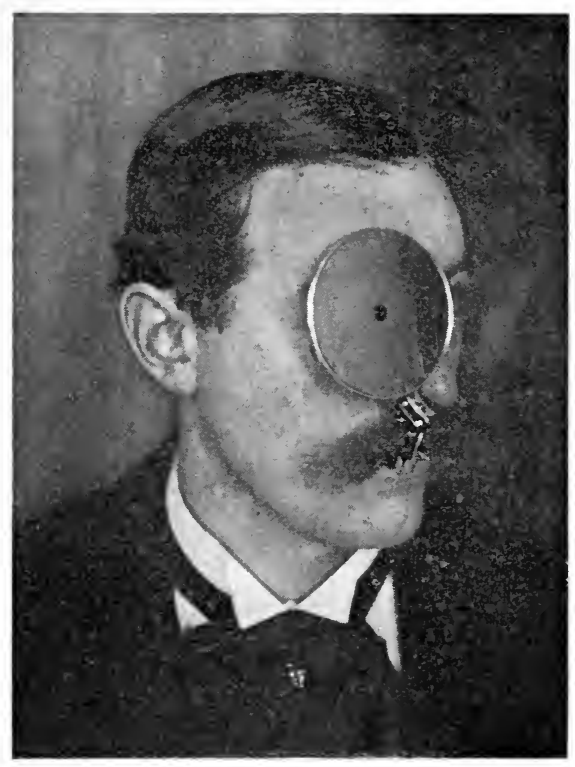

FIG. 22.-Head mirror supported by the teeth. ing a constant light with little heat, has the disadvantage that when its rays are reflected the image of the filament is so prominent that the field of observation is unevenly illuminated. If the globe is frosted the light is more even, although at the same time it loses much of its intensity. If the bulb is inclosed within an ordinary Mackenzie condenser the duration of the carbon filament is short on account of the lack of heat radiation. The lights which are most favoured by laryngologists and in the order of their popularity are the Welsbach, the Argand gas burner, and oil lamps with a circular wick. The burner 
should be easily adjusted as to height, being mounted upon either an adjustable wall bracket or upon a short stand placed upon the operator's table, or upon a taller stand resting upon the floor. The extraneous rays may be cut off and the intensity of the light increased by inclosing the burner within a metal hood provided with a reflector in the back and a plano-convex lens in front. Acetylene gas, recently discovered, furnishes a most intense and easily controlled light, quite satisfactory for these examinations, and seems destined to become quite popular.

Instruments.-In the examination of the anterior nares some form of nasal speculum should be employed to open the vestibular space as widely as possible; of these there is an immense variety. The one

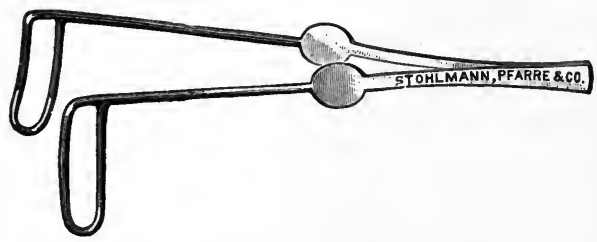

FIG. 23.-Bosworth's nasal speculum.

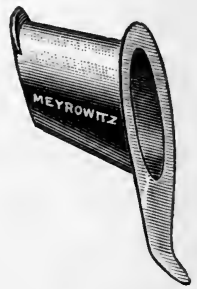

Fig. 24.-Simrock's nasal speculum.

devised by Bosworth is perhaps the simplest. Other specula are Goodwillie's, De Vilbliss's, Frankel's, and Simrock's, and the nasal retractors of Jurasz modified by Dr. Wadsworth Warren. For nasal operations some simple form of self-retaining speculum is advantageous. For cautery operations upon the inferior turbinate the author's speculum with an ivory shield may be used.

Many instruments for depressing the tongue during an examination of the pharynx have been devised; the Fig. 25.-De Vilbliss's selfprinciple in all these is the same: that retaining nasal speculum. of a flat surface, either smooth or roughened, attached to a handle which is bent so as to be out of the line of vision. The simpler the instrument the less likely is it to alarm a timid patient; of the metal depressors, those devised by Miner, Bosworth, and the author serve the purpose very well (see Figs. 26 and 27). Tongue depressors made of glass have the advantage that they can be rendered absolutely aseptic; they look 
clean and bright; they have the disadvantage of not being shaped as easily as the ordinary metal one, so that the handles

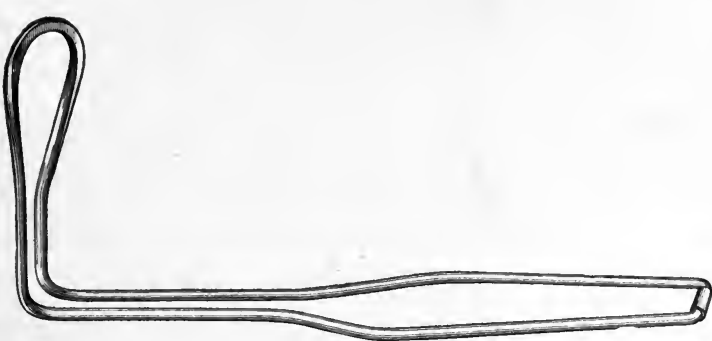

Fig. 26.-De Vilbliss's tongue depressor.

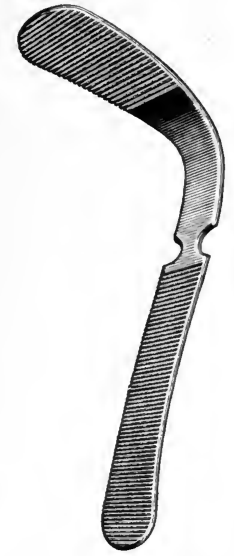

FIG. 27.-Shurly's tongue depressor.

are not so convenient. The wooden spatula is preferred by some since its cheapness permits of its being discarded after one usage. When the patient is a child the tongue depressor should be smaller than the one used for an adult.

The rhinoscopic mirror is comparatively small, being from three eighths to three quarters of an inch in diameter and attached to the stem at an angle of $105^{\circ}$ to $130^{\circ}$. Different sizes should be provided, as the space between the uvula and posterior wall of the pharynx varies in different individuals. Rhinoscopic mirrors have been devised so that the angle which the mirror forms with the stem can be adjusted while the instrument is in position; however, the examiner can easily bend the ordinary instrument so as to adapt it to exceptional cases. The rhinoscopic mirror should be of the best quality, with as brilliant a reflecting surface as possible so that no rays of light may be lost through any imperfection of the instrument. The laryngeal mirrors should also be of different sizes so as to admit of the choice of one as large as possible for each particular case. The circular form is the one most usually employed, although it is convenient to introduce an oval mirror when there is a marked hypertrophy of the tonsils. The angle which the laryngoscopic mirror makes with its stem should be about $120^{\circ}$. These different mirrors can all be used with one adjustable metal handle. 
Anterior Rhinoscopy.-In the examination of the anterior nares the examiner should be seated in front of the patient and the source of light should be adjusted to about the level of the patient's ear. Better results will be

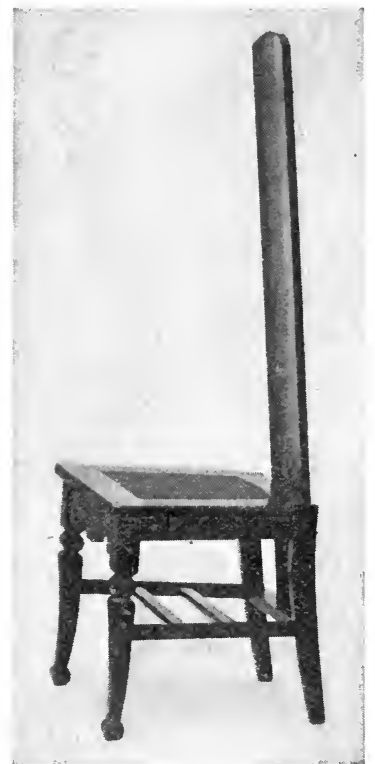

FIG. 28.-Chair for patient in laryngoscopy. obtained if all extraneous light is excluded and the room darkened. The patient should be seated in a chair whose back is straight or even tilted slightly forward (Fig. 28). The examiner may be seated upon a stool, as an ordinary piano stool, which can be raised or lowered. The examiner having assumed an easy, comfortable position, should adjust the head mirror either in front of one eye or on his forehead so that the rays of light will fall upon the patient's upper lip (Fig. 29). The distance of the mirror from the patient's head should be regulated according to its focal length so that the spot of light may be as intense as possible. If the reflector is worn in front of the eye it should be on the side on which the light is placed. After the mirror is once adjusted the examiner's head should remain in about the same position, the position of the patient being changed according to necessity. The opening of the anterior nares should be carefully inspected, excoriations and fissures looked for, while at the same time notice should be taken whether respiration is nasal or buccal. The operator places his first and second fingers upon the patient's forehead and raises the tip of the nose slightly with the thumb while the speculum held between the thumb and the first finger of the other hand is introduced. The hand holding the speculum may be steadied by resting the little finger upon the patient's cheek. Care should be taken that the instrument does not come in contact with the bony parts, as this procedure would occasion considerable discomfort to the examined; also too forcible dilatation, particularly in children, should be avoided, as fissures in the integument are easily produced and are difficult to heal. Gentleness in this 
examination will do much to inspire the confidence of the patient for the more difficult posterior rhinoscopy and laryngoscopy. In this position we can view the anterior inferior part of the septum, the inferior meatus, and the inferior turbinal. By tilting the patient's head slightly backward, the middle meatus and the middle turbinal, with the opposite part of the septum, are exposed. The presence or absence of secretion, the colour of the mucous membrane over the turbinals and the septum, the contour of the septum, the presence or absence of spurs or ridges upon the septal wall, and the roominess of the nasal cavity should be quickly noted. The other nostril is next to be examined in the same manner. If the meatuses are obstructed by secretion, either moist or dry, or their space materially diminished by a swollen membrane, a cleansing spray may be used, followed, if necessary, by a two-per-cent solution of cocaine. The solution of cocaine, however, should not be used before the posterior nares are examined, since, owing to its ischæmic effect, posterior hypertrophies might be unnoticed.

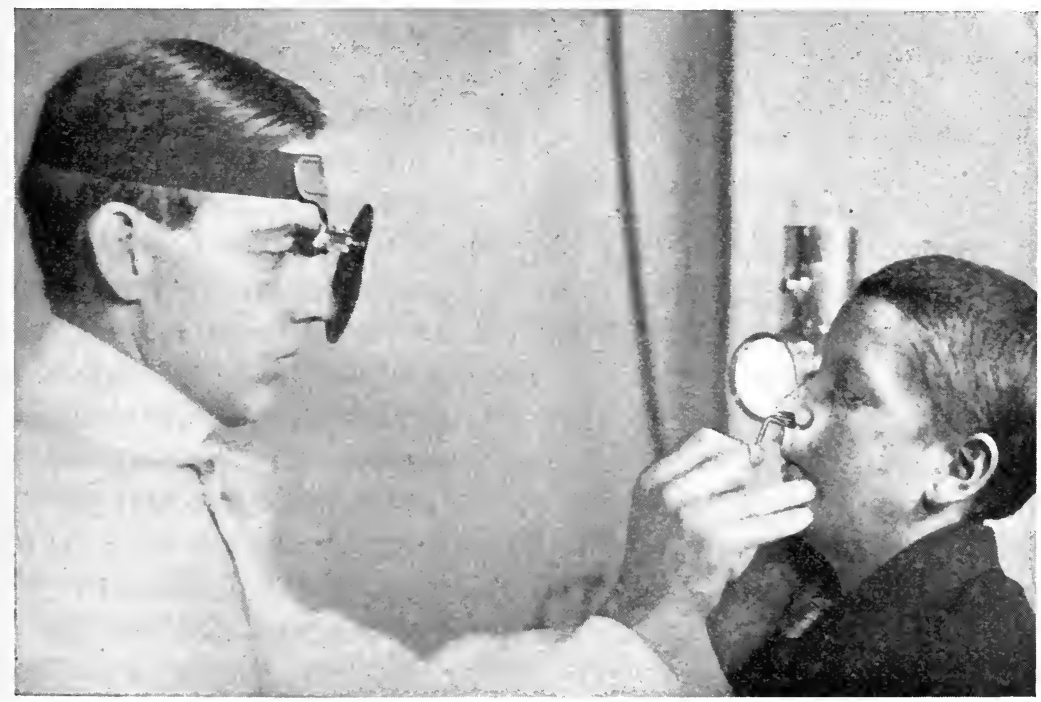

FIG. 29.-Position for anterior rhinoscopy.

Median Rhinoscopy.-This term is applied to an examination of the nares by a small mirror introduced along the floor of the nose. This procedure is limited to those cases where the nasal cavity is very spacious. 
Pharyngoscopy. - The examination of the pharynx should be preceded by a thorough inspection of the lips, gums, teeth, hard palate, and tongue. While a satisfactory view of the structures of the pharynx can be obtained without the aid of any device for depressing the base of the tongue in those individuals, particularly singers, who have well-trained throats, yet the use of some form of tongue depressor is necessary in most cases. The technic of the examination of the pharynx is as follows: The patient having been directed to open the mouth

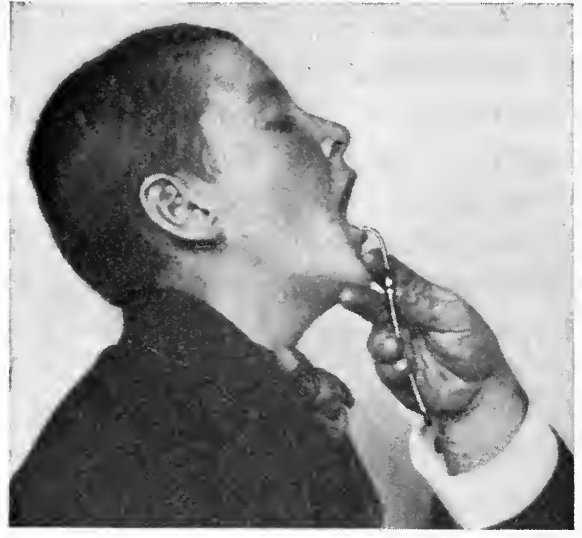

FIG. 30.-Pharyngoscopy. moderately wide, without protruding the tongue, the examiner holding the tongue depressor between the thumb and first finger introduces the instrument, slightly warmed, and with gentle pressure depresses the base of the tongue while the first and second finger steadies the patient's chin (Fig. 30). By this latter procedure the angle of view can be readily altered. Attention should be given that the tongue depressor is not introduced too far and that it does not touch the circumvallate papillæ. If the instrument is not placed in the median line, one side of the tongue will be depressed while the other side will roll up and obscure the view. Depression should not be quick or violent, as a mild, persistent pressure soon tires the muscles of the tongue so that it will lie flat without much danger of inducing nausea. The handle of the depressor should be pulled forward so as to press downward the dorsum of the tongue. By observing these minutiæ the examiner can satisfactorily bring into view the soft palate, uvula, anterior pillars, tonsils, posterior pillars, and the posterior wall of the pharynx. In dealing with children if gentle measures are not successful the patient may be held by the parent or assistant.

Posterior Rhinoscopy.-After the examination of the pharynx, the naso-pharynx may be inspected by the aid of the tongue depressor and the rhinoscopic mirror. The mirror 
selected should be proportioned to the size of the space between the uvula and the posterior wall of the pharynx; it will be found advisable to employ as large a mirror as can be easily introduced and one whose reflecting surface is as bright as possible. Before its introduction the mirror should be warmed by passing it slowly over the flame of the lamp, withdrawing it instantly on the disappearance of the film of moisture which forms on its first heating; the temperature can be tested by touching the back of the mirror against the back of the hand. The tongue should be depressed and the mirror, held between the thumb and the first and second finger, as one would a pen, with the reflecting surface upward, is passed quickly and steadily backward with the shank of the mirror in the angle of the mouth. The mirror should pass between the uvula and one of the anterior pillars until it is posterior to an imaginary line dropped from the lower border of the palate. The head mirror should be so placed that the rays of light are concentrated just below the uvula. The mirror should not touch any part of the throat, either in its introduction or after it is placed, as this will in most patients induce retching at once. As the reflecting surface is small, it is evident that all of the postnasal structures can not be seen at one glance; accordingly, the handle should be manipulated so that all of the parts are brought into view in suc. cession (see Fig. 3I). It is well to adopt some definite plan that will insure a systematic observation. The Eustachian orifice of one side may be used as a starting point, inspecting next the opening of one naris, the posterior end of the septum, the opening of the opposite naris, the other Eustachian tube, and finally the vault of the pharynx. The re-

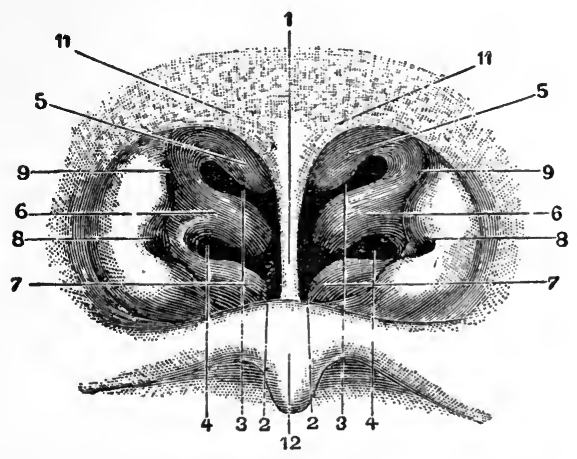

FIG. 3I.-Rhinoscopic image. I, vomer or nasal septum; 2, floor of nose; 3, superior meatus ; 4 , middle meatus ; 5 , superior turbinated bone; 6 , middle turbinated bone; 7 , inferior turbinated bone: 8 , pharyngeal orifice of Eustachian tube; 8, upper portion of Rosenmüller's groove ; II, glandular tissue at the anterior portion of vault of pharynx; I2, posterior surface of velum (Seiler).

$$
\text { stachian tube, and finally the vault of the pharynx. The re- }
$$


flection of the posterior nares will usually show the posterior end of the inferior turbinated body, the middle turbinal, and all or part of the superior turbinal. In case the uvula is so dependent that only one of the choanæ can be seen, the mirror can be withdrawn, and changing with the tongue depressor, introduced on the opposite side of the uvula. During this examination it is necessary that the soft palate be relaxed. Many persons, when the tongue is depressed, involuntarily draw up the soft palate and breathe through the mouth. These individuals, if unable through their own volition to breathe through the nose, should be asked to pronounce some short word ending with a nasal sound, as "hang." If this fails, the patient may be directed to breathe as rapidly as possible so that his attention may be concentrated on the rapidity of his respiration rather than on the examination.

The appearance of the orifice of the Eustachian tube is somewhat funnel-shaped, and its projecting rim is lighter than the surrounding membrane. Between this opening and the posterior wall of the pharynx is the depression known as the fossa of Rosenmiuller. The septum will appear broad above, gradually narrowing as it approaches the floor of the nose. The nasal passages appear as dark spaces with the turbinals projecting into them. The colour of these latter is that of a pale red, the superior turbinal appearing lightest of all. The vault of the pharynx presents a rounded appearance of palered.colour.

Obstacles to Posterior Rhinoscopy. - The obstacles to a successful examination of the naso-pharynx are timidity of the patient, extreme irritability of the pharynx when any instrument is introduced, lack of steadiness on the part of the examiner, and narrowness of the space behind the uvula. The fears of the patient may be allayed by keeping the mirror in his mouth for only a few seconds at a time and diverting his attention during the intervals. The irritability of the pharynx may be diminished by spraying with a four-per-cent solution of cocaine. In some cases it may be necessary for the patient to present himself on several occasions before a satisfactory inspection of the naso-pharynx can be made. The mirror should be withdrawn at once on the least suspicion of gagging, as after the pharyngeal muscles have once contracted spasmodically their irritability is increased for a time. If the 
examiner does not place the mirror promptly and steadily in its proper position, the difficulties of examination are materially increased. In fact, there is no examination of the upper air passages where the technic is more difficult or long practice more necessary than in posterior rhinoscopy. The number of failures becomes less as the examiner becomes more experienced; however, there are cases-perhaps about five per cent at least-in which this examination can not be made. Where the space behind the uvula is small, or where it is desirable to obtain more room for operative purposes, recourse may be had to some of the devices for drawing the palate forward. This may be accoinplished (with cocainization, if necessary) by means of a curved hook, by the White's palate retractor,. or. by means of the elastic tapes. The method of introduction of the White's palate retractor may be summarized as follows: After depressing the tongue the curved hook is introduced behind the palate so that the uvula falls within the wire loop; the two small wire loops are introduced one within each nostril (the necessary curve being obtained by bending the wires for each individual

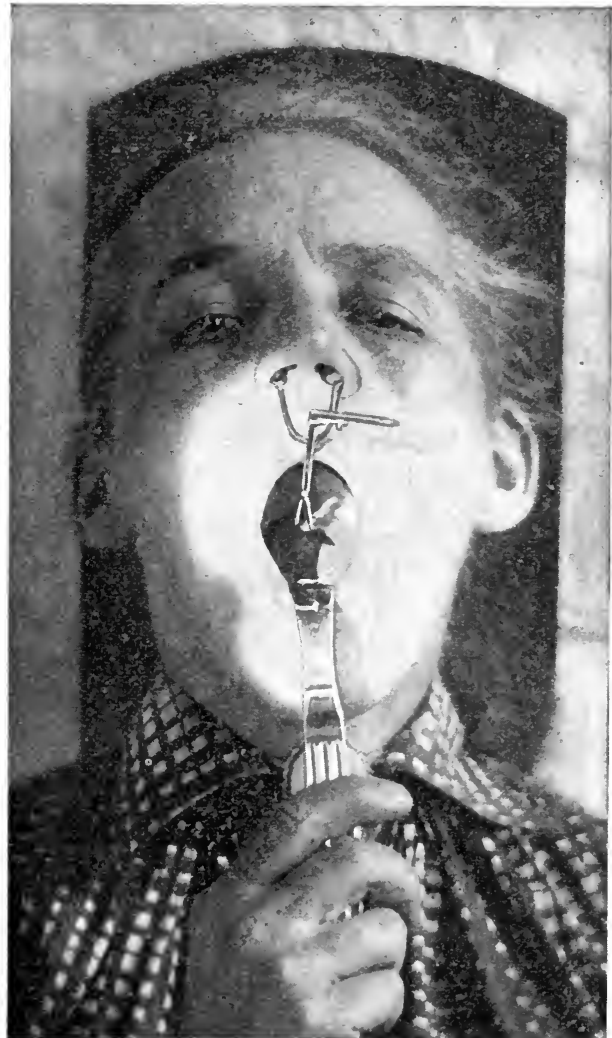

FIG. 32.-White's palate retractor in position. case), the stem of the instrument drawn forward until the palate shows a distinct resistance, after which the small thumbscrew is tightened. The objection to the White's palate retractor is that the patient is unable to swallow when the instrument is in position (Fig. 32). 
The use of the elastic tapes obviates this difficulty. A narrow, flat piece of silk elastic tape, after being thinly coated with vaseline, is introduced through each nostril by means of a soft rubber catheter and withdrawn through the mouth, the uvula lying between the two tapes. The two ends can now be fastened with a small clip after the proper tension has been secured. The tapes, acting as foreign bodies, often produce an excessive flow of mucus which obstructs the view and is most annoying to the patient.

Digital Examination of the Naso-pharynx.-In children, where the obstacles to examination with the mirror are insurmountable, and it is necessary to determine the presence or absence of adenoids, recourse may be had to examination by the finger. If this is made, as it most generally is, without an anæsthetic, the child should be held and a mouth gag introduced, preferably O'Dwyer's, or one can be improvised from a piece of wood or cork. The index finger, the palm of the hand being upward, is passed quickly backward until it meets the posterior pillar, when it can be gently insinuated behind the soft palate. The finger tip is pressed upward and forward until it comes in contact with the septum, which is recognised by its knifelike feel. On either side can be felt the openings of the posterior nares and the condition of the membrane covering the posterior ends of the turbinals. Luschka's tonsil will be felt as a soft cushion in the vault of the pharynx, and the extent and character of any enlargement can

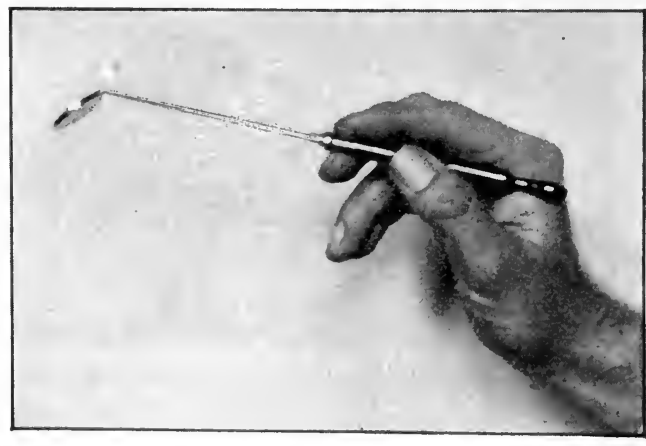

FIG. 33.-Method of holding laryngeal mirror. be accurately noted. The orifices of the Eustachian tubes will be recognised as small, hard, circular projections, and the space immediately surrounding them should be explored to determine the presence or absence of hypertrophied glandular tissue.

Laryngoscopy.-The examination of the larynx is ordinarily made by the laryngoscope, which consists in its simplest 
form of a small mirror mounted upon a handle; the term, how. ever, is usually applied to the throat mirror and concave reflector. The technic of laryngoscopy is as follows: The patient and examiner should be seated, as in the preceding examinations. In order to obtain as much room as possible in the laryngo-pharynx, the tongue is drawn forward by means of a napkin held between the examiner's thumb and forefinger. In this manœuvre the tongue should be supported, or, as it were, rolled over the forefinger, to prevent the frenum from rubbing against the incisor teeth. The laryngeal mirror, held like a pen (Fig. 33), with the reflecting surface parallel to the tongue, should be passed into the oral cavity, and press the uvula up and backward; the handle of the mirror is slightly moved to one side so as to be out of the line of direct vision and slightly depressed. It will be found advantageous to have the patient's head inclined a little backward. The spot of light should be focused as accurately as possible on the throat mirror. Unlike posterior rhinoscopy, if the mirror is large and correctly placed and lighted, the entire laryngeal image can be seen at once. Upon the proper placing of the mirror, upon the maintenance of the proper angle of reflection

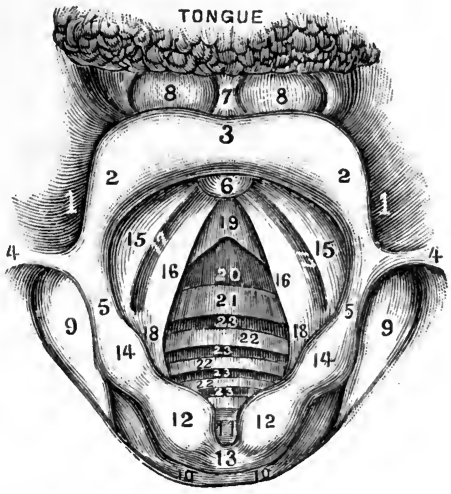

FIG. 34.-Enlarged sketch of normal laryngoscopic image in respiration, slightly forced to exhibit the chief cardinal points of observation in detail. I, lingual surface of epiglottis; 2, laryngeal surface of epiglottis; 3, indented crest of epiglottis ; 4, pharyngo-epiglottic fold; 5, ary-epiglottic fold ; 6 , pad of epiglottis; 7 , glosso-epiglottic ligament ; 8 , lingual sinus; 9 , pharyngo-laryngeal or pyriform sinus ; I0, posterior laryngeal wall, terminating in a line representing the entrance into the œsophagus; II, inter-arytenoid incisure; 12 , supraarytenoid cartilage (Santorini) ; 13 , inter-arytenoid fold ; 14 , cuneiform cartilage (Wrisberg); 15 , ventricular band; 16, vocal cord; I7, ventricle (Morgagni); I8, posterior vocal process ; 19 , thyroid cartilage; 20 , crico-thyroid membrane; 2I, cricoid cartilage; 22, rings of trachea; 23, interspaces between the cartilages of the trachea (Cohen).

depend the success of the examination (Fig. 34). The most prominent object will be the vocal cords, which in their normal condition stand out as two white glistening bands extending antero-posteriorly. In front of them, or, as it will appear in the 
mirror, behind them, will be seen the epiglottis extending upward like a leaf, while in the front of the mirror will appear the arytenoid cartilages. Just above the vocal cords and to either side of them are the ventricular bands, somewhat reddish in
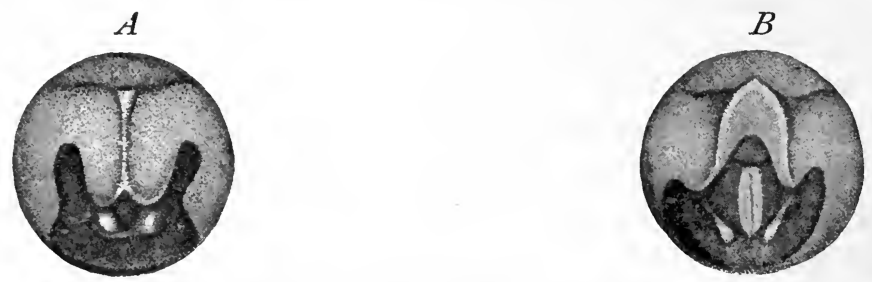

FIG. 35.- $A$, larynx of a child in quiet breathing, nearly covered by the epiglottis; $B$, the same during phonation, with the epiglottis raised and the cords in apposition.

colour. Between these bands and the true cords are the spaces known as the ventricles of the larynx, apparently quite small, yet in reality comparatively roomy. The cartilages of Wrisberg and Santorini show as two nodules on each side of the inter-
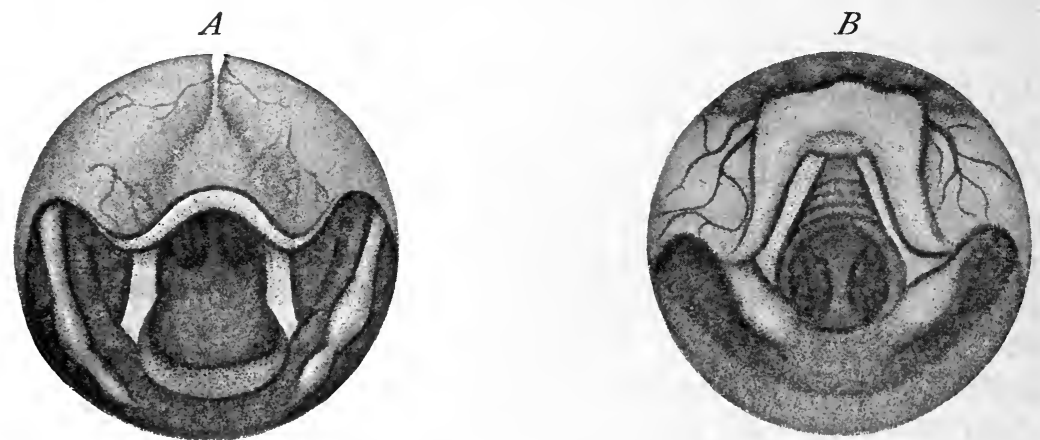

FIG. 36.- $A$, adult larynx and trachea to the bifurcation in deep inspiration, showing the back wall by Kilian's method, with the chin low and the head inclined forward; $B$, usual view of the normal larynx and front wall of the trachea to the bifurcation. (From Krieg's Atlas, with slight modifications.)

arytenoid space; external to these will be the pyriform sinuses, while on either side of the frenum of the epiglottis and adjacent to the tongue will be found the lingual sinuses.

Auto-laryngoscopy.-The difficulties of laryngoscopy are best appreciated, and the practice necessary for the acquirement of a good technic obtained by the observer examining his own larynx. A small plain mirror, mounted upon a suitable support, is required. The light may be allowed to shine from a window into 
the throat, or the concave reflector can be employed, in which case it should be mounted alongside of the plain mirror (see Fig. 37).

Obstacles to Laryngoscopy.-If the mirror has been incorrectly placed against the posterior wall of the pharynx, without pressing the uvula up and backward, the image in the mirror will include only the anterior half of the larynx. The chief obstacle to laryngoscopy is, perhaps, lack of steadiness in the manipulation of the mirror, and the desire to view the image too long. If the patient shows any signs of gagging the mirror should be withdrawn at once. If the pharynx seems irritable it may be sprayed with a solution of cocaine, or the solution applied with a pledget of cotton on an applicator. However, the use of cocaine has the disadvantage of rendering the parts ischrmic, so that the true tints of the picture are lost. If the tonsils are enlarged an oval mirror may be employed, though in cases of extreme enlargement excision may have to be em-

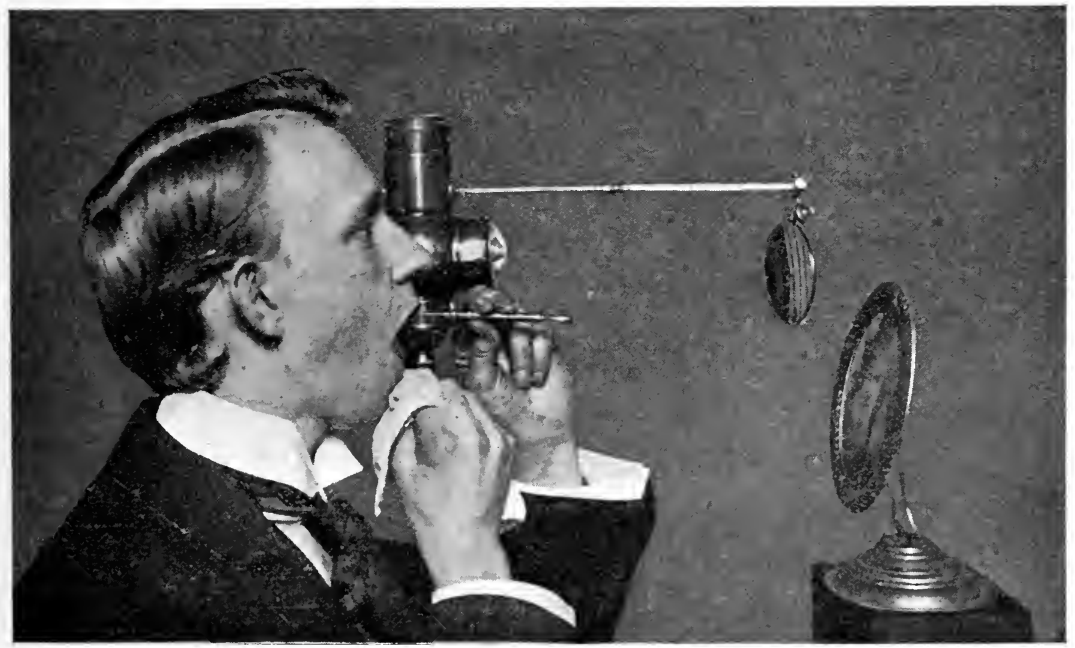

FIG. 37.-Auto-laryngoscopy.

ployed before a satisfactory view can be obtained. If the base of the tongue persistently obstructs the view, the patient himself may hold the tip forward with the napkin while the examiner introduces a small tongue depressor with the left hand. Indeed, in all cases where topical applications are necessary, the patient should be taught how to hold his own tongue forward. 
Attempts at examination should not be prolonged so as to fatigue the examined. The habit of making, as it were, a mental photograph of the laryngeal image should be formed so that the instruments are held in place as short a space of time as possible. While many cases will be encountered in which the examination seems at first almost impossible, yet it is a well-established fact that after a little training the throat becomes very tolerant of instruments, especially of the laryngeal mirror.

Where it is desirable to investigate thoroughly the posterior wali of the larynx the method of Kilian may be employed, which will be understood from Fig. 38 .

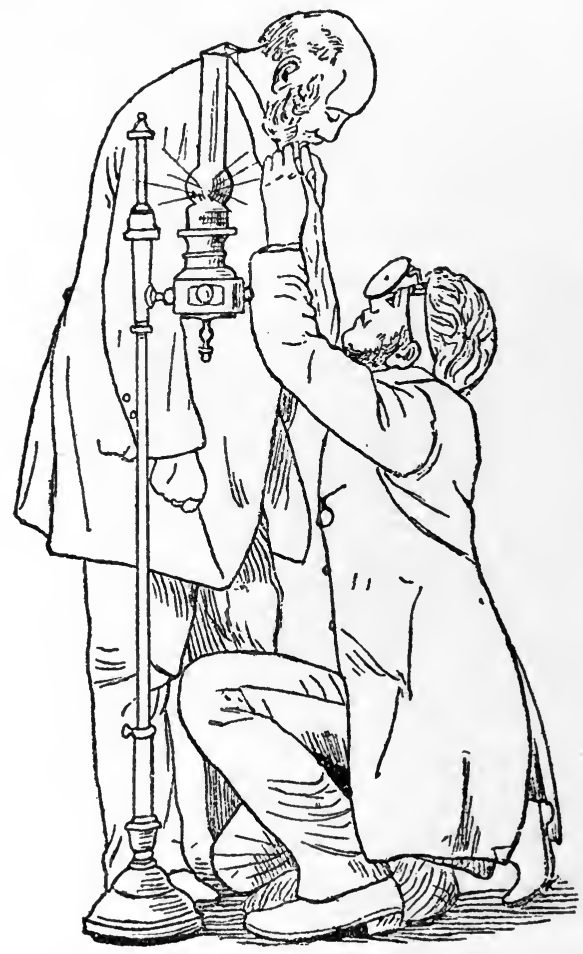

FIG. 38.-Kilian's method of examining the posterior wall of the larynx.

Autoscopy, or direct examination of the larynx without a mirror by forcible depression of the tongue, has been advocated and developed by Dr. Kirstein, of Berlin. The autoscope consists of a spatula, a hood and handle. The patient leans forward with the head strongly flexed backward, the examiner stands up and depresses the tongue forcibly with the spatula, which engages 
the epiglottis. Illumination can be obtained by reflection of the light, or from a prism attached to the handle and a small electric lamp. In those patients where this art can be practised there is certainly an advantage to be gained, as the posterior part of the larynx can be well demonstrated. Autoscopy, however, has not been widely adopted by the laryngologists of this country, and

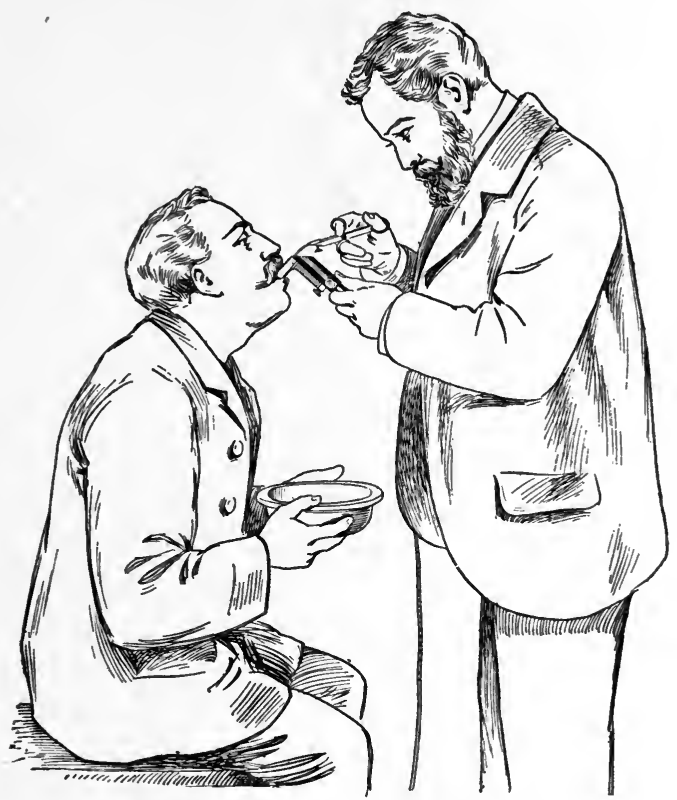

Fig. 39.-Position for autoscopy.

whether its application admits of the extensive use which its advocates claim is still to be demonstrated. In the examination of children under an anæsthetic it certainly offers some advantages over laryngoscopy (Fig. 39).

The use of the Röntgen rays, in laryngeal exploration for the detection of metallic or bony substances, can be employed where the attendant inflammation and irritability are so great as to interfere with the use of the laryngeal mirror. The position of the intubation tube has been well demonstrated by both skiagraphs and the fluorescope. 
Acute rhinitis, acute coryza, acute nasal catarrh, or acute intumescent rhinitis, as its name implies, is an acute inflammation of the Schneiderian mucous membrane. It is one of the most common diseases of the north temperate zone, and is not infrequently met with even in the tropics.

Pathology.--The pathological changes are usually confined for the most part to the Schneiderian portion of the nasal chambers, although in severe cases the upper or olfactory region may be involved. These changes are usually of a transitory character, the first step being an intense fluxion of blood into the turbinated plexuses, which, through pressure upon the peripheral nerve endings and the subsequent vasomotor disturbance, involve the whole vascular apparatus of the nasal passages, including at times even the lining membrane of the side cavities, such as the ethmoidal cells, the sphenoidal sinus, and the antrum of Highmore. Moreover, an extension of the inflammatory process may take place into the naso-pharynx, Eustachian tubes, or even into the pharyngo-laryngeal region. The secondary pathological effects consist of more or less œedema, hyperplasia, and deposition of inflammatory, exudative products. Glasgow, of St. Louis, first called attention to an endemic of acute rhinitis, characterized by an œdematous swelling of the turbinals. Suppuration rarely occurs unless the disease remains active for some time in one of the accessory cavities of the nasal passages. The secretion of the mucous membrane is at first abrogated, but it soon reappears in one or two formsfirst, as an acrid watery discharge, then afterward as a mucous or modified muco-purulent discharge. When the middle turbinated body is particularly involved, thus closing the infundibulum, it will be found that the secretion will contain a larger proportion of pus. On the other hand, if the superior channels 
are particularly affected, the secretion will be of a more serous or watery nature. This is especially so in those cases where the nervous apparatus seems to bear the brunt of the disease. In such an event it will be found that the vascularity and the secretion of the part are variable from hour to hour, while the extreme sensitiveness of the mucous membrane and even of the external nose may continue in a marked degree. The secretion usually consists of serum, lymph, mucin, pus cells, red and white blood-corpuscles, salts of soda, and potash.

Etiology.-The etiology of acute rhinitis may be considered under two heads: First, predisposing causes, and, second, exciting causes. Among the well-recognised predisposing causes of acute rhinitis we will mention first the unsanitary condition of the body, a thing which may be considered, apart from diathesis, as the most important factor in its causation. Many persons, from time to time, have exposed themselves to given exciting causes when the bodily condition was "at or above par" without having suffered from any so-called "cold." Whereas, under similar conditions of exposure with the bodily functions out of harmony, an active rhinitis or some other form of "cold" rapidly supervened. This experience is a matter of such common observation among the profession that it is now a well-recognised element of etiology. Another important factor is that of diathesis, which in some individuals is an inherited, in others an acquired trait. Writers upon this subject have a tendency to pass over this point as unimportant, but when one studies comparatively a large number of cases of this form of disease, this point appears too important to be dismissed without proper consideration. There are families in every community that are sure to have several attacks annually of acute rhinitis, notwithstanding their careful avoidance of every known exciting cause. There are also individuals who display this same predilection in spite of the most carefully regulated habits and regimen. From such clinical observations, which might be enumerated by the thousand, the conclusion can not be avoided that individuality plays an important rôle in this disease; nor can it be accounted for by the existence of permanent lesions of the nasal chambers or adenoids, as some authors avow, since there are many thousand cases occurring where marked permanent lesions of the nose do not exist.

Any occupation which exposes the individual to frequent 
consecutive changes of temperature, or to dust-laden atmosphere, to ammoniacal or other irritating fumes, or necessitates the wetting of the feet or other parts of the body, may become a powerful predisponant. In this connection might be mentioned butchers, grocers, workers in laboratories, machine shops, foundries, ice houses, etc. Exceptionally, it is curious to note, that not all such artisans are especially subject to attacks of acute rhinitis. The practitioner will meet with many such persons who are not usually "subject to colds," excepting during epidemics.

Next, and of greatest importance according to some authors, are the chronic conditions and deformities of the nasal passages, or the presence of enlarged adenoid tissue in the vault of the pharynx.

The frequency of recurring "colds in the head" in subjects of adenoid enlargement and chronic deformities of the nasal passages is certainly a matter of common observation, especially in children.

Sex plays a somewhat important part in the etiology, as it has been observed that males suffer more than females from this disorder.

No age is exempt, although youth and adolescence seem to be the most vulnerable periods of life. In nurslings this disease is especially distressing and dangerous, because of the tend. ency to catarrhal pneumonia, and the inability of the little one to get sufficient nourishment on account of the nasal stenosis. Contrary to expectation, habitual snuff-takers do not seem to be especially predisposed to the disease. This fact probably depends upon the further one that the continual mechanical and chemical effect of the tobacco upon the peripheral nerves produces after a time a permanent obtundity of sensibility and a permanent loss of elasticity in the capillaries and veins.

The most commonly recognized exciting cause of acute rhinitis is the exposure of the body in whole or part to exacerbations of heat and cold-in other words, what is commonly known as "taking cold." Among the many theories advanced in explanation of this process there is one point which is common to them all, and which meets with general acceptancenamely, that there exists an intermediate functional relation between the skin and mucous membranes. The morphological similarity between the mucous membranes and the skin has been well stated by one of the old authors, who denominated 
the mucous membrane as the "internal skin of the body." Since the vascularity of both is regulated by the same vasomotor mechanism, the close relationship must be obvious. Among the many important functions of the skin, as is well known, is the one working co-ordinately with the complex one known as heat production. Thus, heat regulation is largely due to heat radiation taking place through the skin. Hence, any break of equilibrium between the two will be apt to produce vascular derangement and congestions. Besides this, the emunctory function of the skin,

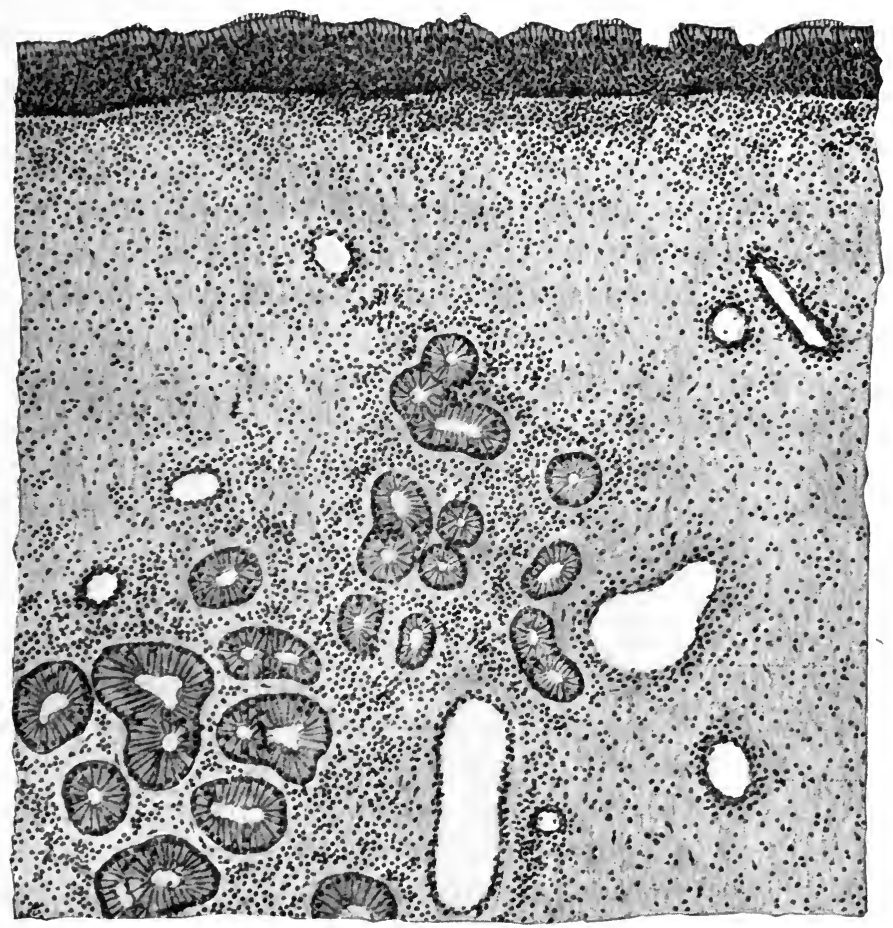

Fig. 39a.-Acute catarrhal rhinitis (Seifert and Kahn), showing erosion of columnar epithelium and infiltration of submucosa with serum and leucocytes.

becoming deranged, may lock up in the body excrementitious substances which thereby become deleterious. A rhinitis is often a local expression of systemic disease, such as influenza, malarial toxæmia, etc. A rhinitis may even pursue a remittent or intermittent course in conformity with the nature of the poison.

Exposure to overheated air, or to air laden with noxious gases, or to air which has been deprived of its proper proportion of oxygen, is a frequent cause of acute rhinitis. A num- 
ber of such cases will follow after an assembly of persons in illventilated public rooms.

Overheated houses and offices are the sources in many cases of this disease, as is also the imperfect distribution of the air of a room or office. Many of the subjects of acute rhinitis will be found among those who sit or sleep close to a window, so that the chilled air by its gravity will flow over the head or neck. If pains be taken to examine with a thermometer the temperature of the air at the floor of a room and at four or five feet from the floor, one will be surprised to discover a difference in the temperature of from five to ten degrees, and sometimes more between the two points. This is especially true of rooms heated by stoves. It is therefore no wonder that persons occupying such rooms, and engaged in some sedentary occupation therein, are the subjects of frequent attacks of acute rhinitis. Inmates of houses or apartments heated by natural gas, as supplied to open grates, or by kerosene or naphtha stoves, are thereby predisposed to attack, unless due care be taken to secure proper ventilation and diffusion of fresh air. From time to time it has been asserted that ordinary acute rbinitis is due to the impact or lodgment of some specific germ or epiphyte, rhizopod, spirillum, or coccus, upon the nasal mucous membrane. This dictum has never been sufficiently verified to stand the test of close observation and investigation, however, although there are some writers who still believe that the initial process is largely dependent upon the mutations in loco of some of the multitudinous aerial micro-organisms.

Casselberry believes that acute rhinitis is an affection caused by pathogenic micro-organisms, and is the result of congestion of the nasal passages occasioned by taking a cold, which evidently favours a microbic invasion of the mucous membrane. He also believes in the direct infection from one child or infant to another by the common use of handkerchiefs and similar articles.

Symptomatology.-One of the earliest symptoms of acute rhinitis consists of malaise, with more or less cephalalgia, myalgia, and chilliness, rarely amounting to a well-marked rigour. This is followed by a rise of temperature of one or two degrees. Concurrently there is a distressing sense of fulness in the region of the nose, forehead, and Eustachian tubes. The 
nasal mucous membrane, upon inspection, appears extremely hyperæmic, while the turbinated bodies are fully distended, completely blocking the nasal passages. For the first few hours - perhaps for the first day-there is little or no secretion, but soon there flows from the nose an acrid, serous discharge which speedily excoriates the skin about the alæ. During this period there is often much photophobia, with an increase in the secretions of the lachrymal apparatus. The discharge from the nose soon changes from a serous to a mucous character, becoming at times muco-purulent, especially if the accessory sinuses are involved. The stenosis of the nasal passages is gradually relieved, or paroxysmally relieved by the activity of the vessels of the mucous membrane.

The active course of an attack rarely lasts longer than three or four days, if the patient remains indoors; but if he continues his vocation or usual daily régime, the duration of the attack may be very much prolonged, and, as a consequence, a subacute inflammation may remain for a considerable period. In this way consecutive acute attacks will be invited, which in the course of a year or so may lead to permanent hyperplasia of the turbinated bodies, constituting a chronic hypertrophic rhinitis. The constitutional disturbance is generally slight, except for twenty-four hours; but in debilitated persons and in young children the pyrexia may last from one to three weeks, on and off; there may be involvement of the cervical lymphatic glands and ear, although such events are uncommon in ordinary non-infectious rhinitis.

Treatment.-The patient should be kept indoors if his temperature be elevated, or if he have much cephalalgia, especially if the weather be at all inclement. Indeed, rest in a warm, well-ventilated room, with light liquid diet for from twenty to forty-eight hours, is often all the treatment necessary. However, at the discretion of the physician, a saline cathartic may be administered, and this followed by small doses of phenacetine or tincture of aconite root (one minim every hour for an adult), together with a hot drink and warm pediluvia at night. Dr. J. Solis-Cohen mentions, as efficient treatment for cutting short such an attack of rhinitis, the administration of several large draughts of moderately cold water during the evening while the patient is well covered up in bed. This proceeding often results in an effectual diaphoresis which may relieve the 
engorged mucous membrane. For the relief of the cephalalgia or the photophobia one or two extra doses of phenacetine may be advisable, or a dose of sulphonal (five to ten grains).

The great majority of cases of acute rhinitis do not come under the care of the physician unless the distress be somewhat unusual or protracted, such as neuralgia or some complication involving the ear, because the laity, generally recognising the absence of danger and the self-limited character of the attack, are prone to rely on domestic remedies or the kindly offices of the nostrum vender. In addition, there exists a sentiment among men that apprehension concerning an attack of ordinary acute coryza betrays a weakness of disposition. I believe, however, that the medical adviser should direct the attention of people to the necessity of modifying their daily life at once and undergoing treatment for the purpose of mitigating the course of the disease. This is especially applicable to those who have deformities of the nasal passages. Without a doubt neglected attacks of acute coryza are the most prolific cause of the various chronic disorders which affect this region. Whether serious deformity is also thus induced is rather questionable.

For rheumatic subjects it will be found that the salicylates are more effectual than quinine or cinchonidia for promoting the general tone of the body. For acute coryza, occurring in malarial districts, the early administration of alterative doses of calomel, followed by full doses of quinine, will be found necessary to restore the function of the parts. In cases showing much excoriation of the nostril, an ointment of resorcin, ten grains to the ounce of unguentum petrolei, or the benzoated oxide-of-zinc ointment, is usually sufficient. Should paroxysms of sneezing continue after the parts have fairly recovered, the patient should be instructed to avoid taking all stimulating condiments with his food for a period of a few weeks. Inhalations of the fumes of carbonate of ammonia or spirits of chloroform are often useful in mitigating temporarily the distress due to the nasal stenosis.

In nervous persons, or those suffering much distress, a few doses of phenacetine (five grains each) may prove sufficient; of course, when this agent is administered the aconite is to be withheld. Cold applications to the forehead are frequently of service when the period of chilliness has passed. It sometimes hap- 
pens, however, that hot applications to the head give relief. Local applications to the nasal passages are of little value unless they contain cocaine hydrochlorate, which should not be used in stronger proportion than a two-per-cent solution, inasmuch as the reactionary effect upon the blood-vessels, by tiring out their contractility, so to speak, is apt to be baneful. Besides which, there is the danger of the patient acquiring the cocaine habit. Should a tendency toward protraction of the course of disease be noted, or should debility supervene, then three grains of quinine (or cinchonidia), with a quarter of a grain of hydrastin, may be administered three or four times daily until such conditions are relieved. In elderly persons the administration of one twenty-fourth to one thirtieth of a grain of strychnine, three times a day, may be indicated. In anæmic children the tincture of chloride of iron, in five-minim doses, with a little glycerine and water, may be taken after or in place of the quinine.

There is a prevalent idea that acute coryza is always a benign affection and does not call for treatment, and that therapy has no influence over it. This is a mistake. Lermoyez thinks that such cases should always be treated, in order to prevent possible suppurative rhinitis, or extension of the disease to the sinuses or lachrymal sac or the middle ear, or an extension to the trachea and bronchial tubes, and that medication will do a great deal toward cutting short the disease. He suggests an excellent solution for inhalation, known as Brandt's remedy, as follows:

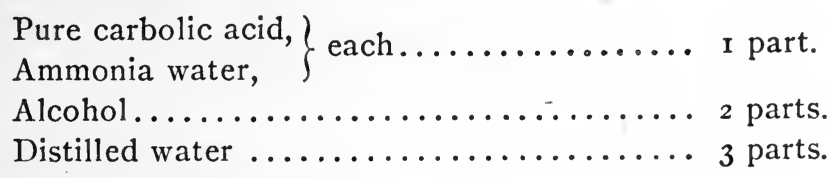

Every hour ten drops of this solution should be dropped on blotting paper, and the vapor inhaled through the nose for several seconds. Among the abortive powders, which should be finely pulverized, Lermoyez prefers the following:

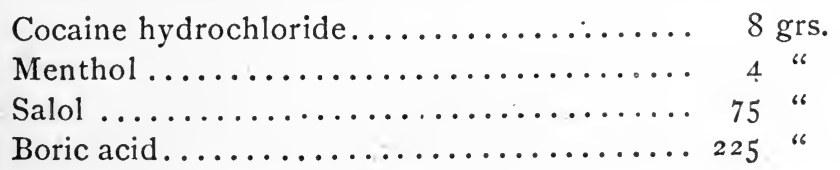


A pinch of this is to be snuffed up every hour. It provokes abundant mucous discharge and affords great relief. According to the experience of the writer, however, all of the snuff powders increase the irritation. Among the internal abortive remedies are quinine, belladonna and camphor combined as Lincoln's formula; there is one that is comparatively reliable, and that is a mixture of equal parts of tincture of belladonna and tincture of aconite root, of which thirty drops are to be taken in divided doses during the day (twenty-four hours). Energetic sweating also, induced by vapor baths, is beneficial. The patient should remain in bed, and abundant perspiration be induced by means of hot drinks and by a potion of acetate of ammonia or Dover's powder. If the coryza itself can not be ameliorated, the most painful symptoms, which are nasal obstruction and headache, may be benefited by palliative treatment. For this nothing is so good as the use of a spray of a boiled and tepid one-per-cent solution of cocaine hydrochlorate. Spraying every two or three hours is sufficient.

A two-per-cent solution of menthol dissolved in pure olive oil can be used as a spray.

A four-per-cent solution of suprarenal extract freely sprayed into the nostrils will often afford much relief, and, unlike cocaine, can be repeated as frequently as desired.

To prevent erythema of the roofs of the nostrils, the entrance of the nose, and upper lip, they should be smeared with vaseline with which boric acid has been incorporated. If there are no general symptoms the patient may go out. If there be fever, he should remain in his room. He must avoid sneezing as much as possible, and refrain from blowing his nose too energetically, as there is danger of projecting septic material into the middle ear.

Irrigation of the nose at the acute period of coryza should be absolutely interdicted, as it results in a constant irritation to the mucous membrane and increases tumefaction. Furthermore, it constitutes a real danger to the ear. On the other hand, it may be indicated afterward when a mucopurulent secretion follows with a tendency to chronic inflammation.

Acute coryza in the newborn is a very serious complication. It disturbs sleep, prevents the child from nursing, and causes the patient to waste away rapidly. In this case nasal obstruc- 
tion should be remedied at once by applying several drops of a two-per-cent oily solution of menthol to the nasal fossæ which detaches the crusts at the opening of the nostril and provokes momentary retraction of the turbinals. The mentholated oil, which is entirely harmless, is preferable to cocaine solution which at that age may cause very serious toxic symptoms. Before the child nurses, the secretions which obstruct the nose should be removed by means of a dry douche given with Politzer's bag or a syringe. When moderate insufflation into each nostril is sufficient this is preferable to nasal irrigation, which should be reserved for cases of purulent rhinitis.

With regard to intubation of the nose, which consists in introducing into the nasal fossæ rubber tubes to establish the air passage, it is considered a dangerous method which wounds the mucous membrane and may give rise to synechia. If the child, in spite of treatment, can not breathe sufficiently through the nose, it must be fed with a spoon.

The best prophylactic treatment for coryza in infants is not to allow them to go out too soon after birth, especially in damp weather. Also in bathing them, soapy water must not be allowed to penetrate the nose.

After an acute attack of rhinitis one should always thoroughly examine the nasal passages and inquire into the domestic history and environment of the individual with a view to ascertaining the causes and adopting proper prophylactic measures. The writer believes that the administration of antipyrine-although recommended by Lermoyez and many other writers-is dangerous as a rule.

Acute Pharyngitis. Acute Faucitis. Acute Uvulitis

The pharynx is commonly involved with the nasal passages, tonsils, uvula, or with the larynx in the acute inflammations constituting "colds." But aside from these, an acute pharyngitis or faucitis frequently occurs as an independent affection.

Etiology.-The disease may be caused by exposure to dust, especially railroad dust, to draughts of cold air, to the inhalation of an unusual amount of tobacco smoke, or to irritating va- 
pours, such as natural gas; also by the imbibition of too much alcoholic liquor, by disorder of the function of the stomach, or constipation, especially in individuals of rheumatic diathesis. In children, the teething process, as well as exposure to sudden changes of temperature, may bring about this malady. In children too young to complain it is frequently overlooked. It has been the writer's experience, as well as that of other practitioners, to discover in many instances an acute faucitis as the basis of what seemed at first to be an obscure illness in these little patients. Either children or adults who are in continual proximity to a case or cases of scarlet fever are liable to acquire acute faucitis, while at the same time showing no other signs or symptoms of scarlet fever. The disease often follows excessive, or an unusual form, of exercise, probably owing to the forced mouth breathing which such exertion may necessitate. In addition to such causes as are enumerated above, many practitioners believe that the principal or proximate cause of acute faucitis is the operation of micro-organisms. It is doubtful, however, whether this disease, like many others, can be reasonably laid at the door of the ubiquitous streptococcus, or other special microbe, because in many of the cases no individual microbe or set of micro-organisms will be found predominant, or apparently bearing any real relation to the pathological process existing.

Symptomatology.-The subjective symptoms complained of are usually a mild degree of soreness of the throat, possibly also of the muscles of the neck, together with a sensation of dryness, and oftentimes slight pain referred to the ears. There is rarely a rigour or much elevation of temperature, or much myalgia complained of, but almost always a feeling of indisposition. Rarely, however, the onset may be marked by a slight chill preceded by a mild degree of general malaise, and followed for a few hours or a day by an elevation of temperature. The writer has never observed any enlargement of the cervical lymphatics.

The objective symptoms consist mainly of more or less hyperæmia, with slight swelling of the mucous membrane of the fauces, including the uvula; as a rule, the faucial tonsils participate less in this abnormal condition than the adjacent mucous membrane. Exceptionally the uvula may be swollen more than any other point about the fauces. 
After a short stage in which the mucous membrane appears dry, striated, and shiny, there is an increase in the amount of mucous secretion, which is disposed to coagulate along the lateral walls of the pharynx and above the free margin of the soft palate on the posterior wall of the naso-pharynx. Once in a while the follicles of the mucous membrane become enlarged, in which case pus may be mingled with the mucous secretion.

Pathology.-The pathological condition in these cases is chiefly one of hyperæmia, suppuration and ulceration never occurring in uncomplicated cases.

Course and Termination.-Occasionally a simple acute faucitis terminates in acute tonsilitis or acute peritonsilitis; but, as a rule, resolution takes place in three or four days under ordinary circumstances.

Treatment.-Usually very little medicinal treatment is necessary. A saline cathartic may be administered at first, and the patient kept indoors and given a warm bath after catharsis has taken place. Should there be any elevation of temperature, a dose or two of tincture. of aconite or of phenacetine may be administered. For the mitigation of the local distress a tablet of biniodide of mercury (one sixtieth to one thirtieth of a grain) may be held in the mouth until dissolved, and the dose repeated every two or three hours until five or six have been administered; or, instead of this, a gargle or spray of a solution of salt and alum (a teaspoonful of each to the pint) may be used every hour or two. When there is much soreness of the muscles of the neck a few doses (five to ten grains) of sodium salicylate are indicated. . Sometimes the inhalation of steam from salt and water gives prompt relief to the local symptoms. In many cases either cold or hot applications to the sides and front of the neck give prompt relief.

\section{UVULITIS}

Inflammation and intumescence of the uvula may occur as an independent affection. Indeed, there are individuals in whom this apparatus is particularly vulnerable. With such persons, for instance, the uvula will become the seat of inflammation and exudation on slight provocation, such as very moderate exposure to cold or wet, chilling of the feet, transient 
indigestion, or excessive use of the voice. The writer has had occasion to observe a singular instance of this tendency in the person of a fireman, who for years has suffered from uvulitis only after attendance at fires where he was obliged to give frequent commands in a loud voice. Strange as it may appear, this individual has never suffered at such times from laryngitis. The writer is also acquainted with a physician who is subject to uvulitis, accompanied by great intumescence, whenever a fit of vomiting is induced from any cause.

The intumescence of the uvula may be very great, the organ being elongated so as to reach even into the vestibule of the larynx or well down into the pharynx, at the same time showing a breadth of half an inch or more.

Symptomatology.-The principal symptoms are very great pain on deglutition, excitation of nausea, and hacking cough through the titillation of the base of the tongue and epiglottis. Besides these, in nervous individuals there is great distress from the sensation of a foreign body in the throat, accompanied by frequent suffocative paroxysms. There is always in these cases an excessive secretion of mucus, with more or less salivation. There is rarely any elevation of temperature or other constitutional symptoms in uncomplicated cases of this disease. The appearance of the uvula varies in shape and contour from a smooth, even elongation and lateral enlargement to a broadly corrugated mass. The colour is usually at first a deep red, which fades as the intumescence increases to a reddish gray. When the exudate is mostly serous the organ appears white and nearly translucent, similar to a simple œdema.

Treatment.-Topical treatment is all that is necessary in uncomplicated cases. The holding of bits of ice in the mouth, alternated, if deemed necessary, with the application of astringents, such as tannic acid, ferric alum, or iron persulphate, is all that is needful. If these measures fail to reduce the swelling, the tip of the uvula should be excised by means of a pair of scissors, the operator first grasping the point to be removed by forceps. This procedure will usually suffice to let the exudate drain out. Scarification, with the point of a bistoury, along the sides more frequently than otherwise fails to relieve the sivelling. 


\section{Acute Laryngitis. Acute Laryngeal Catarrh}

This subject is usually divided into acute catarrhal laryngitis and acute edematous laryngitis. The disease in either form presents several grades of intensity, varying from a mere hyperæmia to an extensive swelling of the mucous membrane, with muco-purulent or even pseudo-membranous exudation. The affections known as "true or inflammatory croup," as well as the non-specific form of "croupous," "fibrinous," or "membranous" laryngitis, are properly varieties of acute laryngitis.

General Etiology.-The most common causes are exposure to cold or heat, over-use of the voice, continuous inhalation of a dust-laden atmosphere, the eruption of the teeth in infants, the operation of several of the micro-organisms, such as the staphylococcus, streptococcus, and bacillus of Friedländer, and trauma. Concerning the above-mentioned micro-organisms, which are often found in cases of non-diphtheritic laryngitis, it is not yet clear whether or not they are primary or secondary causal factors. As elsewhere mentioned, the Klebs-Loeffler bacillus is found in about ninety per cent of the cases of primary membranous or croupous laryngitis, many of which cases do not display other signs of diphtheria, and sometimes the bacillus of Friedländer, the staphylococcus and streptococcus, are found instead of the Klebs-Loeffler bacillus. The etiology, therefore, of primary membranous or croupous laryngitis, as well as that of fibrinous or membranous rhinitis, is often obscure, viewing the subject from a clinical standpoint. Trau$\mathrm{ma}$ is often the cause of acute laryngitis. The inhalation of ammoniacal or other irritating gases, the inspiration of fluid or solid material of a noxious nature, and teething, are common causes. Infants and young children who are taken out without being sufficiently clothed are thus predisposed to an attack.

Season.-The disease is most frequently met with in the spring and autumn seasons, when the atmosphere is damp and sudden changes of the weather are prevalent. It is not confined, however, to the northern climates, but frequently occurs in the southern and tropical climates. It is more frequently associated with pharyngitis, and oftentimes also with catarrhal inflammation of the mucous membrane of the trachea.

Age.-It may occur at any age, although it is less frequently met with in advanced years than during childhood and adoles- 
cence. Children between two and eight years of age frequently suffer from attacks of what is known commonly as false or true croup, although so-called false croup is laryngismus stridulus.

Symptomatology.-The earlier symptoms consist of various degrees of hoarseness, and slight pain upon deglutition sometimes in the neighbourhood of the larynx, especially during attempts at phonation. In the graver cases there is superadded more or less disturbance of the respiratory function, amounting in some cases to stenosis and more or less dyspnœa. There is almost always in adults a sensation of dryness or tickling referred to the pharynx or upper larynx, coupled with a constant desire to clear the throat; and if there is much tumefaction of the tissues there is present more or less cough of a brassy character. In children there are three degrees of intensity recognised under the name of true or inflammatory croup. In the first or milder form the child has a brassy, metallic voice, which may be present only for a while in the morning or at night, the child appearing to feel as well as usual during the day. At night, however, the voice becomes hoarse, or is nearly extinct. There is more or less dry, metallic, "brassy" cough, and the little patient may awake two or three times during the night with slight paroxysms of dyspnœa, characterized as "crowing inspirations." In the severer form the child is hoarse throughout the course of the disease, although the constitutional disturbance may be very slight. There may be slight respiratory disturbance and pain, which render the little patient apprehensive. The paroxysms of dyspnœa at night are more severe, and continue sometimes for several minutes at a time. They are manifested by the usual stridulous "crowing" inspiration, an incessant brassy, ineffectual cough, and more or less exhaustion. In the still severer form the voice is usually extinct. There is a continual obstruction to respiration, the countenance is anxious, the finger tips and lips may be slightly cyanosed, and the skin is more or less moist and cool. In the later stages of the disease the cyanosis may become widespread, the skin covered with a cold, clammy perspiration, while the stenosis of the larynx may be sufficient to cause a sinking in of the epigastrium and the jugular fossæ. In this condition the child, if not relieved, may soon die from the growing asphyxia. The constitutional symptoms in the milder forms are generally slight and evanescent. The pulse is usu- 
ally accelerated in the beginning, and the temperature may rise to $100^{\circ}$ or $101^{\circ}$, but rarely remains at that point for more than a few hours. The prodromal stage may not be at all marked, many cases showing no previous disturbance of either body or voice for more than a few hours before the attack. On the other hand, slight general indisposition, with a very mild degree of hoarseness, may precede the onset by several days. In infants, a period of crying and unusual crossness, with sometimes coryza, may precede the croup, and, in some cases, in older children a coryza and faucitis may precede the actual disease by several hours, or a day or two. Indeed, so-called croup frequently follows a period of faucitis (sore throat).

The laryngoscopic appearance varies with the severity of the disease. In the milder forms hyperæmia only is observed. In the severer forms, however, there is more or less exudation of mucus or muco-pus upon the surface of the mucous membrane of the ventricular bands and the ary-epiglottic folds, or these parts may appear slightly swollen, and of a deep-red colour. In the more severe cases the whole mucous membrane of the upper larynx will be swollen, and more or less thickly covered with a mucous or muco-purulent or fibrinous secretion, amounting in some instances to a thin coating of false membrane upon the parts. The writer has never observed the erosions of the mucous membrane which some authors call attention to in cases of simple acute laryngitis; but they are sometimes observed in the subacute form of the disease (see Plate II, Fig. 3).

Prognosis and Course.-The prognosis is usually favourable in these cases. Indeed, the large majority of them are selflimited, and terminate favourably in from three to eight days; but an attack beginning mildly may become aggravated in its course, so as to reach a condition of gravity after a few days. In such cases the course of the disease is usually ten days, two weeks, or even longer. When a given case of mild acute laryngitis persists after four or five days, especially in a child, some apprehension ought to be felt lest a dangerous amount of swelling and stenosis is approaching or the case be one of diphtheria. Frequent or long-continued attacks of acute laryngitis almost always lead to a chronic form of the disease, and sometimes to the formation of papillomata. 
Treatment. - While the milder types of acute laryngitis, as said before, run a self-limited course, still there is a risk in leaving such cases to the unaided efforts of Nature. On account of the tendency toward infiltration of the tissues in the early stages of the disease, the administration of a mercurial or a saline cathartic, together with the confinement of the patient in a warm apartment, will often be sufficient to bring about speedy recovery. If, however, the signs continue and pyrexia supervene, small and frequent doses of tincture of aconite root, or small doses of phenacetine alone or cornbined with salol, may be employed. In the severer forms of the disease active antipyretic treatment, with the administration of cinchonidia, or quinine and salol, will be indicated. In the medium and grave cases, especially such as occur in children, the administration of the tartrate of antimony and potassium in doses of one sixtieth to one thirtieth of a grain, frequently repeated, will be found beneficial. In very young children, a powder consisting of one half to one grain of cinchoniæ, with one twelfth to one half grain of ipecac, administered every two hours, has been very efficient in the hands of the writer. Also small doses of mild mercurial chloride, one twelfth to one quarter of a grain, triturated with sugar and administered every two or three hours until from six to twelve doses have been given, will prove beneficial. In malarial districts quinine should be freely given as the principal line of treatment. The employment of jaborandi, pilocarpine, or apomorphia will be efficacious in the treatment of some cases, especially those of adults. With children, however, the writer has not obtained as satisfactory results from the use of these agents as with tartrate of antimony and potassium, cinchona salts, and mercurials.

Local Treatment.-The application of the cold pack to the laryngeal region, followed, if necessary, by counter-irritation in the form of mustard sinapisms or tincture of iodine, are very useful adjuncts. Later on, however, when the disease has been prolonged, cold and hot packs are less valuable than counterirritation. Internal local medication, in the form of spray or douches, is of little value. The inhalation, however, of steam alone, or medicated by oil of eucalyptus, menthol, or tincture of benzoin, is often highly useful. Exceptionally, in the graver forms of laryngitis, the persistent application of a spray of a solution of bicarbonate of soda, five grains to the ounce pro- 
jected from a steam atomizer, may be used with benefit. Its efficiency, however, will depend upon its almost continual use for from twenty-four to forty-eight hours.

This method of local treatment is especially applicable to non-diphtheritic, croupous, or membranous laryngitis. When, however, all these means fail and dangerous laryngeal stenosis is impending, intubation or tracheotomy should be promptly resorted to (see Intubation). In cases of excessive subglottic swelling intubation may fail to give relief, in which event tracheotomy should be at once performed. Some of these cases in nervous individuals suffer considerably from spasm of the glottis. This phenomenon is to be met by the judicious administration of anodynes, such as hyoscyamus, codeine, opium, small doses of phenacetine, dilute hydrocyanic acid, or nitrite of amyl. In addition to these, the inhalation of small quantities of chloroform, ethyl bromide, or sulphuric ether may be used, providing the suffocative attacks are not too violent. In such an event morphine or pilocarpine may be administered hypodermically.

\section{Edematous Laryngitis}

Edematous laryngitis consists of a severe inflammation, with marked infiltration of the submucous tissue of the laryngeal lining. It is not a very common disease, and is often confounded with oedema of the larynx (œdema glottidis), which is a dropsy consisting of a simple transudation into the tissues of the watery parts of the blood. Edematous laryngitis may be acute or subacute, and primary or secondary. It is usually an acute affection, and can be considered subacute or chronic only when, from a long-continued irrita-

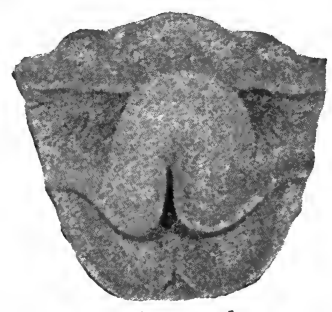

FIG. 40.-Acute edematous laryngitis (Burow). tion, a plastic exudation has slowly taken place. It is less frequently a primary than a secondary affection.

Etiology.-As stated above, primary edematous laryngitis is a very uncommon disease. It generally arises from systemic diseases, such as the exanthemata, severe fevers, sepsis, or other dyscrasiæ, or as an effect of one of the mineral poisons. Smallpox and measles, more commonly than other infectious diseases, give rise to it. Profound malarial toxæmia may also 
cause it. The writer has met with two dangerous cases of edematous laryngitis, one of which occurred in the course of variola, and the other as a result of severe miasmatic poisoning. It may also be primarily caused by the inhalation of the fumes of phosphorus or strong ammonia. It may result from wounds or injuries of the pharynx, as well as of the outer laryngeal structures, or occur as a consequence of operations on the pharynx, larynx, or nasal region. As a secondary affection it is met with usually in connection with syphilis, tuberculosis, carcinoma, myxœdema, and bulbar paralysis. It is also rarely met with in the course of syringomyelia. It may take place in the course of an infectious phlegmonous inflammation of the peritonsillar tissue or any part of the pharynx.

Symptomatology.-The objective symptoms are particularly referable to the larynx. More or less dyspnœa, with extinction of the voice, great pain upon movement of the neck, or upon efforts at deglutition and phonation, are the characteristic signs. There is also more or less salivation. The dyspnœa is usually progressive and continuous, although spasm of the glot. tis often occurs from time to time, greatly increasing the distress of the patient. Cyanosis, mental distress, and afterward hebetude, mark the advanced stages of the disease. The temperature usually remains elevated, except during periods of collapse. The countenance always wears an anxious expression, and there is great restlessness and dyspnœa from an early period of the disease.

Laryngoscopic Appearance.-In the milder degrees of inflammation the swelling may be localized somewhat-confined either to the epiglottis, the arytenoid regions, the ventricular bands, or the subglottic portion of the mucous membrane of the larynx. The parts become variously deformed, of course, according to the situation and extent of the intumescence (see Fig. 40). The colour varies from a bright to a purple gray or mottled red, and there is always a great deal of tenacious mucous secretion adhering to the parts. The mucous character of the secretion may change during the course of the disease to a muco-purulent one. This is especially noticed when erosions of the surface have taken place.

Diagnosis.-This disease may be mistaken for an acute œdema of the glottis, croup, spasmodic croup, laryngeal diphtheria, and the various paralyses of the larynx. The distin- 
guishing features of œdema of the glottis are the suddenness of the attack, its occurrence in connection with some acute systemic or local affection or injury of the pharynx or larynx, and the absence of pyrexia. Besides these points of clinical history, the laryngoscopic appearance of acute odema will usually serve to clear up all doubt, for, instead of an intense inflammatory swelling of the parts being seen, the intumescence will appear white or gray, and its contents thin. It will show a marked dropsical appearance. It will also be soft to the touch, unless it has existed for some time. The laryngoscopic appearances will enable one to distinguish this disease from spasmodic or inflammatory croup, or membranous laryngitis, although it must be admitted that either one of the diseases just mentioned may be complicated by the occurrence of an edematous laryngitis. Simple edematous laryngitis may be distinguished from diphtheria by a review of the clinical history of the latter disease, aided by a microscopic investigation of the exudate. From the various paralyses of the larynx the objective signs as demonstrated by the laryngoscope will constitute our safest guide to a diagnosis. Besides this, it will be remembered that the clinical history of the paralyses rarely includes such constitutional disturbances as elevated temperature, chills, general malaise, etc. It must not be forgotten, however, in this connection that a severe laryngitis may take place in the course of a laryngeal paralysis through the inspiration of food or liquids into the laryngeal cavity.

Prognosis. - The prognosis of edematous laryngitis, when not due to trauma, may be considered unfavourable. Cases occurring in the course of severe systemic poisoning from exanthemata or other dyscrasix are indicative always of a grave condition of the economy, and although the local affection may be effectually relieved, certain local lesions of the larynx of a serious character almost always remain.

Treatment.-The topical treatment should be very active, and consist of the inhalation of steam or other warm sprays, together with the outside application at first of ice, and then counter-irritation and hot fomentations to the neck. The ice should be assiduously applied for from two to three days, if necessary and if well borne. After this, counter-irititation and blisters are useful. Scarification of the affected laryngeal region has never, in the writer's experience, been followed by 
good effects. In two cases scarification produced very transient relief, which was soon followed by an aggravation of the dyspnœa and more swelling. Small and repeated doses of pilocarpine are frequently quite beneficial in these cases. When the stenosis of the larynx becomes marked and retrogression of the swelling does not seem imminent, either intubation or tracheotomy should be made without further delay. Too great hesitation in relieving the dyspnœa by such means should be avoided, for there is great danger to the patient's life from delay when respiration is greatly embarrassed. Intubation is often difficult of accomplishment, and persistent attempts may lead to the sudden death of the patient. It is therefore better, when one or two attempts at the introduction of the tube have failed, to at once proceed to the performance of tracheotomy.

\section{Membranous or Croupous Laryngitis}

Croupous laryngitis, also called membranous croup, is considered to be identical with laryngeal diphtheria, inasmuch as about eighty to ninety per cent of the cases show the presence of the Klebs-Loeffler bacillus in the exudate. It occurs sometimes, however, independently of the diphtheria micro-organism as a result of chemical or mechanical irritation or other traumatism. It is the form of laryngitis which usually follows the inhalation of ammonia, or the deglutition of caustic substances, such as potash, soda, carbolic acid, etc.

It may also follow or accompany the exanthemata, especially measles and scarlet fever.

Diagnosis. - Differentiation from laryngeal diphtheria is not always possible, even when the attack closely follows some slight injury.

Bacteriological examination should always be instituted, unless the traumatism is of such a nature as to leave no doubt regarding the origin of the malady.

Treatment.- The treatment of the majority of cases should be along the lines laid down for laryngeal diphtheria-viz., by hypodermic injections of antitoxine. When, however, as before mentioned, the disease depends upon some other wellknown cause, such as chemical or mechanical injury, perichondritis, etc., the treatment should be of a topical character principally, including inhalations of steam and mild alkaline, 
antiseptic sprays, etc. The general treatment to be adopted will, of course, depend upon the nature of the conditions. Small doses of calomel or iodide of ammonia will be found useful in a majority of the cases.

Intubation or tracheotomy will be indicated whenever the stenosis of the larynx becomes threatening.

\section{Acute Glandular Fever of Children}

Acute glandular fever of children is not a very common affection; it usually comes on suddenly and unaccountably, and is characterized by enlargement of some of the lymphatic glands, general malaise, chills and fever. This condition has been recognised from time to time for a number of years as one of the odd diseases of children. It has sometimes been classified with the exanthemata, and sometimes with mumps, sometimes with whooping-cough; but as it lacks so many features belonging to either of these diseases, it has come to attain a separate place in medical nosology. The reason for considering it in connection with diseases of the throat is because of its frequent occurrence in connection with some of the catarrhal affections of the nose and fauces.

Etiology.-The cause of this disease is problematical. It is supposed, however, that some micro-organism, such as one of the streptococci, is responsible for its occurrence. The theory is, that some infecting micro-organism gains access to a lymph vessel or gland, and by its presence suddenly excites irritation throughout the neighbouring lymph vessels and glands. This being accomplished, the pyrexia and other symptoms occur as reactionary or resistant phenomena. Some cases have been traced supposedly to insect bites, some to ptomaine poisons, some to traumatism, some to infection from the teeth, some to severe catarrhal inflammations of the nose or pharynx; tubercular infection is responsible for many cases.

Course and Termination.-The course of the disease is irregular. It usually lasts from three days to a week; but it may become chronic, lasting for months. The highest temperature occurs usually during the first three days. In some cases the disease is not ushered in by a chill; but, as a rule, either a rigour or spells of chilliness precede or accompany the early swelling of the lymphatic glands. The disease usually ends in recovery at the end of a week or ten days, though sometimes 
two weeks or even more may elapse before convalesence is fully established, or a longer time if deep-seated abscesses or permanent hyperplasia occur. Suppuration and abscess are often terminations of this glandular inflammation, and sometimes the latter process does not occur until some time after convalescence has been established from the primary attack.

Symptomatology.-The glands usually affected are those of the neck (the cervical and auricular), but sometimes the lymphatic glands of the axilla or groin may participate. Abscesses may form in the cervical glands and yet cause very little extra trouble, or, as sometimes happens, infection of the surrounding cellular tissue may supervene, and the aspect of the case then becomes more serious. Retropharyngeal or peritonsillar abscesses may be a result of this acute glandular fever. Suppuration of the middle ear or deep burrowing abscesses in the lower portion of the neck may arise. In many cases a very mild degree of tonsilitis or pharyngo-laryngitis accompanies the disease.

Diagnosis. - The diagnosis is very difficult to make out in almost all cases because of the absence of specific signs. For example, when any of the exanthemata, or diphtheria, or cerebro-spinal meningitis is prevailing, a case of acute glandular fever will at its onset simulate these respective diseases. And in case of a rhinitis or faucitis in any form being present, the diagnosis of the case becomes, of course, always doubtful. Indeed, for these reasons in many cases the diagnosis can not be made out until the disease is well on its way to recovery. Moreover, there are subacute and chronic cases of glandular fever which may be mistaken for tuberculosis and other diseases. The writer saw a case which lasted nearly a year with lymphoadenitis of the groin and neck.

Prognosis. - The prognosis is usually favourable, unless diffused suppuration, abscesses, or consequent secondary sepsis supervenes. If, however, inflammation and suppuration of lymphatic glands, in juxtaposition to important structures, occur, or if tuberculosis supervene, the prognosis, of course, is grave. For example, in one recorded case suppuration took place in the deeper portions of the neck and caused a sudden and fatal termination by hemorrhage on account of ulceration of the carotid artery. Again, in a case which came under the writer's observation, an abscess discharging pus into the cellular tissue of the lower part of the neck, and afterward involving 
the upper portion of the mediastinum, caused death. Retropharyngeal abscess in children (especially weakly and ill-nourished ones) occasionally follows the course of this affection and may contribute to a fatal termination.

Treatment.-The treatment must be entirely expectant. The therapeutic indications being, (I) to limit the febrile action; (2) to remove, if possible, the source of infection ; and (3) to avoid and mitigate complications. For the first indication aconite, small doses of phenacetine, or the cold bath would be in order. For the second, the internal administration of calomel, or bichloride of mercury, or biniodide of mercury, together with saline cathartics or diaphoretics, aided by the local application of ice or cold water to the part affected, is indicated. In addition a mercurial or iodine ointment may be applied. The administration of quinine, salicin, or sodium salicylate, or salol may also give good results. Following the third indication, the early detection of points of suppuration and abscess and their prompt evacuation, including free drainage afterward of the tissues involved, should be accomplished. Besides, when either the nasal or pharyngeal regions are involved, as often happens, it may be necessary to apply mild saline or astringent sprays or pigments in order to modify the hyperæmia or inflammation of these parts. Potassium iodide and iron iodide are indicated for the slower-going cases.

\section{Pharyngitis Herpetica}

Herpetic Sore Throat. Herpes of the Pharynx; Common Membranous Sore Throat; Aphthous Sore Throat; Benign Croupous Angina. (Thorner.)-Herpetic eruptions upon the mucous membrane of the upper respiratory tract are not of very common occurrence. The eruption may appear at the nasal vestibule, but is usually found in the pharynx, on its posterior wall, or on the anterior surface of the faucial pillars.

Etiology and Pathology.-Lermoyez divides the subject into three groups: I: The acute infectious form. 2. Neurotrophic form. 3. The chronic recurring form. Exposure to cold is often given as an exciting cause, but this event is probably not very often a causative factor, for the disease is met with generally in persons of nervous disposition, who are given to much worry rather than to the contraction of "colds." Persons whose general health is "below par," such as ill-fed, 
nursing mothers, etc., and persons who are otherwise healthy, but (through diathesis) suffer more or less with herpetic eruptions in different parts of the body, are liable to suffer from it. Such individuals belong to a class which the old French medical writers denominated "the dartrous." Herpes here, as elsewhere, is undoubtedly chiefly an expression of neurotic disorder. It may depend upon structural changes of the central or peripheral nervous system, or upon functional perturbation only; as a rule, when occurring in the pharynx it indicates a milder type of disease than when affecting the skin.

Olivier, Herzog, and others desire to identify this with other forms of herpes, as originating from the excitation of the spheno-palatal ganglion (Meckel's). Sometimes, if the eruption is located in the domain of the trigeminus, it presents certain irregularities.

Lermoyez and Barozzi report an interesting case, in which the eruption quite closely followed the "bucco-pharyngeal zone":

"An old man, aged seventy-eight, suffered on and off for two years with a sort of buccal pain without general symptoms. The superior part of the cavity of the mouth presented a diffuse red colour on the arch of the palate and soft palate up to the anterior pillars. Upon the spots most highly coloured there were vesicles, some limpid and others yellowish; besides, there were some very small ulcerations, and at the level of the gums and labio-gingival folds there was a ring of vesicles. This eruption came in spots for twenty days, and assumed the form of a semicircle. This was a case within the double buccopharyngeal zone following the course of the anterior palatine nerves. The two lateral zones were not formed at the same time-the right preceding the left. This sort of case is rare. No eruption was seen upon the tonsils (ninth pair) nor on the posterior wall of the pharynx (tenth pair)."

Some writers connect this form of disease with aphthous folliculitis on account of its frequent occurrence with aphthous stomatitis in children. The ulceration is usually not very extensive. Exceptionally, however, extensive and dangerous phagedenic ulceration may ensue, constituting what has been termed "gangrenum oris." Such cases should be considered, however, as ulcerous pharyngitis. Many think that there is some relationship between attacks of this kind and derange- 
ments of the cutaneous apparatus. The cause of the eruption has also been ascribed to rheumatism.

Symptomatology.-Among the earliest principal symptoms is sore throat. The pain is described as of a burning or stinging character. There are no constitutional symptoms in the majority of cases if the patient be otherwise well. Exceptionally, however, the attack is ushered in by a slight chilliness and a moderate rise of temperature, lasting a few hours, and occasionally the disease is accompanied by neuralgia, insomnia, or other nervous phenomena.

Appearance.-Inspection of the throat reveals one, two, three, or more patches of hyperæmia, varying from a ten-cent piece to half a dollar in size. Upon this hyperæmic base may be seen one or more clusters of fine, slightly raised papillæ or vesicles; but, as Cohen remarks, the vesicular stage is seldom observed. Sometimes, if the maceration of the papillæ has not been too rapid, little vesicles may be detected in some of the groups. A thin, pultaceous membranous secretion almost always takes place after the eruption is well established; this may cover the patch pretty well or only partially. Underneath it may be found sometimes a little muco-pus. The membrane falls off easily, or becomes dissolved. It is often removed by the fluids of the mouth, leaving the characteristic red colour, and sometimes a few raw eroded spots at the site of the papular outbreak. This condition usually lasts but a few days, although in some cases it may be continued for many days, or even weeks, on and off. In connection with the appearance of this eruption in the pharynx or on the soft palate, a few papillæ may appear at the upper angle of the nose, or just inside the nasal vestibule, or at the corner of the lips. These eruptions sometimes accompany the disease known as xerosis (dryness of the mouth) pharyngis. The eruption in the pharynx may be coincident with a similar one on the prepuce, or about the mouth or the anus.

Treatment.-Very little local treatment is required, as a rule. Antiseptic and bland mouth washes containing some of the essential oils or oleo-resins generally give the most relief. Sometimes painting the part, when the patches are not too extensive, with very mild solutions of nitrate of silver, two or three grains to the ounce, is highly useful. A pigment of resorcin and glycerine (ten grains to the ounce) is a good 
remedy. The application of a pigment composed of equal parts of compound tincture of benzoin and glycerine is beneficial. General treatment seems to be productive of the best results. First, some saline cathartic or diuretic should be administered. The skin may afterward be stimulated by means of hot baths or salt sponge baths; and, according to the condition of the patient, either tonics or so called alteratives may be given from time to time through the day. Vialle has recommended hypodermic injections of pilocarpine hydrochlorate. For the so-called aphthous or ulcerous variety more active local treatment becomes necessary. For this purpose strong solutions of nitrate of silver, chloracetic acid, carbolic acid, lactic acid, or argyrol, are highly useful. The writer has seen excellent effects from the local application of strong solutions of sulphide of calcium, followed, sometimes, by applications of Peruvian balsam or tincture of benzoin. In addition, the insufflation on the part of a mixture of calomel and calcined magnesia, or of iodoform and naphthalin, aids the healing. (See formulary.)

Eczematous eruptions may occur-within the vestibule of the nose, and may cause a great deal of local trouble from the increase of secretion excited in the lower nasal passages and its collection and desiccation. The eczematous form of eruption is generally met with in children, thus differing from the herpetic, which is more frequently met with after puberty. Eczema affecting the mucous membrane should be treated similarly to such eruptions on other parts of the body. The anterior nares in these cases should be kept clean by means of some bland alkaline solution, common salt being as good as anything, and the cleansing should be immediately followed by the use of some oleaginous preparation, such as petrolina oil or vaseline, resorcin ointment, or benzoated oxide-of-zinc ointment. Internal medication, according to the systemic indications, must not be neglected. Fluid extract of hydrangea and sodium phosphate are excellent remedies.

\section{Local Diseases of the Nose}

The region of the anterior nares, from its exposed position, is frequently the seat of disease, owing to injury, infection, or the operation of impalpable foreign bodies, such as dust, etc. 
Furuncle-Boil.-One of the most common affections is furuncle or boil. These little abscesses usually occupy the upper anterior sulcus of the vestibule of the nose, but may appear anywhere upon the internal lining of the alæ nasi. They vary in size from a large pinhead to that of a pea. Generally there is no constitutional disturbance, nor much if any local rhinitis, induced by their presence; occasionally, however, they give rise to an abscess of the septum which may reach the size of a canary egg. They may also start erysipelas of the face. They may originate in either a sebaceous or hair follicle, which, according to some histologists, are identical. Considerable inflammation and pain, attends the affection, until such time as the contents of the little abscess are evacuated. Usually but one of these occurs at a time, although there may be a succession of them, or they may be multiple.

Etiology.-The causes of the affection are usually not very well known; a streptococcus or staphylococcus infection is supposed to be the cause. The general supposition is that the infective material is carried by the finger of the individual by picking or otherwise clearing the vestibule of the nose. In some instances the affection has been traced to the bites of insects, and in others to the transference from pustules or boils elsewhere on the body of some of the infecting material.

Abscess of the nasal septum is not a very common affection. It is usually unilateral, but may be bilateral. The inflammation giving rise to the abscess is usually confined to the submucosa, but there are instances in which the perichondrium has been the basis of the mischief.

The etiology is often obscure, especially in the absence of any of the well-known dyscrasiæ. Some writers have attributed the development of these abscesses to metastasis of the materies morbi from some other point in the neighbourhood, thus charging its origin to the lymph stream. Some cases are the result of trauma. The writer met with one such case, where the injury was caused by the point of a lead pencil accidentally wounding the mucous membrane of the septum. The site of these abscesses may be either in the region of the cartilaginous or bony septum-by far the larger number occurring in the former situation. The writer has met with but one case which was seated over the bony septum. The symptoms of these abscesses are usually pain referred to the "bridge of the 
nose" or frontal region, more or less muco-serous secretion accompanied by lachrymation and nasal stenosis. As the abscess grows in size the nose becomes more tender to the touch and swollen, while the nasal stenosis and pain are progressive. There are usually no general symptoms accompanying it, such as pyrexia, chilliness, etc., although exceptionally, when an abscess is seated upon each side, or is of considerable size, chilliness and a moderate amount of pyrexia may occur. After the evacuation of the contents of the sac restitution is usually quite prompt; but sometimes, when the abscess is seated over the cartilaginous portion, softening and perforation of the septum follow.

Diagnosis.-Simple abscess of the nasal septum is sometimes mistaken for syphilis of the nose. The latter affection, however, may be distinguished by the diffuse character and extent of the swelling, the gummy nature of the secretion, and the history and course of the case. 


\section{CHAPTER IV}

\section{INFLUENZA}

INFLUENZA may be defined as an acute catarrhal inflammation of the nasal passages, pharynx, trachea, and bronchial tubes -in other words, an acute inflammation of the respiratory lining membrane, reaching from the nasal passages to at least the bronchial tubes of the second class. The disease is very common throughout the north temperate zone during the autumn and spring seasons. It affects mostly males and adolescents, although in exceptionally bad seasons many children also suffer. It is believed to be contagious, since there are so many instances of its introduction into a family by one member. This has become a phenomenon recognised by the laity, who expect that when one member of a household is attacked the disease will not respect the others. It is rarely severe or dangerous, excepting with the aged and the very young.

The specific character of influenza has been established by the discovery and behaviour of the bacillus of Pfeiffer.

Pfeiffer in 1890 isolated a bacillus which he regarded as the specific cause of influenza. He inoculated animals with cultures of this germ, and produced in them the symptoms of influenza. Although his experiments were corroborated at the time by Babes, Cornier, Kitasato, and a few others, yet his work was not accepted by the majority of pathologists and clinicians until within the last three years.

Etiology.-There has been much speculation during the past hundred years in regard to atmospheric vicissitudes as a principal cause of this disease. Consequently, its existence has been attributed in turn to variable winds from one direction or to steady winds from another direction; or to dust-laden atmosphere; or to changes in the amount of ozone; or to changes in humidity; or to alterations in the proportion of nitrogen and oxygen contained in the atmosphere. Such lines of thought 
are not astonishing when we remember how often outbreaks of influenza appear in the wake of changes in the atmosphere or weather. It may often be noticed, in the spring of the year, that a community whose individuals are in ordinarily good sanitary condition will suffer in large numbers from influenza after a sudden change of " the weather" from warm to cold or cold to warm, etc.; while, on the contrary, during spells of "steady weather" cases of influenza do not as frequently appear. This weather theory is explained by many as simply a coincidence when coupled with the fact that at times people are careless about their clothing or regimen, but that no one is liable to an attack of influenza unless the particular microorganism attacks him, and that this morbific agent is about us in a latent state at all times. A refutation of this theory may lie in the fact that the relation between these outbreaks, as registered by the various boards of health in the different States, and the vicissitudes of the weather as recorded by the signal service, show a correspondence. It will be found upon consulting the statistics of these institutions that endemics of influenza follow closely upon marked changes of the weather. Now, whether these changes of themselves contribute toward generating the organic morbific agent by altering the proportional relationship of ozone or moisture to the other constituents of the atmosphere, or whether such outside conditions, by irritation, merely prepare the mucous membrane for the invasion of the bacilli, is not now known. Neither can it be positively stated that a redundance or a diminution of ozone, oxygen, nitrogen, carbon dioxide, or other elements of the atmosphere furnish to the respiratory apparatus an irritating air of such character as to set up alone an inflammatory process. If we were to adopt any of these theories concerning the subject, the most probable one would be that the falling of the upper strata of air-which often constitutes such changes-carries down the floating particles nearer the earth, where, being respired, they serve as local irritants. At the same time the heat-radiating and emunctory functions of the skin being thrown out of equilibrium by the sudden change in temperature, etc., contribute toward an internal congestion as a primary step.

Symptomatology.--Influenza is usually ushered in by general malaise, a chill, or feeling of chilliness, with the symptoms of acute rhinitis, together with a dryness and burning sensation 
of the throat, uneasiness, oppression, or soreness in the region of the sternum, myalgia of the back, chest, and other parts of the body. The temperature soon rises from $101^{\circ}$ to $103^{\circ}$, vacillating irregularly but usually maintaining its greater height during the evening. A dry, painful cough soon comes on with or without hoarseness. A sense of debility out of proportion to the length of the illness is experienced, which in the aged and the feeble amounts to a serious adynamia. The cough at first is apt to be incessant and dry, but after forty-eight hours, tussive effort brings about the expectoration of a small amount of glairy mucus, which increases toward the end of the first week, when free expectoration from the bronchial tubes takes place ; at the same time, in favourable cases, the pain in the chest gradually diminishes, the voice loses its hoarseness, and the respiration becomes easier. The rhinitis by this time usually disappears or greatly abates, but it may continue to annoy the patient for two or more weeks. The physical signs are usually indicative of a swelling of the mucous lining of the bronchial tubes, as evidenced by wheezy, sonorous respiration heard on both sides of the chest. After secretion is well established, however, these signs give way to moist crackling or mucous râles. In children the râles are sometimes so marked that they may be detected by palpation all over the chest. The pharynx usually participates as well as the larynx and trachea and shows a diffuse hyperæmia of its mucous membrane.

Course and Complications. - Although the majority of cases of influenza are not of a serious nature, excepting, as before mentioned, when occurring in infants and the aged, yet serious complications occur often enough in ordinary cases to warrant the practitioner in being always upon guard. Ordinary cases run anywhere from ten days to two or three weeks; then consuming about one week to ten days in convalescence. This is by no means a rule, inasmuch as some patients suffering from a mild type of the disease experience an inordinate degree of general debility as in la grippe. The most frequent complications are the various forms of pneumonia arising often from an extension of the inflammation down the respiratory passages; catarrhal pneumonia is much more often met with than the plastic or lobar form. Adenitis, from involvement of the lymphatic glands of the cervical or other regions of the body, is not infrequently met with, especially in the young; suppuration of 
the glands, however, does not occur as often as might be expected, excepting in those possessing a tubercular tendency. The nervous system is in all cases pre-eminently impressed during the course of the disease and may show the principal effects of the attack. The morbific agent seems to act particularly upon the ganglionic nervous system, and hence transitory derangements of various organs of the body, apparently succeeding each other, are not uncommon phenomena. I' have known several cases of influenza which left the patient with dyspepsia, chronic diarrhœa, and other morbid intestinal or hepatic disorders, requiring months for complete recovery. Cardiac and renal lesions, contrary to expectation, are not very common complications or sequelæ of this disease, although such sequelæ are sometimes observed. Articular rheumatism is not an uncommon complication and may manifest itself early in the second week. Gout also, in those predisposed to lithiasis, is very apt to complicate an attack of influenza; therefore, any feeling of pain or swelling, or sensation of heat in the neighbourhood of joints, should arouse suspicion and attract particular attention. Facial and occipital neuralgia are marked phenomena in some cases, especially in the early course of the disease, or when the rhinitis has been severe. Influenza frequently lays the foundation of hay fever and local neuroses. The writer has seen several cases of paralysis of the superior maxillary and supraorbital nerve as a result of this disease when it had not even been severe in character. Chronic catarrhal affections, with or without suppuration of the nasal passages, of the pharynx, of the larynx, and of the accessory cavities and middle ear, are frequent accompaniments of influenza.

Ewald says that it is now believed, a fact borne out by clinical experience, that influenza is a malady strongly inclined to produce suppurative sinusitis or purulent rhinitis, especially when the mucous surfaces of the respiratory tract are the seat of the disease.

Weichselbaum discovered in sixteen persons dying from influenza that abscess of the antrum and the frontal sinus was present. A case is reported in which death was produced by meningitis as a result of influenza complicated by empyema of the antrum and ethmoidal labyrinth.

Among those who use the voice artistically, a chronic hyperæmia, at least, of the laryngeal mucous membrane may 
continue a long time. Paresis of the laryngeal nerves, however, is much less frequent than in the pandemic form of the disease (la grippe).

Diagnosis.-The diagnosis between influenza, acute rhinitis, and la grippe is not easy to make. In typical cases of acute rhinitis, however severe, we seldom have involvement of the bronchial mucous membrane, while in influenza this is one of the chief features of the attack ; true, in acute rhinitis we seldom find the inflammation confined to the nasal chambers or to the naso-pharynx throughout the course of the disease, but extension from these places of early involvement is slow and gradual, rarely reaching below the larynx; while, on the other hand, in influenza an early and almost immediate implication of the bronchial tubes is characteristic. The differentiation between influenza and measles (also rötheln) is sometimes difficult to make out early in the case of children, especially when both diseases are prevailing in the community at the same time. However, after a few days, it can soon be determined by the clinical history of the case and the appearance of the eruption. Whether these two diseases can occur in the same individual at the same time is a question which has not yet been satisfactorily settled. It must be mentioned, however, that cases of measles occurring during the prevalence of influenza may assume at such times more serious aspects. The differential diagnosis of influenza from la grippe is not always an easy matter, but is frequently dependent upon the pandemic or very widespread and graver character of the one as compared with the milder and endemic character of the other. In its general features, sudden and violent accession, and overwhelming prostration, la grippe is a scourge of the worst type-perhaps influenza and la grippe are only varieties of the same disease. If this is the case, may we not also regard Asiatic cholera and cholera morbus as the same disease from an etiological standpoint?

Treatment.-Asthenic cases must be kept indoors and very quiet (recumbent) from the very accession of the attack until all danger of extension and complication has passed. Even mild cases should not be allowed too much liberty in pursuance of the daily routine, at least during the first four or five days. Should loss of appetite appear as an early symptom, the patient ought to be placed at once upon concentrated 
liquid diet in moderate quantity, such as milk, broths, farinaceous liquids, etc.; indeed, it might be stated as an axiom that in all cases the diet should be restricted to the fluid or semifluid, at least for a time. After the acme has passed and the bodily temperature has assumed its normal state, a moderately full mixed diet may be allowed. The medicinal treatment should begin in the majority of cases (excepting where the bowels have previously been evacuated) by the administration of a few doses of calomel, one tenth to one quarter of a grain every hour until four or five doses have been given, or blue mass (five grains); this may be combined with a little ipecac or sodium bicarbonate to hasten the action, or a saline cathartic may be given instead. Should no catharsis result within three or four hours, some saline, such as magnesium sulphate, potassium and sodium tartrate, seidlitz powder, or a laxative mineral water may be administered to secure free movement of the bowels. It will be useless, until the accomplishment of this design, to expect any action from the administration of diaphoretics or diuretics. After catharsis has taken place, a diaphoretic in the form of a hot drink, frequent small doses of Dover's powder, or the fluid extract of jaborandi (if not contraindicated) will be efficient. Upon the following day, if the pyrexia is not too great, the administration of quinia sulphate, or cinchonidia sulphate, together with capsicum or leptandrine, or hydrastine, may be ordered. Should there be much cephalalgia or myalgia, with pyrexia, small doses of phenacetine (one or two grains), alone or with salol, in three- to five-grain doses every two or three hours for a few doses may be given, or instead, one-minim doses of tincture of aconite root, or ten-minim doses of gelsemium. Salol, sodium salicylate, or salicin may at any time be substituted for the quinine or cinchonidia, if any untoward effect of these drugs be manifested. Calomel has long been much used in the early stages, and is often clearly indicated for its wellknown so-called alterative effect. Felsenthal claims, if it be given before the first three days of the disease, that it is capable of entirely aborting the attack. He recommends that it be given for men in two doses of three grains each; for women two and a half grains in three doses, and children $\frac{15}{100}$ for each year of age. (New York Medical Journal, December 25,1897, p. 882.) If the cough be irritating, and phys- 
ical examination of the chest shows that the smaller bronchial tubes or the lobules are becoming involved, then small and oftrepeated doses of tartar emetic should be carefully employed. This agent should be continued until expectoration appears, unless progressive prostration supervenes, or the skin remains continually or unduly moist and cool. Should such signs be observed, it would be well to substitute for the tartar emetic iodide of ammonia in two- to five-grain doses given, if necessary, in conjunction with quinine, cinchonidia, or salicin. If insomnia or restlessness be persistent small doses of chloral hydrate, codeine, Dover's powder, or sulphonal may be administered without danger; if the restlessness be accompanied by elevation of temperature the chloral hydrate will be found much more efficient than either sulphonal or opium, on account of its selective action on the so-called heat centres. However, if the pain and restlessness, etc., seem to be largely due to the elevated temperature, and if general prostration be not marked, then five-grain doses of either phenacetine or acetanilide will be found a much more efficient remedy for securing rest and sleep.

The crisis or lysis of the disease having passed, such restorative agents as strychnine, picrotoxine, phosphorus (see formulary), alcoholic stimulants, and malt beverages may be carefully administered. With regard to alcoholic stimulants of all sorts, great caution should be exercised in their administration, especially with children, and adults unaccustomed to their use, because there is a liability of inducing the alcoholic habit, and also because the sympathetic nervous system before having recovered its regulating tonus may be unduly sensitive to the effects of stimulants. Indeed, I have observed many cases where the injudicious use of brandy and whiskey has kept up a condition of alcoholic toxicity leading to congestions of various organs and a needless prolongation of the course of the disease.

It seems to me that a good rule to follow in such cases is to make careful observations of the effects, both immediate and remote, upon the patient, of the precise quantity of stimulant taken during a period of from twelve to twenty-four hours. Should ill effects be complained of, or untoward phenomena be observed during such a period, the quantity of stimulant should be regulated accordingly, or, if necessary, entirely withheld, and instead aromatic spirits of ammonia, or the carbonate of 
ammonia, substituted therefor. Frequent bathing of the skin is very useful; but the substitution of alcohol for salt and water or ammonia water is to be interdicted when the temperature is inclined to fall below normal, because, through its tendency to evaporate, alcohol bathing aids in lessening the temperature. When diarrhœa or a tendency to intestinal indigestion remains after the other symptoms have disappeared, the administration of tincture of the chloride of iron (in ten- to twenty-drop doses) in glycerine and water (see formulary) will oftentimes prove efficacious. If, however, one of the forms of dyspepsia accompanies the disturbance, it will be necessary to regulate the diet and perhaps administer pancreatin, bismuth, or other appropriate stomachic remedies; these should be given alone and in full doses, but never in combination with the iron. Should rectal symptoms persist, the organ should be examined and appropriate local treatment adopted, such as enemata of salt and water alone, or with one per cent of carbolic acid, iodoform emulsion, etc. This precaution must not be omitted, for many cases of chronic rectal disease originate in attacks of influenza. Complications affecting the nervous system, the ear, the pulmonary, or circulatory, or renal apparatus, must be met as they arise. No definite directions can be given as to how to meet these various sequelæ, as it is obvious that any disease particularly affecting the ganglionic nervous system must present various phases of disturbance which will require skilful vet conservative plans of treatment.

\section{La Grippe-Pandemic Influenza}

According to Parkes this disease, which has been known since the ninth century, presents essentially the same clinical features as influenza, excepting in the severity and the wide range of its visitations. While influenza is a local or endemic disease most prevalent during the changeable season of the year, and common to the north temperate zone, la grippe has no geographical limitations, but "swoops down," as it were, upon a whole continent without much notice, even attacking persons on shipboard at sea whether the ship is near an infected port or not. It has been repeatedly observed, however, that the disease follows closely the lines of travel or commercial intercourse; hence, the general opinion is that it is both "conta- 
gious and infectious," depending for its spread upon the commingling of human beings. The later pandemics have been supposedly traced to Russian origin; although upon this point there still exists some controversy, many Continental authorities holding that la grippe has its origin in China. One thing, however, is established beyond controversy concerning the origin of the outbreaks during the last century-viz., that each one arose in a cold country and in one which was pre-eminent for the squalor and aggregation of its inhabitants. The disease seems to appear in cycles with from two to fifty years intervening, although history shows one interval of two hundred years between scourges. Notwithstanding the various changes in social life incident to the progress of civilization, history shows that the pandemics or epidemics of ancient times presented very nearly the same clinical features and complications as those of modern times. The different social conditions of the members of a community seem to offer no hindrance to its access and ravages, for as many of the ignorant and squalid, in the United States at least, escape attacks as the more fortunate class of citizens.

Etiology.-Age and sex present no obstacles to the accession of la grippe, for when a pandemic is raging in a community any sort of a mixed community may become its prey; nor is it confined to the human family, since, according to excellent veterinary authority, la grippe is believed to be identical with that similar scourge of the horse known as the epizootic. It is noted that those persons who are subject to confinement indoors are more susceptible to attack.

Little doubt exists now concerning the specific cause of la grippe, for the majority of the profession accept the doctrine that it is essentially caused by the influenza bacillus. The question has arisen, however, whether or not the micro-organisms which cause the pandemic form (la grippe) may not be a distinct or peculiar variety of microbe related to the ordinary influenza bacillus, but not exactly identical with it. La grippe is certainly more communicable than the ordinary influenza. Some important contributions seem to have been made to bacteriological science by the labours of Dr. Reilly and Dr. Wyncoop, who are connected with the Chicago Department of Health. These investigators have been giving considerable attention to the bacillus of influenza, and a preliminary report of their 
labours by Dr. Wyncoop appears in the bulletin of the Chicago Bureau of Vital Statistics for January, I899. The points to which these investigators have given special attention are of great interest to physicians and sanitarians. Is there an influenza vera due to a special germ, the bacillus of Pfeiffer, and is there also a pseudo-influenza, the so-called grippe of the laity, in which this specific germ is absent? The one disease dangerous by its toxic effect upon the vital centres, and the other one comparatively innocuous, much in the same way as a streptococcic tonsilitis differs in virulence from a Klebs-Loeffler infection. Dr. Wyncoop in his preliminary report says it has been occasionally observed by physicians that when influenza was present in the household the eyes of the members of the family suddenly became acutely inflamed, accompanied by severe pain, swelling, and redness of the conjunctiva, with a profuse discharge of pus of medium consistence and cheesy appearance. In that the disorder differed materially from acute conjunctivitis, it was suspected that the bacillus of influenza might have something to do with the diseased condition. Cultures were made from discharges, and the suspicion was verified by finding in great numbers the influenza organism. Experiments are now being made upon the eyes of guinea-pigs with pure cultures of the bacillus, and it is hoped that we will soon be able to report further concerning this additional manifestation of the ubiquity of this maleficent microbe.

Symptomatology.-The symptomatology, considered in its principal features, does not differ from ordinary influenza, excepting by such variation as the rapidity of the course, and the more profound constitutional impression which the disease would naturally exhibit. In this respect the symptoms and signs of la grippe and influenza are analogous in relationship to those of Asiatic cholera and cholera morbus. The extreme virulence of the infection is often illustrated by the death of strong men within a day or two after their seizure. The great and prompt mortality among children and debilitated persons is also an evidence of its strong infective character. The effect upon the nerve centres is marked by an intense degree of debility which is out of proportion to the local expression of the disease, and constitutes a striking feature. Favourable changes in atmospheric conditions seem to have no effect in checking the course of the disease; but it is noticed often, how- 
ever, that undulations of cold, damp weather will serve to aggravate its spread and intensity; but from this fact we must not infer that the prevalence of the disease is more than incidentally related to such atmospheric vicissitudes.

Course.-The disease is really a very dangerous one, and is responsible for a large share of the mortality of large cities. In England, for instance, an increased death-rate, largely due to la grippe, and especially severe upon old people, has of late in London reached as high a figure as 38.5 per cent, and in Liverpool the frightful rate of 55.5 per cent has been recorded.

The above statement will apply equally to the other European countries, especially Germany, Austria, and Russia. In the United States, however, while the disease manifests as intense a virulence, yet, the domiciliary and domestic conditions being better, the amount of fatality is somewhat less.

The course and termination may be summed up as an irregular regularity ; its complications and anomalies are legion. For instance, a case may seem to be progressing with favourable regularity, when all at once may appear signs of relapse owing to serious implication of one of the internal organs or of the nervous system. Therefore, diverse paralyses, secondary pneumonia, hepatitis, pericarditis, endocarditis, pleuritis, nephritis, neuritis, etc., are frequent complications. Among the most common of these may be mentioned a septic diss̀eminated catarrhal pneumonia, which usually proves fatal under any sort of management. In these cases it would seem as if complete paralysis of the pulmonary vasomotor nerves had taken place, since oftentimes the lungs seem to fill up with blood in a few hours to such an extent that life soon becomes extinct. I have known of such a complication running its course in from fifteen to twenty hours in a patient who was just about to sit up and receive the congratulations of friends over the favourable termination of the "hard illness." In other instances such relapses may appear during the first or second week of apparent convalescence, and then "carry off" the patient. Therefore, indeed, the profound impression which the disease makes upon the heart and circulatory apparatus is a matter of common observation, so that it has come to be one of the expected complications. Even in cases of moderate severity the pulse may be feeble, frequent, and irregular- 120 to 140 
per minute-while tachycardia and acardia are frequently met with even during convalescence. The respiration is also usually irregular in rhythm and frequency, even when no extensive pulmonary lesion exists. The period of convalescence is really an unknown quantity, for it will be observed that many patients do not regain their normal condition until after the lapse of weeks or months.

Sequelæ.-The sequelæ of la grippe are also diverse. The most common are paralyses, more or less partial or extensive, affecting the laryngeal nerves (especially the inferior); the ocular and maxillary branches of the fifth or some of the spinal nerves, also neuropathies, affecting the nerve centres or ganglionic nervous system. Many cases of the various forms of mental disease are traceable to an attack of this disease. Chronic catarrhal affections of the pharyngeal and laryngeal mucous membrane not infrequently owe their origin to influenza and la grippe. Pleuritis and empyema are frequent results of an attack of influenza and la grippe, and so are inflammations of the middle ear and mastoid cells. Undoubtedly one of the most alarming of the morbid conditions following la grippe and influenza, and one which has not been sufficiently recognised, is phthisis pulmonalis. During the first part of our late pandemic (1889-'90) I was loath to accept the statements of many patients suffering from phthisis, who invariably attributed the beginning of their disease to an attack of la grippe. But, after my attention had been so frequently and forcibly attracted to this point by so many statements, I took pains to carefully investigate the personal history of a number of cases, and in consequence was led to the conviction that many of these patients' statements were correct. Whether or not this idea comports with the bacillary theory of the origin of phthisis pulmonalis may be questioned; nevertheless, the clinical fact above stated may be so well attested by careful observations as to thoroughly convince any practitioner who may take the trouble to investigate for himself.

Prognosis.-The prognosis of this disease is always uncertain; the careful practitioner ought never to give an entirely favourable prognosis, at any rate during the period of activity of the epidemic. Cases with the most favourable aspect in their earlier stages may suddenly develop the most serious complications. Moreover, no patient having suffered an attack of la 
grippe ought ever to be lost sight of entirely by his physician until full restoration to health has been accomplished.

Treatment.-The practice of giving cardiac depressants in the early stages of this disease is dangerous, since the utmost care should be taken to sustain as far as possible the excitability of the ganglionic nervous system. It is not the direct action upon the cardiac centres which produces so much mischief as it is the repression or alteration of the functions of the vasomotor nerves. Any effect of this sort may result in a paresis and interference with hematosis which might induce a dangerous and perhaps irrevocable pulmonary engorgement. Therefore, with this point in view only such antipyretic measures are to be adopted as will contribute the least toward the vascular derangement of the parenchymatous organs. For this reason, even though the temperature be high, the administration of acetanilide, antifebrine, phenacetine, or antipyrine is inadvisable. Aconite, tartar emetic, or even veratrum viride are to be regarded as preferable, because less prompt in their effects as "cardiac depressants." The idea that any of these agents can be administered with greater efficiency and safety, when guarded by digitalis, strophanthus, nitro-glycerine or other antagonistic cardiac stimulants, is decidedly questionable. A moment's reflection upon the physiological action of the several coal-tar derivatives will show that they are not at all synergistic with the cardiac stimulants mentioned, and consequently if acting at all will neutralize the effect of the latter. The safest way of reducing temperature in these cases is by carefully sponging the whole or part of the body with cold water followed by the applications of heat, if necessary, and the use of saline depressants. In other words, the skin and the intestinal canal should be the battle ground at first in the fight against hyperpyrexia. Phosphorus, quinine, salol and later strychnine will prove to be the most efficacious agents for combating the disease throughout its course; while opium, hyoscyamus, and belladonna will prove, as a rule, the safest anodynes. Hyoscyamus, codeine, and belladonna are usually sufficient to neutralize the distressing symptoms, and can usually be employed with safety. Chloral hydrate may be used with care while there is a rise of temperature, but should never be administered in any considerable dose when the temperature is falling. Alcoholic stimulants may be employed 
early in some cases, especially in those who have been accustomed to their use as a beverage, but the quantity administered should always be carefully scrutinized lest unpleasant toxic effects be produced. Strychnine, digitalis, and nitro-glycerine are essential in cases of advancing or impending depression; if time be an important element these agents should be given hypodermically, but it is not well to administer any of them too early in a case of ordinary severity lest the system become obtunded to their effects. Phosphorus, as a rule, is well borne throughout the course of the disease, and, although somewhat slower in its effect than some of the more diffusible stimulants, may be considered to be one of the most important agents that can be selected.

The iodide, carbonate, and other preparations of ammonia may be freely used to promote expectoration and glandular activity. For the same purpose, in cases showing an intense bronchial inflammation from the beginning, and where the pulse is not too frequent or weak, sanguinaria may be employed, either alone or combined with ammonia muriate, with satisfaction; but if much cardiac depression be noticed, it must not be given. It is a good plan, as a matter of routine practice, to administer large quantities of water, preferably distilled, excepting when it causes distress of the stomach or intestines. Should there be evidence of more than ordinary engorgement of the kidneys, digitalis, preferably the infusion with potassium acetate, will be found highly beneficial.

The diet should be fluid while the disease is active, and above all things an equable warm temperature should be maintained in the sick-room. 


\section{CHAPTER V}

\section{DIPHTHERIA}

Definition.-An acute communicable disease characterized by a croupous inflammation of the throat and severe toxæmia.

History.-History shows that man has suffered from the ravages of diphtheria from a very early period. Perhaps no disease has been more written about by ancient authors than this one. Pythagoras wrote of a disease which consisted of "closing up of the throat." A graphic description of its clinical history was given by Aretæus. Galen, in the second century, wrote exhaustively upon the disease, and it is mentioned in the Talmud. Asclepiades wrote of it also about the end of the fourth century. Paul of Ægina, about 660 A. D., devoted considerable attention to the disease. It was known to the people of Europe in ancient times generally as "Malum Egyptiacum." During the middle ages there was not so much written concerning it. Bryonios wrote of an epidemic in Rome occurring in 856 and 1004 A. D. Van Woerd reports an epidemic which spread in Germany in 15I7, and shortly after appeared in Amsterdam. It seems to have first appeared in Spain at the commencement of the sixteenth century, having probably invaded that country from France, where in 1576 a severe epidemic, especially in the large cities, was described by Baillon. One of the worst epidemics which visited Europe was the one which spread through Spain and Italy at the end of the sixteenth and beginning of the seventeenth centuries under the name of the "Garrotillo." The mortality was very great, inasmuch as authors writing about it distinctly describe a malignant septic form. Many of these ancient authors distinguished and described cases where death was due to septicæmia or general intoxication; they also, especially Villarial and Herrera, described the paralytic sequences. Villarial, Cortesein, and Mercado drew attention to its contagious character. 
According to Francotte, in both Italy and Spain at that early time tracheotomy was performed, and sometimes with success, for the relief of these patients. Its first appearance in Great Britain is supposed to have been somewhere between 1638 and 1663 , at which time it was written upon by John Josselin and others. Its advent into North America was noted in 1735. to I770 by Colden, Douglas, Bard, and others. It was subsequently written upon in England by Home, Fothergill, Grant, Huxham, and Starr. Malouin dates the first appearance of the disease in Paris in 1743 , and described a very severe epidemic in 1748 and 1749 . Francotte states that in 1807 Napoleon I decreed an international congress for the consideration of croup, which was reported by Royer and Collard. There were seventy-nine memoirs presented to this meeting. Jume, of Genoa, and Albers, of Bremen, took the prizes.

In 1847 Virchow first pointed out a distinction between croupous and diphtheritic inflammations. Finally, the accurate observations and succinct contributions by Bretonneau and Trousseau seemed to complete the literature of the subject to modern times; indeed, since the work of Bretonneau, Trousseau, and Virchow very little of importance has been contributed until the present era of bacteriological investigation, noted by the important discoveries of Klebs, Loeffler, Behring, Roux and Yersin, Brieger, Fränkel, and others. Our present knowledge, therefore, regarding the microbic. cause of diphtheria may be said to have begun in 1883 , when Klebs found by microscopic examination of a number of cases the diphtheria bacillus.

During the following year Loeffler succeeded in isolating the bacillus in absolutely pure culture. He was therefore able to describe minutely its morphology, tinctorial, cultural, and biological properties. By so doing he furnished a firm, permanent, and reliable foundation on which subsequent workers could build with confidence. Loeffler affirmed the observations of Klebs regarding the presence of this bacillus in the false membranes of the disease. The fact that the bacilli were always found in guinea-pigs at the point of inoculation, and not within the organs, led Loeffler to believe that the microorganisms produced a powerful poison which was absorbed and carried through the body. This, however, was subsequently established by Loeffler's own researches in 1887 , and 
in 1888 the poisonous products of the bacillus were isolated; the researches of Roux and Yersin in 1888 , and Brieger and Fränkel in 1890 were exhaustive and important. Loeffler also found a bacillus identical to it, morphologically and physiologically, in the mouth of a healthy child. He also observed that the typical diphtheritic paralysis noted in man was not reproduced in animals; also, when applied to the uninjured mucous membrane of animals the cultures had no effect (Novy).

Etiology.-The essential exciting cause of this disease is now demonstrated to be a micro-organism called the KlebsLoeffler bacillus, which generally attacks first the mucous membrane of the fauces or larynx.

The Klebs-Loeffler bacillus is a small organism 2.5 to $3 \mathrm{~mm}$. in length and 0.5 to $0.8 \mathrm{~mm}$. in thickness (Fig. 41). Among its striking features are its variation in form, and the irregular manner in which it takes the stain. The ends of the organism are frequently clubbed, although the form and size vary greatly under diverse circumstances. When it is stained it shows a series of clear spaces with intensely

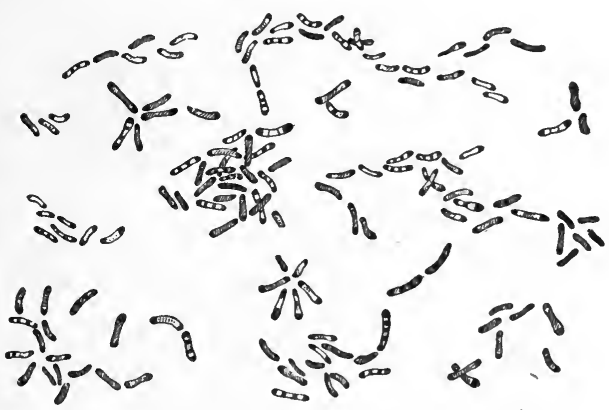

FIG. 41.-Klebs-Loeffler bacillus (Stengel).

stained particles. It grows readily on a variety of culture media, and most readily on the modified blood serum (first introduced by Loeffler), and does not form spores. According to Welch and Abbott, it may be killed in fluids by an exposure of ten minutes to $58^{\circ} \mathrm{C}$. $\left(136.4^{\circ} \mathrm{F}\right.$.). Under favourable conditions it may remain alive for weeks or even months in fragments of dried membrane. The bacillus of diphtheria is best stained with Loeffler's alkaline methylene-blue solution (Rotch).

Contagion.-While the disease has long been recognised as an infectious disease, there has from time to time been much discussion as to the degree of contagiousness-in other words, as to the liability of its spreading through intermediary means, such as by clothing, etc. 
Notable instances have been observed, however, of the contagium retaining its activity in clothing for a considerable time, and afterward, under favourable circumstances, giving rise to the disease in susceptible individuals. The origin of endemics through articles of clothing, etc., in places free of the disease for years is cited as proof of this property of the contagium. On the other hand, testimony of weight has been offered to show that endemics of diphtheria have arisen in comparatively new and isolated places remote from the public highways, without any known introduction of the diphtheria germs through the personal appurtenances of any individual.

Predisposing Causes.-As a rule, there are several wellknown predisposing causes.

Susceptibility:- Some families are more susceptible than others, and persons with chronic pharyngeal or tonsillar disease, or adenoids, are more susceptible. Ravolli points out certain analogies between diphtheria and tuberculosis, and gives a record of observations in twenty-one families who were predisposed to tuberculosis where diphtheria seemed to appear without any special occasion for contagion. He combats the contagiousness of diphtheria, or at least believes it to be exaggerated, and thinks that diphtheria and tuberculosis coexist as special tendencies in the same family.

Hagenbach found on post-mortem examination that ten per cent of the diphtheritic cases were tuberculous. In the twentyone families cited by Ravolli, five had already shown tuberculous manifestations.

Hoppe-Seyler found that twenty-three per cent of the cases he examined were tuberculous. He also found that out of two hundred cases of diphtheria admitted to the hospital there were forty-two in which there was no tuberculosis either in the patients themselves or in their families, which gives a percentage of twenty-one.

Age.-By far the greater number of cases occur in childhood, and heretofore diphtheria has been considered one of the most fatal of the diseases of early life. Adults, however, are not immune, although not as susceptible as children.

Diphtheria in early infancy is very rare. Henoch, in 1,403 cases collected, found none under three months. Feer, in 4,250 cases in Basle, found seven only under three months. Only two and six tenths per cent of the whole number were under 
one year of age. The causes of this exemption are, first, the slight opportunity for infection; second, the antitoxic properties of the blood of infants, as proved by Fishel, von Wunchiem, and other observers; third, the absenoe of catarrhal conditions of the upper air passages.

Sex.-There is no distinction with regard to sex, although after the age of seven years more boys are supposed to be affected than girls, owing to the extra outdoor life and exposure of the former.

Season.-Diphtheria is apt to prevail during the spring, autumn, and winter seasons, although large communities are seldom free from it the year round. The degree of virulence of the disease varies by epochs. Sometimes in a certain community the cases occurring during the spring may run a much more severe course than those occurring in the same community the following autumn, or vice versa. Again, it has been frequently noted that the virulence of epidemics or endemics of diphtheria are cyclical, taking perhaps several years for reaching a certain acme of intensity and then declining for the next few years.

This undulatory character has been observed from the earliest times. Previous acute catarrhal attacks invite the advent of diphtheria. Endemics of influenza and measles in northern climates are frequently accompanied by diphtheria. Likewise cases of acute follicular tonsilitis, in localities where diphtheria is prevailing, invite attacks of the disease, so that we may have, as it were, the two diseases coexisting.

Social Condition. - Social condition seems to have no marked influence as a predisponant; for although in crowded communities a large proportion of the poor and squalid are sufferers from the disease, yet if proper isolation and hygienic care be instituted the number of new cases becomes rapidly lessened, showing conclusively, from an individual standpoint, that the children of the poor are no more liable than those of the well-to-do, excepting as they may be subjected to greater exposure.

Pathology.-The pathology of diphtheria, as might be expected, has drawn to itself considerable talented attention during the last century. Its parasitic nature was predicted by Laycock, of Edinburgh, in 1858. He promulgated the doctrine that diphtheria was caused by a vegetable fungus, 
which he thought to be the oidium albicans. Soon after Jodin also found similar epiphytes, as he supposed. Later Thomas Hillier, Buhl, Hüter, James, Wendeberg, Clawsen, Wardlof, Senator, Eberth, Letzerich, Cohen, Cornil, Negli, Talamon and a few others were working along this line of thought-the parasitic nature of the disease-and each of them contributed from time to time important discoveries of micro-organisms which were variously named Leptothrix buccalis, Diplosporum fuscum, Zygodesmus fuscus, Tellitia diphtherica, Oidium albicans, etc. These were variously described and their nature, habitat, and behaviour well noted (Francotte).

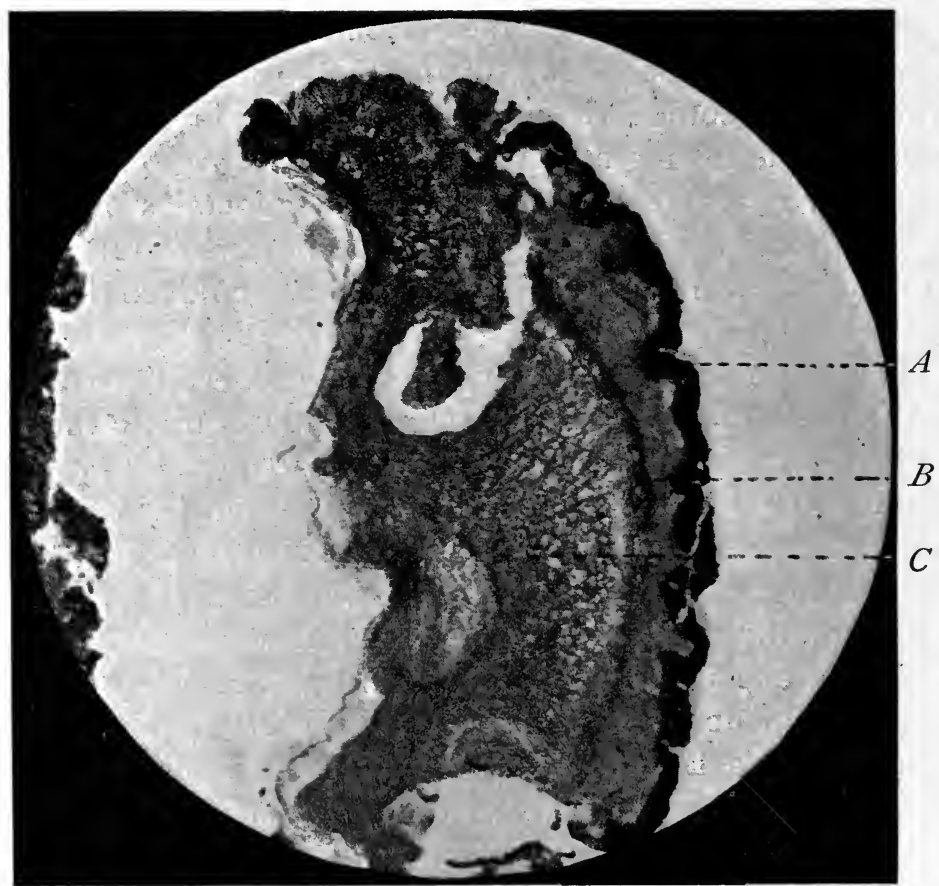

FIG. 4ra.-Photomicrograph of exudate from a case of pharyngeal diphtheria $(\times 60)$. $A$, squamous epithelium undergoing necrosis ; $B$, zone of leucocytes; $C$, fibrin.

Thus it will be seen that the ground has been continuously worked over down to the brilliant bacteriological and biological demonstrations of Klebs and Loeffler, which showed up the specific microbe and its poisonous toxines, so that now the pathology of this dread disease may very properly be said to consist of three principal elements: First, some antecedent 
circumstances for preparing the soil or the part attacked (pharynx, larynx, soft palate, etc.); second, the lodgment of the specific bacterium; and, third, the consequent fermenting process resulting in the development of the toxine, which becomes absorbed and carried through the system by the blood stream. From this, therefore, it appears that diphtheria is at first essentially a local disease, and secondly, that its constitutional or systemic features depend upon the particular effects of the toxine which the local fermentative process induces. Many observers speak of the toxine as secreted by bacteria. This theory, it seems to the writer, is untenable, for the bacterium is not endowed with a specialized apparatus for secretion; it is more likely, in accordance with the behaviour of microorganisms in general, that instead of themselves secreting or manufacturing toxines, they simply alter or split up the biochemic relations of the fluids or cells of the part upon which they lodge, thus inducing a fermentation. Buchner and Hahn have succeeded in obtaining a clear yellow fluid by crushing or compressing the cells, with a pressure of from four hundred to five hundred atmospheres; and after every vestige of the living cell has been removed by filtration, the liquid discloses an important property, that of producing alcoholic fermentation, thus refuting the hitherto accepted idea that fermentation is associated only with the presence of living cells. It would seem highly improbable that specific micro-organisms by themselves, although entities, without the aid of antecedent factors, produce this characteristic disease, inasmuch as the micro-organisms are often present, together with other pathogenic germs, in the mouths and throats of healthy individuals. David and others show conclusively that these germs commonly inhabit the buccal cavity and pharynx of man. Now, whether the disease is precipitated by some antecedent circumstance or accident to the economy which destroys the defensive power of the secretions of the mucous membrane of the throat, and thus gives opportunity for the action of the micro-organism in its special direction, or whether some untoward event connected with the economy of the part arrests the normal phagocytic power of the various leucocytes, or whether some other event that we know not of transpires to bring about a suitable medium for the beginning of the disease (such as inflammation) by bestowing upon the bacterium a pathogenic 
power, are questions which as yet remain unexplained. From a practical point of view, however, it would seem that professional opinion is coming back to the doctrine of Oertel, promulgated in 1868 , that diphtheria is to be considered at first a local disease, and, secondly, a constitutional infection by means of the toxine generated.

Inoculability.-The disease is undoubtedly inoculable. Laboratory experiments show that the blood of an infected animal will produce the disease in another susceptible animal when inoculated. Accounts so far are not clear as to whether in all instances any local manifestation is produced, excepting at the point of inoculation, in animals which have been made to acquire the disease by inoculation (Novy).

Metin inoculated rabbits and guinea-pigs with emulsions in bouillon or serum culture of the diphtheria bacillus. At intervals he examined the blood for organisms, and found that they had completely disappeared in an hour, having been ingulfed by phagocytes.

Reisman reports two cases of direct inoculation. One, a bacteriologist, while using a pipette, drew a few drops of a virulent culture of the diphtheria bacillus into his mouth. The period of incubation was forty-eight hours. About six thousand units of antitoxine were injected, and recovery took place. The patches disappeared on the fifth day. The diphtheria bacillus appeared in cultures from the secretion of the tonsils for about three weeks afterward, notwithstanding the local use of antiseptics. The second case was that of a child eleven days old.

Immunization.-According to Ehrlich's theory, the contaminating toxine operates by overcoming, according to circumstances, the natural antitoxine of the blood. The present methods toward immunization (hypodermatic injection of antitoxine) bid fair to stamp out this dread disease. Undoubtedly many persons are, at least for a time, naturally immune, but a knowledge of the fact before an exposure to the disease is always wanting. Triolo and others have discovered that a filtered saliva has strong bactericidal properties; analogously it may be mentioned that Lassaigne, a French chemist, many years ago discovered that the fluid secreted in the pharyngeal cavity of the herbivora is antiseptic. Immunization against serpent poison, anthrax, and tetanus are further examples of steps taken by preventive medicine in this direction. 
Ehrlich's theory of immunization is highly ingenious, and it is to be hoped that his conclusion may in the not far distant - future be satisfactorily demonstrated.

Symptomatology.-Diphtheria, like all other diseases, presents itself in different grades of severity, from a very mild type, scarcely as severe as follicular tonsilitis, to a grave form of primary blood poisoning which may destroy life within twenty-four hours after its onset. Between these two extremes, therefore, all sorts of clinical aspects are presented. A typical case usually comes on after a day or two of malaise, and presents first a stiffness of the neck, slight soreness of the throat and swelling of the cervical glands, a distinct rigour, or simply sensations of chilliness, followed by aching of the muscles, and elevation of temperature. There may be at this time an attack of vomiting, with more or less headache and suffusion of the eyes, but the principal complaint will be that of weakness, myalgia, soreness of the throat, and fever. The degree of odynphagia, however, varies greatly. Sometimes it is very slight indeed, and at other times it is severe, according to the amount of local inflammation of the fauces.

The period of high pyrexia rarely lasts longer than two or three days, the temperature seldom going above $103^{\circ}$ to $104^{\circ} \mathrm{F}$. After this it falls to a range of between $99^{\circ}$, and $100^{\circ}$, or $101^{\circ}$. The exacerbations of temperature are sometimes quite irregular, coming perhaps in the forenoon or evening, or perhaps only in the evening; but, as a rule, a slight rise at least takes place in the evening, and is followed by a corresponding fall to near the normal or sometimes below, in the morning. There is rarely much, if any, perspiration following the decline of temperature, excepting in severe cases of septic poisoning, when something like a crisis may occur. A characteristic symptom, which usually endures from three days to a week, is pain in the lumbar region, varying in different cases from a slight to an excruciating pain; indeed, some patients complain of this much more than they do of the soreness of the throat. At the end of a week the general symptoms may become of a graver character, according to the amount and severity of the sepsis. The pulse, which perhaps has maintained its tension up to this time, may now become very frequent and small, the countenance may present a leaden hue, the mouth remaining 
open, the fingers cyanosed, the skin moist and clammy, at the same time the patient showing considerable mental hebetude and indisposition to move or talk or take nourishment-in short, very marked inattention to external surroundings. The heart's action may be feeble and become the source of great anxiety.

Hibbard investigated the heart signs in eight hundred cases of diphtheria, and found that when the pulse-rate was above I 50 the mortality was fifty per cent. Irregularity of the pulse showed a mortality of forty-seven per cent. A slow heart did not appear to be dangerous, except in children. Patients who died of cardiac failure showed degeneration of the pneumogastric nerve. Two or three days after the advent of the disease the tenderness about the neck becomes marked, and in a majority of instances of pharyngeal or naso-pharyngeal diphtheria the cervical lymphatic glands are swollen and more or less hard. If the nose or naso-pharynx be involved, a characteristic stoppage of the nasal passages is apparent, and soon an almost constant discharge of ichorous fluid appears, accompanied by attempts on the part of the patient every little while to clear the nose by blowing, hawking, and by screatus.

Objective Symptoms. - The appearances of the throat are quite characteristic. The disease usually manifests itself at first upon one or both tonsils; occasionally, however, its first appearance is upon the uvula, the lateral or posterior walls of the pharynx. The affected mucous membrane appears swollen, and of a peculiar bluish-red colour; upon this there is soon formed grayish-white films of limited area, which rapidly coalesce and thicken. From these points the exudate gradually spreads down over the tonsil, or up toward the soft palate and uvula. If the local process be unilateral at the start, within the first day, or perhaps at the end of the second day, small patches will be noticed on the other side of the pharynx, and from this focus, from time to time, the exudate will gradually spread until the other side is covered.

It is very rare indeed that the exudate spreads into the buccal cavity, but it may gradually ascend to the naso-pharynx (ascending case), or it may descend toward the larynx, invading first the pyriform sinus and afterward the larynx itself (descending case). But when the exudate reaches a low point in the pharynx there is more or less tumefaction of the epiglottis, ary-epiglottic folds, and sometimes of the laryngeal mucous 
membrane, although there may not be any exudate within the cavity of the larynx. The pseudo-membrane, which at first seems of a grayish-white colour, becomes changed to either a buff or a slaty colour. It is closely adherent to the mucous membrane and only with difficulty can be removed, and as layer after layer is deposited the false membrane becomes decidedly thick and tough. If the disease progresses rapidly the exudate may cover a good deal of the region of the fauces in a very short time, successive layers forming rapidly in spots. If exfoliation takes place the spot may soon be covered again. Even after the line of demarcation seems to have been reached, and the disease remains passive for a day or so, an advancing line of exudation may take place after this interval, and continue to extend farther, so that until the disease has run its course the patient may not be considered exempt from a renewal of exudation. If the pseudo-membrane be detached by force, the mucous membrane is observed to be excoriated, showing little bleeding points here and there, which indicate that at least the epithelial layers are included in the false membrane. While the limits and direction of the exudate can not be definitely predicted, yet it may always be expected that when the exudate extends principally upward, that the naso-pharynx and posterior surface of the soft palate, and possibly the nasal passages, will become involved before the disease runs its course; or if, on the other hand, the exudate begins at the lower portion of the tonsil or upon the lower portion of one of the pillars, and tends to spread downward, there will be a probability of the involvement of the larynx. When the exudate, however, seems to be pretty well confined to the faucial tonsil, and after the third day of the beginning of the disease there are no more large patches observed upon other parts of the throat, it is fair to presume that the local process is about to cease.

Varieties. - For clinical reasons certain varieties have been recognised by observers according to location and the severity of the course; thus the disease has been designated as nasal diphtheria when the false membrane has extended to the nasal passages or naso-pharynx. It is designated laryngcal diphtheria or croup when the primary seat of the disease is in the larynx; catarrhal diphtheria when the attack has been so mild that scarcely any distinguishing features, such as false mem- 
brane, could be observed; and grave, malignant, or septic diphtheria when the system seems to be very much overwhelmed by the primary intoxication.

The disease has also been distinguished as endemic and epidemic according to the extent of territory over which it prevails.

Anomalous Cases.-There are various anomalies met with which deserve to be mentioned; for instance, there are cases where the same individual is attacked repeatedly within a year or a season. There are cases of chronic diphthcria where the

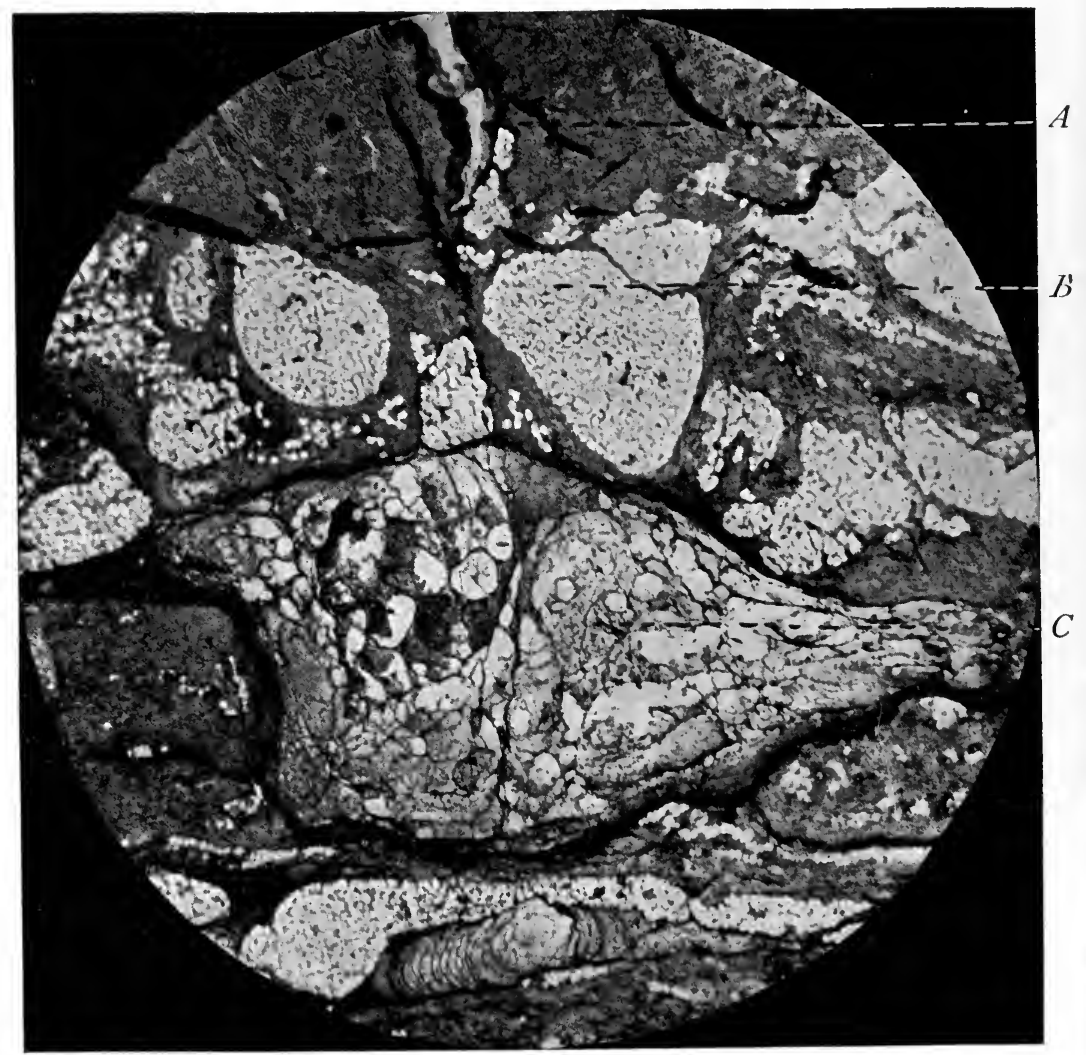

FIG. $4 \mathrm{I} b$. - Photomicrograph of vertical section through tracheotomy wound on the fifth day. $A$, integument ; $B$, fat ; $C$, fibrin and leucocytes.

local diphtheritic process may remain for an unusually long time, producing only slight constitutional disturbance, or at least short acute or subacute recurrences.

C. Meyer has reported two cases. The writer has met with 
a few such cases, three of which were probably primary nasal diphtheria. It is to be noted in all such cases that the process, as a rule, is confined to the anterior and middle portions of the nasal passages. (See Fibrinous Rhinitis.) Nasal diphtheria sometimes insidiously extends to the inside of the Eustachian tubes, producing diphtheritic otitis. It may also follow the nasal duct to the orbit and thus produce diphtheritic conjunctivitis and endanger the eye. There are many cases recorded of diphtheria affecting the mucous membrane of the eyelids. The rectum and vagina may also be the seat of diphtheritic inflammation. The writer has never seen a case of diphtheria of the anterior portion of the buccal cavity. It is well known that a diphtheritic exudation will frequently cover portions of the skin which have been denuded of its epidermis.

Another anomaly met with not infrequently concerns the constitutional phenomena. During virulent epidemics death may take place from the primary impression on the nerve centres made by the initial poisoning. In such cases the exudate may be very slight. Many observers believe that in such cases the naso-pharynx has been the seat of the local process and has not been detected.

Another class of cases not infrequently met with includes a very mild primary toxæmia followed by a virulent secondary poisoning at about the end of the first week. Such cases may show at first little, if any, involvement of the lymphatic glands of the neck, but shortly an increase of exudation, a rapidly growing intumescence of the lymphatic glands, a lowering of the pulse, extreme adynamia, mental hebetude, and other symptoms of approaching collapse may ensue.

Another class of cases exhibits an eruption very similar to a scarlatinal rash. This may appear on the second or third day, but more frequently between the seventh and tenth days independently of the exhibition of antitoxine. When this eruption appears early it complicates somewhat the diagnosis, especially if scarlet fever be prevalent in the neighbourhood. Scarlatinal eruptions in diphtheria were first called attention to by Germain Sée in 1858 , and also about the same time by Dr. Jacobi, of New York.

This class of cases was formerly described more often than nowadays by clinical observers and considered to be a mixture of diphtheria and scarlet fever. Although the idea has 
been combated that these two diseases could coexist, yet a large number of cases have been observed from time to time during the last fifty years in which the clinical signs of each disease-scarlet fever and diphtheria-seemed well marked. Lately by bacteriological examination the diphtheritic character of the local exudate in some of these cases of supposed scarlet fever has been confirmed. The writer has observed several cases in the diphtheria and scarlet-fever departments of Harper Hospital in which the characteristic clinical history of each disease running concurrently in each case seemed to be marked. It will be unnecessary to discuss the clinical history of these cases in detail ; suffice it to say that the high temperature, eruption; desquamation, and other signs of scarlet fever were present, together with the Klebs-Loeffler bacillus. The writer offers no theory in explanation of these facts.

Complications and Sequelæ. - Diphtheria being a general infectious disease, communicating its toxicity to the fluids of the body, may affect almost any of the organs or apparatus through the medium of the circulating fluids. The mouth, as stated before, is seldom attacked, although the older authors cite a few examples. The esophagus and stomach are also rarely involved in the process, although a few cases have been reported. Concerning the stomach, two cases have been reported by Lutz, one by West, and one by Fränkel. Diphtheria of the intestines, especially of the large intestine, has been observed, several cases having been reported by Roche and Bizzozero, while diphtheritic exudate upon the margin of the anus and rectum has been frequently observed. The diphtheritic process has frequently taken place in the vagina, and gynecologists record a similar form of disorder affecting the lining membrane of the uterus. Cases of this character were recently reported by Dr. H. W. Longyear, of Detroit. Williams (J. W.) reported a case of diphtheria of the vulva in which the KlebsLoeffler bacillus was demonstrated. Recovery took place after antitoxine was used. He states that there are on record only two cases of diphtheria of the vulva properly confirmed by bacteriological examination; so-called diphtheritic endometritis and puerperal diphtheria having nothing to do with true diphtheria. Streptococcus pyogenes may cause pseudo-diphtheritic membrane in any part of the genital canal. Freymuth and Petruschky report a case of vulvitis gangrenosa (noma 
genitalia) in which the diphtheria bacillus was found. Recovery after antitoxine took place in this case. They also report a second case of diphtheritic noma, this time of the face. Frederick Smith has demonstrated the presence of diphtheria bacilli in the urine of guinea-pigs inoculated with culture of the bacillus.

The salivary glands have been reported by Balzer and Tobaman to have been affected. Several pathologists have reported that the liver and spleen participated in some cases.

The respiratory tract is frequently the seat of the diphtheritic process in children. Those cases known as descending, in which the exudate occupies the larynx at an early stage, are very apt to terminate in a true diphtheritic bronchitis and pneumonia.

The kidneys are almost always involved in all cases of diphtheria. Of course several degrees of disturbance are observed, accorting to the character and amount of the poison. The vulnerability of the kidneys to this as well as to other infections is very easily explained by the fact that Nature undoubtedly calls upon these emunctory organs soon after the access of the disease to eliminate the poison.

Albuminuria on that account is one of the earliest manifestations of the disease. It was first described by Wood, of Birmingham, in 1857 , while some months later Germain Sée, independently of Wood, called attention to it. From that time to the present it has been considered one of the regular clinical features of the affection.

Next to the kidneys the nervous system seems to suffer more frequently than any other remote organ or apparatus. The most notable lesion of the nervous system is manifested by paralysis, although other perturbations of function, such as hyperæsthesia and neuralgia, have been noticed in some instances. Although the lesions of the nervous system had been recognised casually by the older writers, it was not until 1863 that these lesions were first particularly pointed out by Charcot and Vulpian, and about a year later Oertel called attention to a general muscular atrophy which accompanied some of the severe cases of diphtheria. In 1876 Rieman showed lesions of the medulla oblongata following what he designated as diphtheritic meningitis, and Deegarine demonstrated lesions of the anterior roots of the spinal nerves which he honoured by the 
name pachymeningitis, but he found no alteration of the posterior roots. In subsequent investigations he located very. decided lesions in the axis cylinders of the nerves. Ghisi in I864, and Manigault subsequently, amplified the researches of these observers. During the last thirty years pathologists have frequently confirmed the observations of these earlier observers, although some of the modern ones have severally laid greater stress upon cortical lesions, lesions of the axis cylinders or of the muscular corpuscles. As said before, paralysis is one of the most important sequelæ of diphtheria. It usually appears more marked from three to six weeks after the termination of the attack, although occasionally it comes on earlier. Its more common site is in the throat, the soft palate and the pharyngeal and laryngeal muscles being involved in the order mentioned. Next to these parts the upper extremities suffer, after that the lower extremities, and next in frequency the ocular muscles. In aggravated cases both the upper and lower extremities may be paralyzed. The writer observed a case in Harper Hospital a few years ago, that of a woman in whom almost all the voluntary muscles suffered from paresis. This was an aggravated case and lasted for a period of five months. She could swallow with great difficulty, and was obliged to be fed with care, it being necessary sometimes to introduce the stomach tube. The special senses of vision, hearing, and phonation were also involved. The electro-muscular sense in this woman was very feeble. Her mental faculties, while perhaps a little slow, were not notably impaired. I learned subsequently that she made a tolerably good recovery after a period of eighteen months from the time of the attack of diphtheria. Dr. E. W. Presley, of Clover, S. C., reported a case of a girl eight years old, in whom paralysis followed a severe attack of diphtheria in the following order: First, paralysis of the soft palate, then of the ocular muscles, giving rise to strabismus; afterward the sphincter muscles of the iris became paralyzed, so that the pupils were enormously dilated. In a short time both arms and legs were involved, knee jerk being entirely absent, and the sensibility of the skin was diminished. This case recovered. Indeed, entire recovery from these paralyses is the rule.

The onset of a paresis is usually accompanied by a recurrence of fever, which may last from twenty-four hours to three 
days. One of the earliest symptoms of palatal paralysis is a change in the quality of the voice from the ordinary to the nasal, and the regurgitation of fluids through the nose in the act of swallowing. In many cases semisolid and solid food can be swallowed with much more ease than fluids. A considerable degree of paralysis of the muscles of deglutition-especially if accompanied by paralysis of the upper laryngeal muscles-is dangerous on account of allowing the ingress of food or other foreign material into the larynx. For this reason, the impaction of food in the larynx or trachea may cause death by asphyxia, or a dangerous and fatal traumatic pneumonia may arise from the continued ingress of food into the bronchial tubes.

Dr. Frederick J. Batten has studied carefully the pathology of diphtheritic paralysis in the human subject. He examined five cases of paralysis ranging from forty-three to one hundred days from the beginning of illness. He used Marchi's method, as he found that the characteristic appearance of degeneration as shown by this method is not produced by post-mortem change. In the cases examined he found a parenchymatous degeneration in the myelin sheath of the peripheral nerves, sensory and motor. He found no changes in the nerve cells when studied by Nissl's method. He thinks the changes described by Muraviev in the cells of the anterior horn may have existed earlier in his cases and then disappeared, as they were all of oyer six years' standing. Thomas found changes in the peripheral nerves in diphtheritic paralysis, varying from a granular and broken condition of the sheath up to a disappearance of the sheath and axis cylinder (Thayer, Progressive Medicine, March, I899).

According to Paul Ehrlich's theory of immunization in diphtheria, there are in the diphtherial poison certain substances present along with the toxines, and these are formed by rearrangement of the molecules of the toxines. Some of these substances have less affinity for antitoxine than do the true toxines, and they do not combine with antitoxine until all the true toxines have been satisfied. These substances he calls epitoxoids. If large quantities of poison have been absorbed, these epitoxoids may be present in large amount after the true lethal toxines have all been neutralized. We should therefore expect that if some such substance as this be the cause of paralysis, 
the greater will be the proportion of paralysis as the test dose of the poison becomes larger. Woodhead found that the antitoxine itself did not produce paralysis. Paralysis did not occur in guinea-pigs which were given very large doses of antitoxine. E. W. Goodell says that paralysis has increased since antitoxine was introduced, but it is because patients now live to show paralysis, who without antitoxines would have died at an earlier period. W. A. Macfarlane and many others insist upon giving large doses of antitoxine. J. English reports a case of diphtheritic paralysis of the bladder-a very rare condition. There was incontinence of the urine for fifteen years, no paralysis elsewhere (Thayer, Medical Progress, March, 1899).

Pneumonia may arise, however, from the entrance and activity of micro-organisms, as well as from inspiration of food.

Dr. George Duffey and others state that micro-organisms peculiar to erysipelas, to influenza, to tuberculosis, to enteric fever, the Klebs-Loeffler bacillus or the anthrax bacillus, may each give rise to pneumonia.

Diagnosis.-Acute follicular tonsilitis (membranous tonsilitis), the throat lesions of some of the exanthemata, acute glandular fever of children, and pharyngeal mycosis, in some respects may simulate diphtheria. Acute follicular tonsilitis in its clinical history and symptoms certainly resembles the mild types of diphtheria more than any of the other diseases mentioned. Each one begins with malaise and chills, followed by more or less reactionary fever. Acute follicular tonsilitis is also contagious among children. At first sight the appearances of the throat in the two diseases seem very much alike, but the exudate in follicular tonsilitis will be found in small points located at the mouths of the follicles or crypts of the tonsil. It is white or grayish-white in colour, soft, and very easily removed with a brush or a swab, leaving no erosion of the mucous membrane beneath. Sometimes a clump or two will be more tenacious than usual, nevertheless it can be easily removed with the handle of a spoon, a brush, or a bit of wood, showing the surface of the mucous membrane beneath intact. There are cases, however, a little more severe than the typical ones, the exudates of which may show a decided fibrinous character. Such cases, when occurring during the prevalence of diphtheria, are often difficult to differentiate. Among the exanthemata, 
scarlet fever, variola, and measles are apt to be mistaken for diphtheria, but this mistake is likely to occur only during the very early stages of the illness. In variola the clinical history will serve to reach a proper diagnosis in the majority of instances. The acute glandular fever of children begins very much like diphtheria, by a swelling of the cervical glands and the lymphatic tissue of the mucous membrane of the throat, but inspection will soon reveal the fact that no exudate, or but a very slight coagulation of secretion, is found on the mucous membrane. Pharyngeal mycosis resembles diphtheria only in showing white spots on the tonsils or lateral walls of the pharynx. As no constitutional symptoms of any moment attend this disease, and as there is little or no inflammation of the mucous membrane of the fauces, all doubt may soon be cleared up, especially when the character of the exudate of diphtheria is taken into account. But, aside from the clinical history of the case, a thorough examination of the pharynx will show that the white patches of mycosis are not exudates, but adhering fungous growths. It must be remembered that temperature can not be relied upon absolutely as an aid in the diagnosis of diphtheria. Secondary syphilis may be mistaken for diphtheria while the condylomata are present in the pharynx.

All uncertainty may be cleared up usually by microscopic examination, for, since the discovery of the Klebs-Loeffler bacillus and its acceptance as the specific cause of diphtheria, the diagnosis rests upon the bacteriological examination of the exudates for the diphtheria bacillus, which differs widely from the characteristic mycelia and spores of mycosis. For purposes of examination the material (exudate) may be collected from the throat by means of a little swab of cotton which has been previously sterilized. The swab should be made by tying a little pledget of cotton to a piece of wire just long enough to go into a small test-tube. The wire swab is to be carried inside the test-tube, which has been thoroughly sterilized, the mouth of the tube being stopped by sterilized cotton wool.

The patient's throat should be inspected under good illumination, and the piece of cotton upon the wire brushed firmly across the tonsils, rubbing over any pieces of membrane which may be present. If a culture tube is at hand the solidified serum may be inoculated at once by drawing the swab lightly over its surface, after which the culture tube is plugged with ster- 
ilized cotton and the cotton swab returned to the proper testtube. If the culture is placed in an incubator it will be ready for examination in about eighteen to twenty-four hours.

There are, however, cases occurring from time to time, even during the prevalence of diphtheria, which are very puzzling from the fact that the typical bacillus can not always be found in the exudates. Bacteriologists have, however, isolated another micro-organism called the pseudo-diphtheritic bacillus and xerosis bacillus, to which organism is ascribed the occurrence of many of the atypical cases. There are some clinicians, however, who do not believe that this so-called pseudo or short bacillus is properly classified. W. Jacques, one of the medical inspectors of the Chicago health department, after examination of a large number of cases, arrived at the following conclusions: First, that the short Klebs-Loeffler bacillus apparently produces a toxine of greater virulence than the long one, although the local manifestations may not be so extensive. Second, that the long Klebs-Loeffler bacillus and streptococci, when found alone, give rise to a milder type of disease. Third, that the streptococcus is found associated with the Klebs-Loeffler bacillus in most of the severe cases, the streptococcus thus probably giving rise to the more severe inflammation, opens up avenues for the entrance of more of the specific toxine. Also, that the benefit of the antitoxine from the Klebs-Loeffler bacillus in cases where that bacterium is not present is explained by the similarity of effect of the antitoxines of the various bacilli. This latter conclusion is corroborated by several observers who have used the diphtheria antitoxine for different diseases. According to Atkinson, the bacteriological diagnosis frequently gives negative results. Spronck summarizes the literature relating to the distinction between true and false diphtheria bacilli. He concludes that, first, a microscopic and macroscopic examination of the colonies developed on serum are insufficient for a certain diagnosis. Second, inoculation of an animal is indispensable in all cases of diphtheria, unless the case is severe or occurs during an epidemic. Third, that De Martini's criterion that true diphtheria bacilli, whether virulent or so attenuated as to be harmless, will not grow in anti-diphtheritic serum, has been proved to be wrong by C. Fränkel and himself. The.latter has found that when the hypodermic injection of two cubic centimetres of a 
recent broth culture does not kill a guinea-pig weighing about three hundred grammes but causes a more or less marked local edema, in the great majority of cases one has to do with the pseudo-diphtheria bacillus.

Apropos of this subject, it might be well to call attention to a paper presented to the Paris Academy of Medicine by M. Mégrim, who showed to the academy a specimen of the holothyrus coccinnella of Gervais. He stated that this epiphyte was very dangerous to fowls, and also to children when placed upon the skin, causing irritation and edema, and that these effects were much more pronounced on mucous membranes, thus showing that possibly there are some microbes yet undiscovered which take part in the production of diseases of the mucous membrane. Fritz Franz notes that the Klebs-Loeffler bacillus may vary greatly in its characteristics, for those held to distinguish the pseudo-diphtheria bacillus all lie within the region of variations which the true diphtheria bacillus can show. There are widespread saprophytes which can be distinguished from the true diphtheria bacillus only by pathogeneity. In this connection C. Fränkel thinks that virulence is too variable a quality to be used as a dividing line. The virulence of the diphtheria bacillus can vary according to the kind of medium used, and can be thus completely destroyed. The experiments made by Trumpp, working in Escherich's clinic, are interesting and important. In this connection he obtained pseudo-diphtheria bacilli from pleuritic pus, five cubic centimetres of a bouillon culture of which gave no reaction when injected in a guinea-pig. He inoculated a guinea-pig with a particle of this mixed with diphtheria toxine, then cultivated the bacillus on a media and inoculated it again, mixed with diphtheria toxine. He repeated this process a number of times, and at length obtained a culture which was highly virulent. This would indicate that the diphtheria bacillus and the pseudo-diphtheria bacillus are really one organism. As this bacillus is found in the mouths of half of all individuals examined, Schanz puts forward the view that the Klebs-Loeffler bacillus can not be the only cause of the formation of the false membrane, but that, lodging in the membrane and growing there, it produces a toxine which gives the general symptoms. He holds that the xerosis bacillus and the pseudo-diphtheria bacillus are identical. Slawyk and Manicatide made a careful 
examination of many different stock cultures of diphtheria bacillus with the object of throwing light on the subject of the unity of the two diphtheria bacilli. Of forty-two cultures considered diphtheria, thirty-eight proved to be true diphtheria. Their conclusions thus favour the unity of the bacillus. Myerhof made a study of the morphology of the diphtheria bacillus, and comes to the conclusion that we are not yet in a position to definitely classify them as either the schizomycetes or hyphomycetes.

Relative to the question, Does the streptococcus heighten the virulence of the diphtheria bacillus, or does the streptococcus itself cause the difference? Bernheim found that if animals received antitoxines of both the diphtheria bacillus and the streptococcus, they showed symptoms no worse when inoculated with mixed toxines than when inoculated with the diphtheria toxine alone. Albert claims to have proved that the streptococcus acts by increasing the virulence of the toxine production of the diphtheria bacillus. His animal experiments, however, do not rule out the action of the streptococcus toxine itself.

J. Simon and F. Benoit write of an epidemic occurring in the barracks at Lyons, where 108 soldiers were examined. The Klebs-Loeffler bacillus was found in 32, and, of these, 2 I had symptoms of angina, I I had no symptoms. The more pronounced the inflammation of the mucous membrane the more numerous and virulent were the bacilli. Roux and Yersin found diphtheria bacilli in 26 out of 59 healthy school children. Mead Fulton found them in 4I out of 234 persons examined. Westbrook (in this country) states that in a school in which diphtheria was endemic, 478 persons were examined and diphtheria bacillus found in 172 . Of these, 59 had no sore throat. An atypical bacillus was found, the culture of which took longer to kill a guinea-pig than does the Klebs-Loeffler bacillus. E. Fränkel finds that the xerosis bacillus gives a more abundant growth on the Loeffler-blood serum and on peptone agar, than does the diphtheria bacillus. In bouillon, the diphtheria bacillus causes an acid reaction, the xerosis bacillus an alkaline, while the pseudo-diphtheria bacillus, as found on the conjunctiva, does not alter the reaction. Fränkel could not make a distinction between the xerosis bacillus and the Hoffman-Loeffler pseudo-diphtheria bacillus of the throat. $\mathrm{H}$. 
Heinersdorf examined many cultures of the diphtheria-like bacilli to determine the value of the differential stain recently proposed by Max Neisser.

By this stain granules are brought out in the Klebs-Loeffler bacillus which are not seen in the pseudo forms. Heinersdorf found that nine- to sixteen-hour cultures which show the granules typically in the bacilli or typical form are the KlebsLoeffler bacilli. In nine- to sixteen-hour cultures the xerosis bacilli show no granules. In cultures of over twenty-four hours granules may appear, but not typically. Auckenthaler found Neisser's granules in all cultures of Klebs-Loeffler bacilli. $\mathrm{He}$ found granules also in small number in one culture of pseudodiphtheria bacillus. He finds the Neisser method of great practical value, but not absolute. Richard J. Hewlett found that Neisser's method applied in all cultures examined. Czaplewski's plan of staining fresh smears by Gram's method gave positive results in a third of all cases of diphtheria. Although the pseudo-diphtheria bacilli take the Gram stain, yet they are in such small numbers in the fresh smear that they do not often cause any difficulty. It is therefore shown that a bacillus must not be called pseudo-diphtheria on account of the morphology or even lack of virulence. Unless it is proved that a suspected bacillus does not produce a toxine which is antagonized by diphtheria antitoxine it is safer to regard it as true diphtheria and treat the case accordingly.

\section{Mixed Infection}

So many atypical and puzzling cases of diphtheria are observed from time to time, irregular in their course, clinical features, and behaviour under treatment by antitoxine, that some scientific explanation has been eagerly sought for.

The varying results of bacteriological examination, too, have caused no little surprise sometimes, as, for instance, when cases are found presenting at least many of the typical clinical phenomena of diphtheria, while the specific KlebsLoeffler bacillus is wanting, or, on the other hand, cases presenting the clinical features of acute follicular tonsilitis or influenza, which show the presence of the specific microorganism of diphtheria. Such cases have been generally accounted for by the discovery and isolation of a bacillus 
denominated the pseudo-bacillus, a Klebs-Loeffler bacillus in its tinctorial culture, biological and morphological properties, but yet differing from the Klebs-Loeffler bacillus in pathogenic power. Besides this, it has been found that in some cases the infection has been produced by a mixture of the specific germ with some member or members of the streptococci farnily, and to this could be attributed certain phases in the nature or course of the attack, the predominant features being dependent upon the influence of either one or the other of the microorganisms, according to which gained the ascendency in the infecting process. The mild croupous inflammations, as, for instance, croupous laryngitis and fibrinous rhinitis, are by some authors attributed to the operation of the staphylococcus. Many, however, state that croupous laryngitis is more often due to the streptococcus. According to Jennings (C. G.), eighty-five to ninety-five per cent of the cases of primary pseudo-membranous laryngitis are due to diphtheritic infection. Baumgartner makes it plain that there is no essential difference between croupous and diphtheritic inflammation. The "diphtheritic" in diphtheria is but the extension of the croupous membrane-forming process into the deeper layer of the tissues. "Croup" in diphtheria is nothing but a superficial "diphtheritis" of the epithelial layer. An interesting article published in the Journal of the American Medical Association, by Doctor Jacques, offers a very clear exposition by theory of some of the anomalies connected with this subject, which is freely quoted here.

"The germ or germs which are the chief causative factors of diphtheria are most sensitive to environment, by which is meant all the conditions influencing the life of the germ as well as that of the patient who furnishes the soil for invasion. Environment is continually changing. No two consecutive days have the same amount of sunshine, heat, and moisture. Each season brings conditions which have their influence upon the germs of the membranous anginas. With a knowledge of these facts, it is readily comprehensible why anginas of one year or one season should differ from those of another. If the conditions are favourable to the virulence of certain germs, it will prove the causative factor of the anginas of that year, subsiding into a secondary position with new conditions which favour the virulence of another germ. The bacteriology of the 
anginas of 1896 and 1897 has presented many interesting differences from those of last year. At that time nearly seventyfive per cent of all the inflammations, including those causing stenosis of the larynx, were due to the Klebs-Loeffler bacilli. At the close of this year it is some member of the streptococci family that most often causes invasion. Following its invasion each germ has a definite train of clinical symptoms characteristic of itself, and were it not for the fact that there are so many mixed infections, there would be little difficulty in making a diagnosis without the microscope. But it is seldom the case that a culture reveals only one germ. Others are nearly always present. It may be as accessories in a minor degree to one germ causing the lesion. In every mouth there are a number of pathogenic germs which are impotent except when favourable conditions are presented for multiplication. If constitutional resistance becomes weakened toward any one of the germs present, that one may become an exciting cause of inflammation. When a germ acquires this malignancy it is probably because the patient's antitoxic power is reduced. For instance, the case of a child was diagnosed as KlebsLoeffler diphtheria, and antitoxine administered, followed by recovery. Three weeks later a second inflammation occurred, culture showing mixed infection, with Klebs-Loeffler bacilli present. Antitoxine was again injected. The case followed the usual course of a streptococcus angina. The patient was already immunized against the Klebs-Loeffler bacillus, and although the germ was present it had but little influence upon the inflammation. In laryngeal inflammations the staphylococcus causes simply spasmodic laryngitis. The streptococcus stenosis is of long duration, sometimes requiring reintubation or the substitution of tracheotomy. If the Klebs-Loeffler bacillus causes the stenosis, it is possible to be as positive of the action of antitoxine as of morphine, while the ratio of mortality becomes from five to twenty per cent instead of eighty to ninety per cent. The staphylococcus is nearly always present in the fauces, and it is always present in slight inflammation of the tonsils. The crypts of enlarged tonsils contain them, and a slight cold causes a congestion of the mucous membrane, producing the necessary condition for disease. Occasionally the staphylococcus takes on considerable malignancy. The temperature rises to $104^{\circ}$ and $105^{\circ}$, causing considerable 
constitutional disturbance. The invasion of this germ may produce either spasmodic or exudative laryngitis, whose chief characteristic is a short duration. The streptococcus exhibits a much greater malignancy, and has a wide range of virulence, depending upon the condition under which the invasion takes place. The points of invasion may be in the tonsils, but the entire tissues of the fauces are more likely to be involved, producing a dark-red appearance with little or no membrane for thirty-six or forty-eight hours. The membrane is always small in proportion to the amount of inflammation. There is a tendency for the inflammation to spread back of the tonsils up into the posterior pharynx, invading the Eustachian tube, causing great difficulty in swallowing, also impairing the hearing. While the temperature in staphylococcus is high in the beginning, and reaches a maximum in twelve to twenty-four hours, the temperature in streptococci infection gradually increases to $102^{\circ}$ or $103^{\circ}$, remaining stationary for two or three days until influenced by treatment. The symptoms are neither so apparent nor so alarming as in the invasion of Klebs-Loeffler bacillus. The germs seem to have an anæsthetic action locally, and act as a constitutional depressant. There may be little or no pain. The patient will be quiet, gradually going into stupor. On examination of the fauces, membrane will be present either following the primary inflammation or at the first inception. True Klebs-Loeffler diphtheria is the least painful of all the anginas. The invasion usually takes place in the fauces, spreading to those parts of the mucous membrane which are susceptible, such as the nasal passages, the posterior fauces, and the trachea. During the past year the pseudo-diphtheria bacillus has frequently appeared in the cultures. My observation leads me to think this germ is the Klebs-Loeffler bacillus changed in its morphology owing to the soil on which it grows. The reasons are as follows: I. Cases have resulted fatally with this bacillus as the invading germ, giving every clinical evidence that death was caused by ptomaine poisoning. 2. The genuine Klebs-Loeffler diphtheria has been contracted directly from cases of pseudo-diphtheria. 3. The morphologic change between the pseudo and the Klebs-Loeffler bacillus is no greater than is seen by growing the Klebs-Loeffler bacilli on blood serum in agar-agar, or 
the change which the Klebs-Loeffler bacillus undergoes from the beginning to the end of the case of true diphtheria." Jennings (C. G.) believes that "the pseudo-diphtheria bacillus is not a pathogenic germ, and not a factor in the production of mixed infection. It may be present in both healthy and diseased throats, and its presence is of no pathological importance. The Klebs-Loeffler bacilli may be present without any manifestation of clinical symptoms until the system is permeated with toxin, and evidence of toxin poisoning appears. If the nature of the disease has not been discovered and antitoxine has not been given before this stage is reached the chances for recovery are greatly reduced."

There are also some curious facts and observations contained in the report of Dr. F. E. Wyncoop, assistant bacteriologist of the Chicago Health Department, much of which is here quoted.

Dr. F. E. Wyncoop, first assistant bacteriologist of the Chicago Department of Health, in his recently issued report, states that in several instances the bacillus of diphtheria and that of influenza have been found at the same time in the throat, and the symptoms have usually accorded with this fact. The influenza bacillus has been found present also in cases of scarlet fever, measles, and pneumonia. In all these instances it has been noticed that there were clinical manifestations that were difficult to interpret until the microscopic examination was made. Dr. Northrup, of New York, has reported cases of epidemic measles that were complicated by diphtheria. Dr. Wyncoop states some curious facts in relation to the diagnosis of cases suspected of being diphtheria relative to the obscure symptoms of the disease. In cases that appeared to be diphtheritic at the beginning the presence of the Canon-Pfeiffer bacillus has been demonstrated, and the subsequent course of the disease has confirmed the microscopic diagnosis of influenza. In many of these cases the throat symptoms were so marked-redness, swelling of the tonsils, well-developed membrane-that the physician would not hesitate to pronounce the case one of diphtheria, yet the influenza bacillus was found in a condition almost pure. Cases with little throat disturbance, general depression, rise of temperature, and myalgia, were found to be cases of mild diphtheria, notwithstanding the symptoms were those of influ- 
enza. The influenza bacillus, too, was the sole micro-organism found in certain cases that appeared to be instances of amygdalitis or of severe laryngitis. Dr. Wyncoop also remarks that when there is influenza in a household some member of the family may be suddenly attacked with acute inflammation of the conjunctiva, characterized by severe pain, swelling, redness, and profuse discharge of pus of medium consistence and cheesy appearance. It has been learned also that since the January report was published the Canon-Pfeiffer bacillus has been found in the bronchial mucus of a number of patients presenting the characteristic symptoms of mumps. These symptoms, however, have not lasted more than three or four days, then the swelling and soreness have subsided, and the temperature has fallen to normal. But coincidently with the subsidence of the symptoms of mumps there has occurred, in some cases, an especial intestinal disturbance-peritonitis, or dysentery in a few cases. (New York Medical Journal, March, 1899.)

Treatment.-The treatment may be considered under three heads : prophylactic, local, and constitutional.

Prophylaxis. - The prophylactic treatment embraces immunization, quarantine and the adoption of sanitary measures. As the disease is contagious without doubt, it follows obviously that the more strictly the patient can be quarantined the less liability there is to the spread of the disease. This point is pretty thoroughly understood nowadays by the laity, and, through the efficient co-operation of the medical profession with the sanitary authorities, prophylactic measures are now very successfully and generally carried out. In this connection it may be well to notice the question of spreading the disease through "fomites"-articles of clothing, etc. While the spread of the disease in this manner is perhaps not as notable as is that of scarlet fever, still, there is a vast accumulation of facts which demonstrate without doubt that there is danger, under certain circumstances, of perpetuating and extending the affection in this manner. It therefore behooves the physician in all cases to take upon himself the particular responsibility of informing the household where a case of diphtheria exists, that articles of clothing and upholstered furniture are capable of holding, for a considerable time at least, the infection. Although people are loath to have their household ap- 
purtenances destroyed or damaged, still in the majority of instances the physician will find, after due explanation of these facts, that they will willingly co-operate in the destruction or disinfection of all susceptible articles.

From a sanitary point of view, the question of drainage or sewerage of the house must enter into consideration, inasmuch as it has been found in some instances that defective drainage and accumulated garbage may harbour the disease, although it is not now believed, as it was formerly, that there is any direct connection between the perpetuation of diphtheria and sewer gas.

Local Prophylaxis.-Many practitioners have faith in local applications, and many things have been suggested, such as gargles or sprays of solutions of alum, borax, boracic acid, mercuric chloride and biniodide, hydrogen peroxide, thymol, carbolic acid, calcium bromide and sulphide, liquor sodæ chorinata, potassium permanganate, resorcin, and other agents. While these may be useful, yet the only reliable prophylactic agent is anti-diphtheritic serum. This should be used without hesitation in doses of 500 to I,OOO units-smaller doses for infants.

Local Treatment.-The chief therapeutic indication in the local treatment of diphtheria is to wash and disinfect the seat of the disease. This may be said not only theoretically but practically to be an important part of the treatment. For this purpose peroxide of hydrogen 1 to 4 or 7 , solution of bichloride of mercury I to 2,000 or I to 3,000 , should be used with a syringe, douche, or ordinary atomizer every one, two, three, or four hours, according to the features of the case. Following these ablutions where practicable the fauces and tonsils should be painted with Loeffler's solution, or a mixture of eucalyptus, thymol, and alcohol (see formula), or resorcin, glycerine, and water (see formula) in the early stages of the disease. Astringents and escharotics are of no practical use.

Applications of limewater, solution of sodium bicarbonate, and sometimes the vegetable acids, are used for the purpose of dissolving the false membrane. For this purpose the juice of the carica papaya-Papaine-has been highly recommended. It was introduced by Wurtz and Bouchut in 1879. For the same purpose solutions of pepsin have been found beneficial. 
These agents, however, are not much used where antitoxine and the modern antiseptic remedies are obtainable.

After exfoliation has taken place, however, and raw granular spots are presented, these may be touched with tincture of chloride of iron and glycerine in equal proportions, or a mild pigment of nitrate of silver (see formula), or a mixture of tannic and carbolic acid (see formula), or, in some instances when the tendency to reproduction has ceased, insufflations of iodoform, europhen, or aristol may be substituted. These substances should not be used, however, where there is a tendency to dryness of the throat, or where there appears to be a secondary formation of exudate. When the method of douching the throat by syringe is impracticable, as in the case of young children, or on account of some inflammatory complication, then the frequent use of an atomizer for spraying should be substituted. When using the stronger substances, such as Loeffler's solution, tincture of chloride of iron, nitrate of silver, etc., the application should be made by means of a brush or swab, care being taken to hold the tongue well out of the way and thoroughly touch the lateral and posterior walls of the pharynx. In the case of nasal and naso-pharyngeal diphtheria applications should be made, if possible, by the syringe, the postnasal-syringe in preference, to avoid endangering the Eustachian tubes. Antiseptic solutions, however, such as peroxide of hydrogen, bichloride of mercury, etc., should be persistently used through the nasal passages. As often as may be necessary, and in severe cases, it is better to swab the naso-pharynx with a solution of acetozone or argyrol by means of a pledget of cotton attached to a probe. The small amount of bleeding and the excoriations which follow such a manœuvre are not at all objectionable when compared with the immense benefit to the patient from the application of Loeffler's solution directly to the infected naso-pharynx. An objection has been raised by some to the syringing of the nose from before backward on account of the danger of throwing the infectious material into the Eustachian tube or into the accessory sinuses, and thus inadvertently inviting diphtheritic otitis or diphtheritic inflammation of the antrum or ethmoid cells. The writer is inclined to think that such objection is rather theoretical, as scarcely a well-authenticated case of this sort, as the result of syringing, has been recorded; therefore the practitioner should 
not be deterred from a thorough local treatment, with a view of checking as far as possible the toxæmia by attacking the seat of the generation of the poison.

Constitutional Treatment.-The constitutional treatment which is now in high favour throughout the world and seems to be more successful than any which has ever been adopted is the administration of anti-diphtheritic serum. It is unnecessary to go into the history of the researches leading to the present accurate knowledge concerning this mode of (antitoxine) treatment any more than to refer as a matter of duty to the laudable work of its promoters, viz., Pasteur, Behring, Roux, and Yersin, to whom undoubtedly the world is indebted for the beneficent practical results observed every day around us. With this plan of treatment the dread disease, diphtheria, is robbed of at least one half its virulence.

Besides the specific treatment various complications may have to be met; for instance, it is well to administer, according to the age and condition of the patient, either a good-sized dose or a few small doses of calomel, for the double purpose of evacuating the bowels and obtaining the action of the mercury on the glandular apparatus. This plan ought to be adopted as a routine treatment in almost every case, for it will not in any way interfere with subsequent treatment. After having given two or three injections of the antitoxine, quinine, either by the mouth or hypodermically, should be administered; indeed, in cases of secondary poisoning, which, however, are rare under antitoxine treatment, one or two hypodermic injections of muriate of quinine (from three to five grains) may be administered two, three, four, or five times daily until exfoliation has taken place and the albumen is disappearing from the urine, after which it is a good plan, as a matter of routine treatment, to administer strychnine in suitable doses (not too small, however) through the period of convalescence. The diet should consist of nutritious fluids throughout the course of the disease. In cases of marked adynamia the concentrated foods, together with a moderate amount of alcoholic stimulants, should be administered continuously. Regarding alcoholic stimulants, care should be taken not to increase the doses too rapidly, lest a tolerance to the alcohol be gained by the system at a time when its impression upon the nerve centres is most needed. The writer would therefore recom- 
mend that the stimulant in these cases be regulated with precision, however severe the blood poisoning may be. In critical cases it is far better to use rectified spirits, because the dose can be much more accurately gauged. When the appetite returns, providing there is no serious paralysis of the muscles of the throat, solid food may be given with progressive frequency. When, however, paralysis of the throat supervenes it will be far safer, until the patient gets accustomed to the difficulty of deglutition, to insist upon the administration of soft food, in order to avoid the possible danger of asphyxiation by the impaction of solid food into the trachea or bronchi.

Great attention should be paid during convalescence to the heart. Many patients die from fatal syncope even on the verge of recovery, so that the pulse and heart's action should be watched from day to day, and any "flagging of the circulation" should be met by the administration of digitalis and alcoholic stimulants, while at the same time the attendants should be particularly cautioned against allowing any quick movement or sudden effort on the part of the patient; indeed, in such cases, until the danger point has been passed, it is far safer to keep the patient in a recumbent posture, however irksome it may be.

Physiological Action and Dosage of Diphtheria Antitoxine Serum.-In health there is practically no physiological action from a hypodermic injection of this serum. Its action is supposed to be due to the ordinary globulocidal action on human blood of alien serum. A large majority of observers believe, and accumulated clinical evidence seems to bear testimony to the fact, that antitoxine serum is in no way responsible for albuminuria, or nephritis, or the cardiac depression so frequently accompanying cases of diphtheria. Rashes, such as erythema simplex, urticaria, and erythema multiforma, with or without arthritic pains, frequently follow the injection of antitoxic serum. One or the other of these eruptions may also follow a prophylactic dose. Bazin believes that these rashes occur generally in subjects that are predisposed to frequent attacks of "hives."

The local effect of antitoxine on the exudate, in from six to eighteen hours after a sufficient dose of the antitoxic serum has been administered, is that a red line forms around the diphtheritic exudate, while the patch itself gives the appearance of 
being soft and pultaceous. Soon after this occurs the patches begin to exfoliate in part or wholly, leaving the surface of the mucous membrane red and granular.

Occasionally there will be slight renewals of the exudate in spots, but this secondary film will remain soft and will soon slough off. The process of exfoliation in the milder cases may take place within a day after the physiological action of the serum has been obtained, or the process may consume several days or even a week before the throat thoroughly clears up.

In cases of laryngeal diphtheria it is found that the effects of the serum are often much more prompt; the exfoliation and softening of the false membrane consuming only six to ten hours in some cases, while in others the exfoliation takes place more rapidly and en masse. This may constitute an additional danger to life from suffocation, because, the patch not being wholly freed from the mucous membrane, the patient may not be able to expel it by coughing, and the mass loosening may fall down upon the glottis, or through the glottis into the trachea, and produce death. This is a danger which should always be provided for in cases of laryngeal diphtheria where there is reason to believe that much exudate has taken place.

The general effect of antitoxine is first to produce a reactionary rise of temperature. This, however, does not always take place, while on the other hand, in exceptional cases, the hyperpyrexia is quite marked. It is not usually of long duration, as the temperature is considerably lowered at the end of twentyfour hours. As the body temperature approaches the normal the patient loses the feeling of malaise which has been so constantly present up to this time. The backache and headache are also frequently relieved, and in some instances the return of a feeling of well-being is exceedingly prompt. These changes in symptoms, however, will vary according to the severity of the disease and according to the time in the course of the disease at which the serum has been used. For example, prompt relief of the symptoms, both general and local, need not be expected when the use of the serum has been greatly postponed.

The dosage must vary a little according to the judgment of the physician. It would be safe to follow the tables laid down by the American Pediatric Society. In a case of ordinary severity in a child under one year of age from 300 to 500 units may be 
used; with older children and adults from 2,000 to 4,00o units. In cases of greater severity, or those in which the nose or larynx are involved, I,000 or even 1,500 units may be given to a child about one year of age, and under the same circumstances from 2,000 to 3,000 units to older children and adults. If amelioration does not take place in from twelve to eighteen or twenty-four hours the dose may be repeated, and under certain circumstances a third or fourth dose may be given. In these cases of persistent severity in older children and adults it will sometimes be found highly beneficial to give between the doses of antitoxic serum a hypodermic injection of five grains of the hydrochlorate of quinine. The effect of this drug administered in this way sometimes proves beneficially synergistic to the serum.

The importance of the early administration of the antitoxic serum in all cases should not be lost sight of. As Bazin remarks, it is entirely unnecessary to wait for the bacteriological diagnosis in cases of suspected diphtheria.

The immunization of persons who have been exposed to diphtheria has come to be recognised as a necessary duty on the part of the attending physician. It is due to the zeal and efforts of a few enthusiastic bacteriologists and practitionersKitasato, Roux, Arone, Aronson, and others-that the antitoxic serum is very generally recognised as an efficient immunizing agent. The efficacy of antitoxine is strikingly apparent in the official public health statistics recently published by the German Grovernment for the years 1885 to 1895 -the latter the first year that antitoxine came into general use. The mortality from diphtheria and croup was less by 49.48 per cent than the average of the preceding years. The morbidity also showed a similar decrease, varying with the locality from 31.50 to 64.75 per cent less than preceding years. The reports from Norway are still more conclusive. At Geneva, also, the children in a large asylum were exposed to contagion, and all but two received preventive inoculation. These two promptly succumbed, while none of the rest were affected. It requires a smaller dose for immunizing a healthy subject than when the disease is operating upon the economy. From 100 to 300 units are usually sufficient to confer immunity for a number of days. According to Dr. Bigg's observation such immunity will last for about thirty days. It has also been found by the observa- 
tion of Northrup that the immunizing of children with antitoxine serum while suffering from measles will in a great measure prevent the accession of diphtheria, which, as is well known, is a very common complication in epidemics of measles.

Absorption of Antitoxine in the Alimentary Canal.-Escherich and Chantemesse have claimed that diphtheria antitoxine is readily absorbed by infants if administered per os or per rectum, but that it is not absorbed by older children or by adults when administered in this way.

The Preparation of the Diphtheria Antitoxine Serum

The horse is selected for the artificial production of antitoxic serum, first, because of its size, which consequently yields a larger amount of serum within a given time by the expenditure of the same amount of labour; second, because this animal is naturally predisposed to but three constitutional infectious diseases to which the human race is liable, viz., glanders, influenza, and tuberculosis. Concerning the latter disease, however, it may be added that the horse is not especially susceptible.

The animals used for this purpose are kept in light, airy stables. They are well fed and cared for, and when not sick are given a certain amount of exercise.

Healthy animals are selected, and in some stables are first inoculated with mallein and tuberculin respectively, when if any reaction to these agents be noticed they are rejected. If otherwise, they are treated as follows according to Bazin :

A standardized toxine is used which is prepared by the growth of Klebs-Loeffler bacilli in bouillon, the bacilli being entirely removed by careful filtration, and tests have shown that the toxine produced is of a certain strength-namely, that 0.oI cubic centimetre when injected into a guinea-pig weighing 300 grams will produce death in from twenty-four to thirtysix hours. As an initial dose, r.o cubic centimetre of this solution is introduced hypodermically. This will produce elevation of temperature and irritability lasting about three days. After an interval of a few days, during which the animal recovers, another hypodermic injection containing a larger dose is given, and so on the process is repeated with progressively increasing doses until after about ten or twelve weeks the 
animal may be receiving 300 cubic centimetres at a dose without visible reaction. A small amount of blood is then withdrawn and the serum tested as to its antitoxine strength.

This is done by simultaneously injecting a guinea-pig with o. I cubic centimetre of the standardized serum (which is ten times the fatal dose-the "toxic unit") and a quantity of the horse serum to be examined. The minimum quantity of the serum which will present a lethal result is supposed to contain an antitoxic unit. If the serum does not come up to a certain strength of antitoxic power the horse is subjected to further injections of toxine (Bazin).

Concentrated serums are produced by more prolonged periods of inoculation and less frequent bleedings of the horse. The serum of one horse may vary considerably at different bleedings, so that it is necessary to test the serum at each successive bleeding. A preservative is added to the serum to prevent contamination by the growth of micro-organisms, and it is placed in sterilized bottles, upon which should be placed labels denoting its strength in antitoxic units, together with date of preparation. According to Bazin, trikresol is used most widely as a preservative. Many practitioners prefer the concentrated solutions, because a less quantity can be used, while the effect can be reached several hours sooner.

The relative strength of the various specimens of serum varies greatly as found upon the market. Some serums are supposed to possess but 50 antitoxic units to the cubic centimetre; other serums $100,200,250$, or 500 to each cubic centimetre. There is also some complaint from time to time of a discrepancy between the actual strength of a given specimen and that guaranteed by the manufacturer. Some specimens have been found on the market that were more or less decomposed.

All serums should be up to the standard indicated by the manufacturers, and should be free from all noxious agents, such as those introduced as preservatives or those arising from decomposition of any sort. Manufacturers and their agents should be made to suffer severe penalties for selling impure, inert, or spurious antitoxine. 


\section{CHAPTER VI}

FIBRINOUS RHINITIS.-ACUTE FOLLICULAR TONSILITIS.ACUTE PERITONSILITIS

Fibrinous Rhinitis. Membranous Rhinitis. Fibrinoplastic Rhinitis. Croupous Rhinitis

AN inflammation of the Schneiderian mucous membrane characterized by the development of a pseudo-membrane.

This disease is not frequently met with, or, if occurring often, it is not always recognised. Kyle, of Philadelphia, who has paid considerable attention to the investigation of this disease, recognises two forms from an etiological and pathological basis: one due to the specific diphtheria bacillus, the other, non-specific, not due to any special micro-organism.

Etiology and Pathology.-Professional opinion is somewhat divided regarding the specific origin of this malady, many observers believing that all cases are due to the specific germ of diphtheria, or, in other words, that they are all diphtheritic; while others, on the contrary, believe that a majority of the cases ought not to be regarded in any sense as true diphtheria. Kyle, with others, while leaning to the opinion that microorganisms are usually the cause of the disease, yet speaks of a fibrinous inflammation which may take place from other sources of irritation of the mucous membrane. There is no doubt that chemical agents as well as micro-organisms will bring about fibrinous rhinitis.

We frequently meet with cases following exposure to volatile pungent agents, such as ammonia, chlorine gas, sulphureted hydrogen, and phosphorus. The writer met with one case of persistent fibrinous rhinitis on both sides of the nose which was due to the fumes of phosphorus. While cases due to trauma present, in a measure, the same clinical features as the socalled idiopathic ones, yet it is admitted that there must be 
some peculiarity of the irritant in order to produce this sort of inflammation and exudation. But because the majority of cases present little or no constitutional disturbance and run a very irregular (prolonged or limited) course, many practitioners are loath to classify them as cases of nasal diphtheria. Looking upon diphtheria first as a local and then as a constitutional or systemic affection (through the absorption of toxines), it would seem probable that the majority of these cases are really local diphtheria without toxine infection. It does not require a wide stretch of the imagination to realize that a diphtheritic process without toxine infection may remain localized, as, for example, in diphtheritic croup. Furthermore, in view of the results of recent bacteriological examinations of the nasal secretion, and the anatomical and physiological peculiarities of these parts, it is obvious that a microbic irritation as well as a chemical one of the surface of the nasal septum or turbinates might take place without producing fermentation, and if so without the subsequent generation and absorption of a toxine. Indeed, it is a debated question whether the mucous membrane of the nasal passages can directly absorb any proteid or chemical substances; for the lymphatic vessels are so arranged that absorption from the surface of the nasal mucous membrane is not at all probable; while, moreover, the secretions themselves offer enough resistance to any process of fermentation to probably prevent the development of toxines. The question now arises, Why should the irritation in a given instance produce an exudate of fibrinous character instead of a muco-purulent or serous one? Such a question, of course, can not be definitely answered; but it may be surmised that the true reason will be found in some peculiar reaction which takes place between the irritating agent and the epithelial covering of the mucous membrane or its secreting products, coupled with some temporary abnormal condition of either the membrane itself or its secretions. For no one doubts for a moment that the nasal passages are constantly being invaded by all sorts of micro-organisms afloat, and during endemics or epidemics of diphtheria, certainly all persons coming more or less in contact with such cases must inhale through the nostrils whatever germs are contained in the surrounding air.

Dr. Ravenel contributes a report of his observations upon 
ten cases of typical membranous rhinitis (Rhinitis fibrinosa), none of which proved fatal. In some of them there had been no known exposure to diphtheria. In all of the cases but one the Klebs-Loeffler bacillus was found. In all but three of them there was little if any implication of the fauces; and in none of them were the constitutional symptoms of a grave character. Ravenel believes that these cases are almost always true diphtheria, and cautions the profession against regarding them as non-diphtheritic on account of the absence of constitutional symptoms. He thinks the diagnosis should always be made from bacteriological examinations.

In this country, Park and Abbott have reported some cases, where other micro-organisms were found. Abel mentions a case in which the Micrococcus lanceolatus was found, while many observers have found staphylococci. Very few cases are on record of fibrinous rhinitis being a source of contagion to others, and Ravenel asks the interesting question, Why is it that patients carrying about a virulent type of Klebs-Loeffler bacillus do not more often transmit diphtheria to those with whom they come in contact? In every case observed by Park and Abbott, and in all but one of Ravenel's, the organisms obtained from the nose were possessed of very feeble vitality even when highly virulent. Although kept under most favourable conditions on Loeffler's blood serum, the cultures died in from three to four weeks, with one exception noted. It seems probable that the apparent lack of infecting power is due to this feeble vitality (Ravenel). The literature on this subject is very scanty. It is interesting to observe, however, that when infection does occur the resulting disease is membranous rhinitis rather than the ordinary form of diphtheria. It is not easy to explain why this should be the case, and more extended experience may prove some of these observations to be inaccurate. There have been but few instances in which faucial diphtheria has followed infection from membranous rhinitis: one was reported by Concetti, one by Scheineman, and the one by Ravenel.

Dr. (John) Dunn, of Richmond, Va., reported a case of membranous rhinitis in a child of five years of age who two weeks before observation had suffered from what was diagnosed as amygdalitis, with white patches on the throat. The seat of the membrane was upon the anterior two thirds of the nasal sep- 
tum and the middle turbinate bone in the left nasal passage, a posterior deviation seeming to cause its limit. The membrane was very thick and tough. There were no constitutional symptoms. After removing the membrane by syringing with a solution of biborate of soda and the forceps, it reformed. There was always a raw surface beneath the membrane. The membrane repeatedly reformed, and after ceasing to show itself, the child was troubled for some weeks with a mucous discharge from the nose. At no time was the right side affected. No examination was made for the Klebs-Loeffler bacillus.

Various germs may cause croupous inflammation besides the Klebs-Loeffler bacillus. Von Hoffman's bacillus and the streptococcus, either alone or associated, are common causes. Also a non-specific membranous exudate, as said before, may form after the inhalation of irritating fumes or after cauterization. In croupous inflammations due to specific bacterial causes there is no tendency to organization, while in those not of bacterial origin a capillary budding may take place on the surface, desquamating the mucous membrane (Kyle, Fränkel). Every variety of inflammation necessarily begins in the vascular layer, the degree of alteration in this layer determining the alterations in the basement membrane and epithelial layers. These layers are dependent upon the submucosa for their nutrition. If, then, the blood supply be cut off, the epithelial layer, or the layer farthest from nutrition, will undergo necrosis (Kyle). According to Abbott, considering the question of diphtheria, "it is not always possible to establish a dependence between the clinical course of a disease and the degree of virulence possessed by the organism causing it." The studies of Booker show clearly that the local changes seen in the throats of patients suffering from measles and scarlet fever are anatomically distinct from those seen in diphtheria. Abbott, in his researches, found that a cultivation from the nasal membrane of one little patient suffering from fibrinous rhinitis showed the Klebs-Loeffler bacillus in large numbers. The normal virulence inoculations of guinea-pigs caused death in them within forty-eight hours. The pathological media characteristic of these inoculations were taken from a sister of the same patient also affected with the same disease. The cultivation proved the inoculations to be possessed of decidedly pathological properties. 
The same thing occurred in Park's six cases, the bacilli cultivated possessing only a low degree of virulence. Abbott suggests that the bacilli may vary in intensity. Sometimes there may be complete absence of the Klebs-Loeffler bacilli; but in such cases the difficulty of finding them must be taken into account. Kyle afterward found the Klebs-Loeffler bacillus in three cases observed by himself. Abel found the diphtheria bacillus in the membrane of some cases, and Fränkel the pneumococcus in that of others. Berket found Klebs-Loeffler bacilli in two cases. Sedziak reported cases of fibrinous rhinitis which followed eczema resulting from a wound on the chin through the act of shaving, where the staphylococci were the only micro-organisms found. This observer, basing his opinion upon a case seen in a young man, a colleague, as well as upon an exhaustive study of the literature of the subject, considers that fibrinous rhinitis is an independent pathological process having nothing in common with diphtheria. He was unable to find the Klebs-Loeffler bacillus in the membrane by the use of the Loeffler method, but by a special method of Weigert's found fibrin, lymphoid cells, and a few cocci. He also made a culture on agar-agar which resembled the Streptococcus pyogenes. Under the microscope there were seen different cocci. Inoculations of rabbits were negative. Hartman, Howard, and others, have seen croupous or fibrinous rhinitis occur sporadically after measles and amygdalitis, and sometimes after operations for adenoid growths. Hartman, of Berlin, has reported six cases, and in two others has given a history of direct infection from one subject to the other. In the latter cases, subsequent diphtheritic paralysis occurred, and in five other cases there was a secondary formation of membrane in the larynx. Seiler refers to several cases met with in his practice, two of which subsequently had paralysis of the soft palate. Bosworth speaks of croupous rhinitis as an acute inflammation of the mucous membrane lining the nasal passages (Kyle). Newcombe reported two cases of membranous rhinitis, a microscopic examination of which showed only ordinary fibrinous structure entangling a few epithelial and basement cells here and there, scanty and spherical. Hall states that the exudation resembles in many respects that seen in diphtheria, being of white colour, more or less firmly adherent to the subjacent mucous membrane, and when removed leaving a 
bleeding surface. Occasionally, however, the membrane is loosely attached and can consequently be removed without causing bleeding (Kyle). The exudation is usually limited to the mucous membrane of the nose, and the pharynx does not show signs of disturbance, although Hunter reported cases in which the exudation occurred also on the tonsil. Watson believes that it is analogous to the so-called diphtheritic membrane of scarlet fever and due to some form of micrococcus. Steel reported four cases showing the presence of the bacillus diphtheriæ, and Berginsky two. The disease often appears without any exciting cause being discoverable. Potter estimates that a fibrinous rhinitis occurs in about two per cent of all cases of rhinitis. Of the two cases which Kyle reported, and which he saw at his clinic in Philadelphia, one, a girl, without any exposure to diphtheria, had nasal hemorrhage, and one, a boy, complained of much pain of the nose. There was no history of diphtheria in either case. There were three children in the family, and two were affected. A history given in conversation showed that both were affected about the same time; yet they were in the house three days before the date of attack, a sufficient time to warrant the suspicion that the second child became infected from the first. The hygienic surroundings were not good. Serum tubes inoculated from the infected area showed the presence of Staphylococcus aureus and Staphylococcus pyogenes albus. There were no Klebs-Loeffler bacilli present, nor any germs resembling them. Hardened sections of the exudate showed organized and unorganized material-fibrin entangling in its meshes leucocytes and epithelial cells. The membrane was laminated.

Twelve cases of fibrinous rhinitis were reported by Dr. Eaman (Laryngoscope, March, I898). The Klebs-Loeffler bacillus was found in the exudates of each case and they all recovered. The conjunctiva may also be the seat of a fibrinous inflammation analogous to fibrinous rhinitis. .Harlan, of Philadelphia, has reported a case in a girl ten years of age, and Howe, of Buffalo, has reported a case in a boy eight years of age. From Howe's case two children occupying the same hospital ward contracted diphtheria and died. Diphtheria bacilli were discovered in these conjunctiva cases only during the acute stage of the attacks. 
Age.-The disease occurs both in children and in adults, although in children it runs a somewhat more protracted course and may be more severe. It is believed by many that fibrinous rhinitis is essentially a disease of early life. Of twenty-six cases collected by Raulin, three occurred during early adolescence and three in adults, while the remaining twenty occurred during infancy and childhood (Kyle, Casselberry).

Symptomatology.-The disease is generally quite limited in extent, rarely attacking both nasal passages simultaneously. It is for the most part confined to the anterior portion of the septum, very seldom extending to the limits of the choana. It may involve one of the turbinate bodies, but this seldom occurs. An existing spur or deflection of the septum will usually limit its extent posteriorly.

At the onset some chilliness may be experienced with cephalalgia and myalgia of short duration, followed by slight pyrexia. These symptoms, however, are usually transient ones, although the patient for a considerable time may feel a little debilitated.

There is always an uncomfortable feeling about the frontal region, sometimes amounting to pain, which varies according to the amount of nasal stenosis.

Local. - The characteristic local signs are more or less stenosis of the affected side, with a constant flow of nasal secretion of sero-mucous or sero-purulent character. The nose is always somewhat swollen and tender, and the edges of the nostril red, tender, and perhaps excoriated.

Examination of the nasal passages will reveal an occlusion caused by a grayish fibrinous deposit which is bathed in mucoserum. The probe shows it to be firmly adherent and rather tough in consistence. It is usually compact, and seems to be well organized. Should any of the exudate be exfoliated, the mucous membrane underneath will appear hyperæmic and perhaps slightly granular.

Diagnosis. - This disease resembles principally nasal syphilis, especially perichondritis of the septum, sometimes (especially in children) polypus, or the effects of injury. From the former it may be distinguished by the clinical history and the smaller size or more patchy appearance of the exudate, which lacks firmness and is of softer consistence. The secretion also 
in syphilis is of a muco-purulent and gummy character. Again, in syphilis both sides of the nose are usually affected, while the odour, especially if the bone be affected, is marked. From polypus, it may be distinguished by the absence of a veritable tumour which is movable, and which shows decided limitation of attachments. From traumatic inflammation, by the thinner pultaceous character of the exudate, together with the clinical history.

Prognosis and Course.-This disease has no definite course; an attack may last from one to six weeks and even longer. The prognosis is usually favourable, excepting in young children, when it is rather unfavourable. Paralytic and renal affections sometimes follow an attack, but not always. In children, albumen may be found in the urine.

Treatment.-General. As the diphtheritic character of many of these cases is called in question the doubt may arise as to whether any systemic treatment is necessary, especially regarding the use of anti-diphtheritic serum. In a majority of cases, especially those occurring in adults, the absence of much, if any, constitutional disturbance would indicate that internal medication is not necessary. Yet as a matter of prophylaxis, if for no other reason, and in view of the facts already alluded to concerning the etiology of the disease, one or two doses at least of antitoxine could with propriety be administered. Besides this, repeated doses of some saline mineral water or a few doses of Rochelle salts ought to be administered for the purpose of assisting the eliminating functions of the body. For weakly persons, especially children, some tonic medication is advisable.

Local.-The local treatment should consist of the free use, by means of anterior or, preferably, posterior douches, of solutions of peroxide of hydrogen, resorcin, Dobell's solution or mercuric chloride ( I to IO,OOO), boric acid, or sodium bicarbonate. The latter agent is very beneficial, particularly on ac- count of its solvent action on the pseudo-membrane. Care should be exercised in the use of the anterior douche, so that the nasal contents may not be lodged around the Eustachian tubes. The so-called nasal douche ought not to be used in place of the syringe. In protracted cases the insufflation of iodoform and naphthaline (see formula), after the cleansing of the nasal passages with a solution of resorcin, will afford excellent effects. When the exudate is firmly seated it is not best 
to forcibly remove it in order to medicate the underlying mucous membrane, but rather to attempt its solution by either alkaline applications, such as sodium bicarbonate, limewater, or by acidulous agents, such as dilute lactic or chloracetic acid. But if the exudate is already exfoliating, then no harm will ensue from assisting its removal by the gentle employment of forceps or swab.

Should erosions or ulceration of the mucous membrane remain after the subsidence of the disease, they should be treated by applications of copper sulphate, zinc sulphate, silver nitrate, argyrol, or some such agent.

\section{Acute Follicular Tonsilitis. Acute Lacunar Tonsilitis. Membranous Tonsilitis}

Definition.-An acute infectious inflammation of the faucial tonsils, sometimes communicable.

Etiology.-For consideration, the causes may be divided into exciting and predisposing.

Exciting Causes.-This disease is considered to be essentially of microbic origin, the micro-organisms entering through the crypts and there setting up the characteristic changes which result in inflammation of the gland. There has been some discussion regarding this point, viz., the place of entrance of the micro-organisms. E. Fränkel and others seem to have shown by trustworthy microscopical and biological examination of the inflamed tissues that the whole current of inflammatory products, leucocytes, etc., goes from within outward, and Fränkel does not believe, on that account, that micro-organisms can enter the follicles of the gland against this outward-flowing stream. He thinks that the micro-organism gains access to the interior of the gland by penetrating some of the neighbouring lymphoid tissue, and not by going directly through the open crypt leading to the follicles.

Many observers, on the other hand, affirm and attempt to demonstrate that the tonsil by its open crypts is frequently a subject of infection, and that, moreover, it serves to retain and extend infectious micro-organisms through the body. However this may be, it seems certain that the disease is, at least partially, the result of the activity of some organic poison, and that this poison must be in the nature of a bacterial one. We 
do not, however, know just what local condition forms the prerequisite for the invasion and operation of the microbe. It is very certain, however, that some preparation of the soil, so to speak, takes place, otherwise persons possessed of aḅnormal crypts and follicles would be constantly suffering from attacks of the disease. Many physicians believe that any circumstance which will induce a congestion of the organ will furnish the requisite conditions for the lodgment and development of the micro-organism. Cases have been reported which followed the use of the galvano-cautery, or chemical escharotics. The streptococcus and staphylococcus are each regarded as the microorganisms which cause this disease, although some writers include the pneumococcus and the typhoid (Eberth) bacillus among the causal agents.

Predisposing Causes.-So-called taking cold is considered the principal predisposing cause. But undoubtedly chronic disease of the tonsil itself and adenoids are the principal causes.

Age.-It occurs more often in adults and older children, although young children are by no means exempt. Casselberry and others believe that it occurs more often than is supposed in young children, but is overlooked. There are many observers who think that it occurs in infants in connection with simple acute fauceitis or laryngitis.

Sex.-The two sexes are equally liable to attacks of the disease.

Occupation and habits have probably but little influence in determining the onset of the disease, as it may occur indifferently in persons following any occupation. 'It is as frequently met with among persons of good habits as well as with persons of bad habits.

The so-called "taking cold" is credited with being one of the principal predisposing causes, and undoubtedly this is true, for the clinical history of a large majority of cases will furnish evidence of some unusual exposure or irregularity of this nature, just preceding the access of the affection.

Diphtheria, scarlet fever, and measles are each supposed to stand in a causal relation to acute follicular tonsilitis. Undoubtedly there are cases which may be attributed to the prevalence of any one of these diseases, especially diphtheria and scarlet fever. It is not uncommon to find a large number of adults becoming affected with acute follicular tonsilitis 
in a neighbourhood where diphtheria, scarlet fever, or influenza is prevailing.

Rheumatism.- It is the opinion of many writers that rheumatism is an important predisposing cause in many cases. While it can not be denied that rheumatism is probably an etiologic factor in some instances, yet its importance in this direction has undoubtedly been very much overestimated. Whether rheumatism be considered one of the infectious diseases (due to a micro-organism) or simply a hæmic disease depending upon irregularities of metabolism, it has only an accidental relation, from a clinical point of view, with acute follicular tonsilitis. Violent exercise in the open air by those unaccustomed to it is also a predisposing cause.

Symptomatology.-The period of incubation varies from twenty-four hours to three days; in all probability it is not definitely known, and the period is more often one day than three. Its prodromal symptoms are similar to those of other infectious diseases, consisting of a feeling of general malaise. The access of the disease is usually sharp, and marked by a rigour or chilliness covering a period of four to twelve hours, during which time, however, the temperature begins to rise, until at the end of the first day it may reach $103^{\circ}$ or $104^{\circ}$ Fahr. The pulse, of course, under such conditions is frequent, from I00 to 120. Cephalalgia and general myalgia continue, as a rule, until about the latter part of the second day, when they gradually disappear; as the temperature becomes lower the myalgia may be superseded by lumbar pain for a while. Coincidently with these symptoms there is painful deglutition, with moderate salivation and tenderness of the lymphatic glands of the neck. The urine voided is lessened in quantity and concentrated in character, and the bowels are usually constipated and the skin dry.

Objective Signs. - Inspection of the throat shows at first hyperæmia and intumescence of the tonsil affected. Soon around the crypts of the organ a fibrinous exudate, white in colour, rather thin and pultaceous in character, takes place, and, as the disease progresses, they coalesce with each other to form larger membranous patches. These patches are easily removed by a brush or a swab, and, as a rule, leave no erosion of the membrane beneath. The pillars of the fauces are usually hy. peræmic and somewhat tumescent, and rarely they may be 
studded with one or two membranous patches also. The soft palate and sometimes the uvula become swollen, and occasionally small patches of mucus may be seen upon their surfaces. More frequently than otherwise both faucial tonsils may be affected, although the disease may be confined to one of them only (see Plate V, Fig. 6).

Course and Termination.-Acute follicular tonsilitis is not usually a dangerous affection. It runs its course in about three days, terminating in the restitution of the parts without suppuration. It often seems to be communicable; particularly among children. Albumen may sometimes be found in the urine, at least during the first forty-eight hours. Atypical cases, however, occur in which the course of the disease may be lengthened to a week, or even two weeks. The disease may be confined entirely to one side, and at the end of its course a peritonsilitis, with resulting suppuration and abscess, may take place. When diphtheria is prevalent, follicular tonsilitis may give rise to a veritable attack of diphtheria. The tonsil, and sometimes the pharynx, of the affected side may be left for a considerable time in a state of subacute inflammation, and in some instances a more or less extensive lympho-adenitis, affecting the cervical lymphatic ganglia, may also take place. The lympho-adenitis may be acute or subacute in character.

Diagnosis. - This affection more frequently simulates diphtheria than any other disease ; indeed, when diphtheria is prevalent, it is sometimes impossible by clinical signs, or even sometimes by bacteriological investigation, to absolutely differentiate between the severe types of this disease and diphtheria. Dr. Roaldes and many others believe that the bacterial examination should be almost entirely relied upon for the diagnosis of these anginas. Usually, however, the short duration and mild course of acute follicular tonsilitis, together with the negative bacterial characteristics and typical character of the exudate (being soft, pultaceous, and more or less confined to the crypts), will serve to distinguish the affection. From chronic follicular tonsilitis it may be distinguished by the absence of constitutional disturbance, if it be the former affection. As this disease is sometimes associated with croup in children, it is very apt to be mistaken in such instances for diphtheria, but a bacteriological examination of the exudate will usually suffice to determine the question. 
Ordinary acute (catarrhal) tonsilitis, herpetic pharyngitis (herpetic angina), syphilitic angina, traumatic angina, aphthous angina, mycosis, muguet, ulcerous angina, may each simulate acute follicular tonsilitis. Besides a bacteriological examination which may at once determine the real character of the disease, the following clinical characteristics, presented in tabular form, may serve to eliminate any doubt:

Acute follicular tonsilitis............ Rarely any real pseudo-membrane; usually also pharyngeal hyperæmia.

Herpetic angina..................... Groups of vesicles. Thin, soluble, white membrane, showing upon removal pinhead ulcerations or vesicles.

Syphilitic angina............... No real pseudo-membrane. History of case. No constitutional disturbance.

Traumatic angina from escharotics, etc.... Limited to region of injury. History.

Aphthous angina................. Implication of buccal cavity, ulceration of mucous membrane.

Mycosis....................... Chronic. No pyrexia. Microscopical examination shows presence of leptothrix, etc.

Muguet buccalis................. No pyrexia. Microscopical examination shows oidium albicans.

Ulcerous angina.................. History. Well-defined ulceration not confined to the tonsil.

Prognosis.-As indicated above, the prognosis is usually favourable, unless some complications, such as acute edematous laryngitis, acute retro-pharyngeal abscess, septic lympho-adenitis, or acute otitis, should supervene. As a consequence of this disease we occasionally see a very mild degree of paresis of the soft palate. While this is uncommon, nevertheless it can not be denied that such an event may transpire, even in the absence of diphtheria toxin.

Treatment.-As this is a mild and self-limited disease, it is seldom necessary to adopt any vigorous means of treatment. If diphtheria be prevailing and the case be unusually aggravated, it is well to give one or two injections of diphtheritic antitoxine; but in typical cases such a precautionary measure will be scarcely necessary. At the outset a few small doses of calomel, followed, if necessary, by a saline cathartic, will almost always prove beneficial. This may be followed by the internal administration of salol or aspirin in doses varying according to the age of the patient. During the period of pyrexia a few doses of phenacetin or acetanilid may be administered, 
both for the purpose of mitigating the distress of the patient and for lowering the temperature. However, in diseases of this character, which naturally have a limited course and are not dangerous to life, it is not a good plan always to interfere too much with the reactionary fever, which undoubtedly serves an excellent purpose in the process of natural resistance. As defervescence takes place, the administration of quinine, either alone or in conjunction with the tincture of chloride of iron, will be found advisable. Should the disease leave the patient in a debilitated state, of course suitable tonic and nutrient treatment must be adopted. For the throat, the careful use of a weak solution of cocaine hydrochlorate by means of an atomizer will be found highly beneficial. Besides this, either by gargling or spray, the application of bichloride of mercury (one to two or three thousand), or resorcin (ten grains to the ounce), or carbolic acid and alum (in the proportion of two grains of the former and five to ten grains of the latter to the ounce), may be found very useful. For gargling or douching of the parts a solution of bicarbonate of soda, either alone or with alum, should be selected. Spraying the parts with a two-per-cent solution of oil of eucalyptus or a one per cent solution of thymol is beneficial. Any complications, such as lymphadenitis or peritonsilitis, etc., should be treated as the circumstances demand. It will be unnecessary to add that, should suppuration take place in the peritonsillar tissue, the pus should be evacuated as soon as formed. Should the affected tonsil remain intumescent or otherwise diseased after the course of the affection is finished, then appropriate treatment should be directed toward the organ as soon as practicable.

Acute Peritonsilitis. Peritonsilitis Abscedens. Acute Amygdalitis. Phlegmonous Tonsilitis. Quinsy

Acute Peritonsilitis and Acute Follicular or Lacunar Tonsilitis are often confounded. But the two types of acute inflammation differ from each other chiefly in the fact that the former is confined mostly to the peritonsillar tissue, and the latter to the tonsil itself.

Peritonsilitis.-This disease may be defined as an acute inflammation of the peritonsillar lymphatic tissue, leading to suppuration and abscess. It usually commences, and may remain in 
the glands occupying the space above the faucial tonsils"spatium pharyngo maxillare" of Chiari.

Etiology.-The causes of this disease are usually grouped into predisposing and exciting. The predisposing causes are usually described as age, sex, diathesis, and the anatomical conditions of the throat, occupation, climate, hygienic surroundings, habits, and the exciting cause as an infection by micro-organisms, principally the Streptococcus pyogenes.

Predisposing Causes.-Age.-The period of adolescence seems to be the susceptible one for attacks of this affection-a majority of the cases occurring between the ages of fifteen and forty. It is seldom met with in children under ten years of age, or in persons over fifty, although a few cases have been recorded of the disease being met with in infancy. Lennox Browne and Cohen have seen cases in persons aged seventyone and eighty respectively. Males suffer more frequently than females from peritonsilitis. According to Bean the proportion is three males to two females. This predisposition on the part of males is attributed to the particular habits and occupations of the male sex.

Diathesis.-The strumous diathesis is still credited by some as being one of the principal predisposing causes. Even those who believe that the strumous diathesis is identical with "tubercular tendency" still believe that such conditions act as a predisposing cause. That there are certain individuals possessing a tendency to inflammation and suppuration of their lymphoid tissues must be admitted. Some observers believe that an undue enlargement of the tonsillar or other pharyngeal glands constitutes an expression of such an inherent tendency to suppuration and glandular disease. Many writers emphasize the rheumatic and gouty diathesis as strong predisposing causes of not only this, but of the other acute and subacute diseases of the throat. It is believed that peritonsilitis has been more especially connected with the rheumatic diathesis than with any other. Harrison Allen, Bean, Hinkel, Hope, Ingalls, and many other writers have called especial attention to the causal relation between acute peritonsilitis and rheumatism, and also illustrated the idea by the report of a number of cases. While undoubtedly the observations referred to have been perfectly correct in all particulars, still, the writer does not understand how it can be conceded on general principles that any special 
connection exists between the rheumatic diathesis and acute peritonsillar inflammation (quinsy) any more than between the rheumatic diathesis and other acute diseases, such as pneumonia, etc., for it is frequently observed that persons liable to attacks of rheumatism may also suffer from any of the other acute diseases. Concerning the point of direct alternation or metastasis, so to speak, between rheumatic affections and acute tonsillar and other acute throat affections, all that can be said is that events of that kind are too few to furnish any basis for correct generalization. The writer, in over twenty-five years of practice, has seen only one case of acute articular rheumatism directly connected with acute peritonsilitis. On the other hand, it must also be admitted that a person having undergone some extraordinary exposure might possibly have both diseases developed almost simultaneously or consecutively, but it seems to the writer that such an occurrence ought to be viewed in the light of an accident rather than an etiologic correlation.

It must be conceded, however, concerning this point that there is an individual tendency to peritonsilitis as yet undefined. It is a well-known fact that certain individuals, some of whom are usually healthy-if not perhaps robust-will suffer from two or three attacks of this disease every year. In such instances it will be found that they are not all attacked at a particular season, when the disease might be expected to prevail-namely, during the spring or autumn, but perhaps in the summer time-July or August. These individuals do not seem to be otherwise susceptible to prevailing infectious acute diseases, but apparently prone to attacks of peritonsilitis, principally whenever their physiological balance is lost from any cause whatever, thus showing a marked individual tendency or abnormal anatomical conditions.

Occupation.-Any occupation which subjects the individual to an attack of acute rhinitis or slight faucitis may serve as a predisposing cause of this affection-for example, occupations which subject the individual to frequent alternations of temperature, or which entail confinement in overheated rooms or workshops, or expose the individual to noxious gases or to draughts of air. Notwithstanding this, there is no particular occupation which can be named as necessarily predisposing to the disease. 
Climate.-Climate certainly has a marked influence, inasmuch as the majority of cases occur in damp, changeable climates, and yet the disease is not infrequently seen in warm or even dry climates.

The Season. - The majority of cases undoubtedly occur during the autumn, winter, and spring months, although there are many exceptions to this rule, for the writer has often met with cases of these acute affections in the summer season.

Hygienic Surroundings. - The hygienic surroundings have an influence in preparing susceptible individuals for the access of the disease by lowering the powers of resistance, perhaps, but in no other way, since it is not found among the poor and squalid any oftener than among the "well-to-do" portion of the population. In conclusion, it may be safe to assume that we know of no particular predisposing cause or causes of this disease, and that an attack may follow any general derangement of the bodily functions, whether it be an undue use of the voice, the effect of indulgence in the use of tobacco or alcoholic stimulants, or unusual exposure to cold, heat, or draughts while the skin is unduly sensitive, or to mental perturbation, due to excitement, depression, or fatigue. In fact, any one of the above-mentioned conditions, or a combination of one or more of them, may be causal elements in any case. Dr. J. W. Park, for instance, relates a case illustrative of the relationship between amygdalitis and the cerebro-spinal centres in the case of a perfectly healthy mill girl, having had no exposure to cold, who developed acute amygdalitis four hours after being struck in the back by a loose belt. He also relates another case, that of a lady who suffered from an attack of amygdalitis after reading a newspaper account of a fire in which two people were horribly burned to death. The patient had never left her room nor in any way been exposed to any exciting cause. $\mathrm{He}$ accounts for this by the fact that the naso-pharyngeal nerves arise from nuclei in the floor of the fourth ventricle, and are very closely connected with the pneumogastric, sympathetic, and faucial nerves, and with the superior cervical ganglion. "It touches like a key battery the whole sympathetic system, both giving and receiving impressions; and, being a branch of the eighth pair, also extends an intimate relation to the spinal accessory, which receives filaments from the lateral tract as far down as the sixth cervical, while its action with 
the vagus renders the circuit complete and gives ground for the peculiar pathological phenomena referred to."

Habits.-Great emphasis was formerly laid upon the habits of the individual, and consequently the excessive use of tobacco, alcoholic beverages, gluttony-especially regarding nitrogenous food-were all regarded as predisposing to attacks of quinsy. A careful survey of the subject, however, leads to the conclusion that the inferences regarding the causal relation of bad habits are often erroneous, for one would be astonished to find that persons living the most unphysiological lives imaginable never suffer from peritonsilitis, while others, who are notoriously. careful and abstemious, living under good hygienic surroundings, are frequently victims of the disease.

Exciting Causes.-The general opinion is that the exciting and perhaps essential catise of the disease is the invasion of the lymphoid tissue around the tonsil by micro-organisms, such as the Streptococcus pyogenes, or the Staphylococcus pyogenes -albus and aureus. There is some difference of opinion concerning the method of invasion, some observers and pathologists believing that the entrance to these tissues is from the outside through the follicular glands of the mucous membrane, or those of the tonsil, while others think that the invasion comes from within, the microbe arising from some neighbouring lymph node, and proceeding thence by means of the lymph stream and the tissue around the tonsil under consideration. Other observers again regard rheumatism as an exciting rather than a predisposing cause, and charge to it the origin of the attack. In addition to the microbic invasion above mentioned, it is well to emphasize as an exciting cause anything which produces a sudden disturbance of the relationship between the external and internal capillary circulation.

Pathology.-The first step in the pathology of peritonsilitis is a determination of blood to the tissues, caused by the action of the toxine upon the blood-vessels.

The succeeding changes of the inflammation are similar to those encountered elsewhere, being exudation of serum and emigration of red and white cells.

If the toxins are in excess, so that resolution does not take place, necrosis ensues, with liquefaction of the area attacked. 
Symptomatology.-The symptomatology may be considered under two heads: subjective and objective.

Subjective.-The period of incubation of this disease is really unknown, but it is usually very short in any event. Among the first symptoms complained of are myalgia, cephalalgia, lassitude-in short, general malaise. This is soon followed-at the end of a few hours, perhaps-by a well-marked chill or a period of chilliness. Contemporaneously the patient complains of stiffness of the throat and neck, a little soreness of the throat in swallowing. The temperature after the rigour may rise rapidly to $104^{\circ} \mathrm{F}$. or $105^{\circ} \mathrm{F}$., remaining there for from six to twelve hours, when it usually drops to $10 \mathrm{I}^{\circ}$ or $1 \mathrm{IO}^{\circ}$, and remains at that point with very little fluctuation, until suppuration is established. The symptoms relative to the throat increase in severity so that deglutination becomes very difficult and painful. As the swelling of the parts increases, the saliva, instead of being swallowed, drivels from the half-open mouth; phonation is avoided, as also all movements requiring action of the muscles of the neck, on account of the pain entailed by such movements. The lymphatic glands on the affected side are apt to be swollen and tender. The pulse, which was at first full and hard, at the end of a few days becomes softer as the weakness of the patient increases. This goes on for from five to seven or eight days, when suppuration of the inflamed tissues takes place with the formation of abscess. The abscess will discharge spontaneously soon after its formation, as a rule, but may remain quiescent for several days.

Objective.-In the early stages of the disease the soft palate, uvula, and anterior pillar, and sometimes the posterior pillar of the fauces, are seen to be red and swollen. The velum palati and uvula are sometimes so swollen as to almost completely veil the upper part of the posterior wall of the oropharynx. As the disease advances this tumefaction increases, and not only the tonsil is swollen and red, but the anterior pillar, either at its junction with the tongue or above, is seen to be prominent, shining, and bulging forward (see Plate $\mathrm{V}$, Fig. 5). In some instances the palatal tonsil is also involved, and may be considerably infiltrated. The tongue may also be swollen by the supervention of more or less glossitis; the lingual tonsil at the base of the tongue also frequently participates. On account of the tumefaction of the parts when 
extensive, it is almost impossible for the patient to open the mouth more than an inch. To the sense of touch these parts seem hard and unyielding. When the tonsil itself is not much involved it may seem softer than the surrounding tissue. In some instances the supratonsillar lymphoid tissue seems to bear the brunt of the disease, in other instances the tonsil itself seems to be the focus of inflammation, but in the majority of cases, as before stated, the surrounding glandular tissue seems to be the part chiefly affected. About the end of the first week, or perhaps a little earlier, another rigour or spell of chilliness is apt to take place, although exceptionally this does not occur at all in the course of the disease. This phenomenon betokens the formation of pus in the affected tissues. The peritonsillar inflammation, according to appearances, presents two stages of development : first, showing a marked one-sided hyperæmia of the mucous membrane of the velum, palatine arches, and tonsil ; and second, an aggravated intumescence of all these parts. Some writers describe a third stage denotive of the formation of abscess, but, according to the experience of the writer, it is very difficult, from appearances alone, to discern between this so-called third stage and the condition of intumescence above described. Occasionally the disease may successively attack the other side, in which case the debility of the patient is aggravated. This may lead to a fatal issue through general sepsis or exhaustion.

\section{The Course and Termination}

This disease usually runs a self-limited course, covering a period of from seven to fourteen days. As a rule but one side of the throat is affected, in which case the rupture or opening of the abscess is followed by almost immediate restitution. Exceptionally, however, the disease may attack consecutively the opposite side, in which case it will go through about the same course as the first attack. Necessarily the double attack leads to greater exhaustion of the patient, not only from the effects of the local inflammation, etc., but from the lack of food and drink which the patient has undergone.

It sometimes happens that the inflammation and suppuration extend to the lymphatic glands or connective tissue in the neighbourhood, and retro-pharyngeal abscess, or mediastinal 
implication, or general sepsis may ensue, either of which complications may cause the death of the patient.

Treatment.-Unfortunately, medical art can do but little, excepting to assuage to a certain extent the sufferings of the patient. A great many remedies have been extolled from time to time as prophylactics or special checks to the course of the disease, but experience has demonstrated that all such measures are doomed to failure in the majority of instances. Occasionally, however, some one agent will apparently have the effect of preventing suppuration, or otherwise cutting short the disease. Among the remedies that have been thought especially efficacious may be mentioned oil of turpentine, guaiacum, tincture of chloride of iron, iodoform, tincture of camphor, iodine, Peruvian balsam, carbolic acid, peroxide of hydrogen, formaldehyde, nitrate of silver, sulphate of copper, aristol, europhene, iodide and bromide of calcium, and the salicylates. Besides these agents for topical medication the administration of aconite, quinine, sulphite of sodium, salicylic acid, sulphide of calcium, biniodide of mercury, etc., have been often highly recommended. It is well always to begin the treatment of this disease by the administration of a brisk cathartic, either mercurial or saline, or both, sometimes in conjunction with hot bathing. Ice externally applied and also kept in the mouth is useful. Applications by means of spray or pigment of guaiacol, or carbolic acid and glycerine, or nitrate of silver, or insufflations of iodoform, are each very good remedies for topical treatment. After the disease has become established, however, the best plan is to favour suppuration by means of hot fomentations to the neck, and applications to the throat of steam, or hot gargles of flaxseed or slippery elm tea. Gargling, however, is often so painful that it may be a question whether more harm than good does not result from it. As an adjuvant remedy which can be used throughout the whole course of the disease, a spray of two to four per cent of cocaine hydrochlorate will be found valuable for mitigating the sufferings of the patient. As soon as suppuration has become established the practitioner should endeavour to relieve the tissues of the pus; but before adopting such a procedure the finger should be carried into the pharynx and a careful examination made of the affected parts, in order to ascertain the location of the pus. This being done, an incision with either a bistoury or gum lancet will be in order. 
The incision may be made at either the upper or lower portion of the tonsil, or preferably through the anterior pillar of the fauces, whichever place seems best. The gum lancet will be found preferable to the bistoury in the majority of instances, because by the sense of touch it may be carried to the parts and an incision made without fear of doing injury to the subjacent vessels or neighbouring parts. When a bistoury is used its blade should be wrapped with surgeons' plaster up to about half an inch of the point. When the anterior pillar is determined upon for the point of incision the cut should be made in a longitudinal direction to the pillar. When, however, the tonsil proper is the objective point, the incision may be made horizontally. No antiseptic precautions are necessary in these cases, inasmuch as the secretions are more or less contaminated, and become more so by the evacuation of the pus. Scarification of the parts before suppuration has taken place was formerly practised, but as it so seldom produced any beneficial results it is not now very often resorted to.

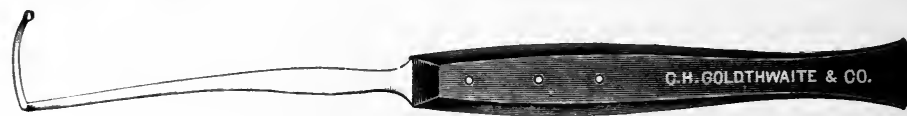

FIG. 42.-Leland's tonsil knife.

Dr. Leland, of Boston, has met with excellent results in cutting short the course of the disease, and also in preventing its recurrence, by thoroughly incising and clearing out the tissues during the stage of exudation. By means of his tonsil knife (see Fig. 42) he incises the tonsil, in a perpendicular direction through its investing membrane, sufficiently to admit the forefinger. The finger is then inserted (after being rendered as aseptic as possible) and made to thoroughly gouge out, as it were, the surrounding glandular tissue. This is a very painful and somewhat heroic proceeding, but nevertheless well worth trial on account of the results which it produces-shortening of the course and preventing recurrence. Recurrence may also sometimes be prevented by either thoroughly curetting or cauterizing the plica triangularis (supratonsillar fossa), a spot where the initial inflammation of acute peritonsilitis is generally found. This should be done soon after an attack. 


\section{CHAPTER VII}

ERYSIPELAS OF THE UPPER AIR PASSAGES-ERYSIPELAS OF THE NOSE, THROAT, AND LARYNX

\section{Nose}

So-CALLED idiopathic facial erysipelas is generally looked upon as an infectious disease of the skin, and is commonly first seen upon the outside of the nose or face, notwithstanding that the majority of such cases actually originate within the vestibule of the nose, or at the inner canthi of the eye. It begins usually in a small papule or furuncle, whence it spreads generally toward the outside. Although erysipelas may be confined to the mucous membranes, yet, as is well known, its predilection is for the skin. The disease may, however, appear primarily upon one of the mucous membranes and run its course there, but usually it occurs secondarily in these tissues, unless we class with it the various phlegmonous or so-called infectious inflammations of the nasal and faucial regions, which some do not regard as erysipelatous.

Etiology.-It is generally conceded that erysipelas is a specific disease caused proximately by a specific organism called the streptococcus erysipelatis. This micro-organism was first isolated and cultivated by Fehleisen. These microbes are sometimes difficult to differentiate from the other members of the streptococci family, but biologic experimentation shows that, when properly introduced into the tissues of the lower animals, an artificial disease identical in all of its characteristics with erysipelas is engendered. Just what the antecedent condition required for the pathogenic operation of the specific organism is has not yet been made out. The disease when affecting the mucous membranes presents different degrees of virulence, from a mild type to one that proves rapidly fatal.

Age.-It seldom attacks the young, although a few cases in infants have been reported. The more susceptible period of life, however, is that of adolescence. Traumatism will 
sometimes give rise to it, and especially an injury to one of the mucous membranes from some chemical agent, such as caustic ammonia, soda, potassa, etc. Flies and other animate objects sometimes carry the infectious material. A case of erysipelas following the lodgment of the Texas screw worm in the nose has been reported.

Symptomatology.-One of the earliest symptoms is a rigour or chilliness followed by pyrexia, the temperature rising to about $101^{\circ}$ or $102^{\circ}$, and in severe and rapidly spreading cases the temperature may reach $104^{\circ}$ or $105^{\circ}$. Adynamia is usually well marked-the pulse being soft and very frequent. Cephalalgia and myalgia are usually attendant symptoms. Albumen also appears in the urine between the third and fifth day. If the disease be located mostly in the nose, stenosis is complete, and this organ, together with the eye-lids and perhaps the adjacent skin of the face, is more or less swollen and tender. The lymphatic glands of the neck are also swollen and tender, and frequently the headache is intense, betokening an impending meningitis. The disease may also involve the accessory. sinuses of the nose.

When the disease affects the fauces there is intense pain of the throat (odynophagia), which is aggravated by attempts at swallowing; and if the lymphatic glands be involved the respiration is more or less embarrassed, according to the amount of swelling of the parts. In such cases fatal edematous laryngitis is very apt to terminate the life of the patient. Inspection of the fauces shows the mucous membrane to be intensely hyperæmic and very much swollen. The soft palate, uvula, and faucial pillars are exceedingly swollen, the former hanging down into the cavity of the mouth upon the dorsum of the tongue, while the latter projects out in columnar shaped folds into the cavity of the throat. The surface of the membrane is usually covered with more or less ropy mucus. If the disease be at all extensive the epiglottis is also very much swollen and may be edematous. The base of the tongue and lingual glands also participate and are very much swollen. The disease may extend from the pharynx along the Eustachian tubes into the ears, when a dangerous inflammation of these organs, even followed by meningitis, may result. Should inflammation of the lymphatic and cellular tissue reach the point of suppuration, then additional symptoms pertaining to sepsis, 
such as rigours, chilliness, pyrexia, delirium, and marked depression of the vital powers ensue. (Plate II, Fig. 5.)

Diagnosis. - The diagnosis of this disease in the earlier stages is sometimes very difficult. If erysipelatous inflammation of the skin in the neighbourhood is going on concurrently, or the disease be endemic at the time, the diagnosis becomes very much simplified. Its local manifestations, as well as the general symptoms attending its access, resemble scarlatina, severe acute pharyngitis (or phlegmonous pharyngitis), and phlegmonous or peri-tonsilitis. As erysipelas is not often met with in children, it may be usually excluded from consideration in cases of sore throat occurring in childhood; especially can this be done if scarlatina is prevailing in the neighbourhood. But from acute phlegmonous pharyngitis it cannot always be distinguished, in the case of infants and delicate children. Scarlatina usually presents itself at first in the faucial tonsil, around which the inflammation may be located for a considerable time, whereas in erysipelas the hyperæmia at a very early period is spread over nearly the whole of the pharyngeal mucous membrane. The course of erysipelas is usually shorter in most cases than phlegmonous pharyngitis or tonsilitis. Indeed, it is a fortunate thing for the human family that erysipelas of the mucous membranes is of such infrequent occurrence. This disease could scarcely be mistaken for spasmodic croup (laryngismus stridulus) because of the absence of marked antecedent symptoms, but it may easily be mistaken for edematous laryngitis arising from other causes. Erysipelas of the mucous membrane of the nose, which is very rare, may be mistaken for syphilitic or tuberculous perichondritis or for glanders, but the history of the case, in view of the slower course of perichondritis, will serve to clear up the doubt. In the severe form of glanders destruction of the tissues comes almost immediately after the onset of the disease. The tissues, in the latter affection, do not seem to be hyperæmic from inflammation, for there is an intense intumescence with gangrenous foci almost from the beginning. Besides this, the previous history of the case may aid in the differentiation. Abscess of the septum nasi is sometimes mistaken for erysipelas, but the limited degree of constitutional disturbance, together with the purely local character of the affection, will serve to distinguish this form of disease. 
Prognosis. - The prognosis in this disease is always grave. There are especially three sources of danger. One is edematous laryngitis, another is septicæmia, and another is meningitis. One of these conditions, or all combined, may be present in a given case. Should the type of the disease be mild the chances of the patient's recovery are usually good, although a protracted convalescence, with more or less chronic inflammation of the mucous membrane affected, will ensue. The usual course of the disease is from ten days to two weeks, when no such complication as suppuration ensues; otherwise the illness may cover a period of one to two months.

Treatment.- The therapeutic indications are, first, to limit the local process, if that be possible, and, secondly, to assist nature in resisting the general effects of infection. Unfortunately, local treatment of erysipelas of the nose or fauces accomplishes very little beyond palliative effects, inasmuch as the stronger medicinal agents cannot be freely used, owing to the situation of the disease; nor can they be as thoroughly applied, owing to the nature of the tissues involved. However, frequent spraying of the parts with a solution of muriate of ammonia (five to ten grains to the ounce) will usually prove beneficial. After the spraying, applications of creolin or ichthyol, tincture of chloride of iron and glycerine, or oil of turpentine mixed with olive oil (see formulæ), may be applied by means of a camel's-hair brush or cotton wad. When the tissues, however; are very much swollen, all treatment, save the use of spray, may prove impracticable. Throughout the course of the disease small pieces of ice held in the mouth are usually soothing and may accomplish some good. Also the ice-pack around the throat or over the nose will be found highly beneficial during the active stage of the disease. The constitutional treatment adopted should be active. Hypodermic injections of quinine hydrochlorate, or hypodermic injections of anti-streptococcus serum, are the principal agents to be selected. The latter has proved of marked benefit in cutting short and mitigating erysipelas when affecting other parts of the body.

Concerning complications, if suppuration is about to take place the process should be favoured by warm applications in the form of gargles or outside poultices, and pus should be 
evacuated as soon as found. If edematous laryngitis or cedema of the larynx threatens life, then intubation or tracheotomy should be performed, unless the swelling be confined mostly to the vestibule of the larynx, in which case the pent-up fluids in the epiglottis or ary-epiglottidean folds can be released by scarification of the parts. (See chapter on Acute Laryngitis.)

\section{Acute Retropharyngeal Abscess}

This disease, which fortunately is not of frequent occurrence, has attracted a great deal of attention both on account of its fatal tendency and the variety of its clinical characteristics. It consists of an active inflammation and infiltration of the lymphatic and connective tissue of the retropharyngeal region, with a rapid formation of either circumscribed or diffuse abscess. On account of the marked implication of the adenoid tissue, Casselberry suggests the name "lympho-adenitis" for these cases. According to Hencke, the disease was first discovered by Fleming in I850. Hencke, Bokai, Gautier, Gougenheim, Burkhardt, Roe, Mayer, Bosworth, Knight, Cohen, Koplik, and other writers have since furnished valuable literature upon this subject.

Etiology. Age.-The disease generally affects infants and children. The opinion of those who have investigated the subject is that the disease is found oftener in infancy and childhood before ten years of age. In infancy the majority of cases occur before the age of two years (Koplik, Mayer, Roe, Gougenheim). A few cases have been seen in adults. Dr. R. H. Herrold, of Chicago, reported a case of retropharyngeal abscess in a young woman twenty-two years of age, who was previously healthy. It apparently ran a short course of about thirty-six hours from the time of the beginning of alarming symptoms. There was spontaneous rupture of the abscess. No known cause for the attack was discovered. Moore reported a case in a man aged forty years; Dunn, a man aged sixty-two; and Fischer two cases, one a boy aged eighteen years, and the other a man aged thirty-eight years, respectively. The writer has seen one case in an adult, aged thirty, and but four cases in children, one of which was a fatal "burrowing abscess" which finally reached the mediastinum. In children and infants the retropharyngeal space, so far as its length is concerned, is not 
so large comparatively as in the adult. Symington shows that although the whole neck is relatively longer in children than in the adult, the larynx in infants is fully one vertebra higher than in the adult. The retropharyngeal space in infants is thus encroached upon, and in the presence of abscess gives rise to symptoms of pressure on the larynx sooner than would be the case in an adult. The retropharyngeal space is, according to the investigation of Gillette, the seat of several lymph nodes which are intimately connected with the system of lymph vessels belonging to the tonsils and the soft tissues of the palate. These in turn are connected with the glandula faciales profunde and the glandula cervicales profunde. All processes, therefore, which are going on in these organs, such as simple angina, diphtheria, or the infections of scarlet fever or measles, or any lesions of the mucous membranes of the mouth, such as aphthæ, are apt to affect in sympathy these retropharyngeal glands (Karewski, Bokai, Koplik, Mayer). Some writers believe that the origin of the disease is generally found in some throat infection, such as peritonsilitis or the anginas connected with the exanthemata-scarlatina or measles, or with diphtheria-while others who have seen a number of cases deny this relationship so far as diphtheria and the exanthemata are concerned. To caries of the vertebræ was formerly ascribed the origin of many cases, but in the light of recent investigations caries or other disease of the vertebræ is found to be responsible for a very small proportion of the cases. Bokai, as quoted by Casselberry, found only seven cases out of two hundred and four that were secondary to caries of the vertebræ. There are a few cases in which syphilis has been the cause. The prevailing opinion is that the essential cause of the disease is an infection by bacteria-some one of the streptococcus family.

Koplik has isolated four distinct species of the streptococcus, which he says are the micro-organisms preșent in the pus of these abscesses.

The streptococci classify themselves into two varieties, first, Streptococcus brevis, and, second, Streptococcus longus. By animal experiments with both streptococci varieties it was shown that they produce no effects similar to what is seen in experiments with streptococci taken from virulent pus; they were not virulent, and the animals were not visibly disturbed in health by the investigation. But one animal, a young rabbit, contracted 
pleurisy from infection of Streptococcus longus (a) pharyngis, and died in three days. The Streptococcus longus (b) produced no visible effect on the health of the animals experimented upon. In no animal was tuberculosis produced from the injection of any pus in the pure crude state. This is important by showing the statement of some observers, that acute retropharyngeal abscess in some cases is tuberculous, is unsupported by investigation. It must be concluded, therefore, from a study of the bacteriology of acute retropharyngeal abscess, that the abscess in these cases is purely an acute pyogenic infection, and unconnected with any previously existing tuberculous diathesis. The affection of the lymph nodes or bones of the spinal column must be taken apart from those cases which constitute the infantile sepsis of scarlet fever. Regarding the abscess also, it is a benign disease, with a tendency, when aided by treatment, toward rapid recovery. Koplik's experience is based upon seventyseven cases of retropharyngeal abscess taken from cases in infancy and childhood below ten years of age.

Symptomatology.-The physical signs of acute retropharyngeal abscess, obtained by inspection and careful digital examination of the fauces, give the most positive information. The elevated temperature, the anxious expression of the child, the pain evinced upon movement, especially of head and neck, and the peculiar, changed piping voice constitute valuable clinical signs. The head of the patient is sometimes thrown back, and there is a peculiarly nasal, metallic noise given forth by the infant, leading to a suspicion of pressure on the larynx. The infant refuses to nurse, and wakes up with a start at intervals, breathing with difficulty if the abscess be formed. The temperature ranges from $101^{\circ}$ to $104^{\circ}$, and the pulse rate is usually 120 to 140 .

Diagnosis. - The diagnosis is not difficult if one has met with a few cases, yet these cases might be mistaken for acute laryngeal disease (croup) or diphtheritic paralysis of the fauces. In a great number of infants, not in any way suffering from pharyngeal disease, the fauces may appear swollen; and when the head is thrown back there may be seen a prominence in the fauces to one or the other side, which is caused by an abnormal development of the vertebral bodies in this region. In such a case the only way to find out positively is to carefully explore the walls of the pharynx with the index finger. Kop- 
lik has sometimes met with cases where there was retropharyngeal adenoids without abscess, and where the enlarged node was hard. Sometimes "the abscess is so low down, opposite the larynx, as not to be visible through the opening in the fauces," or sometimes low down and laterally situated behind the pillars. Therefore by digital exploration alone can one make the diagnosis. Mayer's table for differential diagnosis is an excellent one, and is as follows:

\section{IN CROUP}

Voice, soon extinguished.

Dysphagia, none.

False'membrane, present.

Bacilli, present.

Tumefaction, none.
IN RETROPHARYNGEAL ABSCESS

Only altered in intonation.

Very prominent.

None.

None.

Always present.

To this may be added the suggestion of Cohen, that if retropharyngeal abscess be present, the breathing is relieved by bending the head of the child forward, and increased by bending the head backward, or placing the child on its back.

The restlessness of the child is often so extreme that examination is nearly impossible. Geralde and others have suggested chloroform narcosis, in order to allay this restlessness and to permit examination.

It is needless to add that the administration of chloroform is fraught with great danger, and that with the use of a properly constructed mouth gag an examination of the most intractable subject may be made. While examining with the finger the abscess may be ruptured, when the pus is apt to flow into the larynx; therefore severe pressure should be avoided. Accidents of this sort are recorded by Bokai and Hickey in one case.

Prognosis.-Many of these abscesses, if left alone, will naturally burst and thus bring about recovery. There are several cases recorded where these abscesses have burst and discharged gradually, and no case has been known of an infant having been suffocated during the natural course of the case (Koplik). Bokai, however, records such a fatality having happened during sleep. He recorded a case of prostration and continuance of pressure symptoms of the larynx after the evacuation of the pus, and also a similar case in which râles were heard in the chest, indicating the possible inspiration of pus into the lungs. Laryngeal spasms, and one case complicated by basilar menin- 
gitis, have been reported in connection with this affection. In another case diphtheria caused death. In another, pneumonia. Platot and Variot have recorded the death of an infant suffering from retropharyngeal abscess at the moment of incision. Death did not occur from invasion of the air passages with pus, but from reflex syncope. Bokai records one case of burrowing retropharyngeal abscess which caused death by hemorrhage, from its opening into a large blood-vessel. The pus may burst into the interior of the ear and thus discharge externally. The majority of these cases end in recovery. Of Bokai Sr.'s one.hundred and seventy-nine patients, only six died; and of Bokai Jr.'s one hundred and thirty-eight cases, only eight died.

Treatment.-The chief aim is to evacuate the abscess as soon as possible, either by internal or external incision. Burkhardt advocates operating from without always, because of the difficulty of keeping the abscess open. He also advocates general anæsthesia. Mayer also endorses the ideas and method of Burkhardt. Bokai says that in one hundred and six cases in which he operated internally he was compelled to reincise two or more times in seventeen cases only. He favours strongly internal incision. He thinks the demand for antiseptic treatment in children is less urgent than it is with adults.

The following is the method of incising internally: The infant, having been freed from articles of dress, is held in the lap of an assistant in such a manner as to face the light (unless it be deemed advisable to use a head mirror). The head is held firm by a second assistant. The operator, standing in front of the patient, depresses the tongue firmly with a tongue depresser, or a mouth-gag is introduced, so as, if possible, to expose the abscess. A bistoury, with the blade covered by rubber plaster so as to leave only half an inch of the point exposed, is inserted into the abscess, and the incision made longitudinally from above downward, inclining, if at all, toward the median line. The assistant is instructed to quickly turn the infant forward, face down, as soon as the abscess is incised, so that the pus may escape from the mouth. By exerting gentle pressure at the side of the neck the flow of pus is often facilitated. Sometimes blunt headed compressing forceps (guided with the finger) may be inserted afterward into the incision and then opened a little in order to aid the speedy escape of the abscess 
contents. It is not well to incise the swelling before it has suppurated, for in this way the symptoms will not be relieved. It is desirable therefore to wait until you can discover deep fluctuation before incising. In cases where the abscess can not be seen it can be opened, guided by the sense of touch. In cases where the cervical glands seem to be extensively swollen and softened, external operation is preferable. Tuberculous abscesses also might better be opened from without in many cases (Koplik). When the abscess is in full view through the mouth, there is no question about the propriety of opening it within instead of from the outside of the fauces. The use of chloroform or ether should be avoided if possible, but in cases of external operation, where much careful dissection from outside the neck is required, general anæsthesia should be adopted without hesitation.

\section{Acute Infectious Phlegmonous Pharyngitis (Pharyngitis} Phlegmonosa Diffusa-Cellulitis Phlegmonosa Pharyngis)

An acute, dangerous, and diffuse phlegmonous inflammation of the adenoid and cellular tissue of the throat or neck, resulting in suppuration.

Etiology.-Acute infectious phlegmonous pharyngitis, or primary acute pharyngitis, was first described as a new affection by Senator. It is believed by some to be of the same nature as peritonsilitis ascendens. Some authors believe that it is simply an extension of the common acute septic phlegmon of the pharynx or tonsils to the cellular tissue of the neck. The disease is generally believed to be of undoubted bacterial origin, although no particular micro-organism has been charged with its causation. Virchow and other pathologists look upon it as identical with the other diffuse phlegmonous processes. Some authorities believe it is identical with erysipelas of the pharynx, and others that it is the same disease as angina Ludovici (Ludwig's disease) ; others, again, that it is a form of retropharyngeal abscess. Hamann thinks the term "Ludwig's angina " should be dropped, as it is applied to several affections. It may occur in connection with infection of the tongue or mouth. A fatal case has been reported of streptococcic infection of the tongue in the course of a puerperal affection with severe acute glossitis and great swelling of the anterior part of 
the tongue and submaxillary region; streptococci were found after three days. It was supposed in this case that infection had taken place by the mouth. This disease generally attacks healthy persons.

Symptomatology.-The prodromal stage is very short and active. The principal early symptoms are soreness of the throat, muscles of the neck and back, a long rigour or several rigours followed by high fever, the temperature rising to $103^{\circ}$ or $104^{\circ}$. The temperature may not reach the normal point or below during the course of the disease except at the approach of death. The amount of hoarseness and other laryngeal symptoms will of course depend upon the location of the disease and whether or not the upper larynx be involved. The laryngeal as well as the pharyngeal mucosa may participate in the hyperæmia and general swelling, or, on the contrary, the internal structures of the throat may be but comparatively slightly affected. The submaxillary and cervical glands are usually very much swollen and tender. Indeed, the whole cervical region rapidly swells and feels doughy to the touch. The prostration becomes great in a comparatively short time, and as the disease advances the symptoms become very grave, the clinical history being analogous to that of any other ease of acute and overwhelming sepsis. The face has an anxious expression; the skin is cold, and covered with clammy perspiration; the neck on the affected side, even to the clavicle or below, is greatly swollen and sodden. The temperature remains high ; tongue, lips, and gums are dry and covered by sordes. There is stupor or a muttering delirium, and sometimes active delirium. The pulse grows very weak and "thready," and the signs of approaching dissolution are marked. Sometimes the initial faucial symptoms are quite insignificant. The author has seen two cases of this sort, in which death ensued on the sixth and eighth days respectively, in which the angina was apparently very mild. Should the case be prolonged, the course of the disease simulates that of typhoid fever, and death may come through exhaustion. The urine, usually scanty, always contains albumen, and in some cases may be suppressed. The usual course of the disease is short and sharp; death may ensue in from six to ten days. The tissues are enormously swollen. There is a diffuse purulent infiltration of the deeper parts of the neck, including sometimes 
the cellular tissue over the thorax. The contents of one of these diffuse abscesses is an abundant and ichorous pus of greenish colour and fetid odour. The streptococcus pyogenes aureus and tetragenus have been found in the pus from these abscesses, and to the invasion of the latter the disease has been ascribed by some pathologists.

Diagnosis.-This affection may be mistaken for retropharyngeal abscess, erysipelas, or scarlatina anginosa. The rapid course and violent systemic and local disturbance of this disease will serve to differentiate it from the others.

Treatment.-Treatment is usually quite unavailing. Mercurial preparations, such as the mild chloride or the corrosive chloride, may be used at first, and after that strychnia and quinine, digitalis, and nitroglycerine may be given, or boracic acid in five-grain doses up to the point of physiological tolerance. At the very earliest appearance of suppuration, however, the parts should be freely incised, drained, and the abcesss cavity thoroughly washed with peroxide of hydrogen or mercuric bichloride. Antistreptococcus serum may prove beneficial and should be tried. The quinine, as well as the strychnia, should always be administered hypodermically; quinine hydrochlorate may be given in five-grain doses in this way every four hours.

Mangen and Reynold have used normal saline solution in such cases of sepsis-subcutaneous injections in quantities varying from two hundred to five hundred grammes-with excellent results. 


\section{CHAPTER VIII}

DISEASES OF THE NASAL PASSAGES, PHARYNX, AND LARYNX DEPENDING UPON THE EXANTHEMATA AND THE ESSENTIAL FEVERS

Scarlet Fever (Scarlatina).-Scarlet fever, perhaps more than any other of the exanthemata, is responsible for the development of inflammation, suppuration, or ulceration of the pharynx. It is well known that in some forms the pharynx bears the brunt of the disease (scarlatina anginosa). Slight inflammation of the pharynx and soft palate, with more or less intumescence, attends nearly every case of scarlet fever, and in the severer types of the disease destructive ulceration may ensue. A rash (redness and furriness) upon the soft palate may precede the appearance of the characteristic rash upon the skin. At first the eruption appears as red blotches upon the posterior wall of the pharynx and anterior surface of the velum palati. Then the mucous membrane within twelve to twenty-four hours afterward begins to swell, and continues to do so until extreme tumefaction of the whole of the fauces may supervene, including sometimes the faucial tonsils. As the disease advances, in some instances the swollen tissue begins to break down, and phlyctenulæ appear, which soon become points of ulceration. The amount and continuance of the ulceration will depend largely upon the type and course of the disease. In severe cases, especially those occurring in weak and broken-down subjects, the points of ulceration may coalesce into large patches, and the subsequent sloughing and destruction of tissue may become very great. The writer has seen cases which resulted in the loss of portions of the soft palate, the uvula, parts of the faucial pillars, or portions of the palatal tonsils. Fortunately, such grave cases are not frequently met with. The larynx, in such cases, is involved to a certain extent so that the epiglottis may participate in the ulceration, and a dangerous œdema glottidis may arise before the local affection becomes 
checked. Tumefaction, ulceration, or sloughing may attend any severe type of the disease. In such cases vigorous local treatment will be found necessary.

Treatment.-In the first place, the throat should be kept as clear as possible of the accumulating secretions by the free use of the syringe with suitable antiseptic solutions, such as peroxide of hydrogen, carbolic acid, resorcin, permanganate of potassium, diluted chlorine water. (See formula.) After the cleansing just referred to has been accomplished, applications of nitrate of silver, iodoform, aristol, Loeffler's solution, or oil of turpentine should be made to the ulcerating spots at least twice daily. The local treatment should be assiduously carried out until healthy granulation of the abraded surfaces is brought about.

In case ulceration of the epiglottis or ulcerative laryngitis ensues, insufflations of iodoform mixed with powdered gum acacia (see formula) should be frequently used.

Measles.-With measles the lining membrane of the whole pulmonary tract is usually more or less involved. On the third day the symptoms of an intense rhinitis, such as lachrymation, nasal stenosis, and extra nasal secretion are usually present in all cases. When the frontal sinus or ethmoid cells are involved, the headache, photophobia, or neuralgia may be very much aggravated, and may be accompanied in young children by convulsions. If infection of one of the accessory cavities has taken place, a great increase in the pyrexia is apparent, and soon the nasal secretion may take on a purulent character, while the frontal and nasal regions will show marked tenderness to the touch. The larynx is also the seat of more or less inflammation in this disease, which may amount to a serious complication through the swelling of the mucous membrane, which in turn may interfere with respiration to a dangerous degree. Thus an œdema of the epiglottis or glottis, or an acute edematous laryngitis, is likely to arise. In such conditions there is extinction of the voice, with stridulous respiration, brassy cough, or, worse, dyspnœa and impending asphyxia.

The nasal mucous membrane, fauces, and larynx usually suffer most in this disease. There may be considerable sinusitis or laryngitis. Among the accessory cavities the frontal sinus and ethmoid cells are the ones most frequently involved, although exceptionally in older subjects the maxillary sinus is 
affected. A purulent rhinitis, and occasionally abscess of the septum nasi, may be produced; also a catarrhal inflammation of the middle ear or disease of the mastoid cells may accompany and complicate the affection.

Treatment.-Warm saline solutions (preferably a solution of common salt), one drachm to the pint, should be injected into the nasal passages in all cases which show a severe degree of rhinitis. This may be followed by a spray of a weak solution of cocaine (one half to one grain to the ounce) for children, and (two grains to the ounce) a somewhat stronger one for adolescents. Besides this, mild oleaginous antiseptic solutions of eucalyptus oil, menthol, etc., may be used. Mercury bichloride and thymol, as a rule, seem to be too irritating for continued use in such cases, but a one-half to one-per-cent mixture of creolin in water is sometimes well borne. In addition, cold compresses may with advantage be laid over the frontal and nasal regions.

After the subsidence of the disease the nasal passages should be thoroughly examined, and if there be evidence of a purulent rhinitis, treatment for such a condition must be adopted at once. Hypertrophic rhinitis, chronic tonsilitis, or enlargement of the adenoid tissue at the vault of the pharynx may be induced by an attack of measles.

A mild laryngitis may need no particular treatment, but when it is observed that the patient is very hoarse and has a very brassy cough, inhalations of steam, or steam medicated with compound tincture of benzoin, or sodium bicarbonate or sodium benzoate, should be prescribed; at the same time compresses of cold water may be laid over the outside of the larynx. The inhalations may be used every hour or two throughout the day and night, if necessary. In addition, the patient should be kept in a warm room, the air of which is constantly charged with steam, and a teaspoonful of

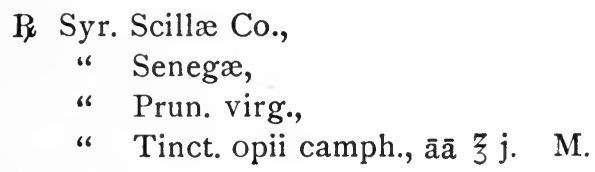

may be given every two, three, or four hours. Counter-irritation may also be made over the larynx. Should these measures fail to give relief and the symptoms indicate approaching 
laryngeal stenosis, then intubation or tracheotomy should be resorted to without too much delay.

Rötheln.-Although in this disease the eruption may be detected upon the mucous membrane of the fauces and a mild degree of fauceitis be present, yet it is very seldom necessary to institute any local treatment. The writer, however, has seen one case of rötheln with severe inflammation of the larynx which caused for a short time a great deal of anxiety, and required vigorous local treatment for its relief. But as a rule the faucial or laryngeal inflammation is very slight.

Variola and Varicella. - The throat complications sometimes give rise to a great deal of trouble. The eruption may occur upon the soft palate or posterior wall of the pharynx before showing itself upon the skin. Excepting very severe cases, however, a few only of the papules or vesicles appear in the throat, generally not more than a dozen or so. Exceptionally, however, the mucous membrane takes considerable part in the disease, in which event the attendant inflammation and tumefaction may become dangerous. The larynx may also be the seat of some of the papules or vesicles, in which event there is more or less inflammation and swelling of the parts, with disturbance of respiration, amounting perhaps to alarming dyspnœa, if not fatal asphyxiation, as a result of the great amount of swelling and consequent contraction of the lumen of the larynx and trachea. In one case which came under the writer's observation the laryngeal stenosis was so great that tracheotomy was necessary. The operation, however, did not save the patient, on account of the virulence of the sepsis attending the attack. Rhinitis of a severe type has also been observed in this disease. The writer is not aware of any case of disease of the accessory cavities having been attributed to variola.

Typhoid Fever.-The principal throat complications of typhoid fever consist of various hemorrhages from the pharynx or larynx, or adenopathies. Pharyngitis, laryngitis, or various forms and degrees of paresis, affecting the constrictor muscles of the pharynx or the laryngeal muscles, may occur. Dr. Kresilian has reported four consecutive cases of typhoid fever in which throat lesions had been presented. These consisted for the most part of severe pharyngitis, with ulceration and swelling of the mucosa extending into the ears. Paresis of the adductor muscles of the larynx is the usual form, occurring in 
severe types of typhoid fever. Although the paresis is usually temporary, passing off at the end of a tedious convalescence, yet it may remain for a long time. The writer has met with three cases of paresis of a vocal band due to typhoid fever in which the lesion was permanent. In each case the laryngoscopic mirror showed an adductor paresis or paralysis. In one case, however, the immobility of the vocal band seemed to be partially due to some hyperplasia of the arytenoid region. The cause of the vocal disability is usually either a neuritis, a temporary cerebral lesion affecting the nutrition of the ninth pair of nerves, or pressure from some adenopathy.

Typhus Fever.-Laryngeal complications have been reported in connection with typhus fever, but as that disease is so rarely met with nowadays a mere mention will be sufficient.

Malarial Fevers.-The various malarial fevers, and especially chronic malarial toxæmia, frequently give rise to various pharyngeal and laryngeal derangements, chiefly inflammations or neuroses. The character of the throat lesions is usually neurotic, consisting of various spasmodic affections of the pharyngeal constrictors or supraglottic muscles. Generally, a paresis and not a paralysis has been observed in connection with malarial diseases. These lesions may be permanent or temporary, although as a rule they are transient, disappearing with the permanent subsidence of the malarial disease. The writer met with one very interesting case of chorea of the vocal cords, and one of laryngeal epilepsy, which (as the antimalarial treatment showed) depended upon chronic malarial toxæmia.

Rheumatism.-Congestion and inflammation of the mucous membrane and muscles of the pharynx, and morbid conditions of the tonsils and larynx, may occur in connection with rheumatism and gout. Many observers believe that rheumatism, or at least an excess of uric acid, etc., in the system, plays a prominent part in the etiology of diseases of the fauces and larynx. Many observers have studied and reported cases of rheumatism affecting wholly the fauces or larynx. Up to recent years very little had been written regarding this peculiar form of throat lesion. Dr. Ingals and the writer presented papers before the Ninth International Medical Congress upon different phases of this subject in which the theories and observations up to that time were gathered and set forth. Undoubtedly there are instances of articular rheumatism affect- 
ing the arytenoid joints, and of muscular rheumatism affecting the muscles of the pharynx and larynx. There are also perhaps cases of acute tonsilitis of rheumatic origin, yet they are not very frequently met with as distinct forms of disease. But because a person possessing a "rheumatic diathesis" is the subject of attack by one of the diseases in question, it is not therefore to be inferred that the case is one of rheumatism. In many of the cases supposed to be rheumatic affections of the fauces, tonsils, and larynx the rheumatic element might be logically excluded. The gouty diathesis is held responsible for a certain number of cases of pharyngeal and laryngeal disease, especially those of a chronic nature.

Ingals, Allen, Knight, Langmaid, Bishop, and others who have paid particular attention to these forms of throat disease in relation to the development of uric acid, etc., have adduced a great many facts to show that a given number of cases have originated from the faulty metabolism expressed by the rheumatic and gouty diathesis. Now and then cases are met with of acute as well as subacute or chronic inflammations of the mucous membranes or muscles of the throat which are undoubtedly of a rheumatic or gouty. nature.

Treatment.-Whenever it seems plain that there is a connection between the throat affection and the development of uric acid or lithiasis, and the subject is known to be prone to attacks of either acute or chronic rheumatism, of course appropriate antirheumatic treatment should be adopted, especially in those cases in which the hyperæmia of the mucous membrane, soreness, and other symptoms seem to be prolonged and otherwise unaccounted for. The same may be said with reference to gout, which undoubtedly in rare instances may constitute the principal cause of the throat affection. Therefore mineral waters, baths, the salicylates, and other suitable treatment should be adopted. The writer has met with several cases of typical "sore throat" which presented objectively little or no abnormality of the mucous membrane which proved to be of a rheumatic nature, and yielded to appropriate treatment for subacute rheumatism. Rarely, cases of rheumatism affecting the arytenoid joints principally have been observed. 


\section{PART II}

\section{CHAPTER IX}

NEUROSES OF THE UPPER AIR PASSAGES

THE nervous diseases of the upper air passages may be conveniently considered according to the following classifications :

I. Disorders affecting the nerves of special sense.

2. Disorders affecting the sensory or vasomotor nerves.

3. Disorders affecting the motor nerves.

The first group comprises mainly disturbances of the function of olfaction-parosmia, hyperosmia, anosmia, and phonation.

The second group comprises perversions of ordinary sensibility-paræsthesia, hyperæsthesia, anæsthesia, neuralgia, and vasomotor disturbances.

The third group comprises paresis, paralysis, and the various spastic affections.

Any of these abnormalities may be due to a cerebral, bulbar, spinal, or peripheral lesion, or may be the result of either a direct or reflex irritation. According to Kirk and Pfluger, reflex movements occurring independently of sensation are excito-motor. Those guided or accompanied by sensation (feebly perceptive) are called sensori-motor. All reflex movements are involuntary, although subject to some control.

Recent physiological research has shown that the sympathetic nerve elements are directly connected with every part and tissue of the body, and therefore that peripheral effects are not entirely dependent upon communicating branches.

Dr. Carpenter believes that there are intimate relations existing between morbid conditions of the nose and diseases of the central nervous system. He considers many cases of neuralgia, partial paralysis, hyperæsthesia, analgesia of the extremities, epilepsy, and chorea as accompaniments of chronic naso- 
pharyngeal catarrh. They are very readily explained through reflex irritation, and subside on proper treatment given to the nasal affection.

\section{Neuroses of the Nose and Nasopharynx}

One of the best classifications of this subject is the following, given by Dr. Joseph White in Burnett's System: “ Neuroses of the nose and nasopharynx may be divided into the neuroses of olfaction, or alterations of the sense of smell, and reflex neuroses, which comprise the highest group of phenomena seen in motor, vasomotor, and trophic disturbances, which at times emanate from impressions made on the olfactory or the trigeminus and its sympathetic connections. Under this classification we may have parosmia or parosphresia, hyperosmia or hyperosphresia, anosmia or anosphresia."

\section{GROUP I}

\section{Parosmia, Hyperosmia, Anosmia}

Parosmia.-Parosmia consists of a perturbation of the sense of smell without complete loss or without exaggeration, through which a person perceives odours which are not at the time presented. The consequent sensations may be agreeable or disagreeable, independently of the nature of the objects from which they arise. There are many different examples of this condition, such as a perverted sense of smell perceived from flowers or perfumes, those which are agreeable or disagreeable to other persons producing the opposite effect upon the subject of this affection. I once met with a young lady whose perception of the odour emanating from nearly all of the garden flowers was to her like that of illuminating gas; this person did not suffer from any known structural disease, but was a highly nervous individual with an unusually keen intellect. A similar state of olfaction is a frequent accompaniment of insanity, and may be conjoined to a perturbation of the other special senses. I remember to have met an insane patient who perceived the most delightful sensations from the odour of fæces; and another who delighted in the odour of onions; indeed, the latter person would procure and hide onions about her room and under her bed. These perturbations may also be temporarily incident to many of the acute diseases, such as typhoid fever, pneumonia, la grippe, etc. In such instances they are undoubtedly depend- 
ent upon irregular excitations and depressions of the central nervous system. Parosmia is rarely due to local disease of the nasal chambers. It is usually met with in persons of a highly sensitive nervous organization, and may be congenital.

Hyperosmia.-Hyperosmia, as its name denotes, is a condition of undue sensitiveness of olfaction. It is commonly met with among refined and sensitive people. Indeed, I have never met with an instance in any other sort of person. It may be a result of an excessive æsthetic cultivation. Such persons are always sensitive, and easily shocked by emotions of any sort. I knew a gentleman who was so sensitive that his appetite was often annihilated by the ordinary odour of food when placed upon the table. It was such a marked habit of his economy that in his home the dining table had to be supplied with a clockwork fan in order to dissipate the odour from the hot food as it was put upon the table. He could hardly sit down to a meal supplied with onions, cabbage, or any of the decomposed cheeses (so delicious to many palates). We meet with many persons who seem to be endowed with a very acute sense of smell for the recognition of all, especially disagreeable, odours. This abnormality can scarcely be considered a morbid condition in many instances, but rather an exaggeration of physiological perception approaching the higher specialization of the sense of smell belonging to the lower animals. This exaggerated sense may not be confined to olfaction alone in some individuals, but applies to those of sight and hearing as well. These conditions may be congenital or acquired, and the cause local or central. Among the local causes most frequently tending to their production are undoubtedly inflammatory or structural changes in the nerve or nerve endings in the olfactory passages. Such obliquities of function may come from continuous irritation or pressure, or trophic disturbances, perhaps, induced by glandular atrophy or by neuritis. Hyperosmia, however, more frequently accompanies acute or subacute local inflammations or systemic affections than it does chronic structural changes of the nasal chambers. The most frequent causes, however, must be sought for in individual peculiarities, either local or general. In most of the cases met with it will be found that this sensitiveness has existed either with or without excessive sternutation from childhood, and if the patient be otherwise healthy, and there be no structural change in any 
of the nervous apparatus of the part or adjacent parts, it must be placed to the credit of that field of unaccountable phenomena called idiosyncrasy. In other. cases, and indeed in many of them, it can be traced to some abnormal condition of the sensory nervous system itself.

Anosmia.-Anosmia is by far the most common derangement of the function of olfaction. The subject has been variously divided by different authors for consideration; by some it is spoken of as anosmia inspiratoria or anosmia expiratoria, according as the act of smell is inspiratory or expiratory with the subject ( $Z_{\text {waardemaker}}$. It is also divided into anosmia essentialis or anosmia intercranialis, according as the nerve endings or nerves themselves are altered, or the central olfactory apparatus in the brain is affected (White). The disease may be unilateral or bilateral, partial or complete. It is always accompanied by more or less loss of the sense of taste. The only difference between anosmia inspiratoria and anosmia expiratoria consists in whether the access to the olfactory nerves is effected anteriorly from the vestibule of the nose or posteriorly through the nasopharynx. Anosmia essentialis comprises all those cases depending upon a local cause, and occurs almost always, at least temporarily, during an attack of acute coryza, whether such attack be primary or secondary. It is due in this instance usually to the closure of the attic of the nose by the swelling of the turbinals. When the swelling is chronic the same effect ensues, and exists with more or less interruption throughout the duration of the swelling, but generally alternates between the two sides of the nose. Another frequent cause of anosmia is the presence of nasal polypi, the effect of which, by blocking the upper nasal passages, is the same as when the turbinals are swollen; it practically shuts off the olfactory region from the air, and thus prevents any odoriferous particles from reaching the olfactory region. Next in frequency may be mentioned deformities of the nasal septum or superior turbinated bones which operate likewise toward closing the olfactory fissure, the degree of anosmia being in proportion to the completeness of the closure. Besides the mere stenosis, it is supposed by many that the pressure exerted by polypi or turgescent superior turbinals or a septal deflection is an important element in the production of anosmia through the degenerative or nutritional changes induced by 
the pressure exerted upon the nerve ends. Anosmia is also one of the most persistent accompaniments of atrophic rhinitis, and is undoubtedly due in such instances to degeneration of the nerve apparatus. Such degeneration, however, must be slow of accomplishment, for it is astonishing how long the sense of smell may be lost and yet afterward recovered. Bosworth states that a continuous suspension of this function for eight years is sufficient, under ordinary circumstances, to produce its utter annihilation; but there are cases on record of a restoration of the function after a suspension of fifteen or even eighteen years. In one case related by White the suspension existed twenty-four years. The sense of taste, which so materially depends upon that of smell, is also lost in a proportional degree to that of olfaction. It seems rather curious that the gustatory and hypoglossal nerves, although so intimately related in function and primary origin to the olfactory, are so impotent to reciprocally aid the disabled olfactory nerves in maintaining their functions. Yet it is possible that there may be some imperceptible trophic assistance responsible for preserving the olfactory terminal twigs from utter degeneration in those cases where the olfactory function has been suspended a long time.

Anosmia intercranialis may be looked upon as purely a secondary affection, depending, as before mentioned, upon either disease of the nerve trunk or of the brain in the neighbourhood in which the olfactory bulb or its primordial nucleus lies. It may occur from injuries, such as fracture of the skull or penetrating wounds, and also from various degenerative changes taking place in the brain. Bibard thinks it often occurs after blows upon the head, falls, etc. We are sometimes astonished, however, to observe that extensive disease of the brain in the neighbourhood of the olfactory bulbs or their nuclei may exist (when taking place slowly) without the destruction of this special sense. This point was well exemplified a few years ago by a post-mortem examination upon a patient who died at St. Luke's Hospital (Detroit, Mich.) of extensive chronic abscess of the right frontal lobe of the brain, involving the region about the olfactory bulb as well (the suppuration having originated in the middle and internal ear); no loss or perversion of the sense of smell had been observed during life. In many cases no cause can be discovered. 
Prognosis. - The prognosis of these affections will depend generally upon the cause. Those cases depending upon some general disturbance or idiosyncrasy will hinge entirely upon the course of such conditions. The cases of local origin, so to speak, will of course depend upon the nature of the local disease. When polypi are observed they may be removed, and the anosmia will consequently be relieved; but in those where structural disease of the olfactory fissure has become permanent, the prognosis will of course be less favourable. This is also true of cases depending upon degeneration of nerve trunks, either through deposits in their investing membrane or in the bony foramina through which they pass. The same may be said of accidents to the region.

Treatment. - No special line of treatment for perosmia and hyperosmia can be laid down, inasmuch as these conditions depend so often upon abnormal states of the system. Anosmia of a permanent character depending upon local structural disease must be met by a careful rational treatment having for its object the removal of the local cause. If synechia or bony deflections or polypoid growths or suppuration of the ethmoid cells be the cause, prompt surgical methods for the relief of these conditions must be instituted. In some cases it may be impossible to remove all of the polypoid excrescence with a snare. When such is the case, the curette, either sharp or dull, should be carefully used. Much circumspection is necessary in doing this lest the mucous membrane, containing the olfactory nerve ends and trifacial nerve ends, be unduly injured. The practice of using the galvano-cautery high up in the méatus is a dangerous one, excepting in selected cases with a very wide nasal attic, inasmuch as an amount of destruction may be easily produced which can not subsequently be remedied. Projecting spurs or ridges of the septum should always be removed. In short, the plain indication is to clear the attic of the nose thoroughly so as to render the admission of air for the purposes of olfaction easy. In cases presenting hyperplasia of the membrane itself there is little to be accomplished by surgical interference. In such cases a persistent local medication with such agents as iodine, tannin, creosote, tannate of cannabin, and muriate of ammonia constitutes the better plan of procedure. Likewise in atrophic rhinitis escharotics of all sorts should be avoided, and an 
attempt be made to stay the degenerative changes of the mucous membrane by prudent stimulation with either the galvanic current, the faradic current, massage, or medicinal excitants, in accordance with the treatment recommended for atrophic rhinitis. When hypertrophy of the turbinals exists it should be reduced by one or another of the methods suggested in the chapter upon that subject.

Bibard recommends for anosmia, besides irrigation and faradization, a snuff composed of quinine and bismuth.

\section{GROUP II}

Disorders affecting the Sensory or Vasomotor Nervous Apparatus of the Upper Air Passages. Hay Fever, Hypercesthesia, Anesthesia, Analgesia, Parcesthesia, Neuralgia

The sensory and vasomotor are undoubtedly the most important of the nervous mechanisms, especially in their relation to disease, because of directly supervising the circulation of the blood and fluids, the amount and to a certain extent the character of the internal secretions, the co-ordination of the manifold automatic activities of the economy, and finally the relationship between voluntary and involuntary impulses.

\section{Hay Fever. Idiosyncratic Coryza \\ Synonyms : Estival catarrh, rose cold}

This disease is characterized by an intense coryza, coming on periodically at a particular time or season, running a more or less definite course, usually embracing from one to three months, and often terminating as abruptly as it appears, usually at the advent of frost. There are two kinds-spring and autumn.

Etiology.-The causes of hay fever may be divided into exciting and predisposing.

Exciting Causes.-Owing to the seasonal character of the attacks-appearing in August, the beginning of autumn, or in June-the principal exciting cause for a long time has almost universally been attributed to the ingress and lodgment in the nasal passages of pollen from the estivating plants, such as the graminaceæ, ambrosia artemisiæfolia ragwee(i), and the solidago virga aurea (golden-rod). Hence has arisen the vernacular name of the disease, "hay fever," while the 
attacks occurring in June (the rose month) have been attributed mainly to emanations from the rose, and hence have been denominated by the laity " rose cold." Besides these commonly recognised exciting causes exceptional cases have been observed to arise apparently from emanations from horses, cattle, cats, and other animals. Many of these cases, however, have been confounded with neuroses of similar character (to be mentioned later on), which have no relation whatever to the seasons, and which are not hay fever proper. The pollen explanation of the cause of hay fever has been regarded as inadequate, yet it gains support from the fact that many susceptible individuals when removed from these vegetable sources of irritation are immune from the attack, as, for instance, while at sea or even on the American great lakes. Against this, it must be admitted that change of location plays only a part in modifying or preventing the access of the disorder, because many persons when sojourning in particular (land) localities lose, or nearly lose, their susceptibility, notwithstanding the presence of some of the inimical plants.

Bostwick, himself a sufferer from hay fever, gave a detailed account of this disease in I8I9. Pollen was first suspected to be the exciting cause of hay fever by Elliottson, of London, England, in I831. In I869 Helmholtz promulgated the doctrine that the symptoms were produced by vibrios existing in the nasal passages. In 1873 , Blakely, by carefully conducted experiments, was led to believe that the pollen of grasses and flowers was the existing cause of hay fever. In I883, John N. MacKenzie and Roe advocated the idea that a local hyperæsthesia is associated with it and occasioned by a diseased condition, either latent or active, of the nasal mucous membrane. Daly and Harrison Allen also attributed it to that cause, and to obstruction of one or both nasal chambers. John N. MacKenzie believed that the disease. was dependent upon some functional derangement of the nerve centres as a predisposing cause. He pointed out also that there were sensitive areas in the nasal passages. Sajous advanced the view that it was due to idiosyncrasy; that certain individuals became affected by certain circumstances, as hypertrophy of the nasal mucous membrane with increased irritability. Three conditions are essential factors in the production of hay fever: first, an external irritant; second, predisposition on the part of the subject to be- 
come influenced by this irritant; and, third, a vulnerable or sensitive area over which the system becomes influenced by the irritant. The presence of pollen, the neurotic habit, and the morbid condition of the mucous membrane are therefore held to be the principal causes.

Predisposing Causes-Heredity.-Since only certain individuals are susceptible, and since the phenomena incident to the attack of vasomotor perturbation point out its essentially neurotic character, there can be little doubt about the propriety of classifying the disease among the neuroses. The investigations of Beard long ago, and others since, have clearly shown that either this disease or various other nervous disorders have existed to a greater or lesser degree in the families of a large number of hay-fever sufferers. Indeed, the direct line of such inheritance is often shown in parent and child. Heredity was believed by Baird to have an influence. In Wyman's experience there was heredity in 20 per cent, and in Baird's 33 per cent. In forty cases recorded by Sajous, 35 per cent had near relatives with a clear history of hay fever, and 42 per cent had asthmatic relatives. Baird also established the fact that the prevailing tendency was in the direction of the nervous diseases, such as chorea, epilepsy, and other kindred affections.

Dr. Alexander Haig, Seth Scott Bishop, and many others, attribute the disease chiefly to the presence of an excessive amount of uric acid in the blood or other body fluids. Many practitioners lay great stress upon the causative influence of this product (uric acid). It would seem probable that it is not the presence of this agent (uric acid) which constitutes the causal factor, but the general perversion of the chemical functions of which uric acid may be at times an indication. Indeed, we are uncertain as to just how an excess of uric acid arises and circulates in the blood. Dr. Strangways emphasizes the condition of alkalinity of the nasal secretion as an exciting cause, and for that reason recommends acidifying the same by means of injections of the mild vegetable acids into the nasal passages. Pre-existing disease of the nasal passages, such as hypertrophied turbinals, neoplasms, and deformities of the septum, are to be considered prominent predisposing causes. This view was first really brought out by Daly, and has been strenuously advocated by many laryngologists. Bosworth has re- 
ported that about 80 per cent of cases of hay fever which came under his observation showed either neoplasms, hypertrophies, or deformities of the septum. Dr. Price Brown firmly believes in a strong causal relation between hypertrophic rhinitis and hay fever, which belief is partially based on the clinical history of the disease as manifested upon himself. Edema of the nasal mucous nembrane, Glasgow thinks, especially when occurring in the olfactory region, may induce such structural change in the nerve filaments as to constitute wholly the cause. The theory of the intranasal causation of hay fever is a plausible one, and will undoubtedly explain in a satisfactory way the origin of many cases, but a review and generalization of the clinical facts do not support it. The instructive statistic arrangement of facts given by Bosworth should certainly be accepted without reserve, coming from so careful and reliable an observer, but from a broader clinical view (that of a general practitioner) his data seem inadequate for the following reasons: First, a majority of the cases of hay fever do not fall into the hands of the laryngologist ; second, very many cases of neoplasm, structural changes, septal and other deformities of the nose, do not show any signs or symptoms of hay fever from one year to another; third, general practitioners who meet with the largest number of cases of hay asthma fail to notice obstruction of the nasal passages or other abnormality during the interim between paroxysms. In very many cases it is therefore fair to conclude, although Bosworth and others who believe with him are perfectly correct in their observations as far as they go, that if we make an extensive collective investigation of nearly all of the cases we shall find that the morbid conditions of the nasal chambers play but an inferior part in the etiology of this disease.

Age.-Adolescence, or more particularly the second decade of life, is the period at which most people are attacked. There are many cases on record of children between the ages of six and twelve years suffering from attacks of this disease, and a few in nursing infants.

Sex.-Males are more susceptible than females.

Race.-Race is an important etiological factor. The AngloSaxon, German, and French are more frequently subjects of this disease than other nationalities.

Location.-Hay fever appears more often in northern than in southern climates-more often in low flat countries border- 
ing on large bodies of water than in dry or elevated regions. A steady cool atmosphere or an elevated country not only mitigates the severity of the attack, but frequently confers perfect immunity, while a cool, damp locality favours its occurrence. Until recent times the disease was not frequently met with west of the Mississippi river or in the elevated regions of the east, such as the White Mountains, Adirondacks, or Catskills, but nowadays it is noted that persons dwelling in these places do not enjoy the same immunity as formerly. Bosworth accounts for this fact by the probable changes in the mode of life of the inhabitants, or rather by the change in the character of the inhabitants, comparing the present mode of living with that coincident to pioneer life. This undoubtedly constitutes a rational explanation, although it may reasonably be inferred that changes in the vegetation incident to a more settled civilization may have had some influence also, because many plants which are now found in these regions did not formerly grow there. The destruction of our forests must also be taken into consideration.

Social Condition.-Social condition seems to be an important predisponant, because the disease is almost always met with among the educated and the so-called well-to-do classthose of highly sensitive nervous organization.

Symptomatology.-The disease may be less severe and of shorter duration in its earlier than in its later visitations. Usually the first symptoms are a feeling of more or less malaise, a slight elevation of temperature, and frequent attacks of sneezing. Soon cephalalgia makes its appearance, with occlusion of the nostrils and suffusion of the eyes; there is also a sense of formication or itching which is quite persistent, and generally attributed to the region of the soft palate, vestibule of the nose, or both. The secretion of the nasal membrane is watery, copious, and so irritating that after the lapse of a day or two it excoriates more or less the alæ of the nose and the upper lip. This secretion, however, soon becomes sero-mucous in character, and in some cases contains traces of pus. The extreme stenosis or stuffiness of the nose may abate a little during sleep, but the dependent side of the nose will remain closed because infiltrated by the fluid gravitating from the upper side of the nose. In the severer attacks there is so much headache and confusion of thought that patients are unable to pursue their 
accustomed vocations. The throat, and in the majority of cases the nasopharynx and pharynx, become involved, and the sufferer complains of heat, dryness, tickling, painful deglutition, and even hoarseness and tinnitus aurium.

The appetite in many cases is very much impaired for a considerable time, in consequence of which there may be loss of flesh and marked debility before the attack is over. It must not be thought that the intense symptoms are continuous from hour to hour and from day to day throughout the whole period, for as a rule patients are relieved from time to time for many hours, and may perhaps for a whole day experience a remission of the general and local symptoms. The examination of the nose during the height of an attack discloses a highly intumescent and anæmic condition of the turbinated bodies, with greatly increased sensibility of the mucous membrane; these bodies are so swollen in the majority of cases that the nasal passages are absolutely occluded. The soft palate and pharynx are almost always hyperæmic, and show a varying amount of swelling and hypersecretion; the palatal tonsils, however, are rarely involved to any extent, excepting in rare instances when a patient in this condition contracts an acute follicular tonsilitis. The later accessions of the disease are apt to be accompanied by asthma, which sometimes becomes established and constitutes the principal feature. I have known many old hay-fever patients with whom the symptoms relating to the upper air passages have become transitory and insignificant, while asthma had become the principal manifestation. During its course the skin over any or all parts of the body, except the hands, is apt to become very sensitive, so that a draft of air, either cold or hot, or a few drops of water striking the surface, may quickly bring on an exacerbation of the nasal symptoms. I have known a slight bruise likewise do it.

Pathology.-The pathology of this disease has been until lately lodged in the realm of speculation. There are those who believe that no structural change takes place in any of the parts involved, and that there is no inflammation, in a pathological sense, of the nasal tissues. Others, again, consider the disease simply a neurosis, and essentially an irritation of the ganglionic centres, spheno-palatine, and Gasserian. John N. MacKenzie and others consider it a vasomotor paresis from peripheral irritation only. 
We are greatly indebted to the recent investigations of Dr. Otto Scherer (of Detroit) and Dr. Dunbar (of Hamburg) for a clear exposition of the pathology of this disease. The spring hay fever, which prevails in Europe, arises from the action of the toxine of the graminæ, while the autumn hay fever, of the United States and Canada, arises from the action of the toxine of the ragweed (artemesia).

Dr. Scherer gathered the pollen of the variously accused plants during the season from August 6th to September I 5th, and made various tests after the season, beginning October 27 th, both upon hay fever subjects and immunes.

$\mathrm{He}$ found that the pollen and extract (toxine) of the ragweed only produced an attack readily, and this on hay fever subjects only. To avoid any error from suggestion, the subjects of his experiments were not informed of the object sought by his manipulations. Eleven of his subjects were well-known hay fever sufferers (some having asthma) and 56 were immunes. The preparations used were the pollen itself, the solutions of the toxin extracted (which in every instance of the immunes proved negative), and an etheral extract (in order to ascertain whether there was a volatile potent substance present).

These various preparations were applied either to the mucous membrane of the eyelid, nose, or by subcutaneous injection. In all instances (excepting the etheral extract) the susceptible patient was affected, while the immune was not affected. The hypodermic injections in the immune produced local irritation and swelling of the arm, lasting about forty-eight hours, but no hay fever.

The experimentally induced attack lasted from twenty-four to forty-eight hours, giving all the typical signs. A high temperature destroys the potency of the toxin.

These experiments would therefore demonstrate that autumnal hay fever is caused by a toxin contained in the pollen of the ragweed, which is in some way liberated by maceration in the nasal passages, and thus absorbed into the blood. To account for the peculiar neurotic or systemic effects, Dr. Scherer concludes that the absorbed toxin acting on susceptible nerve centres produces a state of hyperæsthesia similar to the poison of rabies or tetanus, which thus renders the person very susceptible to all sorts of external irritants-such as light, dust, foul air, etc. 
According to Bosworth, there are from eight to sixteen ounces of fluid secreted in the nasal cavities of the healthy subject every day, and under these morbid conditions the amount is obviously greatly increased.

Complications.-One of the most frequent complications of hay fever when the attack is severe is chronic bronchial catarrh and emphysema. Hernia and cerebral apoplexy sometimes occur in consequence of acts of violent sneezing. The "side cavities" of the nose, the ethmoid cells, the frontal, sphenoidal, or maxillary sinuses, may become involved and be the seat of inflammatory processes, which may terminate in suppuration or lead to cerebral meningitis or other disease of the brain. The middle ear also frequently suffers from extension of the local disturbance through the Eustachian tube; violent sneezing or inordinate and incessant blowing of the nose may rupture the tympanum, which in turn may lead to suppurative otitis media. I am unable to find any recorded case of tuberculosis having been induced by hay fever, although not long ago a medical friend in the course of conversation stated that he had met with one such case. Although the paroxysms are usually progressive, for three or four weeks at any rate, yet there are cases in which a decline in the severity of the symptoms gradually takes place after the first week up to the time of their disappearance. There are cases also which continue in mitigated form for a considerable time after the advent of frost; one patient of mine uniformly suffers from about the middle of August until the latter part of December, and as yet $I$ have been unable to find any reason for such a protracted course. A rare complication of this disease is facial erysipelas. I have seen three cases of this sort during a practice of twenty-five years; in one of these patients there has been no return of the hay fever, although fifteen years have now elapsed, so that the erysipelas may in this instance be considered "a blessing in disguise."

Treatment.-The treatment naturally resolves itself into prophylactic, general, and local. The prophylactic treatment should be undertaken from two to three weeks before the expected accession of the disease. During this period great attention should be paid to the general regimen. Exercise in moderation for those of sedentary habits, modification of the amount of labour of brain workers, including a reason- 
able amount of rest, restriction of the habits of those addicted to the pleasures of life, especially in regard to alcoholic beverages and stimulating food, frequent bathing of the body with saline solutions, and cold douches with subsequent friction, the relief of constipation and impaired or tardy digestion, should be carefully ordered with a view to promoting as far as possible a healthy state of the body. At this time any nasal or pharyngeal catarrh should be attended to by mild methods of treatment. By so doing it will be found that, although the attack may not be averted, its course will be very much mitigated. When for any reason the patient cannot migrate to a more salubrious climate, the use of quinine in three to five-grain doses, with or without codeine, will be found a useful treatment from day to day. When there is a great deal of cephalalgia and general nervous perturbation arising from excessive tumefaction of the turbinated bodies, small doses of acetanilid or phenacetin (five grains) may be administered once or twice a day, or, instead of this, an application of cocaine may be made to the nasal passages, either by a spray or pigment (Bosworth). It must be remembered, however, that while this latter agent excels all others in giving prompt relief to the distressing symptoms, it soon loses its efficacy, requiring an increase of the dose. Permanent benefit from this drug is rarely observed, yet there are many cases in which the use of cocaine would seem to be required. For palliation of the local distress, menthol, taken in the mouth in the form of troches, is useful (Price Brown). When quinine is not well borne, salol, salicylate of sodium, or salicin may be substituted. 'In persons of nervous temperament the administration of one-sixtieth grain of phosphorus in oil three times a day, or five minims of Fowler's solution of arsenic in a teaspoonful of tincture of wormwood, will be found beneficial.

Dr. N. J. Wilson, from an examination of the blood, observed that when the patient suffered most the blood was most strongly alkaline (at which time it is richest in uric acid). He found the urine in some cases loaded with uric acid and urates, and in others it contained but little. Many cases did badly on salicylate of sodium. He believes with Haig that a great deal depends upon the selection of the medication. While small doses of quinine are apt to produce uricacidæmia, a large 
dose of six to fifteen grains of the hydrochlorate will have the action of the sulphate, which clears the blood of uric acid and thus benefits the patients. He has frequently benefited asthmatics by giving ten grains of quinine.

"The acidity of the urine bears a fairly constant relation to the urea, both tending to rise and fall together, and the relation given by Haig, I of acid to 6.6 of urea, is very constant. Whatever raises urea raises acidity, and vice versa."

Of course it is unnecessary to remind the practitioner that during this period, from accession to acme, the surroundings of the sufferer, both from a psychic and physical standpoint, should be conducive to rest. I have frequently observed during even mild courses of the disease intense relapses from mental perturbation incident to untoward events, or extra mental requirements arising in the domestic and commercial life of the patient.

The local treatment should be of the most conservative character in all cases. It has become almost a general practice to cauterize the turbinated bodies either with the galvanocautery, trichloracetic acid (Gleitsman), chromic acid (Jarvis), or other agents of an escharotic nature. Such procedures have given good results in a great number of cases, while on the other hand some patients have been made worse through the induction of ulceration, perichondritis, inflammation of the accessory cavities, or septic poisoning. It is therefore obvious that the greatest care and judgment should be exercised before any destructive agents are applied to these parts after the accession of the disease.

It seems to me that discretion would dictate that the proper time for the adoption of any one of these plans of treatment is a month or two previous to the anticipated attack. I have seen one very distressing case of suppurative inflammation of the antrum of Highmore, involving also the lachrymal sac and the nasal duct, and leading to almost fatal septicæmia, from the application of the galvano-cautery after infiltration of the nasal tissues had fully taken place. I have also seen, on the other hand, a few examples of notable curtailment of the course of the disease following a superficial application of the galvanoelectrode to the turbinated bodies during an attack; but such results, of which there are many recorded instances, may possibly be explained by the derivative effect of the cautery, in 
accordance with the therapeutic law of counter-irritation. If such be the case, it might do equally well to select some portion of the skin instead; indeed, it may be stated that the application of a small blister to the upper part of the cervical region, or just behind the ears, or the frequent application of tincture of iodine to these regions, has been observed to bring about a diminution of the severity of the symptoms. It is stated that this practice is more efficacious with old than with young persons. It seems to me that the value of the galvano-cautery in reducing the turbinated bodies must be considered as established. In many instances, although, it will not entirely prevent the expected attack for the ensuing season, and may fail in preventing a recurrence of attack the following seasons, it is apt to modify their severity. For these reasons it would seem that either the galvano-cautery or trichloracetic acid (preferably the former) should be used in nearly every case when the application can be made at least three weeks before the individual's "hay-fever time." The art of its application is no small factor in its therapeutic success. Theoretically, the objects to be gained by its application are, first, the reduction of the size of the turbinated bodies, and, second, the destruction or alteration of the nerve terminals. Sometimes it is sufficient, in order to produce a profound impression on the membrane or nerve endings, to merely singe or scorch, as it were, more or less extensively the surface; this must not be done, however, in cases presenting hypertrophying turbinated bodies, as it might stimulate still further hyperplasia, notwithstanding the probable mitigating effect upon the approaching local disturbance. Inasmuch as we cannot hope to bring the electrode in contact with all the superficial nerve ends, since too many of them are inaccessible from the outside, it therefore seems desirable that the deeper destruction of the turbinated tissue should be the prevailing object of this treatment in the majority of cases. Accordingly the operator should draw one or two lines across the turbinated body with the incandescent electrode, pressing well into the deeper tissues, with the view of their being pinned down, so to speak, by the subsequent cicatricial contraction. One side only of the nose is to be operated on at a séance; the interval elapsing between the manipulation of the two sides will depend, of course, upon the character of the phenomena resulting from the first application. 
The modern treatment by the application of various preparations of the suprarenal gland known as adrenalin compounds, in mixture with salt solution, is highly useful.

The particular action of these preparations is upon the vasomotor filaments, resulting in a decidedly astringent and analgesic effect. These phenomena are ofttimes so marked that an operation under the influence of the agent may be made without pain or much, if any, hemorrhage. The untoward effects of the drug must, of course, be mentioned. On account of probably the idiosyncrasy of the patient the application of

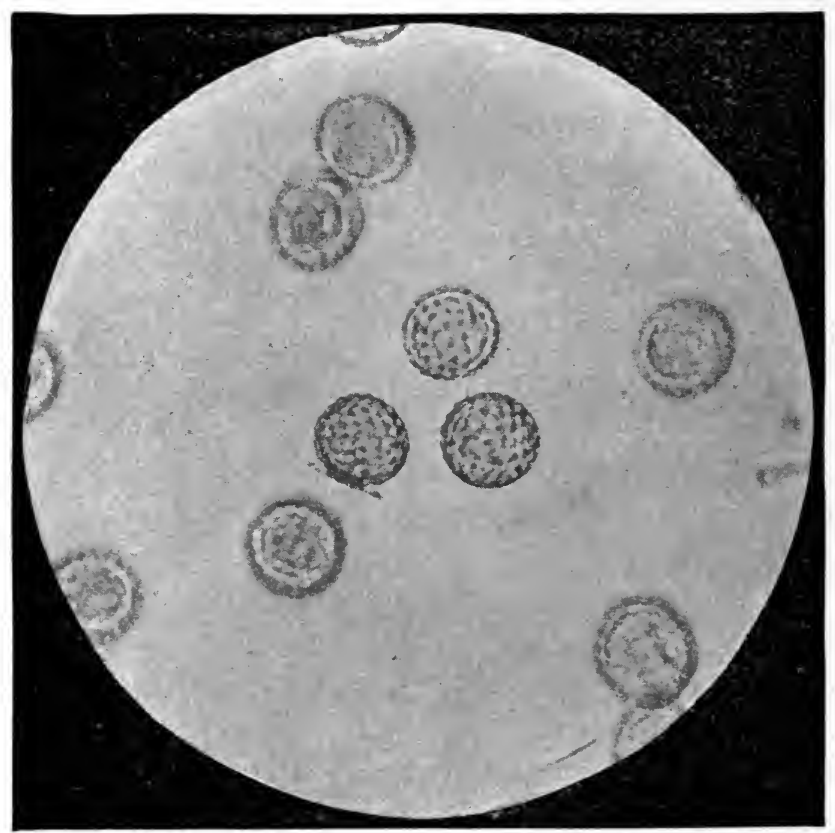

FIG. 42a.-Pollen of ambrosia artemisiæfolia (ragweed), $\times 400$.

adrenalin may produce instead of analgesia persistent irritation and a great amount of coryza. These untoward effects may be greatly modified by changing the strength of the solution-for instance, using I to 20000 or I to 30000 parts instead of I to I0000, which is the common standard. A further modification may be made by substituting for the salt solution bicarbonate of soda. The evil effects of adrenalin solution are far from common, but are met with sufficiently often to warrant their consideration. 
The following is a most useful formula for adrenalin spray:

Sol. adrenalin chlorid $(\mathrm{I}-\mathrm{I} 000) \ldots \ldots \ldots \ldots \ldots$. $\mathrm{I}$ part

Normal salt solution................. parts

There has been placed upon the market recently extracts of the suprarenals which only need dilution to be ready for application, and being freed from the albumins are not liable to decomposition.

Sprays have little or no effect in the majority of cases, unless, perhaps, we except the oleaginous sprays and the suprarenal and cocaine sprays which sometimes relieve at least the distressing symptoms of formication or dryness referred to the soft palate and vestibule of the nose. I think, however, that in the very beginning a thorough cleansing of the nasal passages with a spray or injection of a solution of chloride of sodium, chlorate of potash, or biborate of soda will prove highly beneficial in modifying the subsequent distress. Helmholtz advocated the injection of a solution of hydrochlorate of quinine, upon the theory. that the disease depended upon the existence of certain micro-organisms which he thought could be thus destroyed; this treatment, though very generally used for a time, was soon found to be inefficient.

The injection of various antiseptics, as bichloride of mercùry, oil of eucalyptus, creosote, creolin, carbolic acid, dilute alcohol, thymol, acetic and citric acid, etc., have all been tried, and after a time gradually abandoned. Various snuffs having for their base starch, talcum, subnitrate of bismuth, gum acacia, and containing opium, morphine, atropine, daturin, antipyrine, or cocaine, have been from time to time advocated, but excepting in special cases no great reliance can be placed upon them; indeed, the mechanical irritation resulting from this form of medication is obviously objectionable. I must say, however, that I occasionally have seen benefit resulting from the use of a snuff composed of gum acacia, starch, and daturin (formulary); a small pinch of this mixture may be used frequently as a snuff, or when this is impracticable, owing to stenosis, it may be blown into the nostrils from a small insufflator or from a quill; if this excites sneezing or increases the discharge from the nasal passages it should be promptly 
discontinued. The use internally of tincture of stramonium, or tincture of belladonna, or tincture of lobelia, is at times found to be good palliative treatment.

\section{Nasal Hydrorrheea and Sialorrheea}

Nasal hydrorrhœa as an independent affection is rare; of the number of cases reported, the majority were associated with some local structural lesion of the nasal passages. Hardic reports seven cases in which atrophy of the optic nerve existed. Kyle gives an account of twenty-seven cases (sixteen of which were collected by Bosworth) in a very instructive article on the subject. Bosworth, C. E. Bean, T. M. Hardic, Wood, A. R. Anderson, G. F. Keiper, E. Jones, and Baker have published reports of cases. Miles reported a similar condition of the mucous membrane of the intestine.

Etiology.-The causes of nasal hydrorrhœa may be local or systemic, or a mixture of both. Among the local causes usually enumerated may be mentioned nasal polypi, hypertrophic or atrophic rhinitis, or diseases of the accessory sinuses of the nose.

Among the systemic causes may be mentioned diseases of the central nervous system, or lesions of the nerves (fifth pair), malarial toxæmia, or injury to the head. Some authors do not regard those cases which are characterized by the escape of cerebral fluid alone as cases of hydrorrhœa. Kyle states that carious teeth may be a cause of the affection.

Two of Bean's cases were associated with hay fever, asthma, and polypus, respectively, and one of Hardic's and Wood's suffered from asthma and one from malaria, while several of the cases reported by others have been accompanied with some intranasal or cerebral disease. Scheppegrell reported a case of recurrent headache relieved each time by the discharge through the right nostril of a fluid from the cranial cavity.

Trifacial paralysis (Althaus, Hardic) and hydrocephalus internus (Lehr) are also suggested as causes of the affection. Bosworth reported two cases which did not show any local cause. He thinks that polypus is the sequence rather than the cause of the disease.

Cases are also met with in which the etiology is very ob- 
scure. The writer has seen four cases of persistent hydrorrhœa, in three of which the cause could not be traced out. In one of them there were marked myxomatous changes going on in the nasal mucous membrane. Several theories have been put forth to account for the phenomena. Some authors believe that hydrorrhœa is only a symptom at all times; others think it is a manifestation of vasomotor paresis, and allied to hay fever and asthma. Again, others think it is connected with some mechanical derangement (pressure) or accident to the arachnoid ramifications of the ethmoid region.

Kyle believes that hydrorrhœa is a local manifestation of a constitutional condition, and due to some circulatory lesion in which the venous return is impeded, producing in the nasal mucous membrane a cyanotic condition analogous to congestive lesions in such organs, as the lungs, liver, and kidneys. Mucous membrane, he says, when repeatedly congested, tends to remain so, thus allowing leakage of serum into the perivascular tissue, which when near the surface becomes edematous, and the epithelial cells undergo, in their effort to elaborate the excess of fluid, hydropic degeneration, the membrane relieving itself and becoming infiltrated, accounting for the recurring attacks.

Microscopical examination usually shows water-soaked epithelial scales and leucocytes. Tube inoculations made from the secretion by Kyle did not show any particular microorganism as belonging to it. Sections of a piece of the nasal mucous membrane in this condition, made by the same author, showed round-celled infiltration of the submucosa, and dilated vessels with thinned walls. The epithelial layer was desquamated.

It appears to the writer that in every case some special irritant, either direct or reflex, to the terminal nerve filaments lying in the tunica propria must exist. It will be remem. bered that a limited secretion, serous in character, naturally covers the surface of the olfactory region, a secretion which is produced in a delicate manner from the ultimate layers (see Physiology). This would be increased through an irritation of either the trifacial or olfactory nerves. Undoubtedly also the phenomena in question may proceed from an oversecretion of arachnoid fluid which finds its way down through the perineural sheaths. Nerve lesions of the fifth pair would alter 
both nerve influence and blood supply, and the condition would constitute a reflex neurosis (Kyle).

Myles suggests that the dripping is due to overdistended lymph vessels of the pituitary membrane, which by bursting cause fistulous openings. Hardic thinks that the disease is due to vasomotor paresis. Bean says it only occurs in subjects of nasal catarrh, but the number of cases met with is too small to consider nasal catarrhal affections as an important causal factor.

Dr. St. Clair Thomson, of London, reported "a case of cerebro-spinal rhinorrhœa" in a woman, who made little or no complaint excepting from the annoyance due to the continual dripping. Vague pains about the head of this patient were relieved by the discharge. Dr. Thomson thinks that it is probable that the fluid finds its way out from the subarachnoid space at the base of the skull within the perineural sheaths of the branches of the olfactory nerves.

He also thinks that the condition is always associated with increased intracerebral pressure. He reports that of twentyone patients, including his own, no less than seventeen presented some cerebral symptoms, and eight of them showed retinal changes.

The writer has met with four cases, but in none of them could any special cerebral pressure or retinal changes be detected. The theory that a serous infiltration into the perineural sheaths of the olfactory nerves takes place would seem untenable for two reasons: first, there is little or no space in these sheaths for the collection of or trickling through of fluid; and, second, the pressure from even a very small amount of fluid in these nerve tracts, if it were possible, would lead to serious disturbance in the neighbourhood. The more feasible explanation of its occurrence, as given by Kyle, Bean, and others, is that from repeated congestions or stasis, etc., a transudation of serum from the vessels takes place.

Symptomatology. - These cases occur usually in healthy persons beyond middle age or in old age. The attacks usually appear suddenly without much premonition. The discharge of fluid comes on paroxysmally, sometimes two or three or four times a day. The discharge of watery secretion may be preceded by headache, sneezing, or a sense of obstruction of the nostrils. When the discharge starts it usually relieves the 
headache or other pain. The secretion is apt to flow continuously from one nostril only for a considerable time-an hour or more. The patient experiences the same sensation as if water had been insufflated. The discharge is sometimes very irritating, excoriating the upper lip. An examination of the nasal passages usually shows no hyperæmia, but a swollen edematous condition of the parts. The mucous membrane may be of a dull bluish-red or pink tinge. On examination between the attacks the mucous membrane is usually pale and edematous. The sense of smell may be unimpaired. The attacks may last for months at a time, then disappear and recur.

Treatment.-The treatment is quite unsatisfactory. Of course, in cases of intranasal disease the principal treatment should be directed toward relieving such abnormal conditions as may be prominent.

Systemic treatment by such agents as the bromides, hyoscyamus, strychnia, phosphorus, belladonna, etc., should be instituted.

Dr. F. Keiper recommends a spray of a solution of atropine sulphate (gr. j to water $\xi \mathrm{iv}$ ), to be used morning, noon, and night. H. C. Baker controlled a case by the application of galvanism to the outside of the nose. Sprays and snuffs of cocaine, morphine, etc., have been recommended. According to the writer's experience the best local applications are atropine, daturine, and quinine used in the form of sprays.

\section{SiAlorRhGeA}

A similar condition arising from the salivary glands has been written about by a few observers. Klippel and Lefas studied the affection experimentally. The submaxillary and parotid glands were submitted to two sorts of nervous influences, one excito-secretory (chorda tympana) and the other moderating (sympathetic).

The parotid gland they found to possess excito-secretory filaments originating in the facial nerve, and a salivary centre, so to speak, was found to exist at the floor of the fourth ventricle.

A pathological state of salivation (sialorrhœa) is observed in various neuroses, such as epilepsy, hysteria, hydrophobia, labio-glosso-pharyngeal paralysis, certain hemiplegias, in neuritis 
of the facial nerves, and in facial neuralgias. It is often a reflex phenomenon accompanying stomatitis, dentition, Riggs's disease of the gums, dyspepsia, and frequently follows the use of toxic agents such as mercury, iodine, or jaborandi; finally it may be due to inflammation or other disease of the salivary glands themselves.

Treatment.-The treatment will vary according to the cause. Sialorrhœa is more often symptomatic of some disease outside of the salivary glands, and therefore will rarely call for any special treatment.

Troches containing belladonna or atropine, or mouth washes containing gallic or nitric acids, are frequently beneficial. In those cases depending upon disease of the mouth, teeth, or salivary glands themselves, the treatment must of course be directed toward these parts.

\section{Xerostomia (Dry Mouth)}

Xerostomia is characterized by a gradual repression of the secretion of the mucous membrane of the mouth, tongue, fauces, and salivary glands, sometimes including the nasal passages and the conjunctivæ.

It is a rare disease, and according to W. F. Chappell was first described in 1868 . The name xerostomia was given to it by Hutchinson and Hadden. Dr. Fraser, of Edinburgh, collected and reported the histories of nineteen cases a few years ago (Chappell). Three interesting cases have been reported more recently by W. F. Chappell, Beverly Robinson, and C. H. Richardson respectively. (New York Medical Journal, vol. lxiii, p. 277.)

Etiology.-The etiology of this affection is not as yet clearly understood. It is regarded, however, as a neurotic disorder, affecting principally the trophic nerve branches. Mental or physical overwork, worry, and malnutrition may give rise to it. It may also accompany diseases of the spinal cord, such as locomotor ataxia, syringomyelia, etc. Chappell's case terminated in paralysis. The writer has observed one case connected with locomotor ataxia.

The disease is generally met with in persons of middle or advanced age, and probably more frequently in males than in females. Habits and social condition are predisposing causes. 
The writer has met with one case which apparently preceded the development of carcinoma of the lower end of the esophagus. When occurring as an independent affection it may be transient, and connected with insomnia, so-called dyspepsia, "Riggs's disease," or the excessive use of tobacco and alcoholic liquors.

Symptomatology.-The chief complaint of the patient is a continual sensation of dryness, or "pricking dryness," of the mouth and throat, which can be but partially relieved by the frequent use of watery, oleaginous, or mucilaginous drinks. Impairment of the voice, dry cough, more or less indigestion, flatulence, nervousness, and considerable general debility are observed in cases of long standing. Whatever the status or complications of the case may be, the patients usually complain of insomnia and restlessness, and there is always a display of great anxiety. The stomach is generally deranged, and the teeth crumble and are finally lost.

The patient may complain much of soreness or tenderness of the parts, especially during acts of deglutition or hawking. But distressing dryness, or "pricking dryness," is the chief symptom.

Diagnosis.-This disease may be mistaken for buccal or pharyngeal syphilis, or herpes of the fauces. Its very chronic course, appearance, and symptomatology, however, will serve to distinguish it from these affections.

Course and Duration.-It may occur incidentally to various nervous or gastric disorders of a transient nature, or it may continue for a long time, finally producing a degree of exhaustion leading to death, or its career may end by the advent of paralysis, or some other affection denotative of structural cerebral disease.

Appearances.-The inside of the mouth, fauces, and possibly the upper larynx, present a uniformly bluish-red or pale pink colour. The surface of the mucous membrane appears smooth, dry, and glistening, with no enlarged follicles or collection of secretion about any of the lymphoid glands. The mucous membrane may be very sensitive to the touch in some places, while other places seem quite insensitive.

In the case reported by Chappell the roof of the mouth was very sensitive, and there was a small ulcer at the interarytenoid space. 
Treatment.-The treatment is unsatisfactory. The therapeutic indications will depend mainly upon whether the disease in a given case is an entity, or only a local expression of some neurotic or stomachic disorder. In the latter event, of course, it is only necessary to adopt therapeutic measures looking toward the relief of the diseased structures upon which the xerostomia depends. If, on the other hand, no remote lesion of consequence can be found, then a vigorous local treatment is in order. The daily application of galvanism, by means of a naked electrode, is one of the best remedies. At the same time the patient may be directed to use every two or four hours through the day a spray of a three-per-cent solution of either menthol, oil of eucalyptus, or camphor in oil of petrolina, and at night to hold a tablet of pilocarpine ( $\mathrm{gr} .1 / 8$ ) in the mouth, allowing it to gradually dissolve. A gargle consisting of one teaspoonful of compound tincture of benzoin in one half cupful of hot water is also a useful remedy. The chewing of chamomile flowers is also beneficial.

For the further relief of the sensation of dryness the writer has observed good effects from bits of chloride of sodium frequently taken into the mouth and held there, and the bismuth boroglyceride mixture applied with a camel's-hair brush (see formulary). Stimulating washes containing capsicum, xanthoxylin, etc., do not generally afford relief. In some cases gum acaciæ, gum tragacanth, and balsam of Peru, with the chewing of chamomile flowers, will prove beneficial.

Besides topical applications, it will be necessary to institute a general treatment, and the administration of strychnia, fluid extract of piscidia erythrina, tincture of chamomile, etc., will be useful. The writer has observed good effects from the use of arsenic and phosphorus also. Much may be accomplished by systematic bathing, with friction to the skin. Acid and alkaline preparations when taken internally are usually harm$\mathrm{ful}$, unless the malady is depending upon a disordered stomach.

When in a given case a neoplasm is discovered it should be removed, if possible, for it may be the principal cause of the affection.

\section{HyPERASTHESIA}

Hyperæsthesia of the upper air passages is not very common as an independent affection, but is usually a concomitant 
of hay fever, neuralgia, or the acute inflammatory diseases of this region. When occurring, however, independently of these diseases it is usually of reflex origin, and may be traced to irritation, by pressure or otherwise, of some proximate or remote ganglion or plexus, such às the cervical or sphenopalatine ganglia, or the solar, carotid, or splanchnic plexus. It is generally associated with paræsthesia when not directly due to inflammation or disorder of the part concerned. It is commonly met with in adults, especially women, although other persons of nervous temperament may be affected.

\section{Hyperasthesia of the Nasal Passages and Nasopharynx}

When occurring in this locality independently of hay fever and influenza it usually takes the form of frequent sternutationparoxysmal sneezing. The patient sneezes upon slight provocation, such as inhalation of air slightly laden with dust, gas, or smoke, or the sudden exposure to bright sunlight, upon fatigue or indigestion, upon getting out of bed in the morning, or upon entering a place where many persons are assembled, etc. Gradenigo reported a case of vertigo and pseudo-angina pectoris of nasal origin.

W. S. Renner thinks that this abnormality bears a relationship to asthma and rose cold; also that the name coryza instead of rhinitis should be applied to it, because often no inflammation of the nasal passages exists. This condition seems to be entirely independent of season, and therefore in this respect differs from hay fever.

Rhinoscopic examination reveals a variety of appearances. Sometimes there is evidence of intranasal disease, but often no particular abnormality can be observed. The mucous membrane may be sensitive to the touch, especially in the vasomotor sensitive areas pointed out by John N. MacKenzie, or it may show no extra sensibility. It may be pale and somewhat swollen, or of a pink or red colour, and not swollen.

Mulhall very aptly compares the different appearances of the nasal mucous membrane under the influence of vasomotor disturbance to that of the face under the emotion of anger-one person's face will be pale while another's will be flushed.

A transient coryza and lachrymation, and perhaps headache, may follow the sneezing fit. With some persons a sensation of 
pleasure, and with others slight pain, may accompany the sneezing.

Treatment.-When the affection is symptomatic, depending upon some general disorder, of course no special line of treatment will be indicated. When, however, the cause is found to be of a local nature, attention should be chiefly directed to the nasal passages. In such cases, therefore, any abnormal growth, hyperplasia, or deformity should be at once corrected.

If particularly sensitive spots are found they should be treated by the galvano-cautery, or pigments containing the vegetable astringents, such as tannic acid, tannate of cannabin, etc., anodyne pigments containing menthol, carbolic acid, morphine, iodoform, belladonna, aconite, together with the boroglyceride mixture, suprarenal extract, or cocaine, which may also be applied. Regarding the latter agent, great care should be exercised lest the patient become habituated to the use of the drug. A spray of mucilage of acaciæ will sometimes prove beneficial. Likewise, sprays of olive or petrolina oil may give great relief.

\section{Hyperosthesia of the Pharynx}

Hyperæsthesia of the pharynx may be defined as an exaggerated sensitiveness to either mechanical, chemical, or thermic irritation. It is frequently a congenital condition, and may be due to brain disease. There are many degrees of extra sensitiveness belonging to the pharynx, varying from a slight irritability to a painful susceptibility to the slightest touch. This hyperæsthesia is almost always accompanied by more or less motor irritability which adds to the annoyance of the patient. I have seen three cases in children in whom the abnormality could be traced to the period of weaning. The presence of tape-worm will sometimes cause it. The condition is generally met with, however, among nervous females, spirit drinkers, and excessive smokers, and sometimes has its origin in neuralgia. The main characteristic consists of the inability of the patient to bear anything save food to touch the pharynx. In some cases even anything hard in the bolus of food will cause pain and retching. Such patients are very susceptible also to hot and cold fluids or to anything highly spiced. They are also susceptible to cold air. I saw one patient who could with com- 
fort swallow only foods or fluids at a lukewarm temperature, and who was obliged to have the food thoroughly moistened before attempting to masticate and swallow it. With the majority of such patients the process of taking a meal always leaves a sense of soreness of the pharynx, lasting from a few minutes to an hour or so. The inadvertent deglutition of something too hard, too hot, or too spicy will necessitate a suspension of eating for a time. In a few instances the disease has been connected with epilepsy. Epileptic seizures might prove fatal during eating through the impaction of food in the larynx. I knew of one such case which terminated fatally before a physician arrived. It is often very difficult to make a thorough examination of the pharynx owing to the apprehension of the patient. As a rule nothing is observed excepting a slight hyperæmia. Often the first manifestation of the disease is an impulse to leave the table once or twice during a meal on account of slight regurgitation of food, or the fancied or real collection of mucus in the throat. Such persons when questioned closely will undoubtedly state that they have been suffering from an unusual sensitiveness or slight soreness of the pharynx for a considerable time, with a tendency to vomit on slight provocation. The tendency to vomiting may indicate disease of the central nervous system.

Treatment.-Among beer, wine, and coffee drinkers it is obvious that the first therapeutic indication points to a modification of such habits. Among the remedial measures, pepsin preparations, either alone or in connection with dilute hydrobromic and phosphoric acid, will usually be found sufficient, while the strictest dietary regimen must be instituted. If a rheumatic or gouty diathesis be manifested suitable saline mineral waters together with a non-nitrogenous diet must be prescribed. In persons of irritable temperament or those prone to insomnia and neurasthenia general treatment toward modifying such conditions must be instituted. While it is generally reprehensible to prescribe anodynes for such patients, it may be necessary in some cases to resort to such agents temporarily until the nervous functions have been sufficiently regulated. For this purpose hyoscyamus, cannabis indica, sodium and potassium bromides, codeine or hydrobromic acid will be found most efficient. Besides, frequent bathing with salt water followed by friction will be of great service. Any cause of in- 
digestion is to be carefully avoided, and the use of tobacco, coffee, or alcoholic stimulants or malt beverages must be prohibited. It is obvious that local treatment will accomplish little good if such habits are maintained. Attention must be paid to the other senses, such as sight and hearing, because abnormalities of these functions will often need treatment, inasmuch as the wearing of suitable spectacles to relieve eye strain in a case of this sort will be of paramount therapeutic value.

\section{Hyperesthesia of the Larynx}

Hyperæsthesia of the larynx as an independent affection occurs very rarely. An undue sensitiveness may be expected in connection with pharyngo-laryngeal hyperæmia and inflammation, and, moreover, in hyperæsthesia of the pharynx from gastro-intestinal or other disease. It may appear to some extent in cases characterized by prolonged periods of cough, such as hooping-cough, or reflex spasmodic cough from pleuritic adhesions, etc. Occasionally it may arise from disease of the lymphoid tissue at the base of the tongue.

Treatment.-Anodyne sprays, inhalations, and lozenges are the most efficient agents for local or palliative treatment. Generally, however, internal medication according to the cause of the malady will be the most efficacious method of treatment.

\section{PARASTHESIA}

\section{Paresthesia of the Nasal Passages}

The nasal passages are comparatively free from this form of disorder, excepting when some lesion of the spinal cord or brain has affected the sensory nerves of the whole of the upper respiratory tract. The varieties of itching or burning sensations referred to the external nose, or just within the nasal vestibule, are examples of it. The irritation in question is usually reflex, often depending upon some malady of the stomach, intestines, or other organ sympathetically connected. The most notable examples are the itching of the nares in connection with hemorrhoids, entozoa, or aural disease. It may be directly brought about, however, by injuries, small submucous growths, or tumefaction of the septum. C. H. Knight and Kafeman cite cases of paræsthesia depending upon enlarged 
lymphoid tissue at the vault of the pharynx. Bosworth aiso says that adenoid growths may become the cause of such reflex symptoms. Chorea and laryngismus have been attributed to nasal disease, especially to adenoids. The writer has met with many cases in general practice which were dependent upon derangements outside of the nose and throat, although presenting at the same time either hypertrophic rhinitis or hypertrophy of the pharyngeal tonsil.

Treatment.-The most efficient symptomatic treatment consists in the application of menthol or chloroform. Of course, the treatment of the remote cause is the principal object.

\section{Parcesthesia of the Pharynx}

This is by far the most common seat of abnormal sensations. The sensations may be confined to one spot, one side, or to one of the anatomical regions involving the whole pharyngeal cavity, or they may be referred to the surface of the neck or the anterior laryngo-tracheal region of Gottstein.

Symptoms. - The sensations usually complained of are pricking, choking, burning, pinching, itching, tickling, gnawing, aching, dryness, soreness, or pain, a sensation of the pressure of a foreign body in the pharynx or upper larynx, such as a bristle, pin, tack, sliver, or a smooth object, such as a cherrystone, grape-seed, etc. These sensations are usually quite constant excepting during sleep, eating, or the voluntary control of respiration. For instance, a patient will say that she is not free from the itching or burning in her throat excepting during and just after swallowing. There is generally excessive secretion of the pharyngeal and buccal mucus, saliva, and persistent hawking. There is also unnecessary mental apprehension, even amounting to hypochondriasis or hysteria.

Age.-Paræsthesia pharyngis is seldom met with in the very young, but more commonly in persons ranging in age from eighteen to fifty, although I have met with a few cases in persons over sixty years of age.

Sex.- It is more frequently met with in women than men; in fact, a large proportion of the cases occur in women.

Etiology.-The patients are usually of highly sensitive or emotional temperament, for it is seldom met with among the male labouring population. The illiterate are by no means exempt, however, as its frequent occurrence among ignorant 
women will attest. The cause is essentially either a direct or reflex irritation affecting the sensory nerves of the throat, the reflex probably predominating. Among the direct or local causes may be particularized injury to the pharynx, larynx, or nasopharynx, from foreign bodies such as fish-bones, pins, needles, toothpicks, spears of hay, etc., the lodgment of which produces a certain degree of irritation with or without abrasion. After the removal of the foreign body the lesion is followed by a continued sensation of pain or annoyance, as if the foreign body still remained. Lethal doses of belladonna, aconite, or other drugs having a selective action upon the throat are said to be followed in some persons by a period of pharyngeal paræsthesia. Also the swallowing of capsicum, acids, or large hard boluses, such as fruit stones, will produce the affection in susceptible individuals. Disease of the pharynx itself, especially atrophic pharyngitis and neoplasms situated upon its lateral walls or upon the soft palate, or neoplasms in the external auditory canal and enlarged lymphatic glands of the neighbouring region, or carious teeth, may bring about the affection.

Kafeman has seen a number of cases where no apparent abnormality of the pharynx, larynx, or soft palate could be detected by the naked eye and characterized by persistent hawking and gagging, which were due evidently to the lodgment and inspissation of secretion in the crypts or sulci of the pharyngeal tonsil, the removal of which by a probe produced permanent relief of the symptoms. Moritz-Schmidt thinks that often some overlooked lesion of the lymphoid ring of the pharyngo-laryngeal region is the real cause, and also hardened plugs of secretion in the tonsillar lacunæ.

Diseases of the central nervous system (bulbar disease), or of the trunks of the trigeminus, facial, or dental nerves, may also give rise to it. Enlarged follicles of the mucous membrane of the pharynx or glosso-pharyngeal region, or hardened secretions, in their cavity, may also be included as a cause, probably operating through the pressure exerted upon the terminal nerve twigs, for these twigs contain both sensory and motor filaments.

Newcomb, C. H. Knight, Gottstein, and others report that paræsthesia pharyngis may sometimes constitute a pretubercular symptom. This is true, and the same may be observed as a symptomatic element of other systemic diseases. The 
writer has frequently observed paræsthesia in connection with the early stage of phthisis pulmonalis and laryngeal phthisis. Dana emphasizes the influence of occupation and personal habits as exciting causes. He also calls attention to the occurrence of heat and cold paræsthesia (psycho-æsthesia) in connection with local neuritis or other irritation of sensory nerves which contain mixed heat fibres.

However, by far the greater number of cases met with show no lesion or abnormality of the pharyngeal mucous membrane, excepting perhaps a merely transient hyperæmia. There is generally an unusual degree of mental apprehension and depression, amounting almost to insanity. These cases may therefore be mostly regarded as reflex. The general disturbances which lie at the bottom of so-called throat symptoms are legion, as every practitioner can attest, inasmuch as any derangement of the economy may in certain individuals give rise to paræsthesia pharyngis, especially if such persons be suffering from neurasthenia or undergoing the menopause. Diseases and disturbances of the generative organs, the stomach, intestines, rectum, liver, and bladder, together with those states known as hysteria and hypochondriasis, are frequently causes of paræsthesia pharyngis.

We meet with many cases which seem to depend upon anæmia or other abnormal conditions of the blood, such as malarial toxæmia. I would call particular attention to the connection between the symptoms under consideration and dis. eases of the ovaries and rectum. I have frequently been consulted by persons complaining of one or more of the morbid sensations in the throat already alluded to (sometimes accompanied by the globus hystericus), in whom no abnormal appearance, save a fleeting hyperæmia, could be found in the fauces, pharynx, or larynx, but whose malady was subsequently found to be a fissure of the rectum, or perhaps some morbid condition of the ovaries. However, judgment in this particular should not be formed too hastily, since cases of paræsthesia which have been diagnosed as due to hysteria or neurasthenia have subsequently proved to have been caused by local disease of the tonsils or of the lymphatic tissue of the throat, or even to a deeply impacted and overlooked foreign body, such as a sliver or small seed. I once had a patient who insisted that a spicule of straw was in her throat, notwithstanding my asser- 
tions to the contrary, based upon repeated thorough examinations. She subsequently had a peripharyngeal abscess, which, upon being opened by Dr. Walker (a colleague), discharged with the pus the spicule of straw which the patient had all along insisted was imprisoned in her throat.

Paræsthesia as well as other neuroses may bear a causative relationship to meteorological conditions. It is a well-known fact that some persons are so sensitive as to be affected by changes of the weather physically as well as mentally.

Prognosis.-The prognosis when the disease is not the accompaniment of some serious lesion of the central nervous system is generally favourable, but the course is tedious. These patients generally suffer a great deal from mental anxiety, insomnia, and malnutrition. They are frequently imbued with the idea that some cancerous growth is taking place. Indeed, cancrophobia and syphilophobia are often marked features in the course of the affection. In such patients the prognosis is of course very much more unfavourable. I have observed a few cases of this sort which passed through hypochondria into insanity. It therefore behooves the practitioner when meeting with any case of paræsthesia pharyngis to give the most guarded prognosis until the real cause or complications of the case are thoroughly made out, on account of the many perplexities associated with all derangements of the sensory nervous system. The art of the general practitioner is needed for the treatment of these cases, for the very best laryngologist by local means may not be able to manage the case successfully, unless he has had more or less experience in general practice and applies his therapy from a broad view of the case. Therefore each one must be subjected to thorough investigation before any line of treatment can be decided upon.

Treatment.-For cases of local origin, of course, topical treatment must be adopted, and sometimes for mental effect merely such a course becomes necessary for a time. Glandular diseases, neoplasms, inflammations, or deformities or atrophic changes of any portion of the nasopharyngeal or laryngeal apparatus must be looked for and actively treated by suitable medication or operation; either astringent, antiseptic, or escharotic applications may be sufficient. The pharyngeal mucous membrane must be kept clean and lubricated in all cases by the use of some mild spray of a solution of borax, 
boracic acid, or some other fluid used every three or four hours. If any lesion be suspected of the lacunæ of the faucial or pharyngeal tonsils, a thorough examination of these organs with a probe will be necessary. For the nasopharynx the palate hook or the palate retractor of White or Jarvis, or the rubber cords of Wales, should be applied previous to examination, because digital examination will not be sufficient for ascertaining the state of the recesses of these glands. Should any collection of secretion be found, of course its removal will be necessary. Should the patient's general system be out of order, this, of course, is to be corrected. The treatment of the reflex forms of the disease is necessarily very diverse, and will depend largely upon the nature of the disease in the remote organ or apparatus of the body which excites the paræsthesia. I may mention, however, that among those agents having selective action on the sympathetic nervous system the following will be found efficient in paræsthesia : codeine, monobromate of camphor, arsenic bromide, chloral hydrate, asafœtida, cannabis indica, scutellaria, hyoscyamus, belladonna, aconite, salol, and phenacetine. Any one of these agents may be combined with some tonic or nerve stimulant. Some of them will be found also useful for local medication when such is deemed advisable. A very weak solution of cocaine, menthol, acetanilid, or coniine hydrochlorate will be found serviceable in subduing local sensations. Great caution must be observed in the use of cocaine, lest the patient become addicted to the cocaine habit. Fortunately, however, this drug does not serve to essentially mitigate the affection in the majority of cases.

The application of a mild galvanic current will sometimes produce very marked relief, especially in two classes of cases, namely, those accompanied by mental apprehension and those which show some local trophic change in the mucous membrane. The galvanic or faradic current may be used with benefit also upon those cases of paræsthesia following the impaction of a foreign body, as well as upon those who imagine the presence in the throat of some foreign material. Permanent relief, however, for cases of reflex origin cannot be expected until the real seat of the morbid irritation is traced out. Above all, in those cases which are not dependent upon structural local disease the psychic element of treatment must not be lost sight of. 


\section{Parcesthesia of the Larynx}

Dr. Felix Semon, referring to the observations of Zeissl and Gottstein, summarizes that the sensations arising from the different organs of the neck are usually expressed at the anterior laryngo-tracheal region (the sphere of common sensation of Zeissl).

Etiology.-Paræsthesia of the larynx is usually associated with paræsthesia of the pharynx, or a general paræsthesia and hyperæsthesia incident to bulbar or some other central nervous disorder deranging especially the sensory nerves. When of local origin, it may be the sequel of laryngitis, of influenza, of measles, of the impaction of a foreign body in the larynx or its pyriform sinuses, or of some direct injury, leaving a contusion or abrasion. It may also arise from irritation of or pressure upon the superior laryngeal nerve or its branches, by bronchocele, or by enlarged lymphatic glands in the neighbourhood.

A few cases have been known to follow or accompany a long course of facial neuralgia; however, the latter malady is usually characterized by more or less pain and hyperæsthesia.

Symptoms. - The principal complaint is of the sensation of a foreign body, either smooth or sharp, in the cavity of the larynx, together with sensations of burning, pricking, or choking. The latter symptom is particularly referred to the laryngo-tracheal region over the front of the neck, and is similar in every respect to what is known as globus hystericus. These symptoms may be more or less continuous or paroxysmal during waking hours. Many patients complain of inability to breathe cool or cold air, or to eat any spicy food, without exciting exacerbations of the annoying sensations. Stridulous, ineffective cough is often added to the other complaints, and sometimes also disturbance of the function of deglutition. Mental apprehension of choking is usually a symptom, and may be connected with signs of insanity.

Laryngoscopic Examination.-Laryngoscopic examination in the majority of cases will reveal nothing abnormal. Sometimes a slight hyperæmia, an unusual jerkiness of the laryngeal muscles, or a slight enlargement of the glosso-laryngeal glands, will be observed. An undue irritability of both pharynx and larynx, however, is generally present. Indeed, in some cases 
the sensitiveness is so heightened that even the examination is made with difficulty. Touching the posterior wall of the pharynx or any part of the inside wall of the larynx with a probe may be followed by sensations of burning pain, choking, gagging, or even induce vertigo, syncope, or an hysterical attack.

Prognosis.-The course of the affection varies greatly according to the general condition of the patient on the one hand, and the ability of the physician to locate and attack the real source of the malady; in short, whether or not the disease is essential, or merely symptomatic. I met with a case which followed the temporary impaction of a fish-bone in the right pyriform sinus, characterized by sensations of choking, burning, tickling, and stridulous cough, which continued with varied remissions for about eight months after the removal of the foreign body. I have had also a case of moderate paræsthesia under observation during the last seven years (burning sensation and feeling of foreign body in the larynx, accompanied with severe and sometimes frequent paroxysms of cough), which followed an attack of influenza complicated with acute pleuritis. This woman is now about fifty-seven years of age, and in all other respects seems to be healthy. There is very little if any psychic perturbation observed. I might add that there has been no aphonia, nor much hoarseness, and no abnormal condition of the larynx observed, except hyperæmia for a time now and then following paroxysms of cough. The inhalation of very cold air, or air containing illuminating gas, tobacco or other smoke, is very apt to bring on exacerbations of all the sensations, together with spasmodic cough. The prognosis in most of the cases of a peripheral or reflex origin is favourable.

Treatment.-In the absence of any rational understanding of the cause in a given case the treatment of course can only be empirical, and directed toward the mitigation of the more prominent annoyances. The patient will very soon discover what excites suffering and will avoid such causes. Topical treatment in those cases depending upon local lesion is of little use excepting for relief of the sensibility of the part, and even this desideratum can often be better reached by a judicious general treatment, such as the administration of phosphorus and the application of salt baths, aided by the cool or cold 
shower bath. Strychnia and quinine may be selected for some cases, although they will be found inadequate for others. The bromides are probably the most useful. Tartar emetic also has proved highly beneficial in my experience when given in solution in small doses frequently repeated. It is especially indicated when spasmodic cough is present. Bromoform and gelsemium are also valuable, and may be found more efficacious than either of the other agents mentioned, especially where neuralgia also attends the case. Carefully used inhalations of chloroform or nitrite of amyl may be prescribed for those cases characterized by severe cough or paroxysmal recurrences of the paræsthetic symptoms.

\section{Anæsthesia And Analgesia}

Anæsthesia and analgesia usually occur as local disturbances in connection with special cerebral or spinal lesions, and may not be limited to this locality ; but when following the frequent imbibition or application of toxic agents, such as aconite, cocaine, belladonna, creosote, menthol, etc., or the systemic diseases, such as diphtheria, the disability in question may be confined to the pharynx, larynx, and buccal membrane. Generally the pharynx (fauces) is the principal seat. The nasal passages are not often involved. The larynx is always affected with anæsthesia when the superior laryngeal nerve is paralyzed, because the superior laryngeal is the nerve of sensation of the laryngeal mucous membrane.

Analgesia, and also anæsthesia, may constitute a congenital physical defect, as illustrated in those individuals called freaks, who eat and swallow glass, or stick pins and sharp blades through their skin and mucous membranes, or swallow swords, knives, etc., without the production of pain or other noticeable sensation. I do not know that physiologists have as yet determined whether the defect in such individuals lies in the posterior roots of the spinal cord, in the cerebrum, or both. In a person of this sort who fell under my observation there seemed to be complete analgesia of all the mucous membranes and skin, but the tactile sense and sphincter reflexes seemed to be normal.

Treatment.-The treatment will be indicated readily by the nature of the case, for when either of these conditions 
are merely symptomatic no treatment apart from that for the causative lesion will be in order. But when of local origin and existence, due to the improper use of chemical agents or otherwise, the indication is for the employment of suitable galvanic, faradic, or mechanical (massage) measures of treatment.

\section{Spasmodic Affections of the Upper Air Passages}

\section{(Kinesioneurosis or Hyperkinesis)}

Evidently for the guardianship of those important portals, the pharynx and larynx, there exists a physiological irritability of the muscles of the fauces and larynx which renders them peculiarly liable to sudden or spasmodic action. This action, when occurring co-ordinately, even though excessively, gives but little annoyance to the subject, and therefore but rarely calls for modification. But when occurring incoordinately, especially upon too slight a provocation, it becomes a manifestation of disease, denoting an abnormal irritation somewhere.

The morbid spasmodic action may depend upon either structural or functional disease of the affected part or some remote part of the body, and consequently may be of direct or reflex origin.

Although, as a rule, morbid muscular spasm is indicative of an irritative lesion of nerve centre or nerve, yet it is frequently associated with some form of paresis or paralysis of neighbouring parts, and is generally accompanied with more or less hyperæsthesia of the overlying mucous membrane.

The spastic action may be limited to a portion of the throat -perhaps to one group of muscles only-or, on the other hand, may affect all the pharyngeal, palatal, and laryngeal muscles at once. In the latter event, however, it will be found that the several groups of muscles contract in rapid succession during a paroxysm, rather than simultaneously.

Etiology.-A clinical classification would group together, I. Those cases depending upon structural lesions of the brain, medulla, spinal cord, or ganglionic centres.

2. Those cases depending upon functional diseases of the above-mentioned centres.

3. Those cases depending upon peripheral or local lesions, 
embracing changes in either nerve, muscle, gland, or mucous membrane. action.

4. Those of reflex character-exhibitions of abnormal reflex

5. Those cases arising from toxæmias of various sorts.

Sex.-The female sex, especially those least capable of controlling their emotional faculties, are more susceptible to such attacks, also those who suffer more or less from anæmia. There are, however, notable exceptions to this rule, for even the strong and robust may become victims of these disorders.

Age.-The majority of cases are found in persons from puberty up to about thirty years of age.

Social Condition.-Persons living in idleness and affluence, and those especially engaged in satisfying their personal wants and appetites, are prone to this as to other forms of nervous disease.

Occupation.-Inordinate and faulty use of the voice, particularly by neurotic persons, may lead to either ordinary or choreic spasm of the larynx and pharynx.

Nasal Passages.- Sternutation is about the only spasmodic affection of the nasal passages proper. It occurs physiologically from an excitation by such agents as dust or noxious emanations or draughts of air, and soon passes off when the obnoxious excitant is out of the way. It sometimes, however, appears as a veritable neurosis of acquired or hereditary origin, for there are many persons who suffer from long and violent paroxysms of sneezing without obvious provocation by any external excitant, and whose nasal passages, as far as one can ascertain by observation, are normal. Such persons are very susceptible to slight drafts of cold air, especially upon the neck or feet, or to the entrance into the nose of even imperceptible quantities of dust or smoke, while nearly all of them violently sneeze upon sudden exposure to the sun's rays. So far as the writer's observation is concerned, nearly all of these subjects, whether predisposed to hay fever or not, exhibit an hereditary predisposition, and are especially susceptible to exposure to the sun. Inordinate sneezing is sometimes, though rarely, a symptom of disease of the brain or medulla. Two cases of this kind have come to the writer's knowledge, one of cerebral syphilis, and the other of progressive dementia. Of 
course, in the above consideration of sternutation local diseases of the nasal passages are excluded.

Pharynx and Soft Palate.-Spasm of the pharynx and soft palate alone is not very common, and usually happens in connection with some disease of the neighbouring parts. It is more often met with in connection with general affections, such as tetanus, hydrophobia, epilepsy, locomotor ataxia, general chorea, or some toxæmia, such as mercurial or antimony poisoning. It may be a prominent symptom, however, in the course of progressive bulbar paralysis, locomotor ataxia, syringomyelitis, insanity, hysteria, or so-called hypochondriasis. As a phenomena of hysteria or hystero-epilepsy it is of common occurrence and may be persistent, in which event the constrictor muscles of the pharynx usually participate. It constitutes often the most annoying phase of globus hystericus. Ovarian disease is a common cause, and so is pregnancy sometimes.

As a purely local affection, it is more often an accompaniment of an irritation due to the presence of neoplasms in the naso- or oro-pharynx, or of highly inflammatory conditions of the pharyngeal mucous membrane itself, or of traumatism. It may be combined with neuralgia of the second division of the fifth or of neighbouring nerves, and sometimes it arises from neoplasms or other disease of the ear. The writer has seen one case which accompanied a suppurative disease of the ear and mastoid cells, which entirely abated after surgical measures had been adopted for the relief of the diseased ear. Again, cases infrequently arise as a sequence of diseased teeth or gums. I have been informed by reliable dentists that they have observed spasm of the soft palate and pharynx in cases of caries of the teeth, jaw, and in Riggs's disease of the gums.

There is a functional form of pharyngeal and palatal spasm occurring apparently as an independent affection, without presenting any inflammation of the pharyngeal mucous membrane which has been given by some authors the title of dysphagia.

Sometimes, but seldom, these spasmodic phenomena are found in connection with dry cough, or hyperæsthesia, or both, whether the cause of the disturbance be systemic or local. An inordinate sensitiveness of the posterior wall of the pharynx 
may always be noticed at a point just above the epiglottis in these cases.

The majority of cases of reflex spasm of the pharynx are observed in connection with diseases of the generative organs, especially the ovaries, and male urethra, or with the stomach, esophagus, intestines, and with disturbances of the brain. Porcher states that in cases of stricture of the esophagus with diverticula, cough with spasm of the pharyngeal muscles is frequently observed. The writer had a case under observation in which an incessant desire to swallow, accompanied by annoying cough, would follow any even moderate movement of the neck, or attempts at forcible phonation, or a full voluntary inspiration. These symptoms had been gradually increasing in this patient during a period of about three months, and had reached a point of very serious annoyance. Up to this time, however, no central lesion had been discovered, but soon after this the patient was attacked by a very pronounced and painful eruption of herpes zoster, which was followed (after three weeks) by paraplegia. The case after a time passed from under the writer's observation, on account of the patient's removal to her home in Ohio. It was subsequently learned that she died from "myelitis spinalis."

Larynx.-Transient laryngeal spasm is a very common occurrence when arising from local irritation of a mechanical or chemical nature, such as a caustic application to the pharynx or larynx, or the temporary lodgment of a foreign substance in either of the pyriform sinuses of the larynx or in the larynx proper. Although usually the removal of the offending substance secures prompt subsidence of the nervo-muscular excitement, yet occasionally the spasmodic action, even amounting to "spells of choking," will remain for a considerable time thereafter.

The writer was once called to a case which illustrated this odd feature. The patient, a man, had accidentally drawn a chlorate of potash lozenge into his larynx, and although he had expelled it a few minutes after its entrance, the laryngeal spasm and cough did not subside until about three days afterward, notwithstanding the administration frequently of various antispasmodic agents. The fearful apprehension of the patient probably aided materially in producing this anomalous result, inasmuch as laryngoscopic examination failed to 
show any local lesion except a moderate degree of hyperæmia. Laryngeal spasm may be due either to a direct irritation or to pressure operating on the laryngeal structures-especially the nerves-or to an irritation at some remote part of the body which is reflected to the nerves and muscles of the larynx, constituting a reflex spasm.

When of reflex origin it may almost always be attributed to one or more of the following abnormal conditions: Ovarian and uterine disease, hysteria, hystero-epilepsy, anæmia, chorea, hyperacidity of the stomach, uric-acid toxæmia, any of the various chronic structural diseases of the brain and spinal cord, chronic alcoholism, or lassophobia. The writer has twice witnessed alarming paroxysms of spasm of the glottis in cases of hystero-epilepsy, and once in a case of lassophobia, where the patient was bitten by a dog which she thought to be rabid.

Among the most common of the immediate or direct causes may be mentioned slight pressure upon one of the nerves of the larynx, especially the superior laryngeal or its branches. This may come about by enlargement of the thyroid, thymus, or lym. phatic glands, or from the pressure of an aneurism of the superior laryngeal artery. When the pressure exerted is continuous and severe, a paralysis of the nerve, of course, must finally supervene. In cases of injury or pressure upon the inferior laryngeal nerve, a period of spasmodic action may accompany the irritation at first thus preceding the loss of motion. Again, an apparent spasm or overcontraction of the adductors may be observed when paresis of the abductor muscles has taken place, because the balance of muscular tonus, so to speak, between the adductors and the abductors is disturbed, the adductor tonus remaining while the abductor tonus is enfeebled. Normal spasm of individual muscles of the larynx sometimes occurs without producing very much embarrassment of respiration, but the function of phonation may be thereby disturbed. This condition is usually due to purely local causes, such as a neoplasm, injury, syphilitic, lupous, or tuberculous ulceration, together with the consequent cicatrization. In such cases the spasm is never severe, and resembles choreic muscular movement. It is often confounded with fixation of one or the other vocal cords or ventricular bands by hyperplastic processes. A survey of the subject leads to the conclusion that laryngeal 
spasm is generally a secondary or symptomatic disorder, and therefore in dealing with every case this fact should be kept in view.

The larynx, however, probably on account of its specialized nervo-muscular mechanism, seems much more liable to spastic affections than other portions of the upper air passages. Porcher and Bosworth divide the clinical history into two divisions, viz., spasm of the glottis in children and spasm of the larynx in adults. These and other authors include in the latter division chorea laryngis, reflex epileptiform neuroses of the larynx, and cases denominated by Lefferts and others laryngeal vertigo. While this division of the subject answers the purpose very well, yet it would seem preferable to make a distinction between chorea laryngis, epileptiform spasm, and laryngeal vertigo, because vertigo is simply a phenomena which may accompany any perturbation of respiration of sufficient intensity and duration to interfere with the circulation of blood in the brain; besides, vertigo with glottic spasm is frequently an expression of a crisis in locomotor ataxia, hemiplegia, or neurasthenia, and is therefore significant as a symptom only of any form of laryngeal or general spasm.

Although this term, laryngeal vertigo, is used by some authors to designate a particular group of cases, the writer does not believe that it deserves such particularization, because vertigo, as said before, is merely a symptom of many diverse disorders, especially those involving sudden suspension or perturbation of the circulation and respiration. Undoubtedly many of the cases regarded as either laryngeal vertigo, laryngeal epilepsy, or laryngeal chorea are cases of aggravated spasm of the glottis only.

Laryngeal chorea may be considered as a choreic affection manifesting itself in the laryngeal muscles-a local chorea. In a few cases the choreic movements are confined entirely to the laryngeal muscles, but generally there are also other muscles involved, especially those supplied by neighbouring nerves of the same group.

Choreic movements of the vocal cords (or bands) are frequently met with as an intercurrent phenomenon in various acute or subacute conditions, but under such conditions these manifestations are transient only, and cannot be properly con- 
sidered as constituting chorea laryngis. The reason for this local predisposition of the larynx is not always plain, but it may arise from the overtaxing of the laryngeal muscles by vocal effort, the presence of some slight structural change, such as trachoma or papilloma, or a chronic laryngitis. It is met with in vocal teachers and vocal students more frequently than in other persons. The writer has met with one case in a stevedore, one in a seamstress, one in a female clerk, and one in a physician. The two latter were suffering from oxaluria also, the correction of which condition entirely relieved the chorea.

Rheumatism, syphilis, and alcoholism have been charged with the production of some cases of this affection.

The laryngoscope shows little if any abnormality, as a rule, in the colour or structure of the larynx, but the vocal cords are seen to be in motion almost constantly, the movement consisting in their approach and receding from each other in a desultory and irregular manner. Whenever any attempt at phonation is made the tremor and convulsive contractions of the vocal cords increase very much.

Laryngeal Epilepsy.-Epileptic spasm confined to the laryngeal muscles is rare. The writer has seen two cases which at first sight might be so regarded, but which subsequent observation showed to be the beginning of a lateral epileptic paroxysm only. When the seizure was destined to be mild the aura would first develop in the larynx. Both cases were syphilitic, and possibly some cicatricial tissue in the larynx may have accounted for the commencement of the spastic action in the larynx.

There are cases, nevertheless, in which the larynx, or larynx and pharynx, seem to bear the whole brunt of the seizures. Such cases are undoubtedly laryngeal epilepsy, and their course is not without danger to the patient on account of the possibility of asphyxia or cardiac failure supervening upon an exceptionally long attack.

Under the heading, Spasm of the Glottis in Adults, as given by some authors, are included the several forms of laryngeal spasm usually affecting adults, and comprising transient attacks of a functional character representing perturbations arising from irregular excitation of nerves, ganglionic centres, or the great sympathetic; also spasm resulting from direct 
irritants, and, lastly, those particular forms of laryngeal spasm which have received the name of laryngeal epilepsy and chorea laryngis. The latter, however, having a distinct symptomatology, ought not to be confused with laryngeal epilepsy; in other words, one disease should be classified with epilepsy, while the other should be classified with chorea.

Diagnosis. - The diagnosis is very easily made by the clinical history and laryngoscopic appearances. These affections are to be differentiated from laryngeal tumours, subglottic hypertrophy, œdema of the glottis, neoplasms, tuberculosis, paralysis, etc. The absence of preceding laryngeal disturbances will serve to differentiate them from odema glottidis. However, the laryngoscope will enable us to differentiate between these several affections. See illustrations of the laryngoscopic appearance of hypertrophy of the larynx, œdema glottidis, and papillomata. All of these may be distinguished, by laryngoscopic appearance, from laryngeal spasm, because the larynx in spasm shows a normal or nearly normal appearance. Next in order it will be necessary to differentiate these diseases from the various laryngeal paralyses. This will not always be easy, because so many cases of abductor paralysis in the early stage are mistaken for spasm of the glottis on account of the more powerful action of the adductors. In this affection, as also in functional aphonia, there is no spasmodic action present; the muscles of the larynx are seen to be partially immobile and slow to action, but with little or no spasm unless it be a slight tremor, while in chorea laryngis attempts at phonation are usually attended by rapid spasmodic actions of the vocal cords upon each side simultaneously.

Symptomatology in Adults.-Ordinary spasm of the glottis in adults is usually preceded by a tickling or choking sensation referred by the patient to the posterior wall of the pharynx or the external sensitive area of the neck just in front of the larynx. Short acts of swallowing following each other in rapid succession, interrupted only by gagging or slight attempts at retching, usually precede, a gasp or two lasting for a few moments, when the real strangling or prolonged stridulous inspiratory efforts take place, accompanied by the crowing or interrupted noise which is peculiar to this action. Expiration is almost suspended for a time, as the patient's whole effort is 
directed toward drawing air into the lungs. The patient now with anxious expression, suffused, wide-open eyes, livid lips and face, and stretched neck, rushes about aimlessly or toward a door or window in search of relief. If the stridor is not maintained too long a time there is no loss of consciousness nor extreme cyanosis; but, on the other hand, if the paroxysm be prolonged, the surface becomes cold and purple, the eyes glassy, consciousness becomes suspended, and the patient falls to the floor either limp or with more or less convulsive movement. The paroxysm seldom leads to a fatal result, excepting when there exists some cardiac lesion or arterio sclerosis. More or less vertigo, exhaustion, and an inordinate apprehension over a return of the frightful paroxysm are inevitable results of a severe spasm of the glottis. The spasm in such cases is undoubtedly confined to the adductors, because spasm of the abductors, unless the diaphragm be involved, would not produce this clinical picture. Spastic affections confined to the abductors are rare, and when occurring usually disturb the function of phonation, and not respiration. The laryngoscopic appearance of the glottis during a paroxysm is sure to show almost complete closure, by apposition of the vocal cords up to the posterior vocal processes. From this point, posteriorly embracing the membranous portion of the glottis, a slight divergence of the vocal cords leaves a small triangular chink only through which air can gain access to the trachea.' It would almost seem impossible to make a laryngoscopic examination during a severe paroxysm, unless a great amount of outside force were used to restrain the patient; but one familiar with the physiology of the larynx can easily imagine that this might be the condition of the glottis at such a time.

Prognosis.-The prognosis will depend entirely upon the course and gravity of the disease upon which these manifestations depend.

Treatment.-From the diverse causes of the affection it is evident that no regular line of treatment can be laid down. Hence the general principles for the treatment of the various acute or chronic maladies which give rise to the glottic spasm must be followed, and exceptionally a well-directed local treatment of the larynx may become essential for those cases which show decided local abnormalities. Harsh applications, how- 
ever, are always to be avoided. When neoplasms or other morbid structural changes appear, proper surgical measures for their correction should be adopted. Laryngeal epilepsy or chorea should be treated the same as epilepsy or chorea when affecting other parts of the body, by systemic and not by local medication.

\section{Spasm of the Glottis in Children}

\section{Laryngismus Stridulus, "False Croup"-Spasmodic Croup}

This affection, which is quite common, depends upon so many different conditions that it may be looked upon as symptomatic generally, instead of a disease by itself. There are undoubtedly two distinct forms of laryngismus stridulus, the one a transient affection accompanying a slight catarrhal laryngitis (in healthy as well as unhealthy children), and the other a more persistent neurotic manifestation depending upon some seated constitutional disease. The disease has been written about very much during the past. As long ago as I830, Kopp, who wrote exhaustively upon it, believed that the disease was always due to pressure upon the laryngeal nerves by an enlarged thymus gland. His theory, however, was disproved in I 852 and I 858 by Bednar and Friedleben, who determined by careful experiment that a constant connection between the phenomena of laryngismus and hypertrophy of the thymus gland did not exist (Strubling). Grawitz, Marfan, and Lange believed that the sudden death so often terminating certain cases of stridulus was really due to a snapping off or breaking of the trachea which was held so tightly by the enlarged gland (thymus) (Strubling). The majority of authors believe now that the disease is produced by irritation of the recurrent laryngeal nerve through direct pressure by enlarged glands or other tissues lying along its course, or through disease affecting the central nucleus of the spinal accessory, or by an irritation about the floor of the fourth ventricle. While many observers believe that nearly all of these cases are reflex, the result of some irritation of the central nervous system, still much discussion has taken place as to whether or not all cases depend upon disease of the cortex or bulb (medulla). Spastic contractions of the glottis may be the precursory sign of 
recurrent paralysis, for, according to Semon's law, the abductors of the glottis are the first to be affected in paralysis of the recurrent. Semon is of the opinion that neither spasm nor paralysis has been shown to depend upon disease of the cortex alone, while Rethi and R. Russell believe there is a centre for even an abduction of the lips of the glottis as well as a separate cortical centre for adduction and respiration. This seems to be supported by the experiments made upon animals by Landois, who by chemical irritation of the cerebral meninges of animals caused not only an initial cry. analogous to that observed in epilepsy and hydrocephalus, but a spastic condition of the adductors with croaking inspiration. Physiologists tell us that cough as the result of normal reflex action can only take place by irritation of the respiratory passages, yet clinical observations show that cough and glottic spasm may arise from irritation reflected from various parts of the body -as, for example, during the eruption of teeth.

Symptomatology.-The advent of the spasm is usually sudden, oftentimes coming on while the little patient is asleep or at the breast, but, on the other hand, sometimes preceded by a period of restlessness. It may be ushered in by a sharp, short cry, followed by stridulous, croaking, inspiratory efforts, interrupted by short ineffectual expiration, the lips and skin soon become bluish or purplish in colour, the eyes suffused and glassy, and the skin cool and clammy. The respiratory struggle may merge into a partial or general convulsion of greater or less intensity, or, instead, the paroxysm may subside without such an event. The little patient afterward shows great exhaustion, and falls into a stupor which may last an hour or more, or, if the paroxysm be very light, it may after a little time resume its nursing or playing. From the stupor it wakes up in a fit of crying, which may last for an hour or more. In some children the nervous irritation will continue for a day. In the case of healthy children, where the laryngismus is of short duration and due to slight laryngeal catarrh, the exhaustion will of course be much less pronounced. In such instances, according to Cohen, the laryngismus is probably induced by the collection and desiccation of a little mucus about the rima glottidis during sleep or laryngeal rest; this acts as a local mechanical irritant, exciting spasm of the glottis before it can be dislodged. It may also be due to the swelling of the mucous 
membrane of the larynx. It may also occur in nervous children after an attack of intestinal disturbance of any sort, perhaps without the absorption of any toxin from the intestine. Children, however, who are affected with rickets, craniotabes, syphilis, or other chronic wasting disease, may suffer frequent and prolonged paroxysms of laryngismus. Chronic disease of the larynx, either inflammatory or neoplastic, is not so apt to excite laryngismus, because the nerves of the organ have time to become tolerant of the abnormality.

Prognosis. - When depending upon an acute laryngitis, teething, or gastro-intestinal disturbances, the prognosis is generally favourable. But, on the other hand, when the subject is suffering from tabes, syphilis, or other serious constitutional diseases, the glottic spasm may be regarded as an untoward sign of the general disorder.

Treatment.-Laryngismus stridulus, when incident to an acute laryngitis or some other acute affection, does not usually need much special treatment. It may betoken an irritable condition of the nerve centres, which will demand the use of such sedatives as chloral hydrate, potassium or sodium bromide, phenacetine, or hot baths. When catarrhal laryngitis is the cause of the spasm, hot baths frequently repeated and small doses of either aconite, tartar emetic, or pilocarpine, together with sinipisms applied to the front of the neck, will usually be all the treatment required. In addition to this, however, it is advisable to keep the child quiet in a warm room, the air of which is kept moist by steam. When gastro-intestinal disturbance is the predominant concomitant, two or three good doses of oil of turpentine (five to ten minims) may be administered in draughts of milk; or, instead, the turpentine may be given (in half-drachm doses) well mixed with white of egg and milk, by the rectum.

Paralyses of the Muscles of the Upper Air Passages

The paralysis may be limited to any one of the regions of the throat, or may affect muscles or groups of muscles in one or more portions of this region. A glance at the anatomy and physiology of the upper air passages will serve to remind one of the intimate and co-ordinate nervous and muscular relations existing between these several parts, and will also make 
it apparent that no territorial limitation, so to speak, of a general functional character can strictly belong to any one anatomical or clinical division. Besides, when it is remembered how closely related anatomically and histologically the nuclei and the bulbar connections of the cranial nerves are, it seems remarkable indeed that their insulation, and that of their communicating branches, is so perfect as to exempt any of them from pathologic participation, when any considerable lesion of the brain, cortex, medulla, or nerve trunks has occurred. From this point of view it might be expected, therefore, that cortical or bulbar lesions, or severe systemic toxæmia, would invariably produce very general paralysis of the muscles of at least one side of the pharynx and larynx; but this is not always the case, for in many instances such lesions may paralyze one side or one group or a set of muscles only, as, for instance, diphtheria paralyzing the muscles of the soft palate. On the other hand, if one of the contributory nerve trunks, or some of its branches, be the seat of a lesion through pressure or injury, then, as might be expected, the paralysis will be limited usually to that particular nerve and its ramifications. Moreover, we meet with a class of paralyses which may be termed reflex, because showing no particular central or peripheral nerve or muscle lesion to account for their occurrence. Such reflex action may be either excito-motor or sensorimotor. In addition, there is another class in which the lesions are at least predominantly in the muscles, called myopathic; so that we may properly classify these several affections in regard to their essential etiology into cerebral (or cortical), bulbar, peripheral, reflex, and myopathic.

The cerebral lesion may be extensive, including the greater part of the brain, a hemisphere, or a circumscribed lesion located in the neighbourhood of the nucleus of the pneumogastric or spinal accessory, such as tumour, gumma, abscess, sclerosis, softening, hemorrhages, etc. ; or it may be functional, depending upon $(a)$ neurasthenia, hysteria, concussion, psychic perturbances, etc., or $(b)$ depending upon the effects of toxic agents, such as those developed by diphtheria, pneumonia, erysipelas, influenza, syphilis, the exanthemata, or lead, arsenic, etc.

The structural bulbar lesions causing paralysis may likewise be of the same nature as those enumerated for cerebral or cortical lesions. But special mention might be made of 
progressive bulbar paralysis, progressive muscular atrophy, and syringomyelitis; also any marked disturbance of the respiratory centre will often produce some pharyngeal or laryngeal paralysis or paresis. The peripheral structural lesions usually consist of injury or pressure by tumours, enlarged glands, morbid growths, tumefactions, inflammations, and ulcerations affecting some portion of a nerve trunk or its branches between its distribution and its emergence from the cranium.

The peripheral functional causes consist generally of purely local changes in the mucous membrane, producing irritation of the peripheral nerve ends. This irritation may be of an inflammatory or trophic nature, such as local inflammation, tumefaction, hypertrophy, or atrophy, etc.

\section{Paralysis of the Muscles of the Nose, Soft Palate, AND PhaRYNX}

Paralysis of the external muscles of the nose is usually due to injuries of the facial nerve, or is symptomatic of some more widespread lesion. When the dilator or compressor naris muscles are predominantly affected, the cause is usually of myopathic nature, and may be either congenital or the result of traumatism.

Soft Palate and Pharynx.-There are three classes of paralysis or paresis of the soft palate and pharynx, met with, depending upon (a) some systemic poisoning; (b) injury to the motor nerves; $(c)$ disease of the muscles (myopathic). Diphtheria is by far the most common cause of paralysis of the soft palate, although the disease may rarely follow a severe attack of acute follicular tonsilitis or phlegmonous tonsilitis. A paralysis arising from toxæmia is usually bilateral.

Injuries to the pharyngeal nerves are sometimes the cause of a paralysis which is usually one-sided. This must not be confounded, however, with congenital atrophy of the palate muscles, which may simulate paralysis unless the observer be careful to note that the relaxed side is not immovable. The pharyngeal muscles do not often participate in the toxic paralyses, and when they do it is generally the palato-pharyngeus, and not the constrictors, which are so affected. The latter muscles are involved early in cases of progressive bulbar paralysis. 
Myopathic paralyses are generally the result of either severe inflammations, excessive use of the throat, rheumatism, gout, or injury. The latter diseases are only occasional causes, and in such instances the condition may be looked upon as a purely muscular affection (myositis) rather than a true paralysis.

Symptoms. - The subjective symptoms are quite characteristic, consisting of more or less inability to swallow-liquids especially returning through the nasal passages-and a peculiar nasal tone of the voice with inability to enunciate the nasal sounds. The articulation is slow and deliberate. Many patients also complain of dryness of the throat and inability to free the nasopharynx readily of secretion by nasal screatus and hawking.

Treatment.-When paralysis follows such a toxæmia as diphtheria it will usually disappear after a period of four to eight weeks. The recovery may often be hastened, however, by the local application of the faradic current and the internal administration of strychnia or picrotoxin. The same treatment will apply to the other varieties, except those known as myopathic, in which often the faradic current is inadvisable, while the galvanic may prove beneficial. In such cases a spray of a solution of arg. nit. ( $\mathrm{gr} . \mathrm{x}$ to $\tilde{\mathrm{J}} \mathrm{I}$ ) or argyrol ( Io to 20 per cent) applied once daily will be useful.

\section{Paralysis and Paresis (Akinesis) of the Larynx}

Of the many classifications published, that of Masucci, which is here given, seems the most comprehensive:

I. Paralysis of the tensors of the vocal cords.

2. Paralysis of the constrictors of the glottis.

3. Paralysis of the dilators of the glottis.

4. Paralysis of all the muscles of the vocal cords innervated by the recurrent.

(I) Paralysis of the crico-thyroid (superior laryngeal nerve).

Symptoms. - Anæsthesia of the mucous membrane; deglutition impaired and uncertain; voice hoarse and monotonous, with inability to emit sharp tones.

Laryngeal Image.-Epiglottis immobile and standing against the base of the tongue; movements of the vocal cords normal; the opening of the glottis undulated. 
(2) Paralysis of the adductors (inferior laryngeal nerve). Symptoms.-Dysphonia and aphonia; respiration normal.

Laryngeal Image.-Different degrees of incomplete closure of the glottis, taking various forms as follows:

A. In paralysis of all the adductors the vocal cords assume inspiratory or median position (see Fig. 50) upon attempts at phonation.

B. In paralysis of the lateral crico-arytenoid muscles the glottis may open up to the anterior vocal processes (see Fig. 44 and No. 2, diagram).

C. In paralysis of the transverse arytenoid the intercartilaginous portion of the glottis forms a triangular opening, while the interligamentous portion is closed (see Fig. 46 and No. 3, diagram).

D. In paralysis of the internal thyro-arytenoid the vocal cords appear straight, but their free borders show a concave line, making the rima glottidis of oval form (see Fig. 46 and Nos. $2 \times 4$, diagram).

E. In paralysis at the same time of the arytenoideus and internal thyro-arytenoid both the interligamentous and intercartilaginous portions of the glottis remain open; but if the paralysis is unilateral the image of course is asymmetrical, and the sound vocal cord passes beyond the median line to meet its fellow during efforts at phonation, while the paralyzed cord either remains stationary or moves but feebly toward the median line (see Figs. 43 and 49).

(3) Paralysis of the abductors.

Symptoms.-Strong inspiratory dyspnœa, expiration free, voice normal, or nearly so, but accompanied by a stridulous noise.

Laryngeal Image.-Very small glottic chink, which is closed more completely with inspiration (see Fig. 47 and No. I, diagram).

(4) Unilateral paralysis of the posterior crico-thyroid.

Symptoms. - The respiratory difficulty is light, unless the patient makes some effort in moving around, etc.; the voice is not clear, but somewhat hoarse.

Laryngeal Image.- The glottis is only half open, because the paralyzed vocal cord remains in the median line (see Fig. 43 and No. 5, diagram). 


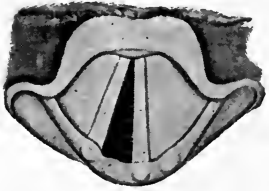

Fig. 43.-Left recurrent paralysis. Abduction.

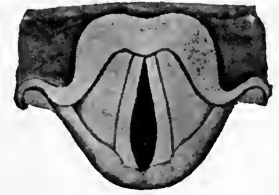

FIG. 44.-Bilateral paralysis of lateral crico-arytenoids.

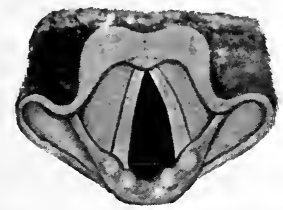

FIG. 45.-Left recurrent paralysis. Abduction.

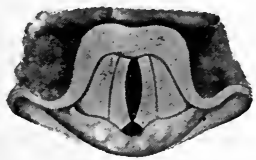

Fig. 46.-Bilateral paralysis of internal thyro-arytenoids and arytenoids.

(From De Schwinitz and Randall.)

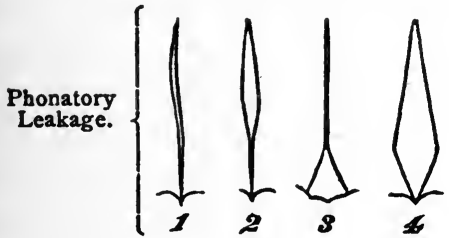

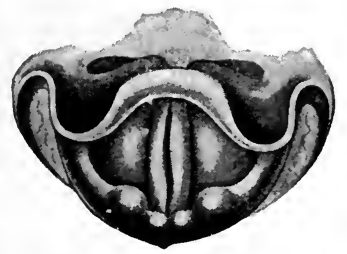

Fig. 47.-Bilateral paralysis of external tensors (Mackenzie).

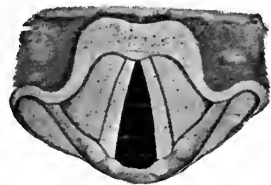

FIG. 48.-Bilateral recurrent paralysis.

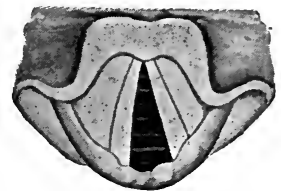

Fig. 49.-Paralysis of left posterior crico-arytenoid. Inspiration.

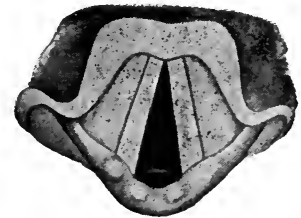

FIG. 50.-Relaxed or cadaveric position.
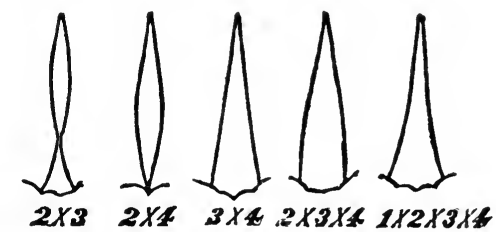

FIG. 5I.-The schematic diagram shows the outline of the rima glottidis during phonation in the paresis or paralyses affecting the vocal cords. Nos. I, 2, 3, and 4 represent the various forms of adductor paresis, and Nos. $2 \times 3,2 \times 4$, etc., represent combinations of the disabilities illustrated by Nos. I, 2, 3, and 4 . The shape of the rima glottidis in respiratory insufficiency, resulting from abductor paralysis or paresis, is represented by No. 5, $a$ and $b$ (Elsberg). 
(5) Paralysis of all the muscles of the vocal cords; complete bilateral paralysis of the recurrent.

Symptoms. - Absolute aphonia.

Laryngeal Image.-Cadaveric position of the vocal cords (see Fig. 50).

(6) Paralysis of all the muscles of one vocal cord; complete unilateral paralysis of the recurrent.

Symptoms.-Voice harsh, hoarse; respiration lightly panting during exertion, such as fast walking, etc.

Laryngeal Image.-Paralysis of the vocal cord in cadaveric position; crossing of the arytenoid cartilages and oblique position of the glottis, because the arytenoid and vocal cord of the opposite side pass the median line (see Fig. 45).

It will be seen that the most dangerous of all these paralyses is that affecting the abductors of the glottis, for this necessarily interferes with respiration to such an extent as to render death imminent at any time when both sides are affected. This form of paralysis involves the most important nerve connected with the larynx, the inferior laryngeal, which arises from the vagus and spinal accessory. There has been a great deal of discussion ever since the time of Longet and Claude Bernard regarding the physiology of this important organ, the larynx, and within recent times only has the question approached anything like a settlement. The principal discussion has hinged upon whether or not there was an independent cortical centre for the innervation of the larynx, or whether all the nerve force required for its complex functions is derived from the medulla oblongata.

There can be no doubt now, it would seem, that the nerves of the larynx are connected with a cortical as well as a bulbar nerve centre. The function of the larynx is plainly a double one, a respiratory automatic function (which consists of the opening and closing of the glottis for respiration), and a distinct voluntary action comprising voice production, which is not automatic. It is obvious, then, that for the exercise of these two functions, co-ordinately a cerebral as well as a bulbar connection is necessary, for the one is automatic and the other not entirely so, but voluntary. Analogues of this double innervation are to be found in other parts of the body, as, for instance, the centre in the lumbar region for defecation and mic- 
turition, the centres for cardiac and respiratory activity and inhibition, all of which are automatic and to a certain extent volitional. There can therefore be no question regarding the necessity of the nervous mechanism governing the larynx being of a mixed character, with nerves carrying motor and sensory filaments of specialized character for the consummation of the delicate and intricate operations of phonation, respiration, and deglutition.

Space will not allow of a complete history of the very many experiments which have been made upon the lower animals, together with observations upon disabled human subjects, relating to this interesting subject, but a few words outlining the several steps may not be out of place here.

Lermoyez states that there are two experimental facts and two clinical facts upon which the discussion of the subject of the innervation of the larynx principally rests, as follows:

I. Section of the recurrent laryngeal nerve produces a complete paralysis of the movements of separation and approach (abduction and adduction) of the corresponding vocal cord.

2. Excitation of the recurrent laryngeal nerve brings and maintains the corresponding vocal cord in the median line, thus showing the predominance of action of the constrictor muscles over the dilator muscles. It might be added, also, that this clearly shows the double function of the larynx. The clinical facts are, first, complete suppression of the action of a recurrent nerve immobilizes the vocal cord on that side in the cadaveric position; second, incomplete suppression of the action of a recurrent nerve immobilizes the vocal cord on that side in the median position.

These conditions hold good whether depending upon lesions of the bulb or of the peripheral nerves. He excludes, of course, in the above statement those cases where the median position of the vocal cord is due to a myopathic paralysis.

Gerhardt, in 1863 , was the first who called attention to the fact that either the pneumogastric or the recurrent laryngeal nerves could give rise to diverse symptoms, according as the different filaments were affected. He called this paralysis of the abductors, and considered the causes myopathic, and seated in the posterior crico-arytenoid muscles.

Etiology and Pathogeny.-As might be surmised, the pathogeny will depend upon a great many diverse causal 
factors. The general classification given by Lermoyez seems to be the clearest; he divides the causes in a general way into

1. Lesions at the level of the trunk of the recurrent laryngeal.

2. Lesions at the level of the pneumogastric trunk.

3. Lesions at the level of the trunk of the spinal accessory.

4. Lesions at the level of the bulb.

5. Lesions at the level of the cervical plexus.

Statistics upon the causes as given by Avellis, and based upon one hundred and fifty cases of recurrent paralysis, show that, of the one hundred and fifty cases, in eighty-five times only was the etiology thoroughly recognised. In sixty-five cases the latent or remote causes were inexplicable. The pathogenic influences which were determined are tabulated as follows :

Thoracic aneurism.............. 24 times.

Tumours of the esophagus .......... 5

Pericarditis.................... I "

Pleuritis, dry or plastic............. 2 "

Tuberculosis of the right lung........ 7

Tuberculosis of the left lung......... 5

Cervical adenopathies ............. 7 "

Goitres ..................... I4 "

Extirpation of goitre $\ldots \ldots \ldots \ldots \ldots \ldots \ldots$ I "

Tracheotomy.................. I "

Pott's disease of the cervical spine....... 2 "

Wounds of the axillary plexus.......... I "

Furuncle of the neck ............. I "

Diphtheria................... r r "

Paralysis of nervous origin........... 2

Cerebral affections................ 5 "

Syphilis.................... 3 "

Hereditary syphilis $\ldots \ldots \ldots \ldots \ldots \ldots \ldots \ldots$ I "

Congenital paralysis .............. I "

Sex.-Relating to the sex of the one hundred and fifty cases, one hundred and six were males and forty-four females.

Location.-Relating to the seat, it is noticed that the paralysis was forty-six times on the right side, ninety-two times on the left side, and twelve times on both sides. Although these 
statistics are far from perfect on account of the small number of cases collected, still Lermoyez believes that three important points are brought out by them : first, the great frequency of peripheral lesions which belong to the recurrent (neck and thorax) in relation to the central ones of the bulb and spinal cord ; second, the predominance of peripheral paralysis of the left recurrent, which may be explained by the fact that this nerve takes a much longer intrathoracic course than does the right, and is therefore more exposed in its course on that account; and third, the predilection of the masculine sex for recurrent paralysis.

Aortic aneurism and tabes are very frequent causes of this affection, and it may be said without doubt that in most of such cases an antecedent syphilis constitutes the primary cause. This would show still more prominently if it were not that goitre, which is more common in females, is also one of the common causes of recurrent paralysis.

To particularize the causes of recurrent paralysis, we may mention, first, lesions of the trunk of the recurrent by traumatism, compression, or neuritis. The traumatism is rarely accidental, but often surgical. Lefferts reports a case of accidental traumatism in a woman who was stuck in the neck by a pair of scissors. The recurrent is not infrequently wounded in operations made for thyroidectomy (Lermoyez, Tillaux, Richelot), external esophagotomy, extirpation of the lymphatic glands of the neck, ligature of the common carotid, oftentimes in tracheotomy from the pressure of the tube (Massini and Wagner), or compression from a thoracic aneurism, and adenopathies of the ganglionic chain about the larynx, trachea, or bronchi. M. Rosenberg reported a case of partial paralysis of the vocal adductors following an operation for removal of tubercular glands from the neck. These causes may operate together in the production of paralysis (Halevy). Aneurisms, which are more often capable of pressure upon the recurrent laryngeal, are seated ordinarily upon the arch of the aorta, at the trunk of the brachiocephalic, carotid, and left subclavian, and exceptionally the right subclavian and the pulmonary artery (Riegel). Sometimes there are two aneurisms, one of which may escape detection. Onodi reports a case of this kind. Sometimes the aneurism cannot be detected until after death. Schmidt and Oliver relate cases where the aneurism 
during life had, even after careful stethoscopic examination, been undetected.

Many observers believe that aneurism is the most common of all the causes of recurrent paralysis, especially of the left side. Botey believes that almost all cases are due to this cause. In cases where both recurrents are paralyzed the pressure is supposed to be exerted upon each side, unless it be a case of extensive cerebral lesion. When syphilis produces recurrent paralysis it is generally due to changes in the tracheo-bronchial glands, and the same may be said when tuberculosis lies at the bottom of the attack (Gougenheim and Tissier). Secondary cancer of the lymphatic glands may produce it. Leukæmia and pseudo-leukæmia may produce paralysis of the left recurrent, in consequence of the tumefaction of the lymphatic glands, which they induce.

Cancer of the esophagus is frequently a cause of paralysis of not only the left recurrent, but the right as well (Schmidt). Tumours and pleurisy confined to the mediastinum may produce this paralysis. Hajek ascribes some cases of paresis to the effects of pneumonia. The writer has met with four cases of moderate paresis of the adductor of one side (three right and one left) following acute pneumonia.

The following table of Moncorge summarizes the relative causes of lateral and bilateral paralysis.

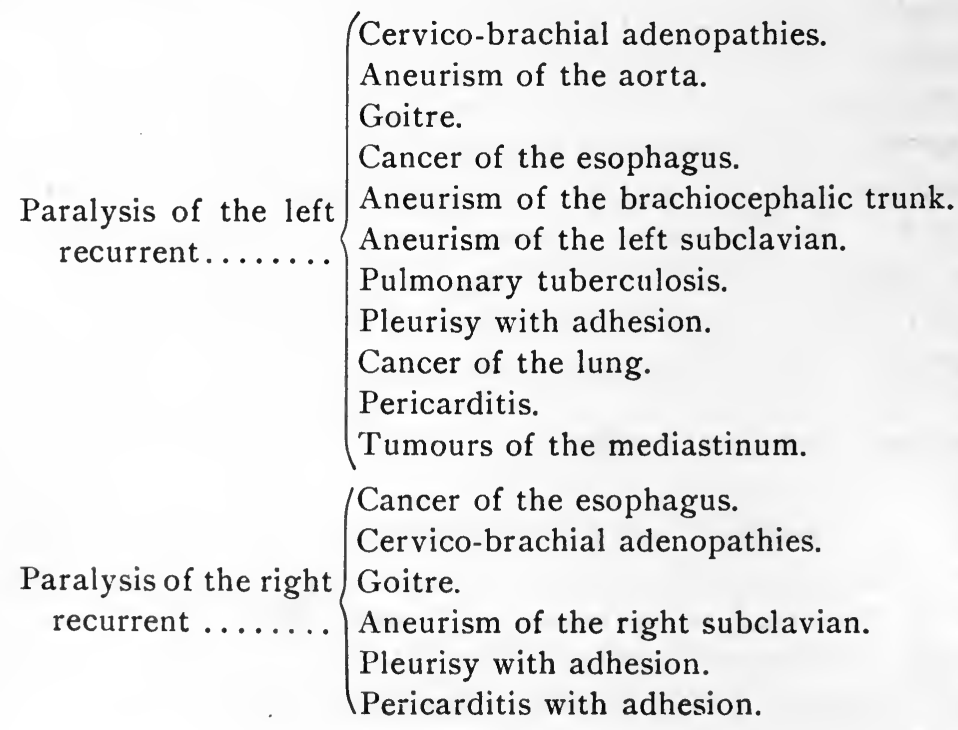


Bilateral recurrent $\left(\begin{array}{l}\text { Cervico-brachial adenopathies. } \\ \text { Garalysis...... } \\ \text { Multiple aneurisms. } \\ \text { Cancer of the esophagus. } \\ \text { Pericarditis with thickening. }\end{array}\right.$

There are, besides cases of toxic neuritis, infectious neuritis, neuritis consecutive to bulbar lesions, and primary neuritis of the recurrent itself.

A variety of partial paralyses may occur in accordance with the nature of the extrinsic or intrinsic causes; for instance, the pressure on a nerve trunk, or the degree of pathologic change in it, will determine, of course, the resulting interference with its function.

Diagnosis.-The diagnosis of paresis or paralysis is to be made by the laryngoscopic appearances principally (see Figures on page 223), although, of course, the clinical history is necessary in order to determine the nature of the case.

Prognosis. - The prognosis will depend entirely upon the nature of the cause- $(a)$ whether the phenomena depend upon lesions of the great nerve centres, (b) upon local disease of the larynx or its neighbourhood (and its curability), or (c) upon some dyscrasiæ, such as syphilis, tuberculosis, or chemical poisoning with lead, antimony, belladonna, antipyrine, etc.

Cases depending upon syphilis, removable tumours, or 'enlarged glands are usually quite amenable to treatment.

Treatment.-The general treatment wholly depends upon the nature of the cause. The therapeutic indications, therefore, will accordingly vary greatly.

Local Treatment. - Local treatment must necessarily be mostly expectant. For keeping up the nutrition of the parts, however, nothing will surpass the effects of electricity. The galvanic is preferable for the treatment of the majority of cases, as a rule, yet there are many in which the faradic or static currents will be found more efficient. In using either the galvanic or faradic current, one electrode should always be applied inside the throat. 


\section{Functional Aphonia}

The term functional aphonia covers a variety of cases characterized by more or less loss of voice, which cannot be traced to any particular structural disease of the larynx.

Etiology.-For obvious reasons the etiology is very obscure. It has generally been attributed, even by observers in prelaryngoscopic days, to hysteria or allied emotional disturbances. Some authors believe that the condition always depends upon some change, perhaps temporary, in the nervomuscular apparatus of the larynx, while others account for its appearance through transient derangements of the blood circulating in the brain or medulla, while not a few consider it always reflex, and attribute it entirely to functional derangements of the sensory centres.

Clinical observation would indicate that the majority of cases of functional aphonia arise from disturbances affecting the brain and other sensory centres, such as mental depression or excitement, and shocks of diverse character to the nervous system, fatigue from overuse of the voice, ocular disturbance (eye strain), anæmia, malarial and other toxæmias. H. S. Straight and F. B. Eaton report cases of aphonia due to hypertrophic rhinitis.

The writer has met with several cases in women who have been suffering with a subacute pharyngitis, associated with mild stomatitis. They were somewhat hysterical, and complained of globus hystericus. The affection occasionally co-exists with aural disease. Ingals relates a case of deafness with hysterical aphonia. Arslan reports five cases, four of which were suffering from nasopharyngeal disease, and one had had influenza.

Besides these causes it is sometimes observed as a sequence of measles or pneumonia, and rarely it has been observed to follow a common cold or slight traumatism; in these cases, however, there is probably some slight local structural alteration of muscle or nerve fibres. Previous disease, however, does not seem to play an important part in inducing this form of aphonia, for it is a striking fact that very many of the cases are found among people in health. Aphonia is not often observed as a sequence of the acute diseases, excepting diphtheria and cerebro-spinal meningitis. 
Age.-It is seldom met with in childhood, and may be considered as a disease belonging almost exclusively to adult life.

Sex.-It is more common in females than in males.

Pathology.-The pathology is but very imperfectly understood, because the majority of such cases recover; indeed, it may be said that our pathological data rest more upon clinical observation and physiological experiment than upon morbid anatomy and histology. The muscles usually at fault are the adductors of the glottis, especially the thyro-arytenoidei, the arytenoidei, and less frequently the crico-thyroidei. Therefore one is forced to look upon the co-ordinating nervous mechanism as the basis of the disease. It is probably in many instances due to a failure of the conducting filaments to carry to the proper destination impulses generated by the will. This, of course, is a mere theory based upon the anatomic and physiologic knowledge of the complex co-ordinate qualities (inhibitory, conducting, and excito-motor) belonging to the pneumogastric and spinal accessory. If it be true that the pneumogastric and spinal accessory respectively inclose specialized insulated conducting filaments for the larynx, which have a central connection both with the cortex and medulla and give off no communicating branches until well nigh their distal destination, then an explanation of the various types of functional paresis might be found in supposing-

a. The existence of a transient or habitual derangement (either chemical or physical) of the nerve cells of the centres, so that the impulse is either too feeble or is not properly generated.

b. Short circuiting or switching, so to speak, of the impulse or current, so that sensory impulses traverse motor filaments, or motor impulses traverse sensory filaments. Or,

c. Deflection of the entire current through the peripheral communicating branches to the wrong destination.

The writer, with many others, has long believed that in the majority of cases of habitual hysteria or allied exhibitions of loss of mental control there must be some sort of structural or at least physical derangement of the nerve elements, either in the nerve centres or ganglia or conducting nerve fibres. Although the so-called functional nervous disorders and their relation to the nerve cells of the cortex and spinal cord have 
not yet been precisely demonstrated, there is reason for hope that this great desideratum will soon be reached, for the recent researches of Nissl, Benda, Flemming, Dercum, Huber, Golgi, Rámon y Cajal, Von Lenhossèk, Lewellys Barker, Mills, and others are rapidly bringing to comprehension the hitherto hidden histologic and biologic activities of the elemental nerve cell and the nerve fibril. Dercum, in a paper On the Functions of the Neuron with especial Reference to the Phenomena presented by Hysteria and Hypnotism (published in the American Journal of the Medical Sciences, August, 1896), says in the course of his discussion: "Can it be that the neuron is not an absolutely fixed morphological element? Can it be that it possesses a certain though perhaps limited movement? Realizing the practical value and the wide application of this idea, I have examined the literature to see whether a similar interpretation of nervous phenomena had occurred to others." $\mathrm{He}$ found that, independently of one another, the same idea nad occurred to three others who had published memoirs containing their researches and conclusions in 1890-viz., Rabl-Ruckhard, Lepine, and Mathias Duval. In this connection it may be mentioned that Rámon y Cajal and Nansen contend that nerve cells do not move, although the former author, according to Dercum, maintains that the neuroglia cells possess a great degree of mobility. Just why the laryngeal apparatus should be selected for the expression of such derangements, especially where the vocal apparatus has not been undergoing any particular degree of training or exercise, cannot be explained any more than can be explained analogous affections of the eye, ear, tactile, or other special senses suffering from similar nervous conditions.

The writer had occasion to observe in the same family one member suffering from true functional aphonia consequent upon sudden grief, and another member a loss of the special tactile sense in one hand from a similar cause. It would therefore seem probable in all cases of functional aphonia, especially of sudden onset and not connected with local disease in the larynx, that the pathogenesis of the affection may depend upon the cortex or ganglion centres. It is a notable fact that, unlike paralyses depending upon recognised structural disorders, the nervo-trophic function is not lost, for although a vocal cord may be impassive for a number of months, no great 
amount of degeneration either of nerve or muscle takes place; for when the proper functions are re-established, phonation also either suddenly or gradually returns. . Porcher and Bosworth state that the muscular integrity undoubtedly suffers in some cases, and may be responsible for the continuance of the aphonia. Infiltration, either interstitial or interfibrillar, as well as chemic changes of the muscle fluids, may affect the ultimate corpuscles so that the lesion may be purely a peripheral one. Hajeck thinks that aphonia following pneumonia may be due to pressure upon nerve filaments by effused products in bronchial glands, but considering the frequency of acute pneumonia and the infrequency of subsequent partial or complete aphonia, such an explanation would seem insufficient; neither will the fact of laryngeal paralysis, sometimes depending upon apical tuberculosis of the right side, furnish analogously an adequate support for such a theory. It may sometimes be observed that partial or complete aphonia does follow a pneumonia which occurs in the course of influenza, but in such cases, besides the toxæmia, the larynx, trachea, bronchial glands, and indeed the whole respiratory tract have been subjected to a great amount of damage, so that intumescence of the laryngeal as well as the neighbouring lymphatic tissues may have produced a sufficient amount of local lesion, temporarily at least, to cause partial or complete loss of the voice; its endurance, however, after the disappearance of the effects of inflammation, etc., can then only be ascribed to neurotic disorder. It sometimes affects females in the very early stages of phthisis. Bonney, of Colorado, gives a number of such instances.

Symptomatology.-The main symptom is complete extinction of the voice, although sometimes through great effort of the patient some sound may be emitted. The power of whispering is not abolished; neither is the respiration embarrassed in uncomplicated cases; a slight cough, which is phonetic, is usually observed. Dr. Lennox Brown thinks that the accompaniment of dumbness is an indication of structural lesion. The patient rarely experiences any pain about the throat or any difficulty in swallowing. The onset of the disorder is usually sudden, although in persons who have been suffering from some profound toxæmia or nervous perturbation, such as insomnia, etc., for a long time, the onset may be gradual. Many of the patients are apparently healthy, however, and are 
attacked coincidently with some great mental excitement or strain, such as fright or trouble coming to them suddenly. In such cases the aphonia may be immediately preceded by fainting, vomiting, diarrhœa, or a sharp pain of short duration in the region of the neck. There is usually more or less anæsthesia of the lips, tongue, buccal cavity, or throat. Sometimes numbness of the hands, feet, or face is complained of.

Laryngoscopic examination may not reveal either hyperæmia or intumescence of the tissues, excepting in those persons who have suffered from a local disorder. Frequently, however, the mucous membrane will appear anæmic, or at least paler than normal. This pale appearance is especially noticeable in cases which may have followed a variety of influenza, first described by W. C. Glasgow. The vocal cords are seen to be movable, but unable to meet one another during attempts at phonation, or, if meeting, it is only for an instant. There is also a notable lack of co-ordinate action. The arytenoids are not rotated inward so far as to form the proper position for phonation. The vocal cords may be in a cadaveric position-i. e., completely relaxed. If only the anterior crico-thyroid be involved, the vocal cord will appear relaxed or sagged throughout its length. This feature, however, is more often noticed in cases brought on by accident (blows upon the neck) or by fatigue through extraordinary use of the voice on some particular occasion.

Diagnosis.-This disorder may be mistaken for laryngeal phthisis, the early stages of which it sometimes accompanies; for paralysis due to structural disease; for immobility due to rheumatism of the arytenoid joints; or the first stages of syphilitic disease, or to infiltration of the glandular tissue in the laryngeal ventricles, or for small neoplasms situated on a vocal cord, or for invisible subglottic infiltration or for pachydermia. Laryngoscopic examination alone will not always enable one to diagnose the disease from the abovementioned and other affections, without the aid of the clinical history.

The disease usually comes on suddenly and may be intermittent. Bilateral paralysis of the abductors is rare, and causes intense and dangerous dyspnœa, especially on slight exertion. The whisper in hysterical aphonia is not at all laboured, and a hacking cough is usually present in hysterical aphonia. In 
some cases whispering may be impossible owing to the lack of will power. In nearly all cases the clinical history will furnish important diagnostic points.

Prognosis is generally favourable, although many cases endure for a long time, yet the majority ultimately recover their voice. The writer has observed recovery in several cases after the continuance of aphonia from two to five years, although all authors state that degeneration of nerve or muscular tissue is likely to take place from disuse.

Treatment. - In very many cases no plan of treatment, either general or local, seems to hasten recovery. Sudden mental impressions, especially of a joyous type may bring about a sudden return of the vocal function, as also may fright and apprehension. The perilous situation of a child or other relative, for instance, may induce the recovery of the function. A woman under the writer's observation, who had suffered from aphonia for many months, suddenly regained her voice through the excitement incident to an accident to her child. Indeed, there are many instances recorded where the approach of danger to the person afflicted, or to some dear relative, calling for sudden and perhaps extraordinary action, has proved sufficient to bring back the voice. In some cases the influence of suggestion has been successful in restoring the voice. Dr. Sanger Brown induced restoration of the voice by giving a number of detailed instructions to the patient, and finally directing that she speak on the fourth day. Dr. A. C. Follett has reported a similar successful experience with a case. Dr. J. A. Bach recommends that the patient be directed to vocalize while coughing. Dr. C. H. Knight and others have reported cases of hysterical aphonia which have been relieved promptly by cauterization of the posterior ends of the inferior turbinals. Arslan reports five cases treated with success by anæsthetization with ethyl bromide. The patients were rapidly anæsthetized with full doses (ten grains) of the drug, and as soon as they were partly insensible it was suggested that they shout loudly their names and count numbers.

The general treatment must be based upon the previous history of the patient as well as upon the present condition. Strychnia, chloride of gold and sodium, iron, sulphate of copper, salicin, and phosphorus given by the mouth, are among the most efficient remedies of the tonic class. The cold bath-pref- 
erably shower baths-is very often successful. The writer knows of a very obstinate case, in a hypochondriacal and hysterical man, which was quite promptly relieved by repeated cold shower baths. In very nervous persons, especially the so-called hysterical, relief may be secured by the administration freely of asafœtida, camphor monobromate, hyoscyamus, scutellaria, or the bromides.

Occasionally, when there is much hacking cough, a little antimony and potassa tartrate ( $\mathrm{I}-30 \mathrm{gr}$.), frequently repeated, may be serviceable. In cases of malarial or other toxæmia, of course the main treatment must be directed toward the relief of the general condition.

Local Treatment.-Local treatment is often beneficial. Its usefulness depends probably upon the excitement or stimulation which follows an application and the mental impression produced. It is sometimes a matter of indifference what the nature of the agent may be, inasmuch as a spray of a solution of salt and water, ammonium muriate, or the introduction of a brush or pledget of cotton into the larynx, may be equally efficient. However, when such means are unavailing, it is well to resort to applications of more stimulating agents, such as arg. nit. (gr. $\mathrm{x}-\mathrm{xx}$ to $\mathrm{oz}$.), chloride of zinc (gr. $\mathrm{v}-\mathrm{x}$ to oz.), Lugol's solution of iodine, olei carophylli et ol. gossyp. in the proportion of $3 \mathrm{j}$ of the former to $3 \mathrm{iij}$ of the latter. When no good results follow this line of treatment, then the application of electricity, especially the faradic current, will be indicated, and will usually be found beneficial. It seems to make little difference whether the cathode or the anode is applied to the inside of the larynx, but one or the other of the poles should be so applied by means of a suitable laryngeal electrode, while the other is placed on the outside of the neck, preferably at the auriculo-maxillary fossa. The duration of the séance will depend, of course, upon the toleration of the parts and the amount of self-control exercised by the patient; one half minute for each application, and from two to four of them at a séance, will usually be sufficient. The séances may be repeated two to four times a week, according to circumstances. In the writer's experience the application of electricity wholly to the outside of the laryngeal region is in the majority of cases of little benefit, except, perhaps, for the production of psychic effects only. 


\section{Neuralgia of the Upper Air Passages}

This is usually symptomatic only, being associated generally with hyperæsthesia, paræsthesia, or one of the spastic affections of this region.

Nose and Nasal Passages.-It is rarely confined to the nose except in the form of successive twinges of pain, a symptom often accompanying any acute inflammation of the nasal structures.

The nasal passages, however, may sometimes participate in the pain or neuralgia emanating from the facial and trigeminal nerves, their dental or other branches, or from disease of the Gasserian ganglion, or the trunks emerging from it. The nose and sometimes the pharynx are affected in disease of the ganglion when the pain takes the temporal, petrosal, or supra-orbital route.

Supra-orbital neuralgia, which (as will be mentioned in Chapter XIII) so often accompanies disease of the nasal accessory sinuses, may include the nasal twigs of the corresponding side.

Dr. William M. Copp, of Philadelphia (Medical News, vol. lxvi, p. 665), states that the insufflation of sodium chloride into the nasal cavity gives almost instant relief to many cases of severe pain (neuralgia) about the face or ears. He cites several examples to this effect. The method of using it is as follows: Twenty-five grains of finely powdered salt is loaded into a glass nose insufflator with short rubber tube attached by which to blow by the mouth the mixture into the nasal cavity. The charge is blown just after the patient has emptied the air from the lungs. It may be blown in from a tube of paper. This method of treatment was first suggested by Mr. George Leslie, of Edinburgh.

Neuralgia of the Pharynx.-Neuralgia of the pharynx is an uncommon affection, and is usually a symptom only of a group signalizing disease or injury of some neighbouring nerve trunk, plexus, or ganglion. Cases, however, occur occasionally in which disease of the ear, inflammations or ulcerations of the deeper structures of the pharynx (especially about its lateral walls), or affections of the cervical spine, give rise to it. The paroxysmal darting, stinging pain is generally referred by the patient to one or the other of the districts traversed by the pharyngeal branches of the facial nerves. 
Larynx.-Neuralgia of the larynx of a recurrent or paroxysmal character is seldom met with, if the sometimes severe perichondritis attending ulceration or phlegmonous inflammation be excluded. Of course, like other parts of the throat, it may participate in attacks of neuritis, etc., of the neighbouring nerves.

Treatment.-No special treatment of the part affected is indicated when the neuralgia is merely symptomatic, for the treatment directed toward the cause or origin of the pain will be sufficient. Applications of tincture of aconite or tincture of aconite and morphia or cocaine, by means of a brush or swab, to the locality pointed out as the particular seat of pain (if that be accessible), may be beneficial, but generally it will be found that systemic treatment or treatment directed elsewhere than the nose, pharynx, or larynx will be necessary to alleviate the pain. The case is different, however, when the neuralgia is due to local disease of a part of the respiratory tract within reach, such as ulceration, pressure, abscess, or disease of the bone, etc. Under these circumstances prompt relief may follow the necessary applications, manipulation, or operation upon the offending locality. Thus the opening of a suppurating gland, the covering of an ulcerated or abraded surface, or even cauterizing the same, or removing a piece of necrosed bone, or an enlarged adenoid mass, may constitute the necessary therapeutic measure. 


\section{CHAPTER $\mathrm{X}$}

CHRONIC INFECTIOUS DISEASES OF THE UPPER AIR PASSAGES

Syphilis of the Nose, Larynx, and Pharynx

SyPHILIS, either congenital or acquired, may manifest itself as a primary, secondary, or tertiary lesion in any of these regions.

\section{Congenital and Hereditary Syphilis}

Hereditary syphilis, properly speaking, comprises only those cases where infection has taken place at the time of conception; but for clinical reasons syphilographers classify cases with the congenital ones, where the patient is infected subsequently to conception. Rules laid down by various authors for distinguishing purely hereditary cases from the early acquired ones in infants are often difficult to follow. The tardy development of congenital syphilis (syphilis hereditaria tarda) is denied by many authors, who think that in such reported cases the early symptoms have been overlooked, but there are undoubtedly many examples of such delay. Dr. J. H. Bryan relates such an example with a very unique history. The mother of his patient contracted a chancre of the lip from kissing her lover, whom she afterward deserted for her present husband, a man who had always been healthy. The chancre on this woman was excised, and no secondary cutaneous or other symptoms ever made their appearance. She had, however, "some throat and bronchial trouble," which was relieved after taking " a course of iodide of potassium." The result of her marriage was three girls; the oldest, between sixteen and seventeen years of age, has been an invalid bordering on imbecility a number of years; the second daughter, who is fifteen years of age, a fine-looking girl, has thus far shown no signs of syphilis; the third daughter, the subject of this narrative, is now twelve years of age. 
When she was about nine years of age she began to show signs of "failing health." Both eyes were the seat of interstitial keratitis to such an extent as to cause almost total blindness. She also suffered at this time from deafness and inflammation of the nose. Under the judicious employment of mercury the opacity of the right cornea cleared up entirely, and that of the left is rapidly disappearing. She recently came under the doctor's care for a severe sore throat and persistent cough, which prevented her from getting any continuous rest at night. On examination, the nose was found to be filled with a thick, muco-purulent secretion which, when removed, showed a pale thickened membrane lining its cavity. The teeth were regular, not at all nicked. On inspection of the throat, a small laceration on the anterior surface of the soft palate was observed; while in the post-nasal space there was a large ulceration extending down to the soft palate, involving the right posterior palatine fold, and destroying the right tonsil. Examination of the larynx revealed also an ulceration which had destroyed the right half of the free margin of the epiglottis. There was enlargement of the arytenoid cartilages and aryepiglottic folds to such an extent that it was impossible to get a view of the interior of the laryngeal cavity. She rapidly improved, however, under treatment by mercury and iodide of potassium.

Congenital syphilis usually manifests itself between the second and sixth weeks, but may appear earlier, even at birth. J. N. MacKenzie found, upon an investigation of this subject a few years ago, that fifty-five per cent of the cases occurred within the first year, and about thirty-three per cent during the first six months. It occasionally happens, however, that its appearance is delayed until about the time of puberty. When the symptoms occur at birth it denotes a stronger infection, so that the patient usually succumbs to the disease in a little while. Catarrhal rhinitis (snuffles) and pharyngitis are almost always the early symptoms, although the disease may manifest itself in some other form at first. A frequent manifestation is a deep ulceration of the soft palate, which extends to the hard palate and posterior nasal space, with a tendency to attack the bones and produce caries or necrosis. The larynx, although sometimes the seat of congenital syphilis, is not nearly so often affected as the nose, soft palate, and pharynx. According to Bumstead, the 
laryngeal lesion is always secondary to the pharyngeal affection-that is, the pharyngeal ulceration precedes or closely coincides with the laryngeal. MacKenzie, on the other hand, believes that the laryngeal lesions occur much more frequently than is generally supposed, and states that we may look for the invasion of the larynx in congenital syphilis with as much confidence as in the acquired variety.

The writer has never seen a case of laryngeal congenital syphilis that did not occur a long time after the appearance of lesions in the pharynx, soft palate, or nose.

Symptomatology.-The infant usually displays hoarseness while crying, although the larynx may not at the time be involved. The nasal passages are obstructed, and mucous patches or ulceration of the pharynx, of the mouth, and possibly also of the nasal passages, soon appear. The discharges from the nasal passages become ichorous and desiccated into brownish-yellow, dry, or gummy crusts, which, together with the swelling of the mucous membrane, almost completely blocks the nasal passages and thus menaces life. Together with the nasal and pharyngeal symptoms, cutaneous eruptions appear generally at first upon the hands, feet, and face, and soon spread more or less to other parts. The little patient progressively emaciates, both from the effects of the disease and its inability to nurse. The skin soon shrivels. Lymph nodes may appear in the auricular and cervical region. The ears may become the seat of ichorous or purulent discharge. The Hutchinson (serrated) teeth may present an additional sign. If the disease has existed for a few weeks, fissures at the angles of the mouth, junctions of the alæ of the nose and face, or at the axillary region, may be observed. The majority of the cases of congenital syphilis are ranked as secondary, yet there are many cases later in development which clinically resemble tertiary forms. The latter are much slower in their evolution than the former class, and the destruction of tissue is more persistent. When affecting older children (from seven to sixteen or eighteen years), the symptomatology and course are about the same as in the acquired form affecting adults. In infancy, however, it must be remembered that nearly all cases of syphilis are apt to produce extensive disease (infiltration and degeneration) of the liver, spleen, intestines, or kidneys, besides the local lesions of the upper respiratory tract. The 
local signs usually precede the constitutional symptoms, but not always. Coryza syphilitica is a constant menace to the infant's life, from the malnutrition ensuing, and from the interference with respiration, which very soon takes place in young infants before the development of the ethmoid cells, and while the sphenoid, frontal, and maxillary sinuses are only in a rudimentary state. At this time the meati are very narrow and the posterior nasal space small, partly because of the membrana prevertebralis, and partly because of the vertical direction which the pharynx takes (Pierce, Zuckerkandle, Allen). Besides this, the secretions in the infant are more apt to become encrusted and hard. Hassing reported a case of nasal stenosis of this sort in which the dyspnœa was so urgent that tracheotomy had to be performed; but Schmiegelow, referring to this same case, attributed the dyspnœa to spasm of the glottis produced by reflex irritation from the coryza syphilitica.

Primary Syphilis (Acquired).-The primary form is seldom met with in the nose or throat, but more frequently on the lips and on the inside of the cheek. Primary chancre of the larynx probably never occurs. Indeed, many practitioners of large experience in venereal diseases have never met with more than one or two cases of primary chancre in other regions than the penis or vulva.

Nose. - Primary syphilis of the nose occurs with great rarity. Dupont found chancre of the nose only once in 1,773 cases (Pierce). Pierce has not observed a single instance in 3,000 cases of nasal disease. Maure has published a case. Spencer Watson reported a case, the point of infection being the septum. O. Seifert, according to Pierce, has lately collected 27 cases of chancre on or in the nose. In the largest number of cases the alæ nasi were the points of infection. In the lesser number the chancre was within the nose, and frequently on the septum. Up to I 89437 cases had been reported with chancre, in most of them on the septum. The finger in the majority of cases is supposed to be the carrier of the infection. Higguet saw two cases where the snuff-box was the medium of distribution. Kissing has been the means of infection in cases reported by Bulkley, Heissler, and Sherrill. Picking the nose and thus excoriating its membranes with the nails after having washed utensils used by the syphilitic 
has several times been the mode of primary infection (Cohen. Pierce).

The clinical appearance of primary chancre in the interior of the nose usually varies according to the age of the patient. In young children the sore frequently appears and runs its course as a simple papule without induration (Massei). In the adult it may also in the beginning appear as a papule or benign ulceration, but it soon becomes indurated, and in both classes of persons interference with respiration may become a prominent symptom.

A case reported by Rasori had an intense frontal headache. In the case reported by Watson there were elevation of temperature and great swelling of the interior of the nose. With Maure's case the patient had an unusual tendency to epistaxis (Pierce). Swelling of the submaxillary, sublingual, and periauricular glands usually takes place, and is an aid in diagnosis.

\section{Mouth, Pharynx, Soft Palate, Tonsil, and Larynx}

Undoubtedly primary chancre on the lip or inside of the mouth occurs more frequently than has been supposed. Bulkley, Blakewell, and other authors cite many examples of infection which have been innocently acquired; and the laryngologist frequently meets with the results of such infection among children who have acquired the disease through innocent contact with some perşon suffering from syphilis. The writer has met with seven extra-genital cases of syphilis in the young which were innocently acquired. The oldest of these was fifteen years of age. In each instance the true nature of the disease had not been suspected until the lapse of considerable time.

In one of these children secondary syphilis appeared in the larynx and made dangerous ravages before the true nature of the malady was ascertained. In this case the disease was contracted from a syphilitic sore on the lower lip of a servant in the employ of the child's family. So many instances of a similar character have come to light of late years that it behooves the practitioner to be constantly on guard when dealing with ulcerative diseases of the throat, mouth, and nose. Indeed, a persistent chronic sore throat which is not tubercular should give rise to a suspicion of syphilis. Dr. Griffin, of New York, reported in the Medical Record, October I, 1892, I,200 cases 
of syphilis of the throat which had come under his observation, 12 of which were cases of initial lesion; 2 of them were situated on the tonsil; 9 were located on either the upper or lower lip, and I on the lower portion of the tongue. Since then he has collected the histories of 20 cases where the location of the primary chancre is unusual. These cases came under observation in both private and clinic practice. Bosworth, out of 753 cases of chancre, found a primary lesion upon the tonsil in two cases. Schadek collected 68 cases; 22 of these histories included a rather doubtful diagnosis; of the 46 remaining, in 34 the lesion was located upon the tonsil, and in 12 in other parts of the fauces.

About twenty other writers have reported cases of initial lesion occurring in the region of the mouth (Griffin). Dr. Griffin reported the cases of three children. The first child, aged nine years, was inoculated on the lip by drinking out of a tin pail that had some syphilitic virus on its edge. His brother had a chancre of the tonsil by sucking a stick of candy which his diseased brother had bitten into; thus, in this case, the inoculation took place directly from the virus of the chancre. A sister of these boys also became inoculated on the lip by kissing her brothers, thus showing another instance of direct inoculation from the chancre. Very often these cases are not recognised until the occurrence of bubo in the neck. The bubo is present in almost every case, although instances are met with of primary inoculation of the mouth without the bubo manifesting itself. Such cases are exceptional, however. The bubo is usually seated on the same side as the chancre. The submaxillary glands are the ones generally involved, although the subhyoid, post-auricular, and post-cervical may be found swollen. Induration appears usually in the first or second week of the chancre, and its duration is variable.

The induration appears earlier when the chancre is situated in the region of the mouth. As the chancre heals, the bubo subdivides and appears like soft glands under the touch (MacKenzie, Griffin). In strumous subjects the glands may become larger than in previously healthy persons. The bubo varies; it may be large or small, and appears on each side of the neck. Indeed, the glands on both sides often participate in the infection. These buboes seldom break down into abscesses if treatment has been commenced early. Keyes speaks of bubo as an 
almost constant symptom of syphilitic chancre, yet, as stated before, there are exceptions where no bubo ever develops. Griffin says the eruption makes its appearance much earlier when the lesion is situated in the vicinity of the buccal cavity. He has seen eruptions develop in six days after the primary chancre has made its appearance in the mouth. The severity of the syphilide here is about the same as if the inoculation had taken place in the usual part of the body, and depends for its development upon the peculiarity of the constitution attacked. Griffin reports a bad case of syphilide in which the spot covered the entire back and front of the chest. It occurred in the fourth week from the date of the primary infection, a chancre on the lip received from a kiss from the patient's husband, who had mucous patches on the tongue and was in the third year of his syphilitic disease. When the sore is located on the tonsil it is liable to be mistaken for a secondary syphilitic ulceration.

The doubt may be cleared up if there be a prompt appearance of a bubo in the neck-a symptom which is generally absent in the secondary lesions, or if there be a cutaneous eruption, this would stamp the case as probably secondary.

Secondary syphilis (which occurs usually from five to eight weeks after the primary infection) is very likely to manifest itself in the throat. It sometimes, however, affects the nose (especially the nasal septum) in conjunction with the soft palate or pharynx, but rarely are the nasal passages alone the seat of secondary lesions. Secondary syphilis manifests itself in the nose as $(a)$ erythema, (b) condylomata, $(c)$ secondary ulceration (J. N. MacKenzie, Pierce).

Erythema manifests itself in the earliest stage as a catarrh (coryza syphilitica simplex). It is often overlooked when it attacks adults. In infancy it is of the greatest importance (Cohen, Pierce). In the greatest number of cases the objective and subjective signs at first differ only slightly from those observed in simple acute catarrh. In syphilitic catarrh the onset is less violent and more gradual, while the secretion, especially in the first stage, is not so profuse; but when the lesion is established it lasts longer than in the simple acute catarrh, and may be ichorous. Disseminated patches of welldefined erythema may be seen, especially on the septum, and on these may be developed the patches which constitute the 
first pathognomonic sign of syphilis. If syphilitic erythema of the pharynx be present also, it aids in the diagnosis. Nasal manifestations are among the surest secondary signs of hereditary syphilis-that is, at the time of or shortly after birth. Later on they become less characteristic.

Condylomata appear in the nose much less frequently than in the mouth, isthmus faucium, lips, cheeks, tongue, and tonsils. According to most observers, it is only in exceptional cases that they are met with in the nose. Michaelson and Scheck have seen them several times in the vestibulum nasi, but nowhere else in the nose. Seifert gives the same experience. Pierce says that he has seen six cases of superficial erosions in the vestibule which were doubtless condylomatous in origin, and in one case there were circumscribed plaques along the inferior turbinated body. Devasei found them within the nose only eight times in one hundred and eighty-six cases, and Bosserau twice in the vestibule in one hundred and ten cases. The condylomata as seen on the pituitary membrane vary somewhat from those seen on other mucous surfaces owing to maceration; are only slightly elevated, and resemble circumscribed thickenings of epithelium. They have a strong tendency to ulcerate and the secretion is active. When they are acted upon by chemical irritants we may have more extensive destruction of the bony parts (Pierce). Such cases are a mixed form of coryza syphilitica and coryza professionalis, according to Toplitz and Maure; but usually when the condylomata ulcerate the ulcer is superficial, presenting a loss of the layers of epithelium only, leaving the base covered by exuberant granulations. In only rare cases do these ulcerations penetrate to greater depth and show a grayish slough. They are usually surrounded by a zone of deeply injected tissue. Syphilis in its latent or granulomatous form, when invading the nose, assumes extraordinary importance, and usually involves the bone, producing insidiously extensive ravages. The time of onset of late syphilis of the nose, according to Michaelson's statistics, varies from one to three years after infection. According to Pierce, in five cases in which the data upon this point was ascertained, the time elapsing varied from one to four, to thirteen, to seventeen and eighteen years after infection. The local lesion, however, may begin with abrupt symptoms, or the commencement and course may be slow and 
insidious. The septum is the part most frequently attacked in nasal secondary syphilis, but all perforations of the septum are not syphilitic by any means. The ulcer syphilitica septinarium perforativa is to be diagnosticated from the common, simple, so-called idiopathic perforation, and from that caused by periostitis or perichondritis following trauma, tuberculosis, scarlet fever, diphtheria, and the application of chemicals. According to Seifert and Schrötter, a syphilitic perforation confined strictly to the cartilaginous septum is extremely rare, although its occurrence is possible, but when the syphilitic process attacks the bone the cartilaginous septum is also involved. Dr. Charles H. Knight reports a case of syphilitic disease of the right inferior turbinated body in a girl fifteen years of age, which on account of its size and rapid growth was supposed to be a round-celled sarcoma, and upon this diagnosis it was contemplated to remove the growth by excision of the superior maxillary. The appearance of a peculiar swelling on the leg led to the supposition of syphilis. The girl was given vigorous constitutional treatment upon this hypothesis, and the swelling of the leg, as well as the tumour in the nose, disappeared.

Fauces and Larynx.-Mucous patches or condylornata upon which the secondary manifestations depend consist of small, flattened, opaque patches with a peculiar moist, silver-gray or glistening surface (Woodhead). To the touch they seem to be slightly indurated, and if of long standing they appear to be quite thickened. The formation of the patch is soon followed by ulceration, beginning at the centre, but in the tonsil, according to Cornil and Ranvier, this course is comparatively rare. The microscopic appearance of the mucous patches here is similar to those in other regions.

The tonsils are not infrequently the site of secondary manifestations. Indeed, next to the soft palate and the pharynx the tonsil is most frequently affected. Next in order of frequency comes the larynx, especially the laryngeal face of the epiglottis, the epiglottic folds, or some other anterior part of the larynx. It might be stated, however, that nowadays (although the disease is as prevalent as ever before) the cases come under observation at a much earlier period, because people are more apprehensive and have a greater knowledge of the effects of the disease; therefore very extensive ulcerations charac- 
teristic of neglected cases are met with less frequently than formerly.

Usually the first local manifestation of secondary syphilis in the throat is an erythema affecting each side quite sym-

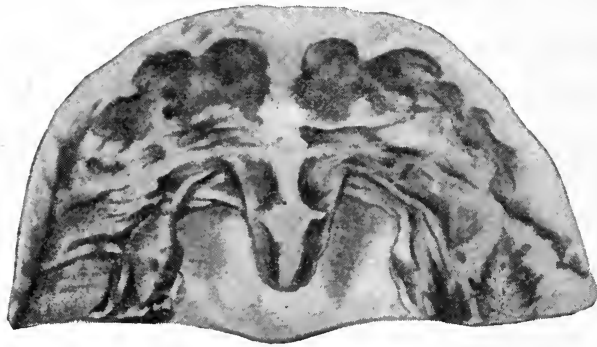

FIG. 52.-Symmetrically crescent-shaped condylomata of soft palate (Lennox Browne).

metrically, and involving in a few days the fauces and soft palate. Condy. lomata, also symmetrically arranged, soon follow, and appear as small, crescent-shaped, slightly raised white patches, that have been very aptly likened to hoar-frost (Fig. 52). They appear as festoons bordered by crenated red lines, which stretch from the raphe of the soft palate to the pillars of the fauces. This appearance is not so frequently observed when the fauces alone are the seat of the trouble as when the soft palate is first affected; for the mucous patches, while yet symmetrical, are much less regular in their outline, and appear more as islands of false membrane, which rise from a deep-red surface, when confined, for the most part, to the pharyngeal walls. The mucous membrane, however, is seen sometimes to be mottled, according to the amount of inflammatory thickening about the several patches. In a few days, if no treatment has been adopted, these condylomata break down into superficial ulcers, at first distinct, but soon running into one another, and forming here and there open abrasions covered with delicate granulations and grayish pasty sloughs, intermingled and surrounded by elevated edges, which in turn are surrounded by a well-defined red areola (see Fig. 53).

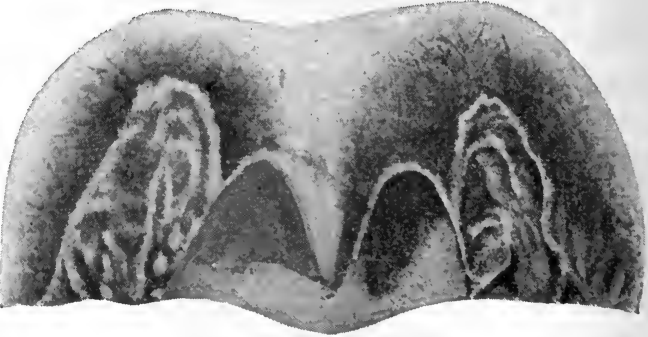

FIG. 53.-Deep secondary syphilitic ulceration of the soft palate and fauces (Lennox Browne).

The ulcers may appear later as if punched out of the tissues, or they may occur as long oval-shaped sulci filled with 
a purulent, whitish, pultaceous matter, the removal of which will show a dirty yellow or grayish floor undermining and excavating the edges (see Fig. 54). Considerable suppuration may accompany the ulceration, especially if a tonsil be the part involved in the disease. In some cases the tonsils are the principal seat of the disease. Dr. Bryan and others have reported cases of severe syphilitic amygdalitis without much involvement of the pharynx. The writer met with a case of secondary syphilitic tonsilitis affecting both palatal tonsils, in which the pain and difficulty of deglutition were intense. The local disease was very persistent, and showed no ulceration of either the pharynx or larynx. This case was also accompanied by ulceration of the buccal membrane and gums, with great salivation.

The pharynx usually escapes any marked ulceration, excepting what occurs from contiguity of tissue with the tonsils and pillars of the fauces. Dr. A. Barogli, of Cincinnati, has reported four cases of ulcerative syphilis of the pharynx which he has met with. He finds little mention in the literature of such lesions. His cases were in men, all of whom drank alcoholic

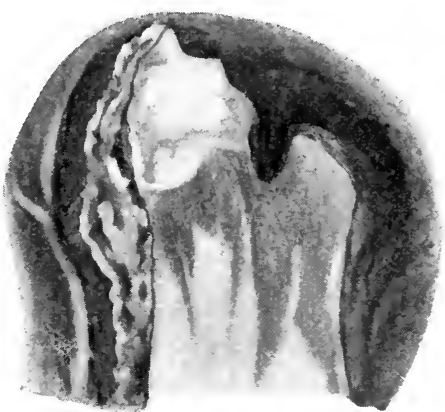

FIG. 54.-Deep syphilitic ulceration of soft palate and fauces (Lennox Browne). liquors and chewed tobacco. J. N. MacKenzie speaks of secondary changes in the pharynx as a result of lesions in the surrounding tissues. In hospital practice, secondary pharyngeal syphilides are frequently met with, but (as stated by MacKenzie) usually in conjunction with other lesions.

Tertiary.-The tertiary form is much more severe and destructive in its local manifestations. The gumma may be single or multiple. These growths are surrounded by a fibrous capsule, from which run through the growth a gelatinous-looking substance of grayish or pinkish-yellow colour occupying the interior; these processes often extend along the course of the vessels. Tertiary lesions may appear within a few months, or not for a year or more after the primary infection, or the poison may remain latent, the local disturbance not developing for years afterward. It is not un- 
common to meet with extensive tertiary ulceration of the pharynx, with perhaps necrosis of the palate bone or vomer, in cases where the local disease has arisen ten to fifteen years

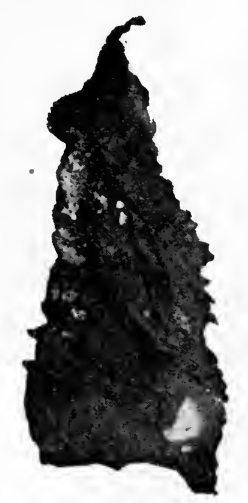

FIG. 55.-Sequestrum of bone from case of nasal syphilis (natural size). Author's case. after the time of the primary infection. Gummata therefore may take a long time to form and break down. The amount of inflammation of the perichondrium and periosteum in the nose caused by a gumma may be quite extensive, and the corresponding necrosis may be considerable (see Fig. 55). The turbinals may also participate, as well as the exterior tissues of the nose, so that the whole organ will be swollen inside and outside and very tender to the touch. The turbinals do not, as a rule, participate unless the case has been a neglected one. There is a great deal of lachrymation, pain, and other distress from the almost total stenosis of the nasal passages. There is constant discharge of an ichorous, gummy matter, with formation of crusts. This discharge excoriates more or less the skin of the alæ and the upper lip, and sometimes emits a bad odour.

There is also present more or less cephalalgia-a boring pain over the nasal or frontal regions; and when the disease is at all extensive there is a considerable amount of constitutional disturbance, such as chilliness, aching of the back or limbs, and pyrexia. The inevitable result of this local inflammation, if unchecked, is more or less loss of the septum, both cartilaginous and bony, and in many cases a further inflammation and suppuration of the ethmoid cells, with sometimes empyema of the antrum and more or less necrosis of the bony parts in the neighbourhood. Even the superior maxillary, together with the nasal bones, may become necrosed, thus allowing the nose to drop, producing "the saddle nose," and otherwise deforming the passage. These cases are pitiable, for an inspection after the ravages of the disease may show that not a vestige remains of either the septum or the turbinated bodies. The writer (as well as other observers) has seen a number of cases which have lost all of the turbinated bodies, the vomer, nasal, and palate bones.

The tertiary manifestations in the throat are quite charac- 
teristic. The gummatous deposit here is usually unilateral. The seat of predilection is the junction of the soft and hard palate near the median line (Fig. 56), although sometimes the tonsils and posterior pillars or lateral walls of the pharynx bear all the local manifestations (see Figs. 57-60). The first indications of the affection are usually a very marked thickening of the soft palate, relaxation of the uvula with tumefaction, pharyngeal erythema, local in extent, and more or less disturbance of deglutition.

There is also an excessive discharge of mucus from the nasopharynx, causing much hawking and spitting. After a while-it may be days or weeks -if the swelling continues, a softened spot will appear toward the centre of the erythematous patch on the soft palate, near the junction of the hard palate, or over the hard palate alone, which will soon be followed by a small perforation, and as the disease continues the perforation enlarges. Change in the voice, nasal intonation, and difficulty in swallowing liquids soon appear as symptoms of this condition. Subsequently, if the disease be unchecked, ulceration and sloughing of the soft palate take place; when the ulceration is bilateral the uvula may be sloughed off, or show a fissure on each side, with the uvula dangling between. This ulceration is more or less rapid according to the constitutional condition of the individual. The ulceration may be deep, serpiginous, and vicious, quickly extending to the deeper parts, thus differing in this respect from the laceration which accompanies the secondary form of the disease. These cases of severe ulceration are not met with often. When the larynx is involved the same characteristic destructive ulceration occurs (see Fig. 6I). A perichondritis, more or less extensive, is soon set up on one

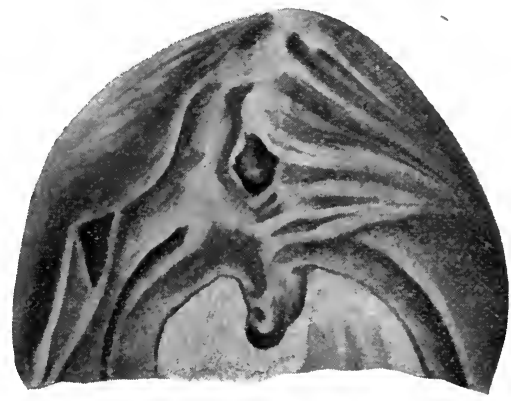

FIG. 56.-Tertiary syphilitic ulceration and perforation at junction of soft and hard palate (Lennox Browne). side or the other, which may result in the necrosis and exfoliation of a portion of the involved laryngeal cartilages. The process of healing, after the ulceration and sloughing of the parts have ceased, is usually rapid, but the extensive and 


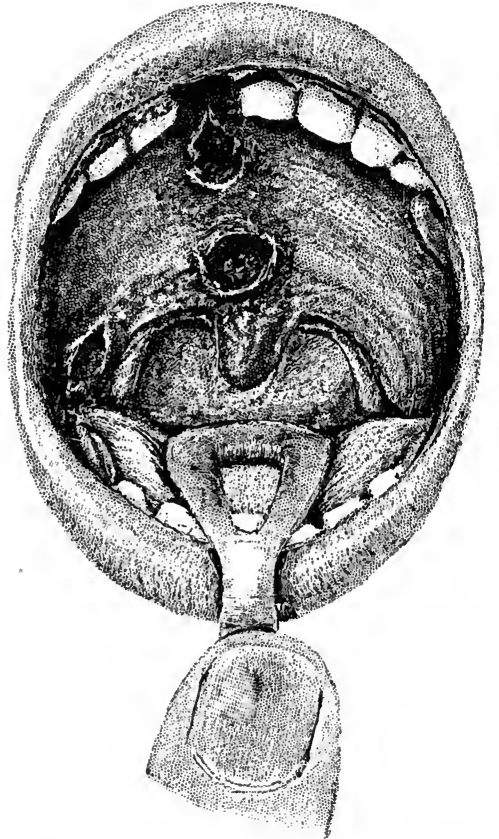

FIG. 57.-Double perforation (syphilitic) of palate (after De Blois).

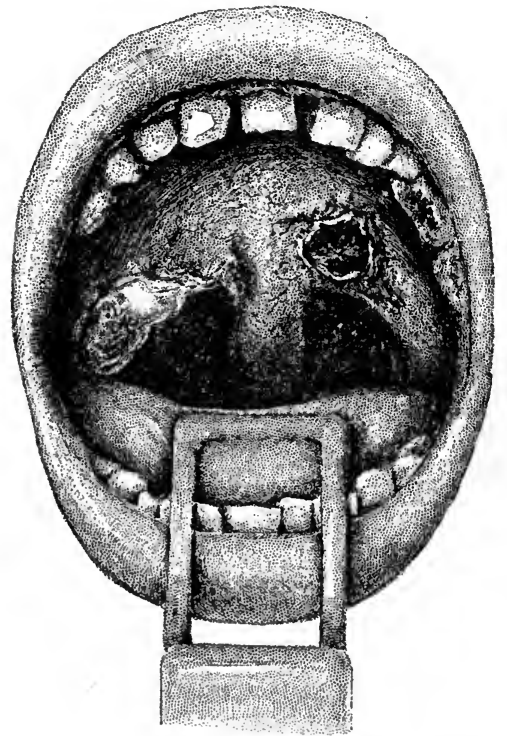

FIG. 59.-Syphilitic ulceration of right pillar and perforation of palate (after De Blois).

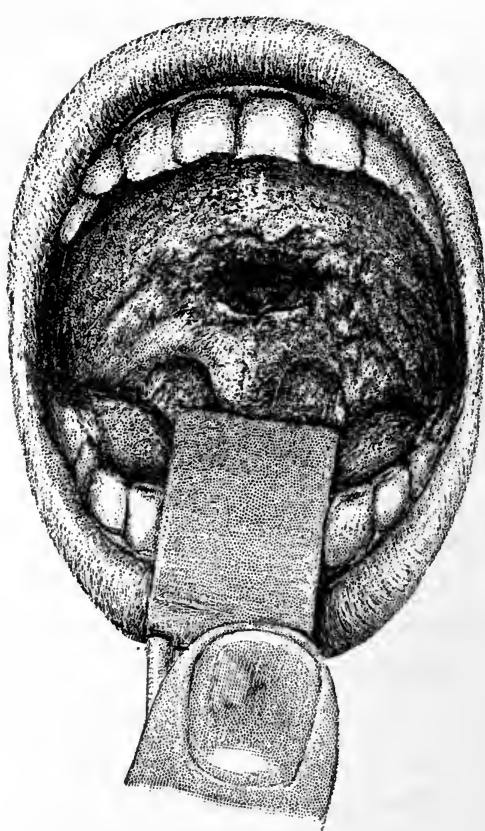

FIG. 58.-Syphilitic perforation of palate (after De Blois).

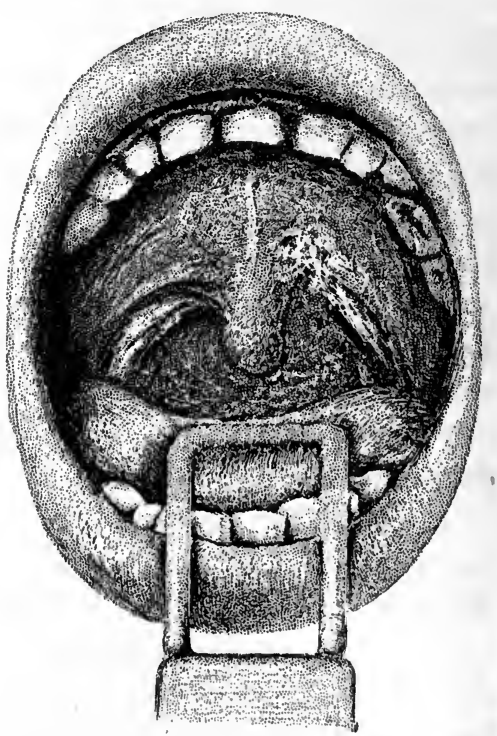

FIG. 60.-Healed cicatrix of case illustrated in Fig. 59 (after De Blois). 
rigid cicatrices (see Fig. 60) which result from their contraction may not only produce great deformity of the parts, but may prove troublesome or even dangerous by closing the whole nasopharynx through the attachment of the soft palate to the posterior wall of the pharynx; also by causing stenosis of the larynx from the formation of cicatricial bands, which contract or obstruct the lumen of the tube or exercise compression upon one part or another. Many authorities believe that this characteristic of the cicatricial tissue of tertiary syphilis is peculiar to this disorder, because, notwithstanding the apparent local recovery of the part, the syphilitic toxine being still present, influences the morphology of the reparative tissue. This theory, of course, must be considered untenable, inasmuch as we observe the same effects from extensive destruction of tissue in these or other regions by burns or escharotics. The probable explanation lies in the fact that the fibroid element preponderates in the reparative tissue whenever there has been a wholesale destruction of the natural tissues.

Symptoms.-The subjective symptoms belonging to these several local manifestations amount to very little, and are simply the result of interference with the functions of the parts, as, for

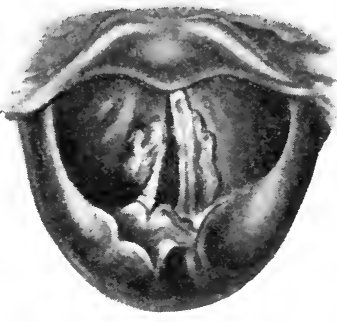

FIG. 61.-Syphilitic ulceration of the larynx with perichondritis (Lennox Browne). instance, from the swelling and inflammation in the nasal passages, soft palate, or pharynx. Aside from the ordinary constitutional symptoms belonging to the disease, we rarely meet with much pyrexia or general weakness excepting in the severe nasal form, or in the advanced tertiary ulceration of the pharynx and larynx, or when the disease is combined with tuberculosis. In these cases there is more or less emaciation in consequences of the inability to take food, and in severe types sleep may be interfered with. In neglected cases, where the nutrition has been impaired very much, patients manifest more or less adynamia. When the patient is suffering from septic poisoning through the secondary development of adenitis or abscess of neighbouring glands, the general depression may become alarming. Extensive suppuration of these glands will sometimes lead to a dangerous condition or a fatal issue. 
When the larynx is extensively involved, as in perichondritis and necrosis, danger to life ensues from impending suffocation as well as from septicæmia. These complications, however, are not commonly met with. Many reflex symptoms may arise from syphilis of either the nose or throat, such as convulsions, vertigo, and rheumatic and cardiac symptoms. Neuralgia, mental disease, and paralysis of various kinds frequently originate from tertiary syphilis. The stomach may get out of order in this disease either from sympathetic irritation, or from the effects of the swallowed products of throat ulceration, or from the ingestion of medicine in too large quantities or badly compounded. No reason can be given for the selection of the throat for the secondary and tertiary manifestation of syphilis, unless it be found in the rich glandular apparatus apportioned to this region, and some special emunctory powers belonging to the same. Regarding the nose as the seat of the local process, nought but mystery surrounds the question, unless we imagine that some hidden antecedent lesion or the feeble resistance of the nasal septum serves as an explanation.

Diagnosis. - The diagnosis is usually not difficult if we have in connection with typical objective symptoms a clear clinical history, but unfortunately in many instances the latter desideratum is lacking, especially in cases of syphilis acquired innocently through the development of a chancre about the lips, mouth, tonsil, or in those cases in which obscure tertiary manifestations come on perhaps years after the primary infection.

NASAL.-Nasal syphilis is usually primary or tertiary. The appearances in the primary form resemble mostly fibrinous or diphtheritic rhinitis, severe localized influenza, abscess of the septum, and injury to the septum. The clinical history of the case will sometimes remove all doubt, but not always. In all these diseases, excepting influenza, one side only of the nasal passages may seem to be affected, for syphilis, as well as fibrinous rhinitis, is generally unilateral. Age is not a determining factor, since children may become inoculated in this region with syphilis as well as with diphtheria. Bacteriological examination may reveal the Klebs-Loeffler bacillus and thus settle the diagnosis, but often in cases of fibrinous rhinitis the specific germ cannot be demonstrated. Constitutional disturbance may be absent in either case. Nasal stenosis, lachrymation, pain, and frontal distress are symptoms of each; also enlarged lymphatic 
glands may accompany the three former. The points upon which differentiation must rest are: (a) History and environment : especially whether or not the patient has been exposed to either syphilis, diphtheria, influenza, or has received an injury. (b) Mode of accession: whether the first manifestation was a papule, and, if so, its character; with or without rhinitis. (c) Course: while the course of either syphilis or fibrinous rhinitis may be slow, in syphilis the inflammation and tumefaction of the mucous membrane take place much slower than in either fibrinous or diphtheritic rhinitis, or from the effects of traumatism. Besides, the syphilitic disease can often be traced from a limited site which will show ulceration, and, later, some destruction of cartilage. The character of the nasal discharge in primary syphilis is more apt to be sero-mucous, ichorous, or gummy ; in fibrinous rhinitis it is membranous, existing in layers and containing pus; and in the traumatic it is muco-purulent or sero-purulent.

In tertiary syphilis of the nose the tumefaction is usually not so great, and the discharge, although abundant, remains and desiccates into large masses within the passages. Besides, the subjects of the disease are usually adults. In abscess there is no fibrinous exudation, the swelling is circumscribed, and there are usually present throbbing sensations together with the external soreness. Rhinitis from influenza is accompanied by great nervous disturbance, abundant serous discharge, tumefaction of both sides, and is in every feature more or less acute.

In many cases the differentiation from tuberculosis is beset with great difficulties, especially if we have to depend upon local appearances alone. The shape of the syphilitic ulceration of the septum is in the large majority of cases longitudinal, while the tubercular is usually round or irregular in form; but we may have a circumscribed gumma in the nose which results in a round ulcer, and we-may have tubercular ulcers which are longitudinal (Massei, Bosworth, Pierce). These are, however, rare, and occur with greatest frequency on the turbinated bodies. The infiltration in secondary and tertiary syphilis spreads beneath the "mucous membrane to within and between the perichondrium and cartilage, causing necrosis of large areas of bone or cartilage in a very short time, which will become manifest from the peculiar stench emitted after probing. This never occurs in tuberculosis. The tubercular perfora- 
tions of the septum take place gradually. The tubercular granulations slowly replace the osseous tissue. Then, too, the tubercular manifestations are in a large number of cases preceded by the same disease on the face and in the lungs. The age of the patient is of some value, for nasal tuberculosis occurs most frequently in the young. Finally, histological examination of the tissue may decide through presence of the tubercle bacillus, although this sign is not absolutely a certain one.

The local appearances of leprosy and anthrax and rhinoscleroma may resemble those of syphilis, but the general appearances of the several diseases are sufficiently distinct. It may be difficult without microscopic examination to differentiate between the primary and some of the late syphilitic manifestations in the nose, especially about the alæ, and malignant disease, but the clinical history will serve to determine it. We may meet with mixed infection of tuberculosis or leprosy with syphilis, or of lupus and carcinoma developing on a syphilitic abscess or scar. All this makes the diagnosis extremely difficult. The postnasal space should always be inspected, though this area is not often the seat of late syphilitic manifestations. In all cases whenever there is bone necrosis or a sequestrum we should institute specific treatment. In some cases difficulty may be experienced in differentiating between necrosing ethmoiditis and syphilis of the middle turbinated body when the latter disease is confined to that region, as in a case reported by Ingals and Moldenhauer respectively:

The case of a child has been reported in which a string in its nose gave rise to symptoms of nasal syphilis, a brown sanious discharge, etc. (Medical News, February 26, I 899).

The slow course, the absence of slough, the relative smallness or absence of the sequestrum in necrosing ethmoiditis, and the clinical history, are distinctive features. Care must be taken not to mistake the syphilitic infiltration for a simple hypertrophy or even a simple deviation, and also to distinguish between a simple and specific coryza in the early stages, especially in infants. In the case of atrophic ulceration within the nose occurring in the course of bulbar tabes, we must rely upon the general nervous symptoms in reaching a correct diagnosis. Nasal diphtheria or fibrinous rhinitis most often presents a train of signs not unlike syphilis. An instructive case 
is reported in the Medical News, February 26, I898. The author has seen two cases in young adults of localized membranous rhinitis which appeared like syphilis. Syphilitic ulceration of the mouth and tongue frequently persists after all signs of other lesions disappear.

Pharynx and Fauces.-During the first or so-called erythematous stage of secondary syphilis the diagnosis may be puzzling, unless the characteristic markings of syphilis are apparent and the clinical history be complete, but there are no ordinary inflammatory or microbic affections of the fauces which produce the symmetrical crenated erythema so characteristic of secondary syphilis.

When the typical clinical history is wanting or the local appearance of the fauces is anomalous, then secondary or tertiary syphilis may be mistaken for chronic pharyngitis, acute or chronic follicular tonsilitis, mycosis faucium, septic ulcerative pharyngitis, tuberculosis, lupus, epithelioma, or sarcoma. The chronic pharyngitis of excessive drinkers or smokers, or the effects of the application of nitrate of silver or other caustic application to the throat, may simulate syphilis by reason of the persistent erythematous inflammation caused in the one case, and the likeness of the cauterized patch to a condyloma in the other. An erroneous diagnosis, therefore, can easily be made if the data attending the course of the malady are not carefully considered. The dictum that all cases of persistent erythematous or ulcerative pharyngitis are probably syphilitic should be kept in mind, although it must be remembered that there are many exceptions to such a rule. Syphilitic disease will show in a short time its peculiarities unless checked by efficient treatment. When caustics have been used upon the mucous membrane, more or less of a slough will soon appear, exfoliate, and leave healthy granulations beneath instead of an ulcer. If the case be one of severe subacute or chronic pharyngitis only, no ulceration need be expected, whatever may have been the duration of the attack. The location of the inflammation, however, on the soft palate, especially near its base or upon the hard palate, will always constitute strong presumptive evidence of the syphilitic nature of the disease. The appearance of syphilis of the tonsil in many respects resembles chronic follicular tonsilitis and mycosis of the tonsil. Cleansing of the parts and further examination by the probe will 
reveal in syphilis a general tumefaction of the tissues, accompanied by ulceration either in the mouths of the crypts or just around them, while in the case of chronic follicular tonsilitis and mycosis there is no ulceration and no general tumefaction. In mycosis the white exudate is more or less fixed to the surface of the mucous membrane, and is not accompanied by any surrounding inflammation. Septic ulcerative pharyngitis presents small, irregularly shaped ulcerations, more or less scattered, which come and go, as it were, disappearing from a place in a day, while fresh ones appear in other places. These cases are frequently associated with an ulcerative stomatitis. In tuberculosis the diseased points are scattered, appearing as grayish or mottled deposits just beneath the surface of the mucous membrane, which is usually pale. They slowly break down, leaving small, superficial, circular, or irregularly shaped ulcerations. Besides this, there is more or less pyrexia, anorexia, etc.

Lupus.-The main distinguishing features of lupus are its slow growth-slowness of the changes from the formation to decadence of fungoid granulations at each step of the process ; also its limitation for a long time to a small district. Besides this, when ulceration is taking place, the ulcers are irregularly linear or serpiginous in form, and the cicatrices are striated, and of a peculiarly white or bluish-white colour.

Epithelioma and Sarcoma.-Advanced tertiary syphilis, characterized by tumour formation, may sometimes simulate either epithelioma or sarcoma, but a history of the local growth, together with the distinct neoplastic character of the local affection, will generally serve to determine the diagnosis. Whether the tumour be an epithelioma or sarcoma, it will be ascertained that the beginning was probably marked by one or several painful papules, which rapidly increased in number until aggregating to become a veritable tumour before ulceration began, thus differing from the evolution of a gumma, which usually starts below the surface of the tissues, remaining quiescent for a time, and then progressively breaking down from centre to periphery.

There are, however, many complex cases in which one or another of these simulating affections occur in syphilitic subjects. In such cases the true condition can only be arrived at either by a careful process of reasoning by exclusion, or by the crucial test of a course of constitutional antisy philitic treatment. 
Larynx.-An early invasion of the larynx in secondary syphilis is not common, for usually the parts above suffer first. This may be accounted for by the disparity existing between the number, character, and function of the glands of the larynx as compared with the fauces. When the larynx becomes the seat of condylomata, primarily, which is rare, or simultaneously with the faucial region, it may be attributed to either the intensity of the infection or to a previously morbid condition of the larynx. The condylomata or mucous patches in this region are usually small. The ulcers maintain a crescentic or oval shape, are not deep, are surrounded by the characteristic red areola, and bounded by the same sort of elevated border common to the ulcerations of this disease. In neglected cases the ulcers may coalesce, causing great destruction of tissue and resulting in extreme cases in perichondritis, erosion, or necrosis of the laryngeal cartilage. (See Fig. 6r.) The pain when the ulcers are small and scattered is not usually great, unless there be extensive infiltration. The site of the ulceration is commonly the epiglottis, its lingual face, ary-epiglottic folds, ventricular bands, and vocal cords. The early and sometimes the late development of secondary syphilis in the larynx may resemble tuberculosis or a benign ulceration of the mucous membrane following an intense acute laryngeal catarrh or a membranous laryngitis. Diphtheritic croup of mild character may be mistaken for syphilis. But the clinical history of the case will serve to clear up any doubt. Occasionally, however, the lapse of time and the effects of treatment may have to be relied upon to distinguish a persistent erythematous stage of secondary syphilis from a chronic or subacute laryngitis. The tertiary lesions of the larynx may resemble tuberculosis, carcinoma, and lupus, and their differentiation is not always easy, especially by local signs alone.

Tertiary.-In the tertiary form of syphilis the diagnosis is sometimes a little difficult to make out when the disease is advanced, because of its resemblance in part to tuberculosis, lupus, or carcinomatous affections. When the characteristic changes have taken place in the gummata the diagnosis is very readily made out, but when the tonsil or lateral wall of the pharynx bears the burden of the local manifestation it is sometimes a little difficult to differentiate it from the affections already mentioned. Granular tuberculosis is rather rare and is of slow 
growth in the fauces; likewise, lupus grows by slow stages, and manifests itself by large granulations that have not been preceded by any ulceration. The lymphatic glands of the neck and neighbourhood are much more likely to be involved in syphilitic disease. In the various malignant diseases the extreme pain and slow accession by new growth may serve to differentiate the two affections. In advanced stages of these diseases, when the heterologous tissue is breaking down, it is then often difficult to diagnose syphilis from carcinoma or sarcoma when the clinical history is defective. The tendency to hemorrhage, the presence of a lobulated growth, the pain attending movements of the muscles of the throat and neck, and the slowness of the breaking down of the tissues, will often serve to exclude tertiary syphilis from the problem. In syphilis of the larynx, the small isolated ulceration upon a red patch, the sharp-cut edges of the ulcer, the whitish or granular bottom of the excavation, will serve to distinguish it from tubercle, which manifests itself in the shape of mottled papules (some soft and others not), mingled with scattered superficial ulcers covered with sticky mucus and perhaps having no red areola unless by accident, and all lying upon either a uniformly hyperæmic or upon a swollen anæmic grayish-pink mucous membrane. The swelling of the parts in tuberculosis is also quite significant as compared with syphilis. In the former the arytenoids and posterior parts of the larynx are involved, while in the latter form of disease the anterior parts are usually the ones affected. In the tertiary form the character of the syphilitic ulceration is quite irregular-may be deep and serpiginous. This may also be the case in advanced tuberculous laryngitis, but in this disease the general history of the case and the pulmonary involvement will settle the diagnosis. Syphilitic ulceration of the mouth and tongue occasionally persists after all signs of lesions elsewhere disappear. Undoubtedly in such cases buccal ulceration is kept up by the action of the non-specific micro-organisms which abound in the mouth. The tongue is apt to participate actively in both primary and secondary syphilis when the tonsils are the seat of the disease. The diagnosis between sprue and chronic local syphilis is sometimes difficult.

Dr. Joseph S. Gibb gives a very interesting table of the signs for differential diagnosis in ulceration of the pharynx and 
larynx in syphilis, carcinoma, tuberculosis, and lupus, which is copied on pages 262 and 263.

Pathology.-Whatever the character of the lesion, be it infiltration or ulceration, it is always due to one factor, the granuloma (John N. MacKenzie, Pierce). The infiltration may be circumscribed or diffuse, the borders of which are more or less sharply defined from the surrounding tissue, but the tendency of the infiltration is to become general, especially in secondary syphilis. In such cases the new cell elements are absorbed and the mucous membrane deformed by a true cirrhotic process. It produces the appearance in the nose of rhinitis or pharyngitis atrophica, as described by J. N. MacKenzie, or of shrinking of the wings of the nose without previous ulceration, as described by Lange, but the usual result is uiceration. This takes place from the surface inward, or vice versa (Pierce). The ulcerative process is progressive and invades the healthy tissue, and the spreading of the ulceration is always preceded by infiltration. There are great variations in the appearance of the syphilitic ulcerations within the nose. This is due both to the degree of virulence of the process itself and to the structure of the particular part of the nose in which it occurs. The ulcer is usually covered with grayish-white, green, or dark brown coating. The base is found to be more or less granulated. The granulations are pale, glassy, and possessed of a low degree of vitality. The borders of the ulcer are usually elevated and more or less sharply cut. The shape of the ulcer varies. The longitudinal form of a syphilitic ulcer of the septum has come to be regarded as characteristic, but in a number of cases the ulcer and perforations have been quite round (Pierce). The collateral œdema accompanying late syphilitic disease of the nose varies in different cases and in different stages. The osteo-periostitis syphilitica simplex or neoplastica can attack any of the bones which go to form the nasal skeleton (Massei). The infiltration may result in exfoliation, rarefying osteitis, or plastic osteitis. According to the observations of Scheck, Sanger and Schuster, Bosworth, and others, the entire septum may be absorbed with the discharge of a sequestrum. Lennox Brown has seen a case of this kind in which a large part of the bony septum was transformed into an apparently membranous partition with a pin-head perforation, but at no time was the 


\section{Ulceration of the Pharynx}

\begin{tabular}{c|c|c|c}
\hline Syphilis. & Carcinoma. & Tuberculosis. & Lupus. \\
\cline { 1 - 3 } Pain usually slight. & Pain lancinating, & Pain severe; not & Pain slight, if any.
\end{tabular}
severe, constant, constant ; aggravatoften excruciating. ed by efforts at degIn many cases re- lutition or clearing ferred to ear.

Ulcer clear cut. Destruction of tissue great. Appears very early in the course of the disease-within two or three weeks.

Profuse purulent discharge and necrotic tissue cover surface of ulcer.

Borders of ulcer indurated and hyperæmic.

Ulcer rapidly destructive, and extends deeply.

Ulcer confines itself to pharynx, rarely extends to nasopharynx, and never to larynx.

Cicatrices often present.

General condition unimpaired.

Often evidences of specific disease in other organs.

No fever.

Rapidly improves under iodides.

Sputum contains no distinctive morbid product.

Microscopic examination of excised tissue reveals large number of small round cells.
No clear-cut ulcer. Normal tissue replaced by morbid growth. Ulceration does not occur for 2 or 3 mos. after appearance of growth.

Very little discharge covers the growth. When ulceration occurs the surface is covered by a thin sanious discharge.

The growth is of stony hardness. An areola surrounds the growth, but no induration occurs until the parts are encroached upon by the growth itself.

Quite rapid in its course ; extends in all directions.

No anatomic boundary confines the growth ; extends in all directions and attacks all tissues.

No cicatrices.

Early in the course of the disease the general condition is good ; later, however, the health fails rapidly.

No manifestations of previous disease.

\section{No fever.}

Disease is uninfluenced by iodides.

Examination of sputum negative.

Microscopic examination of growth shows characteristic cells of the various forms of carcinoma. the throat of inspissated nucus ; sometimes referred to ear.

Ulcer shallow and not clear cut, shading imperceptibly into the normal tissue ; ulceration occurs very early.

The surface of the ulcer is covered by muco-purulent secretion and agglutinated mucus.

No areola or indu. ration.

Erodes slowly and laterally, not deeply.

Confines itself to mucous membrane of pharynx ; extends laterally.

\section{No cicatrices.}

General condition poor from the outset, indicating some grave constitutional disease.

Pulmonary and laryngeal manifestations.

\section{High fever.}

Is not influenced by iodides.

Tubercle bacilli found in sputum.

Microscopic examination shows giantcell, tubercle bacilli. and other evidence of tuberculosis.
Ulceration rarely seen, the process being rather marked by the absorption of tissue.

Little or no secretion.

Disease consists of indurated nodules.

Exceedingly slow in course.

May extend to larynx.

\section{Cicatrices numer-} ous.

General condition very slowly impaired.

Cutaneousmanifestations previous to and coincident with the pharyngeal.

No fever.

Not influenced by iodides.

Examination of sputum negative.

Microscopic examination very similar to that of tuberculosis. 
Ulceration of the Larynx

\begin{tabular}{l} 
Syphilis. \\
\hline Pain usually slight. \\
Attacks any por- \\
tion of larynx, and \\
ulcerates rapidly. \\
Is rarely seen in \\
the stage of indura- \\
tion, the first evi- \\
dence being a clear- \\
cut, deep ulcer.
\end{tabular}

Some induration around the ulcer, but usually very little œdema.

Ulcer extends deeply, often involving cartilage.

Surface of ulcer covered by mucopurulent secretion and necrotic tissue.

Mucous membrane hyperæmic and injected.

Laryngeal stenosis is not common until cicatrization occurs.

General health unimpaired.

Frequent evidences of syphilitic disease in other tissues.

Rapidly improves under the iodides.

Sputum contains no distinctive morbid product.

Microscopic examination of excised tissue reveals large number of small, round cells.

\begin{tabular}{|c|c} 
Tuberculosis. & \multicolumn{1}{c}{ Lupus. } \\
\cline { 1 - 2 } $\begin{array}{c}\text { Pain in deglutition } \\
\text { severe. }\end{array}$ & No pain. \\
$\begin{array}{l}\text { The favourite site } \\
\text { is in the interaryte- } \\
\text { noid space of the }\end{array}$ & $\begin{array}{l}\text { Attacks any por- } \\
\text { tion. Ulcerates very } \\
\text { slowly. }\end{array}$
\end{tabular}

base of arytenoid cartilages ; ulcerates slowly.

The first appearance is that of a new growth occupying the laryngeal cavity; no clear-cut uicer.

The growth fills or encroaches on the laryngeal cavity.

Growth extended in all directions, involving all tissues in its course.

Surface of growth covered by discharge.

Mucousmembrane hyperæmic.

Laryngeal stenosis quite common.

Early in the disease no impairment of general health ; later, marked cachexia. In primary laryngeal carcinoma no other involvement until later in the disease.

Iodides have no influence on the course of the disease.

Examination of sputum negative.

Microscopic examination of tissue shows characteristic cells of various forms of carcinoma.
Usually the first appearances are small spots of induration, rapidly followed by great œdema.

Great œdema of arytenoids.

Ulceration extends laterally, but not deeply.

Surface of ulcer

muco-purulent secretion and agglutinated mucus.

Mucous membrane pale.

Laryngeal stenosis rarely occurs.

Health impaired previous to laryngeal involvement.

Previous and coincident pulmonary

Nodular masses.

\section{Little or no œedema.}

Very slow in progress ; ulcer rarely observed.

charge.

Mucous membrane injected.

Slight stenosis.

Very slight imp̀airment of general health.

Frequently cutaneousmanifestations. trouble.

Iodides have no influence.

Tubercle bacilli found in sputum.

Microscopic examination of tissue ination ver shows giant-cell, tu- to that of tuberculobercle bacilli, and sis. other evidences of tuberculosis. 
sequestrum thrown off. Fournier, in his lectures on nasocranial syphilitic osteitis, has called attention to the manner in which nasal syphilis may be fatal. In such cases the ethmoid or the sphenoidal bones are rarely involved. There may be, however, extensive necrosis of the nasal and palate bones without any meningeal or cerebral disease, if there be no gummata.

Secondarily the results may be:

I. Meningitis in its various forms.

2. Thrombosis of the sinuses, especially the sinus cavernosa, which lies close to the sella turcica.

3. Thrombosis of the vena ophthalmica, which empties into the sinus.

4. The escape of pus, or extension of inflammation into the neighbouring parts, as the orbit.

5. Implication of the optic and olfactory nerves, or the third, fourth, and sixth pairs.

6. Encephalitis circumscripta or diffusa, with abscess. Lermoyez, Dupley, Lallemond, and others report cases of this sort.

Psychic symptoms may depend upon the presence of a sequestrum.

Syphilitic nasal manifestations may give rise to reflex phenomena, and may increase the gravity of a mental disorder. The various deformities which result from nasal syphilis are well known. Cases have been reported, and the author has also seen one where the bones of the nose were gone entirely, in which the antrum, nose, orbit, and mouth apparently formed one cavity. The best known deformity is the so-called "saddle nose," but this is not always due to syphilis. Michaelson has reported a case in which a traumatic phlegmon of the structures composing the bridge of the nose produced this deformity, and Seifert observed a similar case. Pierce also adds a case occurring in a woman who had osteomyelitis. Seifert declares that stenosis may result when the atrophic rhinitis occurs in the first years of life through the contraction of scar tissue.

Sequelæ of Syphilis.-The consequences of syphilis of the upper air passages may be considered as immediate and remote, and vary in significance according to the amount of destruction of the parts and impairment of the function produced. Among the immediate sequences may be mentioned 
hemorrhage, suffocation, malnutrition, and various intercurrent diseases; and among the remote effects are loss of tissue and bone, adhesions between contiguous parts, and deformities due to the contraction of cicatrices. Occlusion of the nasal passages, both anteriorly and posteriorly, may result from syphilis. M. Lenhardt reports a case in a man aged forty-five, of complete occlusion of the nares from syphilitic cicatrices. Almost complete occlusion of the naso-pharyngeal opening may occur from the same cause. Cohen, Bosworth, MacKenzie, and almost every author on laryngology, report having seen such cases. The writer has seen several; in one (a woman), a slight open-

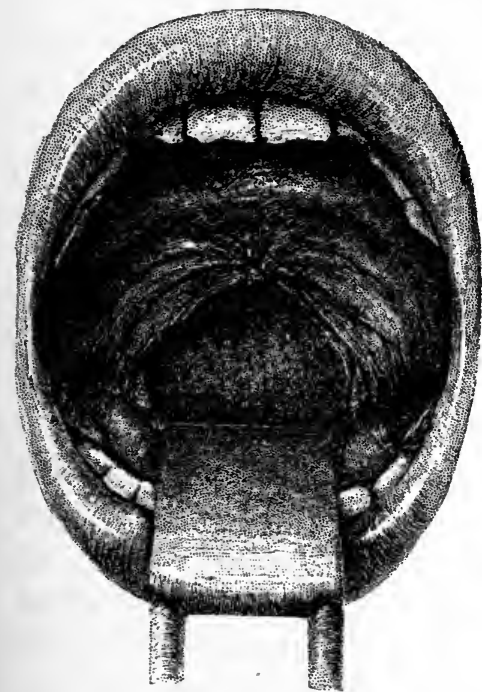

FIG. 62.-Cicatrix after loss of part of soft palate and uvula (T. A. De Blois).

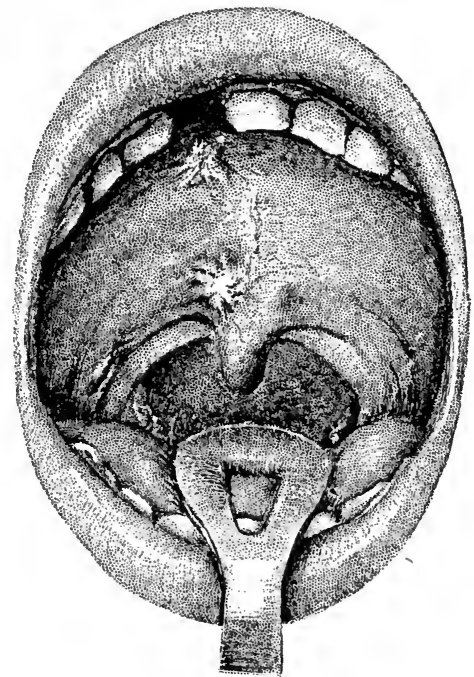

FIG. 63.-Healed cicatrix of case illustrated in Fig. 62 ('T. A. De Blois).

ing in the cicatricial adhesion between the oro- and nasopharynx was maintained by the permanent presence of a loop of silk ribbon. (See Figs. 62, 63.)

Treatment.-The treatment consists of both local and general measures. The general treatment, as is pretty widely known, consists essentially in the administration of mercurials and preparations of iodine. In the primary and secondary forms the mercurial element of treatment should predominate. There should be administered blue mass, calomel, mercury protoiodide, etc. In the later stages of the secondary form 
the mixed treatment has been found by experience to be most serviceable. This consists of the combination of a mercurial salt such as corrosive chloride of mercury, or the mercury biniodide, with iodide of potassium. (See Formulæ.) In the tertiary forms of the disease, while in many instances the combination with a mercurial is advisable, yet our sheet-anchor consists of thorough dosing with iodide of potassium or some form of iodine given with adjuvant treatment, such as bathing and the use of saline cathartics. The local treatment for any suspected primary infection should consist of mild topical applications, with a view of correcting the character of the secretions and modifying the character of the local disturbance. It is rarely that we meet with a case of this sort early enough to be able to modify the breaking down of the initial chancre. For this purpose mild sprays of a solution of the corrosive mercurial chloride, such as $\mathrm{I}-3000$ or $\mathrm{I}-4000$, are useful, as are also sprays of peroxide of hydrogen, one part to seven, or a solution of borax or boracic acid. In the severer ulcerations much benefit will be received from the application of solutions of permanganate of potassium (one, two, or three grains to the ounce), solutions of corrosive sublimate; insufflations of iodoform, aristol, or orthoform, or of biniodide of mercury mixed with sugar and powdered gum arabic, in the proportion of one grain to the ounce of the excipient, are found quite useful for topical treatment; but of all these, the insufflation of iodoform is perhaps the most efficient where there is ulceration. Nitrate of silver and chloride of zinc may be judiciously applied in cases of obstinate ulceration. The parts should first be thoroughly cleansed with peroxide of hydrogen or solution of borax, after which a pigment of nitrate of silver or chloride of zinc, twenty to forty grains to the ounce, may be applied to the ulceration. Lactic acid and chloracetic acid may likewise be applied in some cases. This may be easily accomplished by dipping a pledget of absorbent cotton in a saturated solution of either of these agents and applying it to the part. When there is very much pain and tumefaction, a pigment of carbolic acid will be found highly useful, besides affording some antiseptic effect. This may be used as a pigment in the proportion of ten to twenty grains to the ounce, or as a spray mixed with glycerine and water in the proportion of five grains to the ounce. The general hygiene of the patient is to be kept in 
view, and frequent bathing, either in simple or some saline water, is to be insisted upon in cases of tertiary syphilis. The diet is to be regulated as it would be for a manifestation of the disease in any other region. Neumann thinks that mercury and iodine are not specific in preventing the course of syphilis, but rather in removing the products of the disease. Professor Boeck, of Christiana, thinks that he has seen benefit from hypodermic injections of serum obtained from the cavity of the tunica vaginalis of syphilitic patients who were suffering from hydrocele. He has used this treatment in primary, secondary, and tertiary cases. He does not, however, think that this plan of treatment is always superior to mercury and iodine. Dr. R. Kopper has treated with good results sixty-nine hospital and fifty-eight ambulant cases of syphilis with hypodermic injections of corrosive sublimate. The solution is made of corrosive sublimate, 5 parts; sodium chloride, 5 parts; distilled water, 100 parts.

The injections were given every five to eight days, deeply into the gluteal region.

Iodine has been recommended by Dr. Pierce and others in one-twelfth grain doses three times a day.

Benzoate of mercury and cyanide of mercury are preferred by some practitioners, especially for hypodermic medication. The benzoate is just now popular. The writer has obtained quick results from the hypodermic injection of one-twentieth to one-fortieth grain of cyanide of mercury in cases of extensive secondary ulceration with sloughing.

Dr. Lane (British Medical Journal, March, I897, p. 35I) advocates the intravenous injection of cyanide of mercury in syphilis. He uses about twenty minims of a one-per-cent solution. He first bandages the arm as for ordinary venesection, sterilizes the skin, needle, etc., and introduces the fluid into one of the protruding veins, quickly withdrawing the needle and closing the opening as one would for venesection. $\mathrm{He}$ claims that the manipulation is painless to the patient, and therefore preferable to injection into the cellular tissue anywhere. This practice will not probably become popular on account of the trouble attending the preparation and the dangers arising from its careless execution.

The various vegetable alteratives, such as stillingia, trifolium, sarsaparilla, cohosh, etc. (see formula), are very useful 
adjuvants of mercury and iodine. There are individuals in whom untoward effects promptly appear under the use of the old-time specifics, mercury and iodine. With such patients the vegetable alteratives (so-called) will be efficient substitutes. Frequently, hot or warm saline baths in conjunction with potassium iodide will be found very useful, if not necessary in some instances, to favour elimination. Under the use of hot saline baths much larger doses of both mercury and potassium or sodium iodide may be better borne by the system. This is undoubtedly the key to the important part played by the hot mineral bath treatment of syphilis. For patients who are abnormally susceptible to the effects of mercury or iodine when taken by the mouth, a course of mercurial fumigations or inunctions may be prescribed, or the drugs may be administered hypodermically, and thus relieve the stomach of the offending doses.

For the treatment of congenital syphilis no agent is better, as a rule, than mercury, either in the form of mercurial chalk, calomel, or mercury bichloride. To an infant between six and twelve months of age, one to two grains of mercurial chalk or one half to one grain of calomel; or one-sixtieth to one-fortieth grain of mercury bichloride (in solution) may be given three times a day. The old method of inunction by mercurial ointment is a good one. The infant's body band may be smeared well every day, or every other day, with mercurial ointment, which will then become rubbed in, so to speak, by the movements of the child. Besides this, mercurial fumigation may be applied if the case warrants it.

In all cases of nose and throat syphilis it must not be forgotten that the constitutional treatment is the most important plan, the local treatment ranking as auxiliary only.

The treatment of the sequences of syphilis of the upper air passages will vary with the requirements of the case. Impending stenosis of the nose, nasopharynx, or oropharynx must be attended to by daily (or oftener) dilatation by means of properly constructed mechanical devices. Likewise stenosis of the larynx or trachea may require the prompt performance of tracheotomy or frequent intubation. Deformities of the pharynx and oropharynx due to cicatricial contraction may be relieved sometimes by operative procedure, but in the writer's experience such operations have invariably failed to 
better the condition of the parts. Schadle, of St. Paul, successfully relieved a case of cicatricial stenosis of the anterior nares by incision, followed by persistently keeping in each nasal passage a split strip of iodoform gauze passed through and tied over the front of the nose.

Regarding the relief of palato-pharyngeal contractures and adhesions, but few authors offer much encouragement. Elsberg's method was to separate the parts with a blunt staphylorrhaphy knife, force the finger through, and have the patient use daily a hard-rubber palate retractor to prevent readhesion. Dieffenbach, after incision, drew the soft palate forward as far as possible by means of a ligature passing out of the mouth and fastened to the ears. Sponge tents have been used, spring clips, tubes and rings of lead, metal, rubber, etc. When there is a sequestrum of necrosed bone so large or hard that it cannot be removed through the nasal passage, an attempt should be made to break it in situ with forceps; that failing, recourse should be had to the operation described by Rouge in 1873 . By this method the soft parts encircling the external nose are detached by an incision along the gingivo-labial furrow, and the dissection carried upward close to the bone until the nares are freely exposed. If necessary, additional space may be gained by chipping off with bone forceps the osseous margins of the introitus nasi.

This operation is especially adapted to cases of syphilitic necrosis in which the size and shape of the sequestrum preclude its removal through the natural passages without producing extreme pain to the patient and damage to the soft parts. Bone exposed through ulceration of the soft parts can always be readily distinguished from necrosed bone by the mobility of the latter and the different sensation conveyed by the probe. The blind use of the drill and the burr, especially in the ethmoid region, cannot be too strongly condemned. Nasal polyps seldom complicate cases of syphilis, although such neoplasms are occasionally met with. C. H. Knight has never seen a genuine polyp in a case of nasal syphilis except in one giving a clear history of nasal polyp previously.

The overcoming of the deformity known as "saddle nose" (which results from more or less destruction of the cartilaginous and bony supports of the nose) is often a difficult problem. The method first suggested by Lerievant consists of inserting 
beneath the depressed nose a supporting framework of metal. Aluminum was first used, and subsequently C. Martin, of Lyons, made an artificial bridge of platinum with lateral arms to be imbedded in the superior maxilla on either side. This operation was introduced into this country by Dr. R. F. Weir in 1892. The length and shape of the bridge and of the arms must be adapted to each individual case. One removed by the writer was worn by a patient for seven months, and was then replaced by a larger one. It was put in place by the Rouge operation. The difficulty is that the bridge becomes destroyed or the edges so eroded that it has to be removed, or the skin over the dorsum of the nose becomes very sensitive and finally ulcerates, allowing the metal to protrude.

Laryngeal and tracheal stenosis from cicatricial constriction generally requires operative interference sooner or later. The obstruction arising from either laryngeal or tracheal syphilis may be caused by a growth instead of cicatricial tissue. In the trachea the growths are generally gummatous, while in the larynx the obstructive growth is generally papillomatous. In either case, however, if there be much obstruction to respiration, intubation or tracheotomy ought to be performed without delay, and the obstruction attended to afterward, either by removal, or, if cicatricial, by forcible dilatation with a laryngeal bougie. Formerly the laryngeal bougie (Wagner's and Schrötter's being the best) was extensively used for overcoming laryngeal obstruction due to cicatricial tissue and contraction of the tube, but of late the use of O'Dwyer's laryngeal tubes, by the manipulation known as intubation, offers a much more efficient and a safer method.

In some cases intubation renders tracheotomy unnecessary. In a case treated by my associate, Dr. W. S. Anderson, dilatation of the larynx was successfully accomplished by the frequent introduction and wearing of an O'Dwyer laryngeal tube covering a period of three months.

Tracheal stenosis, if persistent, will eventually require tracheotomy. Oftentimes, however, mechanical dilatation and the faithful ingestion of potassium iodide by the patient will secure relief without operation. Bryan reports an interesting case of syphilitic tracheal stenosis relieved by large doses of potassium iodide. Developing gummata in the trachea may 
give rise to alarming stenosis, and during their growth may take on the characters of angioma. The writer has had two cases of this sort in which there was alarming hemorrhage from the inside after the opening into the trachea was made. The first case, a man thirty-two years old, died about four hours after the tracheotomy from exhaustion due to recurring hemorrhage. The second case, a woman, was saved only through the skilled attendance of my associate, Dr. Hickey, aided by the hospital house staff and trained nurses for a period of ten hours. In both these cases the swelling inside of the trachea could be seen by the aid of the laryngoscope, but the extremely vascular character and wide extent of the swelling over the anterior wall of the trachea was not thoroughly recognised by laryngoscopic examination. Syphilitic ulceration and its consequences are undoubtedly very frequently the causes of tracheal stenosis, and sometimes tubercular granulomata are to blame. Tumours of other sorts are sometimes situated in the trachea, and sometimes the stenosis is due to pressure from without, from enlarged mediastinal glands, gummata, or from aneurism. Any of these conditions may co-exist with syphilis.

Hence the conditions producing tracheal stenosis may be divided into external and internal. Among the former may be mentioned aneurism of the innominate artery; thoracic bronchocele, the cystic or fibroid variety; tumours of the thyroid gland, more especially those of a malignant nature; gummata, lympho-adenoma, and growths in the mediastinum and esophagus. By pressure upon the trachea, these conditions may cause severe dyspnœa; tumours sometimes find their abode on the inside also. The trachea may be pushed from its normal and anatomical relations, or flattened by compression. Aneurism of the transverse arch of the aorta, growing backward, has been known to compress the trachea to two thirds of its normal size. Tubercular disease of the bronchial glands in children sometimes gives rise to these symptoms. Garảult distinguishes between stenosis of the larynx and that of the lower air passages as follows: When the obstruction is laryngeal, the head is thrown back as far as possible; when it is tracheal, the head is stretched forward and the chin slightly depressed, so as to relax the trachea. 


\section{Tuberculosis of the Upper Air Passages-Nasal Pas- Sages, Pharynx, Larynx, Pharyngeal, Palatal, and Lingual TONSILS}

Definition.-A particular infiltration with productive inflammation, nodular formations, and necrosis of the tissues, supposed to be due invariably to the invasion of the tubercle bacillus of Koch.

General Etiology.-Although there are etiologic features of the disease peculiar to each of the iocalities mentioned above, yet the general points are applicable to all varieties of so-called tuberculosis, wherever its site. Perhaps no field of medicine has attracted more thought and research during the last century than tuberculosis in general, and the upper air passages in particular, and certainly with the exception of carcinoma no subject has brought forth such diverse explanations concerning its pathological, clinical, and etiological characters. The consensus of opinion at the present time is that all forms or varieties of tuberculosis originate entirely through the presence in the tissues of the tubercle bacillus of Koch, and that to its action, aided subsequently by other pathogenic bacilli, may be attributed all the phenomena constituting the pathogenesis of the disease or diseases in question. This doctrine seems to be so well sustained by a multitude of credible bacteriologic and biologic experiments as to leave no room for doubt, and certainly if it were not for the irregularities and discrepancies in the natural history of the tubercular process as observed in human beings (especially when the upper air passages are affected), there could be no objection whatever to placing the whole responsibility upon this ubiquitous bacillus. But, unfortunately for a strict interpretation of the doctrine, the expected "tissue reactions" do not always regularly follow upon the evidence of bacillary invasion, nor do the clinical data in many cases confirm either the presence or operation of the tubercle bacilli. For example, tuberculosis of the upper air passages (nose, pharynx, and larynx) constitute but a small proportion of the whole number of cases of tuberculosis. Although from a physical point of view these regions offer particular inducements for the lodgment of tubercle bacilli, yet, in many cases of purely localized tuberculosis of this region, 
the presence of tubercle bacilli cannot be demonstrated at all, so that we must agree with the many clinicians who declare that our knowledge on the subject is far from complete. Kafeman says that, in spite of the existing knowledge of the subject, a multitude of questions concerning tuberculosis of the upper air passages await solution. Jonathan Wright says: "It is yet an unsolved mystery how tubercle bacillus reaches the most favoured and chosen seat of its selected acts in the air passages to the lungs, and how pneumonia occurs. To a bacteriologist it is almost inconceivable how microbes, entering the nasal passages with the tidal air and going through the tortuous moist passages of the nose, pass by the surface of the palate and post-pharyngeal wall into the larynx, between the false and true cords, down along the tube of the trachea and bronchi, and finally finds a lodging on the walls of the bronchi or air cells. The chances of their being arrested before thus reaching such places seem almost infinite, especially since the tidal air must stop at a comparatively high point in the respiratory channel and flow forward again on expiration."

The writer is inclined to believe, with Unterberger, that the rôle of the tubercle bacillus in spreading disease has been overestimated, and that the independent powers ascribed to it have been also exaggerated. The tubercle bacillus is slow of growth, and requires a proper nidus or soil for development. To these prerequisites have been credited much of the pathologic and clinical ambiguity observed in the course of bacilli, and for similar reasons the bacillus of diphtheria also has been suspected of belonging to, or at least as closely related to, the streptothrix group of micro-organisms (see plate in Sajous's Medical Progress, p. 225). "Babes and Levaditi describe an actinomycotic form of the tubercle bacillus, the development of which they traced after injecting pure cultures of slight virulence in the meninges of rabbits" (Sajous's Med. Prog.). Lubarsch also obtained actinomyces-like growths of tubercle bacilli by placing a loopful of bacilli in small wounds in the liver, kidney, or mammary gland. "The bacilli assumed a radiating arrangement with club-shaped free ends." "They assume that this peculiar form of growth results when many bacilli become crowded into a small space, so that further growth is hindered. The club formations are probably the result of degenerative changes" (vide Med. Prog.). Vagedes shows by 
suitable experiments that human tubercle bacilli from different sources have varying degrees of virulence. From thorough comparative studies of bacilli of mammalian tuberculosis, Theobald Smith (Med. Prog., March, 1899) reaches the following conclusions: "Bovine and other animal bacilli grow less vigorously for a number of generations than the sputum bacilli. They are less influenced by modifications in the culture media, and they tend to remain short. Cattle inoculated by bovine bacilli generally lose weight and develop a more or less existing tuberculosis, while cattle inoculated with sputum bacilli gain some in weight, and there develops only a purely local, non-progressive, atypical tuberculosis around the seat of puncture into the lungs." Occasionally the sputum cases fail to develop any disease. This establishes a distinctively human and bovine variety of tubercle bacilli, the human having but slight virulence for cattle. This view has also been confirmed by Koch. Askanazy reports two cases in man which had the form of pearly disease. The true interpretation of such cases will remain obscure until the etiological agent is studied further; the variable character of tissue reaction to tubercle bacilli according to species, deprives pathological study, when taken alone, of any decisive value, in view of the probable existence of distinct bacillary varieties. The question raised by Smith of the transformation of one variety of tubercle bacillus so as to correspond with the cultural and pathogenic peculiarities of another, is one of great practical importance in relation to the source of infection, and it has received an important contribution in Nocard's valuable study of the bacilli of mammalian and avian tuberculosis. These microbes are generally regarded as distinct because of well-defined cultural and pathogenic differences. Nocard took advantage of the peculiar conditions offered by cultivating bacilli in small collodion sacs in the bodies of animals. "These sacs permit of a ready change in the composition of the fluid they contain, thus preventing autointoxication from the bacteria while the fluids of the animal incubator enter the sac gradually. Sacs containing bacilli of human tuberculosis were inserted into the abdomen of chickens, and after four months cultures of the now thick fluid in the sac presented colonies which resembled greatly those of the avian type of bacilli, being soft and unctuous. After six to eight months the bacilli had become pathogenic for chickens, 
the lesions produced being identical with those of natural avian tuberculosis."

"It appears that by this method it is possible to change the bacillus of mammalian tuberculosis so as to make it very similar to if not identical with the avian. It will be recalled that Koch's bacillus is not originally pathogenic for fowls. Nocard concludes that these two bacilli, apparently so different, are but varieties of the same species. Spontaneous and experimental pseudo-tuberculosis-that is, a disease characterized by nodules in which the causal agent is not the bacillus of tuberculosis-has been described in guinea-pigs, rabbits, and other animals by Malasey, Vignal, Nocard, Pfeiffer, Kutscher, and others as due to a non-liquefying bacillus occurring in zöoglea masses or discretely." " Robert Muir describes an identical or closely related disease in singing-birds characterized by the appearance in the spleen and liver of small yellow nodules com. posed chiefly of masses of bacilli whose growth starts from the capillaries and results in but slight reactive changes. The bacillus is readily cultivated. On gelatine it resembles the bacillus of typhoid." Experimental inoculations were successful in birds as well as in rodents, the lesions in the latter being more cellular, but in both the spleen and liver were mostly affected. Paul Courmont would apply the term tuberculosis to all diseases characterized by nodules, and would reserve pseudo-tuber. culosis for the proliferations which occur around inert foreign bodies. Whether this is more logical than to limit tuberculosis, as at present, and to designate other tubercular diseases as pseudo-tuberculosis, may well be questioned. In one case stress is laid more on the character of the lesion, and in the other on the causal agent. In neither is the nomenclature, although convenient, a very desirable one. Courmont studied a fibrino-hemorrhagic and productive synovitis with typical caseous tubercles without the presence of the tubercle bacillus. From the nodular lesions that developed in guinea-pigs after subcutaneous injection of fluid from the joint, he isolated and cultivated an immobile bacillus which formed chains and produced in animals a nodular, exudative, and quite often hemorrhagic lesion, which in a nodular form presented a striking histological similarity to experimental miliary tuberculosis. Alfred Moeller isolated from timothy hay, vegetables, and the fæces of cows, horses, goats, hogs, and mules, a bacillus which resem- 
bles very much the tubercle bacillus in cultural properties. Inoculated into rabbits and guinea-pigs, a picture resembling miliary tuberculosis often developed; in some cases pulmonary lesions with cavities resulted. Microscopically, such lesions resembled very closely tubercles; they contained giant cells and bacilli which stained like tubercle bacilli. Cultures from the lesions in the animals did not grow readily for a time, again resembling tubercle bacilli. Cases where the lesions closely resembled tuberculosis were, however, exceptional; in most cases one accustomed to tuberculosis in these animals would not recognise it as tuberculosis, but in many cases the resemblance was bewildering. Moeller thinks that this is a new, not previously described, bacillus. It is different from those found in butter; it belongs to the same class as does the tubercle bacillus of mammals and birds.

The experiments of Drs. Babi, Perron, and Gimeno serve to show that tuberculosis and phthisis are not always the same disease (New York Medical Journal, June 4, I898, p. 805).

The clinical observations of the writer are confirmatory of the above-mentioned conclusions; also the experimental observations of Dr. Gibbes and the writer upon monkeys would seem to show that these animals may suffer from two different types of pulmonary disease-tuberculosis and phthisis-when the illness is not acquired by inoculation.

Ledoux-Lebard concludes that there are more grounds for placing the bacillus of tuberculosis in the genus cladothrix than in the genus streptothrix. He proposes to keep Metschnikoff's new name for the tubercular bacillus-viz., sclerothrix Kochii-but to transfer the bacillus to the genus cladothrix. Anomalous, branching, club-shaped forms of the tubercle bacillus in cultures in the sputum and in the tissues have been described, and on this account the view has been advanced by some that the tubercle bacilli are not all of one family.

Latency.-Tubercle bacilli are said to be capable of existing in a latent state in the tissues for a long time without losing their potency. According to Bollinger, tubercle bacilli have remained latent in the bronchial glands for twenty years without losing their vitality. He (Bollinger) also reported to have found tubercle bacilli in the bronchial glands of non-scrofulous children who died of measles. Its latent property is supposed to be illustrated also by cases of tuberculosis which have 
developed after injuries acting as exciting causes. Dr. O. Schroeder reported a case of a healthy man having shown symptoms of tuberculosis after a severe fall. Tubercle bacilli were not found, however, until the fortieth day after the injury, while he was suffering from fever and showed the physical signs of "upper lobe pneumonia." After the abatement of the fever, etc.-at about the end of the second monththe patient recovered, and there were no tubercle bacilli in the sputum. Other similar reports of cases, as well as those reported as terminations or complications of typhoid fever and such diseases, were probably not tuberculosis, although the tubercle bacilli may have been found in some specimens of sputum. Valuable as this sign is (the presence of tubercle bacilli), it will not be safe to base a diagnosis upon their presence alone, because tubercle bacilli are not always present in certain stages of veritable pulmonary tuberculosis. Meigs believes that all forms of tuberculosis are, morphologically, only a growth of fibro-cellular tissue and its subsequent destruction, and that the tubercle bacilli are not the cause of the process. Tubercle bacilli are supposed to gain access to a given part in one of the following ways:

A. By direct implantation from outside sources.

B. By indirect implantation from some active or latent focus within the body.

C. From without, and conveyed by the air through the respiratory system.

D. By the food and drink.

E. By inoculation through abrasions of the integument or mucous membrane.

F. From within, conveyed through the blood-vessels, or

$\mathrm{G}$. Conveyed through the lymph vessels.

The tubercle bacillus probably acts as an enzyme, and its action is a biochemical one, similar to other ferments, while the toxins, which are the result of its presence, are probably derived from the tissues acted upon, and are not secretions of the microorganism itself. In a majority of tuberculous processes the tubercle bacilli are associated with other micro-organisms, some of which (staphylococci), according to Michaelis, always circulate in the blood of some phthisical patients. Hirschlaff found staphylococci in the blood of four phthisical patients out of seven whose blood he examined. Indeed, pathologists now 
ascribe much of the later phenomena to other bacteria, such as streptococci. The appearance of local tuberculosis in parts of the body remote from the orifices is accounted for by the migration of the tubercle bacilli through the blood or lymph vessels. In this way osteo-arthritic tuberculosis, tuberculous herniæ (Sprengel), meningeal and peritoneal tuberculosis, tuberculosis of the cranial nerves, etc., are accounted for, as well as tuberculosis of the upper air passages. The defence which Nature offers against tubercular as well as other infections may be formulated as

a. General : A natural immunity arising from an individual physiological character; metabolism.

b. Local cell action (leucocytosis, phagocytosis); glandular secretions, or antitoxins.

Regarding the efficiency of phagocytosis there is some difference of opinion among observers. This is also true concerning the alleged part played by leucocytes. Hahn concludes from experiments with typhoid bacilli and staphylococci that the body is protected against infection by the products of metabolism, and not by the product of disintegration of leucocytes. The effects of the glandular secretions of the several localities will be discussed under their appropriate headings, but it may be stated here that considerable power of resisting infection is attributed to the secretion of the mucous membrane, saliva, etc. The defensive effects of the internal secretion, blood, or blood serum against tuberculous processes has been, as far as tried, very disappointing. Dr. Gibbes and the writer carried on a few experiments upon monkeys a few years ago with a view of immunizing the animals against tuberculosis by injections of the blood serum of affected monkeys, and also another series of experiments in an attempt to modify the disease by similar injections of the serum from other affected animals; but the work proved negative. Much more of such work has been done since, which has also been more or less unsuccessful, although it is true that many cases of successful treatment have been reported by the use of tuberculin, tuberculocidon, Paquin's immunizing horse serum, and other serums.

Heredity.-Heredity was formerly considered as the principal predisposing cause, but since the discovery by Koch of the tubercle bacillus many practitioners pay little if any attention 
to heredity as a causal factor. The fact, however, that so many cases of tuberculosis occur in given families, even following two or three generations, despite most favourable hygienic surroundings and their individual separation, ought to convince any liberal-minded practitioner of the importance of heredity as a predisposing factor in the causation of the disease. The writer can cite an instance (one of many similar ones) which came under observation in a family where six children (of healthy parents) out of a family of seven died of phthisis pulmonalis after reaching about twenty years of age respectively. They did not live in the same house during all this time, were well cared for, were more or less separated from one another-in fact, two of the male victims were taken ill while living away from home in Kansas. Indeed, the question of contagion might properly be eliminated here. A close scrutiny into the family history of the European antecedents of this family revealed the fact that for three generations tuberculosis had been a veritable scourge among the paternal ancestry, while a few cases had occurred also upon the maternal side. Modern writers, while acknowledging this factor are nevertheless prone to minimize it too much by using the term "susceptibility." Among the several theories concerning the modus operandi of heredity, the one given by Dr. Buckmaster is perhaps the most striking. He says that "the evidence that bacteria may be transferred from parent to offspring seems incontestable, and the routes by which this is possible appear to be two, viz., by a direct bacterial invasion of the essential reproductive cells, or by the egg cell or the embryo receiving micro-organisms from the female, in which case the blood stream is the channel of infection. Bacterial infection then may be either germinative or placental, and in mammals the latter mode is not infrequently observed." The observations of Pasteur in regard to pebrine-a disease of silkworms-are well known. They showed that in this malady definite sporocyst forms were transmitted from the imago, or perfect insect, by way of the egg cell, and that the larva was directly infected in this manner. Gärtner also has absolutely demonstrated the fact that ova may contain pathogenic organisms. Infection from the male, however, stands on rather a different footing, and both on theoretical and experimental grounds it may be considered "that a germinative infection of the ovum never 
occurs by the conveyance of micro-organisms in the male reproductive cells." This theory, it is thought, may account for congenital tuberculosis, but it is insufficient to explain the condition known by the term " hereditary predisposition" or " tendency," or to account for the origin of laryngeal phthisis.

Contagion and Inoculation.- The propriety of classing tuberculosis with communicable infectious diseases-such, for example, as small-pox, scarlet fever, etc.-is still an open one, although many of the so-called sanitary experts consider it settled beyond a doubt that tuberculosis is, conventionally speaking, a communicable and infectious disease, and that it is spread through the medium of dried sputum containing tubercle bacilli. It is true that there are a number of apparently indisputable proofs on record of the transmission of the disease (the pulmonary form) directly from one individual to another, but the truth is that the idea of its communicable nature has been evolved more from inoculation experiments in the lower animals than by clinical observation. There is no doubt that artificial inoculation or the artificial insufflation of tuberculous sputum will produce the disease in susceptible lower animals. Dr. Gibbes and myself produced the disease in monkeys by oft-repeated insufflations of dried sputum. However, it must be remembered that the conditions in such experiments are favourable to the disease, and are not at all analogous to the ordinary accession of the disease in the human subject. In determining precisely the rôle played by contact with the dried sputum or tuberculous contagium, so to speak, in a given case, all other natural causative factors should be eliminated; for if, for instance, the patient has had some previous attack of acute disease, such as influenza or measles, or has been exposed for a long time to such deleterious influences as overwork, anxiety, unhygienic occupation, or habitat, etc., and in addition possesses a hereditary predisposition, then the fact of close exposure to a tuberculous individual will lose some of its significance. Even the convincing examples reported of transmission of tuberculosis from husband to wife should be taken with due allowance, in view of the fact that phthisis pulmonalis has overtaken many a faithful wife who has nursed her husband through long spells of sickness which were not of a tuberculous nature. What is needed to clear up this question, it seems to me, is a knowledge of the number of instances of potent exposire that 
have not resulted in the acquirement of the disease. A number of cases apparently showing the unmistakable acquirement of the disease by contact have been reported from time to time. Indeed, the writer has met with seven such cases, all of which were unmistakably pulmonary phthisis, and whose origin could not be otherwise accounted for. Wasserman reported a remarkable case of an infant acquiring the disease by contact, but these cases are so few compared to the whole number extant that sufficient proof of its contagiousness is still wanting. Various writers have ascribed the transmission of the disease through utensils and articles of clothing. One writer, in an article in the New York Medical Journal for June II, I898, has endeavoured to show that the holy water used in Catholic churches is a medium for spreading the contagium, while several others have indicted the communion cup and our handshaking custom for spreading tubercle bacilli and other micro-organisms. The question of the spreading of tuberculosis in hospitals through contact has been touched upon a little by some writers, and while it is quite generally agreed that such a danger probably exists, yet no definite conclusions on the details of the subject have been reached. The British Medical Association, through a committee, made an investigation of this subject several years ago. The committee reported a belief that pulmonary phthisis might be communicated from man to man, but offered no definite conclusions.

Pursuant to the idea of its communicability, especially in hospitals, many of the large institutions in Europe have adopted the plan of isolating tuberculous patients, while others have not. Debove claims that this practice has greatly diminished the mortality by tuberculous diseases in England. Jaccoud reported the cases of four previously healthy men who were in the hospital with non-tuberculous diseases and who were lying in proximity to a "consumptive" patient, and within a short period afterward they all returned to the hospital suffering from acute phthisis. It might be inferred from this, he says, that these men had derived the contagion from their consumptive ward mate, but Jaccoud doubts the truth of such a conclusion from the present state of our knowledge. He thinks it more likely that auto-infection in many cases of traumatism, or such diseases as measles or la grippe, may awaken to virulence some latent germs. Terrier asserted that hospital employees in 
France become tuberculous in considerable proportion, while a large number of students who were hardest worked and spent most of their time in hospitals contracted tuberculosis. On the other hand, Jaccoud and others are inclined to attribute the onset of the disease to the evolution from latent tubercle bacilli which have been ensconced in bronchial or other mediastinal glands. Dr. A. L. Loomis, of New York, a few years ago made a study of "glandular bacillosis" without tubercular lesions, and found that in forty per cent the bronchial glands of individuals not tuberculous contained active tubercle bacilli capable of infecting animals. Carl Spengler found the same condition in an autopsy upon four children dead of diphtheria. The writer has never known of a case of pulmonary or laryngeal phthisis arising unmistakably from contact in any of the hospitals in which he has served during the last thirty years.

Inoculation.-The transmission of tuberculosis by inoculation in the lower animals of course is a well-known fact, and cases have been reported of similar results in humans from accidental auto-inoculation. The famous case of the woman being inoculated while emptying a spittoon of a consumptive, which went the rounds of the medical journals in 1889 , is an example. I have known a few medical men who have stated that they had been inoculated accidentally with tuberculous material without effect. There have been several reports of tubercular inoculation of persons through the operation of circumcision. M. Ware published a report of fifty recorded cases of such inoculation, twenty-one of which number were infants.

The origin of tuberculosis in this way is undoubtedly rare, notwithstanding the great opportunities afforded for it in daily life. Many instances may be observed in the large cities of children whose hands and faces are freely bedecked with scratches every day, fondling and playing around a phthisical mother, without becoming inoculated. This question, however, will have to be considered again in reference to local tuberculosis.

Predisposing Diseases.-In practice, any of the various forms of tuberculosis may be observed to follow diverse acute affections. Influenza, measles, acute pleuritis, acute plastic and catarrhal pneumonia, and typhoid fever are often the forerunners of phthisis pulmonalis or laryngeal tuberculosis. Besides these, many of the chronic affections, as well as severe injuries, such as compound fractures, seem to lay the founda- 
tion for the development of the disease. Of all these, perhaps influenza, measles, and the acute pulmonary affections are most frequently followed by tuberculosis. Indeed, according to the experience of the writer, the first two named are particularly liable to engender some form of tuberculosis. The nasal, pharyngeal, and laryngeal forms, however, do not occur as often in this connection as the pulmonary. Another disease which acts frequently as a predisposing cause of laryngeal tuberculosis is syphilis, especially the tertiary form. Indeed, the two affections (syphilis and tuberculosis) sometimes coexist in the same individual (see Plate III, Fig. 6).

Social Condition.-The social condition of the individual, including habitat, habit, food, and clothing, certainly plays an important part in the induction of tuberculosis, especially pulmonary and laryngeal. A damp, illy ventilated, and badly warmed domicile, by favouring the accession of so-called "colds," is very likely to induce and maintain chronic catarrhal affections of the upper air passages. Likewise insufficient or excessive clothing, together with poorly or over-heated houses, by disturbing the nervo-vascular function of the skin, may sooner or later lead to chronic catarrhal affections, and then to some form of phthisis. Improprieties of this sort make the spring, autumn, and winter seasons of our northern climates notable for the development of tubercular affections.

Food.-It is admitted by many, even by some of the radical bacillary advocates, that disturbances of the normal metabolism are necessary to the full production of tubercular disease. Hence the question of proper nutrition for persons residing in northern climates is an important one, because indispensable for maintaining the physiological tone of the system. A good, wellcooked mixed diet (meat, vegetables, besides fruit) constitutes a good defensive measure. In this connection arises the question of tuberculous food, and how often, if at all, the disease is really spread by the ingestion of such food. "Tuberculous meat" and "tuberculous milk" are the particularly offensive articles noted. Many interesting reports have been made of the origin of cases of tuberculosis of the intestines, in infants especially, and of the larynx and lungs in adults, from the ingestion of tuberculous meat or milk. I confess to entertain grave doubt as to the accuracy of conclusions based upon these occasionally appearing reports. If there is the great 
danger lurking in meat and milk which the assertions of advocates of the doctrine would have us believe, then why has not this greatest of meat-eating and milk-drinking nations been almost annihilated by the "white plague"?

In the early days of bacteriology, Sir W. Roberts showed that milk cells and blood were in the living body absolutely free from bacteria, and if drawn through sterilizing tubes into sterilized flasks plugged with sterilized cotton wool, would remain unchanged for months or years without even having been themselves boiled. Not only putrefaction, but so-called spontaneous coagulation in milk cells, was brought about by the access of bacteria from without, but now it is shown that myriads of non-pathological bacteria are present in milk and cream as they are sold. After the best possible precautions against external contamination are taken they are invariably present in the milk by the time it reaches the market. Guinther and Thierfelder came to the conclusion that the spontaneous coagulation and lactic fermentation of milk are the work of one particular species of bacteria which is always abundant in milk when first drawn. They think the poisons are not in the caseous or peptone form, but in the bodies of the bacilli themselves. Gronig examined seventeen vessels of butter, and injected portions of the butter into fifty-one guinea-pigs. The result was that eleven guinea-pigs were found post-mortem to present the pathological appearances of tuberculosis. Of the seventeen specimens of butter, eight produced tuberculosis; but as no culture experiments were made the work is somewhat invalidated. Obermuiller also examined fourteen specimens of butter and found tubercle bacilli each time. Rabinswitch examined eighty specimens and found no tubercle bacilli. Interesting discussions upon this topic may be found in the Transactions of the Congress for Tuberculosis, held in Paris, France, during the last five years; also papers published by Brush, of New York, and Ernst, of Boston.

The writer fed three monkeys for a period of three months almost entirely upon milk from cows which were pronounced to be tuberculous by a reliable veterinary surgeon, without producing general or intestinal tuberculosis. Dr. Troy and myself subsequently fed some healthy guinea-pigs (which are supposed to be as susceptible to bovine as to human tuberculosis) for two months with food always mixed with tuberculous milk 
without observing any positive result. I knew of a case of an infant (now grown to adult life) who was fed for months principally on milk obtained from at least one tuberculous cow. No unusual illness accompanied the development of this child. However, Strauss succeeded in infecting guinea-pigs and rabbits through the ingestion of tubercle bacilli. Tuberculous poultry, I believe, is not considered as dangerous, because the experiments of Strauss, Koch, Gilbert, and others have shown that the avian differs materially from the human tuberculosis in biologic and pathologic character, and that birds, as a rule, are immune to mammalian tuberculous material While Strauss and other experimenters claimed to have infected guinea-pigs and rabbits with avian tubercular material, others have met with negative results from similar work. Among them, Matteucci found that tubercle bacilli of avian origin have only slight pathogenic properties in mammals. Several articles have been published against the use of pâté de foie gras as food on account of the supposed tuberculous character of the liver, as shown by the alleged discovery of tubercle bacilli in it. Other bacteriologists have declared that the bacilli found in this substance, while resembling tubercle bacilli in colour reaction, do not meet the biologic tests. It is probable that we eat a goodly quantity of tubercle bacilli as well as other pathogenic micro-organisms daily, but receive no particular damage thereby when we are in a physiological state.

Habits.-Many cases of laryngeal and some cases of pharyngeal phthisis have been induced by the bad habits of the individual. Excessive smoking of tobacco and drinking of alcoholic liquors may be regarded, therefore, as an adjunct cause of the disease in some cases. Loss of sleep and mental worry are both calculated to diminish the power of the economy to resist disease. These two causal elements are too frequently overlooked.

Occupation.-Occupation is an important predisposing cause. Bad hygienic surroundings in a workshop or office will have a bad influence upon the system generally, while local draughts of cold air, especially in a place overheated, also favour attacks of catarrhal inflammation of the larynx, and thus may lead to tuberculosis. Occupations subjecting a person to the inhalation of dust or to sudden change of temperature are favourable to the development of the disease. 
Age.-Laryngeal tuberculosis may occur at any age, although the most susceptible time of life is the period of adolescence. It is a striking fact, however, that childhood is particularly exempt from tuberculosis of the upper air passages. Old age is not exempt, although when the upper air passages are affected it is usually secondary to a long-continued chronic bronchitis.

Sex.-Tuberculosis of the upper air passages is more frequently met with in males than in females. This fact is accounted for by the difference in the mode of life of the sexes. Of late years the difference has become lessened owing to a change in the social condition of female life, whereby women are exposed alike with men to the vicissitudes attending business occupations of all sorts.

\section{Nasal Tuberculosis}

Nasal tuberculosis was formerly considered an extremely rare affection, but of late years a relatively greater number of cases has been reported. It may be primary or secondary, although the primary form is more rarely observed. Chiari, Rethi, Hajek, Herzog, Moritz-Schmidt, Kafeman, Farlow, Wright, and others, have published reports of several cases of the primary form. Farlow publishes two cases, Kafeman two, Chiari four, and Rethi one, in which there was no tubercular disease of the lungs or of any other locality of the body; neither were tubercle bacilli found in Farlow's, Kafeman's, or Rethi's cases, while Chiari found them in only four of his six cases.

Dr. A. Cartaz lately published an article based upon the collection of the histories of eighty cases of nasal tuberculosis, seven of which had been under his own observation. Of this number he found that the great majority were coincident to pulmonary phthisis. This accords with the experience of the majority of observers, who report to have generally seen the disease associated with either pulmonary or laryngeal phthisis. The writer has never met with a case of the primary form. Herzog has collected twenty cases of primary nasal tuberculosis. He reports ten cases of both forms under his own observation. It is rarely secondary to primary laryngeal phthisis (Herzog). 
The disease usually occurs under one of two forms, viz. :

a. An ulceration.

b. A tumour formation (granuloma).

The former is much more intractable, as a rule, although both forms are obstinate. A third mixed form has been suggested by Kocher presenting both granulomata and ulcers, but such a mixture may be observed in the course of any case. The tumour formation is usually found in the primary cases, while ulcers are found in cases of advanced pulmonary tuberculosis.

Etiology.-Its etiology, especially that of the primary form, has been the subject of much speculation. There have been several theories advanced to account for the disease, all of them, of course, presuming that tubercle bacilii are the essential cause. The presumption is that the bacilli are conveyed to an abraded spot in the mucous membrane by the finger, the air, or by sputum, through acts of vomiting and coughing, or that the bacilli are conveyed through the lymph or blood stream from some tuberculous focus elsewhere in the body to the part. While some observers feel satisfied that the exposure of the nasal passages to bacilli carried in the air, together with the almost continual abrasion of its mucous membrane from one cause and another, offers a sufficient explanation, others believe that the disease probably occurs from the conveyance of the tubercle bacilli to the nose from a distant gland by the lymph stream.

The correctness of these theories is based upon the affirmative results obtained by some bacteriological examinations on the one hand, and overthrown by the negative results obtained by similar examinations made by others. Strauss, in 1894, demonstrated the presence of tubercle bacilli in the nasal cavity, and proved their virulence by the results of inoculation in animals; and attention has also been called by Hildebrand to the amount of bacteria-laden dust which lodges in the vestibule of the nose during ordinary respiration. The nose has long been looked upon as a filter for the air about to be inspired. It has been found capable of filtering out about three fourths to four fifths of the bacteria contained in the air, passing at a rate of one litre a minute. Wright, however, believes that, as about nine litres in a minute pass, the nose probably does not collect all the bacteria. Von Besser found various 
pathogenic bacteria in the nose as well as in the larynx and trachea (Diplococcus pneumonia, Streptococcus pyogenes, and Staphylococcus pyogenes aureus). Strauss and Kafeman made twentynine examinations of the secretions from the interior of the nasal passages of healthy individuals who had been frequenting the rooms of phthisical patients, and found large numbers of tubercle bacilli in the nasal secretions of nine. On the other hand, St. Clair Thompson and Hewlett found that the inside of the nose only exceptionally showed the presence of bacteria. Ferni and Bretschneider also came to the same conclusion, and asserted that the nasal passages were quasi sterile. Wurz and Lermoyez maintain that the mucus of the nasal passages is sterile and germicidal. They say that it will even annihilate anthrax spores contained in broth. Besides this, Kafeman suggests that there may be an element of prevention in the movement of the cilia of the nasal mucous membrane. It must be remembered, however, that when the mucous membrane is in a state of inflammation its natural secretions may be entirely arrested, giving way to an excessive mucous secretion only, or it may be altered so that its distinctive chemical properties are lost, and its defensive power thereby lessened or destroyed.

Age.-The disease may occur at any age, excepting probably in the earliest months of life, but usually between ten and forty years of age.

Sex.-It shows no predilection for sex.

Pathological Anatomy.-Infiltration and tubercle formation are the principal characteristics. Owing to the nature of the tissues the infiltration is usually limited, unless the lesion be a manifestation of miliary tubercle. The little disseminated nodules or granulations usually show the histologic characters of tubercle (large cells, epithelioid and lymphoid). In the ulcerating form the tissues are so soon necrosed that the histologic characters may be lost. It is seldom that tubercle bacilli are found. The lesion is very persistent, recurring again and again in the parts.

Appearance.-Symptomatology.-The ulcers may be flat, quite large, and have a floor resembling fresh-cut bacon; or they may be small, multiple, and covered with scabs or crusts, which when removed show a red granular base, with a redder coloured border. The flat form is more often met with on the middle portion of the septum or on the turbinated bodies 
(Kafeman). The latter appearance is found when the site of the disease is near the anterior nares.

In Herzog's eighty tabulated cases, wherever mentioned, the disease was upon the cartilaginous septum in nearly all, in some ascending from the floor of the nose and anterior end of the turbinated bone. In six of the cases described by Chiari the disease affected also other parts of the nose. In two cases the lower turbinated bones alone were involved. Rethi's case extended to the antrum of Highmore.

Ulceration may extend inward along the nasal duct, involving the eyes, or to the accessory sinuses, especially the frontal and ethmoid, or outward, attacking the upper lip. The multiple ulceration is very apt to work toward the outside (Hajek).

There may be no constitutional disturbance, unless the lesion occurs in a person already suffering from tuberculosis elsewhere; hence the primary form of the disease may for a long time remain a local process.

The tumour form, already mentioned, is met with more often as a primary disease. It may appear as an isolated papular growth, varying in size from a kernel of wheat to a good-sized pea or it may consist of an aggregation of several very small papules, either placed closely together or separated by a tiny ulcer or ulcers. In the latter case the granulations seem to spring more often from the edge. They are red or purplish red, and bleed easily when the mucus or scabs covering them are removed. If these granulations break down, more or less ulceration supervenes.

Diagnosis.-The diagnosis of secondary and ulcerating nasal tuberculosis is usually an easy matter on account of the clinical signs of tuberculosis abounding elsewhere, but the tumour, nodule, or granulation form so called may be easily mistaken for syphilis, lupus, papillomata, angiomata (mulberry), or carcinomata. Koschier described a tuberculous tumour of the roof of the nose which might be mistaken for carcinoma. Lupus of the nose may complicate nasal tuberculosis (Herzog). The difficulty is increased by the knowledge of the fact that neither the histologic nor bacteriologic examination can invariably be depended upon, because tubercle bacilli are more often absent than present in the diseased tissues, as in Farlow's and Kafeman's cases, while giant cells, the aggregation of round cells, and a reticular formation are not absolutely confined to 
tubercular overgrowths; besides, in some cases even the histologic characteristics of tubercle may be absent. Herzog reports ten cases, some of which might be regarded as lupus. Indeed, Kafeman reported two cases, either or both of which might be considered lupus. Moreover, it will be found that in many cases of pulmonary phthisis there is more or less nasal discharge of a catarrhal nature, sometimes attended with slight ulceration about the nose, which is not really tubercular, but only a coincidental phenomenon. Payson Clark shows that the condition of the nasal membrane in pulmonary phthisis is generally either anæmic or more or less hyperæmic; and Solly, in an analysis of two hundred cases of phthisis pulmonalis at Colorado Springs, found that twenty-eight per cent of the cases had nasal disease, which in many instances he found was one-sided, corresponding to the side of the diseased lung.

Nasal tuberculosis may be distinguished from syphilis by differences in the clinical history, also by the appearance of the local lesion, which in syphilis is attended usually by much more tumefaction, absence of nodules, and a more active ulceration; besides, where doubt exists, it may be cleared up by the test of an antisyphilitic treatment. The appearance of the tubercular granulomata may resemble epithelioma, especially where enlarged lymph nodes are present in the auriculo-cervical region. The small size of the tubercular nodules, their apathetic, painless course, together with their histological features and the clinical history, will serve to distinguish them.

From lupus the diagnosis is difficult, especially when the tubercular lesion is rather extended. One differential sign of importance is that tuberculous ulcers show but slight tendency to heal, while lupus ulceration does heal. The ordinary course of each may be very slow. Lupus, however, usually shows some skin lesion, and is more apt to involve the vestibule and outside of the nose than tuberculosis.

Prognosis and Course.-Its course is very chronic, and relapses are prone to occur after surgical interference (Kafeman's case). While not dangerous to life in itself, it is apt to lead to complications, such as involvement of the nasal duct, the antrum of Highmore, or the other nasal sinuses. It may be complicated with tuberculosis of the pharynx, larynx, tonsils, palate, tongue, or cervical glands. 
Nasopharynx.-Several cases of tubercular disease of the nasopharynx, especially the adenoid tissue at the vault, have been reported of late years, yet the disease may be considered as uncommon. Dieulafoy, I think, was the first who pointed out this region as a point of tubercular infection. The neoplastic or nodular form has been the one usually found in this situation.

Etiology.-The exciting cause has been more often ascribed to abrasions from traumatism, such as operations, than to contiguous infection or extension from neighbouring parts or glands. Lermoyez believes that in these cases the infecting materia1 (tubercle bacilli) has invaded the adenoid tissue from some of the outlying glands where the micro-organism had been present in a latent state.

Some laryngologists attribute the development of the disease to direct infection by means of the finger of the practitioner, or from instruments used in operations for the removel of enlarged adenoid tissue. 'Two classes of cases of tuberculosis of this region have been reported: one in which the disease develops after an operation in previously tubercular subjects, and the other in which the disease appears in healthy subjects after operations at the pharyngeal vault.

Pharynx.-Primary tuberculosis of the pharynx is not frequently met with, although it is an admitted fact that tubercle bacilli are often, if not constantly, present in the pharynges of healthy individuals. Willigk, in autopsies of thirteen hundred and seven cases of tuberculosis, found the pharynx affected in one case. The case he reported was that of a girl, nineteen years of age, who complained of slight accumulation of mucus which she hawked out of her throat in the morning. No cough was present at that time; examination of the larynx was negative; no internal congestion; pharynx showed slight congestion, with here and there grayish white spots scattered over the membrane. The patient had no cough, did not feel sick, was in fair physical condition, her appetite and sleep good, no history of tuberculosis in her family, examination of the lungs negative. The temperature ran unusually high, even marking $104^{\circ}$ and over. Examination of the sputum showed tubercle bacilli in large numbers. For weeks she had this high temperature in the afternoon. She died in four months (Griffin). Levy, of Colorado, believes that pharyngeal tuberculosis occurs 
in about one and a half per cent of all cases of phthisis. Bosworth, Mackenzie, Lennox Brown, Wright, Heinze, Delavan, Schifferowitsch, Chappell, Theisen, Hajek, and other writers, all agree as to its infrequent occurrence. It usually occurs as a secondary manifestation of miliary tuberculosis. Pharyngeal tuberculosis usually runs a rapid course-from three to four months. DeBlois reported a case which ran a course of but two months. It seldom occurs in children. Ulceration in the oropharynx may appear early, although the tumour or granulation form usually precedes ulceration, especially in miliary tuberculosis. The writer has met with but one case of the primary form of the disease. In this, the lateral walls of the pharynx and back portion of the cheeks seemed to be sprinkled with little nutmeg-coloured nodules, which successively, by amalgamation into patches, broke down into small ragged ulcers. The posterior and side walls and surface of the posterior pillars are the favourite sites, unless the mouth be also involved, in which event the surface of the anterior pillars becomes chiefly implicated. Oftentimes a large ulcer is formed by the coalescence of a number of small ones. The granulation form, which may show itself in one spot or a few spots only, soon gives way to small ulcers by the degeneration of the nodules. These nodules are similar in appearance to tubercular nodules which invade mucous membranes elsewhere. They are small flattened or cone-shaped granulations, with red and usually ragged borders, the whole covered by a cheesy mucus mixed with small crusts. There may be considerable swelling (œdema) of the surrounding tissues. Rethi reports a severe case which lighted up suddenly, commencing in the left wall of the pharynx and extending to the Eustachian orifice.

Dr. Walter F. Chappell reported three cases of pharyngeal tuberculosis, one of which was primary. The first case was that in a man twenty-four years of age, who had marked pulmonary involvement before the appearance of the disease upon the pharynx. From the time the pharynx was first invaded the disease ran a very rapid course. The mucous membrane had previously presented a pearly gray or yellowish appearance, and within forty-eight hours these yellow spots resembled those seen in cases of follicular amygdalitis. These spots had a thin covering of epithelium, but within a few hours of their breakdown bright yellow curdy secretions dotted the 
surface. These were readily brushed off, leaving an extremely small opening covered with the same curdy secretions. The pharynx had become also edematous prior to the appearance of the yellow spots, and as soon as they all discharged the swelling subsided and gave the mucous membrane a wrinkled appearance. The minute spots of ulceration soon coalesced and poured out a mass of thick tenacious secretion. The disease followed the usual course of acute cases, making deep, irregular excavations in the soft palate. The patient survived only about six weeks after the pharynx was attacked.

In the second case reported, the disease seemed to confine itself throughout its course to the right posterior pharyngeal pillar adjoining the soft palate. This case survived only five months after the beginning of the pharyngeal disease. $\mathrm{He}$ met in this case with one large tuberculous ulcer, extending from the anterior commissure of the larynx upward to the cushion of the epiglottis, with considerable infiltration of the arytenoid cartilage and epiglottis.

The third case, that in a woman nineteen years of age, showed no pulmonary lesion until the pharyngeal disease was well advanced. She had taken care of a sister who died of acute tuberculosis, and after the sister's death had slept in the room occupied by the sister, using the same bed-clothing. She had taken no care whatever to prevent infection. This case also ran a rapid course. Examination of mucus from the nasopharynx contained tubercle bacilli. Several pieces of tissue were cut from the right pharyngeal fold, which were examined by Dr. Jonathan Wright and Dr. Parke. The latter inoculated two guinea-pigs with this material, one of which, although dying of septicæmia, showed tubercular spots in the spleen, and other internal organs which contained tubercle bacilli. Four months later the second guinea-pig, which had presented signs of the disease, was killed, and found to be suffering from disseminated tuberculosis. Other portions of the same specimens were hardened, and examination microscopically of sections showed the presence of tubercle bacilli, but few, however, were found in two or three sections out of about forty examined. The bacilli were in more than one section, and the tubercle granula, and sometimes the giant cells, were situated usually in the centre of the section. In the case of the woman, injections of creosote and lactic acid, also curetting, were thoroughly 
employed, with little benefit to the right side, but producing considerable improvement in the left side. The right tonsil and right pillars of the fauces became involved, but not until over four months from the date of the pharyngeal disease was any pulmonary lesion discovered. Soon after this time the disease spread rapidly through the pharynx, larynx, and pulmonary tissue.

The Mouth, Tongue, Soft, and Hard Palate.-These parts are sometimes also involved with the oropharynx. The appearance of the lesion is about the same as when located in the pharynx. Carrière reports a case of ulceration confined to the inner surface of the cheek in a patient thirty-seven years old, which was regarded as tuberculosis because of the presence of tubercle bacilli.

First, there was a slight abrasion for a few days. This healed, leaving a somewhat elevated scar. Soon afterward ulceration in the scar supervened, extended, and presented the appearance of a raised lesion with whitish edges. The tubercle bacilli were found in the margin.

Tuberculosis of the mouth and palate is extremely painful after ulceration has taken place. The site of the tubercular development may be on one side only of the mouth, but usually both sides are involved at nearly the same time. The lymphatic glands are generally swollen, although they may not be painful to the touch. The gums are sometimes the principal seat of the disease, and from this situation the process of infection and ulceration may extend to the maxillary bones, especially if aided by the presence of a carious tooth, or if a carious tooth be extracted while the disease is in progress a rapid extension may take place (Kafeman).

Tongue.-The ulceration of the tongue is quite obstinate, as well as being very painful.

Usually there is no appearance of disseminated nodules, but fissured ragged ulcers with granular edges quickly form. Sometimes the whole coating of the organ is involved almost to the muscle. The tubercular development may often take place from the bottom of the follicles of the mucous membrane and extend upward toward the surface.

If the hard palate be invaded destruction of the bone rapidly takes place. The velum palati is seldom affected alone, but is involved along with the pharynx. M. Barbier recently reported 
a case of tubercular perforation of the velum palati in connection with tuberculosis of the tonsils.

Tonsils and Lingual Lymphatic Glands.-From the numerous pathological reports of tuberculous tonsils one would be led to believe that the tonsils are much more commonly affected than is generally supposed.

Professor Dieulafoy, of Paris, calls attention to a variety of "pharyngeal tuberculosis," the favourite site of which is the adenoid tissue of the nasopharynx. It manifests its presence by a glandular growth in that region-in other words, by hypertrophy of either the pharyngeal or faucial tonsils.

The reports of Strassman, Dmochowsky, Schlenker, Hannan, Kafeman, and Moritz-Schmidt and Schech would indicate that tuberculosis of the tonsils is a common accompaniment of pulmonary phthisis, and that it is very often overlooked. Dmochowsky found tonsillar tuberculosis in fifteen cadavers dead from tuberculosis. The tubercular growths, as well as the ulceration, are said to be sometimes too small for macroscopic recognition. The tubercle develops mainly in the cavity of the crypts, the ulceration not coming to the surface at all. The infection is said to take place from the outside, mainly through epithelial abrasions at the mouth of the crypts or follicles, or through the epithelial chasms of Stohr. Bacilli are present sometimes in enormous quantities (Kafeman). Dmochowsky describes two forms pathologically. In one, the tissues show effects analogous to common inflammation, and while bacilli are present there are no epithelioid nor giant cells. In the other, the tissues show a number of widely dispersed tubercles extending into the lymph spaces, accompanied by tubercle bacilli also.

Krückman believes that the entrance to the lymphatics may be through the tonsils, or through the pharyngeal or the buccal membranes. Proof has been lacking of this, however, and attempts at producing tuberculosis by introducing tubercle bacilli into the tonsils have failed. Kruickman found upon post mortem examination of the bodies of twenty-five tubercular patients that in twelve he could demonstrate tubercle in the tonsils, and in every case the lymph glands of the neck were also affected. In sixty per cent of the cases of pulmonary tuberculosis he states that similar inflammation of the tonsils was present. 
Jonathan Wright states that Dieulafoy had inoculated sixtyone guinea-pigs in the peritoneal cavity with the tissue of palatal tonsils taken from apparently non-tuberculous children. Of these, eight (or a little less than one in eight) contracted tuberculosis. In thirty-five guinea-pigs inoculated with lymphatic tissue from the nasopharynx in the same manner, seven (that being one in five) developed tuberculosis. Lermoyez had previously reported two cases of tuberculosis, one apparently dependent upon an operation for adenoids. Günther also made examinations of tonsils, and found, as he claimed, that no tubercular infection had taken place. Dr. Wright says, "A well-known physician of Brooklyn, a skilled bacteriologist, told me that he had found tubercle bacilli in his own nose after a prolonged stay in the phthisical wards of one of our hospitals. He was and still remains, after the lapse of five or six years, a perfectly healthy man." Investigations of Baumgarten, Simms, Woodhead, Krückman, and others, would prove conclusively that the bacilli pass through the epithelium of the throat into the cervical lymphatics. Dr. Hoedenpyl examined about two hundred sections of this tissue and found none with anything like tubercle in the tonsils. Dr. W. Howie put into the peritoneal cavities and subcutaneous tissues of several guinea-pigs diseased faucial tonsils and adenoids, five of the former and seven of the latter. This series of experiments by Dr. Howie resulted negatively; no tubercle bacilli were found in sections and no tubercle developed in the guinea-pigs, though three died of septicæmia. "Giant cells were found in the tonsils by Dr. Ricardo Botey in two cases of amygdalar hypertrophy with 'larval tubercle.' He stated he had also seen giant cells in the sections of the tonsils. In nineteen other tonsils specially examined he found giant cells in two only, and never found bacilli. In two cases which he saw later he discovered no tubercle bacilli in tonsillar sections, although he obtained positive results by animal experiments, but in both cases the histories gave ample evidence of pre-existing tuberculosis elsewhere. After this, Botey inoculated ten guinea-pigs with tonsils from non-tuberculous cases and obtained negative results, except in one case, which died of pulmonary and secondary tuberculosis, but there was no tubercle at the site of inoculation. From his experience he deduces the following conclusions: First, that there exist certain forms of anygdalitis or hypertrophy of a 
primary tuberculous character, impossible sometimes to positively distinguish from the common hypertrophy of these organs with which every one is familiar. Second, this condition, which at first is of the 'larval tuberculosis' type in some cases, and latent in others, is a serious menace to the organism. Third, this 'larval tuberculosis' of the tonsils is presented, especially in adolescence, without the least pulmonary or intestinal lesion, except in some rare cases. Fourth, it is possible to diagnose it at any time with the help of the microscope and inoculation. Fifth, it is probable that the majority of cases of hypertrophic amygdalitis are not tuberculous, for although we know that the experiments vary greatly, nevertheless we do not know the proportion of one to the other. Sixth, this does not signify that all cases of 'larval amygdalar tuberculosis,' even supposing that they are diagnosed opportunely, are followed by different cervical adenopathies terminating in pulmonary phthisis. On the contrary, it is probable that in many cases the disease subsides in the lymphatic glands without tubercular germs reaching the pulmonary parenchyma later. Wright further says that he is inclined, both from clinical and pathological evidence, to agree with Dr. Hoedenpyl that tuberculous amygdalitis is a rare affection, and that the tonsils are seldom the seat of primary inoculation. Tubercle bacilli have been found in the bronchial and mesenteric glands of apparently non-tuberculous people. They have been shown to exist in these lymphatic structures by animal inoculation, when they could not be demonstrated by the microscope. That the same thing should be demonstrated in tonsils would not be a matter of surprise, but we should expect it to occur more often than it appears. Regarding the question of the return of tubercle bacilli through intact mucous membrane, he furnished a plate demonstrating that the epithelium in such cases is very thin. It is said by Lake and others that pyogenic cocci create points of suppuration through which tubercle bacilli find their way. This would appear not to be the case in the specimen shown. That tubercle bacilli can penetrate the laryngeal epithelia of the living individual, may still be considered a matter of some doubt."

This lymphatic infection is often started by an oscurrence of measles, scarlet fever, hooping-cough, etc. Glandular tuberculosis may in its turn remain local, and finally end in 
recovery. The third stage of tonsillar tuberculosis, it is said, is part of the progress of the illness caused by the bacilli reaching these organs from the cervical glands by way of the lymphatics, thoracic duct, and the right heart. M. Chaveau stated that in animals fed on tuberculous matter infection may take place by inoculation of the adenoid tissue at the base of the tongue and the pharynx, this inoculation being proved by the swelling of the cervical and submaxillary glands; sometimes it takes place through a minute erosion, but often the surface has been found intact. The autopsy table has shown only two or three complete cases where examination gave evidence of bacilli. Now, there must be a good many cases in which the tubercle bacillus has perished without leaving a trace behind-on the skirmish line, as it were. Krückman has lately shown by a number of carefully conducted examinations that in adults the tonsils and cervical glands are usually affected by tubercle bacilli, but that the lungs are really the seat of the disease. On the other hand, in children he believes that the cervical lymphatics are infected before the pulmonary tissues. We have clinical evidence that it is in the late stage at which enlarged cervical or lymphatic glands are discoverable. Corbin has shown that he can produce a lesion similar to tuber. cle formation without connection with the living tubercle bacillus; and a French author has said that the bacillus is not enough-we must have the reactionary lesion from the tubercle. As clinicians, we may say boldly that neither tubercle nor the tubercle bacillus, nor both together, are enough. The tubercle bacilli are at one end of the chain, and tubercle ison the autopsy table-at the other end of it. "Tuberculosis itself does not mean either of these. It means to us both of these plus the vital energy of the bacillus, on the one hand, and that of the resisting human organism on the other."

It seems incredible that there can be many cases of tubercular disease of the tonsils which entirely escape observation during life, as some pathologists intimate. It is more likely that either caseous material which has accumulated in the mouths of crypts or follicular glands, more from mechanical than vital action, during the later days of life, may have been sometimes mistaken for a tubercular infiltration, or possibly, as has been more than surmised, there are other bacilli which may so closely resemble tubercle bacilli in tinctorial reaction as to be 
mistaken for them. It has been demonstrated that at least one species of bacillus, the smegma bacillus, is analogous in its tinctorial reactions with the tubercle bacilli. The writer has never met with a case of primary tuberculosis of the tonsil, although his observations cover a long period of time, and include the observations of several thousand cases of various forms of tuberculous disease.

The lingual glands, or, as they are sometimes termed, the lin. gual tonsil, as well as any of the other glands comprising the lymphoid ring of the pharynx, may be the seat of tubercular disease, but such events are rare, excepting as anomalous secondary or intercurrent invasions.

Theoretically, the various surface irregularities and open ducts of the glandulæ, etc., which stud the surface of the tonsils and pharynx, together with the rich supply of lymph and blood-vessels, would seem to offer a well-prepared field for the origin and development of tubercular disease. Hence the comparative rarity of such disease in this region is one of the inexplicable facts which appeal to us, unless we accept in explanation the observations of B. Fränkel, who thinks that there is a stream of leucocytes going from within the tonsil outward which washes disease germs away.

Diagnosis of Tuberculosis of Pharynx, Tonsils, Palate, and Tongue.-On account of its infrequent occurrence, tuberculosis in this region may easily be overlooked. It may be mistaken for syphilis, lupus, stomatitis, chronic pharyngitis, carcinoma, sarcoma, mycosis, actinomycosis, chronic follicular tonsilitis, or for either of the following acute diseases, follicular tonsilitis, uvulitis, or pharyngitis.

A thorough consideration, first of the general clinical history, including the previous personal history of the patient, the accession of the disease, and the form of constitutional disturbance present, is necessary for a correct diagnosis; next, a thorough examination of the parts affected; and finally, a histologic examination of the mucous membrane when practicable.

If there be much constitutional disturbance, it is probable evidence of coexisting tuberculosis of the lungs, intestines, or some other internal apparatus, for in the beginning, at least, the local manifestations of tuberculosis do not give rise to excessive pyrexia and adynamia.

In tuberculosis of the mucous membranes the infiltration is 
usually moderate, the granulations are small, and either gray, yellow, nutmeg, or reddish in colour, without any particular areola of inflammation. The ulcerations are small, sometimes elongated, with a slightly granular or baconlike floor. There may be a few small granulations shooting out from flattened edges, like buds. There is usually little or no scar tissue. There may be no areola of hyperæmia around the patches. The surrounding tissues are generally pale, sodden, or edematous. When the ulcers are large they are very ragged and irregular, and partly covered by thickened and tenacious mucus or muco-pus.

In tonsillar tuberculosis the ulceration may be more or less hidden, but with a probe the character of it can be ascertained, so that it may be differentiated from syphilis, mycosis, and chronic follicular tonsilitis. The ulceration of carcinoma comes on late in the course of the disease, after a regular tumour formation. There are large, soft granulations, which can be easily distinguished from reparative tissue. Lymphosarcoma usually appears in large nodes irregularly lobulated, and set in greatly tumefied and hyperæmic tissue.

Tuberculosis of the hard palate, when there is necrosis of bone, is often difficult to distinguish from tertiary syphilis. It may require the test of treatment to differentiate it. The acute throat affections mentioned are to be distinguished according to the symptomatology given in discussing those diseases.

Course and Prognosis.-The course of tuberculosis in these localities is usually rapid. There is always an extreme amount of pain, unless the disease be very much localized and of slow development. The disease, therefore, will necessarily reduce the vital powers rapidly through the inability of the patient to get the proper amount of food, drink, and sleep. "In the nasopharyngeal variety the constitutional disturbance is very great, hyperpyrexia predominating throughout its course. The prognosis is always grave. Tuberculosis of the faucial tonsils is also rapid in its course.

Larynx.-Tuberculous laryngitis may occur as a primary or secondary affection. Much discussion has taken place upon the question of its primary origin, but it must be admitted that it often develops primarily. Were the disease particularly a secondary or coexistent feature of pulmonary phthisis, 
we should meet with it in nearly every case of the pulmonary affection instead of in only about thirteen per cent of such cases. It is nearly always a concomitant of acute pulmonary tuberculosis, but less seldom coexists with chronic phthisis. It is stated by the majority of authors that it occurs in about one third of the cases of pulmonary tuberculosis. Bosworth, however, thinks that the statistics of Willigk (giving about thirteen per cent) are more nearly correct. It seems to the writer, however, that no definite conclusion as to the relative frequency of "laryngeal tuberculosis" can be reached until the clinical discrepancies surrounding the terms "phthisis" and "tuberculosis" are settled.

The symptomatology varies according to the severity of the disease and the extent of tissue involved. In the early stages of those cases in which the larynx is the principal seat of the morbid process constitutional symptoms, such as pyrexia, debility, anorexia, diaphoresis, etc., may be absent or present to a slight degree, but in cases of severe general or pulmonary infection, of course, both general and local symptoms will be accordingly severe. Between these two extremes great differences will be observed, especially as the disease is quite irregular in its course and subject to remissions and exacerbations. Dyspnœa from either hyperplastic formations in the larynx or immobility of the arytenoids due to ankylosis of their joints usually accompanies the latter stages, and with this, of course, utter extinction of the voice and dysphonia.

The local subjective symptoms consist chiefly of hoarseness, dysphonia, a sense of tickling and dryness referred to the throat, more or less hacking cough, and painful deglutition. These symptoms become aggravated as the disease progresses until the patient suffers pain and distress almost continually. The pain in deglutition, however, varies very much according to the site and extent of the local disease. Ulcerations of the epiglottis and arytenoid processes generally give rise to more distress than if situated within the laryngeal cavity.

The laryngoscopic appearance also varies very much. There are two types of the disease met with in practice, the one, described by some authors as the inflammatory, in which the pharyngeal and laryngeal mucous membrane is hyperæmic and swollen, and the other, described as the anemic, in which the mucous membrane is pale and intumescent (see Plate III, 
Figs. I and 5). Between these two types various conditions are observed. The usual site of the tubercular process is at the posterior wall of the larynx, or at one or the other of the arytenoid regions, although tubercular formation may be limited to the epiglottis. The susceptibility of the posterior wall, especially that portion known as the interarytenoid space, is probably due to its peculiar anatomical formation and the structure of its mucous membrane made necessary to allow of its folding mobility. Fig. 64 , representing a section of a normal interarytenoid space, shows the folds of the surface, which are well cal-

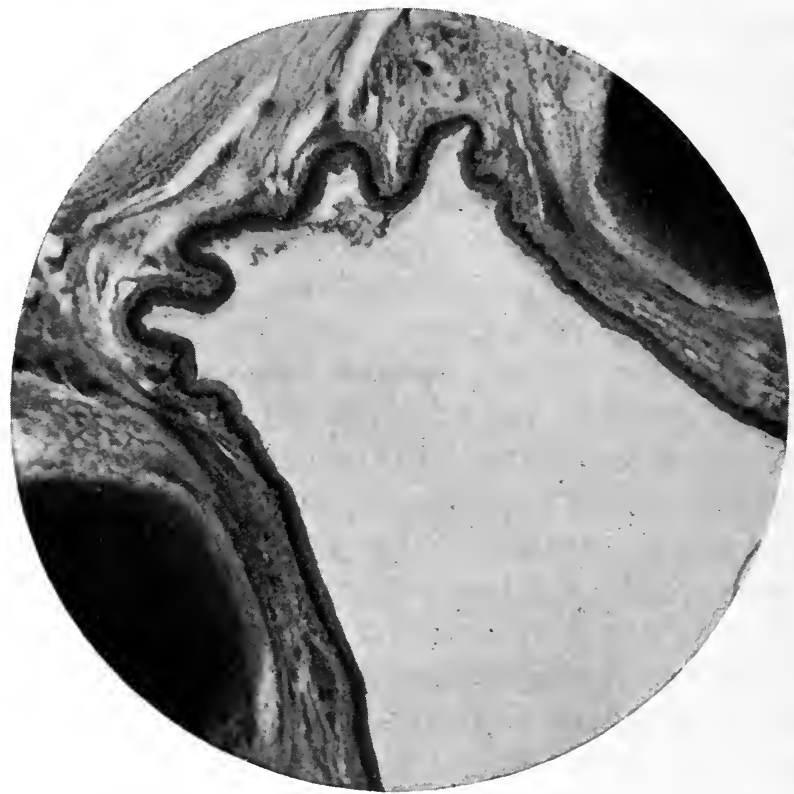

FIG. 64.-Section through interarytenoid space of normal larynx, showing the folding or corrugation of mucous membrane (photomicrograph by Dr. P. M. Hickey).

culated to give lodgment to extraneous material. This space appears swollen early in the disease, as a rule, together with either or both of the arytenoid bodies, giving to the latter a "clubbed" appearance. When the tuberculosis has followed a series of attacks of subacute or a chronic laryngitis, the swelling will appear quite diffused over the whole vestibule (or upper part) of the larynx, and sometimes, when the attack is sharp, the arytenoid bodies will take on the appearance of edematous laryngitis (see Fig. 65). A similar edematous swelling of the epi- 
glottis may occur, giving the so-called "turban" appearance to it. The swollen tissue soon breaks down, presenting a variety of pictures. When the ulceration begins it may proceed

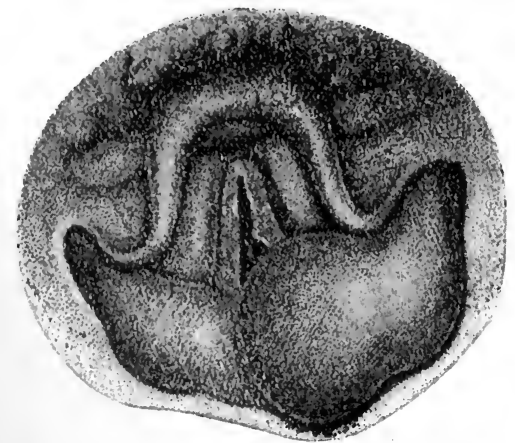

FIG. 65.-Acute inflammatory œdema of tuberculous larynx (Grünwald).

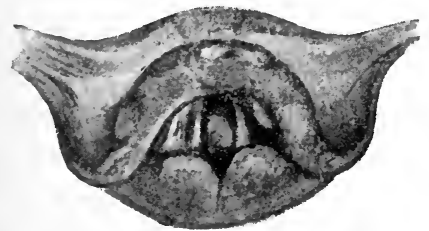

Fig. 66.-Superficial tubercular ulcers of the vocal cords and of the free edges of the epiglottis (Grünwald).

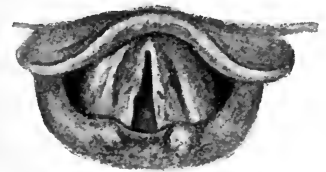

FIG. 67.-Tubercular ulceration of the glottis in the form of a girdle (Türck).

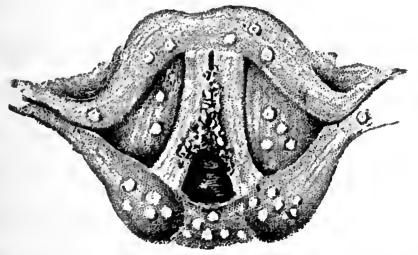

Fig. 68.-Unusual manifestation of tuberculosis of larynx (Rice).

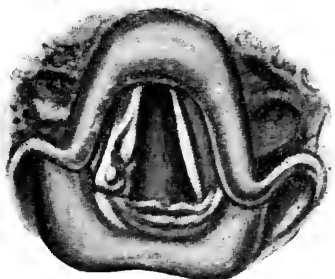

FIG. 69.-A pale larynx with swollen and roughened posterior wall and cicatricial changes about the left vocal cord, while the destructive process is proceeding in the right (Burow).

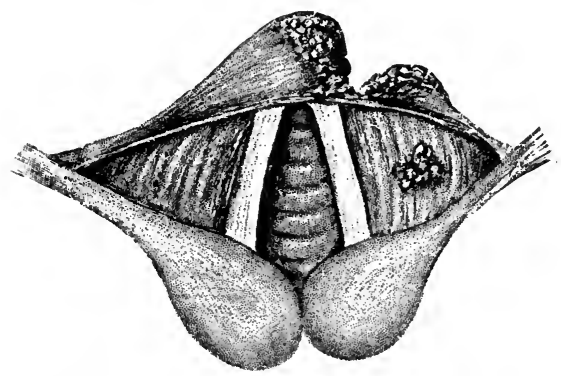

FIG. 70.-Unusual manifestations of tuberculosis of the larynx (Rice).

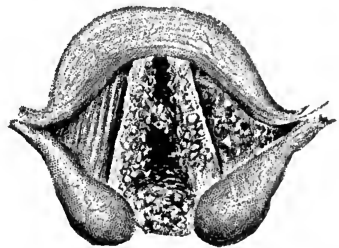

Fig. 7I.-Tuberculosis of larynx-granular hyperplasia (Rice).

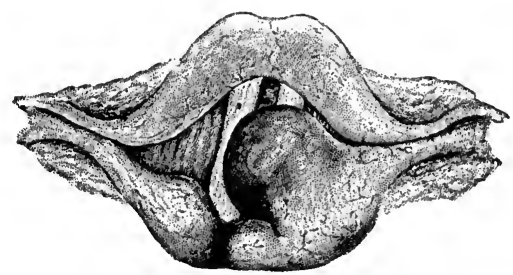

Fig. 72.-Tubercular tumour (Rice). 


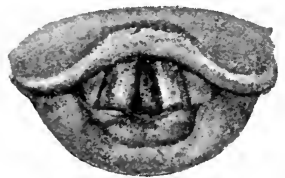

FIG. 73.-Tubercular ulceration and granulomata on the posterior wall of the larynx (Türck).

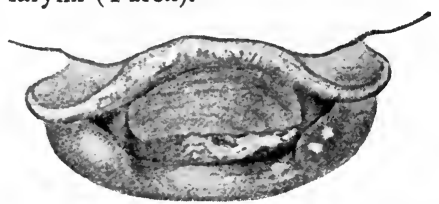

FIG. 74.-Tubercular ulceration of the posterior wall of the larynx (Türck).

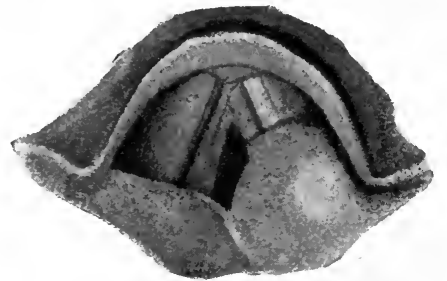

FIG. 75.-Tubercular perichondritis of the arytenoid (Burow).

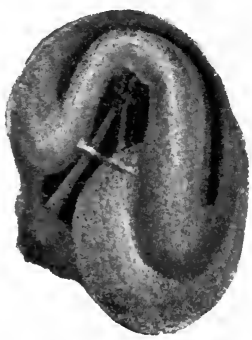

FIG. 76.-Tubercular perichondritis of the arytenoid (Burow).

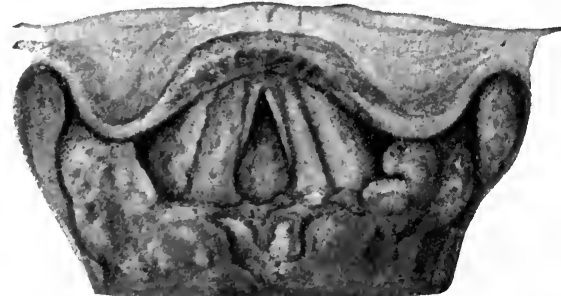

FIG. 77.-Extensive tubercular ulceration of the larynx - necrosis of cricoid with considerable new tissue formation (Türck). rapidly in one or a few spots (see Fig. 66 and Plate III, Fig. 4 ), or become quite general around the glottis (see Fig. 67). Occasionally the tubercular deposit is scattered in small nodules over the epiglottis or glottis, giving a nutmeg appearance to the parts (see Fig. 68). This appearance is more often met with in the so-called anæmic form of the disease where the tissue changes are usually slower. In such cases it is not uncommon to see ulceration, cicatrization, and granulomata forming, going on at once in the same subject (see Figs. 69 and $7 \mathrm{I}$, and Plate III, Fig. 3). Occasionally in the early stages the ulceration may be unusually deep in one or two places (see Figs. 70 and 71). The granular hyperplasia which attends the slowgoing cases may be comparatively transient, breaking down almost as soon as formed (see Fig. 7I), or remain organized into veritable tumours (see Figs. 72 and 73, and Plate IV, Figs. 2 and 3). Hyperplasia confined to the posterior wall of the larynx is frequently seen in the later stages of the disease (see Figs. 73 and 74). The appearances attending the late stage are usually quite characteristic, although sometimes presenting anomalies. Tubercular perichondri- 
tis of one or more of the laryngeal cartilages frequently occurs, those commonly involved being the arytenoid and cricoid; still, in very chronic cases and those occurring concurrently with syphilis all of the laryngeal cartilages may become affected. Necrosis and exfoliation of pieces of cartilage occasionally take place. Figs. 75 and 76 represent the laryngeal image of cases of perichondritis of the arytenoid cartilages, and Fig. 77 that of perichondritis of the cricoid cartilage with considerable hyperplasia.

The amount of destruction of tissue which may take place within the larynx is indicated by Fig. 78 .

Diagnosis. - The subjective symptoms of laryngeal phthisis, such as evening pyrexia, anorexia, small and rapid pulse, adynamia, cough, etc., will usually suffice to determine with certainty the diagnosis; but in the early stages of the disease, especially in the type known as inflammatory, when there are only slight symptoms present, it may be mistaken for subacute or chronic laryngitis or the early stage of secondary syphilis. Laryngoscopic examination will generally reveal the true character of the malady, however. The site of the swell-

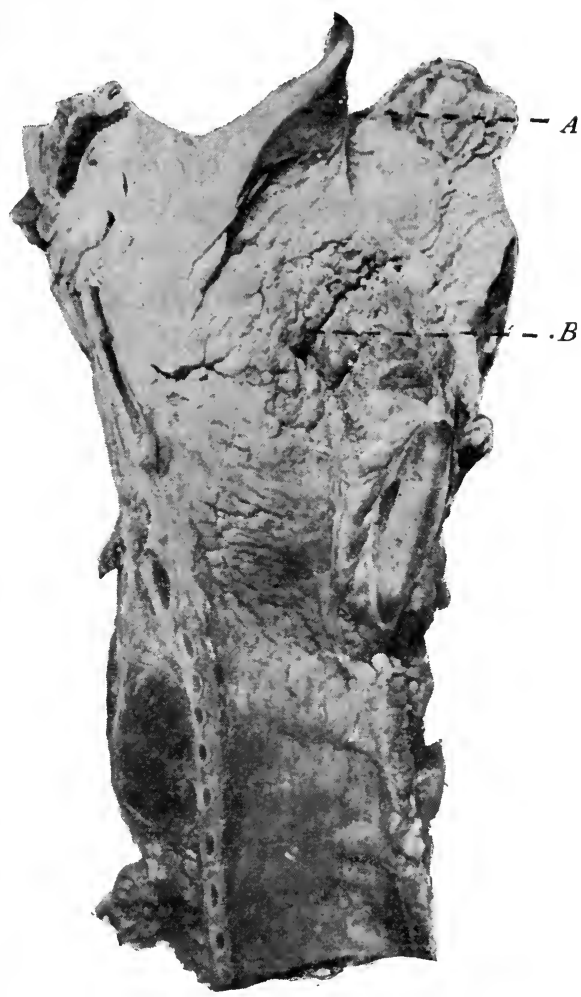

Fig. 78. - Lateral half of a tubercular larynx. $A$, epiglottis; $B$, tubercular infiltration of the interior of the larynx (author's case). ing (infiltration) in the posterior wall (arytenoid commissure) or arytenoid bodies of the larynx and the club-shaped character of the swelling in the latter situation are almost "pathognomonic signs." In the so-called anæmic type of the disease the yellowish-gray colour of the intumescent membrane, either homogeneous and smooth, or studded with lighter or deeper-coloured spots (mottled), is characteristic of phthisis (see Plate III, Figs. I and 2). 
The ulceration of the later stages is also quite characteristic, for the ulcers are small, irregular, flat, and indolent in appearance, with bacon-like floors, and with little or no areola or hyperæmia. A worm-eaten appearance is observed, especially of the epiglottis. In the late stages, when sloughing and gran. ulation are going on, it is sometimes difficult to distinguish by the appearance laryngeal phthisis from tertiary laryngeal syphilis, lupus, or malignant disease (see Plate III, Fig. 4, and Plate IV, Fig. 4). The clinical history, however, in such cases will usually settle all doubt. In this connection the reader is referred to the excellent table of Dr. Gibb, on page 262 , for the points of differential diagnosis between the diseases just mentioned.

Course and Prognosis. - The course is variable, according to the extent of the disease and the resisting powers and environment of the patient; the prognosis is likewise somewhat uncertain, although the majority of persons suffering from this malady in a severe form do not recover. The so-called inflammatory form and cases of undoubted miliary tuberculosis usually run a very rapid course to death. The writer has met with many cases in which the disease reached a fatal termination in from four to six months, although the disease was recognised early, before marked constitutional symptoms had made their appearance.

Treatment.-The treatment of this affection is usually classified as general and local. The general treatment may be considered under the head of climatic, hygienic, specific, and symptomatic; and the local treatment may be discussed under the following captions: local medication by inhalation, sprays, or insufflations, and surgical treatment.

The influence of climate in modifying all the tuberculous diseases is well known, and need not be discussed at length. However, in no form is it more disappointing than in that of active laryngeal tuberculosis. Usually a warm, moist climate is the most beneficial in the majority of cases, provided it is not too enervating, such as a tropical or subtropical one. The dry, warm climates of Arizona, New Mexico, and some parts of California, do not seem to suit all of these cases, perhaps on account of the dryness of the air, for in many instances the laryngeal symptoms seem to grow worse in such localities. When a dusty or windy feature is characteristic of the climate 
of the locality, the discomfort and distress are sure to be increased (Ingals). With regard to the altitude, it may be pointed out, as a rule, that only a moderate elevation is well tolerated by many subjects. A large majority of those who dwell above three thousand feet are apt to suffer extra distress, if not exacerbations of inflammation of the larynx; this is especially true of the well-marked inflammatory and hyperplastic cases. There are, however, many exceptions, especially when the local lesion is in the early stage, or when much infiltration and ulceration are present. Strange as it may seem, cases showing but little involvement of the lungs are not as much relieved as those in which there is a notable involvement of those organs, such as softening or excavation. A cold, dry climate, with plenty of sunshine, has been found generally to be more beneficial than a highly elevated, dry, or dusty one. Sometimes, however, when the cold is very intense, the laryngeal mucous membrane seems to be irritated thereby, especially if there be much hyperæmia of the throat. In short, according to experience, a windy, dusty locality is not suitable for cases of laryngeal phthisis, except when there is more or less ulceration of the larynx or caseation of the lungs going on. A moist, warm climate, or ocean voyages, would therefore seem preferable as a rule. Of course it will be understood that the individual, and the individual circumstances, may furnish valid exceptions to any rule. The foregoing remarks are applicable also to pharyngeal, nasal, and tonsillar tuberculosis.

The hygienic treatment consists for the most part in proper clothing, not too frequent bathing, and the administration of nourishing but digestible food, out-of-door life as far as possible during the day, with a cool, but not too cold, sleeping apartment. Patients with this disease should be very careful about exposure to coal gas for any length of time. The use of tobacco, either chewing or smoking, ought to be prohibited; also the too frequent use of cold and hot drinks in close alternation, such as ice water and hot tea or coffee at meal-times. The full quota of sleep each twenty-four hours should be obtained. Patients should be enjoined to spare the voice as much as possible in conversation; singing and public speaking ought to be prohibited. I have known several instances where patients in the early stages of laryngeal phthisis, presenting certainly good prospects for at least temporary recov- 
ery, have been thrown into almost a fatal exacerbation by the strenuous use of the voice. One of these cases, I may briefly relate was that of a clergyman who was making an apparently good recovery. He had been away for some months from his duties, and had returned to his residence in a district containing a great many friends among the congregations of several churches where he was well known. About the time of his return there was considerable church activity and public agitation of special moral questions concerning the district in which he lived. As he had been a popular and efficient pulpit orator, lecturer, and worker, he was persuaded to take an active part in these events. After making three prolonged and rather vehement orations in three adjacent towns within a period of a week or so, he contracted a violent inflammation of the larynx, which renewed the exudation into the tissues, and ulceration affecting permanently the integrity of the larynx ensued, necessitating his immediate removal to the South, where he expired in about nine months. A similar effect occurred in the case of a prominent lawyer whose case bade fair to end in recovery. He was induced to return from a sojourn in the West Indies to take part in some important cases, and after finishing this task a severe inflammation of the larynx with great tubercular infiltration followed. Many like instances could be given to show the necessity of rest, as absolute as may be, in the management of laryngeal phthisis or tuberculosis.

The specific treatment of laryngeal tuberculosis has not yet arrived at the perfection which we would wish. A great many agents have been brought forward from time to time which have undoubtedly been attended by a modicum of good results, but owing to numerous failures they have been gradually put aside. The seekers of truth in this field of discovery are numerous, however, and we may with reason hope that, guided by past failures and partial successes, the right therapeutic measures may yet be reached. Among the more modern agents of supposed specific value may be mentioned creosote, guaiacum, tuberculin and its various modifications, dog serum, goat serum, horse serum, plain and tuberculized, nuclein, chloride of gold and sodium, chlorine water with chloride of sodium, oil of cloves, cinnamon and other essential oils, chloride of zinc, the formates, iodine, etc. There are many cases 
on record of moderate and even brilliant results following the use of each of these agents, both in laryngeal and pulmonary tuberculosis, but, as said above, the effects have been short of general expectation. The writer is led to the conviction, from experience with these several agents, that they are perhaps less useful in a specific sense in laryngeal than in pulmonary tuberculosis. These agents have all been used in the general belief, of course, that all cases of laryngeal tuberculosis were simply the expression of a general infection. But undoubtedly some cases reported to have been cured have been aggravated forms of ordinary chronic inflammation of the larynx, while some may have been cases of chronic laryngitis complicated by syphilis. The writer has been deceived once in a while by such an erroneous diagnosis, and undoubtedly others have met with the same misfortune. These remedies have been used both hypodermically and per os. While the hypodermic use of such remedial agents is manifestly more desirable, the attending pain and difficulty of properly carrying out this plan of medication renders its general adoption of doubtful practicability, especially in private practice.

I am still of the opinion that, among the agents mentioned, iodine when conjoined with some proteid substance furnishes the best results, so far as specific medication is concerned. It seems to make some difference in its effects whether or not the drug is administered in solution with iodide of potassium, in glycerine, and water, and mixed with some proteid material, such as beef tea, for I have found repeatedly that the administration of the drug, either hypodermically or by the mouth, in a mixture with bouillon, will produce more lasting benefit than when given without it; so that we have adopted the habit in hospital practice of administering it in bouillon or milk. Iodine may also be given advantageously in combination with glycerine and extract of malt. I have also used creosote and guaiacol in the same way with apparently better effects than when given with water; we may therefore be pardoned for the suggestion that perhaps the majority of remedies administered for constitutional effects (if there are no chemical contra-indications) would be more efficacious when combined with some proteid substance. Whether a chemical combination actually takes place between these agents and a proteid of the animal fluid used, I am unable to say, but possibly at 
some future time this point may be satisfactorily demonstrated. Guaiacol and oil of cloves are both very useful when administered in an emulsion in suitable doses three or four times daily, or (sometimes a better way) in very small doses repeated every hour for a certain number of times, according to the tolerance of the patient. The former agent as "benzosol" has given very good results. Guaiacol may also be combined with iodine and olive oil. In the more chronic cases, characterized by anæmia of the mucous membrane, phosphorus administered in a solution with olive oil in capsule is highly beneficial. This combination may be found on the market in the form of capsules containing from one one-hundredth to one thirtieth of a grain of phosphorus in ten to fifteen minims of oil. It should be administered always just after food has been taken, and if irritation of the stomach, urticarious eruption, or aphrodisiac effects follow its use, the dose must be materially modified, or the administration of the drug stopped. Arsenic, especially arsenate of iron, chloride of gold and sodium, salicin, and strychnine are very valuable tonic agents. Strychnine and salicin are among the most useful agents for general effects. When there is much hyperpyrexia, salol or sodium salicylate may be substituted for salicin. For the temporary repression of temperature, acetanilide or aconite or judicious bathing may be resorted to. The somewhat prevalent practice of combining either of these agents with other things, sometimes not synergistic, is certainly a bad one. Of course this criticism does not apply to mixtures containing sedatives such as codeine or hyoscyamus, but, as a rule, it is preferable to administer all drugs designed for temporary effect, alone. The use of alcoholic beverages in this disease is fraught with perplexity, for while in many instances alcohol in one form or another is indicated, yet the condition of the throat may be such as to interdict its use, either on account of the local pain attending its deglutition, or on account of its decidedly deleterious effect upon the diseased pharynx or larynx. It will be found better generally to select the malt beverages and preparations rather than the spirituous. The administration of a little rectified spirits with milk, however, will be found useful when large draughts of whisky, brandy, rum, etc., cannot be consumed without distress or evil effects.

The symptomatic treatment of laryngeal tuberculosis can 
scarcely be formulated, and will depend upon a variety of circumstances which will indicate the agents to be selected for each individual case; they are chiefly agents to allay cough, pain, hyperpyrexia, diarrhœa, mental perturbation, etc.

As laryngeal tuberculosis, when advanced, is perhaps one of the most painful affections to which flesh is heir, the practitioner may be sorely taxed to meet the constant demand for relief without incurring other mischief. However, opium and its salts, for internal medication in such cases, still retain their popularity; codeine, morphine, or the powdered drug may be employed. Cough may be mitigated by the milder anodynes, such as codeine, tincture of opium, hydrochlorate of cocaine, hydrate of chloral, etc., together with the various expectorants and balsams (see formulary). Care should be taken that sirups are not used too freely, lest nausea, vomiting, and anorexia are produced. Chloride of ammonia and other chlorides may cause severe pain when the mucous membrane is ulcerated, otherwise they may be used. Sleep may be secured by the administration of sulphonal, trional, chloral, and bromides, or some preparation of opium, alone or in combination with one or the other anodynes mentioned.

The local treatment consists of the inhalation of gases or medicated air, sprays, and powders. Inhalation of medicated air is of little permanent benefit excepting in the early stages, or when there is an unusual amount of salivary or other expectoration. There are many suitable inhalers on the market from which volatile substances may be inhaled, either with or without the aid of hot water. A simple instrument may be made of tin, with a perforated receptacle at its distal end for holding a piece of sponge, upon which may be dropped preparations of creosote, carbolic acid, menthol, camphor, creolin, tincture of benzoin, tincture of myrrh, etc., combined with chloroform, ether, alcohol, or spirits of ammonia (see Fig. 215). These agents may also be dropped into hot water contained in an inhaler (see Fig. 214). These instruments can be used by the patient several times a day, with sometimes very good effects in the early stages of the disease. When, however, this form of inhalation increases the sensation of "dryness," "stiffness," or pain referred to the throat, they should be discontinued. A preferable method of inhalation in general is accomplished by spraying the medicaments into the throat from either a 
steam or a hand-ball atomizer. The spray may be projected directly into the throat, or (especially if it be oleaginous) into a globe inhaler (see Fig. 213), from which it can be inhaled through a face shield completely inclosing the nose and mouth. This is a much more thorough method of local treatment for laryngeal phthisis, especially when the agent is in the form of oleaginous solution. All such agents as creosote, camphor, menthol, carbolic acid, guaiacol, creolin, phenol, oil of eucalyptus, etc., may be dissolved in a pure fluid petrolatum or olive oil and projected into the inhaler, whence they can be carried into the larynx by the ordinary process of respiration. Acetozone can be dropped into hot water and inhaled. Although this means of local medication may fail in producing radical change, it nevertheless affords efficient relief. Watery solutions are best used by spraying directly, as near as possible, upon the parts. In this manner there may be used with tolerable efficiency solutions of formate of soda, biborate of soda, alum, boric acid, carbolic acid, cocaine, morphine, hydrochlorate of coniine, tannic acid, sulphate of iron, sulphate of zinc, nitrate of silver, chloride of zinc, etc. These chemicals may be used in varying quantities, according to the requirements of the case (see formulary). Insufflations of powders upon the larynx, although of great value, especially when ulceration has taken place, are sometimes not well tolerated, on account of the desiccation of the secretions or the prolonged and distressing paroxysms of cough which they may produce. Among the agents most useful as insufflations in laryngeal tuberculosis may be mentioned iodoform, iodoform with boric acid or bismuth, naphthaline, aristol, europhen, boric acid, tannic acid, ammonio-citrate of iron and potassium, stearate of zinc or alum, subnitrate or tannate of bismuth, morphine with gum acacia, etc. The insufflations should always be preceded by a thorough spraying of the parts with some detergent solution such as biborate of soda or phosphate of soda, and care should be taken that the amount of powder propelled upon the larynx is not too great, otherwise distressing spasm and cough may supervene, lasting a considerable time, and producing not only extra congestion and irritation of the parts, but perhaps vomiting and exhaustion. It is often desirable to apply to ulcerations certain medicaments in the form of pigments. This may be done preferably by using the Wagner 
laryngeal brush, or a pledget of absorbent cotton, either held by forceps or wound around the roughened end of a probe or applicator. In this way solutions of nitrate of silver, chloracetic acid, lactic acid, carbolic acid, chloride of iron, chloride of zinc, formaldehyde, creosote, formate of sodium, ichthyol, argyrol, etc., may be carried to the parts.

The range of surgical measures for the relief of this disease is necessarily limited. The papillomatous excrescences which sometimes accompany progressive ulceration may have to be removed by evulsion, snaring, or escharotics. Especially is this the case when-such protuberances occur about the vocal cords or ventricular bands, and by their presence obstruct respiration or produce great mechanical irritation of contiguous parts. A few years ago Dr. Heryng promulgated the plan of thoroughly curetting tuberculous ulcers, and afterward applying to the surface so abraded lactic acid in twenty-, thirty-, or fifty-per-cent solutions, or stronger, according to effects (see Chapter XVIII). This met with success in the treatment of some cases. There is no doubt that it may be a very efficient and practicable measure in cases of isolated ulceration in the upper part of the larynx, but for a large proportion of cases of laryngeal tuberculosis presenting no limited lesions, but a simultaneous breaking down of many spots separated from each other, this plan of treatment will necessarily be limited in its application. Besides this, many times the lesions will be found out of reach; then, too, there are individuals in whom for some reason these parts are too sensitive for interference of that sort, even under the influence of cocaine, without exciting a general disturbance which is not easily allayed. This was my experience in two favourable cases, when I was obliged to desist after very little manipulation. The application of lactic acid without the curettement is often well borne and efficient. Chappell advises curettement, and the application of pure lactic acid or pure ichthyol, or twenty grains of silver nitrate afterward. Scarification of the larynx in the chronic forms of the disease is a measure which is not practised, perhaps, as often as it ought to be. The writer called attention some twelve years ago to this plan of relieving the tension of the parts, and many practitioners who have adopted it have reported good results. It ought not, however, to be indiscriminately adopted, because the objection 
urged against the practice, viz., that it opens up the deeper tissues to further infection, holds good in many instances. However, as ulceration in any event is inevitable, especially after great infiltration, it will be found that the anticipation of Nature's step in this direction by scarification will greatly modify the subsequent necrosis of the tissues, and at the same time enable the practitioner to meet the prospective condition by more thorough medication. I would therefore still advise, in some cases of inordinate swelling, even though it be not considered edematous, a reasonable amount of scarification over the tumefied parts, whether it be the arytenoid cartilages, interarytenoid space, ary-epiglottic folds, or epiglottis.

Hypodermic injections of creosote or other agents into the laryngeal mucous membrane have been in my hands very disappointing, although the practice, according to Chappell, has given some good results. See Chapter XVIII, Methods of Local Treatment.

It must be confessed that local treatment in many cases is of little value excepting for the relief of pain and other phases of distress, mainly, perhaps, because the lesions are more or less out of reach and affect too deeply the mucous membrane. On the other hand, in some of the slower-going cases, characterized by limitation and accessibility of the lesion, a judicious local treatment constitutes our principal means of assisting Nature to resist the progress of the disease. We must agree with Gleitsman that, theoretically, the thorough removal of the diseased tissues by surgical means and the induction of a healthy reparative process is the ideal desideratum, but, unfortunately, there are as yet practical difficulties in the way of successfully carrying out such aims. So we are still, as it were, obliged to drift along empirically, without exact principles of therapy to follow in the management of this disease. It will therefore be impossible to lay down any rules for the application of this, that, or the other agent or preparation, and the question of the selection of topical medication must be left to the skill and experience of the practitioner. It may be added, however, in conclusion, that of all agents for general use, perhaps none surpasses iodoform, aristol, resorcin, tannin, mercury protochloride, used either by insufflation, or in solution, or in a mixture with petrolatum oil. Next in value, especially when ulceration has begun, may be ranked lactic, chloracetic, and 
carbolic acids, and after these chlorine water, sodium formate, silver nitrate, zinc chloride, mercury bichloride and biniodide, creosote, argyrol, and oil of eucalyptus. The inhalation from a face-shield inhaler of one ounce of a solution of mercuric chloride ( 1 to 3000 ), immediately followed by an inhalation of from two to four ounces of a solution of (one to two per cent) iodoform in petrolatum oil, will oftentimes prove highly beneficial. The inhalation of chlorine water combined with a solution of salt in water is also very efficient when it does not cause much pain or coughing. Usually from one half to one ounce of each (mixed) is as much as ought to be given at a séance.

One of the leading therapeutic indications is the assuagement of pain and laryngeal distress. For such purposes we must resort preferably to the local application of either cocaine, coniine hydrochlorate, bromoform, morphine, formanilide, atropine, aconitine, chloroform, menthol, ethyl bromide, creolin, or similar agents. These substances are best used in the form of spray or pigments, excepting ethyl bromide and chloroform, which are volatile (see formulary).

In cases where much soreness is generated from a more or less continued muscular action of the pharyngeal and laryngeal muscles, coniine hydrochlorate, menthol, or ice (held in the mouth until melted) will be found beneficial. For promoting - an antagonistic effect on the sensations of burning and rawness complained of, either cocaine, aconitine, atropine, or hyoscine, will be found useful in addition to other treatment.

Cocaine hydrochlorate, or morphine alone or in combination with either iodoform, aristol, naphthaline, or carbolic acid, will be found to be the "sheet-anchors" for the relief of pain. The frequent cleansing of the throat with sprays containing peroxide of hydrogen, boric or carbolic acid, when well borne, is a good practice, and one which greatly promotes the comfort of the patient, to say the least (see formulary).

\section{Lupus of the Upper Air Passages}

\section{Nasal, Pharyngeal, and Laryngeal}

Lupus consists in the development of small peculiar nodules (lupomata) in the mucous membrane, which afterward undergo absorption (resolution) or ulceration, leaving in their 
site a peculiarly striated cicatrix. There are several varieties of lupus described by dermatologists, according to location and extent, but clinically they are all embraced in either one of two well-known types, viz., lupus vulgaris and lupus erythematosus. Lupus vulgaris may cause deep-seated destruction of tissue or be quite superficial. The former condition is known as lupus exulcerans (or lupus exedens), and the milder form as lupus nonexedens. It is usually lupus vulgaris which affects the mucous membranes.

Lupus erythematosus received its name from Cazenauve, on account of its healing with the formation of scar tissue similar to lupus vulgaris. Many modern dermatologists and pathologists, however, think that it should not be classed with lupus because tubercle bacilli have not been found in the tissue formation of its eruptions. Unna, for instance, suggests that it be called "seborrhœa congestiva," and not classed with the tubercular affections; but Besmier, of Paris, and some others, maintain that it is a tubercular affection, and therefore should retain its present place in classification. Leloir also believes that it is a tuberculosis, and capable of reproducing itself by inoculation (see his classification).

Andry reported a second case of erythematous lupus in which the lesions presented the particular histological features -i. e., tubercles were found. The case was one of erythematous lupus in character, observed in a young woman twentyseven years of age. The lesions found were not characterized anatomically as tubercular, and were few in number, it being necessary to examine a large number of sections to find them. Tubercle bacilli were sought for without success. In spite of the absence of bacilli, he believed the case to be of tuberculous nature, and an ordinary ery thematous lupus. The disease commonly attacks and is confined to the skin, but when the site is in the neighbourhood of any of the orifices of the body, such as the nares, mouth, ears, vagina, or rectum, it is apt to extend and involve the contiguous mucous membrane. Exceptionally, however, it affects the mucous membrane principally, in which case the nasal or buccal membrane, soft palate, pharynx, larynx, conjunctiva, rectum, or vagina may be the part affected. Leloir's statistics show that, out of a total of three hundred and twelve cases of lupus, the mucous membrane was involved one hundred and nine times. He believes that primary lupus 
is more common than is supposed, and escapes notice. Neisser concludes that lupus of the face is generally caused by extension from adjoining mucous tracts, especially from the nose. In the last seven or eight years carefully studied cases have made it probable that from twenty to forty-five per cent of all cases of facial lupus really begin in the contiguous mucous membrane (Jonathan Wright, Carrier, Bendar). Fifteen years ago Lefferts predicted that more careful observations would reveal many cases of lupus of the throat. For a number of years it was thought that lupus of the mucous membrane of the nose was always an extension from the outside. Cases of lupus confined to the throat, pharynx, and larynx have been reported since Von Ziemssen's case in 1876 by Harland and Obertlischen, and of the tongue by Clarke and Leloir. Chiari and Riche, in 1883 , reported thirty-eight cases of lupus of the larynx. A case of primary lupus of the pharynx was reported by Jonathan Wright.

Such regions as the gastric, intestinal, or bronchial are not subject to invasion, although it is reported that the disease has been observed on the mucous membrane of the trachea.

Undoubtedly primary lupus of the nasal passages, pharynx, and palate is quite rare, the disease when present in these regions usually having extended from the outside.

Nose.-The nasal mucous membrane is sometimes the starting point (Jonathan Wright). It is usually confined to the neighbourhood of the external nares, but occasionally extends along to the retro-nasal region. The disease when located on the septum soon causes perforation of that structure.

Primary lupus laryngis is rare, many laryngologists of large experience not having seen a case, while others have seen very few; among these M. Mackenzie mentions having seen two, Lefferts four, and Rice three. As the invasion is insidious, and the signs of the same very obscure, many observers believe that it occurs in the larynx oftener than is supposed, especially as the disease generally attacks the young. Among them R. De la Sota y Lastra and Rice make this suggestion. The epiglottis is generally the part attacked. In thirty-eight cases reported by Chiari and Riche the epiglottis was found free in three only.

Symptomatology.-The constitutional disturbance coincident to this affection is practically slight, unless the disease be 
so situated or so advanced as to interfere with the functions of deglutition or respiration, or unless complications attended by unusual inflammatory or septic processes going on in the neighbouring glands or tissue supervene. Such complications are, however, rare until the disease has run a long and aggravated course with great destruction of tissue, for usually there is little tendency to general infection, although Leloir and other authors state that infection may occur at any time during suppuration. Of course, if pulmonary phthisis complicates the disease constitutional disturbance will occur. As a rule, the subjects of lupus feel quite well, although a little weakened.

The subjective symptoms, when the disease is located in the fauces, are principally more or less difficulty and pain in deglutition, and frequent desire to hawk and expectorate. Difficulty in the enunciation of words is also experienced when the swelling of the parts is at all considerable, or if there be much ulceration. Of course, if the invasion of the fauces be extensive, there will be a notable aggravation of these symptoms. When the larynx is involved, unless the disease be limited to a small portion of the epiglottis, there will be some hoarseness and a sense of tickling, dryness, or swelling complained of, together with some pain or difficulty in deglutition. When the disease is advanced or extensive, dysphonia, or aphonia, and increased pain and soreness of this region will become marked symptoms. In such an event, more or less pyrexia and an increased general weakness will be observed, to which may be added more or less dyspnœa.

Appearances. Nose.-Lupus generally appears at first in small patches, like an erythema, but on close inspection the patch will show a granular or papular surface, the granulations being small, about the size of a millet seed, and either of a yellowish-gray or red colour. There may be two or three of these patches at one time, about the size of a split pea. They may continue discrete for a time, or soon coalesce at some portion of their edge. The tissues around are somewhat swollen, although in the nasal passages, perhaps, there is at first less infiltration than when the disease is upon the pharynx, soft palate, or larynx. The swollen tissues of the parts are usually of a grayish colour, and appear not unlike sodden turbinate tissues. The secretion is not very great, and soon desiccates into crusts ; indeed, in some cases there seems to be a lack of secretion (see 
Fig. 79). As the disease advances the whole nose becomes more swollen and tender, and possibly an erythematous inflammation of the skin of the ala may appear, while the nasal passages on the affected side become blocked with crusts and the swelling of the surrounding mucous membrane. The granulations (lupomata) appear larger and soft, and soon they seem to melt away by a process of slow suppuration, and leave sinuous, glistening tracks of cicatricial tissue. If the disease be advancing circumferentially, fresh patches will be seen in proximity to the ones ulcerating, which of course will go through the same process. Oftentimes the course of the disease is so slow that for weeks there will be little change noted in the appearance of the patch from day to day. On the other hand, if the disease take a rapid course, serpiginous prolongations may be seen jutting out from day to day, or numerous independent patches will be coalescing and breaking down, so that the destruction of tissue will be considerable. Dr. L. Mackall, Jr., of Washington, reports a case in the Medical News (vol. lxvii, p. II) of advanced lupus vulgaris of the face occurring in a girl eighteen years of age. It apparently began in the nose, and after six years attacked the outside. At the time of her visit the nose was

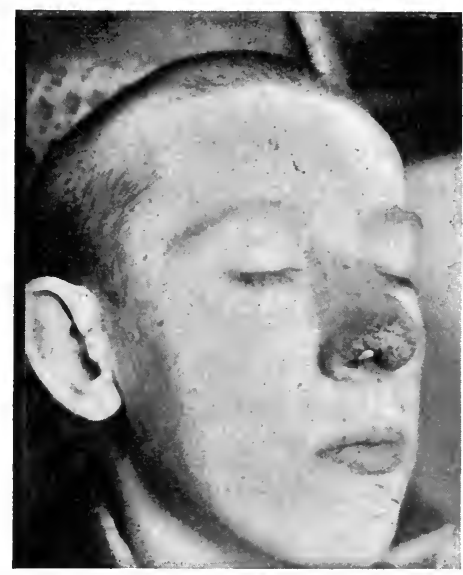

FIG. 79.-Lupus vulgaris of right nasal passage and ala of nose. entirely gone (being represented simply by a cavity), as well as some portion of the eyelids. Usually, however, the exacerbations are of short duration, giving place to long intervals of cicatrization.

Pharynx and Soft Palate.-Lupus may invade any place about the fauces. The favourite sites for the disease are the posterior wall, the faucial arches, and either the outer or posterior surface of the soft palate. Jonathan Wright thinks that primary lupus of the soft palate and pharynx is more common than is supposed. He reports an interesting case of lupus of the pharynx in a male seventeen years of age, in which there was no history of hereditary tuberculosis, cancer, or syphilis, 
nor of acquired syphilis. The local appearance of lupus is generally much less marked on the mucous membranes than on the skin.

The lupus nodules are usually quite hard in this region, and of a yellowish colour at first. There is more infiltration of the surrounding tissues, however, which gives the appearance of great anæmia. When the nodules break down the ulceration is apt to be irregular and serpiginous. There is considerable difficulty in deglutition if the disease be upon the velum palati or faucial arches. The tonsils usually escape invasion altogether. There is generally enlargement of the cervical glands, the diseased part is hard, and is not very sensitive to the touch. The contraction of the resulting cicatrices in this situation is quite great, and cause the patient much annoyance when fully developed. The course of the disease here, as elsewhere, is about the same-slow and intermittent.

Larynx.-The lingual face of the epiglottis is usually at first affected, and presents a slightly swollen hyperæmic condition with a few papular projections like granulations. In some cáses the free border of the epiglottis appears whitish or gray, thickened and indented here and there with dark-red papillomatous formations. After a while the laryngeal vestibule becomes, from infiltration, etc., thickened and misshapen, presenting circular folds or rugæ similar to a slightly prolapsed rectum, and studded here and there with grayish glistening fissures and dark-red papules, which may be coalescent in places, forming a patch. These are not very painful to the touch. At the later stage these spots become softened, and soon show ulceration, with ragged edges and reddened granular areola (see Plate II, Fig. 2). According to Leloir, and contrary to Baumgarten, suppuration of the lupous patch is not an essential characteristic of lupus, but is due to the confined action of the agents of suppuration and the bacillus of Koch. If the seat of the disease be the border of the epiglottis, it may appear as if worm-eaten (Lastra). The lupoma, or elemental lesion, may take the nodular form, or be quite diffuse. Even now the swelling and hyperæmia of the surrounding parts may be quite insignificant, for the infiltration seems undulatory and chronic, and the general condition of the patient may remain good in every particular. The duration of the disease is slow, may cover a number of years, and may terminate in pul- 
monary or meningeal tuberculosis or epithelioma (Morrow), although not necessarily. At the same time the local process is subject to great variations of exacerbation and quiescence, when affecting the mucous membrane as well as the skin. A retrogression is marked mainly by a gradual healing of the ulceration with the formation of a thin, bluish-gray, glistening cicatrix, somewhat more moist and duller, however, than the lupous scar tissue of the skin, and surrounded by thickened striæ of mucous membrane. Then, after a period of quiescence more or less prolonged (which in some cases leads one to the conclusion that the cure has been effected), there is a recrudescence which at the time cannot be accounted for by any event in the domestic or clinical history of the patient. It will be observed to start by the development of two or more foci of papillary swelling and inflammation, starting from the periphery of the old lesion, which perhaps go through a more aggravated course of softening, tumefaction, and ulceration, etc., accompanied with more or less extensive infiltration of surrounding tissue as before. Indeed, some cases present a greater severity of local action at each exacerbation, so that the ulceration and inflammation may become alarming, and perhaps assume the serpiginous form spoken of by some writers, an event which is quite dangerous, and apt to be extensive in its ravages, simulating, to a certain degree, lupus exulcerans (lupus exedens) of the skin. Besides this, the contraction of the cicatrices from such an amount of tissue destruction leaves troublesome and sometimes vicious deformities, which may endanger life by interference with the laryngeal or esophageal openings (stenosis) (see Plate IV, Fig. 6).

Etiology.-Lupus was formerly regarded as related to "scrofulous cancer," if not really cancerous, mainly from the observation that some cases became cancerous; it was also thought to be a sequence of syphilis. Ricord held that it was a manifestation of inherited tertiary syphilis. Kaposi, however, says positively that syphilis in a parent has no connection with lupus in the children. Moreover, Hebra and Kaposi have found recent syphilis and lupus in the same individual. Some authorities have believed, and still do, that lupus vulgaris, especially in the skin, belongs to the scrofuloderma-that it is essentially a scrofulous disease. Kaposi and others combat this idea, and say that scrofula is entirely absent in many cases 
of lupus. The latter view is to some extent prevalent nowadays, but as so-called scrofula is generally considered to be of a tuberculous nature, this view would coincide with the prevailing opinion that lupus is a local tuberculosis, and therefore allied to both scrofula and tuberculosis. Indeed, it is so called by a majority of modern dermatologists and pathologists, and may be found in the category with tuberculosis, verrucosa cutis, scrofuloderma (tuberculosis of the subcutaneous tissue), tuberculosis cutis, etc. The tubercular nature of lupus was suspected for years before the discovery of the tubercle bacilli of Koch, on account of the histological analogies between these several affections, as persistently pointed out by Friedländer and others, but the hematogenetic origin of lupus was not assailed until the discovery of tubercle bacilli in lupous tissue (lupoma) by Demme, and afterward by others, when all doubt ceased in the minds of the majority of observers, and the tubercle bacilli (as in other tuberculous diseases) became the recognised essential cause of lupus. Moreover, these views have been strengthened from time to time by experimental and other observations, apparently showing the inoculability and infectiousness of lupous tissue, for J. Jadassohn, Leloir, and others, have cited instances of the production of lupus by inoculation, and Leloir and several others claim to have demonstrated this by the transmission to guinea-pigs and rabbits of tuberculosis by inoculation with lupous tissue or cultures. They regard every case of lupus vulgaris as due to inoculation with the tubercle bacillus, and deny the so-called hematogenous development of the disease as promulgated by Baumgarten. The starting point is the entrance into the skin or mucous membrane of the microbe. It probably takes place in one of the following ways:

I. Direct inoculation from without.

2. Indirect inoculation by continuity from deep tuberculous foci.

3. Inoculation by way of lymphatics or the veins passing through a tubercular focus more or less remote.

4. Infection of hematic origin.

5. Infection by inheritance.

Methods I and 2 are probably the most frequent. Shoemaker says that although the origin of lupus has been generally ascribed to the tubercle bacilli, yet these micro- 
organisms "are present in a confessedly small number of cases." He has often searched for them in vain. He further believes that lupus does occur in a certain proportion of tuberculous subjects, but that such cases are in the minority. "In human beings cases of direct local inoculation of tuberculosis in the skin and accessible mucous membranes have been reported, but they are not very common" (Prudden and Delafield, p. 223).

It is now looked upon by many as a form of cutaneous tuberculosis, although it is admittedly of a different clinical type from other forms of tuberculosis, and its lesions contain few bacilli. In some instances it occurs in connection with pulmonary tuberculosis.

Kaposi says: "No experimental proof has been offered that characteristic lupus vulgaris can be produced by inoculation with tubercle bacilli. The inoculation of lupous tissue and of the bacilli derived from it has given rise to inflammatory products, but not to lupus, nor has any clinical proof been found, unless we accept extremely careless statements. This is less true concerning a few of the published cases, such as that of Besnier (lupus in vaccination scar) and Sachs (lupus after piercing the ear). These and other similar although less carefully observed cases might demonstrate the occurrence of lupus from inoculation of tubercle bacilli." Dr. J. M. Winfield, of Brooklyn, N. Y., reported the occurrence of lupus vulgaris in the wife and daughter of a tuberculous subject. Dr. Winfield believes that the woman and child became inoculated with tuberculous material from the father, who died of phthisis pulmonalis. His belief is based upon the fact that this man was very careless about expectorating on the floor where his child was creeping about, and into a broken spittoon, and wiping his mouth with a towel which was used by his wife and child. Koch demonstrated that lupus tissue and tissue cultures of tubercle bacilli in abrasions of the skin produced a local tuberculosis. Cornil and Leloir implanted lupus tissue in the peritoneal cavity of guinea-pigs, and in five out of fourteen cases induced general peritoneal tuberculosis. In all the cases of Leloir and Cornil, and in four cases of Koch which were mentioned up to the time, the identity of lupus and tuberculosis was proved. Tubercle bacilli were found in every instance. Doutrelepont, Pfeiffer, Koch, and Pagenstecher found 
tubercle bacilli in artificial lupus, caused by implanting tuberculous products. Dr. Bollinger has shown that the more scattered the material and the greater the number of bacilli in the injection used, the more severe and more rapid was the development of lesions caused thereby. It was estimated that about eight hundred bacilli are required to produce fatal tuberculosis in guinea-pigs. The reasons for the identity of tuberculosis and lupus vulgaris are so well stated in Demme's classic brochure upon that subject that his resume from that source, making use of the translation as found in Senn's Surgical Bacteriology, are here given (p. 172): "First, similarity of histological structure. Second, presence of bacillus tuberculosis in granulation tissue of lupus. Third, the production of typical tuberculosis in animals not immune to this disease by implanting lupous tissue, or the introduction of pure culture of bacillus tuberculosis obtained from lupous tissue. Fourth, the fact that patients suffering from lupus are frequently attacked by and die from tuberculosis of other organs. Fifth, the prev. alence of tuberculous affections among relatives suffering from lupus (hereditary predisposition)." These facts are accepted at the present time by the medical world as the correct ones. Besides the foregoing distinct anatomical bacteria landmarks, lupus vulgaris has also definite clinical aspects which belong to it exclusively and serve to differentiate it from every other cutaneous disease. Some authors believe that lupus vulgaris is identical with lupus erythematodes; in other words, that these two maladies are one and the same. There are certain facts tending to show that there may be a connection at least between the two affections. There are certain similar points of resemblance. For many, lupus vulgaris and lupus erythematodes are but two forms of cutaneous tuberculosis which must be differentiated. In France, they are regarded by many as but two varieties of one and the same disease. Wassinger is the most prominent champion of this opinion. However, there are no instances, I believe, where lupus has been the result of contact (contagion) of one lupus patient with another. Kaposi and other European and American dermatologists are rather skeptical as to the identity of lupus with tuberculous affections. Kaposi says: "Nevertheless, the attempted demonstration of the identity of scrofula, tuberculosis, and lupus has not yet proved such identity." Cases of "inoculating tuberculosis" 
are reported in constantly increasing numbers, but it seems to be regarded as immaterial that years may have elapsed between the assumed "inoculation" and the occurrence of the tuberculosis of the skin. "No experimental proof has been offered, however, that characteristic lupus vulgaris can be produced by inoculation of tubercle bacilli." Drs. Morrison and Symington (Journal of Cutaneous and Venereal Diseases, vol. ix, p. 268) examined the tissue from twenty lupus cases without finding the tubercle bacilli, and M. Cornil examined the skin of eleven lupus patients and found only one tubercle bacillus. Dr. Heneage Gibbes believes lupus to be a tubercular affection, but says that the bacilli are sometimes not found in the lupous tissue. Kaposi and others deny the infectiousness and heredity of lupus, but several observers have recited cases where the parent of a patient suffered from lupus and instances where several brothers and sisters of the patient suffered from lupus. Leloir again says that diversity in the phenomena of the several varieties may be accounted for by the mode and seat of the inoculation (inoculation from within outward or from without inward), the development of the virus in parts more or less vascular, the greater or lesser virulence of the virus inoculated, and different degrees of reaction of the tissues.

Moynihan describes an interesting case in the British Medical Journal (November 20, I 896), which he believes was due to local and primary inoculation of tubercle. The patient was a girl aged 20, who three months previously had cut herself on the dorsum of hand. The cut did not heal, and inflammation extended. She had done washing in which there were three handkerchiefs of a tuberculous patient. The ulcer was removed with a wide margin of healthy tissue, and the wound healed permanently and rapidly. The microscopic section of the piece removed showed disturbance of the epithelium, owing to chronic inflammatory exudation in the underlying cutis. The epidermis was thickened and varicosed, and the underlying round-cell infiltration was arranged in foci, resembling tuberculous inflammation. In some of these a giant cell, with numerous nuclei similar to tubercle, were to be seen.

Concerning the exciting causes of lupus, especially as regards the mucous membrane, there seems to be a lack of positive information. Syphilis, and an eczema of the fistula leading to the site of tuberculous diseases of the nose, are cited as 
having induced the formation of lupus in a few cases, but mechanical or chemical injuries are not spoken of as probable causes, unless we accept the theory of those who believe in the direct or indirect inoculation with tubercle bacilli, and who think that a previous abrasion is necessary for the introduction of the germ. Leloir's classification of the varieties of disease which he believes are tubercular, is as follows:

a. True lupus, non-exedens and exedens.

b. Atypical varieties of lupus: I. Lupus vulgaris colloide. 2. Lupus vulgaris myxomatosis. 3. Lupus vulgaris sclerosis and dermisclerosis. 4. Lupus vulgaris erythematoid.

c. Scrofulo-tuberculous gummata, dermic and hypodermic.

d. Ulcerative tuberculosis: I. Secondary. 2. Primary.

$e$. Mixed tegumentary tuberculosis, resulting from a combination of two or more of these varieties.

A review of the literature (somewhat conflicting) upon the etiology of lupus, from a clinical standpoint, might lead to the following considerations :

a. If lupus is due to the presence of the tubercle bacilli, and therefore of the same pathological nature as the other so-called tuberculous diseases, how can its clinical course, which is much at variance with that of the other tubercular affections, be accounted for? Is there in the histological, biological, or chemical constituents of either the skin or mucous membranes at the orifices of the body any special antidotal property in the form of either serum, cell, proteid, ferment, secretion, or tissue, which will so effectually resist the development or growth of the tubercle bacilli, and the extension of its accompanying toxines, as to almost nullify its hitherto known destructive tendencies?

b. What, also, is the reason that the implanting of tubercle bacilli in the mucous membrane of the larynx in one instance produces a slow-going, practically non-infectious local disease (lupus), while in another instance the same micro-organism rapidly develops, extends, and sets up a general infection, with rapidly destructive local changes? It would seem that all the explanations touching these points are inadequate to reconcile these clinical anomalies. As Kafeman says, "we are yet ignorant of the law." The histologic character of the skin is urged by some as an explanation of the very chronic course of lupus and the absence of tubercle bacilli. Their encapsulation con. 
tributes to render them rather latent, according to Unna. Lastra, however, seeks to account for these incongruities by the opinion that the bacilli, or the accompanying toxines, may be attenuated, and, in substantiating this opinion by analogy, he cites the effects of small doses of chemic agents on the system, as compared with larger doses of the same, thus assuming that karyokinesis or mitosis depends upon dosage. According to Unna, the smallest number of tubercle bacilli are able to stimulate productive inflammation and formation of giant cells and sero-fibrinous exudation. But be that as it may, could we, even under conditions of attenuation, expect any such pathological process in the mucous membrane of the larynx, an organ whose susceptibility to tubercle bacilli and tuberculous toxines are continually alleged as a fact?

Age.-All authorities agree that lupus is a disease of early life, although there are instances on record of its origin during advanced life. Leloir thinks it begins in infancy.

Sex.-The female sex seems to be particularly liable. Of seventy-nine cases collated by Bosworth, fifty-one were females and eighteen males.

Heredity.-There have been differences of opinion regarding the hereditary transmission of lupus, but the unquestionable instances of such transmission are rare.

Inoculability.-I think the majority of dermatologists believe that the disease is inoculable, either directly or indirectly, but as that question has been dealt with in the discussion above, there is nothing to be added here excepting to recite the oftquoted verdict of a territorial jury, viz., " Not proven."

Traumatism has been cited as a predisposing cause of the disease in the skin, and by those who accept the doctrine of inoculability of the disease it is believed to be an important etiological factor.

Pathology.-According to Prudden and Delafield, lupus consists in the presence of small multiple nodules of newformed tissue, somewhat resembling granulation tissue, in the cutis, mucosa, or submucosa. By the formation of new nodules and a more diffuse cellular infiltration of the tissue between them, the lesion tends to spread, and by the confluence of the infiltrated portions a dense and more or less extensive area of nodular infiltration may be found. There may be an excessive production and exfoliation of epidermis or epithelium over 
the infiltrated area, or an ulceration of the new tissue (Prudden and Delafield, p. 228). "In the clinical group of diseases called lupus there are other forms of lesion which are not caused by the tubercle bacilli."

Unna lays great stress upon the part played by the large socalled plasma cells of Waldeyer, and thinks the giant cells are of secondary formation. He also points out that the tubercle bacilli are encapsulated in the giant cells, and therefore become



FIG. 80.-Lupus of the mucous membrane (Kaposi). $a, a$, mucous membrane, showing cell infiltration and papillary outgrowths; $b$, lupus nodules; $c, c, c$, mucous glands, infiltrated with lupous growth; $d, d$, cartilage; $e, e$, submucous fat; $f$, artery (cross section), thickening of intima.

latent. Jadassohn thinks the plasma cells are not of any particular importance in lupus, because occurring in other inflammatory processes.

Kaposi says that the pathology of the disease relating to the skin does not differ essentially from that affecting the mucous membrane (see Fig. 80). "In all instances of tuberculosis we find the tubercle the product of the tubercle bacilli, a nodule of so-called granulation tissue composed histologically 
of small round cells deeply stained by colouring agents, together with larger cells possessing a clear nucleus that have been called epithelioid cells, and large cellular elements with peripherally arranged nuclei and homogeneous centre-the Langhaus giant cells. These cells are inclosed between the meshes of connective tissue, and are characterized in distinction from normal cells by their instability. Sooner or later a modification begins in the nodule. Its centre becomes necrotic-i. e., the cell protoplasm is coagulated-the nuclei lose their power of responding to staining agents, the intercellular substance also takes part in the degeneration, and there results a coagulation necrosis in the Weigert sense-a condition that has been called cheesy degeneration."

Diagnosis.-Lupus of the larynx may simulate tuberculosis, syphilis, epithelioma, rhinoscleroma, or chronic glanders. Michaelson has shown that it is almost impossible to trace accurately the limits which separate lupus of the mucosa from tuberculosis of the mucosa, because it is only in the cicatrices that we find the characteristic lupus nodule when the disease attacks the mucous membrane.

Concerning the dependence upon microscopical examination for its diagnosis, Dr. B. M. Ricketts, of Cincinnati, says: "When we depend upon microscopic investigation we must not accept the picture as one to be recognised at all times. While I believe that the bacilli, when found, indicate a certain disease, I cannot accept the statement that it is not that disease because the bacilli cannot be found. In other words, I am not ready to accept the microscopic investigation as infallible. I would depend upon clinical history and appearance in making diagnosis of lupus rather than the statement of any microscopist. Neither do I believe in the infallibility of any diagnostician, and the question arises, When does it become constitutional, when extirpation would be useless? It is also important to determine that degree in any of the malignant growths." He has treated sixty-five cases of lupus, of which forty-two were lupus vulgaris and twenty-three lupus erythematosus (New York Medical Journal, pp. 58, 449). According to Neisser (Morrow) the chief diagnostic points of lupus are its beginning in childhood, and its very chronic course. If the lupus laryngitis be secondary to the same disease of the skin, or an extension from it to the fauces and larynx, then the diag- 
nosis may be easily made out; but when the larynx is pri. marily affected the task may be a more difficult one, at least in some of its stages. The general clinical history, showing an absence of constitutional disturbance of any moment, will serve to distinguish it from laryngeal phthisis, and from syphilis, the clinical history belonging to the latter disease. The absence of much inflammation, the dry, negative, non-suppurating appearance of the ulceration, limited in extent and not clearly defined, with rather coarse, granulated edges, and streaks or patches of cicatricial tissue, will usually serve to differentiate it from the sharply cut suppurating ulcers of syphilis, surrounded as they are with highly coloured and tolerably well-marked areola; and from the shallow, roundish, or lenticular ulcers on a pale and swollen mucous membrane which distinguish this disease and tuberculosis. When ulceration is active the local appearances might lead to some confusion, but in such cases the clinical history, as well as the characteristics of the local formation, will remove doubt. Syphilitic ulceration of the nose often resembles lupus, and the diagnosis in such cases may have to be suspended awaiting developments.

Rhinoscleroma usually begins on the skin of the nose first. Its ulceration is flat, and soon covered by a stiff crust. There is usually no softening or ulceration, nor very much contraction, and no such melting away, so to speak, of the tissues as in lupus.

Epithelioma can only be mistaken for lupus when it is of limited extent in the nose or the larynx. When occurring in the larynx it is situated at first generally either upon the epiglottis or in the laryngeal ventricle. It may be seen as a more or less reddish growth of uneven although unbroken surface, standing out from the tissues.

Treatment.-The treatment should be both constitutional and local. The systemic treatment should proceed upon the tonic and so-called alterative plan. The subjects of lupus more often than otherwise show the effects of malnutrition, therefore a generous diet, out-of-door life, and suitable clothing, together with such tonics as salicin, quinine, Fowler's solution of arsenic, tincture chloride of iron, etc., should be administered. Arsenic in one form or another has always been considered highly beneficial. Indeed, it has been deemed by some as almost a specific for lupus. It certainly is one of the most 
useful remedies in vogue. Iodine and iodide of iron are also of great value, especially if combined with the sirup of the hypophosphites. In my limited experience the use of arsenical preparations and iodine, either hypodermically or by the mouth, have given the best general results. Iodide of arsenic and Fowler's solution are favourite remedies. Cod-liver oil and malt preparations may also be taken. When there is a cachectic, sallow appearance, with tendency to lymphadenitis, phosphorus in oil should be given three times a day. The chief indication for local treatment is the removal of the offending tissue. This has always been a desideratum. The older practitioners sought to accomplish this elimination of diseased tissue by means of powerful caustics, such as caustic soda, caustic potassa, arsenious and nitric acids, chloride of zinc, etc. More recently dermatologists have resorted to either the knife or curette for the speedy removal of lupous tissue. This surgical plan has also been adopted in cases of lupus of the mucous membranes, but, according to Bosworth, with the effect of aggravating the disease sometimes. Undoubtedly, when so situated that the diseased tissues can be wholly cut away at once, such an operation should be performed. Schutz recommends for skin lupus, after curettement, an application of pyrogallic acid, I to 4 , and for mucous membrane a watery solution of zinc chloride, twenty to thirty per cent.

B. Merrill Ricketts (of Cincinnati) reports a case of lupus in a woman, aged fifty-one, who was treated successfully by electrolysis. There were thirty-four lesions destroyed, and but one remains. After previous application of cocaine, the needle should be inserted in different directions over the diseased skin. It is not necessary to insert it into the deeper tissues. The length of time which the needle should remain, and also the length of the several séances, will depend upon the experience of the operator with the patient. There were fiftyeight lesions altogether in this one. A. Ravogli (Jour. Am. Med. Ass'n, Dec. 5, 1896) advocates further trials of electrolysis in the treatment of this disease.

The treatment of lupus by violet or chemical rays of light was reported by A. Ransome (B. Med. J., Jan. 29, 1898, p. 19).

Schiff reported two cases of lupus vulgaris of the skin treated by the Röntgen rays with noteworthy results. The first case, a girl fourteen years of age, had lupus of the skin on the left 
forearm since the age of three. The diseased parts were exposed to the rays daily for two hours at a time. On the tenth day the first reaction appeared; the exposed parts became red and swollen, especially about the lupous infiltrate, and abundant secretion trickled out from beneath the crusts; the ulcerated surface was surrounded by a rosy halo. Under a continuance of treatment the ulcers became clean and granulated; a pinhead-sized wart-like structure appeared at the bottom. At the height of the reaction on the nineteenth day the treatment was interrupted for two months and a half, and cicatrization had not been completed. On the flexor side of the forearm, where the rays were prevented by the bones from reaching the skin, the lupous infiltrate remained unchanged. The second case was one of lupus in the region of the larynx; treated in the same manner as the first case. The use of the galvano-cautery ought in many cases to supersede other escharotic treatment, although strong solutions of chloride of zinc or lactic acid will often give very satisfactory results. The writer has employed with success applications of lactic acid, followed by a spray of a strong solution of resorcin daily in cases of lupous laryngitis. Pyoctanin, topically applied as Bougard's paste, or injected into the parts by the hypodermic syringe, is reported to be efficient. I have used a solution of iodine in this manner, but could not see that the effects were more striking than when introduced elsewhere under the skin. The application of balsam of Peru has been reported as giving good results; also for the nose, applications daily of iodine and oil of cloves. There is danger of producing edematous or phlegmonous inflammation of the larynx by rough treatment; for that reason it is obvious that the same thorough treatment as applied to the skin would be inadmissible for the larynx. For routine local treatment it will be found that resorcin or iodoform, or both, in conjunction with the careful use of the galvano-cautery, lactic acid, or chromic acid, will constitute the most efficient and safest treatment for this disease. Iodine and oil of cloves, or codliver oil and iodoform, may be applied daily.

H. Bergeat (Jour. Amer. Med. Ass'n, March I3, I897) recommends guaiacol locally. Tuberculin and tuberculocidin, which were such popular remedies for lupus, seem to have been abandoned. O'Lassar treated five cases of lupus vulgaris with "lupus coccus," which were probably benefited by this mode of treat- 
ment. The injections were borne without any local reaction. Semifluid oleo-stearate of zinc is recommended by Dr. W. F. Chappell as an application. The accumulated experience of the last two years shows the $\mathrm{X}$-ray to be the most reliable local method of treatment.

\section{RHINOSCLEROMA}

This disease was first described by Hebra and Kaposi. It is exceedingly rare, especially in this country. In the whole United States, according to Dr. Jackson, there had been reported only three cases up to I894. W. Freudenthal has reported a very interesting case, and written an excellent brochure on the subject. Rhinoscleroma has been classed among the skin diseases, but it is not confined to cutaneous tissue ; indeed, two cases have been reported by Cornil and Alvarez respectively to have been confined to the larynx. Chiari, Ganghofner, and Paltauf think that it is identical with the disease known as laryngitis hypertrophica chronica inferior. Freudenthal thinks it is also analogous or identical to Stoerck's blennorrhœa of the larynx. The trachea may also be the seat of the affection.

Etiology.-It is supposed to be due to the invasion of the submucosa by the bacillus of Frisch, a micro-organism which resembles Friedländer's pneumococcus. What, if any, morbid condition pre-exists to invite this bacillus has not yet been pointed out.

Clinical History.-The disease often appears on the inside of the nose or upon the soft palate, and sometimes on the skin of the vestibule of the nose as a small, grayish tubercle. This slowly grows and extends, until the tissues affected become very hard (ivory-like), white, or bluish. The nasal passages are entirely obstructed, as the disease

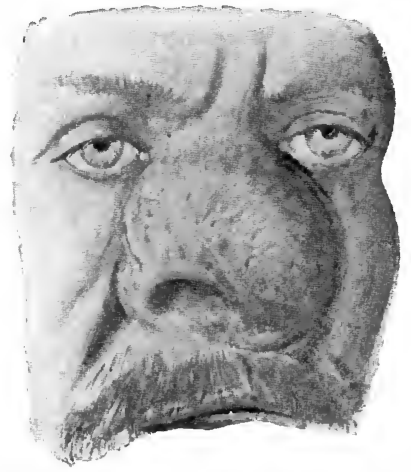

FIG. 81.-Rhinoscleroma (after Freudenthal). advances, from the tumescence of the mucosa and submucosa. In Freudenthal's case (see Fig. 8I) both nasal passages, the pharynx, and larynx were involved. On account of the laryngeal implication, a tracheotomy tube had been worn by the 
patient for a long time. There is not much pain complained of, but considerable general debility. There is no pyrexia, excepting as incidental to intercurrent morbid states. The disease is slowly progressive, and finally produces fatal exhaustion of the vital powers.

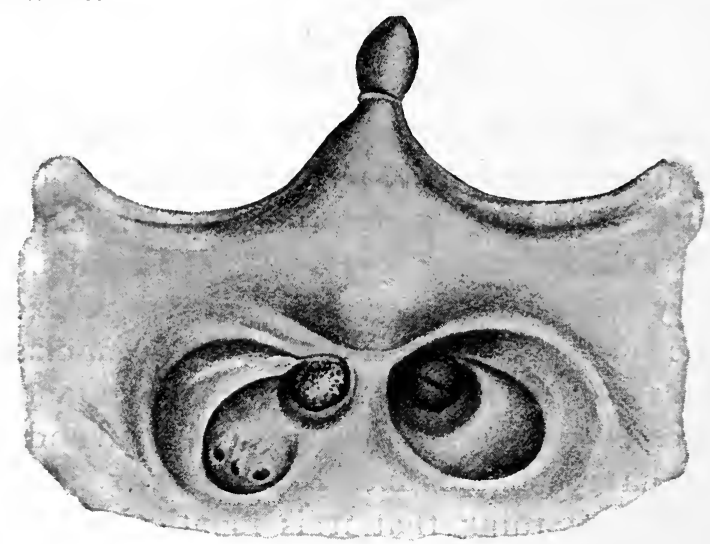

FIG. 82.-Posterior rhinoscopic image of rhino-scleroma.

Diagnosis.-The differential diagnosis lies between syphilis, lupus, tuberculosis, and the hypertrophic laryngitis mentioned above.

The tumefaction in rhinoscleroma does not break down as a gumma would do (Freudenthal), and besides this, the slow course and extreme hardness of rhinoscleroma will serve to distinguish it from the syphilitic disorder. From tuberculosis it may be distinguished by the absence of constitutional disturbance. When beginning in the larynx, it cannot be easily differentiated excepting by the clinical history. From lupus it differs mainly in its local characteristics and the absence of ulceration.

The diagnosis may finally depend upon the microscopic examination, searching for the bacillus of Frisch.

Prognosis. - The prognosis is always unfavourable.

Treatment.-Nothing in the way of medicinal or surgical treatment has heretofore proved curative of this affection. Freudenthal has proposed the hypodermic injection of Coley's fluid, or rhinosclerine, the serum first introduced by Pawlowsky, which is obtained from a culture of the bacillus of Frisch. 


\section{CHAPTER XI}

\section{CHRONIC DISEASES OF THE UPPER AIR PASSAGES}

\section{Chronic Rhinitis, Chronic Nasal Catarrh, Chronic Coryza}

A STRICT definition of the term chronic rhinitis would imply that the disease is simply a chronic inflammation of the mucous membrane of the nasal passages, whereas, as ordinarily understood, the name includes three or four distinct pathological conditions, viz., hypertrophy of the tissues (hypertrophic rhinitis), atrophy of the tissues (atrophic rhinitis), a purulent inflammation of the upper nasal region (purulent rhinitis), and an intumescent rhinitis. However, as they each may depend upon a common cause, the majority of authors group them together.

General Etiology.-Chronic nasal disease is very common in northern climates, and is found in persons of all ages and in both sexes, although, according to Natier, there is a nasal inflammation more common to men than to women. It exists either in the form of hypertrophic or atrophic rhinitis, with adenoid and tonsillar hypertrophy, or in connection with various deformities of the nasal passages.

Childhood.-Many cases occur in childhood which are undoubtedly overlooked. There is an abundance of statistics relating to the etiology of this subject; among them the following give a fair idea of the etiological conditions, so far as children are concerned.

Chappell, of New York, examined the nose and throat of 2,000 children, in order to determine the frequency of certain abnormal conditions; 955 were taken from the New York Juvenile Asylum, 645 from Grammar School No. 49, and 200 from the orphan asylum. Of the entire number, the following abnormal conditions were found: 
Adenoid growth...................... 60

Enlarged tonsils....................... 270

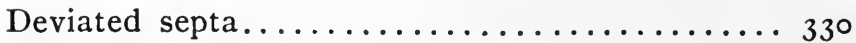

Spurs of septa................... 150

Hypertrophy of inferior turbinated bodies...... 260

Hypertrophy of middle turbinated bodies ....... I6 1

This shows that 1,231 were suffering from some anatomical abnormality, and usually with the accompanying symptoms of respiratory obstruction and catarrh. Of the entire number, I,292 were boys and 708 were girls. Of the enlarged tonsils, (270), I 83 were of both palatal tonsils, and 87 of one only; 160 of these cases were males and I Io females.

Of the deviated septa (330), 270 presented deviation of the cartilaginous septum, 50 of the bony septum, and Io of both bony and cartilaginous.

Of the cartilaginous deviations, 148 were to the right and 122 to the left. Of the bony, 35 were to the right and 15 to the left. Of the cases of deviation, 50 per cent were more numerous in boys than in girls. It is generally believed that deviation of the septum occurs in adults in the proportion of about 35 per cent of those examined. Chappell and others think that a large percentage is due to one of four causes: I, traumatism; 2, hypertrophy of the turbinated body, or some other source of the unilateral pressure; 3 , hyperplasia from long-continued irritation; 4 , difference in the period of the development of the bones of the face and septa; and to this may be added hereditary peculiarity of structures.

HyPERTROPHIC RHINITIS (Hypertrophy of the Turbinate Bodies)

Etiology.-The causes of this affection may consist of repeated attacks of acute coryza ; congenital or acquired deformities of the nasal passages which interfere with their freedom; neoplasms; the continued inhalation of a noxious, irritating, or dust-laden atmosphere; a damp habitat; an occupation which subjects the person to alternations of cold and heat, and other circumstances which tend to maintain an engorgement of the venous or cavernous plexus of the turbinates. Certain irritable conditions of the spheno-palatine ganglia, or its nerves, may also produce this condition in an insidious manner by increasing the activity of the nutrient vessels, thus leading to a more or less permanent vaso-motor paresis (John N. MacKenzie). 
Finally, it may be a sequence of diphtheria, or the exanthemata. Measles also is responsible for many cases, especially those occurring in children, while in some cases the cause remains a mystery, since we often find the disease in healthy persons whose surroundings have always been excellent, whose habits of life have been good, and whose nasal passages are devoid of any marked deformity. Moreover, in such persons there may be no history of repeated attacks of acute coryza. Undoubtedly climatic conditions and our mode of life, especially the practice of living in overheated houses and offices during the winter season, is a strong contributory cause of chronic rhinitis.

The lengthy catalogue of etiological factors given in many memoirs and text-books must appear to any analytical mind illogical and untenable (Seiss). Early cases of nasal catarrh are dependent in part on climatic, in part on anatomical developmental conditions, and largely on factors yet undefinable, which are a part of our civilized life. "The fads of rhinologists," when weighed from the standpoint of comparative pathology and broad observation, are found to be but common sieves (Seiss). With snuff-takers, as might be expected, the turbinated bodies are usually more or less enlarged, and become more or less insensible to touch. Concerning both the acute and chronic forms, Cohen and Bosworth are inclined to attribute the disease to purulent discharge-specific infection. Lennox Brown, Sajous, Seiler, and Robinson have made no special mention of purulent discharge in children, and classed such conditions under other causes, such as hypertrophy of the glandular structures. Bosworth takes the ground that the conditions are purely local in character, and depend on no constitutional taint, consisting essentially in an increased secretion of mucus in the earlier stages, together with a rapid desquamation of the epithelial cells, which finally runs its course as a purulent disease in from five to ten years. In the rhinitis of children, up to the age of twelve to fifteen, epithelial structures and glandulæ are especially liable to take on diseased action. We see this often manifested in the frequent hypertrophic condition of the glandular structures (nasal and pharyngeal) of children and youth, whereas in adults inflammation of the mucous membranes is more apt to result in hypertrophy of the connective tissue. During childhood the lymphatic system undergoes its greatest development; hence it is that the lymph glands are more prone 
to alterations in nutrition than in adults. In children there is a great activity in the development of the epithelial cells, and when they are stimulated extra growth is excited, leading to one of two distinct results: I. Piling themselves up on each other so as to form true epithelial hypertrophy, together with an increase of lymph tissue, and lymph corpuscles, as in the case of adenoid growths and enlarged tonsils-forms which are likely to follow scarlet fever, measles, or diphtheria. 2. The epithelial cells may fail to adhere, and the result is a rapid desquamation, with an increased flow of mucus, thus forming an abundant muco-purulent discharge. Both of these processes may go on in the same case. Many think that children of a strumous or scrofulous diathesis are more prone to take on the second form, or exfoliation of the epithelial structures. Quoting L. C. Kline, he believes that an acute cold in a child differs from that of an adult, in that the inflammation involves the epithelial coat of the nasopharyngeal glands, producing nasal stenosis, while in the adult the inflammation involves the mucous membrane proper and extends into the deeper structures. This inflammatory disease of the nose, as a rule, in children between the ages of three and five years, if not cured, will culminate in one of two results, or be a combination of both; namely, the desquamative or suppurative form, or the generation of the adenoid form of disease. If the case is confined to a strictly desquamative variety it will gradually extend into the mucous follicles, rob them of their epithelial lining, and destroy their secretive power, and in this way lead to the formation of crusts, and finally terminate in atrophic rhinitis at about fourteen or fifteen years of age. A characteristic symptom of this disease is a marked purulent discharge from both nostrils, usually creamy or straw colour. Kline gives twenty-five descriptive cases that had enlarged tonsils and adenoids, all following a fever, scarlet fever, or measles, and five purely catarrhal cases which gave no history of these systemic diseases. The strumous or scrofulous diathesis are even nowadays frequently considered to be elements of these abnormalities. The writer has met with a few cases of excessive mucous discharge in children, without hypertrophy, deformity, or foreign body in the nasal passages.

Symptomatology.- The subjective symptoms come on gradually, the patient finally becomes aware of an obstruction in 
the nasal passages, perhaps accompanied by frequent fits of sneezing and extra secretion, which, gradually increasing, excites particular attention and becomes a painless nuisance. The desire to blow the nose frequently and hawk and sniff grows from day to day, especially during damp, cold weather, while the ability to breathe through the nose progressively decreases. After a while these symptoms become permanent, and the patient is obliged to breathe most of the time, if not all the time, through the mouth, while efforts to clear the nose and throat, especially in the early morning, become more and more urgent. When lying down, especially at night, the dependent side becomes engorged and blocked, owing to the gravitation of blood to the venous plexus of the lower side, and the patient thus passes troublesome nights. The throat, and sometimes the middle ear, may become involved, and symptoms referable to these parts-such as dryness or burning of the throat, and tinnitus aurium, or crackling sounds in the ears-supervene. The secretion may be more or less serous in character for a time, but soon a thicker mucous discharge appears, some of which desiccates in the nasal passages, requiring greater effort for its dislodgment, and thus correspondingly adding to the patient's discomfort. The larynx also frequently becomes involved in long-standing cases. This is because of the alteration of the natural secretion of the nose on the one hand, and the obstruction to nasal respiration on the other, either condition favouring the direct impingement of the air upon the pharynx and larynx, without having been previously warmed and moistened by its passage through the nose as in physiological respiration. A continuation or aggravation of the enlargement of the turbinals and nasal stenosis, together with the morbid changes in the middle ear, are accounted for by Bosworth and others upon the supposition that the air of the nasopharynx is maintained in a rarefied state by the acts of respiration, together with the hawking efforts of the patient to remove the obstruction and secretion. These efforts, acting upon the inclosed air of the nasopharynx like a syringe or air-pump, and drawing the air out of that cavity little by little, while the plugged nasal passages preclude a corresponding supply in the natural way, thus produce a partial vacuum, which in turn favours the further swelling of the turbinates, on the principle of suction, 
very much as the skin is drawn into the cup in the process of dry cupping. Undoubtedly this theory will account for the pathological conditions of some cases, especially those complicated by the presence of large polypi ; but I doubt whether it is worthy of general application, because the law of the diffusion of gases, together with the area and pliability of the palatal opening to the nasopharynx, would seriously interfere with anything like the imprisonment of air and the existence of even a partial vacuum in these parts for any length of time, notwithstanding the nasal passages might be quite permanently closed - that is, unless the soft palate and uvula were kept in pretty close apposition to the posterior wall of the pharynx, an event which could happen only from structural disease or deformity of these parts. Partial or total loss of the sense of smell is a more or less constant symptom of nasal obstruction, and if the nerve filaments be pressed upon for any length of time, pain referred to the nose, eyes, or frontal region may be complained of. If the accessory cavities or ethmoid cells have participated, then pain of a neuralgic character is often complained of. The voice is notably changed by the nasal obstruction, giving rise to what is called the "nasal tone." This nasal tone is due to the breaking off of the natural resonance of the voice by the obstructed nasopharyngeal space. More or less deafness or "hardness of hearing" is a frequent accompaniment, because of the irregular or imperfect manner in which the middle ear is aerated. Various ocular troubles may also be caused. Berger and others find hypertrophic rhinitis the chief cause of ciliary and conjunctival disease.

Anterior Hypertrophies.-The appearance of anterior hypertrophy is quite characteristic. By the aid of a strong light and a nasal speculum the mucous membrane of the turbinated body may be seen projecting out even against the septum, presenting a round, smooth, or pyriform outline, sometimes very much resembling a tumour. The surface may be papillary, resembling the surface of a raspberry. This is sometimes called "the mulberry hypertrophy." The mucous membrane is usually quite red, although in some cases, owing to the character of the intumescence, it may be of a pinkish-gray or gray colour (intumescent rhinitis). This is particularly noticeable in those suffering from systemic derangements. When touched with a probe the parts feel rather firm, but so elastic that they 
soon resume their former contour on the withdrawal of the instrument, unless it be an old hypertrophy. The inferior and middle turbinates are the ones generally affected, and the hyperplasia generally involves more than the anterior ends.

Posterior Hypertrophics.-Posterior hypertrophies, as seen in the rhinoscopic mirror, present themselves at the choanæ as large, grayish, rounded, smooth, or corrugated masses, varying in size from a large pea to a good-sized almond, or larger. These projections do not bleed easily when touched. They are usually softer to the touch than the anterior hypertrophies, and contract more readily after being touched, so that sometimes, after a thorough examination with a probe, further inspection may show them much reduced in size. When a good rhinoscopic examination is impossible a thorough digital examination should be made, as recommended for the detection of adenoid enlargements in the nasopharynx.

Anterior hypertrophies are more often confined to the anterior third of the lower turbinated body, while posterior hypertrophies may be situated on either or both inferior and middle turbinated bodies. In many instances the turbinated bodies are found to be hypertrophied from one end to the other. These swellings may be of moderate dimensions, in which case they do not touch the opposing side of the septum, so that narrow, slit-like channels, either above or below the middle of the swellings, may be seen. Sometimes a physiological swelling of the turbinated bodies, due to exposure to cold or a vacillating temperature, may simulate true hyperplasia. It is therefore better, especially in the winter season, before arriving at a conclusion, to apply a weak solution of cocaine (two to four per cent), or a solution of adrenalin chloride ( $I-6000)$, to the part, when, if the swelling be merely a transient turgescence of the venous plexus, it will soon disappear, leaving the nasal channels patent. If, on the other hand, the swelling be due to a true hyperplasia, only a very moderate contraction will hiave taken place, so that the tissues will still appear swollen, and when touched with the probe will be found firm. Besides this, the nasal obstruction may be aggravated by a swelling on one or both sides of the nasal septum, anteriorly or posteriorly - "ballooning of the septum," as it is called. What is commonly known as polypoid hypertrophy consists of a sort of edematous swelling or intumescence of the turbinated body, 
simulating in colour and consistence a polyp. The appearance of sulci on the surface may give to the swelling a slightly lobulated appearance. There is, however, no outgrowth and no pedicle, as in the case of a polyp. The condition here may be essentially one of diminished rather than excessive vascularity, the interstices and glands being filled with a fluid of a muco-serous nature. The secretion in these cases from the surface of the mucous membrane may be profuse and watery. It has been computed that over seven thousand grains of water are excreted from the upper air passages in twenty-four hours, and Aschenbrandt has demonstrated by a series of carefully conducted experiments that the source of this moisture is largely in the nasal chambers.

According to Zuckerkandl, hypertrophies and atrophies are more pronounced in the respiratory region of the nose, yet by no means limited to that area. They may infrequently be found in the olfactory region.

Pathology.-The three important tissues of the turbinated bodies, viz., the epithelial, the vascular, and the stroma or elastic tissue, may undergo change simultaneously or consecutively, according to some unknown accident or law, so that an inordinate proliferation of epithelial tissue, or active cytosis predominating in the morbid process, may induce a hypertrophy which is soft, vascular, or papillomatous in character.

If the vascular tissue (the cavernous plexus) participates mostly, there is first a weakening of the walls, and then a rapid proliferation of connective tissure about these vessel walls, which gradually impairs their elasticity, or, as Asch states, impairs their ability to recoil, and as the process proceeds may obliterate more or less the channels and cavernous spaces, or leave them more or less permanently open, with the contained blood in a condition of stasis, inducing transudation, by which the spaces are filled with exudates of serum, lymph, leucocytes, round cells, etc., which exudates may perhaps become partially organized. The irritation attending the early conditions may stimulate the progressive proliferation of connective and fibrous tissue in such amount as to replace more or less the other tissues, in which case there is a hyperplasia of considerable density, the pressure of which gradually destroys the lumen of the blood, and lymph vessels, and glands, and furthermore impinges upon the cavernous spaces until perhaps, ac- 
cording to Seiler, there are no cavernous spaces left excepting those next to the bones. In either event the resulting changes destroy so much the function and usefulness of these important bodies as to convert them into pathologic hindrances to respiration, olfaction, and audition. Moreover, from pressure and loss of vitality they may subsequently undergo sclerosis or atrophy. This would be a natural consequence of such morbid action, according to the histo-pathologic processes, which take place under similar circumstances in other parts of the body. But whether this takes place always is a question which seems as yet unsettled. Bosworth, John N. MacKenzie, and others think that atrophy inevitably succeeds hypertrophy of these tissues. Undoubtedly it does take place often, but certainly not always, as may be attested by the large number of cases of various degrees of turbinate hypertrophy, which endure for a number of years without showing atrophic alteration. Undoubtedly the individual character of the trophic nerves and of the glandular apparatus of the part is important in determining the course and final effects of the morbid process.

Although hypertrophic rhinitis is generally looked upon as essentially an initiative inflammation, yet there are many cases in which the inflammatory features are so mild-in fact, almost wanting - that one is led to doubt whether or not these tissue alternations may not oftentimes be classified as trophic affections from the beginning.

The shape, size, site, and extent of the hypertrophy are various. Sometimes the enlargement appears very vascular, in other instances anæmic, of grayish colour or edematous. In some cases the swollen turbinate appears lobulated or papillomatous; in other cases, again, smooth, dry, and glistening. The site of hypertrophy varies also, although the lower and the posterior part of the inferior turbinals are most frequently affected.

There is usually considerable extra secretion of mucus or sero-mucus. The character and quantity of the discharge, however, will depend often upon whether or not there is some antecedent or complicating disease of the nasal passages, such as deflection of the septum, disease of one of the accessory sinuses, etc. In the latter event the discharge may be purulent. 
Classification.-John N. MacKenzie divides the subject of true rhinitis hypertrophica into $(a)$ dilatation with hypertrophy (see Fig. 85) and (b) complete hypertrophy, sometimes called sclerotic rhinitis. The hypertrophies of the middle

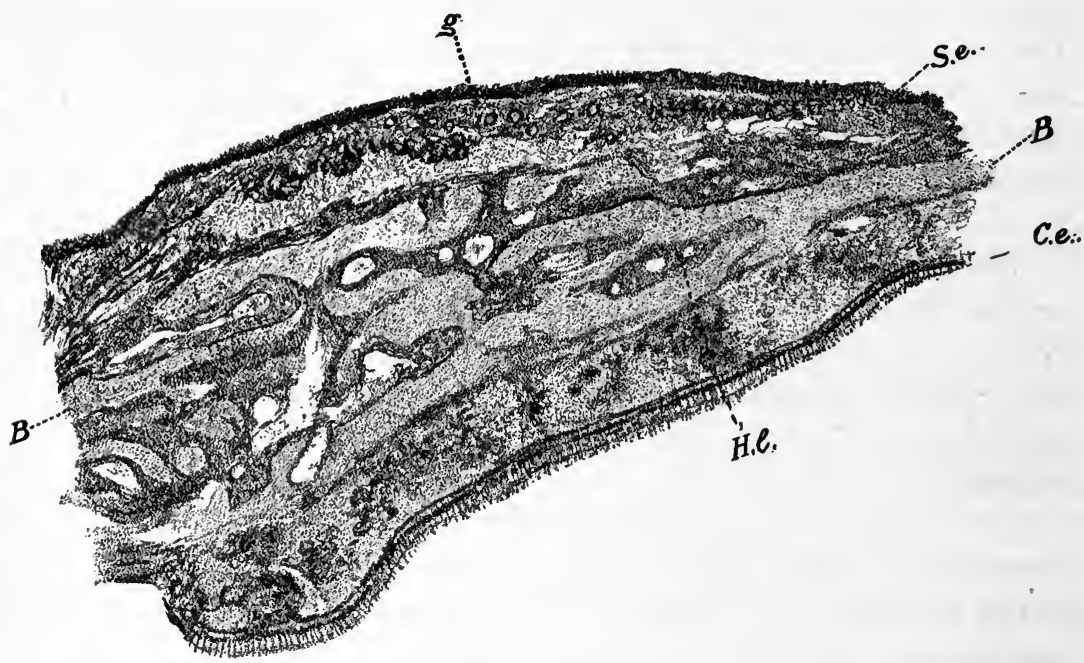

FIG. 83.-Bony cysts (Jonathan Wright). $B$, bone ; H. l., Howship's lacunæ with new connective tissue and blood vessels; $C$. $e$., ciliated epithelial lining of cyst cavity ; $S$. e., surface epithelium; $g$, glands.

turbinated bone are sometimes denominated nasal fibromata. They do frequently undergo a myxomatous degeneration, leading to the formation of myxo-fibromata (see Fig. 86) (Ziegler, J. Wright). They are sometimes thin, soft, and gelatinous, giving the appearance of a sessile polyp. In such instances the membrane is light pink or yellowish in colour, and more or less hard to the touch. The rims of the Eustachian tubes may also appear yellow and harder than normal, and the fossæ of Rosenmüller may appear deeper or broader than normal. The pharynx may likewise present marked changes. In advanced cases the size of the pharyngeal cavity is much enlarged, in consequence of the atrophy resulting from pressure.

As a result of long standing, chronic inflammation, the mucous membrane and submucous cavernous tissue are densely studded with fibroid tissue and leucocytes, which, following a universal law, usually becomes organized into new connective tissue, which contracts and produces pressure upon surrounding tissues. There are four modes of obliteration of 
the erectile sinuses in "sclerotic catarrh," according to John N. MacKenzie : first, by hypertrophy under the formation of connective tissue and contraction of the same; second, by obliteration of the lumen of vessels by masses of round cells and organization of the same; third, by formation of buds on the acinous walls (see Fig. 85); and fourth, by the formation of thrombi in some of the vessels (see Fig. 86). The first two processes are those given under the name of chronic inflammation. The formation of "buds" or fibrous tissue, which more or less blocks the lumen of the spaces by forming parietal thrombi, as described by MacKenzie, might be considered as a special formation of active blood-vessels. They occur probably more commonly in the subacute cases of so-called nasal catarrh, in which the inflammation partakes more of the acute type. All of these phenomena, however, bring about the formation in the affected turbinated tissues of a fibrous mass, the pressure from which induces atrophic processes in the glandular structures, finally destroying them (see Fig. 86). Early in the path-

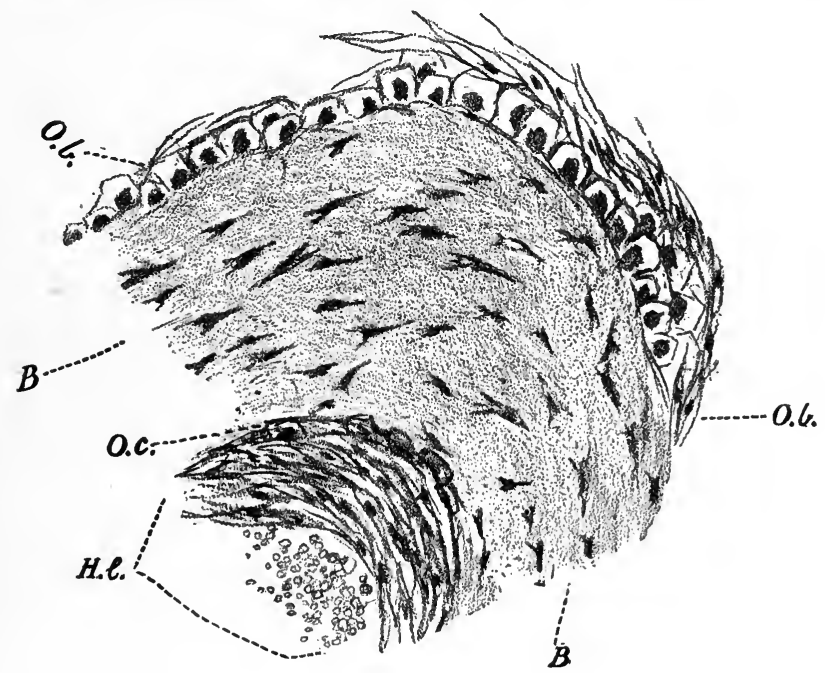

FIG. 84.-Bony cysts (Jonathan Wright). $B$, bone; H. l., Howship's lacunæ with new connective tissue and blood vessels; $O . b$., osteoblasts; $O . c_{\text {. }}$, osteoclasts.

ological history of sclerotic rhinitis changes in the epithelial layer take place, consisting of proliferation, and, later in the process, the formation of hypertrophied papillæ. These multiple papillæ or papillomata are all of very minute size, and are 
spread over the surface of the turbinated tissue. The structure which this produces is like that of so-called typical papillomata. They are formed by vascular connective tissue, which begins by proliferating layers of the endothelium of the bloodvessels, forming vascular loops. These minute papillomata may

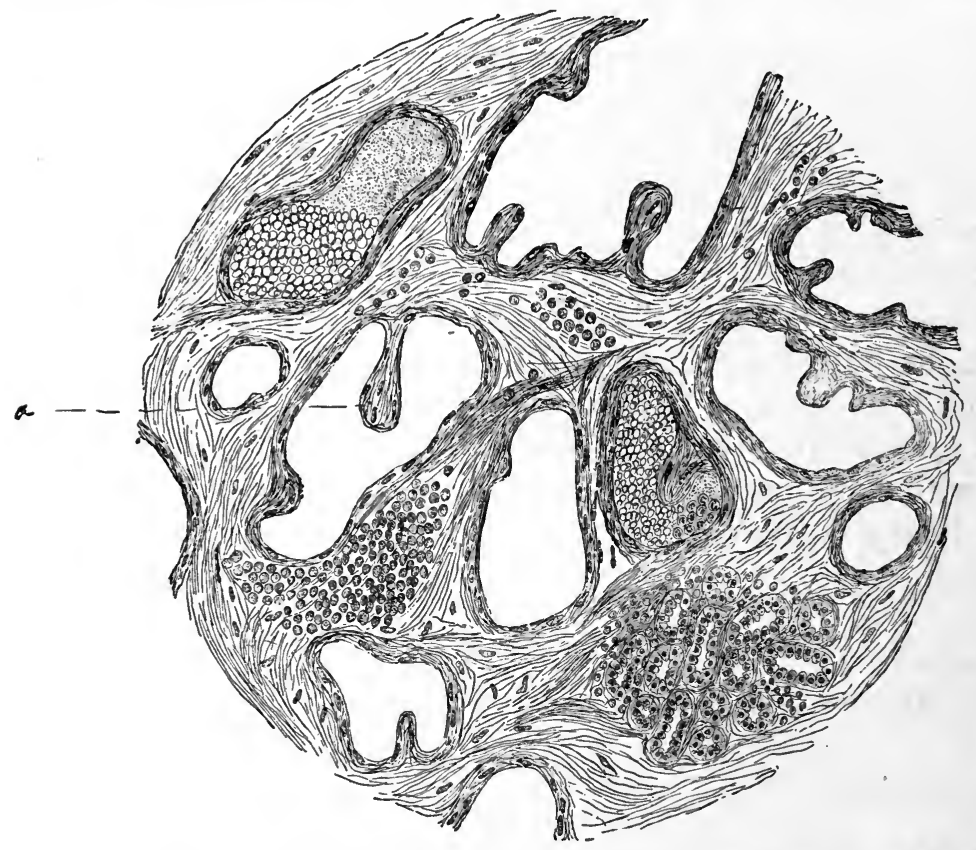

FIG. 85.-Microscopical appearances of tissue in chronic hypertrophic nasal inflammation before complete obliteration of the erectile spaces has taken place (John N. MacKenzie).

undergo atrophy, the surface of the turbinated body becoming smooth and glassy, or they may persist indefinitely. The angiomatous or papillomatous hypertrophy seems softer to the touch by the probe, but is quite as persistent as the other forms where the connective-tissue elements are increased. We might add that there is still another-a so-called edematous form of hypertrophy-in which the venous plexus as well as the mucous membrane proper are rather diminished in their structural integrity, while the interstromal spaces are filled with a more or less dense fluid similar but thicker than that contained in mucous polyps. This condition has been ably described by Dr. Jonathan Wright. The capillaries in any of these forms of hypertrophy may be greatly enlarged and their relation to 
the venous sinuses be very much altered, although the capillaries, normally, have no direct connection with the venous plexi. Changes in the epithelium, such as erosions and ulcerations, rarely occur, except through attrition with a neighbouring part, and even in such cases it will generally be found that some untoward constitutional state is the important etiological factor. The writer has never seen in this disease an ulceration of the mucous membrane. The affection in children, as already stated, is often accompanied by hypertrophy of the pharyn-

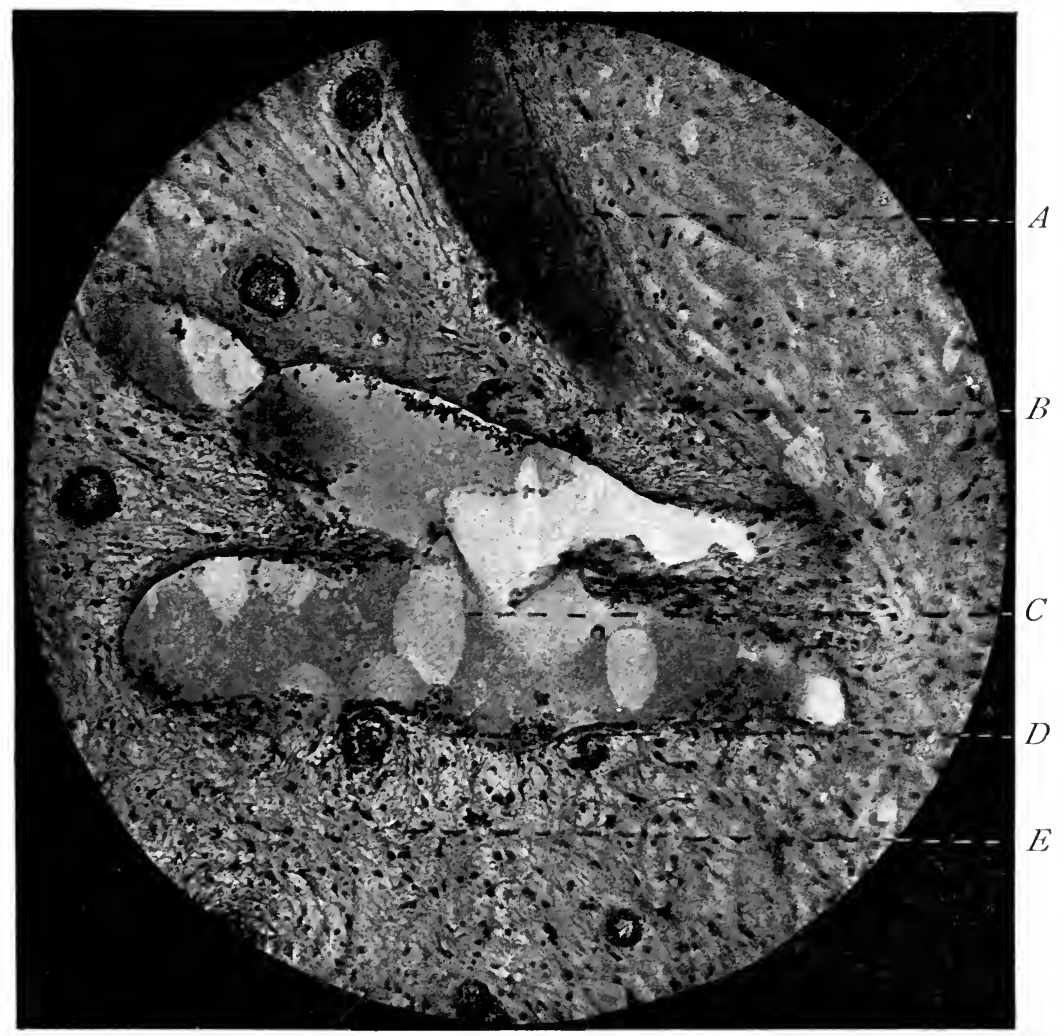

FIG. 86.-Photomicrograph of section from posterior hypertrophy (specimen shown in Fig. 87). $A$, venous sinus filled with serum; $B$, leucocytes along wall of sinus ; $C$, sinus ; $D$, thrombus ; $E$, loose fibrous tissue.

geal and fancial tonșils. It is also often accompanied by an eczematous eruption about the nostrils and just inside the restibule of the nose in children.

Diagnosis.-The diagnosis can usually be easily made. The disease may, however, be mistaken for nasal engorgement 
depending upon a neurotic perturbation, a toxæmia, or other temporary systemic disorder, such as influenza, hay fever, or malarial poisoning. The projection upon the part of a solution of cocaine (two to four per cent) will often assist in diagnosis, for if the swelling be a true hypertrophy, the cocaine will not diminish it very much, while if it be only a congestion or temporary stasis of blood in the cavernous plexus it will be observed to nearly disappear as an effect of the cocaine. It may also be mistaken for the presence of nasal and nasopharyngeal polypi and other neoplasms, such as sarcoma; also for syphilitic disease of the septum nasi or other parts of the nasal chambers. It often resembles acute edematous rhinitis.

In rhinitis cedematosa the swelling of the turbinates may be so great posteriorly as to cause a projection into the nasopharynx simulating myxoma, as in a case reported by Dr. Mulhall, of St. Louis. The swelling may be confined to either one
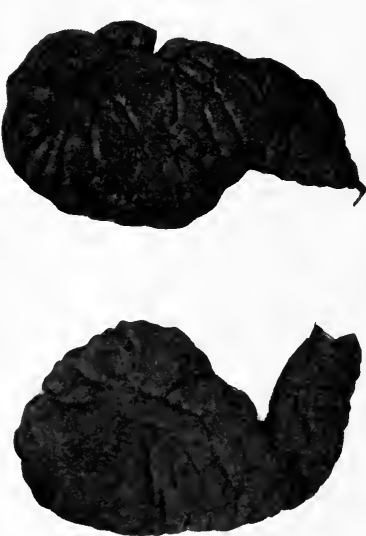

Fig. 87.-Posterior hypertrophies of the right and left inferior turbinals (removed by snare; natural size). or the other of the turbinates, or appear alternately in one and the other nasal passage. It consists of serous infiltration of the mucosa principally, and is pale, and soft to the touch. There is not very much mucous discharge from the nose in such cases, but very great nasal stenosis (Glasgow). Puncture of the swelling relieves the parts temporarily, but the serous fluid soon accumulates again if the cause of the disease continues.

From the systemic affections the clinical history of the case will usually suffice to differentiate it. Dr. Glasgow, who first called attention to this affection, 'regards it as often a form of la grippe, although it may be subacute or chronic, constituting chronic intumescent rhinitis.

The local symptoms and appearance are essentially more or less obstruction to the nasal passages, with mouth-breathing, and extra secretion and discharge of mucous or muco-purulent material. Chronic hypertrophic rhinitis is often mistaken for myxomatous growths, and, indeed, sometimes the swelling, posteriorly, resembles a polyp very much, while in many cases 
the two are associated (see Fig. 87). The swelling is seen to be continuous with the adjacent mucous membrane, without a pedicle, and of the same colour and texture as the surrounding parts (see Fig. 88).

Treatment.-The treatment of this affection should be for the most part local, although systemic medication and climatic changes are often necessary, especially in children who suffer from enlargement of all the pharyngeal, faucial, and lingual lymphoid tissue and other glands. Some cases, but not all, of true hypertrophy of the turbinated bodies, as well as hypertrophy of the faucial tonsils, are much relieved by a residence in warm or dry climates, such as the Northwest, Arizona, Mexico, and Egypt. In another class of cases (children) the internal administration of the iodide of iron, or the

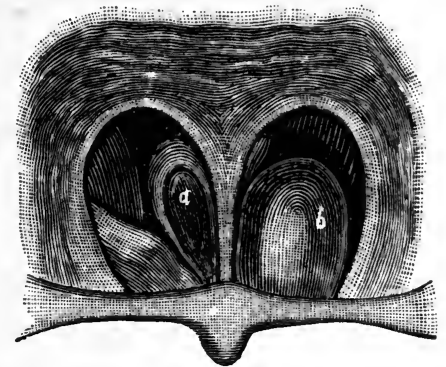

FIG. 88.- $a$, cup-shaped depression on deviated septum, caused by swollen posterior end of turbinal ; $b$, hypertrophied turbinal (posterior end) on opposite side (Jarvis). iodides, may prove very beneficial, especially if the patient be at or about the age of puberty, at which time there is a natural tendency toward the resorption of lymphoid tissue. However, in the majority of cases vigorous local treatment only gives relief.

M. Laverand believes that a residence at the seaside for a certain length of time may be beneficial for many cases of chronic affections of the nose, throat, and ear, with the following exceptions :

I. If there is a constant or intermittent suppuration of the ears.

2. If there is a sclerotic otitis, with buzzing sound in the ears.

3. Diseases of the pharynx and of the larynx in persons subject to congestion, in excitable tuberculous persons, and in arthritics who are predisposed to congestion or acute or subacute attacks of inflammation of the mucous membranes.

The writer does not think that there are many cases of true hypertrophic rhinitis which can be effectually relieved without the adoption of some form of surgical treatment; for the use of sprays or powders of any sort are signally inefficient, excepting 
for palliative treatment, while the same may be said of climatic changes.

Seiler, of Philadelphia, believes that a sort of prophylactic treatment should be carefully adopted, especially for the forestalling of catarrhal affections in children. He recommends for this purpose teaching the child early in life to cleanse the nose daily by the snuffing up of alkaline solutions. The writer thinks that this mode of treating natural nasal passages should be avoided, because the nasal secretions serve an important physiological purpose, and should not be washed away.

Dr. Bates, of New York, recommends the syringing down or through the nasal duct with sweet oil, solution of salt and water, or solution of nitrate of silver, one grain to the ounce, as the case may be. He prefers the oil to be used three times a week. He was led to this form of treatment by finding that patients suffering from dacryocystitis, and also from one of the various forms of nasal catarrh, were promptly relieved by treatment of the nasal duct.

As a rule, there are two main therapeutic indications: first, to relieve the mechanical obstruction to nasal respiration and the function of olfaction; and, secondly, to relieve the pathological condition of the parts and its sequences, such as defective drainage, etc. There are several methods by which this may be accomplished, viz., by the use of escharotics, the snare, the knife, or the scissors. When the bony or cartilaginous framework of the nose presents any considerable deformity in the shape of spurs or elbows or ridges of the septum, or shelving or hooked projections of the turbinated bones, a radical cure cannot be effected without their removal. The writer thinks, however, that the first step should be the destruction of a portion of the offending hypertrophic membrane, providing care is taken to always destroy as little of the mucous membrane as possible.

Escharotics:-A great many chemical escharotics have been used from time to time, such as Vienna paste, arsenious, acetic, lactic, chloracetic, nitromuriatic, carbolic, and chromic acids ; of these, the chloracetic and chromic acids have enjoyed the greatest popularity, on account of their being less painful in their application and more limited in their destructive action.

A submucous linear cauterization of the nasal turbinals has been advocated by Dr. Norville H. Pierce, of Chicago, for 
the reduction of hypertrophied turbinated bodies. The mode of procedure is as follows: A small incision is made in the hypertrophied membrane first; then with a blunt, flat probe the mucous membrane is carefully separated from the erectile tissue underneath; then a sound, the end of which is cup-shaped and upon which has been fused a few crystals of chromic acid, is inserted into the incision, and the track already made by the probe thus cauterized. It is said to be less painful than ordinary cauterization, and that it will not produce sloughs or any bad after-effects.

Killian, Gleitsman, and others advocate the method of using a saturated solution of trichloracetic acid, applied on a cotton wad to the district to be cauterized. It would seem, however, that each and all of these chemical agents are objectionable as escharotic agents for the nasal mucous membranes, because of the difficulty in accurately limiting the range of their action on the surface of the membrane, while securing at the same time the caustic action to a sufficient depth. Hence the galvanocautery is undoubtedly far superior for the destruction of the soft tissues, because its application can be regulated with accuracy, so that one may be certain of destroying the tissues to the desired depth and extent. Besides this, there is usually little or no bleeding, and not much reactionary swelling or inflammation of the tissues if proper care is used. The ulterior effect of any cauterization is not only the destruction of the deeper bloodvessels and tissues (cavernous plexus), but to pin down, so to speak, by cicatricial tissue the redundant projecting surface.

Sometimes an unusual hemorrhage or a reactionary inflammation of the nasal passages, pharynx, or accessory sinuses may follow the use of the galvano-cautery, or, in fact, the use of any escharotic. Dr. Daly and others deprecate the unnecessary use of the galvano-cautery for intranasal treatment. He believes, as also Bosworth and others, that it should not be used excepting by skilled hands. A number of cases of severe inflammation of the tissues of the nose, and also of the middle ear, as a result of its careless use have been reported.

Dr. T. A. De Blois, in speaking of the after-results of cauterization of the turbinates, calls attention to the fact that while the development of cicatricial tissue may show the beneficial result of the operation, that the mucous membrane in many cases above and below the cicatrization becomes subsequently 
hypertrophied, requiring still further operative procedure. $\mathrm{He}$ calls attention also to the errors either of not burning the tissue enough on the one hand or burning it too much on the other. It is undoubtedly true that a too superficial cauterization of the mucous membrane may do harm instead of good, by exciting the development of granulation tissue and hyperplasia; and that too great a destruction of the tissue may not only prove mischievous at the time by producing great irritation and inflammation of the adjacent and subjacent structures, but may prove pernicious through the permanent loss of too much mucous membrane and its substitution with cicatricial tissue. Besides, there arises the possibility of starting and favouring a progressive atrophy by the destruction of too much tissue. The danger of adhesions between the two sides must be kept in mind also.

An article from the pen of Dr. E. Fletcher Ingals, "On Cauterization of the Nares and Accidents that may Follow," presents the subject in such an excellent manner that I quote from it freely as follows :

In his experience Dr. Ingals has never had any serious results from cauterization, and believes that those which have occurred to others have often been due to carelessness. Looking over the records of his private patients, he found that for hypertrophic rhinitis, intumescent rhinitis, and simple chronic rhinitis, cauterizations have been done about one and a fifth times on the average in each patient. Fourteen hundred and fifty patients with hypertrophic rhinitis had been cauterized I,950 times, 450 patients with intumescent rhinitis had been cauterized 150 times, and 700 patients with simple chronic rhinitis had been cauterized I 50 times, making 2,600 patients cauterized 3,000 times. These figures, he says, are not strictly accurate, but are as near as can be computed without actually counting the cauterizations done in each and every case. Excluding the cases of simple chronic rhinitis which have been frequently cauterized, he finds I,900 cases suffering from hypertrophic or intumescent rhinitis that have been cauterized 2,850 times, making an average of about one and one-third times for each patient. An examination of these records, which have been carefully kept, reveals no serious accident in any cases. Frequently, in about twenty per cent of the cases, especially in cold weather, the patients may suffer considerable inconvenience 
for four or five days from reaction, and in a limited number of cases-perhaps five per cent-they feel for ten or twelve days afterward as though they had taken "cold in the head." Frequently a long cauterization will produce swelling and obstruction of the nares, and headache with some fever, for four or five days. In most if not all of these cases, if cauterization of one half this extent were made the unfavourable symptoms would not follow. The annoyance which patients suffer after cauterization, as a rule, depends largely upon the extent of the burn, frequency of its repetition, and care exercised to avoid "taking cold." He has frequently observed slight adhesions following cauterization, especially where it has been done opposite a large spur over the septum. In only one case, however, did he have serious hemorrhage following cauterization. He believes that cases of inflammation of the Eustachian tube or middle ear, as reported, having followed cauterization of the nares, have been due to carrying the electrode too far back. He has not seen any inflammation of the tonsils following cauterization. - Erysipelatous inflammation has been a sequel of this treatment in only 4 persons out of 2,600 . In two of these the erysipelas did not come on until eight or ten days after the operation, and in one of these it recurred about a month after cauterization. $\mathrm{He}$ has seen several persons in whom the use of a spray containing two or three grains of menthol to the ounce would cause inflammation of the nostril and of the upper lip within three or four days. He cites a case of Dr. A. H. Plummer, who reported to him that an acute dermatitis always followed the use of a one-grain solution of menthol and carbolic acid, each in an ounce of liquid albolene, within twenty-four hours after its use. He believes, therefore, that idiosyncrasy has a good deal to do with these cases of erysipelas following cauterization of the nasal passages, etc.

The details of the application of the galvano-cautery are as follows :

The operation is much more easily performed with the patient in the sitting posture, but when for any reason it is desirable to use a general anæsthetic, such as chloroform, of course the patient should be in the recumbent position. Neither ether nor any other inflammable agent should ever be used, because of the danger of the ignition of the fumes by the incandescent electrode. Nitrous-oxide anæsthesia may be 
used when more convenient. The first step consists of thoroughly cleansing the nasal passages with one of the mild saline solutions, by means of either a douche or a spray. Next, a spray of a four-per-cent solution of cocaine should be projected into the nasal passage, and, followed by the application of a ten-per-cent solution of cocaine, carried to the part by means of a pledget of absorbent cotton. At the end of four to seven minutes the mucous membrane ought to look pale, and should have lost its sensibility. If these effects are wanting, another application of the drug should be made.

The part having now been prepared, a nasal speculum or shield should be introduced to protect the opposite surface of the septum from the heat of the electrode. The speculum de.

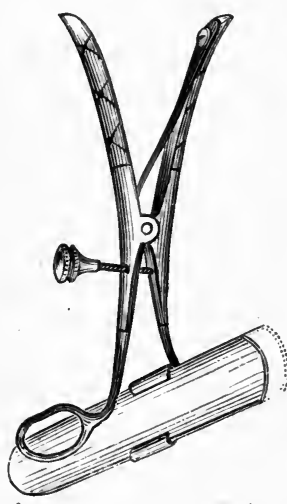

FIG. 89.-Shurly's nasal speculum with ivory slide, right and left. vised by the author usually answers the purpose very well (see Fig. 89); instead an electrode with one side guarded may be employed. This is a necessary precaution in order to avoid the subsequent formation of granulation tissue on each side, which would lead to the development of troublesome synechia or bridges of cicatricial tissue. The speculum should first be introduced into the naris closed, with the ivory shield drawn out, after which it is to be opened a little by moving the set-screw, and the shield then gently pushed in alongside the septum. The speculum being now in position, the electrode is introduced well back with its edge against the region to be touched, the electric current is turned on, and the glowing electrode firmly and slowly drawn forward to the anterior limit of the part to be cauterized, when it is to be turned off and the instrument withdrawn. The electrode should not be withdrawn suddenly, lest it adhere to the tissues. Care must be taken not to touch the vestibule of the nares in the withdrawal. Such a mishap gives the patient pain, and also sometimes leaves a pain. ful abrasion (burn) of the nostril which may last several days. 
The subsequent treatment consists of spraying the part with petrolina oil or cotton-seed oil, and immediately thereafter blowing a little aristol on the part from an insufflator. It is not necessary, as a rule, to introduce absorbent cotton or any such substance into the nasal passage, excepting in cold weather or when there is much hemorrhage. When the weather is cold a small piece of absorbent cotton should be put into the nostril, and the patient directed to remove it immediately upon arriving at home. This may be repeated for three or four days whenever the patient goes out. In case of considerable hemorrhage, a strip of Bernay's cotton may be introduced after having been dipped in carbolized oil. This may be removed on the second day by gentle manipulation after a thorough spraying of the passage with either petrolina or cotton-seed oil, and an insufflation of aristol made. Should too much reactionary inflammation supervene, the part should be treated once or twice daily with a spray of Dobell's or a creoline solution, followed by an insufflation of either aristol or iodoform. Only one side of the nose should be operated on at a sitting. When both sides are to be cauterized there should be an interval of at least a week between the sittings. It is rarely necessary for the patient to remain indoors continuously for more than a few hours or a day after a cauterization. If, however, the patient's occupation entails exposure to "cold," dust, etc., the wounded nasal passage should be protected by the wearing of a pledget of cotton in the anterior naris.' Any of the cautery batteries on the market may be used. In places provided with a public lighting plant, the street current, properly modified by a "rheostat" or "transformer," will be found far superior to the portable or storage batteries, as a rule. Electrodes, of various shapes and sizes, will be found at the instrument makers.

The thermo-cautery is rarely applicable to the treatment of this disease because of its cumbersome mechanism. The red-hot probe, which was formerly used, is only efficient for a mere superficial limited action. Electrolysis has been used of late with apparently good results, some practitioners preferring the monopolar and some the bipolar methods. The writer has had little experience in its application to nasal hypertrophies, but, from observation upon a limited number of cases, concludes that the bipolar method is preferable. For general use it is much inferior 
to the galvanocautery, on account mainly of the uncertainty of its effects. For the removal of posterior hypertrophies there is probably no better method than the one introduced by Jarvis, by means of the wire snare (Fig. 90). This operation is often difficult of accomplishment because it is not easy to engage the distended tissues in the loop, especially if there be any deformity

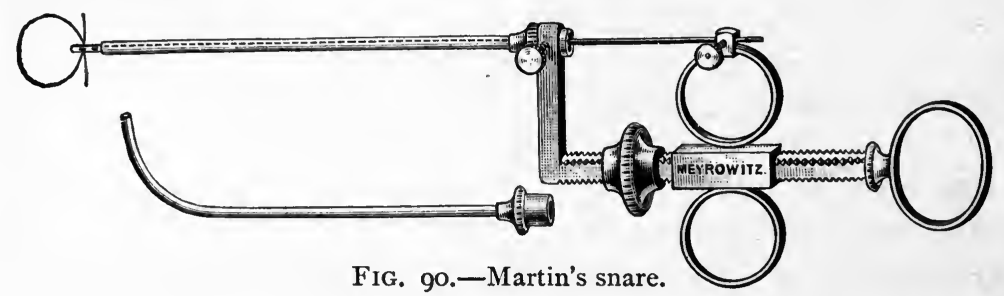

of the nasal septum. The snare of Dr. Hickey (see Fig. 92), which allows the loop to project from the side of the cannula, greatly facilitates this manipulation. In cases of anterior hypertrophies, particularly of the lower turbinated body, this method of operation (by snare) will often be impossible to execute without the use of the transfixion needle. The galvano-cautery loop of platinum wire may be used for the removal of posterior hypertrophies and the turbinated bones when for any reason the cold

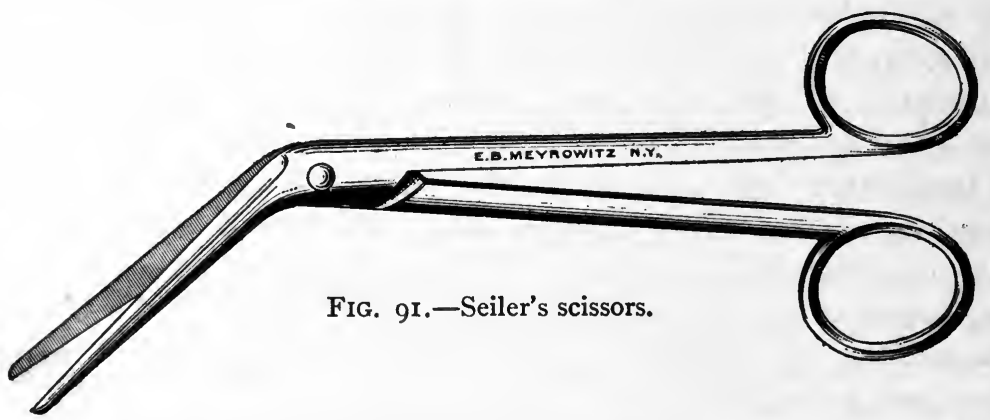

snare is considered inadvisable, as, for instance, with hæmophilia. It is as a rule far more difficult of adjustment than the cold wire snare. The knife and scissors are rarely used nowadays for the removal of exuberant portions of the turbinated tissues, unless portions of the turbinated bones are to be included; for this purpose the serrated scissors are very useful (Fig. 9I).

Dr. A. E. Prince, of Jacksonville, Ill., has devised a pair of cutting forceps for removal of posterior hypertrophies of the turbinals which he recommended to be used in place of 
the snare. The operation is as follows: He anæsthetizes the patient in the recumbent posture with two drams of ethylbromide, or with chloroform or ether. The mouth being kept open with a gag, the index finger of the left hand is passed into

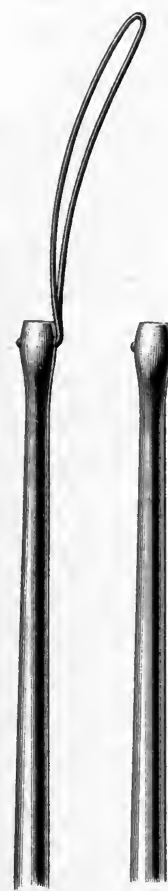

Fig. 92.-Hickey's snare for posterior hypertrophies. The loop on the left shows position for introduction; the loop on the right is the position (produced by tightening the wire) suitable for engaging the growth.

the naso-pharynx, and the forceps (with a cutting edge on each blade) are introduced with convex side down until reaching the finger which is upon the enlarged mass. The forceps are now rotated upward and opened so as to include the mass between their jaws, when, the blades being brought together, a twisting movement outward is made which takes away the mass, partly by cutting and partly by tearing. When necessary, the opposite side is operated upon at the same time. The advantages of this method over the snare are only apparent in those cases where it is impossible to engage the mass in the loop of the snare.

If other appliances are not at hand, the curved serrated scissors, or a pair of dull-edged scissors, may be used for cutting away the hypertrophic tissue. However, as this proceeding is a painful one under any circumstances, and as the resulting wound may be troublesome, this plan cannot be recommended.

Drs. S. Stein, H. V. Wurdeman, and many others, corroborate the observations of Gleitsman regarding the excellent effects of trichloracetic acid, both with and after the galvano-cautery, especially its power for preventing reactive inflammation after the galvano-cautery, or after any other cauterization. A solution of I to 500-2000 once applied will keepall suppurative processes in abeyance for a week. It may be used also in acute rhinitis, or after any operative procedure in the nose as an antiseptic. In coryza from any sort of injury it may be used in the proportion of half a teaspoonful of a one-per-cent solution, 
dropped lukewarm into either side of the nose, two to four times a day, when it will produce an abundant secretion of mucus and improvement of all subjective symptoms. Stein has seldom observed serious complications after intranasal operations when this was used. He believes that it has an antibacteriologic action, and that it imprisons the micro-organisms in the colloid secretions. After cutting operations he usually puts a crystal of trichloracetic acid on the edge of the wound, but he uses it in a different manner upon a galvanocautery wound. In the latter instance the burned area is divested of its water by mopping with a piedget of absorbent cotton, so that the crystals do not liquefy except on the edges of the burn. I believe, here, he uses a strongly concentrated solution. (As this acid is very hygroscopic, if the cork of a bottle containing the crystals be kept open for a few hours such a solution may be obtained.) An important point in the use of trichloracetic acid is that after its use no adhesive inflammation of the neighbouring parts follows, such as often occurs after chromic acid and cauterization by the galvano-cautery. This kindly action has allowed the use of the galvano-cautery, with an after application of trichloracetic acid upon growths of the nasal septum and in very narrow noses, without danger of adhesions; and is a very useful caustic for the softer hypertrophies and enlargements as well as in inflammation of the pharynx and tonsils.

The scarification of the turbinated bodies with a knife, as formerly practised, is, as far as I have observed, useless, excepting for temporary relief. The insufflation of astringent powders, excepting in young children, is of very little benefit; in fact, as a rule, such treatment is pernicious, because it adds to the irritation of the nasal passages. The inefficiency of topical applications, whether astringent or not, becomes obvious when we consider that the pathological changes are below the surface, and cannot be influenced very much by temporary changes in the calibre of the blood-vessels.

For the milder conditions of chronic rhinitis many practitioners use the oleaginous preparations in the form of sprays, such as mixtures of petrolina, olive, or cotton-seed oil, with eucalyptus, rosemary, camphor, thymol, creosote, etc.

Clarence Rice believes that the long-continued use of oily sprays in the nose tends to produce dryness of the surface and 
a contraction of the erectile tissues which simulates atrophy. He thinks that iodoform, iodol, and aristol seem to lose much of their characteristic effects when sprayed in these oily vehicles. These remedies are much more effectively used when in ethereal solution applied by applicators or when insufflated in the form of powder. Many observers think that the effect of throwing watery solutions of medicaments into the anterior nares, excepting in cases of atrophic rhinitis, is harmful. Rice prefers solutions of hydrogen peroxide, from ten to twenty per cent, or mercuric bichloride, I to 4000 ; solutions of boric acid, or potassium permanganate, or carbolic acid for cleansing.

Judicious bathing and exercise frequently prove efficacious for the relief of hypertrophic rhinitis, when the turbinals are not too much enlarged nor too much thickened by connective tissue. Indeed, the writer knows of several cases, especially in children, where frequent rubbing of the skin, out-of-door exercise, and proper feeding seemed to avert the necessity of an operation. In children, however, the removal of enlarged adenoid glands may constitute all the treatment necessary.

Adhesions.-Synechia after cauterizations frequently occur, even when the operator is careful. For the management of such accidents see the chapter on Deformities of Nasal Passages.

\section{Chronic Atrophic Rhinitis}

Synonyms : Rhinitis Sclerosa-Rhinitis Atrophica-Non-fœtida-Dry Catarrh

Atrophic rhinitis non-fœtida is a disease characterized by progressive atrophy of the glandular tissue of the mucous membrane of the nose and turbinated bones. It is usually confined at first to the turbinate bodies, but later on involves the whole of the Schneiderian membrane proper, and the olfactory portion as well.

Etiology.-The etiology of this affection has been a matter of speculation ever since the time of its recognition as a disease. At the present time it is by many observers regarded as the effect of hypertrophic rhinitis, and by others as the expression of an essentially neurotrophic disorder. Again, its causation is laid upon chemical and microbiotic grounds, while other observers think the disease is an effect of purulent rhinitis. The experiments of Thomson and Hewlet, however, seem to show that neither micro-organism nor chemical changes in 
the secretions play much of a part in its etiology. They summarize the results of their observations as follows :

I. In all bacterioscopic examinations of the nasal fossæ and all researches relating to the nasal mucous membrane, etc., a clear distinction must be made between the vestibule of the nose and the mucous cavity proper. The former is lined with skin, and is no part of the nose cavity proper, leading to it only.

2. Tumefaction of the lining of the vestibule is difficult to avoid, even when this source of error has been realized.

3. In the dust and crusts of mucus and débris deposited among the vibrissæ of healthy subjects micro-organisms are never absent, but are usually abundant.

4. On the Schneiderian membrane the reverse is the case, for under normal conditions micro-organisms are never plentiful, rarely even numerous, and in more than eighty per cent of the cases no organisms whatever are found, the mucous membrane being perfectly sterile.

5. The occurrence of pathogenic organisms must be so infrequent that their presence on the Schneiderian membrane can only be regarded as exceptional.

Kyle, I believe, also states that there is no etiological connection between bacteria and this disease (atrophic rhinitis). Some bacteriologists have been able to isolate many germs from cultures made from the exudate in which marked odour existed (ozena). Some of these germs were pathogenic, and others non-pathogenic, but no one germ was considered an important factor, from the fact that no one germ was found to be always present. In cases in which the odour is pronounced there is often found a germ which by macroscopic and microscopic appearance (as well as by the test of sense of smell) is denominated the "bacillus fœetida." Loewenberg, Christovitch, and Fränkel believe in the microbic pathogenesis of the disease. The anæmia which almost all cases exhibit has been attributed to the interference with nasal breathing. That it is a true anæmia has been clearly proved by a series of blood examinations of cases in which there was nasal obstruction, the counts being made before and after relief. Zaufal observes that fetor of the breath is most marked in young subjects, and that after the age of twenty it is much less. Many observers regard this disease and ozena as identical.

Those believing in an antecedent hypertrophic stage say 
that during this stage of the disease small nodular masses are discharged, which are often described by the patient as pieces of flesh. Their presence is indicative of tissue destruction. Microscopically these masses are usually polypoid or adenoid. The fact, however, that fully thirty per cent of the cases of atrophic rhinitis are not preceded by hypertrophy, serves to neutralize the value of that theory. Dr. Clinton, however, reports to have seen a case which passed during several months from the hypertrophic to the atrophic stage.

According to Dr. John N. MacKenzie, atrophic rhinitis always appears as a sequel to pre-existing catarrhal inflammation. The rapidity with which the hypertrophic passes into the atrophic form of rhinitis is proportionate, in all probability, to the possession of some constitutional taint, such as congenital or acquired syphilis (Porcher). Bosworth believes that purulent rhinitis is the cause which in children is a catarrhal process in the first year, consisting essentially in an increased secretion of mucus, together with rapid desquamation of epithelial cells in its early stages, finally running its course as a purulent disease in from five to ten years, and developing into what is known as atrophic rhinitis. The theory that purulent inflammation of the accessory cavities is a cause of atrophic rhinitis was advanced many years ago by Michael. Hypertrophied mucous membrane has been observed in one nostril and atrophic degeneration in the other, by some observers, but this does not prove that either condition depends upon the other (Porcher). Syphilis frequently produces an atrophic degeneration in one or both nasal passages. As a natural result of rhinitis, purulent inflammation may originate in the accessory sinuses, and may likewise end in atrophic degeneration, with more or less complete destruction of the muciparous glands and follicles. It is said that atrophic degeneration almost always begins upon the middle or inferior turbinate bodies, but such is by no means the case, for in very many instances it begins in the superior turbinates.

Porcher and others state that sometimes the nasal passages become occluded, as if a hypertrophic instead of an atrophic process had been established. Such instances, however, are very rare. The atrophy in some cases is supposed to be due to pressure from antecedent hypertrophy, and sometimes it is attributed to simple non-use of the channel. 
Many writers believe that atrophic rhinitis is not a disease per se, but is a result of any inflammation, acute or chronic, specific or non-specific, whether excited by exposure to cold or continuous inhalation of irritating dust, gas, powders, etc., which causes a purulent inflammation that may or may not involve the accessory sinuses, but which must be sufficiently prolonged to wash away the epithelium, etc., from the nasal mucosa of turbinates, etc. (Porcher).

'Atrophic rhinitis may occur at any age, but is found more frequently in youth, adolescents, and the aged; it may occur among the latter as one of the features of senility. The disease is rather more frequent in the female than in the male adolescent. It does not occur in childhood, as a rule, except as a result of a congenital syphilis or tuberculosis, although it is sometimes met with at the age of seven years, or younger. The inhalation of irritating gases, such as ammonia, the fumes of hydrofluoric acid, chromic acid, and phosphorus, is regarded as predisposing to the development of atrophic rhinitis. A dry climate may also predispose to its development.

Dr. H. M. Wilson, Jr. (Denver), speaks theoretically of the effects of the drying of the nasal secretions into films, which make pressure upon the membrane of the nose and lead to its atrophy. Commenting on Bosworth's theory, he remarks that in the dry climate of Colorado this theory, if tenable, should be easily exemplified, inasmuch as the air is almost constantly dry, and very often dust-laden. Although there are many cases of this disease in Colorado still this factor in the causation does not seem sufficient, according to his observation. He thinks that the two elements of dry air and alkali dust do act forcibly in the production of the disease, but in the following way: the dry air essentially, in demanding on the part of the secretory apparatus a large amount of fluid; and the alkali dust, by attracting to the parts a larger quantity of blood. These agencies, unceasing in their action, make their influence felt at the entrance to the respiratory tract, and as long as the turbinates continue to respond to this excitation they must suffer accordingly. There comes a time, however, when the turbinates fail to meet the demand, and when they not only cease to grow, but atrophy. Of course, if this process could be demonstrated step by step, the relation of hypertrophic to atrophic rhinitis might be determined once for all, but in the 
very nature of things this is impossible. He says further, as a result of a careful study of the disease as it occurs in Colorado: "It is certain that the presence of dry discharge has nothing to do with its inception, and very little indeed to do with its continuance, for while wasted turbinals are very often observed in those who have spent a number of years in this State, an almost invariable absence of the indisputable fetor and accumulation of secretion is suggestive of a physiological rather than a mechanical process. The observation of these wasted structures, free from the classic accompaniments of stench and dry discharge, enforces the idea that the hypertrophic stage must have preceded the present, and that as a result of continuous and excessive stimulation has passed into one of exhaustion and of waste."

Artisans whose nasal mucous membrane is constantly impinged upon by the dust from wood, metal, and woollen fabrics, those who take snuff, and who live in their workshops or in cellars, are more liable to suffer from this affection.

For the last fifteen years the idea that the disease in some way depends for its origin upon nutritional failure has been gaining ground, and it was upon this theory that the writer in I 880 suggested and practised the application of galvanism within the nasal passages. The most essential point connected with the etiology of atrophic rhinitis is the question, What induces the initial trophic stages? in other words, What causes the perversion of function in the so-called trophic nerves? The writer confesses inability to answer this question, and knows of no investigations which have solved the problem, unless it be the theory of an antecedent neuritis affecting the trigeminal nerves. The array of various theories which have been advanced from time to time need not detain us because of their failure generally to coincide with clinical observations and facts.

Symptomatology.-The onset of the disease is so insidious that the early symptoms usually escape attention, especially if beginning in childhood. The patient notices first an increasing serous and crusty discharge from the nose, accompanied by a progressive sensation of uneasiness and sense of foreign substance in the nasal passages; then a diminution of discharge, accompanied by a sense of dryness, supervenes, to which is added a continual desire to clear the nose by blowing, acts of screatus, and hawking. The odour from the nasal passages is 
not marked until after considerable atrophy has taken place, at which time, even in spite of frequent cleansing of the passages, the fetor may simulate that of ozena. Fetor is a most distressing annoyance to the patient and his friends. The sense of smell is always very much obtunded, and may be entirely annihilated, or become subjective. From the result of local irritation of the nerve ends more or less cephalalgia or dull aching pain may be experienced, the latter being usually referred to the maxillary bone or occipital region. It is a noteworthy fact that the aching of the parts just mentioned is generally coincident to the amount of accumulation of desiccated secretion in the nasal channels. Different degrees of deafness and tinnitus aurium usually accompany the affection, denoting involvement of the middle ear or Eustachian tubes, and if the case be of long standing the latter symptoms are prominent ones. There is rarely any constitutional disturbance accompanying the disease in uncomplicated cases.

G. E. De Schweinitz (Philadelphia) states that a large number of ocular disorders (blepharitis, or conjunctival phlyctenulæ, kerato-conjunctivitis, and dacryocystitis) may have their origin in intranasal disease of this character. Thus, frequently there is an association between atrophic conditions of the nasal mucous membrane and the conjunctiva. There are quite a number of observations recorded in which serious symptoms concerning the eyes themselves, or the external ocular muscles and refractive media, have depended upon nasal and naso-pharyngeal disease.

The appearances are quite characteristic, and for convenience of description may be presented in three groups: The first comprises those cases which are sequential upon hypertrophic rhinitis, or upon local pressure from spurs, neoplasms, etc., and those in the earlier stages of the disease. In these the mucous membrane is observed to be hyperæmic, not very thin, and plastered here and there with clumps of semi-fluid secretion of whitish or grayish colour; the meatuses seem roomy, though the turbinated bodies show only a moderate degree of atrophy. After removal of the secretion the underlying mucous membrane appears smooth and shiny. The nasopharynx, although containing adherent clumps of dried mucus, appears otherwise quite natural. In the second group the meatuses appear much more roomy; the walls of the nasal chambers are plas- 
tered with a much dryer and more adherent secretion of a yellowish-gray colour. There may be more or less fetor of the secretion and expired air at this time, but not amounting to that of ozena. In the third group these appearances are much more aggravated, for the turbinated bodies are atrophied to mere vestiges projecting into the spacious nasal passages; the septum also seems much thinner, while any previously thickened or prominent spurs are also diminished or almost obliterated. The nasopharynx is generally involved, being roomy, and plastered with hard, dry, yellowish, greenish, or black crusts, especially over the pharyngeal tonsil. On removal of these crusts the parts beneath will appear very thin, shiny, and striated. In this stage of the disease the posterior ends of the turbinated bodies are apparently wanting in the rhinoscopic image, and may be represented by devious crenated lines only. The mucous membrane may glisten, or be of a dull red colour, granular, and speckled, or it may be of a pale buff colour, and also extremely attenuated. No capillaries or veinlets are apparent, as a rule. This condition is sometimes designated " nasal sclerosis."

Although "nasal sclerosis" may differ from atrophy in the rapid and sudden generation of fibrous tissue, they are really different conditions requiring somewhat different treatment (Seiss). In aggravated cases the whole attic of the nose may be open to the vision, and the sense of smell be entirely lost. Deafness and tinnitus aurium are likewise prominent, and almost constant accompaniments. The pharynx, and even the upper larynx, are usually involved in the advanced atrophic process, so that all the features of that form of pharyngeal disease commonly described as chronic follicular pharyngitis or atrophic pharyngitis are present.

The writer has met with several cases, especially in hospital practice, where the turbinals were entirely gone, and the nasal septum likewise nearly obliterated, the whole intranasal region presenting two large cavities not partitioned, excepting by a ridge along the floor of the nasal passages.

Pathology.-The striking pathological change in advanced cases is the almost entire loss of the glandular elements and other structures of the mucous membranes. Many of the glands and acini are replaced by straggling strands of connective or fibrous tissue. Some of the capillaries have been 
obliterated, others have become tortuous, while the epithelial or subepithelial tissues are histologically changed or wanting. The stratum of subepithelial tissue, which is so characteristic of mucous membrane, is usually entirely effaced, while the

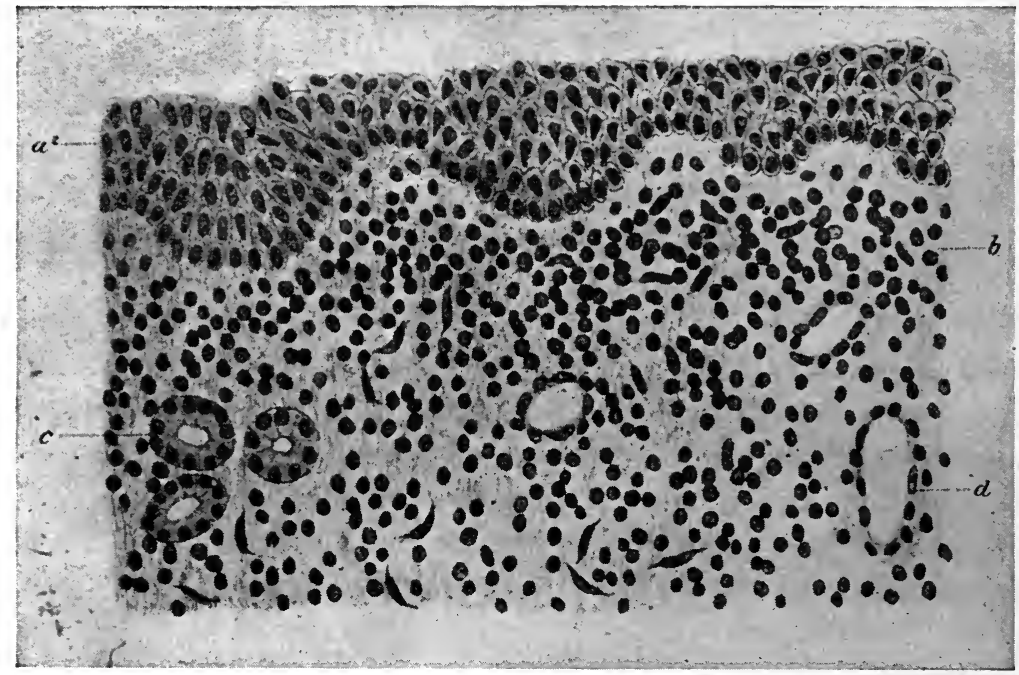

FIG. 93.-Atrophic rhinitis (Seifert and Kahn).

$a^{2}$, cylindrical cells changed to a cuboid form; $b$, plane showing (exceptional) cellular infiltration; $c$, section through atrophied glandulæ; $d$, section through a dilated capillary vessel.

surface epithelium is very thin, of irregular shape, and lies loosely in patches on the surface. Indeed, the histological features of the membrane are practically changed into a layer of loosely woven strands of ibrous tissue, inclosing irregular thick-walled, tortuous or obliterated capillaries and veins (see Fig. 93). The surface of the mucous membranes, in fact, is mostly protected by the film of dried secretion deposited upon it.

Prognosis.-The prognosis, so far as the nasal apparatus is concerned, is almost hopeless, for the wasted parts and their lost functions can never be restored.

A number of cases of the arrest of the disease, and some cures, have been recorded. Dr. John Dunn, of Richmond, Va., reports a case of cured atrophic rhinitis in a woman aged thirtytwo years. There was little or no secretion; the mucous membrane was a little paler than normal, and the seat of numerous 
minute depressions, some smaller, some larger, than others, and irregularly placed.

According to his description, these depressions were visible as minute scars, which had neither the healthy red colour nor moist appearance of the adjacent healthy areas of mucous membrane. They were confined to no one part of the nasal passages, but were spread promiscuously over the turbinals, the septum, and floor of the nose; they were very plainly marked, and were larger than elsewhere near the posterior border of the septum. There was not present any visible sign of an active inflammatory process. She gave a history of having had ozena from the time she was eighteen until she was twenty-eight years of age.

Treatment-General.-The treatment of this affection should be both general and local, the latter chiefly demanding attention. The general treatment should be hygienic, and the patient placed, if possible, amid the most salubrious surroundings. To that end, exposure to extremes of temperature, or to gasor dust-laden air, should be avoided. When consistent, the patient should be advised to live in a warm, moist climate, for oftentimes a residence near the southern sea-shore contributes immensely toward the cessation of the atrophy. Indeed, I have known cases of atrophic rhinitis in its earlier stages to be completely relieved by a change of residence to a subtropical climate-for example, one of the ocean islands. Systemic medicinal treatment often aids materially the effect of the local applications. The administration in moderate doses of iodide of potassium, iodide of sodium, iodide of iron, or iodide of arsenic will often be found of great benefit even when the patient is without syphilitic taint. These agents also are beneficial in the case of obese individuals.

The writer has noticed marked benefit following the internal administration of thyroid extract in one case ; and in another, small doses of pilocarpine, given three times a day, proved efficacious. The cases selected for the above-mentioned treatment were individuals who were otherwise in a pretty good state of health. It may be deemed inadvisable to subject weakly persons to either of these plans of treatment. The oldtime remedy, tartrate of antimony and potassium (Tartar emetic), has also proved beneficial, according to the writer's experience, in several cases. It should be given in small doses, and 
may be repeated three, four, or five times a day, if causing no nausea. The therapeutic value of each of these remedies probably depends upon their particular action upon the glandular apparatus of the mucous membrane.

In cases of atrophic rhinitis accompanied by considerable orbital or facial neuralgia, in which there is no evidence of marked suppuration of the accessory cavities, the continued administration of tincture of gelsemium, in five- to ten-drop doses, repeated four times a day, will give a very satisfactory result.

Strychnine, iron, or phosphorus in any form will be clearly indicated in the management of some cases, especially females, and those affected with chloro-anæmia or functional neurosis of the ganglionic nervous system. Mercurials, quinine, arsenic, or salicin may be necessary adjuvants in the treatment of persons who live in malarial districts. If goitre coexists, the internal use of iodine or its application by the method of electrophoresis should be adopted; the thorough rubbing in of the biniodide of mercury ointment will also oftentimes be advantageous.

Proper stomachic treatment for patients suffering from dyspepsia is necessary; indeed, the proper attention to the regulation of the digestive and assimilative functions will be a valuable adjuvant to the local treatment.

In all cases it is of the greatest importance to overcome habits of constipation. This will usually be best accomplished by the administration in reasonable quantities of the milder mineral waters or other saline laxatives. Another aid in general treatment is frequent bathing, either by sponging or immersion. The temperature of the bath, its kind and frequency, should be prescribed according to the susceptibility and needs of the patient. Friction baths also, and what are known as soap baths, often prove beneficial; for the latter purpose commercial laundry soap should be used. The method of taking a soap bath is as follows: The patient, being stripped of clothing and occupying a very warm room, is thoroughly covered with a thick lather of soap, which is allowed to partially dry, during a period of from three to five minutes; at the end of this time the redundant lather is wiped off with a dry towel (no water being used), the night-clothes are now put on, and the patient directed to go to bed. The following morning, on rising, a warm sponge bath may be taken. This bath may be repeated two or three times a week. 
Local. - Two sorts of local treatment should be instituted in every case, the one consisting of sprays, washes, or douches for the purpose mainly of removing the altered secretions and cleansing the parts, while the other should consist of measures for the promotion or restitution of the functions of the mucous membrane. For the former purpose a great variety of alkaline solutions are applicable, such as biborate of soda,-bicarbonate or chloride of soda, chlorate of potassium, phosphate of soda, etc., to which may be added antiseptics, such as carbolic acid, boric acid, menthol, formaldehyde, thymol, creolin, or chlorine water, etc. Or, instead of these, solutions of peroxide of hydrogen (diluted one to seven or ten), potassium permanganate (one to three grains to the ounce), or mercuric bichloride (I to 3,000) may be used. These solutions should be warmed if introduced by a nasal douche, syringe, or as a snuff water; but if projected upon the parts from an atomizer, previous warming will be unnecessary.

Lichtwitz advises nasal douches to be used only when there are crusts to be removed, because the dangers from its use are, first, disturbance of sense of smell, due to the action of the chemicals used; second, headache; and, third, suppuration of the middle ear. It will sometimes happen that even if properly used these means will fail to cleanse the nasal channels; in which case swabbing out the parts with cotton wads, either held in well constructed forceps or wound upon a cotton carrier, must be resorted to. In this manner the adherent masses can be dislodged. The cleansing process having been completed, the parts will be ready for other topical treatment. For routine applications, oleaginous solutions of thymol, eucalyptus, camphor, menthol, naphthaline and oleum picis, or oleum picis and balsam of Peru, are the safest and best, and for many cases in the earlier stages may suffice to bring about the desired amelioration.

Dr. Clarence C. Rice, of New York, recommends highly ozone in the treatment of some forms of atrophic rhinitis.

Dr. George L. Richards uses a solution of formaldehyde after the removal of crusts, etc. Each nasal passage being thoroughly washed out with a solution containing five to ten drops of a forty-per-cent solution of formaldehyde to eight ounces of warm water. As it is irritating even in a weak solution, a previous spray with cocaine is advisable. The writer has observed ex- 
cellent effects from injecting the nasal passages with very hot water, after the method recommended by Dr. Armstrong, of Brooklyn, N. Y. The water at first should be lukewarm, and then made hotter up to the point of toleration. It may be used twice daily.

In the later stages, however, more energetic local treatment is necessary to rouse healthy action for the delay or checking of the wasting process, and the following means have been found to bring forth good results. First, and probably the most efficient, perhaps, is the use of galvanism or faradism. The current should be conducted through a naked electrode with a ball-shaped distal extrèmity; the electrode should be slender, and long enough to touch almost any part of the walls of the nasal chambers. It is better to commence with galvanism, with a strength of current indicated by from one to five milliampères. One pole, preferably the cathode, should be applied to the auricular fossa, while the other, represented by the nasal electrode, is passed rapidly to and fro over the surface of the membrane, first upon one side of the nose and then upon the other. The duration of the séance will depend upon the susceptibility of the patient and the amount of hyperæmia or reactionary irritation induced. In cases of aggravated atrophy, a brisk faradic current will be found more efficient than the galvanic. At first the current should be a "primary" one, but if the toleration of the parts will admit of it, a "secondary current " may be substituted. In many instances it will be found advisable to alternate the séances between the galvanic and faradic currents. After the application of electricity the membrane should be sprayed with a solution of oil of rosemary, or oil of eucalyptus (one per cent) in petrolina or cottonseed oil. There are some patients who will tolerate the electric treatment daily, while others will not bear the applications oftener than once, twice, or three times a week. With the latter patients, an application may be made daily of a pigment of either resorcin, thymol, balsam of Peru, oleum picis, aristol, europhen, iodine, capsicum, oil of cloves, or extract of xanthoxylin (see formulary). Massage of the atrophic membrane has proved an efficient means of treatment in the hands of many laryngologists. There are two methods of doing this, one by hand and the other by an electric motor. This treatment consists in making rapid strokes with a ball-pointed in- 
strument all over the surface of the mucous membrane. If the patient experiences much pain from the manipulation, the end of the probe may be covered with a thin layer of cotton wadding or thin leather.

Massage for this affection was introduced by Dr. Michael Braum, of Austria, in a communication read at the Tenth International Medical Congress. Dr. Kellgrew, of London, has had an extended experience with this mode of treatment. The movements of Kellgrew consist of two kinds: the one, very light superficial and rapidly-made stroking movements over the parts, and the other, vibratory movements in rapid succession. The whole procedure is characterized by lightness, rapidity, and elasticity of touch. Probably the vibratory movements vary from two to three hundred per minute. The metal sound recommended measures twenty centimetres in length, and has a diameter at the larger end of two millimetres. The end entering the nose is finished with a rubber head, to which cotton may be attached. This cotton may be immersed in various antiseptic alkaline or other solutions. The sound is held in the hand like a pen-that is, between the thumb and first and second fingers. First the floor of the nose is treated, then the meatuses, inferior turbinated body, and then the middle turbinate, the full length, after which the septum is treated.

The Eustachian prominences may also be treated in the same manner, as well as the orifice of the Eustachian tube itself. The number of different sounds used in a single case varies. Kellgrew sometimes uses as many as twenty in one séance. Strict cleanliness must be observed, and the incrustations must be carefully removed. The vibrations should be made to every part of the nose within reach. The séance lasts from half a minute to ten minutes, according to the patient's power of endurance. Various solutions are recommended to be used in connection with this treatment. If there is great sensitiveness of the mucous membrane, it may be first painted with a twentyper-cent cocaine solution; subsequently petrolina oil may be applied, or vaseline ointment or a ten-per-cent iodine and glycerine ointment, or one of corrosive sublimate, or an ointment of balsam of Peru. Kellgrew claims to have treated one thousand patients by this method with marked success. The pharyngeal and laryngeal diseases have been included in this number. The most striking results obtained, it is said, were in fetid 
atrophic rhinitis (ozena). In some instances reported the atrophic turbinated bodies were observed to be returning to their normal proportions. Dr. Pierce, of London, England, reported six cases treated by this method which were taken from Chiara's clinic. In summarizing, Dr. Pierce commends, first, cleanliness, which is essential; second, the vibratory movements.

Dr. W. Freudenthal designed a very good instrument for employing electricity in vibratory massage. Another very efficient means of treating this disease is by the insertion of a tampon of absorbent cotton, as suggested by Gottstein. This should be inserted at night, and allowed to remain, if possible, until the following morning; one side only should be tamponed at a time. The tampon should be made large enough and long enough to snugly fit and impinge against the whole nasal cavity. For purposes of safety a thread should encircle the tampon throughout its extent, and the ends be fixed by means of a small piece of plaster to the cheek. By observing this precaution, the accident of the cotton being drawn into the nasopharynx or larynx may be averted. The rationale of the application of the tampon consists in the slight constant irritation which is bestowed by its gentle, constant pressure against the membrane. The removal of such a tampon several hours after its introduction discloses to the observer a membrane which is moist with secretion. It is asserted by some practitioners that the beneficial effect is partly due to the exclusion of air from the nasal passages, and not to the pressure of the cotton altogether. Porcher recommends saturating the cotton with the following solution: Iodide of potassium, $2^{1} / 2$ drams; iodine, 40 grains; glycerine, I ounce. The tampon is packed below the upper turbinate and the roof of the nose, and allowed to remain there for twenty-four hours. He reports very favourable results in one case so treated. The writer agrees with Seiler in the belief that the cotton tampon is just as beneficial without being saturated with anything, the principal effect being due to the irritation which its presence produces. Aseptic wool may be used as a substitute for the cotton (Seiler). Dr. Hubbard, of Toledo, saturates the cotton with an alcoholic solution of acetanilide. Leland, of Boston, applies a solution of ( 10 to 20 per cent) cocaine first, in order to open the nasal channel, and then introduces pledgets 
of cotton, which are allowed to remain for twenty or thirty minutes. These pledgets are then removed, and the nostrils packed with antiseptic cotton. He also uses resorcin as an application. Roe advocates the use of a solution of nitrate of silver after the parts have been thoroughly cleansed, and follows this often by the use of the galvanic current. MacKenzie and Ingals are opposed to the use of cocaine in these cases, both on account of the toxic effect and because it finally induces a permanent congestion of the parts. There is no doubt that less escharotic and more stimulating applications produce the best effects. The application to the parts of iodine, capsicum, and xanthoxylin, in the form of pigments carefully mixed, will give very good results. The application of iodine mixed with oleum theobromi, in the proportion of two to five grains of the former to one dram of the latter, or with oil of cloves, will be found beneficial and not very painful. It will be understood that the application must be made with a camel's-hair brush, while the cocoa butter is in a melted state. Powders, with the exception of iodoform, aristol, europhen, and orthoform, and the oleates of zinc or alum, ought never to be used in the treatment of this disease. The vegetable astringents, such as tannic or gallic acids, never fail to aggravate the condition of the parts. Iodoform or aristol, when mixed with sugar and powdered gum acacia, will sometimes act beneficially. Resorcin will be found to be an important topical application; being non-toxic, it may be quite freely used as a spray by the patient at home. It exerts a gently stimulat. ing and antiseptic effect.

\section{OZENA}

\section{Rhinitis Chronica Fotida-Rhinitis Caseosa-Fetid Coryza}

Ozena derives its name from the peculiarly offensive odour which characterizes the nasal secretion. It is regarded by many as a symptom, indicative of a morbid state which allows certain chemic or putrefactive changes to take place in the secretions of the nose, rather than as a special disease. This opinion depends upon the fact that fetid odours emanating from the nose may accompany caries or necrosis of the bones, nasal syphilis, the presence of malignant or benign tumours within the nasal chambers, or other morbid, conditions. Not- 
withstanding that it is generally associated with atrophy of the turbinals and pituitary membrane in general, it seems proper to treat of it independently, inasmuch as this odour is not always present in ordinary atrophic rhinitis.

The crusts may form anywhere on the surface of the lining membrane. They are found in greatest abundance usually in the superior and middle meatuses and toward the posterior portion of the nasal fossæ, and in the nasopharynx. The peculiar, penetrating, fetid odour is so disagreeable that it is well-nigh impossible for any one to associate with the patient, or even to be comfortable in the same room. Ozena is also characterized by a secretion, more or less purulent, which dries in the nasal passages in the form of crusts of a brown, yellow, green, or blackish colour. It is to these crusts that the odour belongs. Some writers, notably Moure, Tissier, and others, recognise four or five varieties of ozena. Moure, for instance, describes an adenoid form, a sinuous form, a necrosis form, a purulent form, and an atrophic form. Massei and others recognise a caseous form. While undoubtedly such divisions of the subject may prove convenient, it will be found difficult in practice to follow closely the lines of distinction.

Etiology and Pathology.-There has been much study and research from time to time concerning the etiology of this affection, on account of the obscurity and insidiousness of its early clinical history.

Age.-It is met with more often between the ages of five and nineteen, according to Goodale. Infants, however, are not exempt. Moldenhauer and Bosworth believe that the large majority of cases of this as well as the non-fetid form of atrophic rhinitis originate in a purulent rhinitis in childhood. The writer has never seen a case which originated after twenty years of age that was not syphilitic.

Sex.-Females are more susceptible to the disease than males. Both fetid and non-fetid atrophies are more than twice as common in females than males (Groodale).

It is met with more frequently among the poor, and possibly the ill-fed. It was formerly ascribed to struma, syphilis, or tuberculosis, many believing that it always represented a syphilitic taint, or a mixture of syphilis and scrofula or some other dyscrasia; for this reason the local manifestations were regarded as of little consequence. Schech, Brosman, and 
others believe that dyscrasiæ and scrofulosis are without doubt causative factors; but the majority of writers state that the greatest number of cases occur in constitutionally healthy persons. Rice, Moure, Goodale, Price Brown, and others, however, call particular attention to chloro-anæmia and unsanitary surroundings as favouring its development. According to the writer's observation, a large proportion of the cases are met with in hospital (clinic) practice. Again, it was supposed to depend always upon disease of the accessory cavities (muco-purulent sinusitis), particularly of the frontal and maxillary sinuses and ethmoid cells. One of the most vigorous advocates of this theory was Dr. Carl Michel, of Cologne, and later Grünwald, Bresgen, and others. This idea prevails to a wide extent at the present time, although in the opinion of different authors it is attributed to a "purulent rhinitis," or an antecedent hypertrophy or polypoid degeneration of the pituitary membrane, or to the presence of fungi in the nasal passages, which are capable, like the yeast fungus, of producing special fermentation products. Among the fungi, the mucor mucedo, the aspergillus, the torulæ, etc., have been often found in the nasal passages in old and neglected cases of ozena, where they have remained in a state of more or less passive or saprophytic existence. Blandon, Velpeau, Tissier, and Bosworth, among others, ascribe the disease almost entirely to a purulent ethmoiditis or osteitis. Bosworth thinks that ozena not only has its origin in this purulent osteitis, but that its peculiar manifestations are really the consequence of a purulent inflammation of the ethmoid cells, which has had its beginning during childhood. This same view is also brought forward in explanation of the pathogeny of atrophic rhinitis, non-fœtida, especially by those who regard ozena and ordinary atrophic rhinitis as one and the same disease.

The theory of the bacterial cause of the disease, although for a long time suspected, and perhaps occasionally partially demonstrated, did not attract very general attention until again promulgated by E. Fränkel in 1884. Loewenburg at the time described a bacillus which he called the "bacillus encapsulatus," which he had found present in all cases of the disease which he examined. It was therefore accepted by many as the specific cause of ozena. Guarnania also described a micro-organism-the streptothrix alba-which he, Massei, and 
others regard as the specific cause of the disease. It may be mentioned, however, in passing, that animals which have been inoculated with cultures or products of these micro-organisms have not become similarly affected, nor, as far as the writer is aware, have such animals ever suffered or died from septicæmia as a result of inoculation.

The peculiar fetid odour is generally supposed to be the result of a chemical or bio-chemical action upon the secretions retained in the nasal chambers. This view, however, may be seriously questioned. It seems more likely that the phenomenon (fœetidity) is not alone the result of the retention of the secretions in the nasal fossæ, and the effect of ordinary putrefactive decomposition thereof, but instead arises from some peculiarity of either the secretion of the part or the histologic elements exfoliated therefrom.

The theory that the disease is always due to purulent rhinitis, especially affecting the ethmoid cells, seems to be a plausible one, because of the analogous odour which accompanies the presence of carious or necrosed bone in the nasal passages. But the fact that many cases of purulent rhinitis, in which the source of the process can be traced to diseased (carious) ethmoidal cells, do not exhibit much, if any, odour or particular desiccation of the secretion, rather militates against this explanation of its etiology.

There has been much discussion regarding the part, if any, which antecedent hypertrophy of the turbinate bodies, nasal or pharyngeal adenopathies, polypi, or intranasal deformities may play in its causation. Although opinion is still somewhat divided upon this point, it would seem that the weight of evidence would indicate that only comparatively few cases exhibit any such abnormalities, either antecedent to or concurrent with the course of the disease. Exceptionally, ozena occurs in some cases of partial atrophy denominated technically "pressure atrophies."

The neurotrophic theory of causation must certainly find favour, when it is considered how insidiously the disease approaches and runs its course without marked inflammatory disturbance or sepsis. But just what the initial lesion is, or where and when it begins, are still unsettled questions. However, the conclusion that fibres or communicating branches of the trigeminus are probably concerned, does not seem at all 
illogical when one takes into account the physiological importance of the trigeminus in the nutrition of the nasal apparatus, and compares this fact with the striking process of denutrition which characterizes the course of this disease.

Pathology.-While pathology has not as yet cleared up the early steps of the diseased process, yet it has added greatly to our knowledge of it. Autopsies have been made upon subjects who had suffered from atrophic rhinitis, but, unfortunately, until recent times no particular examinations of the nasal passages had been made. Zuckerkandl, Haberman, and Fränkel have made many autopsies, but the results of their observations have not always been satisfactory. In some of Haberman's subjects the examination of the ethmoid cells proved negative ; and, as remarked by Moure, the examinations of Zuckerkandl having been made more for anatomical than for pathological purposes, the value of his observations becomes somewhat lessened, from a pathological point of view. Schuchardt's histologic studies were made from pieces of membrane taken from living subjects. The extreme atrophy of the turbinated bodies, both the cavernous and the osseous tissue, together with the atrophy, even to extinction of the glandular tissue of the pituitary membrane, are found to be constant pathologic features.

Regarding the microgenetic theory, it is obvious that anfractuous passages such as the nasal fossæ, lined with a mucous membrane which is undergoing atrophy, and the secretions of which are chemically if not biologically perverted, should furnish a good habitat for all sorts of aerial micro-organisms. Nevertheless, it has not been positively demonstrated that either Loewenburg's bacillus, the pseudo-diphtheria bacillus of Lautman, or the streptothrix alba of Guarnania, are the specific and only causes of the disease. Perhaps their presence may be considered as incidental, and depending upon the fermentative process taking place in the secretions, rather than as a causative factor.

Lautman thinks that the fetor depends upon the crusts and secretions entirely, and that there is really no syphilitic or scrofulous ozena, but that in such cases either necrosed bone or ulceration causes the particular phenomena. To summarize, it may be stated that the pathogenesis may be based upon three positive theories, viz.: 
I. The microbiotic theory. This theory probably has the most adherents. According to Abel, atrophic ozena is an infectious malady caused by the "bacillus mucosus," a microbe which is identical with the bacillus of Loewenburg. According to Belfanti and Della Vedova, it is the pseudo-diphtheria bacillus which plays the principal part in causation. This view he justifies by culture experiments. The pseudo-diphtheria bacillus however, is not always found in the cultures, and when found is usually associated with the "bacillus mucosus" of Abel. Microscopic sections of the mucous membrane seem to show that the tissues are not invaded by either the one or the other of these micro-organisms, contrary to Belfanti's belief. Lautman does not believe in the specificity of either of these bacilli in their causal relation to the disease.

2. The nervous theory. According to this hypothesis, trophic changes affect the secretions and the vitality of the pituitary membrane, while the fetidity is due to saprophytic bacilli lodged on the surface of the deteriorated mucous membrane. The alleged proof is shown by sero-therapy. Injections of serum were tried in seven cases, resulting in the disappearance of fetidity in each. There was no change in the mucous membrane, however. Hypodermic injections of a salt solution were also made in four patients with good results.

3. The inflammatory theory (Gottstein and Zuckerkandl), that atrophy follows a period of hypertrophy. Zuckerkandl adheres to the view that atrophy of the mucous membrane and the turbinated bones is always preceded by a hypertrophic condition, and that atrophy of the bones prevails. He distinguishes three stages of atrophy, in the mildest of which only the turbinated bodies are thinner and more fragile; next, the size of the bone is reduced, and from the edge it is no longer convex, but plain, or even concave, until finally, of the bone so affected, only a small projection remains. The external nasal walls also become affected by atrophy, and frequently become so thin that the canals and the lachrymal ducts disappear. The angular nasal cavity gains so in size in many cases that the sphenoidal cavity as well as.the foramina ethmoidea are included. He found in nine cases that the sub-epithelial layers showed considerable infiltration of cells; the condition of the secretory glands in some cases presented a normal appearance, in others they were infiltrated, and in still other cases the num- 
ber of glands was reduced. The increase in the connective tissue was so marked that the degree of atrophic change could be judged thereby. Regarding fetor, he thinks that degeneration of pavement epithelia is the most important factor, the microorganisms being of only secondary importance. Schuchardt says the cause of ozena is the changed ciliary epithelium of the nasal mucous membrane into pavement epithelium. He remarks that in catarrhal conditions of the throat, and also of the uterus, the transformation of columnar into pavement epithelia occurs, and in consequence fetor makes its appearance. Berliner came to another conclusion in regard to the pathology of ozena. He thinks that primarily the cause of the disease is hypertrophy of the middle turbinated bone, and its pressure against the septum thereby produces a stagnation of the secretion, and successively an atrophy of the mucous membrane. The fetor, he thinks, is primarily caused by the contact of the stagnated secretion with the atmospheric air and its microorganisms. H. Krause found that the intima, and E. Bruckel that the adventitia of the nasal arteries, were obliterated. Muldaner claims that ozena is gradually produced by degeneration of the blood-vessels which supply the nose. Zaufaal thinks that the disease is the result of congenital malformation of the turbinated bones. Waterman, agreeing with Schuchardt, found, upon microscopic examination of pieces of mucous membrane, that the ciliated epithelium had undergone a change into pavement epithelium, with here and there a cylindrical epithelial scale apparent. The fibrillary connective tissue was bent, and there were accumulations of the cells, and a few corpuscles. Warren finds in the early stages that only the inferior turbinated bone and the tissue intimately connected with it is atrophied, while the middle turbinated tissue appears hypertrophic. He asks the question, Why, in certain individuals only, does the atrophic follow the hypertrophic form of the disease? He accounts for it by a special lack of resistance or a disposition toward atrophic conditions and neurotrophic disturbances in certain individuals. The nerve fibres (sympathetic) share in the degeneration, and this explains the vasomotor disturbances which Fränkel, Krause, and Muldaner call attention to. The bony parts not being properly nourished by the blood-vessels tributary to them, they also participate in the atrophic condition. The question has been asked, Why do not 
all the secretory glands of a region presided over by a given set of nerves undergo fatty degeneration or infiltration under abnormal circumstances? The probable answer to this query may possibly be found in the fact that peripheral or terminal nervous structures are subject to limited pathological processes independently of the nerve trunks from which they are derived.

Treatment.-Although from time to time cases of recovery from ozena are reported, yet in the majority of cases any plan of treatment affords temporary relief only. Thorough cleansing of the parts is always the first, if not the principal, element of treatment. Next to this come efforts to improve the general physical condition, and to prevent or limit the peculiar secretion and the chemical or microbiotic changes which such unhealthy secretion undergoes.

To accomplish the first desideratum, any of the well-known detergent or antiseptic alkaline solutions in common use should be diligently used by the patient, coupled, if necessary, with the mechanical removal of the crusts. Next, effort should be made to prevent the secretion and its decomposition. The internal administration of agents such as iron, strychnia, arsenic, iodide of iron, cod-liver oil, and phosphorus for improving the general condition are always in order. In this connection the hygiene of the person must be kept in view, and plenty of outdoor life, with good nitrogenous and other nutritious food, should be ordered.

Regarding the special treatment of the disease, as might be expected, many different plans and agents have been suggested. Cozzolino believes in the surgical treatment. He scrapes the accessible membrane once or twice a week with a curette. He does not think much of the specific treatment of the disease by anti-diphtheritic or other serums. On the other hand, Gradenigo, and others who accept the theory of the infective character of ozena, recognise Belfanti's or the other bacilli as its cause. Gradenigo has used hypodermically the anti-diphtheritic serum in thirty-two cases, sixteen of which were practically cured-that is, the disease was mitigated to such an extent that the formation of crusts and generation of fetor were stopped. In the other cases there was also a marked improvement shown. Most of the patients had had the disease for a long time, and had tried other treatment without avail. In the cases that were approximately cured, 
the duration of treatment varied from thirty to sixty-eight days, and the number of units of serum employed in the individual cases ranged from 5,200 to 27,200 . In all cases, œdema occurred at the site of the injection, and sometimes urti. caria supervened after a number of injections had been given but these untoward effects were of brief continuance. In the sixteen cases that were cured, the duration of treatment was from fifteen to seventeen days, and the number of injections ranged from four to thirty-five, the number of units of serum used being from 5,200 to 48,000. Mild local or general reaction occurred in almost all of these cases, and attacks of bleeding from the nose were sometimes observed. Professor Gradenigo has employed the same treatment in two cases of chronic purulent otitis, or, rather, it happened that two of his ozena patients were affected with this ear disease, and the treatment was observed to have had a very favourable effect. F. Cathelin reported a case in which injections of five cubic centimetres of anti-diphtheritic serum were made three times a week for three months, cleansing washes being used in the meanwhile. Later, the dose was changed to ten centimetres once weekly. After seven months' treatment the patient was apparently cured.

Stoker strongly advocates the use of oxygen gas, using equal parts of oxygen and purified air; the latter is prepared by being pumped by means of foot or hand bellows through two wash-bowls or bottles, the first containing some water and the second some of Conday's fluid. The bottles are attached to a bag for this purpose, and when the bag is half full it is then detached from the bellows and filled with oxygen. The nose-piece is passed into one nostril, while the other nostril is plugged with cotton wool; the patient is directed to breathe through the mouth, the stop-cocks are turned on, and the treatment begun. In ear cases the terminal piece is placed in the external auditory meatus. It is desirable to have several different sized terminals, in order to fit different sized orifices. The oxygen should be allowed to pass into either the nose or ear from three to six hours daily. In nasal cases it is best to use the gas for about half an hour to an hour at a time, giving intervals of rest between these times. If used for more than an hour it is apt to cause headache. The only additional treatment to be adopted is the use of warm water for cleansing the 
parts during the day. The necessity for doing so, either seldom or often, will depend on the amount of discharge, but it must not be done less than twice daily. Stoker reports four cases, two of ozena and two of suppurative middle-ear diseases, cured by this method.

Rice recommends ozone for the treatment of this affection, as well as for non-fetid atrophic rhinitis.

Kafeman relies more upon tamponage. He fills completely the nasal cavities with tampons, leaving them in a longer or shorter time, according to the circumstances of the case.

Ichthyol is deservedly becoming a popular remedy for this disease. The writer has seen much benefit follow its use as a pigment when applied every day or two.

Dr. David Phillips reports the treatment of twenty-seven cases of atrophic rhinitis with ichthyol. He uses a solution of from one to five per cent in kerosolene. All but one of the cases reported seemed to improve under this treatment. $\mathrm{He}$ also makes applications of it to the pharynx when necessary. His mode of application is as follows: The nose is sprayed with an alkaline solution, and then cleansed by means of cotton wrapped around an applicator. The passages are then allowed to dry, and a five-per-cent kerosolene ichthyol solution is applied thoroughly by means of a cotton applicator. An oily spray for cleansing is not advisable, because a certain portion of it remains on the surface and prevents the ichthyol solution from coming in contact with the mucous membrane. The patient is instructed to clean the nose night and morning with the alkaline spray (see formulary). After the spraying, a mixture consisting of one third each of kerosolene and ichthyol, and about three to five per cent of liquid albolene or petrolina oil, with a little eucalyptol or menthol added to disguise the fishy taste of the ichthyol, is applied. Dr. A. Muellere, of Australia, speaking of the therapeutic action of ichthyol, says that it must be ascribed to a peculiarly close combination between its sulphur and carbon. The introduction of both elements into the nasal cavities in a pure and at the same time soluble form, he thinks, brings about the contraction of the congested and abnormally distended capillaries or veins.

Much other testimony is also available, showing the prompt and beneficial effects of ichthyol. A thorough cleansing of the nasal passages with some alkaline solution to remove the inspis- 
sated secretion is always necessary. The nasal cavities may be flushed with a two- to five-per-cent aqueous solution of ichthyol by means of a syringe or douche. Then a cotton applicator, supplied with a twenty-five- to thirty-five-per-cent solution of the same agent, may be applied by brush or swab to the mucous membrane of the affected areas, including both the anterior and posterior nares. The same treatment is also applicable to the treatment of pharyngitis sicca.

F. Clemens, and others, highly recommend the use of a solution of saccharine by means of a douche or syringe once or twice daily, after previous cleansing of the nasal passages. He uses from one half to one gramme dissolved in an alkaline solution at each treatment. This treatment is based upon the antiseptic action of saccharine on pancreatic fluid and urine.

One-per-cent solutions of meta-cresol."anytole" has also been recommended (in the proportion of sixty parts of meta-cresol to forty parts of "anytin"); of this a one-per-cent solution may be applied daily. Stein advocates the use of chloracetic acid in both non-fetid and fetid atrophic rhinitis. He says it is very useful in weak solutions applied to the nose in simple atrophic rhinitis. He claims that it is able in these cases to produce even hypertrophy of the tissues. In true ozena he applies it with benefit in much stronger solutions-that is, from one half to ten per cent. He regards it in these cases as a specific. He sometimes combines it with iodine in syphilitic cases. It should be applied in the following manner: Begin with a weak solution-one to two per cent-and use this as long as there is improvement, afterward using stronger solutions. It is not proper to begin with strong solutions, as at first many patients are unable to bear them. In many cases the fetor is stopped in a couple of days. For its application, a probe twenty cubic centimetres long and two and a half millimetres thick, which is curved on each end, is employed. One of the ends is bent, in order to get under the turbinated body better. Upon one of the curved ends hygroscopic cotton is wound in the form of a bead. For each application a small amount of solution is dropped upon the probe, and in this manner the other portion of the solution is kept clear. The hardest part of the treatment lies in thoroughly but gently massaging the medicine into all parts of the nasal passage. This method should be continued until crusts no longer form. This procedure is 
at first executed two or three times a day, if it causes no irritation. As soon as the fetor is removed and the crusts are softened, it should be applied once a day. Hemorrhage, if occurring, is readily stopped by cotton-wool tampons.

The writer has found a two-per-cent solution of creolin, followed by ichthyol, or a mixture of iodine and oil of cloves, very efficacious. Oil of mustard, mixed with either olive oil, cotton-seed oil, or petrolina oil, will be found beneficial in some cases. The mixture should not be too strong of the oil of mustard-about one to ten minims to the ounce.

Massage and electricity are also beneficial methods of treatment for this disease. The manipulation is the same as for non-fetid atrophic rhinitis.

E. J. Moure (France) recommends very highly hypodermic injections of iodine. The writer has used this treatment in a few cases since 1890 , and with an excellent result in one case. It is often difficult, however, to carry out this plan of treatment on account of the pain which the injection of iodine causes. Hypodermic injections of a mixture of iodine, guaiacol, and olive oil are less painful. The iodine and oil of cloves mixture (see formulary), when locally applied by brush or swab, is also very efficacious in some cases. The nasal passages, of course, in all cases should be first well cleansed as already stated. A spray of a solution of bichloride of mercury $(1$ to 3,000$)$ is another medicament which is very suitable to some cases, especially syphilitic ones. The mercuric solution may be used sparingly also as a cleansing medicament. Benzoated oxide of zinc, or resorcin ointment or vaseline, will be found useful in the nares of patients with a delicate skin, or those prone to eruptions of eczema.

\section{Diseases of the Pharynx-Chronic Pharyngitis- HYPERTROPHIC-ATROPHIC}

The diseases of the pharnyx are rather difficult to classify, because of the anatomical and histological limitations peculiar to different parts of the pharyngeal cavity. For anatomical and clinical purposes the region is divided into three parts, viz., nasopharynx, oropharynx, and laryngopharynx, the boundaries of which are mentioned in the chapter on Anatomy. The oropharynx is perhaps subject to a greater variety of 
morbid conditions, and is more frequently attacked than the nasopharynx or the laryngopharynx. Considering the exposure of this region in civilized man to abuses from stimulating foods and drinks (either very hot, very cold, or pungent), and to inspiration of air laden with dust or noxious gases and micro-organisms, it is a wonder that it is not the subject of continual morbid action. Indeed, it may, therefore, be ranked next to the esophagus as a tough apparatus. Again, owing to the extreme variability in the appearance of the pharynx, it becomes very difficult to form a proper conception of its normal standard. Some years ago the writer examined about two hundred pharynges of persons not especially suffering from throat disease, and out of this number twenty per cent only showed such uniform conditions of conformation, texture, and colour as would coincide with the healthy standard, and the greater number of these were females. Hence it goes without saying that very few of the pharynges in the male adult may be considered perfectly normal. In about ten per cent of the cases examined on that occasion there were evidences of marked hyperæmia, or follicular hyperplasia or atrophy, and yet the subjects of these abnormalities were unaware, as far as their sensations were concerned, of any abnormal condition. I might state that the majority of the males used tobacco, and some of them whisky and beer also. They were nearly all of the labouring class of people, mostly hospital patients. Possibly it may be thought that this fact-namely, all of them having some ailment-might lessen the value of the above observations. However, it is a matter of common observation that marked abnormal appearances in the pharynx are not always attended with corresponding subjective symptoms, while on the other hand many complaints of pain, disturbances of deglutition, and various uncomfortable sensations referred to this region by patients are often observed to be unattended by any adequate corresponding abnormal appearance. It is believed by some observers that nearly all of the socalled chronic inflammatory diseases of the pharynx are dependent upon disease either of the nasal passages or of the lymphatic glands of the throat or the stomach. Bosworth thinks that nearly all of the chronic diseases of the pharynx, and many belonging to the larynx, are dependent upon nasal disease. 
The nasopharynix is not especially subject to inflammation, excepting in connection with the nasal passages and the oropharynx. There are but few cases met with, of acute or subacute inflammation confined to the nasopharynx, and when occurring are often passed by as neuroses. The principal symptoms of an acute inflammation are soreness or burning referred to this region, and a constant desire to hawk and spit, together with more or less nervous perturbation. The pharyngeal tonsil, however, is frequently the seat of hypertrophy or hyperplasia constituting the so-called adenoid vegetations, or adenopathies which are so commonly met with in children.

\section{Chronic Pharyngitis}

\section{Chronic Follicular Pharyngitis-Chronic Hypertrophic Follicular Pharyngitis}

The subject of chronic pharyngitis may be divided for consideration into three varieties: the hypertrophic, atrophic, and exudative, the latter of which is often considered a stage in the course of the former two.

Etiology.-The predisposing causes are principally hereditary predisposition, disease of the nasal mucous membrane, and disturbances of the stomach. A few authorities maintain that this disease of the pharynx depends almost entirely upon a predisposition on the part of the patient, while others think that it is nearly always secondary to some disease of the stomach. Therefore the etiology is rather obscure. It is a question whether any form of this disease can be laid to any particular cause.

Age-Chronic follicular pharyngitis (hypertrophic) is met with usually in adolescents. Only exceptionally are the young affected.

Sex.-Males are affected with this disease more often than females-in the proportion of five to one.

Occupation.-Dusty occupations, and those avocations requiring the strenuous use of the voice, predispose to the disease. The prevalence of some of its symptoms among clergymen led to the name of "clergyman's sore throat."

It is very common among bartenders and those who are occupied in a vitiated atmosphere. According to Cohen, those 
who are obliged to inhale air laden with tobacco smoke are liable to become afflicted with it.

Exciting Causes.-Among the exciting causes may be enumerated the use of tobacco and alcoholic stimulants, mouth breathing, inhalation of a dusty atmosphere, chronic disease of the tonsils, dyspepsia, constipation, etc.; but there are many cases met with in practice which cannot be attributed to these or any other known conditions. It is impossible to lay down any fixed principles of etiology worthy of general adoption. Notwithstanding this, however, it must be admitted that numerous cases occur which present an unmistakable causal relation between the tobacco habit or the drinking habit, or some antecedent disease of the nasal passage, or possibly hereditary tendency or chronic derangement of the stomach and alimentary canal. French, Allen, Daly, and others, have published abundant evidence in support of the relation between the chronic diseases of the throat and nose and disorders of the digestive apparatus. The writer has often noticed also a clinical connection between the condition of the large intestine or rectum and subacute and chronic pharyngitis and laryngitisfor example, in cases of hemorrhoids and anal fissure. There are many individuals who possess a tendency for acquiring inflammation, or at least hyperæmia, of the pharynx whenever some slight irregularity of the bodily functions occurs. This is notable in some women who always complain of soreness of the throat, and whose pharynges present more or less hyperæmia at the menstrual epoch if the latter function be in any way unnatural. Such cases are also met with in women during the early or later stages of pregnancy. These cases are undoubtedly due to neuro-vascular disturbance, and might properly be relegated to the neuroses but for the fact that one or more of the several features of inflammation are present. They cannot be classed as cases of acute inflammation of the pharynx because lacking some of its clinical phenomena. The rheumatic and gouty diatheses also act as causal factors in some cases. Harrison Allen and Hinkel have called attention particularly to the influence of gout on the course of chronic throat affections.

Harrison Allen has sharply discriminated between ordinary "acute sore throat" and the lithæmic throat, and has reported a number of instances of both conditions. The frequent depend- 
ence of chronic pharyngitis on diathesis has been long recognised by all writers. Concerning the causation of "chronic catarrhal sore throat," S. Solis-Cohen says the disease is not usually a sequel to acute sore throat, but becomes gradually developed without attracting much attention. It may exist with any diathesis; sometimes it is a mere manifestation of disorder in the intestinal tract. "Sore throat" occurring during paroxysms or exacerbations of fever, if acute, may often be overlooked. Much inflammation, however, is not always a feature of the disease. In the writer's experience, the most prominent symptoms have been sensory ones, such as pain and perverted sensations of various kinds, either quite general or referred to circumscribed regions, often described as spots, confined to the pharynx or referred to the larynx. There are symptoms referable to lithæmic or allied conditions which may be easily relieved by treatment directed against such conditions. The manifestations of lithæmia, however, as shown in the upper air passages, are not uniformly typical nor. sharply defined by the local appearances they present. An appearance occasionally seen in cases of "irritable throat," associated with lithæmia, is a patchy congestion of the laryngeal face of the epiglottis, extending along the ary-epiglottic folds, and over the posterior aspect of the ventricular bands. This is associated with a harsh, dry, explosive cough, and a sense of extreme irritation about the larynx. An extreme sensitiveness of the mucous membrane to astringent or stimulating applications was noted by Allen in cases of "gouty sore throat." A similar form of patchy inflammation of the pharynx is seen in some cases of chronic stomach disorder also.

Pathology.-The hypertrophic form consists of an increase of connective tissue in the submucosa, more marked toward the peripheral portions of the glandulæ. Changes also take place in the walls of the capillaries of a similar character, so that they may present irregular knots (varicosities) along their course, in consequence of which their elasticity is impaired, and their calibre diminished in places even to the extent of bringing about a true varicose condition. The hyperplasia does not take place uniformly, but is irregularly distributed, thus leaving the mucous membrane in a tolerably normal condition throughout the intervening neighbourhood. The epithelium in these cases is usually quite abnormal. It is rapidly 
proliferated and misshapen, and not only short-lived and easily exfoliated, but is thinner. There is also in many instances an additional layer of epithelium which is supposed to consist of young, partly developed epithelial scales, which are aggregated with epithelioid bodies and lie loosely over the papillary tufts of the submucosa, here and there. This, although not a physiological process, is nevertheless a beneficent concomitant of the disease, as it serves to protect the peripheral nerve ends, and thus prevents the patient from suffering considerable pain and distress. The secretion is more abundant than normal, and contains a large additional proportion of mucin, epithelial débris, lymphoid cells, and mineral salts. There are also a large number of saprophytic microbes in the secretion, especially in advanced cases. In the early stages, however, there is a notable absence of organisms either in the secretion or upon the surface of the mucous membrane. The secretion is of a serous or seromucous nature, and saturated with mineral salts derived from the blood; while later on, as mentioned above, the secretion becomes more consistent through the increase of organic matter. The exudative form (pharyngitis exudativa), which is confined mostly to the follicles, presents changes which consist mostly of a thickening of the endothelial lining, and the blocking of the lumen of the tubules by leucocytes, various lymphoid cells, fat globules, etc. The capillaries of the papillæ. however, soon undergo marked dilatation, in consequence of which a greater or lesser degree of stasis prevails, which in turn serves to furnish the exudate. This form of the disease rarely exists alone for any considerable length of time, and should really be considered as a stage or feature of the atrophic variety.

Symptomatology.-The symptomatology may be divided into subjective and objective. The early symptoms consist of more or less hacking, hemming, etc., with a sense of dryness of the throat, and possibly slight hoarseness. There may be very slight and transient odynphagia, which usually disappears whenever the throat is lubricated. Later on these symptoms become more aggravated, and annoy the patient not only in the morning or at night, but after fatigue or the use of the voice. In addition, there may be considerable extra secretion from the pharynx. As the disease progresses impairment of the voice takes place, and more or less hoarseness will follow 
any continuous effort at phonation. There may be also symptoms referable to the ear, such as tinnitus aurium, with more or less deafness. In such instances, extra or dried secretion will often be found at the orifices of the Eustachian tube, in the fossa of Rosenmiuller, or lodged a little distance inside the tube. Besides, there may be thickening of the lining membrane of the tube itself, thus diminishing its calibre. The whole of the middle ear, including the ossicles, are frequently involved in the disease. In some instances more or less cough accompanies the later stages; particularly will this occur if there be relaxation of the muscles of the soft palate and an elongated uvula. Instead of cough, some patients complain of more or less gagging, as if their throats (as they say) were being irritated by a feather. Nearly all of the above symptoms accompany the hypertrophic as well as the other varieties. Bosworth has stated that about five thousand grains of fluid are secreted daily by the nose, nasopharynx, and pharynx, in their physiological state. This large amount of fluid is ordinarily disposed of unconsciously to the patient, but in pathological conditions the quantity of secretion may be much diminished, and so altered in its physical property that nearly every particle of it must be disposed of by the patient through voluntary or conscious efforts of expectoration or glutition. Hence arises the idea that the secretions are absolutely increased. The hawking, spitting, gagging, and hemming practised in some cases is nearly continual. There is very little pain complained of in this disease, excepting when the throat gets very dry or an acute attack supervenes. Indeed, the surface of the mucous membrane is rather obtunded than otherwise. The subjective symptoms belonging to the exudative variety are similar to those just given, and therefore deserve no especial mention.

\section{Objective Signs-Appearances}

Hypertrophic.-In the earlier stages the mucous membrane appears hyperæmic and slightly swollen, looking like velvet or raw beef. This appearance, however, is not uniformly distributed over the whole surface, but in districts. The capillary vessels or veins are not seen, as a rule, but exceptionally two or three of them will appear just beneath the surface. The secretion often appears like a collection of drops or glistening pearly 
or red granules scattered over the surface, and in other cases the membrane will appear bathed with a soft fluid mucus, slightly coagulated in spots. Besides, a few clumps of glairy mucus may be seen on the posterior wall of the pharynx, either slowly coursing down from behind the soft palate or being forced down by the act of gagging. The velum palati and uvula may appear relaxed and swollen, and their colour be heightened. The uvula hangs down as if elongated, so that its extremity may lie upon the posterior part of the dorsum of the tongue. Later on, the uniform hyperæmia is replaced by red granular patches, upon which enlarged follicles jut out. These papillæ-like projections often resemble the fresh granulations of a suppurating wound, excepting that they are in clusters. The intervening strips of mucous membrane may be smooth and of a lighter colour, or may be of a grayish tint. The smoothness may be due to a covering of a thin layer of dryish glistening secretion resembling a coating of mucilage or gelatine. The secretion at this time is more or less inspissated and tenacious, and may adhere in shreds to the mucous membrane. As the disease progresses the enlarged follicles appear in smaller aggregations, and, projecting above the surface considerably, give attachment to small masses of tenacious, almost cheesy mucus, while from three to six quite large red follicles stand out. This is characteristic of what is known as the exudative form. As the disease progresses, atrophy of the mucous membrane begins, and the mucous membrane grows thinner, leaving half a dozen or more groups of enlarged follicles of red colour projecting. Enlarged and tortuous capillaries and veins now appear, and can be seen traversing the intervening districts from group to group. The whole pharynx now, upon inspection, gives the impression of dryness. The surface is shining and pale, and its continuity of surface is broken by the clumps of mucus which adhere to the projecting follicles here and there in stringy masses. It sometimes happens that the usual normal colour of the pharynx in a given person may be of a grayish-pink colour: in such a case the abnormal pharynx will show a parchment-like surface much earlier, and at all events the change in appearance from time to time will be less marked. There are no particular appearances belonging to what is called the exudative variety, excepting the persistent and characteristic secretion from the enlarged follicles 
mentioned above. We rarely observe a veritable ulceration of the mucous membrane in this disease, although some individuals, especially those liable to attacks of stomatitis, may suffer from occasional ulceration in the early stages of the disease. When occurring, the site of such ulceration is usually upon the pillars of the fauces or at the junction of the posterior and lateral walls of the pharynx. The ulcers are shallow, with a white base, and similar to points which have been touched by nitrate of silver (see Plate I, Fig. 5). They are usually oval or lenticular in shape, but transient. The denudation from loss of epithelium should not be considered as an ulceration, but an erosion.

The treatment of chronic follicular or hypertrophic pharyngitis may be divided into,

a. Treatment of the nasal passages when they are diseased.

b. The correction of bad habits of living.

c. Special attention to the stomach and other parts of the digestive apparatus.

d. Regulation of exercise, occupation, and environment.

e. The local treatment of the pharynx itself.

While it does not necessarily follow, as elsewhere stated, that this disease is dependent upon hypertrophic rhinitis, deformities of the nasal passages, etc., yet these conditions frequently coexist. If such be the case, little progress can be made toward arresting the disease in the pharynx until that of the nasal passages has been thoroughly alleviated. Likewise, when the case seems to depend upon bad habits of living, such as excessive drinking of alcoholic beverages or coffee, excessive use of tobacco, irregular sleep, or overeating, etc., little benefit will result from local treatment until these habits are corrected.

It will be found that a large majority of cases of this affection are in one way or another dependent upon or coincident with derangement of the stomach, intestines, or other abdominal viscera. Indeed, so-called dyspepsia is one of the most common concomitants of this form of pharyngeal disease. It is therefore obvious that in such cases the treatment should be directed principally, if not altogether, to the digestive tract, in order that all forms of indigestion, abnormal intestinal fermentation, etc., be relieved. The writer has found in many cases of this sort that the administration of one or the other of 
the several preparations of milk of magnesia alone, or in conjunction with either fluid extract of wahoo (euonymus) or extract of dogwood (piscidia erythrina), will give excellent results.

Concerning occupation and environment perhaps no special rules can be laid down, for, strange as it may seem, individuals will be met with who are over-exercising the voice, inhaling dust, or in some other way committing errors which are supposed to induce this affection without showing any material structural disturbance of the pharyngeal membrane. For this reason the practitioner should thoroughly consider the several factors in each case before deciding upon the course of treatment.

The local treatment in a majority of instances is very disappointing. In fact, the writer more often than otherwise refrains from using much, if any, topical medication. There are cases, however, in which the use of sprays or aqueous solutions of salt, borax, or chlorate of potassium, by cleansing the membrane, seem to produce beneficial effects, while in other cases the same class of remedies similarly employed seem to do more harm than good. Wherever the secretions are inclined to coagulate or collect in patches, either in the naso- or oropharynx, cleansing of the surface by a spray or douche is indicated. Very often it will be found that the use of the syringe or douche will be preferable to the spray. The medicinal agents to be selected for this purpose are peroxide of hydrogen, mercuric chloride, sodium biborate, potassium chlorate, creolin, thymol, etc. (see formulary). After the surface of the membrane has been pretty well cleared of secretion it should then be brushed with fluid extract of prickly ash (xanthoxylum), a mixture of extract geranium and hyoscyamus, or of extract of kino and hyoscyamus; or such vegetable astringents as coto bark, tannic acid, gallic acid, or extract of blackberry may be used; or, instead, if deemed best, a pigment containing a mineral substance such as iron, zinc, nitrate of silver, or sulphate of copper, may be selected. A list of these preparations will be found in the formulary. For the more chronic cases, thymol, or thymol with camphor and menthol, are excellent. It is very difficult to lay down rules for the selection of particular preparations, inasmuch as the adoption of the proper treatment for a given case is really more a 
matter of art or experience than of science, for it is almost impossible to explain why one will apply to a certain case a mixture of prickly ash and hyoscyamus, and to another a mixture of tincture of chloride of iron and glycerine, or nitrate of silver and glycerine. As a rule, however, it will be found that in cases showing the least structural change, where the constitutional condition is good, that the mild astringent remedies will prove more beneficial. On the other hand, in cases that are quite chronic, exhibiting considerable structural change, especially of the follicles, such as we find in the exudative variety of the disease, applications of chloride of iron, sulphate of iron, chloride of zinc, sulphate of zinc, nitrate of silver, etc., will be most apt to relieve the morbid condition. The applications of thymol, menthol, balsam peru, and oil of tar are exceedingly useful in the exudative variety, and sometimes in those cases where atrophy is just commencing, but care should be taken not to stimulate the mucous membrane too much. Again, in cases where the secretion is not abnormally excessive or altered, too much cleansing is apt to be harmful. The insufflation of such powders as carbonate of magnesia, aristol, europhen, or tannate of cannabin will be found highly useful when the follicles, especially those of the lateral walls, are particularly hypertrophied. In some cases the writer has secured signal benefit from the application daily of a pigment containing solution of morphia and chloride of iron (one grain of the former to five of the latter) to the ounce of glycerine water. The practice of touching enlarged follicles or groups of enlarged follicles with escharotics, such as chromic acid, chloride of zinc, the galvano-cautery, etc., has not proved generally beneficial in the writer's practice. The same may be said of the practice of cauterizing veins and capillaries which appear prominently in the pharyngeal mucous membrane.

The judicious limited application of nitrate of silver, in solution (from ten to forty grains to the ounce), or argyrol, to hypertrophied follicles, especially in the exudative form of follicular pharyngitis, will often give excellent results. Occasionally, also, the application of the galvano-cautery point to such abnormal places is equally beneficial. 
Atrophic Pharyngitis, or Pharyngitis Sicca

Dry Catarrh of the Throat.-Atrophic pharyngitis is an affection which is characterized by extreme paucity and inspissation of the secretion, and more or less atrophy of the glandulæ of the mucous membrane of the pharynx. It is generally associated with atrophic rhinitis, although it sometimes appears to be an independent affection.

The cases as usually met with may be classified into

a. Those associated with atrophic rhinitis.

b. Those following chronic follicular or hypertrophic pharyngitis.

c. Those which occur without any recognised previous disease of the oro- or laryngopharyngeal regions.

Etiology.-The disease most frequently follows a period of hypertrophic or follicular pharyngitis. It is rarely confined to the oropharynx, but is found to affect the nasopharynx and the laryngopharynx as well, and frequently also the laryngeal mucous membrane, in which case it has been described as laryngitis sicca or atrophic laryngitis.

Age.-It is more frequently met with during the period from adolescence to old age, although it is not infrequently seen in children and the young between the ages of ten and twenty, in connection with atrophic rhinitis.

Sex.-Females are more frequently affected than males with the independent form of the disease.

Habits.-Habits and climate play somewhat of an important part as an exciting cause of the affection, because those persons following dusty occupations, who use a great deal of tobacco, or who live in dry and dusty climates, are liable to suffer from it. Those addicted to the excessive use of alcoholic liquors, or who drink ardent spirits undiluted, are also quite liable to both this as well as the hypertrophic form.

Heredity.-Heredity and diathesis undoubtedly play an important part as predisponents, for I have known of instances where several members of the same family have suffered from the affection.

Diathesis. - The syphilitic taint is recognised as one of the predisposing causes of the affection, especially if the pharynx has been the seat of tertiary ulceration. Lithiasis is also credited with predisposing to it. The starting point in the etiology 
of atrophic pharyngitis is not always clear. The older view, that it is a scrofulous disease or of the same nature as ozena, still has some adherents, notwithstanding the trend of modern thought toward fastening the etiology of all diseases upon some specific or microbiotic cause. Many observers believe that it is but a sequelæ or a stage of ordinary chronic pharyngitis, and that it is not entitled to be classified as an affection sui generis. While undoubtedly this holds true in some instances, the fact remains that many cases are not apparently preceded by any preliminary stage of hypertrophy of the glandular apparatus of the mucous membrane, but, on the contrary, it is noted that the disease begins insidiously and primarily as an atrophic process and so continues. It is therefore impossible, we believe, to look upon all of these cases as being dependent or consequential upon previous hyperplasia. Another theory advanced is that this affection, like ozena, depends upon some chronic disease of the accessory cavities of the nose, as of the ethmoid cells, the frontal, or maxillary sinuses, but there is a notable lack of evidence showing involvement of the accessory cavities during the course of the disease in many cases. There is often no adequate explanation for the origin of the disease in many instances, unless it be found in the nervous mechanism of the parts, or in some supposed dyscrasiæ. Indeed, every opinion so far advanced for explaining its etiology is hypothetical, and while one theory may be sufficient to explain the origin and course of a certain number or class of cases, it will be found inadequate in accounting for the origin of others. That the initial change is usually atrophic in character there can be little doubt, inasmuch as it is always symmetrical. If in the nasal passages both sides are affected, in the pharyngeal cavity both sides equally are involved from the beginning, and even should a neoplasm be present, the neighbourhood of the site of the neoplasm will be no more affected in the atrophic change than the other parts of the pharynx, so that we are, it seems to me, forced to accept as most rational the neurotic theory (as for atrophic rhinitis), which attributes the disease to some malformation or malnutrition of the nerve ganglia and other physical elements, which essentially preside over the growth, development, and nutrition of the mucous apparatus of the upper air passages. This, of course, applies to those cases which are not the result of traumatism of any sort. 
Pathology.-The pathology is much the same as that of atrophic rhinitis, the glandular apparatus of the mucous membrane, as well as the number and size of the capillaries supplying it, gradually diminish. The epithelium becomes thin and successively undeveloped, while the secretion becomes diminished in quantity and thickened, drying in clumps on the surface of the pharynx. No doubt this is aided by the desiccating operation of the air passing over it. When the atrophy is not confined to the mucous membrane of the pharynx, it also affects the turbinated bones of the nose and mucous membrane of the larynx. In consequence of this change the ordinary sensibility to touch of the mucous membrane is very much obtunded, or sometimes lost, and its vascular supply altered.

Symptomatology.-The subjective symptoms of the earlier stage consist mainly of sensations of dryness, tickling, pricking, or burning, referred to the throat, an irritability including frequent efforts at hawking, spitting, and nasal screatus. The principal efforts at clearing the throat are usually made in the morning upon rising, or just after the ingestion of food, or at both periods. Patients generally begin the meal by the deglutition of more or less water to relieve the dryness; in fact, the desire to moisten the throat is a constant one. In the advanced stages the hawking and spitting become more frequent and imperative, until the comfort of the patient is seriously compromised. Exceptionally we meet with advanced types of the disease in which the person, although having considerable thick semi-desiccated masses of secretion plastered to the pharyngeal wall either of the nasopharynx or oropharynx, will do very little hemming or hawking, while previously the same person may have been attempting to clear the throat almost incessantly. It is necessary for some patients to lubricate the throat artificially once, twice, or even three times during the night; indeed, some of them involuntarily wake up for this purpose at certain intervals. Of course these symptoms vary a great deal, according to the susceptibility of the individual to annoyance. Disease of the middle ear (catarrhal) is not an infrequent accompaniment, and is indicated by more or less tinnitus aurium and deafness. Patients are not troubled by much sharp pain, as a rule, but a burning sensation, sometimes relieved by acts of swallowing, is a common symptom. With 
many of them this burning or raw sensation, superadded to the sense of dryness, is the principal complaint.

Voice.-The voice is frequently affected, either from extension of the disease to the laryngeal mucous membrane, or else from sympathetic irritation. Sometimes, however, it may be attributed to the direct effect of the air, which having passed through a perfectly dry nasal and pharyngeal space reaches the larynx more or less cool, impure, or unmodified. There are no particular constitutional disturbances.

Appearance.-The objective symptoms are quite characteristic, and may very properly be divided into three classes. The appearance belonging to the first or early stage shows perhaps but slight thinning of the mucous membrane, a glazed, reddened surface, with here and there jutting out a bunch of moderately enlarged follicles. Just after the act of deglutition this surface, if examined, will present little rugæ or lines running perpendicularly up and down the pharynx, which are paler in colour than the surrounding mucous membrane. Rhinoscopic examination will show a similar appearance of the nasopharynx, but with more or less of a collection of dry secretion hanging about or plastered upon the pharyngeal tonsil, or sometimes a collection of crusts here and there about the pharyngeal tonsil or hanging about the Eustachian tubes. Later on, the collection of secretion is more marked, especially in the nasopharynx and about the origin of the pharyngeal tonsil, where crusts may be noticed. Also along the pharynx, clumps of partially dried secretion may be plastered here and there to the pharyngeal walls. The striated appearance of the pharynx is more marked, and in some cases there are red rugæ running up and down, alternating with grayish glistening lines, while the translucent, thin, glazed, skin-like covering of the surface shows mottled patches broken by enlarged follicles, with viscid stringy mucous sticking or hanging about them (see Fig. 94). Later on, the third or advanced stage of this affection will show also clumps of secretion, which are drier and more numerous, giving the appearance of stucco work. The posterior pharyngeal wall, from the nasopharynx down to the region of the larynx, is of grayish colour, dull and glistening, with here and there islands of granular follicles bounded by rugæ or varicose veins lying between. In some cases there is a bluish tint to the whole surface. The membrane is often 
so thin that the markings of the muscles beneath can be distinctly made out. There are often clumps of follicles projecting out, which look more like the exuberant granulations of an unhealthy wound than anything else. Here and there one or two varicose veins may be seen crossing the posterior wall running beneath or into these clumps of follicles, which seem to lie in a field of cicatricial tissue which has the appearance of parchment. The act of deglutition may change but little the position of the dried secretion, or it may momentarily increase the fulness of the few capillaries or veins which are in

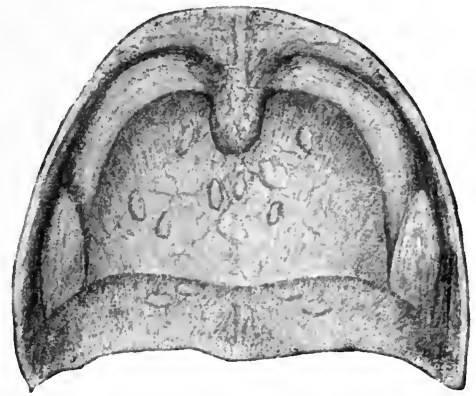

FIG. 94--Atrophic pharyngitis (with enlarged follicles). view, thus giving a redder colour to the membrane. The faucial as well as the pharyngeal tonsils are usually atrophied: in fact, in some instances they seem to be entirely absent, and represented by a few follicles only. The upper part of the larynx may also show the same dry condition, with more or less dryish secretion adherent to the inter-arytenoid region, or lying in the pyriform sinus. A view with the laryngoscopic mirror may disclose the walls of the larynx plastered with small yellowish-gray crusts.

Diagnosis. - The diagnosis is a very simple matter, for this disease is rarely confounded with anything excepting the appearance of the pharynx in some of the acute affections, where from slight engorgement the secretion becomes thickened into a temporary coating, so that the surface seems dry and glistening. This exudate can be easily washed off, however, with spray, when the mucous membrane beneath will be shown to be in a state of simple hyperæmia, smooth, or possibly presenting a finely granular appearance.

Prognosis.-The course of this affection is slow, insidious, progressive, but without danger to life. There is not even a tendency to tuberculosis, as many of the laity believe. Indeed, it is highly exceptional to meet with a case of laryngeal tuberculosis supervening upon this affection. It is sometimes complicated by so-called ozena, especially when the nasal passages are involved, and is often accompanied by very serious de. 
rangements of the voice, varying from a periodical to a continual hoarseness, or even dysphonia.

Spasmodic asthma may coexist, and add to the discomfort of the patient. More or less derangement of the function of the stomach is frequently met with, and has been ascribed by some observers to the influence and presence of the altered secretion which may have been swallowed. This, however, I believe is a matter of speculation rather than a matter of fact, for it is surprising that so many cases of aggravated atrophic pharyngitis are unaccompanied by any particular derangement of the stomach; indeed, the majority of these patients, while not appearing robust or fat, are usually free from any constitutional disturbance after they have passed the worrying stage. After reaching a certain limit the atrophic process seems to stop. Whether the checking of the process is due to a want of further local molecular vitality, or whether there is by nature a physical limit to it, cannot be answered.

Treatment.-The treatment of atrophic pharyngitis has always been notably unsuccessful. While much alleviation has followed different plans of local or constitutional treatment, yet a radical cure has been difficult to obtain. In the case of young people, of course, there is something to be hoped for from judicious treatment in the way of limiting the process. In those along in years, however, nothing can be offered but meagre palliation. In the case of young girls, the physician should be especially stimulated to make great effort, for certainly in such cases the progress of atrophic pharyngitis, especially when accompanied by ozena, constitutes a terrible blight upon their prospects; indeed, it is serious if the voice and hearing be affected. The general treatment should be directed toward improving any defective condition or function of the body that may be observed to be out of order; for instance, habitual constipation must be overcome by diet, or, if necessary, by laxatives. Hasty mastication is to be amended, and all faulty habits of living are to be corrected. Particular attention should be paid to the matter of bathing. If the subject be in the habit of bathing too frequently, this practice should be modified. If the patient, on the contrary, through lack of facilities or for other reasons, has not bathed enough, and the skin is in consequence either too dry or too oily, then more frequent sponging with salt and water, followed by friction, 
should be suggested. In females the condition of the pelvic organs should be kept in mind. Tonics, and especially chalybeate tonics and strychnine (see formulary), are frequently useful in aiding the nutritive processes; sometimes extract of malt has a good effect. Proper exercise and other hygienic measures should be adopted, for the purpose of improving the nutrition. Tranquility of the mind should also be maintained.

Local Treatment.-For local treatment there should be two objects kept in view : first, to keep the parts tolerably free from the accumulating secretion; and, second, to stimulate the nervous and circulatory functions of the part. For the accomplishment of the first object, sprays or douches of solutions of borax, salt and water, chlorate of potassium, sodium bicarbonate, phosphate of soda, Dobell's solution, eucalyptus, creosote or rosemary, or oil of tar with petrolina oil, creolin, camphor, or cinnamon water, etc.; if there be subjective odour or objectionable taste complained of, creosote or cam. phor water with bicarbonate of sodium may be frequently used. The patient should also be cautioned against breathing through the mouth or inhaling much dust. In case of extreme crustation, with fetor, a very weak solution of chlorine gas, or chlorinated soda, or peroxide of hydrogen, may be used as a spray. This cleansing operation should be done at least once daily, and, if necessary, twice or even three times a day. It is well not to allow too much cleansing of the parts, since it must be remembered that the mucous membrane is largely denuded of its epithelium, or at least covered by a thin unhealthy layer only. For the attainment of the second object, stimulating applications, either by means of spray or in the form of pigments, may be used. Before the application of any of the following agents, it will be understood that the parts are to be cleansed with one of the solutions mentioned above. A spray consisting of oleum picis with petrolina oil, oil of eucalyptus with petrolina oil, or thymol with petrolina oil, will be found quite useful. One consisting of a solution of oil of tar and bicarbonate of soda with water, or a solution of ichthyol, may be preferable in some cases. A pigment composed of oleum picis with balsam Peruviana, or balsam Canadensis with alcohol, or thymol with alcohol, or the alcohol alone (see formulary), will be found quite beneficial; but of all the agents, 
perhaps the best is the application of galvanic or faradic electricity through the naked electrode, as recommended for atrophic rhinitis. This method has stood the test of time since the writer first adopted it in 1880 . Gauthier also called attention to the good effects of this treatment some years ago, and it has been found beneficial by Hartman and Delavan. One electrode (see chapter on Instruments) should be applied to the pharynx, moving it from place to place gently, while the other (covered with sponge) may be applied either to the back or side of the neck. Some observers have explained its beneficial effect upon the principle of electrophoresis, supposing that a minute quantity of the copper electrode was dissolved by electrolytic action and diffused through the tissues. Possibly this is true, but careful examination of the electrode by weight, as well as critical inspection by lenses, does not show any loss of the substance of the electrode, even after a long period of service. Undoubtedly the chief therapeutic effect depends upon the stimulation of the nerves and ganglia, including their trophic communicating branches. Much benefit will sometimes result from the operation of massage, either by the hand or with the electric motor. This effect is produced by impinging against the surface of the cleansed mucous membrane some blunt steel instrument, such as the blunt end of a probe, and with rapid, short, striking motion pass it over the surface, much the same as in treating atrophic rhinitis. Should this prove too painful, a little piece of absorbent cotton may be wound around the end of the instrument to relieve the impetus of the blow. Applications of xanthoxylin and lemon juice are quite beneficial. Astringent or caustic applications are strongly contraindicated in the treatment of this affection, as also are insufflations of powders. Much alleviation may result from a residence in a warm, moist climate, such as the Pacific islands, Gulf of Mexico, Bahamas, Florida, or at the Mediterranean sea-shore.

When the larynx needs topical treatment, a two- to five-percent solution of thymol in petrolina oil, or a five- to ten-per-cent solution of oleum picis or camphor, or a three-per-cent solution of oleum terebinth in petrolina oil, will be found highly useful. Either of these may be applied in addition to the sprays recommended to be used every day or three times a week. 


\section{Pharyngitis (Seu Angina) Ulcerosa (Ulcerous Pharyngitis)}

This disease is not common nowadays, owing to the improved hygienic surroundings which prevail in communities and public institutions, but formerly many cases were seen among the poor and squalid and among hospital attendants. It is often seen now among hospital nurses and attendants, or in those who have been confined very closely within the walls of any institution for a long period. M. Mackenzie states that medical students spending much time in anatomical and pathological laboratories and hospitals are liable to become affected with this malady. The affection on this account has sometimes been called "hospital sore throat." The accession of the disease is usually quite rapid, although generally it is succeeded by at least a short period of general malaise. "Sore throat," however, may be the first symptom complained of, and it gradually increases in severity until the act of swallowing becomes very painful and difficult (odynphagia and dysphagia). Coincidently there is chilliness, malaise, and a rise of temperature sometimes as high as $103^{\circ}$ to $105^{\circ} \mathrm{F}$. In such cases there is, as might be expected, a great deal of prostration. The tongue is heavily coated, whitish, and may be dry. It sometimes appears swollen from glossitis, and the breath is generally very offensive; the glands at the angle of the jaw and those of the cervical region are usually enlarged and tender, and the tonsils may also be involved. If so, they are red and swollen; their lacunæ, or crypts, are filled with cheesy secretion, giving the same characteristic appearance as acute follicular tonsilitis. Sometimes the ulceration extends to the faucial tonsil, in which event for a while the disease resembles tubercular ulceration.

Aphthous stomatitis, chronic gingivitis (Riggs's disease of the gums), or caries of the teeth may be associated with this malady, either in an antecedent or sequential relation to it.

In syphilitic, tuberculous, or emaciated children, especially if affected with a severe form of stomatitis, serious ulceration of the pharynx may arise, which may assume gangrenous features.

Rarely, without constitutional disturbance, a small ulcer or two may suddenly appear on the anterior pillar of the fauces in a healthy person. The ulcer is usually superficial, oval, or diamond shaped, and looks like an eschar from the effects of nitrate of silver (see Plate V, Fig. 2). 
The diagnosis can be readily made out, as a rule, but in some cases more or less perplexity may arise in distinguishing this malady from acute follicular tonsilitis, mild diphtheria, or secondary syphilis. Prolonged cases may be mistaken for tuberculosis of the pharynx or tonsils. The peculiar ulceration of the tissues, together with the clinical history, however, will usually enable one to differentiate it from these several diseases. The writer, however, has seen cases of ulceration on the anterior faucial pillar and tonsil which for a time looked like syphilis or tuberculosis. When one or two superficial oval or elliptical ulcers occur, with an erythematous areola on the faucial pillar or on the posterior wall of the pharynx, and the clinical history of any of the above-mentioned diseases is not clear, the diagnosis becomes puzzling. The affection may be due to either herpes or traumatism.

The course of the disease is usually from seven to fourteen days, and the prognosis is generally good, unless the subject of attack has been previously "out of health."

Pathology.-This disease consists of an ulceration of the superficial layers of the mucosa. The inclusion of the follicles causes a fibrinous exudation in which epithelioid bodies, pus cells, micrococci, etc., are entangled. As the sites of predilection seem often to be determined by either the track or peripheral endings of the sensory nerves, it is supposed sometimes to be a neurotic disease.

Treatment.-The treatment consists of the assiduous application by spray, gargle, or pigment of such antiseptics as peroxide of hydrogen, permanganate of potassium, bichloride of mercury, carbolic acid, resorcin, etc. After the cessation of the ulcerative process, mild astringents, such as tannic acid, sulphate of zinc, tincture of chloride of iron with glycerine, etc., may be used. Local applications of boric acid, calomel, biniodide of mercury, are excellent. The constitutional treatment may become quite important. It should consist of the administration of medicinal agents which will improve the bodily strength, such as quinine, strychnine, arsenic, or other systemic tonics, etc. Stimulants when carefully used are of very great utility, especially the malt beverages. The nutrition of the patient, if at fault, should be corrected, and removal from close confinement to fresh air is necessary. 


\section{Hypertrophy OF THE TONSIls-Chronic Tonsilitis-} Chronic Follicular Tonsilitis

An enlargement, of a sub-acute character, of the faucial tonsils is often regarded as identical with chronic hypertrophy ; although the former may lead to the latter condition in time, still the notable preponderance of fibrous tissue and absence of inflammation in hypertrophy are features which distinguish it from a chronic inflammation with exudative enlargement of the gland.

There are two distinct types of hypertrophy of the tonsils, characterized generally by the terms "hard" (fibrous) and "soft" (adenoid). These terms indicate sufficiently the gross nature of the affection. But the true hypertrophy of the tonsil is that variety which is designated "hard." It consists of more than a hyperplasia, it is an augmentation of the stroma of the organ, while the "soft" or "honeycomb" tonsil, though perhaps sometimes larger in dimensions than the other, is not characterized by the growth or increase of the connective tissue, but rather an abnormality of the vessels and the lining of its follicles and crypts. Knight's excellent division of the subject into three classes might be slightly modified so as to include four, viz.:

I. Those very much increased in size, of globular or lobular shape, free, and hard to the touch.

2. Those which are flat, elongated and amalgamated more or less with the neighbouring glands.

3. Those which are adherent more or less to the boundaries of the tonsillar triangular space.

4. The enlarged soft tonsil, either free or adherent, flat, globular, or lobulated.

Etiology. - These conditions are sometimes congenital (lymphoid hypertrophy), but as a rule they follow repeated attacks of acute tonsilitis-either phlegmonous or lacunar-or are sequences of scarlet fever, diphtheria, and measles. A family or hereditary predisposition is an element in the history of many cases. M. Magnan states that hypertrophy of the tonsils does not follow an abscess as a rule. However that may be in Europe, the statement will not apply to our western country, where experience teaches that hypertrophy is often a result of repeated phlegmonous inflammation and abscess (peritonsilitis). 
He observes also that many cases arise from typhoid and scarlet fevers, measles, and diphtheria. In children, enlarged tonsils are frequently associated with hypertrophy of the turbinals, the adenoid tissue of the pharyngeal vault, and the lingual lymphoid tissue (lingual tonsil). There may be, in addition, enlargement of the cervical lymphatic glands. Although intimately connected with the lymph glands and their channels (the lymphatic ring of the fauces so called), the faucial tonsils may be independently affected with any form of chronic disease, which in turn may lead to hypertrophy. M. L. Wilson and others think that it is a question whether "the tonsil is an organ or not," notwithstanding it has a capsule or envelope. Late experiments, however, go to show that the tonsil furnishes one of the internal secretions. The enlargement may be, and more often is, due to a low grade of inflammation, or it may be a non-inflammatory hypertrophy, such as all lymphatic glands are subject to. "Adenoids" are usually an accompaniment. Rheumatism and gout are credited with causing more or less tonsillar irritation-principally through uric-acid formation.

Age.-It is eminently a malady of childhood, and the frequently observed spontaneous disappearance of moderate hypertrophy of the faucial tonsils upon the advent of adolescence has given rise to a common prejudice among the people against abscission, etc.-a prejudice which has been until lately participated in partially by the profession.

Sex.-This condition is seen in children of both sexes about equally; perhaps it occurs a little more often in the male than in the female.

So-called "strumous children" are particularly apt to have enlarged tonsils, even though they have not suffered often from the diseases of childhood mentioned above. However, it cannot be denied that diphtheria, scarlet fever, and other diseases of childhood are the cause of tonsillar and adenoid hypertrophy in the majority of instances.

Successive attacks of acute lacunar or follicular tonsilitis will lead to chronic enlargement of either type in young adults.

Symptomatology.-The most striking symptoms of this affection are a certain thickening or flatness in the quality and amplitude of the voice; a noisy inspiration, especially during sleep, and sometimes during conversation and eating; snoring at night, especially when the tissues of the nasopharynx or 
the nasal passages are also thickened. The obstruction to breathing at night is often so serious that the child cannot rest or sleep quietly, especially if there be also enlarged adenoid glands. Respiration in some cases is greatly impeded by the drawing down of the tonsils with the acts of inspiration. In aggravated cases the "open-mouth breathing," coupled with mental hebetude, contracted thorax, and some deafness, are the prominent and continuous symptoms. All of these signs give to the child the appearance of stupidity. Some patients have difficulty in eating and drinking because of the embarrassment to respiration, while the deglutition is attended with more or less gurgling noise, as the acts of respiration and deglutition become, as it were, "mixed up." The cervical glands are not infrequently enlarged sufficiently to be easily felt. The general health of the patient may suffer from anæmia, indigestion, impaired respiration, and general debility if the enlargement be at all excessive, and especially if the pharyngeal tonsil be also affected. The degree of general disturbance to the economy will always be found in direct ratio to the amount of interference with proper respiration and deglutition. The matter of deglutition is an important point which is frequently overlooked. I have known children who, owing to the trouble of swallowing and breathing while at meals, would go from the table before eating or drinking a sufficient amount for their proper nutrition. Such subjects are also prone to frequent exacerbations of acute inflammation of the tonsils and nasal passages (taking cold), and if the other lymphatic tissues of the throat be hyperplastic as well, they are very sensitive to dampness or changes of weather, which promotes additional swelling of the glandular tissue, and increases the obstruction to respiration. These subjects are often supposed by their families to have "taken cold," when probably no acute inflammation has taken place at all; the increase in size of the tonsils or other neighbouring lymphatic tissue being a result of the humidity and temperature of the atmosphere, which, as is well known, promptly acts on the diseased lymphatic tissue anywhere about the upper air passages.

Appearances-The Hard Tonsil._The appearances are quite characteristic. In the true hypertrophic form (see Plate I, Fig. 6) the tonsils appear as large smooth or corrugated ovoid bodies projecting more or less into the fauces, or perhaps 
elongated and extending from the faucial angle above to the pyriform sinus below. They may not only be elongated, but rounded and lobulated as well, occupying the whole lateral region of the fauces; or they may appear round and almost pedunculated, leaving a space above and below them in the faucial niche, thus resembling tumours (see Fig. 95). Sometimes they appear divided, as if consisting of three distinctly

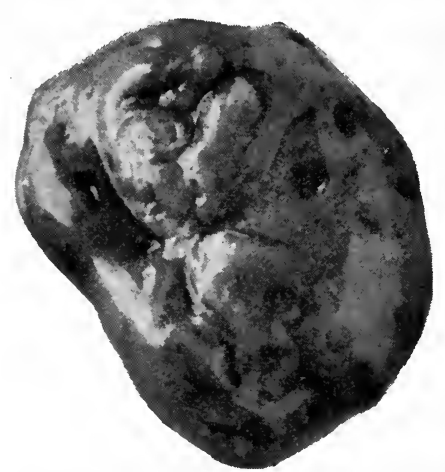

FIG. 95- - Exsected hypertrophied tonsil $\left(x+\frac{1}{2}\right)$. capsulated lobes, the middle mass being larger than the ones above and below. These upper and lower divisions have been occasionally regarded as supernumerary tonsils (Allen). As a rule, however, the masses are quite compact and localized. The colour of the covering membrane is usually lighter or more mottled than normal in specimens of true hypertrophy. If the lingual (tonsil) lymphatic gland participates in the enlargement, its upper part or glandulæ project from under the circumvallate papillæ of the tongue, and meet the lower portion of the faucial tonsil. Exceptionally all of the peritonsillar tissue may be involved, giving to the lateral walls of the fauces a rough mammillated or irregularly lobulated appearance. These tissues are usually very firm to the touch, and the crypts are more or less filled with secretion.

Soft or Adenoid Tonsil.-In the soft variety the tonsils appear spongy or ragged, with their lacunæ or crypts patulous, and more or less filled with a thick, dirty-white, cheesy secretion or concretion of bad odour. The stench from these accumulations in the tonsil is sometimes very fetid. The surrounding glands are also very much enlarged, and the whole mass may be spread through the faucial niche from top to bottom, presenting irregular, reddish, lobulated projections, some of which seem almost pedunculated. According to C. H. Knight and others, this form is always attended with hypertrophy of the adenoid tissue at the vault of the pharynx. The posterior wall of the pharynx frequently participates in the follicular enlargement, and, indeed, all of the surrounding mucous membrane may also be discoloured and apparently thickened. The tonsillar 
tissues are soft to the touch and easily compressible. When compressed, the viscid secretion or cheesy concretion oozes out of the crypts. In some of them the contents become quite hard, almost calcareous, and emit a very fetid odour when crushed.

There is always more or less chronic inflammation of the follicles, and, of course, an abnormal secretion in all of them. In one class of cases the tonsil may seem atrophied rather than hypertrophied, and is hidden behind the anterior faucial pillar. In other instances it is so small that there is only a vestige of the tonsillar tissue remaining. In such cases of atrophy the follicular glands above the tonsil at the upper angle of the faucial niche are often the seat of the abnormal fetid secretion instead of the tonsil itself. This supratonsillar tissue is often the seat of attacks of acute peritonsilitis (quinsy).

Pathology. Hard Tonsil.-True tonsillar hypertrophy presents pathologically a very decided increase in the stroma-

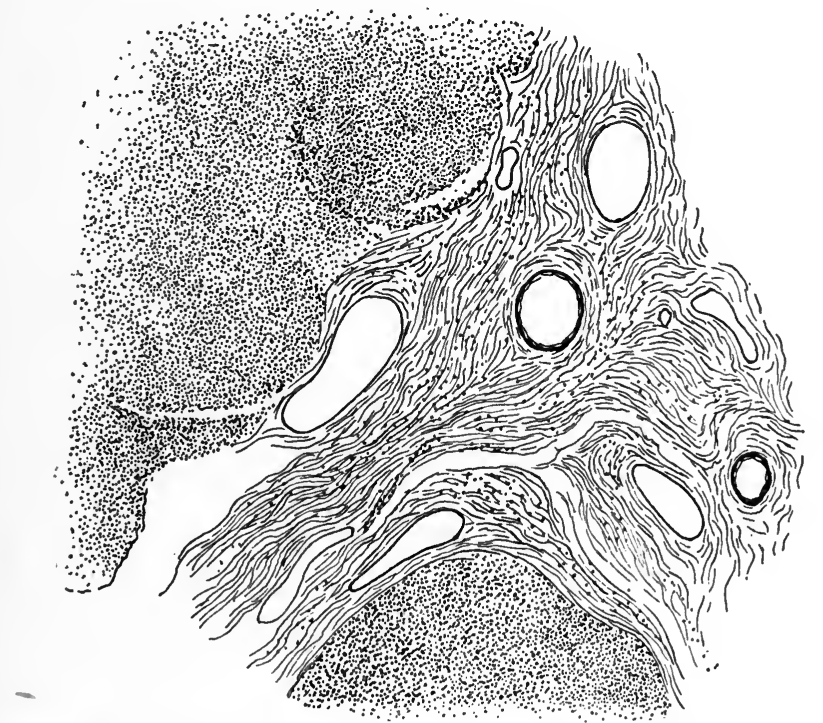

FIG. 96.-Section of hypertrophied faucial tonsil (A. Coolidge, Jr.).

the connective tissue-with a corresponding diminution of the number and size of lymph spaces (see Fig. 96). The increase of the connective-tissue elements is not always confined to the stroma, however, for the lymph vessels and capillaries are also 
reinforced by connective or fibrous tissue, and are thereby contracted. The increase of connective tissue is not readily accounted for, and there are various views among pathologists regarding the initial and subsequent steps of the process. The generally received opinion, however, is that the beginning is an irritation of the vascular tissue, and the result is a hypernutrition of the part. Another hypothesis is that some abnormality of the lymph or of the attendant secretions acts as a primitive irritant. This comports with the modern view that the tonsil can readily absorb to its inner lymph spaces all sorts of foreign material, proteid or crystalline. However, in those cases which follow the exanthemata or diphtheria, the starting point is undoubtedly a specific inflammation of all the structures of the glands. The vascular system, as well as the lymph channels of the part, are thus acutely deranged, and if the irritation continues there will be exudation and interstitial changes in these tissues which may determine an increase of connective tissue about the blood and lymph vessels, with a corresponding destruction or diminution of the surrounding lymph spaces -a permanent hyperplasia. The pathological rule, that wherever there is a continued irritation of the tissues there is a tendency to the increase of connective and fibrous tissue, holds good here as elsewhere in the body.

Soft Adenoid Tonsil.-In the enlarged soft tonsil the pathologic change seems to be of a different character, inasmuch as there is little or no increase of the stroma excepting by the formation of little bands (or trabeculæ) of connective tissue running through the enlarged spaces from one to another. The capillaries are enlarged and varicose and their contractility impaired, while the follicles themselves are also enlarged and their involuted endothelial lining changed and of low vitality. The secretion of the follicles of the tonsil, owing to a continual passive congestion, is more active, but contains other cellular elements, such as altered leucocytes and epithelioid bodies. This is also true of the atrophied "concealed" tonsil.

Diagnosis.-The diagnosis is usually very easily made out. In the adult the disease may be mistaken for a malignant growth, but the history of the case, absence of pain, and enlarged cervical lymphatic glands, together with the characteristic appearance, will be sufficient to distinguish one from the other. The "follicular" or soft variety may be mistaken for 
an acute disease of the throat, if it were not for the absence of pain, difficulty in deglutition, and constitutional disturbance, symptoms which nearly always accompany acute disease.

Prognosis.-The prognosis in these cases is usually favourable if proper treatment is adopted-that is, to relieve the patient of the mechanical obstruction to respiration and glutition, and of the source of the sepsis.

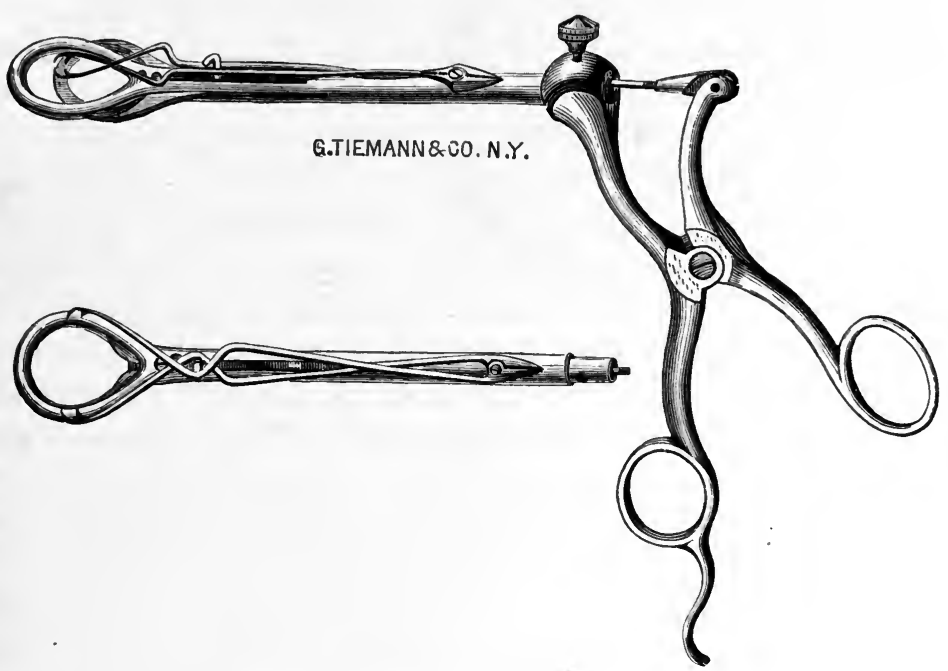

FIG. 97.-Tiemann \& Co.'s tonsillotome.

Treatment.-In the hypertrophic form the redundant portion of the gland must be removed, either with a knife, snare, electrolysis, or some escharotic. The preferable method is with the

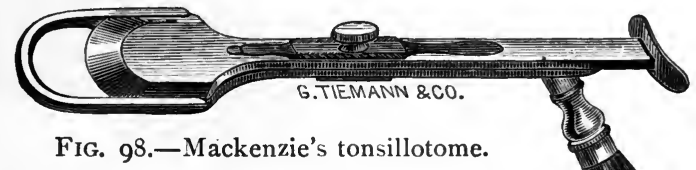
scissors or tonsillotome, of which there are several standard varieties (see Figs. 97 and 98).

Tonsillotomy.-The method of procedure is very simple. The mouth being held open and the tongue held down with a tongue depressor, the guillotine is opened and placed over the projecting portion of the gland, care being taken to hold the instrument close to the anterior pillar, so as to prevent the ring from slipping off or including a piece of the tongue or the uvula. The mechan- 
ism of the tonsillotome is such that its fork or spear pierces or grasps the projecting portion of the tonsil and pulls it out as the knife is pushed or pulled to its place. In the case of children a smaller tonsillotome should be used, for it is often impossible to properly adjust to the tonsil a large ring, owing to the small size of the pharynx. Tiemann's instrument is preferable on this account.

Knife.-When the tonsil is of irregular shape, long, or so lobulated that enough of it cannot be engaged in the ring of a guillotine, the better way will be to use hooked forceps to hold the gland, while with a probe-pointed curved bistoury or tonsil scissors the redundant portion is cut off. This is done as follows: An assistant holding the tongue down with a tongue-depressor, a suitable mouth gag, if necessary, having been adjusted, the operator seizes the tonsil with the volsellum forceps in the left hand or the right, according to the side to be operated upon, and with the knife in the other hand he cuts the projecting portion from below upward by a rapid sawing motion. This is preferable to cutting from above downward, because, as the blood trickles down and the gagging increases, difficulty will be found in following the line of incision previously laid out. The operator will thus also avoid cutting the tongue, an accident which might cause great discomfort to the patient afterward. Oftentimes other methods would

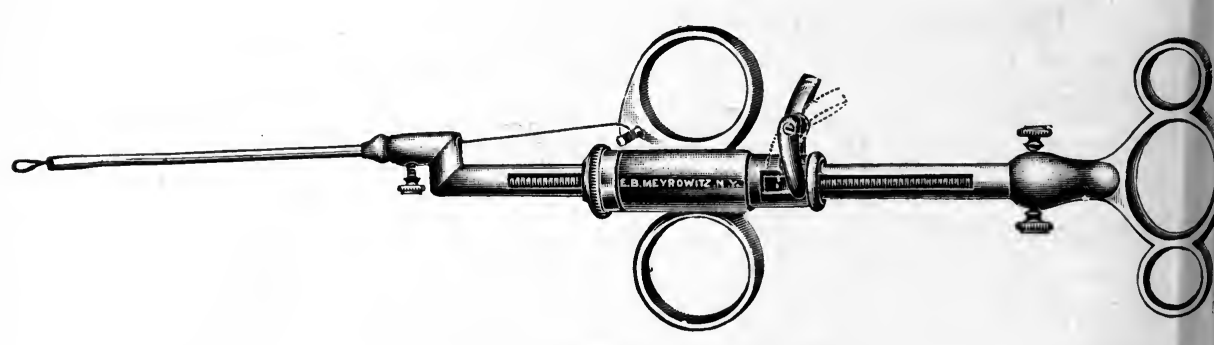

FIG. 99.-Dr. Farlow's tónsil snare.

better be adopted, especially for dealing with the large soft tonsil, because after cutting with guillotine or knife the stump may remain sore and partially unhealed for some time. For this reason many practitioners remove the redundant portion with the cold snare, which is often preferable to any other method (see Fig. 99). 
Wire Snare.-The manipulation of this instrument is as follows: Holding the tongue down with a suitable tongue depressor, the tonsil is seized by an assistant with either the volsellum forceps or a pair of common hook forceps (see chapter on Instruments). The loop of the snare, armed with Nos. 18 to 22 steel piano wire, is now passed over the forceps. The operator now taking the snare in one hand, places the loop over the tonsil, and with a finger of the other hand pushes the wire back and around the tonsil, at the same time by gradual traction closing the loop until it has a firm hold in the desired place. The forceps may now be removed, if desired, and the operation completed by screwing the loop home into the cannula. This operation is usually a bloodless one, but it is difficult, and indeed impossible in some cases, to secure and hold the loop around the tonsil, especially when the surrounding glands are involved in the mass.

Galvano-cautery.-Many practitioners prefer the galvanocautery snare. M. Magnan and others have given up the knife and guillotine excepting for children from three to six years of age for the galvano-cautery or cold snare. The galvano-cautery snare is applied in the same manner as the cold snare, the electric current being turned on after the loop is well adjusted (see chapter on Instruments).

Hemorrhage.-The greatest danger from tonsillotom $y$ by cutting is hemorrhage, which is more liable to occur in cutting the "hard" tonsils, because the coats of the vessels are apt to be sclerotic. It sometimes happens that a branch of the tonsillar artery runs through the central portion of the gland, and if the tissues be sclerotic, of course considerable hemorrhage may ensue from lack of contraction of the vessels. This danger is to be expected, more particularly in adults than in children, although sometimes it does occur with the latter. Dr. Jonathan Wright tabulated some forty cases of alarming hemorrhage which he found on record in the library of the surgeon-general's office, among which two cases proved fatal. Delavan, however, in the literature covering a period of six years, found only twelve cases reported of alarming hemorrhage following tonsillotomy. Wright favours the amygdalotome or galvano-cautery loop. The instrument which he uses for a galvano-cautery loop is very similar to Mackenzie's amygdalotome, except that the wires are inclosed in compressed paper, a non-conducting material. 
Dr. Asch believes the direct causes of serious hemorrhage may be referred to three sources: (I) An abnormal destruction of the tonsils; (2) a venous plexus; (3) profuse capillary bleeding. The tonsils being enlarged, hypertrophied, and sclerotic, the vessels are very liable to remain open when divided, thus causing serious hemorrhage. Farlow uses cutting forceps, and Bliss favours the scissors for removal of the redundant mass. As a substitute for the knife (tonsillotomy), the galvano-puncture (or igni-puncture), the cautery snare, and the cold snare, are preferred by many practitioners. The most alarming case of hemorrhage which the writer ever met with-was in a boy eleven years old. The hemorrhage was finally stopped by holding for some hours a pledget of lint, with the finger, against the stump of the tonsil, this expedient requiring the help of several assistants. The patient became quite exsanguinated. The writer has had several other severe cases of hemorrhage, but succeeded in checking them very soon by the use of Monsel's solution, with or without temporary compression of the parts. There is a great difference of opinion regarding the advisability of tonsillotomy, on account of the liability of excessive hemorrhage following it. Many view the chances of its occurrence with sufficient apprehension to restrain them from resorting to either the knife or guillotine, unless other means of removal fail; while others, who have made a great many tonsillotomies, view with scorn the dangers set forth. Hemorrhage is sometimes secondary and recurrent.

Dr. Price Brown, of Toronto, had a case of alarming recurrent hemorrhage on the fourth day after the operation of tonsillotomy. By the free use of adrenalin, locally, the amount of hemorrhage is greatly lessened.

Dr. N. W. Loeb and others think that the galvano-cautery snare has one advantage by destroying germs which may be in the way, and another in that no hemorrhage results from its use. According to the writer's experience, there is very little liability of a fatality from tonsillotomy if proper precautions are taken. In the first place, both tonsils should not be cut at the same time, if severe hemorrhage ensues, but an interval of sufficient time allowed between the cutting of the first and second tonsil. Secondly, too much of the tonsil should not be cut away-only the projecting portion. In dealing with the elongated lobular or pointed tonsil, 
it usually gives sufficient relief to abscise the lower part, and then, if necessary, take off a piece from the upper part. A regrowth of the tonsil after excision has been reported, but such a phenomenon must be very rare.

The other methods for the reduction of enlarged tonsils, such as ignipuncture with the incandescent galvano-cautery knife, electrolysis, and the application of escharotics, such as Vienna paste, London paste, or chromic acid, etc., may be preferable for certain cases. The use of the latter escharotics, however, merely deserve mention, according to the writer's opinion, as the necessity for their use can scarcely ever arise nowadays.

Ignipuncture.-Ignipuncture and electrolysis, however, are measures which deserve full consideration. Whenever for any reason the danger of hemorrhage may be suspected, as in hæmophilia, the best plan of treatment, next to the cold or galvano-cautery snare, is ignipuncture by means of the electric current or thermal cautery-the former method, however, being preferable. Ignipuncture should cover several sittings, and be applied to one side only at a séance. Before proceeding to the cauterization, the surface of the tonsil should be thoroughly cleansed with some antiseptic spray or solution, after which a four-per-cent solution of cocaine should be applied to the part to be operated upon. Then, after an interval of from three to five minutes, a "cautery knife" of good size is pushed, while incandescent, into the tonsil, if possible between two crypts (or at least not directly into the crypt), and held there for a few seconds. It is then withdrawn, and after an interval of a minute or a couple of minutes another puncture in another portion of the tonsil may be made. If the tonsil be very large, four or five such punctures may be made at a sitting without danger of creating too much reactionary inflammation. If the electrode is put into a crypt, it should be passed to the bottom of the canal and held there long enough to destroy the lining, otherwise the destruction of only the outer portion of the crypt may create a pocket, and so block up the inner follicles as to induce suppuration, or at least considerable inflammation of the deeper tissue of the tonsil; whereas if the crypts are avoided by the electrode there will be no retention of secretion, and but little reactionary inflammation. Next in efficiency to ignipuncture is electrolysis. 
Electrolysis.-There are cases in which this method may be preferable, especially where the hypertrophied tonsil seems to be amalgamated with hypertrophied, lingual, and neighbouring lymphatic tissue. Either the monopolar or bipolar method may be used. Some operators prefer one, and some the other. The writer prefers the bipolar method for most cases. Any galvanic battery giving sufficient electro-motive force can be used for the purpose. The parts having been thoroughly cleansed with an antiseptic solution, and cocaine having been applied and time allowed for it to take effect, the needles are attached to the conducting cords or handles and introduced. Owing to the irritability of the throat in some people it is difficult to keep them inserted a sufficient length of time, but it will be found in such cases that the desired end may be accomplished by making more frequent applications. The writer had one case, a nervous woman, whose pharynx was very irritable, to whom the needles could not be applied for more than half a minute at a time, yet the result accomplished through frequent sittings was a very satisfactory one. Generally it is better to keep the needles inserted for about five minutes, or even ten minutes in very hard tonsils, until there appears the characteristic whiteness and ebullition in the neighbourhood of the cathode. There are many designs of instruments in the market for electrolysis, in some of which both needles are supplied (running parallel) to the same handle. This is preferred by many operators. It is undoubtedly better, however, to have each needle separate, so that the operator may have a wider field, if desired, between the two, as he can do with one in each hand; in this way larger or smaller districts of tissue may be included with greater facility. The use of astringent medicaments for the relief of enlarged tonsils is usually of no benefit. Where the enlargement, however, is only moderate, and in young children, such agents may be tried, and sometimes they are beneficial, if used in connection with the internal administration of the sirup of the iodide of iron. In the enlarged soft tonsils the rubbing of the part daily with powdered alum or sodium bicarbonate has been recommended. Laryngologists are frequently asked to remove tonsils for the fancied purpose of improving the voice. Indeed, it has become a "fad" in some quarters, very like the cutting of the middle finger tendon for people who desire to facilitate 
their piano playing. Undoubtedly, in many instances a tonsillotomy will be necessary to improve the quality of the voice, but I think no self-respecting practitioner will consent to the enucleation of small or normal tonsils, which in no practical way interfere with artistic vocal cultivation.

The Treatment of Chronic Follicular Tonsilitis.-Those cases already mentioned of chronic inflammation as occurring in adults, unaccompanied by much, if any, enlargement of the organ, and characterized mainly by the accumulation of altered and offensive secretions in the follicles, can often be successfully relieved by a thorough washing out of the offending crypts with a solution of bichloride of mercury ( 1 to 3,000), peroxide of hydrogen ( 1 to 9), or a solution of resorcin (Io grains to the ounce), by means of a small pointed syringe or a powerful spray. Besides, from time to time a small probe, the end of which has been loaded with nitrate of silver, should be passed into the crypt, or, instead, a solution of chloride of zinc (Io to 20 grains to the ounce) may be injected once or twice a week. In some cases when the tonsil is soft, hyperæmic, and has patulous crypts, containing all the time foul secretion, débris of food, etc., the galvano-cautery will be found the most useful agent. In such cases, by passing a pointed electrode at a red heat quickly in and out of the crypts, more for the purpose of producing irritation than destruction, one can bring about great amelioration, if not a cure. In some instances one or two crypts may terminate in a sort of a sac or cup with an opening at the top. In such a case a small curved knife may be inserted behind this anterior wall or diaphragm, and a slit made through it for the purpose of securing drainage of the part and partial restoration of the natural conditions; then perhaps no further trouble will ensue, especially if the part be afterward kept cleansed. In those instances where the tonsil is much atrophied and the supratonsillar follicles at the upper part of the faucial arch or niche seem to give rise to almost all of the abnormal secretion, the washing out of this space with a solution of borax, and afterward applying, by means of a pledget of absorbent cotton, a mixture of eucalyptol and menthol, or of oleum picis and balsam Peruviana a few times, will often remedy the evil. Sometimes, however, the relief of this condition will require the application of nitrate of silver, chloride of zinci, the galvano-cautery, or the curette. The excision of 
the soft enlarged tonsil in adults is always a matter for careful deliberation, not on account of the danger of hemorrhage nor the fear of subsequent inflammation, so much as the possibility of leaving a painful, ragged stump, which may be a source of irritation to the patient for years afterward. Therefore the writer prefers for these cases, in general, a treatment by ignipuncture, the galvano-cautery knife, or electrolysis applied as mentioned above. The objection, however, to the latter plan of treatment is based on the length of time involved by the method. When carefully used there is not much danger, but severe inflammation, and even suppuration, with abscess of the neighbouring tissues, may sometimes follow electrolytic applications in this region. With children and young adults it is better to remove the diseased mass by curette or punch.

Enucleation.-Enucleation of the tonsil is not very often necessary, excepting for some neoplastic condition.

The patient should be anæsthetized and the mouth gag properly adjusted; the tonsil is seized with a pair of hooked or volsellum forceps and drawn well forward from its bed, and by means of some blunt instrument, preferably an Addis periosteum dissector and scissors, the gland is gradually worked out. In this way not only much hemorrhage is avoided, but the cutting of tissue will be limited unless the capsular attachments or amalgamation of the parts are extensive. The knife, as a rule, can scarcely ever be used in this region freely without danger of wounding the deeper tissues or vessels of the faucial region. The tonsillar artery is sometimes large, and takes such a course that it cannot be avoided.

Hypertrophy of the Lymphoid Tissue at the Vault of the Pharynx-Adenoids-Hypertrophy of the PharYNGEAL OR LUSCHKA'S TONSIL.- "Adenoid VegetaTIONS"

This affection, also denominated, by Woakes and others, lymphoid papillomata, consists of an enlargement or overgrowth of the lymphoid or "adenoid" tissue situated at the vault of the pharynx. Mayer, Ganghofner, Tornwaldt, and Luschka were the first to describe this mass of glandular tissue and distinguish it by the name of gland or tonsil ; but to Dr. Wilhelm Meyer, of Copenhagen, belongs the credit of 
having first called attention to the evil effects of its overgrowth and pointed out the practical remedy for the same. While the disease is met with in every zone of the earth, it is more commonly seen in the north temperate zone.

Race.-It is not common to any particular race, although most frequently met with among the civilized inhabitants in regions of low altitude. The half-civilized and aboriginal dwellers, however, are not free from the disease, as it is met with in the American Indian, and, according to Meyer, of New York (Medical Record, April 4, 1896), enlarged adenoids may be found in Greenlanders, South Americans, pure Mongols, and among the natives of southeastern Asia.

Frequency.-Notwithstanding that so much literature on the subject has appeared during the last few years, the frequent occurrence of extraordinary hypertrophy of the pharyngeal tonsil is still a mooted question. Ingals, of Chicago, says: "I find, among my records of private patients since $\mathrm{I} 88 \mathrm{I}$, histories of between II,000 and 1 2,000 persons. Of them, about 90 per cent, or, in even numbers, 10,000, came to me because of some disease of the chest, throat, or nasal cavity. Of these, 203, or, in even numbers, 200 , equaling only 2 per cent of the whole, had hypertrophy of the pharyngeal tonsil. It is impossible to determine the relative frequency of diseases of the chest, throat, and nose, but as only 2 per cent of these special cases suffered from enlargement of this gland, it was clear that the percentage must be even smaller when all classes of cases are considèred. Of the 200 cases of hypertrophy of the pharyngeal tonsil, out of I0,000 consecutive cases of disease of the chest, throat, and nose, 60 per cent were residents of the city, and 30 per cent came from the country or smaller towns. In some cases the hypertrophy was of so little importance that it had not attracted the patient's attention, and was discovered incidentally."

Meyer states that of 148 persons who consulted him for throat and ear diseases, I 5, or I in 9, had "adenoids." Robinson, from a large number of examinations, considers the disease rather infrequent. The author's experience coincides with that of Ingals and Robinson, if cases of moderate hypertrophy causing no obstruction to nasal respiration be excluded.

Etiology. Age.-According to all authors, the affection generally develops in early childhood. It is more common between the ages of two and fifteen, 4 per cent being observed 
between two and eight years, and 37 per cent between nine and sixteen, according to Ingals's statistics. The affection may, however, arise in older persons. Meyer observed less than 37 per cent under ten years of age, and less than 62 per cent, all told, under fifteen. Bosworth's statistics show less than 30 per cent under fifteen years of age. In 90 per cent of his cases the disease certainly developed in infancy or early childhood.

In children, the lymphatic tissue is especially susceptible to vascular changes on comparatively slight causes, and the repeated colds from which they suffer keep the lymphoid tissue of the pharynx congested-overfed, so to speak-and overgrowth is to be expected.

In adults, a condition of modified hypertrophy is frequently seen, which may have existed from childhood unnoticed, or may have developed after puberty. As a rule, however, all the lymphoid tissue of the throat has a tendency to atrophy after puberty. Delavan, Jonathan Wright, and others, have met with this enlargement in women about the age of thirty.

In this connection Delavan suggests that the enlargements may be sometimes of a temporary nature, recurring upon the operation of colds or other exciting causes. He mentioned the case of a young woman, the vault of whose pharynx was examined by several physicians, some of whom found enlargement and some equally competent did not. He thinks these enlargements are analogous to that of the faucial tonsils which takes place after taking cold. He proposes the title "acute recurrent enlargements of the adenoid tissue at the vault of the pharynx" for these cases. The writer has seen one case in which the enlargement occurred after the age of twentytwo in a person who had suffered from mumps about three months before coming under his observation. This is verified by the fact that several examinations made of this individual's nasopharynx before the attack of mumps did not reveal any material enlargement of the pharyngeal tonsil.

A. Brindel finds that adenoids are uncommon up to three years of age, but are common between the ages of eleven and fourteen years. He finds that adults furnish about io per cent, and adolescents (fifteen to twenty years of age) furnish I4 per cent of the cases.

It is undoubtedly of infrequent occurrence during infancy, 
especially before the second period of dentition. Jonathan Wright has shown that the nasopharynx of infants is usually smooth, the lymphoid tissue not being developed.

Sex.-According to Bosworth, the affection appears to be more frequent in girls than in boys, 41 per cent having been recorded as affecting males and 59 per cent females. In Meyer's cases a little over 5 I per cent were males. Hajek found the disease about equally in the two sexes.

Heredity.-It is attributed by many authors to hereditary influence. Hajek found that about ro per cent of his cases were apparently of hereditary origin. Price Brown, Delavan, and other authors lay great stress on heredity as an element of causation. It is observed that the high-arched palate characteristic of many "mouth breathers" can be found in several members of a family. Indeed, such conformation in greater or lesser degree may be found to have occurred throughout several generations of a family. Hajek thinks that some cases are due to enlargement of the faucial tonsils. It is true that a great many cases show enlargement of the faucial tonsils, and also of the lingual lymphoid tissue, still it is improbable that hypertrophy of the latter tissues causes hypertrophy of the pharyngeal tonsil. Myles and other practitioners also call attention to the frequent association of hypertrophy of the faucial and sometimes the lingual tonsil with glandular hyper. trophy at the vault of the pharynx (adenoids). Swain thinks that the lingual tonsil is seldom affected before the twentyfifth year. He concludes from his studies in comparative anatomy that in mammals the pharyngeal tonsil is not so extensive as the faucial tonsil in its occurrence, but is at least as frequently seen as the lingual; and in lower forms of life, as in birds and reptiles, it is present in a highly developed form, and therefore of all these collections the lymphatic tissue of the throat is the oldest. Hajek's statistics showed that in 76 per cent one or both faucial tonsils were also enlarged; in 7 per cent one only, and in 69 per cent both of them.

Deformity of the hard palate (high arch) and deviation of the nasal septum have each been considered as contributory or predisposing causes of this affection by many observers, while on the other hand equally competent practitioners have attributed to the overgrowth of the adenoid tissue one or the other of these deformities respectively. 
Inasmuch as deviation of the nasal septum does not usually take place in early life, at least before the seventh year of age, it would seem hardly fair to consider that deformity alone an important factor in the etiology of adenoids.

Körner and Waldow (Gleitsman), after careful examination on the living and dead, concluded that adenoids, or enlarged pharyngeal tonsil, when existing before dentition, was often responsible for the deformity of the superior maxilla, characterized as the "high" or "V-shaped" arch of the palate, and the associated irregularity of the teeth, which usually accompanies such a condition through the obstruction to respiration which the adenoids cause. Gleitsman, after quoting the work of the above-mentioned authors, dissents from that view somewhat. He says: "The assumption advanced, that adenoids produce a change in the bony substance, especially of the upper maxilla, appears to me nothing better than a hypothesis." He is undoubtedly right on this point, for we meet with many cases of deflected septum and high-arched palate (as pointed out some years ago by Jarvis and Delavan), without much, if any, glandular enlargement at the pharyngeal vault. Many such cases are especially met with by general practitioners, who do not refer them to specialists on account of the absence of any particularly embarrassing symptoms.

"A catarrhal condition" of the nasal passages has been considered one of the potent causes of the overgrowth of the adenoid tissue. This was noted by Hajek in 86 cases. In 30 per cent the nasal passages were free, in $12^{x} / 2$ per cent they were obstructed by exostosis of the septum, and in 50 per cent by hypertrophy or swelling of the inferior turbinated bodies. Excessive secretion or other catarrhal symptoms were also noted in 60 per cent of the cases. The experiments of Ascherbrandt, in 1886, show the important part played by free nasal respiration (by warming and moistening the air) in preventing disease of the upper air passages.

The theory of Greville MacDonald is, that a majority of the cases of enlargement of Luschka's tonsil are due to obstruction in the nasal passages, in consequence of which the air in the nasopharynx is kept rarefied by the mouth-breathing which must accompany such a condition. Eames, Hopkins, and others also lay great stress upon nasal stenosis as a cause of lymphoid hypertrophy at the vault of the pharynx. The 
hypothesis is, that as soon as respiration is obstructed through the nose there comes the physical necessity of diminution in the barometric pressure behind the seat of stenosis. This results in the more or less overfilling of the blood-vessels, which in turn leads to hypernutrition and overgrowth. The general opinion is that adenoids commence growing during childhood, but, if left to themselves, shrink and disappear during maturity. There are many exceptions to this rule. There are cases in which the enlargement of the adenoid tissue has not manifested itself until after puberty, and also cases where the adenoids have remained enlarged until after middle life.

The strumous and tubercular diatheses have been credited with inducing overgrowth of this tissue. Very often the subjects of this disease are children of tubercular parents. M. Lermoyez alludes to an experience in which some enlarged adenoid growths in the nasopharynx proved to be tuberculous in structure. Dieulafoy, Broca, Helme, Chiari, Freudenthal, and others, believe that there are many cases which are tubercular in their nature, and that they are often overlooked. M. Gourc has never found the tubercle bacillus in an examination of $20 \mathrm{I}$ cases. Thirty-seven times he found streptococci (never pure), 69 times the staphylococcus (pure), and 69 times associated with other elements; also with cocci (pure), 4I times; with cocci associated, 54 times; the pneumococci, 3 times; and the leptothrix (pure), twice. Moreover, among those examined he noted 30 times a collateral tuberculosis, 18 times hereditary tuberculosis, and 17 times personal or acquired tuberculosis. The author has never met with a case of tuberculosis of the lymphoid tissue at the vault of the pharynx in either hospital or private practice. The lymphoid hypertrophy under consideration may, but does not frequently, occur in children of tubercular parents, and not in tubercular children themselves without the vault of the pharynx being the seat of an active tubercular process. It is generally admitted, however, by clinicians that the tubercular diathesis or tubercular tendency may be a predisposing cause. A damp habitat favours the development of this, as of all other lymphoid hyperplasia, for it is in low, moist regions that the greater number of cases of adenoids will be met with.

Exophthalmic goitre has been credited with being a cause of the disease when found associated with it; but the truth of 
this statement may be questioned, inasmuch as the several varieties of goitre are usually accompanied by more or less hyperplasia of the neighbouring lymphoid or lymphatic tissue, as any general practitioner can attest.

Symptomatology.-The subjective symptoms will vary, of course, with the extent, seat, and character of the enlargement. The most striking sign is the obstruction to nasal respiration, which compels the patient to breathe and speak through the

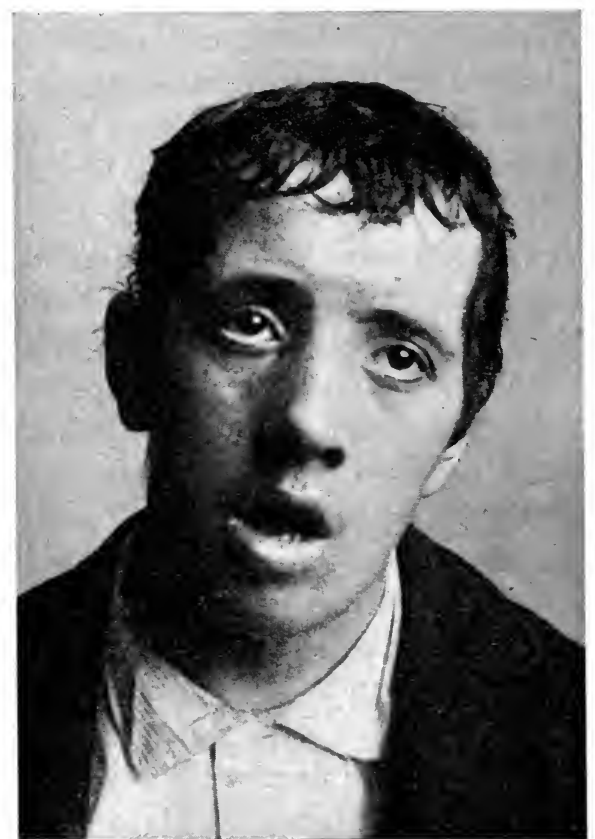

FIG. 100. - Typical facial appearance of a mouth breather. Case of adenoid vegetations and hypertrophic rhinitis. mouth. These patients are often called "mouth breathers." They present a peculiarly stupid facial expression. The pinched nares, open and ofttimes drivelling mouth, noisy respiration, continual acts of glutition, expressionless eye with drooping lid, dulness of hearing, and nasal intonation of voice, form a group of symptoms which are typical of this condition (see Fig. I00). In addition; there is often a lack of development or a deformity of the thorax, with or without asthma; the sub. ject is restless at night, suffering distress in breathing, and intermissions of sleep amounting to nightmare or night terrors. There may be nocturnal enuresis, diaphoresis, or both. The lower pharynx is usually dry from constantly breathing through the mouth, while the faucial tonsils are apt to be swollen more or less, and also dry. The prominence of these symptoms will of course depend upon the amount of nasal obstruction offered by the adenoid growth, for it is obvious that a small amount of enlarged adenoid tissue in the vault of the pharynx may become as formidable an obstruction, if the capacity of the nasopharynx be limited on 
account of congenital deformity, hypertrophy of the turbinates, etc., as would a greater degree of enlargement in a comparatively good-sized nasopharynx.

Neuralgia, chorea, and other neurotic manifestations, are sometimes symptoms of this condition. Dr. Howard S. Straight, of Cleveland, Ohio, reported a case of a boy, sixteen years of age, who had been suffering from headache for three years, which was due to adenoid growths in the nasopharynx. The headaches ceased after removal of the adenoids. "Why the adenoid growth, which had existed probably more than three years, did not produce headache before, is not accounted for." Sometimes acute symptoms and complications accompany enlargement of the adenoid tissue. Langmaid reported to the American Laryngological Association a case of cervical adenitis with development of œdema of the larynx and posterior pharyngeal abscess, resulting in spontaneous opening without septicæmia. Diphtheria was suspected, but no evidence was obtained in support of the suspicion.

Colds, Influenza, etc.-While undoubtedly the origin of adenoids can very often be traced to an attack of measles, diphtheria, scarlet fever, rheumatism, or influenza, it can often be attributed to colds. This, however, is probably an erroneous conclusion, based upon substituting effect for cause. Many children suffering with adenoids are supposed to be taking cold almost continually because they exhibit "catarrhal symptoms," such as excessive nasal discharge and more or less nasal stenosis; but in such cases the so-called colds or catarrhal symptoms can invariably be attributed to the lymphoid hypertrophy. Hajek stated that 3 per cent of his cases appeared to follow measles, 4 per cent scarlet fever, I per cent diphtheria, and 3 per cent were attributed by the parents of the patients to influenza and pneumonia; about 16 per cent were attributed to frequent colds. In 63 per cent nothing could be discovered in the clinical history which would in any way account for the origin of the disease. He also says that about 35 per cent of these patients were subject to diseases of the throat. This, of course, might be expected as a result of the mouth breathing.

Many cases of recurrent spasmodic croup will be found to depend upon hypertrophy of the pharyngeal tonsil and other tissue of the lymphoid ring of the fauces. 
Ear.-Deafness is such a common symptom that it is generally looked for in these cases. A. Brindel published his observations, made upon 6i 8 cases occurring in M. Moure's clinic at Bordeaux. During a period of two years, 280 cases came for treatment of ear disorders ; 127 of these complained of obstructed nasal respiration alone, and 142 complained of some throat disorder-pharyngitis, laryngitis, etc.-and 69 presented no special symptoms. Out of 102 cases of glandular hypertrophy at the vault of the pharynx cited by Meyer, 72 had diseases of the ear. In Swinburne's practice among I79 patients suffering from middle-ear disease, 27 , or about one in six, had adenoid vegetations in the vault of the pharynx. He found the ear diseased in a large proportion of all cases of adenoid vegetations.

Woakes thinks that in England twenty per cent of the persons suffering from these overgrowths have aural disease. He does not believe, however, that non-suppurative disease of the middle ear can arise from occlusion of the Eustachian tubes alone. Beverly Robinson takes exception to the oft-repeated statement that aural disease is a new complication of either deviated septum or lymphoid hypertrophy at the vault of the pharynx. He has frequently observed that the aural disease was on the side opposite the deviation of the septum. Impairment of hearing may be out of proportion to the amount of enlargement. The writer has had three cases in young children who were deaf-mutes in whom the lymphoid hypertrophy was very moderate indeed.

The location of the growth has an important influence in determining the danger to the ear, for adenoid enlargement confined to the pharyngeal tonsil itself may not interfere very much with the function of the Eustachian tube.

Dr. F. E. Hopkins urges that we bear in mind the influence which the lymphoid tissue in the nasopharynx has upon the ear. On account of this mass being in close proximity to the Eustachian tubes, it must have an influence upon the middle ear, and, according to Burnett, Blake, Randall, and Dench, this influence may extend to the labyrinth also. It is a fact of common observation that a large proportion of the cases of deafness is due to the presence of adenoids in childhood. The statistics of Woakes show a striking connection between the presence of adenoids and aural disease. He says that 95 per 
cent of the cases present aural complications, and Meyer in his original 102 cases found over 70 per cent with such complications. This condition is to be found in every country, and history shows that it has existed in all past ages. This condition (adenoids) is said to prevail in most of the cases of deaf-mutism. Sendziak, of Warsaw, quotes several authors who report from $57 \frac{5}{10}$ per cent to 73 per cent of deaf-mutes suffering from the presence of adenoids. In marked cases the child is as stupid as he looks. This stupidity has, according to A. Jacobi and Solis-Cohen, "an anatomical basis in the relation between the lymphatic circulation through the brain to the nasopharynx. The presence of a large mass of hypertrophied tissue interferes with the proper drainage, so to speak, of the brain." The dull expression is still further enhanced by the deafness. Children suffering from this condition are supposed to be more susceptible to the infectious diseases because they possess a lowered vitality. It is also said that as they are "mouth breathers" the inspired bacteria find suitable places of lodgment upon the unhealthy surface, which proves a good culture ground for their propagation. It is undoubtedly true, as mentioned above, that children with adenoids and enlarged faucial tonsils are more susceptible to the acute faucial diseases, to the exanthemata, and more liable to septic infection.

Diagnosis.-The symptomatology given above would seem to render the diagnosis easy, yet a thorough examination of the nasal passages, as well as of the lower pharynx, is necessary to arrive at a positive diagnosis, inasmuch as obstruction to nasal respiration from other causes may give rise to about the same subjective symptoms. The examination of the nasopharynx by the rhinoscopic mirror cannot always be made-indeed, very seldom in the case of young children. Therefore reliance must be placed upon palpation, with the finger carried directly behind the soft palate. It will sometimes be necessary to place the patient under an anæsthetic before the examination is undertaken. It is better to do so with obstreperous children rather than omit the digital exploration. The manipulations should be conducted about as follows: The child being held upon the lap of an assistant or laid upon a table, the mouth is opened and a mouth gag inserted. In its absence an improvised one made of a piece of wood will answer the purpose. It is wise to provide the finger to be used in the 
examination with a rubber or metal shield, or wrap it with a piece of adhesive plaster, in order to avoid chafing the skin against the teeth of the child. The finger is now passed back until it comes in contact with one of the faucial pillars or the posterior wall of the pharynx, and then insinuated behind the soft palate into the nasopharynx, where a careful exploration of the whole of the cavity embracing the parts around the choanæ and the fossa of Rosenmüller should be made. The contour will be found to vary in different individuals; in some cases the sensation conveyed to the finger will be that of a lobulated mass at the vault or along the posterior curve of the pharynx; again, the sensation imparted will be that of small currants, perhaps in clusters, distributed from one side to the other, or along the median line of the posterior wall of the pharynx; while again, vegetations will hang down in stalactite fashion and impart the sensation of velvety tassels. With regard to the consistency of these masses, some will feel firm and encapsulated like sweetbreads, while others may be soft and likened to the feeling of a piece of wet sponge. The objects to be determined in the examination are to ascertain just how much these masses obstruct the choanæ, where they are principally located, and their character. As before mentioned, when the nasopharynx is capacious, a moderate hypertrophy of this glandular mass may not prove to be a very great obstruction to respiration. If so, and the patient is a child, the parts may be safely left to the absorptive operations of Nature.

Pathology.-These growths are composed of lymphoid tissue, formerly termed adenoid from the mistaken idea that it is glandular in nature. The true structure has long been known, but the term adenoid, originally employed by Meyer, still clings to it. The hypertrophied tissue in the nasopharynx and the faucial tonsil is believed by many to be identical in structure, the surface being covered with columnar ciliated epithelium, and the mass made up according to the general structure of lymphatic tissue, with a delicate, highly defined reticulum crowded with lymph corpuscles. The formations under con. sideration, then, are simply a hypertrophy of lymphoid tissue normally present in the pharynx and nasopharynx (see Fig. IOI). The lack of the fibrous element in the pharyngeal tonsil accounts for its friability. The faucial tonsil through constant 
irritation is of greater density. In a patient who has adenoids it is common to find upon the posterior wall of the oropharynx scattered lymph follicles, which give the surface a roughly granular appearance or feeling to the touch. Sometimes the lymphatic tissue of this particular region is gathered in considerable amount just behind and parallel to the posterior pillars.

Prognosis.-The prognosis is always favourable when proper treatment is adopted. When it is not, various diseases of the ear and pulmonary organs may result-asthma and phthisis pulmonalis indirectly, and otitis media and deafness directly. Where the obstruction is very great, thus interfering materially with healthy respiration and with proper sleep, the development of the child in every particular is apt to be retarded, and deformities of the nose and chest will certainly supervene.

Treatment.-The treatment in such cases should

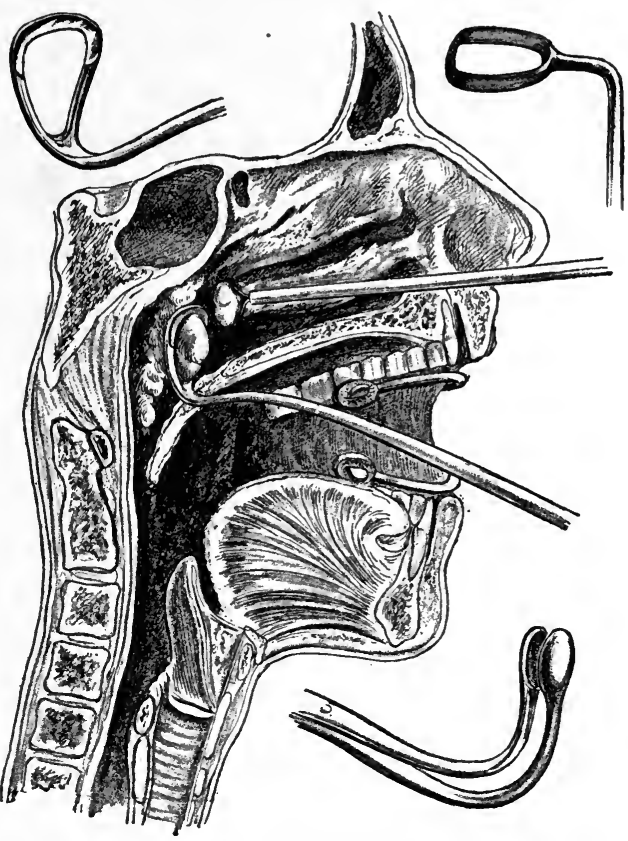

FIG. Ior.--The Gottstein curette (its blade shown also above to the left) applied for the removal of the pharyngeal tonsil, the mouth being held open by a gag. A snare is also shown engaging a posterior turbinal hypertrophy through the naris. The Hartmann curette is shown above and the Löwenberg forceps below, to the right. (From American Text-Book of Diseases of the Eye, Ear, Nose, and Throat.)

be both local and general. The main indication for local treatment is the removal of the obstruction to respiration. This should be done without much delay. In older persons the nasopharynx may be reached for this purpose without the use of a general anæsthetic, but in children it will generally be found necessary to anæsthetize the patient either with chloroform or the A. C. E. mixture; the latter is strongly recommended for weakly, strumous children, especially for those showing marked deformity of the thorax. The writer considers that there is little danger in the administration of chloroform to any young 
subject, provided that it is mixed with plenty of air and the quantity restricted to the actual needs of the operation. As the operation takes but little time, the patient need not be under the full influence of the anæsthetic very long. The writer agrees with Jonathan Wright, and many other operators, that it is never necessary to carry the anæsthesia to the full limit for these operations. It is perhaps unnecessary to add that the patient should always take the anæsthetic in a recumbent position, and never sitting up. Nitrous oxide may often be employed as an anæsthetic for operation upon older children and adults. W. E. Casselberry recommends nitrous oxide, but states that the period of anæsthesia is often too short for some operations. Experience has shown that this anæsthetic, however, is safer than others, and possesses the advantage of rapid revival of the patient; the method of Gardner Hunt or Hewett, of England, and A. Van Arsdale, of New York, who modify the action of nitrous oxide by the addition of oxygen in order to render it more effective, may be followed. Regarding the safety of nitrous oxide, it is estimated by Dr. H. C. Wood that it has been given in 750,000 cases annually, and we have been able to learn of but thirteen deaths fairly attributable to it.* Schleich's mixture is not applicable to these cases, as a rule. With older persons, the patient should be seated before the operator in the position for rhinoscopic examination with the velum palati secured forward, either by the elastic tapes of Wales, or by the self-retaining palate retractor of White or Jarvis (see Fig. 32). A ten- to twenty-per-cent solution of cocaine carried upon a curved applicator is next applied to the field of operation. Should the patient be inclined to close the mouth inadvertently, a gag must be used. The operator takes the rhinoscopic mirror in one hand, and the cutting forceps, or the curette, in the other, and, guided by the mirror, quickly seizes the growths one after the other and removes them.

If considerable hemorrhage follows, have a relay of mirrors; when one is smeared the assistant may hand the operator a clean one. If the adenoid growths are very soft, they may be removed with the finger-nail, or with the adenoid finger curette. When the palate retractor is used, although it may be a self-retaining one, it is advisable for an assistant to hold the instrument in

* Dr. Hickey had a case, a boy aged twelve, who developed an epileptic fit during the nitrous oxide anæsthesia, without a fatal termination. 
place, lest, in the act of retching or gagging, it be dislodged. When a patient is under an anæsthetic, these expedients for the management of the soft palate are unnecessary, for the operative manipulations can be performed entirely by the sense of touch. The patient having been anæsthetized and the mouth gag applied, he may lie either upon the back or upon the side, with his face turned toward the operator, who now passes the forefinger of the left hand, palmar side upward, into the nasopharynx until the growth is felt. The forceps (see Fig. IO2) or curette (see Fig.

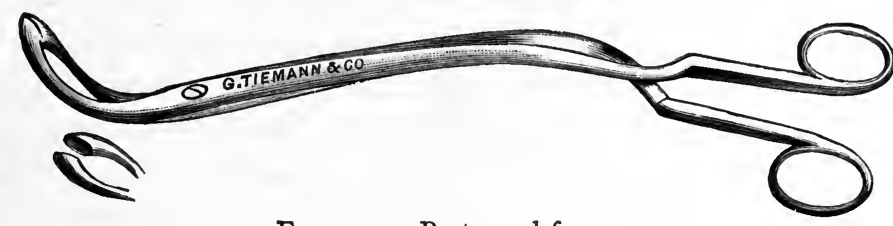

Fig. I02.-Post-nasal forceps.

I03) are now passed along on the palmar side of the finger, and the growths seized or scraped away under the guidance of the finger still in the naso-pharynx. Care should be taken to avoid injuring the prominences of the Eustachian tubes and the margins of the choanæ, or posterior edge of the nasal septum. Enlargements, which are apparently encapsulated, should be crushed with the forceps rather than scraped away with a curette, in order to avoid seriously injuring the nasopharynx by tearing away too much of the mucous membrane or portions of the Eustachian

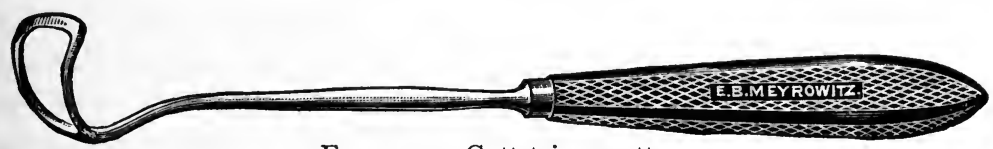

FIG. I03.-Gottstein curette.

tubes, or lacerating the posterior edge of the nasal septum. Rose and Seiler suspend the head of the patient over the edge of the operating table, believing that it facilitates manipulations, and especially prevents the blood from running into the larynx. There can be no objection to the placing of the head in this position; however, the operation can be usually performed so quickly that the ingress of blood into the larynx may be considered a very slight source of danger. A great variety of forceps and curettes to meet different conditions have been devised for the performance of this operation. When the adenoids are quite soft and sessile, many operators prefer the curette, and 
those devised by Gottstein, Löwenberg, Gradle, or Casselberry seem to enjoy the greatest popularity. The forceps of Gradle will do excellent service. The "actionary forceps" of Butts, and the post-nasal scissors of Dunn, are a combination of both cutting and torsion instruments. Myles wraps a piece of gauze around his finger and wipes out the débris after curettement.

In cases complicated by the presence of an unusual amount of fibrous tissue amounting almost to a fibrous growth, the adenotome of Delavan, where it can be used, will be found to be the most efficient instrument; unfortunately, it cannot be applied to any part except the vault and the posterior wall. It is claimed by some operators that the use of the curette is limited, and that all parts of the wall cannot be reached with safety. On the contrary, it is claimed by the advocates of the curette that the forceps are inefficient because of the cutting edge reaching only limited parts of the vault and posterior wall. It seems to the writer that both classes of instruments have their uses, inasmuch as we find such great variations in the size and shape of the nasopharynx, so that in some cases a pair of short-necked forceps will prove inefficient, and one must substitute for it a longer necked instrument. The instrument which will probably prove the most efficient in a given case can be decided upon after a careful examination. In many cases nothing will act better than the curette of Gottstein.

We meet with some nasopharynges which are very low and broad, some which are high and narrow, and some which are deformed by projecting bone either at the sides or at the posterior wall. I have met with two cases where the nasopharynx was almost divided into two portions by the projecting deformity of the cervical vertebra. In one case it was undoubtedly due to an exostosis. For all these reasons it would seem that every operator should be provided with several instruments of different models in order to conveniently deal with cases as they may arise. The local treatment of adenoid growths by topical medication is obviously utterly useless, so that it need not be discussed.

Regrowths of Adenoids.-The question of regrowth of the tissue is almost sure to be asked by the family of the patient. While in a majority of instances there will be no recurrence 
when the growths are thoroughly removed, yet one must agree with Dr. A. A. Bliss, and others, that regrowths do occur. The elementary or non-malignant character of the tissue leads one to believe that such recurrence is hardly to be expected, while clinical experience certainly supports such a view.

There are instances where a return of the original symptoms has indicated that recurrence of the hypertrophy has taken place.

During the last few years I have met nine cases where evulsion operations had been performed, and yet, after a period of relief, the symptoms of obstruction in the nasopharynx had returned. Dr. Harrison Allen, in his Clinical Study of the Skull-according to Jonathan Wright-has demonstrated from a large number of crania of all races that great variation in structure exists in the region of the vault, at the articulation of the vomer with the vaginal process of the sphenoid and sphenoidal processes of the palate bones. These variations are characterized by the failure in the complete union of the parts named, the open areas between showing evidence of having been filled during life with vascular tissue.

Allen terms these openings and passages "canales basis vomeris." He states that in many instances the wings of the vomer are quite free from the sphenoidal and vaginal processes, and that they are not in close articulation with the body of the sphenoid, numerous free openings leading in between the articulating surfaces.

Drs. Bliss and Jonathan Wright have brought out another explanation of interest in regard to the possible recurrence of naso-pharyngeal adenoids-that is, the variations in the character of this lymphoid mass in different individuals. The faucial tonsils show similar variations. There is a very decided difference between the soft, friable variety of lymphoid tissue and that which is infiltrated by fibrous bands, shown macroscopically by trabeculæ which lace through the growth, projecting inward from a more or less completely developed capsule. In such cases the lymphoid structure is thus divided into celllike groupings, more or less completely cut off from similar and neighbouring areas. We frequently meet this condition in the faucial tonsil, and probably an analogous state is not uncommon in the naso-pharyngeal tonsil. In both lymphoid 
structures this condition appears to be the result of inflammatory processes.

Bliss's patients all showed "a certain under-development of the entire naso-faucial region. The nares were small. The hard palate in the older cases was Gothic-arched. The naso-pharyngeal space was very narrow, with, as a rule, a low, flat vault, suggesting the horizontal position of the basilar process of the occipital bone common to early infancy. Indeed, the whole general structure of the naso-faucial area was infantile. An exception to this was found in two instances, young adults, where, instead of the horizontal basilar process, the latter ascended sharply in an oblique direction, and appeared to articulate with the sphenoid considerably above the vomero-sphenoidal articulation. In the recess thus formed a large amount of adenoid tissue was packed.

"Another feature of these cases was the excessive vascularity of the hypertrophied lymphoid tissue."

In a few instances where, in guiding the instrument used for extraction, he scraped deeply with the finger-nail, fine, friable spicules of bone were noticed about the articulation of the vomerine wings and sphenoid, in the roof of that area which Harrison Allen has termed "the posterula." Now, from this experience, and influenced by the ideas briefly suggested, it seems probable that these cases of apparent recurrence were not examples of a true regrowth of the adenoid tissue, but, on the contrary, have been the result of an incomplete removal of the grow ths at the original operations. Under ordinary conditions such incomplete removal would not have resulted in a return of symptoms. In cases, however, where we may have deep infiltration of the lymphoid tissue into fissures about the vomero-sphenoidal articulation; where we have an abnormally rich vascular supply distributed to this tissue and through this area; where we have the tissue itself mapped off into cells or small areas distinct from one another, it appears that we have possibilities for a recurrence of symptoms after an operation which has failed to remove very completely the whole mass of diseased tissue. Such return of symptoms is not due to a recurrence of the original growth, but may be caused by an inflammatory process arising in or continuing in parts which have remained. In course of time these portions gradually enlarge, and cause symptoms of obstruction, 
pressure, or irritation. To avoid such return of these conditions, he (Bliss) would advocate a very complete removal of the hypertrophied adenoid mass in all cases, especially of those portions of it which lie well forward about the vomero-sphenoidal articulation or roof of the posterula, and of those parts which lie in the lateral regions of the nasopharynx, above the upper border of the Eustachian openings and in Rosenmiuller's fossa. The idea has heretofore prevailed that after only a partial removal or trimming away of the naso-faucial lymphoid tissue, a process of absorption, aided by cicatrization and contraction, would induce the further obliteration of the masses. In a fairly normal subject this may be the case, yet it is a question if it be wise to trust that Nature will thus complete our operation for us (Bliss). Dr. F. E. Hopkins, of New York, reported a case of recurrence of lymphoid hypertrophy in the nasopharynx after removal. This subject has been ably discussed also by Dr. Delavan, Dr. Wright, and others. Hopkins reported twelve cases. Dr. Delavan reported an interesting case where recurrence occurred twice. Dr. J. Wright also reported a case. Dr. Leverend, of Lille, reported two cases of undoubted recurrence in children aged respectively eight and nine years. Dr. Barrett has also seen cases of a return where he was satisfied that the removal had been complete. Dr. Gleitsman reported a case in the practice of another which required two operations.

Where there is also enlargement of the turbinals, faucial tonsils, and deformity of the nasal septum, these conditions must also be remedied in order to secure complete nasal respiration. The propriety of operating at the same time upon the tonsils, adenoids, and the nasal passages, will depend upon the peculiarities of the case. It is, however, the opinion of careful practitioners that the galvano-cautery, saw, knife, or chisel should not be used in the nasal cavities at the time of the evulsion of adenoid growths even under the most approved methods of asepsis. As a rule, not much mischief follows the removal of adenoids and the excision of the faucial tonsils at the same time. In some cases, however, in anticipation of hemorrhage or reactionary inflammation, it would be better to postpone either one or the other operation until a future time. In some cases of marked deformity of the vault relief will not be obtained until they are turned over to a skillful dentist. The 
prevalence of the acute infectious diseases should always be taken into account before determining upon any operation.

Hemorrhage.-Drs. Delavan, Raault, Cartaz, Woakes, and others, have reported cases of secondary hemorrhage following the removal of adenoids. This may come on immediately after the operation, or within a few hours. The above-named observers have reported cases of secondary hemorrhage on the fifth, sixth, or eighth day after the operation. One case was reported of removal of adenoids of moderate size from a child of four years of age, which was followed by hemorrhage that ultimately resulted in death. Three instances occurred in the practice of Dr. Delavan of fatal secondary hemorrhage, and a case was reported by Hooper of fatal hemorrhage following a digital examination. The writer has never met with a case of dangerous hemorrhage after removal of the adenoid tissue at the vault of the pharynx. In one case of "a bleeder" (a child) the galvano-cautery was used instead of the forceps or curette. Cases of adenoids in tuberculous subjects should be operated on by the galvano-cautery, as recommended by Lermoyez, Chiari, and others, because this method will diminish the chances of subsequent caseation and infection.

While general treatment is of course ineffectual for the complete removal by absorption of these obstructions, yet in many instances the economy has been disturbed, and Nature should be aided in her efforts to restore proper nutrition. For this reason it may be considered a pardonable sin to adopt the routine treatment of prescribing for such patients tonics consisting of some form of iron, preferably the iodide, together with sirup of hypophosphites or quinine, salicin, or phosphorus. It is well also to prescribe a course of sponge baths, with subsequent friction, to stimulate the function of the skin, and unless there be contra-indications, a highly nitrogenized food should be advised. When entirely practicable, such patients will be found to rapidly improve in health by a sojourn at the seashore or in the mountains. A few cases have been published of tuberculosis having followed operations for the removal of adenoids, which event has been accounted for upon the theory that the tubercle bacillus had been conveyed either by the operator or through the atmosphere to the wounded and abraded parts. Undoubtedly this event is very rare, for, out of the vast number of cases operated on of late years, very few 
cases of actual tuberculosis of the nasopharynx have been recorded. Perhaps some of the reports have been based upon erroneous observations or insufficient clinical data, like a case of supposed local tuberculosis of the pharyngeal tonsil which came under the author's notice-a case of a boy sixteen years old, in which the pulmonary lesion had existed and partly retrogressed eighteen months before the occurrence of the presumed "primary tuberculosis of the pharyngeal tonsil."

The writer has operated upon many children who seemed to be in the pretubercular stage, but has never known of tuberculosis supervening.

M. Lermoyez, alluding to his experiences with some cases which, on examination, proved to be tuberculous and not lymphatic, says that in some cases the removal of the growths gave only temporary relief, and return soon followed. In one case, a rapid development of tuberculosis of the lungs followed the operation. He proposes, in all cases where there may be a suspicion of the tuberculous nature of the adenoid growths, to forestall the operation by histological examination of a test portion, and complete the operation finally by the galvanocautery, in order to hinder the entrance of germs into the open blood-vessels.

\section{Diseases of the Lingual Tonsil}

What is called the lingual tonsil is that portion of lymphatic tissue which is situated at the base of the tongue, between it and the epiglottis, forming the under segment of the lymphatic ring of the fauces. (See the chapter on Anatomy and Physiology.) This small amount of lymphatic tissue is sometimes the seat of disease independently of any other part of the fauces, but it more frequently participates in the faucial diseasese. g., peritonsilitis, tonsilitis, malignant tumours, diphtheria, or other morbid changes in this region. In diseases of the faucial or pharyngeal tonsils or the tongue, the lymphoid tissue is very apt to become secondarily affected. Swain called attention particularly to these diseases in 1886 . Since then, Bosworth, Langmaid, Knight, Hopkins, Price Brown, Bowen, and other writers, have published interesting articles on the diseases of this glandular mass. 
Acute Inflammation.-Sometimes the lingual tonsil is the seat of an acute inflammation which may result in suppuration and abscess. Swain thinks that acute diseases of the lingual tonsil have until lately been quite universally overlooked. He finds acute inflammation frequently. Seifert has reported sixteen cases of inflammation of the lingual tonsil terminating in abscess, and Saminsky reported, in I 893, that he had had twentytwo cases of acute inflammation of the lingual tonsil, eight of which, however, had been peritonsillar. Swain, in 1898 , reported that he had met with twelve cases of abscess of the lingual tonsil. These authors severally believe that this tissue is so situated (at the base of the tongue) that the buccal secretions and other fluids from the mouth flowing over it render it liable to become infected from extraneous material, or microorganisms, so that its frequent implication in disease or its inflammation is not to be wondered at.

Symptomatology.-The principal symptoms of acute inflammation of the lingual tonsil are a feeling of soreness referred to the base of the tongue, and more or less to the whole hyoid region; a constant desire to swallow, or clear the throat, and more or less pain in deglutition and upon movements of the tongue; moreover, a sense of constriction referred to the anterior part of the larynx, and in some cases more or less spasm of the constrictor muscles of the pharynx and those of the upper part of the larynx. As the epiglottis, from its contiguity, is apt to be involved in any considerable inflammation, laryngeal symptoms, such as choking sensations, more or less spasm, paroxysms of apprehension of suffocation, with more or less harassing cough, are not infrequent symptoms. The writer has seen but two cases of particularly acute inflammation of the lingual tonsil-one in a case of glossitis, and the other in a case following injury from the lodgment of a tack in the sublingual sinus. In both instances the lymphoid tissue, circumvallate papillæ of the tongue, epiglottis, and posterior faucial pillar seemed to be involved. The writer has never seen an acute case which was confined entirely to the lingual tonsil. As above mentioned, suppuration and abscess may follow acute inflammation in this region, independently of disease of the neighbouring tissues.

Chronic Enlargement-Hypertrophy of the Lingual Tonsil.Chronic enlargement of the lingual tonsil is of more common 
occurrence; it sometimes projects sufficiently to come in contact with the lingual face of the epiglottis during acts of phonation. In such cases the circumvallate papillæ of the tongue will be found hypertrophied also, and generally there will be hypertrophy of the faucial tonsil, the follicular glands of the pharynx, and the lymphatic glands below. Some observers believe that chronic enlargement of the lingual tonsil is generally, if not always, associated with hypertrophy of the adenoid tissue at the vault of the pharynx. While this may be true in some cases, yet, according to the experience of the writer, hypertrophy of the lingual tonsil is more often associated with hypertrophy of the faucial tonsil or its surrounding glands than with the adenoid tissue at the vault of the pharynx (pharyngeal tonsil).

Symptomatology.-When there are any subjective symptoms at all denotive of this condition, they consist generally of sensations of constriction, or choking, or irritation, referred either to the back of the tongue or the anterior portion of the laryngeal region, accompanied or not by a distressing and persistent tickling cough. The writer had one patient under observation who suffered more or less from a harassing cough, sometimes associated with gagging, for a period of six or eight months. This patient had never shown any constitutional disturbance worthy of note during this time.

Foreign Bodies.-Foreign bodies or concretions are sometimes found in.the lingual tonsil, and may give rise to the same subjective and objective symptoms belonging to ordinary hypertrophy of the glands. (See chapter on Foreign Bodies.)

Treatment.-Acute inflammation will demand active local treatment. About the best local applications consist of pieces of ice, and frequent spraying with peroxide of hydrogen or menthol. Cocain may also be employed to allay pain. When suppuration has taken place, the pus should be promptly evacuated. Regarding enlargement of the lingual tonsil, great circumspection is necessary in every instance before concluding that a pathologic condition exists, for a great many individuals will appear to have, by laryngoscopic examination, an inordinate enlargement of the lingual tonsil, associated perhaps with hyperæmia, who will present no symptoms of disorder or discomfort; in which event it is obvious, upon due reflection and observation, that the condition is probably not really a 
pathologic one, but only an abnormal anatomical condition, so to speak. The writer's attention has been called in a number of instances to what seemed a diseased condition of the lingual tonsil in patients who were really suffering from disturbances of the gastro-intestinal canal or liver instead, and after judicious treatment directed toward the offending organs, the apparent anomalous appearance and symptoms referable to the lingual tonsil disappeared. Likewise, in cases of acute or subacute pharyngitis or pharyngo-laryngitis, the lingual tonsil may often be temporarily swollen as a concomitant of those affections. From such observations, therefore, one should not be too hasty in attributing such symptoms as cough, neurotic or gastro-intestinal disturbances, to this little isthmus of lymphoid tissue without due consideration. Notwithstanding all this, however, there are instances where chronic inflammation or hyperplasia (hypertrophy) of the lingual tonsil gives rise to considerable disturbance and distress, such, for example, as in the case already alluded to of persistent cough. Whenever a causal relation between this tissue and the subjective and objective symptoms is established, of course there can be no question about directing the principal treatment toward the lingual tonsil. For the purpose of reducing chronic inflammatory conditions, gargles and douches, composed of peroxide of hydrogen, carbolic acid, alum, muriate of ammonia, biborate of soda, emulsions of oil of eucalyptus, iodoform, aristol, or orthoform, will be highly useful. Sometimes the painting of the parts with a mixture of tincture of chloride of iron and glycerine, or a solution of nitrate of silver, will give good results. When, however, the affection is a true hypertrophy of sufficient magnitude to impinge upon the epiglottis or encroach upon the glosso-epiglottidean sinuses, then some escharotic should be employed, and for this purpose the galvano-cautery is probably the best. Several of the projecting points may be touched with the incandescent electrode at each sitting, and it may take but two or three sittings to satisfactorily accomplish the reduction of the enlarged glands. When the hypertrophy is of a soft, villous character, the curette, or the instrument of Myles or Chappell, may be used for removing the redundant tissue. This operation should be followed by an application or two of nitrate of silver, persulphate of iron, or sulphate of copper. When the parts are properly cocainized the operation usually 
causes little or no pain. Sometimes, when the manipulation has been too severe, considerable reactionary inflammation, and even œdema of the epiglottis, may follow.

\section{Chronic Laryngitis-Chronic Laryngeal Catarrh}

This disease consists of a chronic inflammation of the mucous membrane of the larynx, embracing its glandular, vascular, and connective tissue.

The several varieties included in the subject have been variously named and classified, according to different authors, as follows :

Simple or primary.

Secondary (to acute laryngitis).

Glandular.

Hypertrophic.

Upon a Clinical Basis:

Primary.

Secondary or consecutive (M. Mackenzie).

Traumatic.

Upon a Pathological Basis :

Simple or hyperæmic.

Hyperplastic (including edematous).

Hypertrophy $\left\{\begin{array}{l}\text { Superior, } \\ \text { Inferior, } \\ \text { Trachoma, } \\ \text { Pachydermia. }\end{array}\right.$

Atrophic.

Hemorrhagic.

Any one of these varieties may be secondary; consecutive, or traumatic. Indeed, many observers believe that all forms of chronic laryngitis are necessarily secondary occurrences.

Germain Sée, Bosworth, Jarvis, and many others, maintain that a chronic laryngeal catarrh is always associated with or preceded by disease of the nasal or naso-pharyngeal regions.

According to Bosworth, there is no such thing as primary chronic laryngitis. He believes that the disease always extends from the nasal or naso-pharyngeal region. John N. MacKenzie also says that "as an isolated affection it is rarely met with." Capart, Cohen, Roe, and others, while not taking so radical a stand on this question, entertain the opinion that it is generally 
associated with disease of the nose or nasopharynx; and so do the majority of authors. However, as the normal anatomico-physiological standard of the nasal and naso-pharyngeal mucous membrane is as yet undetermined upon scientific principles, I do not see how it is possible to argue this point to an exact conclusion.

So far as ordinary clinical observation goes, it must be admitted that we meet with many cases of simple chronic laryngeal catarrh which are entirely independent of disease of adjacent structures, just as we meet with isolated chronic disease of the tonsils which is in no way dependent for its occurrence or continuance upon conditions of the neighbouring parts. This apparent discrepancy of opinion can be accounted for undoubtedly, as suggested by A. H. Smith, Robinson, Langmaid, Cohen, and others, by the fact that a majority of the cases of localized chronic laryngitis are not met with by the specialist until advanced or complicated.

There are a great number of cases called chronic hypercemia or congestion, which properly should be considered as primary or "idiopathic" chronic laryngitis, although I am aware that many authors do not regard such cases as independent forms of disease. These cases are often associated with dyscrasiæ or perverted conditions of the liver, stomach, intestines, kidneys, heart, skin, or other organs; while some are associated with certain functional activities, such as menstruation. Frequently, in persons who use the voice extraordinarily, such as singers, public speakers, and the like, a permanent hyperæmia of the laryngeal membrane is met with, without much, if any, structural change. This class of cases deserves more definite consideration than has been usually accorded to it by the majority of writers.

Langmaid and Seiler, however, have called particular attention to an inflammatory state of the laryngeal mucous membrane observed in singers (a sort of hyperæmia), which they ascribe to faulty methods of using the voice.

Etiology.-Chronic laryngitis is due to a great variety of causes, operating either directly upon the larynx or indirectly through disease or functional disturbance of the economy elsewhere. The greater number of cases undoubtedly arise from previous acute inflammation or other injury to the part. 
Age.-While no age is exempt, by far the greater number of cases occur between the ages of eighteen and fifty. In childhood the acute form of the disease is most frequently met with, especially in connection with croup, diphtheria, scarlet fever, measles, cerebro-spinal meningitis, etc., yet the chronic form is not uncommon as a consequence of the acute affection and as a sequence of the diseases mentioned above.

Michael states that in nearly all the acute infectious diseases of children the larynx is involved.

Boys at the age of puberty, owing to the physiological changes occurring then, are susceptible to chronic laryngeal catarrh (Cohen, von Ziemssen, M. Mackenzie, Mulhall).

Bergeron, after describing the changes which take place in the anatomy of the larynx at puberty, says that, since at this time the organ is in a state of congestion which produces hypernutrition, any pathological condition existing before puberty is apt to persist after this epoch. When met with in old age it is usually due to the vascular and glandular changes incident to senility. It is often associated with chronic bronchial catarrh, and is then often mistaken for laryngeal phthisis.

Sex.-Males have heretofore been considered more liable than females. This has been accounted for by the nature of the habits and occupations of the former. However, modern times have opened up so many hitherto masculine avenues of occupation for women, and furnished so many overheated and badly ventilated offices, stores, and houses, that we meet with a proportional increase in the number of cases in females. The atrophic form, however, is more often found in females than in males.

Occupation.-For many years clergymen and other public orators, singers, actors, street criers, sailors, and such as use the voice extraordinarily, have been considered especially liable to this disease. The reason for it is obvious, even setting aside surrounding circumstances; for any organ of the body upon or about which undue functional energy is demanded will be prone to suffer to a greater or lesser degree, especially when used under particular circumstances, for it is but a step from a physiological to a pathological state of vascularity.

The forms most frequently met with in these persons are the hyperamic and the hyperplastic. However, any of the other 
forms (hypertrophic, atrophic, or hemorrhagic) may be met with.

Persons who work in dust or smoke, such as blacksmiths, stone-cutters, broom-makers, employees of knitting factories, and furriers; saloon-keepers, who are constantly subjected to the inhalation of air vitiated with human emanations and tobacco-smoke; brewers, butchers, and ice-men, who are frequently subjected to sudden changes of temperature by going in and out of ice-boxes or cold vaults; teamsters and street-car conductors, who are exposed to the inclemencies of weather and also to street dust, are each especially liable to acquire this disease. Regarding street dust, however, Dr. J. N. MacKenzie thinks that there is too much stress laid upon it as an etiological factor, while Miguel and others believe street dust to be frequently a cause of the disease.

Luigi Manfredi, who examined the street dust of Naples, found that the number of microbes of all sorts to the gramme amounted on the average to 761,521,000. Remarkable differences were disclosed in the proportion of microbes to dust in different quarters of the city, varying from $10,000,000$ to 5,000 ,000,000 per gramme. Many species of these micro-organisms he found by experiment to be pathogenic. Although little importance may be placed upon the special agency of microbes, yet it is obvious that the inhalation of an undue quantity of dust, more than can be strained out by the nasal passages, must exert a baneful irritating influence upon the mucous membrane of the larynx, where much of it will be deposited. It is not necessary, from this point of view, that nasal or nasopharyngeal disease should pre-exist.

Book-keepers who use the voice considerably (calling off) in overheated offices, and who perhaps sleep in cool rooms, often suffer from chronic laryngitis.

Habits. - The habits and mode of life certainly have much to do with the production and continuance of this affection.

Improper eating and drinking, such as taking large quantities of cold water in close alternation with hot liquids or food, will often exert a deleterious effect upon the larynx independently of any pharyngeal complication.

The abuse of alcoholic liquors is a prolific source of chronic laryngeal catarrh. In fact, there are few immoderate drinkers 
who do not suffer more or less from this disease. The pachydermic form is ascribed to drinkers by some writers.

The habit of smoking tobacco is a very common exciting cause. There are, indeed, very few persons who are addicted to any great degree to this habit who do not show with the laryngoscope more or less hyperæmia or inflammation of the laryngeal mucous membrane.

Other diseases and conditions may give rise to it. Frequent attacks of acute laryngitis (colds) in persons who use the voice much, invariably lead to the chronic form of the disease. Hence public singers and actors, being especially liable to attacks of acute laryngitis on account of their unavoidable exposure in cold theatres, or in travelling, and to other vicissitudes attending their profession, while at the same time being under the necessity of using the voice during the course of such acute attacks, are frequently the subjects of chronic laryngitis.

Naso-pharyngeal and Nasal Diseases. - We have already spoken of the pronounced opinions held regarding these diseases as factors in the etiology of chronic laryngeal catarrh. Whether nasal disease ever leads to such structural changes as characterize the hypertrophic, atrophic, and pachydermatous forms is a question; but that they may be associated is of course a fact. Many observers believe that the atrophic form is always an extension of pharyngitis sicca.

That enlargement of the pharyngeal tonsil or other disease of the adenoid tissue at the pharyngeal vault, by causing mouth-breathing, will in time bring about a catarrhal inflammation of the larynx, as well as of the pharynx, is well supported by ample experience.

Heredity.-A few observers recognise a catarrhal diathesis (Beverly Robinson, Boucheron, Cohen), while many, among them MacKenzie and B. Fränkel, do not think such a particular tendency exists, or that there is sufficient evidence of a socalled catarrhal diathesis.

Although it would be extremely difficult to describe with exactness a catarrhal diathesis, yet it is a matter of almost common observation that members of certain families are usually susceptible to diseases of the mucous membranes independently of prevailing exciting causes. I am familiar with families who show such a weakness. In one of these (consist- 
ing of five), whose members were noted for their good voices, and who were otherwise healthy, not one was able to continue the cultivation of the voice up to an artistic point, on account of the supervention of chronic laryngeal catarrh, notwithstanding all other circumstances were favourable. I am sure that due consideration of such observations would lead one to the conclusion that there is a predisposition in certain individuals to diseases of the mucous membranes, which cannot be explained upon any other hypothesis than that of hereditary proclivity. Such individuals are often classed with those inheriting a so-called strumous or tuberculous tendency, but unjustly so, it seems to me, inasmuch as they are not peculiarly liable to tubercular affections. Only a few years ago some observers (Ziemssen, Cohen, and others) looked upon long-continued chronic laryngeal catarrh, especially if the sputum contained much pus, as in many instances a primary tuberculous stage.

In this connection we would refer to the statements made by several writers, that chronic laryngeal catarrh sometimes occurs from the irritation or infection resulting from the presence in the larynx of more or less secretion which has dropped down from the nasopharynx. The explanation given is about as follows: The vestibule of the larynx being generally open, and the lower pharynx and esophagus closed, any redundancy of secretion above would naturally flow gently down into the open laryngeal vestibule and accumulate there for a time until ejected by cough, the collection taking place mostly during sleep.

However plausible this theory may appear at first sight, it seems fallacious when we consider that the larynx is not so constructed as to receive and hold much mucus or secretion which is not of its own production, without causing decided interference with quiet respiration ; furthermore, it is well known by physiologists that the esophagus automatically opens, or performs the act of glutition, even during sleep, if the accumulation be sufficient to make such a movement necessary.

Enlarged glands at the base of the tongue sometimes lead to chronic laryngeal catarrh (Cohen, Swain), but probably not often. By paying particular attention to this locality in all laryngeal examinations, one will be astonished at the number of individuals who have apparently enlarged glands at the base of the tongue (lingual tonsil) without showing any sign of lar- 
yngeal disease. One will therefore soon become convinced that the pathological import of such formation has been exaggerated. Great care must be exercised also not to overlook a low-lying tonsillar lobe (Allen), which may or may not constitute the source of irritation.

Chronic tonsillar disease (hypertrophic-follicular) is sometimes a cause of this affection. The mechanical irritation of an elongated uvula has been mentioned as one of the causes of chronic laryngitis by Beverly Robinson, Gottstein, and others. While this may be true of a few cases, the absence of such a condition in many cases of laryngeal disease would seem to lessen its importance as a causal factor.

Impaired cardiac power (Beverly Robinson) will sometimes induce a chronic or passive congestion of the larynx, especially in a subject who has been using the voice much.

Dyspepsia and indigestion are frequent precursors of chronic laryngeal catarrh, also intestinal irritation and hemorrhoids, especially in tobacco-smokers and nervous persons, the disease under such conditions probably originating through the mechanism belonging to the pneumogastric nerves. It is a fact well known to laryngologists that few people who suffer for any length of time from gastric derangement fail to exhibit in the laryngoscopic mirror a certain degree, at least, of hyperæmia of the laryngeal mucous membrane.

Acute desquamative nephritis frequently gives rise to chronic laryngitis, either the simple form, or more often the edematous form, the swelling in this case being a veritable hyperplasia which remains for a considerable time, and not a simple œdema such as is often observed in the course of chronic Bright's disease.

Ovarian and uterine disease frequently cause chronic laryngeal catarrh, usually of the simple variety, by first producing muscular spasm and congestion, then hyperplasia, and sometimes hypertrophy, as in the case of a woman with ovarian disease, who came under my observation recently at Harper Hospital, Detroit, who had marked subglottic hypertrophy, and who suffered with so much respiratory obstruction as to cause serious paroxysms of dyspnœa, requiring oxygen inhalations on several occasions, and finally leading to preparations for the performance of tracheotomy. She slowly improved, however, under iodine and iodide of potassium up to a certain 
point, when the ovaries and tubes were removed by Dr. J. H. Carstens, with the result of producing rapid and effectual relief. A few days after the operation her voice returned and the dyspnœa became almost unnoticeable. Just before leaving the hospital, laryngoscopic examination showed the hypertrophy to have greatly subsided. Her voice was tolerably good, although hoarse, and her respiration comparatively easy.

Reflex action and structural nervous diseases affecting other parts of the throat, or the body generally, are frequently causal factors. Without doubt the intimate relationship between the pharynx and the larynx is due to nervous influence rather than to continuity of structure, as illustrated by the effect of a drink of water in allaying laryngeal irritation during the exercise of the voice (Cohen). The water, of course, does not enter the larynx, but relieves the voice by its effect on the pharyngeal nerves. Likewise the influence of distant or neighbouring pathological conditions may affect the larynx.

Chronic congestion leading to structural change may be produced by improper use of the voice, as already mentioned (Seiler and Langmaid). Likewise excessive use of the voice, instead of directly inducing local disease, may lead to uterine disease first, according to Wing, thus demonstrating the truth of the oft-repeated observation that reflex action may play the most important part in pathological processes, especially when the ganglionic nervous system is concerned.

Frequent glottic spasm from lumbricoid worms, leading afterward to chronic laryngeal catarrh, has been reported by M. Maselli. Abnormally continuous tension of the vocal cords (Hooper), or extreme debility from excessive use of the voice (Langmaid), may also directly lead to chronic laryngeal catarrh. Often structural nervous diseases, such as bulbo-nuclear disease and affections of the cortex, produce this affection (John $\mathrm{N}$. MacKenzie, Krause, Delavan, J. Garel).

Although the cortical centre for the larynx is still a matter of discussion, yet it is observed that brain disease may induce chronic laryngitis before paralysis takes place. Neurasthenia and vasomotor disturbances (Daly), ataxia (Scherschewsky), and other diseases of the spinal cord, may be immediate causes. Direct as well as indirect irritation of the recurrent nerves (Livon, Hooper), as in a case of pressure by lymphatic glands, 
cited by Gouguenheim, will produce it. Diseases of the ear of a chronic nature may produce the disease reflexly.

Many other nervous disorders, such as hysteria and chorea, give rise to it; so also may perverted mental function, such as melancholia or even severe grief (Isambert). Hemiplegia and like affections favour its occurrence by allowing the entrance of mucus, salivary secretion, and particles of food, which the larynx, from deficient excito-motor sensibility, fails to eject.

Erysipelas frequently causes this affection by inducing an acute œdema, which is only partially removed by absorption, thus leaving the submucous tissues swollen, and constituting the chronic edematous variety (Delavan, Fred. Massei). The exanthemata, scarlet fever, measles, etc., are prolific causes, not only in children, as before mentioned, but also in adults (Moore, Daly, Milsonneau, and B. Barth). Non-infectious cutaneous diseases may also give rise to it; instances are recorded of chronic laryngeal catarrh following pemphigus, urticaria (Mackenzie, Isambert), and herpes (B. Fischer, Schnitzler, S. H. Chapman, G. Herzog).

Rheumatism and lithæmia may also cause it, especially the former disease, when affecting the arytenoid joints or the laryngeal muscles. Chomel pointed out this relationship years ago. It will be found in practice, however, that persons who are subject to muscular rheumatism, or so-called neuralgia or rheumatic phlegmonous sore throat (A. Mantle, Isambert), are often afflicted with a chronic laryngitis. Laranza and Ingals have not been able to find a single case of rheumatism affecting the laryngeal muscles, however. The disease is exceedingly infrequent in subjects of acute articular rheumatism. Ingals, in a paper read before the Ninth International Medical Congress, cited three recorded examples of an acute form of laryngitis in connection with articular rheumatism, and Elsberg reported a case affecting the crico-arytenoid joint. Ingals further says that he can find no mention in medical literature of a chronic rheumatic laryngitis, but he clearly establishes the characteristics of such an affection. Fauvel and Moure have demonstrated in their clinics a number of rheumatic manifestations in cases of chronic laryngeal catarrh, which they seem to regard, however, as of rather secondary importance. Vierordt cited a case in which there was a combination of goitre and 
chronic hypertrophic laryngeal catarrh, the latter affection getting better as the goitre grew worse.

Small-pox and typhoid fever are very apt to leave the patient with a chronic laryngeal catarrh independently of perichondritis. Schiffers and Dr. Paul Koch have given examples of these disastrous results in connection with chronic laryngitis in such cases. C. Gerhardt reports a case of typhoid fever with striking laryngeal inflammation and ulceration, which he denominates "laryngo-typhoid." He believes such cases occur oftener than is supposed, and that they are overlooked.

Phlegmonous or suppurative inflammation of the larynx very generally leads to a chronic inflammation of longer or shorter duration. Moure and others find in cases of this nature a great similarity to phthisis. In a case of primary phlegmonous inflammation of the right ventricular band, reported by the author in 1879 , there followed for a long time a chronic laryngeal catarrh. Scorbutus and malarial toxæmia may cause the hemorrhagic variety of the disease.

Chronic inflammation of the mucous membrane of greater or lesser extent may follow any direct injury to the larynx. Very severe injuries, however, are usually followed by death, persons often dying suddenly from a blow over the larynx (Marschka). I believe there are only three or four cases on record of recovery after fracture of the cricoid cartilage.

Chronic inflammation succeeding these injuries is usually characterized by more or less extravasation of blood and œdema, subsequently hyperplasia and hypertrophy. But exceptionally a severe injury may leave little or no chronic inflammation.

If contusions or concussions leave any chronic change of the laryngeal mucous membrane, it is usually a general thickening or swelling accompanied by more or less disability in phonation, even amounting to aphonia. The colour of the mucous membrane is not usually an intense red, but more of a grayishred tint. An interesting case of chronic laryngeal catarrh with complete aphonia occurring after an injury has been reported by Packard and one by Hopper.

Symptomatology.-Subjective. The early symptoms of primary chronic laryngitis are usually not well marked, because of the insidious approach of the disease. A sense of fulness, stiffness, tickling, or itching, referred to the larynx, and accom- 
panied by acts of hemming and hawking, constitute the principal early signs. If the person be a public speaker, singer, or in any other way accustomed to extra vocal activity, these symptoms will be more marked and progressive, and there will soon be added more or less hoarseness and hacking cough, especially after vocal effort. These uncomfortable sensations increase in duration. and frequency, until efforts at clearing the throat become necessary perhaps during prolonged conversation, with or without slight coughing or hawking. The voice becomes hoarse and less reliable for prolonged or extra effort, and after a while is "clear" only in the early morning or after a period of rest, while the hacking cough becomes more or less a permanent feature.

Some singers in a moderately advanced stage of the disease show no hoarseness during conversation, but only at the beginning of the singing act, while others are hoarse during conversation, but not after "getting started" at singing (Langmaid). This observation applies also to actors and public speakers. Hoarseness is an exceedingly variable symptom, and, strange to say, does not always represent the extent or gravity of the pathological condition; for instance, a very general involvement and thickening of the laryngeal mucous membrane in one case will give rise to but moderate hoarseness, while a lesser degree of structural change in another case may almost, if not quite, annihilate the voice.

This discrepancy has been accounted for in different ways by authors. It has been attributed to dryness or want of lubrication of the surface of the vocal cords by Sajous, to interfibrillar thickening in the vocal muscles by Cohen and Lennox Browne, to peripheral nerve pressure by Livon, Moure, and others, and to mechanical interference from hyperplasia by Pipping and Brissard. Undoubtedly all of these explanations are in the main correct, but apply to particular cases, so that no one explanation will meet all cases. The character of the individual, the extent, nature, and part particularly involved, will generally account for the amount of functional disturbance exhibited ; for instance, a thickening at the interarytenoid incisure or at the edges of the vocal cords may prevent their approximation and result in great vocal disability, so that the further course of the disease will be marked by relatively greater hoarseness and more decided difficulty in voice pro- 
duction, requiring greater expiratory effort, while perhaps pathological change about the epiglottis will be marked by an increase of cough.

In advanced stages the aphonia may be complete, or there may be a brassy whisper broken by discordant raspy sounds. In this instance there is much complaint of fatigue, referred to the chest and epigastrium, and sometimes to the muscles of the back, due undoubtedly to the extra tax imposed upon the respiratory muscles by forced expiration. The patient may also complain of pain in the larynx during vocal efforts, which induces a disposition to avoid speaking. There is usually little or no odynphagia. Respiration is not very much embarrassed unless there be considerable thickening of the mucous membrane or lack of movement of the vocal cords, such as takes place in the edematous and hypertrophic varieties. In these forms the obstruction to respiration is sometimes dangerous. It is usually not so alarming as in acute edematous laryngitis, because of the slower approach of the local condition in the chronic form of the disease. In a case of chronic edematous laryngitis following small-pox and affecting principally the epiglottis, which I saw some years ago, the patient would experience no suffocative attacks if he held his tongue forward out of the mouth. Occasionally there may be considerable pain of the larynx or neck complained of, sometimes of a lancinating character. This may be paroxysmal, like neuralgia, or continuous; it is observed especially in the hypertrophic form.

In the edematous variety there is generally more or less difficulty of swallowing, especially of liquids, owing to the imperfect closure of the epiglottis; there is also considerable dyspnoa on exertion. The expectoration is at first a little glairy mucus, changing later on to muco-pus, and increasing in quantity to perhaps two ounces or more daily. As the secretion increases the cough may be more harassing.

In the atrophic form the principal complaint is of a dry, burning, tickling, or pricking sensation, with a constant desire to cough, especially after general exercise or after having walked some distance facing the wind. There are also in many cases suffocative attacks, coming on mostly during the night or in the early morning, and simulating spasmodic croup or asthma.

The cough is usually of a wheezy or whistling character, 
and occasionally spasmodic, similar to hooping-cough. The writer saw a case in an adult which was supposed, from the character of the cough, to be an unusually prolonged case of hooping-cough, but laryngoscopic examination revealed at once the nature of the difficulty-an atrophic laryngitis. There may be little or no expectoration in this form, but when the patient can expectorate more freely it affords a sense of relief.

The hemorrhagic form is characterized by the expectoration of blood and bloody mucus. The patient can very often predict from his sensations the onset of a hemorrhage. While there is seldom complaint of pain, there is always complaint of fulness and stuffiness of the larynx, with a sense of something foreign in the organ.

Pachydermia laryngis (warty formation), first described by Virchow, manifests few particular subjective signs, except the persistence of dyspnœa, due to impaired abduction of the vocal bands, and a continual, regular hoarseness, with sometimes odynphagia. The evenness of the hoarseness is quite remarkable. There are no general symptoms especially belonging to this condition, except such as go with the clinical history of alcoholism or syphilis, to which it is often related. The constitutional disturbance depends largely upon the amount of dyspnœea present, which is sometimes alarming. Chiari and others believe the affection to be a result of syphilitic infection, but Virchow, Meyer, and Bergengruin consider it due to the irritation from chronic catarrhal inflammation of the mucous membrane, and, like trachoma, partly the result of friction between the opposite nodular edges of the vocal bands. There have been several cases reported since Virchow's publication on the subject, the observations on which seem to agree in the main.

Laryngoscopic Appearances. Hyperæmia.-This may be general throughout the whole mucous lining of the larynx, or may be confined to the vestibule, the epiglottis, the arytenoid region, the ary-epiglottic folds, or the vocal or ventricular bands. Dr. Price Brown has reported an interesting case of chronic laryngitis confined to the posterior portion of the larynx.

When hyperæmia is partial it is commonly seen in the order of frequency specified above, with the exception, perhaps, that in vocal artists the vocal cords, especially their posterior 
half or free edges, may be the only part displaying abnormal colour. The colour, although heightened, varies very much in different cases, from a deep red to a pinkish or brownish red; while the parts normally appearing white or gray in the laryngeal image (such as the vocal bands) may be pink or pinkish red in streaks, or of a mottled brown or slaty-gray colour (see Plate II, Fig. 6, showing normal colour of larynx).

Although hyperæmia is due to increased vascularity, enlarged blood-vessels are seldom seen, except over the epiglottis.

There may be little extra secretion visible. The surface is usually smooth, and the image may convey an idea either of general moisture or of dryness. In many cases swelling or thickening of the mucous membrane is not apparent (Elsberg, Hooper, Langmaid, Jarvis, Gottstein).

Laryngeal examination from day to day shows great variation of colour independently of treatment, especially in nervous individuals and in persons who use the voice much.

The laryngoscopic appearance in chronic hyperæmia may be accompanied by corresponding subjective symptoms. Indeed, many actors showing this condition are not debarred from the exercise of their voice upon the stage. In other persons, also, we are often astonished to see a notable chronic hyperæmia without much, if any, hoarseness or other uncomfortable symptoms.

Hyperplastic Form.-In this variety the laryngoscope reveals not only hyperæmia, but also a structural change of the mucous membrane, more or less general, according to the extent of the disease, and varying from a slight thickening to a sodden, swollen state simulating œdema (see Fig. I04).

These changes may be quite general, or confined to any one or more of the regions of the laryngeal cavity (see Figs. I05 and 106). The simpler degree is usually limited to the epiglottis, arytenoid region, and ary-epiglottic folds, but may appear saliently in one or both vocal or ventricular bands. Unlike simple hyperæmia, the blood-vessels are prominent, especially over the epiglottis, while in some cases the veins show distinctly, constituting the phlebectasis laryngea described by Mackenzie.

The severer degree shows more marked textural change than mere thickening, for the surface may appear granular 
(chronic laryngitis granulosa), or the posterior wall of the larynx only may be prominent and uneven, as if the exudate had been deposited and organized in layers one upon the other, or as broad projecting papillæ (see Fig. 107).

The vocal bands may appear round or very broad, with roughened surface, while the edges seem loose or longitudinally creased, as if folded up, or show thinnish prolongations, as if fringed on their under surface. Although ulceration or erosion is rare, yet it may take place. Fig. I08, from Türck, shows a case of erosion occurring in the course of an attack of primary syphilis.

The ventricular bands, one or both, may be so swollen as almost to obscure the vocal bands, appearing sodden, of darkred colour, and almost, if not quite, obliterating the appearance of a ventricular space (Fig. I09). One or both arytenoid projections may show marked enlargement with a granular surface.

There is always excessive secretion apparent, either thin or thick in consistence, sometimes enough to form bridges or threads hanging between the vocal or ventricular bands, or appearing as small clumps plastered to the roughened posterior wall, or as stringy masses depending between the arytenoids, or hanging to the ventricular bands, or profusely bathing the arytenoids (Fig. I ro).

Often there is also a collection to be seen below the glottis, sticking to the tracheal wall. The interarytenoid space seems to be a favourite location for the collection of the secretion, for it will be seen in this situation oftener than elsewhere. The mobility of the arytenoids is greatly lessened, and sometimes almost obliterated, simulating paralysis (Fig. I I I).

Edematous Form.-The picture of the so-called chronic edematous laryngeal catarrh differs considerably from the foregoing, in that the swelling is more pronounced and of a dark yellowish or grayish colour, marked by enlarged bloodvessels upon the surface; sometimes these are so numerous and prominent as to give a pinkish or reddish hue to the swelling. This form usually follows an injury when not connected with syphilis. The epiglottis, arytenoids, and ary-epiglottic folds are more often affected, although in many instances either the epiglottis or one or other of the arytenoids or aryepiglottic folds is alone involved in the swelling (Fig. 104). 


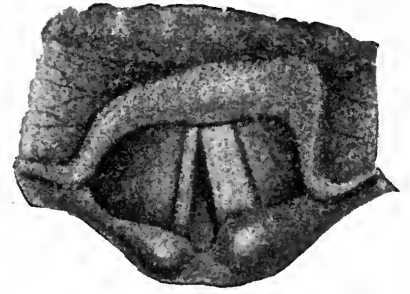

FIG. I04.-Edema of the glottisunilateral (Burow).

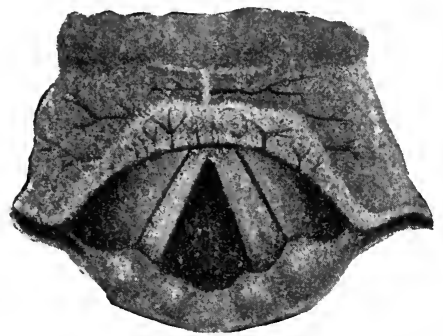

FIG. 105.-Chronic laryngeal catarrh, affecting principally the vocal cord on the left side (Burow).

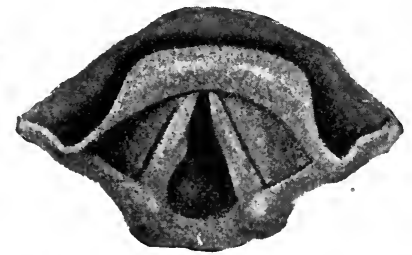

FIG. I06.-Chronic laryngeal catarrh, showing circumscribed swelling of one vocal cord (Burow).
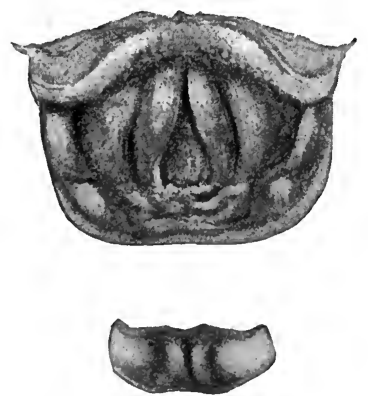

FIG. 107.-Chronic inflammation of the vocal cords, with swelling of the mucous membrane of the posterior wall of the larynx (Türck).

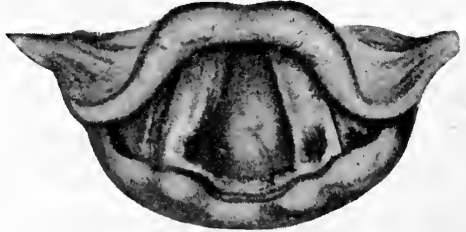

Fig. I08.-Ulcers of the vocal cords, probably catarrhal in their nature, although coincident with a primary syphilitic ulcer of the genital organs (Türck).

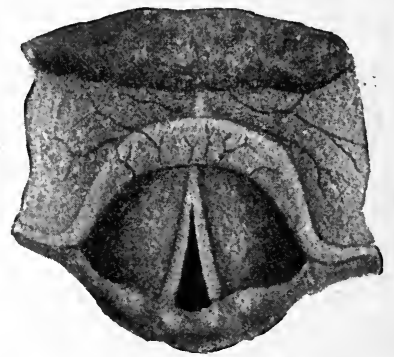

FIG. I09. - Chronic laryngeal catarrh, with swelling of the ventricular bands.

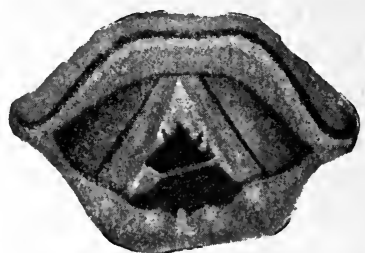

Fig. IIo.-Chronic laryngeal catarrh, with thickened mucus adhering to the vocal cords (Burow).

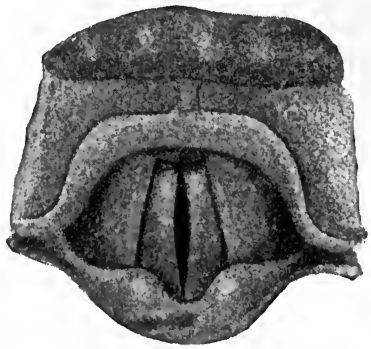

FIG. III.-Chronic laryngeal catarrh, with paresis of vocal cords (Burow). 


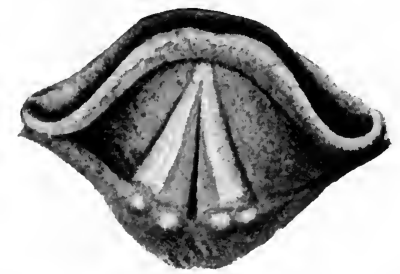

FIG. I12.-Chorditis vocalis hypertrophica superior (Burow).

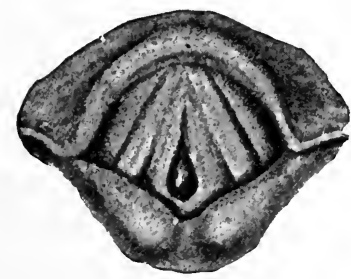

FIG. II3.-Chorditis vocalis hypertrophica inferior (Burow).

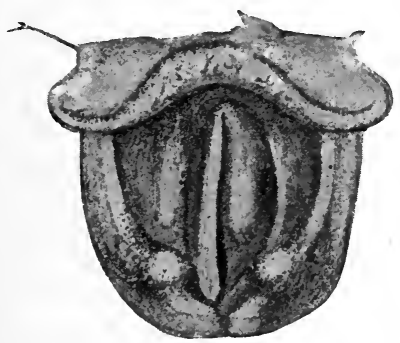

FIG. II4. - Perichondritis of the left half of the cricoid cartilage with unopened abscess (Türck).

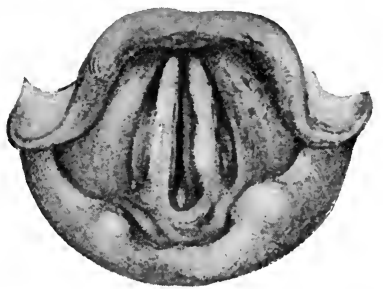

FIG. I15. - Perichondritis of the posterior part and left half of the cricoid cartilage (Türck).

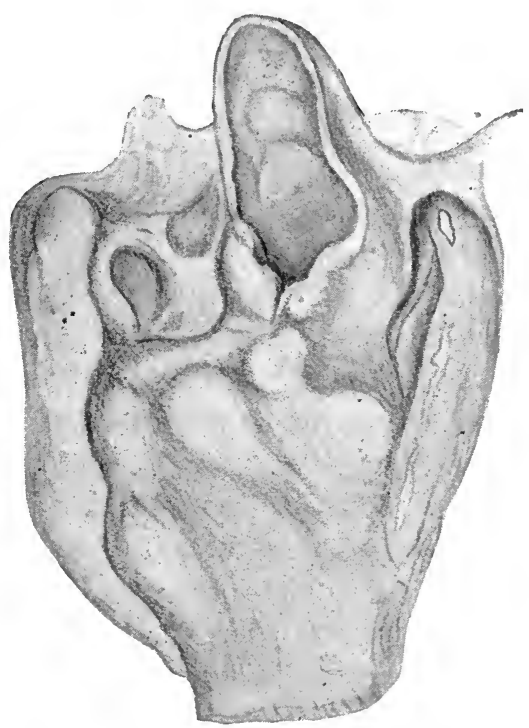

FIG. I I6.-Idiopathic perichondritis (Türck).

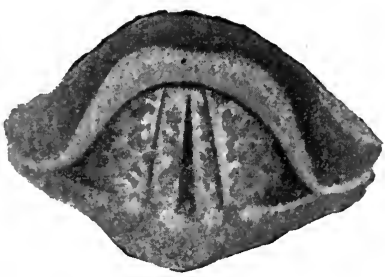

FIG. II7.-Hemorrhagic laryngitis (Burow). 
The swelling differs from acute œedema by its greater firmness to the touch, lesser prominence, and darker colour. There is less glistening and more vascularity of the surface. When the swelling is considerable it is almost impossible to get a view of the underlying region, such as the ventricular or vocal bands, especially in cases where the mobility of the arytenoids is almost entirely lost.

Hypertrophic Form.-There are two principal forms of hypertrophic laryngitis, viz., superior (Fig. I12) or general, and inferior (Fig. II3); besides these there are the nodular proliferations called trachoma, and the warty formation, pachydermia, which are really localized hypertrophies.

The former presents a laryngoscopic image similar to that of chronic œdema (Fig. I04), except that the surface is not very vascular, but is very uneven, presenting ridges, projections, furrows, or prominences differing in colour one from the other, at the same time entirely effacing the natural contour and appearance of the parts. The swellings may be circumscribed, somewhat simulating tumours.

Occasionally the thickening may involve pretty nearly the whole laryngeal lining, but sometimes only one of the ventricular bands or ary-epiglottic folds or the interarytenoid region may be the seat of the change. When secondary, however, to some other disease, as, for instance, perichondritis, it will be limited to the situation of the early lesion (Figs. I I4 and I I5). The "chorditis tuberosa" of Türck, or "trachoma of the vocal cords" of Morell Mackenzie, is an instance of localized hypertrophy confined to the mucous glands of the vocal cords. These appear as white spots projecting from along the middle portion of the vocal bands surrounded by small patches of congestion. They usually appear in cases of chronic laryngitis of long standing in singers and public speakers.

Pachydermia Laryngis. - The laryngeal image shows a sort of non-vascular warty projection of dark-gray or yellowish hue, rough or laminated, issuing from the posterior section of the upper or inner side of the vocal bands. The growths are usually bilateral, and situated opposite each other when affecting both cords (Plate II, Fig. I). The laryngeal mucous membrane is generally hyperæmic and thickened, and sometimes there is also subglottic hypertrophy. The vocal bands are of a dark-red colour throughout their extent, excep- 
tionally dark gray with red lines running longitudinally across them.

Chronic Laryngitis Hypertrophica Inferior.-The laryngoscopic image is quite striking from the appearance of grayish rubber-like rolls situated just beneath the vocal cords and moving somewhat with them. Although seeming to be attached, the swelling is most marked toward the posterior portion of the vocal bands, and apparently extends to the posterior wall of the larynx (Fig. I I3). The swelling is usually covered by inspissated secretion looking like scabs, of a greenish or grayish tint; and not easily removed by acts of hawking or coughing. During a brisk inspiration the swelling is brought to view, and then it is seen to be a scroll-shaped growth beneath the vocal bands, and continuous with their under surface.

Hemorrhagic Form.-The laryngeal appearance may show three local conditions: vaso-motor turgescence, extravasation, and possibly a lesion of the surface.

In the first the whole laryngeal mucous membrane, including the vocal bands, is in a state of high vascular turgescence, and blood issues from numerous spots, giving the characteristic mottled appearance to the upper surface of the ventricular bands, epiglottis, or vocal bands (Fig. II7). These darkish, irregular spots mark the situation of small clots.

Sometimes, as in typhus fever and scorbutus, there seems to be a general transudation of a bloody serum from numerous papular red patches or areas in different parts of the larynx. This liquid does not coagulate easily, but freely bathes the membrane, collecting here and there in drops along the shelving projections of the vocal and ventricular bands.

In other instances the hemorrhage is quite localized, so that the blood may be seen oozing or trickling from its source of exit over the neighbouring surface. Clinton Wagner reports a very interesting observation of this sort, where the blood could be seen oozing in little drops from the left ventricular band or ventricle.

When erosion, or ulceration, or rupture of the membrane has taken place-which, of course, is not common-it will be indicated by the nature and quantity of the blood poured out, if the seat of lesion be out of direct view.

When the hemorrhage has taken place beneath the surface 
-extravasation-it will be indicated by dark blotches, over which the surface will be slightly raised. This is not an infrequent occurrence in typhus and typhoid fevers, scorbutus, and other dyscrasiæ. The extravasation is sometimes quite localized. A. H. Smith, C. E. Bean, Lennox Browne, and Schnitzler have reported cases in which the hemorrhage occurred in one of the vocal bands only.

Clots are often seen in the larynx from hemorrhage of the bronchi or lungs. In such cases the clots will be found most often clinging loosely to the edges of the ventricular or vocal bands in dangling shreds, while similar clots may be seen along the walls of the trachea. Sometimes, however, after a severe hæmoptysis the upper surface of the ventricular and vocal bands will be found well covered with tolerably firm coagula.

A laryngeal hemorrhage, as Rethi says, may not always be associated with an inflammation, especially when occurring during menstruation, or when affecting the so-called bleeders (Lennox Browne).

The laryngeal appearance in the atrophic form is characteristic. In the first place, the laryngeal opening is large. The ventricular and vocal bands are thin, the former looking rather paler than natural, and glistening. Small scales of dried secretion may be observed on the vocal bands, or clinging about the interarytenoid region, even down to the posterior wall of the trachea or below (Fig. I10). There may be a fetid odour to these scales.

Pathology.-Chronic catarrhal inflammation of the larynx, although generally believed to be a sequence of the acute form, may come on so insidiously as never to have shown the pathological steps of an acute inflammation. Green says that "chronic catarrhal inflammations of mucous membranes differ from the acute, inasmuch as the subepithelial connective tissue is often extensively infiltrated with small cells, which may ultimately form an imperfectly fibrillated structure."

Delafield and Prudden, speaking of chronic catarrhal inflammation, say: "This form of inflammation is regularly attended with structural changes which are evident after death. But yet these changes are not always in proportion to the symptoms observed during life. The excessive production of mucus and pus which exists during life is not necessarily at- 
tended with marked changes in the mucous glands or bloodvessels."

Payne says: "Chronic inflammation may begin suddenly or rapidly, like the acute form, or else may come on insidiously and gradually, but differs in this, that, instead of undergoing spontaneous decline, it will remain for a long time at a certain level of intensity, neither getting worse nor entirely going away, like a curve approaching its asymptote. It is obvious that the distinction is one of degree, and that the line is sometimes very difficult to draw. Chronic inflammation in general shows less hyperæmia with its attendant phenomena, while the exudation, cell migration, and possibly other tissue changes, remain."

Although these general descriptions very aptly apply to many cases of idiopathic chronic laryngeal catarrh, yet its course and effects establish several distinct morbid states, which are probably due to the nature of the region or organ involved, and which need special attention. Thus we meet with chronic hyperæmia without much, if any, structural change, which may continue for an indefinite time. This is especially met with in public speakers and singers. It undoubtedly depends upon an extra vascularity not wholly transcending the physiological state. In time it ought to lead to a pathological state-hyperplasia; yet this does not always happen, if the patient be duly careful of his general physical welfare.

I have met with several such cases. One in particular, which I will mention, was in the person of an actor whom I had under observation from time to time during a period of about fifteen years. Although his larynx always presented the appearance of hyperæmia, there was never laryngoscopic evidence of hyperplasia to any extent, excepting during acute exacerbations.

Hyperplasia.-The thickening is usually due to exudation in the submucosa and enlargement of the blood-vessels, with proliferation of connective-tissue elements. These changes may be general, or confined to certain portions of the larynx. The exudate may be so little or be so evenly distributed as to cause little alteration of the physical characters of the membrane, or its absorption so partial as to leave the texture nodular and uneven. 
Edema, so called, is not a serous effusion in chronic laryngitis, as it is in the acute disease, but a veritable hyperplasia undergoing fibrillar changes. The latter, however, becomes gradually absorbed, leaving the other elements to either break down or become organized, thus constituting hypertrophy. Any continued strangulation of the vessels or extra formation of fibrous tissue will suffice to alter these conditions and lead to contraction and atrophy.

Chronic edematous laryngitis is often incident to deep. seated inflammations, such as perichondritis, or semi-chronic suppurative inflammations.

In laryngitis hypertrophica inferior the whole glandular apparatus is very much enlarged and changed, while the perivascular spaces are more or less occupied with new tissue or new vessels. The secretion in this form is also altered. In the condition known as trachoma, or chorditis vocalis tuberosa, there is at first granular (follicular). hyperplasia, and afterward epithelial proliferation. Rice believes this condition to be identical with pachydermia, and he, with others, thinks it is in some way connected with the inferior hypertrophic form.

Pachydermia.-This condition was first described by Virchow, who recognised two forms, one of a syphilitic nature, the other the result of simple chronic inflammation. Dr. Julius Sommerbrodt and Dr. Rheine state that this process undoubtedly occurs under the influence of a chronic catarrhal inflammation in which a thickening of the epithelial layer takes place.

Gottstein speaks of a verrucous and a diffuse form. Under the latter he evidently includes trachoma of the vocal bands, and he is supported in this by Rice. This peculiar formation is accounted for by some (Schrötter) upon the same theory as that of the origin of trachoma of the vocal bands-viz., friction between the opposite edges after nodules have been formed. This, it seems to me, is a more plausible explanation of the origin of pachydermia than of trachoma. It is reasonable to suppose that a small projection or two in the region of the posterior vocal processes would result in further growth by continual irritation from friction, on account -of the great mobility of these parts during phonation.

Chorditis Vocalis Tuberosa of Türck (Trachoma of Mackenzie).-Ziemssen and others consider this condition a "der- 
moid metamorphosis"; and Gougoenheim believes all these cases to be very much the same as papillary development elsewhere. Rice has found chorditis tuberosa associated with subglottic hypertrophy.

S. Gross contends that some cases reported as hypertrophic inflammation are instances of primary "laryngoscleroma," as can be demonstrated by microscopical examination showing the presence of the bacilli of ordinary rhinoscleroma.

E. Meyer reports eleven cases of pachydermia laryngis, all involving the posterior part of the vocal bands. He doubts any association of the lesion with either syphilis or tuberculosis; while C. Chiari, on the other hand, believes he has seen the disease produced by both syphilis and tuberculosis, as well as by chronic catarrh, in alcoholic drinkers. In cases of diffuse infiltration of the interarytenoid region he has found the epithelial layer thickened to five or six times its normal dimensions.

Under the title of A Case of Hard Warts (Verruca Dura) of the Larynx, P. Bergengrün reports a case without a trace of either syphilis or tuberculosis in personal or parental history. The pharyngeal mucous membrane was dry: The larynx showed intense chronic catarrh. The lining of the left ventricle was especially swollen and deeply injected, covering the greater part of the left vocal band. There was intense bilateral subchordal swelling. The mucous membrane over the arytenoids and interarytenoid region was greatly swollen; from the latter arose a number of whitish warty prominences, some of them the size of a small pea, their surface slightly irregular. Palpation with a sound conveyed the idea of very dense consistence. They were sessile and movable, and therefore not attached to the basement tissues. Microscopic examination of the incised masses showed "exclusively the epidermoidal characteristics of hard or corneous warts."

Atrophic Form.-There is a marked diminution in the number of the vessels and glands, and a loss of basement tissue of the mucous membrane. It is generally accompanied by atrophy of the nasal or pharyngeal mucous membrane, but may be seen as an independent condition. The whole lining structure of the larynx is thin and pale, and on section under the microscope shows obliteration of some of the vessels and loss of the glandular elements of the submucosa, with which appear inter- 
woven a few laminations of fibrous tissue, and at times increased pigmentation. Moure considers the epithelial layer as the early seat of the disease.

Green, speaking of the changes after infiltration of the connective tissues in chronic catarrhal affections, says : "The membrane thus becomes indurated and thickened, and the pressure exercised by the new growth may induce atrophic changes in the glandular structures which it contains."

Hemorrhagic Form.-Increased vascularity, in whole or in part, characterizes the condition of the lining membrane of the larynx, with increased number and size of the vessels, the walls of which in very chronic cases are thinner than normal and lie loosely embedded. In other cases the principal change seems to be in the epithelium, which is soft and fragile, easily separating from the subjacent layers. Small erosions or ulcerations are sometimes shown in spots (Schnitzler, Lennox Browne).

Diagnosis. - The diagnosis of the several forms of chronic laryngitis from laryngeal and pulmonary phthisis, syphilis, car. cinoma, lupus, and adenitis, and from some of the benign neoplasms, is not always an easy task.

In the diagnosis from the earlier stages of tuberculosis the clinical history will be of great service. We seldom see cases of a tuberculous nature without more or less constitutional disturbance, rapid pulse, pyrexia, etc. While the laryngoscopic appearances are often similar in phthisis and in chronic laryngitis, there is usually a paleness of the membrane, with or without a pyriform swelling of the arytenoids ("clubbing") in the former. In the so-called hyperæmic or inflammatory form of laryngeal phthisis, however, while the colour is intense the swelling of the mucous membrane is far greater than in simple laryngitis, and is especially observed at the arytenoid projections or interarytenoid space, while small ulcerations and an irregular outline in this situation, or on the sides of the glottis, or on the ventricular or vocal bands, will serve to recognise the latter disease. In the later stages the diagnosis becomes easier, owing to the characteristic structural changes belonging to laryngeal phthisis.

The edematous form may be easily confounded with any of the syphilitic forms of laryngeal disease, because aggravated hyperplasia of this sort is common to both, although it is more often seen in connection with the syphilitic affection. There- 
fore differentiation will sometimes depend largely upon the history of the case.

Hypertrophy, superior or inferior, may be distinguished from carcinoma and lupus by the colour, lupus showing a high degree of redness with a granular surface, carcinoma less redness, but with an uneven surface and a tumour formation, while in hypertrophy the swelling is pale, smooth, and even.

The early (erythematous) stages of secondary syphilis resemble chronic laryngeal catarrh, so that in some instances the clinical history or course of the case will have to be depended upon for diagnosis.

Generally, however, the redness and swelling of syphilis will be confined to a few patches, where perhaps a sharp look may discover the initial softening of ulceration. When ulcers are present they will add to the probability that the inflammation is a specific one.

From tertiary syphilis there will be little trouble in distinguishing chronic laryngeal catarrh, for the structural changes are characteristic.

The differentiation between erosion and ulceration is not always easy, because both lesions may occur in either affection.

The superficial character and the absence of an inflammatory areola, together with the clinical history, will serve to determine the character of these lesions, especially if the patient be kept under observation for a few days. When ulceration occurs with considerable hypertrophy, it is much more stationary than the ulceration of either syphilis or tuberculosis, in which diseases the destructive process is usually rapid and deep-seated.

Chronic rheumatic laryngitis and perichondritis of the arytenoids (Fig. II4) may resemble each other at first, but soon the intensity and extent of the swelling, and the presence of more or less pus or muco-pus about the swelling, together with the clinical history of the case, will serve to distinguish the former from the latter. The constitutional disturbance may be considerable with either affection.

Enchondrosis and chronic edematous laryngitis may appear alike in the laryngoscopic mirror, but the circumscribed contour and smooth surface, together with the sense of hardness conveyed by the probe, will solve the question of diagnosis. 
Inferior hypertrophy may sometimes be mistaken for an accumulation of desiccated secretion below the vocal bands, but the smooth, rubber-like surface of the projection, moving with the vocal bands, together with a certain degree of dyspnœa, will serve to determine the difference. It is sometimes difficult to differentiate between this form of hypertrophy and the membranous formation which is the result of congenital malformation or injury. Organic disease at the roots of the motor nerves of the larynx, causing paralysis of the posterior crico-arytenoid muscles (Cohen, Krause, Semon), may be mistaken for hypertrophic or pachydermic laryngitis, especially as their subjective symptoms are similar; but careful laryngoscopic examination (although hyperæmia and swelling may be present) will serve to show the paralytic character of the affection.

Prolapsus of the ventricular bands (Elsberg, Cohen, Lefferts) may be mistaken for superior laryngeal hypertrophy or some neoplasm, or for angioma laryngis. Laryngoscopic examination, together with the aid of a laryngeal probe, will show the place of attachment as well as the character of the swelling.

Angioma can be differentiated from hemorrhagic laryngitis by the presence of clots in the former, and the firm localized patch of blood-vessels elevated above the surface characteristic of the latter. It differs from the fibrous or cystic growth in its colour and contour, being flat, and, as it were, diffuse, and from lupus and epithelioma by the apparent evenness of its surface.

Such a formation cannot be taken for phlebectasis laryngis, because in the latter condition the veins can be easily made out.

Papillomata can be differentiated from chorditis vocalis tuberosa by their colour, size, and distinct attachment.

Trachomatous projections appear as a line of little white papules projecting above or from the edges of the vocal cords.

Exaggerated dimensions of the laryngeal ventricles may be mistaken for the atrophic variety of laryngeal catarrh, but the absence of dried secretion (crusts) on the surface, the abnormal thinness of the mucous membrane, are distinguishing features of the latter. The presence or absence also of atrophic pharyngitis will aid in the diagnosis. 
Course and Termination.-Idiopathic chronic laryngitis may continue for years without giving rise to serious structural change. Many authors believe that the disease is sure to extend along the respiratory apparatus, and terminate in some form of phthisis pulmonalis or tuberculosis. According to late pathological theories concerning the bacterial origin or cause of pulmonary phthisis, such a result would appear inevitable, because of the suitable soil for the implantation of germs which such a pathological condition would afford, especially in the event of ulceration, which, according to Schnitzler and Fränkel, is said to be good ground for bacterial invasion. However, the clinical experience of the world in laryngology must show emphatically the error of such views by the great number of subjects of chronic laryngeal catarrh who are never affected with either laryngeal or pulmonary phthisis. Nevertheless, such a termination does take place in a given number of cases, the inflammatory or catarrhal form of phthisis pulmonalis usually supervening. In our experiments upon monkeys with dried and pulverized sputum, Dr. Gibbes and myself have not been able to observe any particular laryngeal lesions following insufflation, although some of the sputum must have been lodged on the laryngeal walls.

Ulceration is not frequent in the course of simple chronic laryngitis, and its occurrence is taken by many to indicate the tubercular or syphilitic nature of the case.

Tymowski thinks that statements to this effect are far too sweeping, and believes from abundant careful observation that he is able to refute them.

In the chronic hyperplastic form called edematous, ulceration is not so uncommon, because of the continual pressure and injury to which some of the parts are exposed. This is particularly so with the epiglottis and the arytenoid projections, which not only are the most common seat of exudation, but are also more exposed to injury or irritation.

Erosion or aphthous ulceration, especially of the epiglottis or of the vocal bands, is quite often seen in the course of the disease (Gottstein), though seldom leading to ulceration of the submucous tissues. Fritsche relates a case of ulceration which he thinks was caused by gastric juice.

The abrasions are generally of transitory duration, and are confined to the epithelial layer. They may be observed in 
connection with the so-called "dartrous diathesis" of the older French authors. Gottstein relates a case of laryngeal œdema caused by the formation of phlyctenulæ.

Perichondritis seldom occurs incidentally to chronic laryngitis of non-specific origin, excepting when due to some of the infectious diseases, such as typhus fever, small-pox, or to a mechanical injury, etc. In a case of typhus fever in a Belgian emigrant who came under my observation some years ago, a persistent chronic laryngitis supervened. Hyperplasia continued after recovery from the fever, and about six months thereafter I was called to see him again, and found him suffering from a severe perichondritis of the left arytenoid cartilage. Suppuration took place, and an abscess formed; the pus was liberated by incision. No exfoliation of cartilage ever followed this, but there was eventually almost complete immobility of the part.

Dr. David Newman relates an interesting case of suppurative perichondritis without antecedent laryngeal disease or traumatism. It is said rarely to occur as an independent affection (von Ziemssen). C. Gerhardt reports a case of cricoid perichondritis, supposed to have been caused by long-continued decubitus, in which there was chronic laryngeal catarrh.

L. Brieger gives among the causes of laryngeal perichondritis, chronic laryngitis attended with considerable hyperplasia. Schrötter, Gottstein, Lennox Browne, and others, state that although perichondritis is more often secondary to tuberculosis, syphilis, erysipelas, arthritis, etc., yet it is frequently met with as a primary affection, they believe, as a result of cold or general infection and structural degeneration.

Three cases of perichondritis with ulceration, occurring in the course of chronic laryngitis following convalescence from small-pox and typhoid fever, are reported by Mr. Eve in the Transactions of the London Pathological Society for I88I.

Suppuration and abscess are not common in the course of chronic laryngitis, except in the case of severe exacerbations from cold or intercurrent diseases. It is therefore highly probable that a person in whom chronic laryngeal catarrh exists will be more liable to severe laryngeal complications, such as suppuration, ulceration, or perichondritis, if attacked by any of the acute infectious diseases.

A case of dangerous œdema of the larynx becoming chronic 
came under my observation in a lawyer who had contracted a pernicious malarial fever in the South, and who had been afflicted for several years previously with chronic laryngeal catarrh.

Hypertrophy, either superior or inferior, is apt to supervene in the course of this disease. Any prolonged local irritation in the hyperplastic form may lead to it.

Dissipated or ill-nourished persons furnish the greatest number of examples. More or less stenosis, and consequently dyspnœa, supervene, and tracheotomy may become necessary, although the individual may get along comfortably with a considerable amount of stenosis, provided it comes on gradually.

Atrophy may result as a sequence of hypertrophy (Green, Gibbes). Indeed, many observers say that it is the inevitable result of hypertrophy or hyperplasia here as well as in the nasal passages and pharynx. However this may be in some cases, it is certainly not true of all, for we often meet with all the laryngoscopic appearances of atrophy without any previous history of hyperplasia. Important changes occur in the ventricle both in this and in the hypertrophic form.

Hemorrhagic chronic laryngitis is sui generis, and must not be confounded with laryngeal hemorrhage. The latter condition may attend almost any case of chronic laryngitis, especially if erosion or great localized swellings occur.

Permanent immobility of the vocal bands from interstitial deposit, either in the muscles or about the arytenoid articulations, is a frequent termination of this disease, of course result. ing in more or less loss of voice and embarrassment of respiration. It not infrequently happens that spasmodic asthma results from the long-continued laryngeal inflammation.

Angioma of the larynix (Glasgow), varix, and cystic tumours may result from this disease. Papillomata are frequently the result or accompaniment of chronic laryngeal catarrh, especially in children. Although neoplasms are said to cause this disease, it is questionable whether myxomata are the cause or consequence of it.

Treatment.-The treatment of this affection may be conveniently considered under the head of general and topical.

General-Medicinal.-The selection of medicinal agents for internal administration will depend, of course, upon the sys- 
temic conditions of the patient, keeping in mind the cardinal point of removing as far as possible all causes. It will be frequently observed that general treatment is more efficacious than topical. Therefore any functional derangement of the body must be strictly attended to, especially those belonging to the cholopoietic viscera, the nervous system, the skin, and, in females, the sexual organs. The relief of a subacute gastric catarrh, of a constipated habit, or of some ovarian or uterine disorder, will often be the only treatment required for the milder forms of this disease.

While it cannot be positively asserted that any of the remedies in vogue have a selective action on the laryngeal mucous membrane, yet it is a matter of frequent observation that certain drugs, such as iodine and its compounds, ammonium muriate, belladonna, xanthoxylin, preparations of phosphorus, aconite, mercury biniodide, antimony tartrate, lobelia, hydrastin, the balsams, turpentine and its compounds, do give decided effects in this direction.

Hygienic treatment, it is obvious, is important. The patient ought to be removed from damp, overheated, or other bad domiciliary surroundings.

Improper modes of living should be interdicted, such as the use of tobacco or of alcoholic or malt liquors in excess, late hours and evening air, overeating, especially of highly spiced or rich food, and sedentary indoor life. Dusty and otherwise noxious vocations should be modified. The skin should be kept moderately active by means of exercise, occasional soap baths, and friction. The daily sponging of the neck and chest with cold water to render the skin more obdurate to external impressions of cold air, and the abandonment of the very harmful practice of wearing chest protectors indoors, should be insisted upon.

The beneficial effects of the renunciation of bad habits in cases of chronic laryngeal catarrh are strikingly exemplified by the fact, as related to me by Dr. Lathrop (late physician to the Michigan State Prison), that prisoners suffering from this disease will often recover after being immured for some months, without having been under any particular medical treatment.

A favourable climate is important, but in this respect each individual is "a law unto himself." In general, however, a 
warm, moist climate is beneficial. Those cases which exhibit considerable secretion will do better in a dry, warm climate of moderate altitude, unless it be very dusty. Ingals considers that the untoward effects in these cases may be attributed partly to the dryness of the atmosphere (from its effects on the nervous system) and partly to the irritating dust often found in high altitudes; and, further, that " a high and dry atmosphere is usually injurious to persons suffering from chronic laryngitis, but is beneficial in exceptional cases."

Cold climates, whether dry or moist, are not beneficial, as a rule, to cases of chronic laryngeal catarrh attended with any degree of hyperplasia. But the hemorrhagic and atrophic varieties are exceptions, for I have known of several persons suffering with the latter that were materially benefited by a cold, dry climate.

Mineral waters have been recognised for many years as very beneficial in the treatment of chronic laryngeal catarrh. For plethoric people and those leading sedentary lives, especially after reaching the age of forty-five, no class of remedial agents are more valuable than the saline mineral waters, taken internally and applied externally as baths. Likewise in cases of anæmia the chalybeate waters are of great value. When used locally as spray, however, their value is questionable.

The effects are more noticeable when the patient resides at the springs than when the same water is used at home. Therefore due credit must be given to the other features embraced by the change of scene (including perhaps change of habits) and relief from worry, incident to a sojourn at a watering place.

Galvanic electricity may prove of value. Dr. H. A. Johnson records several cases of chronic laryngitis with aphonia which were greatly benefited by systematic treatment with the galvanic current. The mental effect of this agent in cases characterized by functional nervous disease is marked. I know of the case of a lady suffering with chronic laryngeal hyperæmia, probably due to functional disturbance of the ganglionic nervous system, who was supposed to have recovered through the application of the galvanic current made to the region of the neck daily for a period of ten days. But it was afterward discovered by her physician that, owing to a defect in one of the electrodes, the circuit had never really been closed. 
Topical.-The topical treatment may be carried out by means of sprays (aqueous and oleaginous), inhalations of medicated vapours, pigments, powders, and external applications.

The great difficulty in local treatment consists in the application often enough of medicaments to the laryngeal cavity by the patient or nurse. It is a well-known fact that much of the spray from any sort of apparatus directed toward the laryngeal cavity is condensed upon the fauces, soft palate, tongue, or mouth, and therefore does not gain access to the laryngeal mucous membrane at all. In fact, unless pains be taken to train the patient to quick inspiratory efforts, the only effects to be expected from this mode of treatment are of a purely reflex or vicarious nature. To be effective, the spray should be driven directly into the larynx through a down-spray tube held over the laryngeal opening. These defects are overcome, however, in great measure by the use of a globe into which the spray is propelled, and to which some sort of mask, inclosing the nostrils and face, is attached, as shown by Fig. 2 I3.

With this apparatus more or less of the medicament, especially if it be oleaginous, must enter with the tidal air, since the whole act of respiration is carried on through the globe and attached rubber face shield. In this way oleaginous, balsamic, and aqueous solutions can be easily inhaled by the patient. The steam atomizer (that made by Codman and Shurtleff is the best) will also be of service, if the patient be instructed to close the nose during inspiration. The same instruction should be given in the use of a handball atomizer.

Medicated vapours, as advocated by Lennox Browne, are of great utility. These may be given by means of a hot-water inhaler, consisting of a receptacle capable of holding about a quart, and containing one pint of very hot water, to which has been added the medicament. By acts of inspiration the patient draws air down the little openings of the cover and through the mixture and up into the mouth through the inhaling tube at the top. There are many devices for the accomplishment of this, perhaps the best being a porcelain inhaler which is provided with a thermometer, as recommended by the late Sir Morell Mackenzie. As the porcelain inhalers are expensive and easily broken, cheaper ones, made of tin, as shown in Fig. 214, will answer the purpose quite as well.

For some cases the "dry" inhaler (Fig. 2 I 5), which fits over 
the face like a mask, and contains a sponge at the distal end for holding the medicament, may be used.

Of course, only substances more or less volatile can be used in such an inhaler (the respiratory current of air passing through is supposed to take up the medicament). The application of the inhalation should continue at least twenty or thirty minutes.

Pigments applied in the larynx are undoubtedly the most efficient method of local treatment for a certain class of cases, such as the hyperplastic, hypertrophic, and atrophic.

This can be done through the instrumentality of a piece of sponge, a camel's-hair brush, or a pledget of cotton. Escharotics may be applied in this way, although it is far safer to use aluminum rods or a caustic holder for such a purpose. The brush of Clinton Wagner is probably the best instrument for the purpose, although many laryngologists use equally well the cotton or sponge.

Powders are sometimes propelled into the larynx, but, as a rule, they prove irritating and harmful, while they are rarely dissolved by the secretions. With the exception of iodoform, iodol, aristol, bismuth, morphine, tannic acid, and a few other agents, their use in the treatment of chronic laryngitis has been generally abandoned.

There are many forms of insufflators in the market, from simple glass tubes to elaborate bottle "powder-blowers." The disconnecting hard-rubber insufflator devised by the author some years ago, a modification of the old form of insufflator, will be found very convenient for this purpose (Fig. 2IO).

External applications such as ice, hot water, poultices, blisters, and counter-irritants such as thapsia, croton oil, tincture of iodine, etc., are frequently efficacious when applied over the region of the larynx or at the sides of the neck.

The treatment of the particular forms of chronic laryngitis deserves some attention.

Hyperæmic Laryngitis.-This form, as before mentioned, usually depends upon faulty use or overuse of the voice, or upon some vaso-motor manifestation of remote disease or perverted function.

In the former case, it is obvious that particular attention should be paid to restriction and modification of the use of the voice. Rest, therefore-which, as E. M. Moore says, is one half 
of all therapy - should be, if possible, strictly enforced. Beverly Robinson, Langmaid, and Seiler lay great stress upon this measure (rest of the voice) in all such cases. Exercise of the voice by proper training is often, however, the very best treatment for some cases.

If the hyperæmia be kept up by disease elsewhere, of course the treatment should be directed toward the faulty region or apparatus, together with such special measures as may seem necessary. Benefit will often ensue from the administration internally of a mixture of sirup of wheat phosphates (Phillips's) and dilute hydrobromic acid, a teaspoonful three times a day in water, or fifteen to twenty minims of dilute phosphoric acid in water three times a day, or Inglis's elixir of fir, or Wheeler's compound elixir of phosphate of iron. If there be some relaxation of the bowels, from fifteen to twenty minims of tincture of chloride of iron in glycerine and water three times a day should be prescribed.

Where there is not only loss of appetite but also slight pyrexia, a capsule containing one-fourth grain of hydrastin (white alkaloid) and three grains of cinchonidia may be taken three or four times a day, or Wheeler's compound elixir of phosphate of iron. If there be a rheumatic tendency, salicylic acid or sodium salicylate, or salicin, may be taken three or four times daily instead.

Should the neurotic element predominate and the phosphate and acid hydrobromic mixture prove inadequate, an iron and strychnia mixture may be given, together with a dose at night of asafœtida, ammonium valerianate, camphor monobromide, or hyoscine hydrobromate; or one hundredth of a grain of glonoin, or three to five grains of acetanilide or phenacetine. Instead of these, fifteen grains of sodium bromide may be preferable to allay nervous perturbation.

If the patient is much troubled with cough, codeia, with or without a little rum and sirup, or sirup of yerba santa, or the compound lobelia lozenges (Hancock's or Wyeth's), may prove a needed adjuvant. It is rarely necessary to give opium, morphine, or cocaine internally. Hoffman's anodyne or the compound spirit of chloroform will often answer as well to allay a tickling cough. Frequently the inhalation of a few drops of chloroform or ether will fulfil the purpose better.

Topically only the mildest treatment is admissible. Here 
the meddlesome practitioner gets in some of his worst work. The leading local therapeutic indication is to prevent as far as possible hyperplasia and hypertrophy. Therefore, while occasionally a stimulating application may be advisable, usually all such ought to be avoided, and only the blandest medicaments, if any, used in the larynx.

The sprays of petrolina oil, albolene, or camphorated petrolina (Parke, Davis \& Co.'s) are the best.

It is seldom necessary to brush the larynx, and to insufflate powders.

With professionals who are unable to procure rest of the voice, the excellent recommendation of J. Solis-Cohen to use small doses of tartar emetic, and also small pieces of ice in the mouth, with cold water to the outside of the neck, should be adopted.

Hyperplastic Laryngitis.-A general thickening of moderate degree, as shown by the laryngoscope, calls for steady local treatment in the form of sprays and pigments. A spray of petrolina oil, or oil of eucalyptus and petrolina, or camphorated petrolina, should be used about twice daily, unless the oleaginous nature of the remedy causes nausea. The petrolina oil and camphorated petrolina are especially valuable. Besides this, two, three, or four times a week zinc sulphate, cadmium sulphate, zinc chloride, alum sulphate, ferric alum, silver nitrate, tannic acid, or hamamelis with a solution of potassium chlorate, should be applied directly to the larynx, either as spray or as a pigment. In practice it will be found better to apply both spray and pigment-first the spray, which will clear off the surface of the membrane, and then the pigment. As a preliminary spray, a solution of sodium chloride, Dobell's or Seiler's solution, or a solution of potassium chlorate, alone or with distilled extract of hamamelis, or aqua picis with either sodium bicarbonate or potassium chlorate, will be both grateful and effectual-of course care being taken not to select the potassium chlorate, nor any chloride, if silver nitrate is to follow.

Where there is much secretion with consequent hacking, frequent inhalations of camphorated petrolina or naphthaline, or creolin and petrolina, will be found highly efficacious. If there be much hyperæsthesia or glottic spasm, a solution of cocaine hydrochlorate (from two to five per cent) may be 
applied. This agent, very properly, is not used so much as formerly on account of its untoward effects (J. Solis-Cohen, S. S. Cohen), not only of a systemic character, but because it causes relaxation of the blood-vessels, as pointed out by John N. MacKenzie, Cohen, Asch, and others.

Menthol is a remedy of especial value for diminishing the sensitiveness of the laryngeal mucous membrane, besides being in many cases decidedly curative. A spray of ice water is efficacious in the hemorrhagic variety as a curative agent.

When transient erosions are seen, a spray of tannic acid, followed by camphorated petrolina, or creolin and petrolina, or by hydrargyrum bichloride, will often suffice; but if more persistent, or accompanied by pemphigus, herpes, or eczema, an insufflation of stearate of zinc, stearate of alum or tannin, iodoform, aristol with starch, or resorcin with starch, may be necessary. Silver nitrate (Cohen), although often effectual, will sometimes be of less value than the above-mentioned agents. However, when real ulceration occurs, silver nitrate, chromic or carbolic acid, or peroxide of hydrogen may be applied. The insufflation of iodoform, iodol, aristol, or resorcin with starch is preferable, because entailing less risk of irritation.

Chronic edematous laryngitis requires usually the application of cold or counter-irritation to the outside, and internally the administration of mercury, iodine, or calcium sulphate, with the application of sprays and pigments containing the vegetable or mineral astringents. A spray of an aqueous extract of arbor vitæ is especially useful; also compound tincture of benzoin or creosote, or iodine, by inhalation from a hot-water inhaler. When exacerbations of swelling take place, especially when showing additional deposit of serum, surgical means will be necessary-first scarification, and, if that is ineffectual, tracheotomy.

Atrophic laryngitis demands an active local treatment with stimulating agents, which will, as it were, stir up the flagging trophic nerves. Powders, however, are generally not admissible because of their drying effect, although it is urged by some that the mechanical irritation of powders proves useful. However that may be in theory, in practice it is generally found that most substances so used produce unpleasant effects, by further desiccating the secretions, excepting perhaps silver nitrate and tannate of quinine, which seem to act 
as local stimulants without drying the secretions. Oleaginous inhalations, such as camphorated petrolina, albolene, petrolina oil, eucalyptus oil, naphthaline, thymol, oleum picis, and balsam of Peru, or a solution of mercuric bichloride in cinnamon water, will be found most useful.

In the way of pigments, those containing silver nitrate, copper sulphate, zinc chloride, iodine, balsam of Peru, and oleum picis, or oil of eucalyptus, will be found curative so far as anything can be curative. The use of galvanic electricity, the naked electrode being applied to the parts when practicable, after the plan suggested by the author and Drs. Hartman and Delavan, is probably the most serviceable. Unfortunately, however, there are many patients who cannot bear the treatment thus applied, notwithstanding the usually diminished sensitiveness of the laryngeal membrane in this condition. Frequent applications of a small piece of dry sponge by means of an Elsberg or other sponge holder, and the massage procedure of Bergmann and Weiss, are also deserving of trial in these cases. The treatment of atrophic laryngitis, however, is usually unsatisfactory, for the obvious reason that atrophied tissue cannot be restored.

Hypertrophy.-The treatment, whether general or limited, although non-specific, requires the administration of iodine or mercury, or both, as well as active local treatment. Iodine is best administered in the form of the sirup of hydriodic acid in teaspoonful doses three or four times a day, or potassium iodide, or ammonium iodide. Ammonium iodide will sometimes be preferable on account of its selective action upon the respiratory mucous membrane; ammonium muriate has also been highly extolled; mercurial fumigations are advocated by George E. Law in that form of chronic laryngitis known as pseudo-membranous. Mercuric biniodide may be alternated with the iodine to advantage.

The local treatment consists for the most part in the application of iodine, mercury, or astringent pigments. Dr. Holmes, for the treatment of that form known as chronic laryngitis granulosa, advocates the use of strong mineral astringents (in pigments or powders), such as silver nitrate carried to the part upon a probe. Semon and Cohen also urge the use of strong solutions of silver nitrate in all forms of chronic laryngitis. 
No special treatment seems necessary for trachoma or chorditis vocalis tuberosa, although a spray of aqueous extract of arbor vitæ is very efficient. F. I. Knight states that the proliferations may spontaneously disappear. Cohen, Rice, and others, recommend silver nitrate. Labus recommends flaying the mucous membrane in cases of great hyperplasia, and Heryng advocates curetting in similar cases.

Chorditis vocalis hypertrophica inferior will require about the same treatment as hypertrophy in other situations, especially the administration of iodine or its compounds. The required local treatment, when there is considerable stenosis, is catheterization, as well as the application of strong astringents (Schoetz, Marian). When the obstruction to respiration is very great, of course tracheotomy must be performed without delay.

Schoetz, who gives the histories of three cases, believes that the treatment of chronic subglottic laryngitis should be begun early and actively kept up, so as to prevent hypertrophy.

The general treatment of pachydermia laryngis should be like that of hypertrophy. The local treatment will require the use of escharotics carried to the seat of trouble, or surgical measures such as evulsion. The best and safest escharotics are chromic acid, according to Sajous and Jarvis, and the galvano-cautery. The former is best carried to the part on a Jarvis laryngeal chromic-acid carrier (see Fig. 202). The galvano-cautery loop is used by Meyer. In using either of these agents great care must be taken lest the patient die of spasm of the glottis. In many instances it will not be safe to make such applications without first performing tracheotomy. 


\section{CHAPTER XII}

LOCAL DISEASES OF THE NOSE AND THROAT

\section{Perforation of the Cartilaginous Septum}

THIS is frequently met with throughout the Northern States. It occurs much more often in males than in females. Perforation may vary from a mere pin-head opening to one measuring an eighth or three eighths of an inch in diameter, and in aggravated cases nearly the whole of the triangular cartilage may be missing, so that the anterior columnar and the dorsal ridge continuous with the nasal spine of the maxilla will be all that is left of this region of the septum.

Etiology.-Until recent times perforation of the cartilaginous septurn was almost always attributed to the effect of syphilis, in one form or another. Congenital syphilis especially was considered as one of the principal causes, but as the progress of rhinology has gathered from time to time more accurate data concerning the history of these cases, the formerly accepted idea of causation has become modified. There are many cases where it is almost impossible to trace the cause or causes of the lesion. The majority of them, however, are attributable to the habit of picking the nose with the finger or to the operation of cold. Dr. Straw, of Ashland, in a paper read before the American Medical Association, promulgated the idea that the great prevalence of perforated cartilaginous septa in the northwestern country could be attributed to the effects of cold -the severity of the winter weather. The theory is that there is more or less of a slight freezing and thawing process going on at the extremity of the nose from exposure to the very low temperature which prevails in the winter season there, the result of which upon the capillaries and cartilaginous canaliculi is to diminish the nutritive supply and induce a low grade of inflammation perhaps, which ultimately produces a sort of 
molecular death of the cartilage. This ingenious theory seems to the writer feasible, and, when taken in connection with possible antecedent deflections or thickening of the cartilaginous septum, becomes all the more rational. Taking into consideration the anatomical arrangement of the cartilaginous septum and the natural means for its supply of nutrition, it does not seem strange that the more or less continual pressure or irritation exerted by picking with the finger, or the operation of frost-bites, should result in a gradual process of molecular dissolution. It will be found that by far the larger number of perforations of the cartilaginous septum occur in subjects suffering from deformity of the septum combined with hypertrophic nasal catarrh. Either condition, it is obvious, favours the desiccation of secretion at the lower portion of the nose, which of itself exerts a baneful influence upon the epithelial layers of the mucous membrane.

Treatment.-In the majority of instances the first thing to do is to relieve any existing deformity or hypertrophy of the nasal passage which may exist; at the same time the patient should be directed to frequently anoint the septum on each side with vaseline, or benzoated oxide of zinc ointment, or lanoline. Instruction should be given the patient to refrain from touching the part with the finger. If, however, the areola of mucous membrane around the perforation becomes very dry, this part should be touched two or three times a week with some stimulant, such as Peruvian balsam, oil of tar, or a weak solution of ichthyol. These applications not only favour the restitution of the surrounding mucous membrane and cartilage, but serve to bring about a healing or covering of the edges of the perforation. It will be noticed in nearly all cases that the perforation seems to enlarge by the exfoliation, so to speak, of its circumferential edge. It has been a common practice to treat these edges with the galvano-cautery or other caustics in a vain endeavour to heal them and thus prevent extension. The same treatment has been applied to the septum when observed to be growing thin or about to perforate. But, in the writer's experience, all of these applications are of no benefit; on the contrary, they are harmful. The best local treatment, as a rule, for an existing perforation is to let its edges alone, excepting to apply from time to time some ointment for purposes of lubrication. 
Herpes of the Nose.-Herpetic or eczematous eruptions sometimes take place in the vestibule of the nose or farther in. It is very seldom, however, that they are found farther than an inch in the nasal passages. The favourite site of these eruptions is the nasal septum or the inner lining of the alæ nasi. Herpes may occur in this situation simultaneously with an eruption upon the soft palate, or buccal cavity, or pharynx, or in the neighbourhood of any of the muco-cutaneous junctions of the body.

\section{Mycosis (Pharyngis, Tonsillaris, Laryngis)}

Faucial or.pharyngeal mycosis is a chronic affection of rather infrequent occurrence, and consists in the development of a fungus (epiphyte) at the mouths of the crypts of the tonsils, or about the mouths of follicles elsewhere in the pharynx, tonsils, or larynx. The growth may extend as low as the large or small bronchi. According to Hemenway, the first article published on the disease was in 1893. Bean states that $B$. Fränkel reported the first case in 1873 . Gumbinner wrote of it in 1883 . Since then, articles have appeared written by Newcomb, Jonathan Wright, F. I. Knight, Hemenway, Greiner, Stoerk, and others. Decker and Sieffert reported a collection of thirty-four cases up to i 883 .

Etiology.-This affection is usually found in adolescents. Females are attacked more frequently than males, in the proportion of about nine of the former to one of the latter.' It is not common to any social condition in life. The remote or predisposing causes are still a matter of uncertainty. Many observers believe that the disease is a sequence of acidity of the oral secretions (Damoschino), of mouth breathing, of chronic follicular tonsilitis, or of a chronic folliculitis in some other part of the faucial region, such condition especially favouring the implantation and growth of the spores of either the leptothrix buccalis, aspergillus fumigatus, oidium albicans, saccharomycetes, leptothrix maxima, or bacillus buccalis. Sarcinæ have also been found, as well as other fungi which are common to the mouth. Leptothrix buccalis and bacillus fasciculatus are the fungi usually found in the deposits. Hemenway does not believe that pharyngomycosis is due to the leptothrix, which, he says, grows on the gums but not on the pharynx. Some authors ascribe the disease to a special bacillus, and Schmie- 
gelow ascribes it to the bacillus anthracoides buccalis-the bacillus mycosis. Klebs classifies leptothrix buccalis with the algæ. Many of the lime algæ which occur in sweat and salt water possess special importance as relating to lime formations. These are present on the uncared-for teeth and in perspiration. This fungus, the bacillus mycosis, has not been cultivated outside the body, and is not influenced in water to which lime has been added. It is often found in rhinoliths and tonsillar concretions. Other observers, however, believe that the remote conditions favouring the implantation and growth of the fungus lie more in changes of secretion than in any structural pathological change of the parts. The probability is, however, that both are factors in the predisposing cause, and that neither acidity nor alkalinity of the secretions alone are sufficient to promote the origin and progress of the growth without certain changes in the organic constituents of the secretions due to catarrhal inflammation. That many cases are preceded by some pathologic change in the parts is evident from the clinical history, which shows more or less pathologic change about the tonsils or their crypts or of the follicular glands. It was formerly ascribed to the scrofulous diathesis, and then to the arthritic, but as a majority of the cases do not belong to either class of disease it will be necessary to discard that theory. A few cases may be observed among the anæmic, but there is probably no causal relationship. Dental caries, leptothrix, and chronic enlargements of the tonsils have been ascribed as predisposing causes. Indeed, the topography of the pathologic tonsil favours such growth. Damp, unhealthy surroundings are given as a cause (Hemenway). Lennox Browne mentions an unbealthy state of the skin as favourable to the disease. The writer has met with several cases in the very early stages of phthisis pulmonalis. It is said to occur subsequent to attacks of diphtheria (Vanderpoel). The fact is, that we have no absolute knowledge of just what are the predisposing conditions. It cannot be universally ascribed to chronic folliculitis, or any of the other causes given, because the large majority of such cases are not attended by mycosis. We are therefore forced to the theoretical conclusion that some peculiarity of the part furnishes the determining cause, inasmuch as the spores of these fungi are more or less in contact with the mucous membrane, crypts, and follicles of the fauces of almost every person; 
while with only a very few does a lodgment and growth take place.

Pathology.-The pathology is mainly a history of the growth and extension of the fungus after its lodgment. The growth is slow, usually confined at first to one or two focipreferably the tonsillar crypts-developing slowly, and after a while (perhaps some months) spreading to even the posterior wall of the pharynx, the recesses of the fauces, base of the tongue, or even upon the upper portions of the larynx, trachea, and nose. Dr. John N. MacKenzie reported a case of aspergillus mycosis affecting the maxillary sinus, and Jonathan Wright and Hemenway reported one case each of the occurrence of mycosis in the nasal passage. It seldom, however, spreads to or affects the pharyngeal tonsil, or inside of the larynx, although Price Brown, of Toronto, has reported a case of mycosis of the larynx. Microscopic examination usually shows in the mass distinct well-formed mycelia, with thalia or filaments rising in tufts and inclosing between their meshes more or less of the secretion in various states of degeneration, leucocytes, and distorted or partially disintegrated epithelioid cells, together with inspissated mucin. The filaments are straight or flexed. The mycelia do not penetrate very deeply, but seem to be attached mostly between the epithelial cells at or just inside the mouths of the follicles and reaching behind the first circle of endothelium. The tissues in the immediate neighbourhood are sometimes swollen (edematous) from slight engorgement of the capillaries, transudation of serum, and the presence of leucocytes and bacilli. The lymph channels are not interfered with to any great extent, except when the roots of the growth either clasp or press upon them, in which case additional spaces are formed, to become afterward filled with leucocytes and serum. The capillary loops of the mucosa are of course more or less disturbed, some of them being entirely blocked up with pigment and cells, and in small areas here and there the coadjustment of the epithelial cells is very irregular and broken. In some places, where the growth is thick, filaments of mycelium seem to supply the place of the natural covering almost entirely. This is especially marked in the tonsillar crypts and at the margins of the follicles. Pus cells are rarely found in the débris, but a number of fat globules are almost always present. All the elements of the 
epithelium in the neighbourhood seem to be sodden, as if undergoing necrosis.

Symptomatology.-There are no constitutional symptoms belonging to this affection excepting in some instances where indigestion, either stomachic, intestinal, or both, may be coincident. Indeed, many patients have no special subjective symptoms attending the disease, and if it were not for the discovery of white spots by inspection, they would not know of any pathological condition of the throat. This is true occasionally of cases which show quite an extensive growth. As a rule, however, these patients notice a slight soreness of the throat, which may be localized to their senses on one side or the other, or which may seem to them quite diffuse. The pain, however, is quite insignificant, and does not in any way interfere with their daily routine of life. The observation of this condition before the looking-glass is frequently what leads patients to consult the doctor. They also complain, as a rule, of a bad taste, especially in the morning. They have a coated tongue, and some extra secretion of mucus. The vocal function is rarely affected, although in two cases of singers which have come under my observation it was thought that the vocal faculty was impaired by the disease. How much their mental perturbation contributed to this condition cannot, of course, be estimated. According to Bean, mental depression is a common symptom, and usually quite persistent. When quite extensive throughout the fauces, the irritation is very much augmented, and such subjective symptoms as hemming, hawking, expectoration, sensations of fulness, burning, or obstruction of the throat, may prove very annoying. Besides, frequently recurring attacks of acute sore throat may increase the patient's distress. Coincidently, also, there may be progressive disturbance of the stomach, indicated by nausea, dyspepsia, or pain, and if the growth proceeds upward into the nasopharynx there may be added symptoms of irritation of the nasal passages or ears, the latter symptom being probably due to irritation of the pharyngeal nerves about the Eustachian tube. The appearances are quite characteristic (see Plate I, Fig. 4). Upon inspecting the fauces, white or pearly tuft-like projections, more or less frayed in patches, are to be seen projecting above the surface from the margins of the crypts or follicles of one or the other of the tonsils, or 
about the lingual glands. These masses cannot be easily, if at all, removed with a cotton pledget, probe, or brush. They may be coalescent, but are usually discrete, and appear as dots here and there, sometimes filling the crypts, while the intervening tissue appears natural. Instead of being grouped together, these little white masses or flecks will be scattered over each side of the fauces and down to the base of the tongue. When the disease has progressed to a considerable extent, these masses are found closer together, and arranged in numerous groups all over the tonsils, about the base of the tongue, and sides of the pharynx. When removed by force, the growth is apt to be speedily renewed, sometimes in twenty-four hours. The lingual tonsil and adjacent glandular tissue is frequently the principal seat of the growth.

Diagnosis.-The diagnosis is usually not very difficult. The disease may be confounded with chronic follicular tonsilitis or with mild diphtheritic pharyngitis. In the latter affections the structural change in the tonsil and mucous membrane, together with the fact that the white inspissated secretion is easily removed with probe or spray, will serve to distinguish them. It ought not to be confounded with either acute follicular tonsilitis or diphtheria, because of the wide difference in the character of the deposit, as shown macroscopically and microscopically ; besides, no constitutional disturbance is present unless the mycosis be a coincident of some other disease, such as phthisis or rheumatism.

Prognosis. - This disease is not a grave one, and may exist for years, as said before, without producing any constitutional disturbance. It has been thought by some observers that it might induce the development of lupus or epithelioma, but such effects have not been indisputably observed. The long continuance of the disease and its progressive encroachment upon the fauces, of course, may lead to serious chronic inflammation and atrophy of the structures. On account of its occurrence in the subjects of phthisis pulmonalis, it has been surmised that some pathological relationship existed between the two diseases, but as yet no such relation has been discovered.

Treatment.-It may be thought unnecessary, on account of the benign nature and slow progress of the disease and the infrequency of complications, to adopt any mode of treatment. Although a spontaneous cure may take place, still it is not con- 
sidered a self-limited disease. The presence of an abnormal condition, whether accompanied by distressing subjective symptoms or not, will make some persons unhappy, so that for this reason, if no other, the adoption of prompt and efficient treatment will become necessary. It is obvious that the treatment required is chiefly a topical one, yet in cases where the clinical history points to any disturbance elsewhere, such disturbance demands attention in order to modify any of the errors of nutrition upon which the progress of the growth may in a measure depend. With regard to topical treatment, it is almost useless to apply by spray antiseptics or astringents, or to apply chemical escharotics, until the principal part of the growth, with its mycelia, has been removed. For this purpose the most efficient instrument is the curette-either a spoon or a small fenestrated curette, the former for large and prominent masses, and the latter for clearing the mouths of crypts, follicles, or more hidden places. The fauces should be cocainized with a spray of four-to ten-per-cent solution of cocaine hydrochlorate, and a certain region selected for operation at each sitting, from which locality the growth is to be thoroughly scraped off with a curette, after which a spray of boric acid or an insufflation of a powder consisting of bismuth and powdered chalk should be blown upon the part. Each day, for several days afterward, a pigment consisting of liquor ferrisubsulphatis mixed with glycerine and water, or a solution of nitrate of silver, may be pencilled over the part (see formula). After the lapse of a few days another region may be similarly curetted and treated as before. This method of treatment should be followed up until the so-called white spots fail to reappear. It sometimes requires many weeks of treatment to entirely obliterate the growth. I have known of one case showing a very moderate growth of the fungus in which full restoration of the part was brought about by systemic treatment with saline mineral water in connection with the exhibition of strychnia, iron, and hyoscyamus; and another mild case which, under the care of a homœopathic physician, was apparently cured by the local use of a trituration of biniodide of mercury. However, it must be added that such results are the exception, and not the rule, as a large majority of the cases will require the persistent local treatment indicated above. Tobacco smoking has been advocated as a 
remedy for the disease. The use of the galvano-cautery and other escharotics has been pretty thoroughly exploited by many practitioners. F. I. Knight, Hemenway, Bean, Newcomb, and others, advocate the thorough use of the galvanocautery. According to the writer's experience, the galvanocautery has been inefficient until after thorough curettement has been done. Wagner advocates the application of chromic acid, but, according to the writer's observation, the best results have followed the adoption of curettement first, and then, if necessary, escharotic applications. The use of formic aldehyde in solution will sometimes give excellent results in mild and limited cases.

\section{VARIX AND HÆMatocele of THE Fauces}

Varix and hamatocele of the fauces are not uncommon affections. Varix, however, is much more common than hæmatocele. Varices at the base of the tongue occur very often, frequently without causing any particular inconvenience to the person.

Etiology.-Varix is generally met with in alcohol drinkers, obese and elderly persons, gouty or rheumatic subjects, or those affected with more or less arterio-sclerosis and cardiac lesions, and especially if any of these conditions are accompanied by asthma.

The sites of these varices are usually the base of the tongue, just above the lingual tonsil (or including it), the lower portion of the lateral wall of the pharynx below the palatal tonsils (when they are small), and the anterior and posterior pillars of the fauces near the junction of the velum palati. In the latter situation hæmatocele is usually found; it may be attached to the posterior border of the edge, or upon the anterior border of the faucial pillar. Rarely do these blood tumours occur upon the posterior wall of the pharynx. The writer has seen but one case in the latter situation. Varices here may comprise a part of a general varicose condition of the small veins, a condition often noticed in individuals suffering from cardiac or chronic renal disease. The varices and hæmatoceles of the faucial pillars and upper pharynx vary in size from the barleycorn to a large pea. They may be round or oval, but are usually irregularly shaped and flat, their size and shape depending 
entirely upon the situation and the congeries of vessels involved. Those at the base of the tongue are usually more diffuse and flat, the vessels rarely rising in a papillomatous form unless the formation be a hæmatocele.

Symptomatology.-Either varix or hæmatocele are often observed in the throats of perfectly healthy persons. For example, it sometimes happens that a healthy person will consult the doctor about a "little black tumour" of the throat which has just been discovered before a looking-glass. The patient, recognising the abnormality, becomes alarmed, and hurriedly seeks medical counsel. In the majority of cases, as above mentioned, no subjective symptoms attend this formation; but sometimes frequent attacks of slight hemorrhage, more or less faucial irritation, cough, gagging, irregular or persistent contractions of the constrictor muscles of the pharynx, are predominant symptoms. The hemorrhages are quite insignificant, as a rule, but occasionally they are considerable in amount, especially when the varices occupy the posterior portion of the dorsum or root of the tongue. The writer has several times been consulted by patients suffering from this condition, who thought they were attacked with hæmoptysis. Indeed, the writer has known of three cases of varix of the tongue and fauces which were treated by quacks for pulmonary consumption on account of the blood spitting and cough. These cases are not always free from distressing symptoms, and, like some of those affected by enlarged lingual tonsils, a direct or reflex cough, with gagging, vomiting, and spasmodic action of the constrictor muscles, may become a serious illness, which, if continuing, might decidedly undermine the health of the patient. My associate, Dr. P. M. Hickey, a short time ago met with a very interesting case of faucial varix in a woman who was just undergoing the menopause, and to whom the subjective symptoms were very distressing. The hemorrhages were frequent, and the cough almost incessant. The case was entirely relieved by a few applications of the galvanocautery.

Diagnosis. - The diagnosis can be easily made out by ordinary inspection with the laryngoscope. In all cases of slight hemorrhage from the mouth or throat, examination should be made in order to determine the presence or absence of varicose vessels (Plate V, Fig. 4). 
Treatment.-Varicose vessels at the base of the tongue should be let alone if they give rise to no subjective or objective symptoms. If, however, enlarged follicles seem to be connected with them, or it is found from repeated examination that any of the vessel walls are growing thinner, then, even in the absence of particular symptoms, the practitioner will be justified in bestowing upon this region some local treatment. For small varices or small hæmatoceles, their strangulation by means of a snare of very fine wire is a good plan; or, if that be undesirable, the galvano-cautery snare, or a curved galvanocautery electrode, may be applied as frequently as may be deemed necessary. Applications of chemical agents outside of escharotics are not of much use unless the protuberant vessels be very small, in which case applications of persulphate or perchloride of iron will sometimes be found sufficient. Hæmatocele occurring in the lower part of the pharynx should be treated in the same way. Varices at the base of the tongue may require a number of applications of the galvano-cautery. A knife-shaped or pointed curved electrode should be applied to the larger of the vessels, and carried deep enough to cut clear through the vessel, for the treatment will be ineffectual if these little veins are only hacked into or burned through their upper surface. Styptic collodion, when it is well borne, may be used with benefit in cases showing but moderate formation. When this plan of treatment is determined upon, the surface of the tongue should be thoroughly dried with absorbent cotton first, and immediately thereafter the collodion applied. It is unnecessary to state that this agent will be apt to cause more or less distress if any amount of it touches the epiglottis. In all cases the practitioner must not lose sight of the necessity for adopting such constitutional treatment as may be indicated.

Another plan of treatment perhaps worthy of mention consists in destroying the veins by one or two hypodermatic injections of a solution of tannic or carbolic acid, perchloride of iron, or alum. This treatment is more particularly applicable to hæmatocele than to varix. It must be remembered, however, that it is not entirely devoid of danger even when aseptically done. Of all agents suitable for such a purpose, tannic or carbolic acids are the safest. The dangers alluded to are suppurative inflammation, abscess, gangrene, phlebitis, and thrombosis of some larger veins in the neighbourhood. 
Leucoplakia BucCalis et Lingualis-ICHThyosis LinguÆ

This very uncommon disease has been recognised for a comparatively short time only. It has been described, according to Ingals, as "smokers' patches," "glass-blowers" " or "professional patches," or "psoriasis linguæ," "tylosis," and variously as manifestations of syphilis and epithelioma. The name leucoplakia was suggested by Schwimmer, who wrote very intelligently of it a few years ago. The disease was first brought prominently before the profession by J. W. Hulke, under the title "ichthyosis linguæ." The first paper written upon it was by Alibert, in 1822 , and some time after by Bazin, Schwimmer, Ullmann, Guinand, Debove, W. Fairlie Clarke, and more recently by $\mathrm{E}$. F. Ingals, to whom we are greatly indebted for valuable researches into the literature of the subject.

The definition of the disease given by Ingals is as follows:

Leucoplakia buccalis is a chronic affection of the buccal mucous membrane, characterized by thickening of the epithelium, and the formation of white, opaline, elevated patches, which usually become fissured and painful, and after continuing for a long time are likely to terminate in epithelioma.

Ingals considers it an idiopathic disease, distinct from psoriasis, smokers' patches, and syphilis.

Etiology.-There is still diversity of opinion regarding its etiology and classification. Excessive tobacco smoking and syphilis are regarded as the most common causes of the affection. Highly spiced food and the imbibition of alcoholic stimulants are also listed as causative factors, while some observers have charged to hereditary tendency a good share in its causation. Among the cases reported, however, are some in which the affection cannot be ascribed to any one or two of the causes enumerated. Adult males are more frequently affected.

Pathology and Appearance.-The essential pathological character is that of a chronic inflammation of the mucous membrane with hypertrophy of the epithelial layer. In the first stage of the affection hyperæmia of the deeper layers of the epithelium exists, which is soon followed by exudation and induration. The microscope shows the epithelium thickened, the papillæ enlarged and flattened, and the blood-vessels dilated, 
and with an accumulation of leucocytes about their walls. The superficial layer of the "mucous corium" is infiltrated with embryonic cells, and the deep layer is involved in vascular alterations (Ingals).

Appearance.-The patches are usually found on the dorsum of the tongue or the inner surface of the cheeks and lips, and seldom on the fauces. In a case seen by the writer, however, the right anterior faucial pillar was affected as well as the buccal cavity. The first appearance consists usually of a patch of hyperæmia, which is soon followed by a round white patch with a limited red areola. The white patch gradually thickens and becomes dry and furrowed, and, according to Ingals, sometimes exfoliates, leaving a slightly excavated base simulating an ulcer. Occasionally ulceration of a papillary (warty) character may supervene. The spread of the disease takes place from the periphery, as a rule, and may involve a space two inches in diameter. In the case already alluded to by the writer, the patch on the inside of the cheek was elliptical in shape, and its long diameter about two inches, while the one on the faucial pillar was about one half by one fourth of an inch in size. The surface of these patches occasionally becomes very much raised and hardened, so as to present a horny appearance.

Symptomatology. - There are rarely any constitutional symptoms, unless the disease, through ulceration or a development into epithelioma, so interferes with nutrition as to induce some form of sepsis. The patients are always anxious, however, especially as they generally believe that the malady is a cancerous one. There is little pain at first, but as the disease progresses, and fissures, ulceration, or increased swelling supervene, there arises, of course, a corresponding amount of pain and distress. The patch itself may not be sensitive to touch, or even to incision, but the surrounding parts may become quite sensitive.

Diagnosis.-Leucoplakia may be mistaken for mycosis, for the "professional patches" (Guinand) occurring in glass-blowers, for "smokers' patches," for "mercurial patches," for "psoriasis linguæ," for "syphilitic patches," or for epithelioma unconnected with the leucoplakia (Ingals).

Sometimes syphilis or carcinoma coexists with leucoplakia, in which event the diagnosis, of course, may be very difficult. 
The following table will present at a glance the main points of difference :

\begin{tabular}{|c|c|c|c|}
\hline Leucoplakia. & Mycosis. & Smokers' patches. & Glass-blowers' patches. \\
\hline $\begin{array}{l}\text { No constitutional } \\
\text { symptoms ; usually } \\
\text { single patch, situated } \\
\text { on the surface of the } \\
\text { tongue or inside the } \\
\text { cheeks ; very white, } \\
\text { raised, hard, dry, fur- } \\
\text { rowed, or horny ; } \\
\text { slight areola ; excep- } \\
\text { tionally ulcerated ; } \\
\text { exceptionally multi- } \\
\text { ple ; microscopic ap- } \\
\text { pearance, altered epi- } \\
\text { thelium. }\end{array}$ & $\begin{array}{l}\text { Numerous small, } \\
\text { smooth, white exu- } \\
\text { dates, not much } \\
\text { raised, not hard, no } \\
\text { red areola, situated } \\
\text { at mouths of glan- } \\
\text { dulæ, particularly } \\
\text { the tonsillar crypts. }\end{array}$ & $\begin{array}{l}\text { Usually on lips or } \\
\text { under surface of } \\
\text { tongue; not raised, } \\
\text { and not particularly } \\
\text { white. }\end{array}$ & $\begin{array}{l}\text { Symmetrically sit- } \\
\text { uated at sides of } \\
\text { lower jaw or under } \\
\text { the sides of tongue. } \\
\text { Thin whitish exudate } \\
\text { soon exfoliating. }\end{array}$ \\
\hline Mercurial patches. & Psoriasis. & Syphilis. & Epithelioma. \\
\hline $\begin{array}{l}\text { Large flabby, coat- } \\
\text { ed tongue; fetid } \\
\text { breath; patches gray- } \\
\text { ish, thin, moist, and } \\
\text { situated along sides } \\
\text { of tongue ; generally } \\
\text { much salivation. }\end{array}$ & $\begin{array}{l}\text { Reddish and white } \\
\text { crescentic or stellate } \\
\text { patches, rapidly ex- } \\
\text { foliating, leaving the } \\
\text { surface raw and fis- } \\
\text { sured. }\end{array}$ & $\begin{array}{l}\text { History : Small, } \\
\text { round, oval, or cres- } \\
\text { centic patches with } \\
\text { areola, usually situat- } \\
\text { ed primarily about } \\
\text { the fauces, slough- } \\
\text { ing, and showing } \\
\text { excavation of the tis- } \\
\text { sues; enlargement } \\
\text { of the neighbouring } \\
\text { lymphatic glands. }\end{array}$ & $\begin{array}{l}\text { History : Papular } \\
\text { or granular develop- } \\
\text { ment; whitish patch- } \\
\text { es exceptional, and } \\
\text { only occurring in } \\
\text { connection with } \\
\text { breaking down of } \\
\text { tissue. }\end{array}$ \\
\hline
\end{tabular}

Course and Prognosis.-Leucoplakia is very slow in its course. It is prone to pass into epithelioma, according to Ingals and others. Mauriac thinks there are three varieties of the disease: the dartrous or arthritic, the syphilitic, and the epitheliomatous. The one due to syphilis, he thinks, is curable, while the others are incurable. When the disease is becoming cancerous, a non-inflammatory enlargement of the lymphatic glands, more sharp pain, and exfoliation and ulceration of the patches will be noted. The writer has seen two cases of the disease, one of which recovered, while the other (a case under the care of Dr. Welch, of Grand Rapids, Michigan) developed. into epithelioma, and soon thereafter passed from under observation.

Treatment.-The majority of writers give little hope of recovery by any plan of treatment. Some rely mostly upon systemic treatment, while others recommend topical treatment 
principally for the so-called non-syphilitic cases. Mercury, arsenic, potassium iodide, iodine, and the various saline depurants, have been used for internal medication with varying results. The general treatment will vary according to the indications presented in each case. Local applications of silver nitrate, mercury nitrate, iodine, zinc chloride, bole Armenium, carbolic, nitric, and hydrochloric acids, the alkaline caustics, the actual and galvano-cautery, have each been recommended. Dr. Ingals reported a case before the American Laryngological Association (1885) which was effectually cured by about thirty applications of the galvano-cautery. This agent was used by Dr. Welch in the case alluded to, but without arresting the disease.

In the case of the writer's which apparently recovered, the agent used topically was caustic soda and lime (London paste). It was applied successively for about four months, at intervals of three, five, or eight days, to portions of the patch at a time. Considerable tissue was thus destroyed, as the resulting cicatrices bear witness. According to the writer's observation, the soda escharotic seemed to dissolve the morbid tissue little by little, and to induce the production in its place of healthy connective tissue. 


\section{CHAPTER XIII}

DISEASES OF THE ACCESSORY CAVITIES OF THE NOSE, THE ANTRUM OF HIGHMORE, OR MAXILLARY SINUS-THE FRONTAL AND SPHENOIDAL SINUSES, AND THE ETHMOID CELLS

Inflammation of the Sinuses (Sinusitis Maxillaris; $S$. Frontalis; S. Sphenoidalis; Ethmoiditis)

INFLAMMATION of various grades is the most common of the affections of these sinuses. It may be acute or chronic, primary or secondary, the majority of cases being of secondary origin.

\section{Acute Sinusitis}

may affect either one or more than one of these cavities simultaneously, or one and then another consecutively. For this reason it will be unnecessary to treat of each one separately under this head. Anatomical and pathological discoveries in this connection date as far back as 1883 (Zuccarini), when three cases of erysipelatous suppuration of the accessory sinuses of the nose were observed.

General Etiology.-Influenza and the exanthemata are very often the causes of this affection on account of the almost invariable implication of the pituitary membrane in these diseases. Their involvement during the course of the exanthemata or other infectious diseases is often overlooked, unless some latent or chronic disorder of their interior remains as a sequel to the systemic affection. Infection of one or more of the sinuses as a result of surgical operation or other injury to the nasal mucous membrane or turbinate bones may arise. The retention of secretion or blood as a result of packing or plugging the nasal passages is sometimes the cause of such infection. In hospitals, particularly, àn inflammation from infection of these cavities is liable to occur after operations on 
the nasal passages. The writer has had two cases of acute sinusitis in hospital practice originating from infection of the wounded nasal passage, probably through other patients or dressings. Dr. P. M. Hickey saw one case of severe sinusitis from packing the nasal passages too completely after operation. Operations during cold weather or during the prevalence of diphtheria or erysipelas may give rise to it. External injuries to the nose or to the face, especially in the neighbourhood of the orbits, may cause the disease. Diseased teeth also are frequently the cause of acute maxillary sinusitis. Notwithstanding the indirect and well-guarded connection of these sinuses with the nasal passages (by means of small devious canals and sulci opening into the infundibulum of the middle meatus), it is rather surprising that they are not more often seriously involved in the acute and chronic affections of the nasal passages, inasmuch as they belong to the same lymphatic district and are under the same neurotrophic influences as the nasal passages. Their escape from injury so often during the course of measles, facial erysipelas, and influenza, is especially remarkable.

Symptomatology.-When the whole upper respiratory tract is the seat of acute inflammation, it is difficult to distinguish by the symptoms which one of the accessory sinuses particularly is affected, as the pain, aching, and distress presented can be easily attributed to the swelling and other vasomotor disturbances of the pituitary membrane itself. When, however, during the course of a common coryza or influenza, pain or aching (neuralgia), sense of fulness or other distress seems fixed in the locality of the "side cavities" of the nasal passages, it may be taken as an indication of a sinusitis of that particular cavity. The clinical signs of acute frontal sinusitis are often confounded with infra-orbital neuralgia from other causes. It is always preceded, however, by coryza for some days, and commences by a very clear neuralgic pain seated about the region of the frontal sinus. Outside the period of the paroxysm the pain may be brought on again by sneezing, or rapid movements of the head; an important symptom is the pain produced by tapping with the finger upon the anterior wall of the sinus, though this sign may, however, accompany ethmoiditis or neuritis of the orbital branches of the facial nerve. The rhinoscope may show a little muco-pus in the 
posterior nares or about the hiatus semilunaris, or tumefaction of the middle turbinates. When there is inflammation of either one of these sinuses, there may be no unusual secretion at all, and no visible swelling of the external parts, to indicate the seat of the trouble. Inflammation or irritation of the antrum, the frontal or ethmoid sinuses, may linger for a considerable time after a severe attack of influenza or acute rhinitis without terminating in suppuration-gradual recovery taking place, with or without extra mucous discharge into the nasal passage, yet the possibility of suppuration and empyema should be kept in view in every case, especially when the local symptoms continue, with severe or constitutional disturbances superadded. Dr. F. C. Cobb and many others call attention to the bad results which may follow neglect of the accessory sinuses during the course of and directly after attacks of influenza, measles, and similar diseases.

Diagnosis.-As a rule, in all of the acute catarrhal inflammations of the accessory cavities the symptoms or signs are so similar to those of a severe rhinitis that an exact differential diagnosis becomes almost impossible. No dependence can be placed upon the character or quantity of the discharge as a distinctive sign, for a colloid or coloured mucus may issue from any one of them into the middle meatus or pharynx, when either one is affected. M. Moll reports to have seen cases of empyema even of the antrum maxillaris without any pain about the eye or the orbit, or without a sense of pressure or pain in the canine fossa, or teeth, or without any complaint of supra-orbital neuralgia. The writer has met with similar cases. Such cases may go on to recovery without surgical interference, as the disease, whatever it may be, of the nasal passages subsides. When, however, a neoplasm is the cause of the disorder, the aspect of the case becomes chronic, the discharge from the accessory cavity soon becomes changed to pus or sero-pus, while the new growth, by its pressure upon the surrounding parts, brings on other distinctive signs, such as swelling, deformity, localized tenderness, and pain, together with more or less pyrexia and other constitutional symptoms. 


\section{Chronic Diseases of the Accessory Cavities}

Catarrhal inflammation, suppuration, neoplasms, and various conditions due to injury or deformity, comprise the common chronic affections of these cavities. The disease process is usually confined to the lining membrane of the cavity, which in all of them is internally blended with the periosteum. When their osseous walls are involved, it is usually due to neoplasms or other foreign bodies, the influence of some strong local infection, such as "farcy," or to some dyscrasia, such as syphilis. The ethmoid cells and the antrum of Highmore are more frequently the seat of disease than the others.

Simple chronic catarrhal inflammation gives rise to an excess of secretion which is mucous or muco-serous in character; but if for any reason the drainage of the cavity be obstructed, and some new infecting agent gain access thereto, then suppuration, with formation of pus, may take place. When there is retention of the secretion (muco-serum) in a simple catarrhal inflammation, the case is known as a mucocele; but when the products of suppuration (pus) are retained, the case is known as an empy. ema. Empyema of any of the sinuses may occur, but that of the antrum of Highmore, or maxillary sinus (which is the largest of all), is perhaps the most common. The statistics regarding the frequency of the occurrence of these empyemas, especially those of the antra and the frontal sinus, are probably misleading, inasmuch as there are many cases occurring in the practice of dentists, general surgeons, and ophthalmologists respectively, which are never placed on record. Again, there are undoubtedly many cases operated upon, and reported as empyemas, which are simply sub-acute or chronic catarrhal inflammations. The writer and his associates have met with comparatively few cases of empyema of the accessory cavities in a large clinical experience, although the antrum, as Bryan states, from its close relationship to the nasal passages as well as to the alveolar arch, is necessarily quite liable to suffer from extensions of disease from either of these localities.

General Etiology.-Chronic rhinitis, deformities of the nasal septum and turbinated bodies, neoplasms, and diseases of the teeth, are the most common causes of chronic catarrhal and suppurative inflammation of the accessory cavities. 
Among the neoplasms, nasal polypi, more often than other neoplasms, lead to chronic sinusitis of the ethmoid cells and frontal sinus, on account of the obstruction which they offer to free nasal drainage and the irritation which is maintained by their pressure. Syphilis, especially tertiary, is frequently the primary cause of suppurative inflammation of the ethmoid cells, frontal or sphenoidal sinuses. Injuries which have produced fracture of the nasal bones, or displacement of the turbinates, are also frequently the cause. Phosphorus and other mineral poisons may give rise to disease of the sinuses, inflammation, caries, or even necrosis of bone. Dr. Henry L. Wagner reported a case of sero-purulent maxillary sinusitis from lead poisoning in a patient thirty-two years of age, in whom the disease had existed a long time and had given rise to per. sistent supra-orbital neuralgia.

Age.-Diseases of the accessory sinuses are not confined to any age. Ethmoiditis, however, is perhaps more commonly met with in children as a result of severe or neglected rhinitis. Empyema of the antra may likewise occur in children from errors in dentition, or from supernumerary teeth, one of which may project into the antrum, acting as a foreign body (Wythe). Pedley (as reported by Bryan) saw a case of empyema of the antrum in a child eight years old, the result of caries of a canine tooth. Power met with a case of antral empyema in a child eight weeks old, which it is supposed was caused by an injury inflicted by the obstetric forceps during delivery.

Sex.-Males are more frequently affected than females. This is accounted for by the difference in the mode of life of the sexes. Ethmoiditis, however, is quite as frequently met with in females.

Antrum.-Empyema of the antrum may be open or latent. In the former the discharge is visible more or less in the nasal passages or pharynx, while in the latter it is pent up, and does not flow out into these passages.

There has been considerable discussion upon the question whether antral diseases (especially suppuration) arise oftener from extension of disease from the nasal passages than from the teeth and alveolar process. Heath, Semon, Fränkel, Moritz Schmitt, and Küchenbecker assert that disease of the teeth is the most common source of the affection, while Bosworth, Zuckerkandl, Krause, Hartman, Ziem, Bronner, MacDonald, 
and others, believe that in the majority of cases it results oftener from disease of the nasal cavities. Küchenbecker found that out of $3 \mathrm{I}$ cases, 33 per cent were the result of dental caries; 22 per cent were due to general diseases; Io per cent to tumours; 22 per cent to unknown causes, and only I 3 could be traced to nasal origin (Bryan). Talbot, of Chicago, examined 6,000 antra which showed 1,274 abscesses molares. Of these molar abscesses 76 ( 6 per cent) extended into and apparently discharged into the antrum. He further states that in the treatment of 384 cases of pulpless molars during the past twenty-four years, only 4 cases of diseased antra were found. Although the first and second molar teeth are the ones usually found diseased, yet the writer has observed one case in which disease of a second bicuspid seemed to light up the antral malady, while Zuckerkandl thinks that the extension of inflammation from the nasal passages directly into the antrum is a frequent cause of the disease. Bosworth thinks that such extension through continuity of the tissues is extremely rare, but that obstruction to the ostium maxillare, by causing retention of the secretion (which subsequently undergoes chemical change), is what usually brings about the diseased condition. He therefore ascribes to hypertrophy of the middle turbinates and to polypi of the nasal passages the principal part in the origin of the majority of cases of maxillary disease. Of the cases which have come under the writer's observation, the majority have followed or accompanied dental diseases or operations. Diphtheria of the antrum as an extension of nasal diphtheria is said by Bryan to have taken place. Bryan also describes a phlegmonous form of inflammation of the antrum which may arise from an extension of a similar infective inflammation from the tongue or pharynx to the antrum. He states that a severe inflammation of the antrum may be secondary to laryngeal croup. The prevailing opinion is that the disease often arises from septic infection from the nasal passages.

\section{Eтhmoid Cells}

Etiology.-Chronic diseases of these cells arise in a majority of cases from long-continued rhinitis or polypi. It may be produced by infection, foreign bodies or tumours in the nasal passages, by syphilis, or by traumatism. 
The writer is convinced, however, that measles, diphtheria, and influenza are frequently the causes. Scarlet fever and typhoid fever do not often lead to it, because those diseases only exceptionally affect the nasal passages to any extent. The causal relation of nasal neoplasms is possibly overrated, for, according to the writer's observation, a large majority of cases of ethmoiditis, both in children and in adults, have not been preceded or accompanied by any form of neoplasms whatever (see Fig. I18). On the other hand, Grünald and others

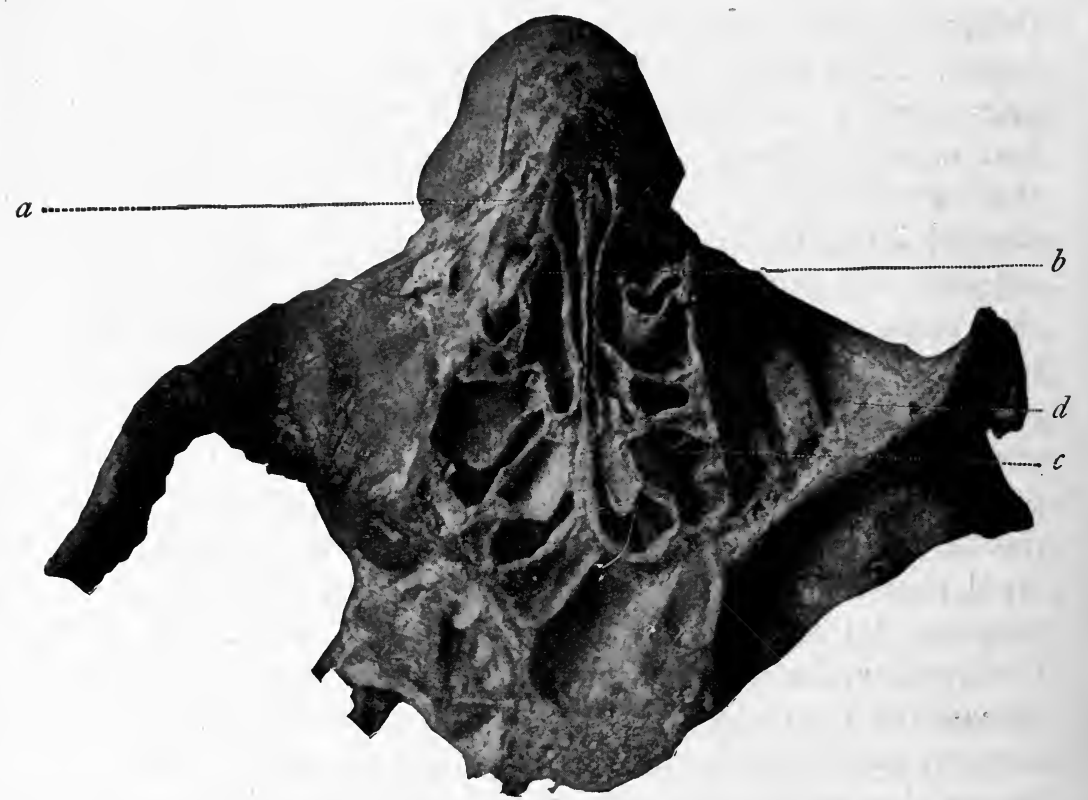

FIG. Ir8. - Horizontal section through ethmoid cells (Hartmann's Atlas). $a$, septum nasi ; $b$, anterior ethmoidal cells ; $c$, posterior ethmoidal cells ; $d$, orbit.

seem to think that nearly all cases of necrosing ethmoiditis are connected with the presence of polypi. There are many instances where chronic suppurative inflammation of the middle and superior meatus or nasal attic accompany or follow diphtheria, typhoid fever, influenza, small-pox, or traumatism, that remain confined to the lining membrane, and do not seem to implicate the bone or the ethmoid labyrinth particularly.

Facial erysipelas may cause suppuration of the ethmoid cells by the extension of the inflammation through the nasal duct or nasal passages. Zuckerkandl, however, believes, on 
the contrary, that the nasal inflammation leads to erysipelas in such instances. Traumatism, especially through meddlesome and unskilful application of chemicals, or instruments such as the chisel, gouge, drill, curette, or the galvano-cautery knife, may cause a purulent inflammation of the nasal attic and lining of the ethmoid cells, either with or without injury to the olfactory filaments. Disease and neoplasms of the orbits are also frequently responsible for inflammation of these cells (Bryan).

A rhinitis described as rhinitis caseosa by Duplay, in 1874 , is considered to be one of the chief causes of chronic ethmoiditis. Potequet, however, denies the existence of such a disease, and states that Duplay's conclusions were based on errors of diagnosis. Cozzolino, on the other hand, recognises it, but says that it is a very rare disease. He considers it a desquamative rhinitis, which is observed principally in "scrofulous subjects." $\mathrm{He}$ thinks it is analogous to a condition of the middle ear, where "cholesteatomatous masses" are found in the tympanic cavity and mastoid cells, and suggests the name of "cholesteatomatous rhinitis." Schleicher believes the caseous secretion is accounted for by a degeneration of mucous polypi. Lennox Browne says that no satisfactory account of the disease has been given, but that it seems to be due to long-retained secretions originating in the superior meatus, or in one or more of the accessory cavities of the nose, and that it is usually associated with caries of the ethmoid bone. He states that Hall has seen the sphenoidal sinuses filled with this caseous material in five instances in dissecting-room subjects of advanced age, in which there was no disease of the bone present. In many cases the writer believes that this affection commences as a chronic rhinitis, developing in the course of an attack of la grippe or influenza, extending to the ethmoid bone subsequently, where it terminates in suppuration and possibly caries of the bony trabeculæ of the ethmoid cells-in other words, a " necrosing ethmoiditis " or "ethmoiditis granulosa."

The author has met with several cases of this sort, the origin of which could be traced to an attack of influenza, and the course of which exhibited the features of an ethmoiditis with caseous discharge, and ultimately caries of the ethmoid cells. The peculiar character of the secretion (known as caseous) is not common, and when occurring is probably due to some constitutional physical trait in the individual rather than to any 
peculiar pathological process. In one case of the writer's the discharge was very great, and composed mainly of fat and pus. The writer observed this caseous secretion once in a case of empyema of the antrum of long standing, in which there was limited caries of the alveolar process of the maxilla.

Diagnosis.-In well-marked cases the diagnosis of abscess of the ethmoid cells is not a difficult matter when the probe is freely used, but in the majority of instances, the subjective symptoms being ambiguous, it is difficult to determine whether or not the neighbouring sinuses are implicated.

\section{Frontal SinUS}

Etiology.-The frontal sinuses are very seldom the primary seat of more than a transient inflammation, excepting from the presence of tumours, which are then generally extensions of malignant disease from the neighbouring nasal passages or orbits. Some cases, however, arise from the development of gummata, or from traumatism. Sometimes these sinuses are the seat of a catarrhal or suppurative inflammation, which has extended to them by contiguity from previously diseased posterior ethmoid cells.

Retention cysts, osteomata, and malignant growths are very uncommon in this situation, but are occasionally met with, the latter, as mentioned above, invading the frontal sinus from neighbouring parts, such as the orbit, nasopharynx, or nasal passages.

Dr. F. B. Eaton lately reported an interesting case of retention cyst of the frontal sinus in a patient twenty years of age.

Sphenoidal Sinus.-This sinus, from its anatomical situation, is less frequently affected with either catarrhal or suppurative inflammation or with neoplasms or injury than its fellows, although Winslow and some other writers suggest that disease in this sinus is often overlooked. When diseased, it is usually in consequence of syphilis, or an extension of inflammation or other disease from the ethmoidal sinuses, the vault of the pharynx, the orbits, or the brain. The continued pressure upon the sphenoid bone of a developing malignant growth situated in the vault of the pharynx, or the growth of a syphilitic gumma arising from the inside of the skull and invading the sinus from the outside, are the most frequent causes of its disease. 
Neoplasms rarely originate in the sphenoid sinus, although a few cases of sarcoma have been found there.

Symptomatology.-The symptoms of chronic disease of the accessory cavities are chiefly local and objective. Exceptionally, however, as in the case of active or extensive suppuration or periostitis, the system may become affected by septicæmia from absorption of the products of suppuration and necrosed tissue, or the general strength may become impaired as an effect of severe and long-continued pain and anxiety. The pain, however, is usually of a dull, aching character, and is referred to the location of the sinus (the maxillary or frontal region principally) affected; exceptionally, however, it may be of a lancinating character and paroxysmal (neuralgia), especially when the frontal sinus is the seat of the disease. When the opening of the sinus is completely obstructed, the distress may be expressed as a sense of excruciating pressure. The writer had a case of frontal empyema in which the patient suffered excruciating pain over the frontal region and through the eyes (requiring large doses of morphine frequently repeated to secure relief) until the pus was evacuated. Tenderness over the inner portion of the orbit and the supra- or infra-orbital region may accompany the pain in cases of disease of the frontal, sphenoidal, or ethmoid sinuses. Pain referred to the top of the head is a symptom of sphenoidal disease. Vertigo and attacks of syncope, or persistent occipital pain, with vertigo and disturbances of vision, may be symptoms of disease in either the frontal or sphenoidal sinuses. Continued pain in the frontal region should always arouse suspicion of a sinusitis, especially if accompanied by ocular disturbance. In many cases where the distention of the cavity is insignificant no pain may be complained of at all. The continuance of pressure symptoms and disorders of vision usually denote neoplasms, whether any change of external contour be presented or not.

Objective Signs.-One of the chief objective signs in cases of chronic suppurative inflammation of any of these sinuses, or when neoplasms are therein, is the discharge of pus, mucopus, or sero-pus into the nasal passages or pharynx. The discharge may be continual or intermittent. The quantity, consistence, and other characteristics of the secretions will vary, of course, according to circumstances, but it will generally be found in the nasal passages or nasopharynx, in the middle 
meatus, on the posterior ends of the middle turbinated bones, or trickling down the posterior wall of the oropharynx. If the maxillary sinus be affected, the quantity of pus may be greater and its consistence thinner. The discharge will usually be intermittent. If the ostium maxillare be closed, the face may become swollen and tender to the touch on the affected side, and there may be slight œdema of the lower eyelid or upper lip, while the gums may appear swollen and tender on the affected side. The patient will complain of more or less pain about the cheek, or suffer from persistent facial neuralgia, with a sense of weight referred also to the affected side. If the secretion be imprisoned for a considerable time by closure of the ostium or infundibulum, the pus may ooze out at the inner canthus of the eye, and the patient will then present the "frog-face" appearance. This feature may be present when there is extensive dental caries, alveolar periostitis, or necrosis of the maxillary bone.

Ethmoid Cells.-The special signs connected with disease in this location are the continual appearance of pus in the middle meatus, enlarged or soggy turbinates, a sense of great obstruction and tenderness referred to the upper nasal region, more or less headache, and possibly weakness of vision. There may be also supra-orbital neuralgia, asthma, hay fever, or diverse reflex disturbances affecting the eyes, ears, or throat.

Frontal Sinuses.-Pain over the frontal region of a dull character (headache) is generally complained of, but when the sinus is distended either from retained secretion or a neoplasm, there is great tenderness upon pressure over the base of the nose or inner wall of the orbit, together with constant frontal headache. External swelling in this region may occur together with exophthalmos and photophobia, and extra supra-orbital tenderness, especially if the neoplasm be present. The objective symptoms pertaining to this locality are rather obscure, and are very like those which might accompany ethmoiditis, a cerebral tumour or other structural cerebral disease, a developing gumma, or a systemic affection involving the nasal cavities. In sphenoidal abscess or empyema the pain is usually persistent and referred to the top of the head, while the vertigo, though mild, is quite a permanent symptom. The character of the pus or discharge is not characteristic in disease of any one of these sinuses. When coming from either of them it may 
be fetid, quite thin, quite thick, or appear desiccated into crusts. It varies in colour through all the shades of yellow and green, or, if containing blood, may be reddish in colour.

\section{The Differential Diagnosis of Diseases of the Accessory Cavities}

As stated before, the differential diagnosis in regard to the location of the disease is difficult in many cases. In all suspected cases a thorough cleansing with a solution of borax, bicarbonate of soda, common salt, or some other mild alkaline solution, applied either with atomizer or syringe (preferably the post-nasal syringe), should precede examination.

As stated before, the appearance of pus in either nasal cavity, or upon the posterior wall of the pharynx, is, as a rule, unmistakable evidence that one or the other of these sinuses is involved, unless there be a foreign body in the nasal chamber. In many instances suppuration of two or more of the accessory cavities is taking place simultaneously. In others the antrum may have become diseased through being the receptacle of the drainage from the suppurating ethmoid or frontal sinuses above. Empyema of the antrum, with closure of the ostium maxillare, may occur, however, without any discharge of pus being visible in the nasal passages or pharynx. Likewise, if occlusion of the passage from the ethmoid cells or frontal sinus to the infundibulum takes place, there may be no pus or muco-pus seen in the nasal passages. In such an event the differential diagnosis will depend upon other less distinctive signs, and a thorough examination with the probe. It is a matter of common experience, as stated by Watson, Bryan, and others, that in empyema of the antrum the discharge is generally intermittent, taking place after the cavity is filled up to the level of the ostium maxillare (so to speak), and is therefore ready to overflow. For this reason the principal discharge may take place two, three, or four hours after rising in the morning. In suppuration or empyema of the ethmoidal or frontal sinuses, on the other hand, the discharge is continuous, and not dependent upon the position of the head, and of the sphenoidal sinus the discharge is seen mostly on the walls of the nasopharynx; hence, for purposes of differentiation, it has long been recommended that after the thorough cleansing of the nasal passages by spray or douche, the patient be directed 
to throw the head to one side for a minute or so, or assume the position recommended by Fränkel, to bend the body forward and place the head down between the knees. After either of these movements, if the antrum be the site of the trouble, a quantity of pus will be seen at once in the middle meatus, whereas, if the ethmoidal or frontal sinus be the site of the disorder, no particular increase in the amount of discharge will be observed either in the nasal passages or upon the posterior wall of the pharynx. Schech, and many dentists, recommend tapping the first and second molar teeth of the affected side, in which event not only will the patient experience sensations of soreness if the antrum be affected, but the tapping may be followed by a slight pouring out of discharge through the ostium maxillare. In subjects with wide and otherwise favourable anatomical formation of the nasal passages, or with a patulous ostium maxillare, the pus may be seen issuing from the ostium maxillare almost continuously, whether the antra or the ethmoidal or frontal sinus be affected; such cases are, of course, puzzling.

In doubtful exigencies, probing the antrum has been recommended. This is done by passing a small probe with its point bent at almost a right angle along the middle meatus, until the ostium maxillare is felt, whereupon the instrument is given a little twist downward and forward, in order to penetrate the opening into the antrum; if pus be present in any considerable quantity in the cavity, it will be seen oozing out through the opening upon the withdrawal of the probe. Krause recommends, in cases of doubt, that the antrum should be perforated by a trocar and, if necessary afterward, the cavity injected through the opening thus made with a solution of peroxide of hydrogen, so that if pus be present in the cavity, the characteristic foam always observed to take place when peroxide of hydrogen and pus are mixed will be displayed by the fluid flowing into the nasal cavity. Often, owing to the difficulty (from an overhanging or balloon-shaped middle turbinated bone) of either finding the ostium with the probe or perforating it with a trocar or drill, this plan will be found impracticable without removing the end of the turbinate.

Krause, Oaks, and Mikulicz recommend perforating the antrum from the inferior meatus instead of the middle meatus. This is, however, more painful and more difficult, owing to the 
thickness of the wall in this situation, and is also oftentimes impossible, owing to the narrowness of the nasal passages of some subjects. When this course is decided upon, however, the trocar of Krause or Myles's cannula needle (see Fig. I i9) will be found most adaptable. In place of this instrument the electric drill may

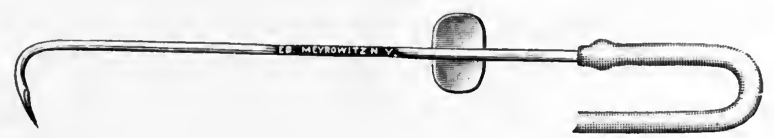

FIG. II9.-Dr. Myles's curved cannula needle for perforating the antrum of Highmore through the middle nasal meatus.

be used if the opposing septum does not interfere. The use of Hajek's straight cannula needle (see Fig. I2I) is probably the simplest, and can be introduced easily.

Illumination.-Voltini, Heryng, and Krause were among the first to suggest and practice transillumination for the diagnosis of empyema of the antrum. For this purpose a small electric lamp (see Fig. 120) is to be introduced into the mouth and held in the median line, after which the lips are closed around it. In this way the two antra are illuminated under normal conditions; but if pus or a tumour be present in one or the other of them, the illumination of the antrum on the affected side is obscured by a shadow or dark patches. The method of Renult consists of placing the lamp in the lateral part of the mouth, instead of centrally on the tongue. By this method, under normal conditions, there will be seen on each side, at about the level of the nasal bone, a clear zone of illumination irregularly oval, and not as bright as the corresponding sub-

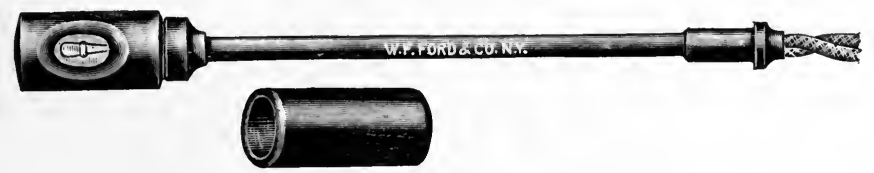

FIG. I20.-Lamp for transillumination.

orbital region, but clearly distinct from the darker parts (J. F. Oaks). In addition, there may be observed also the pupillary reflex of Davidsohn, which consists of an illumination of the pupil of the eye on the sound side. Another illumination sign pointed out by Garel is the absence of luminous perception on 
the affected side. The Röntgen ray is coming to be one of the most certain methods of examination to one who has practised the art, for by it abnormalities of the antrum may often be made out, as well as its contents. In cases of doubt, however, perforation through the nasal passage by means of the cannula should be adopted. Dr. D. V. Kyle, of Philadelphia, introduced an instrument for illuminating the posterior nares, which consists of a small incandescent lamp of four voltage power, placed on the end of two light insulated copper wires, similar to an electrode. This is covered with an aluminum cap, which is fenestrated so as to allow the passage of the light. The instrument is very small. The aluminum cap protects the part from the heat which constitutes such an objection to many other electrical devices of this sort. The cap will remain cool for at least half a minute, and by interrupting the current frequently it may be kept cool for a longer period. Although very valuable as a means of diagnosis in diseases of the antrum, transillumination cannot always be depended upon entirely for differentiation, for in subjects where either of the antra are occupied more or less with bony projections or partitions, a cystic tumour, or a mucous polyp, the results of illumination will prove inadequate. Talbot, of Chicago, in his examination of 6,000 antra, found septa in 963 of them. There are also recorded instances of failure to obtain pus from antra where transillumination had furnished signs of its presence. The illumination signs referred to the orbits as recommended by Davidsohn and Garel may be also unreliable, on account of the normal variation in the anatomical formation of the skulls of human beings. Nevertheless, there are instances where these methods of illumination may furnish the only reliable guidance as to the location of an empyema, whether in the maxillary or frontal sinus, or even in the anterior ethmoid cells. In the writer's experience, however, this method of diagnosis has been very often unreliable regarding the antrum of Highmore. For instance, in a case where the darkness of the antrum of one side, under illumination, seemed unmistakable to all of those present, repeated perforation failed to obtain any fluid or give evidence of any disease whatever. In both instances the site of disease proved to be in the ethmoid cells. Where the diagnosis cannot be made out after a careful observance and comparison of the clinical signs and symptoms, the practitioner is 
then justified in opening the antrum; and if the result of this proceeding be negative, then it will be fair to assume in almost all instances that the disease is located in the ethmoid cells or the other sinuses. If polypi be observed anywhere in the nasal passages, it is unnecessary to state that their removal should be effected as a preliminary step.

It has been proposed, as a means of diagnosis for disease of the antrum in cases where the ostium maxillare is very much narrowed (after first closing the nasal passages), to blow a current of air through the nares with considerable force by means of the Politzer air bag. The effect of this will be to draw out through the ostium some of the discharge into the middle meatus. Should this happen, of course it will serve to locate the disease in the antrum. It is also suggested as a means of treatment for emptying the antrum in cases where the discharge is very thick and of a colloid nature. Bryan, however, points out a danger in this method by the propulsion of some of this discharge into the mouth of the Eustachian tube. The same author (Dr. J. B. Bryan), in an interesting report of a case of suppurating ethmoiditis terminating in caries (in the New York Medical Journal, vol. lvii), thinks that the diagnosis of ethmoiditis can usually be made out from the following symptoms when they are present; viz.: a swollen condition of the middle turbinate body; the presence of pus in the middle meatus, and, if the posterior surfaces are involved, the presence of pus flowing over the posterior extremity of the middle turbinated body into the posterior nasal space; the absence of the "pulsating light reflex" which is observed in abscess of the antrum; swelling at the inner angle of the orbit; exophthalmous; narrowing of the field of vision; pressure over the inner angle of the orbit, causing pain in the orbit and along the infraorbital ridge. The presence of pus in the middle meatus is common to inflammation of the frontal and maxillary sinuses as well as of that of the ethmoidal sinus, and it is very difficult at times to trace out its source; but according to Max Schaefer, we have in pain a much more reliable symptom in discriminating between abscesses of the respective sinuses. In the case of the frontal sinus, pain is felt at the right or left of the nose, and extends along the infra-orbital ridge. In the writer's experience, pain is generally absent in chronic abscess of the antra. The complications of suppurating ethmoiditis most frequently 
met with are abscess of the antrum, abscess of the orbit, and meningitis. Abscess of the antrum frequently complicates suppuration of the ethmoid cells. The frequency of this complication is probably due to the proximity of the two openings and the continuity of their lining membrane in the infundibulum, so that inflammation of the nasal passages affecting one cavity is liable to extend to the other. Besides this, any mechanical obstruction, either from polypi or hypertrophic rhinitis, causing retention of mucus in one or both sinuses, may thus pave the way for infection and suppuration. Again, pus accumulating in the middle meatus may work its way subsequently into the antrum ; and sometimes, owing to an anomalous condition of the ethmoid bone, in which the anterior and posterior ethmoid cells communicate directly with the antrum, both sinuses may be simultaneously affected. We do not know how often this anomaly occurs-perhaps oftener than we suppose. In some instances where the neighbouring sinuses thus directly communicate with each other the openings may be pathological; but in others they may be natural, as illustrated in some specimens in the Army Medical Museum, in which the frontal sinus opens directly into the summit of the antrum. Bryan and other writers prefer, after opening up this region, to use the snare and the sharp curette rather than the drill.

Concerning the detection of suppuration and abscess of the upper sinuses-ethmoid, frontal, and sphenoid-much reliance may be placed upon skilful probing. Grünwald and other authorities seem to consider such manipulation easy of accomplishment, and of paramount value in nearly all cases. While the great value of the probe is admitted by every practitioner, still its usefulness over every other method of investigation has been exaggerated by many writers. Any sort of a probe will not answer the purpose nicely. The instrument should have a dull, flattened extremity, be ductile enough to admit of bending at various angles, and yet firm enough to hold its shape under moderate pressure. With such an instrument in dexterous hands the upper nasal passages can be thoroughly explored, and any denudation, caries, or granulation tissue detected. Rhinoscopic examination is also necessary in order to ascertain the condition of the posterior portions of the meatus. In this way so-called caseous ethmoiditis of the posterior cells may be at once discovered. The ocular and psychic symptoms 
are to be carefully weighed in cases of doubt, as their presence will often indicate the location of the disease in the proper sinuses. Neuralgia affecting several branches of the trigeminus may be a symptom of the disease located in one only or more than one of the accessory cavities. Mental disturbance, such as melancholia with homicidal or suicidal tendency, has been known to depend upon empyema of the frontal, ethmoidal, or sphenoidal sinuses. Indeed, meningitis and cerebral abscess may follow disease of the walls of either the frontal, ethmoidal, or sphenoidal sinuses. The differential diagnosis will often require a careful consideration of the following symptoms: Suppuration, ocular disturbances, and neuroses, such as headache, neuralgia, etc.; and the employment of the following manipulations, such as rhinoscopy, transillumination, percussion of teeth, probing, and exploratory puncture.

Treatment.-Acute Sinusitis. - The acute diseases affecting these cavities usually need no other local treatment than the careful application of mild detergent and antiseptic lotions to the nasal passages by means of spray or posterior douche. The application externally of ice bags or cold lotions may be of service where there are no contra-indications, especially for the relief of cephalalgia and frontal distress. These applications may be placed over the nose, cheeks, or frontal region, as indicated. When cold applications are inadmissible, relief may be obtained from warm or hot external applications, or douches through the posterior nares of a hot solution of salt and water (one dram to the pint). Sometimes a hot emollient douche of flaxseed or slippery-elm tea, or an infusion of dried peaches, to which has been added salt or bicarbonate of soda in the proportion of a teaspoonful to the pint, will afford great relief. Either one of these medicinal douches may be used every two or three hours, if necessary. When the distress is not relieved by these simple measures, inhalations of menthol, alone or in solution with alcohol or spirits of camphor, may be tried. Finally, inhalations of chloroform or ether, or a spray of a solution (five per cent) of cocaine, may be required. If suppuration takes place the pus must be promptly evacuated.

\section{The Chronic Affections}

Chronic catarrhal inflammations of the accessory cavities require carefully studied plans of surgical treatment and medicated 
douches. When decided hypertrophy of the turbinals, neoplasms, deformity of the cartilaginous or bony septum of the nasal passages are present, these defects should be corrected at once by means of the galvano-cautery, the snare, the saw, the chisel, the drill, or such other surgical measures as the judgment of the practitioner may dictate. If, however, such abnormalities do not exist, excepting perhaps in a minor degree, and the case is not a very chronic one, then topical medication, by douches, will usually be sufficient. Even in cases of suppurative inflammation at the attic of the nose, topical medication will often be sufficient to bring about recovery. The most efficient means of medicating this region will of course depend somewhat upon the anatomical formation of the parts, but, as a rule, the writer would beg to emphasize the fact that douches or sprays, whatever be their character, projected into the anterior nares, are far less efficient than the same medicaments injected through the posterior choanæ. A moment's reflection upon the anatomy of the nasal passages will suffice to point out the reason for this. The curling turbinals convert each meatus into a sort of cone, whose apex presents anteriorly and base posteriorly. Thus in the majority of subjects the turbinals shield the infundibula and meati from intrusion anteriorly, but, opening out broadly toward the posterior nares, expose the superior and middle meatus posteriorly, so that they are accessible to agents which may be carried through the nasopharynx to the parts. This point was well emphasized by Carl Michell. Besides this, the aurists have demonstrated the danger to the Eustachian tube and middle ear of medication by anterior douches, sprays, and insufflations. The use of powders in different forms of sinusitis is rarely useful, excepting in purulent ethmoiditis with exposure of the cells, when insufflations of aristol, resorcin, europhen, iodoform, or orthoform will be found very beneficial.

Among the medicinal agents which will be found very efficient for local application may be mentioned solutions of biborate of soda, acetate of lead, tannic acid, chlorate of potassium, formate of sodium, carbolic acid and glycerine, boroglyceride with bismuth, nitrate of silver, zinc sulphate, permanganate of potassium, chloride of calcium, mild solutions of creolin, camphor, eucalyptol, creosote, oil of cinnamon, balsam of Peru, menthol, thymol, or oil of tar in petrolina oil or cotton-seed 
oil. Among the most convenient of these various applications for general use the writer would mention resorcin, bismuth and boroglyceride, tannic acid and acetate of lead (see formulary).

When it has been determined that an empyema or abscess is established in any one of these sinuses, of course medicinal medication is useless. Nothing but efficient surgical treatment will be of service; therefore the practitioner must fall back upon the old surgical maxim, "When pus is found, evacuate it."

\section{Surgical Treatment of Chronic Sinusitis and Empyema}

Antrum of Highmore.-Considerable discussion has taken place as to whether the old method of surgical procedure introduced by Hunter, Sir Astley Cooper, Desault, and the older surgeons, ought to be followed, namely, that of opening the cavity of the antrum through the alveoli or the canine fossa, or whether the more modern method of perforating the lateral wall of the middle or inferior nasal meatus is preferable. There are objections urged against each of these methods. The principal objections stated against opening through the alveolar arch are: I, the necessity of sometimes sacrificing a good tooth ; 2 , the probability of contamination of the inside of the cavity from secretions and food from the mouth; 3 , the discomfort which comes to the patient by a trickling discharge into the mouth along the inside of the lips. Besides these, Miller, Freeman, and others, have objected to this plan of operation (the alveolar plan) on account of the danger to the stomach and general system, which the constant presence of pus in the mouth might offer. These objectionable features are supposed to be obviated by opening the antrum through the nasal cavity, so that the discharge may take place into the nose, "its natural receptacle," without causing much discomfort to the patient, while at the same time greatly diminishing the possibility of infection. Besides, it is believed that the antrum can be kept open and syringed much better when the opening is made through the nasal passage. "There are, however, strong objections to the general adoption of this plan for the relief of antral empyema, because ( 1 ) it is very difficult in some subjects to reach the antrum through the nasal fossa; (2) there is quite as much, if not more, danger of infection from the nasal passages than from the mouth or canine fossa; and (3) 
the lowest point of the abscess cannot always be reached by an opening through the nasal passages without danger either from hemorrhage or subsequent inflammation. Perforation through the canine fossa has the advantage of permitting of a larger opening for subsequent treatment, and the possibility of affording better drainage. However, many surgeons believe that the matter of drainage is inferior to the object of obtaining free access to the cavity for thorough after-treatment. Opening through the alveolar process has the advantage of evacuating the abscess from its lowest point and thus more thoroughly emptying it.* Besides this, the operation is a much simpler one, entirely devoid of danger and hemorrhage, and one that can be performed by any good dentist. It therefore seems far preferable, excepting, perhaps, in special cases, to the other ones. Again, some observers object to the alveolar operation, on account of the difficulty of keeping the artificial opening patent, because of the formation of little plugs of coagulated blood and lymph which close the opening after each irrigation, while others think that this is no objection, but an advantage. Many practitioners advise the closing of the opening by an artificial plug of cotton or metal after each irrigation or treatment. Several operators and dentists have proposed the adaptation of a plug and artificial tooth combined, where it is desired to keep the alveolar opening patent. Bosworth, however, thinks a plug is unnecessary. Perforation through the canine fossa is rarely necessary, although a few surgeons prefer it. It does not seem to offer any advantage over the other plans for purposes of evacuating the abscess, while there is a possibility of unskilled operators wounding the infra-orbital nerve, or even perforating the floor of the orbit. Another method which may be resorted to is perforating the roof of the mouth into the antrum.

The Method of operating through the Alveolar Process.-The patient may be anæsthetized with nitrous oxide or chloroform. If either the first bicuspid or first or second molar alveolus be empty, one of these spots should be selected for the entrance of the trephine or drill. If, on the other hand, either of these teeth are not absent, then the first or second molar tooth should be drawn, and a small drill or trephine (propelled by a dental foot engine or an electromotor) is placed in the alveolus and directed upward, inward, and slightly backward, until the 
bone has been perforated. Usually pus will follow the withdrawal of the drill. Sometimes, however, when the collection is more or less inspissated, it will not flow until the cavity has been subsequently injected with fluid. This having been accomplished, the cavity should be washed with a mild solution of peroxide of hydrogen and water (one part to seven) or a 2-per-cent solution of biborate of soda, or 3-per-cent solution of carbolic acid. In either case the solution should always be warm when injected. A small stylet, similar to that used in dacrocystitis, or a probe, may now be inserted and exploration of the cavity be made, in order to ascertain whether a neoplasm be present, or whether there be any disease of the bony wall. After this, the opening may be closed with a little plug of absorbent cotton, or with a stylet and cannula. Usually there is no discomfort to the patient from wearing such plug or cannula. The patient may be directed to inject the cavity two or three times a day, if necessary, by means of a little syringe, having a very small but long cannula extremity. This treatment can be conducted from day to day, until no sign of pus or mucus is observed in the fluid returning from the cavity. The sinus to the antrum may be enlarged at any time, if desired, by the use of a larger trephine.

When all evidence of disease has disappeared, the opening may be allowed to close by granulation. If, on the other hand, there be evidence of caries, or the presence of a neoplasm, a large opening at the canine fossa or the operation of Luc-Caldwell will become necessary.

Perforation through Canine Fossa.-This is an old method of operation, having been practised over a hundred years ago. The operation of Desault and Küster has been the one adopted of late years, and consists essentially of opening the antrum freely with a trephine through the canine fossa, having first severed the overlying soft parts with a knife. This operation has recently been modified by Luc-Caldwell, and De Roaldes, who, after making a large opening into the sinus in this manner, curette the inside and then cauterize it with a strong solution of zinc chloride. A counter opening is also made from the sinus into the nasal passage, and a gauze drain, together with a curved stylet, inserted, so that the sinus can be flushed and disinfected through the nose. After this is done, the mucous membrane covering the canine fossa is sewed together and ailowed to heal at once. The healing usually takes place 
in about three days. This operation is highly extolled by De Roaldes and others, and is sometimes necessary for the relief of some cases. Zuckerkandl and others, however, have pointed out certain dangers from hemorrhage, the wounding of the infraorbital nerve, and severe reactionary inflammation from these operations at the canine fossa. Drilling through the fossa, either with a hand drill, such as that of Freeman, or with an electromotor drill, is usually effectual, excepting in the cases where growths in the antrum or disease of its bony walls exist.

Perforation through the Nasal Cavity.-The nasal wall of the antrum may be perforated near the floor of the inferior meatus, higher up or just under the middle turbinated bone.

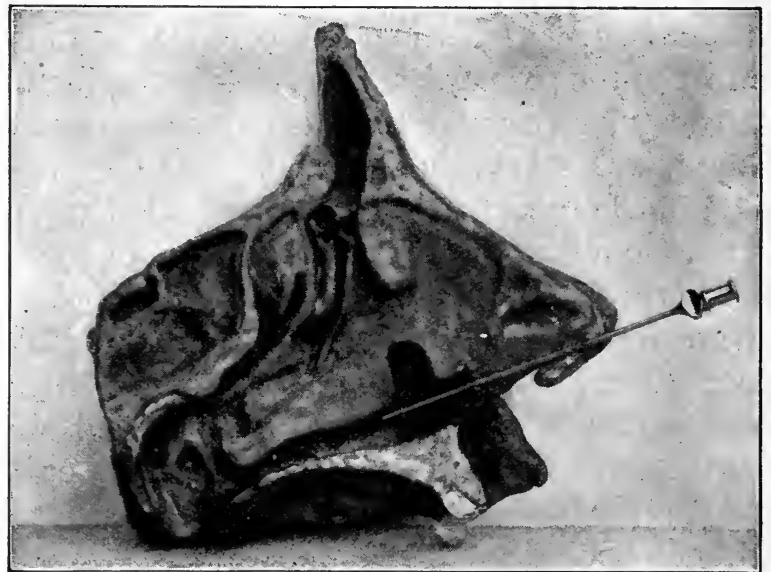

FIG. I2I.-Hajek's straight cannula inserted for puncture of the antrum wall in the inferior meatus (in a cast of the nasal chamber, dissected to show the lachrymal duct, the infundibulum, etc.). (From An American Text-Book of Diseases of the Eye, Ear, Nose, and Throat.)

The former method was proposed by Mikulicz, and the latter operation by Krause, who uses a curved trocar which is pushed up under the middle turbinated bone, quite far back, until reaching the point which is to be penetrated. The perforation is then made by a movement of the handle forcibly toward the septum, at the same time keeping up a slight rotary or boring movement. Krause advises an opening $3 / 8$ of an inch wide and $5 / 8$ of an inch long to be made. He then uses freely insufflations of iodoform. Considerable hemorrhage usually attends either of these operations, and they are often difficult of accomplishment, especially where the meatus is nar- 
row or deformed. Sometimes, for its accomplishment, it is necessary to first remove the anterior portion of the inferior

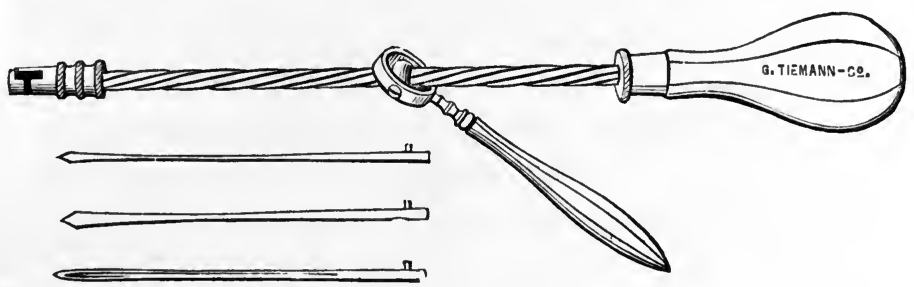

FIG. I22.-Hand drill, with guard.

turbinate body. Where an extensive opening of the antrum is necessary, as for curettement or removal of a growth, an opening through the canine fossa or roof of the mouth is preferable. If the perforation has been made through the lateral wall of the nasal passage, that passage must be washed out from one to three times daily, as well as the antrum, according to circumstances, in order to prevent infection of the antrum by nasal secretion, etc. When the disease seems to be obstinate, and mild detergent solutions prove inefficient to correct or stop the discharge, a weak solution of iodine ( $\mathrm{t} / 2$ per cent), 2- to 5-per-cent solution of nitrate of silver, $1 / 2^{-}$to 1 -per-cent solution of formate of sodium, or 2- to 5-per-cent solution of resorcin, may be injected after each irrigation. Should the discharge become fetid, then I- to 2-per-cent solution of carbolic acid, or 1 to 3,000 bichloride of mercury, or $/ 2^{-}$to 1 -per-cent solution of permanganate of potassium may be used instead.

Treatment of Empyema, or Abscess of the Ethmoidal Sinuses.In this event evacuation of the pus will also be the leading indication. If, upon examination, some portion of the ethmoid bone be denuded, this spot may be drilled into by a small drill or trephine propelled by an electric engine (Fig. I2I) or a hand drill (Fig. I22), and the opening subsequently enlarged by means of the ethmoid curette or spoon (Fig. 123). Very

(

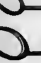

TIEMANN 800 .

Fig. I23.-Buck's nasal and ear curette.

often drilling may be unnecessary, for with the curette a sufficient perforation may be made to evacuate the pent-up pus. In those cases where the diseased region is inaccessible from enlargement or irregular formation of the middle turbinated 
bone, this body, in whole or in part, should be removed either with a snare, the saw, trephine, or drill. In many instances it will be found necessary to remove a portion of the middle turbinated bone with the snare or cutting forceps, or to clip off the anterior portion only. Occasionally, however, it may be necessary to remove the whole of the bone, and possibly a portion of the inferior turbinated bone. There seems to be a fallacy regarding the relation of the turbinated bones to the ethmoid cells. The anatomical fact, is that while the paraethmoid cells are often continuous with the cancellated structure of the middle turbinates in a large majority of instances, the communication between the cancellated tissue of the turbinates and the anterior and posterior ethmoid cells is only accidental; therefore, according to this view, the principal benefit arising from the removal of the middle turbinated bone, as a whole, is one of expediency for clearing the field in order to gain access to the ethmoid cells. When the posterior cells are the seat of disease, a more extensive mutilation of the turbinate, in order to reach them, may be necessary. When the curette is insufficient for restoring the drainage of the diseased tissue, the drill will be found a most efficient auxiliary. Not only one, but sometimes four or five perforations with the drill will have to be made before the pus can be thoroughly evacuated and the parts placed in a condition to heal. For the modus operandi of removing the turbinals, see Chapter XI, on Hypertrophic Rhinitis.

In necrosing ethmoiditis a thorough curettement of the diseased tissue is generally all that is necessary. Care should be taken, however, not to scrape away too much of the mucous membrane.

The after-treatment consists of the thorough cleansing of the parts once or twice daily, by means of the post-nasal syringe, with one of the detergent solutions already mentioned (see formulary), and the insufflation upon the parts of either iodoform, aristol, europhen, or orthoform. Should suppuration continue, despite these measures, for a considerable time, say three weeks or a month, then further operative procedure should be adopted, as it probably will be found that more extensive disease of the ethmoid bone, with possibly involvement of the frontal sinuses, exists. Great conservation in the surgical treatment of these parts should be practised. The 
writer has met with some cases of wanton destruction of the internal nasal apparatus not only by forceful and radical tyros, but by experienced operators. The time has come, therefore, for the flaunting of caution signals in operations on the nasal passages.

Frontal Sinuses.-Empyema of the frontal sinuses is to be treated similarly to the other empyemas, by opening the sinus. These sinuses may be opened from the outside, by trephining out a small disk of the frontal bone covering them, or they may be opened from the inside of the nasal cavity (see Fig. 2). The latter operation is best performed by means of the drill. The drill should be carried along the upper edge of the middle turbinated bone, directly upward and a little backward to the attic of the nose, and, when in position, the motive power applied. More resistance to the drill will be felt in this operation than when the ethmoid cells are being perforated. Care should be taken lest the cranial cavity be entered.

Sphenoidal Sinuses.-The sphenoidal sinus is rarely the seat of empyema, perhaps never primarily. Many difficulties arise in opening this cavity. It is claimed by some rhinologists that it can be opened through the nares by drilling through the body of the sphenoid bone. I quite agree with Bosworth, however, in questioning the accomplishment of this result from this direction, although it is reported to have been done in many instances. The drill or trocar should be carried along the back of the middle turbinated body until meeting the wall at the posterior part of the nasopharynx, when it is pushed backward and upward. Another method, and sometimes a feasible one, for opening the sphenoidal sinus is through the vault of the pharynx. This is accomplished by the insertion of a curved trocar of such shape that it may go around the soft and hard palate with the point directed upward. Being placed in position, it is worked directly upward by a rotary motion through the wall of the sphenoid until the sinus is reached. It is unnecessary to state that great care is required in either method of operation, lest the cranial cavity be perforated. The operation is rarely necessary, excepting when syphilis or malignant disease attacks the neighbourhood (see Bryan, Jour. Amer. Med. Assoc.). Indeed, it is usually through the encroachments of malignant disease situated in the nasal passages or nasopharynx that this sinus is affected. 


\section{CHAPTER XIV \\ NEOPLASMS OF THE UPPER AIR PASSAGES}

THE subject of neoplasms of the upper air passages for the purpose of consideration may be divided into two classes: benign and malignant. Either class may occur primarily in the air passages, or secondarily after invasion of the other parts. The benign growths $(a)$ include myxomata, fibromata, cystomata, adenomata, lipomata and angiomata, papillomata, osteomata and enchondromata, and teratomata (mixed), according to Lennox Browne.

Malignant growths (b) include sarcomata (mucoid, lymphoid, and fibroid) and epitheliomata (alveolar, stratified, and columnar).

The mixed growths, so-called $(c)$, commonly met with are myxo-sarcomata, fibro-sarcomata, and those occurring in connection with some dyscrasiz, as syphilis or tuberculosis, which include condylomata, gummata, tuberculosis, and lupus.

Certain forms of these growths are commonly found in particular regions, as, for instance, the myxomata in the nasal passages, the papillomata in the larynx, etc.

General Histology.-The general histology of the neoplasms found in the upper air passages differs but little from that of similar growths in other situations.

General Etiology.-Excepting those neoplastic forms evolved from syphilitic, tuberculous, or traumatic lesions, the essential etiology of the neoplasms, both benign and malignant, found in the upper air passages, is largely a matter of speculation; indeed, the words of Marcus Aurelius might well be applied here: "Remember that all is opinion"; likewise, regarding malignant growths, unless we accept the dictum of Virchow, that continual irritation of normal tissues may lead to malignancy, the same lack of definite knowledge obtains. Regarding laryngeal growths, especially papillomata, some ob- 
servers adhere to the belief (among these particularly Lennox Browne) that irritation of benign neoplasms by mechanical or escharotic means may convert them into malignant growths. Lennox Browne, quoting his own and the statistics of M. Mackenzie and Semon, states that a change from a benignant to malignant nature may occur in the same neoplasm. J. L. Goodale reported a case of cancerous degeneration of a laryngeal papilloma having taken place in a man sixty-four years of age. The first tumour was removed by the mouth, examined microscopically, and pronounced benign. There was recurrence of the growth, and the larynx was removed. Microscopic examination of this growth showed it to be an "epidermoid cancer." He also reported a case of carcinoma of the nasal passages in a man fifty-one years of age, which had apparently developed from polyps, with which the man had been troubled for fifteen years, having had them repeatedly removed (Jour. Amer. Med. Assoc., February 3, I900). Dr. Charles Phelps, of New York, cites a case of fatal and rapidly growing epithelioma of the larynx, which, from previous observation and the clinical history, was probably benign in the beginning. The conclusion which he draws from this and other cases of neoplasms coming under his observation as a surgeon is, that early and radical removal of neoplasms, whether benign or malignant, should be the aim of the practitioner. Recently, Bryson Delavan reported a case in which malignant disease seemed to supervene in a case of catarrhal laryngitis with pulmonary phthisis. J. SolisCohen mentions a case in which, after thyreotomy for a simple laryngeal papilloma, epitheliomatous disease developed in the cicatrix of the operation wound, while the interior of the larynx remained free. An example is reported by Schnitzler of a papilloma, which had been removed and the base burned with caustic, being followed by carcinomatous disease and perichondritis. He could recall only three such instances in his experience. Storck believes that this malignant transformation is hastened by endolaryngeal operations, especially by burning. Bohmer narrates a most interesting case of this malignant degeneration. Schnitzler also reports a case of what may be called double transformation-that is, an example of a benign papilloma which changed first into a carcinoma and then into a sarcoma. Other instances of this sort are reported from time to time in the medical journals. 
Dr. Delie, in the American Journal of the Medical Sciences, I898, p. 235, relates a case of apparent transformation of adenoids into round-cell sarcoma. The patient was a boy aged thirteen, who was operated on for removal of adenoids. They recurred several times, and at last microscopical examination showed the removed tissue to be sarcoma, although the microscopical examination of the tissue removed in the earlier operations showed it to be adenoid. There was considerable hemorrhage, and finally death took place.

The writer's experience and observations concerning this question accord with the majority of observers up to this time, that papillomata and adenomata, perhaps, are liable to become malignant in the course of time, not alone by excitation from escharotics, electric or mechanical irritation, but also when no such irritation is applied. This is manifestly the case with adenomata affecting the mammary gland, as any practitioner can attest ; and it is equally true of papillomata and adenomata of the pharynx and larynx, as the writer has had occasion to observe in five cases, the metamorphosis of which was exemplified by microscopic examination; yet it must be admitted that the number of recorded cases in which benign growths have unmistakably become converted into malignant ones by local irritating treatment is too small to justify the promulgation of a law to this effect. It must be remembered, however, that a wide field for error opens up in this connection when we consider that the histologic characters of papillomata and squamous epitheliomata are not very diverse; and the same statement may apply to the characteristics of fibro-myxomata and sarcomata, as well as all forms of fibro-cystic and myxomatous growths, many of which are often found of such ambiguous features, to say the least, that the histologic features peculiar to each are difficult of differentiation even by the most skilled microscopist.

Jonathan Wright also says: "The difficulty in distinguishing lymphoid growths of the mucous membrane from smallcelled sarcoma by microscopic examination is often very great." He says: "In the last year I have been puzzled in several cases, and submitted sections to several experienced microscopists. In every instance I met with a diversity of opinion as to the character of the growth. I have quite a number of such sections in my possession, and the similarity between the 
simple lymphoid growths and those which afterward proved to be malignant is striking. In one case, that of a small-roundcelled sarcoma of the nose, which I followed by repeated microscopic examinations of fragments removed at different times, the first specimen was universally regarded as of benign lymphoid character. It gradually changed in appearance to that of undoubted sarcoma of the small-round-cell variety. The clinical history and situation made the diagnosis of sarcoma probable even at first."

It would therefore seem impossible to dispose of this question until such time as the initial biologic and chemic changes have been revealed. The hope that the development of bacteriology might bring to light the initial causes of these neoplasms in the form of a microbe or some definite cystogenetic metamorphosis, while not yet realized, ought not to be given up, for recent investigations in this field of science are certainly encouraging, especially with regard to the pathology of carcinomata.

\section{Benign Neoplasms of the Nasal Passages and NASOPHARYNX}

Myxomata and Fibromata.-The etiology of these neoplasms (polypi) and cysts has long been a subject of discussion, especially nasal polypi. Boerhaave, in 1818 , promulgated the theory that nasal polyps originated from irritation caused by the retention of secretion in a mucous follicle. The follicle expanding from the pressure behind and attracting additional nutrition finally developed into a pouch filled with mucin, etc. (known as a nasal polyp). On the same principle the earlier pathologists sought to explain the origin of cysts, with the exception that the initial stoppage of the duct was due to an accidental calcareous deposit or the inspissation of the secretion"retention cyst." The varying character of the enveloping structure in each was attributed to an accidental growth of more or less connective or fibrous tissue, determined by the nature of the part and the quality of the irritation. Some such explanation seemed to be acceptable until recent times, when opportunity for more definite observation has led to the belief that inflammation of the tissues in one way or another is responsible for these neoplasms. Woakes explains their forma- 
tion upon the hypothesis that in cases of long-continued nasal catarrh an osseous caries and cleft of the middle turbinated bone gradually takes place, spicules of bone being thereby deflected upon which there is unduly proliferated layers of connective tissue which finally become the envelope of the polyp ; in other words, his theory is that nasal polypi are secondary to purulent or inflammatory disease or necrosis of the middle turbinated bone or ethmoid cells. This theory has been accepted by many rhinologists. The writer has been unable to verify the theory advocated by Woakes, having examined a large number of cases both in hospital and private practice without being able to find more than two cases showing a particular cleft or split of the middle turbinated bone, and in each of these cases in question no polypi had developed, while in a large number of cases, after thorough removal of polypi, no such cleft as described by Woakes could be perceived, nor in many instances any necrosing ethmoiditis. It is true, however, that in some cases what seems to be a spicule of bone may be felt with a probe after the removal of the polypi, but this condition is undoubtedly (as subsequent observation will show) due to mechanical injury of the covering of the bone caused by removal of the neoplasm. A majority of observers have recently connected the growth of nasal polypi with an antecedent ethmoiditis (Bosworth, Casselberry, and Grünwald). Undoubtedly there are cases in which these two conditions coexist, but it is very doubtlul whether their causal relation has been firmly established. Jonathan Wright is inclined to attach much importance to a previous inflammatory action (an edematous rhinitis). The writer has no theory to offer, but is inclined to regard the theory of inflammation and hypertrophy of the mucous structures coexisting with a budding process of the same as the more plausible explanation of their origin. If we consider the normal histology of the mucous membrane of the middle turbinated bone, and especially the middle meatus, where these growths so often take their origin, it will require no wide stretch of the imagination to conceive of such an outgrowth taking place from distorted cell proliferation in these simple or tubular glands. Besides, this theory would seem to coincide with their multiple formation, inasmuch as these mucous tubules (follicles) are formed by a series of involutions, and, moreover, are in close juxtapo- 
sition. Attention might be called to the fact that the contents of polypi, whether mucoid or fibrinous, are largely composed of mucin, which is the principal element of all Schnei-

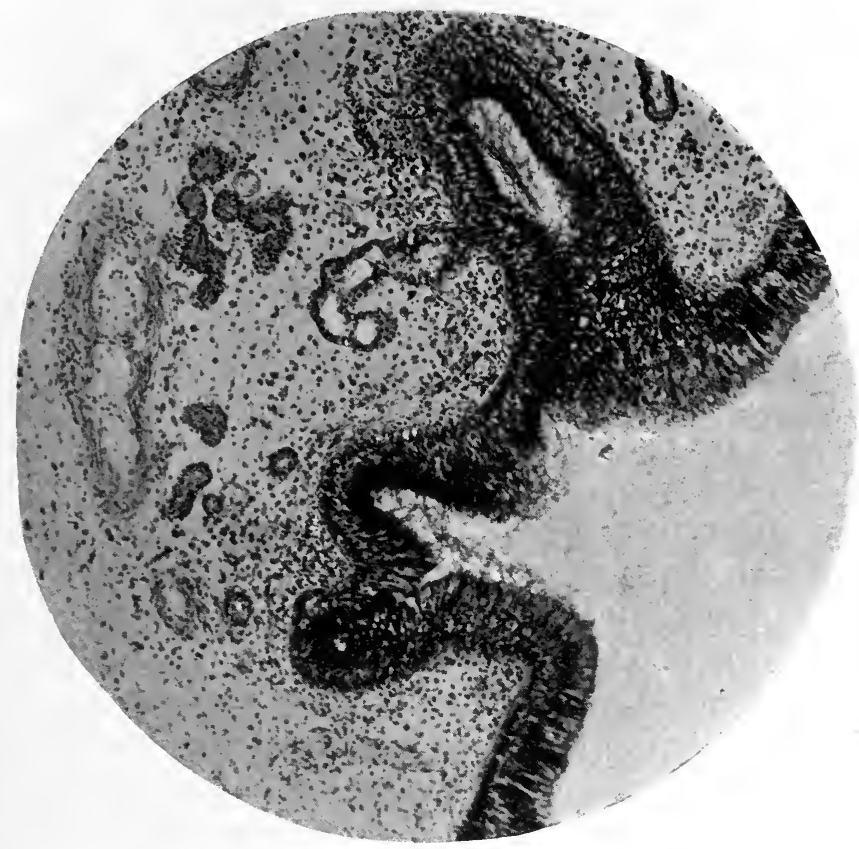

FIG. 124.-Section of nasal polypus (from microphotograph by Dr. P. M. Hickey).

derian gland secretion (see Fig. I24, which represents a section prepared by Dr. P. M. Hickey; it shows the involution process).

Cysts.-Cysts are apt to grow from any mucous or serous membrane, and also, though rarely, from bone. They usually originate through a permanent occlusion of a follicle, gland, or duct, and develop first through the accretion of normal secretion, and then by its alteration, irritation, and the specialization of the lining membrane. The exciting cause may consist in the first place of a subtle external injury, an inflammation, or the development of an irritating element in a lymph space, capillary, acinus, or duct, or a foreign body. The formation of bony cysts has attracted considerable attention since the publication of the works of McDonald and Woakes on the nasal passages. Jonathan Wright, commenting upon Greville Mc- 
Donald's work, On the Muco-periosteum of the Nose; Struc. ture of Mucous Polypi, Caries, and Suppuration, says: "I have been surprised to see how nearly the conclusions I have arrived at through my own studies have been anticipated in McDonald's work. This is especially true in regard to bony changes and edematous polypi within the nose, but I must confess I have not been able to see any evidence to prove McDonald's theory correct in regard to the formation of these bony nasal cysts.

"In order to discuss the subject more intelligently, I have had a drawing made by camera lucida of the bony wall of one of these cysts, taken from a specimen sent me by Dr. Butts.

"It will be seen here that it is in a process of bone formation and of bone absorption by means of the osteoblasts and osteoclasts, which are plainly shown here, as they were in the drawing by Van Giesen in Dr. Knight's paper. The Howslip lacunæ, the new connective tissue, and the blood-vessels are seen arranged with the same relation to the bony structure itself as one sees to some extent both in the turbinated bones of infants and in the form of bone disease met with in Woakes's so-called necrosing ethmoiditis, associated in many instances with edematous inflammation or polypoid degeneration of the mucosa.

"The chief interest in these cases lies in their pathogenesis. Hypertrophy of bone is nearly always accompanied by a rarefaction-i. e., small cavities are hollowed out of the newly formed bone tissue by absorption. The middle turbinated bone is especially prone to hypertrophic change, in contradistinction to the inferior turbinated bone, whose mucous membrane is the part of the structure which is enlarged in hypertrophy. Knowing this, it would be natural to suppose that rarefaction following hypertrophy of the middle turbinated bone would account for the cyst cavity. As a matter of fact, however, it will be observed in this drawing, as well as in the one in Dr. Knight's paper, that the bony cavity is lined by a scanty stroma without glands or many blood-vessels, and by columnar ciliated epithelium. It becomes evident, therefore, that the interior of this cyst must at some time have been in communication with the external surface. To account for this, Dr. McDonald (quoting at second hand from Dr. Knight) 'supposes the lesion to have its origin in an osteophytic periostitis, doubtless secondary to a hypertrophic rhinitis involving the 
soft parts.' The margin of the bone begins to curve outward and upward until it meets the body of the bone at the point where at length adhesion takes place. The objections to this are :

"I. It is very difficult to conceive of such a peculiar twist to a hypertrophic bone process.

" 2. Intermediate states of the bone are not observed, or at least not reported, either clinically or post mortem.

"3. Finally and most conclusively, there is no way of accounting for the disappearance of the mucous glands, which are tolerably abundant on the under surface of the middle turbinated bone.

"Wagner suggests again the theory advanced by Zuckerkandl, that these cysts are but the extension of the cavity of the bulla of the ethmoid, or of one of the other ethmoid cells, in the body of the middle turbinated bone.

"While this would seem more acceptable, it may be urged that the cavities do not seem to communicate at all with the ethmoid cells. Steida makes a suggestion which seems to be a valuable one, and which had often occurred to me. We may suppose that these cysts have their starting-point in physiological cavities left by the gradual closure of their outlets by processes in embryonic development similar to the cells of the ethmoidal labyrinth, only not so large. The mucous membrane lining these cells has no glands, or but few, and but a scanty stroma.

"Supposing the pre-existence of such cavities, we may understand how the osteophytic bone process figured here may enlarge them into cysts, recognised clinically by symptoms of obstruction and intranasal pressure.

"Steida's specimen was examined by Professor Lubarsch, who, after describing the external coverings and the internal cylindrical epithelium, says :

" The greatest interest lies in the bony wall, which externally and internally is limited by the dense connective tissue of the periosteum. It shows lamellar structure, and is of varying thickness at different points. The rich development of osteoblasts is especially observable ; they are often in several layers, and are pushed in between the periosteum and the bone substance, and are especially abundant at the outer edge of the bony layer. Corresponding to the bone-forming activities are 
to be found absorption processes, although in general not so active, in the form of osteoblasts, which lie in the Howslip lacunæ. In some places they are more abundant, and have caused a marked thickening of the bony layer.'

"In other words, we have a hyperplastic osteitis and a rarefying osteitis coexisting, it being said by pathologists that osteoblasts may be transformed into osteoclasts, and these subsequently changed back to osteoblasts. These cuboidal cells, then, which exist along the bordering line of the periosteum and the bone, possess the power of depositing bone salts, and at other times acquire the power of absorbing them. Both of these processes are physiological in the development of bone, but go on in a more irregular fashion during inflammatory changes. In Woakes's book on Nasal Polypus, the illustration he gives of his necrosing ethmoiditis corresponds exactly with this process, as may be seen by turning to the works of Delafield and Prudden, Ziegler, Birch-Hirschfeld, or Cornil and Ranvier. Woakes was led into this error by confounding the loose connective tissue which occurs in the Howslip lacunæ with the analogous structure of edematous nasal polypi, and calling both myxomatous.

"This histological bone change exists in the nose in connection with chronic inflammation very much more frequently than is commonly supposed. It is nearly always seen in the pieces of bone that come away with nasal polypi. It is found in suppurative ethmoiditis combined with edematous inflammation of the mucous membrane. I have lately found it in the bone underlying the chronic hypertrophy of the posterior end of the inferior turbinated bone.

"It is a curious fact that these bony cysts have been reported almost exclusively in women. The three or four cases that I have observed myself have all been in females. They occur, too, at an age (twenty to forty) when hypertrophic inflammations of the mucosa are most frequent. There are a great many clinical as well as pathologic facts which go to show that the process in the bone is an inflammatory one, having its origin, as McDonald says, in a chronic inflammation of the nasal mucous membrane. This, spreading to the bony walls of these closed cells of embryonic origin, may, as has been shown, cause their growth by hyperplastic and absorptive bone changes, and possibly by the pressure of exudations. 
"Casteneda says that in many of the cases there is a history of traumatism.

"After all is said, there are many points about this puzzling subject that still remain obscure, and what I have advanced above, it must be admitted, is more or less conjectural, so far as its etiological significance goes, although the facts, I believe, have been correctly stated.

"Dr. Berens, after hearing some of the remarks above published, was kind enough to show me the ethmoid bone, in which the middle turbinated bone was dilated in its anterior portion so as to form a large cavity which communicated freely above, in the dry specimen, with the ethmoid cells proper. Evidently the origin here is either as stated by Zuckerkandl or as modified by Steida. There were plain evidences of osteophytic action at the lower border of the bone."

Nasal Passages and Nasopharynx.-By far the most frequent neoplasms found in the nasal passages are the myxomatous and fibromatous polypi. Jonathan Wright, however, doubts the occurrence of true myxoma here. Next in frequency are found the papillomata, angiomata, chondromata, osteomata, and cystomata; of the malignant growths, the sarcomata and epitheliomata are most frequently seen.

Regarding polypi, Zuckerkandl describes five different forms. Bosworth and Seiler recognise but three varieties: the mucoid, fibroid, and cystic. Of these, the mucoid polyp is the most frequently met with.

Zuckerkandl's researches on cadavers, as summarized, demonstrate that two thirds of all nasal polypi originate from the middle meatus, beneath the middle turbinated body, and that approximately two thirds of this number take origin from the edges of the hiatus semilunaris, which is a concentric opening in the infundibular space, whose upward and downward deviations enter respectively the frontal and maxillary sinuses, and which is located high up beneath the middle turbinated body. He thinks the muco-purulent secretion present, by decomposing in the middle meatus and around the middle turbinated body, excites irritation, and furnishes a certain soil favourable to the growth of polypi. Hopmann believes that the primary lesion which leads to the development of mucous polypi lies in some impairment of the venous escape, resulting in an edema- 
tous condition of the membrane. True vasomotor paresis also, by favouring edematous transudation, exerts a like effect. Casselberry believes in the myxomatous diathesis, and recommends in some cases the removal of the antero-inferior part of the middle turbinated body. They very frequently occur in both nasal passages, and are usually multiple, several growths emanating, perhaps, from one or two pedicles. They may occur at any age, although rarely met with in childhood. A few cases which were of congenital origin have been reported by Dr. Dunbar Roy, Dr. Price Brown, of Toronto, and others. Moure (France) found only 5 cases of polypi in children, out of a total of 10,590 cases examined. Several observers, among whom are Hopmann, Heymann, Michael, of Hamburg, report a few cases occurring under the age of seven years. Natier was unable to find a single instance in I,200 cases examined at his clinic. Cardonae, of Italy, and Dunbar Roy have seen cases of congenital polypus in which the superior deflection of the septum, as pointed out by Jarvis, was present in these cases. They grow very rapidly, soon filling the nasal passages, and perhaps also protruding through the posterior choanæ, or possibly appearing at the nares. The surrounding parts may be softened and deformed by their continued pressure; thus the nose, maxillæ, and lower floor of the orbit may become softened or displaced, especially when the tumours arise in clusters. Individual polypi may attain great size. Dr. Samuel Johnson, of Baltimore, reported to the American Laryngological Association a case of a large myxomatous tumour having its origin in the nose and projecting into the pharynx. The tumour measured two inches in length and three quarters of an inch in the thickest part.

Dr. W. W. Ashhurst, of Philadelphia (Med. News, vol. lx), reports having been called to see a lad, seventeen years of age, suffering from extreme dyspnœa reaching the point of suffocation, which was caused by the projection into the pharynx of a large mucous polyp, which was attached by a pedicle to the inferior turbinated bone. At his first visit he performed tracheotomy, to avoid impending death. The growth was afterward removed. According to the clinical history, this boy had suffered from the presence of this growth for about five years. No reason was given in the report for its sudden augmentation. 
Appearance.-In appearance, nasal polypi are smooth, pyriform or round in shape, and pedunculated (see Plate I, Fig. I). They vary in size from a cherry to that of a pigeon's egg, or larger; they are of a gray or pinkish-gray colour, soft and pliable to the touch of the finger or probe, and not very vascular. They usually originate from the middle or posterior border of the middle turbinate bone, although they sometimes take their origin from the upper border of the choanæ.

Fibromata.-These are generally single, and confined to one side of the nose only. Their deep origin is in the periosteum; they are much slower in their growth than the mucoid variety. They are also usually pyriform in shape, and pedunculated. The pedicle, however, is generally much broader. A few cases have been reported of fibroid polyps having appeared to be sessile. The shape, however, will vary somewhat according to their situation and the amount of compression they have undergone. They have a more pinkish colour than mucous polyps, and in some instances show a capillary network on their outside. They are more vascular, and to the touch they are considerably firmer than the mucoid variety, on account of the extra amount of fibrous tissue which enters into their composition. The contents of the fibroid polyp, like that of the mucoid, consist largely of mucin, with a little serum and sometimes blood. Very often their cavity is divided into a number of spaces by fibrous partitions, which lends additional firmness to the tumour. Fibrous polyps are prone to start from the margin of the ostium maxillare, or the posterior end of the middle turbinate bone. From the latter situation the growth is usually downward and backward into the nasopharynx. The fibroid growth is more destructive to the surrounding parts, on account of its greater firmness.

The fibro-myxomata, as the name denotes, are histologically a mixture of these two varieties. While these do not vary in their outward appearance to any great extent, still the internal formation partakes of the character of the two varieties mentioned above. Their progress is also mixed, inasmuch as they usually grow slower than the pure mucoid polyp, and develop faster than a purely fibroid polyp. There seems to be a strong tendency for them to recur and to become malignant, the writer having seen two examples of fibro-myxomata-which subsequently became malignant, and finally, by repeated recru- 
descence and extension, caused the death of the patients. They may have been malignant from the beginning, if we assume that microscopic examination is not infallible for the differentiation of histologic characteristics. Some surgeons believe that all fibroid and fibro-myxomatous growths are quasi-malignant. The following cases illustrate these tendencies of fibroid neoplasms:

Dr. Charles H. Knight, of New York (N. Y. Med. Jour., vol. lii, p. 342), reports a case of unusually rapid recurrence of a fibro-sarcoma of the right nasal fossa in a man aged forty-two. The tumour was thoroughly removed by Dr. Weir at the New York Hospital, by Chassaignac's operation, but soon recurred, and the man died from profuse hemorrhage. Butlin says it is almost always necessary to remove some of the surrounding tissues-in some instances a very wide area-in order to prevent a local recurrence of the disease. In cases of this class it is often difficult to determine beforehand the exact origin or situation of the neoplasm. Dr. Knight discourages partial and palliative operations involving the sphenoidal and ethmoidal sinuses.

Dr. Price Brown, of Toronto, Canada (Jour. Am. Med. Assoc., vol. xxv, p. 859), reports a case of recurrent naso-pharyngeal fibroma occurring in a bleeder, aged twenty-two years, who had a good family history. The tumour had been probably growing for about five years. He had snared off pieces at different sittings. The bleeding was always very severe. The tumour also seemed to grow as fast as it was removed. Microscopic examination showed it to be a fibro-sarcoma. It was finally removed piecemeal, but returned within four years afterward and produced occlusion of the posterior part of the right nasal passage. The patient subsequently went to Boston, where he was operated upon in a homœopathic hospital. About three years afterward he came under the observation of Dr. Brown again, and showed almost complete occlusion. It seemed to be an extensive sessile growth. It was finally removed by repeated applications of the galvano-cautery.

Dr. J. H. Shorter, of Macon, Georgia (Jour. Am. Med. Assoc., vol. xxvii, p. 644), reports a case of malignant tumour of the nasal cavity, which was removed by external operation in the New York Hospital, but soon recurred, and finally caused the death of the patient at the expiration of three months after 
operation by extension of the growth to the cranial cavity. Several diagnoses were made in this case: first a diagnosis of syphilis, then another of benign tumour, both opinions being based upon microscopic examination.

Dr. Charles W. Shields, of Richmond, Virginia (N. Y. Med. Jour., vol. lxiv, p. 354), reports two cases of naso-pharyngeal fibromata, occupying both the nasal passage and upper pharynx ; in one, a man twenty-three years of age, the tumour was removed by several applications of the irido-platinum wire; the other case occurred in a negress after the age of fortyeight years. There is probably no other report of a case occurring in the negro. Bosworth says: "When we consider the great frequency of uterine fibroids in the coloured race, we might naturally anticipate the same frequency of fibromata of the upper air passages; and yet, as a matter of fact, I know of no recorded instance of fibrous tumour occurring in a negro." Of 66 cases reported by Bensch, only one was in a female, a girl of fourteen. One writer's collection of 58 cases does not include a single woman, and Morell Mackenzie thought instances of the disease occurring in women must be looked upon as altogether exceptional, but subsequent examination of the reported cases shows that the female does not possess immunity.

The cystic variety is quite rare, the first instance of the kind having been reported by Seiler in 1882 . Since then a few cases have been reported by American and European làryngologists. It usually occurs as a single tumour upon one side of the nose only, and is always pedunculated. The common site is the lower portion of the lower edge of the middle turbinate bone. These tumours may grow to large proportions and present themselves in the naso-pharyngeal cavity. H. E. Strachan, of Kingston, Jamaica, reports a case in a boy, suffering from a left exophthalmos, in the inner wall of the orbit, encroaching on the orbital cavity. No symptoms of nasal disturbance had been complained of, but on examination there was found some obstruction of the passage. Forcibly exploring the cavity from the front through the left nostril, a tumour was found high up in the posterior part of the left nasal cavity, which was compressing the inner orbital wall. It was removed, and proved to be a cyst, containing about one ounce of mucoid material. 
Cysts are usually more spherical than pyriform in shape, excepting in situations where the pressure of surrounding parts may modify their shape. In appearance they are much whiter than either the mucoid or fibroid variety, and offer considerable resistance to the finger or probe, although much less resistant than the fibroid. They also give a sense of fluctuation. They consist simply of a sack of connective tissue containing for the most part leucocytes with muco-serous fluid. Dr. J. N. MacKenzie has reported a case of blood cyst (hæmatoma) of the posterior nares. Dr. W. K. Rogers, of Columbus, Ohio, has reported a case of echinococcus cyst of the nasal passages (Jour. Am. Med. Assoc., February 3, I900).

Dr. J. W. Dunn, of Richmond, Virginia (N. Y. Med. Jour., vol. lix, p. 238), reported a case of cystic tumour occurring in a woman twenty-six years of age, extending from the internal choanum about three fourths of an inch along the floor of the nose. It reached from the septum to the external wall of the nose. The greatest depth of the tumour was about one fourth of an inch. There was no communication between the teeth and the antrum of Highmore. The tumour was opened with the knife, the contents flushed out, and the cavity packed with cotton saturated with I to I 500 of bichloride of mercury. After a few days the sac was washed out with tincture of iodine. It gradually diminished in size, and a complete cure resulted.

Dr. C. W. Richardson, of Washington, D. C. (Jour. Am. Med. Assoc., vol. xvii, p. 502), reports two cases of nasal cystomata. The treatment consisted in puncturing the cysts and afterward removing the cyst walls.

Spencer Watson also reported a case to the Medical Society of London. It is important to bear in mind that there is a marked difference between true retention cysts and cysts formed by the degeneration of solid cartilage. As Watson says, it is not uncommon to meet with small adenomata which have been undergoing cystic degeneration. The same is true of gelatinous polyps, but, including Lefferts, Ingals, and Horsley's cases, which are reported in full, and Seiler's, of three of which he only makes mention, we have found only seven of these interesting neoplasms.

The usual seat of these growths is on the outer wall of the nasal cavity. The pathological history of these growths within the nasal cavity cannot differ from that of true retention cysts 
occurring upon mucous surfaces in general, being formed from retention of the secretions within the normal glands of the mucosa. The pressure of the retained secretion becoming too great, an abnormal activity takes place within the gland, and slowly or rapidly becomes transformed into a true cystoma.

Dilatation, or cystic degeneration of the middle turbinated bone, may obstruct the middle meatus to such an extent as to cause considerable disturbance. An osseous cyst of the turbinate or its adjacent bulla seems to have its origin in an ethmoidal cell, which is in direct communication with the spongy bodies involved. When irritation or inflammation of the ethmoid cells takes place their contents flow into the body with which it is connected, and in time may form a cystic tumour, the walls of which are composed of osseous substance, thin and shell-like in consistence. These bodies, from long-continued chronic inflammation, often enlarge, and cause the same obstructive symptoms as the cyst just described. They are probably, as Seiler says, merely retention cysts. If ruptured or emptied by puncture they soon refill.

Papillomata.-Papillomata are not common in the nasal passages, although Hopmann, Wright, Hinkel, Dickerman, Knight, and others, have reported them. Bosworth reports only one case in two hundred persons examined. Hopmann describes two varieties, the epithelioid (benign) and the soft papilloma. Papillomata are usually the result of an irritation which is specific (syphilis, tuberculosis), although any morbid process giving rise to granulation tissue may lead to their development. In many instances, however (such as papilloma of the pharynx), it is difficult to understand the cause of their appearance when no great amount of irritation, inflammation, or ulceration has preceded.

Dr. Richard McKinney, of Memphis, Tennessee, reported a case in the New York Medical Journal, March 4, I899, and reviews the literature of the subject. Bosworth states that in 200 cases of benign tumours of the nose, but I of them was a papilloma. Williams, of Bristol, reports having seen but 2 cases of nasal papillomata in his entire experience. Hopmann found in a total of 430 cases of benign nasal tumours that 78 were papillomata. Schaefer observed 20 instances of papillomata. This large percentage seemed offset by the report of 
Schmeigler, who found in I 7 cases of nasal tumours only i case of papilloma, while Zuckerkandl, in 39 cases of polypi, also found but I case of papilloma. Wright and Douglass, of New York, have also reported 3 cases. Jonathan Wright protests against Hopmann's declaration relative to the frequency of papillomata in the nasal fossa. Out of 700 nasal tumours and hypertrophies removed he saw but I case which he regarded as true nasal papilloma. This was a small tumour, a velvet-like papilla, about half the size of a split pea, situated at a point above the centre of the cartilaginous septum in the left nasal fossa (Fig. I25). Lacarret speaks of having seen 2 cases with the aspect of papillomata, which proved upon microscopic examination to be hypertrophies of the mucous membrane and granulation tissue. Hopmann classed them in two varieties, hard and soft, the classification being seemingly based upon the character of the epithelial covering of the growth; those growths which appear near the margin of the nostril usually partaking of the hard variety, having squamous epithelial covering and only rarely attaining large size, while those which occur farther back and higher up in the nasal cavity, being covered with columnar epithelium, are said to be softer in consistence and to grow much larger. But the more recent investigations of Dr. Jonathan Wright completely upset this classification of Hopmann, it being clearly shown by Wright that Hopmann had described as one of his varieties the ordinary polypus formation, which from pressure had taken on a false papillomatous appearance. Dr. Wright's investigations have been confirmed by later observers. Recently, Dr. Beaman Douglass, of New York, in reporting a case of papilloma of the nasal septum, clearly explained the difference in the microscopical structure between a papilloma, fibroma, and the Hopmann polypus. We thus, then, as Dr. Wright states, are unable to say how many cases of true nasal papillomata have been observed. The general appearance of a nasal papilloma is that of a warty growth, being of a grayish-pink or reddish colour, and lobulated, the surface being irregular and likened to that of a raspberry. Nasal papillomata may be found ranging in size from that of a pea to that of a filbert, and may be either single or multiple.

They usually occur singly, and grow from either the anterior portion of the septum or from the inferior turbinated bone. 
The epithelioid variety is usually of slow growth, and shows little tendency to spread beyond its original site. The soft or what is called by some the "mulberry papillomata" or "angioma," on the other hand, is of very rapid growth, and shows quite a tendency to spread. This is why some observers have

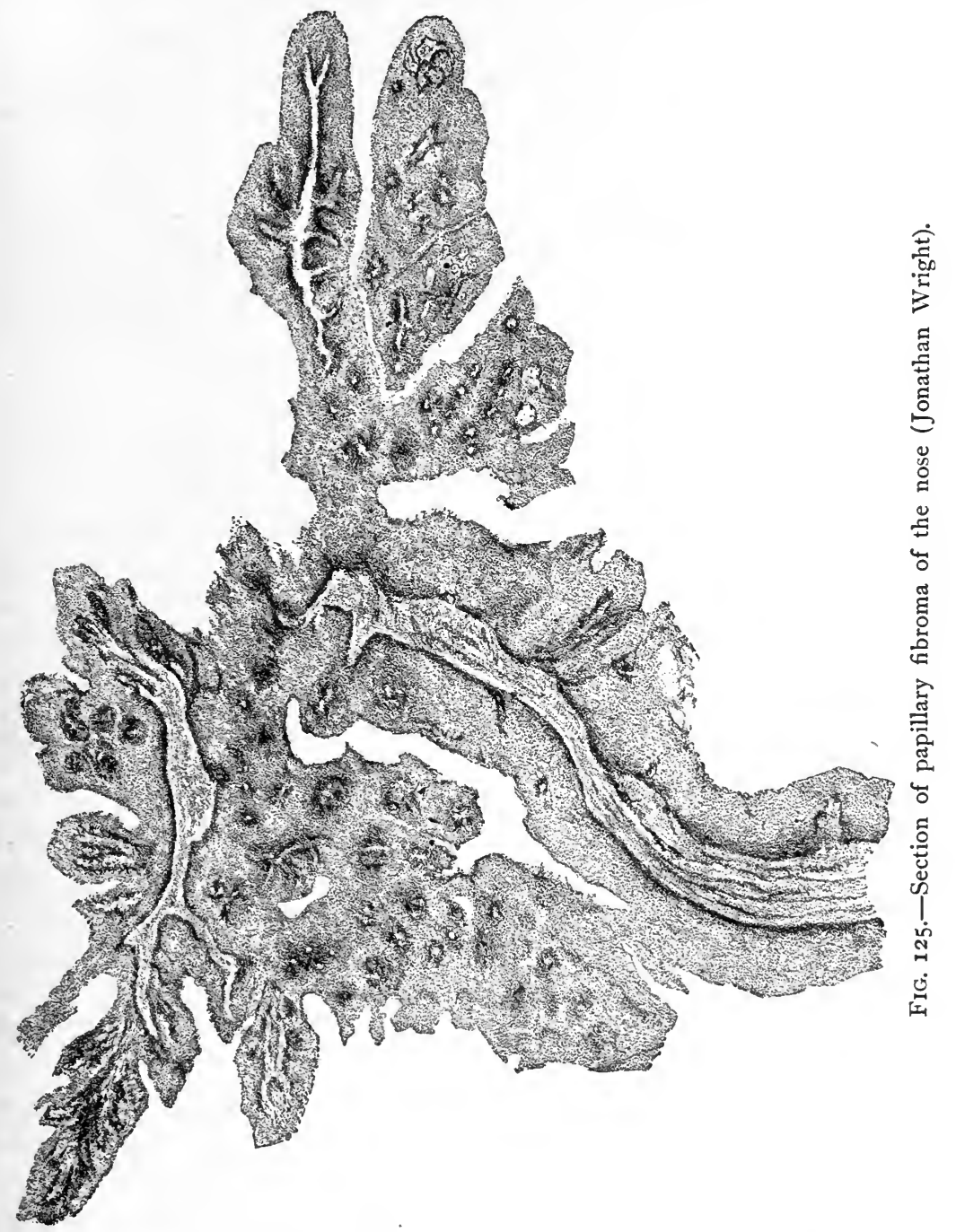

considered it malignant. On account of their site, which is within the vestibule of the nose, Seiler and others have believed that the most common cause of their origin is exter- 
nal irritation, especially with the finger-nail in picking the nose.

True angiomata, or erectile tumours, are said to be very rare in the nasal passages. Cases have been reported, however, by Wagner, Glasgow, Delavan, Roe, Jarvis, Casselberry, and others. Glasgow's case was reported to be an angiofibroma. Dr. George H. Godson reported a case of false angioma due to a blood clot, as proved by microscopical examination, which afterward underwent absorption. Seiler was

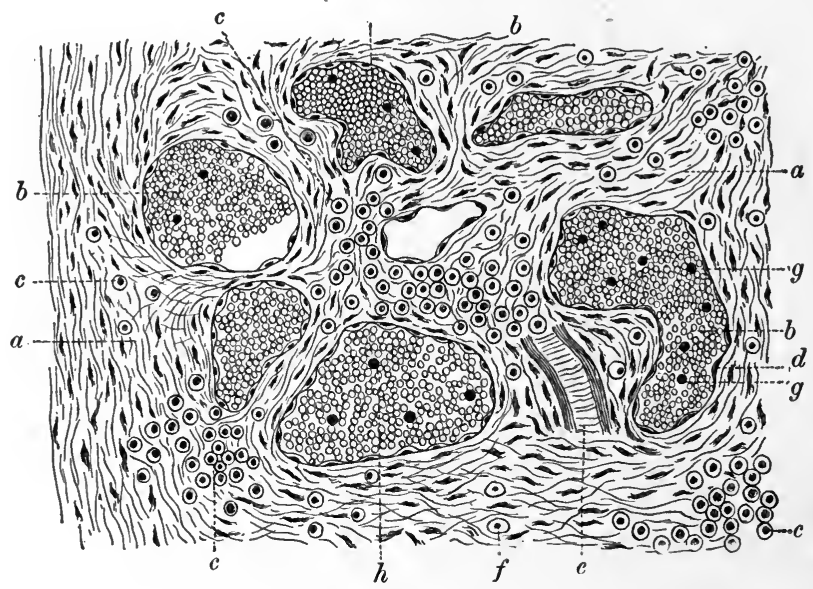

FIG. I26.-Angioma of the nose. $a$, fibrous connective tissue ; $b$, angiomatous vessels (blood sinuses); $c$, small-round-cell infiltration; $d$, endothelial lining of blood sinus ; $e$, arteriole ; $f$, plasma cells ; $g$, leucocytes ; $h$, red blood-corpuscles.

able to collect the reports of ro cases only. They are usually small, about the size of a pea, of dark-red colour, and always pulsate synchronously with the arteries. At first they resemble hypertrophy of the turbinated tissue of extra colouration, but more thorough examination, especially later on, will show a distinct circumscribed limit to them. They are smoother than so-called papillomata (Fig. I26).

Chondromata.-These growths are very rare in the nose, and are said by Morell Mackenzie to occur generally at or near the age of puberty, when cell development is most active. They usually spring from the cartilaginous septum, and are sessile. They may resemble the fibromata at first, although examination with the probe will show very much less resiliency than in fibroma. 
Osteomata of the nasal passages are extremely rare. Seiler was the first to give a good description or differential diagnosis between these growths and exostoses. These tumours usually arise by a pedicle from the mucous membrane of either the septum or turbinated bodies. They present a smooth surface, are pinkish in colour, and hard to the touch, although movable sometimes. When of long standing they are apt to present lacerated spots upon their surface, and may show little spicules of bone. The odour from the nose is fetid, and characteristic of decaying bone. When the tumour presents itself, more or less erosion of the surrounding part is sure to occur. There are two varieties described in the text-books, the eburnated and the cancellated, each presenting the microscopical characters of compact and cancellated bony tissue respectively. They vary in size from a cherry to a pigeon's egg.

\section{Benign Neoplasms of the Pharynx, Soft Palate, and TONSILS}

Benign growths of the oropharynx, soft palate, and tonsils are not very frequently seen in this country. Although each variety may be met with, they are generally found in the following order of frequency: Papillomata, fibromata, cystomata, lympho-adenomata, angiomata, and so on. The site of these growths varies greatly; although the pharynx and soft palate are the parts from which they more often spring, still they may be seen elsewhere in this region, as, for instance, in the laryngopharynx. The writer met with one case of fibroma which sprang from and occupied the right pyriform sinus.

Papillomata are not so very rare in this situation. Accord. ing to the writer's experience, papillomata are found usually on the margins of the faucial pillars and along the edge of the soft palate, uvula, and in the region of the palato-pharyngeal folds (Fig. I27). They may occur at any age. They are frequently multiple, sometimes growing in clusters along the edges of the fauces. Dr. Delie, of Paris, reports a case in a child two and a half years of age, in which the entire isthmus on the palate, lateral walls of the pharynx, and tonsils were occupied by papillomatous vegetations. Clinton Wagner reported a case of papilloma on the extreme tip of the uvula, and another case where the growth was situated upon the posterior 
arch of the fauces. It was of large size, and looked more like a fibroma. He thinks that papillomata in these situations occur more often than is generally reported, because they are

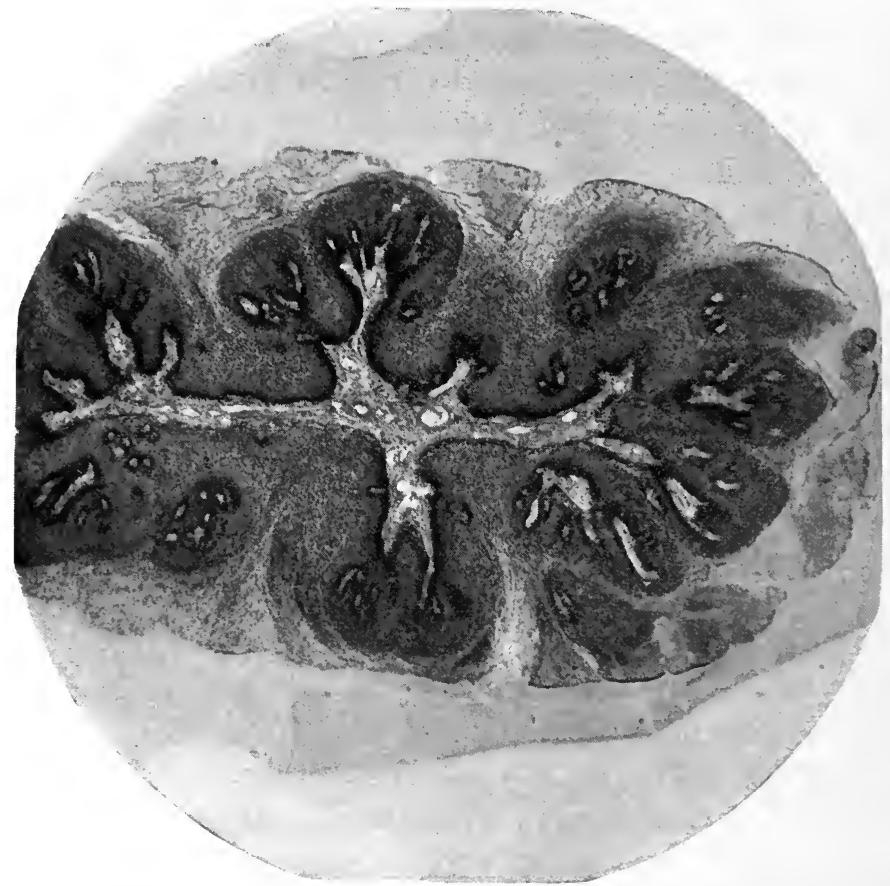

FIG. 127.-Section of papilloma of the uvula (author's case). Photomicrograph by Dr. P. M. Hickey.

frequently overlooked. Lefferts reported a case of papilloma of the soft palate of large size. Dr. Scheppegrell, of New Orleans, La., has reported two cases of pedunculated papillomata arising from the soft palate.

Dr. John Dun, of Richmond, Va., has seen five cases of pedicled papillomata growing from the soft palate, uvula, or pillars of the fauces.

Dr. H. T. Machell, of Toronto, Canada, reported a case of papillomatous disease of the tonsils in a girl ten years of age. He had operated upon this girl three years previously for the removal of a lipomatous tumour of the neck. The patient had made no complaint of acute attacks of sore throat. There was well-marked enlargement of both lobes of the thyroid gland, and excessive enlargement of both tonsils, which were studded 
with closely packed papillary bodies, almost closing the faucial entrance, and presenting, of course, the ordinary symptoms connected with such mechanical obstruction. This is a rare affection. The patient subsequently died of scarlet fever.

Fibromata are met with more often in the nasopharynx than in the oropharynx. They may also take their origin from the choana, the tonsils, or from the posterior pillars of the fauces, or fossæ of Rosenmüller. They usually occur singly, are of pyriform shape, and may grow to large proportions. Indeed, the tumour may fill the entire nasopharynx, and invade the cranium.

Lincoln, Price Brown, and others, have reported interesting cases of this sort. Both these practitioners finally removed them piecemeal, so to speak, with the galvano-cautery. The writer has met with three cases only of fibroid tumours of large size in this situation. The tumours may be pedunculated or quasi-sessile. When distinctly pedunculated they are movable.

Ingals reported a case of a large naso-pharyngeal fibroid tumour in a boy eleven years of age, from whom the protruding portion of the tumour was removed from the nasopharynx by the galvano-cautery platinum loop, and the remaining portion, which had grown up behind the palate bone to the zygomatic arch, was removed by frequent hypodermic injections of dilute lactic acid, following the use of cocaine.

Naso-pharyngeal fibromata bleed quite readily. Lincoln collected and reported 58 cases; 38 were removed by severe operations, such as resection of the maxillary bone. Only ro cases were cured. Fourteen were operated upon by the galvano-cautery, with II cures and 3 relapses. About one third of the cases succumbed to pneumonia, another one third from the immediate consequences of pneumonia and septicæmia, and about one third escaped death after long and complicated runs of septicæmia. Wutzer, of Bonn, had 17 cases of relapses out of a total not stated. Middeldorf always applies the electrocautery in the treatment of these cases. Hippocrates used a loop, and extracted them either by evulsion or the knife. Vibrisi had a curved bladed forceps for seizing the growth. Celsus seized the growth with the tong-like forceps. Smye and Mott would remove the superior maxillary (Cheatham).

Myxo-fibromata sometimes occupy the nasopharynx, but arise by a pedicle from one of the posterior turbinates. Fibrom- 
ata usually arise from the pharynx, and the mixed form from about the choana. Myxo-fibromata are usually pedunculated. Cheatham reported a case of a large fibro-myxoma in a boy about ten years of age, which from pressure had caused suppuration of both ears with mastoid periostitis. He also reported a case of pure polypus of the pharynx in a young man twentyeight years of age.

Fibro-lipomatous tumours of the pharynx are very rare. Roe reports a fibro-lipoma situated on the posterior wall of the pharynx alone. The fatty portion of the tumour had become quite soft, making, as the author states, a combination of lipoma-molle and a fibro-lipoma. Owing to its situation and form, and its attachment to the deep tissues below, it was with considerable difficulty removed. The principal inconvenience suffered by the patient was in deglutition, and in respiration in certain positions of the body.

Cystomata.-Cystic growths in this region are usually seated somewhere along the posterior pillar or spring from the tonsil or the sulcus above the tonsil, or the posterior wall of the velum palati. The writer has had two cases of cysts springing from just behind the lateral margin of the velum palati. Cysts in this region do not assume very large proportions, as a rule. As mentioned above, they may originate from the nasopharynx or margin of one of the choanæ, but they are not very common in this situation. An interesting case of a large retropharyngeal cyst is reported in the Laryngoscope for March, 1898, p. I48.

Lymphomata or lympho-adenomata are sometimes met with in the pharynx, usually occurring as a pedunculated single lobulated tumour. This form of growth may arise from any portion of the pharynx, because this region is richly endowed with glands throughout (L. Browne). They are usually found, however, springing from the lateral wall, and may involve the posterior portion of the tonsil. In the only case which the writer has seen there was considerable enlargement of the cervical lymphatic glands. The case alluded to was supposed to be of tubercular nature, but for five years after the removal of the tumours (during which time the patient was under observation) no tubercular manifestations appeared.

Angiomata of the pharynx or tonsils are occasionally met with. Dr. William Martin, of Bristol, Pa., has reported a case 
of angioma of the nasopharynx. Dr. J. H. Hartman also reported a case of angioma of the left tonsil in a man thirty-two years of age.

Myxomata.-The myxomata are rarely found in the oropharynx, although, according to Lennox Browne, mucoceles are not so infrequent. The writer has never met with a case of true mucous polyp which originated in the oropharynx or laryngopharynx.

Osteomata.-Osteomata are of very infrequent occurrence, either in the pharynx or tonsils. Dr. Alexander W. Sterling, of Atlanta, Ga., reports three cases of bony growth on the palatal tonsils, two of the cases being brother and sister. They were not calcareous degenerations in the tonsillar crypts, but growths occurring in the deeper tissue. He thinks that they were adenoidal prolongations of the transverse processes of the vertebræ and styloid processes of the parietal bone.

Etiology.-The etiology of neoplasms of this region is still uncertain. All of them, excepting perhaps the cystic variety, are apt to occur during the period of adolescence, although cysts and papillomata frequently appear in advanced life. One peculiarity in connection with pharyngeal neoplasms (if we except, perhaps, the fibromata and lymph-adenomata) is the slowness with which they augment in size. There are many instances where small growths of this kind have been carried by patients for many years without notable increase in size.

Symptomatology and Appearance.-The symptomatology depends very largely upon the situation of the growth and its size. When so situated as to interfere with the function of respiration or deglutition, either by pressure or movement, a considerable amount of distress may follow ; for instance, neoplasms in the lower pharynx will always interfere more or less with the function of respiration or deglutition, so that more or less dysphagia, hacking cough, or laryngeal spasm may harass the patient. Besides this, these growths are apt to produce an increase of secretion, an unpleasant sensation of dryness of the pharynx, and also pain of a neuralgic character, referred to the front or side of the neck or darting into the ear. Difficulty of nasal respiration and the provocation of considerable hawking may result from their presence in the nasopharynx; while constant efforts at swallowing and changes of intonation of the voice, together with more or less soreness 
of the throat, may accompany the presence of any of them when seated upon the velum palati or upper portion of the lateral wall of the pharynx. Thorough inspection and examination of the pharynx by palpation will reveal the presence of these tumours, and when such symptoms as have been narrated are persistent, a thorough examination should always be made. The presence of enlarged lymphoid tissue at the base of the tongue (the lingual tonsil) is said to give rise to a similar train of symptoms. These hypertrophies, however, will be treated of in connection with adenoid vegetations at the vault of the pharynx, and need not detain us here. The writer, however, would like to say, in passing, he does not consider that hypertrophy of the lingual or sublingual lymphatic tissue should be classed with the neoplasms.

Malignant Growths.-The malignant growths which are usually found in the upper air passages are sarcomata, lymphosarcomata (round cell and spindle cell), myxo-sarcomata, and epitheliomata (squamous and alveolar). These may be classified into the primary-those springing primarily from either the nasal passages, pharynx, or larynx-and those which involve these regions secondarily, having originated outside in contiguous parts. The question often arises, whether malignant growths in the upper air passages can develop by metastasis. Such a mode of origin is considered doubtful, but instances of the kind have been reported.

Several cases have been recorded of secondary deposits in the mediastinal glands, in the lungs, and in the abdominal cavity. As a rule, glandular involvement is a late feature of malignant disease of the larynx; it usually does not make its appearance until after ulceration has ensued. Extrinsic laryngeal carcinoma is more likely to occasion glandular infiltration than intrinsic. Ample explanation for this is found by reference to Sappey's demonstrations of the peculiarities of the lymphatic distribution in this region.

Dr. Charles P. Grayson, of Philadelphia, reported a case of intrinsic carcinoma in a man thirty-four years of age, of negative family history, in which a secondary deposit (epithelioma) made its appearance upon the lower lip, the laryngeal growth remaining intrinsic.

Malignant disease may originate in any portion of the upper respiratory tract. It is a common affection of the larynx, 
but rare in the trachea. It is moderately prevalent in the thyroid gland. According to Hunter Mackenzie, in 750 cases of malignant new growths reported by him, 6 were carcinoma of the larynx, 4 of the pharynx, and 5 of the thyroid gland. Within the larynx, epithelioma (squamous-celled carcinoma) is more frequently met with. Sarcoma of the larynx is a rare disease. Sir Morell Mackenzie met with only 5 cases of it. Butlin mentions 25 cases as having been collected by him. Cylindrical- or columnar-celled carcinoma is also extremely rare, and only I case is reported by Butlin. Sir Morell Mackenzie never met with a case of secondary cancer of the larynx originating by infection, and only knew of one case in which, the original disease having been in the larynx, cancer was developed secondarily in other parts of the body; but a case has been reported by Rollier in which metastasis to the lungs, liver, and brain followed a sarcoma and suppurative perichondritis of the larynx; and Hermann mentions a case of simple lymphosarcomatous metastasis of the larynx and trachea (Hunter Mackenzie).

Of 50 cases of malignant disease of the larynx collected by Butlin, 3 had metastatic deposits in the kidneys, liver, and lungs. The glands usually affected in carcinoma of the larynx are the cervical, laryngeal, and tracheal glands; but there are cases recorded of metastasis to distant glands, such as the lumbar. Butlin asserts that sarcomata do not affect the glands, for the reason that sarcomata, owing their origin to the proliferation of the cellular elements of the connective tissue, the solid structures swell and cause a diminution of the calibre of the lymphatic vessels, which proceeds to a complete obliteration and renders them incapable of transmitting the infective material of the tumour. Lennox Browne controverts this theory, for in 3 out of 4 cases under his notice glandular enlargement with complications were present. Glandular enlargement may be present and may not be detected during life.

Sarcomata and Lymphosarcomata.-This form of malignant growth is most frequently found in the nasal passages, in the tonsil and lower pharynx, or on the epiglottis. The lymphosarcomata are perhaps more frequently found in the tonsil and lower pharynx, and the myxosarcomata more frequently in the nasal passages and nasopharynx. In the nasal regions the site of origin is usually the middle turbinated body 
and external wall (bone or infundibulum). Occasionally the growth has its origin in the nasopharynx. The writer has seen one case of this sort originating at the vault of the pharynx,

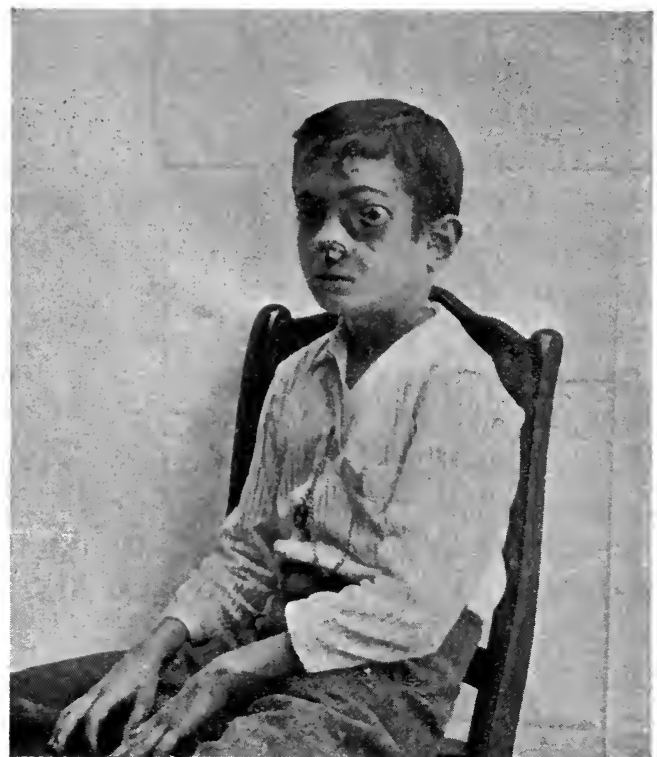

FIG. I28.-Sarcoma in the left nasal passage (Bliss). and another which apparently originated in the fossa of Rosemüller. In their growth from the nasal passages they usually proceed upward and backward into the superior meatus, and as they progress they involve in their progress the cartilaginous and bony parts, following the superior meatus by preference to the orbit, or through the ethmoid cells into the frontal sinus and foramina, spreading in the direction of least resistance. Occasionally they invade the antrum of Highmore instead, and sometimes follow the middle meatus to the choanæ, and thence spread to the lateral walls of the nasopharynx. As they progress, however, the several accessory cavities, orbit, and even the cranial cavity, are invaded.

The myxosarcomata usually appear at first like ordinary polyps, but soon show their malignant character by assuming a sort of cauliflower, lobulated contour, and by a rapid development with a high state of vascularization. 'Initially they may appear as pediculated tumours, but soon the pedicle is lost, and we find them with a broad base, which in turn finally disappears as the mass increases and grasps the tissues about it. It usually occurs upon one side of the nose only, which it soon completely fills. Outside deformity, such as flattening of the nose, prominence or deflection of the eyeball, a projection of the facial bones ("frog-face"), indicates the extension of the growth. The recurrence of these growths after removal is usually very prompt. 
Bosworth, in summing up the results of 42 cases of sarcoma of the nasal passages found in literature up to 1889 , concludes that sarcoma of the nose does not apparently present the same malignant tendencies as it does when found in other locations. Unfortunately, all of the cases of sarcoma which the writer has met with have shown a high degree of malignancy. Dr. J. E. Boylan, of Cincinnati, reported a case of nasal spindle-celled sarcoma, which he removed by means of a snare, curetting the base, which up to two years afterward had not recurred. The 2 I cases enumerated by Boylan since Bosworth's tabulation do not include several to which reference was found, but which could not be obtained in a detailed report, nor do they include cases in which the growth did not originate in the nasal passages. Excluding 2 of these 2 I cases in which no operation was attempted, and 2 others in which it was admittedly incomplete, there remain 17 cases in which an attempt was made to extirpate the growth. Of these, but 2 are recorded to have died from recurrence, I of them four months after operation. In 5 cases no subsequent history is referred to. The remaining Io cases were reported as either recoveries or no recurrence within a few months. Bryan thinks there is a hope of complete eradication by early removal, especially where they are thoroughly exposed.

William Porter reported 2 cases of sarcoma of the nasal passages and accessory sinuses without recovery. A. A. Bliss reported 2 cases (received at the German Hospital, Philadelphia) in children, one four years of age and the other nine years, who were suffering from large sarcomatous growths (Fig. I28). He thinks in both cases the growth originated in the middle turbinate body of the left side and within the ethmoidal sinuses, and then followed paths of least resistance downward to the floor of the nares, forward and backward, and then upward. They were unusually rapid in their development. The writer has met with 2 cases in children, originating in the nasal fossæ, which were of very rapid growth.

C. H. Knight published an interesting case of fibro-sarcoma of the right nasal fossa of great extent, which ultimately caused the death of the patient. The tumour not only filled up the whole nasal passage and nasopharynx, but extended into the orbit. It was finally removed by Dr. Weir, who made Chassignac's operation. The growth invaded the ethmoidal cells 
and sphenoidal sinus. The patient was forty-two years of age. Cheatham mentions having seen 2 cases of sarcoma of the pharynx with enlarged cervical glands, I of which, in a man, sprang from the left lateral wall and completely blocked the nasal passages.

J. E. H. Nichols reported a case of sarcoma blocking the nasal passages and attached to the sphenoid bone. It was finally removed, together with a portion of the wall of sphenoid bone, by a chisel. There was very little hemorrhage. There were several recurrences, and finally an extensive external operation was made. The growth extended, involving the antrum, orbit, and pharynx, and reached the dura mater before death. $\mathrm{He}$ also reported another fatal case in which he had used Coley's fluid. A case of sarcoma of the nasopharynx, in a child six years old, was reported by A. W. McCoy, of Philadelphia. Lennox Browne thinks that this form of disease is extremely rare in young children, especially infants. McCoy saw one case a few years ago in an infant a little over one year of age. Greene, of Grand Rapids, reported the case of a very large sarcomatous growth which originated in the nose and spread throughout the nasal passages to the orbit and cranial cavity in a comparatively short time, although several operations for removal were made.

Dr. J. E. Boylan, of Cincinnati (New York Med. Jour., vol. lxiv, p. 43), reports a case of spindle-celled sarcoma of the nasal passage completely filling the left nasal cavity almost to its anterior opening. This patient suffered severely from bleeding. The tumour was removed by the cold wire snare and with flat forceps, and subsequent hemorrhage stopped by plugging the nasal passage with iodoform gauze. The site of the growth was afterward thoroughly curetted. The growth seemed to be a single tumour springing from the middle of the left turbinated body, having its attachment over about an inch and a half or an inch and three quarters of the outer wall, originating in the lower turbinated body. No recurrence has been reported.

Dr. Horace Clark, of Buffalo (Med. News, vol. lxiv, p. 716), reports a case of sarcoma of the nose in a woman seventy-nine years of age, whose family history was negative. When she first came under his observation the tumour was situated in the left nostril, was about the size of a small cherry, and bled 
upon the slightest touch. It was hard, but not sensitive. It rapidly grew in size, filling out like a bladder, and pressing out the ala of the nose. She had been subject to severe epistaxis. The left nostril had become almost entirely obliterated. The growth was removed by the galvano-cautery loop.

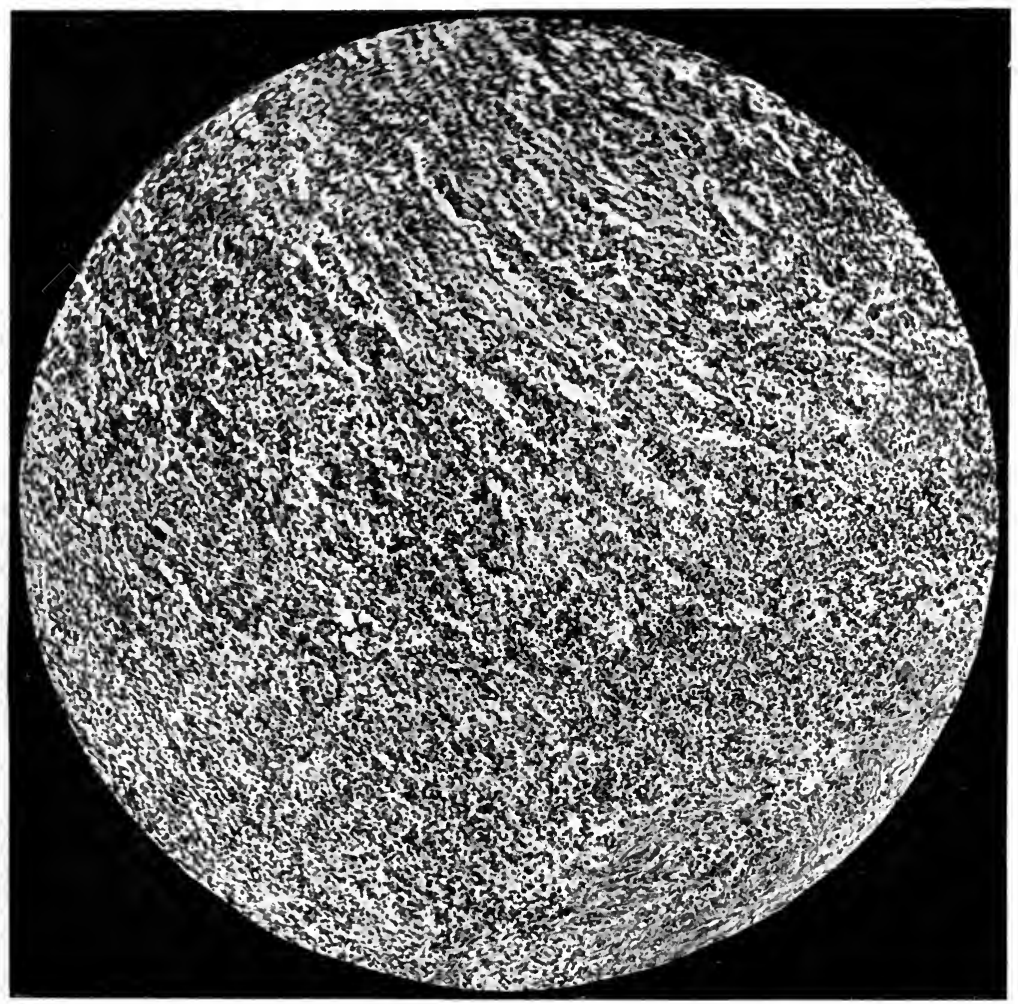

FIG. I28a.-Photomicrograph of round-celled sarcoma of nose.

Dr. Boylan urges that persons meeting with these cases, especially those operating, should report accurate information concerning them covering a period of several years.

Sarcoma of the pharynx is said to be more common in the male. It is not a common seat of the disease, however. In I9 cases, I4 of them were males. Such growths usually spring from the basilar process of the occipital bone. If it is in the nose the cervical glands do not often become involved. Small- 
celled sarcoma extend rapidly, large-celled slower. As the disease progresses, of course, it gradually destroys all the surrounding lymphatic tissues, and a proportionate degree of suppuration distinguishes its march.

Sarcoma of the Tonsil.-Next to the nasal passages, the tonsil is most frequently the seat of sarcoma; and, according to Berger, the soft palate is most frequently the seat of mixed neoplasms. He thinks the latter form a well-defined class, having marked anatomical and clinical characters, resembling either sarcoma or epithelioma. Dr. Lamphear, of St. Louis, reported 2 cases, I of whom died after ligature of the carotid artery, and the other from a recurrence of the disease. He published a table of $7 \mathrm{I}$ cases, 2 of which had been reported by Newman, of Glasgow.

According to Morell Mackenzie, in the examination of 8,289 cases of tumours of the tonsil, only 3 were of a malignant character. According to Warren (surgical pathology), sarcoma of the fauces is a much more common disease than is generally supposed. He says quite a number of operations have been performed for this affection in Boston. He mentions 4 cases of Cheever's, I each of Homans, Richardson, Porter, and himself.

Dr. William Gray, microscopist of the Army Medical Museum, reported a case of alveolar sarcoma of the tonsil which came under his observation for microscopic examination. He also gives a report of the collection of 18 cases of round-cell and lymphosarcoma of the tonsil, of which 12 were males. In one of these cases the duration of the disease is stated to be two years.

J. Solis-Cohen reported a case of sarcoma of the tonsil about the size of a hen's egg occurring in a spinster fifty-eight years of age. The soft palate was closely adherent to the surface of the tumour. It was removed by evulsion after loosening the adhesions between the soft palate and the tumour. Throughout the course of its growth, estimated at three and a half years, the patient had complained of no pain, but some difficulty of deglutition and articulation was present owing to the mechanical obstruction to the fauces. There was no enlargement of the cervical glands.

Ingals reported a case to the American Laryngological Association of sarcoma of the right tonsil, about the size of a pigeon's 
egg, in a man fifty-nine years of age. No hereditary or other cause could be assigned for the origin of the growth. The cervical glands were also enlarged. The growth was removed with the steel wire écraseur, and a month later a suspiciouslooking portion of the stump was treated with a 6o-per-cent solution of lactic acid, applied every three or four days for three weeks. This completely removed the unhealthy remnants, and the patient made a good recovery. The remaining large portion back of the soft pillar was treated by injections of 5 minims of a 25-per-cent solution of lactic acid. The strength of the solution was gradually increased to 60 per cent, which, however, on account of occasional sloughing, had to be stopped. The solution was reduced to 50 per cent, and from 8 to io minims of this was injected three times a week. This resulted in materially diminishing the size of the growth. Free bleeding immediately followed these injections. At the time the report was made the patient was greatly improved in general health, and the growth, although having extended to the tongue, seemed to be limited.

A. H. Cleveland reported a case of malignant neoplasm involving considerable of the soft palate, and extending well into one of the faucial arches, in which the growth was removed by the galvano and Paquelin cautery. The patient made a good recovery, and no subsequent operation of the cleft of the palate was necessary. This patient, like some other similar cases, had little or no pain. The writer has never succeeded in so removing epitheliomatous or sarcomatous growths from the soft palate and lateral walls of the fauces that recurrence did not promptly take place.

Symptomatology.-Sarcomata bleed very easily, and the loss of blood is quite considerable upon slight provocation; for instance, the touching of the tumour with the probe in examination may induce a free hemorrhage. Sneezing or violent blowing of the nose, or coughing, when the diseased mass is situated in the tonsil, may bring about a severe hemorrhage. This, together with signs of accruing nasal obstruction, are often the prominent symptoms. As the disease progresses, however, more or less pain of a lancinating character about the face, with or without headache, and vertigo, usually supervene. When the orbit or Eustachian tubes are reached, then ocular symptoms or perturbations of the sense of hearing, with earache, soon mani- 
fest themselves. After the growth has reached a considerable size, whether situated mainly in the nasal passages or the pharynx or nasopharynx, a certain degree of anæmia and extreme debility occurs.

This is not always in proportion to the actual loss of blood, the pyrexia, or the pain, but is apparently the result of a per-

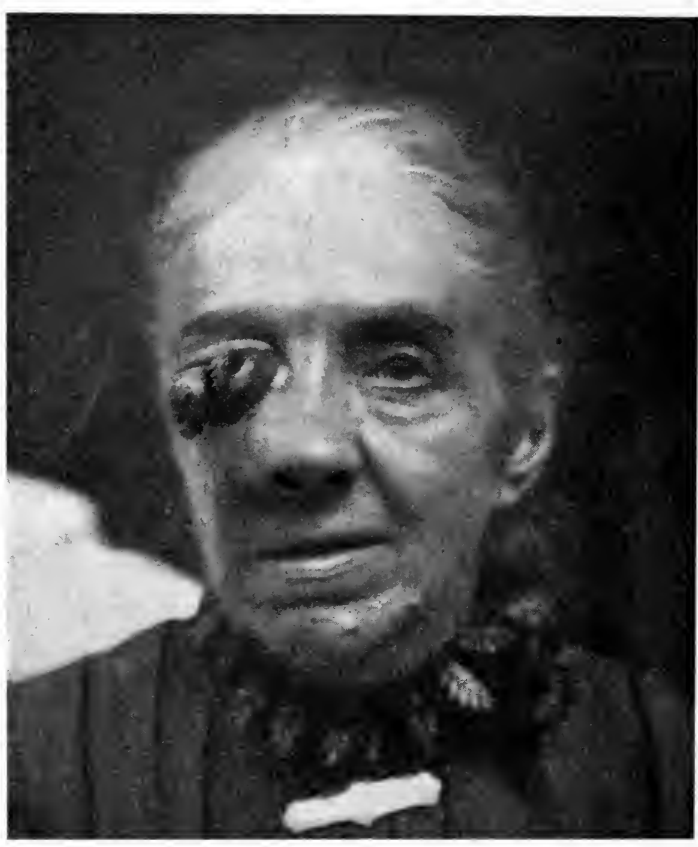

FIG. I29.-Carcinoma of antrum and secondary growth in orbit. turbation of the general nervous system from perhaps slight and continuous septicæmia. The discharge from the nose or from the throat is usually of a serous nature, or an acrid, sometimes thick mucous secretion, containing some blood and a little pus. This disease (sarcoma) usually occurs in the young, and is seldom met with in middle life. Epithelioma of the nasal passages is extremely rare as a primary affection (Fig. I29). There are cases on record which seemed to follow lupus of the nose. The common sites of these growths are the lymphoid tissue at the base of the tongue, the epiglottis, the tonsil and peritonsillar glandular tissue, and the laryngeal ventricles. Many cases, however, of laryngeal epitheliomata are found upon autopsy to have been in all probability secondary invasions from the esophagus.

The squamous epithelioma may be said to occur more frequently in the larynx than the alveolar form, while the alveolar form arises more frequently from the tonsils and glandular tissue of the pharynx. It usually begins on one side and spreads thence to the opposite side. 
Males seem to be more frequently affected than females, according to the statistics of von Ziemssen, Morell Mackenzie, Lennox Browne, Butlin, and Delavan.

Symptomatology.-Epithelioma in these parts usually has a small beginning. It is difficult frequently to discern the neoplasm in the early stages. Especially is this the case at the linguo-pharyngeal junction or in the tonsil. It may remain as a small growth for several weeks, or even months, before becoming distinct. The appearance of the parts, however, will arouse suspicion from the fact that there is a very gradual and progressive tumescence or vascularity, while the lingual glands in the neighbourhood will become more prominent. The writer remembers to have seen a case in a man aged sixty-two, in whom an epithelioma originated in the linguo-pharyngeal sulcus and base of the tongue, which was for several months difficult to recognise, excepting by the gradual tumefaction of the tissues and the symptoms evolved therefrom. Sooner or later, however, a distinct neoplasm will appear, red, creased, or lobulated, showing lacerated tufts, and finally small suppurating sinuses and sloughs, all of which will indicate the development of the growth. The symptoms will vary according to the situation of the growth; for instance, in the nasopharynx or pharynx, dysphagia is an early symptom in addition to the peculiar lancinating pains which accompany

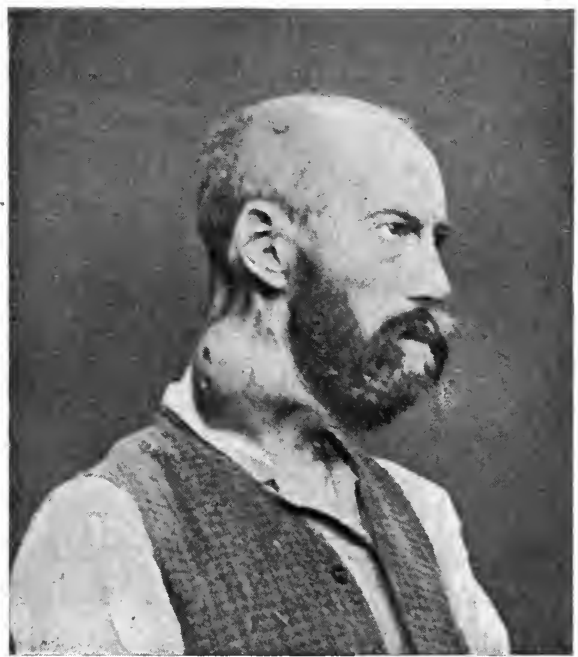

FIG. 130.-Carcinoma of the larynx and the pharynx, involving cervical glands.

the development of such malignant growths. The pain at first is fugitive in character, and radiating sometimes in one direction and sometimes in another (Fig. I 30).

When the surrounding lymphatic tissue begins to participate in the process the pain of course becomes more constant and harassing. If the growth be situated anywhere about the oro- 
pharynx or about the tonsil, there will be added to the dysphagia or pain more or less salivation, dysphonia, and disinclination to swallow. Hot, salty, or acid articles of food will cause more or less additional suffering. If the growth be quite confined to the larynx the early symptoms are hoarseness with increasing dysphonia. These symptoms may continue for a long time before aphonia actually supervenes. Indeed, complete aphonia may not occur at all, even when the disease is well advanced. Should the epiglottis be the particular site of the growth, much difficulty and pain in deglutition will occur upon contact of the bolus of food and the movement of parts. Glottic and pharyngeal spasm may occur from the entrance into the larynx of particles of food. The growth at this situation and at the base of the tongue gives rise to much more pain than if situated upon the lateral walls of the pharynx or confined to the larynx itself. Lancinating pains running into the ear are also common symptoms. Attacks of dyspnœa will come on, at first only after considerable exertion, but later upon the slightest movement of the body, especially if there be laryngeal stenosis or pressure upon the terminal filaments of the vagi or inferior laryngeal nerves. As the disease advances in the larynx respiration can only be carried on by great attention on the part of the patient, and the use of the extra respiratory muscles. The cachexia is at this period most marked, the body has become wasted, the pulse feeble and small, the facial expression extremely anxious, and the skin always bathed with a clammy perspiration. The mental faculties are somewhat dulled, although unfortunately not enough to be obtuse to the excruciating agony which each hour of life brings. To such patients death indeed is a relief. Scirrhus and the other forms of malignant disease not mentioned above are almost never met with in the upper air passages, excepting as an extension of the process from contiguous parts. The importance of an early diagnosis is very important indeed, for the fate of the patient may depend entirely upon it; for example, an early manifestation of malignant growth, if overlooked, may soon become inoperable.

Diagnosis.-Histological examination nowadays furnishes the main reliance for differential diagnosis concerning the nature of these several growths. The writer, however, is in full accord with what has been said by various authors, such 
as Cohen; Bosworth, Jonathan Wright, Lennox Browne, Schech, and others, that sometimes in the earlier stages these neoplasms can only with difficulty be made out; for often the appearances under the microscope of some of these growthsnotably papillomata and epitheliomata, myxosarcomata and fibromata, sarcomata and angiomata-are similar. The general histological characters of these several growths have been only briefly alluded to, and for a fuller account of these characteristics the reader is referred to the text-books on pathology. The clinical points of differential diagnosis between papillomata, epitheliomata, lupus, sarcomata, etc., are often extremely obscure. If situated at the base of the tongue, the lingual tonsil, epiglottis, or thereabouts, suffocative symptoms soon supervene, and there is always more or less hemorrhage from acts of coughing and deglutition. Pharyngeal mycosis has been mistaken for malignant disease, but the clinical history and appearance, even without histological examination, should suffice to remove all doubt.

\section{Neoplasms of the Accessory Cavities of the Nose}

These may be classified as extrinsic or intrinsic, the former term including all growths which have originated outside the cavity, and the latter those which have commenced within the cavity.

They may be either benign or malignant, the same as in other regions of the body. Intrinsic neoplasms of the accessory cavities are very rare; the majority of growths found there spring from either the nasal passages or the nasopharynx. The writer has met with five cases only which undoubtedly had their origin in the accessory cavities, one of which was in the frontal and four in the maxillary sinus. Of these neoplasms, two were malignant (sarcomata) and three were benign (myxomata and fibromyxomata). In two of the cases previous injury was credited with being a causal factor.

The early symptoms of a neoplasm in one of these accessory cavities are similar to those of latent empyema, but later on, as the growth of the neoplasm impinges on the surrounding walls, constant pain and progressive tenderness and deformity of the part ensue. In the case of the maxillary and frontal sinuses the bulging deformity of the external parts 
becomes marked; in the case of the frontal sinus the eyeball and nasal walls are pressed out of position, presenting the appearance technically called exophthalmos, and when the sphenoidal sinus is the seat of a growing neoplasm, alarming neurotic or cerebral symptoms will soon appear.

The prognosis and course of the case will depend, of course, upon the nature of the growth and the probability of its entire removal. If it be malignant, no procedure will probably give more than temporary relief. Malignant tumours of the nasal passages or naso-pharyngeal space usually extend to these sinuses, sometimes rapidly, and on account of the inevitable tendency to recurrence are usually inoperable. Dr. Levy, of Denver, Col., reported (in the New York Medical Journal, vol. lix, p. 335) a typical case of sarcoma of the nose in a man aged sixty-two years, involving the antrum of Highmore. The tumour was removed with the cold snare. The antrum was also operated upon and relieved of a collection of fetid, greenish fluid. Microscopic examination showed the tumour to be a large-celled sarcoma. At no time were any of the adjacent glands enlarged or affected. On account of the large size of the tumour, pressure symptoms affecting the facial, second division of the fifth, palatine branches, Meckel's ganglion, and other branches of the fifth were quite prominent.

Treatment.-The only treatment, so far as the accessory cavity is concerned, is to lay it open by one of the various methods given in the surgical text-books and thoroughly remove the growth. This should always be done in the case of intrinsic neoplasms as soon as the diagnosis is made.

\section{Treatment of Neoplasms of the Upper Air Passages}

\section{Nasal Passages, Nasopharynx, and Fauces}

The therapeutic indication in all of these cases is, of course, to remove the morbid growth.

Benign tumours can generally be removed without much difficulty, but malignant ones cannot always be so disposed of. However, there are many cases on record of recurring fibromata, sarcomata, or epitheliomata which have been effectually and permanently removed and the patient's life thereby saved. Hence in all cases which are operable-that is, where there is 
not too much of the contiguous tissue involved-an attempt should be promptly made to remove the morbid growth. But, for instance, where a pillar of the fauces, together with the neighbouring tonsil and glands, are involved in a malignant growth, there will be no hope of accomplishing thorough removal, and consequently palliative treatment only can be adopted.

The removal of the neoplasms from this region may be accomplished by one of the following methods:

a. Excision, dissection by means of the knife, scissors, or dissector.

b. Evulsion, by means of forceps, écraseur, snare, or curette.

c. Immediate or gradual destruction of the tissue by means of chemical escharotics, the electric or thermal cautery, or electrolysis.

$d$. Injection of the tissues hypodermically with various inorganic or organic fluids for the purpose of arresting or destroying the growth.

Before proceeding with any of these measures it will be necessary to obtund the sensibility of the part by the application of cocaine, eucaine, bromo-ethyl, chloretone, or the adoption of general anæsthesia.

Excision is often the best method for removing papillomata or cystomata of the fauces, soft palate, or uvula. For this purpose a pair of serrated scissors will answer the purpose well. Cysts of the nasal passages or fauces, if irremovable by the snare, can also be taken away by the scissors. Osteomata and enchondromata, however, will best be removed by the saw or gouge.

Evulsion.-Various forms of forceps for this purpose have been devised, also various patterns of snares and curettes. For

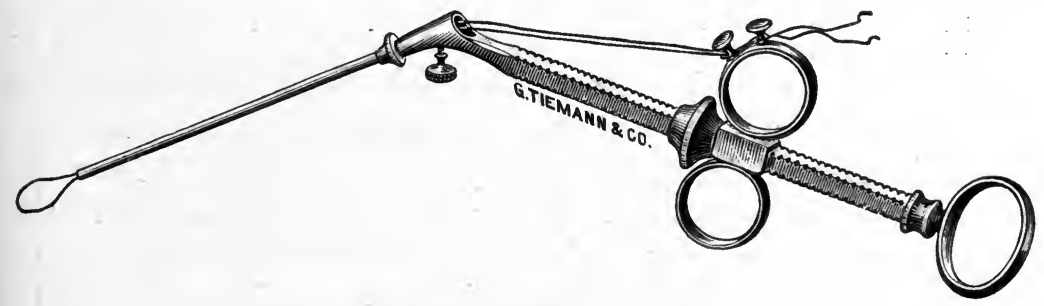

Fig. 131.-Bosworth's snare.

all-around work, however, the Bosworth snare (Fig. I3I) will be found preferable, excepting, perhaps, for the removal of fibroid 
tumours or tonsils, when the heavier Jarvis's snare may be necessary (Fig. 132). There are various spoon and ring curettes of different shapes and sizes on the market, each of which will be found useful. Myxomata and fibromata can usually be re-

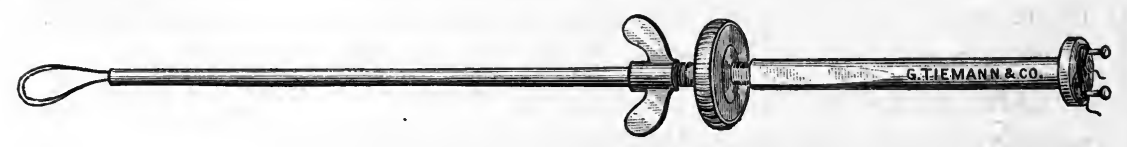

FIG. I32.- Jarvis's wire snare.

moved by the wire snare better than with any other instrument. Piano wire (Nos. 18 to 22) should be used, as a rule, although for operating on fibroid or myxomatous polyps in the nasopharynx, annealed steel, or platinum wire, which is ductile, may be more convenient.

Method of Operating with the Snare in the Nose.-The wire should be cut of such length that the loop will be large enough to encompass the growth, and yet not so large that when pulled home the loop will not entirely close at the distal point of the cannula, otherwise the tumour may slip away and not be cut through. Having the snare loaded. with the wire loop, which is properly twisted around the posts at the handle, the nostril is dilated with a nasal speculum, and the loop slowly introduced flatwise along the nasal septum until the desired point is reached, when by slowly twisting, ducking the point a little, and otherwise manœuvring, the tumour is engaged in the loop. Now, by gently depressing the handle, the loop is pushed up toward the attachment of the polyp, and a firm hold is secured, after which the loop may be slowly pulled into the cannula, either by the thumb slide or the screw. If the tissue be tough, it will be necessary to use the screw after the loop is tightened around the growth.

When operating in the nasopharynx it may be necessary to pass the loop through the nasal passage, and while holding the instrument in position, with the left hand, carry the forefinger of the right hand behind the soft palate, and with it adjust the loop around the neoplasm. When the growth hanging in the nasopharynx is large, a soft ductile wire will sometimes be found preferable to the steel spring piano wire. When the polyps are small, and there are several, it is better to introduce smaller loops and fish out, as it were, each one, rather than attempt to get out the mass. It often happens that, although 
there are several polyps growing from one pedicle, you cannot introduce a loop large enough to encompass the whole mass at once. Sometimes, when the growths are pretty well back, it is a good plan to pull them forward with the forceps just before trying to engage them in the snare.

The nasal evulsion forceps (Fig. 133) may in some cases be used to advantage in twisting off some of the smaller neoplasms. Formerly nasal polyps were always removed by evulsion with forceps. The hemorrhage is usually insignificant, although at times there is considerable loss of blood. Nasal polypi usually recur again and again. To endeavour to prevent this, it may be necessary to remove with snare or cutting forceps the middle or anterior end of the inferior turbinated bone, or to curette

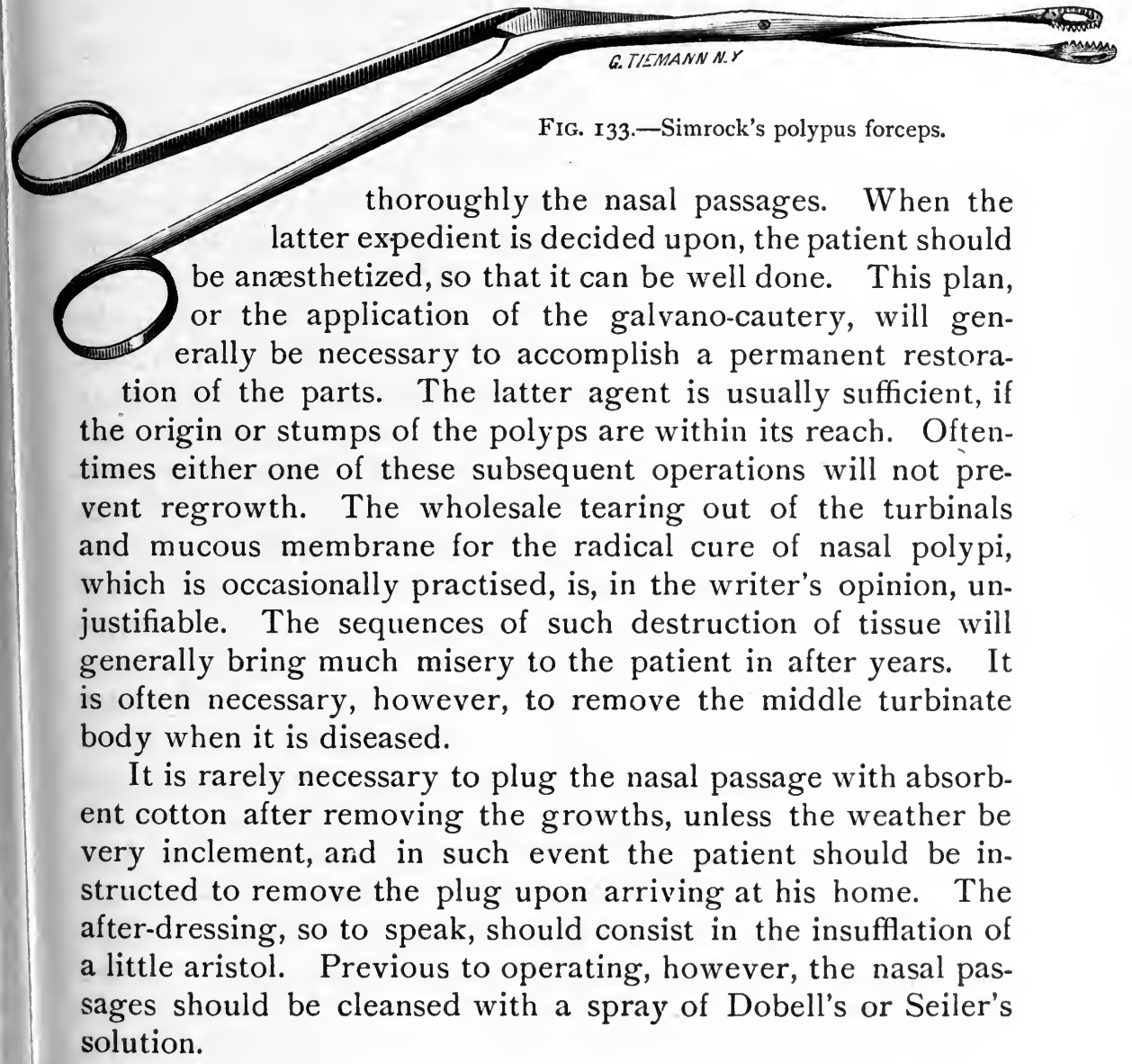


Curettement.-This manipulation should be carefully done, the instrument being introduced flatwise, with the convex surface against the septum; it is carried up under a strong light, if possible, to the seat of the polypus or edematous tissue, and gently but firmly moved through it with a see-saw motion, so as to tear away only the soft granulomatous tissue. Care should be exercised also not to wound the tissue at the attic of the nose any more than possible.

The galvano-cautery loop may be necessary for the removal of some tumours, such as fibromata or angiomata, malignant growths, or neoplasms occurring in bleeders. In such cases it will be necessary to adjust the loop either with the finger or with slender forceps before the growth can be inclosed. For large nasopharyngeal growths this is the preferable instrument. The doubled wire should be introduced along the floor of the nose into the nasopharynx and there caught, as it were, with the finger of the other hand, as it lies against the posterior wall of the pharynx. It is now opened out into a loop with two fingers in the pharynx, and passed around the tumour, while from the other end it is being gently drawn forward through the nose. This being done, and the tumour engaged, the ends of the wire are put through the cannulæ fixed to the handle, drawn up firmly and gently, and the current turned on, during which time the loop is gradually tightened.

Destruction of the growth by chemical caustics or galvanocautery is not often resorted to for benign growths, but for malignant ones it is the principal means of treatment.

The galvano-cautery loop, when applicable, is the most expeditious and safest means of removing or destroying malignant growths. But often the tumour is of such shape, extent, or in such a position, that the loop cannot be used, in which event the flat electrodes must be substituted.

When used, the electrode should be made to destroy as much tissue as possible at a sitting - all of it, if practicable-for light superficial touching of an epithelioma or sarcoma does more harm than good, by stimulating its growth. The thermocautery is not equally as efficient, because it cannot be thoroughly applied in the nose or throat, nor is its distinctive action as perfect as the electro-cautery.

In many of the fibromata and sarcomata electrolysis is a 
valuable and safe agent to use. Dr. Kwrasberg, of Copenhagen, recommends electrolytic treatment for fibrous tumours of the nasopharynx especially. He has reported four cases successfully treated in this way. Dr. J. Solis-Cohen reported the cure of a case of rhino-pharyngeal fibro-sarcoma by electrolysis after between twenty and thirty sittings. The writer has been successful in two cases of fibromata.

Many practitioners report the successful reduction and cure of sarcoma, epithelioma, and fibroma by means of galvano-cautery.

Dr. Charles L. Leonard, of Philadelphia, reports the results of Röntgen-ray treatment. The patient was forty years old and had a sarcoma of the left tonsil that nearly filled the pharynx. X-ray treatments were applied both internally and externally. The patient's general condition improved rapidly, the tumour gradually decreasing. The tonsil has nearly become normal in size. He is still receiving occasional treatments, but has attended to his business for the past six months.

The recurrence of malignant growths, even after apparently complete removal, is discouraging. It has been the writer's misfortune, as a rule, to observe the recurrence of the growth within a greater or lesser length of time in nearly every instance, even after its apparently complete destruction or removal, and in every case of sarcoma of the nasal passages or nasopharynx recurrence has taken place either within a few weeks or months, or at the end of one or two years.

Chemical Escharotics.-Of all the chemical escharotics, chromic acid seems to be the best. Even this agent, however, is far inferior to the galvano-cautery or electrolysis.

Injection into the substance of the neoplasms is not a new plan of treatment, at least for benign ones, for it was the early practice to inject polypi with solutions of tannic acid, nitric acid, tincture of iodine, tincture of the chloride of iron, alcohol, etc. ; and twenty-five years ago some surgeons-among them Dr. E. M. Moore, of Rochester, N. Y.-injected carcinomatous tumours with alcohol and tincture of iodine (Fig. I34). Solutions of lactic acid have been recommended by Ingals, and solutions of chloracetic acid by Gleitsman; also chloride of zinc and persulphate of iron by others. Recently our hopes were raised by the idea advanced by Dr. Coley of injecting a sterilized culture of streptococci for the cure of malignant growths. The writer and his assistants used Coley's fluid hypodermically 
on eight patients at St. Mary's and Harper Hospitals, and also in private practice, but without in any instance observing any permanent benefit. The cases were sarcomata and epitheliomata. The writer used a ten-per-cent solution of o sodium formate in two cases of epithelioma, in one of

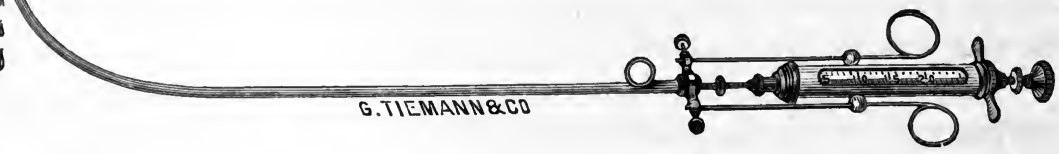

FIG. I34.-Upson's syringe for hypodermic injections of various substances for the destruction of polypi.

which the growth seemed to retrogress for a short time. In one case of lymphosarcoma of the tonsil and faucial arch, in a woman fifty years of age, injections of dog serum and iodine were used alternately every other day for a period of four weeks without any beneficial effect. Injections of carbolic acid have also been used to some extent.

It seems, therefore, that for the treatment of malignant growths of the nasal passages, pharynx, and fauces, the best plans of treatment, as a rule, are the galvano-cautery and electrolysis.

General Treatment.-No specific constitutional treatment has proved efficacious for malignant neoplasms. Tonics and good food are about all that it is necessary to recommend. Arsenic, iodine, and iron are still recommended as beneficial adjuvants. If bacteriologists can establish with certainty that cancer is a microbiotic disease, that fact may lead to some potent addition to our therapeutic armamentarium ; at present, however, we can see but one certainty in the therapeutic field, and that is extirpation by knife or cautery.

\section{NeOplasms OF THE LARYNX}

The neoplasms most frequently found in the larynx are papillomata and fibromata, and next to these epitheliomata, but cystic tumours, myxomata, sarcomata, angiomata, lipomata, adenomata, and gummata are occasionally found. Malignant growths may occur and remain confined to the inside of the larynx, when, according to Picqui's classification, they are said to be intrinsic, and when originating outside the cavity of the 
larynx they are said to be extrinsic. Excepting epitheliomata, the majority of the malignant growths are usually extrinsic.

The relative frequency of the several neoplasms is shown by Massei's table, based on the collection of reports of five hundred cases, as follows:

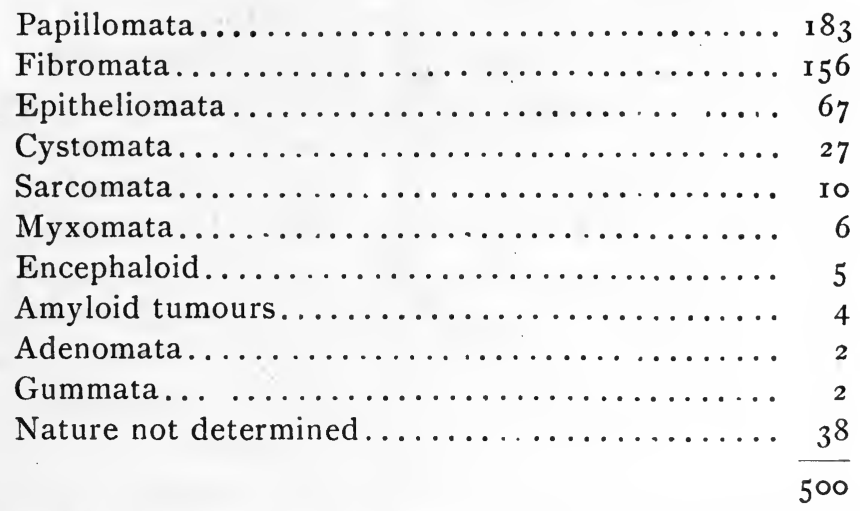

Etiology.-Laryngeal neoplasms are not as frequently met with as formerly, either in the United States or in Europe. The reason given by Massei, of Naples, for this fact is undoubt-. edly a good one, namely, that owing to the development of laryngology during the last two decades, acute and subacute affections of the larynx, which so often constitute an important etiological factor, are more promptly attended to and relieved. This is also true concerning syphilis. Fox, of New York, has stated that, owing to the education of the people regarding this disease and the facilities for treatment offered to the poor, the later features of syphilis, especially as manifested in the skin, are much more rarely seen than twenty-five years ago. This statement, I think, can be corroborated by the majority of practitioners. Any portion of the intralaryngeal surface may furnish a site for neoplasms, but, as a rule, they are more often found upon the vocal bands, especially their anterior and posterior portions, than in other places.

The susceptibility of one portion of the larynx more than another to become the seat of a neoplasm may be accounted for in great measure by the different histologic character of the several parts of the organ, the greater mobility of one part over another, and the greater liability to irritation and injury of one part more than another. For instance, the vocal cords, so rich in epithelial tissue, and also so mobile, are especially the 
seat of the epithelial growths, both benign and malignant. Of I, IOO cases of laryngeal neoplasms collected by von Bruns, 836 were on the vocal cords. They are less frequently seen in the subglottic space. According to Fauvel's statistics as quoted by Wright, out of 300 cases of laryngeal neoplasms collected, only 9 were subglottic. The writer has met with 4 cases of subglottic laryngeal growths out of an estimated total of 50 cases. Two cases were gummata, probably, and 2 were papillomata. One of them was a case of multiple papillomata in a child four years of age, some of which grew from the ary. epiglottic folds, and some (5) were subglottic. A thyreotomy was done and the subglottic neoplasms were removed. The child made a good recovery and has a tolerably good voice. The growths of the upper larynx were removed by evulsion with forceps after many attempts, the parts having been sprayed each time with a 2-per-cent solution of cocaine.

Many laryngologists have held that tumours found upon the anterior portions of the vocal bands were more likely to be of benign nature than those situated posteriorly. However, Delavan and others state that they have met with enough exceptions to invalidate any such rule.

Papillomata.-Papillomata-or warts, as they are sometimes called-are the most frequent of the new growths met with in the larynx. While, as a rule, they spring out from the vocal bands, yet they are frequently seen attached to the ventricular bands, epiglottis, or posterior portions of the ary-epiglottic folds. They may grow in clusters when the case is denominated a diffuse or multiple papillomata, or

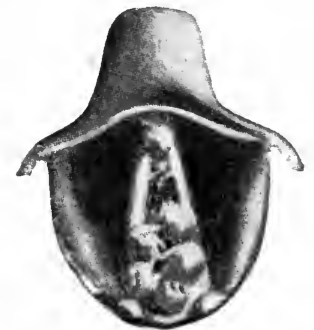

FIG. I35. - Congenital papillomata (W. F. Chappell). they may occur singly. In the multiple or clustered variety pedicles are not so distinctly formed as in the discrete or single variety. Very interesting cases of the clustered form of grow th have been reported by Jarvis, Chappell, and others, where nearly the whole upper laryngeal region was occupied (Fig. 135). When occurring singly the pedicle may be of considerable length, so that the tumour is quite mobile, in which case it is usually round or pyriform in shape.

Etiology.-The etiology is ascribed to irritation, frequent attacks of laryngitis, or excessive use of the voice. Speaking 
of the etiological factors of papilloma, Fauvel, J. N. MacKenzie, Cohen, Lennox Browne, Semon, and Sajous regard the principal etiological factor in the development of the simple papillomatous growths as hyperæmia or chronic laryngeal catarrh, while Bosworth, on the other hand, believes that many of them grow from a healthy membrane. . Gerow and Fauvel believe that a papilloma is not a tumour but merely a proliferation of the mucous membrane, and that this proliferation will be produced, for example, under the direct irritation of the membrane, due to a purulent, viscid, or dry secretion, such as one sees in the various catarrhal affections. Dr. A. B. Thrasher reported a case of papilloma of the epiglottis in connection with adenoid hypertrophy at the vault of the pharynx, and two small, almost sessile, papillomata of the vocal cords. The epiglottic tumour was attached very near the free border, was about the size of an English walnut, and rested upon the laryngeal surface. It gave rise to many attacks of coughing and more or less dyspnoa. The writer met with one case of multiple papillomata in which two of the neoplasms were situated upon the laryngeal face of the epiglottis. Indeed, in multiple papillomata the epiglottis is frequently the seat of one or more growths.

It has been observed that these growths have a progressive and a retrogressive stage. The periods of growth and retrogression, however, have no particular limit, so that one cannot predict the length of time occupied by either stage. They may recur after removal, and may even disappear spontaneously. This is not an uncommon event in the case of children.

Dr. Dunn, of Richmond, Va., reports a case of intralaryngeal tumour (New York Med. Jour., vol. lvi, p. 568) which was, from the clinical history, characterized by severe pain in the ear and by the presence of a bulging over the left laryngeal ventricle, and which was diagnosed as carcinoma. This patient was a hay-fever subject, and as it was about decided to adopt operative procedures for the removal of the growth, the usual hay-fever attack came on, during which time the laryngeal symptoms passed away, and also the laryngeal growth. This is cited as an example of the difficulty of exact diagnosis in endolaryngeal growths, and also as another example of unexpected, almost spontaneous, disappearance of the tumour.

Dr. R. F. Lincoln, of New York (New York Med. Jour., vol. iii, p. 78), reported to the American Laryngological Asso- 
ciation a case of laryngeal tumour the size of a small egg, which was thoroughly removed by the late Dr. Elsberg, by the operation of thyreotomy. The patient remained free from any symptom of difficulty for twenty-two years, when she came under the observation of Dr. Lincoln, having a tumour about the size of a large kernel of corn growing from the posterior third of the right vocal cord. The former tumour was attached to the anterior portion of the larynx. Microscopical examination of the tumour showed it to be a simple papilloma.

Dr. Jos. A. White, of Richmond, Va., reported a case that he saw of multiple papillomata of the larynx in a child, with the anomaly of a distinct papilloma on the epiglottis.

Dr. J. Solis-Cohen, of Philadelphia (Medical - News, vol. lxiii, p. 664), reported a case of multiple papillomata in a child (in whom tracheotomy was performed) at four years of age. Laryngeal inspection revealed the presence of multiple neoplasms filling the upper portion of the larynx and extending along the laryngeal face of the epiglottis almost to its very tip. There were likewise a few multiple neoplasms at the base of the tongue and along the pharyngo-epiglottic and glosso-pharyngeal folds. There was very slight repullulation after the last removal of the growth.

Papillomata of the larynx often coexist with similar growths in other regions, such as the nose, pharynx, etc.

Dr. Wright reports the case of a housemaid, aged twenty years, who presented several papillary growths in the nasal passages, and a subglottic tumour of considerable size attached on the right side of the cricoid cartilage. There was no history of syphilitic disease. Subsequently thyreotomy was performed, and the growth was found to extend two and a half inches below the vocal cords and to involve three fourths of the circumference of the trachea at the point of greatest growth. It had the microscopic appearance of an ordinary papilloma.

Sex.-These neoplasms are more commonly found in the male than in the female sex.

Age.-They may occur at any age; indeed, a large number. of cases are met with in childhood, sometimes in very young children.

There have been many reports of congenital cases. Chappell met with one in an infant four weeks old.

Dr. Jos. A. White, of Richmond, Va. (Jour. Amer. Med. 
Assoc.), reports a spontaneous cure of multiple papillomata of the larynx after a tracheotomy in a child aged five years. The diagnosis was made by laryngoscopic examination. The growths were at first removed with forceps by the endolaryngeal method, but two months afterward the child returned with a recurrence of the growth, and suffering from great dyspnœa. It was also noticed that a papilloma was growing from the lower face of the epiglottis on the right side. The child was operated upon again by the forceps with the result of removing most of the growth, but the following November it was brought back with a further recurrence in the shape of a large cauliflower mass in the larynx, and a pedunculated tumour of the epiglottis. A tracheotomy was then made. He recommends tracheotomy in the place of thyreotomy in these cases, and thinks the tracheotomy tube ought to be worn for a long time until all danger of recurrence of the growth has passed. This, however, cannot always be carried out on account of the weakening of the tracheal wall, and because the tube excites the growth of dangerous granulations.

Many practitioners, among them Lennox Browne, believe that adenoids in the nasopharynx are a factor in the production of papillomata of the larynx in children. The writer has never met with the coexistence of laryngeal papillomata and marked adenoid enlargement at the vault of the pharynx. They are apt to grow slowly, progressively, and reach a considerable size. In a case which came under the writer's observation a few years ago, there were four or five papillomatous growths in a cluster around a fibroma, the body of which was the size of a large pea. This hung down between the vocal cords, being attached to the upper surface, while the papillomata seemed almost sessile, and gave one the idea of a small cauliflower. The multiple variety is more frequently met with in children, while, on the other hand, the discrete or single form is usually found in adults (Plate II, Fig. 4).

Fibromata.-Fibromata occur next in order of frequency, according to general statistics. They usually occur singly, although sometimes they are met with as lobulated growths, and are attached to either the ventricular bands or vocal bands (Fig. 136). They are prone to spring from the edges of the vocal bands, especially at the posterior part (Fig. 137). They 
are sometimes associated with papillomata and sometimes with adenomata of the larynx, and at times they undergo cystic degeneration.

Chiari believes that fibromata of the vocal cords are inflammatory, and not neoplastic, in origin. They are frequently edematous, and sometimes contain hemorrhagic extravasations. According to Clinton Wagner and M. Macken-

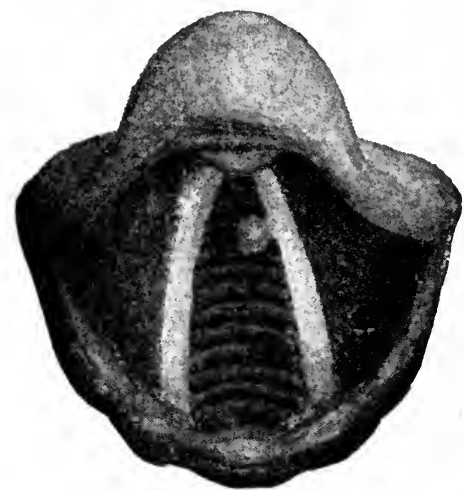

FIG. I36.-Fibroma of the vocal cord (W. F. Chappell).

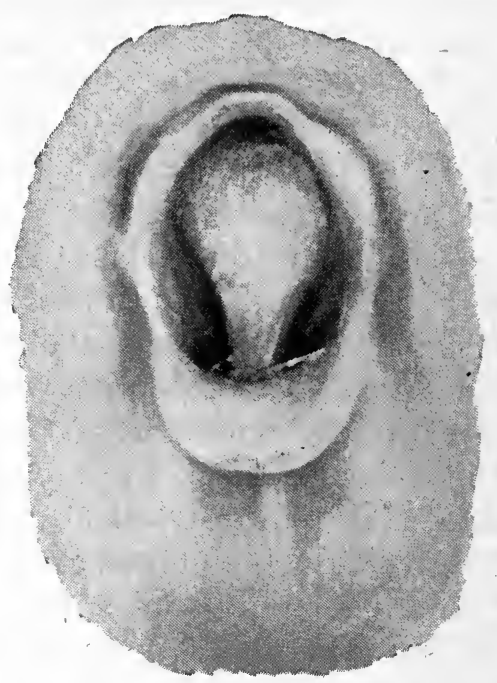

FIG. I37--Fibro-myxoma (H. L. Swain).

zie, fibromata occur in about eleven per cent of the cases of laryngeal neoplasms, and according to Fauvel, in about five per cent of these cases.

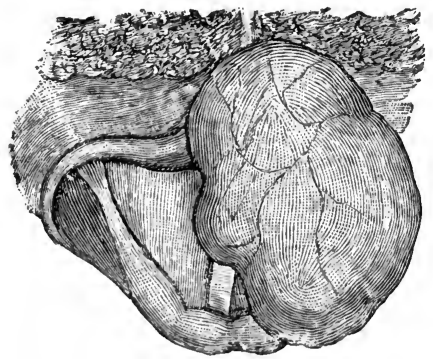

FIG. 138.-Myxoma of the epiglottis (Vander Poel).

Myxomata are rarely found in the larynx. A few cases have been recorded (Fig. I38). C. H. Knight reported a case of subglottic myxomata which was at first diagnosed as a papilloma (Fig. I39). Dr. Alexander IV. McCoy, of Philadelphia (New York Med. Jour., vol. lvii, p. 126), reported a case of myxoma of the larynx, one of those exceedingly rare grow ths. He also alludes to a remarkable case of multiple myxomata of the larynx reported by Clinton Wagner. The writer has never met with a case.

Lymphomata are occasionally met with in the larynx, but all authors regard them as of rare occurrence. Jonathan Wright 
believes that these neoplasms are not so uncommon as is generally supposed, and that if some of the papillary tumours were more carefully examined they would be found to consist largely of lymphoid tissue. Wright says that more than one

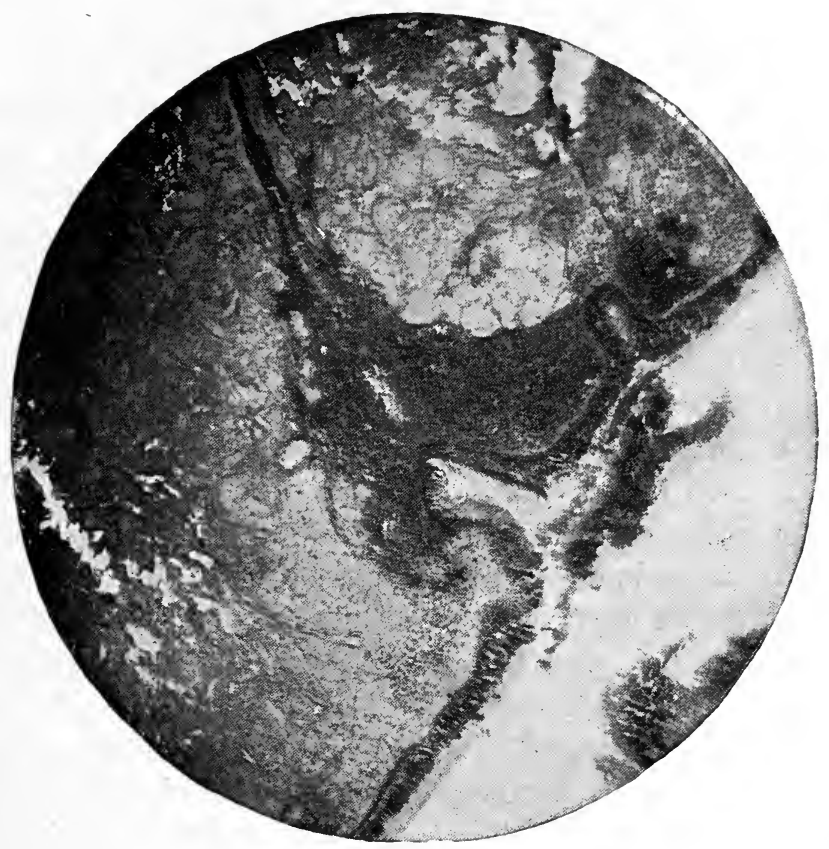

FIG. 139.-Subglottic myxoma.

half the cases of benign laryngeal neoplasms have been reported as papillomata, which is suggestive of insufficient pathologic discrimination. He reported a case which was shown by histologic examination to be a lympho-papilloma. He mentions two or three cases only of this form of growth-one case referred to by Gottstein and one by Beal (in London Lancet), both of which were connected with general lymphomatosis. Wolfenden and Martin also, in their Studies in Pathologic Anatomy, allude to and give an illustration of lymphoma confined to the larynx.

Lipoma is also very rarely seen in the larynx. J. H. Farlow reported to the American Laryngological Association an interesting case of polypoid lipoma of the larynx, and Dr. P. McBride has reported a case of fatty tumour of the epiglottis. 
Gummata and Condylomata.-These syphilitic outgrowths but rarely appear in the larynx and trachea independently, and, when occurring, indicate that some previous chronic lesion has preceded them. They may be accompanied by gumma of the mediastinum. Moreover, they are often classed or recognised under the terms laryngitis hypertrophica (superior and inferior), or pachydermia laryngitis. E. H. Griffin (in the New York Med. Jour., vol. lxii, p. 627) reported a case of condylomata in the trachea. The writer met with a case of severe laryngeal stenosis from gummatous swelling in the larynx, appearing like laryngitis hypertrophica, necessitating tracheotomy. The patient recovered.

Gummata in the form of isolated tumours are not very frequently seen upon the laryngeal mucous membrane. When occurring, they are usually found upon the anterior or posterior wall or in the subglottic region. The case of subglottic hypertrophy, related in the chapter on Syphilis, which came under the writer's observation, was undoubtedly a gummatous growth at first, as was also the angiomatous case. These gummata may break down very rapidly, or may pursue a more chronic course of gradual absorption under the administration of suitable antisyphilitic treatment.

Dr. S. K. Merrick, of Baltimore, reported a case of a gummatous tumour of the left laryngeal ventricle closely simulating an everted ventricle (a condition first brought to notice by Dr. Lefferts, of New York). Although the stenosis was considerable, the administration continuously of antisyphilitic treatment, together with the local application of dilute lactic acid, was quite sufficient to secure the dispersion of the growth.

Angioma.-Angiomata of the larynx are exceedingly rare, but few writers claiming to have seen them in their own practice. Fränkel reports two cases, Heinze one, Elsberg two, Kidd one, MacKenzie one on the ventricular band and one in the hyoid fossa. All those reported were of soft consistency when situated at or near the anterior part of the larynx.

The writer has met with but one case which occurred in a syphilitic subject, and which was diagnosed as a gumma. The growth almost entirely encircled the lower laryngeal and upper tracheal region. Tracheotomy was done to relieve the urgent dyspnœa, which had gradually developed in spite of antisyphilitic treatment. The growth, of course, was incised in opening 
the trachea, which gave rise to uncontrollable hemorrhage, which finally resulted in the death of the patient.

Ferreri reports a case of angioma the size of a hazelnut, in the inferior portion of the left vocal band. It was crushed with forceps. Alarming hemorrhage took place at night, which was with difficulty controlled. Tracheotomy was performed, when alarming hemorrhage again took place. The patient died forty-eight hours after from infectious pneumonia.

Dr. W. C. Gibson, of St. Louis (Amer. Jour. Med. Sciences), reported a case of cavernous angioma, about the size of a large pea, attached to the anterior part of the left vocal cord. The tumour was lobulated, and a portion of it reached the vocal commissure, while another portion was attached by a pedicle to the surface and edge of the vocal cord. This permitted it at times to fall below the cord. The growth was removed by Schrötter's tube forceps and the base of the growth cauterized. There was very little hemorrhage.

Cysts.-Cysts of the larynx are also rare. They usually occur as a single growth springing from one of the vocal cords, the ventricle of the larynx, or the epiglottis-more often from either the epiglottis or laryngeal ventricle. Moure found that a large percentage of these cysts were attached to the vocal cord. Schwartz collected the statistics of ${ }_{1} 38$ cases. Of this number, 67 were extralaryngeal, 61 being of the epiglottis; while of the intralaryngeal, 48 were of the vocal band and only 9 of the ventricle (C. H. Knight).

Cysts in this situation are usually of primary origin (not being degenerated adenomata). Casselberry saw one springing from the left arytenoid body. C. $\mathrm{H}$. Knight, Noquet, the author, and others have reported cases of cysts of the epiglottis. The contents of these tumours are usually seromucous. They present a characteristic pearly colour and translucent appearance, seem very movable, and are, as a rule, attached by a pedicle (Fig. I40). Moure, Gavel, and others

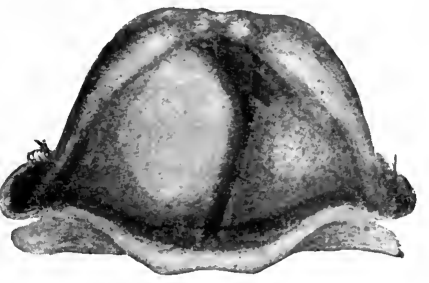

FIG. I40.-Cyst of the larynx (Ingals). have collected and reported a number of these cases. The writer on one occasion removed a laryngeal cyst from a man who undoubtedly had carried the growth for a number of 
years. He had experienced very little inconvenience from it, excepting when the tumour happened to remain between the vocal cords long enough to obstruct respiration for a few moments. It of course embarrassed phonation some. So little did it inconvenience the patient, however, that he was with difficulty persuaded to submit to its removal.

Tubercular Tumours.-Tubercular tumours (independent) sometimes develop in the larynx and reach a good size before breaking down. They often resemble papillomata or other laryngeal neoplasms. Tobold, Hennig, Ingals, J. N. MacKenzie, and J. P. Clark, of Boston, have reported such cases. Clark's excellent brochure on the subject cites a number of cases (13) reported by various authors. (See chapter on Tuberculosis.) The writer has never met with tubercular tumours of the larynx larger than a small pea (granulomata), although having examined many hundred cases. Notwithstanding that the histologic examination of some of the reported cases may show small tubercles and giant-cell formation, as in Heryng's and Clark's cases, yet the possibility of the neoplasm being an independent growth incident to tuberculosis only is very great. In other words, a laryngeal fibroma or papilloma may coexist in a tuberculous subject, as well as in a case of Bright's disease of the kidneys.

\section{Malignant NeOplasms}

Carcinoma.-Among the malignant growths, epithelioma is more commonly met with, although there are cases on record of glandular carcinoma and sarcoma, arising in the larynx. Indeed, it may be stated that epithelioma of the larynx frequently occurs as a primary affection. Malignancy here does not differ very much from that of any portion of the body. Massei, in tabulating his 500 cases of laryngeal neoplasms, mentions having met with epithelioma in 67, sarcoma in 10 , and encephaloid carcinoma in 5 of them.

Primary cancer may originate at any point within the lar$\mathrm{ynx}$, but is rarely infraglottic. Butlin found, of 50 cases of laryngeal cancer, that 3 only were infraglottic. The poverty in lymphatics of this region as compared with the ary-epiglottic folds and the supraglottic area is generally supposed to be the cause of this rarity. Regarding the seat of origin, Morell Mackenzie found that in 50 out of 100 cases one of the ventricular 
bands was the part first affected, while Schwartz affirms that the epiglottis and the vocal cords are most frequently attacked. Cancer of the larynx may exist with tubercular and syphilitic disease.

Sokotowski has reported two cases of laryngeal cancer with pulmonary tuberculosis. Semon also has seen this combination of cancer and tuberculosis. Cheatham has also written of having seen a case of undoubted cancer of the larynx accompanying tertiary syphilis.

Sex.-Males are more susceptible to malignant neoplasms than females.

Age.-Malignant growths of the larynx are rarely met with under forty years of age, exception being made in the case of sarcoma, however. While published statistics vary a little as to the age limits of the latter, still its appearance is rarely met with under forty years of age. Sarcoma of the larynx in a child seven years of age and epithelioma in a child three years of age have been reported.

Sarcoma.-Dr. Garnet, from a study of 60 cases, states that sarcoma of the larynx is much more rare than epithelial tumours in this region. The etiology is obscure, as it is of sarcoma in general. It has been found at all ages, even in children, and, like epithelial tumours, it is much more frequent in males than in females. The majority of cases observed are very dissimilar. But two clearly defined types exist. First, the infiltration form, characterized by extralaryngeal encroachment-its eccentric progress invading the neighbouring organs-and by the absence of ulcerations of the sides of the laryngeal cavity. Second, the vegetating form, characterized by the formation of numerous intralaryngeal polypi. All of the histological varieties have been observed in the larynx.

Dr. John A. Thompson, of Cincinnati, reported a case of sarcoma of the larynx in a blacksmith thirty-five years of age, which was finally removed by laryngectomy, with recovery of the patient. The growth was unusually rapid in its course, although the lymphatic glands were not involved. The tumour was not very painful except on pressure. It seemed to spring from the inner portion of the left side of the larynx. Portions of the growth were removed from time to time during a period of a month. As microscopic examination failed to clear up the diagnosis as between sarcoma and syphilis, the patient 38 
was put upon antisyphilitic treatment, but with no effect in hindering the growth of the tumour. Finally laryngectomy was performed.

Site.-The early site of primary laryngeal epithelioma or encephaloid carcinoma is usually the laryngeal ventricle, ventricular bands, or epiglottis. Perhaps the epiglottis is as frequently attacked as any other portion of the larynx. It often happens, when the growth is observed to be occupying the posterior wall or either arytenoid region, that it will be found to have taken its start in the esophagus, or in the lymphoid tissue between the esophagus and larynx. It usually begins as a small excrescence, appearing very much like papilloma, although showing a more uneven or mammillated surface and a brighter red colour. It appears to be sessile. Sarcoma and encephaloid cancer of the larynx are much more rapid in their course than epitheliomata; especially is this true of sarcoma, which may involve, even in a few weeks, the whole of one side of the larynx, including the deep lymphatic tissue of the neck.

Prolapse or eversion of the laryngeal ventricle was first pointed out by Lefferts in I882. This morbid condition is exceedingly rare, and is the result of rapid swelling of or the entanglement of foreign material in the laryngeal ventricle, or traumatism. It closely simulates a neoplasm.

The diagnosis, however, can be arrived at from the clinical history of the case, if not by laryngoscopic examination.

Symptomatology.-The symptomatology may be considered under two heads, viz., subjective and objective.

The subjective symptoms during the earlier growth of laryngeal neoplasms are not always very marked, unless the growth be so situated as to interfere with respiration or phonation. Alteration (hoarseness) or extinction of voice (aphonia) may be the principal difficulty. One of the earliest symptoms, however, is hoarseness, the amount of which will depend, of course, upon the position and mobility of the tumour. Laryngeal neoplasms may occur with little or no disturbance of the voice and respiration when the neoplasm is out of the way of the vocal cords or glottis. When, however, the edges of the vocal cords are impinged upon, phonation is at once seriously interfered with; the voice is not only hoarse, but irregular, altered in its quality, and characterized by discordant interruptions or instantaneous changes of tone: for example, if the patient 
be asked to say, "The good deeds of men live after them," the first two words may be uttered in a low-pitched voice, the next two in a high-pitched, piping tone, and the utterance of the next may be interrupted by either a silent or a whispering interval, while the last word or two will be brought out in an expulsive or explosive manner under stress of a strong expiratory effort. This uncertainty and unevenness of phonation is quite characteristic, although it may occur in other diseases, such as insanity, bulbar paralysis, chorea, or other neuroses. Arthritic inflammation of the arytenoid joints and deafness may be accompanied by similar peculiarities of phonation. When the growth is large, more or less extinction of the voice and dyspnœa necessarily supervene. There is generally no pyrexia or other particular systemic symptom excepting in cases of tubercular tumours.

In a case of epithelioma of the larynx reported by Dr. George R. Fowler, of Brooklyn, the aphonia which was present during the early stages of the growth was afterward supplanted, as the tumour grew in size, by a return of the voice. This was accounted for by the new position of the growth allowing the right vocal cord to approach its fellow.

The benign neoplasms usually give rise to very little pain or difficulty of deglutition, but, on the other hand, malignant neoplasms, here as elsewhere, are apt to give rise to considerable suffering almost from the beginning. The pain with deglutition will depend somewhat upon the location of the growth, and whether the surrounding tissues are in a state of ulceration or inflammation. For instance, an epithelioma of the epiglottis, or one situated in the region of either of the arytenoids, gives rise to considerable suffering, especially upon acts of deglutition. Dyspnœa may be a prominent symptom if the growth be large enough to mechanically interfere with respiration by pressure, or otherwise encroach upon the nervous apparatus of the larynx. Enlarged lymphatic glands which may press upon the branches of the superior laryngeal nerve will oftentimes give rise to marked disturbances of the respiratory function, although there be no endolaryngeal obstruction whatever to the glottis. 
The Objective Symptoms-Laryngoscopic Appearances

The laryngoscopic mirror will usually bring to view endolaryngeal or intralaryngeal neoplasms. When growths are situated upon the posterior wall and well down into the larynx they may escape observation for a time, unless one adopts the method of Killian (described elsewhere) for examining the posterior wall. Sometimes when a very small growth (such as a papilloma) is seated upon the edge of the vocal cord, very near the anterior commissure or upon the under side of it, it may, unless great care be taken, also escape observation. In the case of children it is often impossible to make a laryngoscopic examination, and the nature and seat of the growth, therefore, cannot be made out. Several times the writer has had under observation children who were suffering from laryngeal growths, in whom, notwithstanding the exercise of great patience (and even with the aid of an anæsthetic), scarcely a glimpse of the anterior of the larynx could be secured. The new method of examination (direct) recently promulgated by Dr. Kerstein, has not, in the writer's practice, proved more successful with children than the old way. The failure, however, may be ascribed not so much to the method as to our lack of manipulative skill.

According to Dr. Fränkel, the most frequent form of laryngeal carcinoma seen is that of a tumour on the vocal bandpolypoid carcinoma. At first there is no other symptom than hoarseness, which is not due to any immobility of the vocal band, but rather to the mechanical interference with the tonal qualities of the vocal membrane, and this is not peculiar to carcinoma. These polypoid carcinomata of the vocal band appear in the commencement as a flat, broadly sessile elevation, and grow into the tissues more than out into the free space, as is the case with benign growths. As the epithelioma breaks down, the surface appears either flat or rugose, and grayish or whitish. This form is usually a keratoid or horny carcinoma (Fränkel). In some cases carcinoma of the vocal cords is diffuse, and has the disposition to extend in the superficies instead of forming a circumscribed tumour. At first it can hardly be distinguished from any other thickening of the mucous membrane, but soon small hemispherical nodules, the size of a pinhead to that of linseeds, project from the diffuse 
tumefaction, sometimes one only, sometimes several. Some portions appear lardaceous, while others appear hyperæmic. Almost invariably one band (or cord) only is affected. The growth is slow. There is less tendency to extension in depth than on the surface, until finally the entire vocal band becomes involved. Even then the band may maintain its general contour and motility. In the keratoid carcinoma the "cornification" is the prominent feature; in simple carcinoma it is the papillary formation. The impaired motility of the vocal band, to which Semon has recently called attention, has been noted by Fränkel only when the growth occupied the posterior portion of the vocal band where the vocal process penetrates the soft parts. In such cases the median position of the vocal cords soon takes place. Polypoid and diffuse carcinomata occur in the ventricular bands or ary-epiglottic folds, but the chalky-looking masses do not appear. Tumefaction is red, sometimes redder than the healthy tissue surrounding. The polypoid form is more distinctly dendritic than when on the vocal band. The extension, though slow, is rather more rapid than where there is pavement epithelium. Carcinoma of the posterior wall of the larynx soon causes impaired motility of the vocal bands; sometimes it causes painful dysphagia at the commencement. Carcinoma of the ventricle causes at first no other change than to force the ventricular band or ary-epiglottic fold toward the free space of the larynx, and remains for a long time covered and concealed by this structure. It soon produces mechanical impairment in the outward excursion of the vocal bands, and finally the ventricular band is forced so far forward into the anterior of the larynx as not only to cover the vocal band but also a portion of the opposite half of the larynx. Fränkel thinks that ventricular carcinoma frequently begins in the glandular structures. Its diagnosis from inspection is very difficult, but frequent observation eventually shows that the ventricular band and ary-epiglottic folds are not swollen, but are pushed outward. About the only other tumour occurring in the ventricle is a gumma. The diagnosis of gumma is usually practicable by its absorption under antisyphilitic treatment. Diffuse carcinoma does not produce stridor, even though both rocal bands are implicated throughout their entire length. When a circular carcinoma extends to the ventricular bands these structures become covered with red nodules 
the size of small peas. The ventricular bands swell and cover the vocal bands so that mere stripes remain visible. Carcinomata extending to the ventricular bands and ary-epiglottic folds, and the epiglottis, usually become dendritic and cauliflower-like in their development. This is the form most frequently described and depicted. Laryngeal carcinoma takes sudden stages of recrudescence after intermittent intervals of apparent quiescence, and gradually produces stridor, which may proceed to asphyxia.

Glandular swelling is less frequent, and is later in appearance than with carcinoma of the majority of other organs; sometimes it is absent in cases that have been fatal. Minute glandular swellings of the neck are difficult to feel in the healthy subject, but there are instances in which the glandular swelling of the throat is the most prominent symptom complained of ; thus it is difficult to estimate the value of the tumefaction of the lymphatic glands in the diagnosis of laryngeal carcinoma. The carcinomatous tissues have a tendency to undergo disintegration at certain stages. The process is ulcerative at first, gangrenous later. This period depends upon special conditions. Despite the friction to which the parts are subjected in the performance of function, ulcerous destruction is a late manifestation. It does not take place until the carcinoma has existed for a year at least. With the appearance of ulceration the period of tumour growth closes. When the ulcerated process becomes more extensive it may present an appearance similar to the ulceration in syphilis or tuberculosis. Rapid disintegration gives rise to peculiar fetor, which sometimes proclaims the diagnosis before laryngeal inspection has been made. This ulceration is considered the second period after extending perhaps over a number of months, then the third period begins. This is characterized by deep-seated disintegration, especially necrosis of the cartilage and gangrene of the surface, extending to the neighbouring organs. The penetrating carcinoma attacks the connective tissue, the elastic membrane, the muscles, and the glands. As to the penetration of the cartilage, while Fränkel has not yet investigated Schottelein's observation that carcinoma penetrates into the medullary and vascular cartilage only, he confirms the observations that it readily attacks ossified cartilage, especially of the thyroid, and the plate of the cricoid as well as the lower portion 
of the arytenoid. Healthy cartilage in the neighbourhood of carcinoma undergoes calcification. Additional necrosis of cartilage is produced by perichondritis. Purulent inflammation

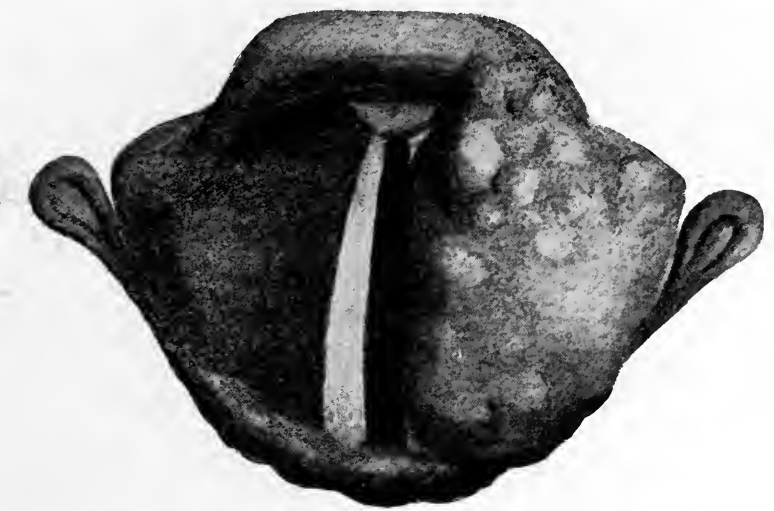

FIG. I4I.-Epithelioma of the larynx (W. F. Chappell).

of the perichondrium is a frequent complication of laryngeal carcinoma. Apart from the evidences of carcinomatous infiltration in the vicinity, there is nothing characteristic in the laryngoscopic image. The perichondritis at its commencement is usually recognisable by the consistence of the tumour, sometimes ulcerated, which has produced it. Fränkel lays great stress on the fetor. Perichondritis, especially when complicated with edematous tumefaction, may produce sudden asphyxia, even when there has been

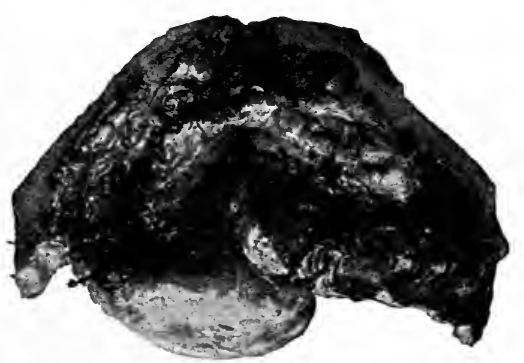

FIG. I42.-Author's case of epithelioma of the larynx removed by the operation of laryngectomy. no precedent stenosis. $\mathrm{He}$ especially mentions cases in which carcinoma developed in the thyroid cartilage. It can often be felt from the outside, and it may be mistaken for an abscess (Figs. I4I, I42, and I43).

Diagnosis.-During the early stages of the development of laryngeal neoplasms the diagnosis is not always easy. As before mentioned, histological examination frequently fails to help us out in this particular, owing to the similarity of the structural arrangement of these several growths. It must be 
said, however, to the credit of the microscopist, that sometimes the operator is at fault in not being able to obtain enough of the growth to allow of a fair examination; for instance, a mere

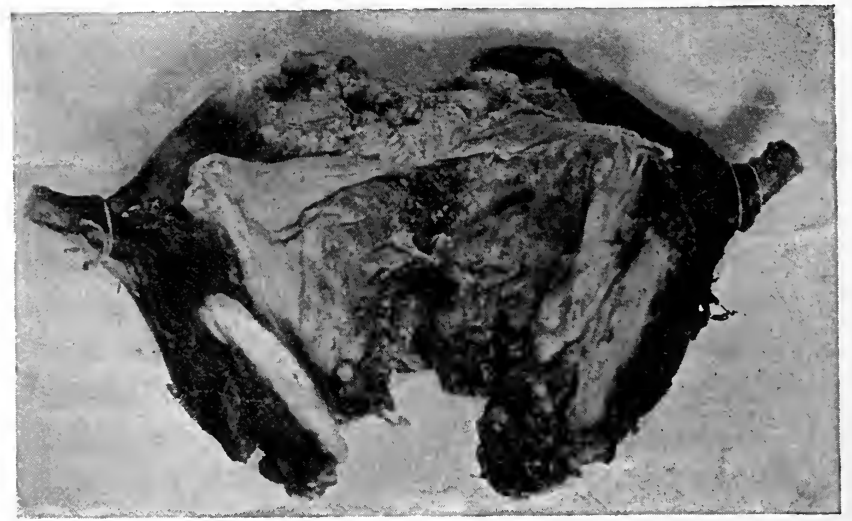

FIG. I43. - Epithelioma of the larynx-laryngectomy (Swain).

scraping from the top of a neoplasm may be quite insufficient for the determination of its real histologic character. Enough of a piece should be obtained to allow of sections being made of the specimen in different directions.

Papillomata and fibromata are usually of slower growth, and are not accompanied by any infiltration of the surrounding tissues or glands; whereas malignant growths, even when they are small, soon excite infiltration of the surrounding parts, and, as they develop rapidly, the lymphatic glands in the neighbourhood may soon become involved. This, of course, will be of material aid in arriving at the character of the neoplasm. However, the earlier and exact diagnosis can sometimes be made only by observing the results of treatment.

It will generally be necessary to thoroughly consider the laryngoscopic and microscopic examination together with the clinical history. The diagnosis between papillomata and epitheliomata is sometimes difficult. When occurring in clusters, the papillomata will present the same non-vascular appearance, will be soft to the touch, more or less pedunculated, and may be unaccompanied by much, if any, tumefaction of the surrounding parts or other inflammatory signs; whereas in epithelioma the growth appears sessile, and seems to include in its formation the immediate tissue around it. There is always: 
some tumefaction and hyperæmia in the neighbourhood. There is a progressive growth in epitheliomata which may be observed from week to week, whereas with papillomata little or no increase in size is observable. The subjective symptoms also differ materially ; for instance, in papillomata no inconvenience is experienced, excepting to the vocal or respiratory function; while with sarcomata and epitheliomata a great deal of pain, distress, immobility of the laryns, with aphonia, dysphagia, pain, and salivation, are early signs. The sarcomata are distinguished with the laryngoscope from the epitheliomata more by their intensely red, velvety appearance, their bleeding points and surfaces, jellylike consistence, and rapid spread by contiguity (see

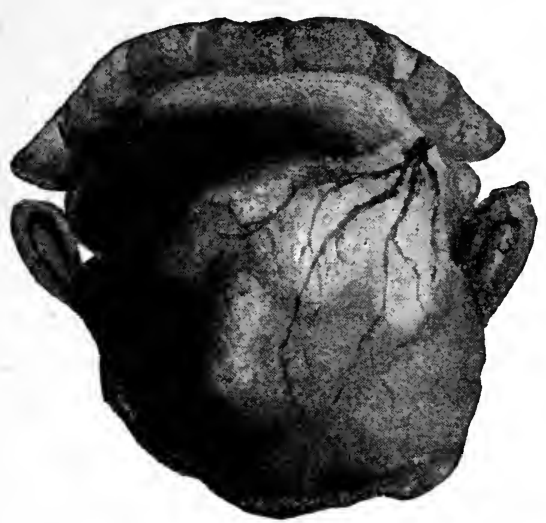

FIG. 144.- Sarcoma of the epiglottis (W. F. Chappell).

\section{Fig. 144). Sometimes ma-}

lignant neoplasms are confounded with the neoplasms arising from tuberculous or syphilitic (gummata) disease of the larynx, or with lupus (see Fig. 145); indeed, it is often extremely difficult to differentiate between lupus and epithelioma, especially

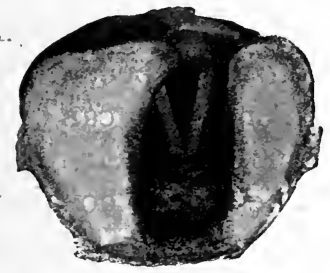

FIG. 145.-Primary lupus of the larynx (E. Mayer).

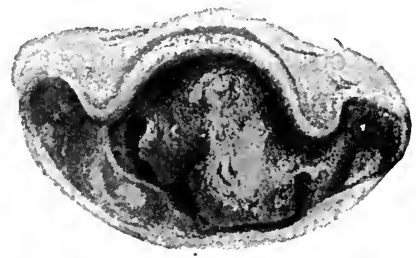

FIG. 146.-Supposed case of enchondroma (Türck).

when the epiglottis is involved. Enchondromata sometimes resemble epitheliomata (see Fig. 146). The differential diagnosis of the gumma is sometimes difficult-as, for instance, when the swelling is quite circumscribed and is low in the larynx. From epithelioma, however, it (gumma) is distinguished by its 
smooth surface and submucous character, as well as by the history of the case. Gerhard, according to J. H. Wright, has stated that a differential diagnostic point between syphilis of the trachea and tracheal carcinoma is that cough is a constant symptom of the former.

Prolapse of the laryngeal ventricle may be mistaken for a neoplasm. Dr. William S. Jones reported a case of prolapse of the laryngeal ventricle (in the Medical News, February 2, I895) which presented at marked intervals symptoms of stenosis, the dyspnœa at such times being very severe. A case of unusual laryngeal growth was reported by Gleitsman (New York Med. Jour., October 3, 1896), very much resembling mycosis or malignant disease. "It appeared as if a piece of cotton wadding had been inserted into the laryngeal ventricle, jutting out between the ventricle and vocal cords. It extended the whole length of the vocal cord from the anterior commissure." The exact diagnosis was not made out, but it was supposed to be probably malignant and perhaps "carcinomatous." Some laryngologists have laid down the rule that a growth situated on the anterior portion of the vocal band is generally benign, while at the posterior part of the larynx it is apt to be malignant; that papillomata of the vocal cords are usually discrete, while in the upper larynx they occur in clusters.' Papillomata are usually of a grayish or pinkish-gray colour, and more or less rounded or pyriform in shape. They do not show an inflamed base nor any swelling of the surrounding parts, and rarely present a broken surface.

Prognosis.-In the case of benign neoplasms the prognosis may be considered almost always favourable, because with modern methods of endolaryngeal treatment they can always be thoroughly removed. Even if they do recur their removal can be effected with certainty again. The use of cocaine for such manipulations is certainly an important aid to treatment. Where formerly laryngologists often failed in thoroughly removing these neoplasms, nowadays, with the aid of cocaine, such failure, except in the case of children, is seldom necessary. Besides, when endolaryngeal methods fail, laryngofission can be almost always safely resorted to. The prognosis in malignant growths is always grave. Even when they are circumscribed and of such size that thorough removal can be effected, they are very apt to recur. In these cases death 
usually comes through starvation and exhaustion, but sometimes the ulceration of the growth or of the adjacent tissues begets a sepsis which proves fatal. In other instances death takes place by the involvement of some important blood-vessel or vital apparatus. A case was reported in the Rev. Internat. de Rhin. Otol. et Laryn. for November I I, I897, of a man who died from hemorrhage of the carotid artery as a result of the presence and ulceration of a tumour in the trachea. Chronic stenosis of the larynx, caused by neoplasms or their ulceration, may be endured for a long time by the patient. More or less dyspnœa is the most urgent symptom, but there may also be present dysphonia, dysphagia, pain, and cyanosis, from improper aëration of the blood. This promotes exhaustion by requiring forced respiration, thus bringing into action the accessory respiratory muscles. There may be also anxiety of the countenance, engorgement of the venous system, and clammy perspiration. When these symptoms appear and seem to continue, an effort should be promptly made to at least relieve the patient by the adoption of some operative procedure, such as tracheotomy.

Recovery of the voice after the complete removal of benign tumours is the rule, unless the vocal apparatus has sustained injury through ulceration by pressure, or been injured by operative proceedings. After thyreotomy or partial laryngectomy, however, such result can not always be expected, although a hoarse quality of voice may be restored.

\section{The Treatment of Benign Neoplasms of the Larynx}

The removal of benign neoplasms can generally be accomplished by endolaryngeal methods of treatment, such as evulsion, crushing or cutting off of the growth with forceps, snaring, curetting, or by the application of escharotics. In endolaryngeal methods the instrument is introduced directly into the larynx through the mouth and pharynx. When the growths are too large or out of reach, or dyspnœa is urgent, or for any other reason this plan is impracticable, then the external operations of subhyoid pharyngotomy, thyreotomy (laryngo-fission), tracheotomy, or laryngectomy are to be resorted to, as in the treatment of malignant growths. The preferable method of dealing with these neoplasms, especially papillomata, is by evul- 
sion with forceps. There are a great variety of these instruments to choose from. They are usually modelled after one of two types, namely, tubular forceps, in which the blades are short and worked by a mechanism in a tube (see Fig. 147),

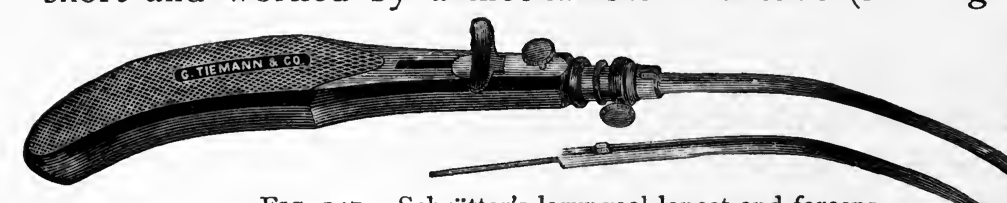

FIG. 147.-Schrötter's laryngeal lancet and forceps.

or two-bladed ordinary forceps, opening either anteroposteriorly or laterally. The latter instruments may be found of different shapes and sizes, with the blades, shanks, and handles adjusted at different angles or curves for reaching different parts of the interior of the larynx (see Figs. 148, I49, and 150). The Schrötter, Mackenzie, Stoerck, Semeleder, or De Blois forceps are probably the best of the tubular ones. The writer has been more successful in removing laryngeal growths with the ordinary pivoted instruments (of which there are a great

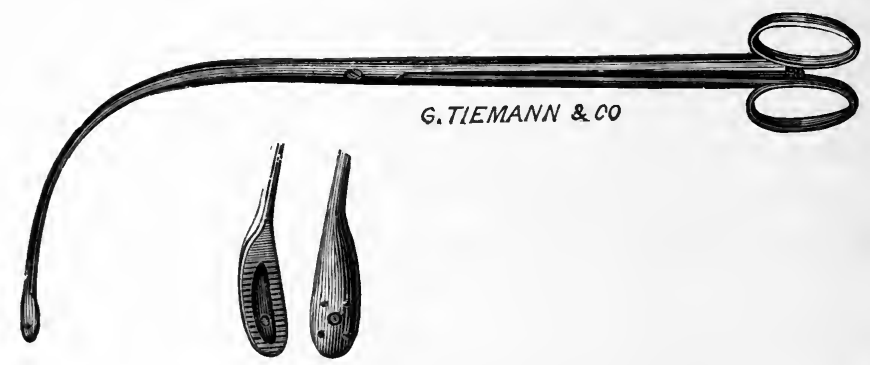

FIG. I48. - Fauvel's laryngeal polypus forceps.

variety) than with the tubular ones. For the removal of growths situated about the anterior commissure of the vocal cords or anterior wall of the larynx, those forceps having the blades and handle set at an acute angle are the most convenient. The method of manipulation with forceps or snare is as follows: The operator, seated before the patient as for laryngoscopy, first applies, if deemed advisable, a spray of a four-percent solution of cocaine, followed by the application, by means of a pledget of cotton or laryngeal brush, of a ten- to twentyper-cent solution to the pharynx and upper larynx. After waiting four or five minutes for the drug to take effect, the 
laryngeal mirror held in the left hand is placed in position as for laryngoscopy, and the forceps (closed) introduced directly into the larynx, using the reflection in the mirror for a guide ; when reaching the neoplasm the blades are quickly opened

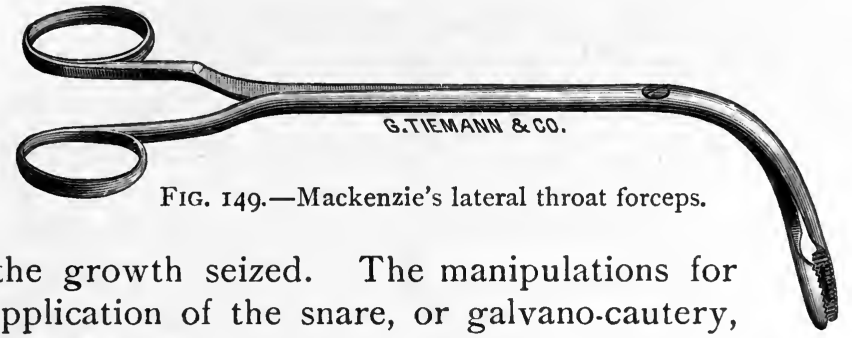

and the growth seized. The manipulations for the application of the snare, or galvano-cautery, or chemical escharotics, are about the same. It will be found necessary, as a rule, to train the patient for a considerable time in order to secure a tolerance of the throat before endolaryngeal manipulations will be successful. Persistent intubation may be tried, especially in children, for the relief and cure of papillomata. The tube by its pressure is supposed to cause either the death or atrophy of the growths. The writer has not been successful in the two cases in which this plan was tried. Levy, of Denver, has reported a successful case in a child four years of age. The tube was worn one hundred and eleven days without removal for any length of time. Tracheotomy may be an expedient measure in some cases, not only for the relief of dyspnœa, but to give a better opportunity for any endolaryngeal manipulation. With a tracheotomy tube inserted, a more persistent effort with for-

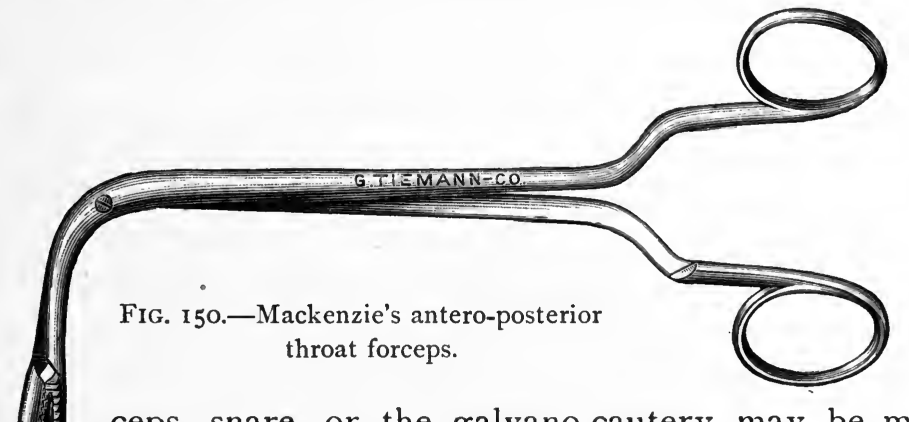

ceps, snare, or the galvano-cautery may be made without danger to the respiratory function. The writer has been able in this way to remove growths which otherwise would have required a thyreotomy. 
The snare, as a rule, is to be preferred for cystic or goodsized fibroid or lymphomatous growths, although sometimes difficult of introduction, especially at first. The snare of Gibbs or Jarvis is the best for practical purposes (Fig. I5I). In any case a séance should not be too long, because it will induce too much irritation of the pharynx and larynx and discourage the patient. The curette is recommended by Massei, Garel, and a few other practitioners, especially for the removal of multiple and soft growths. This, however, offers no advantages over the forceps.

Among the escharotics the most reliable will be found the galvano-cautery, although it is difficult to use it without doing harm, excepting for growths at the vestibule of the larynx. Electrodes of various shapes and size may be kept on hand for

G. TIEMANN \& COO.

FIG. I5I.-Gibbs's laryngeal ecraseur or snare.

that purpose. By the aid of the mirror the manipulation is conducted in much the same manner as the use of forceps; the electrode is to be carried to the desired point, and the current turned on by means of the thumb switch or by an assistant. In the use of this instrument great care should be taken to withdraw it as speedily as possible, so as not to singe other parts of the throat in its passage out. Considerable practice will be required to enable the operator to accomplish this. Where the tumour is conveniently located and the patient has a tolerant throat, it may be found expedient to cauterize the stumps of the neoplasms after their removal. However, as recurrence so infrequently occurs with benign neoplasms, this operation is seldom necessary. For fibromata and multiple papillomata, however, this instrument is.often preferable to the others.

Dr. Robert Gibson, of Youngstown, Ohio, advocates the general use of the galvano-cautery instead of the forceps for the removal of papillomatous growths of the larynx; excepting in cases where the growth is isolated and quite accessible. The cautery handle should be provided with a cut-off, so that 
the operator may have absolute control of the current at all times, otherwise unintentional burning of tissues, entailing perhaps unpleasant consequences, may take place.

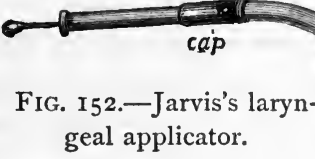
The electrode. should always be well insulated up to its point.

Among the chemical escharotics chromic acid is undoubtedly the best. This agent should be carried into the larynx by a specially devised carrier composed of an outer sheath or tube within which a probe moves (Fig. 152). On the end of the inner probe or wire within the cannula chromic acid is fused and drawn back inside the cannula to be ready for introduction. It is now carried into the larynx, and whenever near the desired spot or neoplasm it may be suddenly projected by means of the spring or other mechanism, and quickly withdrawn from the larynx, the probe being in the cannula. In this manipulation great care should be taken not to overload the end of the probe, because if that be done more or less of the acid is scraped off by the edge of the cannula and falls into the larynx as a fine powder, causing unnecessary suffering and possibly destruction of tissue. When nitrate of silver or other escharotics are used they should be carried into the larynx on a probe in the same way, and never without a proper guard or sheath to protect the adjacent parts from injury during introduction or withdrawal. The local use of cocaine, eucaine, holocaine, etc., is generally a necessary adjuvant in promoting the success of all endolaryngeal manipulations on account of the anæsthetic effects produced upon the mucous membrane. But exceptionally it will be found to induce such a degree of uncontrollable gagging and hawking that the manipulation will be very much hindered, if not made impossible. The writer had such an experience a few months ago in attempting to operate on a case of multiple papillomata in a man. The growths were finally removed without cocaine. Papillomata may sometimes disappear under the use of astringent me- 
dicinal agents, especially in children. The writer has found the aqueous extract of arbor vitæ, in the form of spray or pigment, an excellent agent, and one which is sometimes successful in causing the disappearance of papillomata in children. Tannic acid applied by spray or insufflation has been successfully used for the treatment of benign laryngeal neoplasms. Ichthyol in solution ( $I$ part to Io of distilled water) has been recommended by Massei. It may be used as a spray or injection by means of a laryngeal syringe (see chapter on Instruments). Insufflations of salicylic acid, diluted with powdered acacia in the proportion of I part of acid to 3 parts of acacia, is recommended by Drs. Sherwill and Raynor for reducing epithelial and warty growths on the mucous membranes as well as the skin.

Fibromata, and other benign neoplasms, should be treated in the same manner as papillomata. Their early removal is always desirable. The tolerance of the larynx to neoplasms when of slow formation and small size is quite astonishing. The writer knows of two cases, one a single small fibroma of the right vocal cord, and the other a small cyst about the size of a large pea emanating from the right laryngeal ventricle, existing in persons who will not allow of any operative procedure for their removal. These growths were first recognised by the writer fifteen and ten years ago respectively. These persons have little or no dyspnœa or pain, but more or less cough and hoarseness, which at times are troublesome. For large fibromata, etc., when situated at the upper larynx, electrolysis, or injections of alcohol or lactic acid, may prove beneficial, if for any reason their removal by evulsion or snare be interdicted.

If, however, the diagnosis be satisfactorily made, and attempts at removal by endolaryngeal manipulation prove unsuccessful, it will be advisable then to perform laryngo-fission or pharyngotomy, and remove the growth through the artificial opening thus made.

Treatment of Malignant Neoplasms.-Spontaneous disappearance of malignant growths does not take place as in the case of benign ones, although sarcomata have been reported to disappear under treatment by hypodermic injections of Coley's fluid, antidiphtheritic serum, and through the access of other diseases, such as erysipelas, or the influence of injuries 
or wounds. A number of practitioners have reported cases of sarcoma, and a few of epithelioma, which have been apparently cured by the use of Coley's streptococcus toxin. The writer and his associates, as stated before, have tried this fluid in cases of malignant disease of the throat (sarcoma and epithelioma) without success. Mitigation was observed, however, in two of the cases.

Sarcoma and epithelioma are best treated by extirpation, if that be possible, although Dr. Coley and others report some successful results from the injection of the Coley fluid and the micro-organism of erysipelas into the tissues surrounding these tumours.

Mr. Battle (Med. News, December Io, I 898) presented a case before the Medical Society of London, England, of inoperable sarcoma of the shoulder which had been treated by Coley's fluid. After three months' treatment the tumour entirely disappeared. The diagnosis was thoroughly established by repeated microscopical examination.

Dr. Maurice Richardson, of Boston, has reported a case (in the December number of Annals of Surgery) of the disappearance of a tumour which microscopic investigation had proved to be an infiltrating round-cell sarcoma by a severe wound infection. Operation for its removal had been commenced and abandoned on account of the extent of the growth. Infection of the wounds afterward took place, and the growth disappeared during suppuration.

Dr. McCallum, of Toronto, has used diphtheria antitoxin in multiple sarcoma with good results (Brit. Med. Journ., December I I, I 897, p. I 708).

Zeinmacki (Brit. Med. Jour., October 30, I 897, p. 72) treated twenty cases of inoperable malignant new growth (six were sarcoma and fourteen carcinoma) with streptococcus toxin with favourable result.

Injections of alcohol and tincture of iodine into carcinomatous growths have been used occasionally with benefit.

Concerning the larynx, the main object here, as in other situations of the body, is the removal as thoroughly as possible of the malignant growth. Unfortunately, such a desideratum is often more difficult of accomplishment than in other portions of the body. The two difficulties which stand in the way of the general application of such a rule are the anatomical nature 
of the parts concerned, and the almost hopeless probability of such thorough eradication of the growth that recurrence will not take place. These points have been most admirably brought out by Dr. Delavan in a paper before the British Medical Association, also in a paper before the American Laryngological Association.' He says that, while operations for the cure of malignant disease of the larynx have generally resulted in shortening rather than lengthening the lives of patients so operated upon, the experience of the past few years in certain parts of the world has been somewhat encouraging. Indications are beginning to appear which suggest that for this class of patients there exists a not unpromising future. The improvements which have been offered he divides into three groups, relating, first, to thyreotomy, with or without partial laryngectomy; second, complete laryngectomy, by the method adopted in J. Solis-Cohen's case ; and, third, complete laryngectomy in cases of extensive laryngeal disease with glandular involvement. The first method has been studied and practiced mostly by Dr. Clinton Wagner, of New York, and by Mr. H. T. Butlin and Dr. Semon, of London. The propositions advanced by Mr. Butlin are: I. Every malignant growth of the larynx the intrinsic origin of which can be dealt with, should be treated by an operation in the absence of a decided indication to the contrary, and the operation should be performed with the least possible delay. 2. Every tumour of the larynx suspected of being malignant, of intrinsic origin, of limited extent, and apparently within reach of free removal, justifies an exploratory thyreotomy in a suitable patient, in the absence of infiltration of the surrounding structures, and involvement of the lymphatic glands.

If it were always possible to make the diagnosis between benignancy and malignancy when the growth is purely local, before the invasion of the surrounding parts has taken place, then operative procedure ought certainly to be the rule, and not the exception; but, as already pointed out, it is very often extremely difficult to differentiate by either histological or laryngological examination between benign and malignant growths. This being the case, the question arises, Why not operate and radically extirpate intralaryngeal growths, notwithstanding they may be of doubtful nature? Indeed, some surgeons, among them Butlin, believe this to be the proper 
course, inasmuch as the patient in such instance gets the benefit of the doubt; for if it be malignant, the patient's life may thereby be saved, and though it appear benign, and susceptible of removal by endolaryngeal methods, but little harm is done to the vocal apparatus or the economy by prompt action. This stand would certainly be a justifiable one if malignant growths of the larynx did not show such an inevitable tendency to recurrence, and if one could be certain always that the surrounding tissues were not involved in the disease process. Every surgeon knows that this is one of the difficulties that cannot with certainty be overcome. In many cases of mammary cancer, for instance, where the surrounding lymphatic glands are apparently free, a recurrence shortly after the operation may appear. In the few cases of laryngeal carcinoma which have come under the observation of the writer, there was probably an invasion of the surrounding lymphatic tissue very early in the course of the disease, as the subsequent examination showed. It would seem, therefore, that the question of operating or not becomes a matter of judgment for each individual case. The writer has no doubt that some cases have been allowed to go from bad to worse for want of surgical interference, while others have been assisted in a course from bad to worse through untimely surgical operations. The writer knows of one case of malignant disease of the larynx which had thoroughly invaded the structures outside (extrinsic), and which a gentleman of strong surgical instinct had boldly and unsuccessfully attempted to extirpate, undaunted by torrents of gore and the frequent occurrence of temporary asphyxia. This brilliant operation was rewarded by the extirpation of the larynx, the wounding of one vagus, and the relief of the patient's earthly misery on the spot. Of course, operations performed by experienced and careful surgeons are certainly justifiable in many instances, if there be a prospect of relief.

Malignant growths, when small and circumscribed, may often be removed by endolaryngeal methods. Care should be exercised, however, lest the irritation coincident to the frequent application of forceps, snare, or caustics may promote the growth of the neoplasm.

There are three plans of operation: Subhyoid pharyngotomy ; laryngo-fission, or thyreotomy ; laryngectomy, partial or complete (see chapter on Operations). 
For growths affecting the upper part of the larynx, the subhyoidian incision is usually preferable because less dangerous in its performance, and much less serious in its consequences.

Thyreotomy and laryngectomy have been performed successfully a number of times in the last few years. Dr. Clinton Wagner's work in this line probably compares very favourably with that of any one else. Of the fifteen thyreotomies reported by him, five of the patients are now living at periods of one year, and twelve years, and seventeen years respectively. Of those not now living, one survived two years, one three months, and one ten weeks, and in none of the cases was death due to the operation of thyreotomy. Of these cases, four of them were epithelioma, one enchondroma, three papilloma, and two cicatricial stenosis. Of the five cases of epithelioma reported by Semon, five patients died from the operation within periods varying from one to six years. One showed probable recurrence within four months. Of the remaining four, one operated upon by Hahn, a complete laryngectomy, lived six years and a half without recurrence, and died of heart disease. Of those operated upon by Butlin, there are at least one living and well after six years, one at over three years, and one at over two years. One operated upon by Semon survived more than two years. Two patients operated on by Cohen lived over six and three years respectively, and the same may be said of other operators.

Delavan says: "Comparing recent results with the general statistics lately reported by Powers, namely, eleven per cent of recoveries from complete laryngectomy, and thirteen per cent of recoveries in partial laryngectomy, it will be seen that, as to thyreotomy, such a distinct advance has been made that its value as at present performed cannot be questioned."

The majority of surgeons are very cautious about recommending total extirpation of the larynx for carcinomatous diseases, especially when there is a probability that the surrounding glands and other tissues are involved. On the other hand, there are some who entertain what would seem to be very radical opinions in favour of laryngectomy. Mr. Butlin, whose experience has certainly been very extensive, is in favour of complete removal of all the diseased tissue within reach in every case where it is at all practicable. $\mathrm{He}$ believes in operating without delay after the diagnosis is made. 
Hunter Mackenzie and others advise partial over total laryngectomy when practicable. Mackenzie thinks laryngectomy is absolutely contra-indicated only in the presence of extreme exhaustion and in subjects over seventy years of age, and that neither enlargement of the cervical glands nor the spread of the disease over the structure adjacent to the larynx can be regarded as absolute contra-indications.

Foulis advocates total in preference to partial extirpation, and also that it be done as soon as the diagnosis is definitely made. Czernay also believes that extirpation is certainly indicated as soon as diagnosis of malignancy is made out. Enlarged cervical glands and old age, however, must be recognised as contra-indications. Out of 26 cases reported by Foulis of total extirpation, 14 died of the effects of the operation within fourteen days; of the remaining 12,9 had succumbed to a recurrence, and of these 1 only lived nine months after the operation, while the majority died within four to six months.

In I 878 Paul Brunn published an account of 19 cases of carcinoma which up to that time had been treated by thyreotomy; the result was, 2 deaths shortly after the operation, 16 local recurrences soon or within a few months, I after a year and a half, and I case that ended fatally twenty-two months after the operation, with cancer of the kidney, but without a return in the larynx. On this account Brunns and others denounced thyreotomy. This had such an influence on the medical public that only I I cases of laryngeal cancer were treated in this way during the next decade (Semon):

According to Schwartz, who in I 886 reported 95 cases of total extirpation, 12.6 per cent died directly from the results of the operation, 33.6 per cent succumbed to complications within the first two weeks, and 26 died within two years; of I 5 nothing is known after the first year. Schwartz considers that out of the whole 95, 8 cases only were cured. Schwartz also reported 34 cases of partial extirpation with the following results: 8 cases died from the effects of the operation, I 7 of a recurrence of the disease, 4 lived a year and a half, I lived six years, I was under observation but a short time, and the fate of the others is unknown; only 5 of the whole number lived through the first year after the operation.

Scheier reports the cases of total extirpation published 
from 1880 to 1888,68 in number. Of these, 18 died from the effect of the operation, in 17 recurrence took place, 6 died of intercurrent diseases; others were under observation too short a time. Finally, I3 per cent were supposed to have been cured. Partial extirpation, according to the same author, was performed in 23 cases. Of these, 5 died within six weeks as a result of the operation or of complications, in 5 recurrence took place, and 8 cases were not under observation long enough; 2I per cent were definitely cured, taking a period of six months.

Bartoux gives statistics of 106 patients with similar results -a mortality of 52.8 per cent as a result of the operation or immediate complications. Of partial extirpation Bartoux collected 31 cases, considering the duration of one year a sufficient period. One-sided (partial) extirpation gives the best results ; in 25 cases, 20 were cured (Semon). Much difference of opinion regarding the adoption of laryngectomy and thyreotomy for the removal of malignant disease will probably continue to exist as long as there are differences in individuals and cases. The writer entertains a strong conviction that extirpation of the larynx for malignant disease which has invaded or originated in the pharynx or other outside structures of the larynx is rarely justifiable. It must be admitted, however, that it is often difficult to determine, by the most careful laryngoscopic examination, whether or not a given laryngeal growth is strictly confined to the inside of the larynx, as in the case of epithelioma and laryngectomy reported by the writer in the New York Medical Journal (February, I899), which illustrates the fallibility of laryngoscopic examination for determining the absolute intrinsic situation of the growth. In this case it was discovered during the operation that a portion of the pharynx was involved and had to be removed. But malignant growths confined to the larynx should be removed by the operation of laryngectomy if there are no special contra-indications.

Wendell E. Philips has reported two successful cases of partial laryngectomy.

A case of successful laryngectomy, including a part of the anterior wall of the pharynx, part of the tongue, and glands in the neck, was reported lately by the operator, Dr. Lambert Lack, of London. In this case there was no previous trache- 
otomy. The trachea was stitched to the wound in the skin. Result not known.

Successful laryngectomies made after J. Solis-Cohen's plan have been reported also by, Fowler, Myles, Swain, W. G. Spencer, and others.

Thyreotomy is usually a safe operation and should be resorted to whenever necessary. De Santi has published seventeen cases with favourable results. In the case of a child four years of age suffering from multiple papillomata (both supra-and infra-glottic) the writer performed thyreotomy and removed the neoplasms; the child recovered and has a good voice.

Schnitzler reported a case of multiple sarcoma of the larynx and trachea which microscopic examination showed to be of the round-cell variety. From seven to ten small growths were scattered along the right side of the larynx and in the trachea, while the larynx on the left side was very much infiltrated from the epiglottis down. Tracheotomy was performed and the tumours removed by scraping (curettement), and the application afterward to their base of the thermo-cautery. The cannula was removed on the fourteenth day. Two of these growths were the size of hazel-nuts, one situated on the left and one on the right side of the larynx, about the level of the cricoid cartilage.

Better results have been obtained of late, owing to better facilities for performing surgical operations. The adoption of thyreotomy instead of laryngectomy, together with improvements of surgical technique, methods of asepsis, etc., have contributed much to the success attending these operations recently. The progress in this field of surgical art is largely due to Clinton Wagner, Cohen, Butlin, and Semon. They have greatly improved the method of after-treatment of thyreotomy by discarding the tampon cannula, giving water and fluid food by the mouth soon after the operation, and by simplifying the after-dressing of the wound. Semon first suggested the use of cocaine upon the inside of the larynx after the organ is opened (in thyreotomy), in order to prevent hemorrhage, diminish local sensitiveness, and thus facilitate a thorough removal of the growth; he also first suggested the dusting of the wounds with iodoform-the "dry method," which is a great improvement over former methods of dressing the wound. 


\section{INTUBATION FOR RemOVAL OF NeOplasms}

Dr. R. Levy, of Denver, after speaking of the spontaneous cure of laryngeal neoplasms, by quoting Massei, Lennox Browne, Garrel, and Lowman, alludes to the interesting results of treatment by intubation. Seifferts reported a case in which intubation was done after thyreotomy for papillomata. Massei used it in one case. Lichwitz recommends a fenestrated tube to assist in intralaryngeal treatment of these growths. Peterson met with failure in one case treated by intubation. Waxham reported an interesting case, and also Thrasher, of Cincinnati, of the failure of intubation to cure papillomata. The case reported by C. H. Knight, and that of Reynor, are also noteworthy in this connection. Among the various chronic conditions for which intubation has been mentioned are ankylosis of the laryngeal articulations from cancer, abductor paralysis, syphilis, subglottic neoplasms, and to assist in the removal of foreign bodies; in tuberculosis, after tracheotomy, stenosis following diphtheria, and stenosis following fracture of the larynx. Cohen reported a case of paralysis and tumour, and Dr. J. M. Bleyer, of New York, reported eight cases of syphilitic stenosis of the larynx caused by the formation of a web, which he operated on by a combination of the knife and tubage. Lennox Browne employs a cutting dilator for cicatricial stenosis, and directly after this introduces a tube of hard rubber, which should be left in from two to three days. It may then be taken out and cleansed, and a larger tube inserted in its place. It usually takes at least seven days for the healing of the edges of the cuts. The intubation, however, should be kept up for at least two weeks afterward. 


\section{CHAPTER XV}

\section{INJURIES TO THE UPPER AIR PASSAGES}

Injuries to the Nose.-The nose is frequently the subject of external injury. The displacement of the cartilages is the most common effect of the milder injuries, from falls and blows. The triangular cartilage, on account of its anterior position in the structure of the septum, is the one most prone to dislocation, and is also the most difficult to replace. More severe injuries, especially when impinging higher up on the dorsum of the nose, produce fractures of the vomer and the perpendicular plate of the ethmoid, or dislocation and fracture of the nasal bones, the result of which is flattening of the nose, with more or less obliteration of the meatus. Severe injury directed toward one side of the nose has the same effect, although the flattening or concavity will show upon that side mainly; the meatus in such instances, however, suffers obliteration to as great a degree. The swelling of the soft parts, which occurs a short time after the injury, will often mislead the practitioner to the belief that the organ is not broken or its parts dislocated, and therefore many permanent deformities of the nasal passages are allowed to occur without proper attempt at restitution having been made. It therefore behooves one to always make a thorough examination of the nasal passages after an injury of the nose has been received, in order to ascertain exactly the intranasal condition. After blows upon the tip of the nose, the triangular cartilage is sometimes dislocated to one side or to the other, showing, upon examination, a short distance inside of the vestibule, as a more or less globular red body, which is apt to be mistaken for swollen membrane. The same appearance farther into the passage upon one or both sides is often mistaken for simple infiltration of the membrane. Sometimes the dislocated cartilages will return to their normal position without aid. This is not very strange when one considers how very resilient 
these cartilages are. An instance of this is reported by Dr. L. S. Somers, of Philadelphia-a case in which there was separation of the nasal cartilages from their attachments. They returned to their position and became united. No surgical treatment was instituted. However, as said before, a thorough examination should always be made before the patient is dismissed, because after the parts have become fixed in their new positions it is often difficult, if not impossible, to relieve the deformity.

Treatment.-If the examination has elicited the information that there is a dislocation or deformity or fracture of the bony or cartilaginous part of the septum, the practitioner should at once proceed with the finger, forceps, a large, firm gum catheter, or an esophageal bougie, to bring the parts into proper apposition, after which measures are to be taken to retain them. For this purpose the perforated rubber tubes, or the hard-rubber plugs of Asch and Wagner, or soft-rubber bags inflated with air or injected with water, may be introduced. It sometimes happens, especially where the nasal process of the maxillary bone is involved, that considerable force has to be exerted to return and keep the fragments in position. For this purpose, plugs made of slippery elm, punk, sea tangle, or Brazil wood, or Bernay's cotton, may be advantageously employed; oftentimes the purpose can be accomplished by the insertion of a kite-tailed tampon made of either cotton or, preferably, antiseptic gauze. Plugs of this character should be introduced very slowly into the middle meatus, care being taken that they are not placed too far posteriorly, as they are then liable to pass into or irritate the nasopharynx. When plugs of wood are employed, it will be found more convenient to use two or more strips, since by putting one strip above the other they can be adjusted so as to relieve the pressure upon the turbinated bones; they can also be more easily placed so as to give support in the precise location desired. This manipulation is very important, because a haphazard manner of placing plugs along the inferior and middle meatus often does no good in sustaining the fragments, and may cause unnecessary pain by impinging against the turbinated bones. Outside splints often have to be used, especially where there is a fracture of the nasal bones. The best material for this purpose is gutta percha; it should be prepared by taking a piece of 
proper dimensions and inclosing it entirely in a layer of surgical gauze; it should then be softened by immersion in hot water, and moulded to the outside of the nose, where it is allowed to remain until cool. It is held in place by strips of surgeon's plaster passed over the cheeks from side to side.

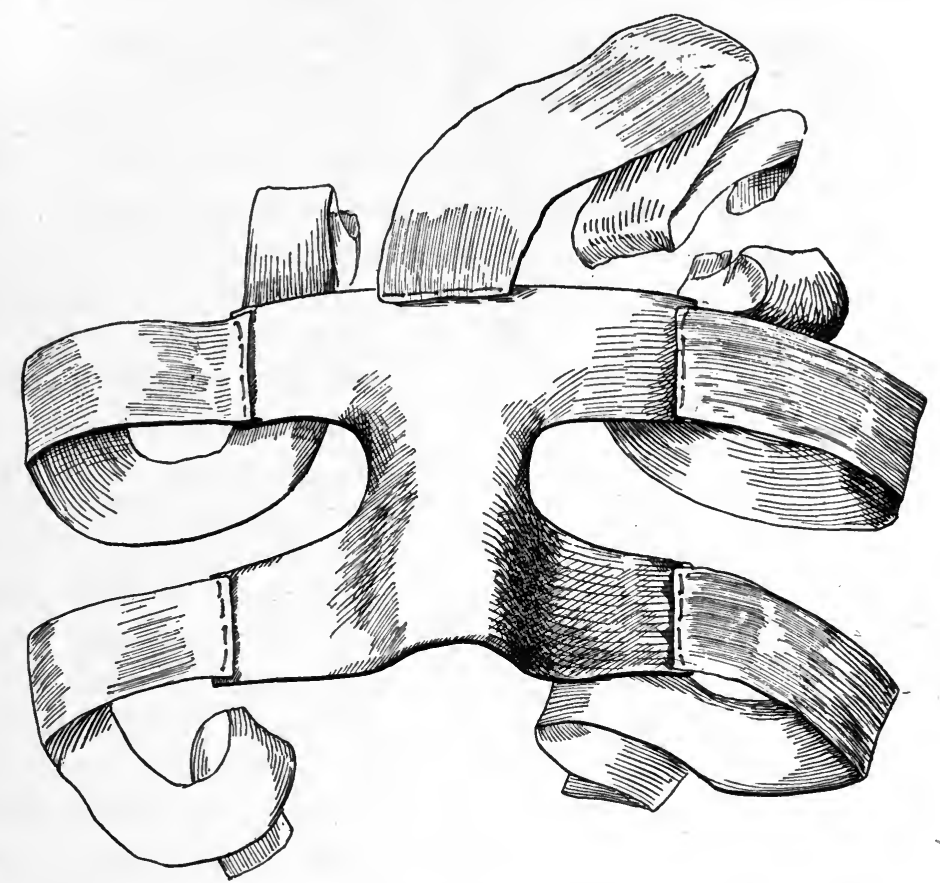

FIG. 153.-Apparatus for repairing a broken nose (W. H. Daly).

There are on the market various wire and tin splints which have been devised by surgeons from time to time, many of which will prove useful in special cases. Dr. Daly (New York Med. Jour., vol. lvi, p. 508) strongly recommends splints made of sheet zinc moulded to the nose and lined with a layer of felt (see Figs. 153 and 154). Dr. Frank C. Cobb, of Boston, has devised a steel band encircling and exactly fitting the head. The band is prevented from slipping down by a strap going across the head, and from slipping up by a band going underneath the chin. To this band there is fitted a splint with a spring, which can be so adjusted as to make pressure on any part of the nose. Injuries or fractures involving the lachrymal canal need special attention for keeping that canal open during 
the process of repair. This can usually be accomplished by the frequent passage of lachrymal probes. The unfortunate concomitants of the plugging of the nose for the injuries in question are the acrid, offensive character of the secretion which is induced, and the intense pain caused by the pressure

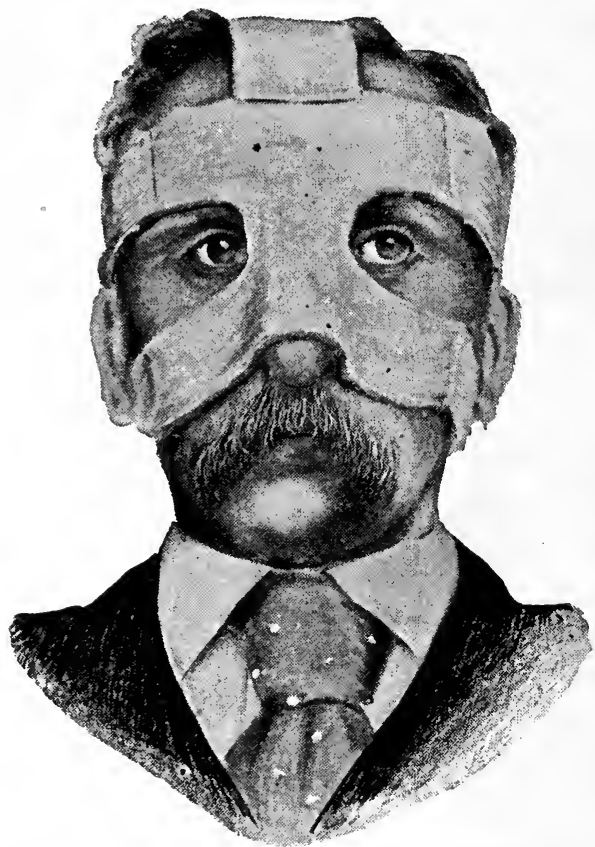

FIG. 154.-An eligible method of repairing a broken nose (W. H. Daly). upon the internal parts. Where the juxtaposition of the fragments can be maintained by a cylindrical softrubber tube, or by an air or water bag, there is much less suffering.

All plugs, especially the harder ones, should be removed as soon as practicable, although it is hardly safe in any case to remove them temporarily before the third day. Asch's perforated rubber cylinders are excellent when they can be used. The permanent removal, as a rule, cannot be made inside of two weeks, and in some cases four or even six weeks must elapse before their final withdrawal.

After ten days or two weeks it will be found that the plugs can be removed for several hours each day, and later on, when practicable, it is well to insert them for the night or day only. In all cases the nasal passages are to be irrigated thoroughly, after each removal, with some bland antiseptic solution (see formulary). Severe fractures of the frontal or facial bones will not enlist our attention, as such injuries belong to the domain of general surgery. One or more of the accessory sinuses of the nose may suffer also by these injuries and require particular treatment afterward. The ethmoid cells and antrum of Highmore, especially, are liable to be left in a state of chronic inflammation from the effect of these injuries, or their proper drainage may be interfered with. The management of such 
conditions is discussed in the chapter on The Accessory Sinuses.

Injuries to the Soft Palate, Tonsils, and Pharynx.--Injuries to the soft palate, tonsils, and pharynx usually occur either from contact with corrosive liquids or gases or from wounds made by wooden sticks or pieces of metal which, being held in the mouth, are accidentally or forcibly thrust backward upon these parts. The author had one case of severe wound of the pharynx in a child, caused by the end of a small piece of wood. The child was running along with the stick held in and protruding from the lips, when she fell forward upon the ground, with the effect of shoving the other end of the stem backward against the pharynx. The writer also had another case of severe laceration of the soft palate and lateral wall of the pharynx in a child, from the entanglement of a button hook.

Wounds of the pharynx sometimes result from the efforts of the patients and bystanders to remove foreign bodies from the throat. These parts usually recover very readily from injury, even when corrosive substances are the cause, unless there is carried with it some septic material, in which case the result may be fatal.

Hemorrhage after wounds of the pharynx is not usually profuse, unless one of the pharyngeal or palatine arteries be punctured or cut, in which case it may be alarming and indeed fatal, unless the carotid artery be tied. Dr. W. M. Baker, of London, England, reported a fatal case from a wound of the ascending pharyngeal artery caused by a pipe stem. Corrosive substances, especially concentrated lye, carbolic acid, etc., sometimes produce extensive sloughing of the parts, and incidentally alarming hemorrhage, by the ulceration of arteries or good-sized veins.

Injuries to the Larynx.-The larynx is so well situated and guarded that it is not very liable to injury either from within or from without, yet it may be subjected to injury internally from the entrance of foreign substances of either solid, liquid, or gaseous nature, or suffer more or less severe injury from external violence, such as garroting, and knife or bullet wounds. Perhaps the most common cause of internal injury can be attributed to the inspiration of corrosive liquids or gases. Among the former, liquid lye, carbolic acid, ammonia, and mineral acids, are often swallowed with suicidal intent. These 
substances are apt to produce violent reactionary inflammation of the epiglottis, arytenoid region, and perhaps the deeper parts of the laryngeal cavity, if inspired. Among the gases may be mentioned ammonia, chlorine, sulphurous acid, and phosphorus fumes. Any of these, if inhaled in sufficient quantity or in a state of sufficient concentration, may produce dangerous injury to the larynx. Besides these, the inhalation of smoke and superheated air or steam may.induce a dangerous laryngitis with œdema of the glottis.

Fractures of the larynx are not very common. The thyroid cartilage is probably more often fractured than its fellows. Henocque collected and published the observations in 52 cases of fracture of the larynx; of these, 23 were of the thyroid, 7 were of the cricoid, and 7 were of the cricoid and thyroid simultaneously. There were I9 deaths in all. Those cases, where the cricoid and thyroid were both implicated were fatal. Fracture of the cricoid is usually fatal. So far as ascertained, the cause of these deaths was asphyxia or general emphysema.

Dr. H. Fussell collected and tabulated 70 reported cases, showing 54 deaths.

Compound fractures of the larynx are sometimes met with, and are always grave cases. They are usually much oftener fatal than incised wounds communicating with the neck.

Dr. E. L. Bell (New York Medical Record, March 28, i896, p. 458), of New Hampshire, reported an interesting case of compound fracture of the thyroid cartilage (the result of an attack by a bull) which recovered. The patient was twentynine years old. The wound was a large, lacerated one, in which all the muscles of the neck covering the front and right side of the larynx were very much torn." The two alæ of the thyroid cartilages were entirely separated. The right ala had a longitudinal fracture, the crico-thyroid membrane was lacerated, and the cricoid cartilage badly bruised. The hemorrhage at first was quite severe. The wound was thoroughly cleansed with sterile solutions of water and a solution of mercuric bichloride, and the outside parts aseptically dressed. The wounds healed nicely. There was not much dyspnœa, and the patient recovered his voice. No trachea tube was used.

Dr. C. M. D. Goldos, of Havana, reported an interesting. 
case of fracture of the laryngo-trachea ending in recovery with a good monotone bass voice. He thought the phonation was produced by the ventricular bands taking the functions of the vocal cords. Singing and crying were impossible in this

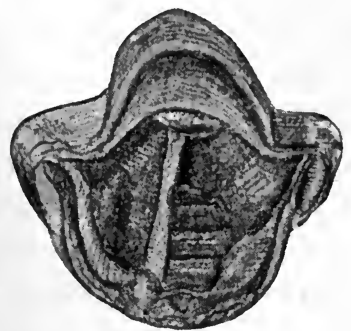

FIG. 155.-Gunshot wound of larynx (case of W. H. Daly, M.D.).

case. An interesting case of knife-stab wound of the larynx is recorded by Dr. Lefferts, and a case of gunshot wound reported by Dr. Daly (Fig. I 55). Knife wounds of the larynx and trachea are often met with in persons who have attempted suicide by cutting the throat. The writer has attended several such individuals in whom the upper front part of the trachea had been badly cut, two of whom recovered from the injury. When the trachea is only slightly incised, and the parts have been carefully stitched up, recovery without any troublesome consequences will usually take place. But when the larynx or trachea is well gashed, the subsequent infiltration, internal hemorrhage, or inspiration pneumonia will often prove fatal.

A case of complete rupture of the trachea was reported in the New York Medical Journal for October 22, I898, of a man, fifty-three years of age, who was struck by an elevator. His sternum was broken, but he survived the accident fourteen days. At the autopsy it was found that there was complete rupture of the trachea. According to Scheppegrell, there are several cases of laryngo-tracheal wounds on record, in which the principal symptoms were urgent dyspnœa and much subcutaneous emphysema, and the result being usually death. He reports three cases of recovery on record, two by Lany and Wagner and one by Lauenstein.

Contusions of the larynx are often met with, and sometimes produce serious effects. Cases reported by Dr. Packard and others show a great amount of

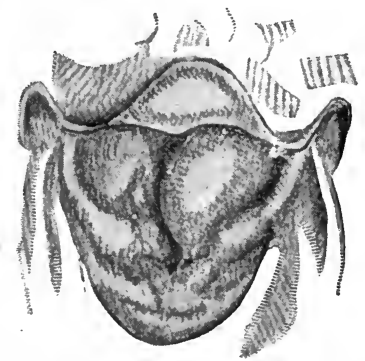

FIG. 156.-Injury to larynx from contusion (J. H. Packard). swelling and structural change of the soft parts of the larynx following such injuries (Fig. I56). Indeed, a severe blow on 
the larynx may cause immediate death (Marchka) even without fracture.

Fractures and injuries to the hyoid bone are sometimes productive of serious disturbance of the larynx, such as more or less œdema and laryngitis, although usually the consequences of such injury are mainly manifested in the mucous membrane and glands of the lower pharynx and the base of the tongue. Dislocation of the hyoid bone is a very rare occurrence. It seems almost incredible that such an injury could occur when one realizes the anatomical relations and adaptability of the hyoid articulations for free movement. - Yet luxation does sometimes occur. Dr. B. R. Shurly has met with one person who has suffered a succession of such luxations.

Dislocation of the laryngeal cartilages occur sometimes, although rarely, without an accompanying fracture. Those on record are luxations of the cricoid.

Effects.-The effects of these injuries to the larynx vary with the extent ${ }^{\circ}$ and nature of the cause. Instant death, as stated before, may occur even from a slight injury. The immediate effects usually consist of swelling of the soft parts (œdema glottidis), more or less deep-seated inflammation of the soft

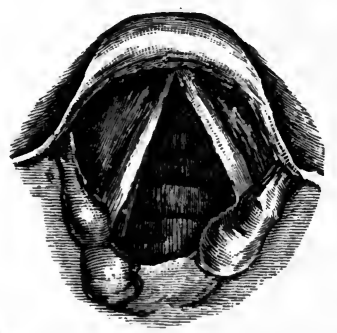

Fig. 157.-Case of paralysis of left vocal band following an incised wound of the neck. (Forced inspiration.)

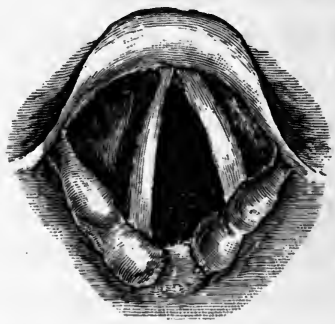

FIG. 158.-Case of paralysis of left vocal band following an incised wound of the neck. (Forced expiration.)

parts, hemorrhage, either immediate or remote, pain, and dyspnœa. Among the remote effects may be mentioned hyper. trophy of the glandular tissue of the mucous membrane (laryn. gitis hypertrophica superior vel inferior), pachydermia laryngis, hemorrhagic laryngitis (see chapter on Chronic Laryngitis), and the various forms of paralysis of the laryngeal muscles (see Figs. 157 and I 58). Stenosis may also occur in consequence of the varying effect of the inflammation (Fig. 159), and should 
the injury cause any considerable loss of tissue, the subsequent cicatrices will certainly impede the function of the organ, if not lead to a more or less permanent stenosis of its lumen.

Treatment of the Injuries of the Pharynx and Larynx.-The treatment of injuries to this region may be considered under two captions :

I. The immediate treatment of the injury.

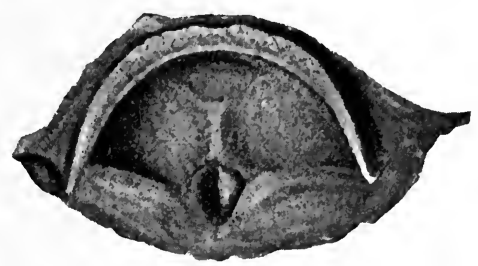

FIG. 159.-Stenosis of the larynx following typhus fever (Burow).

2. The after-treatment or management of the effects of the injury.

In all cases of wounds, whether affecting the pharyngeal cavity or the larynx alone, the first consideration should be to ascertain the amount of injury and the source of the hemorrhage. If hemorrhage be at all free, it must be suppressed. In case the faucial tonsils or pharynx be injured, the application of a styptic spray, such as ferric alum or bits of ice, or a solution of liquor ferri persulphatis, may be sufficient to effectually stop the hemorrhage. Should this fail, applications of very hot water by means of a small piece of sponge to the part may be tried. Should these measures after a thorough trial fail, then an attempt to make pressure upon the bleeding points should be made, for it is very seldom that the bleeding vessels can be tied. This may be accomplished by means of a firmly folded pledget of gauze upon which has been sprinkled a little powdered (Monsel's salt) ferric alum. The pledget can be held in place by the fingers or by suitable forceps. As such action may have to be continued for perhaps several hours, a relay of assistants may be necessary. Should this procedure prove unsuccessful in permanently stopping the hemorrhage, then the most accessible blood-vessel from which the bleeding branches arise must be tied. Should the parts be very much torn, the question of bringing them into apposition under suitable aseptic precautions will arise. In the case of the soft palate or the anterior pillars of the fauces, something may be accomplished in this direction by judicious stitching; also in the case of incised wounds extending from the outside, such as razor or knife wounds of the throat and trachea. But, as a rule, wounds (either incised, bullet, or contused) confined to the inside of the 
throat may be left to heal by granulation. The parts should be frequently treated by antiseptic lotions or sprays, and also dusted with antiseptic powders, such as aristol, iodoform, europhen, boracic acid, etc. Unless the wound be inoculated by septic material or be the result of some severe corrosive agent or a burn, the reparative process, under ordinary care, will generally proceed without any untoward event. When, however, extensive sloughing, abscess, or sepsis occurs, a more thorough treatment of the wounds and parts will be necessary, such as frequent douching with solutions of mercuric bichloride, permanganate of potassium,-formaline, peroxide of hydrogen, etc., and, in addition, the free use of the knife, to open up the diseased tissues, and release pent-up material so as to secure free drainage. A suppurative adenitis following wounds and injuries of this region should never be neglected, lest a fatal septicæmia supervene.

Fractures of the hyoid bone and of the larynx will have to be treated somewhat upon the expectant plan, for no rule of action can be laid down. The application to the outside of strips of surgeon's plaster, covered by starch or shellac bandages, will be found very serviceable, although from the nature of the parts complete immobility cannot, of course, be secured.

Dyspncea, or any degree of obstruction to respiration, must be met by intubation or tracheotomy. Either of these proceedings will frequently be found necessary on account of the tumefaction of the parts inside. Injuries to the laryngeal nerves, resulting in paresis or paralysis, should be treated judiciously by electricity, if there are no signs of neuritis, in order to forestall their degeneration. Burns, and sometimes contusions, will leave bad cicatrices, which in turn will cause troublesome contractions or deformities of the parts, or more or less laryngeal stenosis. Such cases must be treated according to the principles laid down elsewhere. Luxations of the hyoid bone and of the cartilages of the larynx are usually easily reduced. The after-treatment consists in securing to the parts as great a degree of immobility as possible by external mechanical applications, enjoined with abstinence from acts of swallowing and speaking. 


\section{Epistaxis (Nose-bleeding)}

Epistaxis is a hemorrhage from the nose. The blood usually flows from the anterior nares, unless they are obstructed. It varies in amount from a few drops at a time to a continuous flow. When persistent, it becomes dangerous to life, and there are many records of death from it. It is astonishing, however, how often the system will endure the great loss of blood which takes place in some cases of epistaxis.

Etiology.-The causes of epistaxis may be conveniently considered under two heads, viz., local and systemic.

The local causes may be arranged, according to Vansant, into

I. Tumours-angioma, fibroma, sarcoma, carcinoma, etc.

2. Varicose enlargement of the veins of the septum.

3. Perforating ulcers of the septum.

4. Trauma-injuries and operations.

5. Local ulcerations caused by syphilis, tuberculosis, lupus, etc.

6. Deformity of the septum, associated with erosion of the mucous membrane.

Of all the intranasal growths, sarcomata are most apt to produce more or less recurring epistaxis. For that reason, in such cases the practitioner should be very careful about probing or making other manipulations. The writer has observed excessive epistaxis follow the examination of such growths with a probe, and also following the application of the galvanocautery to them. Varicose enlargement of the veins or enlarged capillaries are usually met with on the anterior portion of the cartilaginous septum. It is here that the vessels of the septum, the anterior artery of the septum (a branch of the superior maxillary), and the ascending branch of the palatine connect with numerous communicating branches. Ulceration or abrasion of the mucous membrane may take place anywhere in the nasal passages, but experience shows that in a large majority of cases the site of such a process is upon the cartilaginous portion of the septum, not far from the vestibule of the nose, as pointed out by Lefferts and others. Casselberry says that in ninety per cent of the cases the bleeding point will be found just within the vestibule. Danscher records a case 
in which the patient, a healthy man of forty-eight, of somewhat full habit, was suddenly attacked with bleeding from the left nostril. This was stopped by a tampon, but recurred next evening, when the left conjunctival sac was filled with blood. On clearing this out no cause could be seen for the hemorrhage, until at last blood was observed issuing, drop by drop, from the aperture of the left lower lachrymal canal. On treating this, the bleeding ceased, but two days later a fresh recurrence took place, and was very severe, the blood trickling over the left cheek. The posterior nares were plugged by means of a Bellocq's sound, and on removing the tampon, forty-eight hours later on, bleeding took place again. Subsequent examination of the nostril and lachrymal apparatus revealed no new growth, nor, indeed, anything else abnormal. Danscher has found no other case in literature in which epistaxis was associated with the lachrymal canal so that the blood poured down the cheek. In many instances the ulceration is brought about through the habit of picking the nose with the finger to remove the dried secretion which is apt to collect there. Sometimes this collection is favoured by a deflection of the septum. Bosworth ascribes many cases of ulceration to deflection of the septum, the projecting point of the deformity becoming vulnerable, and thus liable to erosion from picking with the finger and from other causes. Syphilitic and tuberculous ulceration do not often cause severe hemorrhage, unless the disease is progressing rapidly or is extensive. We sometimes meet with a small ulcer or two giving rise to recurring hemorrhage in perfectly healthy persons, especially in children between the ages of eight and twelve years, which cannot be accounted for by the clinical history elicited. Foreign bodies lodged in the nasal passages may give rise to ulceration and consequent epistaxis. Traumatism is probably more often the cause of epistaxis than anything else. Blows upon the external nose, and injuries inside to the mucous membrane through accident or as a result of surgical procedures, may cause alarming hemorrhage.

The effect of removing polyps and redundant tissues by the snare, saw, curette, drill, etc., may be followed by severe hemorrhage for a short time, but it usually subsides spontaneously. The writer has observed persistent and alarming epistaxis after operations on the posterior ends of the turbinates and after 
removal of necrosed bone more often than after other intranasal surgical operations, excepting when dealing with sarcomata or fibromata. Severe epistaxis from the presence of adenoid vegetation at the vault of the pharynx is reported by S. Jankelwitch and others.

Systemic Causes.-Among the systemic causes of epistaxis, the hereditary tendency (hæmophilia, "bleeders") is the most interesting of morbid tendencies. Just what comprises this condition is not definitely known, but whether it is due to local vaso-motor paresis or the lack in the blood of one of the "internal secretions," this form of epistaxis is nearly always severe and persistent, although none of the structures may show any particular lesion. The family history is not always completely to be ascertained in these cases, as the following instance will illustrate: T. F., fifty years of age, and generally healthy, came under the writer's professional care several times with attacks of severe epistaxis, which in each instance required thorough plugging of the nasal passages to check. He had been subject to mild attacks during boyhood. Examination showed no lesion of the nasal mucous membrane at any time, neither did the patient know of any hereditary tendency to bleeding among other members of his family. He has not had an attack for about ten years, but of his three children two are bleeders. Therefore, either the tendency originated with him, or an unknown break in the family history had occurred.

Epistaxis occurring in females at the menstrual period or about the time of the menopause is often called vicarious menstruation. Such an event is usually considered salutary when the flow of blood is not too great. Fränkel cites a case (Ziemssen's Cyclopædia) of this kind, however, which proved fatal. Epistaxis may occur at intervals during the period of pregnancy. It may also occur in persons suffering with hemorrhoids, when the loss of blood from the hemorrhoids has been checked. It frequently occurs in plethoric individuals, especially during a "rush of blood to the head," or in any person suffering from a venous turgescence, the result of violent exercise or excitement. Epistaxis is a frequent accompaniment of various dyscrasiæ, such as scorbutis, purpura, chloræmia, and leukæmia. It frequently precedes or accompanies diphtheria, scarlet fever, variola, typhoid fever, dengue, and malarial poisoning. It is sometimes a particular symptom of nasal diphtheria or fibrin- 
ous rhinitis, and may occur to an alarming extent in these diseases. Cardiac, renal, or hepatic diseases, especially Bright's disease, may give rise to it on account of the passive congestion of the venous system which these affections may produce. Certain of the diseases of the central nervous system may give rise to it.

Habel reports a case of " tic convulsive" accompanied with severe and frequent epistaxis, in which two years thereafter apoplexy occurred. He thinks the hemorrhage was due to direct irritation of the facial nerve at the base of the brain, or to the reflex action.

It is sometimes an alarming accompaniment of arteriosclerosis in the aged, and in such persons may be regarded as a bad sign, although undoubtedly it is a salutary event sometimes by warding off an attack of cerebral apoplexy. Dr. de Roaldes, of New Orleans, has reported four cases of alarming epistaxis accompanying influenza.

In many instances a premonition of an impending hemorrhage may be recognised by such symptoms as noises in the ears, sense of fulness in the head, disturbance of vision, vertigo, etc. Fatal epistaxis in young children may occur in the course of syphilis, diphtheria, or some other constitutional disease. Swoboda reports four cases of fatal epistaxis in nurslings less than one month old, who exhibited typical pictures of purpura -black stools, vomiting of blood, and blood in the mouth. Two had hereditary syphilis, and at the autopsy were found to have had nasal diphtheria. In the third child there was buccal hemorrhage and purpura following a purulent rhinitis and hemorrhagic ophthalmia; at the autopsy there was found an almost complete destruction of the nasal mucosa, laying bare the bone. In all cases the bleeding had not been external, but through the nasopharynx.

Treatment.-In all cases, when practicable, the practitioner should examine the nasal passages carefully by means of a reflector, nasal speculum, and a good illumination, in order to ascertain, if possible, the situation of the bleeding point or points, to which the treatment ought to be applied. If, however, the bleeding is too profuse, and is partially checked by clots already formed in the nasal passages, then it may be unwise to clear them out for a thorough examination.

The treatment indicated will be either local or general, 
according to the nature of the case. For abrasions and ulcerations on the septum, the local application of a styptic by means of a small piece of cotton or lint will usually suffice to check the bleeding. Monsel's salt or Monsel's solution (persulphate of iron), or perchloride of iron, will be found preferable. It should be used, however, in small quantity, and only to a limited extent, because the hard coagula which it makes in combination with blood will prove very troublesome afterward to the patient, and will be very difficult to remove. For a styptic douche or spray, a solution of alum, tannic acid, or ammonio-sulphate of iron and alum will be found most efficient. When the nasal passage is not filled with blood, powdered matico or tannic or gallic acid can be effectually blown in by means of a glass tube or insufflator. In cases of diffuse hemorrhage from systemic causes, or from the presence of tumours, these measures will usually be sufficient. Ice may also be used, either within the vestibule of the nose or on the outside; it may also be applied to the back of the neck. Compression for a time of the ala of the nose against the septum on the affected side, or compression of the upper lip with the finger against the maxilla, just below the base of the anterior column of the nose, will often promptly check the hemorrhage.

Cold water injections into the nose are advocated by some practitioners, but this treatment is apt to produce a troublesome rhinitis afterward. Injections of very hot water are recommended highly by Hamilton, Armstrong, and others. - The water should at first be lukewarm, and then gradually made hotter until reaching a temperature of $127^{\circ}$ or $140^{\circ} \mathrm{F}$., according to the tolerance of the patient. The application of a solution of adrenalin and normal salt solution ( $\mathrm{I}$ to 6,000 or I0,000) is highly efficient in many cases. When the bleeding point can be discovered, the part should be treated directly with the styptic, and if the case is a recurring one, the ulcer or abrasion should finally be touched with either a galvano-cautery electrode or some one of the chemical escharotics, such as chromic, acetic, or carbolic acicl, or nitrate of silver. Gelatine has been used as a hæmostatic with good effect. The nasal passage should be first washed out with a warm watery solution of salt or sodium bicarbonate, and immediately afterward injected with the gelatine, which has been made liquid by heat. One to two ounces can be thus used and allowed to solidify. 
If these several measures fail, and especially if the patient has lost considerable blood, plugging of the nasal passages should be at once resorted to. Any practitioner who allows a patient to die from epistaxis without plugging the nasal passages is decidedly culpable, notwithstanding the various objections offered to the presence of plugs in the nasal passages. The objections referred to are mainly that severe inflammation, with perhaps destruction of the mucosa or dangerous sepsis, may result from the presence of plugs in the nasal passages, and, furthermore, that posterior plugs are apt to cause troublesome disease of the middle ear, or inflammation of the lining membrane of the accessory cavities of the nose. Now such things may happen if tampons are left in too long at a time, or if the passages are not properly cleansed after each removal. Yet I think apprehension on this score may be exaggerated, for I have known of severe cases of epistaxis with which no precautions were taken, and in which the plugs were left in the nose for a week or ten days without any bad result ensuing. Still the writer would not recommend negligence in cases requiring tamponing of the nose. As a rule, the plugs, of whatever nature they may be, should not be left in the nasal passages without removal for more than twentyfour or thirty-six hours.

For tamponing the nose a variety of material has been suggested. Iodoform, or ordinary aseptic gauze, or absorbent cotton, are generally used; some surgeons prefer pieces of sponge, lint, etc.

D. Hayes Agnew's plan was to use tampons of ham fat. A tampon consisting of a thin rubber bag, which is inserted and then filled with air or water, has been suggested by Veeder and others (see Fig. I60). An envelope of oiled silk or cotton filled with absorbent cotton or some other very soft material has been used successfully; also some such envelope or bag filled to the necessary extent with vaseline has been recommended. One of the plans, and a good one, is to push well into the nasal passage a fold of glycerine paper or oiled silk in the shape of a glove finger, and when it is in situ, fill it with bits of gauze or cotton until the nasal passage is packed. It may be removed by a string attached.

The object of all these devices is to fill completely the passage with a tampon which will be soft, smooth, and yet exert 
the necessary amount of pressure. A long strip of cheese-cloth bandage (previously soaked in a solution of peroxide of hydrogen) can be folded and pushed into the passage by a probe, and then either smaller pieces or one of its ends can be packed into this fold. One advantage of this plan is its ready removal.

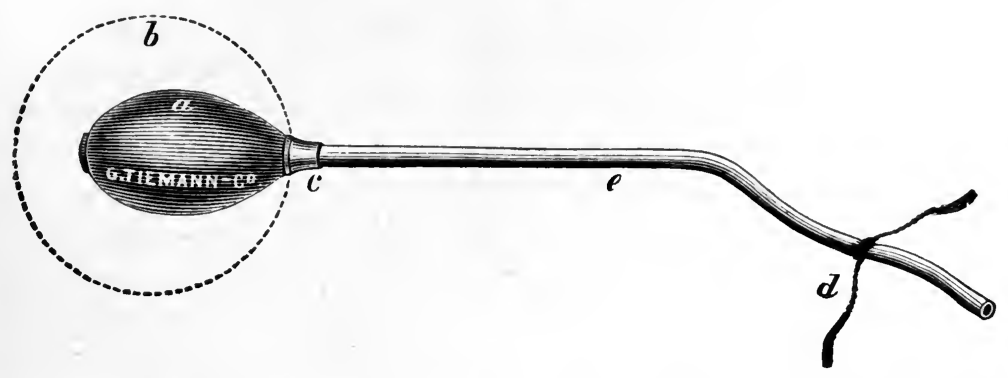

FIG. 160.- Rubber tampon for plugging the nose. $a$, rubber bulb ready for introductíon; $b$, bulb dílated; $d$, string for closing tube.

The writer has for years used tampons of gauze or absorbent cotton arranged as a " kite-tail tampon," for insertion anteriorly, and introduced piece by piece. The pieces of gauze or cotton should be tied on to the string about one and a half to two inches apart. Instead of this, the insertion of a strip of gauze by means of a tampon cannula is easier and as efficient. The first few pieces should be carried well back to the choana, both in the inferior and middle meatus; then each succeeding piece is to be carried in until gently touching its predecessor, and so on until the passage is packed.

The tamponing of the posterior nares or choance is not always an easy manipulation, for sometimes difficulty will be found in getting the plug through the nasopharynx, and, after "landing it," in keeping it there against the uncontrollable and vigorous action of the pharyngeal muscles. Care should be taken, however, to introduce the tampon well into the choana, and not leave it in the nasopharynx (unless the hemorrhage is from the latter cavity), a thing which the inexperienced operator is very apt to do. One way to avoid this error is to make the plug small enough to enter well the choana, but not small enough to easily pass forward into the nasal passage. The direction as to size usually given by writers on the subject is "about the size of a thumb." When one realizes, however, like "Pud- 
d'n-Head Wilson," that all thumbs are not alike, it can be easily imagined what a standard for calculation is thus set up. The "walnut-sized" rule for the size of a tampon is also fallible, so that the best guide, perhaps, is to make the plug for an adult of such size that when compressed it will measure about an inch and a quarter by seven eighths of an inch.

The operation consists in passing a string or cord along the floor of the nose into the pharynx, where the end is caught by a pair of forceps and drawn forward through and out of the mouth, where the tampon is tied to it. The tampon is then drawn into the mouth by pulling the string which is running through the nose, and, assisted by the forefinger of the other hand, it is guided behind the velum palati to its place in the choanum. The string may be introduced through the nose by means of a Bellocq's sound, a small, soft male catheter, a piece of round or square rubber cord about an eighth of an inch in diameter, a piece of large-sized common cord which has been stiffened by having been dipped in glue or shellac and allowed to dry, a long piece of solt (good sized) wire bent double at the middle like a hairpin, to the bend of which the string may be attached, or by means of a Eustachian catheter. Both ends of the string holding the tampon should be left long, the one to protrude through the nose, and the other long enough to reach from the tampon through the nasopharynx and mouth to be fixed externally. Without this latter string there might be great difficulty in removing the tampon. The ends of these strings are to be fixed by pieces of surgeon's plaster to the cheeks.

In cases of persistent epistaxis, necessitating a repeated introduction of the tampon posteriorly, a long piece of silk tape should be introduced and the two ends sewed together. With this the tampon can be taken out every day and a new one inserted without each time introducing a guiding instrument.

For hemorrhage from the nasopharynx, a tampon large enough to fill that cavity will be required. It should be introduced in the same manner as one for the posterior nares, and the two ends of the string fastened to the cheeks by pieces of plaster in the same way.

Constitutional Treatment.-Cases of systematic or idiopathic epistaxis will require constitutional treatment according to the 
condition presented-for instance, vascular turgescence or plethora, depending upon cardiac, hepatic, or renal disease, will be eventually relieved by constitutional treatment directed toward those conditions. Likewise, conditions of anæmia, malarial poisoning, purpura, etc., should be treated accordingly, or the hemorrhage will recur at short intervals.

Hæmophilia also requires systemic treatment. The iron preparations, ergot, and lead acetate have proved useful in these cases. Lately the substance and preparations of the thyroid gland have been suggested for the relief of this condition.

Dr. De Guss has reported the case of a woman (hæmophilia) who had suffered for two years from the loss of blood, which had reduced her to a condition of extreme anæmia. Her face was bloodless, and the mucous membranes absolutely colourless. The gums bled profusely at the least touch, and the legs and arms were covered with spots of purpura. The patient's appetite had remained rather good. She had been using all sorts of hæmostatics, with repeated injections of ergotin, but without avail. His treatment was based upon certain observations made in regard to the action of the thyroid substance in the treatment of metrorrhagia. He prescribed for this woman 3- and 5-grain capsules of thyroid substance. The patient took regularly three capsules a day, beginning on the 9th of October. On the 27 th the weight had diminished only a little over twelve ounces. She stated that she had had no hemorrhages since taking them. The purpuric spots had disappeared, the gums had become hardened, the face had regained its colour. She had not exceeded the dose of three capsules a day. This observation showed, according to the opinion of Dr. De Guss, that the thyroid substance exercises an action as yet unknown upon the plasticity of the blood.

Many of the experiments upon the influence of the thyroid gland upon metabolism have shown some remarkable results.

Schondorff has made long-continued experiments on the dog, and he finds that feeding with the thyroid gland or its active principle (Bowman's thyroidinin) exerts a decided influence on metabolism. There is loss of weight, but the albumin of the body is not attacked until the fat is all used up. 


\section{CHAPTER XVI}

DEFORMITIES OF THE UPPER AIR PASSAGES

External Nose

Deformities of the external nose may be congenital, and may constitute part of a general facial irregularity; but usually the cases coming under observation are the result of fracture or syphilitic disease. Irregularities of the dorsum of the nose may be classified into $(a)$ those presenting one or more protuberances, $(b)$ those presenting depressions, and $(c)$ those cases which have undergone more or less loss of the bony framework, such as the so-called "saddle nose."

Patients are usually very desirous of undergoing treatment for the correction of these external deformities, and submit to surgical proceedings very willingly. There are no rules which can be laid down, however, for the correction of these conditions. The kind and extent of operation must therefore be left almost entirely to the ingenuity of the surgeon. Many very satisfactory results have been obtained from plastic and subcutaneous operations by Jarvis, Roe, Weir, W. B. Johnson, and others. Roe has from time to time published reports of cases showing excellent results. His methods of operation for the small prominences and depressions have been chiefly confined to intranasal manipulation by the drill, saw, and gouge, which for obvious reasons offers many advantages over extranasal methods. In flat nose-saddle nose-external incision is usually required, unless paraffin is used.

Several methods have been recommended. König and Israel's operation is the one that has been most often adopted. It consists in making a deep longitudinal incision along the dorsum of the nose and then taking a flap from the forehead containing not only a little skin, but periosteum with a thin plate of bone attached, which has been chiseled out from the forehead. This flap is now laid into the sunken place and stitched 
to the lower portion of the nose, after which the gap in the forehead is stitched up. A second operation is made to close the lateral gaps.

M. Le Tievant first successfully used a metallic framework for holding up the nose. He employed aluminum as the material for the supporting bridge. M. Claud Martin afterward constructed one of platinum. Modifications of these have been used in this country by Dr. R. F. Wier and others. There is great liability to displacement of this bridge, and to overcome this difficulty Hopkins has devised the form as seen in Fig. 16I. It is cut from a single sheet of platinum one fiftieth of an inch in thickness, and then moulded to fit the individual case. This greater breadth allows of better opportunity for fitting the nose. A V-shaped piece is cut from the centre of the upper end, which reduces the weight and allows of more ready moulding, and relieves the thin skin over the nasal bones from pressure. Oval perforations are made in the bridge, which again reduce the weight, and at the same time the uniting of the

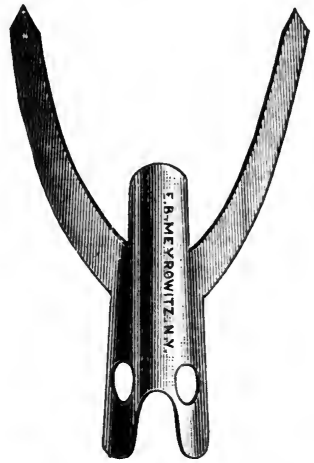
tissues through these openings adds materially to its stability. The supporting arms are made much broader and thinner than in the original model, so that they may hold the alæ in position with the least irritation from pressure. These arms are pointed at the $\epsilon$ nds, and when set into the openings drilled in the superior maxillary bone the bridge is certainly very firmly fixed in position. The arms are to be made longer than is absolutely necessary, and then cut to a suitable length for the individual case at the time of operation. By doing a Rouge's operation (see p. 269) one secures ample room to place the bridge properly, and it is retained in position with surprisingly little irritation. The fact that this splint is made entirely of one piece of metal greatly reduces its cost.

French uses also plugs of cork covered by collodion for holding the nose up.

The great difficulty is that these plates will ulcerate through the skin. The writer was obliged to remove one from a patient upon whom the operation had been well performed by a sur- 
geon in another city, because the edge of the bridge had ulcerated through the skin. Dr. C. H. Knight has reported a similar experience. For refracturing and adjusting the septum or nasal bones, the method and apparatus of Dr. W. B. Johnson is excellent. The instruments and operation proposed aim at a refracture and adjustment in proper position of traumatically displaced nasal bones. The instruments used are an elevator, a retentive apparatus, and a plaster cast to assist in retaining the parts in their proper position after the operation. These conditions, however, offer abundant opportunities for the ingenuity of the operator, since the conditions are so various that no absolute rules of procedure can really be laid down. This will apply also as well to the selection of the material to be used for any apparatus.

Nearly all of the methods for the relief of these deformitiesespecially saddle nose-hitherto adopted will probably be superseded by the newer one consisting of subcutaneous injections of paraffin. Although several instances of ill effects (some serious) have been reported in consequence of this procedure-such as embolism, sloughing, and abscess, reddish discoloration of the skin covering the nose, and deformity by hyperinjection-yet when carefully and aseptically performed, it has been harmless and successful in many cases recorded. The paraffin should be mixed with sufficient "petroleum jelly" to bring its melting point to $\mathrm{IIO}^{\circ} \mathrm{F}$. It should then be sterilized in a closed tube by being kept on a sand bath at a temperature of about $300^{\circ} \mathrm{F}$. for at least ten minutes. The mixture may now be gradually cooled by immersion of the tube in sterilized water at $80^{\circ} \mathrm{F}$. (according to Harmon Smith) until it cools to a temperature of about $120^{\circ}$ $\mathrm{F}$. It is then drawn into the syringe, which has been thoroughly sterilized. The air bubbles are to be expelled by tipping up the syringe, and the mixture allowed to cool until becoming semisolid, when it will be ready for injection. The needle of the syringe should be further heated a little before puncturing the skin to avoid the projection of a more solid plug at first. A piston syringe is not really suitable because of the danger of hyperinjection therefrom. A syringe made on the principle of the "dope cup" (attached to engines) is the proper thing, for then a small, thready mass of the paraffin can be continuously and gradually propelled into the tissue in a semi-solid condition and moulded to the parts simultaneously without the danger of pushing too much out, as may happen through the use of a piston syringe. 
The best instrument to use is that devised by Dr. Harmon Smith, of New York (described in his excellent paper on this subject read before the American Medical Association in 1903), which is so arranged that the paraffin is pushed out by working a screw piston on the principle of the " dope cup."

The parts to be operated upon should be thoroughly sterilized and adhesions between the cutaneous and subcutaneous tissue loosened up, if practicable. Local anæsthesia, by injecting a few drops of 4-per-cent. solution of cocaine, is sufficient for this operation. The moulding of the paraffin should be simultaneous with the injection. Too much should not be injected. It were better to make two or more injections rather than inject too much at one time, for a redundancy cannot be withdrawn and it may cause sloughing or embolism or deformity. Experiments on the lower animals have shown that aseptic paraffin and oils are tolerated when incorporated with the animal tissues. Embolism will be avoided if the mixture be injected slowly and in a semisolid condition.

\section{Deformities of the Nasal Cavities}

Deviations of the Septum.-Deformities of the nasal chambers may affect the septum (either cartilaginous or bony), the turbinated bodies, the palate bones, or the maxillæ. These deformities usually consist of such changes of contour and outline as to present projections or ridges of different angles and shapes, localized hypertrophies, or outgrowths. Deformities of the nasal septum are very common. Stoker thinks that no such thing as a perfectly straight septum exists among civilized people (Roe). According to the statistics gathered and published by Roe, Martin, Mackenzie, Bosworth, and others, septal deviation is certainly the rule, at least among civilized races. Collier estimates that about 80 per cent of savages (aboriginals) are exempt. According to Harrison Allen, fully 20 per cent of negroes are affected with deviated septum. Zuckerkandl found deviations in 37.8 per cent of the specimens (dry skulls) examined by him. Delavan found deviated septum in 50 per cent of the cases examined; M. Mackenzie, 76 per cent; Jarvis, 8I per cent; Sedziak, 83 per cent; Simanskey, 95 per cent; P. Heyman, 96 per cent; Von Kline, 98 per cent; Lowenburg, 86 per cent. 
The largest proportion of these deviations, as shown also by statistics, is found in the cartilaginous and anterior osseous portion of the septum. Attempts at their classification have given rise to a great variety of tables, based largely upon either the anatomic or pathologic principles, according to the fancy of the writer. The table of Stoker is very simple, and seems to be descriptive enough for practical guidance. First, angular; second, curved; third, sigmoid or double curved. These

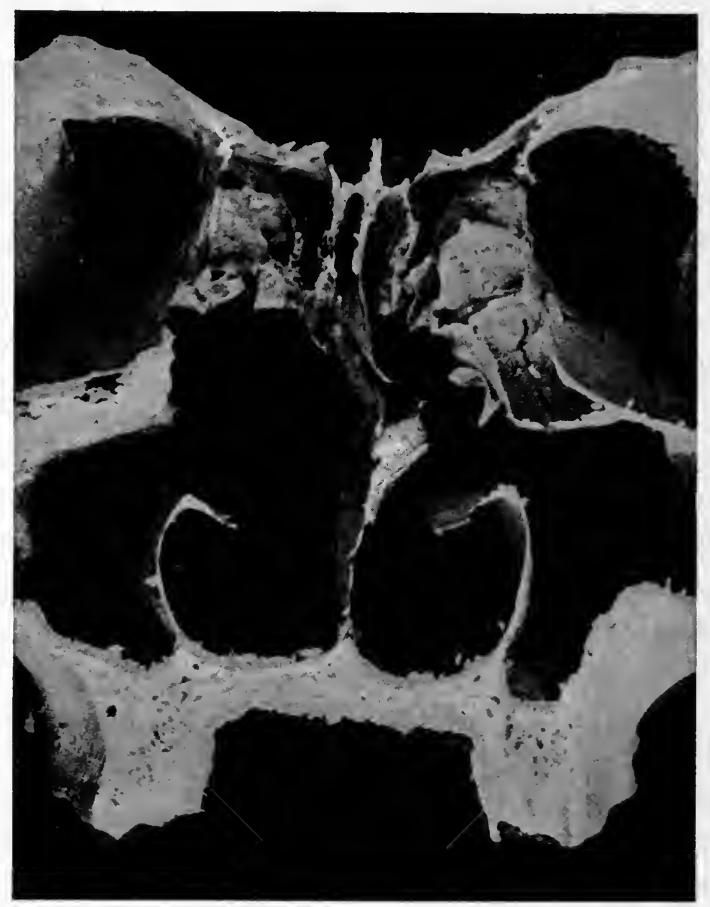

Frg. 162.-Coronal section of dry specimen, showing bony spur (photographed by Dr. P. M. Hickey).

deflections present a great variety of shapes, however, as met with in practice. The deformity usually shows upon one side mostly, although both sides may be affected; this is almost always true of the crenated and ridge-like deformities, for generally the deflection of the anterior curve of one side is very apt to be compensated for by a posterior curve on the other side in the opposite direction, unless there is thickening (see Fig. 162). The deformities of the turbinated bodies consist 
mostly of hypertrophies of one or the other of the turbinated bones, or such irregularities of construction as to form horizontal or spiral-like ledges projecting at angles into the nasal cavities. Spurs may also project from the floor of the nose; if so, they are, of course, usually bony. The size of a ridge or projection from the septum may vary from a slight unevenness of surface to a protuberance of nearly an inch. The writer has seen a congenital deformity of this extent in a broad nasal passage of a patient with a high arch of the palate. Deformities of the vomer and palate bone usually occur in connection with cases of general deformity of the facial bones as well, or in those who have suffered an extensive fracture or injury of the whole region. Sometimes great deformity coexists with either soft or osseous tumours situated in the nasal passages or in their neighbourhood.

Etiology.-The causes of septal deviation may be properly considered under the head of predisposing and exciting. The predisposing causes may be considered as evolutionary-racial, heredity, diathesis (Roe), trauma, and previous disease.

Racial Causes.-It is conceded that deviations of the septum are vastly more frequent among civilized than among savage races. Among Europeans and Americans the percentage varies from 60 to 80 per cent, while among Mongolians, Africans, and Polynesians the deviations are found in about 20 per cent. The frequency of deviation of the septum among the civilized, particularly among the Europeans and Americans, is ascribed to two causes: first, to the increase in the cranial development and the enlargement of the facial angle; and, second, to the admixture of the several races (Martin). Ecker and Lissauer have demonstrated that under the influence of increased development of the cranial mass, the schematic line formed by the cranial vertebræ has a tendency to curve in proportion as we advance in the evolutionary transformation. The septum changes according to the degree and amount of rotation from above downward and from backward forward, around the body of the sphenoid bone taken as a centre. Thus it will readily be seen that as this process of rotation takes place the angle formed between the frontal, the superior maxillary, and the sphenoid bones for the accommodation of the septum becomes proportionately greater, and as the posterior portion of this angle widens, the anterior portion becomes cor- 
respondingly narrowed, which gives less room for the septum toward the anterior portion. This would account for the seeming redundancy of the septum in many cases, there being apparently insufficient room for its accommodation, requiring it to become more or less incurvated or wrinkled, especially the cartilaginous portion (Roe). These studies have been pursued by Potiquet, whose researches were conducted on 400 skulls in the museum in Paris. "He shows that the nasal septum is more prone to lateral curvature as the angle of the face is more inflected in its antero-posterior direction, and that this angle is more and more inflected in an ascending series as we rise from the anthropoid to the European" (Roe). Martin also observes "that these anomalies of the face, due to a mixture of the races, are more efficiently explained by the fact of the blending of the different racial types than by evolutionary or devolutionary changes from a prognothus to an arthognothus type." Delavan points out that the aquiline type of nose, as illustrated in the Slav, the Hebrew, and the ancient Roman, is particularly apt to be associated with deflections; a notable exception of this observation is the fact that the North American Indian, while possessing the aquiline nose, rarely has deflection of the septum. "This is, however, explained by the fact that the Indian belongs to a primitive and pure race, and also by their higher physical development on account of their outdoor life, and, as has been pointed out by . Catlin, by the habit of many Indian mothers of closing the mouths of their children, compelling them to breathe through their noses from their earliest infancy, all of which conduces to the proper development of the nose." Therefore evolutionary and devolutionary changes seem to be very probable causes of septal deformities, although septal deformities are seldom seen before the age of seven years. This doctrine is advocated by Zuckerkandl, Potiquet, Hewittson, and Martin, who think that the abnormal condition results from a change from prognothism to arinothism, as already suggested by Zuckerkandl. McCoy, of Philadelphia, reports several cases of "inversional dental germ of the upper incisor teeth," so that the eruption took place through the floor of the nose into the nasal fossa. Zuckerkandl, De Haviland Hall, and others, also report cases of this abnormal development. There are also analogous cases reported of the eruptional development of teeth through the 
floor of the orbit and into the antrum. In all of these cases of tooth development into the nasal passages there has been fetid discharge. They may also give rise to other deformities secondarily. Such cases may be mistaken for osseous or cartilaginous growths or rhinoliths. Hewittson found that nasal stenosis was often associated with the crowding of the teeth and high arch of the palate. "This failing of the modern face or ultra-refined face," as he terms it; " is attributed to the fact that civilized man partakes habitually of much softer food than fell to the lot of the savage man. He examined large numbers of natives on the Zanzibar coast, Hindoos, and Malays, and a large number of Arabs in Morocco, and did not find any nasal stenosis. "There being no stenosis in these people examined, the septum was found quite or nearly symmetrically placed."

Dr. Potiquet, of Paris, thinks that the lateral curvature of the septum appears to be an evolutionary phenomenon only to the degree of antero-posterior inflection of the axis of the face. Martin does not believe that the axis of the face in the European is greater than in the Hindoo or Arab; but Hewittson claims the latter are seldom troubled with stenosis, and Potiquet believes it is a law with very illiterate and crude people. In this way he excludes all except Europeans and their immediate descendants, but Nature's laws are not elective. Martin states it might be accepted as a law that a straight or symmetrical septum is the distinction of a pure race, while a deviated septum occurs in a mixed race (Roe).

It therefore seems tolerably certain that among the devolutionary capitulations incident to the development of that grand artificial animal, civilized man, the folding of the septum nasi is not one of the least. Undoubtedly the changes in the brain and skull, and the progressive atrophy of the olfactory apparatus which is taking place in us (for it is far inferior to that of the anthropoids and aboriginals) from want of use, may be strong contributory factors in septal deviation. Another predisposing cause may be mentioned, the increasing difficulty and prolongation of parturition among our so-called refined women, a circumstance which might often subject the infant's nasal region to extra pressure, and possibly temporary distortion, either of which events would influence very much the lines of subsequent development of the nose. In Roe's excellent article 
is given succinctly the details of the development of the septum, which is corroborated by Zuckerkandl. He first calls attention to the composition of the septum, consisting of two bones-the perpendicular plate of the ethmoid and the vomer-and one cartilage, the triangular; and that the perpendicular plate of the ethmoid bone is inclosed within the flattened lamella, which descends from the under surface of the cribriform plate, or the horizontal portion of the ethmoid bone forming part of the anterior fossa of the base of the skull, while the lower, broader posterior half articulates with the vomer. Ossification commences in the vomer at about the same period as in the vertebræ (about the age of eight weeks of fotal life), and is not completed until after puberty, and the perpendicular plate of the ethmoid does not begin to ossify until about the first year after birth. The coalescence of the laminæ takes place from behind forward, beginning at about three years. The cartilage of the septum, extending forward from between the two plates of the vomer, is somewhat triangular in form, thicker at its margin than at its centre part, and completes the separation between the nasal fossæ in front. Bearing in mind this fact, that the vomer is composed of two parallel laminæ which do not fully coalesce until after puberty, and in some instances do not coalesce at all (Lefferts described a case of double septum in a man aged twenty-five), we can readily see the effect that would be occasioned by the slightest imperfect development of either of these plates. Hypernutrition of one lamina would cause the other lamina to be pushed out of its normal line of growth, and conversely any lack of full development of one lamina would occasion corresponding deviation. Any such deflection in the vomer would naturally be accentuated in the cartilage of the septum. The probability of this view, that deflections result from defective development of the plates of the vomer, is increased when we take into account the fact that scarcely, if ever, do we find two bones with precisely the same degree of development. The fact that ossification begins in the posterior end of the septum, and coalescence takes place from behind forward, explains why that end is rarely observed to be markedly deflected. Walcker has failed to observe deflection of the septum before the fourth year, while Zuckerkandl states that he has not observed it in children under seven years of age. 
Anton, however, has shown that these deviations may be congenital, by the examination of fifty-six cadavers of newborn infants, in which he found deviations in nine cases, equal to sixteen per cent (Roe). The peculiar forms which deflections of the septum may assume can readily be explained in the light of the fact that the plates may develop unevenly.

Heredity is an important predisposing cause. Deflection of the septum of nearly the same type may be found frequently in father and son. This fact would seem to lend support to the evolutional or devolutional theory of causation. The projections from the septum consist for the most part of ordinary cartilaginous and bony tissue, usually somewhat condensed. The spurs, properly speaking, are almost always cartilaginous, being given off from the cartilaginous septum, although they are often veritable exostoses or ecchondroses. The exostoses may become condensed (eburnated).

Histologically, the cartilaginous spurs consist of condensed or compressed cartilage, with more or less obliteration of the spaces at the base. In the ridge-like spurs the cells at the apex are more contorted and much smaller than in normal cartilage, the canaliculi are more or less obliterated, so that the surface is to a large extent nourished by the submucosa, which is scarcely distinguishable from the attenuated perichondrium. Occasionally osseous tissue is found in these spurs near the base, especially in patients of advanced years, but I believe that bony cells are never found near the surface.

Hypernutrition of one side of the septum from irritation or disease is considered a cause by Roe and other observers. Among them is Greville Macdonald, who thinks that it may account for a deviation, either perpendicular or horizontal. Ingals, on the other hand, thinks that a local malnutrition is often the cause. He believes that the deflection of the septum usually begins in the cartilaginous portion. Jarvis believed that a highly arched palate was associated with many of these deflections, and that such conditions were hereditary, as he had traced many of them through several generations.

Malformation of the superior maxilla is often associated with deviated septa. M. Collier contends that paralysis or paresis of the muscles of the nose is also responsible for an enormous number of these deflections. 
Trauma is undoubtedly in many cases the cause of deviation and of the formation of spurs, exostoses, and ecchondroses. In children, the effect of injury is usually a dislocation of the triangular cartilage, which, if it remains out of place, assumes various shapes during the development of the parts. These dislocations are more easily accomplished in childhood on account of loose attachments, and in consequence the deformity of the nasal passages will vary, of course, with the degree of the injury which has been sustained. In some cases the effects of injury are such as to almost completely efface the anatomical characteristics of the nasal cavities. In aggravated cases the passages may be mere slits between bony and cartilaginous projections and irregularities.

Previous disease, such as catarrhal affections and hypertrophy of the turbinals, is undoubtedly a common cause of deviation of the septum, and of irregular developments of the turbinated bones as well. Many observers believe that septal deviations are predominant causes of hypertrophic nasal catarrh, as well as of inflammation or suppuration of the accessory sinuses. Indeed it is often debated whether the septal or the turbinate abnormality is the cause or the effect of such diseased conditions. It would seem highly probable that a larger number of cases of chronic hypertrophic rhinitis are dependent on deviations of the septum or deformity of the turbinated bones than contrariwise. Other diseases, such as goitre and cretinism (Allen), are sometimes causal factors.

Sex.-Females seem to be almost equally with males affected with nasal deformities.

Age.-Septal deviations, as already stated, are not found in children as often as in adults. Collier states that only about ten per cent of children show deviated septa.

Diathesis.-Roe and others state that deviations of the septum are common among those suffering from a strumous, syphilitic, tubercular, or rachitic diathesis. Löwenberg has especially emphasized the predisposing influence of rickets. It would seem, however, that these dyscrasiæ can only exert an accidental influence, inasmuch as these abnormalities are so commonly observed among persons who do not show in any way signs of these dyscrasiæ.

Symptomatology.-Considering the great number of deviated septa among civilized men, one may well wonder that no sub- 
jective symptoms arise in a vast number of cases, even when more or less mechanical obstruction to the nasal passages would seem inevitable. But it is a fact that many persons go through life with little or no subjective trouble referable to their nasal passages, whose septa, present upon examination, marked deviations or spurs, or even extensive ridges. Just why this should happen in certain instances is surprising. Every practitioner has seen examples of persons whose nasal passages (perhaps only accidentally examined) showed considerable deflection, or spurs of the septum, or deformed turbinals, and who apparently have suffered no inconvenience whatever.

Subjective.-The principal subjective symptoms in connection with these pathologic conditions are more or less interference with nasal respiration (mouth breathing), hypersecretion, defective nasal drainage, interference with the sense of smell, myalgia, and other pain, frequent attacks of sneezing, cephalalgia, neuralgia, or reflex nervous symptoms, middle ear disease, with perhaps deafness and asthma. Meyer states that he has seen maniacal attacks which depended upon nasal stenosis from deviation. Allen, Roe, Hajek, Snow, and others, have made valuable literary contributions to the subject of the association of headaches and other neurotic phenomena with nasal stenosis. While in many cases the operation of turbinectomy, or the removal of septal deformity, will promptly relieve the subjective symptoms in question, yet it will often be found that there are other causal factors of a systemic nature which will need attention. Dr. W. G. Spencer, of London, reported the case of a carpenter, aged forty-six, who after an attack of influenza suffered from trigeminal neuralgia for five months, and who was entirely relieved by a turbinectomy. Many similar instances of relief from neuralgia and other neuroses by the correction of intranasal diseases could be adduced, still, before formulating principles or rules of action on this evidence, we must remember that accidents to or operations upon other parts of the body, even when intranasal disease exists, may produce similar fortuitous results. For example, the writer met with a case of long-continued facial neuralgia, with intranasal disease, that was apparently cured by the accidental cutting of the neck with broken glass, thus frustrating a contemplated operation. Dr. J. Solis-Cohen, of Philadelphia, reported three cases of persistent headache with intranasal dis- 
ease which were apparently not relieved by the correction of the intranasal condition, but were subsequently restored to health by general rational treatment.

Objective.-The objective signs of deviation, or spurs, etc., are varied, of course. There is always more or less stenosis observed, according to the degree of deformity. The projection may entirely occlude one meatus, or both lower and middle meatus. Therefore it is well to examine every case carefully with the probe, as well as by inspection, under a good light. The secretion will vary in character. In cases of doubt, cocaine or adrenalin should be used. As a rule, pretty complete obstruction of the lower meatus by cartilaginous or partly cartilaginous and bony deflection will be associated with extra discharge from the nasopharynx, and possibly with considerable desiccation of the secretion.

Diagnosis.-There is usually little trouble experienced in making the diagnosis. Deviations and spurs of the septum nasi, however, may sonetimes be mistaken for ecchondroses, exostoses, necrosed bone, or abnormal eruption of teeth. These latter formations, however, will present distinct bases of origin, which can usually be made out by careful examination. Meyer suggests, as a point of differential diagnosis between deflection and ecchondroses, the presence of a cavity on the opposite side of the septum, in cases of deflection.

Treatment.-As a rule, when these signs are present and persistent, it is necessary to adopt some measures for the relief of the deformity, although, in those cases resulting from traumatism, it may require several severe operations. Occasionally, however, operative procedure may add to the subsequent sufferings of the patient, or even fail to restore the functions of the nose and the accessory cavities. In one case the writer made several operations upon a patient whose nose had been kicked in by a horse. The patient at the time of the first operation was twenty years of age. By drilling and sawing, a passage on each side of the nose, representing at least a meatus, was effected, and the patient was relieved for a short time. However, a renewal of inflammation, extending to the maxillary sinus on both sides and to the frontal sinus, resulted in considerable distress and supraorbital infiltration. We next endeavoured to make a channel higher up at the same time, perforating both antra. This also relieved the patient a second 
time, but necrosis subsequently supervened and progressed, until what was left of the turbinated bones and the inner plate of the maxillæ on each side became necrosed, and had to be removed. After a time one of my colleagues removed the left superior maxillæ, and the patient died of meningitis two weeks afterward. As the boy had lived for nine years with his nose "smashed in" before my well-meant interference, one might conclude that the endeavour to "open up" the nasal passages must have contributed to the final disastrous result. The writer has since tried on two occasions to relieve enormous deformity and stenosis resulting from severe fractures, but in each case with indifferent success so far as the restoration of the function of the nose was concerned. If any of the deformities in question exist in a person and yet no complaint relating to the nose is made, it would seem discreet to let the nasal passages alone, at least for a time. When, however, as said before, the functions of the nasal passages are very much compromised or the comfort of the patient very much disturbed, then surgical measures should be immediately adopted to procure relief. It was formerly the custom to treat such patients with oleaginous and watery sprays or insufflations of powders, but in the light of our present knowledge and experience such therapy is entirely inadequate. Our aim should be to execute such surgical measures as will reduce or remove the existing deformity.

The removal of the deformity may be accomplished by the use of either knife, cutting forceps, saw, chisel, snare, drill, or by electrolysis; therefore it will be well to take up the different classes of deformities and indicate the methods for their relief. It may be premised that all such operations should be performed with conservatism, remembering that as little as possible of the mucous membrane is to be destroyed, and that, as far as possible, antiseptic precautions, such as thoroughly cleansing the part with antiseptic solutions, etc., be taken.

Cartilaginous Deformities. - Where there is a deformity of the columnar cartilage, so that the vestibule of the nose is occluded, or is unsightly on account of it, it should be corrected. For this purpose an incision should be made with a knife along the prominent convex line at the junction of the mucous membrane and skin, taking care not to wound the lower commissure of the nostril; a small spud (see Fig. I63) 
is now inserted into the incision and the mucous membrane thoroughly loosened up from the underlying cartilage, thus exposing it. By the aid of a delicate pair of toothed forceps the projecting cartilage can now be pulled out a little through the opening thus made, and the necessary amount of it cut off from below upward. The operator must be careful not to wound the commissure or any part of the edge of the vestibule, for by so doing troublesome cicatrices, which will contract the opening, are apt to form. The edges of the cut can

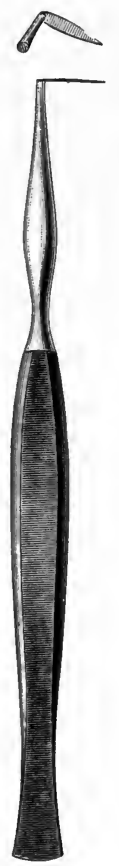

FIG. I63.

Spud for dissecting up the septal mucous membrane (Whitehead). now be brought together with fine stitches, or, what is, perhaps, better, by the application of a little collodion. In this manner no danger will arise of producing either contraction of the nostril or a visible scar.

Deflections and Spurs within the Nose.-When the ridge or spur is situated farther back and wholly upon the cartilaginous septum (it is usually within the limits of the anterior two thirds), the projecting piece may be taken off by the insertion of a probe-pointed bistoury or a smaller probe-pointed knife, and cutting with a sawing motion from below upward, thus taking off cartilage and mucous membrane. The precaution should be taken of placing one finger in the other nostril, to avoid cutting clear through to the opposite side, thus causing a permanent perforation, inasmuch as such perforation adds to the discomfort of the patient afterward.

Although many operators prefer to use the knife for these operations on the cartilaginous septum, yet Bosworth's method of cutting with the saw seems preferable, because attended with less hemorrhage. The objections raised against the use of the saw are that there may be a little more destruction of the mucous membrane and the wound may be more ragged. But according to the writer's experience, the saw has given better results than the knife in these operations, notwithstanding the objections offered. It must be conceded, however, that the result of cutting off the protuberant cartilage wall in this simple manner is sometimes disappointing, on ac- 
count of the resiliency of the cartilage, allowing the curling out of the incised edges, so as to offer nearly as much obstruction as did the original protuberance. This will be found especially in cases where the deflection is considerable through the redundancy of cartilage, the septum, as it were, being too large for the space it occupies. When this tendency of the part is observed, it is necessary to keep the nares plugged under strict antiseptic precautions, so as to press the edges back until the healing of the wound is well along. Unless some pressure is maintained in this way, an angular perpendicular projection may remain in the passage. For this purpose an Asch nasal tube is one of the best things to use. If that be impracticable, then a strip of Bernay's compressed cotton or a plug of iodoform gauze may be inserted. Still better are rubber plugs, as suggested by Dr. Price-Brown, made from sheet rubber (from $1 / 16$ to $1 / 4$ inch in thickness) fashioned to suit the case; the edges, made smooth by filing, should be oiled. They are more efficient than absorbent cotton or gauze tampons, on account of the ease of application and the better result. The Bernay's plugs swell rapidly and more quickly by stripping off the outer layer. They are useful generally for the application of antiseptics or cocaine as well as for splints in septal operations. The degree of compression, of course, depends upon the relative size of the piece introduced into the nasal cavity. In order to obviate the curling or projection of the edges of the cartilage, as just mentioned, many methods have been devised, having for their object the removal of more cartilage and less mucous membrane, and also loosening and repositing the septum toward the median line, and to do this without resulting perforation, or leaving deformities upon either the side operated upon or the opposite side has constituted the chief aim of all operators.

Of those operations that attempt the straightening of the septum, the most important are the Adams's, forcibly breaking the septum, and inserting metal plugs or splints; Steele's incision by stellate punch, followed by the use of ivory plugs; Roberts's multiple incisions by the knife, and the retention of the fragments in place by pins; Sajous's and Ingals's excision of a wedge-shaped piece and afterward using the suture; Roe's crushing operation by means of special forceps, followed by the insertion of antiseptic plugs; Asch's operation, by which the septum is cut with scissor forceps and the fragments held 
by perforated plugs; Allen's operation, in which the base of the septum is detached beneath the mucous membrane, and the septum moved toward the opposite side and retained there by a plug; Watson's operation of dividing the septum, after dissecting up the mucosa and then causing the divided edges to override. Watson thinks that these operations do not aim at reducing the deviation, but simply equalizing its encroachment on the nasal chamber. There is no doubt, as observed by Watson and others, that in many of these cases the curved septum is forced into a straight line, for which it is obviously too large,
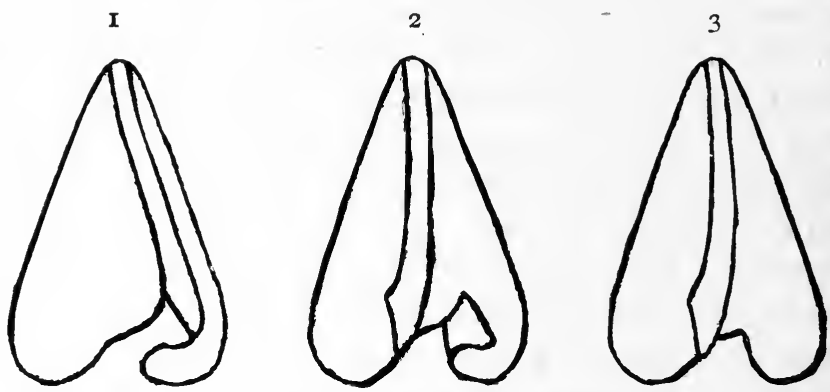

FIG. I64.-Operation for removal of spur of septum (Watson). I, Deviated septum with line of incision ; 2 , septum replaced with spur standing; 3 , projecting spur removed.

and for that reason the results can be but temporary in the majority of cases where the deviation is at all marked. The requirement of the operation may be considered as twofold: I, the operation proper, or the method of reducing the deviation; 2, the means for holding the parts in position until healing has taken place (see Fig. 164).

Escat resects a C-shaped septum without perforating it completely by injecting, after cocaine, enough water to separate and distend the mucous membrane from the cartilage on the convex side. (The membrane is easily detached in the young.) He then resects the convex side, including the protuberant cartilage and part of its membrane at one stroke, leaving the mem. brane on the concave side intact. A gauze tampon for twentyfour hours controls hemorrhage, and the operated side is soon regenerated, having the membrane for support. Ingals, of Chicago, makes an oblique incision through the membrane of the convex portion of the prominence. "He then detaches the membrane for certain distance on each side of the cut from 
the underlying cartilage, exposing the latter. A triangular piece is then cut out, the base of the triangle being at the floor of the nose. Care should be taken in this operation to detach the cut piece of cartilage from the lining membrane of the other side of the nasal cavity without tearing or cutting through it. The first incision is closed by stitches, and the cartilage is pressed into line and supported by means of tampons."

An operation very frequently performed in Philadelphia is described by Sajous as follows: "The least difficult operation, and one which has always given me great satisfaction in simple cartilaginous deflections, is an incision through the protuberance, following its long axis. A smart hemorrhage occurs when the incision is made, but it soon ceases. The end of the finger being introduced into the nostril, the septum is forcibly pushed beyond the centre, and maintained there by packing the previously obstructed nostril with carbolized oakum. The cut edges of the cartilage override each other, and after a couple of weeks are firmly united."

Roberts makes a long incision, oblique or horizontal, according to requirements, through the septum from back to front along the line of deviation or projection with a knife, and with a chisel through the bony septum if it be deviated. A pin is then thrust through the septum above and in front of the incision. By manipulation of the pin and the finger introduced into the obstructed nares the upper or movable portion of the septum above the cut is brought into the middle line, and held in position by forcing the pin forward through the tissues in such a manner that it crosses the incision. A second pin is

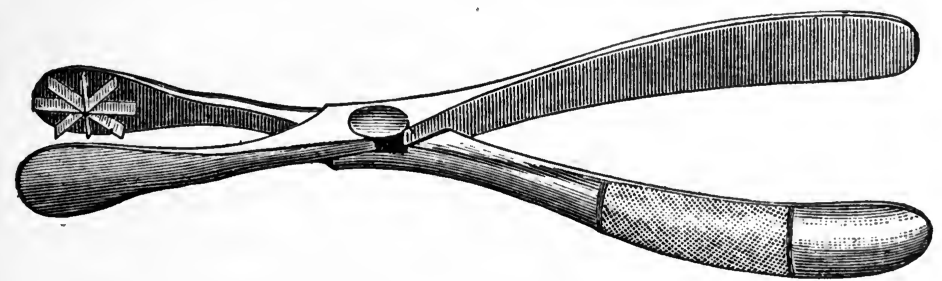

FIG. I65.-Steele's septum forceps.

sometimes introduced through the skin at the lower portion of the nasal bone parallel to the septum, in order to assist in retaining the parts in position. If the deflection of the septum 
is a general rather than an abrupt one, Roberts weakens the septum after the primary incision by multiple incisions with Steele's stellate punch (see Fig. 165), "because the operation will be useless unless the incisions are very free, so as to take away all resiliency of the cartilage."

The operation for cutting and replacing or straightening the nasal septum, as devised by Dr. Morris J. Asch, of New York, has met with great favour by the majority of operators. In his first paper, read before the American Laryngological

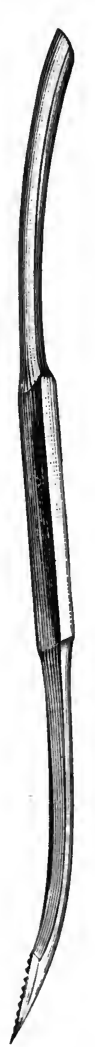

FIG. 166.-Asch's curved gouge for breaking up adhesions between septum and turbinate.

Association in 1890 , he reported 6 successful operations. In a subsequent paper on the subject, Mayer, of New York, reported 20 successful operations by this method. Since then Mayer has published a report of 200 cases, all successful. A synopsis of this (Mayer's) report shows that in 85 per cent a perfectly straight septum was the result, while in the remainder the septum retained some degree of curvature, but never sufficient to cause any stenosis or discomfort whatever. Perforations occurred in about 2 per cent of the operated cases. They were usually about the size of a pin-head, and were caused by the curling up and sloughing of the edge of one of the segments. In no single case was there any alarming hemorrhage either at the time of operation or subsequently. No septic conditions resulted, no disagreeable after-effects, no disturbance traceable to the operation occurred, nor was there at any time any danger to life. These results are the more remarkable in that most of these operations were performed upon dispensary patients, whose physical conditions are rarely of the best.

The instruments devised by Dr. Asch (see Figs. 166 and 167 ) are two separators, one with a blunt edge and the other sharp, to break up any adhesions existing between the septum and the turbinal on the affected side, as also to destroy any posterior obstructions, and the scissors, which are of two sizes. These are powerful instruments, curving outward from the point of crossing and meeting again 
in front. One blade is blunt, while the other is sharp, with a triangular blade, the apex of which is at the tip. The other scissors are made with the blades bent at right angles, and are occasionally necessary for those deviations near the floor of the nose. There are two compressing forceps, one having a long and the other a short beak. Hollow tubes of vulcanite are made with a slight curve on the under surface, in accordance with the normal curve at the entrance of the vestibule of the nose. These tubes have perforations to prevent their slipping out of the passage, and to allow of drainage. They are of different sizes and shapes. Those known as the Asch tubes are of five sizes, some-

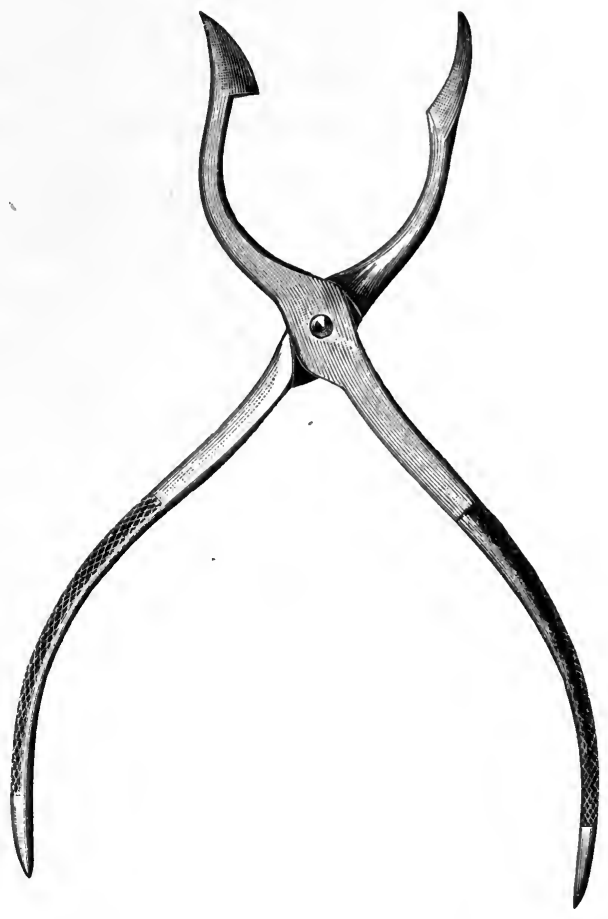

FIG. 167.-Cartilage scissors for operation of deflected septum (Asch).

what oval in shape, and those known as the Mayer tubes are of six sizes, more oval and considerably flatter on the sides. These vulcanite tubes are so prepared that they retain their shape when subjected to the heat of a sterilizer. The operation is best performed under complete anæsthesia, as cocaine does not appear to produce sufficient amount of analgesia; it is necessary that the patient be recumbent. The nose is to be sprayed out immediately preceding the operation, for which purpose an alkaline antiseptic like the Dobell solution is used. The usual preparations for anæsthesia are made, ether or chloroform being the anæsthetic used. All instruments are to be carefully sterilized. The use of an antiseptic spray and the sterilization of instruments for an operation upon a part from which the air cannot be excluded, either 
before or after operation, is nevertheless advisable, for we know that by such care we are surely not adding any infection by our operative procedure. Complete anæsthesia is required, so that the inhaler being once removed it should not be necessary to have recourse to it again, as the operation can be rapidly performed and completed before consciousness is regained.

The following description of the operation is freely quoted from E. Mayer. The head is drawn well backward, in order that blood may not enter the larynx, the table being so placed that either direct or artificial light may be used.

The blunt separator is now introduced sideways on the deviated side, in order that any adhesions existing between the septum and its adjacent mucous membrane may be broken up, enabling us at the same time to ascertain the presence of bony obstructions posteriorly. Should these latter exist, or the adhesions be non-yielding, the separator with sharp edges is required; this, however, is rarely necessary. There may be some hemorrhage after the adhesions are broken up, but it is readily checked by an iced spray, or by pressure of cotton applicators. The open scissors are now introduced parallel to the floor of the nose, the blade in the concavity, the blunt edge over the point of greatest convexity. They are then firmly closed, the blade cutting through the cartilage into the opposite side with a distinct snap. The scissors are then opened and completely withdrawn. They are immediately reintroduced, the direction of the instrument being upward this time, pointing to the frontal bone, the blade again in the cavity and crossing the line of the first incision at as nearly a right angle as possible and at its centre. In this way the incisions will intersect each other; the scissors are now firmly closed and the second incision is made, after which the scissors are opened and withdrawn. We now have four segments as the result of this crucial incision. The finger is then introduced in the deviated side, and the segments are forcibly pushed into the concavity, effectually breaking them at their base.

The powerful blunt forceps is now introduced, one blade in each nostril, and firmly closed, thus straightening the septum and forcing the broken segments to override each other in the concavity. The compression aids also in controlling 
hemorrhage, and the parts are firmly held in apposition. An iced antiseptic solution is sprayed in both nostrils, the forceps being withdrawn. The sterilized tubes are then introduced in the nose, a snugly fitting one on the stenosed side and a smaller one in the opposite nostril, thus causing equable pressure, their presence usually being all-sufficient to control hemorrhage. The operation is now completed. The amount of blood lost varies with each individual, but is invariably promptly checked when the pressure is applied. The patient is placed in bed, cold antiseptic sprays are used half hourly, and iced cloths are applied externally.

Twenty-four hours after operation the tube in the former concave side is withdrawn and not replaced. The antiseptic spray is used once every hour or two, and the compresses are continued. Twenty-four hours later the tube on the stenosed side is removed and thoroughly cleansed. The nose is freely sprayed and cleansed with cotton pledgets, cocaine being used if it be sensitive. The tube is reinserted, unless too large for comfort, when a smaller one is used, the rule being to use a tube large enough to be introduced without force and yet to fill the cavity, no part of it protruding from the nostril. On this day patients are permitted to arise from the bed and assume other than a recumbent posture, the compresses being discontinued. On the third day the tube is again removed, cleansed, and reinserted, and the patient allowed to go home. For the next three or four days he presents himself daily for the removal, cleansing, and reinserting of the tube. At each time of removal the nose is thoroughly cleansed and freed from secretion and clots. Within this first week the patient has learned to extract and reintroduce the tube himself, in the presence of the surgeon, and thereafter performs it once or twice daily at home. The directions are given to remove the tube, spray out the nose, or insufflate warm salt water to which an alkaline antiseptic is added, and then to reintroduce the cleansed tube. The extraction and reinsertion of the tube are quite painless after the first week, and it is remarkable how easily young people become accustomed to it. The patient is now seen once or twice a week for the next four weeks, at the end of which time-five weeks from the day of operation-the tube is finally withdrawn for all time.

The mass of bent cartilage has disappeared, the septum has 42 
become straightened and remains so, and very often the external deformity no longer exists.

Sometimes during the five weeks that the tube is in use it may appear that the septum is bulging greatly on the stenosed side, but it is well to leave it quite alone, as the constant pressure reduces. it finally, for there seems to be a resorptive process going on.

The window resection methods of Ingals, Krieg, and Bonninghaus have been ingeniously modified by Otto T. Freer, who has designed a set of eighteen delicate instruments to perfect the technique. Up to the removal of the triangular cartilage a typical Ingals operation is performed. Its continuance, as far as the cartilage is concerned, is practically the method of Krieg. $\mathrm{He}$ does not continue his resection into the bony wall as do Krieg and Bonninghaus, who cut away large portions of the vomer or perpendicular plate, if deflected; but he fractures the bony septum with Roe's forceps after a preliminary chiseling or trephining, or, where a bone is thin, he removes piece by piece with the Gruenwald forceps, He prefers powdered cocaine applied to both sides of the deflection to the hypodermic method. One to I,000 adrenalin is then applied. The first incision is made vertically along the summit of the cartilaginous deflection and through the mucosa only. It is joined by a horizontal one beginning about three-fourths of an inch posteriorly and brought forward along the bottom of the septum beyond the beginning of the deflection. An anterior and a shorter posterior flap are thus obtained. The mucosa is then completely detached from the convex side by specially designed instruments, and a vertical slit is made through the cartilage in front of the beginning of the deflection without piercing the mucosa of the opposite nostril. A dental spatula is pushed through this opening and the mucosa of the concave side is separated entirely throughout the region of the hollow. Ingals's pointed cartilage knife is now inserted through the slit. It is turned outward, and passed to the posterior border of the hollow and made to cut through the entire length of the cartilage along the base. The free edges are drawn out with rat-toothed forceps. The deflection is removed entire with little sharp-pointed cartilage knives. Any remaining deviated portion of the cartilage may be removed with a Gruenwald forceps. If the deflection is only cartilaginous the flaps are replaced, smoothed down.with a damp cotton swab, and packed evenly with a strip of lint $\frac{1}{4}$ to $\frac{5}{8}$ inch 
wide, which has been rubbed full of bismuth subnitrate. The bony deviations are removed piece by piece with the Grüenwald forceps, chisel, and trephine. Freer reports sixty operations, with ten perforations.

The Watson operation, which has the same object in view, is preferred by some operators. It may be more advantageous for some cases, inasmuch as it saves the cartilage.

Cases will be met with which cannot be properly dealt with by following precisely any particular operation. In such instances the judgment, skill, and experience of the operator must be the guide. The infinite variety of deformities met with, together with the many individual peculiarities of respective cases, must modify the course of manipulation. Hence, it will often happen that no dogmatic rules ought to be followed.

The writer believes that many of the disappointments following the performance of Dr. So-and-so's operation (especially with unskilled operators) may justly be ascribed to the too rigid adherence to rules laid down, without a proper survey of the case.

There is no doubt of the superiority of the Asch, Ingals, Freer, Watson, and other such operations for the correction of extensive deflection or ridges of the cartilaginous septum, but for small spurs or ecchondroses, and aiso for ridges and spurs of the bony septum, the use of the saw is preferable. In the latter case, any method of fracturing and reposition of the bony septum is unsatisfactory, according to the writer's experience.

Saw.-This instrument may be propelled by the hand or an electric or foot motor. The writer prefers the former method of propulsion, especially when operating under chloroform or ether anæsthesia, in which event the patient must be recumbent, and after the insertion of the instrument, as a rule, the manipulation will be conducted chiefly by the sense of touch by the operator. One of the difficulties in the use of the saw comes at the very start, for the operator will frequently find that the resilient smooth tissue will almost effectually resist the engagement of the instrument along the line which he has contemplated.

Drill.-Drilling operations were promoted and improved by Goodwillie, of New York. The instrument (drill) may be worked by the hand or by means of an electro-motor. The latter method has been found preferable in the writer's expe- 
rience, principally because it is more expeditious and gives more precise results. It is not applicable to all deformities, but for the correction of rather straight ridges or shelves of the bony septum, and of shelving or bullous deformities of either the inferior or middle turbinates, it is very convenient. It often happens, however, that the completion of the operation by the conchotome will be desirable. In some of these cases it does not seem necessary to actually remove much of the bone, as the breaking of it excites a process of resorption that finally removes the difficulty.

Dislocation of the Triangular Cartilage.-The forcible dislocation of the triangular cartilage, either by fingers or forceps, for the relief of septal deformity, has not been followed by satisfactory results in the writer's hands.

Galvano-cautery.-The galvano-cautery for the reduction of spurs or projections, either cartilaginous or bony, is not generally satisfactory unless they be very small, because of the great amount of inflammatory action, and sometimes ulceration, which follows.

Snare.-The use of the wire snare and transfixion pins (see Fig. 168), as suggested by Jarvis, is very difficult of accomplishment, because in the majority of instances the pin tears

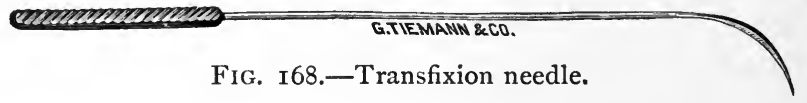

out when the snare is tightened sufficiently; besides that, the operation is tedious, and very painful to the patient unless general anæsthesia is employed.

Electrolysis for correcting these deformities is in favour with many practitioners, but the writer has not observed any superior effects over other methods in the employment of electrolysis for the removal of spurs or deviations. Besides the uncertainty of the results obtained in respect to the removal of the object, there is danger of producing a destruction of tissue far beyond all anticipation. It must be remembered that there are some individuals whose tissues seem to be more susceptible to electrolytic action than others, and the employment of this agent upon patients possessing such an idiosyncrasy may be disastrous. This method has been used and is recommended by a number of American and European laryngol- 
ogists, and some excellent effects have been reported. Casselberry, Newcomb, Ballinger, and others, have written interesting articles upon the subject. The writer has abstracted from one of Casselberry's papers the following:

He says: "Miot, of Paris, was the originator of the method in I888. Following him, Colwer, of Lyons, reported his results in thirty cases. Moure reported cases at the Ninth International Medical Congress, at Berlin, in 1890 . Moritz-Smith also reported cases. Of American authors, Newcomb, of New York, and Ballinger, of Chicago, have reported successful results. All of these operators have employed, as a source of electricity, a primary galvanic battery of about thirty cells. A current strength for electrolysis of nasal spurs should be from I 5 to 40 milliampères, measured with the resistance of the spur in the circuit, and to supply this current from fifteen to twenty cells of a galvanic battery would ordinarily be used, with a corresponding electric motive force of from 12 to 20 volts or more. A current suitable for electrolysis should be characterized by a moderately high tension of voltage and a comparatively low current strength or ampèrage."

The Edison "street current" has an electric motive force of I Io volts, which must be reduced by a current controller. The ampèrage of course depends upon the amount of resistance in the circuit, and it also can be correspondingly reduced by the resistance of lamps and the controller, so that with the spur in the circuit it measures the requisite number of milliampères. Casselberry recommends and describes the MacIntosh current controller for this purpose. He has used exclusively the bi-polar method, as it is conceded by all to be equal to if not more effective than the monopolar, and it is more convenient to the operator and less disagreeable to the patient. Not more than two needles, one representing the positive and the other the negative pole, should be employed, as there is no advantage in the use of multiple points, and, besides, it is thought to be more difficult to escape a certain amount of destruction when more than two points have been inserted. The parts of the needles intended for insertion in the septum should be from 15 to 20 millimetres in length and about half a millimetre in thickness. They should lie parallel, and distant from each other about 3 millimetres. The material which he has found best adapted to the purpose is iridio-platinum, in which enough iridium is 
placed to make the composition very hard and stiff. A very sharp point can be given this substance, and its penetrating power is almost equal to steel. It is not oxidizable. Moure and others recommend steel needles especially, such as are used by sailmakers, but the one connected with the positive pole will oxidize.

Casselberry has reported this treatment in ten cases with satisfactory results.

For general anæsthesia, chloroform, ether, or nitrous oxide may be used. Of these, perhaps chloroform is preferable, unless the patient shows some cardiac or pulmonary lesion. Even in such cases chloroform, if carefully administered, will do no harm. Ether is more difficult of administration and is attended by more excitement of the patient, and, according to the writer's experience, with more hemorrhage. Of course ether should never be employed in any case where the galvanocautery will be used. For short operations nitrous oxide will answer the purpose very well, and if modified by the admixture of oxygen it may answer the purpose well for any of these operations. In all cases the patient's head should be low, to prevent the blood from entering the larynx. The Rose position may often be advisable, or the position suggested by Dr. Carl Seile:, of Philadelphia, of placing the patient in a recumbent, ventral position, with the head projecting over the edge of the operating table. In this way the blood flows continually out of the mouth and nose, avoiding the necessity of plugging the posterior nares or of making a previous tracheotomy. When the operator cannot proceed by the sense of touch, he is to lie on his back under the table, facing the patient. Dr. Seiler also offers another device for getting an image of the parts, by placing a looking-glass on the floor (New York Med. Jour., vol. lxii, p. 635). For many operations, if there be no objection, the plugging of the posterior nares should be done before proceeding to the operation.

Cocaine, eucaine, holocaine, chloretone, ethyl bromide, or ethyl chloride may be selected for local anæsthesia, but cocaine applied to the parts is usually preferred. 'Some operators, however, prefer eucaine or ethyl bromide. The use of adrenalin in connection with cocaine has simplified these operations by keeping the field comparatively free from blood. In removal of posterior nasal growths with the cold snare or an electric 
snare, eucaine is much more desirable, as the contractile power of cocaine frequently renders it impossible to grasp the growth with either instrument. Peroxide of hydrogen is excellent for antiseptic and hemostatic purposes. It is better than bichloride of mercury for extensive operations, because the latter agent will sometimes keep up more or less irritation of the parts.

\section{Results and Complications of Operations for the Correction of Deviated Septa}

The untoward results of such operations may be summed up as hemorrhage, erysipelas, inflammation of the neighbouring accessory sinuses, fauces, or larynx, unintentional injuries to neighbouring parts, such as perforation of the septum, wounds of the orbit, frontal sinus, antrum, or ethmoid cells, synechia (or atresia) of the nasal passages, abscess of the septum, and neuralgia.

Hemorrage, although profuse directly after all cutting operations, is generally arrested readily by the insertion of a tampon of cotton saturated with adrenalin. Exceptionally, however, it may be profuse and continuous, in which event one or more of the expedients recommended in the section on Epistaxis should be adopted. Serious inflammation following these operations must be combated by the assiduous application of mild antiseptic douches or powders to the nasal passages, combined, if necessary, with outward applications of ice, and such systemic treatment and regimen as the case may require. Should suppuration of one of the accessory cavities supervene, an opening for the purpose of securing drainage should be made at once. If proper care and skill be exercised, no serious injury to neighbouring parts will be inflicted. Such accidents, however, do occur in the hands of ambitious and inexperienced surgical rhinologists. The writer has met with several cases where the orbit and frontal sinus had been unintentionally perforated, and one case where the operator had drilled clear through to the outside of the nose. Besides this, the writer has met with cases where the intranasal tissues had been terribly lacerated by saws, drills, chisels, ploughs, and curettes.

It is sometimes astonishing how well nature bears intranasal wounds. As Simpson says, the repair of the intranasal parts after operation is wonderfully prompt. 
Synechia, or atresia, is not an uncommon result of wellplanned and skilfully executed intranasal operations. It should be guarded against in every case by keeping the patient under observation, and passing a probe daily between the walls of the passage; or, what is better, passing a straight Addis's periosteum elevator. The latter instrument is preferable, because it is thin, smooth, flat, and broad, while its distal extremity, being rounded, broad, and bevelled, will not wound the parts or cause the patient much pain by its introduction. When the opposite walls do become united, then an operation for the removal of the bridge should be made.

Sometimes after operation untoward events may take place, such as inflammation and suppuration, more or less extensive, involving neighbouring glands or other parts. Max Thorner mentions instances of lacunar tonsilitis, or pharyngitis, following intranasal operations, also acute purulent otitis, with or without complications, adhesions forming in the nose, epistaxis, etc., as common accidents. He reported several cases of unusual accident in this connection: one of functional aphonia following cauterization of the pharynx for chronic follicular pharyngitis in a young lady eighteen years of age, who was studying elocution; also a case of "temporary amaurosis," following cauterization of the nose, in a man forty years of age; a case of "loss of memory" following an insignificant operation in the nose, in a boy sixteen years of age; a case of intubation in an adult, followed by fatal odema of the larynx; and one case of spasmodic cough and neuralgia after a nasal operation.

The writer has been unfortunate enough to have had three cases of severe faucitis, one of laryngitis, two of sinusitis, and two showing a mild degree of sepsis-"surgical fever"-following operations on the septum and turbinates.

The caution promulgated by Delavan and others against intranasal operations during the prevalence of influenza and other general infectious diseases cannot be too strongly emphasized.

\section{Synechia (Atresia Nasi)}

These bridges of tissue uniting portions of the opposite walls of the nasal passages or vestibule are not uncommon. They may be composed wholly of mucous membrane and car- 
tilage, or mucous membrane and bone (bony synechiæ). They may occur anywhere in the nasal passages. Synechiæ are usually found upon one side of the nose only, but may be on both sides. Some of these formations are of congenital origin, although the majority of them are the result of injury of some sort. Those of congenital origin may be either membranous or bony, although they are usually bony. They may be partial or complete, and situated either at the anterior, middle, or posterior third of the nasal passage; finally, the occlusion may be wholly at the posterior nares. Dr. Payson Clark, of Boston, has published a report of an interesting case of complete bony occlusion of the posterior nares, which was congenital. Some previous disease, such as hypertrophic rhinitis, or the pressure effects of nasal tumours, may lead to ulceration or abrasion, and finally to cicatricial synechia; especially is this liable to occur if the nasal passages are naturally very narrow. Many cases of synechia result from applications of escharotics or the galvano-cautery to the nasal mucous membrane. Inflammation and ulceration of the mucous membrane caused by such systemic diseases as measles, scarlet fever, small-pox, etc., sometimes lead to synechia. Dr. Loeb, of St. Louis, reported a case of double nasal atresia, caused by small-pox, in a young woman aged thirty. The symptoms are sometimes very distressing where there is almost complete occlusion, especially in nursing infants.

Treatment.-Membranous bridges inside the nasal passages should always be excised, if possible. In order to do this effectually, a pair of dressing forceps should be introduced, so that one blade passes above and the other below the bridge, which is thus seized by bringing the blades together. This having been done, the knife, or preferably the saw, is used first upon one side of the forceps and then on the other, thus taking out the middle portion of the bridge. If simply a separation is made, the walls will soon cicatrize together and span the nasal passage again. It is unnecessary to say that the parts must be thoroughly cocainized before operating. The after-treatment will consist of thorough cleansing once or twice a day and the introduction of one or two pieces of sheet gelatine (Hickey's plan) or the placing of a light pledget of absorbent cotton (preferably Bernay's) or gauze between the divided surfaces, in such a way that very jittle pressure will be exerted. The 
formation of synechia after operations may often be prevented by the daily insertion between the surfaces of a probe or a straight Addis dissector. Posterior atresia, if bony, is best relieved by the use of a very narrow chisel, or in favourable cases the drill, followed by the insertion of plugs to keep the surfaces apart. Besides this it will be found very efficient, and perhaps necessary, to apply daily between the separated surface some blunt instrument-such as an Addis dissector, to iron off the granulations.

Atresia of the vestibule of the nose is sometimes met with. It is usually the result of injury, especially of burns or scalds, but may be met with in infants or young children, as a result

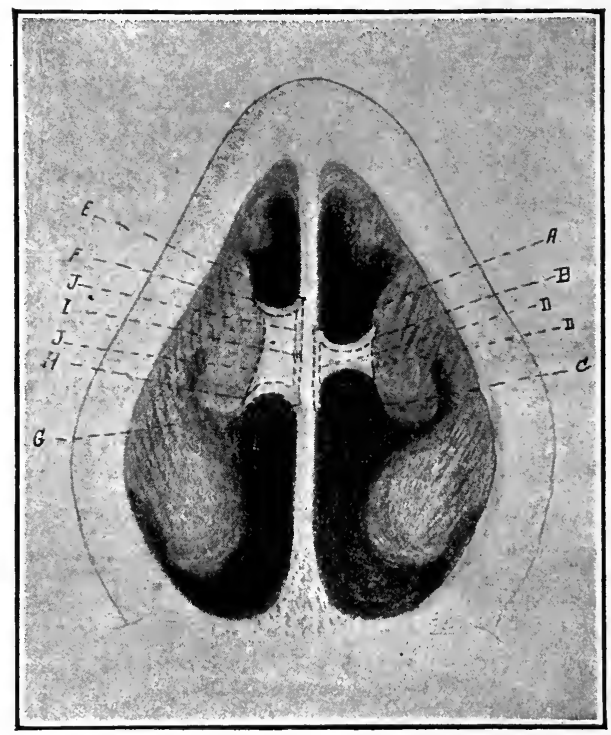

FIG. I69.-Synechia of the nasal passages. (From Roe.)

of injury received during birth or just after birth. It is sometimes the result of severe abrasion or ulceration resulting from some cutaneous disease or syphilis.

The occlusion may be complete or partial. It is usually partial, sometimes leaving but a very small opening for the passage of air.

Operations for the correction of this deformity are usually unsatisfactory, because each successive cicatrization closes the opening more, if that be possible. Enlarging the opening with 
the knife and following this by the insertion of hard-rubber nasal plugs is the preferable method. The treatment is always tedious, and requires the exercise of persistence both on the part of the patient and the surgeon. Oftentimes more or less cicatricial tissue will have to be removed at the time the nostril is enlarged. The writer succeeded in one case in getting a pretty good opening by loosening up the skin a little, both externally and internally, from the line of incision, and then stitching these two edges together. This manœuvre, however, cannot always be accomplished, nor is it always successful when it can be done, as the writer can attest.

\section{Pharynx, Soft Palate, And Uvula}

Pharynx.-The shape and size of the pharyngeal cavity sometimes presents more or less deviation from the normal. The nasopharynx may be very shallow antero-posteriorly on account of the projection of the cervical vertebræ. The oropharynx may likewise be shallow for a similar reason, or one side may be shallow and the opposite side deep, owing to either a lateral displacement of the pharyngeal sac or a deflection of the underlying vertebræ.

The pharynx may be abnormally narrow or abnormally wide. The natural peculiarity of the pharynx in children has already been mentioned in the chapter on Retro-pharyngeal Abscess.

The soft palate may also present an asymmetry of formation which may give rise to difficulty in deglutition or phonation. This may be the result of malformation or paresis. Such a paresis may be permanent and the result of an attack of diphtheria, or be associated with facial paralysis. The writer saw one case in which the left half of the velum palati was evidently undeveloped. The deformed side was neither due to paralysis nor contracture, for it was soft and apparently relaxed, and consisted of a mere membranous band attached like a fringe to the hard palate. The uvula of that side was also notably deficient. The raphe of the velum, however, seemed to be present and gave attachment to the muscles.

The uvula may present great variations in shape and size, the most common of which is a division in the median line, thus really forming two uvulæ, and known as bifid uvula (see 
Fig. I74). The uvula is sometimes seen curled toward one side or the other, and sometimes it is abnormally large or hard (hypertrophy of the uvula). The correction of these abnor-
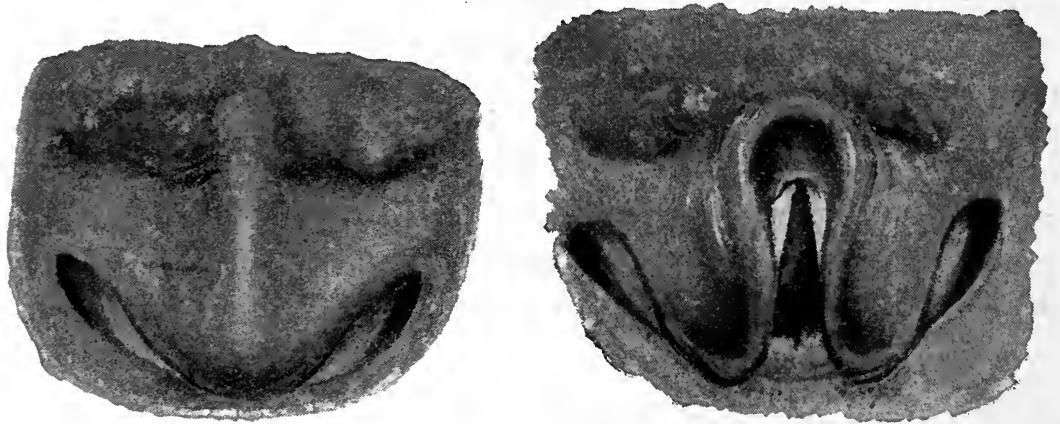

FIGS. I70 and I7I.-Deformity of the epiglottis (Rice).

malities can be easily accomplished with scissors, knife, or wire snare. Hypertrophy and elongation of the uvula calls for its partial abscission.

Larynx.-Deformities of the larynx are not very common, if we except the epiglottis. The epiglottis is found in all forms and styles in apparently otherwise normal larynges. It may be very long or pointed, or flat or bifid, or lobulated. It may appear unusually thin and mobile, or unusually thick and immobile.

The abnormalities of the epiglottis, however, may give rise to troublesome interference with the function of respiration or

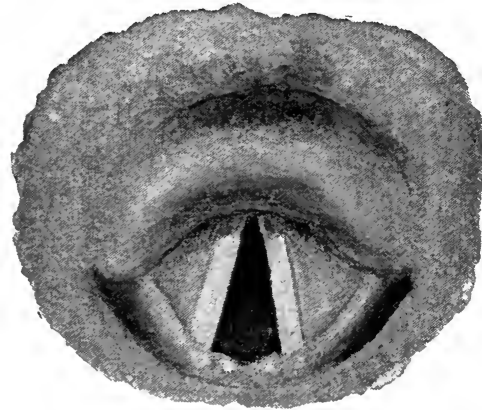

FIG. 172.-Deformity of the epiglottis (Rice). deglutition. Rice has reported cases of persistent disturbance as the result of an abnormally elongated and enlarged epiglottis (see Figs. I70-I 72).

Price Brown, of Toronto, also reported an interesting case of malposition and elongation of the epiglottis in a woman aged twenty-three. In this case the epiglottis took an almost horizontal position. He removed the redundant tip by several applications of the galvano-cautery. He recommends, however, for the treatment of cases not suited for the galvano-cautery, 
that the projection of the epiglottis be abscised by means of a rectangular epiglottome, constructed similarly to a tonsillotome.

Great variations in the shape, length, and position of the epiglottis are often observed. It may be abnormally erect, or supine, or curled, or pointed, or long, without causing the person any particular annoyance under ordinary conditions.

Deformities of the structure making up the body of the larynx are very rare, and usually give rise to more or less stenosis and alterations of phonation. In infancy and early childhood partial synechiæ of the ventricular or vocal bands are sometimes observed (see Figs. I73 and 174). They are usually the result of syphilitic or some other ulceration, although they may occur as congenital defects. The writer met with one such case in an infant a month old, who died,

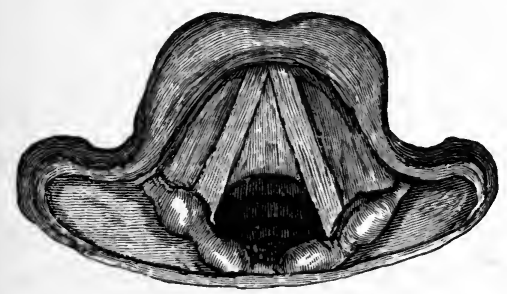

FIG. I73.-Congenital web of larynx (De Blois).

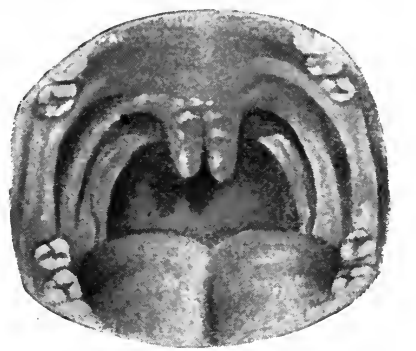

FIG. I74--Bifid uvula.

owing to the refusal of the parents to allow a tracheotomy to be made. The necropsy showed that the anterior portion of the ventricular bands were united, forming a membranous diaphragm in the larynx. 


\section{CHAPTER XVII}

\section{FOREIGN BODIES IN THE UPPER AIR PASSAGES}

\section{Foreign Bodies in the Nasal Passages}

FOREIGN bodies, such as pebbles, small buttons, flies or other insects, fragments of matches, pledgets of cotton or gauze, peas or beans, corn, spears of grass or straw, tablets, pills, etc., are frequently found in the nasal passages; these cases occur mostly in children who, with mischievous design and blissful ignorance of consequences, push such things into their noses. Through circumscribed swelling of the tissues they become more or less permanently lodged. Pledgets of cotton are frequently found in adult noses, where they have been introduced for purposes of medication some time previously. Foreign bodies are sometimes found in the posterior nares, where they have been lodged by acts of coughing, regurgitation, or vomiting. The writer once found, in a case of continual nasal irritation in a man forty years of age, that the irritation was due to the lodgment of a compressed tablet of muriate of ammonia in the posterior part of the middle meatus. The tablet had been thrown there by muscular action from the lower pharynx during a fit of coughing. The patient thought at the time that he had swallowed it.

The effect of the lodgment of a foreign body, as might be supposed, is inflammation, swelling, hypersecretion, and sometimes suppuration in the side of the nose involved. The secretion, which is for a time mucous or sero-mucous, changes to a purulent character. The amount of pain varies in different individuals, and depends upon the character and location of the object ; very often in the case of children there is little or no complaint of pain, and there are many instances of foreign bodies having remained in the nasal passage a long time without giving rise to serious symptoms.

Dr. C. W. Carruthers, of London, reports a case of the re650 
moval of a small stone from the middle meatus of the nose of a woman thirty years of age, which had been there undetected for twenty-three years. The patient had also had polyps removed from time to time. There had been a moderate discharge of muco- and sero-pus, which at times was fetid, and there had been considerable stenosis of the nose, although the voice was not very much affected. There was a very thin incrustation of the stone. The size of the stone was not given. A case was reported at the last meeting of the French Laryngological and Otological Society where a cannula had been inserted in the left nasal passage and forgotten, remaining there forty-two years. It was partially removed, and the symptoms continuing, the remainder was detected by radiography and also removed.

In Dr. Handford's case, a foreign body was in the nasal passage about twenty-seven years, and in Dr. Hebertus's case one was in the nasal passage forty years.

Whenever a persistent muco-purulent discharge from one side of the nose is manifested, especially in children, it may be suspected that a foreign body or a piece of diseased bone is the cause of the discharge, whether pain or sneezing be an accompaniment or not. The foreign body itself may produce caries or necrosis of the neighbouring bones if allowed to remain. Therefore a very thorough examination should be made with the probe if inspection through the speculum reveals no object, for it is often impossible to recognise by inspection alone a small embedded foreign body. The writer has on two or three occasions failed to detect a foreign body in the nose when subsequent events proved that one was there. With children, when a thorough examination is desired, it is usually necessary to administer an anæsthetic before making the attempt at thorough examination.

Rhinoliths.-Rhinoliths are not of very common occurrence, but have been long recognised. Cases of rhinolith were described in the sixteenth century by Mathias von Carboi, Bartilini, Claider, and others, and a collection of such cases was made in 1845 by De Marguay (Scheppegrell).

Etiology.-Rhinoliths usually consist of organic matter and calcareous concretions embedded in the mucous membrane. They generally originate in the anfractuosities between the turbinated bodies, although rarely they may occur in the mu- 
cous membrane of the septum and elsewhere. Dr. Scheppegrell reported a case occurring in a girl thirteen years of age, which was situated upon the floor of the left nasal passage. It was too large to be removed entirely, weighing $24^{\mathrm{T}} / 4$ grains. Their most frequent site, however, is in the middle meatus or the upper part of the nose; they rarely occur anterior to the infundibulum, and are not observed in children. The nidus is often supposed to be a spicule of bone, but the origin may be in a racemose gland, from the retention and inspissation of its secretion, and growing from this by accretion until reaching a good size. It may originate from a blood clot, as in the case reported by Dr. Scheppegrell, of New Orleans. Rhinoliths are supposed sometimes to arise from the retention of medicinal powders, which have been too freely insufflated into the nose. Sajous believes their occurrence is connected with the gouty diathesis. Some observers, however, think that rhinoliths are of microbic origin, while the majority are of the opinion that the nucleus is always some inorganic foreign body or substance. They are usually oblong, porous, and slender, and may present a uniform cast of the part on which they rest. They vary in size, and are sometimes very large, as in the case reported by Dr. Price Brown, of Toronto, which was due to a foreign body, and the cases reported by Dr. M. Mackenzie and Zuckerkandl, all of which were very large.

Symptoms. - Rhinoliths may give rise to more or less irritation and persistent mucous secretion, with or without much sneezing, and signs of nasal stenosis. The nose is not generally very painful to the touch. They may be detected by inspection in a well-illuminated nasal passage, but generally the observer must depend upon exploration with the probe for their detection, because they are often pretty well enveloped by the soft parts.

Parasites in the Nose.-Flies, gnats, and other insects may invade the nose or deposit their ova there. In tropical or southern climates, especially, such cases are most frequently met with. There are certain insects which deposit their ova inside the vestibule of the nose, and their larvæ thence invade the nasal passages, and rapidly multiply. Two able articles on The Texas Screw Worm (Sarcophaga georgina), have been written lately, one by Dr. C. M. Robertson, of Davenport, Iowa, and the other by Dr. J. P. Kimball, setting forth the clinical 
course and the effects of these parasites in the nose. M. Mackenzie described the fly under the name of the Lucilia hominivora.

Symptoms.-Persons suffering from ozena are usually the ones who attract the flesh fly and other insects, and it is while the individual is asleep that the ova are deposited in the nose. From twenty-four to thirty-six hours afterward violent frontal headache, coryza, sneezing, and high fever come on, the temperature going as high as $104^{\circ}$ and $105^{\circ}$. The nasal discharge is a bloody serum, becoming muco-purulent and of offensive odour. The tissues about the eyes, and, indeed, all over the face, become swollen. Sometimes serious epistaxis occurs. From blowing the nose and sneezing from time to time, some of the maggots will be ejected. In some cases two hundred to three hundred have been expelled at a time. The affection may last from a few days to two or three weeks (Kimball and Robertson), according to whether the parasites can be easily destroyed or removed. There is afterward considerable inflammation, and perhaps sloughing of the nasal mucous membrane and even of the external parts of the nose. In consequence of the marked constitutional disturbance, a long period of debility may supervene in those cases which recover. The accessory sinuses are affected, and the worms may even bore their way into the brain from the ethmoidal or frontal sinuses. In fatal cases the meninges have been found inflamed, and the patient has died either from cerebral meningitis or septicæmia. This fly is described by Dr. M. Mackenzie as follows (The Laryngoscope, vol. iv, page I $5 \mathrm{I}$ ):

"The fly is an ovo-viviparous insect, the larvæ, the Sar. cophaga georgina, being hatched within the oviduct. It is I2 millimetres in length, with rather small head and plumose antennal bristles. The face is silver white, with a black spot between the copper-coloured eyes; the thorax light gray, with seven longitudinal black stripes; the satiny silver-gray abdomen checkered with black lines; black feet, and gray, translucent wings. The larva is 18 millimetres long, acephalous, white, cylindrical, tapering to a point at the mouth, and surrounded with a spiral ridge like a screw, whence its popular name of screw worm. The posterior three fourths of the body, up to the point at which it begins to taper, is 3 millimetres in diameter; the mouth is formed by a sort of lip, on 
which are two small protuberances, from the centre of the base of which protrude two black, very sharp corneous mandibles or hooklets, united at their origin in the lip, but separate outside. On the upper side of the body, back of the mouth, and underneath the transparent skin, is a brown patch. To ascertain the time occupied in their development, the larvæ were expressed from a fly upon a piece of tainted meat, and inclosed in a widemouthed bottle and placed in the sun. In twelve hours it was estimated that the mass had increased in bulk forty fold. At the end of twenty-four hours the maggots were half grown, and in forty-eight hours they were fully developed. The sarcophaga, or flesh fly, is found very commonly here around the butcher shops from about the first of March until the last of October. The habit of this fly is to deposit its larvæ on putrid flesh." Dr. Kimball states that he has reliable information of seven cases having occurred around Fort Clear, Texas, within the last ten years. Ozena existed in all the cases, so far as could be ascertained. Undoubtedly attracted by the strong odour, the insect flies into the nostril when the victim is asleep, to drop its larvæ. It seems to be established that this fly deposits its larvæ only on unsound mucous membrane. It may do this in other situations than the nasal fossæ.

The tsetse fly, which is so fatal to cattle in South Africa, is reported to have sometimes produced grave if not fatal inflammation of the nasal mucous membrane, by stinging the inside of the vestibule of the nose and introducing its flagellated infusoria or hæmatozoon (trypanosoma).

Diagnosis.-As before mentioned, the detection of foreign bodies of long standing is not always easy. Therefore, when such a condition is suspected because of persistent nasal stenosis, pain, or muco-purulent discharge, a thorough examination by means of strong illumination, probing should be made; if necessary, a radiograph should be taken. The symptoms and signs of foreign bodies may simulate those arising from caries or necrosis of the bony framework or suppuration of either of the accessory cavities. When the case of foreign body is of long standing, the substance being partially or completely enveloped, it may not at first be felt by the probe, and in such an event, if the history be obscure, great perplexity may arise. Usually, however, the real cause of the stenosis can be made out by thoroughly cocainizing the parts, and slightly incising 
the soft parts covering the apparent swelling. In addition to this, a thorough probing should be made in order to clear up the doubt.

Treatment.-Of course, the only rational treatment is the removal of the offending substance. In cases of recent origin this is usually very easily accomplished by means of blunt hooks, small blunt curettes, forceps, or bent wire. The bent wire, forming a small loop similar to a hairpin, is a very useful instrument for the removal of such things as shoe buttons, peas, beans, kernels of corn, and small objects from the nasal passages. But small nails, tacks, and small elongated pieces of metal will usually require the employment of forceps. With children, more often than otherwise, full anæsthesia will be necessary. It sometimes happens, even in recent cases, that the foreign body is pushed or wedged into the upper meati by the fruitless efforts of the friends to remove the body, and occasionally mischief is done thereby. The writer had a case in a child, where the point of a small screw had been thrust into the antrum by the little patient's parents in an endeavour to remove it from the nose. Much difficulty was experienced in removing it even with the child under chloroform anæsthesia. Sometimes a thorough douching of the nasal passages with a solution of salt and water may be all that is required to remove soft substances. For the removal of rhinoliths, an incision of the soft parts is generally necessary before the forceps can be applied to the concretion. When it is very large a previous crushing will be necessary in order to thoroughly remove it. The writer (who has never met with a very large rhinolith) usually passes a curette over the bed of the calculus after its removal, in order to remove all particles, and then uses a douche in the nose in order to further clear the part of every bit of concretion; for if any particles remain they may constitute the nucleus for another formation. Sufficient anæsthesia can usually be secured by the use of cocaine. For the destruction and removal of parasites, such agents as carbolic acid, formaline, peroxide of hydrogen, alcohol, chloroform, and permanganate of potassium are generally used in conjunction with free douching with warm saline solutions. Dr. Kimball, already quoted as having reported an interesting case of screw worm, found the injection of pure chloroform (as recommended by M. Mackenzie in 1884 ) the most efficacious 
agent for their destruction. It was first used, he states, by Morel, a French army surgeon, in 1862, the remedy having been originally recommended by an apothecary named Dauzats. According to his experiments, a 50-per-cent solution of carbolic acid does not seem to be capable of killing these larvæ, neither immersion in a solution of corrosive sublimate, $\mathrm{I}-500$. Oil of turpentine, after ten minutes' immersion, seemed to kill them. Olive oil and balsam of Peru seemed to produce no effect. Calomel was without effect unless the maggot was buried in it for several minutes. Chloroform, when brought in contact with a half-grown larva, caused almost instantaneous death; the full-grown larvæ were killed by it in from five to seven seconds. The vapour sufficed to stupefy them in a few seconds, but their recovery followed its withdrawal.

\section{Foreign Bodies in the Fauces, Pharynx, Tonsils, AND LARYNX}

The lodgment of foreign bodies in the upper air passages is not of very common occurrence. While the majority of healthy persons are never so afflicted, yet there are a few who are every now and then subjects of such an accident, in whom there is undoubtedly some peculiarity in the anatomical formation of the throat or some physiological peculiarity of the nervo-muscular apparatus to account for it. The majority of laryngologists testify that, considering the bulk of the cases of throat diseases presented for observation, cases of foreign bodies are comparatively infrequent. Dr. Rupp quotes from Professor Juaraz's clinic, of Heidelberg, that of 4,048 patients applying for treatment, I06 (ranging in age from four to sixty-eight years) applied for the removal of foreign bodies from the throat, but in only 4 was a foreign body to be found after careful examination. At the clinics of St. Mary's and Harper Hospital (Detroit) but few cases of this sort have been encountered. The articies which most commonly lodge in the throat are fish bones, chicken bones, and the bones of small game birds, pins, needles, toothpicks, tacks, coins, pieces of thread or string, bristles from tooth brushes, and small pieces of hay or cereals, kernels of corn, and seeds. Any of these articles may lodge in the pharynx, lacunæ of tonsils, behind the faucial arches, in the larynx, trachea, esophagus, or bronchi. 
The nasopharynx, on account of its conformation and situation, is seldom subject to the lodgment of foreign bodies, although occasionally in persons having large or unusually lobulated pharyngeal tonsils a foreign body may lodge or a concretion form there. The writer met with one case where a fragment of toothpick had lodged and remained for several days in the pharyngeal tonsil. There have been a few instances reported of the lodgment of sharp spiculæ of bones, pins, etc., in the pharyngeal tonsil, having been thrown up and caught in the sulci by acts of regurgitation, coughing, sneezing, or vomiting.

Foreign bodies in the pharynx and esophagus may consist of noxious fluid or solid substances. Fish bones, needles, pins, coins, pieces of glass, seeds, small sticks, small toys, wire, and, in fact, any of the small objects handled by man, are liable to become lodged in these passages, and the symptoms attending such accidents will of course vary according to the size, contour, and nature of the object impacted and its location.

In discussing the physiology of deglutition, Dr. Rupp, of New York, asks the question, How come these foreign bodies to be left or fixed in or at the particular places where they are found? The answer to this question involves problems of deglutition as well as secondary questions of pathology, anatomical peculiarities, and personal habits. "Fish bones, needles, etc., so lodged in the throat as to demand surgical assistance in their removal, are, comparatively speaking, very rare circumstances." At the New York Eye and Ear Infirmary during five years (1885 to 1890 ), of 7,840 patients who applied at the throat department for treatment, none of these patients had any foreign body in the pharynx, palate, fauces, or tonsils-that is to say, in the throat. Fifteen of them had foreign bodies in the larynx and in sixteen of them foreign bodies were found in the nasal cavities. Dr. Rupp further says that he is frequently called upon to remove foreign bodies from throats when there are none present. In such cases the pain and discomfort will be found to be due to local trouble of some kind. People addicted to the use of tobacco and alcohol in excess are more often the subjects of these symptoms.

O. Chiari narrates a case of a man having swallowed a piece of wooden toothpick which gave rise to swelling of the left half of the velum. After a great deal of trouble and careful search he gave up the hunt, having been unsuccessful. The next day 
the patient coughed out the splinter spontaneously. Patients have been known to go to as many as half a dozen different doctors before the seat of the foreign body was located. Schaeffer tells of such an instance in his experience, where the foreign body was in one of the folds of the mucous membrane. The following case of an eighteen-months' old child is reported by George White, of Chicago, in which a pin remained in a child's throat after doing serious damage for a considerable time. Several physicians successively had been called in to see the case before Dr. White discovered the location of the pin. Edema of the pharynx had been made the excuse of failure to find and locate it. In this case the pin had been in the child's throat about eight months when Dr. White was called in to see the case. On depressing the child's tongue with his finger, the little one retched, and in looking into the throat at the same time he saw the pin lying across the pharynx just behind the tonsils. Regarding fish bones, more than one may be present at a time. Fish bones are apt to get into the tonsils as well. White relates the case of a dressmaker who often went to bed with dozens of pins in her mouth, as she expressed it, and always awoke in the morning without having got any of them fixed in her throat. Indeed she passed through a long life without any such accident. Carpet layers, upholsterers, and lathers, whose work compels them to keep their mouths full of tacks or nails for hours at a time, are not bothered by these things getting into their throats and becoming fixed there.

Pharynx and Esophagus.-Owing to the shape, musculature, and size of the pharynx, smooth and good-sized foreign bodies are not apt to become lodged there, but passing through are more likely to become lodged at the beginning of the esophagus opposite the cricoid cartilage, or farther down at the lower end of the tube; therefore the foreign bodies which are found in the pharynx are usually small, linear, pointed objects, such as pins, needles, tacks, fragments of bone, small pieces of glass, etc. The pyriform and sublingual sinuses and the crypts of the faucial tonsils frequently give lodgment to foreign bodies. In these situations there are sometimes found fish bones, needles, tacks, seeds, fruit pits, fragments of bone, straws, heads of grain, kernels of corn, and so on. According to the writer's experience of all these situations, the pyriform and sublingual 
sinuses suffer mostly from such invasions. The writer has seen different cases where the pyriform sinus contained a piece of chicken bone, kernels of corn, threads, needles, and fish bones, although, with regard to the latter objects (fish bones), they will more often lodge in the crypts of the tonsils or in the lateral walls of the fauces. Large, soft, smooth, globular, or pyriform objects, as mentioned before, usually descend into the esophagus. While it is a notable fact, as mentioned above, that persons who are accustomed to carry objects in their mouths, such as seamstresses, lathers, and carpet layers, are seldom the subjects of these accidents, still such accidents do occur occasionally. Dr. Rupp (in the New York Medical Journal) quotes Obani's experiments on young sucking animals. Obani forced these animals to swallow 127 needles and pins. The number swallowed by each animal varied from i to 58. Deglutition took place with ease, provided the needles and pins were placed on the tongue longitudinally and the mouth kept shut. One hundred and ten needles passed away from the anus, one needle was found in the rectum above the sphincter, one transfixed the rectum, one was seen fast in the walls of the stomach, and two were found in the appendix or cæcum. Three animals, after having been made to swallow the needles, were given emetics. Two of them vomited the needles. In all three, needles were found transfixed on the walls of the stomach. Obani thinks that the tongue, after preparing the bolus and before sending it to the pharynx, gives it a twist, a gyratory or rotary movement, which movement is continued by the pharyngeal muscles.

Pins, needles, straws, fish bones, and small sticks usually lodge back of the faucial pillars, or sometimes across the pharynx, reaching from one side to the other, or may stick into the tonsils. The writer met with one case where a nail had become impacted with its head back of one tonsil and its end behind the other. The tolerance of these parts to foreign bodies in some instances is remarkable. Roe and Poulet give a number of examples of the retention of foreign bodies in the lower pharynx for days without producing very grave symptoms. However, such cases must be looked upon as exceptional, for, as a rule, inflammation and abscess are pretty sure to follow the retention of a foreign body in this situation for any length of time. Concretions in the tonsils sometimes 
reach large dimensions, and give rise to inflammation and suppuration, if not encysted.

Various sorts of large objects become impacted in the esophagus. There are many instances on record where plates of false teeth, toys, such as tin whistles, coins of all sorts, large pieces of meat, and pieces of bone and gristle, have become arrested in the esophagus.

Larynx.-Considering the well-guarded entrance to the air passages proper, it is not strange that so few cases of the impaction of foreign bodies in the air passages come under observation. The tolerance to foreign bodies exhibited sometimes by the larynx and trachea is remarkable.

According to the writer's experience in the clinics of Harper and St. Mary's Hospitals, very few cases of foreign bodies in the larynx or trachea have applied for relief during twenty years.

Symptoms. - The symptoms of foreign bodies in either of these situations may be classified as immediate and remote.

Fauces and Esophagus. - The immediate symptoms presented are usually the sensation of a foreign body, pricking, or a sharp pain, referred with tolerable accuracy by the patient to the location involved, together with acts of gagging, hawking, futile glutition, and sometimes hacking cough. It has been the experience of the writer that in nine cases out of ten the sensations of the patient as to the locality constitute a reliable guide. Oftentimes, however, examinations have to be made repeatedly, especially in the case of threads, fish bones, or little pieces of straw, before the object is discovered. Pieces of thread, small needles, small fish bones, etc., are sometimes very difficult to observe, especially if the patient be nervous and the pharynx irritable. On several occasions the writer has been about to give up the search, when a little fine, shining line, appearing more like a string of mucus than anything else, has proved to be the offending object (a small fish bone). These little fish bones are very apt to stick into the side of the phar$y n x$, or into one of the tonsillar crypts, or into one of the sulci of the lingual tonsil. In this latter situation it is almost impossible to discover and remove them without the aid of the laryngoscopic mirror. The writer has seldom succeeded in removing from this situation small fish bones, or little pieces of straw or hay or thread, without the aid of the laryngeal mirror. 
Once in a while a foreign body will become lodged in the supratonsillar niche, in which situation, if it be small, it is very apt to escape detection. The symptoms arișing when it is in this situation are usually referred by the patient to the "upper part of the throat," or " back of the nose," or " right back of the palate."

The remote symptoms will also depend upon the seat, size, nature of the foreign body, and the length of time that it has occupied its position. If situated for any time in the pyriform sinus or lingual tonsil, or in the lateral walls of the pharynx, considerable inflammation, and possibly abscess, may develop. Indeed, in some instances its retention will bring about dangerous suppuration, sepsis, or more or less destruction of the neighbouring parts. This is especially true of the lodgment of a hard insoluble body in the pyriform or sublingual sinuses, in which event the cervical glands may become enlarged and suppurate, with the advent of more or less fever and general sepsis. In almost all cases painful glutition is complained of, and in some instances also considerable difficulty in deglutition is experienced. This may in part be due to pain, or in part to the swelling of the neighbouring tissues. The writer met with one case of the impaction of a small piece of pork bone in the right pyriform sinus, which became impacted, through the unwillingness of the patient to submit to its forcible removal, in which suppuration and an abscess in the tissues outside of the pharynx ensued. Owing to the great distress which overtook him and the imminent danger which faced him, the patient finally submitted to an operation for its removal. In another instance, a piece of straw was supposedly lodged in the right sublingual sinus of a woman. After two or three days it either became dislodged, or ulcerated its way through into the submaxillary gland. An abscess finally formed, which was opened by Dr. H. O. Walker, who removed from the bottom of the sac the lost piece of straw. The writer had made repeated examinations of this woman without detecting the foreign body. Another case which came under the writer's observation was that of a woman in whom a piece of thread had lodged in one of the sublingual sinuses, and had created considerable inflammation and swelling about the base of the tongue, with slight œdema of the epiglottis. The object was finally removed under general anærthesia by means of a cu- 
rette. There must have been a small abscess around the foreign body, as there was a discharge of a small amount of pus at the time of its removal. In all such cases, unless there is some swelling or implication of the larynx, the principal symptoms will consist of pain, tumefaction, and signs of suppuration, with possibly the formation of abscess, unless the object becomes encysted. There may be also more or less functional dyspnœa or respiratory distress, which, however, in the majority of such instances, unless the epiglottis be swollen, are nervous phenomena.

The Larynx-Symptomatology. - The symptoms of a foreign body in the larynx may be also classified as immediate and remote, depending upon the nature of the foreign body, and also whether it be fixed or movable.

Immediate Symptoms. - At the ingress of any foreign body, either gaseous, fluid, or solid, a persistent and distressing spasm and cough take place. This may last long enough to amount to positive dyspnœa, interfering with circulation, and causing cyanosis, or, worse still, asphyxia and sudden death. There have been cases in which death has occurred almost at the beginning of the spasm, probably from sudden and absolute asphyxia. As a rule, however, if the object be not too large, the irritability of the larynx ceases after a time, and the respiration goes on, although perhaps more or less irregular and difficult. There is always more or less pain and distress referred to the laryngeal region. The quiescence, however, is only temporary, for at varying intervals successive fits of coughing and dyspnœa will occur, through Nature's continued effort to get rid of the foreign body. When the object is movable, and situated in the trachea, its movements, by displacement, are the cause of the recurring paroxysms of cough, laryngeal spasm, and retching.

The Remote Symptoms.-Objects may sometimes remain in the larynx without producing very serious symptoms, or even damage, although, as a rule, Nature does not tolerate a hard foreign body for any great length of time without setting up considerable inflammation, suppuration, or ulceration. In the case of movable bodies in the trachea rising and falling between the bronchi and larynx, the inflammatory action will be mostly confined to the lining membrane of the tracheal tube. If, however, the object becomes impacted in a bron- 
chus, pulmonary symptoms of a grave character may take place, such as pneumonia, abscess, or gangrene. Traumatic bronchitis, or pneumonitis, with consequent abscess, may result fatally, or the abscess may be discharged, together with the foreign body, either into a bronchus or externally through the chest walls. There are some astonishing instances on record of the tolerance of the larynx to foreign bodies, in some of which the cure was spontaneous. Roe and other authors relate instances of unusual tolerance of this sort. Dr. Langmaid reported a case of the removal of a pin from the larynx two years after having been swallowed (see Fig. I75).

Instances are recorded of

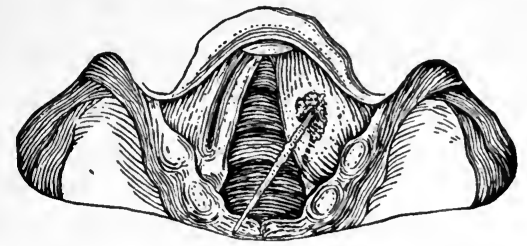

FIG. 175.-A pin which was removed from the larynx two years after having been swallowed (Langmaid). large coins (de Roaldes), a toy locomotive (Johnston), and other such objects becoming lodged and remaining for a time in the larynx without producing death.

An interesting instance occurred in the practice of Dr. S. G. Miner, of Detroit, who met with a case of retained suture in a person who had two years before, in a fit of insanity, attempted to commit suicide by cutting the throat. The wound was stitched at the time, and healed up nicely. Aphonia and dysphonia, however, remained, and gradually grew worse, with the addition of more or less cough and dyspnœa. Laryngoscopic examination showed what appeared to be a glandular growth arising from the left laryngeal ventricle. It was decided to perform thyreotomy, which Dr. Miner did, and removed from the laryngeal ventricle a piece of silk-worm suture about two inches long, which was partly embedded in granulation and connective tissue, and was partly entangled in the laryngeal walls.

Noxious or caustic fluids and gases, such as ammonia, chlorine, phosphorus, etc., or the inhalation of flame, superheated air, steam, or smoke, immediately set up laryngo-tracheitis, bronchitis, or pneumonitis, with or without more or less subsequent destruction of the mucous membrane lining these passages, often resulting fatally.

Diagnosis. - It would seem at first sight that remarks upon the diagnosis were unnecessary, but when we take into con- 
sideration the possibility of a spontaneous removal of the object, and also our inability sometimes to detect it in either the pharynx, esophagus, or trachea, in addition to the possibility of the migration of the object from place to place, through ulceration of the tissues, it will be seen that the diagnosis is not always easily made out. Many patients apply for the removal of foreign bodies when none exist, the case being one of paræsthesia, either temporary or permanent. Paræsthesia of the part may continue in some individuals for a considerable time after the actual removal of the object, while in others (as will be seen by a reference to that subject) paræsthesia may arise when no foreign body has ever been impacted, or at least only temporarily. It sometimes happens, also, that by the ordinary means of manipulation or inspection it is impossible to find or locate a foreign body, and yet the persistence of the symptoms will render it certain that there must be something abnormal in one of these passages.

In children these cases are sometimes mistaken for asthma, croup, or tuberculosis. The writer had a case in a child, four years of age, in whom a piece of chicken bone was extracted by tracheotomy, etc., after having been in the trachea and bronchus for nearly three months. This case had been treated for asthma, and also for tuberculosis. The writer saw another case in a child, of supposed croup or laryngitis, which proved to be due to the lodgment of a small nail in the upper larynx. As there was really no clinical history, the true nature of the case had been overlooked by the writer as well as by the family physician, until the offending nail was ejected during a strenuous coughing spell.

Dr. Tappey, of Detroit, had an interesting case with an ambiguous clinical history in a child two and a half years old, who had swallowed a silver quarter of a dollar. The child was anæsthetized, and a thorough examination made by the careful introduction of esophageal probes, etc., but with negative results, as nothing could be felt with any degree of certainty in the esophagus; but the symptoms continuing-such as difficulty of deglutition, regurgitation of food, cough, etc.-it was decided to try the effect of the $\mathrm{X}$ rays.

The child was taken to Mr. Mansfield, of the Michigan Electrical Company, where a very satisfactory radiograph was obtained, which showed absolutely the location of the object 
in the esophagus. After this Dr. Tappey made an esophagotomy, and extracted the coin. A small fistulous opening remained for a short time, but finally closed up, and the child made a good recovery. It was found that the pressure of the object had caused ulceration, and subsequent hyperplasia of the esophago-tracheal wall, in which the coin had become embedded. If it had remained there much longer it would undoubtedly have produced an esophago-tracheal fistula. Cases of fistulæ between the esophagus and the trachea have been reported occasionally. They are usually serious, and require repeated operations for their closure. The fact of their occurrence in these cases, moreover, emphasizes the necessity for removing a hard foreign body as soon as practicable. In a case which was brought into Harper Hospital a short time ago, and which proved fatal, a bone had been lodged in the middle portion of the esophagus for eighteen days. Esophagotomy was made, the long forceps passed, and the object finally removed. It was covered with a pultaceous mass of pus and mucus. The coughing, expectoration, and difficulty of respiration gave evidence before the operation that an esophago-tracheal fistula existed.

The patient, however, soon afterward died of inspiration pneumonia, and a post-mortem examination revealed an elliptical opening with ulcerating edges between the esophagus and trachea.

Treatment.-The rational treatment for cases of palpable foreign bodies is the removal of the foreign body with as much expedition as possible. This may be done frequently by simple instruments, such as a shoe button-hook, hairpin, long piece of wire bent in the middle like a hairpin, as for removal of foreign bodies from the nose. Such instruments will be found useful in getting small smooth objects out of the pyriform or lingual sinuses. Forceps of different shapes may be used for the removal of these objects when situated in the tonsils or the lateral walls of the pharynx. For the esophagus the esophageal forceps (Fig. I76), pharyngeal probang, and esophageal hook may be used. For this purpose the head is to be thrown back and the instrument carefully introduced, and, if possible, passed by the foreign body. In case of the impaction of meat or cartilage, it has been proposed by some authorities to carry down to the part by a tube a solution of pepsin and hydrochloric acid 
for the purpose of softening it by digestion; but, as a rule, this process will be too slow, and an attempt at mechanical removal should be made at once. These manipulations, it is perhaps unnecessary to add, should not be conducted too persistently without giving the patient rest, for evil in the way of increased local mischief may result from a too long-continued use of

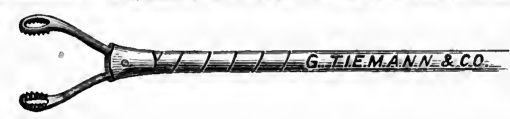

FIG. I76.-Esophageal forceps.

pharyngeal and esophageal instruments; besides, the operator should refrain from the use of too much force, lest the esophagus be ruptured. The practice of pushing the object through the esophagus into the stomach by bougies, etc., is to be deprecated, because of the risk of lacerating or puncturing the esophagus. Unless the object be of a soft character, such as a piece of meat, pudding, bread crust, bean, dried apple, peach, or other vegetable substance, the "shoving of it down " should not be practised. These objects, of course, will usually at first swell by the imbibition of the fluids in the esophagus, but will soon begin to disintegrate. They may be broken up often by carefully and gently pushing them with a probang, passed down into the stomach without danger, but when dealing with meat, cartilage, pieces of bone, or wood, such manipulations become hazardous, as said before, on account of the liability of rupturing the tube.

Larynx and Trachea.-The same rule holds good with regard to the larynx and trachea as with the pharynx and esophagus. Efforts should be made to remove the object at once. When it is impacted in the larynx an effort should be made to remove it by endolaryngeal methods. This may usually be accomplished if the throat be thoroughly cocainized and the right sort of forceps employed. When, however, this plan fails, or if the object has become dislodged from the larynx and has fallen into the trachea, then it is advisable to perform a tracheotomy at once (see Chapter XVIII). If the foreign body be so lodged in the larynx that it cannot be reached by the ordinary opening of a high tracheotomy, then the cricoid ring 
should be split, or, if need be, the thyroid cartilages should be separated (thyreotomy). In one instance in the writer's experience, a child five years of age had a small safety pin impacted in the right laryngeal ventricle. It was found necessary to extend the tracheotomy into a thyreotomy before the object could be reached and removed. Sometimes the foreign body may be removed by turning the patient upside down, directing the subject (if old enough) at the same time to expire, and not inspire. This will sometimes succeed in aiding the escape of any smooth or globular body from the larynx or trachea, especially if it be movable. The escape of coins, kernels of corn, seed, etc., has often been accomplished in this way. These manœuvres, together with the administration of emetics, are sometimes efficient for dislodging foreign bodies from the larynx and trachea; after reasonable trial, however, such expedients ought not to be persisted in to the neglect of operative procedures. Spontaneous removal of foreign bodies from either of these situations may take place. But too much time should not be wasted in this Micawber-like policy, lest the serious consequences already mentioned supervene. The foreign body may ulcerate into the neighbouring tissues, vessels, or organs, and leave behind diseased conditions from which the patient may never recover, or it may set up disturbances which will prove fatal in a short time.

A remarkable case of almost spontaneous removal of a foreign body is reported by Dr. William J. Gillette (New York Medical Journal, March 25, I 899) in the case of a physician who accidentally swallowed a probang made of brass wire (No. 2), six inches in length, the end of which was wrapped with cotton. It soon passed out of reach into the stomach. The patient suffered no inconvenience from the accident for nearly three months, when he began to have distress in the epigastrium, and in the January following he experienced a good deal of sharp pain in the region of the cardiac end of the stomach. At this time it was found that the end of the probang was protruding under the skin in the ninth intercostal space, near the costal cartilages. He was admitted to the hospital, an incision was made through the skin, and the probang extracted with very little trouble, after which he made a very speedy recovery.

Dr. J. A. Wassinger, of Ann Arbor, Mich., reports a case of 
a boy, fourteen years of age, who, while tacking up a lambrequin, drew one of several brass-headed tacks which he was holding in the mouth into his trachea and into the left bronchi. This remained in the bronchi about eighteen months, until it was coughed up with considerable purulent expectoration.

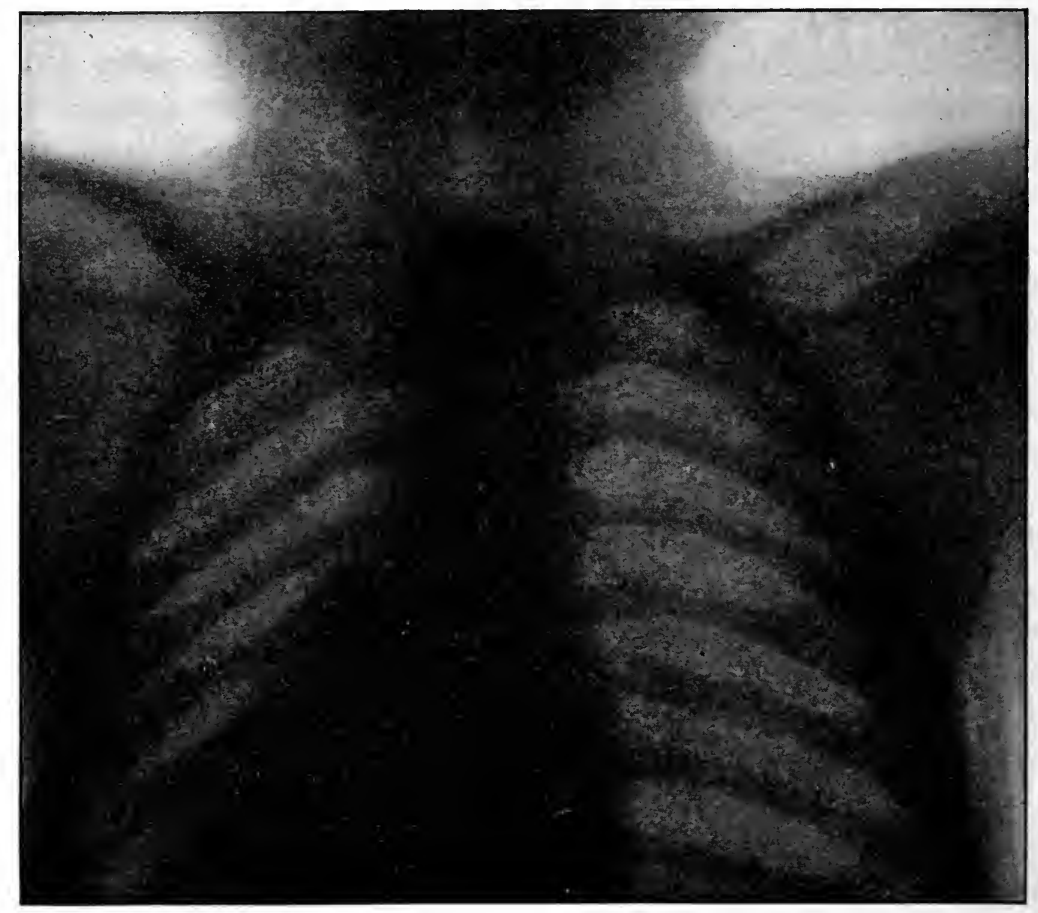

FIG. I $76 a$.-Radiograph of thorax of five-year-old child showing coin impacted in the esophagus. 


\section{CHAPTER XVIII}

PHARYNGOTOMY, ESOPHAGOTOMY, INTUBATION, TRACHEOTOMY, THYREOTOMY, LARYNGECTOMY-METHODS OF LOCAL TREATMENT OF THE UPPER AIR PASSAGES

Preliminary Measures.-For each of these operations, with the exception of intubation, a good light, either natural or artificial, is a prerequisite. Sunlight, of course, is preferable, but in its absence good illumination can be secured by either electricity, gas, or kerosene oil. Semon and other operators prefer a small two- to four-candle-power electric light attached to a headband worn by the operator, but according to the writer's experience this is not as handy as the reflected illumination obtained by means of the concave head reflector, such as is ordinarily employed for laryngoscopy. These, together with other necessary instruments, are generally found in every wellregulated hospital, where all such operations should be done when possible.

Position of the Patient.-Ordinarily, the patient should lie on his back on the operating table, with the shoulders raised by a narrow, hard pillow, so that the head may fall back of it, thus stretching and raising the neck. Some operators advocate the adoption of the Rose position, which consists of allowing the head to fall below the edge of the table, because such a position will more effectually prevent the entrance of blood into the trachea and bronchial tubes during the operation.

Others prefer the Trendelenburg position for similar reasons. While undoubtedly there are cases in which these positions (the Rose and the Trendelenburg) may seem preferable, yet in the majority of cases the commonly adopted one mentioned above will be found most agreeable to the operator, and not more dangerous to the patient. The Rose and the Trendelenburg positions are sometimes objectionable, because serving to distend the veins of the neck and head with blood by the force of gravitation. 
Aseptic Precautions.-Although it is impossible, as a rule, to perform any of these operations in a strictly aseptic manner, yet as far as possible the rules of aseptic surgery should be carefully observed; hence the hands of the operator. and his assistants should be clean, the instruments and appliances sterilized, and the region of the prospective incisions thoroughly cleansed and treated with some antiseptic lotion, such as mercuric bichloride, alcohol, carbolic acid, etc., the same as if an abdominal section were to be made.

Ancesthesia.-Excepting in cases of emergency and great urgency, or when there is a forbidding heart lesion, the patient should be thoroughly anæsthetized before any such operation is undertaken. Chloroform and ether are the anæsthetics chiefly in use for producing profound anæsthesia, and the person to whom they are administered should always be lying down. There have been, however, many other substances brought forward from time to time as substitutes, among which may be mentioned ethyl-bromide, ethyl-chloride, the A.-C.-E. mixture, nitrous oxide alone or in combination with oxygen or ether or chloroform, the Schleich mixture, hypodermic injections of cocaine hydrochlorate, eucaine, holocaine, chloretone, etc. Ethyl-chloride and beta-eucaine are of limited usefulness only. The writer's experience has been confined mostly to chloroform, ether, and nitrous oxide for producing general anæsthesia, because satisfactory results have usually been obtained from the use of these agents. Nitrous oxide and chloroform are to be preferred for short operations, while chloroform followed after a short period of time by ether is to be preferred for longer operations. Chloroform, however, is safe enough when prolonged anæsthesia is required, if it be given carefully, slowly, and mixed with air. The inhalers in common use answer the purpose very well, but sometimes an advantage is gained by the use of an inhaler which will limit the amount of the drug used, and at the same time mix and regulate the quantity of air consumed. One of the best of these instruments is the Gailey inhaler, which was very satisfactorily used in the writer's case of laryngectomy, the details of which were published in the New York Medical Jour., July 16, I898.

Hemorrhage and Other Accidents.-Preparations must be made for dealing with hemorrhage by having on hand a number of catch forceps, ligatures, and the actual cautery. Besides, the 
operator's assistants should be provided with a few pairs of long slender forceps with which to hold small pledgets of gauze, cotton, or pieces of sponge for mopping out the trachea or larynx, or bottom of the wound, if necessary; also a softrubber tube, with a syringe, to be used when swabbing fails to remove the blood or fluids.

It is a good plan always to have a hypodermic syringe loaded with nitro-glycerine or strychnine on hand, in case signs of shock or syncope appear; also oxygen gas in a suitable apparatus for prompt administration in case any untoward signs of chloroform narcosis or apnœa occur. Ice water, hot water, and one or two chemical styptics should be near at hand, in case of being needed to control local capillary hemorrhage or oozing, where ligature or torsion are impracticable.

\section{PhaRyNGOTOMY}

There are two forms of pharyngotomy usually described, distinguished from each other by the situation of the external incision. They are denominated lateral and subhyoid pharyngotomy respectively. The former operation (lateral) is the one usually selected for the removal of foreign bodies which become impacted in the lower pharynx or pyriform sinuses. The latter (subhyoid) operation is selected usually for the removal of growths or portions of the epiglottis.

Lateral Pharyngotomy.-With the head bent to the opposite side, the external incision should be commenced at a point just behind the angle of the inferior maxilla, and continued down parallel with the anterior border of the sterno-cleidomastoid muscle and about one inch away from it, for at least two inches. The further dissection should be carefully carried on by means of a director, care being taken not to wound the parotid gland or sever the digastric or omohyoid muscles, both of which may easily be held aside. The sheath containing the carotid artery and the pneumogastric nerve must also be avoided. After section of the skin and fascia, the dissection should be made principally with the handle of the scalpel or with an Addis dissector until the pharynx is reached. The pharyngeal sac should be incised first with the point of the knife and then the incision enlarged, as may be necessary, with a blunt-pointed scissors or knife carried along a director. It 
will be found necessary usually to tie a few vessels, but the bleeding from the wounded muscular tissue may, as a rule, be checked by the application of hot water or ice water.

Subhyoid Pharyngotomy.-For subhyoid pharyngotomy the external incision should be made in the space between the lower border of the hyoid bone and the upper border of the thyroid cartilage upon either side, according to the object of the operation. For removal of a large portion or the whole of the epiglottis, or a neoplasm, it will sometimes be found more convenient to carry one extremity of the incision farther along in the median line of the neck. The dissection should be carefully made (layer by layer being incised) as mentioned above, care being taken not to wound the thyroid gland. The pharynx having been opened, the growth, the epiglottis, or such portion as is destined for removal, can now be taken hold of with a pair of forceps passed through the external wound and carefully severed from its attachments by means of serrated bladed scissors or a probe-pointed knife. There will be much less hemorrhage,.as a rule, if the serrated scissors are used. A good light is always necessary for this manipulation. Oftentimes it is best to make a tracheotomy first; especially is this advisable before operations on the epiglottis, on account of the liability of the supervention of swelling, or œdema glottidis, which might compromise restoration.

The after-treatment will vary according to the nature of the case. When the operation is made for the removal of foreign bodies, unless there has been considerable suppuration or ulceration the wound may be at once completely closed by sutures and aseptic dressing; but if the operation has been made for the removal of neoplasms or diseased tissue, it may be desirable to leave the lower portion of the wound open, or insert therein a tube for the purpose of securing proper drainage. In either case, before closure the wound should be cleansed with either sterilized water, boric-acid solution, or a solution of mercuric chloride, and then thoroughly dusted with Semon's mixture of iodoform and boric acid, equal parts (care being taken not to use too much powder, lest iodoform poisoning ensue). In severe cases the feeding of the patient should be accomplished by the rectum for two or three days, in order to keep the parts quiet, and the wound free from contact with extraneous material. When eating is resumed, the 
food should be of a liquid or semisolid character, until the wound is pretty well healed and the act of deglutition ceases to be very painful.

\section{ESOPHAGOTOMY}

The position of the patient on the table should be the same as for pharyngotomy, except that the head is to be turned so that the left side of the neck lies uppermost, because the esophagus lies nearer the left side and is therefore easier of access.

The external incision should be about three inches in length, beginning at the lower border of the cricoid cartilage and extending downward along the anterior border of the sterno-cleido mastoid muscle. After the skin and superficial fascia have been divided, the underlying cellular tissue is to be divided upon a director until the digastric and omohyoid muscles are brought in view.

The muscles should be held aside by means of blunt hooks, instead of being cut. A bougie is now introduced into the esophagus through the mouth and pharynx, to serve as a guide for the further dissection of the tissues and as an index of the exact position of the esophagus. Care should be taken not to divide any of the important vessels or nerves of this region. Having reached the esophagus, an incision is made through its wall down to the bougie within, after which the bougie may be withdrawn and the forefinger of the left hand inserted through the incision into the tube. The finger will now serve as a guide for the use of forceps or other instruments which it may be necessary to introduce for the removal of a foreign body or for the performance of any other necessary manipulation. In removing a foreign body, especially if it be of rough configuration, it is far better to enlarge the external opening sufficiently so as to avoid tearing the edges of the wound by forcibly pulling the body through it.

After-treatment.-After cleansing the wound the edges of the incision in the esophagus may be brought together by a number of small eatgut sutures. Care should be taken not to include too much of the tube in the suture, so that it may not become narrowed at this point any more than is necessary.

The external parts may be next sutured also with catgut, and properly dressed with iodoform or bichloride of mercury gauze. The dressing should be removed at the end of twenty- 
four hours, the wound inspected, and redressed after having been dusted over with powdered iodoform or a mixture of iodoform and boric acid.

Owing to the impossibility of absolute quiescence of the parts in this region and the difficulty of fixing the dressings, the wound cannot, as a rule, be left without frequent dressing. No food or drink should be given by the mouth for at least three days after the operation, nutrition being supplied during this time by rectal injections. In the meantime the patient should be instructed to avoid swallowing as much as possible. At the end of three days or thereabouts sterilized water may be given, and if there be no trickling of the water through the wound, then boiled or sterilized milk may be administered, at first in small quantities. If this does not come out of the wound, it may then be given more freely. Should there be any evidence of infiltration of fluid into the tissues of the neck, the external wound should be immediately reopened, a drainage tube inserted, and rectal feeding resumed.

It sometimes happens, when a foreign body has been impacted for a considerable time in the esophagus in such a position as to make strong pressure at some one or two points, that ulceration and perforation will take place, establishing a fistula into the trachea or into the surrounding cellular tissue. The former event may be suspected if persistent cough follows the act of deglutition, and the latter if sharp cervical or mediastinal pain, with or without obvious swelling, follows acts of deglutition. Although there may be a fistulous opening from the esophagus into the trachea, the esophagotomy wound may be entirely closed, unless the fistula be a large one. But when the opening is into the deep cellular tissue of the neck, the external wound should not be entirely closed, unless the surgeon can reach and clear out the extraneous material at once. If this cannot be satisfactorily done, the external wound should be provided with a drainage tube.

In all such instances, after the wound is healed, an esophageal bougie should be passed from time to time for a period of from four to six months, in order to prevent as much as possible the narrowing of the esophagus by the contraction of the cicatricial tissue. 


\section{TRACHEOTOMY}

Opening the trachea is one of the oldest procedures of surgical art. Much ingenuity has been displayed in devising various instruments, called tracheotomes, for opening the trachea and inserting a cannula simultaneously. These instruments, however, have never found favour for any length of time, owing to the danger and the impracticability attending their use.

Operation.-The trachea may be opened either above or below the isthmus of the thyroid gland-the former operation being called the high and the latter the low operation. The position and preparation of the patient for operation has already been described. The external incision for the high operation should be made in the median line of the neck, and should be about two or three inches in length, extending downward from the cricoid cartilage of the larynx. For the low operation, the external incision should commence somewhat lower and extend to the suprasternal notch. The skin and superficial fascia may be cut through by a few sweeps of the bistoury, after which the dissection should proceed more carefully with the aid of a director. The veins, both superficial and deep, will usually be found distended, especially in cases which have been characterized by much dyspnœea. It is therefore advantageous to avoid cutting them in order to prevent annoying hemorrhage. The different layers of fascia and cellular tissue covering the parts are to be taken up on the director and severed by either the knife or scissors, while the large turgid veins are to be held aside. When the thyroid muscles are reached they can be separated with the handle of the scalpel so as to bring into view the deeper fascia covering the trachea. Take up each layer of the same by a pair of forceps, cut a button-hole slit, and insert therein the director upon which the tissue is to be divided by the knife. Before finally opening the trachea, the operator should see that every layer of tissue covering the tube has been severed and that all hemorrhage is quite or nearly stopped. It may be necessary to tie large veins and small arteries before opening the trachea, so as to prevent the blood from flowing or trickling into it from the bottom of the wound. Attention to this point is important, because the presence of much blood in the trachea may become 
a dangerous feature. Sometimes the hemorrhage arises within the trachea, as in subglottic hypertrophy or angioma of the mucous membrane. The writer has had an alarming experience in two such cases, in one of which (an angioma) the patient died from the effects of the intratracheal hemorrhage in about four hours after the operation. The other case would also have proved fatal but for the persistent vigilance of Dr. Hickey, and Mrs. L. E. Gretter, principal of the Harper Hospital training school. The hemorrhage having been checked and the objective part of the trachea fully exposed, the point of the bistoury is now entered at the desired place at the lower end of the wound (with the cutting edge of the knife directed upward) and the anterior wall divided in the median line the desired length, care being taken that the point of the blade does not go far enough through to injure the posterior wall. The perforation having been made, the incision may be more

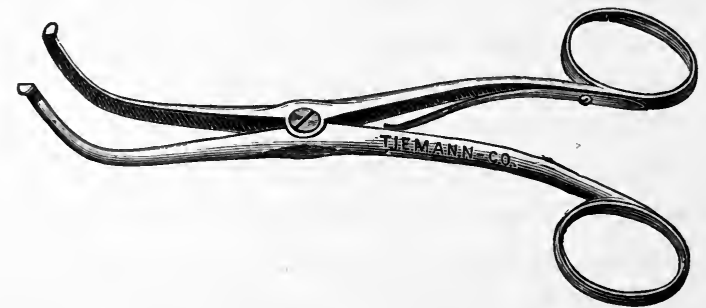

FIG. I77.-Trousseau's dilator, to facilitate the introduction of cannula.

safely completed by the substitution of scissors or a probepointed bistoury for a sharp-pointed bistoury. Usually only two or three rings of the trachea need to be severed. The knife is then withdrawn and the retractors or dilator (see Figs. 177 and 178 ) inserted with which to separate the edges of the incision in order to introduce the cannula or tracheotomy tube into the trachea. Finally, the upper and lower edges of the

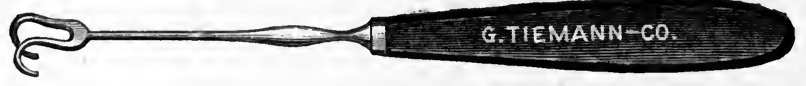

FIG. I78.-Pilcher's retractor.

incision in the soft parts may be brought together by sutures, if advisable, and the wound treated by an insufflation of iodoform. The trachea tube (see Fig. I79) should have a double cannula, so that the inner one can be removed and cleaned fre- 
quently. It should be prepared by being first thoroughly cleansed in very hot water, and a piece of narrow tape should be sewed to each slot at the sides of the flange, one piece being shorter than the other, so that when encircling the neck the two may be tied at the side instead of the back. A small piece of gauze or lint, about two inches square, with a piece of oiled silk, both perforated so as to allow the passage through them of the trachea tube, should be placed between the flange of the tube and the skin of the neck, the oiled silk being outermost, for the purpose of protecting the wound from the discharges coming through the tube.

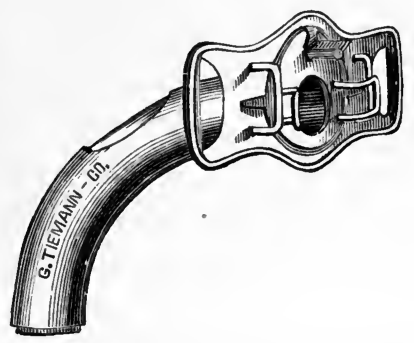

FIG. I79.-Double silver trachea tube, with movable plate. The surface of the gauze or lint in apposition to the wound should be smeared with vaseline, either plain or carbolized. The cannula having been inserted and secured by the tying of the tapes around the neck, a couple of layers of cheesecloth or gauze should be lightly laid over the opening in order to filter the inspired air. The air in the neighbourhood must be kept moist either by means of an atomizer playing upon the parts or by the evolution of steam from some sort of a vessel. Unless this is done a great deal of difficulty will arise from the desiccation of the secretions about the mouth of the tracheal cannula. In all cases the cannula should not be inserted until the trachea has been freed of blood, mucus, or, in cases of diphtheria, of pseudo-membrane. This can be done generally by exciting cough while the edges of incision are held well open, but sometimes it may be necessary to employ a syringe to suck the material out through a catheter or rubber drainage tube. It is not advisable to use the mouth for this purpose in cases of diphtheria or syphilis, on account of the liability of becoming inoculated by the noxious material; several instances of dangerous and even fatal diseases to medical men and nurses have arisen from this practice. When dangerous or continued hemorrhage takes place from the inside of the trachea, the cannula should be removed and the opening in the trachea maintained by retractors until the bleeding has been checked; at the same time the cavity of the trachea must be kept as clear as possible by drawing or sucking the blood 
out frequently with a syringe, preferably a soft-rubber one. If the thyroid gland or its isthmus has been wounded, the bleeding points should be secured by ligature, if possible, or, if ligation be impossible, by the application of the actual cautery to the bleeding points. The operator must be careful to surely introduce the tube into the trachea before leaving the patient. It will sometimes happen that the distal end of the tube lies under the cellular tissue of the anterior wall instead of inside of the trachea. It happens sometimes, on account of the nature of the disease or the conformation of the parts, that the trachea tubes in common use cannot be introduced, or, if introduced, will lie so as to meet the posterior wall of the trachea, causing it to ulcerate or become inflamed, or perhaps so lie that the distal opening is obstructed by the posterior wall itself, or by granulations springing out from the mucous membrane of the posterior wall. Occasionally a cannula of proper diameter will be found to be too short to fairly reach the inside of the trachea. To meet these conditions, cannulæ of special length and shape will have to be made, although there are usually in the market jointed and rectangular cannula. Temporarily in such cases good thick-walled rubber tubing may be made to do service, being held in place by safety pins in front, to which tapes can be tied.

After-treatment.-The after-treatment of tracheotomy, especially in cases of diphtheria, and when trachea tubes must be worn a long time, is very important. Keeping the surrounding air moist is one of the most important points, and next is the attention to the inner tube, in order to keep it clean and free from desiccated gummy secretion. The wound should also be kept clean and as aseptic as possible, by dressings of iodoform, carbolic acid, etc.

The whole trachea tube (i. e., outer as well as inner) should be removed, if possible, by the third or fourth day after operation, in order to cleanse the parts, and as often thereafter as seems necessary, according to the amount of suppuration, etc., going on.

When cannulæ have to be worn a long time, trouble is sometimes experienced from softening of the anterior wall of the trachea, so that when the outer cannula is removed the trachea collapses. This condition may have to be met by stitching the edges of the opening to the external skin. 
Growing granulations are sometimes the cause of stenosis, and if such prove to be a persistent difficulty it may become necessary to reopen the trachea, including division of the cricoid cartilage, so as to remove the adventitious tissue with a curette.

The number of days or weeks that the cannula should be left in will vary, of course, entirely according to the case. There are many instances in which a trachea tube has been worn for years without producing notable difficulty. Indeed, the writer knows of one woman who has worn a trachea tube without much inconvenience for the last fifteen years. The cannula, if made of metal, is apt to become corroded after three weeks, if worn continuously.

\section{INTUBAंTION OF THE LARYNX}

Intubation is the name given to the operation of introducing a tube through the mouth into the larynx and upper trachea for the maintenance of respiration.

History.-Hippocrates practised intubation at the beginning of the Christian era by means of sounds which he passed through the glottis. Bouchut, in 1858 , brought before the Academy of Medicine at Paris two observations upon a new method of treatment of croup by tubage of the larynx. His tubes were made of silver, almost cylindrical ( 5 millimetres to 2 centimetres in length). They were carried into the glottis by means of a sound, analogous to the laryngeal tube of De Paul; the tube was retained outside by aid of a thread of silk, by means of which it was extracted. It was about 2 centimetres in length, and 6 to I m millimetres in diameter. These tubes had two projections at the superior end, after the fashion of movable buttons, intended to maintain them in the glottis upon the vocal cords. After this, Bennati, Garin, Bergman, Brown-Séquard, Fournie, Collin, and Chiari practised "tubage" or "catherization" of the larynx for croup and similar conditions. Frank E. Waxham, in this country. in I 885, practised "tubage" in a child sixteen months old, which was reported in the Archives of Pediatrics, I885, vol. ii., p. 657 .

In 1858 Listen dilated constrictions of the larynx, after performing preliminary tracheotomy, and about the same time 
Bouchut and Horace Green began to operate in a similar way, believing that all forms of stenosis were amenable to treatment by dilatation, by means of tubes passed through and held between the stenosed parts. Their theories and practices met with much support by surgeons of that day. Trousseau thought of practising it, but somehow let it fall into disfavour. The discovery of the laryngoscope was largely responsible for the revival of Bouchut's and other forms of operations. The first disciples of Bouchut and Green were Marduel in 1863 , and Delore, who took up tubage for stenosis laryngis a year

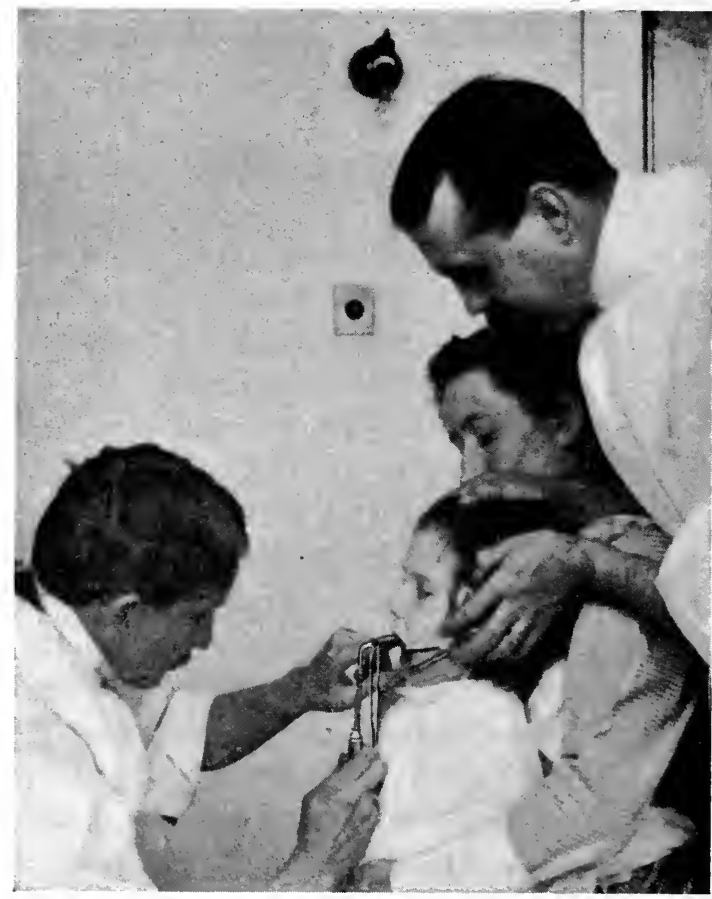

FIG. 180.-Position of child, operator, and assistants, for intubation.

later, and gradually such famous laryngologists as Stoerck, Mackenzie, Türck, Schrötter, Wanlechmer, Herring, and in our own country O'Dwyer and Waxham began the study of the pathology of stenosis of the larynx and its treatment. The great honour and credit of perfecting this treatment for stenosis of the larynx belongs to O'Dwyer, who made intubation a feasible, safe, and successful treatment for diphtheria, croup, and other forms of laryngeal stenosis. 
Indications. - The indications for intubation depend, of course, upon the conditions producing stenosis of the larynx and the amount of such stenosis. This method of relief has

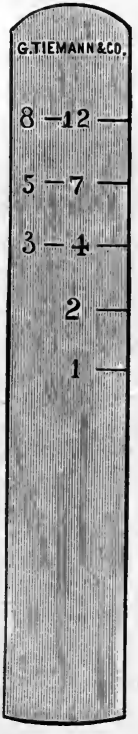

FIG. I8I.

Scale of lengths for O'Dwyer's intubation tubes. steadily grown in usefulness, until in a large majority of cases it has become, very properly, a substitute for tracheotomy and thyreotomy. It may be said, in general, that any condition of laryngeal stenosis calls for intubation, yet its greatest efficiency will be found

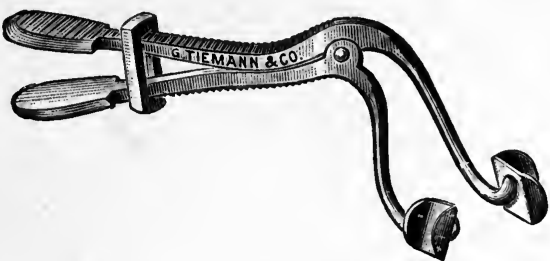

FIG. 182.-O'Dwyer's mouth gag

in cases of diphtheritic laryngitis. As already mentioned, it can be adopted for the relief of dyspnœa and endo-laryngeal tumours, and sometimes in extra-laryngeal tumours, which compromise the lumen of the tube. It is also sometimes highly useful as a measure of relief in cases of stenosis, produced by cicatricial tissue in the larynx, following ulceration from syphilitic or lupus disease, burns, or other-injuries. Intubation, however, is contra-indicated in cases of subglottic hypertrophy, sub-glottic tumours, phthisis pulmonalis, or carcinomatous diseases, where active ulceration may be

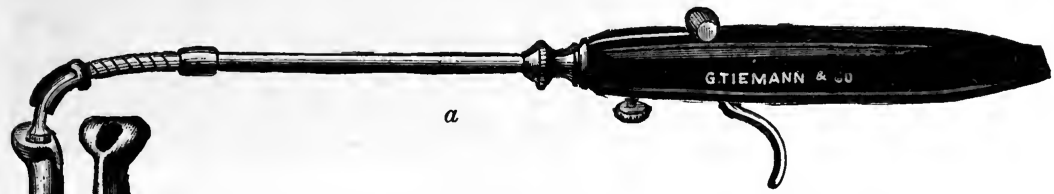

FrG. I83.-O'Dwyer's intubation tube and introducer.

$b$

going on, or where there is much coughing and expectoration. Even in such cases, though, intubation may be of temporary benefit, until such time as tracheotomy or other operative procedure can be adopted. A great deal of the success of this procedure will 
depend upon a selection of the proper sized tube. The instruments most commonly used are those perfected by Dr. O’Dwyer (see Fig. I83).

Operation.-The child is placed in a sitting posture in the lap of an assistant, the arms having been pinioned to the side of the body by means of a sheet thoroughly wrapped around

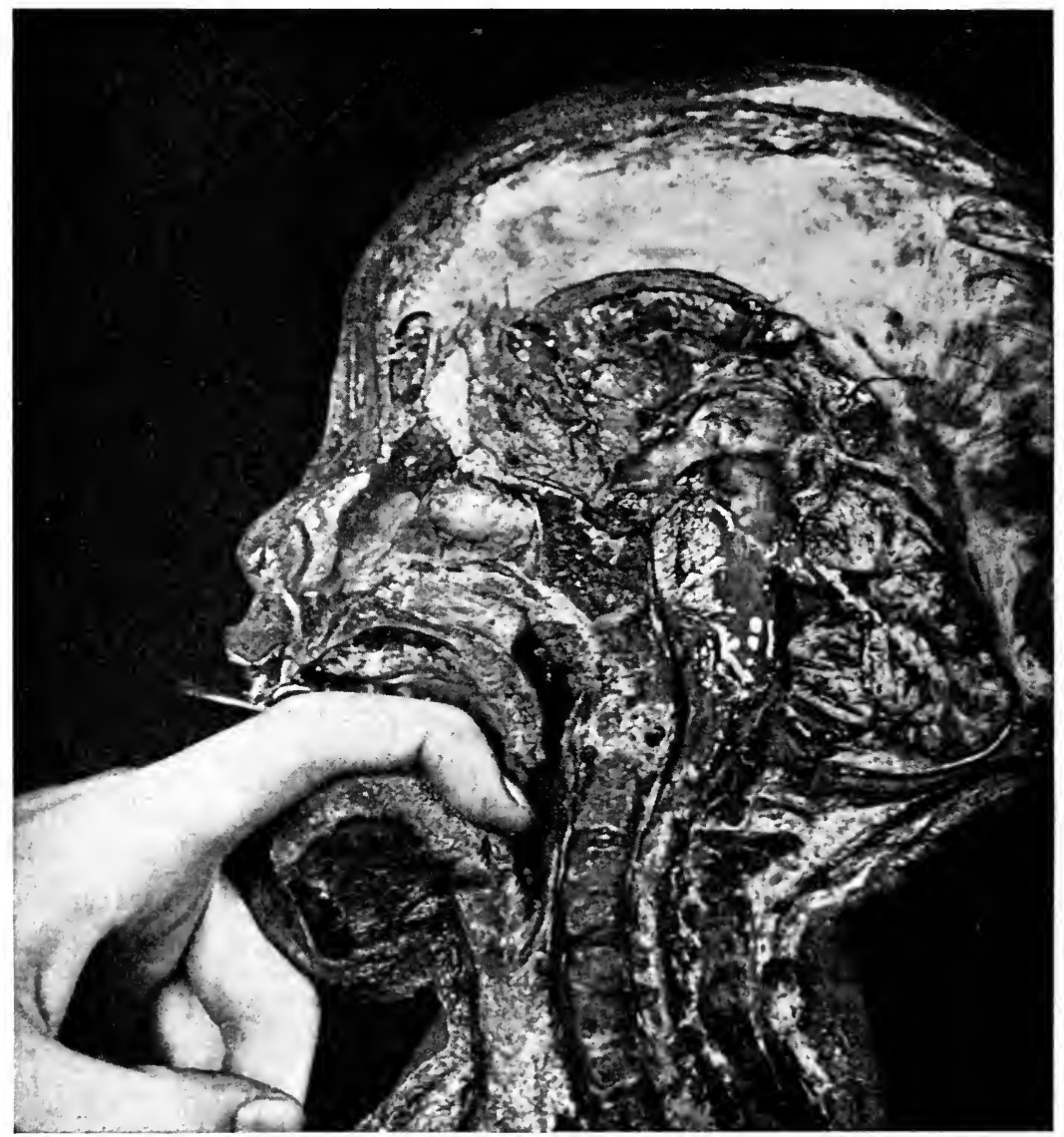

FIG. I84.- Insertion of finger to locate epiglottis and arytenoids.

the thorax (see Fig. I80). The proper sized tube, according to the age of the child, is selected by means of the gauge (see Fig. I 8 I). A thread about two feet long is passed through the eyelet of the tube, and the two ends tied so as to form a loop, and the tube then attached to the introductor (see Fig. I83). Everything being in readiness, the operator sits in front of the 
patient, and first introduces the mouth gag (see Fig. I82) between the jaws, either on the right or on the left side, according to circumstances. The mouth now being held open by the gag, the extended forefinger of the left hand, palmar side down, is carried through the mouth to the base of the tongue, until the epiglottis is reached, or the tips of the arytenoids are felt;

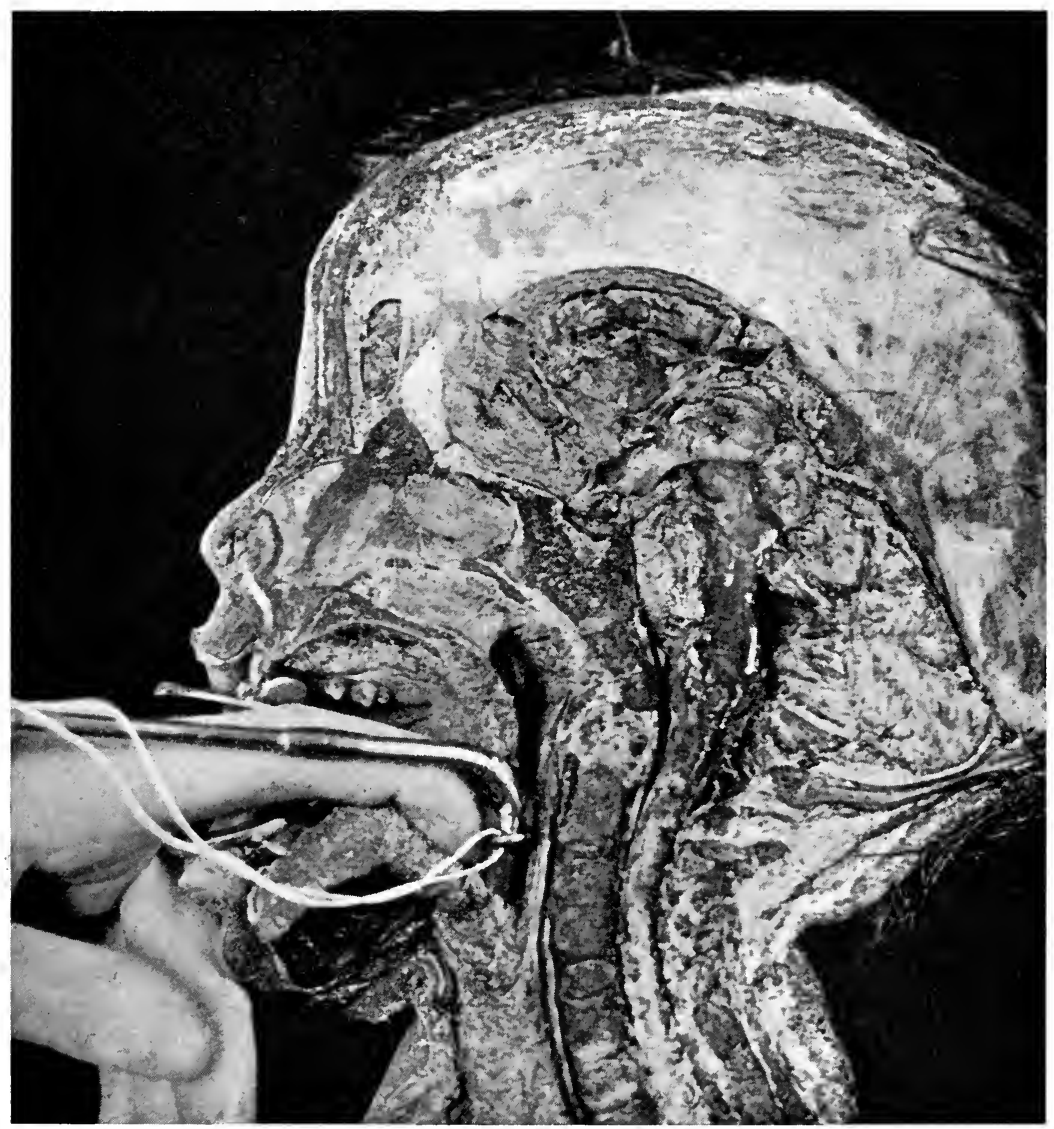

FIG. I85.-Tube entering larynx.

the epiglottis is now pulled gently forward, while the introducer, with the tube attached, following closely the finger as a guide, is quickly introduced, the handle being elevated. The tube having reached the larynx, the applicator is quickly detached and withdrawn, leaving the tube in the larynx, with the loop of thread, which has been attached, hanging out of the 
mouth. More or less gagging and coughing attends and follows the introduction of the tube for a minute or two, but this soon subsides if the tube has reached the proper place-a thing which can soon be determined by the peculiar brassy nature of the cough and the relief of the dyspnœa. The thread at-

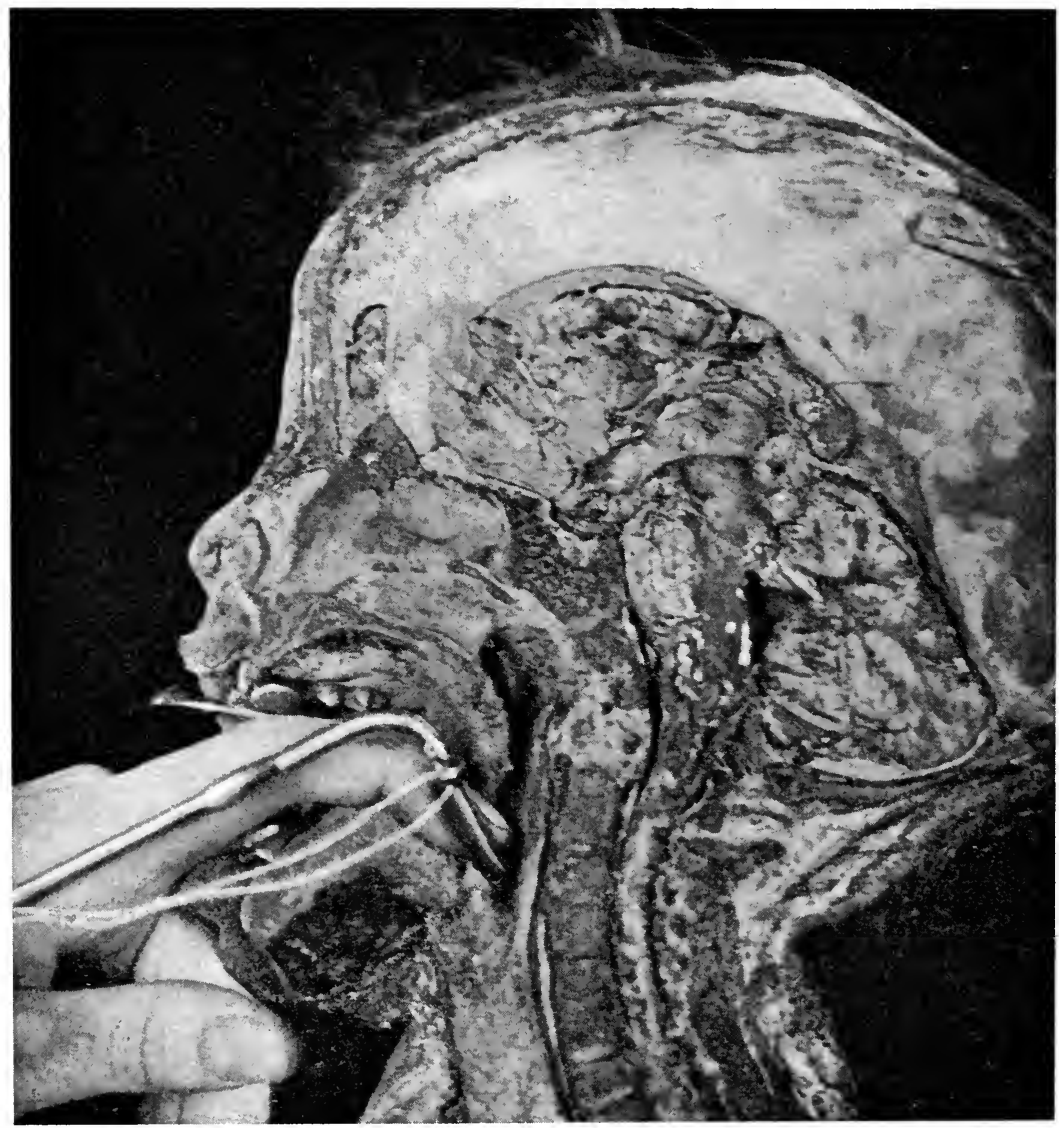

FIG. I86.-Illustrating the error of depressing the handle of the introducer, resulting in the tube entering the esophagus instead of the larynx.

tached to the tube can now be cut and pulled out. It is advisable, however, before removing the thread, to pass the finger once more into the larynx, to ascertain positively the whereabouts of the tube. The various steps in the operation of intubation are illustrated by Figs. I 84 to I9I, after photographs by Dr. P. M. Hickey. 
Difficulties.-The principal difficulty arises from the limited space afforded by the child's pharynx in which to make the necessary turn for reaching the vestibule of the larynx, for in a great many instances it will be found that the tube passes into the esophagus instead of the larynx. Once in a while it happens that the patient does not retain the tube very well,

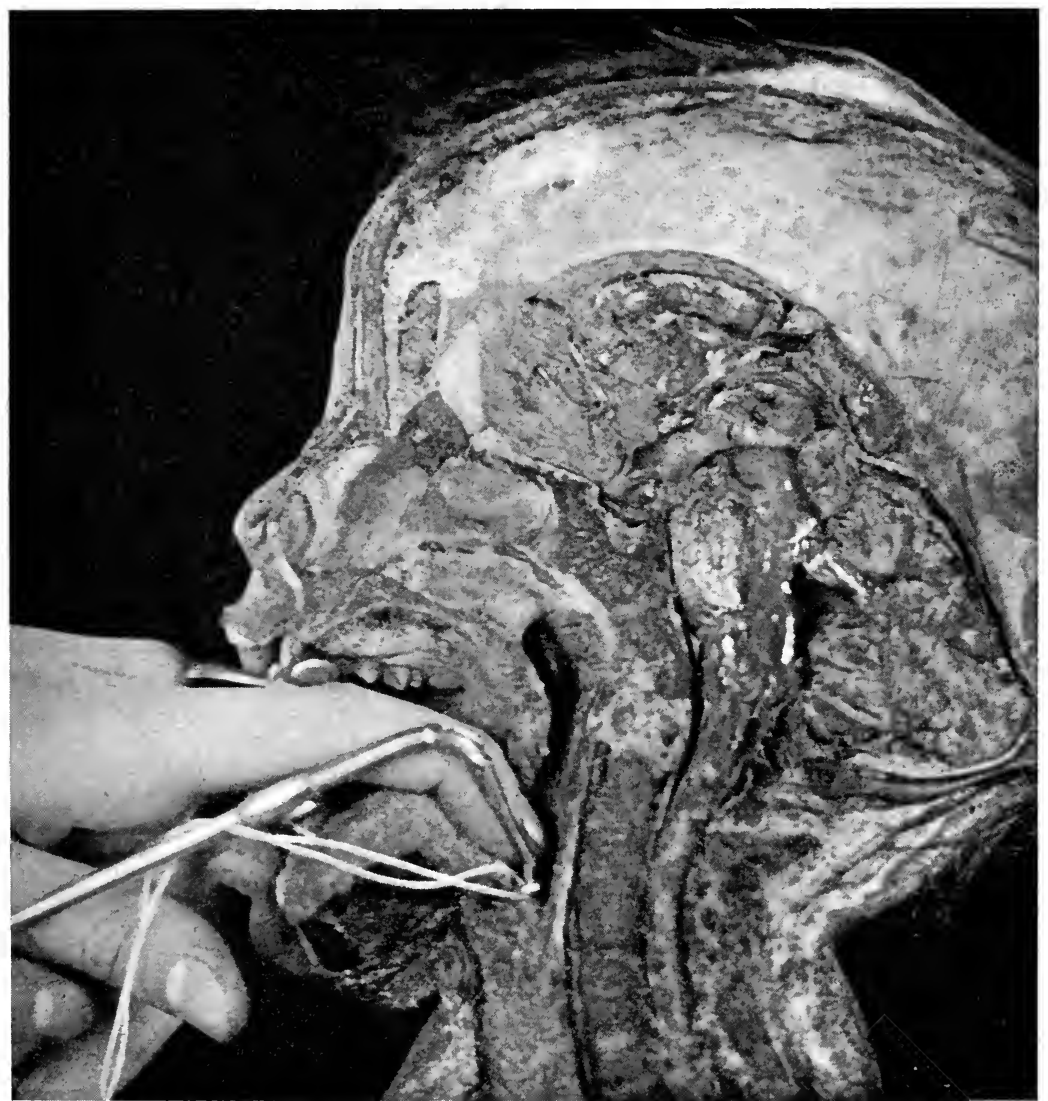

FIG. I87.-Removal of introducer with the finger holding tube in the larynx.

and the act of coughing may dislodge it. This difficulty, however, may be overcome by using a little larger tube. In cases of laryngeal diphtheria, characterized by extensive formation of pseudo-membrane in the larynx and upper trachea, a serious complication may ensue by pushing the loosened exudate down before the end of the tube as it reaches the larynx; fatal 
asphyxia may result from such an accident, unless the tube is immediately withdrawn, when, if the membrane is loose, it will be expelled by acts of coughing (see Fig. 192).

When the tube is lodged in the esophagus instead of the larynx, it is usually expelled by acts of gagging. When such an event does not transpire, the tube may be swallowed, and

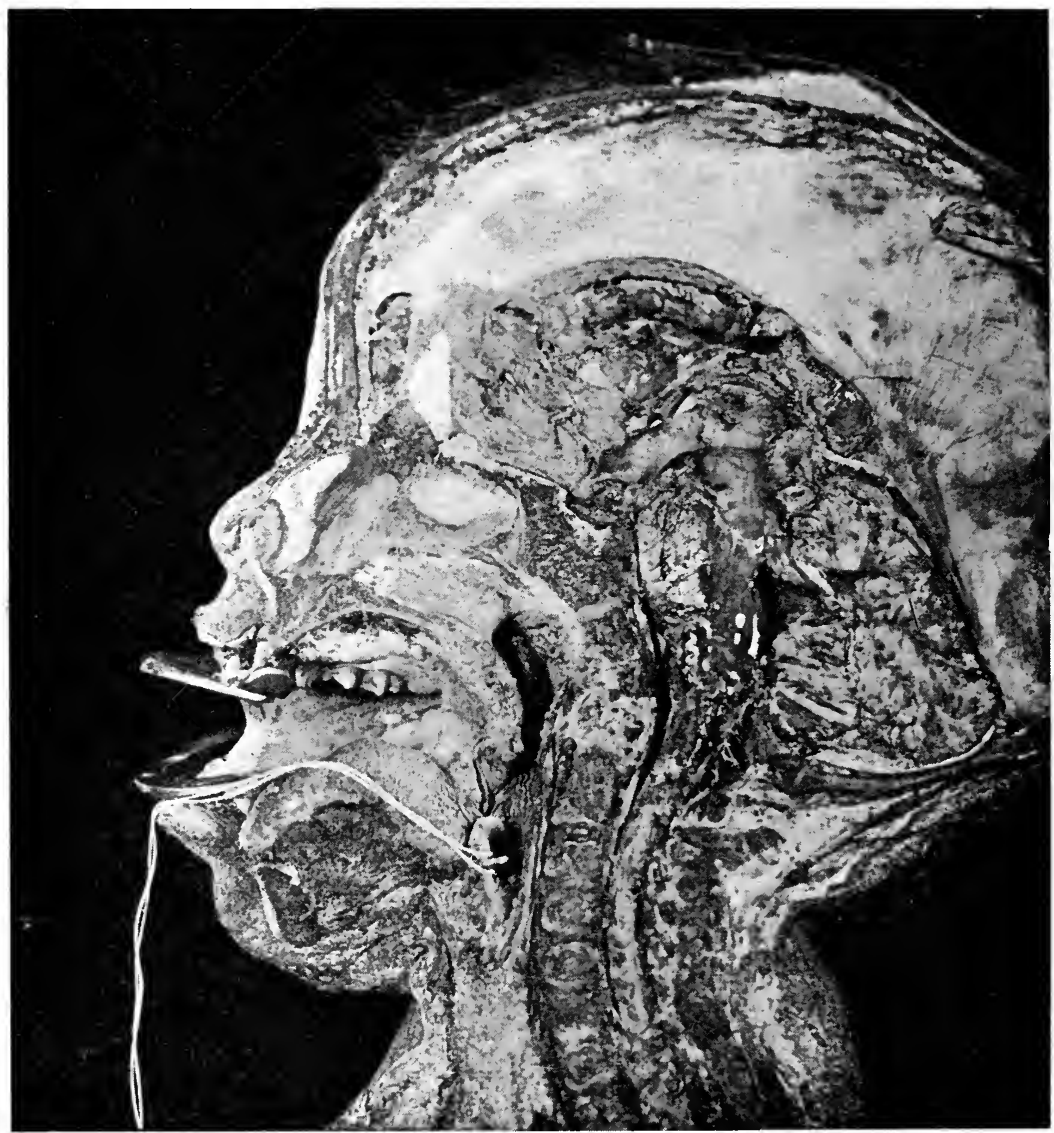

Fig. 188.- Tube in the larynx, with the string attached.

passed through the intestinal tract out of the body. The writer has known of two such instances, in neither one of which were any untoward results noticed. Care should be taken to quickly loosen the holder as soon as the tube is introduced, so that the tube may not be unwittingly removed with the holder. 
Withdrawing the Tube.-When the tube is to be removed, the position and management of the child is the same as indicated for its introduction. The extractor (see Fig. 193), with its jaws closed, is introduced by the guidance of the finger into the lumen of the upper end of the tube; when the beak is

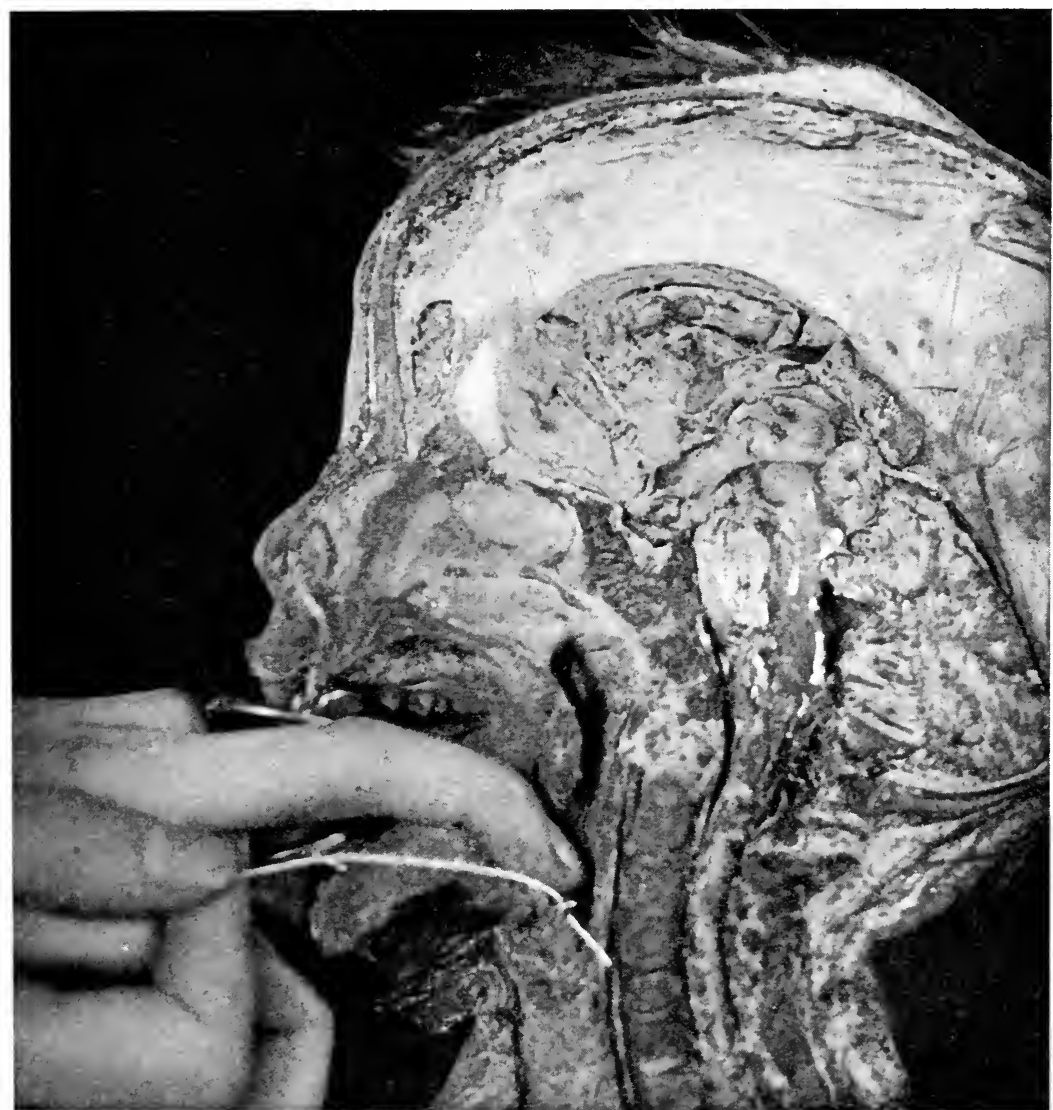

FIG. 189.-Withdrawal of the string, with the finger on the tube to hold it in position.

engaged the jaws of the extractor are forcibly separated and the tube withdrawn. This manipulation will perhaps be rendered easier by remembering that it need not be attended with haste.

After the tube is in position, the action of the epiglottis will be usually so interfered with by the head of the tube that 
attempts at swallowing solid food or liquids will at once bring on violent coughing, and perhaps, later, inspiration pneumonia. If, however, the child be placed in a horizontal position, with the head somewhat lower than the shoulders (see Figs. I94 and 195), liquids may be administered either from a

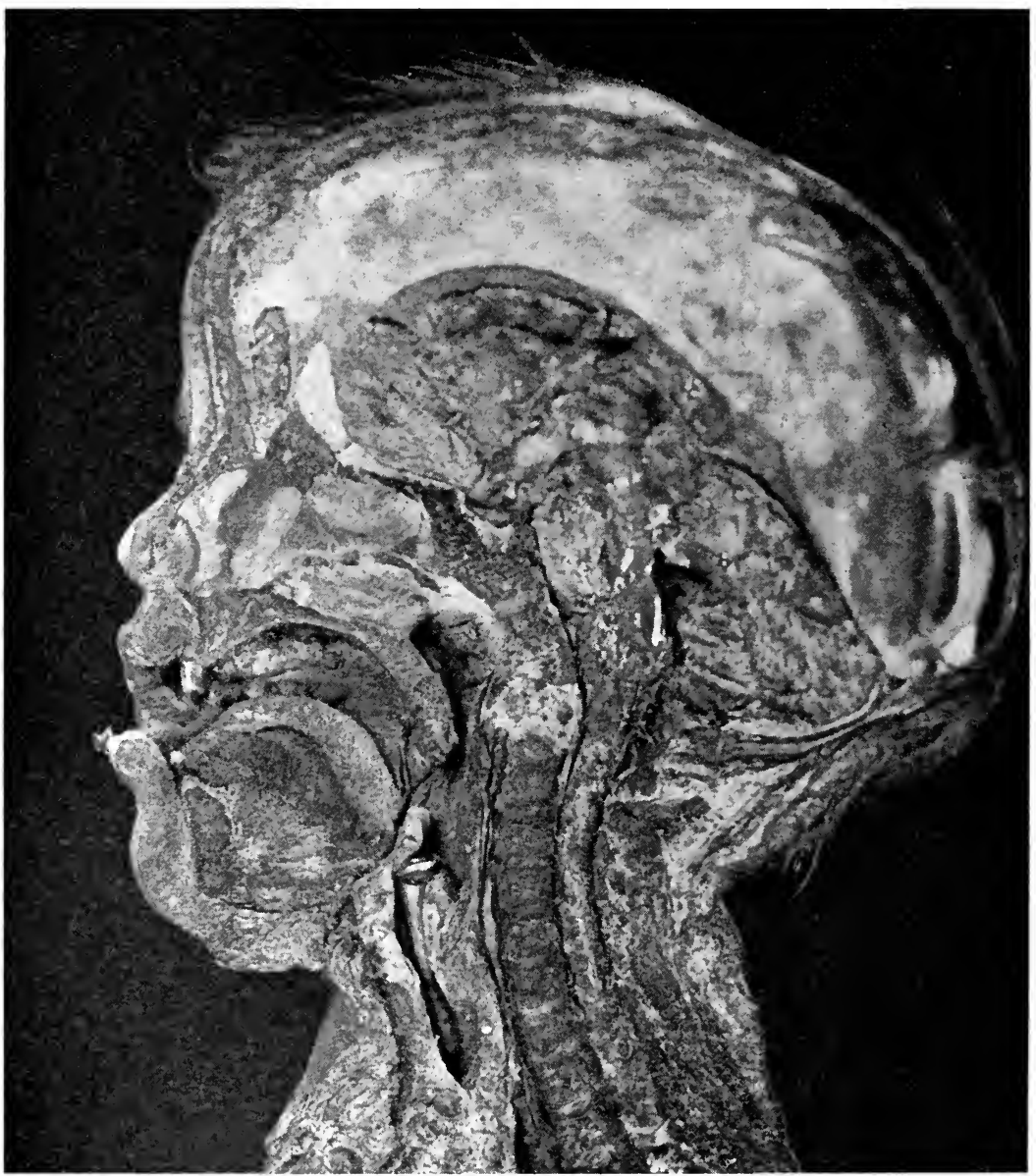

FIG. I9O.-Tube in situ. Showing the head of the tube upon the vocal cords, and the position of its distal end in the trachea.

spoon or nursing bottle, because the fluid follows the roof of the mouth instead of the tongue. If the tube is retained for many days, the habit of swallowing while erect may be reacquired.

The length of time the intubation tube may remain in the larynx without doing serious, if any, harm does not seem to 
have as yet been settled. In fact, continual surprises in this respect confront us (Levy). In one of O'Dwyer's cases the patient did not suffer from retention of the tube for a period of ten months and four days. A case of Waxham's retained the tube four years; of Schmeigelow's, one year; Dillon Brown's, nine months; C. H. Knight's, three months; Brothers's, fifty-

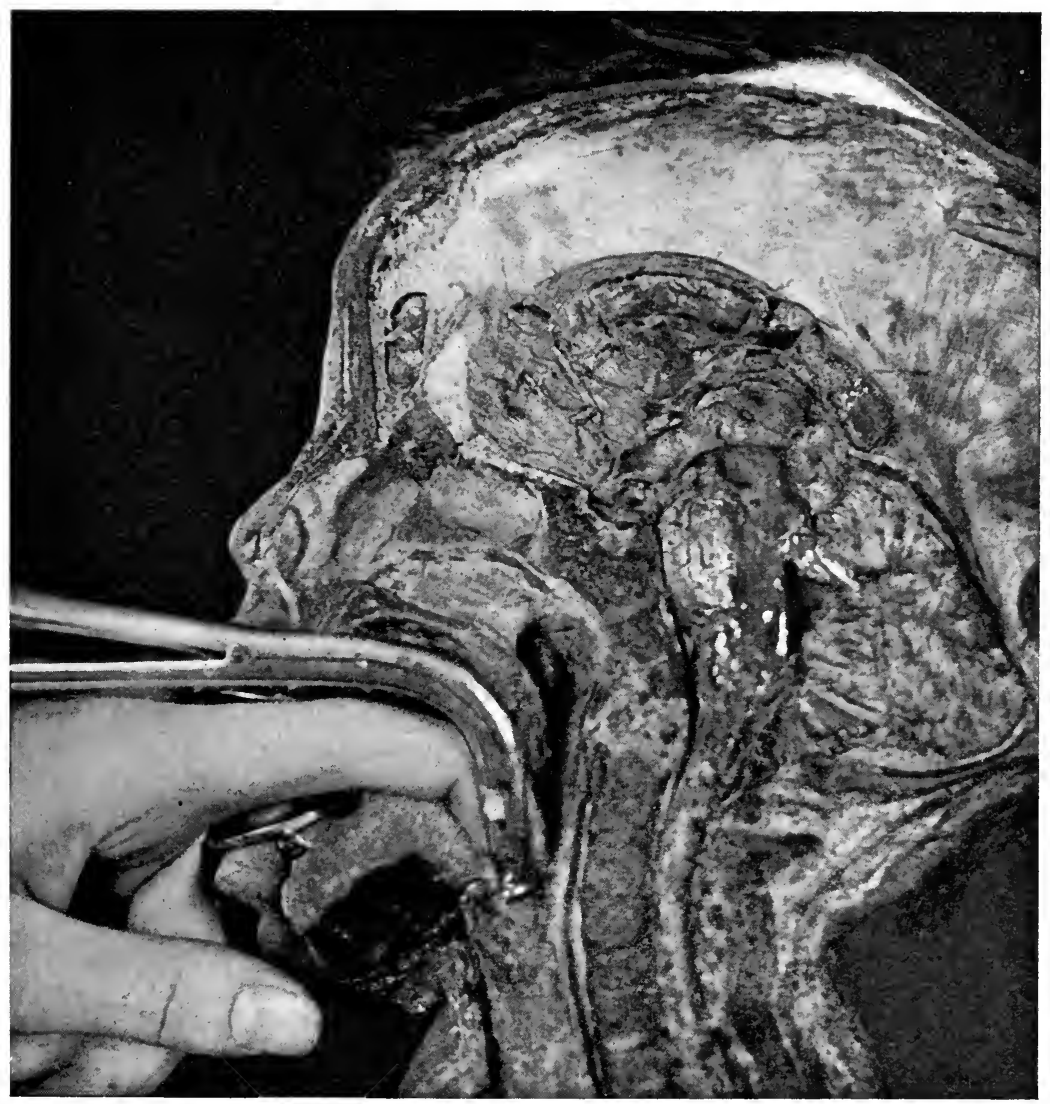

FIG. r91.-Method of removing the tube. The beak of the extractor, guided by the finger, is just entering the tube.

eight days; McCurdy's, forty days. In most of these cases the tube was removed every month or two. In Dr. O'Dwyer's case, however, the tube remained continuously ten months and four days. In Levy's case the tube was retained continuously for sixty-eight days, then removed for twelve hours, and again 


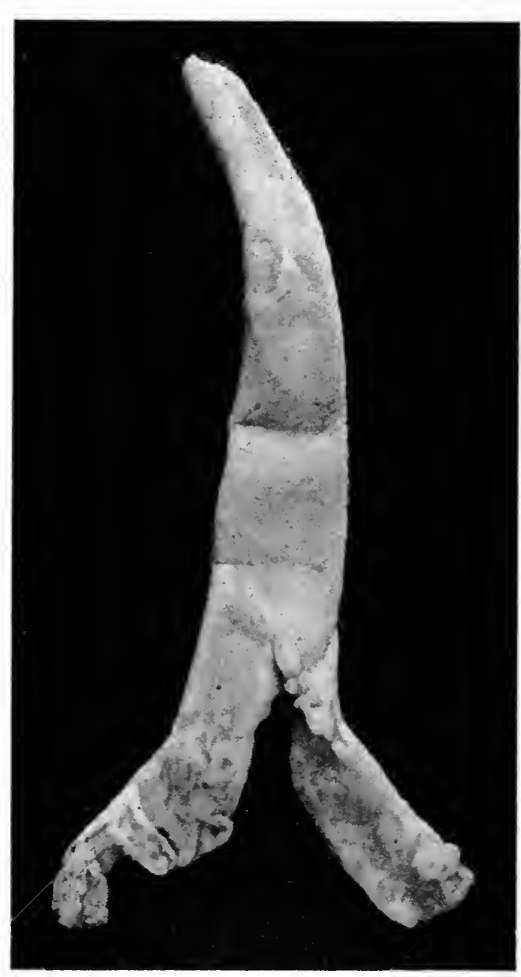

FIG. I92.-Diphtheritic cast of trachea and bronchi (natural size), from a child five years of age, expelled by coughing after removal of intubation tube. Subsequent recovery (case of Dr. B. R. Shurly). retained for one hundred and eleven days, a total of three months and twenty-one days. Feeding was normally performed after a few days' retention of the tube. There was observed no discomfort, except attacks of colds and coughs. This case is noteworthy in occurring in a child eight months old.

In one of the author's cases (a boy five years old) the tube was retained almost continuously for two months, and in another case of Dr. Chittick and the author's (a boy nine years old) the tube was worn almost continuously for six months.

\section{THYREOTOMY}

Thyreotomy, sometimes called laryngo-fissure, as its name denotes, is an operation consisting of the division of the thyroid cartilage in the median line, so as to expose the inside of the larynx. Much credit is due Dr. Clinton Wagner, of New York, and Mr. Butlin, of London, England, for developing and simplifying the method

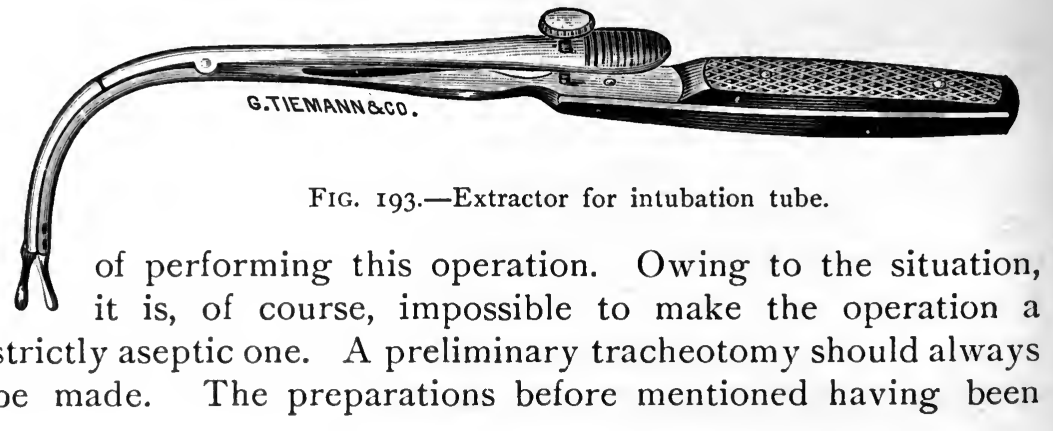



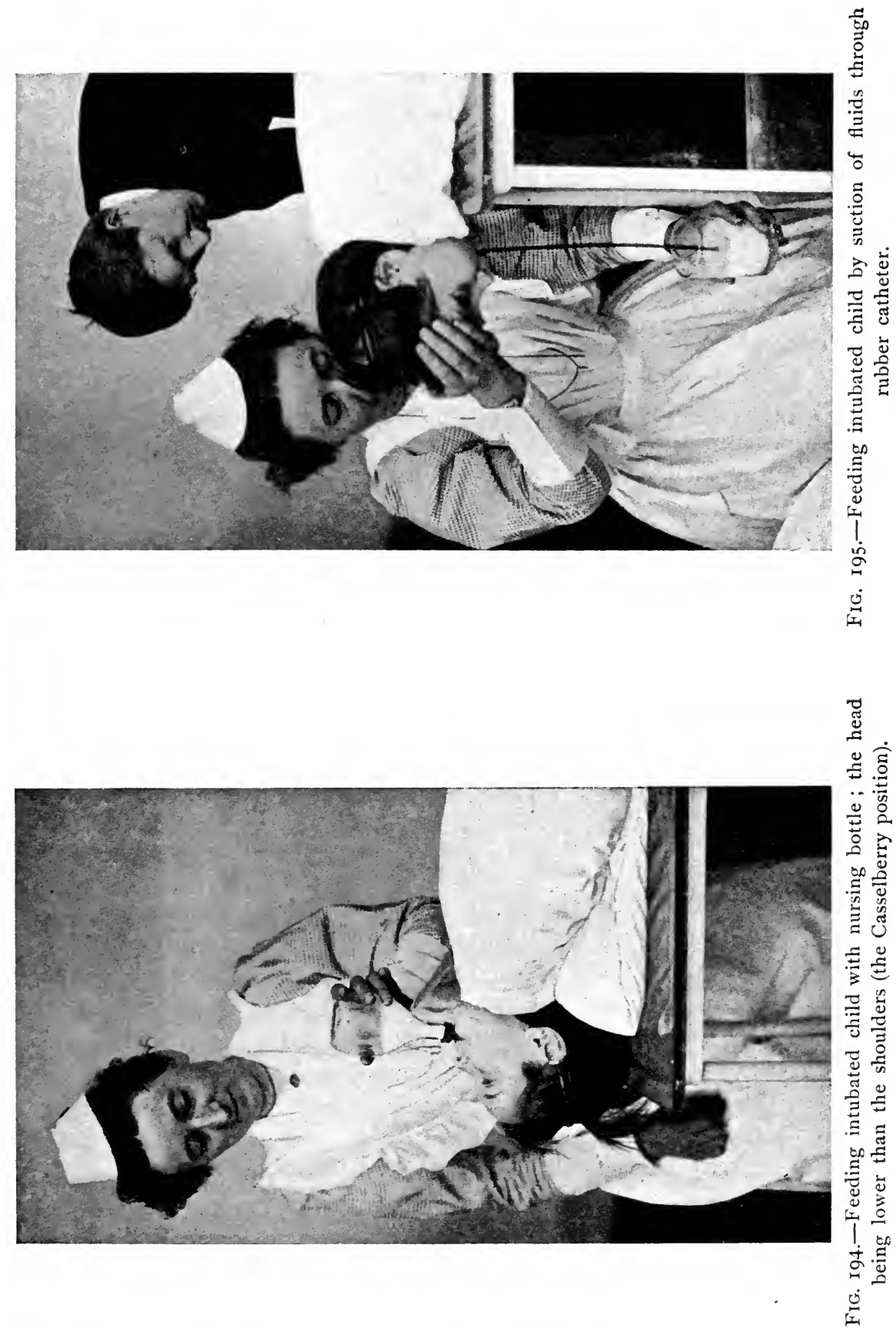
completed, the first incision is to be made by a bistoury along the median line of the larynx, from the lower border of the hyoid bone, down to the upper border of the cricoid cartilage, dividing the skin and superficial fascia. The larger veins should be pulled aside, if possible, instead of being ligated and cut. Now carefully divide, by the aid of a director, each layer of tissue, avoiding the blood-vessels as much as possible. Divide and push to each side the thyro-cricoid muscles with the handle of the bistoury. Having done this, the underlying cartilage, with its covering of perichondrium, will be reached. Now divide the perichondrium in the median line, and insert beneath it an Addis's dissector, or other raspatory, and complete the dissection by peeling, as it were, the perichondrium and all overlying tissue back from the median line for about an inch or an inch and a half on each side, thus leaving the bare cartilage exposed.

This having been accomplished, the thyroid cartilage itself is gently divided exactly in the median line, from its upper to its lower border, by means of a strong knife. Retractors may now be introduced into the incision thus made, and the two sides gradually pulled apart, in order to expose the interior of the larynx. Instead of retractors, the two sections of cartilage may be held apart by means of a stout thread passed through each half. Should it be necessary to obtain more space, the method suggested by Dr. J. Solis-Cohen may be adopted, which consists of cutting off a strip of cartilage about one eighth of an inch wide from each edge. This can be done readily with a stout knife or scissors, without seriously complicating the operation, although it must be remembered that such a step produces more injury to the vocal and ventrical bands. At this stage of the operation it will be necessary to have a strong light projected into the cavity of the larynx, especially if the object of the thyreotomy be the removal of growths. As suggested by Semon, this may be accomplished by means of a small electric head light, such as is found in connection with the so-called electric laryngoscope (see Fig. 196). As before mentioned, it will be just as convenient to use the head mirror with reflected light. The suggestion of Semon, to paint the interior of the larynx with a solution of cocaine and adrenalin immediately after opening it, in order to diminish the sensibility, cough, and capillary hemorrhage, is an excellent 
one. After the removal of the growth or foreign body by forceps, curette, galvano-cautery, etc., the parts may be brought into apposition, and held by stout catgut sutures, or, if necessary, by pin sutures. In some cases it may be better to follow the plan suggested by Clinton Wagner and Butlin, and allow the wound to close entirely by granulation. Formerly, a feeding tube was used, but Wagner, Butlin, and other operators dispensed with that entirely, and sometimes even remove the tracheotomy tube in a very short time after operation. Before

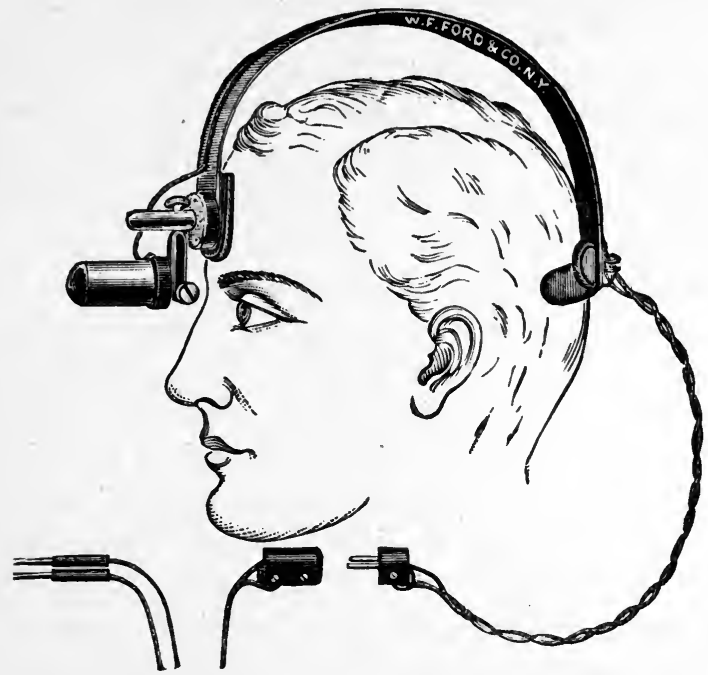

FIG. I96.-Phillips's electric photophore, with adjustment for focusing light.

closing the wound, the inside of the larynx is to be treated by an insufflation of equal parts of iodoform and boric acid. After thus treating the inside of the larynx, the external parts are brought together by suture, the lower part of the wound being provided with a small drainage tube, to avoid the possibility of the retention of septic material, and the whole region finally dressed with iodoform or bichloride gauze. The patient is to be kept lying strictly upon his side for the first two or three days, at which time, usually, the tracheotomy tube can be removed. Sometimes it will be found necessary to retain the tracheotomy tube for a longer time, according to the swelling of the larynx.

In a case of thyreotomy, made by the writer upon a boy four years of age, for the removal of neoplasms, the tracheot- 
omy tube could not be finally removed until about two months after the operation. The taking of food or drink by the mouth may be resumed as soon as the patient can swallow easily, provided none of the food or drink comes through the wound during glutition. Generally the patient is able to take fluid and semisolid food soon after the operation without much difficulty, unless the epiglottis or upper larynx has been injured very much, when the difficulty in deglutition may be very great.

\section{LARYNGECTOMY-LARYNGOTOMY}

An operation for the removal of the larynx. This may be partial or complete, according as the whole or only a part of the larynx is removed. The early steps of the operation are the same as those employed in thyreotomy-namely, preliminary tracheotomy and the stripping of the soft parts from the laryngeal cartilages by means of a dull instrument-dissector. Regarding the preliminary tracheotomy, however, it is maintained by many operators that tracheotomy should be made from ten days to two or three weeks before the operation for the removal of the larynx, because in so doing the patient is relieved partially of the shock of the subsequent operation, has a respite from dyspnœa by free respiration through the trachea tube, and therefore can be more liberally nourished, all of which contributes toward establishing a better vital state for undergoing the subsequent operation (laryngectomy). These are undoubtedly good and sufficient reasons for performing the tracheotomy at least ten days before the laryngectomy. It sometimes happens, however, that the gravity or particular features of the case will not admit of such a preliminary operation, and consequently both must be done at the same time. The tracheotomy should be made as low as possible, so that the first ring of the trachea may be removed if it be found necessary. A good-sized tube ought always to be introduced in order to allow of the insertion afterward of the tampon cannula, which is rather large. The tracheotomy tube should be replaced by the tampon cannula after anæsthesia is accomplished and before making the first incision, and the anæsthetic further administered through it.

The Trendelenburg cannula (see Fig. 197) is provided with a tight-fitting thin rubber investment, which can be inflated with 
air after its introduction, so that it completely fills the lumen of the trachea, thus preventing the ingress of blood. Cohen and Swain have used a cannula surrounded with sponge, which answers the same purpose, and which they like better. It

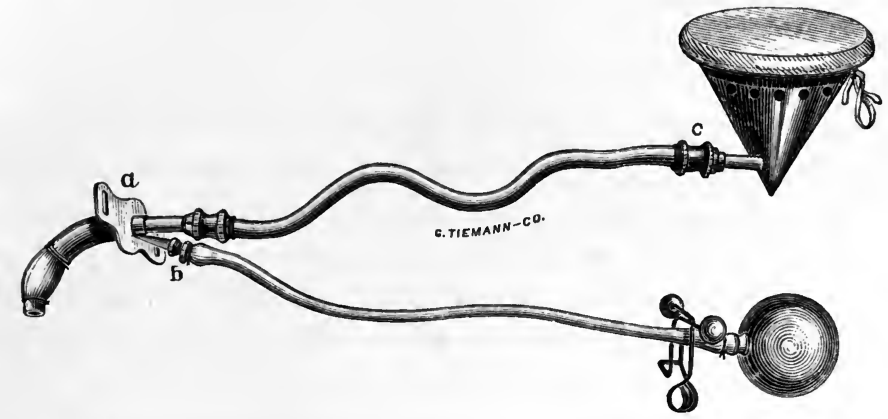

FIG. 197.-Trendelenburg's tracheal tampon. $a$, cannula; $b$, tube with bulb for inflating rubber tampon; $c$, tube with inhaler for anæsthetic.

appears, however, to the writer that the sponge cannula would be more difficult to remove than the rubber one, although perhaps less liable to get out of order than the former when not carefully adjusted.

After the operation the tampon cannula may be replaced by an ordinary trachea tube, when the upper open end of the trachea is stitched to the skin, according to Dr. J. Solis-Cohen's method. It is advisable not to remove the cannula for at least two or three days, if it can be avoided. The soft parts having been peeled away from the larynx by means of the dissector or raspatory down to the posterior borders of the thyroid and cricoid cartilages, and the hemorrhage having been checked, the lower part of the larynx may now be separated by a transverse incision, just below the cricoid cartilage or between the first and second ring, from the trachea, and dissected out from below upward in a very careful manner, with knife and dissector, keeping the instruments always close to the larynx. The arteries are to be caught as they are cut by catch-forceps and tied afterward. Great care should be taken to save as much of the esophagus as possible, and when practicable the attachments of the esophagus and inferior constrictor fibres to the posterior surface of the cricoid should be left. It may happen, as with the case of the writer's, that it is more convenient to separate the upper part of the larynx first, and dissect down- 
ward or from side to side. This may easily be done by clipping with scissors the hyo-thyroid membrane as close as possible to the upper border of the thyroid, leaving the superior cornua of the thyroid and part of the epiglottis.

In making the dissection downward, the wall of the pharynx should be mutilated as little as possible, and the same care should be exercised to save the esophagus after the larynx has been removed. The fragment of thyro-hyoid membrane holding the epiglottis should be carefully stitched to the anterior wall of the pharynx afterward. 'The vessels having been tied and the oozing of blood stopped, a feeding tube may be introduced into the esophagus through the upper angle of the wound, and held there close under the inferior maxilla. It is well not to introduce the feeding tube entirely into the stomach, but allow its distal extremity to remain at least from two to four inches above the cardiac orifice. This will lessen or avoid subsequent nausea and efforts at vomiting. The cut extremity of the trachea should now be brought forward and stitched to the skin, after the method of Brandelberg and Cohen. This plan is certainly far superior to any other, as is well illustrated by the cases of laryngectomy performed by Dr. J. Solis-Cohen and Swain. The wound is to be packed with iodoform gauze. The tampon cannula ought to remain until the upper tracheal opening is closed, or at least until the surrounding swelling has subsided. Temporarily, however, the cut end of the trachea may be closed with a piece of sponge tent. This procedure will effectually prevent any drainage into the trachea. The lower part of the wound should be left open, so that drainage may readily take place, and the patient should lie upon the side.

\section{PARTIAL LARYNGECTOMY}

Partial laryngectomy consists in the removal of a part of the larynx only. It usually includes one side of the thyroid with one of the arytenoid cartilages. Many laryngologists prefer this to total laryngectomy for the removal of malignant and other growths which involve one side only, because, as they contend, it saves for the patient a tolerably good vocal apparatus, and guards him from much future danger, such as inspiration pneumonia, by preserving the natural course of 
respiration through the mouth and pharynx, whereas after total extirpation of the larynx the air and anything suspended therein must go directly from the outside into the trachea.

According to the statistics of Butlin, of the cases reviewed by him, 66 partial extirpations were performed for cancer and 14 times for other causes. A comparison of the results between partial and total extirpation, as given by him, shows a little in favour of partial extirpation, namely, 29 per cent of cures in the latter operation as against 18 per cent in total extirpation; but the mortality from the two operations has been nearly the same, namely, 43 per cent in total and 42 per cent in partial extirpation. Fauvel and St. Hillaire think it advisable to ask if the operation of extirpation of the larynx has not been abused, and if in many cases tracheotomy would not have been preferable. Fauvel concludes that in very many cases the removal of the cartilages and bones of the framework of the larynx in intrinsic carcinoma is wholly unnecessary, and that it is sufficient to expose the affected cartilage or bone and cut away or scrape off the softened parts. "Carcinoma invades bone with difficulty and cartilage with still greater difficulty, and the chance of recurrence in these parts is small."

So far as the operation is concerned, however, it differs but little from that for total extirpation. The manipulation is about the same, excepting that the thyroid cartilage is first divided (thyreotomy), and then dissected out from before backward. The after-treatment is about the same, excepting that the tampon cannula should be left in place until the wound is granulating well. Likewise, the drainage tube should be left in until the wound below is well healed.

Sequences.-Aside from the direct hemorrhage and the dangerous results from pressure upon or injury of the vagus, and the consequent shock of such an operation, the most dangerous sequences in these cases is what is called inspiration pneumonia, which occurs from the drainage of the secretions down the trachea into the lungs. It is found usually to take place, if at all, within a couple of weeks after the operation, although it may take place later from the inspiration of noxious agents through the artificial opening. Next to this comes the danger of septicæmia or pleuritis or mediastinal suppuration. The danger of inspiration pneumonia is said to be as imminent after partial as after total extirpation of the larynx. 


\section{Methods of Local Treatment of the Upper Air Passages}

The local treatment of diseases of the upper air passages may be divided into medicinal and surgical. For the medicinal treatment any of the following methods may be adopted:

I. The application of the medicament in the form of a pigment (paint) by means of a pledget of cotton, a brush, or piece of sponge.

2. The application of the medicament in the form of a spray or douche.

3. The application of the medicament in powdered form by insufflation, or blowing it upon the parts.

4. The injection of the medicament into the cavity of the pharynx, larynx, or trachea by means of a suitable syringe, or beneath the surface of the mucous membrane by means of a hypodermic syringe.

5. By the inhalation of (a) spray, (b) medicated air, (c) gas, and $(d)$ fumes (fumigation).

The ordinary methods of surgical treatment are by curettement, scarification, evulsion, abscission, incision, electrolysis, and cauterization.

Pigments (medicinal painting) may be applied to the upper air passages by means of small pledgets of absorbent cotton wound around the end of a probe or piece of steel wire which is roughened at the proper end for the purpose, called an appli-

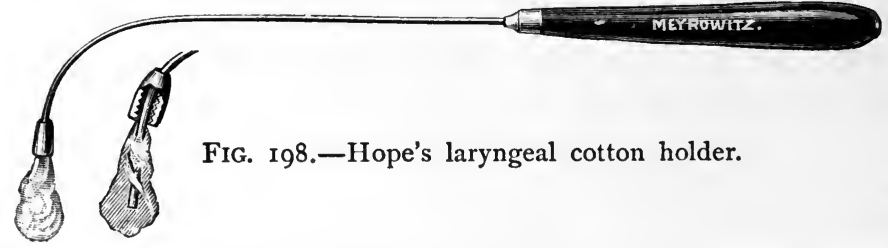

cator (see Fig. I98), by the aid of delicate spring forceps made for holding between its jaws either a piece of cotton or sponge, or by means of a camel's-hair brush mounted on a handle. Instead of either of these, a swab improvised by winding a piece of cotton or cloth on the roughened end of a little stick may be employed. The applicator is preferred often, in the belief that brushes which are repeatedly used become contaminated and may thus possibly convey from one person to another some septic material, whereas the bit of cotton or 
sponge can be thrown away or destroyed immediately after having been used. There is undoubtedly a probability that many brushes may become septic from frequent use unless the greatest care is exercised toward maintaining their cleanliness and sterilization. Another objection urged against the

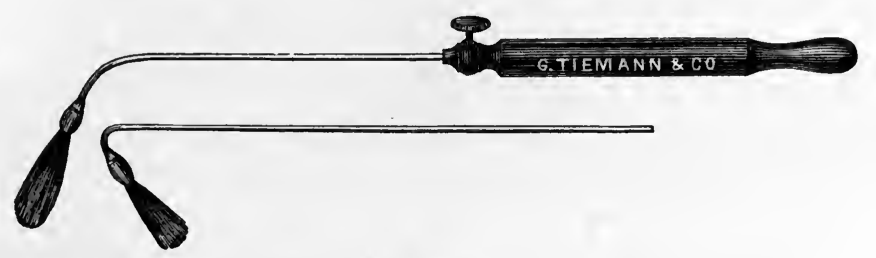

FIG. I99.-Wagner's laryngeal and post-nasal brushes.

brush for the throat or nasal passages is the possible loosening of a hair or two and their deposit upon the parts. For applications to the nasopharynx, pharynx, and tonsils the cotton pledget is greatly to be preferred in all cases, but for the application of pigments to the larynx there is no instrument better than the camel's-hair brush when properly made and shaped. The handle and brushes devised by Dr. Clinton Wagner, of New York, have never been surpassed in the writer's estimation (see Fig. 199).

These brushes are removable from the shank and handle, so that a particular one can be applied successively for a given patient and finally destroyed. The shanks and handles being made of metal, no difficulty will be found in keeping them properly aseptic. Besides, they are so well made that by careful use the hair need never become loosened. The writer has used them for years, and has never known any of the hairs to have broken or become loosened. In every instance the brush should be thoroughly cleansed with hot water and afterward dipped in a five-per-cent solution of carbolic acid directly after being used, while a special set should always be kept on hand for use in syphilitic or other communicable diseases. Delicate spring forceps will be found more convenient for holding pieces of cotton or sponge than probes or applicators, as a rule, because the pledget can be thrown away without handling after being used by just pressing the jaws of the instrument apart and tossing the cotton out. Besides this, a certain firm hold on the cotton or sponge can always be maintained during the manipulation. However carefully a piece of cotton 
is wrapped around the end of an applicator, it may slip off during the process of application. Although such accidents are of rare occurrence when the proper instrument is employed, yet they have happened occasionally, and under some circumstances such an event might produce disastrous consequences. Any forceps of proper shape to reach the part desired may be used, but a pair of spring-handle forceps with small beaks slightly corrugated on their inside face, and bent at about three-quarters of an inch from the distal extremity at an angle of about one hundred and twenty degrees, will be found very convenient. Those de-

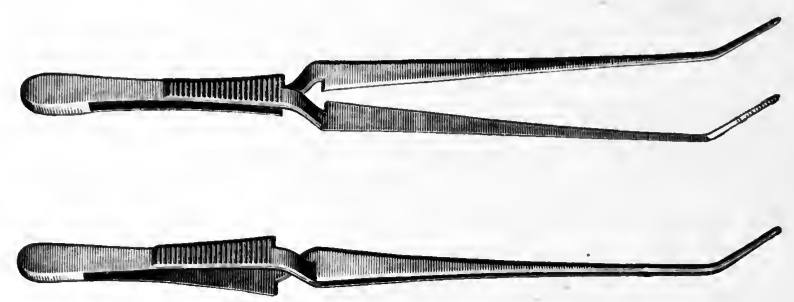

FIGS. 200 and 20r.-Shurly's cotton forceps.

vised by the author (Fig. 200) will answer this purpose. They can be used for making applications to any part of the pharynx, to the nasopharynx and laryngopharynx. Care should be exercised in applying pigments to the pharynx so that a redundance of the medicament is not carried and allowed to fall suddenly into the larynx, for such an occurrence may cause distressing laryngeal spasm and pain. A great variety of applicators especially adapted for various parts of the nasal, pharyngeal, and laryngeal regions may be found in the market. For the application of either lunar caustic or chromic acid to any well-exposed part these agents should be fused on the end of an aluminum probe. This may be readily done by melting the drug in an old-fashioned porcelain mustard spoon or Lente's platinum cup held over an alcohol lamp. These applications, however, have given way to solutions borne by cotton pledgets saturated with argyrol and other such preparations of silver, and the various vegetable and oleaginous mixtures. When the chemical is melted, the end of the probe, previously heated, is thrust into it and rotated while the liquid cools upon it. A coating of the probe as thick as desired may be thus secured. When it is desired to apply either of these substances to some concealed part, such as the nasopharynx, 
laryngopharynx, or larynx, a special instrument (caustic holder), with a shield for protecting the surrounding parts, must be used. Fig. 202 represents Jarvis's laryngeal chromic-acid applicator. In this instrument the caustic carrier is concealed within its sheath until reaching the part to be treated, when it is thrust out by the action of the operator's thumb and then quickly drawn back again into the sheath before removal of the instrument from the throat. In using these instru-

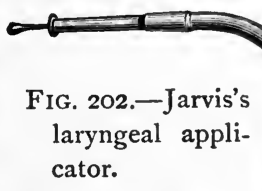
ments within the larynx the operator must be careful that the carrier is not too heavily coated, lest a small piece of the escharotic be chipped off by the edge of the shield and fall into the larynx or trachea.

In using the galvano-cautery within the laryngeal cavity it is necessary to have the electrode provided with an insulated sheath up to the platinum point in order to protect the surrounding parts from the heat. When general anæsthesia is required for operation with the galvano-cautery or thermo-cautery, ether should never be employed, because the glowing electrode would set fire to the ether vapour in the throat and produce disastrous burning which might lead to death.

\section{Spray or Douche}

The application of a medicated spray to either the nasal, pharyngeal, or laryngeal cavities may be accomplished by means of any one of the great variety of "atomizers" in the market. Some of the instruments are adapted for the nebulization of oily solutions, some for the nebulization of watery solutions, and some are so constructed as to nebulize solutions of either an oily or watery character. Many of them are provided with various tips for adaptation to different parts of the throat, but as a rule such distal extremities are superfluous for the patient's use, inasmuch as an instrument provided with a straight tip will meet nearly all requirements. It is necessary that the substance to be atomized should be always cleared of solid particles by filtration. Atomizers may be divided for consideration into three classes: 
I. The steam atomizer, an instrument comprising a receptacle for holding water which is to be converted into steam by boil. ing over an alcohol lamp. The steam issues through a horizontal tube, drawing the fluid up through a perpendicular one, and constitutes the motive power of the apparatus. 2. The

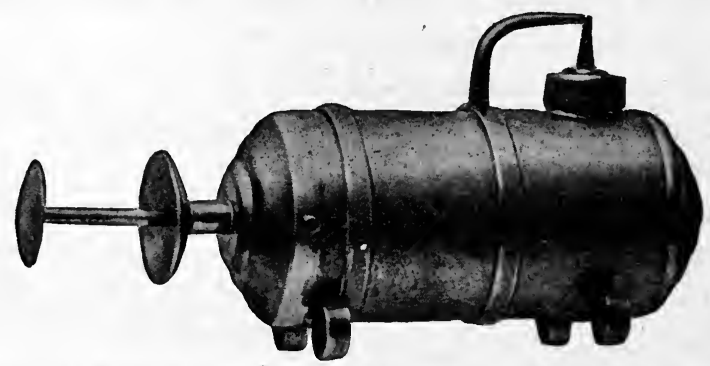

FIG. 203.- Japanese atomizer made in the early part of the eighteenth century, obtained by Father Reanney, chaplain, U. S. N., and presented to author by St. Mary's Academy, of Monroe, Mich.

hand-ball atomizer, in which the motive power is furnished by the compression of the air contained in a rubber bulb. The compression is made by either the hand, foot, or some artificial mechanical device and agency, such as an electric or water motor. 3. The mediate nebulizating instrument, through which the spray is projected first into some globular vessel or cavity, and thence conveyed into the nasal or pharyngeal cavities by acts of inhalation.

The treatment of diseases of the upper air passages by spray is not a recent method, but, indeed, an ancient one. It was not only extensively employed in France in the early part of this century, but evidently by the Japanese in the previous century. Through the kindness of St. Mary's Convent, Monroe, Mich., the writer is enabled to present an illustration of a Japanese atomizer made of brass in the early past century, which was obtained and presented to St. Mary's Academy in I 898 by Rev. Father Reanney, chaplain of the United States steamship Olympia (see Fig. 203). It will be seen that the chimney principle of producing the spray was recognised in the construction of the instrument, and that the motive power is furnished by the operation of the hand upon a piston arranged similarly to an ordinary syringe.

In the use of sprays, of course, no fast rules can be laid down, but undoubtedly there is no department of therapy 
where more blundering is done than in the selection and application of sprays for the relief of diseases of the upper air passages. Here routinism seems to be too often practised, to the detriment of the patient. Sprays cannot be very well applied warm without a rather complicated apparatus, and therefore those of a watery nature should not be injected into the nasal passages in too large a quantity, nor too often, nor for too long a time at a séance, excepting in cases such as ozena, where there is more or less obtundity of the parts, or in cases where there is considerable structural change. Likewise great care should be exercised to avoid propelling upon these parts strong solutions of any sort, especially astringents, unless the peculiar condition of the case will warrant it. Even the mild detergent sprays, such as Dobell's or Seiler's solution, or common salt solution, may do harm if persistently used in cases showing untoward results.

In short, it should be borne in mind that the nasal passages are not naturally designed for the reception of watery solutions, even in the form of spray. The mild oily sprays are usually quite non-irritant, although there are many exceptional instances where even petrolina or olive oil will produce a state of irritation akin to an acute coryza.

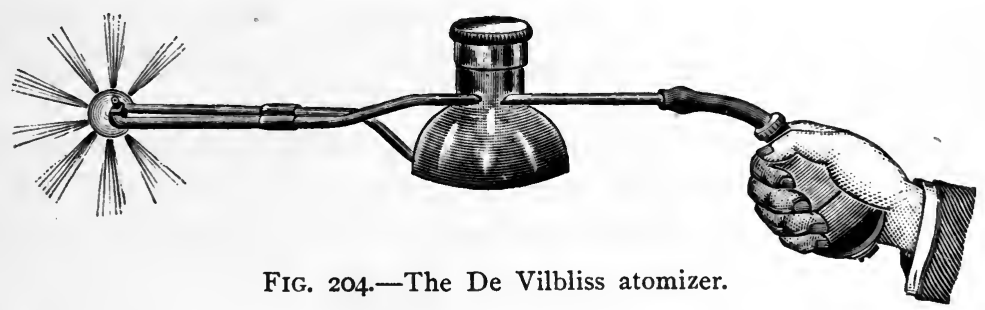

The writer has met with cases where the nasal passages seemed to be so intolerant that almost any sort of topical application was followed by great distress. The same care should also be exercised in the application of pigments or powders to the nasal passages anteriorly. For a long or continuous application of a spray to the pharynx or larynx the steam atomizer will be found preferable. Dr. Frank C. Todd, of Minneapolis (N. Y. Med. Jour., vol. lxiv, p. 760), has devised an apparatus for keeping spray solutions warm for application to the nose. It consists of two parts-a metal bucket just large enough to stand upon one of the small gas stands physi- 
cians frequently have in their offices, and a cover perforated with holes each of the size to hold a Davidson spray bottle. Fastened to this cover at the rims of these holes are hollow metal cylinders closed at the bottom. These are of sufficient length and diameter

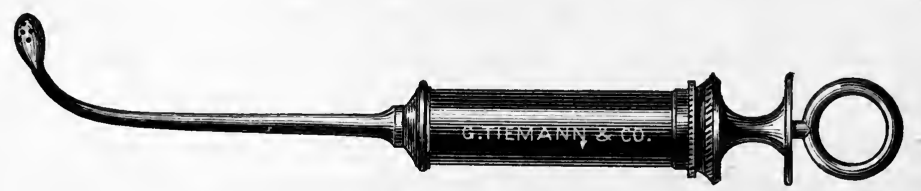

FIG. 205.-Hard-rubber pharyngeal and posterior nares syringe.

to permit the bottles to extend into the pails up to the rims of the bottle. The cover can be rotated. When the heater is used, the pail is filled with hot water, or cold water and heated. It is not necessary to remove the cover or bottles.

When it is desired to reach the larynx with the medicament, the nose may be closed, or one of the globe and face shield attachments may be employed. It must be remembered that unless some such device is used very little of the spray will reach the larynx and trachea, on account of the ready condensation which takes place in the buccal cavity and pharynx. The tongue may also prevent much of the spray from going beyond the pharynx.

Douches are not frequently employed nowadays on account of the ill effects upon the nasal mucous membrane and the middle ear, which often follow their use.

In cases of ozena, chronic syphilis, or other diseases of these passages, where desiccation and collection of the secretion takes place, the use of the douche may become necessary. An ordinary

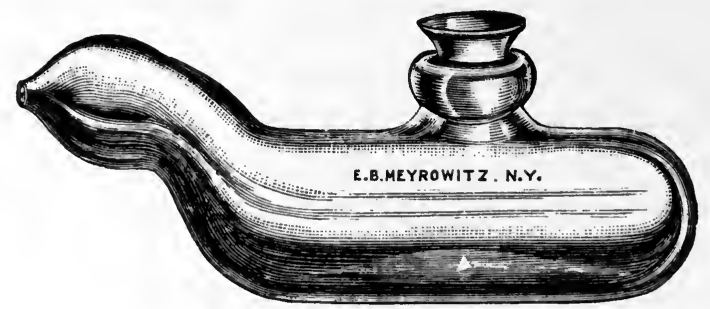

FIG. 206.-Nasal douche.

syringe, or the small pocket douche (Birmingham) found in the drug stores, will answer the purpose usually much better than the old-fashioned large douche which is operated through gravity. 
In all instances the fluid should be introduced into the nasal passages warm. This may be a little difficult in applying peroxide of hydrogen, unless accomplished by diluting the peroxide solution with hot water when just ready to make the application.

The douching of the nasopharynx and posterior nasal passages is often of greater efficacy, as before mentioned. This is accomplished by means of the post-nasal syringe or douche (see Figs. 205, 206, and 207). It is, indeed, very seldom that any evil follows this practice when carefully done. Care should be taken not to wound the mucous membrane of the posterior wall of the palate or the pharyngeal vault with the point of the syringe. Very little force should be exercised in introducing the beak of the syringe behind the palate. The instrument should be gently carried backward through the mouth, then upward behind the velum and a little forward, following the

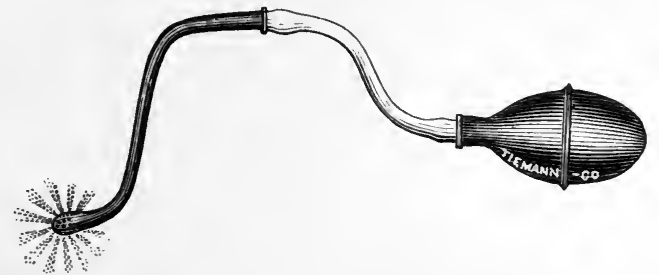

Fig. 207.-Warner's post-nasal douche.

curve of the parts, and when the end is felt to be free, gently push the piston or press the bulb, as the case may be, and the contents of the instrument will be discharged into the nasopharynx and flow out through the anterior nasal passages. This being done, remove the instrument quickly by an upward movement of the hand to release the end of the syringe from behind the soft palate and then withdraw it from the mouth. These movements should be made quickly and almost simultaneously, so as not to catch the soft palate with the hooked end of the syringe; the head of the patient may at the same time be thrown forward so that the fluid will run out through the anterior nares. Sometimes a portion of the stream will run immediately down into the pharyngo-laryngeal region, or into the larynx, causing more or less coughing, gagging, or laryngeal spasm. In such cases, where the parts are intolerant, it is advisable to make the earlier appli- 
cations small in quantity, and introduce the syringe but once at a sitting.

The application of powders (insufflation) is also a very old method of local treatment. Considering the prevalence of the practice and the careless method of using powders years ago, it is a wonder that history has not recorded many serious accidents from the insufflation of powdered drugs into the pharynx and larynx. Powdered zinc sulphate, copper sulphate, silver nitrate, etc., were often blown forcibly into the pharynx or larynx, or allowed to be inspired by the patient. The fact that the recording of experience and the writing of medical articles were not as commonly done as nowadays will, I suppose, account for our failure to see many accidents recorded as a result of treatment of the respiratory passages by insufflation.

There is a great variety of instruments on the market for insufflating powders, each of which, perhaps, has advantages in certain particulars. Any instrument, however, which can be loaded with little trouble, and which is not so large as to allow too much powder to be expelled at once, will answer the purpose (Figs. 208, 209, and 210). Great care should be taken to keep the barrel or distal end of the instrument from becoming

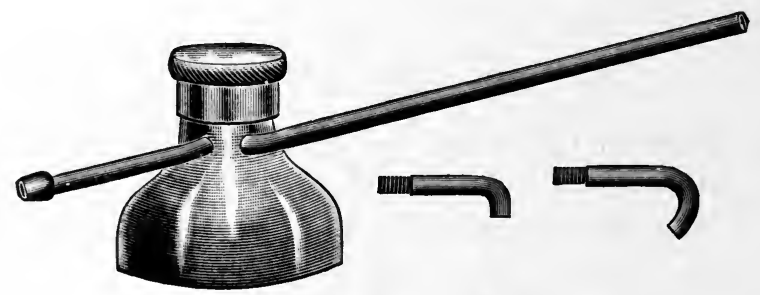

FIG. 208.-De Vilbliss's powder blower.

foul by the collection of the secretions. To this end the pressure on the bulb of the insufflator should be kept up after the discharge of the powder, until the end of the instrument is quite clear of contact with the mucous membrane of the part-either the nasal passages or pharynx-otherwise some of the secretion may be drawn in by suction.

Dr. Emil Amberg, of Detroit, has devised a powder blower which can be thoroughly sterilized. The powder blower is of metal, and the tube connecting the powder blower with the air-supplying apparatus is made of durit. Durit can be boiled 
without any damage. Thus the powder blower can be rendered aseptic (see Fig. 209).

An instrument provided with a removable end is excellent, for the reason that after its use each time the end can be taken
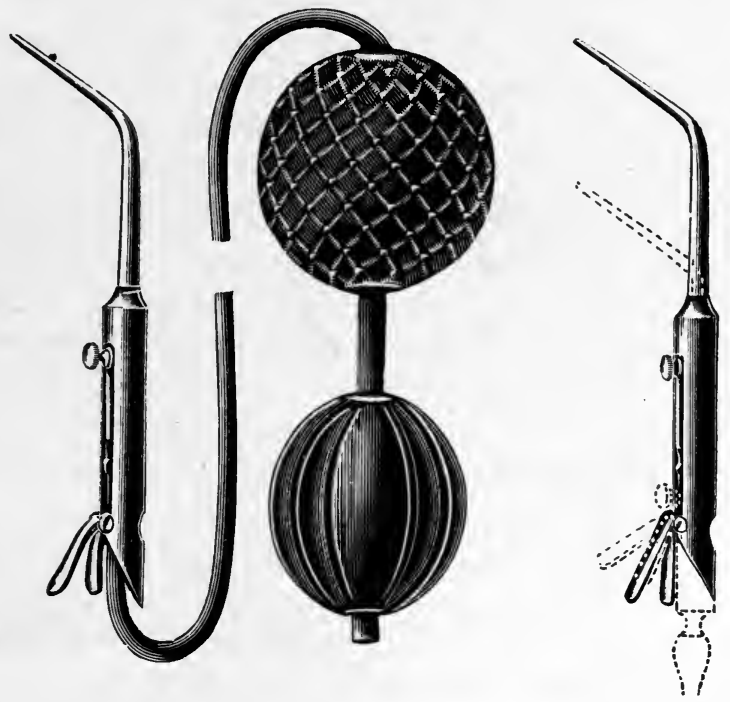

FIG. 209.-Amberg's powder blower.

off and cleansed. Such an insufflator (devised some years ago by the author) was found highly useful in treating contagious throat diseases, like diphtheria (see Fig. 210).

Care should be taken that the powder to be used is very finely triturated and afterward passed through a sieve, otherwise the insufflator tube will soon become clogged by the collection in its lumen of the larger particles.
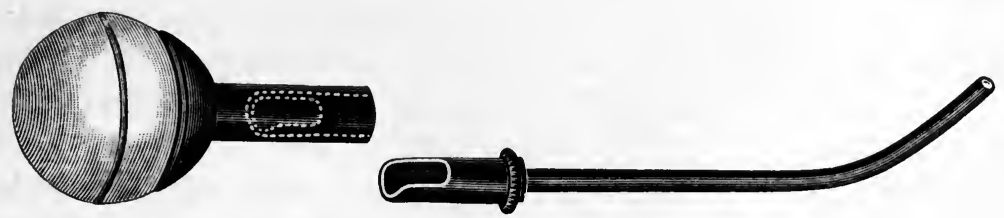

FIG. 210.-Shurly's powder blower.

The manner of injection of medicaments into the nasal passages and pharyngeal cavity has already been spoken of under the subject of douches. Any suitable syringe may be used for this purpose. 
The "submucous injection" (hypodermic) of medicinal substances has been practised more or less since the introduction of the hypodermic syringe. Hypodermic injections of carbolic acid have been advocated for the treatment of peritonsilitis and the angina of scarlet fever by Wales, Chappell, H. J. Lipes, Watson, and others. Up to the present time, however, it has not been a very popular method of treatment. The writer has employed this method in three cases of laryngeal phthisis, but without observing strikingly beneficial effects.

Dr. W. F. Chappell, of New York, is an advocate of this method of local treatment of laryngeal phthisis especially, and has observed some very beneficial effects in a few cases which he has reported to the New York Academy of Medicine. Undoubtedly the method deserves further trial, not only for the treatment of laryngeal phthisis, but for neoplasms, hyperplasias, and the acute inflammations of the mucous membranes. It is often difficult, if not impossible, to use the ordinary hypo-

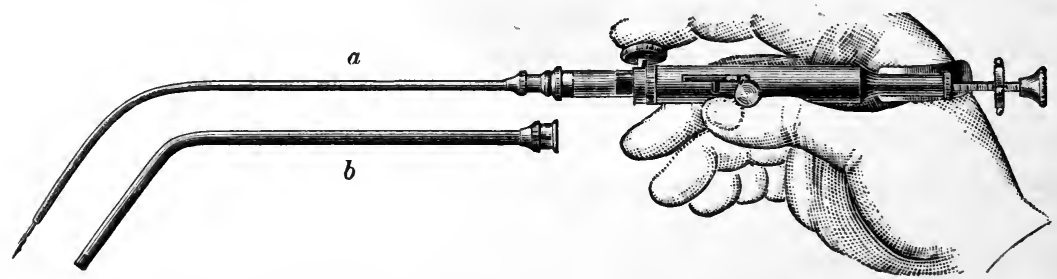

FIG. 2II.-Hypodermic syringe. $a$, laryngeal needle for submucous injection. $b$, laryngeal cannula, with gum-elastic tip and cover, for injections into the larynx and tràchea.

dermic syringe, with its short needle, in the fauces or upper larynx; therefore a long curved needle is absolutely necessary. Dr. Chappell has devised an instrument for the purpose which is excellent (see Fig. 2II), the description of which, together with the method of using it, is here quoted: "A long needle with a laryngeal curve can be used with any hypodermic syringe; but there are some drawbacks to this, as the movement of the hand in pushing down the piston is apt to make the patient gag, and conceals from view the point we wish to inject.

"To obviate this I have had an automatic syringe made, as shown in the cut, which allows the operator to disengage the piston with his thumb, and still keep the point of the syringe in view in the mirror. 
"The shank of the needle is a hollow tube about six inches long, which may be given suitable curves for laryngeal work. The needle is about half an inch in length, and corresponds to the large-sized hypodermic needle, with the opening close to the point.

"The depth to which we desire to introduce the needle is regulated by a small piece of solid rubber ligature, which is drawn over the needle, and may be shortened or lengthened accordingly.

"The rubber casing on the needle makes it easy and safe to employ pressure, and owing to its suction the rubber clings to the mucous membrane and prevents the creosote solution from swelling up around the needle after the injection. The barrel of the syringe is partly glass and partly metal; the latter contains a spiral spring, which is attached to the piston, and a longitudinal opening on the side of the barrel is notched to receive the catch of the spring. The piston goes the entire length of the syringe, and is graduated and furnished with a set screw to regulate the number of drops injected. The solution for injection may be warmed or not. The glass barrel should be filled, and the piston catch pushed into the top notch of the metal barrel. The laryngeal needle, being already sterilized, is screwed on to the syringe, and the piston dropped to the second notch of the barrel, thus filling the needle. After regulating the set screw for one drop (which is the amount usually employed) the syringe is ready for use. It should be passed into the larynx with the right hand, under the guidance of the laryngeal mirror held in the left. The point selected for the injection will of course depend on the situation of the tubercular deposit, and with a little experience any part of the larynx can be reached without much difficulty. When the point of the needle has punctured the mucous membrane the catch of the spring is dislodged with the thumb without chang. ing the position of the hand, and the piston springs down as far as the set screw and makes the injection. If possible, the needle should be held in position for a few moments. Little pain or reaction follows the injection of oily solutions, but pure creosote causes a burning sensation and considerable soreness, which lasts a variable time. Much depends upon the locality of the injection; the posterior surface of the arytenoids seems to be specially sensitive. There is little or no 
hemorrhage after the needle is removed, and the following day the mucous membrane is more tense, and possibly somewhat redder. This condition subsides in the course of a few days, leaving the tissues in a wrinkled condition, as though the mucous membrane was too large for the subjacent parts. This is most noticeable around the arytenoids. Careful judgment is required to determine how often the injections should be made, but as a rule it can be done once in five or six days. If ulceration is proceeding rapidly, one injection may be given daily until three or four have been administered. This number would usually include the amount of tissue involved and promote its more rapid resolution and stimulate granulation. After several injections it is well to wait for a time and see if the circle of resolution will not spread from the point of injection to the neighbouring tissues."

In the treatment of neoplasms of the pharynx or nasopharynx, local medication by this method will be found of great utility in some cases.

The injection of watery or oily solutions into the cavity of the larynx and trachea has been practised from time to time during the last fifteen or twenty years. Indeed, it is authentically stated that this scheme had been practised during the early part of the century. The treatment of the larynx, trachea, and bronchial tubes by intralaryngeal injections has been advocated and practised especially by Botey, Stoerck, Schmatz, Brommer, Natier, and others. Botey claims that comparatively large quantities of fluid may be safely injected. Brommer uses a five-to twenty-per-cent solution of menthol in paroleine. He sometimes adds ten to twenty per cent of water or rectified spirits, ten to fifty per cent of terebene, or three per cent europhen, or two per cent ol. pimenta. He uses two or three per cent sodium bicarbonate or ten per cent boric acid, if there be much secretion. Campbell recommends the injection of terebene in cases of hemoptysis.

A few drops of a five-per-cent solution of cocaine is to be injected first. A dram syringe is the proper instrument to use; it should be previously sterilized.

Professor Stoerck, of Vienna, devised a laryngeal syringe for injecting the cavity of the larynx, which for a time was quite extensively used by laryngologists. Of late, however, this practice has fallen into desuetude, owing to the improve- 
ment of the spray apparatus, the use of pigments, and the distress so often attending the injection of a considerable quantity of fluid into the larynx.

In this country syringing the larynx was also practised extensively by the older laryngologists. Indeed, it is said to have been done by Horace Green in pre-laryngoscopic days. Of late the injection of the trachea for tuberculosis has been again advocated by a number of observers, but the practice has not appreciably grown.

The laryngeal syringe of Stoerck and Tobold (see Fig. 212) will hold about a dram of fluid. Its distal end is a small

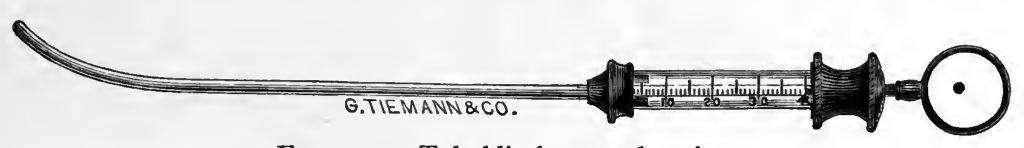

FIG. 212.-Tobold's laryngeal syringe.

hard-rubber tube, curved so as to easily enter the larynx. If the trachea is to be injected, a small, firm, soft-rubber tube should be added to the laryngeal instrument long enough to reach through the glottis.

It is unnecessary to say that the fluids to be injected should always be rather bland, and only a very small quantity should be injected at a sitting. For the larynx the fluid should always be of thin consistence, for if mucilaginous or oily it may produce serious spasm of the glottis. The immediate effects of this procedure vary greatly. Patients suffering from chronic structural disease of the larynx bear the treatment very well indeed, whereas those suffering from the acute or subacute affections are apt to experience great distress and pain from these injections.

By the advocates of tracheal injections it is said that very little distress or coughing follows the injection of mixtures of the essential oils, creosote, etc., into the cavity of the trachea; but according to the writer's limited experience with this method of treatment, the suffering of the patients from cough and other distressing feelings has been very great, excepting in cases of extensive ulceration and exudation in the larynx, or where there was profuse thick secretion on the walls of the trachea. 


\section{Inhalations of Spray and Medicated Air}

There are many cases in which the inhalation of either a nebula (spray) or medicated air will be indicated. For deeper inhalation, an apparatus provided with a globe or vessel into which the spray is propelled, and from which it can be inhaled, is necessary. This instrument, in order to be efficacious, should be provided with a closely fitting shield of rubber for inclosing the face (see globe inhaler, Fig. 213). Oleaginous mixtures are generally preferable for use in this apparatus.

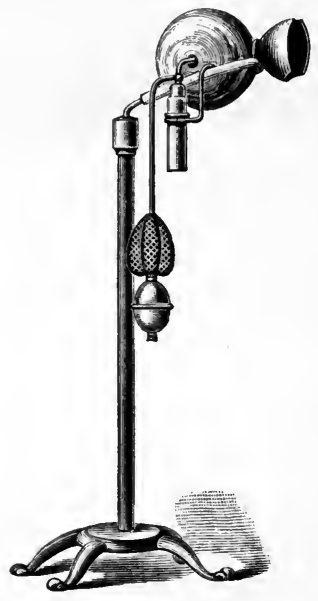

FIG. 213.-Shurly's globe inhaler with rubber face shield (Hartz \& Co.).

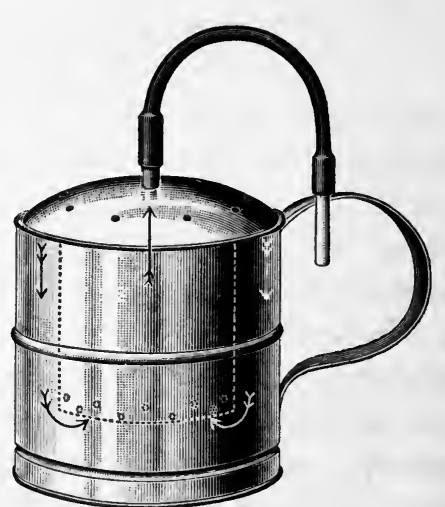

FIG. 214.-Shurly's hot-water inhaler (Hartz \& Co.).

Medicated Air.-Medicated air for inhalation may be obtained either by the use of a hot-water inhaler (see Fig. 214) or a dry inhaler applied over the nose and mouth. The former may be made of tin, in the shape of a can with a removable cover, the margin of the cover being perforated with small holes for the admission of air. On the inside of the cover a cylinder about three inches in diameter is soldered, so that it dips down into the mixture of hot water and medicament.

The centre of the upper surface of the cover is provided with a nipple, over which is fitted a piece of rubber tubing about one eighth of an inch in diameter and sixteen inches long, to the distal end of which is fitted a piece of glass tubing for a mouth-piece. 
The inhaler should be large enough to hold about a quart of water. To prepare this inhaler for use, pour in about a pint of very hot water, then add the medicament, and put on the cover with the rubber tubing and mouth-piece attached. The inhaler may now be placed upon a stand, and the patient, seating himself before it, winds a few strips of cloth (which have been soaked in cold water) around the mouth-piece to keep it cool, places the latter in his mouth, firmly closing the lips around it, holds the nostrils closed between the finger and thumb of one hand, and slowly inhales through the tube; then freeing the nostrils, exhales through the nose, and so on alternately. The patient must be made to understand that he is not to inhale steam, but the air which bubbles through the fluid in the inhaler. He should not inhale too forcibly, lest he draw hot water into his mouth. The patient should not go out of doors for at least half an hour after a period of inhalation from a hot-water inhaler, a steam atomizer, or steam inhaler. Each inhalation treatment should consume from ten to twenty minutes, or until the fluid in the inhaler becomes cool.

The dry inhaler (see Fig. 215) is provided with a sponge at its back part upon which the medicament is dropped in the

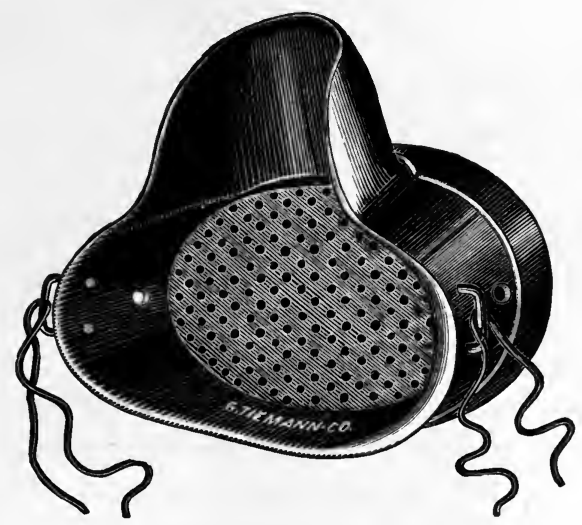

FIG. 215.-Tyndale's antiseptic oro-nasal respirator.

prescribed quantity, after which the instrument is held over the nose and mouth for from ten to fifteen minutes or longer, while the patient breathes through it. Any volatile substance can be used in this form of inhaler (see formulary). 
For the inhalation of gases, oxygen, nitrogen, compressed air, etc., a special apparatus is usually employed (see Fig. 216).

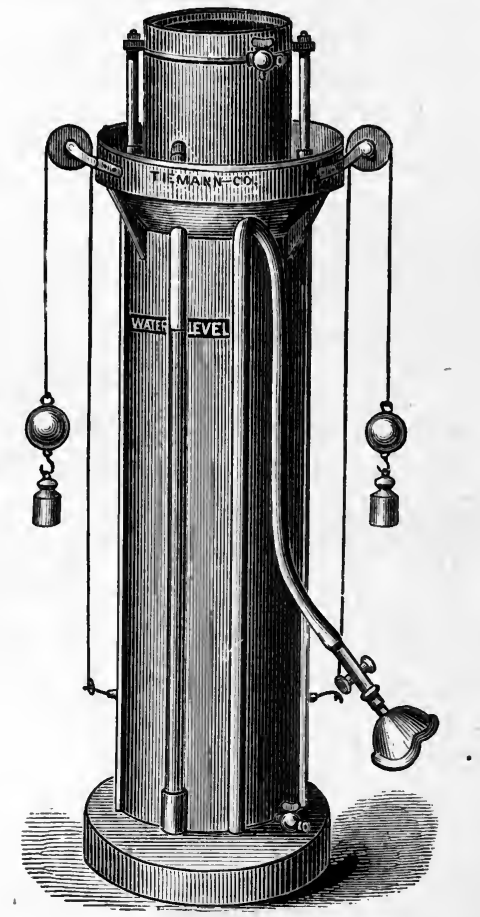

FIG. 2I6.-Tobold's pneumatic apparatus.

An apparatus can be easily improvised, however, by the employment of two large cans or cylinders, each with one open end, one having a little greater diameter than the other. The larger one is set on the floor, open end up, and partially filled with water. The smaller one is passed into it, closed end up. There should be a nipple and stop-cock on the top of the inner cylinder provided with a piece of rubber tubing through which the patient is to inhale. Weights of any desirable density may be placed on the top of this inner cylinder, which will serve to compress the air or gas contained therein and drive it out through the inhaling tube.

Fuming inhalations are sometimes used in throat diseases, as well as for the treatment of asthma and other pulmonary affections. This is usually accomplished by burning the medicament in an iron or porcelain kettle which has been placed in a pan of water. The patient may inhale the fumes as mixed with 
the air of the room, or he may inhale them through a cylinder made of paper which is held closely to the vessel. Oil of turpentine and tar are often inhaled in this manner, as well as other oils or inflammable substances. In syphilis it is sometimes necessary to apply the fumes of mercury. A little calomel is placed in a porcelain receptacle, and the alcohol lamp below is lighted, and as soon as the fumes from the calomel are liberated they are to be blown into the nostril or pharynx by means of a double rubber bulb, or drawn in by the breath.

\section{Surgical Methods of Local Treatment}

Curettement.-Curettement of tuberculous or other ulcers is frequently resorted to. Curettement of the larynx for the relief

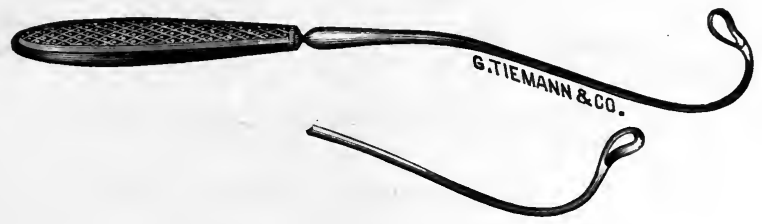

FIG. 217.-Cohen's laryngeal curette.

of laryngeal phthisis has been steadfastly advocated by Heryng, Cohen, Krause, Gleitsman, and others. Specially shaped or curved instruments for this purpose have been devised by the above-named observers and others, and are kept at the instrument makers (see Fig. 217). Before the use of any of these in-

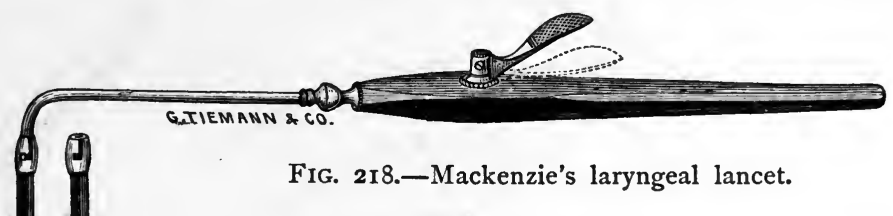

struments the parts should be anæsthetized, as a rule, by the application of a solution or pigment of cocaine (ten to twenty per cent).

Scarification may be done in the fauces or nasal passages by any sort of a small bistoury, the edge of which has been covered to near its point by surgical plaster or cloth. But for scarification of the epiglottis or arytenoid region of the larynx a small curved blade will be necessary or specially devised laryngeal knives (see Fig. 218). 
Evulsion, abscision, and incision are performed by means of forceps, scissors, or knives of particular design, according to the region to be operated upon. These instruments are mentioned in the foregoing chapters.

5
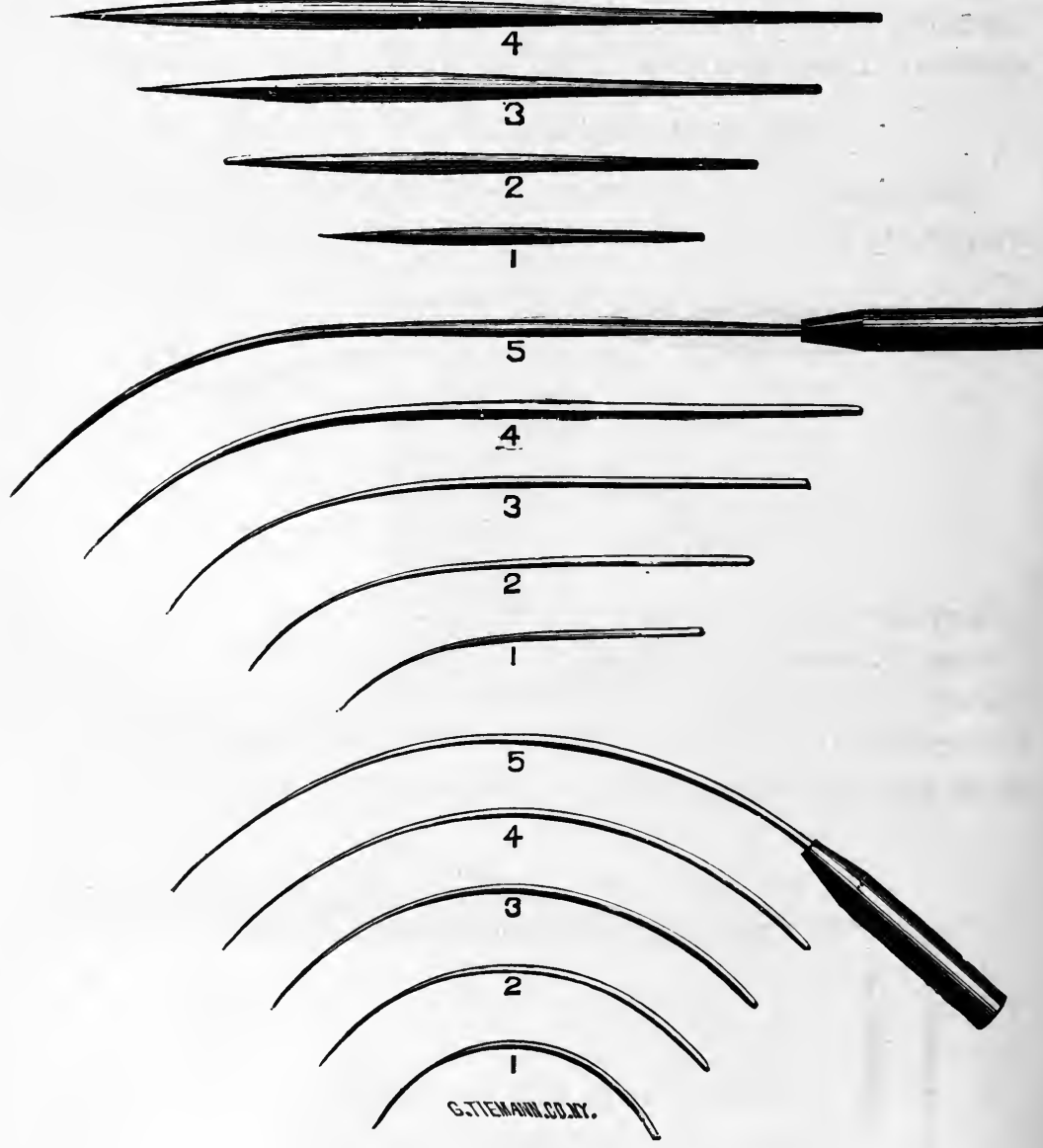

FIG. 2I9.-Needles for electrolysis.

Electrolysis is usually accomplished by needles carrying a current of electricity which are inserted into the tissues; one represents the positive and the other the negative pole of a battery (see Fig. 219).

There are two methods of operating, the monopolar and the 

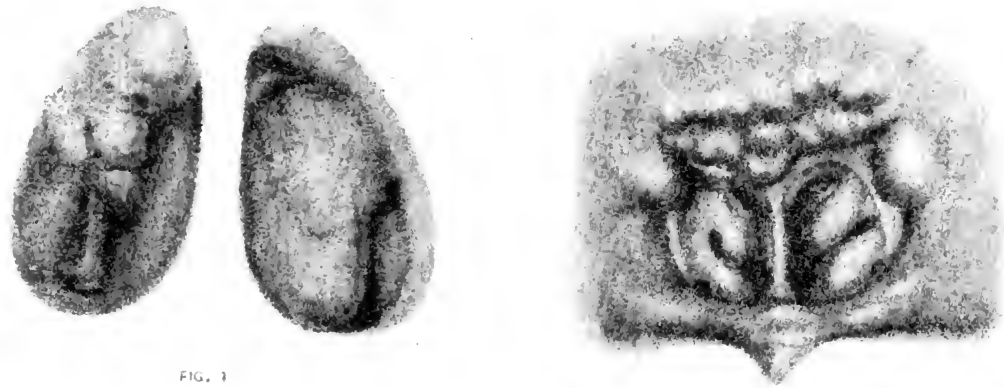

\section{Plate I.}

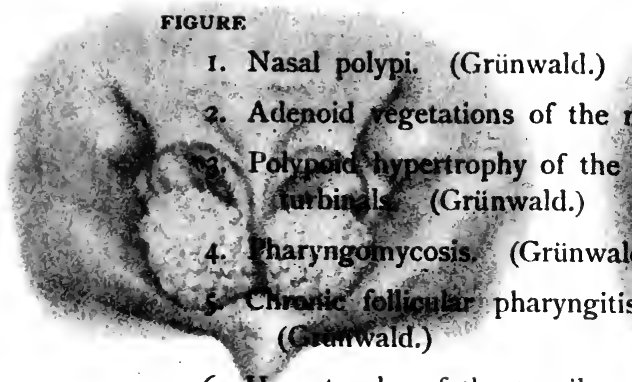

6. Hypertrophy of the tonsils.

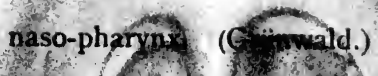

posterior end of the inferior

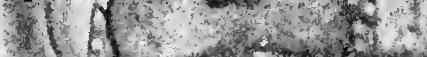

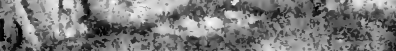
Wh lateral hypertrophy. Wo

(Grünwald.)
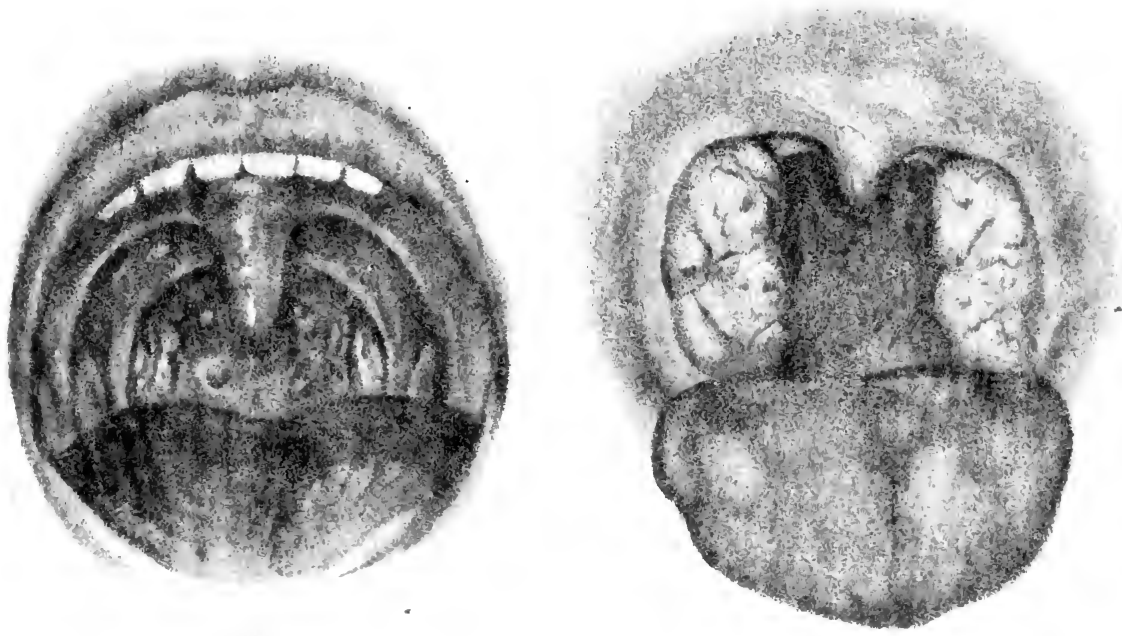
Evulsion, abscision, and incision are performed by means of forceps, scissors, or knives of particular design, according to the region to be operated upon. These instruments are mentioned in the foregoing chapters.

5
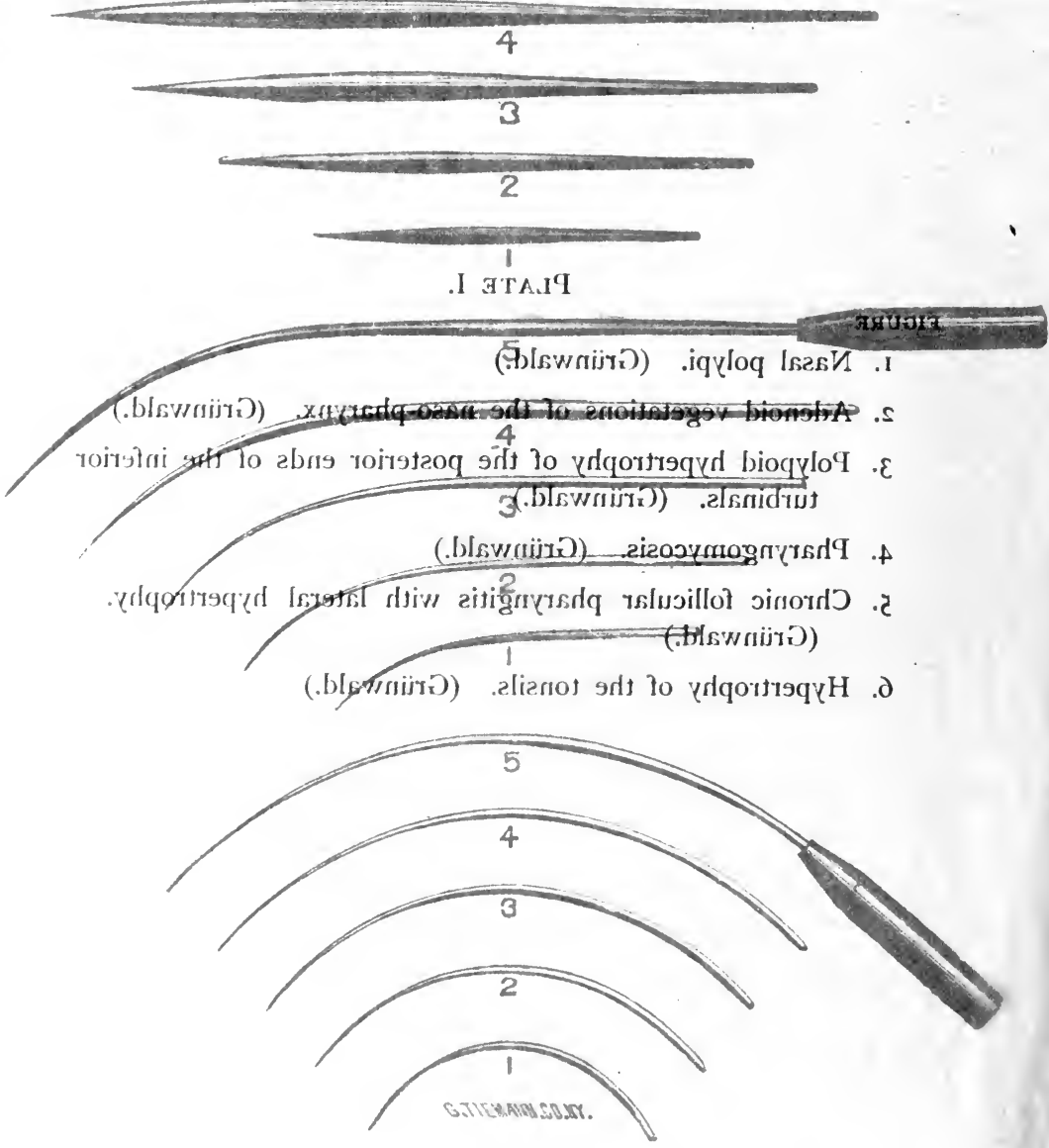

FIG. $219 .-$ Needles for electrolysis.

Eictrolyes is usually accomplished by needles carrying a current of electricity which are inserted into the tissues; one represents the positive and the other the negative pole of a battery (see Fig. 219).

There are two methods of operating, the monopolar and the 


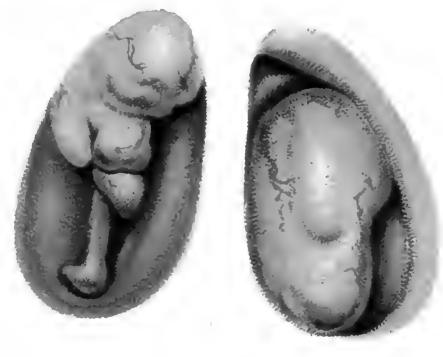

FIG. 1 .

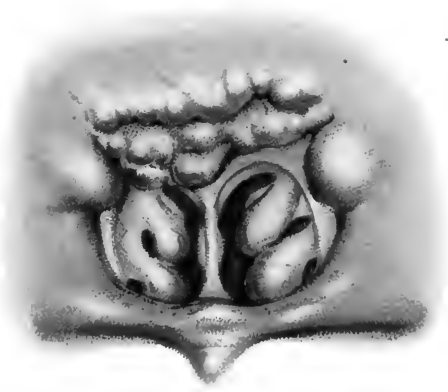

FIG. 2.
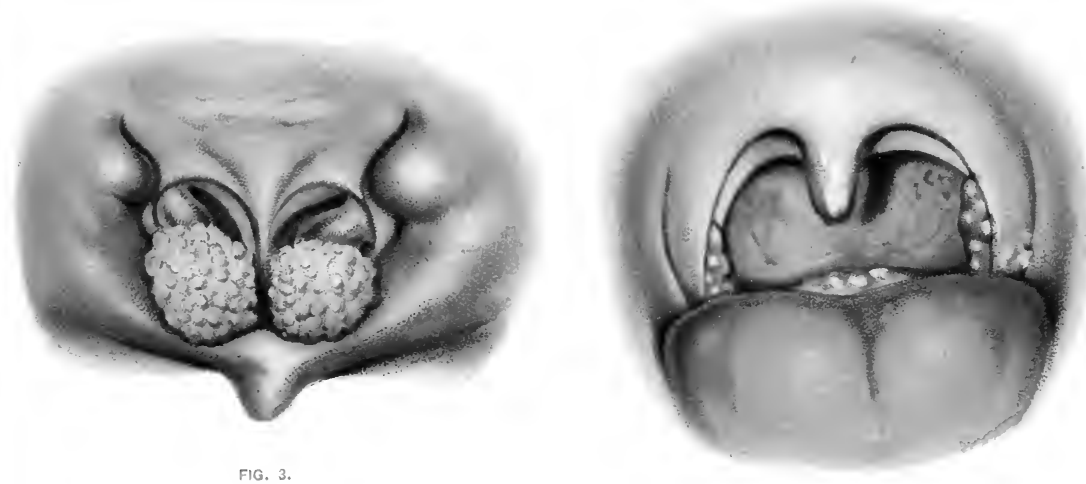

FIG. 3 .
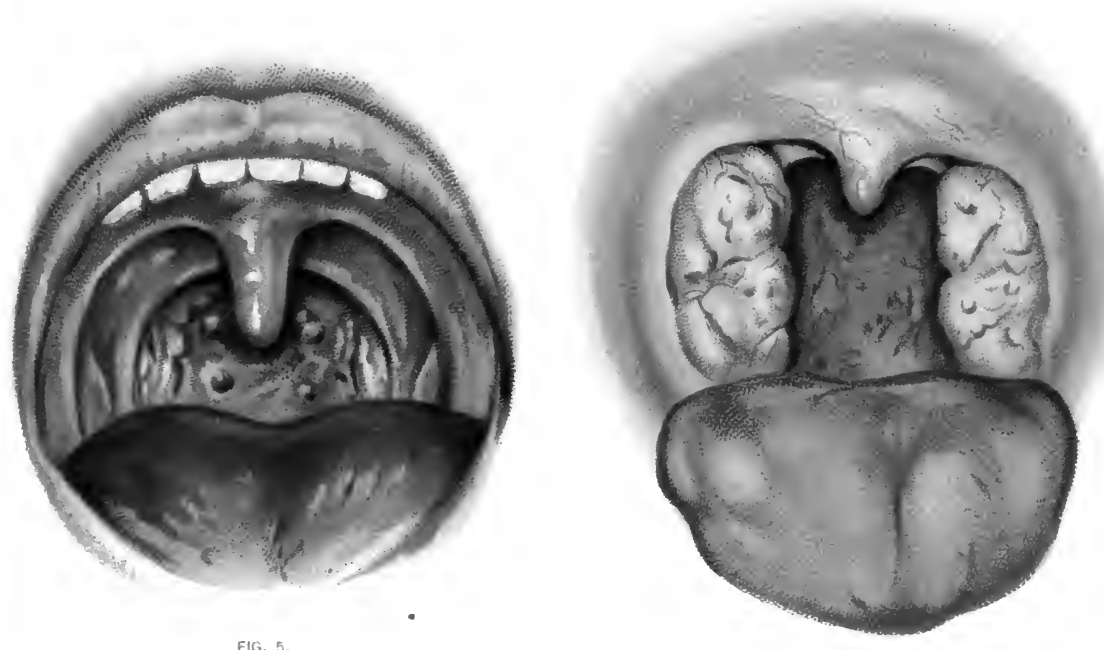

FIG. 6. 



\section{PLATL}
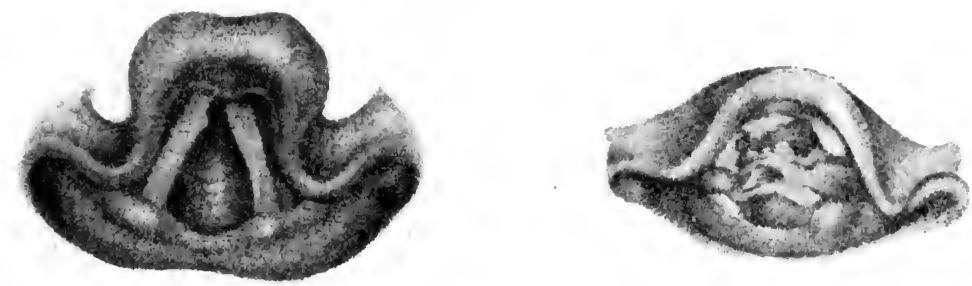

frg, 1.

\section{Plate II.}

FIGURE

I. Hard wart. (Grünwald.)

2. Lupus, cicatrized loss of substance of epiglottis growths on

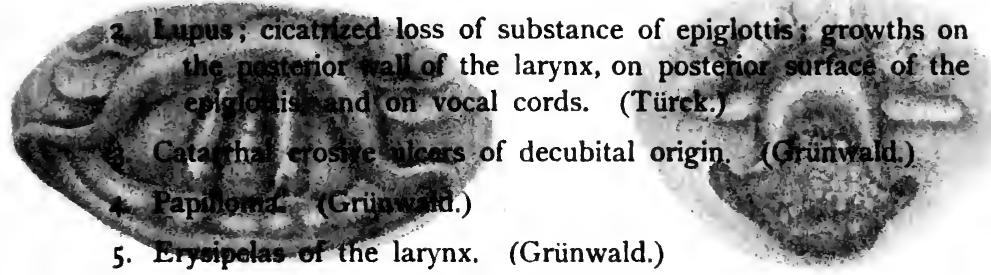

6. Normal larynx during deep respiration. (Schnitzler.)
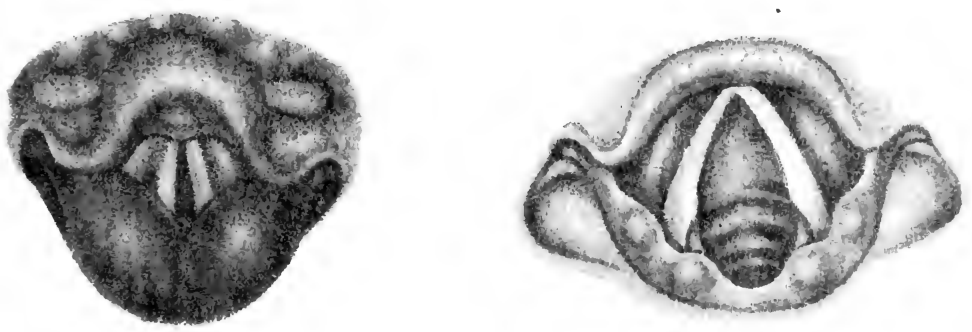
.II GTA.IT

ฐяบอเส

(.blswnijy) .trow broH .I

no adfworg : aitfolgiqg to sonstedue to 2201 b9sintsois ; auquJ $s$

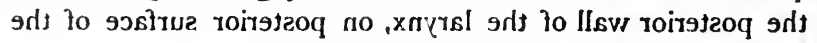

(.ฟวrij T) .ebros lssov no bns, 2ittolgiqg

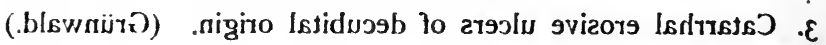

(.blswniujz) .smolliqsq .t

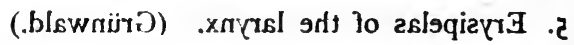

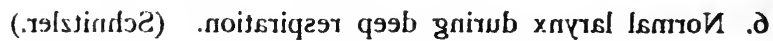


PLATE .II.

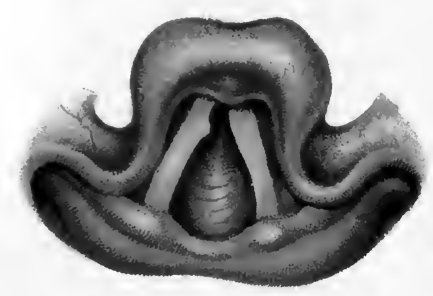

FIG. 1.

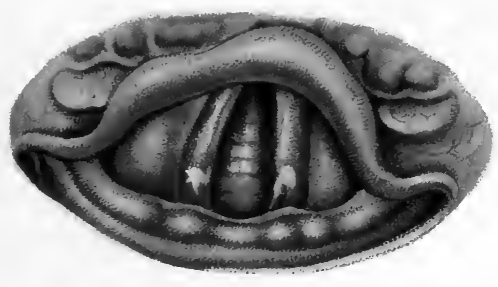

FIG. 3.

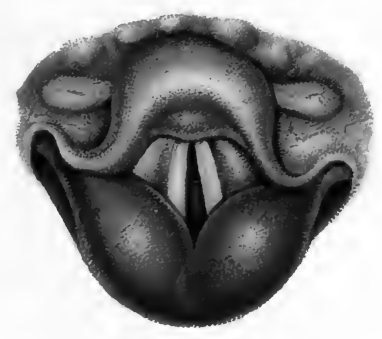

FIG. 5.

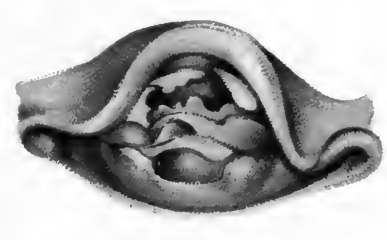

FIG. 2.

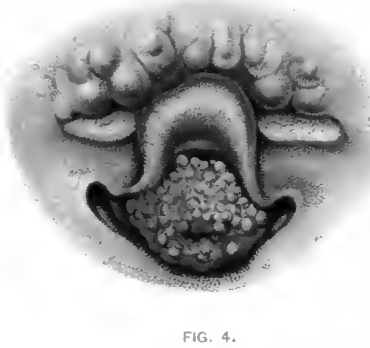

、

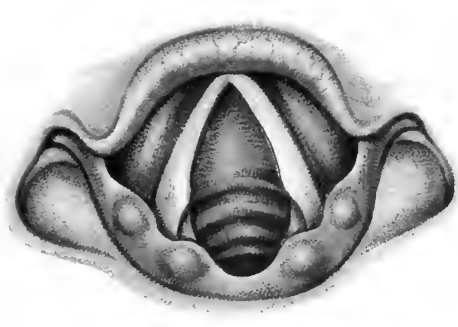

FIG. 6. 



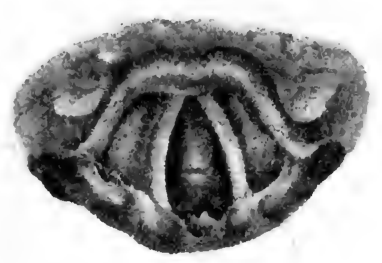

16.

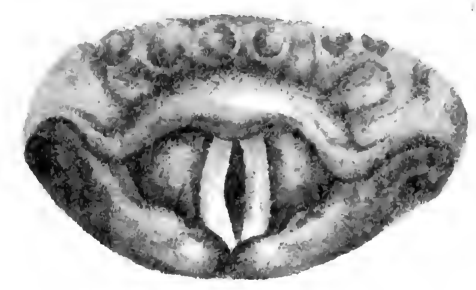

i. . .

Plate III.

FIGURE

I. First stage of tubercular infiltration. (Grinwald.)

a Multhie tubercle infiltration in early stage. (Gruinwald.)

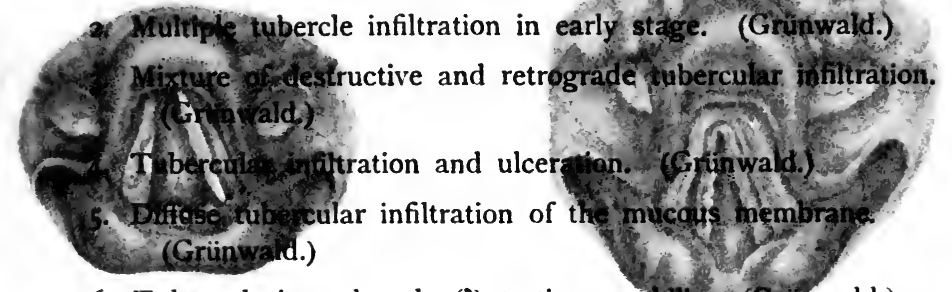

6. Tuberculosis and early (?) tertiary syphilis. (Grinwald.)
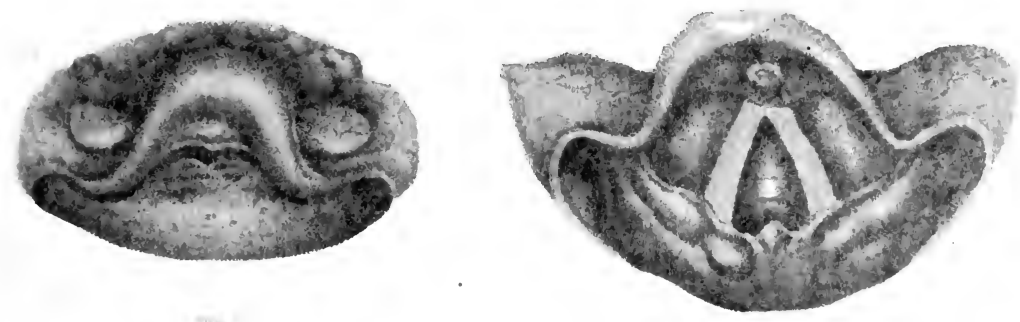


\section{.III GTAJT}

สหบบวา

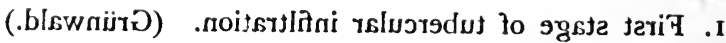
(blswnijy) .9gste үhss ni noifstslini glorsduf slqitluM s

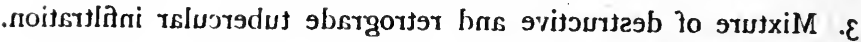
(.blswrijizD)

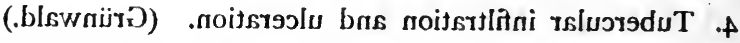
כ. (.blswniugz)

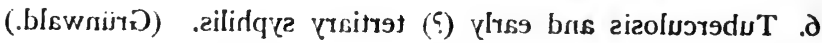




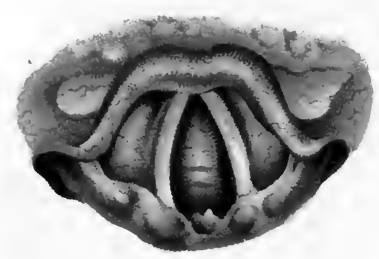

FIG. 1.

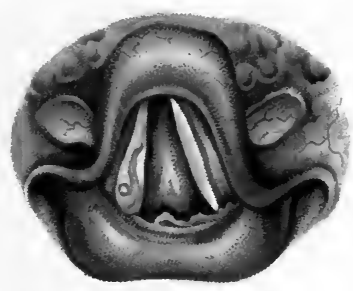

FIG. 3.

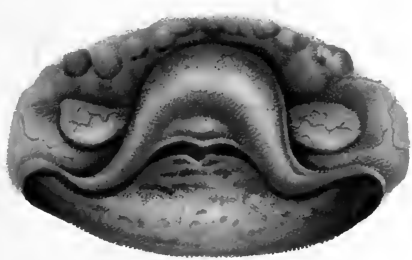

FIG. 5.

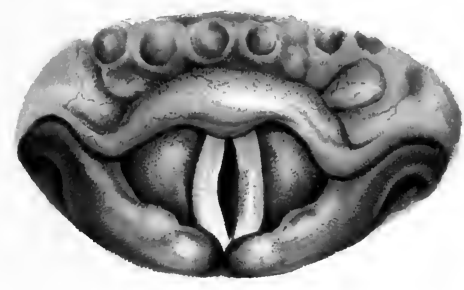

FIG. 2 .

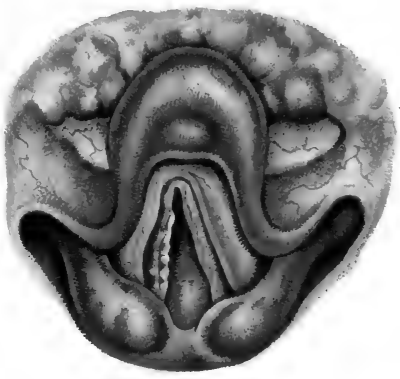

FIG. 4.

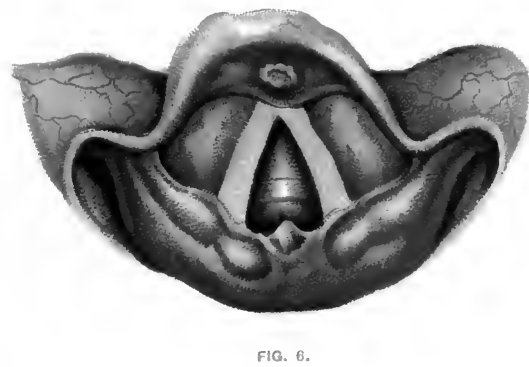




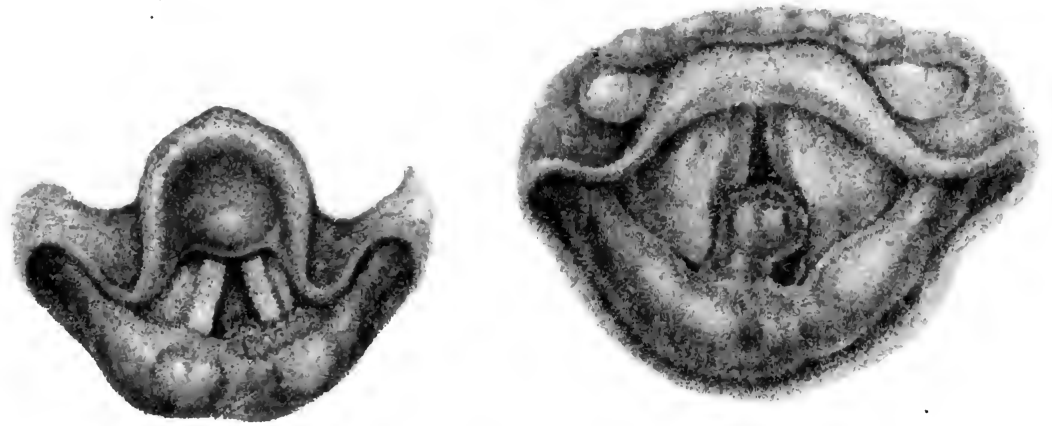

Plate IV.

FIGURE

I. Hyperplastic tuberculosis. (Grïnwald.)

2. Diffuse tubercular infiltration of the whole laiginx and tumour. (Grünwald.)

3. Tubercular fumours: (Griinwald.)

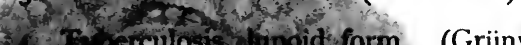

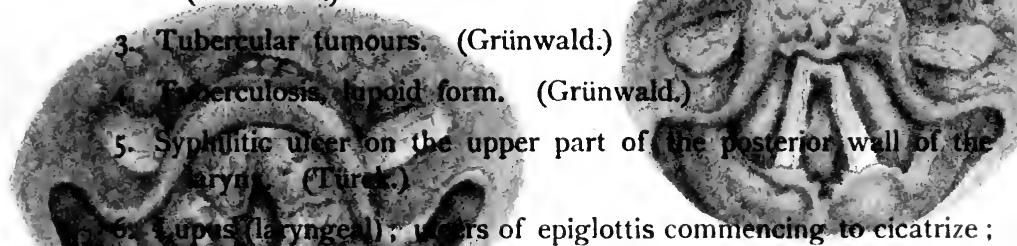

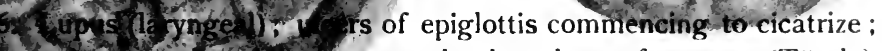
Whephy of nucous glands at base of tongue. (Türck.)
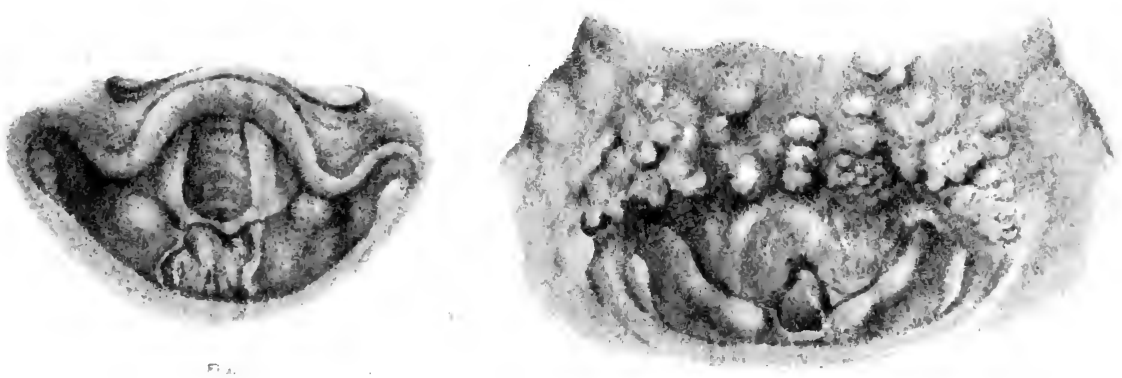


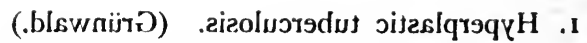

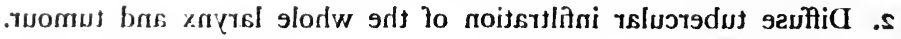
(.blswnijid)

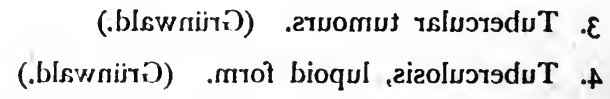

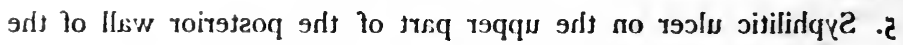

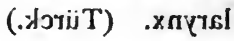

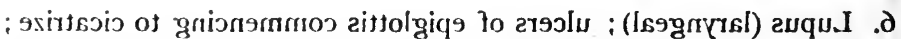

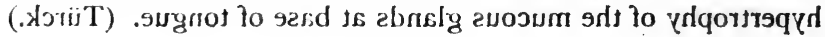




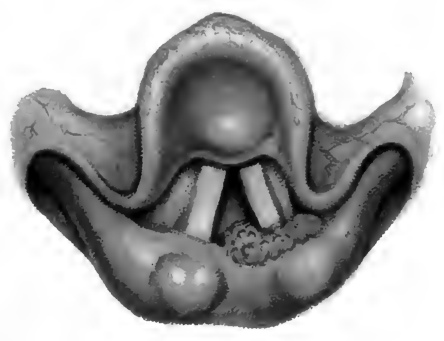

FIG. 1.

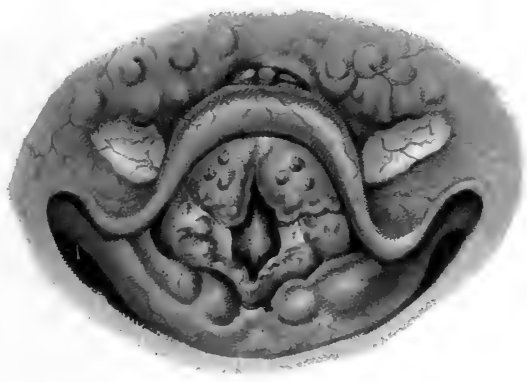

FG. 3.

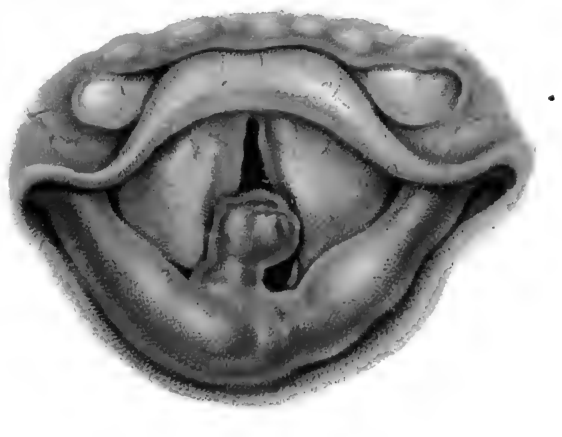

FIG, 2 .

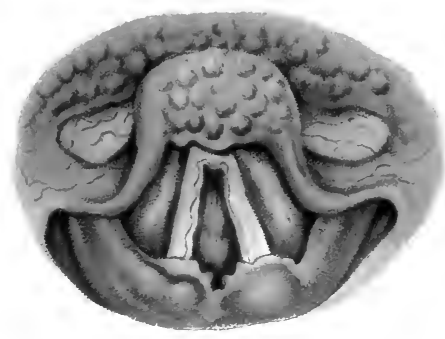

FIG. 4.
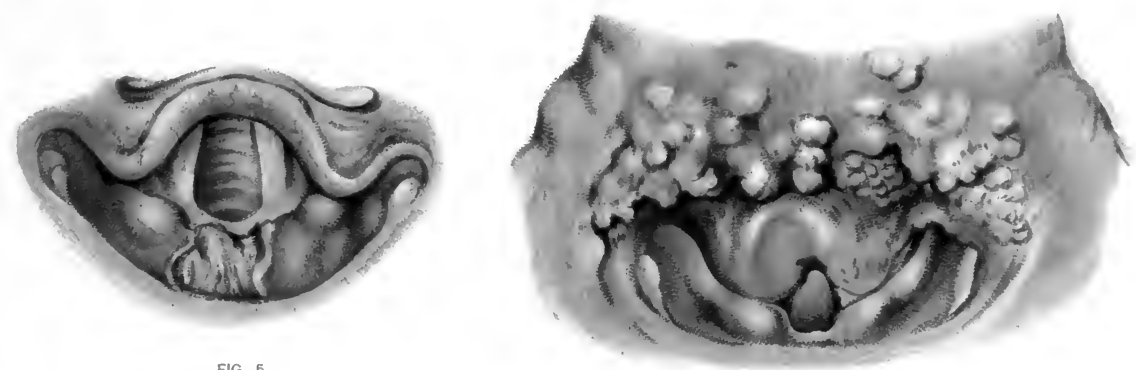

FIG. 5 . 


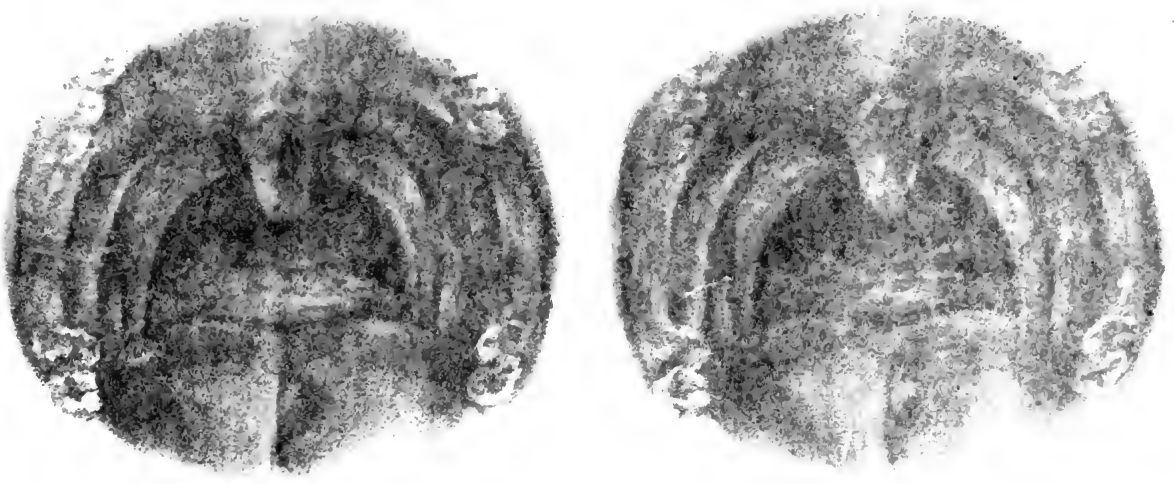

Plate $V$.

FIGURE
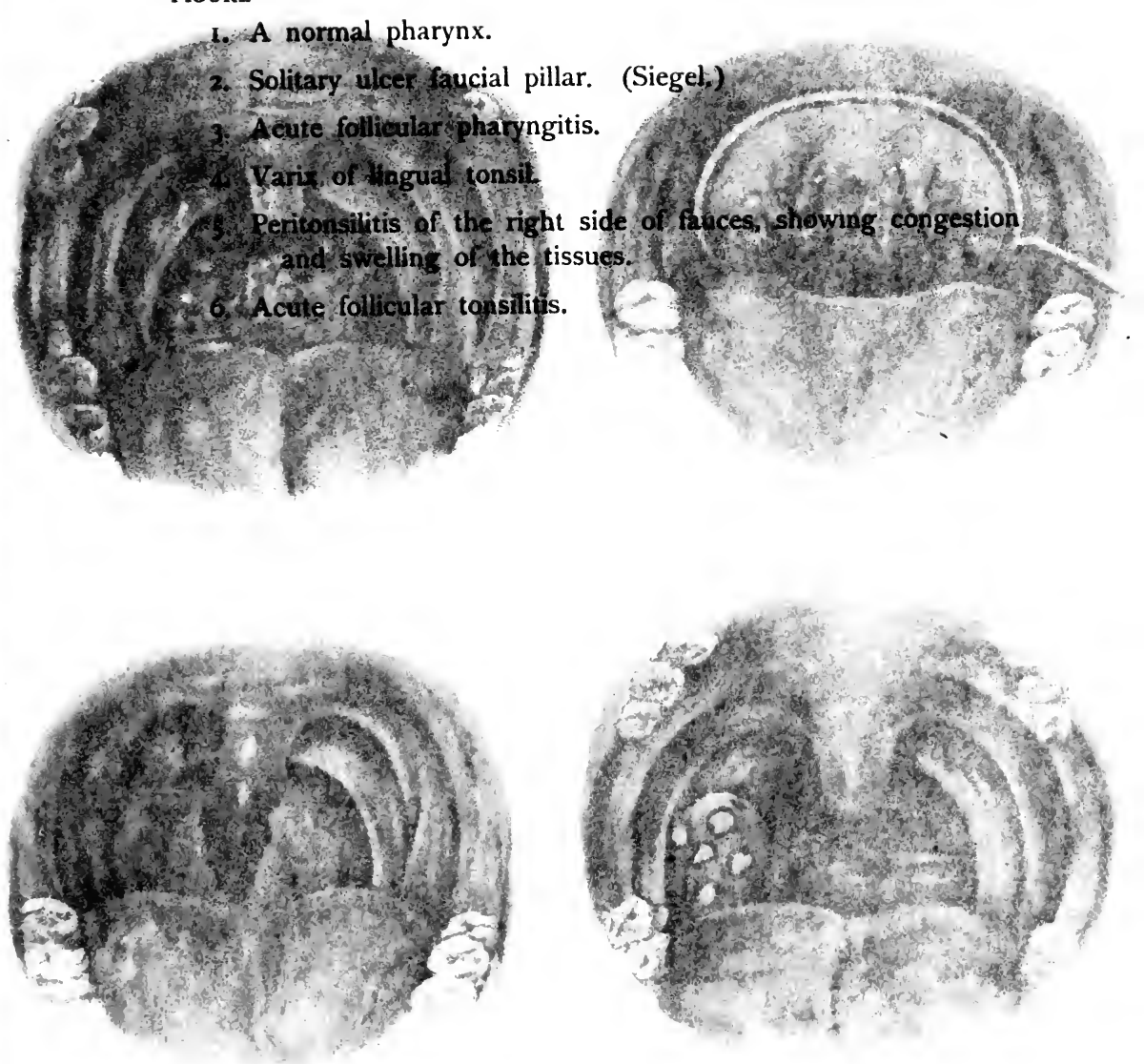
YTAI

उяบอเ7

.xnyтshq Ismion $A$.I

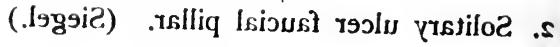

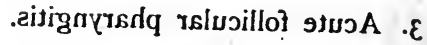
lianot Isugnil to xirs $\mathrm{V}$.

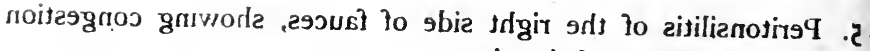
.esuezis snts to gnillswe bns

.aifilianof тslusillol gfuวA.d 

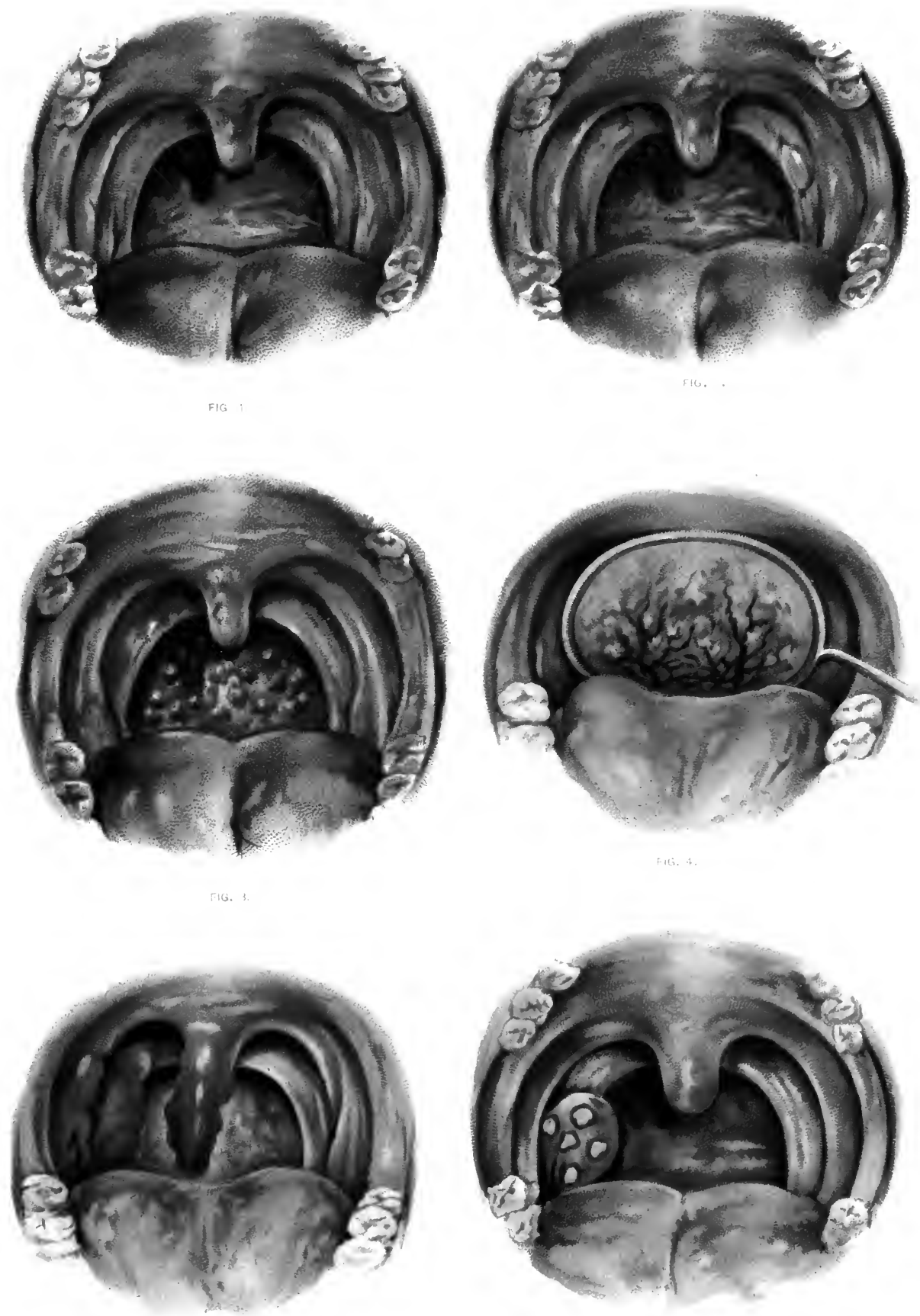


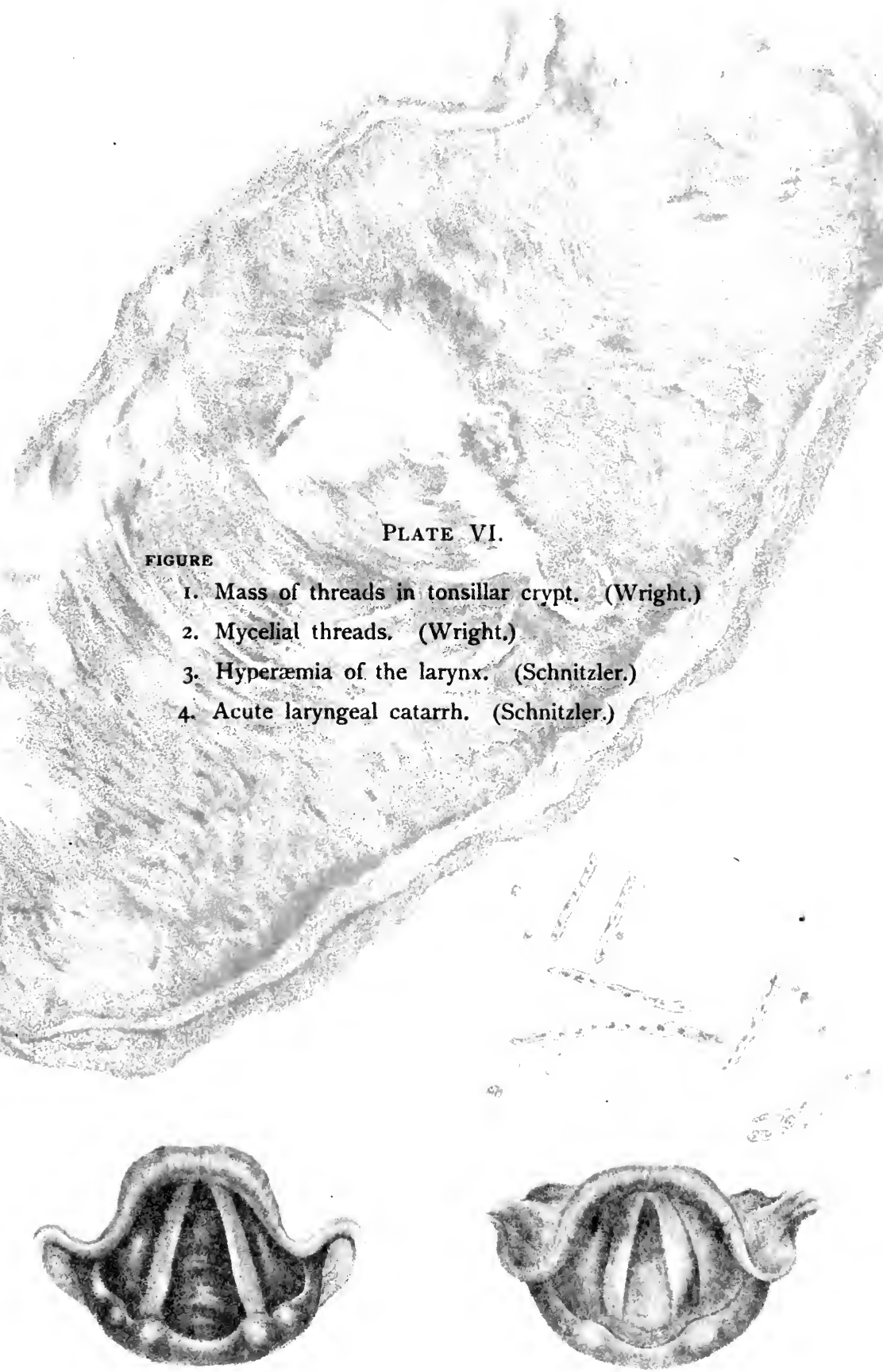


\section{GTAI}

สหบอเศ

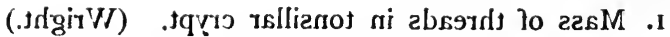

(.thgirW) .absordt Isilsoy $\mathrm{M}$.s (19lstindoद) . .

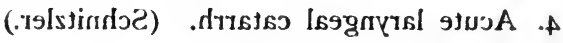



bipolar. The former consists of the insertion of one needle representing the negative pole into the tissues, while the other pole, represented by an electrode instead of a needle, is placed somewhere on the surface of the body, preferably the neck.

The bipolar method consists of the insertion of both needles into the tissues. There are handles made which conveniently hold both needles. In lieu of this, however, the operator can hold.a needle in each hand.

Cauterization.-The galvano-cautery is generally used for nose and throat operations, because the thermo-cautery is too cumbersome an instrument. The apparatus for this purpose

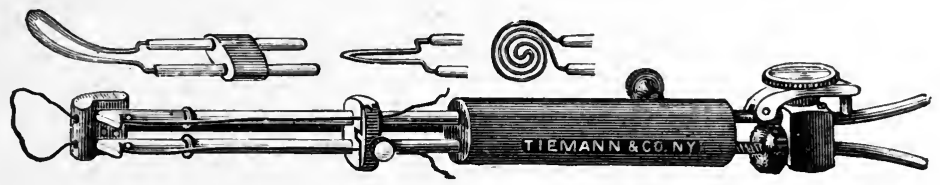

Fig. 220.-Dawson's universal cautery electrode.

is described in the article on hypertrophic rhinitis with the exception of a universal electro-cautery handle (see Fig. 220), and the electric snare devised for operating on the tonsils or neoplasms, which is shown at Fig. $22 \mathrm{I}$.

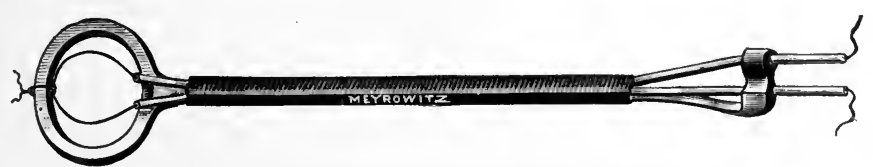

FIG. 221.-C. H. Knight's electric tonsil snare.

For the application of the galvanic or faradic current to the upper air passages specially devised electrodes are required.

GTIEMAMGONY.

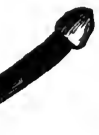

Fig. 222.-Nasal electrode.

For the nasal passages and fauces an electrode, about four inches long, with a globular or ball-like extremity, will answer the purpose (see Fig. 222). For the larynx, one shaped like 
laryngeal forceps, provided with a cut-off at the handle, will be necessary. The one devised by M. Mackenzie is among the best for this purpose (see Fig. 223).

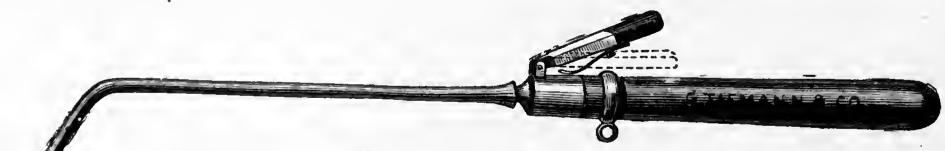

Fig. 223.-Mackenzie's laryngeal clectrode.

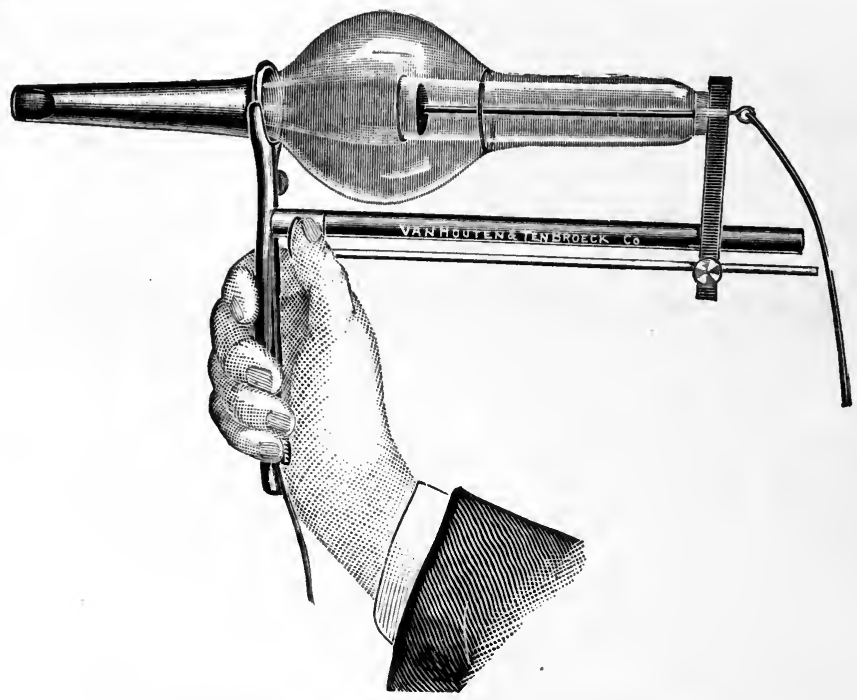

Fig. 224.-Caldwell's throat X-ray tube.

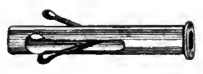

A. Canula.

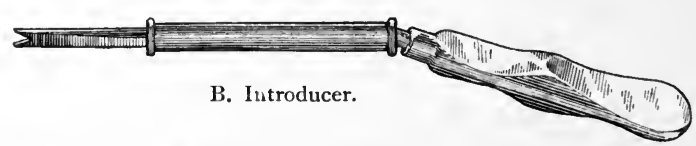

FIG. 225.-Dr. B. R. Shurly's self-retaining metal drainage tube for antrum.

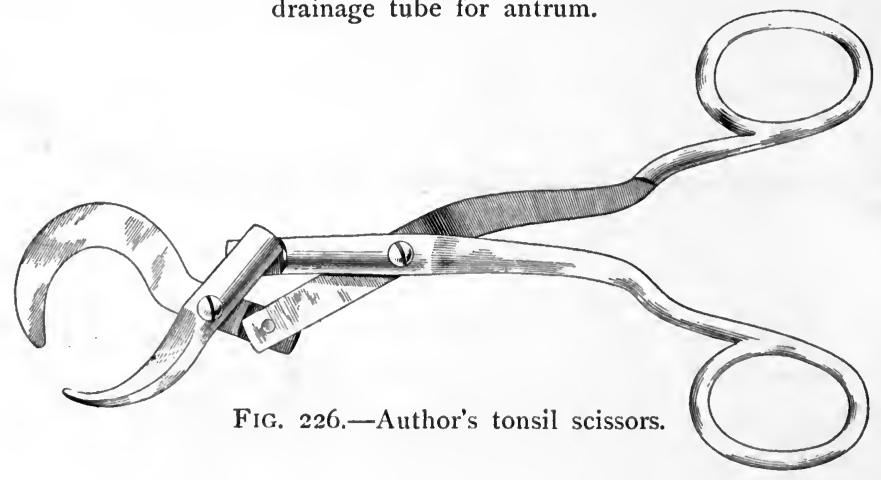




\section{FORMULARY}

\section{SPRAYS}

All aqueous solutions for use in atomizers should be filtered.

Dobell's solution :

R Sodii biboratis.... 3 iv;

Sodii bicarbonatis.. 3 iv ;

Acidi carbolici..... gr. xlviii;

Glycerini ........ $3 \mathrm{j}$;

Aquæ.......q. s. ad Oij.

M. (Cleansing.) This ought to be diluted.

Seiler's solution:

R Sodii bicarb....... 3 viij;

Sodii bibor....... 3 viij;

Sodii benzoatis,

Sodii salicylatis...āā gr. xx ;

Eucalyptol,

Thymol.........āā gr. x ;

Menthol .......... gr. v;

Ol. gaultheriæ...... gtt. vj;

Glycerini......... $\xi$ viijss. ;

Alcoholis.......... $3 \mathrm{ij}$;

Aquæ.......q. s. ad Oxvj.

Re Ammonii chloridi, gr. $x 1-1 \times x x$;

Aquæ destillatæ vel,

Aquæ mentholis, 3 viij.

M. (Cleansing and mildly stimulating.)

R Sodii benz............. gr. v;

Acidi carbolici ........ gr. j;

Glycerini.......... $3 \mathrm{j}$;

Aquæ destillatæ...... $3 \mathrm{vij}$. ing.)

M. (Antiseptic and cleans-
R Sodii benz...........gr. xl;

Aquæ calcis......... ₹ viij.

M. (Cleansing; useful in all forms of acute coryza.)

R Hydrargyri bichloridi ........... gr. $\mathrm{j}$;

Aquæ cinnamomi.. 3 viij-xvj.

M. (Antiseptic; beneficial in syphilitic lesions and all ulcerations.)

R Sodii bicarbonatis... gr. $x \times v$;

Aq. destillatæ...... $\xi \mathrm{v}$;

Ext. hamamelidis dest $\xi \mathrm{j}$.

M. (Cleansing and astringent.)

R Sodii bicarbonatis.. gr. xl-lx; Aquæ destillatæ,

Aquæ picis......āā $\bar{z}$ iv.

M. (Cleansing and stimulating.)

Aqua menthol:

R Mentholis........... gr. j;

Alcoholis........... m $\mathrm{m}$;

Calc. phosphat. prec... gr. v;

Aquæ ............. s. ad $\xi \mathrm{ij}$.

M. (Anodyne.)

R Cocainæ............... gr. v;

Alumnol............. gr. $x$;

Aquæ menthol ....... $3 \mathrm{j}$.

(Astringent for pharynx or $\operatorname{larynx}$.) 
R Lead acet............ gr. v; Potass. acet.......... gr. $\mathrm{x}$ Aquæ destillatæ...... § iv. M. (Strong astringent.)

R Aquæ.............. $\xi$ viij; Creolin ............ $3 \mathrm{j}$.

M. (Antiseptic; for spray or douche.)

Re Ichthyol......... 3 ss.-j; Aquæ............. 3 iv.

M. (Stimulant and antiseptic, beneficial for ozæna.)

R Iodi................ gr. ij;

Pot. iodidi........... gr. v ;

Aquæ............. $\xi \mathrm{ij}$.

M. (Stimulant.)

R Extracti opii aq...... 3 j-ij; Glycerini .......... 3 iv; Aquæ ..........q. s. ad $\mathrm{Oj}$.

M. (Anodyne.)

R Menthol........... gr. v;

Glycerini........... $\xi \mathrm{ij}$

Cocainæ.......... gr. xx ; Aquæ............. $3 \mathrm{ij}$.

M. (Analgesic and astringent.)

B Cocainæ hydrochloratis............ gr. $\mathrm{ij}-\mathrm{v}$; Aquæ menthol...... $\overline{3} \mathrm{j}$.

M. (Anodyne.)

R Ferri et ammonii sulphatis....... gr. $x 1-1 x$; Aquæ destillatæ... 弓 viij.

M. (Strongly astringent ; should not be used in the nose, excepting for hemorrhage.)
R Zinci sulphatis, gr. $v-x-x v-x x$; Aquæ destillatæ...... $\xi$ viij.

M. (Astringent; not to be used in the nose.)

R Acidi tannici.... gr. $\mathrm{v}-\mathrm{x}-\mathrm{xv}$; Alcoholis ....... z ss. ; Aquæ destillatæ. 3 viij. M. (General astringent.)

B Potassii chloratis..... $3 \mathrm{j}$; Aquæ destillatæ vel, Aquæ cinnamomi...... $\xi$ viij. M. (Cleansing.)

Re Potassii chloratis..... gr. xl; Aquæ destillatæ...... $\xi \mathrm{v}$; Extracti hamamelidis, destillati......... $\xi \mathrm{j}$.

M. (Cleansing and slightly astringent.)

R Sodii chloridi........ $3 \mathrm{j}$;

Creosoti........... 3 ss.; Aquæ............ $\xi \mathrm{xv}$. tic.)

M. (Cleansing and antisep-

R Sodii phos......... $3 \mathrm{j}$;

Glycerini........... $\xi \mathrm{j}$;

Aquæ............. $\xi \times v$.

M. (Cleansing.)

R Acidi carbolici....... gr. $\mathrm{xvj}$; Glycerini ......... $3 \mathrm{ij}$; Aquæ destillatæ..... 3 xiv. M. (Antiseptic.)

R Resorcini............ gr. xl; Glycerini........... $\xi \mathrm{j}$; Aquæ.............. $\xi$ vij. M. (Antiseptic.) 
B Resorcini......... 3 ijss.; Potassi chloratis.... 3 jss. ; Aquæ........... s. ad Oj.

M. (Antiseptic and cleansing.)

R Extracti arboris vitæ aquosi.

(Astringent ; useful for applications to papillomata and granulation tissue.)

R Aluminis......... gr. $\mathrm{xl}-1 \mathrm{x}$; Acidi carbolici..... gr. $\mathrm{xv}$;

Glycerini ........ $\xi \mathrm{ij}$;

Aquæ destillatæ... $\xi \mathrm{vj.}$

M. (Astringent.)

R Sodii format.......... gr. $\mathrm{x}$;

Aquæ destillatæ...... $\xi \mathrm{j}$.

M. (Antiseptic.)

R Potass. permang..... 3 ss.; Aquæ destillatæ...... Oj.

M. (Antiseptic.)

R Xanthoxyli, fluidi extracti........... $3 \mathrm{j}$;

Spts. rectific....... $3 \mathrm{ij}$;

Sodii phosphatis..... $3 \mathrm{j}$;

Glycerini.......... 3 iv ;

Conin hydrochloratis.. gr. $\mathrm{j}$; Aquæ destillatæ, q. s. ad $\xi$ viij.

M. (Stimulant ; beneficial for atrophic pharyngitis.)

\section{OLEAGINOUS SPRAYS}

(The following sprays should, if possible, be used in atomizers especially designed for spraying oils. It is not necessary, as a rule, to filter these solutions.)

R Olei picis.......... $3 \mathrm{i}-\mathrm{ij}$; Olei gossyp........ $z^{\mathrm{viij}}$. M.
R Olei eucalypti....... $3 \mathrm{j}$;

Olei petrolinæ....... $\xi$ viij. M.

R Naphthalini ........ gr. $\mathrm{xxx}$; Olei petrolinæ..... $\xi$ viij. M.

R Olei rosemarini, Olei eucalypti...... āā $3 j$; Olei petrolati..q. s. ad $\mathrm{Oj}$. M.

R Iodoformi......... gr. $\mathrm{xxx}$; Olei petrolati......... Oj. M.

R Ol. terebinthi........m $m \mathrm{xl}$;

Ol. petrolinæ........ $\xi$ viij. M.

B Ol. eucalypti....... $3 \mathrm{ij}$;

Ol. gossyp......... 3 viij. M.

R Camphoræ.......... 3 ij;

Ol. petrolinæ....... 3 viij. M.

B Thymol........... gr. $x x$; Ol. petrolinæ........ $\xi$ viij. M.

R Creosoti........... 3 iij; Ol. petrolinæ........ 3 viij. M.

R Ac. carbolici........ 3 ss.; Ol. petrolinæ........ $\xi$ viij. $\mathrm{M}$.

Kyle's formula:

R Camphoræ........... gr. j; Menthol........... gr. iij; Ac. carbolici ....... mij; Albolini............ $\xi \mathrm{j}$. M. 
"Chloretone inhalant" (McClintock's) :

R Chloretone........ gr. vijss.;

Camphoræ........ gr. lxxv;

Menthol.......... gr. 1xxv;

Ol. cinnamoni...... m vijss.;

Ol. petrolinæ..... 3 xxvi.

M.

R Creosoti.........m $\mathrm{m}$;

Spts. chloroform..... 弓 ss. ;

Iodoformi.......... gr. xvj;

Olei gossyp .....q. s. $\bar{z}$ viij. M.

Alum emulsion :

R Aluminis.......... 3 ss.;

Ol. petrolinæ....... $3 \mathrm{vij}$;

Ol. olivæ.......... $3 \mathrm{j}$;

Pulv. acaciæ........ 3 iv;

Aq. calcis........... $\xi \mathrm{j}$;

Aquæ.............s. ad $\xi$ iv.

M.

\section{INHALANTS}

R Acidi carbolici...... gr. $x \times x$;

Am. carb......... $\xi \mathrm{j}$;

Pulv. carb.-lig...... $3 \mathrm{j}$;

Olei lavandulæ...... $m \mathbf{x x}$;

Tr. benz. co...... 3 iv;

Camphoræ........ 3 ij.

M. (Smelling salts.)

Carbo-ligni mixture :

$\mathrm{R}$ Carbonis ligni.......... $3 \mathrm{j}$;

Ammonii carb...... 3 iij;

Tincturæ myrrhæ.... $3 \mathrm{j}$;

Olei limonis.........mij.

M. (Smelling salts.)

R Tinct. benzoin comp.,

One drachm to a pint of hot water.

$\mathrm{R}$ Olei eucalypti,

Half drachm to a pint of hot water.
R Creosoti,

Ten to fifteen minims to a pint of hot water.

Bartholow's formula:

R Acidi carbolici........ $3 \mathrm{j}$;

Tr. iodi............ 3 ss.

Ten to fifteen minims to a pint of hot water.

R Olei picis,

Twenty minims to one half drachm in a pint of hot water.

Re Spt. chloroformi,

Spt. ammonii.......... àā $\bar{z} j$.

M. Twenty to thirty minims in a pint of hot water.

R Acetozon......... gr. $\mathrm{v}$ ad $\mathrm{x}$.

In a pint or quart of hot water.

Be Ether. sulph.,

From twenty to thirty minims in a pint of hot water.

\section{OINTMENTS}

B Ac. carbol........... gr. ij ;

Iodin............. gr. iij;

Atrop. sul........... gr. ss.;

Ung. petrol. ........ $3 \mathrm{j}$.

M.

R Ac. carbol.......... gr. $\mathrm{xx}$;

Ung. petrol........ $3 \mathrm{j}$.

M.

R Iodoform........... gr. xx ;

Bal. Peruvian...... 3 ss.;

Ung. petrol........ $3 \mathrm{j}$.

M.

R Cannabin tannat..... gr. xv;

Vaseline ......... $\xi \mathrm{j}$.

M. 
R Hydrarg. oxid. flav.... gr. $\mathrm{x}$; Ung. petrol......... $3 \mathrm{j}$. M.

\section{DOUCHES}

R Ichthyoli.......... gr. $x x$; Sodii bicarbon....... gr. $\mathrm{xx}$; Aq. mentholis (sol. sat.) ........... $\xi^{\mathrm{ij} \text {; }}$ Aq. creosoti........ $\overline{3} \mathrm{ij}$; Aq. destill.......... 3 iv.

R Bismuth subcarb ...... $3 \mathrm{j}$; Liq. hydrastis, colourless............. $\mathrm{j}_{\mathrm{ij}}$; Boro-glyceride....... $\overline{3} \mathrm{j}$; Aqua destillatæ.....ad $\bar{\xi} \mathrm{xvj}$.

\section{PIGMENTS}

R Acidi tannici ... gr. $\mathrm{v}-\mathrm{x}-\mathrm{xxx}$; Acidi salicylici....... gr. $v$; Glycerini........... $3 \mathrm{ij}$; Aquæ destillatæ...... $3 \mathrm{vj}$. M.

R Aluminis......... gr. $\mathrm{x}-\mathrm{xx}$; Glycerini........... $3 \mathrm{ij}$; Aquæ destillatæ...... $3 \mathrm{vj}$. M.

B Zinci sulphatis, gr. $\mathrm{v}-\mathrm{x}-\mathrm{xv}-\mathrm{xx}$; Glycerini ........... $3 \mathrm{ij}$; Aquæ destillatæ...... $3 \mathrm{vj}$. M.

R Sol. argyrol. 10-20 per cent.

R Creosoti puri......... z j; Glycerini............ $3 \mathrm{j}$. M.
R Argenti nitratis,

\section{Glycerini,} gr. $\mathrm{v}-\mathrm{x}-\mathrm{xv}-\mathrm{xx}-\mathrm{xl}$; Aquæ destillatæ āā 3 iv. M.

R Ferri et ammonii sulphatis, gr. $\mathrm{x}-\mathrm{xv}$; Glycerini........... 3 ij ; Aquæ destillatæ...... $3 \mathrm{vj}$. M.

R Olei picis.......... $\xi \mathrm{j}$;

Balsami Peruviani.... gr. xx.

Mix, with gentle heat, and stir while cooling.

R Tincturæ ferri chloridi.. $3 \mathrm{j}$; Glycerini........... $3 \mathrm{j}$; Aquæ destillatæ...... $3 \mathrm{vj}$. M. Filter.

R Acidi tannici....... gr. $\mathrm{x}$; Acidi borici....... gr. ivss. ; Aq. destillatæ...q. s. $\xi$ j.

R Zinc. chloridi....... gr. $x x$; Glycerini.......... 3 iv ; Aquæ destillatæ..... 3 iv.

R Mentholis.......... gr. $x v$; Ol. eucalypti....... m $\mathbf{x}$;

Ol. petrolinæ...... $3 \mathrm{j}$.

R Ichthyoli........... $3 \mathrm{j}$; Glycerini ............ $3 \mathrm{j}$; Aquæ ............. $3 \mathrm{ij}$. M.

R Iodi......... gr. $\mathrm{v}-\mathrm{x}-\mathrm{xv}-\mathrm{xx}$; Potassii iodidi........ q. s.; Glycerini........... 3 vj; Aquæ destillatæ...... $3 \mathrm{ij}$. M. 
Loeffler's solution :

R Menthol,

ro c. c., about 3 ijss.;

Toluene, q. s. ad,

Creolin, $3^{6}$ c. c., about 3 ixss. ;

2 c. c., about 3 ss.;

Ferric chloride solution,

Alcohol, q. s. ad,

4 c. c., about $3 \mathrm{j}$;

M. IO0 c. c., about $3 \times x v j s s$.

P Resorcini .......... 3 ss.;

Glycerini ......... 3 iv;

Aquæ .............. 3 iv.

M.

Ingals's formula :

R Menthol............gr. j;

Guaiacol........... gr. $x$;

Ol. olivæ......... $\pi \mathrm{x}$.

M. Very beneficial in early stages of peritonsilitis.

R Acidi tannici.......... gr. xl;

Acidi carbolici........ gr. ij;

Glycerini .......... $3 \mathrm{j}$

Aquæ.............. $3 \mathrm{j}$.

M.

Rx Iodoformi.......... 3 ss. ;

Olei eucalypti,

Olei olivæ..........āā $\bar{z}$ ss.

M.

Re Zinci sulphatis..... gr. $\mathrm{v}-\mathrm{xv}$; Glycerini,

Aq............. āā $\bar{s}$ ss.

M.

\section{POWDERS}

R Iodoform ...... two parts; Boracic acid..... three parts; Naphthalene ... five parts; Ol. bergamot.... q. s.
R Hydrarg. chlor. mit... $3 \mathrm{j}$;

Pulv. sacchar. alb..... $3 \mathrm{ij}$;

Pulv. morph. sulph..... gr. j;

Bismuth subnit........ $3 \mathrm{ij}$.

M.

R Acid. tannici......... gr. v;

Cannabin tannat..... gr. $\mathrm{x}$;

Bismuth subnit...... $3 \mathrm{j}$;

Pulvis. amyli...... 3 iij.

R Bol. Armen....... one part;

Sacch. alb....... one part;

Boracis......... two parts.

Re Resorcini,

Pulv. amyli......... āā 3 j.

M.

B Quininæ tannat...... $3 \mathrm{ij}$;

Pulv. amyli........ $3 \mathrm{vj}$.

M.

R Argenti nitratis.... gr. v-x; Pulv. talci........ $3 \mathrm{j}$.

M.

R Ferri subsulph..... gr. $x-x x$;

Pulv. talci. ....... $\xi \mathrm{j}$.

M.

R Anæsthesin.

R Aluminis stearat.

Daturine mixture :

R Daturine ........... gr. j;

Bismuth subnitratis... gr. $\mathrm{xx}$;

Magnesii carbonatis.. $3 \mathrm{ij}$;

Pulv. acaciæ........ gr. xx.

M. bene. Sig. Use as snuff.

Pulvis bismuth et acaciæ :

R Bismuth subnitratis.... $3 \mathrm{j}$;

Pulv. acaciæ ........ 3 ss.

Sig. Use as snuff. 
R Pulv. iodoformi...... $3 \mathrm{ij}$;

. camphoræ...... $3 \mathrm{j}$;

" ac. tannici...... gr. v;

" g. acaciæ....... $3 \mathrm{ij}$.

M.

R Bismuthi subnit...... $3 \mathrm{ij}$;

Pulv, amyli........ 3 ss.;

" ac. tannici...... gr. $x$.

M. bene.

R Bismuthi subnit ..... 3 ss.; Pulv. g. acaciæ...... $3 \mathrm{ij}$; “ argent. nit...... gr. $x$. M.

R Hydrargyri chloridi mitis, Pulv. amyli.........āā $3 \mathrm{ij}$. M.

Bu Morph. sulph....... gr. j; Bism. subnit ..... $3 \mathrm{j}$-ij-iv. M.

R Iodoformi.

Re Iodoformi, Bismuthi subnit., Pulv. amyli.........āā $3 \mathrm{j}$. M.

B Aristol.

R Aristol, Pulv. amyli.........āā $3 \mathrm{j}$.

Re Orthoform.

\section{TONICS, ETC.}

Mistura iodi et ol. caryophylli : R Iodi.............. gr. xij; Olei caryophylli.... $z \mathrm{j}$.

M. May be given internally in milk in doses of from five to ten minims.
R Pulv. ossis (P. D. \&

Co.'s)......... 3 jss. ;

Acidi phosphorici (U.

S. P.)......... 3 ivss.;

Acidi lactici........ $3 \mathrm{j}$;

Pepsin............ gr. $\mathrm{xl}$;

Pancreatin.......... gr. viij;

Glycerini ......... $z^{i j}$;

Ext. malti ......... $\xi^{3}$ viij;

Aquæ........q. s. ad $\mathrm{Oj}$.

M. Dose, two teaspoonfuls three or four times a day.

R Wheat phosphates (acid) (Phillips's).. 弓 viij;

Syrupi........... $\bar{z} \mathbf{v}$;

Alcoholis......... $3 \mathrm{ij}$;

Quininæ........... gr. xvj ;

Cinchonidinæ,

Cinchoninæ.......āā gi. viij;

Tinct. persionis...... $3 \mathrm{j}$.

M. Dose, two teaspoonfuls three or four times daily.

R Syr. wheat phosphates

(Phillips's) ........ $\mathrm{iij}_{\text {; }}$

Ac. hydrobrom, dil..... $\xi \mathrm{j}$.

M. Sig. Dose, teaspoonful in water three times a day. Beneficial in the treatment of chronic pharyngitis and chronic laryngitis.

R Iodi................ gr. iv ;

Ol. olivæ,

Guaiacol.......... āā $\bar{z}$ ss.

M. May be used also hypodermically. 
Mistura iodi et guaiacol:

R Iodi............... gr. iv ; Guaiacol........... 3 j.

M. Beneficial in early stages of peritonsilitis; has been recommended for hypodermic injection and also to be given internally through the mouth.
R Strych. cryst........ gr. j; Ac. acetici......... q. s.; Ferri citratis....... 3 jss. ; Ext. hyoscyami ..... gr. viij; Syr. tolutanis ....... $\xi \mathrm{j}$; Aquæ......... q. ad $\xi$ iv. M. Dose, teaspoonful three times a day. 


\section{N D E X}

Abscess, acute, retropharyngeal, 155 .

Accessory cartilages, nasal, I.

cavities, acute diseases of, 495 .

chronic diseases of, 497 .

sinuses, 4 .

Acetylene gas, 36 .

Acid, trichloracetic, 357 .

Acute amygdalitis, 142. (See Peritonsilitis, acute.)

Adam's apple, $\mathbf{r} 6$.

Adenoid vegetations, 418.

Adenoids, 418.

causative of chorea, I99.

causative of laryngismus, 199 .

Agger nasi, 3.

Air, compressed, inhaler for, $7 \mathbf{1} 4$.

medicated, 7 I2.

Air passages, upper, examination of, 34 .

Alæ of the nose, $\mathrm{r}$.

Amberg's powder blower, 707 .

Amygdalitis, acute, I42.

syphilitic, 249.

Anæsthesia, 670.

for nasal operations, 642 .

pharyngis, 206.

Analgesia pharyngis, 206.

Anatomy of the upper air passages, $\mathrm{I}$.

Aneurism as a cause of laryngeal paralysis, 227.

Angina, 403.

benign croupous, 73 .

Angioma laryngis, 466 .

Angioma of the larynx, 469, 570 .

of the nose, 537,538 .

of the pharynx, 542 .

Anosmia, 169, 172 .

accompaniment of atrophic rhinitis, 173 . essentialis, $\mathbf{1} 72$.

expiratoria, I72.
Anosmia, inspiratoria, 172. intercranialis, 172,173 . nerve degeneration in, 173 .

Anosphresia, I 70. (See Anosmia.)

Anterior ethmoidal cells, 4 . nares, $\mathbf{I}$.

lining of, 6 . palatine nerve, 8 . pillars, Io, II. rhinoscopy, 38 .

Anthrax, differentiated from syphilis, 256.

Antrum of Highmore, 4, 5 .

empyema of, 497 .

opening of, $5 \mathrm{I} 4$.

probing of, 506 .

surgery of, $5 \mathrm{I} 3$.

Antrum, transillumination of, 507.

Aphonia, functional, 230.

Aphonia, hysterical, 230.

diagnosis of, 234 .

etiology of, 230.

laryngoscopic appearance in, 234 .

local treatment of, 236.

pathology of, $23 \mathrm{I}$.

prognosis in, 235 .

symptomatology of, 233.

treatment of, 235.

Aphthous sore throat, 73.

Applicator, Bosworth's, 698 .

laryngeal, Jarvis, 7OI.

Shurly's, 700 .

Arches, palatine, II.

Arterial supply of the pharynx, 15 .

Artery, crico-thyroid, I8, 2 I.

inferior laryngeal, $2 x$.

inferior thyroid, 21.

internal maxillary, 8.

posterior laryngeal, $2 \mathrm{I}$. subclavian, 2 I. 
Artery, superior laryngeal, $2 \mathrm{I}$.

Articulation, cerebral centre for, 33 . nasal, 27.

Ary-epiglottic folds, 18, 20, 21.

Aryteno-epiglottic muscles, 20. folds, I8, 2 I.

Arytenoid cartilages, 15, 16, 17, 20. clubbed appearance of the, 302 .

Arytenoid muscles, 20.

Asch's operation, 632, 634 . tubes, 635 .

Asthma, spasmodic, in atrophic pharyngitis, 400 .

Atomizer, DeVilbliss, 703. steam, 702 .

Atresia, nasi, etiology of, 644 . operation for, 646.

Atresia of the nasal vestibule, 646 .

Atrophic rhinitis, chronic, 359.

Auto-laryngoscopy, 46.

Autoscopy, 47.

Azygos uvulæ muscles, I3.

Bacillus fœtida, 360 .

of Frisch, 333.

of Klebs-Loeffler, 95.

of Loewenburg, 377, 378.

mucosus, 378 .

of Pfeiffer, 79.

Bands, ventricular, 20, $21,29$.

Basal cells of olfactory membrane, 23.

Bernay's cotton sponge, $63 \mathrm{r}$.

Blennorrhœa of the larynx, Stoerck's, 333.

Bodies, foreign, in the upper air passages, 650.

Boils of the nose, 77. (See Furuncles of the nose.)

Bone, ethmoid, $\mathbf{I}$.

hyoid, II, I5, I6.

hyoid, fractures of, 604 .

occipital, basilar process of, 9 .

vomer, $\mathbf{x}$.

Bones, nasal, $\mathbf{I}$.

Bosworth's applicator, 698 . snare, 557.

Bowman's glands, 24.

Bridge, supporting, Hopkins's, 6r 7 .

Brushes, Wagner's, 699.

Bulla ethmoidalis, 4 .

Bursa of the thyroid cartilage, $\mathbf{1 6}$.
Canine fossa, perforation through, 5 I5.

Cannula, Trendelenburg, 694 .

Carcinoma of the larynx, 572 . pharynx, 553.

Cartilage, cricoid, II, I5, I6, I7, I8. quadrilateral, $\mathbf{I}$.

Santorini, I5, 2 I.

septal, I.

thyroid, $15,16,17,18$.

triangular, $\mathrm{I}$.

triangular, dislocation of, 640 .

Wrisberg, 15, 21.

Cartilages, accessory, $\mathbf{I}$.

arytenoid, 15, 16, 17, 19, 20.

arytenoid, clubbed appearance of the, 302 .

dislocation of the laryngeal, 604 .

lateral, $\mathbf{I}$.

of the larynx, 15.

of the nose, I.

Catarrh, dry, 359.

chronic laryngeal, 44I.

chronic nasal, 335.

estival, 175. (See Hay Fever.)

laryngeal, acute, 63 .

nasal, acute, 50.

Cauterization, galvano-, 354, 7I 7 .

Cells, anterior ethmoidal, 4 .

basal, 23.

olfactory, 23.

posterior ethmoidal, 4 .

sustentacular, 23.

Cellulitis phlegmonosa pharyngis, 160.

Chair for laryngoscopy, 38 .

Choanæ, 9. (See Posterior Nares.)

Cholesteatomatous rhinitis, 501 .

Chondromata of the nose, 538 .

Chorditis vocalis tuberosa, 462 .

Chorea laryngis, 212, 213.

Chronic infectious diseases of the upper air passage, 239.

Ciliated epithelium, 6, 9.

Circumvallate papillæ, 40.

Cohen's laryngeal curette, 7 I5.

Colds, 52.

Compressed air, inhaler for, 7I4.

Concretions in the tonsils, 659.

Condenser, Mackelizie, 35.

Condylomata of the nose, 246. of the pharynx, 247 .

Constrictors of the glottis, paralysis of the, 22I, 
Constrictors, pharyngeal, action of, 14 .

Contusions of the larynx, 603 .

Cords, vocal, 16, 18, 29.

in phonation, 30.

Cornicula laryngis, 18.

Coryza, acute, 50.

chronic, 335 .

fetid, 372.

idiosyncratic, I75. (See Hay Fever.)

syphilitica, 242.

syphilitica simplex, 245.

Cribriform plate of ethmoid, 2, 7 .

Crico-arytenoid muscles, I7.

ligaments, 18.

Crico-thyroid arteries, I8, 21.

ligaments, 18.

membrane, 18.

muscle, $18,19$.

muscle, paralysis of, 221 .

Cricoid cartilage, I5, I6, I7, 18.

Croup, "false," 216. (See Laryngismus Stridulus.)

membranous, 70

spasmodic, 216. (See Laryngismus Stridulus.)

Croupous rhinitis, 129. (See Fibrinous Rhinitis.)

Crypts, tonsillar, I I.

Curette, Gottstein, 431.

laryngeal, 7I5.

nasal, 517.

Cystoma of the pharynx, 542.

Cysts of the larynx, 571 .

of the nose, 525 .

Deformities of the nose, 616 .

Deformity of the pharynx, 647 .

Depressors, tongue, 36 .

Deviation of the septum, 6rg. appearance of, 628 .

subjective symptoms of, 627 .

treatment of, 628

De Vilbliss atomizer, 703.

Diaphragm in phonation, 30 .

Diathesis in acute rhinitis, 51 . gouty, in laryngeal diseases, $\mathbf{I} 68$.

Digital examination of the nasopharynx, 44.

Dilator, Trousseau's, 676 .

Dilator tubæ muscle, I2.

Dilators of the glottis, paralysis of, 22 I.
Diphtheria, 93.

anomalous cases of, 104.

antitoxin doses of, 125.

general effect of, 125 .

immunization by, 126 .

local effect of, I24.

complicated by influenza, IIg.

complications of, 106.

contagion of, 95 .

constitutional treatment of, 123.

diagnosis of, 110.

diet in, 123.

etiology of, 95 .

history of, 93.

immunization against, 100.

inoculability of, roo.

local prophylactics, $\mathbf{1 2 1}$.

local treatment of, I2I.

method of taking culture, III.

mixed infection, II5.

objective symptoms of, I02.

paralysis following, 107.

pathology of, 97.

predisposing causes, 96 .

preparation of the antitoxin, 127.

secondary poisoning, 123.

sequelæ, 106.

stimulants in, 123.

symptomatology, ror.

treatment of, 120.

treatment, prophylactic, $\mathbf{I} 20$.

varieties of, I03.

Dislocation of laryngeal cartilagès, 604 .

Douches, 705.

Drill, hand, 517 .

nasal, 639.

Dry catarrh, 359.

inhaler, 713.

mouth, 192. (See Xerostomia.)

Duct, lachrymal, 4.

Dunbar's theory, 18r.

Edematous laryngitis, 67 .

Elastic tapes, 44.

Electrode, Mackenzie's laryngeal, 718. nasal, 717.

Electrolysis, bipolar, 717.

for spurs, 640 .

monopolar, 716 .

needle for, 716 .

Empyema of antrum of Highmore, 497. 
Epiglottis, I5, I7, I8, 2 I.

deformity of, 648.

frenum of, 21 .

function of, 29.

Epileptic spasm of the larynx, 2 I3.

Epileptiform neuroses of the larynx, 212.

Epistaxis, 607.

after operations, 608 .

constitutional treatment for, 6 I4.

etiology of, 607 .

systemic causes of, 609 .

treatment of, 6ro.

Epithelioma, 552.

Epithelium of the larynx, 20.

of the vocal cords, $2 \mathbf{I}$.

Erectile tissue, 7 .

Eruptions, eczematous, 75 .

Erysipelas of the larynx, I5I.

of the nose, $15 \mathrm{r}$.

of the throat, $I_{5} \mathrm{I}$.

age, $\mathrm{I}_{5} \mathrm{I}$.

diagnosis of, 153 .

etiology of, I5 I.

prognosis of, $\mathrm{I} 54$.

symptomatology of, 152.

treatment of, 154 .

Esophagotomy, 669, 673 .

Esophagus, foreign bodies in, 658 .

foreign bodies in, symptoms of, 660 .

Estival catarrh, I75 (See Hay Fever.)

Ethmoid bone, I, 8.

Ethmoid cells, chronic inflammation of, 499.

diagnosis of inflammation of, 502 .

etiology of chronic inflammation of, 497.

inflammation of, 494.

Ethmoidal cells, anterior, 4, 5.

posterior, 4,5 .

sinus, 4,5 .

Ethmoidalis, bulla, 4 .

Ethmoiditis, chronic, complications of, 509.

necrosing, treatment of, 518 .

symptomatology, 504 .

Eustachian tubes, 9.

Examination, digital, of the nasopharynx, 44.

Examination of the upper air passages, 34.

Exanthemata and inflammations of the upper air passages, 163 .

External nose, I.
False vocal chords, 2 I.

Falsetto voice, 32.

Faucial tonsils, II.

Faucitis, acute, 59 .

pathology of, $6 \mathrm{r}$.

symptomatology of, 60 .

treatment, $6 \mathrm{I}$.

Female voice, range of, 32 .

Fever, acute glandular, of children, $7 \mathrm{I}$.

course of, $7 \mathbf{I}$.

diagnosis of, $\mathbf{7 2}$.

etiology of, $7 \mathbf{r}$.

prognosis of, 72 .

symptomatology of, 72 .

treatment of, 73 .

hay, 175 .

rose, I75. (See Hay Fever.)

Fevers, essential, and the upper air passages, I63.

Fibrino-plastic rhinitis, I29.

Fibrinous rhinitis, 129.

Fibro-lipoma of the pharynx, 542.

Fibromata of the larynx, 567.

naso-pharyngeal, $54 \mathrm{I}$.

of the nose, $523,53 \mathrm{I}$.

of the pharynx, 54I.

Fibro-myxomata of the nose, 53I.

Flat nose, 6 I6.

Fœtida, bacillus, 36o.

Folds, ary-epiglottic, I8, 20, 21.

aryteno-epiglottic, $\mathbf{I 8 , 2}$.

Follicular pharyngitis, 15, 386. tonsilitis, 137 .

Forceps, laryngeal, $5^{84}$.

polypus, 559.

post-nasal, 43I.

Foreign bodies in esophagus, 658 .

in the larynx, 656 .

in the larynx, symptoms of, 662.

treatment of, 666.

in the nose, treatment of, 655 .

in the pharynx, 656,658 .

in the pharynx, diagnosis of, 660.

in the upper air passages, 650 .

effect of, 650.

symptoms of, 660 .

Fossæ of Rosenmüller, 9; nasal, I.

Fractures of the hyoid bone, 604.

of the larynx, 602 .

Freer's operation, 638.

Frenum of the epiglottis, 2 I. 
Frisch, bacillus of, 333 .

Frontal sinus, 4 . inflammation of, 494.

Fuming inhalations, 7I4.

Function of smell, 25. of the turbinated bodies, 26 .

Functional aphonia, 230.

Furuncle of the nose, 76 . etiology of, 76 .

diagnosis of, 77 .

\section{Galvano-cautery, 7 7 .}

application of, 353 .

in the treatment of hay fever, 185 . snare, 560,717 .

Gas, acetylene, 36 .

Glands, Bowman's, 24. enlarged lingual, 446.

lingual, tuberculosis of, 295, 299. associated with chronic laryngitis, 446. of the larynx, 2 I.

olfactory, 24 .

Glandular fever, acute, of children, $7 \mathrm{I}$.

Glass-blowers' patches, 492.

Glottis, 22.

constrictors, paralysis of the, $22 \mathrm{I}$.

dilators, paralysis of, $22 \mathrm{I}$.

openers, 19.

spasm of, in children, 216.

in adults, 213 .

Glosso-epiglottic ligament, I8.

Gottstein's curette, 43 I. tampon, 372.

Gouty diathesis in laryngeal diseases, I68.

Gummata of the larynx, 570 .

Hæmatocele of the fauces, 487 .

Hay fever, I 75 ; age, I78.

alkalinity of the blood in, 183 .

a vasomotor paresis, $\mathbf{I} 8 \mathrm{I}$.

complications of, $\mathrm{I} 82$.

Dunbar's theory, $\mathrm{I} 8 \mathrm{I}$.

etiology of, $\mathbf{I} 75$.

exciting causes of, $\mathbf{I} 75$.

galvano-cautery in the treatment of, 185 .

hereditary, 177.

local treatment, 184 .

location, 178 .

morbid conditions of the nasal cham-

bers in, 177 .

objective signs in, 179 .
Hay fever, pathology of, I80.

predisposing causes of, 177 .

race, 178 ; sex, 178 .

Scherer's investigation, $\mathbf{1 8} \mathbf{I}$.

social conditions in, 179 .

suprarenal extract in, 186.

symptomatology of, I79.

treatment, general, 183 .

treatment of, $\mathbf{I} 82$.

uric acid in, 177,183 .

Head mirror, 35.

Hemorrhage after tonsillotomy, 4I 3 .

nasal, see Epistaxis.

of the pharynx, 6or.

Hemorrhages, provision against, 670.

Hereditary syphilis, symptomatology of, 24 I.

Herpes of the nose, $48 \mathrm{I}$.

of the pharynx, 73 .

Hiatus semilunaris, 4.

Highmore, antrum of, 4,5 .

empyema of, 497 .

probing of, 506.

surgery of, $5 \mathrm{I} 3$.

Hook, palate, 43 .

Hopkins's bridge, 6 I 7 .

Horns of the thyroid cartilage, I6.

Hydrorrhœa, nasal, 188.

etiology of, 188.

symptomatology of, I9o.

treatment of, I9I.

Hyo-epiglottic ligament, I8.

Hyoid bone, II, I5, I6, I8.

Hyperæsthesia of the larynx, 198.

treatment of, $19^{8}$.

of the nasal passages, 195 .

treatment of, 196.

of the nasopharynx, 195 .

of the pharynx, 196.

etiology of, I96.

treatment of, 197.

of the upper air passage, 194.

Hyperkinesis, 207.

Hyperosmia, I69, I7I.

treatment of, $\mathbf{1 7 4}$.

Hyperosphresia, I 70.

Hypertrophic rhinitis, 236.

Hypertrophies, posterior, treatment of, 356 .

Hypertrophy of the tonsil, 405 .

of the turbinated bodies, 336 .

Hypodermic syringe for the larynx, 708 . 
Ichthyol in the treatment of ozena, 382 .

Ichthyosis linguæ, 490 .

Idiosyncratic coryza, 175. (See Hay Fever.)

Illumination for examinations, 34 .

Image, laryngoscopic, $45,46$.

rhinoscopic, $4 \mathrm{I}$.

Infectious diseases (chronic) of the upper air passages, 239.

Inferior constrictors of the pharynx, 13 .

Inferior laryngeal artery, $2 \mathrm{I}$. nerve, 21.

Inferior meatus, 4 . thyroid artery, 21. vein, 2 I. turbinated bone, 3 .

Influenza, 79. course and complications of, $8 \mathrm{r}$.

diagnosis of, 83 .

etiology of, 79 .

symptomatology, 80 . treatment of, 83 .

Influenza, pandemic, 86. (See La Grippe.) course of, 89 .

etiology of, 87 .

prognosis of, 90 .

sequelæ, go.

symptomatology of, 88 .

treatment of, 9I.

Infundibulum, 4.

Ingals's operation, 632 .

Inhaler, dry, 713.

oxygen, 714 .

Shurly's, 712.

hot water, 712.

Inhalations, 7I2.

fuming, $7 \mathbf{1} 4$.

Injection into the larynx, 7Io.

Injuries to the larynx, 6oI.

treatment of, 605 .

of the nose, 597 .

treatment of, 598 .

of the pharynx, 6or.

treatment of, 605 .

Interarytenoid space, section of, 302.

Intubation, 669, 679 .

contra-indications for, $68 \mathrm{r}$.

difficulties of, 685 .

for neoplasms, 596 .

history of, 679 .

indications for, $68 \mathbf{r}$.
Intubation, position for, 680 .

position for feeding after, 691 .

technic of, 682.

tube, O'Dwyer's, 68r.

withdrawal of tube, 687 .

Jarvis's laryngeal applicator, 7or. snare, 558.

Kilian's method of examining the posterior wall of the larynx, 47 .

Kinesioneurosis, 207.

Kirsten's method of examining the larynx, 48.

Klebs-Loeffler bacillus, 95 .

Knight's electric tonsil snare, 7I7.

Lachrymal duct, 4 .

Lacunæ, II.

La grippe, 86. (See Pandemic Influenza.) followed by parosmia, I 70 .

Lancet, laryngeal, 584 . Mackenzie's, 7I5.

Laryngeal applicator, 587.

cartilages, dislocation of, 604 .

catarrh, acute, 63 .

chorea, 212, 213.

curette, 715 .

electrode, Mackenzie's, 718.

epilepsy, 2 I 3.

epithelium, 20.

forceps, $5^{84}$.

glands, $2 \mathrm{I}$.

image in paralysis of the crico-thyroid muscle, 22 I.

lancet, 584 .

Mackenzie's, 7I5.

mirror, 37.

method of holding, 44 .

mucous membrane, 20 .

paralysis, 221.

snare, 586 .

spasm, 210.

etiology of, 2Ir.

stenosis syphilitica, 270.

syringe, Tobold's, 7II.

vertigo, 2 I 2.

vestibule, 18.

Laryngectomy, 592, 669, 694.

partial, 696 .

sequences of, 697 . 
Laryngis cornicula, 18. sacculus, 22.

Laryngismus stridulus, etiology of, 216. prognosis in, 218.

symptomatology of, $2 \mathrm{I} 7$.

treatment of, 2 I 8 .

Laryngitis, acute, 63.

etiology of, 63 .

laryngoscopic appearance, 65 .

prognosis of, 65 .

symptomatology of, 64 .

treatment of, 66.

atrophic, 395 .

treatment of, 476 .

chronic, 44I.

age, 443 .

appearance of, 453 .

associated with hypertrophy of the tonsils, 447.

atrophic form, 452, 462.

caused by previous nasal trouble, 445 . caused by rheumatism, 449 .

classification of, $44 \mathrm{I}$.

climatic treatment of, $47 \mathrm{I}$.

course of, 467 .

diagnosis of, 464 .

differentiated from syphilis, 464 .

differentiated from tuberculosis, 464 .

edematous, $452,455,476$.

etiology of, 442.

hemorrhagic form, 453,459, 464 .

heredity in, 445 .

hyperæmic form, 443.

hyperplasia in, $46 \mathrm{I}$.

hyperplastic form, 443, 454 .

hypertrophica inferior, 459 .

influence of habits, 444.

occupation, 443.

pathology of, 460 .

reflex, 448.

sex, 443 .

symptomatology of, 450 .

tobacco in, 445.

topical treatment of, $\mathbf{4 7 2}$.

treatment of, 469 .

croupous, 70.

edematous, 67 .

diagnosis of, 68 .

etiology of, 67 .

laryngoscopic appearance of, 68 .

prognosis of, 69 .
Laryngitis, edematous, symptomatology of, 68.

treatment of, 69.

hyperæmic, treatment of, 473.

hyperplastic, treatment of, 475 .

hypertrophica chronica inferior, 333.

membranous, 70 .

diagnosis of, 70 .

treatment of, 70 .

sicca, 395 .

túberculous, 300.

Laryngo-pharyux, Ir.

functions of, 27.

Laryngoscope, electric, 692.

Laryngoscopic image, 45,46 .

Laryngoscopy, 34, 44.

chair for, 38.

Laryngotomy, 694.

Larynx, angioma of, 570 .

carcinoma of, 572 .

cartilages of, I5.

contusions of, 603 .

cysts of, $57 \mathrm{I}$.

deformity of, 648 .

enervation of, 32 .

erysipelas of, I $5 \mathrm{I}$.

fibromata of, 567 .

foreign bodies in, 656 .

symptoms of, 660 .

treatment of, 666.

fractures of, 602 .

function of, 28.

gummata of, 570 .

hypodermic syringe for, 708 .

inferior hypertrophy of, 466 .

injection into, 710 .

injuries of, 6or.

in respiration, 30.

lipoma of, 569 .

lupus of, 320 .

lymphomata of, 568 .

muscles of, i8.

myxomata of, 568 .

neoplasms of, 562 .

appearance of, 576 .

symptoms of, 574 .

nerves of, 2 I.

neuralgia of, 238 .

pachydermia of, 453,462 .

papillomata of, 564 .

paresis of, $22 \mathrm{I}$. 
Larynx, sarcoma of, 573 . scarification of, 715 .

secondary syphilis of, 259.

sphincter of, 29.

Stoerck's blenorrhœa of, 333.

structure of, 29.

syphilis of, 259.

syringe for, 7II.

tertiary syphilis of, 259.

tubercular neoplasms of, 572 .

tuberculosis of, 300 .

diagnosis of, 305 .

prognosis in, 306.

treatment, climatic, 306 .

treatment, hygienic, 307 .

treatment, local, 3 II.

treatment, specific, 308 .

treatment, surgical, 3I3.

veins of, $2 \mathrm{I}$.

ventricles of, 22.

vestibule of, 29.

whistling, 3I.

Lateral cartilages, I.

crico-arytenoid muscles, I8, I9.

hyoid ligaments, 18.

pharyngotomy, $67 \mathrm{I}$.

Law, Semon's, 217.

Laws, physical, of laryngoscopy, 34 .

Leffert's syringe, 704 .

Leprosy differentiated from syphilis, 256.

Leucoplakia buccalis, 490.

appearance of, $49 \mathrm{I}$.

diagnosis of, 49I.

etiology of, 49 o.

pathology of, 490 .

symptomatology of, $49 \mathrm{I}$.

treatment of, 492.

Levator-palati muscle, $\mathbf{I} 2$.

Levator-uvulæ muscle, I3.

Ligament, glosso-epiglottic, 18 .

hyo-epiglottic, $\mathbf{} 8$.

median, I8.

thyro-epiglottic, 18 .

thyro-hyoid, I8.

Ligaments, crico-arytenoid, I8.

crico-thyroid, 18.

lateral hyoid, $\mathrm{I} 8$.

superior thyro-arytenoid, 20.

thyro-arytenoid, 22.

Light, calcium, 35 .
Light, electric, 35 .

Lingual glands associated with chronic laryngitis, 446.

tuberculosis of, 295, 299.

Lingual sinuses, $2 \mathbf{I}$.

tonsil, 437.

tonsils, tuberculosis of, 295, 299.

Lipoma of the larynx, 569 .

Lithiasis, pharyngeal manifestations of, I68.

Loewenburg, bacillus of, 377,378 .

Lupus erythematosus, 316 .

differentiated from syphilis, 258.

laryngis, 320.

primary, 317 .

pharyngis, 258.

of the nose, 318.

appearance of, 318 .

of the pharynx, 258, 319 .

of the soft palate, 3 I9.

of the upper air passages, 315 .

diagnosis of, 329 .

differentiated from epithelioma, 330 .

differentiated from rhinoscleroma, 330.

differentiated from syphilis, 330.

differentiated from tuberculosis, 330 .

electrolysis in, 33I,

etiology of, 32I.

exciting causes of, 325 .

heredity of, $\mathbf{3 2 7}$.

inoculability of, 327 .

pathology of, 327 .

symptomatology of, 3I 7 .

traumatism in, 327 . .

treatment by galvano-cautery, 332 .

treatment by Röntgen rays, 331 .

treatment of, 330 .

vulgaris, $3 \mathrm{I} 6$.

Luschka's tonsil, 9, II, 4I8.

Lymph vessels of the mucous membrane of the nose, 26.

Lymphatics, nasal, 8.

pharyngeal, I4.

Lymphomata of the larynx, 568.

of the pharynx, 542 .

Mackenzie's condenser, 35 .

laryngeal electrode, 718 .

laryngeal lancet, 7I5.

Malarial fever, laryngeal epilepsy in, 167.

pharyngeal complications of, $\mathbf{I} 67$. 
Maxilla, superior, malformation of, 625 .

Maxillare ostium, 4.

Maxillary sinus, 4,5 .

inflammation of, 494.

Measles, laryngitis accompanying, treatment of, 165 .

rhinitis accompanying, 164, 165 .

Meatus, inferior, 4.

middle, 3 .

superior, 3 .

Median ligament, 18.

rhinoscopy, 39 .

Medicated air, 7I2.

Membrana limitans olfactoria, 23, 24.

Membrane, crico-thyroid, I8. mucous, of the nose, 23.

Schneiderian, 6.

Membranous rhinitis, r29. (See Fibrinous Rhinitis.)

Mercurial patches, 492.

Methods of local treatment, 698 .

Middle constrictors of the pharynx, 13 . meatus, 3 .

thyroid vein, 21 .

turbinated bone, 2.

Mirror, head, 35 .

laryngeal, 37.

rhinoscopic, 37.

Morgagni, ventricles of, 22.

Mouth breathers, 424 .

dry, 192. (See Xerostomia.)

gag, O'Dwyer's, 68I.

tuberculosis of, 294.

Mucus, amount secreted in the nose, 27.

Muscle, arytenoid, 20.

azygos uvulæ, I3.

compressor nasi, I.

crico-arytenoid, $\mathbf{I 7}$.

crico-thyroid, 18 , I9.

paralysis of, 221.

depressor alæ nasi, $\mathbf{r}$.

dilator tubæ, 12.

lateral crico-arytenoid, I8, I9.

levator labii superioris alæque nasi, I. palati, I 2.

uvulæ, 13.

nasal dilators, $\mathbf{I}$.

palato-glossal, $\mathbf{I}$.

palato-pharyngeal, II.

posterior crico-thyroid, paralysis of, 222.

salpingo-pharyngeus, $\mathbf{I} 2$.
Muscle, stylo-pharyngeus, I4. tensor palati, I2.

Muscles, aryteno-epiglottic, 20.

(external) of the nose, I.

inferior pharyngeal constrictors, 13 .

middle pharyngeal constrictors, 13 .

of the larynx, 18.

of the pharynx, 13.

of the soft palate, $\mathbf{I} 2$.

of the upper air passages, paralysis of, 218.

posterior crico-arytenoid, I9.

superior pharyngeal constrictors, 13,14 .

thyro-arytenoid, I8, I9.

thyro-epiglottic, 20.

Mycosis, pharyngeal, 48r.

differentiated from syphilis, 258.

pharyngis, diagnosis of, 485 .

etiology of, $48 \mathrm{I}$.

pathology of, 483.

prognosis of, 485 .

symptomatology of, 484 .

treatment of, 485 .

Myxo-fibromata, naso-pharyngeal, 54I.

Myxomata of the larynx, 568 .

of the nose, 523.

of the pharynx, 543 .

Myxo-sarcoma of the nose, 546.

Nares, anterior, $\mathrm{r}$.

posterior, $\mathbf{I}$.

Nasal articulation, 27.

bones, I.

catarrh, acute, 50 .

chronic, 335 .

curette, 517.

electrode, 7I7.

fossæ, $\mathbf{r}$.

function of, 26.

hydrorrhœa, 188.

lymphatics, 8 .

(external) muscles, I.

nerve, 8 .

parasites, 652 .

diagnosis of, 654 .

treatment of, 655 .

processus, $\mathbf{I}$.

sclerosis, 365 .

secretion, amount of, 182 .

septum, I.

specula, 36 . 
Nasal splints, 599 .

syphilis, 254.

tuberculosis, 289 .

vestibule, $\mathbf{I}$.

Naso-palatine nerve, 8.

Nasopharynx, 8.

digital examination of, 44 .

function of, 27.

secretion of, 27.

tuberculosis of, $29 \mathrm{I}$.

Needle for electrolysis, 716.

Needle, transfixion, 356, 640 .

Neoplasms, benign, of the larynx, treatment of, 582 .

of the nose, 523 .

of the pharynx, 539 .

intubation for, 596 .

malignant, of the upper air passages, 544 .

of the accessory cavities, 555 .

classification of, 555 .

prognosis of, 556 .

treatment of, 556 .

of the epiglottis, 554 .

of the larynx, 562 .

diagnosis of, 579 .

etiology of, 563 .

prognosis in, 582 .

of the upper air passages, 520 .

classification of, 520

general etiology of, 520 .

treatment of, 556

pharyngeal, diagnosis of, 554 .

etiology of, 543.

symptomatology of, 543 .

treatment by escharotics, 56 I.

Nerve, anterior palatine, 8.

inferior laryngeal, 21 .

nasal, 8.

naso-palatine, 8 .

olfactory, $7,23$.

branches of, 24.

recurrent laryngeal, 2 I.

spheno-palatine, 8.

superior laryngeal, $2 \mathbf{I}$.

trifacial, 26.

Vidian, 8.

Nerves, laryngeal central, 2 I.

laryngeal motor, $2 \mathrm{I}$.

of the larynx, 21 .

of the mucous membrane of the nose, 25.

of the pharynx, 15 .
Nerves of sensation (nasal), 8.

pharyngeal, 15 .

Nervous diseases of the upper air passages, I69.

Neuralgia of the larynx, 238.

of the nose, 237.

of the pharynx, 237.

of the upper air passages, 237 .

treatment of, 238 .

Neuroses of the nose and nasopharynx, I 70.

of the upper air passages, $16 \mathrm{~g}$.

classification of, 169 .

prognosis of, 174 .

treatment of, 174 .

Non-ciliated epithelium, 7 .

Nose, benign neoplasmis of, 523 .

bleed, 607. (See Epistaxis.)

cartilages of, $\mathbf{I}$.

erysipelas of, $\mathbf{I} 5 \mathrm{I}$.

external, $\mathbf{I}$.

fatal syphilis of, 264 .

fibromata of, 523, 531.

flat, 6 I6.

function of, 22.

furuncle of, 76 .

herpes of, $48 \mathrm{I}$.

injuries of, 597.

local diseases of, 75 .

lupus of, 318.

lymph vessels of the mucous membrane of, 26.

mucous membrane, vascular arrangement of, 26.

myxomata of, 523 .

myxo-sarcoma of, 546.

nerves of the mucous membrane of the, 25.

neuralgia of, 237.

olfactory region of, 23.

primary syphilis of, 242.

respiratory function of, 26 .

saddle, 616 .

sarcoma of the, 545 .

secondary syphilis of, 245 .

tamponing the, 612.

tertiary syphilis of, 254 .

treatment for injuries of, 598 .

venous sinuses of, 26 .

Notch, thyroid, 16, 18.

Nuclei, zone of oval, 23. 
Obstacles to laryngoscopy, 46 .

to posterior rhinoscopy, 42.

Occipital bone, basilar process, 9 .

O'Dwyer's intubation tube, 681 . mouth gag, 68I.

Olfaction, 22, 23.

theories of, 25.

Olfactory cells, 23.

epithelium, 23.

glands, 24 .

nasal tract, 3,7 .

nerve, $7,23$.

branches of the, 24 .

region of the nose, 23.

Openers of the glottis, 19.

Ophthalmic artery, anterior ethmoidal branches, 8 .

Oropharynx, 9.

function of, 27.

Osteomata of the nose, 539 .

of the pharynx, 543 .

Ostia ethmoidalia, 5.

Ostium maxillare, 4, 5 .

Oxygen inhaler, 714.

Ozena, 373.

bacteriology of, 375 .

cause of fetor, 379 .

etiology of, 374 .

ichthyol in, 382.

inflammatory theory of, 378 .

neurotrophic theory of, 376,378 .

oxygen in the treatment of, $38 \mathrm{I}$.

pathology of, 374,377 .

serum treatment of, 380 .

treatment of, 380 .

Pachydermia laryngis, 453, 458, 462, 478 .

Palate, hard, tuberculosis of, 294.

hook, 43.

retractor, White's, 43 .

soft, 9 , 10.

deformity of, 647 .

lupus of, 3 I 9.

muscles of, $\mathbf{1 2}$.

tuberculosis of, 294.

tuberculosis of, diagnosis of, 299.

Palatine arches, II.

Palato-glossal muscle, Ir.

Palato-pharyngeal muscle, II.

Papillæ, circumvallate, 40.

Papillomata of the larynx, 564 .
Papillomata, etiology of, 564 .

"mulberry," 537.

of the nose, 535 .

of the pharynx, 539 .

Paræsthesia laryngis, 204.

etiology of, 204.

prognosis in, 205.

symptoms in, 204.

treatment of, 205.

Paræesthesia, nasal, 198.

etiology of, Ig8.

treatment of, I99.

Paræsthesia pharyngis, I99.

age in, I99.

electrical treatment in, 203.

etiology in, 199.

following local injuries, 200.

local appearance of, 201.

pretubercular symptom, 200.

prognosis in, 202.

reflex symptom, 201.

sex in, 199 .

symptoms of, I99.

treatment of, 202.

Paraffin injection, 6I8.

Paralysis of the abductors of the vocal cords, 222.

in diphtheria, ro7.

of all the muscles of the vocal cords, 224 .

of one vocal cord, 224 .

of the adductors of the vocal cords, 222. of the constrictors of the glottis, 221 .

of the crico-thyroid muscle, $22 x$.

of the dilators of the glottis, $22 \mathrm{r}$.

of the external muscles of the nose, 220.

of the larynx, 221 .

diagnosis of, 229.

etiology of, 225.

location of, 226.

prognosis of, 229.

treatment of, 229.

of muscles of upper air passages, 218 .

general etiology of, 2 I8.

of the pharyngeal muscles, 220.

etiology of, 2 I9.

symptoms of, $22 \mathrm{I}$.

treatment of, 22 I.

of the posterior crico-thyroid muscle, 222.

of the tensors of the vocal cords, 22 I.

Parasites in the nose, 652 . 
Parasites, nasal, diagnosis of, 654 . symptoms of, 653 .

treatment of, 655 .

Paresis of the larynx, 22I.

Parosmia, I69, I70. definition of, 170 . etiology of, 170 .

Parosphresia, I 70. (See Parosmia.)

Paroxysmal sneezing, I95, 208.

Peritonsilitis, acute, I42.

abscedens, I42. (See Peritonsilitis,
$\quad$ acute.)
course of, I48.
diathesis in, I43.
etiology of, I43.
exciting causes of, I46.
habits, I46.
hygienic surroundings, I45.
occupation, I 44.
pathology of, I46.
predisposing causes of, I43.
rheumatism in, I 43.

symptomatology (objective and subjective), 147 .

treatment of, I49.

Pfeiffer, bacillus of, 79.

Pharyngeal constrictors, action of, I4.

lymphatics, 14 .

lymphoid ring, 9, II.

muscles, I3.

mycosis, $48 \mathrm{I}$.

Pharyngeal neoplasms, etiology of, 543 .

diagnosis of, 554 .

symptomatology of, 543 .

Pharyngeal spasm, 209.

etiology of, 209.

reflex, 2 IO.

Pharyngeal syphilis, primary, 243.

Pharyngeal tonsil, 9, I5.

hypertrophy of, $4 \mathrm{I} 8$.

Pharyngitis, acute, 59.

acute, etiology of, 59 .

pathology of, 6r.

symptomatology of, 60.

treatment of, $6 \mathrm{I}$.

infectious (phlegmonous), 160 .

diagnosis of, 162.

etiology of, I6o.

symptomatology of, I6I

treatment of, 162.

atrophic, 395 .
Pharyngitis, atrophic, appearance of, 398 . asthma in, 400.

diagnosis of, 399.

diathesis in, 395 .

etiology of, 395.

neurotic theory for, 396 .

pathology of, 397 .

prognosis of, 399 .

symptomatology of, 397 .

treatment of, 400 .

treatment, electrical, of, 402.

voice in, 398 .

chronic, 386 .

follicular, 386.

hypertrophic follicular, 386 .

exudativa, 389,391 .

follicular, 15 .

herpetica, 73.

appearance of, 75 .

pathology of, 73.

symptomatology of, 75 .

treatment of, 75 .

hypertrophic, appearance of, 390.

diathesis in, 388 .

etiology of, 386 .

exciting causes of, 387 .

influence of occupation in, 386 .

pathology of, 388.

secretion of, 389 .

symptomatology of, 389 .

treatment of, 392.

phlegmonosa diffusa, 160. (See Pharyngitis, Acute Infectious Phlegmonous.) sicca, 395.

ulcerous, 74, 403 .

diagnosis of, 404 .

pathology of, 404.

symptomatology of, 403 .

treatment of, 404.

Pharyngoscopy, 34, 40.

Pharyngotomy, 669, 671 .

lateral, 671 .

subhyoid, 672 .

Pharynx, appearance in secondary syphilis, 248.

arterial supply of, 15 .

benign neoplasms of, 539 .

carcinoma of, 553 .

diseases of, classification of, 384 .

erysipelas of the, $I_{5} \mathrm{I}$.

foreign bodies in, $656,658,660,663$. 
Pharynx, function of, in deglutition, 27, 28. in voice production, 27.

hemorrhage of, 601 .

herpes of, 73 .

lupus of, 3I9.

neuralgia of, 237 .

oro-, 9.

sarcoma of, 549 .

secondary syphilis of, 257 .

tertiary syphilis of, 250 .

tuberculosis of, 29I, 299, 300.

veins of, 15 .

wounds of, 6or.

Phillips's electric photophore, 693 .

Phlegmonous tonsilitis, 142. (See Peritonsilitis, Acute.)

Phonation, cerebral centre for, 33 .

cortical centre for, 32 .

Photophore, electric, Phillips's, 693.

Physiology of the upper air passages, 22.

Pigments, application of, 698 .

Pilcher's retractor, 676 .

Pillars, anterior, Io. posterior, IO.

Plexuses, venous, 7 .

Plugging of the posterior nares, 613 .

Pneumonia followed by parosmia, I 70 .

Polypi fibromatous, 529 .

myxomatous, 529 .

nasal, appearance of, $53 \mathrm{I}$.

of the nose, 523 .

Polypoid hypertrophy, 34I.

Polypus forceps, 559 .

Pomum Adami, 16.

Posterior crico-arytenoid muscles, I9. paralysis of the, 222.

Posterior ethmoidal cells, 4 . laryngeal artery, $2 \mathrm{I}$.

nares, I, 9 .

pillars, Io.

rhinoscopy, 40.

Post-nasal forceps, $43 \mathrm{r}$.

syringe, 704 .

Powder blower, Amberg's, 706.

Shurly's, 707.

Powders, application of, 706 .

Processes, nasal, I.

Prolapse of the laryngeal ventricle, 574, 582.

Psoriasis linguæ, 490, 49I.

Pyriform sinuses, 20.
Quadrilateral cartilage, $\mathrm{r}$.

Quinsy, I42. (See Peritonsilitis, Acute.)

Recurrent laryngeal nerve, $2 \mathrm{I}$.

Registers of the voice, 30.

Respirator, Tyndale's, 713.

Respiratory function of the nose, 26 . nasal tract, 3,6 .

Respired air, warming and moistening of, 26.

Retractor, palate, White's, 43.

Pilcher's, 676.

Retropharyngeal abscess, acute, 155 .

diagnosis of, 157 .

differentiated from croup, 158.

etiology of, 155 .

micro-organisms of, 156.

prognosis of, 157 .

symptomatology of, 157 .

treatment of, 159 .

Rheumatism affecting the larynx, $\mathbf{I} 68$. pharyngitis in, 167 .

Rhinitis, acute, 50.

acute, diathesis in, $5 \mathrm{I}$. etiology of, $5 \mathrm{I}$.

occupation, 52.

pathology of, 50.

secretion of, $5 \mathrm{I}$.

sex, 52 .

symptomatology of, 54 .

treatment of, 55 .

atrophica, 359 .

anosmia in, 173 .

appearance of, 364 .

etiology of, 359 .

pathology of, 365 .

prognosis in, 366 .

micro-organisms in, 360 .

symptomatology of, 363 .

treatment, electrical, 370.

general, 367 .

local, 369 .

massage, 370 .

caseosa, 373, 501 .

cholesteatomatous, $50 \mathrm{r}$.

chronic, 335 .

atrophic, 359 .

fotida, 373 .

general etiology of, 335 .

symptomatology of, 338 .

fibrinous, I 29, 254. 
Rhinitis, fibrinous, appearance of, I 35 . diagnosis of, 135 . etiology of, 129 . idiopathic, I29. micro-organisms of, I3I. pathology of, I29. prognosis of, 136 . symptomatology, I 35 . treatment, general, 136 . local, 136.

hypertrophica, 336 . appearance of, 347 . classification of, 344 . diagnosis of, 347 . etiology of, 336 . pathology of, 342 . treatment of, 349 .

by escharotics, $35^{\circ}$.

by galvano-cautery, $35 \mathrm{I}$.

non-fœtida, 359 . œdematosa, 348 . purulent, $36 \mathrm{I}$. sclerosa, 359.

Rhinoliths, $65 \mathrm{I}$. etiolugy of, $65 \mathrm{I}$. symptoms of, 652 . treatment of, 655 .

Rhinoscopy, 34 . anterior, 38 . image, 40, 4I. median, 39 . mirror, 37. posterior, 40. obstacles to, 42.

Rhinoscleroma, 333 .

clinical history of, 333 .

diagnosis of, 334 . differentiated from syphilis, 256. etiology of, 333 .

Rima-glottidis, 22, 29.

Ring, pharyngeal lymphoid, I I.

Robert's operation, 633,638 .

Röntgen rays in laryngeal explorations, 49.

Rose cold, I75. (See Hay Fever.)

Rose position, 669 .

Rosenmüller fossæ, 9.

Rotheln, pharyngitis in, I66.

Rouge's operation, 269.

Sacculus laryngis, 22,
Saddle nose, 250, 6r6. operation for, 269.

Salpingo-pharyngeus muscle, 12.

Santorini, cartilages of, 15, 18, 21.

Sarcoma of the larynx, 573 .

of the nose, $5+5$.

of the pharynx, 550 .

of the tonsil, 550 .

Sarcophaga georgina, 652,653 .

Scarification of the larynx, 7 I5 .

Scarlet fever, pharynx, complications of, 163.

treatment, 164 .

Schneiderian membrane, 6 .

Sclerosis, nasal, 365 .

Sebaceous glands, 6 .

Semilunaris hiatus, 4 .

Semon's law, 217.

Septal cartilage, $\mathbf{r}$. operations, complication of, 613 .

hemorrhages after, 643.

Septum, abscess of, 77 .

cartilaginous, treatment of deformity of the, 629 .

deviation of the, 619 .

appearance of, 628 .

diagnosis of, 628 .

etiology of, 621 .

heredity in, 625 .

subjective symptoms of, 627 .

treatment of, 628 .

nasal, $\mathbf{I}$.

perforation of, 479 .

treatment of perforation of, 480 .

Sequelæ of syphilis, 264 .

treatment of, 268.

Shurly's applicators, 700 .

hot water inhaler, 7I2.

inhaler, 712,

powder blower, 707.

Sialorrhœa, 188, I91.

etiology of, I9I.

treatment of, 192.

Sinus, ethmoidal, 4.

frontal, 4 .

maxillary, 4 .

sphenoidal, 4.

Sinuses, accessory, 4 .

lingual, $2 \mathbf{I}$.

membrane lining, 7 .

pyriform, 2 I. 
Sinuses, venous, 7 .

Sinusitis, acute, 494.

acute, diagnosis of, 496 .

etiology of, 494 .

symptomatology of, 495 .

treatment of, 5 II.

chronic, 497 .

differential diagnosis of, 505 .

ethmoidal diagnosis, 504 . treatment of, 517 .

frontal, symptomatology, 504 .

frontal, treatment of, 519 .

general etiology of, 497 .

local treatment of, $5 \mathrm{II}$.

sphenoidal, symptomatology of, 504 .

surgical treatment of, 513 .

symptomatology of, 503 .

treatment of, 5 I9.

sphenoidal, 502.

Smell, function of, 25.

Smoker's patches, 492.

Snare, galvano-cautery, 560 .

laryngeal, 586 .

nasal, Bosworth's, 557 .

use of, 558 .

Sneezing, paroxysmal, I95, 208.

Soft palate, 9, ro.

function of, 28.

muscles of, 12 .

Spasm of the glottis, 213.

in adults, diagnosis of, 214 .

etiology of, 213.

prognosis of, 215 .

symptomatology of, 214 .

treatment of, 215.

in chịldren, 216. (See Laryngismus Stridulus.)

of the larynx, 210.

of the pharynx, 209.

Spasmodic affections of the upper air passages, 207.

etiology of, 207.

of the nasal passages, 208.

Specula, nasal, 36 .

Sphenoidal sinus, 4,5 .

inflammation of, 494 .

Spheno-palatinè nerve, 8 .

Splints, nasal, 599 .

Sponge, cotton, 631 .

Sprays, application of, 7or.

Spurs, electrolysis in, 640 .
Spurs, treatment of, 630 .

Steam atomizer, 702.

Stenosis of the larynx, following syphilis, 270.

Stenosis, tracheal, syphilitic, 270.

Sternutation, 208.

frequent, 195.

Stoerck's blennorrhœa of the larynx, 333.

Stylo-pharyngeus muscle, I4.

Subclavian artery, 21 .

Subhyoid pharyngotomy, 672 .

Superior constrictors of the pharynx, 13 .

laryngeal artery, $2 \mathrm{I}$.

nerve, $2 \mathbf{r}$.

maxilla, malformation of, 625 .

meatus, 3 .

thyro-arytenoid ligaments, 20.

thyroid vein, 21.

turbinate bone, 2 .

Suprarenal extract in the treatment of hay fever, 186.

Sustentacular cells, 23.

Syphilis, hereditary, 239.

hereditaria tarda, 239.

nasal, 254.

fatal, 264 .

objective symptoms of, 253.

of the larynx, secondary, 259.

tertiary, 259.

of the nose, tertiary, 255 .

differentiated from tuberculosis, 255 .

of the pharynx, 257.

differentiated from lupus, 258.

simulating malignancy, 258.

of the upper air passages, 239.

pathology of, 26r.

pharyngeal, primary, 243.

primary, of the nose, 242.

secondary, of the nose, 245.

of the pharynx, appearance of, $2+3$.

of the tonsils, 247 .

sequelæ of, 264.

treatment of, 268.

tertiary, of the nose, 249 .

of the pharynx, 250.

treatment of, 265 .

Synechia, 359, 644 .

of the vocal cords, 649 .

operation for, 645 .

Syringe, Lefferts's, 704 . 
Syringe, post-nasal, 704 .

Tobold's laryngeal, 7II.

Tamponing the nose, 612 .

the posterior nares, $6 \mathrm{I}_{3}$.

Tapes, elastic, 44 .

Tensor palati muscle, I2.

Tensors of the vocal cords, paralysis of, $22 \mathrm{I}$.

Texas screw-worm, 652 .

Thyreotomy, 18, 592, 669, 690 .

Thyro-arytenoid ligameuts, 22.

muscles, 18, r9.

epiglottic ligament, 18 .

muscles, 20.

hyoid ligament, I8.

Thyroid cartilage, 15, 16, 17, 18. notch, $16, \mathrm{x} 8$.

Timbre of voice, 30 .

Tissue, erectile, 7 .

Tobold's laryngeal syringe, 7II.

pneumatic apparatus, 7I4.

To.ngue depressors, 36 .

Tongue tuberculosis of the, 294.

diagnosis of, 299.

Tonsil, enucleation of, 418 .

lingual, 437.

hypertrophy of, 438 .

inflammation of, 438 .

symptomatology of hypertrophy of, 439 .

treatment of hypertrophy of, 439.

Luschka's, 9, II, 418.

pharyngeal, 9,15 .

hypertrophy of, 4I8.

causing aural disease, 426 .

causing colds, 42 .

etiology of, 4I9.

frequency, 4I9.

heredity, $42 \mathrm{I}$.

sex, $42 \mathrm{r}$.

sarcoma of, 550 .

snare, 4I2.

electric, $7 \mathrm{I} 7$.

Tonsils, blood supply of, 15 .

concretions in, 659 .

faucial, Ir.

function of, 28.

hypertrophy of, 405 .

age, 406.

appearance of, 407 .

hard tonsil, 407.

soft tonsil, 408.
Tonsils, hypertrophy of, classification of, 405.

diagnosis of, $4 \mathrm{ro}$.

electrolysis in, $4 \mathrm{I} 5$.

etiology of, 405 .

ignipuncture in, $4 \mathrm{I}_{5}$.

pathology of, 409.

prognosis of, $4 \mathrm{II}$.

symptomatology of, 406.

treatment of, $4 \mathrm{Ir}$.

pharyngeal, hypertrophy of, associated with deformities, $42 \mathrm{I}$.

diagnosis of, 427.

diathesis in, 423 .

hemorrhage after removal, 436 .

pathology of, 428.

prognosis of, 429 .

regrowth of, 432 .

symptomatology, 424 .

treatment of, 429 .

tuberculosis after, 436 .

secondary syphilis of the, 247 .

tuberculosis of the, 295.

diagnosis of, 299.

Tonsilitis, age, 138 .

acute exanthemata and, 138.

acute follicular, $13 \%$.

course and termination, 140 .

diagnosis of, I 40 .

differential diagnosis of, $\mathbf{r} 4 \mathbf{I}$.

etiology of, I37.

exciting causes of, 137 .

objective signs, I39.

prognosis of, $\mathbf{I} 4 \mathbf{I}$.

rheumatism and, I39.

symptomatology of, 139 .

treatment of, $\mathrm{I} 4 \mathrm{r}$.

chronic, 405 .

follicular, 405 .

treatment of, $4 \mathrm{r} 7$.

lacunar, 137. (See Acute Follicular Tonsilitis.)

membranous, 137. (See Acute Follicular Tonsilitis.)

phlegmonous, 142. (See Peritonsilitis, Acute.)

predisposing causes of, 138 .

sex, 138 .

syphilitica, 248, 249.

Tonsillotomy, $4 \mathrm{Ir}$.

causes of hemorrhage after, 4I 4. 
Tonsillotomy, hemorrhage after, 4I3.

Tracheal stenosis, syphilitic, 270.

Tracheotomy, 669, 675 . after treatment, 677 . tube, 677 .

Trachoma, 462.

Transfixion needle, 356,640 .

Transillumination of the antrum, 507 .

Treatment, local, methods of, 698.

Trendelenburg position, 669. cannula, 694 .

Triangular cartilage, I. dislocation of, 640 .

Trichloracetic acid, application of, 357 .

Trifacial nerve, 26.

Trousseau's dilator, 676 .

Tube, intubation, O'Dwyer's, 68I. tracheotomy, 677 .

Tubercular neoplasms of the larynx, 572.

Tuberculosis of the hard palate, 294. of the larynx, 300 .

laryngoscopic appearance of, 3or. symptomatology of, 301 .

of the lingual glands, 295, 299.

of the mouth, 294.

of the nasopharynx, 291.

etiology of, 291.

of the nose, 287 .

appearance of, 288.

diagnosis of, 239 .

differentiated from lupus, 290.

differentiated from syphilis, 290.

etiology of, 287.

pathology of, 288.

prognosis of, 290.

symptomatology of, 288.

palate, 294.

diagnosis of, 299.

of the pharynx, 291.

diagnosis of, 299.

of the soft palate, 294.

of the tongue, 294.

diagnosis of, 299.

of the tonsils, 295.

diagnosis of, 299.

of the upper air passages, 272.

age, 286.

contagion of, 276 .

general etiology of, 272.

heredity of, 276 .

influenza and, 90 .
Tuberculosis of the upper air passages, inoculation of, 282.

latency of, 276 .

predisposing diseases of, 282 .

predisposing habits, 285 .

sex, 286.

Tuberculous laryngitis, 300 .

pharyngeal paralysis in, 166.

Tubes, Eustachian, 9.

Tubular mucous glands, 7 .

Tunica propria, 24.

Turbinals, nasal, 2.

Turbinated bodies, anterior hypertrophies, 340.

function of, 26.

hypertrophy of, 336 .

posterior hypertrophies, $34 \mathrm{I}$.

Tylosis linguæ, 49 J.

Tyndale's respirator, 713 .

Typhoid fever followed by parosmia, I70.

laryngeal paralysis in, $\mathbf{I} 66$.

pharyngeal hemorrhages in, $\mathbf{I} 66$.

Typhus fever, laryngeal complications of, I67.

Ulcer, syphilitica, perforating, of the nose, 247.

Ulcerous pharyngitis, 403.

Uric acid causative of hay fever, 177 .

Uvula, 9, ro.

bifid, 647 .

Uvulitis, 61 .

symptomatology of, 62 .

treatment, 62 .

Variola, I66.

laryngitis in, $\mathbf{I} 66$.

pharyngitis in, $\mathbf{I} 66$.

rhinitis in, 166.

Varix of the fauces, 487.

diagnosis of, 488.

etiology of, 487 .

symptomatology of, 488 .

treatment of, 488.

Vegetations, adenoid, 4I8.

Vein, inferior thyroid, 2 I.

middle thyroid, $2 \mathrm{I}$.

superior thyroid, 2 I.

Veins of the larynx, 2 I.

pharyngeal, 15 .

Velum palati, 9. 
Venous plexuses, 7 . sinuses, 7. of the nose, 26.

Ventricles of the larynx, 22. eversion of, 574,582 . of Morgagni, 22.

Ventricular bands, 20, 21, 29.

Vertigo, laryngeal, 212.

Vestibule of the larynx, 29. laryngeal, $\mathbf{I} 8$. of the nose, $\mathbf{I}$.

Vibrissæ, 6, 26.

Vidian nerve, 8 .

Vocal cords, 16, 18, 29. appearance of, 22. epithelium of, 2 r. false, 21 . in phonation, 30. paralysis of, $22 \mathrm{I}$. tension of, $3 \mathrm{I}$.

Voice, falsetto, 32. female range of, 32 . musical range of, 32 . production, 30 .

Voice, register of, 30. timbre, 30.

Vomer bone, I.

Wagner's brushes, 699.

Watson's operation, 632.

Whistle, definition of, 31 .

Whistling larynx, 3I.

White's palate retractor, 43 .

Whitehead's spud, 630 .

Word-forming, centre for, 33 .

Worm, Texas screw, 652 .

Wounds of the pharynx, 6oI.

Wrisberg, cartilage of, $15,18,21$.

Xerostomia, 192.

appearance of, 193.

course of, I93.

diagnosis in, 193.

etiology of, $\mathbf{r} 92$.

symptomatology of, I93.

treatment of, 193.

Zone of the oval nuclei, 23. 


\section{THE LAW IN ITS RELATIONS TO PHYSICIANS}

By ARTHUR N. TAYLOR, LL.B.

Of the New York Bar

NEW EDITION, REVISED AND ENLARGED

I 2mo. Cloth. Price, $\$ 2.00$

"Rarely has it been our privilege to examine a book which so clearly seems to just fill a demand as this one does. The physician must ever and again recur to the thought that by some slip he may expose himself to a suit whose loss will mean his financial ruin. The average physician does not know in any adequate way just what can legally be expected of him by his patient. Nor can he find out without great effort and probably expense. Neither, on the other hand, does he know what rights he has in the courts. If called into court he must submit utterly to the advice of his attorney. It is to meet these conditions that this book is issued. The author quotes fully of cases, decisions and opinions of leading courts and jurists everywhere, but especially in the various states. It is information such as the physician needs in his ordinary practice in order to protect himself, his reputation, and his family. Of course, unusual cases will come up, but if we know the principles underlying them we may act much more wisely. We believe the book will have a very wide circulation here in New England, a success which it has already achieved in New York."

-Annals of Gynacology and Pediatrics, Boston.

"Every physician should, unless conversant with the law, avail himself of the contents of this book, and he will find himself better prepared to meet the conditions and circumstances involving legal complications into which any of us are liable to find ourselves drawn."-Fournal of XNervous and MMental Diseases, New York.

"This volume is indeed a most valuable one, because physicians should have an idea of the law as it regards them. For often it is found that physicians are ignorant of the most elementary principles of the law ; and this is not all, they do not understand the law which refers to them. The book is an excellent treatise and one which brings out all the facts which it is important that a physician should know. We advise all physicians to buy this book and read it through. In a thousand and one instances it will be found of the greatest value."-Medical Progress, Louisville.

D. A P P L E T O A N D C O P A N Y, N E W Y R K. 


\title{
Leube's Medical Diagnosis.
}

\author{
By Dr. WILHELM v. LEUBE,
}

Professor of Medicine and Physician-in-Chief to the Julius Hospital at Würzburg.

\section{Authorized Translation from the Sixth German Edition.}

\section{Edited, with AnNotations, By JULIUS L. SALINGER, M.D.,}

Late Assistant Professor of Clinical Medicine in the Jefferson Medical College, and Physician to the Philadelphia Hospital.

\section{Colored Plates and 74 Illustrations. Cloth, \$5.00; Half Leather, $\$ 5.50$.}

\section{SOLD ONLY BY SUBSCRIPTION.}

"Leube's book is A1. I shall adopt it in the school ; it is an old favorite."

-William Osler.

"Leube's work on 'Diagnosis' has long been known by German and German-reading physicians as the best of its class in that language. The publishers are to be congratulated on presenting it to the English-reading medical public through the medium of an excellent translation by Dr. Salinger. Six editions in twelve years attest the high opinion of its merits held by the author's confrères. The unquestioned and marked value of this work consists in the excellence of its discussions of differential diagnosis-this, indeed, is its main motive, the plan of the book not contemplating any but passing references to clinical examinations and methods. It is to be hoped, in the interest of accuracy in diagnosis and because of its great value in this respect, that Leube's ' Special Medical Diagnosis' will find its way into the hands of every practitioner of internal medicine."

-Glentworth R. Butler, author of Butler's " Diagnostics of Internal Medicine."

“'Of Leube's 'Diagnosis,' now in its sixth edition, it need only be said that it takes front rank among the works of its class, covering in a most thorough manner the wide range of subjects comprised in the field of internal medicine, and having regard for established modern methods of clinical investigation. Numerous additions have been made by the American editor, adding to the completeness of the text. There is a copious index of 50 pages. The book is well printed, moderately illustrated, and neatly bound."

- New York Medical Record.

" Every physician will extend a hearty welcome to Leube's well-known book. It is an excellent book well translated. It describes diseases and their differential points in a clear and concise way, and besides this imparts a knowledge of the course, complications, and termination of each.

"These are the fundamental principles of the art of diagnosis, and lay a firm foundation for rational treatment. The original work in German has passed through six editions in about twelve years-a conclusive proof of the intrinsic merit of the work.

"After a brief introduction the subject of diseases of the heart is given that careful consideration which its importance demands. Next follow chapters on diagnosis of diseases of the respiratory organs, then diseases of the liver and other abdominal organs. The urinary organs are next considered, then the diseases of the peritoneum and nervous system, and lastly the infectious and contagious diseases. That part of the book devoted to diseases of the nervous system is exhaustive and especially well written, and the chapters on the infectious diseases are alone worth the price of the book. A full index adds to the value of the work."- Fournal of Medicine and Science.

"The editor has served a good cause in translating this sixth edition of Dr. Leube's work in a practical way. The work is certainly very complete and of great value to the busy practitioner of medicine in helping him to clear up a doubtful case. And knowing Dr. Leube's clinical experience is embodied in the work, with a clear and full description, lends great value to the book."-The New York State Fournal of Medicine.

D. APPLETON AND COMPANY, 436 Fifth Avenue, New York. 


\section{NORMAL

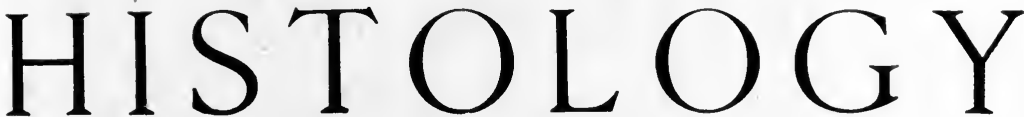

BY

\section{JEREMIAH S. FERGUSON, M.Sc., M.D.}

Instructor in Histology, Cornell University Medical College, New York City

$$
\begin{aligned}
& \text { With } 462 \text { Illustrations, many in colors } \\
& \text { Cloth, } \$ 4.00 \quad \text { Half Leather, } \$ 4.50
\end{aligned}
$$

SOLD ONLY BY SUBSCRIPTION

"This is an eminently practical and useful book, and one in which the subject is treated exhaustively. The author has that all too rare faculty of making things plain which comes from full knowledge and an aptitude for teaching. Since histology is at the basis of our comprehension of physiology, pathology, bacteriology, and clinical medicine unusual space has been devoted to all those organs which serve as a field for the specialist in medicine. This is especially true of the chapters on the central nervous system, which are comprehensive and exhaustive. What is true of this department is more or less true of all, and specialists, practitioners, and students will find a sufficiently adequate treatment of the histology of every tissue of the body."-Medical and Science Fonrnal.

"The subject-matter in this excellent book is thoroughly modern, and is presented in clear and readable fashion, while the unusual profusion and quality of the illustrations contribute a great deal to the attractiveness of the volume. An interesting feature is the free use of drawings made from plastic reconstructions of organs, which should be of great assistance to the student in understanding such structures as the adrenals, various glands, the blood, and lymphatic systems of different regions, etc. . . . The minute anatomy of the eye and of the ear is discussed at much greater length than is customary, and the section devoted to the nervous system is also worthy of note, both on account of its completeness and of the character of its illustrations. The bibliography is conveniently grouped by topics at the end of the volume and presents a wellselected list of the more important contributions to histological literature."

- Neze York Medical Record.

" Both as to text and illustrations this work is the most comprehensive and best-arranged text-book on the subject which has as yet appeared in this country."-Albany Medical Annals.

“This recent claimant to favor should easily dispel existing impressions as to the superiority of foreign works, for Dr. Ferguson has written a work of which nothing but praise can be spoken."-Medical Monthly, Memphis, Tenn.

D. APPLETON A N C OMPANY, PUBLISHERS 436 Fifth Avenue, New York. 


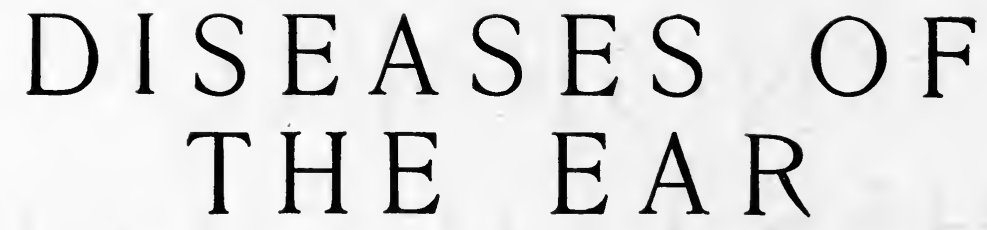

\section{By EDWARD BRADFORD DENCH, Ph.B., M.D.}

Professor of Otology in the University and Bellevue Hospital Medical College ; Aural Surgeon to the New York Eye and Ear Infirmary; Consulting Otologist to St. Luke's Hospital ; Consulting Otologist to the New York Orthopedic Dispensary and Hospital

\section{THIRD EDITION, REVISED AND ENLARGED}

\section{With a Colored Plates and 170 Illustrations in the Text. $8 \mathrm{vo}, 700$ pages. Cloth, $\$ 5.00$}

SOLD ONLY BY SUBSCRIPTION

In this work the author has endeavored to supply the needs of the general practitioner as well as those of the special surgeon. To this end considerable time has been spent in the consideration of pathological changes which may occur in the auditory apparatus. In those portions of the work devoted to the treatment of various diseases an effort has been made to describe in detail the various manipulative procedures employed in the local treatment of the different conditions. The importance of a thorough functional examination of the ear is emphasized, and the results of the most recent and reliable investigations are placed before the reader in a manner best suited to make them available and helpful in diagnosis.

The illustrations are numerous and well executed, and contribute largely to a clear understanding of the text. All the illustrations delineating operative procedures have been carefully made from the author's dissections.

"Impartially considered, we regard this text-book as the best exclusively otological classic published in America."-The Laryngoscope.

"This new edition of the well-known text-book deserves the same success its predecessors have had."-The American Fournal of Ophthalmology.

"The advances of the last few years have required the rewriting of a considerable portion of Dr. Dench's treatise. Particularly is this true with reference to the operative treatment of chronic suppurative otitis media and the various intracranial complications of middle ear suppuration. These receive full and discriminating consideration in the present edition. The author notes his failures as well as his successes with each procedure, and his pages bear the imprint of frankness as well as of a detailed knowledge of the questions discussed. His standing in the field of otology is too well known to call for any perfunctory praise. The typographical appearance of the book is, in our judgment, more attractive than that of the former editions."-Medical Record.

D. APPLETON AND COMPANY, NEW YORK.





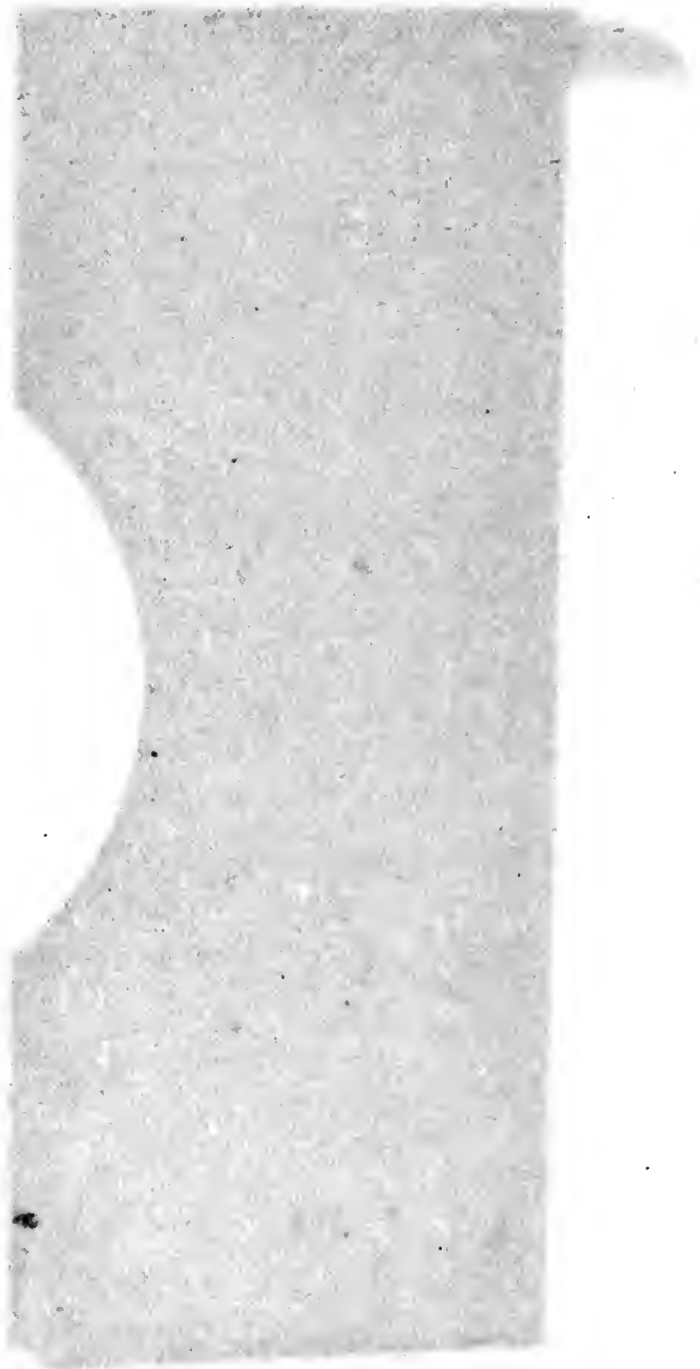




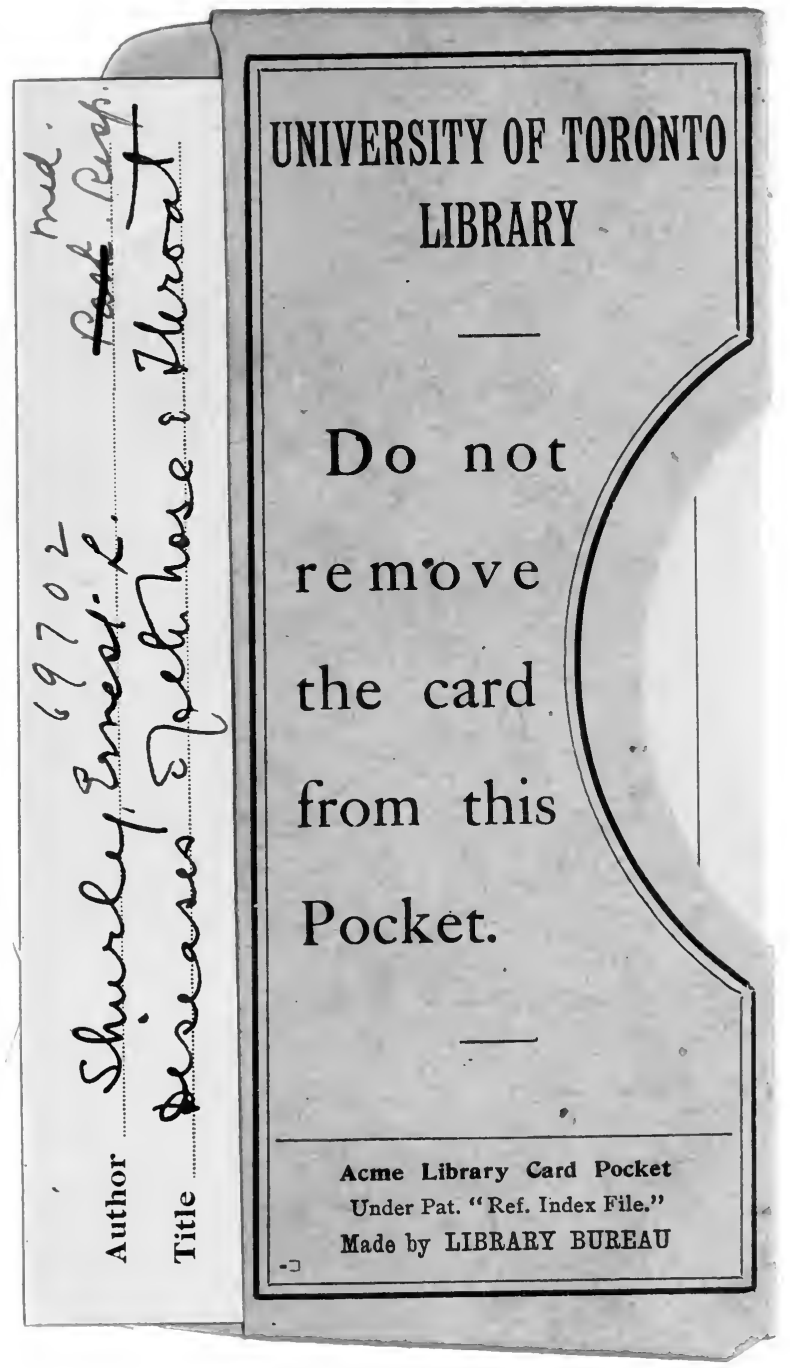


